MAROLO \& LEE LITHW

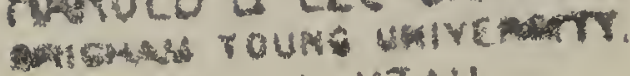

ODAVI UTAH 





Ny ? 


\subsection{5 \\ 762}

- hrasce 271916
A TEXT-BOOK OF
B A C T E R I O L O G Y 



\section{A TEXT-BOOK OF \\ BACTER IOLOGY}

\section{A PRACTICAL TREATISE FOR STUDENTS AND PRACTITIONERS OF MEDICINE}

\section{BY \\ PHILIP HANSON HISS, JR., M.D.}

LATE PROFESSOR OF BACTERIOLOGY, COLLEGE OF PHYSICIANS AND SURGEONS, COLUMBIA -UNIVERSITY, NEW YORK CITY

AND

\section{HANS ZINSSER, M.D.}

PROFESSOR OF BACTERIOLOGY, COLLEGE OF PHYSICIANS AND SURGEONS, COLUMBIA UNIVERSITY, NEW YORK CITY; FORMERLY PROFESSOR OF BACTERIOLOGY AND IMMUNITY, STANFORD UNIVERSITY, CALIFORNIA

WITH ONE HUNDRED AND FIFTY-FIVE ILLUSTRATIONS IN THE TEXT, SOME OF WHICH ARE COLORED

SECOND EDITION
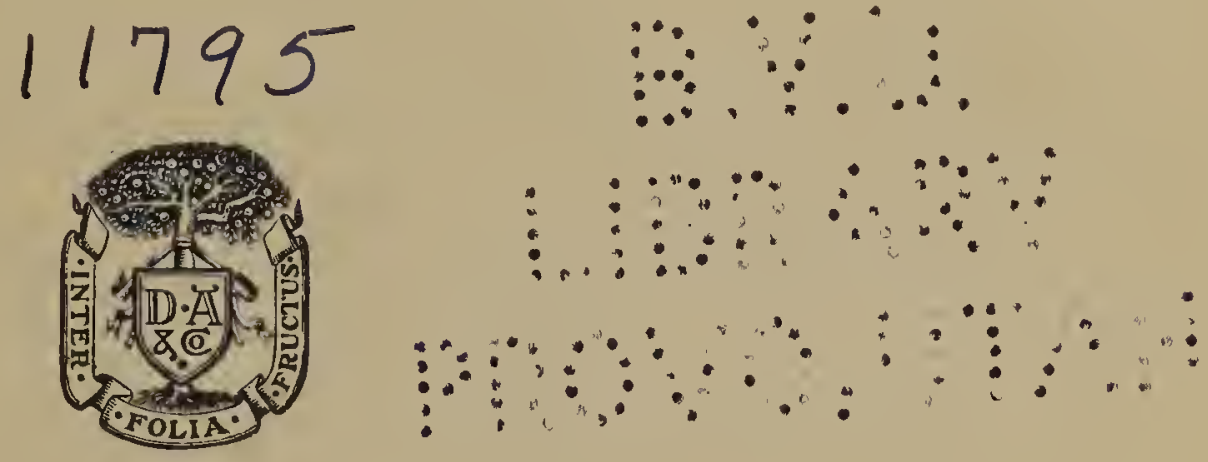

N E W Y ORK A N D L O N D O N

D. A P P LETON A N C COMPAN Y 
Copyright, 1910, and 1914, By

D. APPLETON AND COMPANY

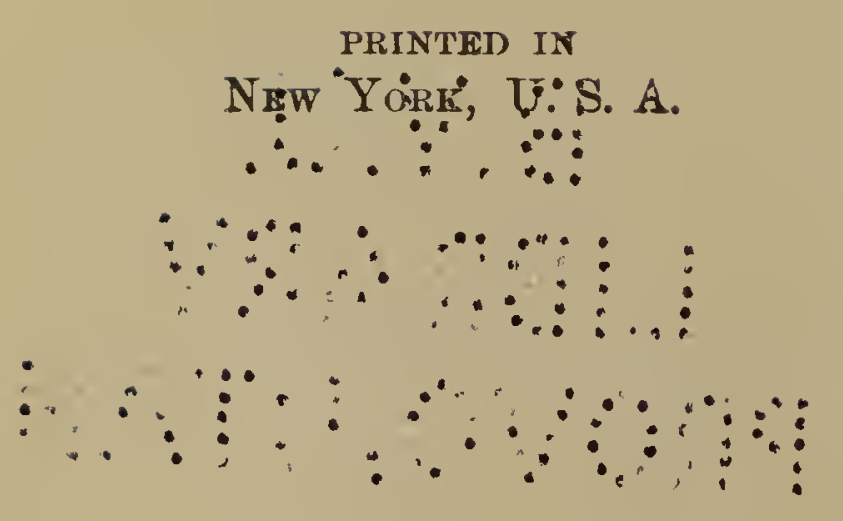




\section{PREFACE TO THE FIRST EDITION}

The volume here presented is primarily a treatise on the fundamental laws and technique of Bacteriology, as illustrated by their application to the study of pathogenic bacteria.

So ubiquitous are the bacteria and so manifold their activities that Bacteriology, although one of the youngest of sciences, has already been divided into special fields-Medical, Sanitary, Agricultural, and Industrial-having little in common, except problems of general bacterial physiology and certain fundamental technical procedures.

From no other point of approach, however, is such a breadth of conception attainable, as through the study of bacteria in their relation to disease processes in man and animals. Through such a study one must become familiar not only with the growth characteristics and products of the bacteria apart from the animal body, thus gaining a knowledge of methods and procedures common to the study of pathogenic and non-pathogenic organisms, but also with those complicated reactions taking place between the bacteria and their products on the one hand and the cells and fluids of the animal body on the other-reactions which often manifest themselves as symptoms and lesions of disease or by visible changes in the test tube.

Through a study and comprehension of the processes underlying these reactions, our knowledge of cell physiology has been broadened, and facts of inestimable value have been discovered, which have thrown light upon some of the most obscure problems of infection and immunity and have led to hitherto unsuspected methods of treatment and diagnosis. Thus, through Medical Bacteriology - that highly specialized offshoot of General Biology and Pathology-have been given back to the parent sciences and to Medicine in general methods and knowledge of the widest application.

It has been our endeavor, therefore, to present this phase of our subject in as broad and critical a manner as possible in the sections 
dealing with infection and immunity and with methods of biological diagnosis and treatment of disease, so that the student and practitioner of medicine, by becoming familiar with underlying laws and principles, may not only be in a position to realize the meaning and scope of some of these newer discoveries and methods, but may be in better position to decide for themselves their proper application and limitations.

We have not hesitated, whenever necessary for a proper understanding of processes of bacterial nutrition or physiology, or for breadth of view in considering problems of the relation of bacteria to our food supply and environment, to make free use of illustrations from the more special fields of agricultural and sanitary bacteriology, and some special methods of the bacteriology of sanitation are given in the last division of the book, dealing with the bacteria in relation to our food and environment.

In conclusion it may be said that the scope and arrangement of subjects treated of in this book are the direct outcome of many years of experience in the instruction of students in medical and in advanced university courses in bacteriology, and that it is our hope that this volume may not only meet the needs of such students but may prove of value to the practitioner of medicine for whom it has also been written.

It is a pleasure to acknowledge the courtesy of those who furnished us with illustrations for use in the text, and our indebtedness to Dr. Gardner Hopkins and Professor Francis Carter Wood for a number of the photomicrographs taken especially for this work.

P. H. H., JR.,

H. Z. 


\section{PREFACE TO THE SECOND EDITION}

INQUIRY in the field of bacteriology is so active at the present day that no general text-book can maintain its usefulness long without frequent revision. In preparing the second edition of this book it has been our purpose to correct omissions and to incorporate the more important researches of the last three years, rather than to alter extensively the plan of the text. From the wealth of material which these years have brought, we have attempted to glean those facts which have seemed to us most important and most directly bearing upon medical problems, since this book was planned, from the beginning, to meet especially the needs of the student of infectious disease.

The most extensive changes and additions have been made in the chapters on streptococci, tuberculosis, plague, leprosy, syphilis, rabies, and poliomyelitis. Short sections on typhus fever, on the plaguelike disease of rodents, and on rat leprosy have been added, and we have inserted a tabulation of our knowledge of filtrable virus, adapted largely from the summary recently published by Wolbach. The Anderson and McClintic method for the standardization of disinfectants, and Churchman's recent work on anilin dyes and bacterial growth, have been added. Many minor corrections and additions have been made throughout the text. In preparing these changes, valuable assistance has been given us by Dr. J. Gardner Hopkins, Associate in Bacteriology at Columbia University, and many helpful suggestions have been made by Drs. Dwyer and Bliss.

It has been gratifying to note how much of the work which seemed to us particularly valuable and enlightening has emanated, during these three years, from American laboratories.

We have purposely omitted making any extensive changes in the section on immunity. The function of this part of the book is to give the beginner a basis for further reading and introduce him, as simply as possible, to the difficult problems of the field. We have felt that the addition of much more detail and theory would render this section unsuited to the needs of a general text-book.

It is a sorrowful necessity that this revision must be put forth withvii 
viii

out the wise counsel of one of its authors. Since the first edition of this book was published Prof. Philip Hanson Hiss, Jr., has died. By his death we have lost a dear friend and a valued teacher, and American bacteriology has been deprived of a worker who was entering into the most brilliant period of his scientific maturity.

H. Z.

New York, 1914 


\section{CONTENTS}

\section{SECTION I}

THE GENERAL BIOLOGY OF BACTERIA AND THE TECHNIQUE OF BACTERIOLOGICAL STUDY

CHAPTER

I. The Development and Scope of Ba Cteriology . . . . . 1

II General Morphology, Reproduction, and Chemical and Physical Properties of the Bacteria. . . . . . . . 9

II. The Relation of Bacteria to Environment, and Their Classification . . . . . . . • . . . . . . 25

IV. The Biological Activities of Bacteria . . . . . . . . 40

V. The Destruction of Bacteria . . . . . . . . . . . 62

VI. Methods Used in the Microscopic Study and Staining of BACTERIA . . . . . . . . . . . . . 93

VII. The Preparation of Culture Media . . . . . . . . . 113

VIII. Method Used in the Cultivation of Bacteria . . . . 141

IX Methods Determining Biological Activities of Bacteria . 164

X. The Bacteriological Examination of Material from Patients . . . . . . . . . . . • . . . 174

\section{SECTION II}

\section{INFEC'IION AND IMMUNI'IY}

Chapter

XI. Fundamental Factors of Pathogenicity and Infection . 181

XII. Defensive Factors of the Animal Organism . • • • • . 189

XIII. Toxins ANd Antitoxins . . . . . . . . . . . . 203

XIV. Production and Testing of Antitoxins . . . . . • . . 216

XV. Lysins, Agglutinins, Precipitins, and Other Antibodies , 224

XVI. The Technique of Serum Reactions . . . . . . . . 249 
CHAPTER

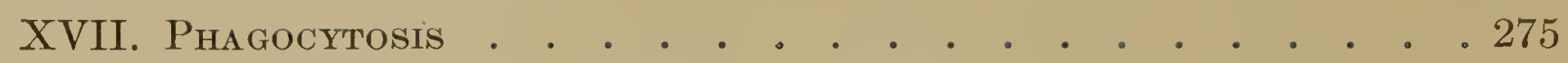

XVIII. Opsonins, Leucocyte Extract, and Aggressins . . • . 281

XIX. Anaphylaxis or Hypersusceptibility . • • • • • • . . 295

XX. Facts and Problems of Immunity in their Bearing upon the Treatment of Infectious Diseases . . . . . . 305

\section{SECTION III}

\section{PATHOGENIC MICROORGANISMS}

CHAPTER

XXI. The Staphylococci (Micrococci) • • • • • • • • • • . 321

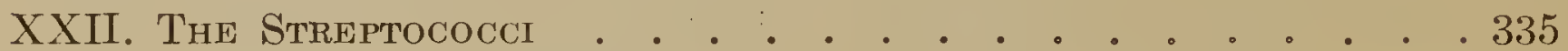

XXIII. Diplococcus PNEUMONiA . . . . . . . . . . . . 352

XXIV. Micrococcus intracellularis meningitidis (Meningococcus) . 371

XXV. Diplococcus Gonorrhoe (Gonococcus), Micrococcus CAtarrhalis, and Other Gram-Negative Cocci . . . . . 380

XXVI. Bacilli of the Colon-Typhoid-Dysentery Group-Bacillus

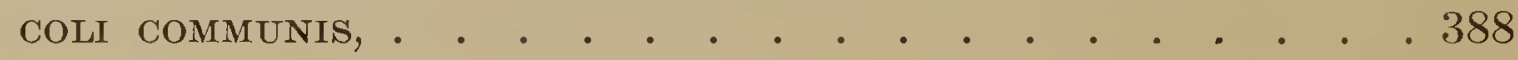

XXVII. Bacilli of the Colon-Typhoid-Dysentery Group (continued) -Bacillus of Typhoid Fever • . . . . . . . . . 399

XXVIII. Bacilli of the Colon-Typhoid-Dysentery Group (continued) -Bacilli Intermediate. Between the Typhoid and Colon Organisms . . . • • • . . . . . . . . . 428

XXiX. Bacilli of the Colon-Typhoid-Dysentery Group (continued) -The Dysentery Bacilil . . . . . . . . . . 435

XXX. Bacillus mucosus capsulatus . . . . . . . . . . . 447 XXXI. Bacillus tetani . . . . . . . . . . . . . . 4456

XXXII. Bacillus of Symptomatic Anthrax, Bacillus of Malignant Edema, Bacillus ä̈rogenes capsulatus, Bacillus botu-

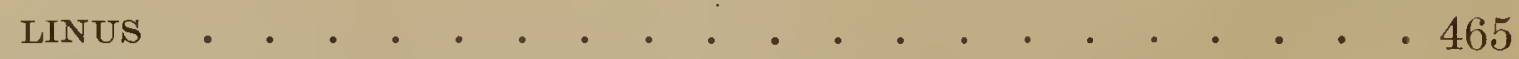

XXXIII. The Tubercle Bacillus . . . . . . . . . . . . . 479

XXXIV. The Smegma Bacillus and the Bacillus of Leprosy . • 503 XXXV. Bacillus diphtheriae, Bacillus Hoffmani, and Bacillus XEROSIS •. . . . . . . . . . . . . . . . 512

XXXVi. Bacillus mallei . • . . . . . . . . . . 528 XXXVII. Bacillus influenz and Closely Related Bacteria • • 536 
XXXVIII. Bordet-Gengou Bacillus, Morax-Axenfeld Bacillus, Zur Nedden Bacillus, Ducrey Bacillus . . . . . . . 543 XXXIX. The Bacilli of the Hemorrhagic Septicemia Group and Bacillus pestis • • • • • • • • • • • • . 551

Xl. Bacillus anthracis and Anthrax • • • • • • . . 563

XLI. Bacillus pyocyaneus • • • • • • • • • • • • • 577

Xlit. Asiatic Cholera and the Cholera Organism • • • • • 582

Xliti. Diseases Caused by Spirochetes . • • • • • • • . 592 XliV. The Higher Bacteria • . • • • • • • . . . . 618

XLV. The Yeasts • • • • • • • • • • • • • • 629 XLVI. HYрномчсетеS • • • • • • • • • • • • • . 635

\section{SEC'IION IV}

\section{DISEASES OF UNKNOWN ETIOLOGY}

CHAPTER

XLVII. Rabies • • • • • • • • • • • • • • • • . 646

XLVIII. SmallPOX • • • • • • • • • • • • • • • • 657

XLIX. Acute Anterior Poliomyelitis • • • • • • • • • • 664

L. Yellow Fever . • • • • • • • • • • • • • 668

LI. Measles, Scarlet Fever, and Foot-and-Mouth Disease • • 675

\section{SECTION V}

BACTERIA IN AIR, SOIL, WATER, AND MILK CHAPTER

LII. Bacteria in the Air and Soll • • • . • • • • • . 683

LIII. Bacteria in Water • • • • • • • • • • • • 689

IIV. Bacteria in Milk and Milk Products, Bacteria in the INDUSTRIES • • • • • • • • • • • • • • • 699 INDEX OF AUTHORS • • • • • • • • • • • 719 INDEX OF SUBJECTS • • • • • • • • • • 731 



\section{LIST OF ILLUSTRATIONS}

FIGURE

PAGE

1. Types of bacterial morphology . . . . . . . . . . . . 10

2. Bacterial capsules . . . . . . . . . . . . . . 12

3. Arrangement of bacterial flagella . . . . . . . . . . . 15

4. Various positions of spores in bacterial cell . . . . . . . . . . . 17

5. Germination of spores . . . . . . . . . . . . . . . . 17

6. Degeneration forms of Bacillus diphtheriæ . . . . . . . . . . . 19

7. Degeneration forms of Bacillus pestis . . . . . . . . . . 20

8. Hot-air sterilizer . . . . . . . . . . . . . . . 69

9. Arnold sterilizer . . . . . . . . . . . . . . . 70

10. Low-temperature sterilizer . . . . . . . . . . . . 71

11. Autoclave . . . . . . . . . . . . . . 72

12. Lentz formalin apparatus . . . . . . . . . . . . . . 90

13. Breslau formaldehyde generator and section of same . . . . . . . 91

14. Hanging drop preparation . . . . . . . . . . . . . . . 94

15. Florence flask . . . . . . . . . . . . . . . . 114

16. Erlenmeyer flask . . . . . . . . . . . . . . . 114

17. Petri dish . . . . . . . . . . . . . . . . . . . 115

18. Test tubes, showing method of stoppering . . . . . . . . 116

19. Burette for titrating media . . . . . . . . . . . . . . 117

20. Tubing media . . . . . . . . . . . . . . . . . . . . 118

21. Media in tubes . . . . . . . . . . . . . . . 119

22. Berkefeld filter . . . . . . . . . . . . . . 120

23. Berkefeld filter . . . . . . . . . . . . . . 121

24. Reichel filter . . . . . . . . . . . . . . . . . 122

25. Kitasato filter . . . . . . . . . . . . . . . 123

26. Maassen filter, for small quantities of fluid . . . . . . . . . 124

27. Platinum wires . . . . . . . . . . . . . . . . 142

28. Taking plugs from tubes before inoculation . . . . . . . . 143

29. Inoculating . . . . . . . . . . . . . . . . . . . 144

30. Pouring inoculating medium into Petri dish . . . . . . . . . 145

31. Streak plate . . . . . . . . . . . . . . . . . . . 147

32. Deep stab cultivation of anaërobic bacteria . . . . . . . . 148

33. Deep stab cultivation of anaërobic bacteria . . . . . . . . . 149

34. Cultivation of anaërobes in fluid under albolin . . . . . . . . . 150

35. Wright's method of anaërobic cultivation in fluid media . . . . . . 151

36. Novy jar . . . . . . . . . . . . . . . . . . 152

37. Wright's method of anaërobic cultivation by the use of pyrogallic-acid solution . . . . . . . . . . . . . . . . . 153

38. Jar for anaërobic cultivation . . . . . . . . . . . 154 
FIGURE

PAGE

39. Apparatus for combining the methods of exhaustion, hydrogen replacement, and oxygen absorption 155

40. Simple apparatus for plate cultivation of anaërobic bacteria . . • . 156

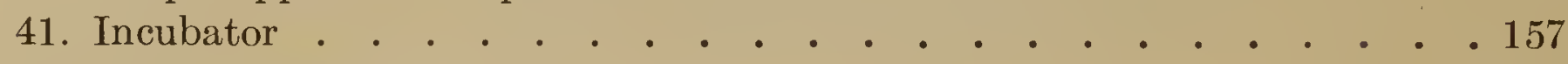

42. Thermo-regulator . . . . . . . . . . . . . . . . . 158

43. Thermo-regulator . . . . . . . . . . . . . . . . . 158

44. Moitessier gas-pressure regulator . . . . . . . . . . . . . 160

45. Variations in the conformation of the borders of bacterial colonies . . 161

46. Wolffhügel counting-plate . : . . . • . . . . . . . . 162

47. Types of fermentation tubes . . . . . . . . . . . . . 165

48. Types of gelatin liquefaction by bacteria . . . . . . . . . . 169

49. Intraperitoneal inoculation of rabbit . . . . . . . . . . 170

50. Intravenous inoculation of rabbit . . . . . . . . . . . 170

51. Intraperitoneal inoculation of guinea-pig . . . . . . . . . . . 171

52. Guinea-pig cage . . . . . . . . . . . . . . . . . 171

53. Rabbit cage . . . . . . . . . . . . . . . . . . 172

54. Blood-culture plate showing streptococcus colonies . . . . . . . . 179

55. Toxin and body cell . . . . . . . • . . • • • . . 206

56. Toxin and antitoxin . . . . . . . . . . . . . . . . . 214

57. Ehrlich's conception of cell-receptors, giving rise to lytic immune bodies 227

58. Complement, amboceptor or immune body, and antigen or immunizing substance . . . . . . . . . . . . . . . . . . . 227

59. Microscopic agglutination reaction . . . . . . . . . . . . . . . 229

60. Macroscopic agglutination . . . . . . . . . . . . . . . . 230

61. Ehrlich's conception of the structure of agglutinins and precipitins . . 238

62. The structure of cell-receptors and immune bodies, according to Ehrlich's conception . . . . . . . . . . . . . . . . . . . . . 239

63. Neisser and Wechsberg's conception of complement deviation . . . . 245

64. Schematic representation of complement fixation in the Bordet-Gengou reaction . . . . . . . . . . . . . . . . . . . . 247

65. Capillary pipette for removal of exudate in doing the Pfeiffer test . 256

66. Wright's capsule for collecting blood . . . . . . . . . . . . 284

67. Pipette for opsonic work . . . . . . . . . . . . . . . . . . 285

68. Pipette with three substances,-corpuscles, bacteria, and serum, as first taken up . . . . . . . . . . . . . . . . . . 285

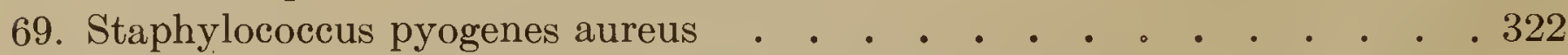

70. Staphylococcus colonies . . . . . . . . . . . . . . . . . . . 323

71. Micrococcus tetragenus . . . . . . . . . . . . . . 334

72. Streptococcus pyogenes . • . • . • • . • . . . . . . . 336

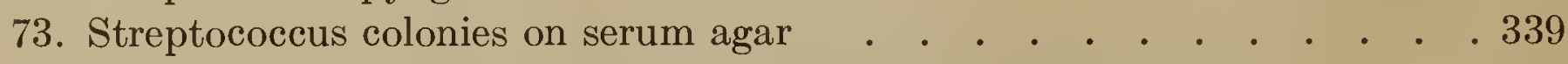

74. Streptococcus colonies from blood culture on blood-agar plate . . . 345

75. Pneumococci . . . . . . . . . . . . . . . . . . 354

76. Pneumococci . . . . . . . . . . . . . . . . . . . 354

77. Meningococcus, pure culture . . . . . . . . . . . . . . 372

78. Meningococcus in spinal fluid . . . . . . . . . . . . . 373

79. Meningococcus culture (streak plate) . . . . . . . . . . . 375

80. Gonococcus pus frorn urethra . . . . . . . . . . . . . 381 
FIGURE

PAGE

81. Gonococcus

382

82. Gonococcus colony

383

83. Bacillus coli communis

390

84. Bacillus coli communis on various media . • • • • • • • • . . 396

85. Bacillus coli communior on various media . • • • • • • • • • • 397

86. Bacillus typhosus . . . . . . . . . • • . . . . . . . 400

87. Bacillus typhosus, showing flagella . . . . . . . . . . . . 401

88. Surface colony of Bacillus typhosus on gelatin . . . . . . . . . . 402

89. Bacillus coli; deep colonies on Hiss plate medium . . . . . . . . 407

90. Bacillus typhosus; deep colonies in Hiss plate medium . . . . . . 408

91. Bacillus typhosus; colony in Hiss plate medium, highly magnified . . 409

92. Colon and typhoid colonies in Hiss plate medium . . . . . . . . 410

93. Scheme of fermentations of the dysentery-typhoid-colon-bacilli in carbohydrate serum-water media . . • . . . . • . . . . 445

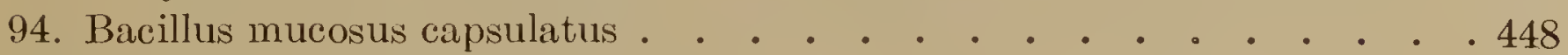

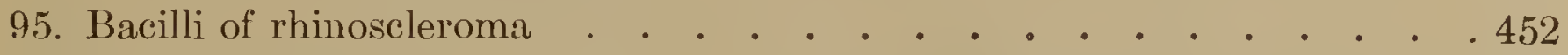

96. Bacillus tetani . . . . . . . . . . . . . . . . . . . 457

97. Young tetanus culture in glucose agar . . . . . . . . . . . . . . 458

98. Older tetanus culture in glucose agar . . . . . . . . . . . . . 459

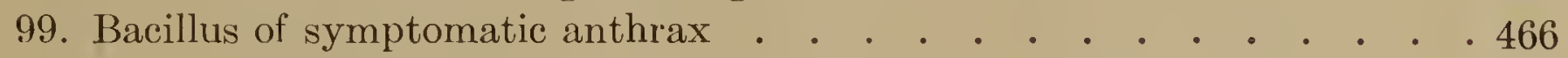

100. Bacillus of symptomatic anthrax, culture in glucose agar . . . . . . . . . . . . . 467

101. Bacillus of malignant edema . . . . . . . . . . . . . . . . . . 469

102. Bacillus of malignant edema, culture in glucose agar . . . . . . 470

103. Tubercle bacilli in sputum . . . . . . . . . . . . . . . . 480

104. Culture of Bacillus tuberculosis in flask of glycerin bouillon . . . . 485

105. Bacillus diphtheriæ . . . . . . . . . . . . . . . • . . 513

106. Colonies of Bacillus diphtheriæ on glycerin agar . • . • . . . . 518

107. Bacillus Hoffmanni . . . . . . . . • . • . • . . . . . . 523

108. Colonies of Bacillus Hoffmanni on agar . . . . . . . . . . 524

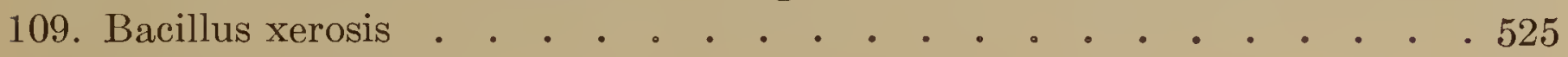

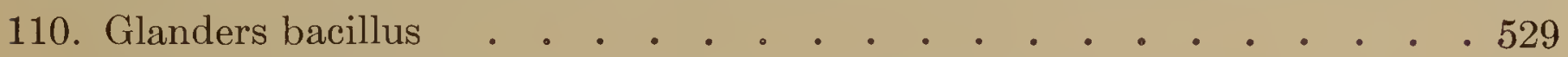

111. Glanders bacilli in tissue . . . . . . . . . . . . . . . . . 531

112. Bacillus influenzæ; smear from pure culture on blood agar . . . . . 537

113. Bacillus influenzæ; smear from sputum . . . . . . . . . . 538

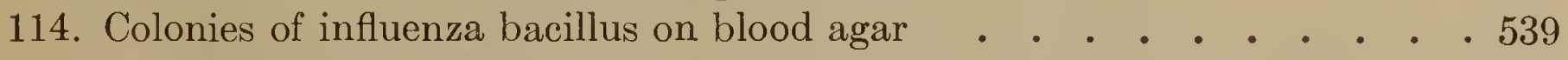

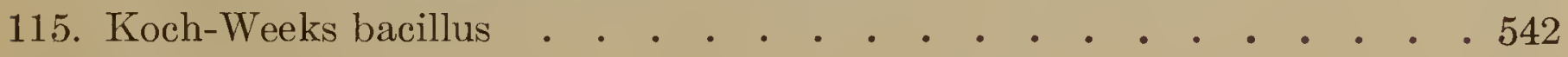

116. Bordet-Gengou bacillus . . . . . . . . . . . . . . . 544

117. Morax-Axenfeld diplo-bacillus . . . . . . . . . • . . 546

118. Bacillus pestis . . . . . . . . . . . . . . . . . 555

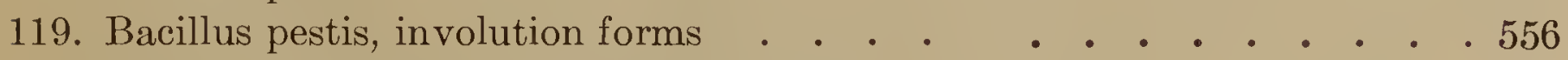

120. Bacillus anthracis; pure culture on agar . . . . . . • • • . 564

121. Bacillus anthracis, in kidney tissue . . . . . . . . . . . 565

122. Bacillus anthracis, in spleen tissue . . . . . . . . . . 566

123. Anthrax colony on gelatin . . . . . . . . . . . . . 567

124. Anthrax colony on agar . . . . . . . . . . . . . . 568

125. Bacillus subtilis . • • • • • • • . • • • • • • • . 569

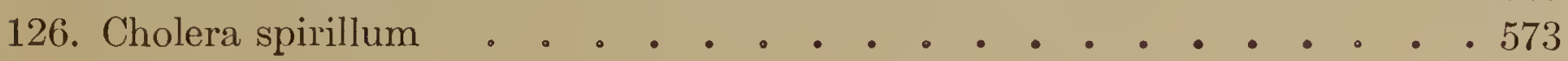


127. Cholera spirillum; stab cultures in gelatin, three days old . . . . . . 586

128. Cholera spirillum; stab culture in gelatin, six days old . . . . . . . 586

129. Spirochrte pallida; smear from chancre . . . . . . . . . . . . 594

130. Spirochrte pallida, in spleen of congenital syphilis . . . . . . . 600

131. Spirochæte pallida, in liver of congenital syphilis . . . . . . . . 601

132. Spirochæte of relapsing fever . . . . . . . . . . . . . . 605

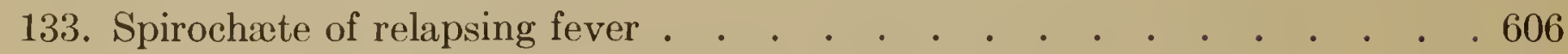

134. Spirochrte of relapsing fever . . . . . . . . . . . . . . 607

135. Spirochæte of Dutton, African tick fever . . . . . . . . . . . . 609

136. Smear from the throat of a case of Vincent's angina . . . . . . . . 611

137. Throat smear, Vincent's angina . . . . . . . . . . . . . . 612

138. Spirochæte gallinarum . . . . . . . . . . . . . . . 616

139. Cladothrix, showing false branching . . . . . . . . . . 620

140. Streptothrix, showing true branching . . . . . . . . . . . . 621

141. Actinomyces granule crushed beneath a cover-glass . . . . . . . . 624

142. Actinomyces granule crushed beneath a cover-glass . . . . . . . . 625

143. Branching filaments of actinomyces . . . . . . . . . . . . . 626

144. Yeast cells . . . . . . . . . . . . . . . . . . . 630

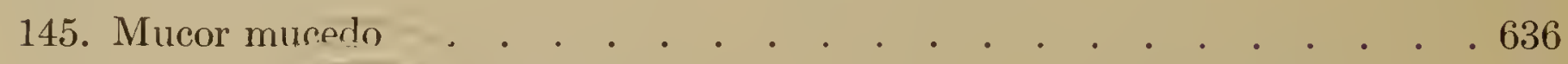

146. Mucor mucedo . . . . . . . . . . . . . . . . . . . . . 637

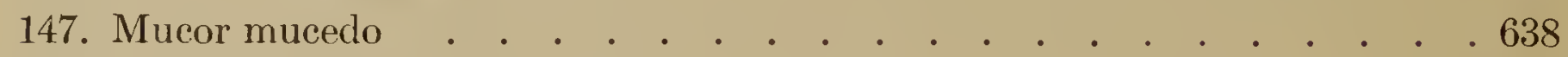

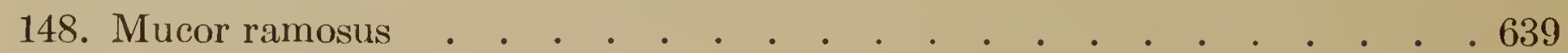

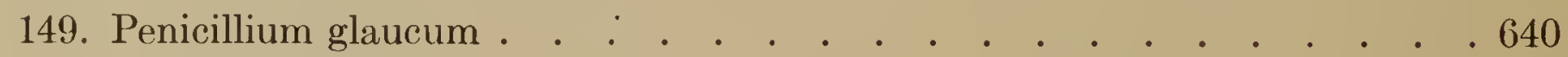

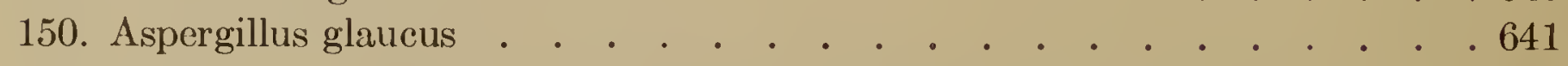

151. Thrush . . . . . . . . . . . . . . . . . . . 642

152. Achorion Schoenleinii . . . . . . . . . . . . . . . . 643

153. Method of drying spinal cord of rabbit for purposes of attenuation . . 653

154. Stegomyia fasciata . . . . . . . . . . . . . . . 671

155. Bacillus bulgaricus . . . . . . . . . . . . . . . . . . . . . . . . . . . . . . 


\section{SECTION I}

\section{THE GENERAL BIOLOGY OF BACTERIA AND THE TECHNIQUE OF BACTERIOLOGICAL STUDY}

\section{CHAPTER I}

\section{THE DEVELOPMENT AND SCOPE OF BACTERIOLOGY}

As we trace back to their ultimate origins the lines of development of living beings of the animal and plant kingdoms, we find them converging toward a common type, represented by a large group of unicellular organisms, so simple in structure, so unspecialized in function, that their classification in either the realm of plants or that of animals becomes little more than an academic question. However, even such microorganisms, in which the functions of nutrition, respiration, locomotion, and reproduction are concentrated within the confines of a single cell, and in which adaptation to special conditions more readily brings about modifications leading to the production of a multitude of delicately graded transitional forms, fall into groups which, either in structure or in biological attributes show evidence of a tendency toward one or the other of the great kingdoms.

Most important of these unicellular forms, for the student of medical science, are the bacteria and the protozoa.

The former, by reason of their undifferentiated protoplasm, their occasional possession of cellulose membranes, their biological tendency to synthetize, as well as to break down organic compounds, and because of the transitional forms which seem to connect them directly with the lower plants, are generally placed in the plant kingdom. The latter, chiefly on the basis of metabolism, are classified with the animals.

Knowledge of the existence of microorganisms as minute as the ones under discussion, was of necessity forced to await the perfection of instruments of magnification. It was not until the latter half of the seventeenth century, therefore, that the Jesuit, Kircher, in 1659, and the Dutch linen-draper, van Leeuwenhoek, in 1675, actually saw and 
described living beings too small to be seen with the naked eye. There can be no doubt that the small bodies seen by these men and their many immediate successors were, at least in part, bacteria. And indeed the descriptions and illustrations of several of the earliest workers correspond with many of the forms which are well known to us at the present day.

During the century following the work of these pioneers, the efforts of investigators lay chiefly in the more exact morphological description of some of the forms of unicellular life, already known. Conspicuous among the work of this period is that of Otto Friedrich Müller. In the generation following Müller's work, however, a marked advance in the study of these forms was made by Ehrenberg, ${ }^{1}$ who established a classification which, in some of its cardinal divisions, is retained until the present day.

Meanwhile the regularity with which these "animalcula" or "infusion animalcula" were demonstrable in tartar from the teeth, in intestinal contents, in well-water, etc., had begun to arouse in the minds of the more advanced physicians of the time a suspicion as to a possible relationship of these minute forms with disease. The conception of "contagion," or transmission of a disease from one human being to another, was, however, even at this time, centuries old. The fact had been recognized by Aristotle, had been reiterated by medieval philosophers, and had led, in 1546, to the division of contagious diseases by Fracastor, into those transmitted "per contactum," and those conveyed indirectly "per fomitem." It was for these mysterious facts of the transmissibility of disease, that clinicians of the eighteenth century, with remarkable insight, saw an explanation in the microorganisms discovered by Leeuwenhoek and his followers.

In fact, Plenciz of Vienna, writing in 1762, not only expressed a belief in the direct etiological connection between microorganisms and some diseases, but was the first to advance the opinion that each malady had its own specific causal agent, which multiplied enormously in the diseased body. The opinions of this author, if translated into the language of our modern knowledge of the subject, came remarkably near to the truth, not only as regards etiology and transmission, but also in their suggestion of a specific therapy for each disease.

The conception of a "contagium vivum" was thus practically established with the work of Plenciz and many others who followed in 
his train, but the astonishingly shallow impression which the acute reasoning of these men left upon the medical thought of their day furnishes an excellent example of the futility of the most penetrating speculation when unsupported by experimental data.

The real advancement in the scientific development of the subject was destined to be carried on along entirely different lines. In 1837, Schwann, a botanist, showed that the yeasts, found in fermenting substances, were living beings, which bore a causal relationship to the process of fermentation. At almost the same time, similar observations were made by a French physicist, Cagniard-Latour. The opinions advanced by these men on the nature of fermentation aroused much interest and discussion, since, at that time and for a long period thereafter, fermentation was ascribed universally to proteid decomposition, a process which was entirely obscure but firmly believed to be of a purely chemical nature.

Although belief in the discovery of Schwann did not completely master the field until after Pasteur had completed his classical studies upon the fermentations occurring in beer and wine, yet the conception of a "fermentum vivum" aroused much speculation, and the attention of physicians and scientists was attracted to the many analogies existing between phenomena of fermentation and those of disease.

The conception of such an analogy, however, was not a new thought in the philosophy of the time. Long before Schwann and CagniardLatour, the philosopher Robert Boyle, working in the seventeenth century, had prophesied that the mystery of infectious diseases would be solved by him who should succeed in elucidating the nature of fermentation.

Nevertheless, the diligent search for microorganisms in relation to various diseases which followed, led to few results, and the successes which were attained were limited to the diseases caused by some of the larger fungi, favus (1839), thrush (1839), and pityriasis versicolor (1846). During this time of ardent but often poorly controlled etiological research, it was Henle who formulated the postulates of conservatism, almost as rigid as the later postulates of Koch, requiring that proof of the etiological relationship of a microorganism to a disease could not be brought merely by finding it in a lesion of the disease, but that constant presence in such lesions must be proven and isolation and study of the microorganism away from the diseased body must be carried out.

It was during this period also that one of the most fundamenta? 
questions, namely, that of the origin of these minute living beings, was being discussed with much passion by the scientific world. It was held by the conservative majority that the microorganisms described by Leeuwenhoek and others after him, were produced by spontaneous generation. The doctrine of spontaneous generation, in fact, was solidly established and sanctified by tradition, and had been applied in the past not alone to microorganisms. ${ }^{1}$ And it must not be forgotten that without the aid of our modern methods of study, satisfactory proof for or against such a process was not easily brought.

Needham, who published in 1749 , had spent much time in fortifying his opinions in favor of spontaneous generation by extensive experimentation. He had placed putrefying material and vegetable infusions in sealed flasks, exposing them for a short time to heat, by immersing them in a vessel of boiling water, and had later shown them to be teeming with microorganisms. He was supported in his views by no less an authority than Buffon. The work of Needham, however, showed a number of experimental inaccuracies which were thoroughly sifted by the Abbé Spallanazani. This investigator repeated the experiments of Needham, employing, however, greater care in sealing his flasks, and subjecting them to a more thorough exposure to heat. His results did not support the views of Needham, but were answered by the latter with the argument that by excessive heating he had produced chemical changes in his solutions which had made spontaneous generation impossible.

The experiments of Schulze, in 1836, who failed to find living organisms in infusions which had been boiled, and to which air had been admitted only after passage through strongly acid solutions, and similar results obtained by Schwann, who had passed the air through highly heated tubes, were open to criticism by their opponents, who claimed that chemical alteration of the air subjected to such drastic influences, had been responsible for the absence of bacteria in the infusion. Similar experiments by Schroeder and Dusch, who had stoppered their flasks with cotton plugs, were not open to this objection, but had also failed to convince. The question was not definitely settled until the years im-

1 Valleri-Radot, in his life of Pasteur, stated that Van Helmont, in the sixteenth century, had given a celebrated prescription for the creation of mice from dirty linen and a few grains of wheat or pieces of cheese. During the centuries following, although, of course, such remarkable and amusing beliefs no longer held sway, nevertheless the question of spontaneous generation of minute and structureless bodies, like the bacteria, still found learned and thoughtful partisans. 
mediately following 1860, when Pasteur conducted a series of experiments which were not only important in incontrovertibly refuting the doctrine of spontaneous generation, but in establishing the principles of scientific investigation which have influenced bacteriological research since his time. ${ }^{1}$

Pasteur attacked the problem from two points of view. In the first place he demonstrated that when air was filtered through cottonwool, innumerable microorganisms were deposited upon the filter. A single shred of such a contaminated filter dropped into a flask of previously sterilized nutritive fluid, sufficed to bring about a rapid and luxuriant growth of microorganisms. In the second place, he succeeded in showing that similar, sterilized "putrescible" liquids, if left in contact with air, would remain uncontaminated provided that the entrance of dust particles were prohibited. This he succeeded in doing by devising flasks, the necks of which had been drawn out into fine tubes bent in the form of a $U$. The ends of these U-tubes, being left open, permitted the sedimentation of dust from the air as far as the lowest angle of the tube, but, in the absence of an air.current, no dust was carried up the second arm into the liquid. In such flasks, he showed that no contamination took place but could be immediately induced by slanting the entire apparatus until the liquid was allowed to run into the bent arm of the U-tube. Finally, by exposing a series of flasks containing sterile yeast infusion, at different atmospheric levels, in places in which the air was subject to varying degrees of dust contamination, he showed an inverse relationship between the purity of the air and the contamination of his flasks with microorganisms.

The doctrine of spontaneous generation had thus received its final refutation, except in one particular. It was not yet clear why complete sterility was not always obtained by the application of definite degrees of heat. This final link in the chain of evidence was supplied, some ten years later, by Cohn, who, in 1871, was the first to observe and correctly interpret bacterial spores and to demonstrate their high powers of resistance against heat and other deleterious influences.

${ }^{1}$ In a letter to his foremost opponent, at this period, Pasteur writes: "In experimental science, it is always a mistake not to doubt when facts do not compel affirmation."

The critical spirit pervading the scientific thought of that time in France is also well expressed by Oliver Wendell Holmes, who said that he had learned three things in Paris: "Not to take authority when I can have facts, not to guess when I can know, and not to think that a man must take physic because he is sick." 
Meanwhile, Pasteur, parallel with his researches upon spontaneous generation, had been carrying on experiments upon the subject of fermentation along the lines suggested by Cagniard-Latour. As a consequence of these experiments, he not only confirmed the opinions both of this author and of Schwann concerning the fermentation of beer and wine by yeasts, but was able to show that a number of other fermentations, such as those of lactic and butyric acid, as well as the decomposition of organic matter by putrefaction, were directly due to the action of microorganisms. It was the discovery of the living agents underlying putrefaction, especially, which exerted the most active influence upon the medical research of the day. This is illustrated by Lister's work. The suppurative processes occurring in infected wounds had long been regarded as a species of putrefaction, and Lord Lister, working directly upon the premises supplied by Pasteur, introduced into both the active and prophylactic treatment of surgical wounds, the antiseptic principles which alone have made modern surgery possible.

There now followed a period in which bacteriological investigation was concentrated upon problems of etiology. Stimulated by Pasteur's successes, the long-cherished hope of finding some specific microorganism as the causal agent in each infectious disease was revived.

Pollender, in 1855, had reported the presence of rod-shaped bodies in the blood and spleen of animals dead of anthrax. Brauell, several years later, had made similar cbservations and had expressed definite opinions as to the causative relationship of these rods to the disease. Convincing proof, however, had not been brought by either of these observers. Finally, in 1863, Davaine, in a series of brilliant investigations, not only confirmed the observations of the two author's mentioned above, but succeeded in demonstrating that the disease could be transmitted by means of blood containing these rods and could never be transmitted by blood from which these rods were absent. Anthrax, thus, is the first disease in which definite proof of bacterial causation was brought.

Speaking before the French Academy of Medicine at this time, Davaine suggested that the manifestations of the disease might in reality represent the results of a specific fermentation produced by the bacilli he had found. This, in a crude way, expresses the modern conception of infectious disease.

Within a few years after this, 1868, the adherents of the parasitic theory of infectious diseases were further encouraged by the discovery, by Obermeier, of a spirillum in the blood of patients suffering from 
relapsing fever. It is not surprising that the successes attained in these diseases, fostering hope of analogous results in all other similar conditions, but without the aid of adequate experimental methods, should have led to many unjustified claims and to much fantastic theorizing. Thus Hallier, at about this time, advanced a theory as to the etiology of infectious diseases, in which he attributed all such conditions to the moulds or hyphomycetes, regarding the smaller form or bacteria as developmental stages of these more complicated forms. Extravagant conjectures of this kind, however, did not maintain themselves for any length of time in the light of the critical attitude which was already pervading bacteriological research.

Progress was made during the years immediately following, chiefly in the elucidation of suppurative processes. Rindfleisch, von Recklinghausen, and Waldeyer, almost simultaneously, described bodies which they observed in sections of tissue containing abscesses, and which they believed to be microorganisms. Notable support was given to their opinion by similar observations made upon pus by Klebs, in 1870. In view, however, of the purely morphological nature of their studies, the opinions of these observers did not entirely prevail. Satisfactory methods of cultivation and isolation had not yet been developed, and Billroth and his followers, with a conservatism entirely justified under existing conditions, while admitting the constant presence of bacteria in purulent lesions, denied their etiological significance. The controversy that followed was rich in suggestions which greatly facilitated the work of later investigators, but could not be definitely settled until 1880, when Koch introduced the technical methods which have made bacteriology an exact science. By the use of solid nutritive media, the isolation of bacteria and their biological study in pure culture were made possible. At about the same time the use of anilin dyes, developed by Weigert, Koch, and Ehrlich, was introduced into morphological study and facilitated the observation of the finer structural details which had been unnoticed while only the grosser methods employed for tissue staining had been available.

With the publication of Koch's work, there began an era unusually rich in results held in leash heretofore by inadequate technical methods. The discovery of the typhoid bacillus in 1880, of the bacillus of fowl cholera and the pneumococcus in the same year, and of the tubercle bacillus in 1882, initiated a series of etiological discoveries which, extending over not more than fifteen years, elucidated the causation of a majority of the infectious diseases. 
Coincident with the elucidation of etiological facts began the inquiry into that field which is now spoken of as the science of immunity. The phenomena which accompany the development of insusceptibility to bacterial infections in man and in animals, first studied by Pasteur, have become the subject of innumerable researches and have led to results of the utmost practical value.

The problems which were encountered were first studied from a purely bacteriological point of view, but their solution has shed light upon biological principles of the broadest application. Investigations into the properties of immune sera, while making bacteriology one of the most important branches of diagnostic and therapeutic medicine, have, at the same time, inseparably linked it with physiology and experimental pathology.

By the revelations of etiological research, and by the study of the biological properties of pathogenic bacteria, contagion, an enemy hitherto unseen and mysterious, was unmasked, and rational campaigns of public sanitation and personal hygiene were made possible. Upon the same elucidations has depended the development of modern surgery-a science which without asepsis and antisepsis would have been doomed to remain in its medieval condition.

Apart from its importance in the purely medical sciences, the study of the bacteria has shed beneficial light, moreover, upon many other fields of human activity. In their relationship to decomposition, the conditions of the soil, and to diseases of plants, the bacteria have been found to occupy a position of great importance in agriculture. Knowledge of bacterial and yeast ferments, furthermore, has become the scientific basis of many industries, chiefly those concerned in the production of wine, beer, and dairy products.

The scope of bacteriology is thus a wide one, and none of its various fields has, as yet, been fully explored. The future of the science is rich in allurement of interest, in promise of result, and in possible benefit to mankind. 


\section{CHAPTER II}

\section{GENERAL MORPHOLOGY, REPRODUCTION, AND CHEMICAL AND PHYSICAL PROPERTIES OF THE BACTERIA}

BACTERIA are exceedingly minute unicellular organisms which may occur perfectly free and singular, or in larger or smaller aggregations, thus forming multicellular groups or colonies, the individuals of which are, however, physiologically independent.

The cells themselves have a number of basic or ground shapes which may be roughly considered in three main classes: The cocci or spheres, the bacilli or straight rods, and the spirilla or curved rod forms.

The cocci are, when fully developed and free, perfectly spherical. When two or more are in apposition, they may be slightly flattened along the tangential surfaces, giving an oval appearance.

The bacilli, or rod-shaped forms, consist of elongated cells whose long diameter may be from two to ten times as great as their width, with ends squarely cut off, as in the case of bacillus anthracis, or gently rounded as in the case of the typhoid bacillus.

The spirilla may vary from small comma-shaped microorganisms, containing but a single curve, to longer or more sinuous forms which may roughly be compared to a corkscrew, being made up of five, six, or more curves. The turns in the typical microorganisms of this class are always in three planes and are spiral rather than simply curved.

Among the known microorganisms, the bacilli by far outnumber other forms, and are probably the most common variety of bacteria in existence. Many variations from these fundamental types may occur even under normal conditions, but contrary to earlier opinions it is now positively known that cocci regularly reproduce cocci, bacilli bacilli, and spirilla spirilla, there being, as far as we know, no mutation from one form into another.

The size of bacteria is subject to considerable variation. Cocci may vary from $.15 \mu$ to $2 . \mu$ in diameter. The average size of the ordinary pus coccus varies from $.8 \mu$ to $1.2 \mu$ in diameter. Fischer has given a graphic illustration of the size of a staphylococcus by calculating that one billion micrococci could easily be contained in a drop of water hav- 
ing a volume of one cubic millimeter. Among the bacilli the range of size is subject to even greater variations. Probably the smallest of the common bacilli is the bacillus of influenza which measures about .5 $\mu$ in length by $.2 \mu$ in thickness. The limit of the optical possibilities of the modern microscope is almost reached by some of the known microorganisms, and it is not at all out of question that sorne of the diseases, for which, up to the present time, no specific microorganisms have
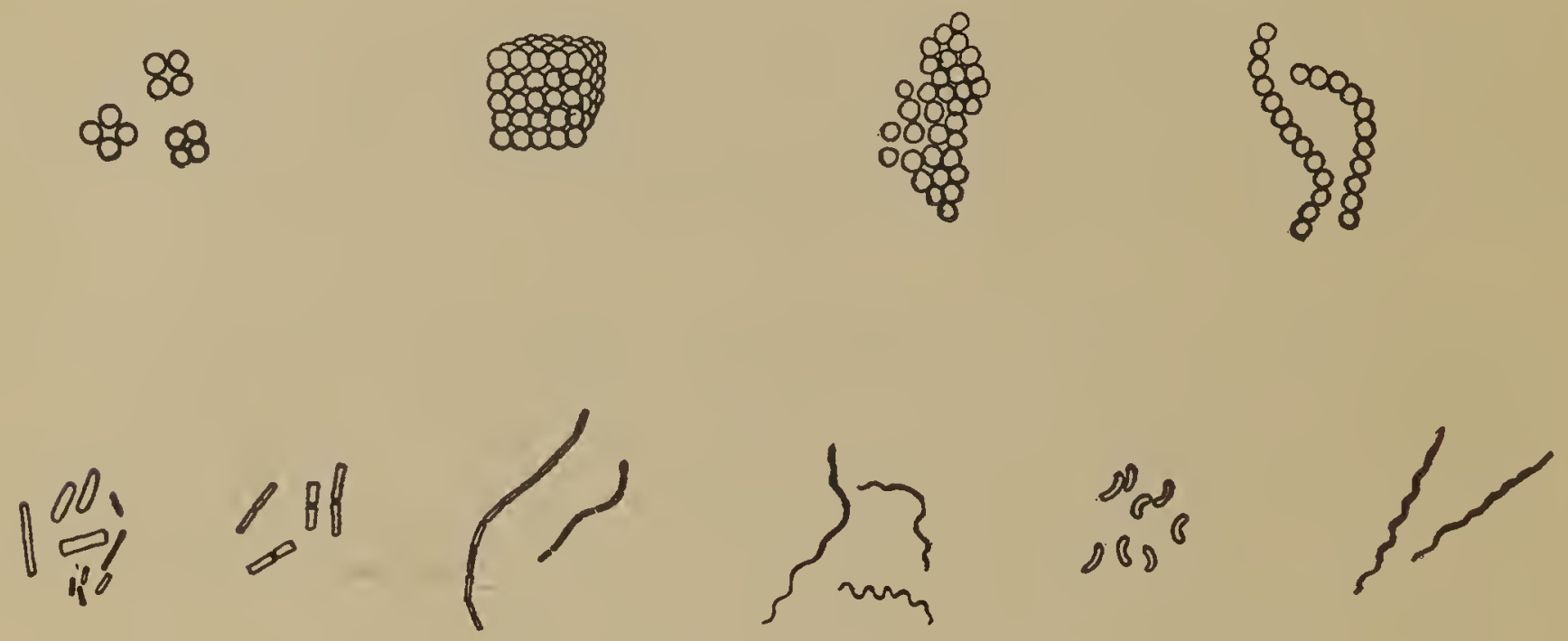

Fig. 1.-Types of Bacterial Morphology.

been found, may be caused by bacteria so small as to be invisible by any of our present methods. In fact, the virus causing the peripneumonia of cattle has been shown to pass through the pores of a Berkefeld filter, which are impenetrable to the smallest of the known bacteria. ${ }^{1}$

\section{MORPHOLOGY OF THE BACTERIAL CELL}

When unstained, most bacteria are transparent, colorless, and apparently homogeneous bodies with a low refractive index. The cells themselves consist of a mass of protoplasm, surrounded, in most instances, by a delicate cell membrane.

The presence of a nucleus ${ }^{2}$ in bacterial cells, though denied by the earlier writers, has been demonstrated beyond question by Zettnow, Nakanishi, ${ }^{3}$ and others. The original opinion of Zettnow was that the entire bacterial body consisted of nuclear material intimately intermingled with the cytoplasm. The opinion now held by most observers

${ }_{1}$ Nocard and Roux, Ann. Past., 12, 1898.

${ }_{2}$ A. Fischer, Jahrbücher f. wissen. Botanik, xxvii.

3 Nakanishi, Münch. med. Woch., vi, 1900. 
who have studied this phase of the subject favors the existence of an ectoplasmic zone which includes cell membrane and flagella, but is definitely a part of the cytoplasm, and an entoplasm in which is concentrated the nuclear material. Bütschli ${ }^{1}$ claims to have demonstrated within this entoplasmic substance a reticular meshwork, between the spaces of which lie granules of chromophilic or nuclear material. Confirmation of this opinion has been brought by Zettnow ${ }^{2}$ and others. Nakanishi, working with a special staining method, asserts that some microorganisms show within the entoplasmic zone a well-defined, minute, round or oval nucleus, which possesses a definitely characteristic staining reaction. ${ }^{3}$

In the bodies of a large number of bacteria, notably in those of the diphtheria group, Ernst, ${ }^{4}$ Babes, ${ }^{5}$ and others have demonstrated granular, deeply staining bodies now spoken of as metachromatic granules, or Babes-Ernst granules, or, because of their frequent position at the ends of bacilli, as polar bodies. These structures are irregular in size and number, and have'a strong affinity for dyes. They are stained distinctly dark in contrast to the rest of the bacterial cell with methylene blue, and may be demonstrated by the special methods of Neisser and of Roux. ${ }^{6}$ Their interpretation has been a matter of much difficulty and of varied opinion. Those who first observed them held that they were a part of the nuclear material of the cell. Others have regarded them as an early stage in spore formation, or as arthrospores. ${ }^{7}$ Again, they have been interpreted as structures comparable to the centrosomes of other unicellular forms. As a matter of fact, the true nature of these bodies is by no means certain. They are present most regularly in microorganisms taken from young and vigorous cultures or in those taken directly from the lesions of disease. It is unlikely that they repre-

1 Bütschli, "Bau der Bakterien," Leipzig, 1890. ${ }^{2}$ Zettnow, Zeit. f. Hyg., xxiv, 1897.

${ }^{3}$ The method of Nakanishi is carried out as follows: Thoroughly cleansed slides are covered with a saturated aqueous solution of methylene blue. This is spread over the slide in an even film and allowed to dry. After drying, the slide should be of a transparent, sky-blue color. The microorganisms to be examined are then emulsified in warm water, or are taken from the fluid media, and dropped upon a cover slip. This is placed, face downward, upon the blue ground of the slide. In this way, bacteria are stained without fixation. Nakanishi claims that by this method the entoplasm is stained blue, while the nuclear material appears of a reddish or purplish hue.

4 Ernst, Zeit. f. Hyg., iv, 1888.

${ }^{5}$ Babes, Zeit. f. Hyg., v, 1889.

${ }^{6}$ See section on stains, p. 107.

7 See section on sporulation, p. 16. 
sent structures in any way comparable to spores, since cultures containing individuals showing metachromatic granules are not more resistant to deleterious influences than are others. Their abundant presence in young vigorous cultures may indicate a relationship between them and the growth energy of the microorganisms. There is no proof, however, that these bodies affect the virulence of the bacteria.

Cell Membrane and Capsule.-Actual proof of the existence of a cell membrane has been brought in the cases of some of the larger forms only, ${ }^{1}$ but the presence of such envelopes may be inferred for most

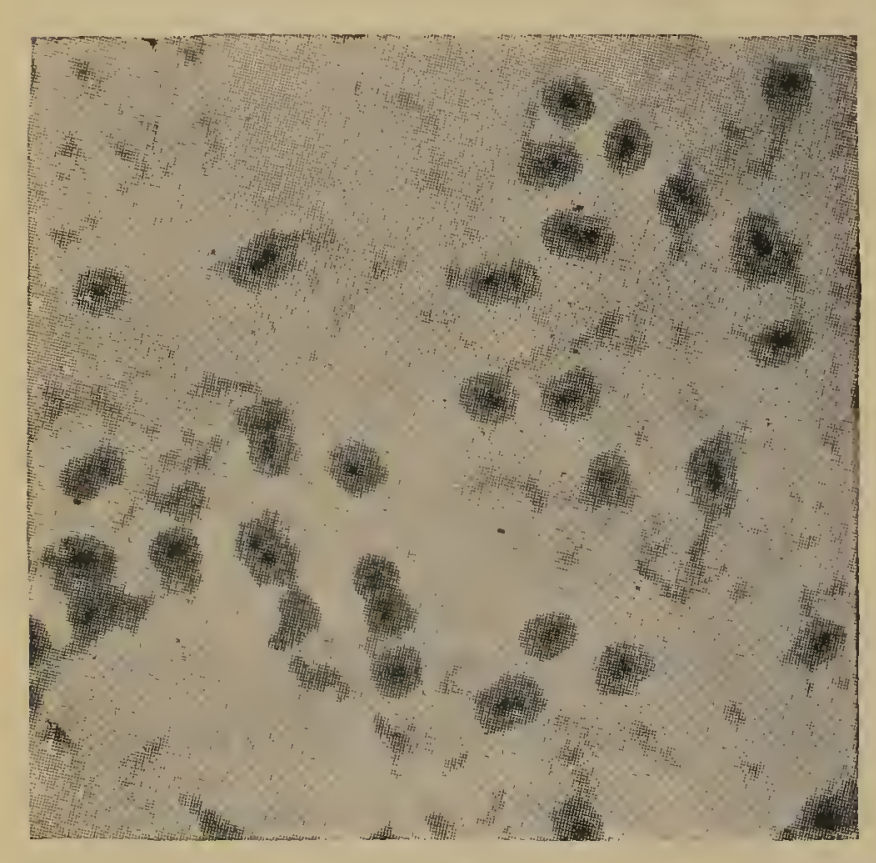

Fig. 2.-Bacterial Capsules. bacteria by their behavior during plasmolysis, where definite retraction of the protoplasm from a well-defined cell outline has been repeatedly observed. The occurrence, furthermore, of so-called "shadow forms" which appear as empty capsules, and of, occasionally, a well-outlined cell body, after the vegetative form has entirely degenerated in the course of sporulation, make the assumption of the presence of a cell membrane appear extremely well founded. Differing from the cell membranes of plant cells, cellulose has not, except in isolated instances, been demonstrable for bacteria, and the membrane is possibly to be regarded rather as a peripheral protoplasmic zone, which remains unstained by the usual manipulations. Zettnow, ${ }^{2}$ who has carefully studied the structure of some of the larger forms, takes the latter view, and regards the "ectoplasmic" zone as a part of the cell protoplasm devoid of nuclear material. Zettnow's opinion is borne out by the greatly increased size of the bacterial cells as seen by means of special stains.

Many bacteria have been shown to possess a mucoid or gelatinous envelope or capsule. According to Migula, ${ }^{3}$ such an envelope is present on all bacteria, though it is in only a few species that it is sufficiently well developed and stable to be easily demonstrable and of differential

\section{${ }^{1}$ Bütschli, loc. cit.}

${ }^{2}$ Zettnow, loc. cit.

${ }^{3}$ Migula, "Systeme d. Bakterien," 1, p. 56. 
value. When stained, the capsule takes the ordinary anilin dyes less deeply than does the bacterial cell body, and varies greatly in thickness, ranging from a thin, just visible margin to dimensions four or five times exceeding the actual size of the bacterial body itself. This structure is perfectly developed in a limited number of bacteria only in which it then becomes an important aid to identification. Most prominent among such bacteria are Diplococcus pneumoniæ, Micrococcus tetragenus, the bacilli of the Friedländer group, and B. aërogenes capsulatus. The development of the capsule seems to depend intimately upon the environment from which the bacteria are taken. It is most easily demonstrable in preparations of bacteria taken directly from animal tissues and fluids, or from media containing animal serum or milk. If cultivated for a prolonged period upon artificial media, many otherwise capsulated microorganisms no longer show this characteristic structure.

Capsules may be demonstrated on bacteria taken from artificial media most successfully when albuminous substances, such as ascitic fluid or blood serum, are present in the culture media, or when the bacteria are smeared upon cover slip or slide in a drop of beef or other serum. ${ }^{1}$ Most observers believe that the capsule represents a swelling of the ectoplasmic zone of bacteria. By others it is regarded as an evidence of the formation of a mucoid intercellular substance, some of which remains adherent to the individual bacteria when removed from cultures. It is noticeable, indeed, that some of the capsulated bacteria, especially Streptococcus mucosus and B. mucosus capsulatus, develop such slimy and gelatinous colonies that, when these are touched with a platinum wire, mucoid threads and strings adhere to the loop. Exactly what the significance of the capsules is cannot yet be decided.

There is, however, definite reason to believe that there is a direct relation between virulence and capsulation; capsulated bacteria are less easily taken up by phagocytes than are the non-capsulated members of the same species. Also, as Porges and others have shown, capsulated organisms are not easily amenable to the agglutinating action of immune sera. Many bacteria (plague, anthrax) which are habitually uncapsulated on artificial media acquire capsules within the infected animal body. Also in some species (pneumococci), the loss of capsule formation as cultivated on the simpler media is accompanied by a diminution of virulence.

Organs of Locomotion.-When suspended in a drop of fluid many bacteria are seen to be actively motile. It is important, however, in 
all cases to distinguish between actual motility and the so-called Brownian or molecular movement which takes place whenever small particles are held in suspension in a fluid.

Brownian or molecular movement is a phenomenon entirely explained by the physical principles of surface tension, and has absolutely no relation to independent motility. 'It may be seen when particles of carmine or any other insoluble substance are suspended in water, and consists in a rapid to and fro vacillation during which there is actually no permanent change in position of the moving particle except inasmuch as this is influenced by currents in the drop.

The true motility of bacteria, on the other hand, is active motion due to impulses originating in the bacteria themselves, where the actual position of the bacterium in the field is permanently changed.

The ability to move in this way is, so far as we know, limited almost entirely to the bacilli and spirilla, there being but few instances where members of the coccus group show active motility. In all cases, with the exception of some of the spirochetes, where motility may occasionally be due to an undulating membrane marginally placed along the body, bacterial motility is due to hair-like organs known as flagella. These flagella have rarely been seen during life, and their recognition and study has been made possible only by special staining methods, such as those devised by Loeffler, van Ermengem, Pitt, and others.

In such stained preparations, the bacterial cell bodies often appear thicker than when ordinary dyes are used, and the flagella apparently are seen to arise from the thickened ectoplasmic zone.

The flagella are long filaments, averaging in thickness from one-tenth to one-thirtieth that of the bacterial body, which often are delicately waved and undulating, and, judging from the positions in which they become fixed in preparations, move by a wavy or screw-like motion. In length they are subject to much variation, but are supposed to be generally longer in old than in young cultures. Very short flagella have been described only on nitrosomonas, one of the nitrifying bacteria discovered by Winogradisky. ${ }^{1}$

As to the finer structures of flagella, little can be made out except that they possess a higher refractive index than the cell body itself, and that they can be stained only with those dyes which bring clearly into view the supposedly true cytoplasm of the cell. Whether they penetrate this cytoplasmic membrane or whether they

1 Winogradsky, Arch. des sci. biologiques, St. Petersburg, 1892, I, 1 and 2. 
are a direct continuation of this peripheral zone of the bacterial body, can not be decided.

The manner in which bacteria move is naturally subject to some variation depending upon the number and position of the flagella possessed by them. Whether bacteria exercise or not the power of motility depends to a large extent upon their present or previous environment. They are usually most motile in vigorous young cultures of from twentyfour to forty-eight hours' growth in favorable media. In old cultures motility may be diminished or even inhibited by acid formation or by other deleterious products of the bacterial metabolism.

At the optimum growth-temperature motility is most active, and a diminution or increase of the temperature to any considerable degree diminishes or inhibits it. Thus actively motile organisms, in the fluid drop, may be seen to diminish distinctly in activity when left for any prolonged time in a cold room, or when the preparation is chilled. Any influence, in other words, chemical or physical, which
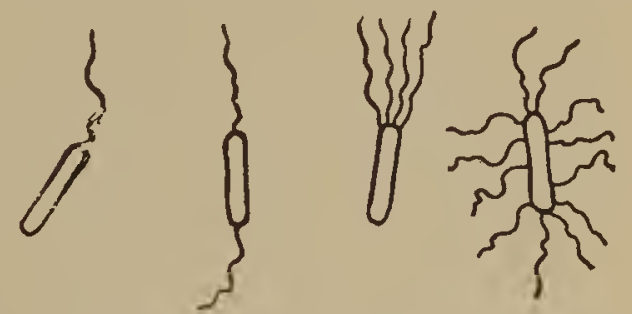

Fig. 3.-Arrangement of Bacterial Flagelila. tends to injure or depress physiologically the bacteria in any way, at the same time tends to inhibit their motility.

Messea ${ }^{1}$ has proposed a classification of bacteria which is based upon the arrangement of their organs of motility, as follows:

I. Gymnobacteria, possessing no flagella.

II. Trichobacteria, with flagella.

1. Monotricha, having a single flagellum at one pole.

2. Lophotricha, having a tuft of flagella at one pole.

3. Amphitricha, with flagella at both poles.

4. Peritricha, with flagella completely surrounding the bacterial body.

Bacterial Spores.-A large number of bacteria possesses the power of developing into a sort of encysted or resting stage by a process commonly spoken of as sporulation or spore formation. The formation of spores by bacteria depends largely upon environmental conditions, and the optimum environment for spore formation differs greatly for various species. It is usually necessary that a temperature of over $20^{\circ} \mathrm{C}$. exist in order that spores may be formed. Unfavorable factors, like acid formation, accumulation of bacterial products in old cultures, or 
lack of nutrition, frequently seem to constitute the stimuli which lead to sporulation. In the case of some species, notably the anthrax bacillus, spores are formed only in the presence of free oxygen and are therefore never formed within the tissues of infected animals. It is claimed that some of the pathogenic anaërobes, like B. tetani and the bacillus of malignant edema, may form spores anaërobically. Nevertheless it has been observed that when an absolute exclusion of oxygen is practiced in the cultivation of these bacteria, vegetative forms only are seen in the cultures. ${ }^{1}$

The process of sporulation is by no means to be regarded as a method of multiplication, since it rarely occurs that a single bacillus produces more than one spore. In some species of bacteria the formation of several spores in one individual has occasionally been observed, but there can be no question about the fact that such a condition is exceptional.

Varieties of spores are often recognized, the so-called arthrospores and the true spores or endospores. It is seriously in doubt whether the structures once spoken of as arthrospores should be considered as in any way comparable to true spores. They are represented by the granular and globular appearances occasionally observed in old cultures of some bacteria, notably streptococcus, cholera spirillum, diphtheria bacillus, and others. It was believed that they were due to a transformation of certain individuals of the cultures into more resistant forms. It is probable, however, that such structures are merely to be regarded as evidences of involution or degeneration, since it has never been demonstrated that cultures containing them are more resistant either to disinfectants or to heat, than cultures showing no evidences of such forms. The true spores or endospores are most common among bacilli, and are rarely observed among the spherical bacteria. They arise within the body of the individual bacterium as a small granule which probably represents a concentration of the protoplasmic substance. Nakanishi ${ }^{2}$ claims that there is a definite relation between these sporogenic globules and the nuclear material of the bacterial cell. At the time at which sporulation occurs there is usually a slight and gradual thickening of the bacillary body. After the formation of this thickening, a spore membrane appears about the same thickened area. The completed spore is usually round or oval, has an extremely high refractive index, and a

1 Zinsser, Jour. Exp. Med., viii, 1906, p. 542.

${ }^{2}$ Nakanishi, Münch. med. Woch., 1900, p. 680. 
membrane which is very resistant. Muhlschlege ${ }^{1}$ believes that the spore membrane is a double structure, and, as stated before, Nakanishi believes that the spore contains nuclear material.

The position of the spore in the mother cell is of some differential importance in that it is usually constant for one and the same species. Thus, the spores of the tetanus bacillus are regularly situated at the extreme ends of the bacillary bodies, while those of anthrax are situated at or near the middle.
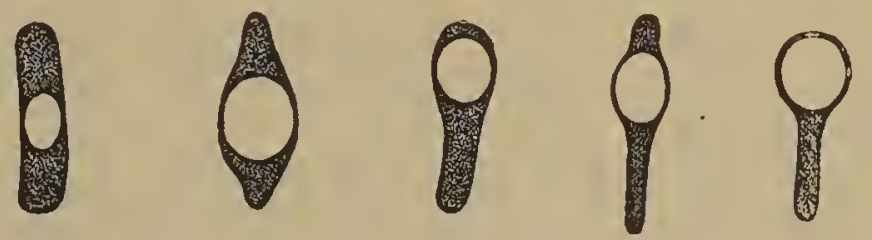

Fig. 4.-V.arious Positions of Spores in Bacterial Cell.

Physiologically, sporulation is probably to be regarded as a method of encystment for the purpose of resisting unfavorable environment,

A
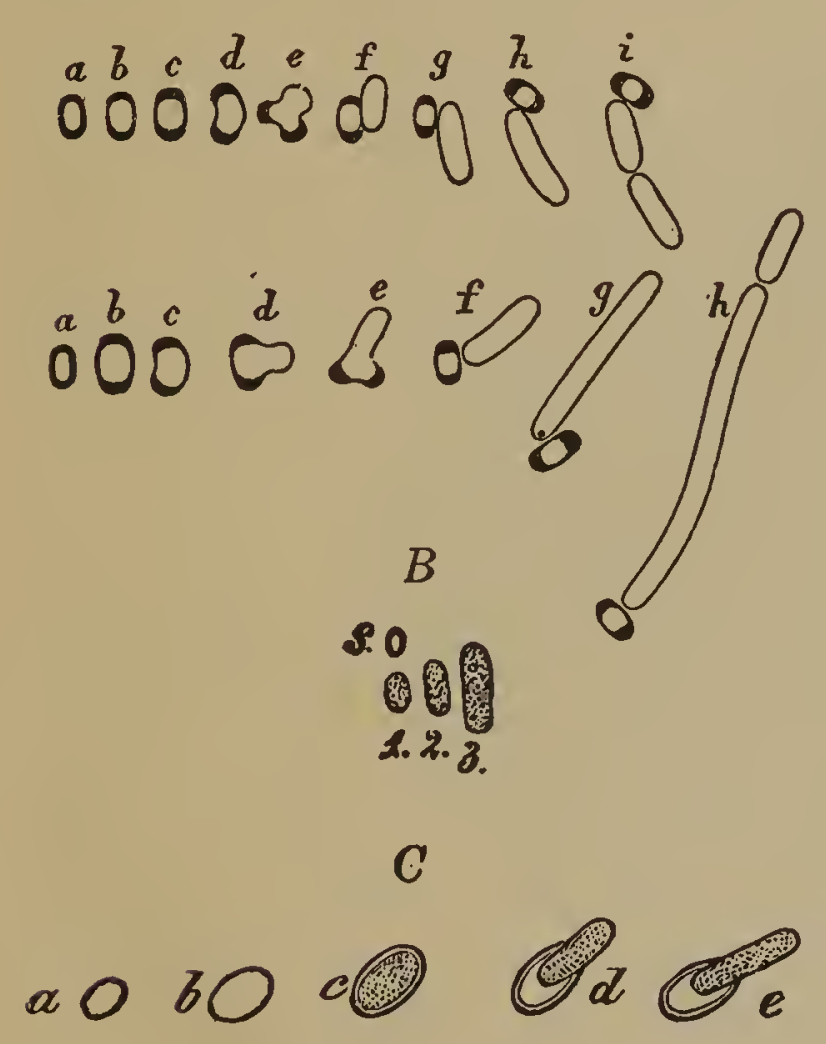

Fig. 5.-Germination of Spores. $A$, Bacillus subtilis, equatorial spore germination; $B$, Bacillus anthracis, germination by simple transition; $C$, Clostrydium butyricum, polar germination.

vironment suitable for bacterial growth as to temperature, moisture, and nutrition, the spores develop into vegetative forms. This process differs according to species. In general it consists of an elongation of and it is indeed true that species of bacteria the vegetative forms of which are rather easily injured by heat, light, drying, and chemicals have a comparatively enormous resistance to these agents after the formation of spores. Thus, while a 10-per-cent solution of carbolic acid will kill the vegetative forms of anthrax bacilli within twenty minutes, anthrax spores are able to resist the same disinfectant for a long period in a concentration of over 50 per cent; and while the vegetative forms of the same bacillus show little more resistance against moist heat than other vegetative forms, the spores will withstand the action of live steam for as long as ten to twelve minutes and more.

Whenever the spores of any microorganism are brought into an en- 
the spore body with a loss of its highly refractile character and resistance to staining fluids. The developing vegetative cell may now rupture and slip out of the spore membrane at one of its poles, leaving the empty spore capsule still visible and attached to the bacillary body. Again, a similar process may take place equatorially instead of at the pole. In other species again, there may be no rupture of the spore membrane at all, the vegetative form arising by gradual elongation of the spore and an absorption or solution of the membrane which is indicated by change in staining reaction. Division by fission in the ordinary way then ensues.

\section{REPRODUCTION OF BACTERIA}

Bacteria multiply by cell division or fission. A young individual increases in size up to the limits of the adult form, when, by simple cleavage, at right angles to the long axis, without any discoverable process of mitosis or nuciear changes, it divides into two individuals. In spite of the claims of various bacteriologists, notably Nakanishi, ${ }^{1}$ traceable analogy to the karyokinesis of other cells has not been definitely established. In the case of the spherical bacteria a slight change to the elliptical form takes place just before cleavage and this cleavage may occur in one plane only, in two planes, or in three planes. According to the limitations of cleavage direction, the cocci assume a chained appearance (streptococci), a grape-like appearance (staphylococci), or an arrangement in packets or cubes having three dimensions (sarcinæ). In the cases of bacilli and spirilla, cleavage takes place in the direction of the short axis. The individuals, after cleavage, may separate from each other, or may remain mutually coherent. The cohesion after cleavage is pronounced in some species of bacteria and slight in others, and, together with the plane of cleavage, determines the morphology of the cell-groups. Thus among the cocei diplo- or double forms, long chains and short chains may arise and furnish a characteristic of great aid in differentiation. Similarly among the bacilli there are forms which appear characteristically as single individuals and others which form chains of varying length.

The rate of growth varies to a certain extent with the species, and also with the favorable or unfavorable character of the envirorment. A generation, that is, the time elapsing in the interval between one

${ }^{1}$ Nakanishi, Cent. f. Bakt., I, xxx, 1901. 
cleavage and the next, has been estimated by A. Fischer ${ }^{1}$ as being about twenty minutes for the cholera spirillum and 16-20 minutes for bacillus coli communis, under the most favorable conditions. The same author has calculated that under these conditions a single cholera spirillum would yield 1600 trillions in a single day. Such a multiplication rate, however, is probably not usual under natural or even artificial conditions, both on account of lack of nutritive material and because of inhibition of the growth of the bacteria by their own products.

\section{VARIATIONS OF BACTERIAL FORMS}

Variations from the basic forms considered in the preceding section may occur, but are not common among bacteria under normal conditions. Thus the formation of club shapes by a thickening of the

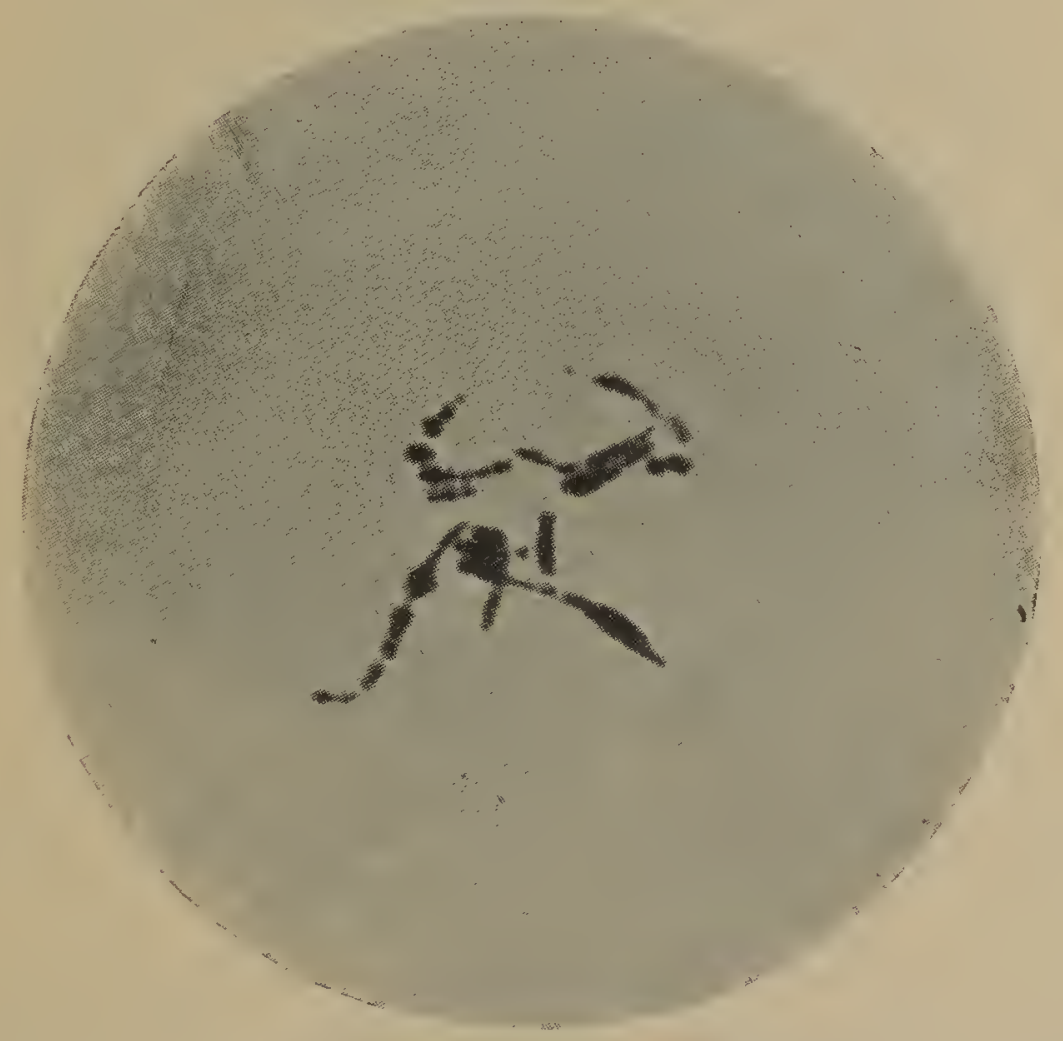

Fig. 6.-Degenneration Forms of Bacillus Diphtheriat. (After Zettnow.)

bacillary body at one or both ends has been frequently observed among bacteria of the diphtheria group, and in the glanders bacillus, and an irregular beading is not infrequently observed in tubercle bacilli under normal conditions. Such pictures can not, in these cases, be regarded as degeneration or involution forms, since they are visible in young, actively growing cultures under ordinary conditions. It is a well-known 
fact, furthermore, that the sizes and contours of bacteria may vary to some extent according to the medium on which they are grown. This may, to a degree, be due to osmotic relations. On fluid media, for instance, many bacteria may appear larger and of a less dense consistency than do members of the same species cultivated upon solid media.

Degeneration Forms.-When bacteria are grown under conditions which are not entirely favorable for their development, or when they are grown for a prolonged period upon artificial culture media without transplantation, there may occur variations which often depart considerably from the ground type, known as degeneration or involution forms.

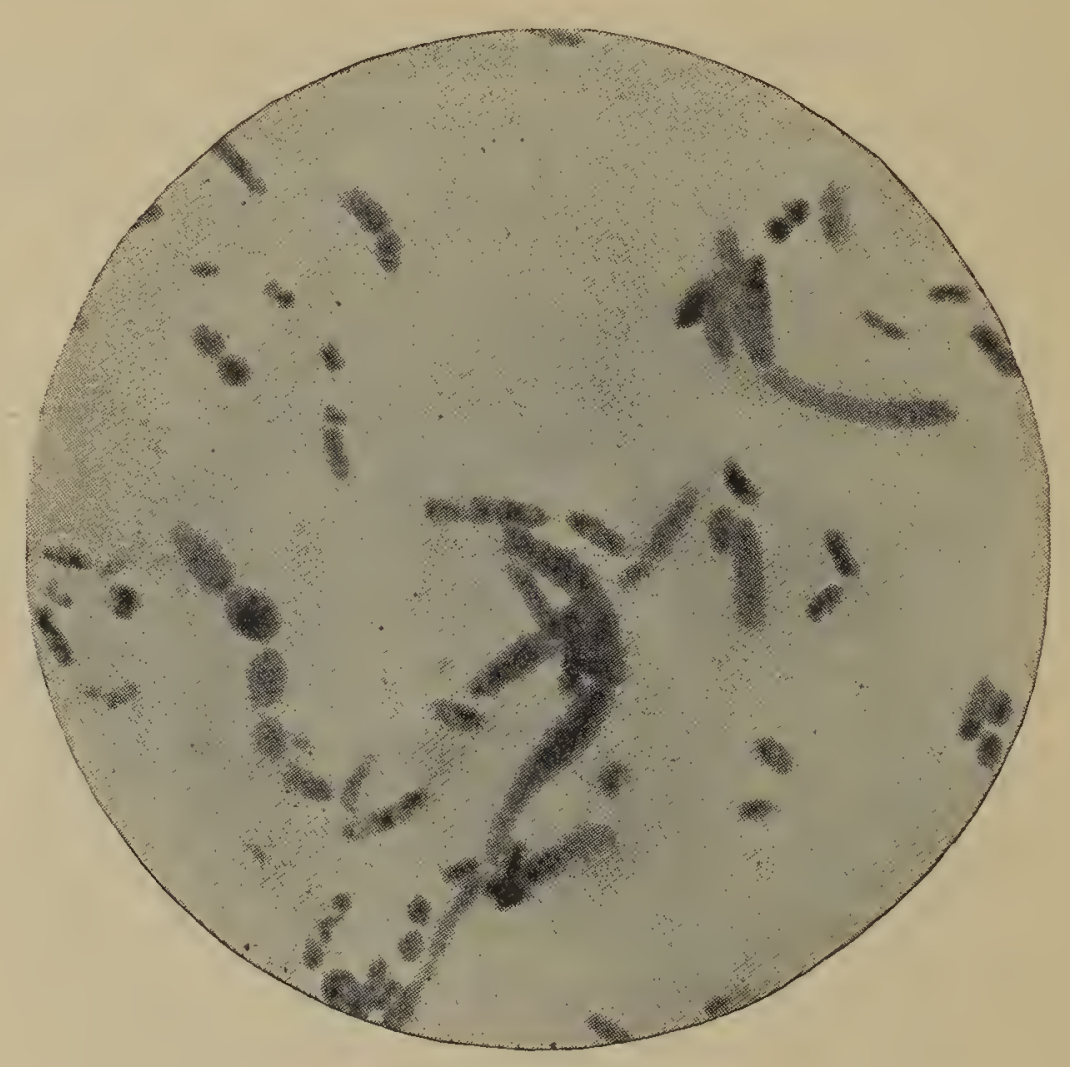

Fig. 7.-Degeneration Forms of Bacillus Pestis. (After Zettnow.)

Thus, in the case of the diphtheria bacillus, old cultures may contain long, irregularly beaded forms with broad expansions at the ends. In the case of $\mathrm{B}$. pestis the fact that large numbers of oval, vacuolated bodies in old cultures are formed regularly has become of differential value. ${ }^{1}$ These degeneration forms are shown most characteristically when the bacteria are cultivated on agar containing 3 to 5 per cent $\mathrm{NaCl}$.

Among the cocci, marked evidences of involution are often seen in cultures of the meningococcus in the form of large, swollen poorlystaining spheres, and in the case of the pneumococcus in the so-called shadow forms which have the appearance of empty capsules. There are

${ }^{1}$ Hankin and Leumann, Cent. f. Bakt., I, xxii, 1897. 
few microorganisms indeed, in which prolonged cultivation on artificial media or other unfavorable influences do not produce variations from the ground type which may often make the cultures morphologically unrecognizable. In the case of many of the spirilla (spirillum Milleri, spirillum Metchnikovi, etc.) the degeneration forms may appear within so short a time as two or three days after transplantation.

\section{CHEMICAL AND PHYSICAL PROPERTIES OF THE BACTERIAL CELL}

Chemical Constituents. - The quantitative chemical composition of bacteria is subject to wide variations, dependent upon the nutritive materials furnished them.

Approximately 80 to 85 per cent of the bacterial body is water. The remainder consists chiefly of proteids which constitute roughly from 50 to 80 per cent of the dry substances. Remaining, after extraction of these, are fats, and in some cases true wax (fatty acid combinations with higher alcohols), traces of cellulose (in some bacteria only), and the ash which makes up usually about 1 to 2 per cent of the dry substances.

The extensive researches of Cramer ${ }^{1}$ have shown how widely at variance quantitative analyses may be when made of cultures of the same species of bacteria grown upon different media. Thus the dry substances of the cholera vibrio were found to be made up of 65 per cent of proteids when the microorganisms were grown upon nutrient broth as against 45 per cent when the same bacteria had been grown upon the proteid-free medium of Uschinsky. Analyses made by Kappes ${ }^{2}$ of B. prodigiosus and by Nencki ${ }^{3}$ and Scheffer of some of the putrefactive bacteria, may serve to illustrate the approximate proportions of the substances making up the bacterial body.

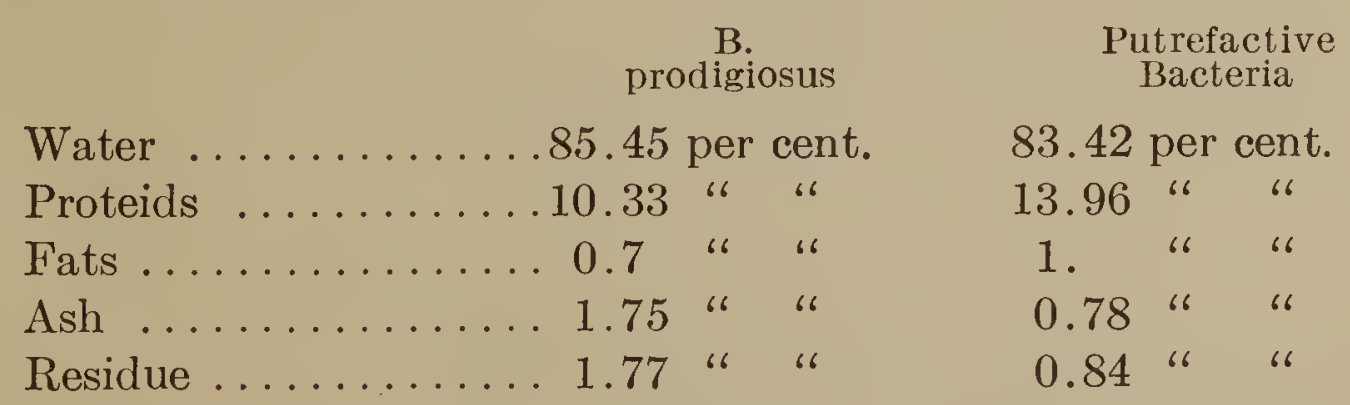

- Cramer, Arch.f. Hyg., xii, xiii, xvi, xxii, xxviii.

2 Kappes, "Analyse der Massenkulturen," etc. Diss., Leipzig, 1889.

3 Nencki und Scheffer, Jour. f. prakt. Chemie, new ser. xix, 1880. 
Analyses of the tubercle bacillus by Ruppel, ${ }^{1}$ Hammerschlag, ${ }^{2}$ Weyl, ${ }^{3}$ and others, have yielded the following approximate results (calculated from results of above-mentioned authors).

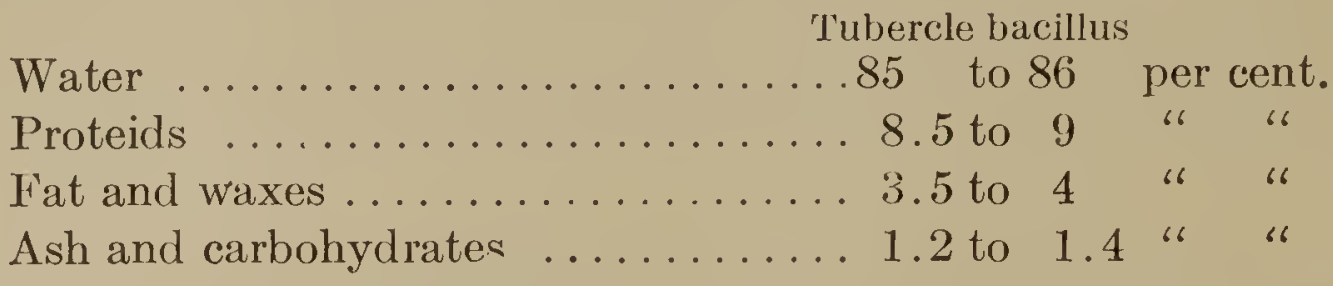

The proteids which are contained in the bacterial dry substances consist partly of nucleoproteids, globulins; and proteids differing materially from those ordinarily met with. Ruppel, in an analysis of the tubercle bacillus, obtained the following values, for 100 grams of dried tubercle bacilli:

Nucleic acid....................8.5 grams.

(Tuberculinic acid)

Nucleoprotamin ...................25.5 "

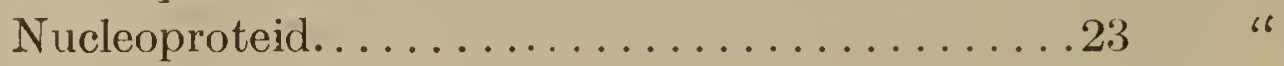

Albuminoids.................... 8.3 "

(Keratin, etc.)

Fat and wax.....................

Ash......................... 9.2 "

A true globulin has been isolated from bacteria by Hellmich, ${ }^{4}$ and true proteids, coagulable by heat, have been demonstrated by Buchner, ${ }^{5}$ in the "Presssaft" or juice obtained by subjecting bacteria to mechanical pressure. In this connection, too, we should not fail to consider the thermolabile toxic substances contained in many bacteria, the endotoxins, which though of uncertain chemical nature, are probably proteid in composition. ${ }^{6}$

The fats which are demonstrable both by microchemical methods, staining with Sudan III., Scharlach R., Osmic acid, and by alcoholether extraction, consist of fatty acids, true fats, and, in the case of the tubercle bacillus at least, of waxy substances. ${ }^{?}$

${ }_{1}^{1}$ Ruppel, Zeit. f. physiol. Chemie, xxvi, 1898.

${ }^{2}$ Hammerschlag, Zeit. f. klin. Med., 1891.

Weyl, Deut. med. Woch., 1891.

- Hellmich, Arch. f. exper. Pathol., etc., xxvi.

- Buchner, Münch. med. Woch., 1897.

'Shaltock, Lancet, May, 1898.

\& De Schweinitz and Dorset, Cent. f. Bakt., I, xxii, 1897. 
The carbohydrates isolated flom various bacteria consist chiefly of small quantities of cellulose and allied bodies, presumably concerned in the formation of the bacterial cell membrane. The demonstration of these substances has been successful only in isolated cases and has not found universal confirmation.

Glycogen-like substances have been demonstrated, according to A. Fischer ${ }^{1}$ in B. subtilis and B. coli. These bacteria stained a reddish brown color when stained with iodin, and after treatment with weak acids were shown to contain dextrose.

The bacterial ash, remaining after removal of other substances, consists largely of phosphates and chlorides of potassium, sodium, calcium, and magnesium.

Osmotic Properties of the Bacterial Cell.-Like all other animal and vegetable cells, the bacterial cell forms in itself a small osmotic unit which reacts delicately to differences of pressure existing between its own protoplasm and the surrounding medium. The perfect and normal morphology of a microorganism, therefore, can exist only when the osmotic pressure within the protoplasm of the cell is isotonic or equal to that of its own environment. The changes produced in the morphological relations of a cell when transferred from one environment into another of varying osmotic pressure, depend intimately upon the "permeability" of the cell membrane for different substances. When such a membrane is permeable for water and not for substances in solution, it is technically spoken of as "semi-permeable." Now, as a matter of fact, the bacterial cell membrane is easily permeable for water, but its permeability differs greatly in various species of bacteria for other substances. Thus, for instance, the cholera vibrio shows great permeability for common salt and B. fluorescens liquefaciens shows a lower permeability for potassium nitrate than do many other bacteria. ${ }^{2}$

When a microorganism is suddenly removed from an environment of low osmotic pressure into one showing a high pressure, say, from a dilute to a concentrated solution of $\mathrm{NaCl}$, an abstraction of water from the cell occurs, with a consequent shrinkage of the protoplasm away from the cell membrane. This process is spoken of as "plasmolysis." Cell death does not usually occur with plasmolysis, but by slow diffusion of the salt itself into the protoplasm, the equilibrium may eventually be restored and the normal morphology of the cell resumed. In all cases

' A. Fischer, "Vorlesungen über die Bakt.," Jena, 1903.

"Gottschlich, in Flügge, "Mikroorganismen," i, p. 91. 
the speed and completeness of the return to normal depends upon the permeability of the cell membrane for the dissolved substances. There is no evidence to support the view that the internal pressure of a cell may be in any way increased by an inherent power of the protoplasm independently of the laws of diffusion. As a general rule, old cultures are more susceptible to plasmolysis than are young and vigorous strains. Spores and, according to A. Fischer, ${ }^{1}$ flagella are much less susceptible. to osmotic changes than are the vegetative bodies.

When, on the other hand, bacteria are suddenly removed from a medium possessing a high osmotic pressure to one comparatively low, say, from a concentrated salt solution to distilled water, a bursting of the cell may occur, a process spoken of as "plasmoptysis." Plasmoptysis leads to cell death, and is probably the cause of the death of microorganisms so often observed in distilled-water emulsions of bacteria.

Other Physical Properties of Bacteria.-The refractive index of the vegetative bacterial body is low, in contrast to the highly refractive character of the spores and flagella. According to Fischer, the ectoplasm or cell membrane shows a higher index than does the endoplasm.

The specific gravity of various microorganisms has been investigated by Bolton, ${ }^{2}$ Rubner, ${ }^{3}$ and others. Some of Rubner's results are the following:

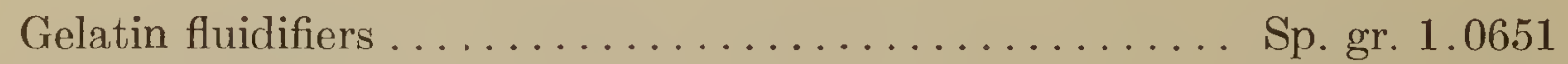

Gas formers.......................... " " 1.0465

Cultures from potato .................... " " 1.038

M. prodigiosus..................... " " 1.054

1 A. Fischer, quoted from Gottschlich in Flügge, "Mikroorganismen," I, p. 91.

${ }^{2}$ Bolton, Zeit. f. Hyg., i, $1886 . \quad{ }^{3}$ Rubner, Arch. f. Hyg., xi, 1890. 


\section{CHAPTER III}

\section{THE RELATION OF BACTERIA CO ENVIRONMENT, AND THEIR CLASSIFICATION}

\section{NUTRITION OF BACTERIA}

LIKE all protoplasmic bodies, bacteria consist of carbon, oxygen, hydrogen, and nitrogen, to which are added inorganic salts and varying quantities of phosphorus and sulphur. In order that bacteria may develop and multiply, therefore, they must be supplied with these substances in proper quantity and in forms suitable for assimilation. To formulate definite laws based on chemical structure as to the compounds suitable, and those unsuitable for use by the bacteria, is obviously impossible owing to the great metabolic variations existing within the bacterial kingdom, and notable attempts to do so, such as those by Loew, ${ }^{1}$ have not successfully withstood critical inquiry.

Carbon.-The carbon necessary for bacterial nourishment or anabolism may be obtained either directly from proteids, carbohydrates, and fats, or from the simpler derivatives of these substances. Thus, the amido-acids, leucin and tyrosin, ketons, and organic acids, like tartaric, citric, and acetic acids, glycerin, and even some of the alcohols, may furnish carbon in a form suitable for bacterial assimilation. A limited number of bacterial species, furthermore, notably the nitrobacteria of Winogradsky, are capable of obtaining their required carbon from atmospheric $\mathrm{CO}_{2}$, and possibly from other simple carbon compounds added to culture media. ${ }^{2}$

Oxygen.--Oxygen is obtained, by the large majority of bacteria, directly from the atmosphere in the form of free $\mathrm{O}_{2}$. For many microorganisms, moreover, the presence of free oxygen is a necessary condition for growth. These are spoken of as the "obligatory aërobes." Among the pathogenic bacteria proper, many, like the gonococcus, bacillus influenzæ, and bacillus pestis, show a marked preference for a well-oxygenated environment. Probably there is no pathogenic micro-

${ }^{1}$ Loew, Cent. f. Bakt., I, xii, 1892.

2 Muntz, Compt. rend. de l'acad. des sciences, t. iii. 
organism which, under certain conditions of nutrition, is entirely unable to exist and multiply in the complete absence of this gas. Tho conditions existing within the infected animal organism cause it to seem likely that all incitants of infection may, at times, thrive in the complete absence of free oxygen.

There is another class of organisms, on the other hand, for whose development the presence of free oxygen is directly injurious. These microorganisms, known as "obligatory anaërobes," obtain their supply of oxygen indirectly, by enzymatic processes of fermentative and. proteolytic cleavage, from carbohydrates and proteids, or by reduction from reducible bodies. Among the pathogenie microorganisms the class of "obligatory anaërobes" is represented chiefly by Bacillus tetani, the bacillus of malignant edema, the bacillus of symptomatic anthrax, Bacillus aërogenes capsulatus, and Bacillus botulinus.

Intermediate between these two classes is a large group of bacteria which thrive well both under aërobic and anaërobic conditions. Some of these, which have a preference for free oxygen but nevertheless possess the power of thriving under anaërobic conditions, are spoken of as "facultative anaërobes." In others the reverse of this is true; these are spoken of as "facultative aërobes." These varieties of bacteria are by far the most numerous and comprise most of our parasitic and saprophytic bacteria.

The relation of microorganisms to oxygen is extremely subtle, therefore, and not to be biologically dismissed by a rigid classification into aërobes, facultative anaërobes, and obligatory anaërobes. Both Engelmann, ${ }^{1}$ by a method of observing motile bacteria in the hanging drop as to their behavior in relation to the oxygen given off by a chlorophyllbearing alga, and Beijerinck, ${ }^{2}$ by a macroscopic method of observing similar bacteria as to their motion away from or toward an oxygenated area, were able to demonstrate delicately graded variations between species, favoring various degrees of oxygen pressure.

The discovery by Pasteur that certain bacteria develop only in the absence of free oxygen, produced a revolution in our conceptions of metabolic processes, since up to that time it was believed that life could be supported only when a free supply of $\mathrm{O}_{2}$ was obtainable. Pasteur's original explanation for this phenomenon was that anaërobic conditions of life were always associated with some form of carbohydiate fermenta-

1 Engelmann, Botanische Zeitung, 1881.

2 Beijerinck, Cent, I. Bakt., I, xiv, 1893. 
tion and that oxygen was obtained by these microorganisms by a splitting of carbohydrates. As a matter of fact, for a large number of microorganisms, this is actually true, and the presence of readily fermentable carbohydrates not only increases the growth energy of a large number of anaërobic bacteria, but in many cases permits otherwise purely aërobic bacteria to thrive under anaërobic conditions. ${ }^{1}$ On the other hand, the basis of anaërobic growth can not always be found in the fermentation of carbohydrates or in the simple process of reduction.

The favorable influence of certain actively reducing bodies, like sodium formate or sodium-indigo-sulphate, upon anaërobic cultivation is probably referable to their ability to remove free oxygen from the media and thus perfect the anaërobiosis. ${ }^{2}$ A number of strictly anaërobic bacteria, however, may develop in the entire absence of carbohydrates or reducing substances, obtaining their oxygen supply from other suitable sources, some of which may be the complex proteids. Thus the tetanus bacillus may ${ }^{3}$ thrive when the nutritive substances in the media are entirely proteid in nature. (See p. 28.)

As Hesse ${ }^{4}$ has shown, the respiratory processes of aërobic bacteria consist in the taking in of oxygen and the excretion of $\mathrm{CO}_{2}$. The $\mathrm{CO}_{2}$ excretion has been shown, in these cases, to be markedly less than is represented in the intake of oxygen.

Anaërobes, likewise, show an excretion of $\mathrm{CO}_{2}$ which must, in these cases, be a result of bacterial katabolism.

Certain bacteria, like the red sulphur bacteria, have the power of utilizing atmospheric oxygen in the same way in which this process takes place in the chlorophyll-bearing plants.

While a profuse supply of oxygen absolutely inhibits the growth of most anaërobes, a number of these may, nevertheless, develop when only small quantities of oxygen are present. Minute quantities of free oxygen in culture media have been shown by Beijerinck ${ }^{5}$ and others not to inhibit the growth of Bacillus tetani and Theobald Smith ${ }^{6}$ has recently demonstrated that when suitable nutritive material in the form of fresh liver tissue is added to bouillon, a number of anaërobic bacteria may be

1 Theobald Smith, Cent. f. Bakt., I, xviii, 1895.

${ }^{2}$ Kitasato and Weyl, Zeit. f. Hyg., viii, 1890.

${ }^{3}$ Chudiakow, Cent. f. Bakt., Ref., II, iv, 1898.

a IIesse, Zeit. f. Hyg., xv, 1897.

s Beijerinck, Cent. f. Bakt., II, vi, 1900.

* Th. Smith, Brown, and Walker, Jour. Med. Res., ix, 1906. 
induced to grow in indifferently anaërobic environment. Ferran, ${ }^{1}$ moreover, succeeded in gradually adapting the tetanus bacillus to an aërobic environment. In this case, however, the virulence of the bacillus was lost.

Nitrogen.-The nitrogen required by bacteria is taken, in most cases, from proteids. Most important in this respect, of course, are the diffusible proteids; but many of the non-diffusible albumins may be rendered assimilable by the proteolyzing enzymes possessed by many microorganisms. Among the pathogenic, more strictly parasitic bacteria, moreover, a delicate specialization may be observed as to the particular varieties of animal albumin which may be utilized by them. Thus the gonococcus grows more readily only upon uncoagulated human blood serum; the Pfeiffer bacillus requires hemoglobin, and the diphtheria bacillus outgrows other bacteria upon a medium composed for the greater part of coagulated beef serum. For bacteria that do not absolutely require native animal proteid for their development, the most common nitrogenous ingredient of culture media is pepton, added in solutions of varied concentration.

A large number of bacteria (pathogenic and saprophytic), on the other hand, may thrive on media containing absolutely no proteid, in which case, of course, a synthetic proteid production by the microorganisms must be assumed. A medium which has been extensively used to demonstrate this phenomenon is that devised by Uschinski, ${ }^{2}$ containing ammonium lactate, glycerin, asparagin (the amide of amidosuccinic acid), and inorganic salts.

Creatin, creatinin, urea and urates, and even ammonia compounds and nitrates, may serve as adequate sources of nitrogen for many of the less parasitic bacteria. A limited number of species, moreover, the bacilli in the root tubercles of the leguminosæ and the nitrogen-fixing organisms of the soil, possess the power of obtaining their supply of nitrogen directly from the free $\mathrm{N}_{2}$ of the atmosphere.

Although the sources of carbonaceous and of nitrogenous food supply have been separately treated in the preceding paragraphs, it should not be forgotten that, in many instances, both elements are taken up within the same compound, and that separate supplies are a necessity in isolated cases only.

Hydrogen.-Hydrogen is obtained by bacteria largely in combina-

Ferran, Cent. f. Bakt., I, xxiv, 1898.

2 Uschinski, Cent. f. Bakt., I, xiv, 1893. 
tion as water and together with the carbon and nitrogen containing substances.

Salts.-The phosphatic constituents of the bacterial body are taken in, chiefly, as phosphates of magnesium, calcium, sodium, or potassium. The phosphates seem to be necessary constituents of culture media, while chlorides, on the other hand, according to Proskauer ${ }^{1}$ and Beck are not absolutely essential. Sodium salts, as a rule, seem to be more advantageous for purposes of bacterial cultivation than potassium salts.

The uncombined sulphur, which is a constituent of the bacterial body in many cases, is usually supplied by soluble sulphates. In the case of the thiobacteria of Winogradsky, however, the presence of free $\mathrm{H}_{2} \mathrm{~S}$ is necessary for its formation. ${ }^{2}$

The iron contained in some of the higher bacteria is taken in in the form of ferrous compounds, and is oxidized in the bacterial body into ferric compounds. •

The relative quantities of the various nutritive substances in culture media are of importance only in so far as too high concentrations may have a distinctly inhibitory influence. In this respect, however, separate species may show widely divergent tastes.

The development of bacteria in any given medium, it may be noted, is far oftener arrested by the accumulation of waste products than by an exhaustion of nutrient materials.

\section{PARASITISIM AND SAPROPHYTISM}

When we speak of bacteria as parasites or as saprophytes, we classify them, primarily, according to their relationship to the bodies of higher animals. "Parasites" are those bacteria which are capable of living and multiplying within the human or animal body, whereas the term "saprophytes" refers to the multitude of microorganisms which are unable to hold their own under the environmental conditions found in the tissues of higher animals, but are found, almost ubiquitously, in air, soil, manure, and water. The separation is by no means a sharp one and carries with it other implications, which the use of these terms always conveys. While parasites are usually very fastidious as to nutritional and temperature requirements, most saprophytes are easily cultivated. upon the simplest media. Thus certain parasitic bacteria, such as the

1 Proskauer and Beck, Zeit. f. Hyg., xviii, 1895.

2 Voges, Cent. f. Bakt., I, xviii, 1893. 
bacillus of influenza, the gonococcus, and others, are dependent upon specific forms of animal proteids for their food supply, while typical saprophytes, like Bacillus proteus, may thrive and multiply upon even the simplest organic proteid derivatives.

Between the strict parasites and the saprophytes, however, there is a large class of bacteria, to which the majority of our pathogenic varieties belong, the members of which are capable of developing luxuriantly under both conditions. These bacteria are often spoken of as facultative parasites.

More recently the question of parasitism and saprophytism has become closely interwoven with our conceptions of virulence. Bail (see section on Aggressins) has classified parasites into strict parasites and half parasites. By the first term he designates bacteria like Bacillus anthracis, which actually invade all the tissues of their host, while, by the term "half parasites," he refers to microorganisms like the spirillum of cholera which gain a foothold upon some part of the body of the host, but do not actually penetrate into the general circulation.

All pathogenic bacteria, therefore, must be grouped as parasites, strict or facultative, while the saprophytes, as a class, perform the far more thankful task of breaking up organic matter outside of the animal body, by putrefaction and fermentation. Absolute separation between the two classes, however, can not be maintained, since many ordinarily saprophytic bacteria may display parasitic qualities if administered in large numbers to animals or man in whom resistance to bacterial invasion is at a low ebb.

\section{ANTAGONISM AND SYMBIOSIS OF BACTERIA}

The ubiquity of bacteria in nature naturally carries with it the simultaneous presence of many species in all places where special conditions have provided a favorable environment for growth. Thus bacteriological investigation of water, milk, manure, soil, or organic infusions, always reveals the presence of a large number of different varieties within the same substance. If the food supply in such a natural culture is at all limited in quantity, or the removal of waste products is prohibited, it will usually be found that gradually the numbers of varieties will diminish and a few species, or even only one, will prevail. In the case of milk, for instance, after standing for three or four days at a suitable temperature, two or three varieties will be found to have taken the place of the twenty or thirty, which may have been present originally. 
This behavior is due to the influences which various microorganisms exert upon each other and is known as antagonism. Such antagonism probably depends upon the fact that the metabolic products of the predominant species (the one or ones for whom the special cultural conditions are most favorable) inhibit the growth of the less vigorous varieties. Many examples, experimentally supported, of such antagonism, can be given. Thus, the gonococcus is distinctly inhibited by the soluble products of Bacillus pyocyaneus, ${ }^{1}$ while in the presence of pyogenic cocci it develops luxuriantly, and the bacillus of plague is completely inhibited when streptococci are present in the culture. ${ }^{2}$

Mutual inhibition may also be due to the monopolizing of the nutrition in the medium by the predominating species or to the change in reaction produced by its growth. This last consideration is probably the secret of the frequently noticed inhibitory effect exerted by acid-producers upon bacteria of putrefaction, and has received practical therapeutic application in Metchnikoff's lactic-acid bacillus therapy, which see.

When the simultaneous presence of two bacterial species within the same environment favors the development of both species, the condition is spoken of as symbiosis. Such dependence is not so frequent as antagonism, but it does occur. Examples of such a condition have been observed in cultures containing diphtheria bacilli and streptococci ${ }^{3}$ and have been frequently observed in cultures containing both aërobic and anaërobic bacteria, where the former favor the development of the latter by monopolizing the supply of free oxygen. Symbiosis may also take place in cultures in which complex food products are split up by one species, furnishing substances for ingestion by species with a lesser digestive ability.

\section{RELATIONS OF BACTERIA TO PHYSICAL ENVIRONMENT}

Relation to Temperature.-Like all other living beings, bacteria develop and multiply by virtue of a series of chemical and physical processes, by means of which growth energy is obtained by destruction or catabolism, and the lost tissues resupplied by absorption of nutritive materials. It is natural, therefore, that the conditions of external

1 Schafer, Fortschr. d. Med., 5, 1896.

2 Bitter, Rep. Egypt Plague Com., Cairo, 1897.

${ }^{3}$ Hilbert, Zeit. f. Hyg., xxix, 1895. 
temperature should intimately affect the metabolic processes. The range of temperature at which bacteria may grow is subject to wide variations among different species. Each species, on the other hand, may thrive within a more or less elastic range of temperature, each one having an optimum, a minimum, and a definite maximum temperature. When the optimum temperature is present in the environment, the functions of absorption and excretion keep pace with each other, and the chemical balance is well preserved. When the temperature is lower than the optimum, all metabolic processes take place more slowly, and the bacterium gradually enters into a resting or latent stage, at which actual growtin may be exceedingly slow or entirely inhibited. When the temperature is higher than the optimum, the destructive processes are carried on more rapidly than the substitution of waste products by absorption, and a gradual weakening of vital energy, or even a gradual death of the bacterium, may take place. When certain bacteria form spores, they become very much more resistant against both high and low temperatures, probably because a true resting stage has been reached, during which metabolism has been reduced to a minimum, there being practically no nutritive material taken in and correspondingly little destruction taking place within the body of the microorganism.

The optimum temperature for various bacteria depends upon the habitual environment, in which the particular species is accustomed to exist. Thus, for the large majority of bacteria pathogenic for human beings, the optimum temperature is at or about $37.5^{\circ} \mathrm{C}$. There are a large number of bacteria common in water, however, which grow hardly at all at the body temperature, but thrive most luxuriantly at temperatures of about $20^{\circ} \mathrm{C}$. F. Forster, ${ }^{1}$ moreover, described certain phosphorescent bacteria, isolated from sea-water, which grow readily at $0^{\circ} \mathrm{C}$, , or a little above. On the other hand, Miquel ${ }^{2}$ has described nonmotile bacilli, which he isolated from the water of the Seine, which grew rapidly at temperatures ranging about $70^{\circ} \mathrm{C}$, and the so-called "mucedinées thermophiles," described by Tsiklinski, ${ }^{3}$ develop most readily at temperatures very little above this. It is thus plain that the temperatures favored by various bacteria depend to a large extent upon an adaptation of these bacteria through many generations to specific environmental conditions. A good illustration of this is furnished by the bacillus of avian tuberculosis, a microorganism differing essentialiy

1 F. Forster, Cent. f. Bakt., ii, 1887.

2 Miquel, Bull. de la Stat. Munic. de Paris, 1879.

3 Tsiklinski, Ann. Past., 1889. 
from the bacillus of human tuberculosis in that its optimum growth temperature lies at $41^{\circ}-42^{\circ} \mathrm{C}$., a temperature which exceeds the optimum temperature for the human type by as much as the normal temperature of birds exceeds that of man. The same principle is illustrated by the facts that the bacteria which have a very low optimum temperature are usually those isolated from water, and the so-called thermophile or high-temperature bacteria are obtained from hot springs and from the upper layers of the soil, where, according to Globig, ${ }^{1}$ occasionally temperatures ranging from about $55^{\circ} \mathrm{C}$. occur.

As stated before, one and the same species may develop within a wide temperature range, and it may be possible, by persistent cultivation at special temperatures, to adapt certain bacteria to grow luxuriantly at temperatures removed by several degrees from their normal sptimum. In such cases it may often occur that special characteristics of the given species may be lost. An example of this is the loss of virulence and of spore-formation which takes place when anthrax bacilli are cultivated at $42^{\circ} \mathrm{C}$, , or the loss of the power to produce pigment when bacillus prodigiosus is grown at temperatures above $30^{\circ} \mathrm{C}$.

The vegetative forms of most of the pathogenic bacteria may grow at temperatures ranging between $20^{\circ} \mathrm{C}$. and $40^{\circ} \mathrm{C}$. This can, however, by no means be regarded as applicable to all of the pathogenic bacteria, as some of these, like the gonococcus, the pneumococcus, the tubercle bacillus, and others, are delicately susceptible to temperature changes and have the power of growing only within limits varying but a few degrees from their optimum. Others, on the other hand, like bacilli of the colon group, Bacillus anthracis, Spirillum choleræ asiaticæ, etc., may develop at temperatures as low as $10^{\circ} \mathrm{C}$. and as high as $40^{\circ} \mathrm{C}$., or over. The range of temperature at which saprophytic bacteria may develop is usually a far wider one. When temperatures exceed in any considerable degree the maximum growth temperature, the vegetative forms of bacteria perish. Thus, ten minutes' exposure to a temperature of between $55^{\circ}$ and $60^{\circ} \mathrm{C}$. causes death of the vegetative forms of most microorganisms. Death in such cases is due probably to a coagulation of the protoplasm, and since all such processes of coagulation take place best in the presence of water, the thermal death point of most bacteria is lower when heat is applied in the form of boiling water or steam, than when employed as dry heat. (See section on Sterilization.)

When spores are present in cultures, the resistance to heat is enor- 
mously increased. Exactly what the explanation of this is can not be at present stated. It may be that the high concentration in which the protoplasmic mass is found in the spores renders it less easily coagulable than is the protoplasm of the vegetative body. A more detailed discussionof these relations will be found in the section on Heat sterilization.

The thermal death points of a large number of bacteria have been very carefully studied by Sternberg, ${ }^{1}$ by a special technique described elsewhere.

The thermal death points ascertained by him in this way, with an exposure of ten minutes in a fluid medium, for some of the more common non-sporogenic bacteria are as follows:

Spirillum choleræ asiaticæ......................... $52^{\circ} \mathrm{C}$.

Diplococcus pneumoniæ ....................... $52^{\circ} \mathrm{C}$.

Streptococcus pyogenes $\ldots \ldots \ldots \ldots \ldots \ldots \ldots \ldots \ldots \ldots \ldots \ldots \ldots \ldots 4^{\circ} \mathrm{C}$.

Bacillus typhosus ........................... $56^{\circ} \mathrm{C}$.

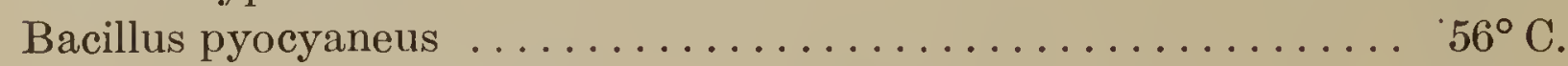

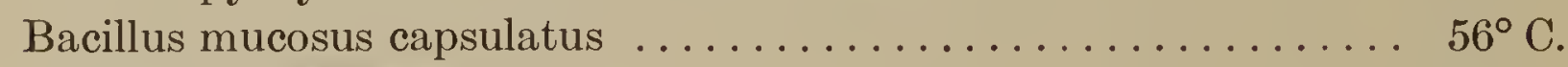

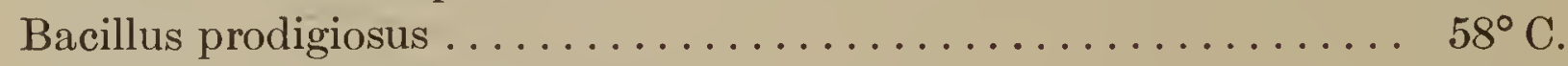

Staphylococcus pyogenes aureus .................. $58^{\circ} \mathrm{C}$.

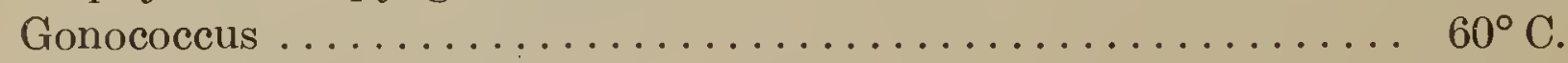

Staphylococcus pyogenes albus $\ldots \ldots \ldots \ldots \ldots \ldots \ldots \ldots \ldots, 62^{\circ} \mathrm{C}$.

The bacillus tuberculosis, though not a spore bearer, seems to be slightly more resistant to heat than other purely vegetative microorganisms. Thus, according to the researches of Smith ${ }^{2}$ and others, ten and twenty minutes' exposure to a temperature of $70^{\circ} \mathrm{C}$. is necessary to destroy tubercle bacilli in a fluid medium. For the effectual destruction of spores by moist heat, a temperature of $100^{\circ} \mathrm{C}$., or boiling point, is usually necessary.

Low temperatures are much less destructive than the high ones, and are even in a number of cases useful in keeping bacteria alive for long periods, inasmuch as metabolic processes are inhibited and life is maintained without actual development in a sort of resting state. Actual destruction by low temperatures rarely takes place. The exposure of diphtheria, typhoid, and other bacilli to temperatures as low as $200^{\circ} \mathrm{C}$. below zero has been carried out without destruction of the microorganisms, a fact which is of great importance in considering the possibility of infection by the vehicle of ice. Meningococci and gonococci, on the other hand, die out rapidly when exposed to $0^{\circ} \mathrm{C}$.

${ }^{1}$ Sternberg, "Textbook of Bacteriology," New York, 1901.

2 Th. Smith, Jour. of Experimental Med., No. 3, 1899. 
Relation to Pressure.-High pressure does not exert any noticeable effects upon bacteria. In the experiments of Certes, ${ }^{1}$ a pressure of two atmospheres seemed to have no influence upon the growth and motility of anthrax bacilli suspended in blood.

Relation to Moisture.-For the growth and development of all bacteria, the presence of water in the culture medium is necessary. It is self-evident that nutritive materials can not be absorbed by an osmotic process unless in a state of solution. While complete dryness does not permit growth, its destructive action upon various bacteria is subject to great differences. The effect of complete drying upon bacteria will be found more fully discussed in the section upon the destruction of bacteria by physical agents. (See page 62.)

In the same section may be found a discussion of the effects of light, electricity, $x$-ray, and radium rays upon bacteria.

\section{THE CLASSIFICATION OF BACTERIA}

Too simple in structure, too varied in biological properties to be definitely identified with either the vegetable or animal kingdom, the bacteria are placed at the bottom of the scale of all living beings. Closely linked on the one hand to the plant kingdom by the yeasts and the molds, and on the other to the animal kingdom by the protozoa, they themselves combine, within one and the same division, attributes so widely divergent as to structure, metabolism, and biological activity that their grouping is more a matter of working convenience than of actual scientific classification. Thus, for instance, all stages of metabolic activity fill in the gap between the synthetizing sulphur and nitrifying bacteria and the purely katabolic activities of some of the aërobic and anaërobic microorganisms which cause putrefaction. Growth takes place within the limits of a wide temperature range, and the specific modes of life and cultural conditions are subject to the widest variations, from those of an indisputably useful saprophytism to those of the most exquisite parasitism. Although, therefore, strictly speaking, the bacteria can be classified as a whole neither in the animal nor in the vegetable realms, being nonchlorophyll-bearing, they are for convenience classified with the fungi or colorless plants.

The relationship of the bacteria to other simple plants may be graphicaily represented by the following scheme:

${ }^{1}$ Certes, Compt. rend, de l'acad, d, sc., 99, Paris, 1884. 


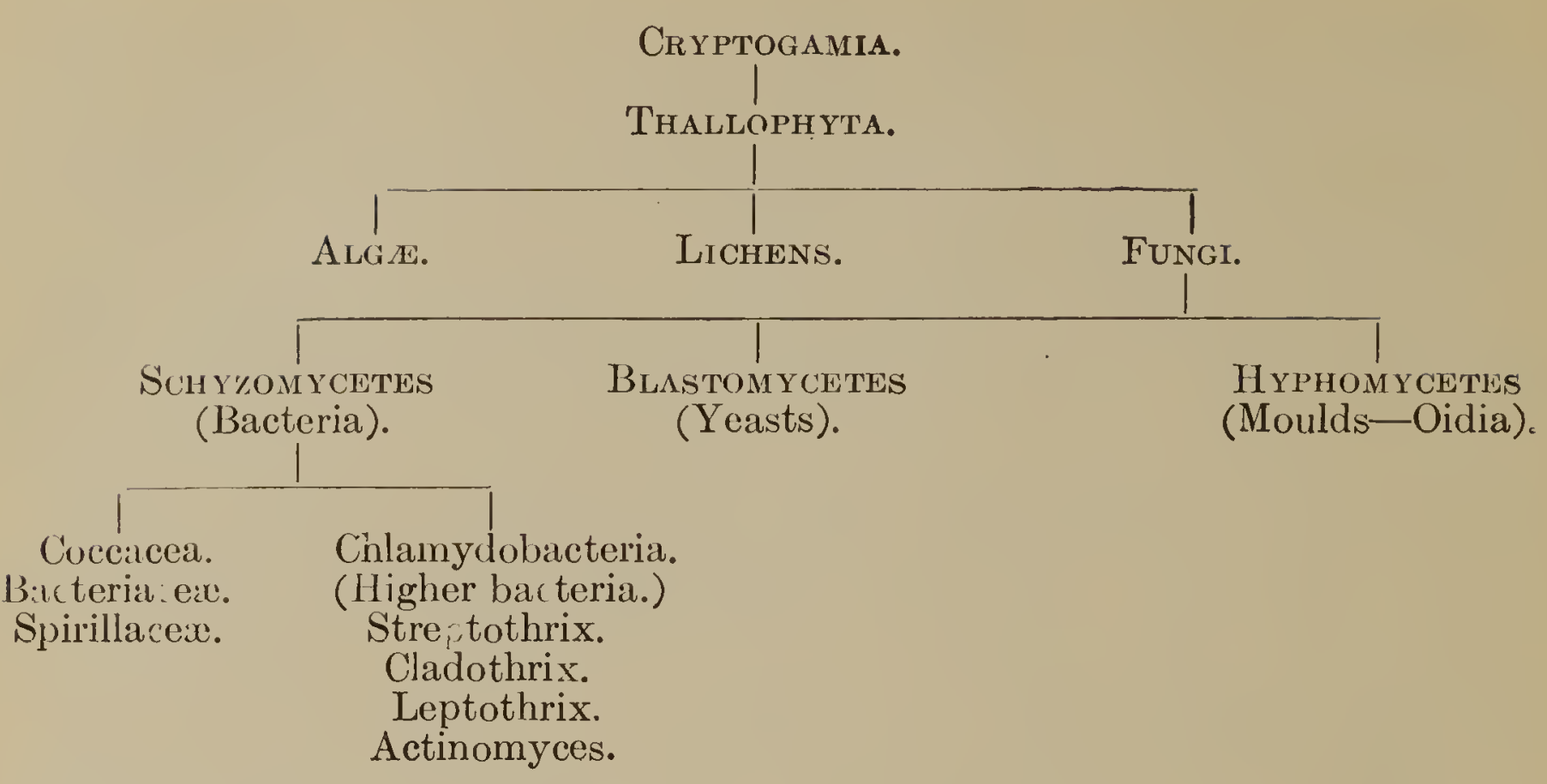

The special classification of the bacteria has offered still greater difficulties, for the lower we proceed in the phylogenetic scale of living beings, the less specialized the morphological and biological characteristics of any group becone, and the more difficult it is to establish a classificaiion which can in any way be regarded as final. It is, therefore, quite impossible to classify the bacterial varieties or species on any basis which can hope to satisfy all the demands of scientific accuracy and it is necessary to resort to the expedient of utilizing some one characteristic which remains constant for the individual genus and to base upon this an attempt at grouping. When bacteria were first discovered, and for many years following, numerous observers contended that the form of the microorganism observed was not a constant one for each genus, but that cocci could be converted into bacilli or spirilla according to environmental conditions. It was Cohn ${ }^{1}$ who, in 1872 , first recognized the constancy of the morphology of bacteria and established, upon morphological basis, a classification which, with minor changes, has been retained until the present day. Such classifications can not, however, be regarded as anything more than a convenient make-shift pending the day when the finer structure and true biological relations of the various bacteria shall have been more accurately inves: tigated. The scheme most commonly accepted at present is the one given below, proposed by Migula ${ }^{2}$ :

1 Cohn, "Beiträge zur Biol. đ. Pllanzen," Heft 1 u. 2, 1872.

2 Migula, "System d. Bakt.," Jena, 1897. 
Bacteria (Schizomycetes).-Fission fungi (chlorophyll free) with cell division in one, two, or three directions of space. Many varieties possess the power of forming endospores. Whenever motility is present, it is carried on by means of flagella, or, more rarely, by undulating membranes.

Family I. Coccacez.-Cells in free state perfectly spherical. Division in one, two, or three directions of space, by which each spherical cell divides into two, four, or eight segments, cach of which again develops into a perfect sphere. Endospore formation rare.

Genus I. Streptococcus.-Cells divide in one direction of space only, for which reason, if they remain connected after fission, beadlike chains may be formed. No organs of locomotion.

Genus II. Micrococcus (Staphylococcus).-Cells divide in two directions of space, whereby, if the cells remain connected after fission, tetrad and grape-like cluster's may be formed. No organs of locomotion.

Genus III. Sarcina.-Cells divide in three directions of space, whereby, if they remain connected after fission, bale-like packets are formed. No organs of locomotion.

Genus IV. Planococcus.-Cells divide in two directions of space, as in micrococcus, but possess flagella.

Genus V. Planosarcina.-Cells divide in three directions of space as in sarcina, but possess flagella.

Family II. Bacteriace ж.-Cells long or short, cylindrical, straight, never spiral. Division in one direction of space only, after preliminary elongation of the rods.

Genus I. Bacterium.-Cells without flagella, often with endospores.

Genus II. Bacillus.-Cells with peritrichal flagella, often with endospores.

Genus III. Pseudomonas.-Cells with polar flagella. Endospores occur in a few species, but are rare.

Family III. Spirillaceж.-Cells spirally curved or representing a part of a spiral curve. Division in one direction of space only, after preceding elongation of cell.

Genus I. Spirosoma.-Cells without organs of locomotion. Rigid. Genus II. Microspira.-Cells rigid, with one or, more rarely, two or three polar undulated flagella. 
Genus III. Spirillum.-Cells rigid, with polar tufts of five to twenty flagella usually curved in semicircular or flatly undulating curves. Genus IV. Spirochcete.-Cells sinously flexible. Organs of locomotion unknown, perhaps a marginal undulating membrane.

Familly IV. Chlamydobacteriace .-Forms of very varying stages of evolution, but all distinguished by a rigid sheath (Hülle) or covering, which surrounds the cells. The cells are united in branched or unbranched threads.

Genus I. Streptothrix.-Cells united in simple, unbranched threads. Division in one direction of space only. Reproduction by nonmotile conidia.

Genus II. Cladothrix.-Cells united or pseudodichotomously branching threads. Division in one direction of space only. Vegetative multiplication by separation of entire branches. Reproduction by swarming forms with polar flagella.

Genus III. Crenothrix.-Cells united in unbranched threads, at first with division in one direction of space only. Later the cells divide in all three directions of space. The daughter cells become rounded and develop into reproductive cells.

Genus IV. Phragmidiothrix.-Cells at first united in unbranched threads, dividing in three directions of space, thus forming a rope of cells. Later some of the cells may penetrate through the delicate sheath, and thus give rise to branches.

Genus V. Thiothrix.-Unbranched, non-motile threads, inclosed in fine sheaths. Division of cells in one direction only. Cells contain sulphur granules.

Family V. Beggiatoace Division in one direction of space only. Motility by undulating membrane as in Oscillaria.

Genus Beggiatoa.-Cells with sulphur granules.

It will be seen in reviewing the classification just given that the subdivisions are based upon questions of form, motility, and situation of flagella. While these characteristics, so far as we know, are constant, there are, nevertheless, many instances in which types entirely similar in these respects must be differentiated. This can be done only by careful study of staining reactions, finer structure, cultural characteristics, and biological activities. 
As a matter of fact, while the botanical classification of the bacteria offers almost insurmountable difficulties, actual identification is not so complicated a task as this would indicate. Identification, once roughly made on a morphological basis, is further carried on by the aid of cultural characteristics, such as the conditions favorable and unfavorable for growth, appearance of growth on different media, and pigment formation, by biochemical reactions and by pathogenic properties. The bacteria occupy so important a place in agriculture, in medicine, and in hygiene, that it rarely becomes necessary for a worker in any paricular field to survey the entire group. The habitat of a large number of pecies is so well known that this consideration alone often gives a clew nvaluable for actual identification. 


\section{CHAPTER IV}

\section{THE BIOLOGICAL ACTIVITIES OF BACTERIA}

While the bacteria pathogenic to man and animals largely usurp the attention of those interested in disease processes, this group of microorganisms is after all but a small specialized off-shoot of the realm of bacteria, and, broadly speaking, actually of minor importance. Surveying the existing scheme of nature, as a whole, it is not an extravagant statement to say that without the bacterial processes which are constantly active in the reduction of complex organic substances to their simple compounds, the chemical interchange between the animal and vegetable kingdoms would fail, and all life on earth would of necessity cease. To understand the full significance of this, it is necessary to consider for a moment the method of the interchange of matter between the animal and vegetable kingdoms.

All animals require for their sustenance organic compounds. They are unable to build up the complex protoplasmic substances which form their body cells from chemical elements or from the simple inorganic salts. They are dependent for the manufacture of their food-stuffs, therefore, directly or indirectly, upon the synthetic or anabolic activities of the green plants.

These plants, by virtue of the chlorophyll contained within the cells of their leaves and stems, and under the influence of sunlight, possess the power of utilizing the carbon of the carbonic acid gas of the atmosphere, and of combining it with water and the nitrogenous salts absorbed by their roots, building up from these simple radicles the highly complex substances required for animal sustenance.

These products of the synthetic activity of the green plants, then, are ingested by members of the animal kingdom, either directly, in the form of vegetable food, or indirectly, as animal matter. They are utilized in the complex laboratory of the animal body and are again broken down into simpler compounds, which leave the body as excreta and secreta.

The excreta and secreta of animals, however, are, in a small part only, made up of substances simple enough to be directly utilized by plants. The dead bodies, moreover, of both animals and plants would 
be of little further value as stores of matter unless new factors intervened to reduce them to that simple form in which they may again enter into the synthetic laboratory of the green plant. Agents for further cleavage of these compounds are required, and these are supplied by the varied activities of the bacteria.

On the other hand, bacteria are also important in the process of synthesis. The main supply of nitrogen available for plant life is found in the elementary state in the atmosphere-a condition in which it can not be utilized as a raw product by the plant. This gap again is bridged by the bacteria found in the root bulbs of the leguminous plants -bacteria which possess the power of assimilating or aiding in the assimilation of atmospheric nitrogen and its preparation for further use by the plant itself. Another bacterial activity which may be classified as an anabolic process is the oxidation of the ammonia, released by decomposition. into nitrites and nitrates. This is carried on by certain bacteria of the soil. These are to be treated of in greater detail in another section.

There is a constant circulation, therefore, of nitrogen and carbon compounds, between the plant and the animal kingdoms, by virtue of an anabolic or constructive process in the one, and a katabolic or destructive process in the other, rendering them mutually interdependent and indispensable. The circuit, however, is not by any means a closed one; there are important gaps, both in the process of cleavage and in that of synthesis, which, if left unbridged by the bacteria, would effectually arrest all life-activity of plants and eventually of animals.

Far from being scourges, therefore, these minute microorganisms are paramount factors in the great cycle of living matter, supplying necessary links in the circulation of both nitrogenous and carbon compounds.

\section{KATABOLIC ACTIVITIES OF BACTERIA}

The katabolic activities of bacteria, then, consist in the fermentation of carbohydrates and in the cleavage of proteids and fats.

Fermentation is carried out to a large extent by the yeasts, but also to no inconsiderable degree by bacteria. Proteid decomposition and the cleavage of fats are carried out almost exclusively by bacteria.

For our knowledge of the fundamental laws underlying these phenomena of fermentation and proteid decomposition, we are indebted to the genius of Pasteur, ${ }^{1}$ who was the first to prove experimentally the

' Pasteur, "Étude sur la bi re," Paris, 1876. 
exclusive and specific parts played by various microorganisms in these processes. While the observations and deductions made by Pasteur have not been greatly modified, a large store of information has been gained since his time, which has thrown additional light upon the chemical details and the more exact manner of action of the factors involved.

The actual work of cleavage in both fermentation and proteid cleavage is carried out by substances known as enzymes or ferments, the nature of which we must further discuss before their manner of action can be fully comprehended.

Bacterial Enzymes or Ferments.-A ferment or enzyme is a substance produced by a living cell, which brings about a chemical reaction without entering into the reaction itself. The enzyme itself is not bound to any of the end products and is not appreciably diminished in quantity after the reaction is over, although its activity may be finally inhibited by one or another of the new products. The action of bacterial enzymes is thus seen to be closely similar to that of the chemical agents technically spoken of as "katalyzers," represented chiefly by dilute acids. Thus, if an aqueous solution of saccharose is brought into contact with a dilute solution of sulphuric acid, the disaccharid is hydrolyzed and is decomposed into levulose and dextrose.

Thus:

$$
\begin{gathered}
\underset{12}{\mathrm{C}_{12} \mathrm{H}_{22} \mathrm{O}_{11}}+\mathrm{H}_{2} \mathrm{O}=\underset{\text { contact with }}{\text { dilute } \mathrm{H}_{2} \mathrm{~S} \mathrm{O}_{4}} \\
\text { Dextrose }
\end{gathered}
$$

During this process, which is known as "inversion," the concentration of the sulphuric acid remains entirely unchanged. While theoretically the changes brought about by enzymes and katalyzers are usually such as would occur spontaneously, the time for the spontaneous occurrence would be, at ordinary temperatures, infinitely long. The definition for enzymes and katalyzers is given by Ostwald, therefore, as "substances which hasten a chemical reaction without themselves taking part in it." Exactly the same result which is obtained by the use of dilute sulphuric acid is caused by the ferment "invertase" produced, for instance, by B. megatherium. Were a solution of saccharose subjected to heat, without katalyzer or ferment, a similar change would occur, but by the mediation of these substances the inversion is produced without other chemical or physical reinforcement.

This analogy between enzymes and katalyzing agents is very striking. Thus, as stated, both katalyzers and enzymes bring about 
changes without themselves being used up in the process, both act without the aid of heat, and the reactions brought about by both have occasionally been shown to be reversible. While this last phenomenon has been variously shown for katalyzers, the process of reversibility has been demonstrated for bacterial enzyme action only in isolated cases. Thus, it has been found that by the action of the yeast enzyme maltase upon concentrated dextrose solutions, a re-formation of maltose may occur. In both cases, moreover, the quantity of enzyme or katalyzer is infinitely small in proportion to the amount of material converted by their action.

There is a close similarity, furthermore, between the bacterial enzymes and the ferments produced by specialized cells of the higher animals and plants. For instance, the action of the ptyalin of the saliva or of the diastase obtained from plants is entirely analogous to the starchsplitting action of the amylase produced by many bacteria.

The action of all enzymes depends most intimately upon environmental conditions. For all of them the presence of moisture is essential. All of them depend for the development of their activity upon the existence of a specifically suitable reaction. Strong acids or alkalies always inhibit, of ten destroy them. Temperatures of over $70^{\circ} \mathrm{C}$. permanently destroy most enzymes, whereas freezing, while temporarily inhibiting their action, causes no permanent injury, so that upon thawing, their activity may be found almost unimpaired. Direct sunlight may injure, but rarely destroys, ferments. Against the weaker disinfectants in common use, enzymes often show a higher resistance than do the bacteria which give rise to them.

The optimum conditions for enzyme action, then, consist in the presence of moisture, the existence of a favorable reaction, weakly acid or alkaline, as the case may be, and a temperature ranging from $35^{\circ}-$ $45^{\circ}$ C. ${ }^{1}$

Proteolytic Enzymes.-In nature, the decomposition of dead animal and vegetable matter occurs only when the conditions are favorable for bacterial development. Thus, as is well known, freezing, sterilizing by heat, or the addition of disinfectants will prevent the rotting of organic material.

In the laboratory, the presence of proteolytic enzymes is determined chiefly by the power of bacteria to liquefy gelatin, fibrin, or coagulated blood serum. These ferments are not always secretions from the bac-

1 Oppenheimer, “Die Fermente," etc. Leipzig, 1900. 
terial cell, but in some cases may be closely bound to the cell-body and separable only by extraction after death. In such cases they are spoken of as endoenzymes. Whenever they are true secretory products, however, they can be obtained separate from the microorganisms which form them by filtration through a Berkefeld candle. From such filtrates they may, in some cases, be obtained in the dry state by precipitation with alcohol. When obtairied in this way the precipitated enzyme is usually much more thermostable than when in solution, for while soluble enzymes in filtrates are usually destroyed by $70^{\circ} \mathrm{C}$., and even less, the dried powder may occasionally withstand $140^{\circ} \mathrm{C}$. for as long as ten minutes. ${ }^{1}$

Apart from the general conditions of temperature and moisture, the development of these enzymes seems to depend directly upon the presence of proteids in the culture media. The number of bacterial species which produce proteriytic enzymes is legion. Among those more commonly met with are staphylococci, B. subtilis, B. proteus, B. fæcalis liquefaciens, Spirillum choleræ asiaticæ, B. anthracis, B. tetani, B. pyocyaneus, and a large number of others. The inability of any given microorganism to liquefy gelatin or fibrin by no means entirely excludes the formation by it of proteolytic enzymes, since these ferments may often be active for one particular class of proteid only.

In order to study the qualitative and quantitative powers of any given bacterial proteolyzing enzyme or protease, it is, of course, necessary to study these processes in pure culture in the test tube with media of known composition. In the refuse heap, in sewage, or in rotting excreta, the process is an extremely complicated one, for besides the bacteria which attack the proteid molecule itself, there are many other species supplementing these and each other, one species attacking the more or less complex end-products left by the action of the others.

Exactly what the chemical reactions are which take place in these cleavages is not entirely clear. It is believed, however, that most of the cleavages are of an hydrolytic nature.

In general, the action of the proteid-splitting ferments is comparable to that of the pancreatic ferment trypsin, and they are most often active in an alkaline environment. They differ, among themselves, chiefly in the form of proteid which they are competent to attack, and in the extent to which they are able to reduce it toward its simple radicles.

A distinction is occasionally made between the terms putrefaction and decay, the former being used to refer to the decomposition taking 
place under anaërobic conditions, that is, in the absence of oxygen, a process usually resulting in incomplete cleavage of the protcid medium; the latter being used to signify decompositions under aërobic conditions and leading to a more complete splitting, the end-products often being represented by such simple compounds as carbon dioxide, water, and ammonia. In general, the products of putrefaction are largely represented by the amino-acids, leucin and tyrosin, fatty acids, mercaptan, indol, and skatol. The gases generated in such decomposition are largely made up of $\mathrm{CO}_{2}$, hydrogen, $\mathrm{NH}_{4}$ and $\mathrm{H}_{2} \mathrm{~S}$. The coincident presence, furthermore, of the carbohydrate-splitting bacteria and of denitrifying microorganisms renders the actual process of putrefaction a chaos of many activities in which the end-products and by-products are qualitatively determinable only with much inexactitude, and which completely defies any attempt at quantitative analysis.

Ptomains.-There are certain products, however, resulting from the proteolytic action of bacterial enzymes upon proteids which claim more than a purely chemical interest because of their toxic action upon the animal organism, and their consequent importance as incitants of disease. Pre-eminent among these are the ptomains. The word ptomain (from $\pi \tau \tilde{\omega} \mu \alpha$, a dead body) is used to designate organic chemical compounds produced by the action of bacteria, which are basic in character; that is, are able to combine with an acid to form a salt. They should be definitely distinguished from the so-called leucomains, a term employed to designate similar substances formed in the course of proteid metabolism within the animal body, and not bacterial in origin. Both in their basic characters and in their nitrogenous constitution, the ptomains resemble the vegetable alkaloids, and for this reason are sometimes spoken of as "animal alkaloids."

The ptomains must be sharply distinguished from the bacterial toxins, which are products of the bacterial growth ilrespective of the medium in which they are grown, except in so far as this hinders or abets the development of the microorganisms. Thus, toxins may be developed by diphtheria organisms, for instance, in proteid-free media. As will be scen in a subsequent section, the true toxins are comparable to the enzymes themselves, rather than to their cleavage products, represented in this instance by the ptomains.

A great number of ptomains are chernically known. Many of these possess little or no toxicity. Others, however, like putrescin (tetramethylenediamin, $\left.\mathrm{C}_{4} \mathrm{H}_{12} \mathrm{~N}_{2}\right)$ and cadaverin $\left(\mathrm{C}_{5} \mathrm{H}_{14} \mathrm{~N}_{2}\right)$ are very highly poisonous. It is to one or another of these ptomains that most 
cases of so-called meat poisoning (kreatoxismus), cheese poisoning (tyrotoxismus), or vegetable poisoning (sitotoxismus) are due.

In each individual case the variety of ptomain resulting from a bacterial decomposition varies with the individual species of microorganism taking part in the process and with the nature of the proteid upon which its development takes place.

In breaking down animal excreta, the task of the bacteria is rather a simpler one than when dealing with the cadavers themselves, for here a part of the cleavage has already been carried out either by the destructive processes accompanying metabolism, or by partial decomposition by bacteria begun within the digestive tract. This material outside of the body is further reduced by bacterial enzymes into still simpler substances, the nitrogen usually being liberated in the form of ammonia. One example of such an ammoniacal fermentation may be found in the case of the urea fermentation by Micrococcus ureæ, in which the cleavage of the urea takes place by hydrolysis according to the following formula:

$$
\left(\mathrm{NH}_{2}\right)_{2} \mathrm{CO}+2 \mathrm{H}_{2} \mathrm{O}=\mathrm{CO}_{2}+2 \mathrm{NH}_{3}+\mathrm{H}_{2} \mathrm{O}
$$

Similar ammoniacal fermentations are carried out, though perhaps according to less simple formulæ, by a large number of microorganisms. Perhaps the most common species which possesses the power is the group represented by B. proteus vulgaris (Hauser).

From what has been said it follows naturally that, so far, the decomposition of the proteid molecule from its complex structure to ammonia or simple ammonia compounds is an indispensably important function, not only for agriculture, but for the maintenance of all life processes. It is clear, on the other hand, that a further decomposition of ammonia compounds into forms too simple to be utilized by the green plants would be a decidedly harmful activity. And yet this is brought about by the so-called denitrifying bacteria which will be considered in a subsequent section.

Lab Enzymes.-There are a number of ferments produced by bacteria which, although affecting proteids, can not properly be classified with the proteolytic enzymes. These are the so-called coagulases or lab enzymes, which have the power of producing coagulation in liquid proteids. Just what the chemical process underlying this coagulation is, is not known. If Hammarsten's ${ }^{1}$ conclusions as to the hydrolytic

'Hammarsten, "Textbook of Physiol, Chemistry," Translation by Mandel. 
nature of the changes produced by them are true, these enzymes are brought into close relationship to the proteolyzers, although a coagulation can hardly be regarded as a true katabolic process. In milk where the lab-action becomes evident by precipitation of casein, a strict differentiation must be made between this coagulation and that brought about by acids or alkalies. In the former case, casein is not only precipitated and converted into paracasein, but is actually changed so that when redissolved it is no longer precipitated by lab. ${ }^{1}$

Coagulating enzymes for milk proteids, blood, and other proteid solutions are produced by a large variety of bacteria. They have been observed in cultures of the cholera vibrio, B. prodigiosus, B. pyocyaneus, and several others. ${ }^{2}$

The lab enzymes are easily destroyed by temperatures of $70^{\circ} \mathrm{C}$. and over, and are very susceptible to excessive acidity or alkalinity.

Fat-Splitting Enzymes (Lipase). - The fat-splitting powers of bacteria have been less studied than some of the other bacterial functions and are correspondingly more obscure. It is known, nevertheless, that the process is due to an enzyme and that it is probably hydrolytic in nature. The following formula represents the simplest method in which some of the molds and bacteria produce cleavage of fats into glycerin and fatty acid.

$$
\mathrm{C}_{3} \mathrm{H}_{5}\left(\mathrm{C}_{\mathrm{n}} \mathrm{H}_{2 \mathrm{n}-1} \mathrm{O}_{2}\right)_{3}+3 \mathrm{H}_{2} \mathrm{O}=\underset{\text { Glycerin }}{\mathrm{C}_{3} \mathrm{H}_{5}\left(\mathrm{OH}_{3}\right)}+\underset{\text { Fatty acid }}{3 \mathrm{C}_{\mathrm{n}} \mathrm{H}_{2 \mathrm{n}} \mathrm{O}_{2}}
$$

Some of the bacteria endowed with the power of producing lipase are the spirillum of cholera, B. fluorescens liquefaciens, B. prodigiosus, B. pyocyaneus, Staphylococcus pyogenes aureus, and some members of the streptothrix family. The methods of investigating this function of bacteria, originated by Ejkmann, ${ }^{3}$ consists in covering the bottom of a Petri dish with tallow and pouring over this a thin layer of agar. Upon this, the bacteria are planted. Any diffusion of lipase from the bacterial colonies becomes evident by a formation of white, opaque spots in the tallow. Carrière ${ }^{4}$ was able to demonstrate a fat-splitting ferment for the tubercle bacillus. Apart from the importance of these enzymes in nature for the destruction of fats, they are industrially important be-

1 Oppenheimer, "Die Fermente u. ihre Wirkung," Leipzig, 1903.

${ }_{2}$ Torini, Atti dei laborat. d. sanita, Rome, 1890.

3 Ejkmann, Cent. f. Bakt., I, xxix, 1901.

${ }^{4}$ Carrière, Comptes rend. de la soc. de biol., 53, 1901. 
cause of their action in rendering butter, milk, tallow, and allied products rancid, and are medically of interest for their action upon fats in the intestinal canal.

Enzymes of Fermentation (The Cleavage of Carbohydrates by Bacteria). - The power to assimilate carbon dioxide from the atmosphere is possessed only by the green plants and some of the colored algæ, and the sulphur or Thiobacteria. All other living beings are thus dependent for their supply of carbon upon the synthetic activities carried on by these plants to the same degree in which they are dependent upon similar processes for their nitrogen supply. The return of this carbon to the atmosphere is, of course, brought about to a large extent by the respiratory processes of the higher animals. The carbon, which, together with nitrogen, forms a part of proteid combinations, is freed, as we have seen in a previous section, by the processes of proteid cleavage. That, however, which is inclosed in the carbohydrate molecule, is set free by the action of yeasts, molds, or bacteria, by an enzymatic process simila: in every respect to that described above for the process of proteid cleavage.

Fermentation.- The power of carbohydrate cleavage is possessed by a large number of the yeasts and bacteria. The process, as has been indicated, is of great importance in the cycle of carbon compounds for the return of carbon to its simplest forms, and is, furthermore, as will be seen in a 'ater section, of great utility in the industries. In each case the power to split a particular carbohydrate is a more or less specific characteristic of a given species of microorganism, and for this reason has been extensively used as a method for the biological differentiation of bacteria. In the course of much careful work upon this question it has been ascertained that the specific carbohydrate-splitting powers of any given species are constant and unchanged through many generations of artificial cultivation. Thus, differentiation of the Gram-negative bacteria, the members of the pneumococcus-streptococcus group, and the diphtheria group, can now largely be made by a study of their sugar fermentations.

In most of these cases, as far as we know, the cleavage is produced by a process of hydrolysis. A convenient nomenclature which has been adopted for the designation of these ferments is that which employs the name of the converted carbohydrate adding the suffix "ase" to indicate the enzyme. There are thus ferments known as amylase, cellulase, lactase, etc.

- Amylase (Diastase or Amylolytic Ferment).-Amylases or starch- 
splitting enzymes are formed by many plants (malt) and by animal organs (pancreas, saliva, liver). Among microorganisms amylase is produced by many of the streptothrix group, by the spirilla of Asiatic cholera and of Finkler-Prior, by B. anthracis, and many other bacteria. A large number of the bacteria found in the soil, furthermore, have been shown to produce amylases. By cultivating bacteria upon starchagar plates, amylase can be readily demonstrated by a clearing of the medium immediately surrounding the colonies. ${ }^{1}$

Since, of course, there are several varieties of starches, it follows that the exact chemical action of amylase differs in individual cases. The determination of the structural disintegration of starch by these ferments is fraught with much difficulty, owing to the polymeric constitution of the starches. Primarily, however, a cleavage takes place into a disaccharid such as maltose (hexobiose), and the non-reducing sugars and dextrin. Beyond this point, however, the further cleavages are subject to much variation and are not entirely clear. The dextrins upon further reduction yield eventually dextrose.

Cellulase.-Cellulose is fermented by a limited number of bacteria, most of them anaërobes. The chemical process by which this takes place is but poorly understood. ${ }^{2}$

Gelase.-An agar-splitting ferment has been found by Gran. ${ }^{3}$

Invertase.-The enzymes which hydrolytically cause cleavage of saccharose into dextrose and levulose are numerous. The chemical process takes place according to the following formula:

$$
\begin{aligned}
& \mathrm{C}_{12} \mathrm{H}_{22} \mathrm{O}_{11}+\mathrm{H}_{2}^{\prime} \mathrm{O}=\mathrm{C}_{6} \mathrm{H}_{12} \mathrm{O}_{6}+\mathrm{C}_{6} \mathrm{H}_{12} \mathrm{O}_{6} \\
& \text { Saccharose Dextrose Levulose }
\end{aligned}
$$

Invertase is produced by many of the yeasts. It is one of the mosit common of the enzymes produced by bacteria, and has been found in cultures of B. megatherium, B. subtilis, pneumococcus, some streptococci, B. coli, and many others. Invertase is usually very susceptible to heat, being destroyed by temperatures of $70^{\circ} \mathrm{C}$. and over. A slightly acid reaction of media abets the inverting action of these enzymes. Strong acids and alkalies inhibit them. Inverting enzymes may be precipitated out of solution by alcohol. Antiseptics even in weak concentrations will inhibit their action.

1 Ejkmann, Cent. f. Bakt., xxix, 1901, and xxxv, 1904.

2 Omelianski, Lafar's "Handb. d. techn. Mykologie," Bd. iii, Chap. 9.

${ }^{3}$ Gran, Bergens Museum Aarbog, 1902, Hft. I. 
Lactase.-Lactose-splitting ferments are extremely common both among bacteria and among the yeasts. The process is here again a hydrolytic cleavage resulting in the formation of the monosaccharids as dextrose and galactose.

Maltase.-A maltose-splitting ferment has also been found in the cultures of many bacteria, leading to the formation of dextrose.

Lactic Acid Fermentation. - Lactic acid (oxyproprionic acid, $\mathrm{C}_{3} \mathrm{H}_{6} \mathrm{O}_{3}$ ) is one of the most common substances to appear among the products of bacterial activity, both in media containing carbohydrates and in those consisting entirely of albuminous substances. In most of these cases, the lactic acid is formed merely as a by-product accompanying many other more complicated chemical cleavages. In some instances, however, lactic acid is produced from carbohydrates, both disaccharids and monosaccharids, as an almost pure product due to a specific bio-chemical process. The reactions taking place in this phenomenon may be briefly expressed according to the following formulæ:

$$
\begin{aligned}
& \mathrm{C}_{12} \mathrm{H}_{22} \mathrm{O}_{11}+\mathrm{H}_{2} \mathrm{O}=\underset{\text { Lactose }}{4 \mathrm{C}_{3} \mathrm{H}_{6} \mathrm{O}_{3}} \\
& \text { Lactic acid }
\end{aligned}
$$

or

$$
\begin{aligned}
& \mathrm{C}_{6} \mathrm{H}_{12} \mathrm{O}_{6}=2 \mathrm{C}_{3} \mathrm{H}_{6} \mathrm{O}_{3} \\
& \text { Dextrose Lactic acid }
\end{aligned}
$$

In the same way lactic acid may be produced by bacteria from levulose.

Examples of lactic acid formation are furnished by the streptococcus lacticus, and B. lactis aërogenes. In the case of the former, the fermentation may indeed proceed by the simple chemical process indicated in the formulæ, since the action of the bacillus is entirely unaccompanied by the evolution of gas.

Numerous other bacteria produce large amounts of lactic acid from lactose, possibly by chemical processes less simply formulated. Among these are bacilli of the colon group, B. prodigiosus, B. proteus vulgaris, and many others. Although lactic acid is usually the chief product in the bacterial fermentation of the simpler carbohydrates, acetic, formic, and butyric acids may often be found as by-products in variable amounts. ${ }^{1}$

Oxydases (Oxydizing Enzymes).-The most common example of oxidation by means of bacterial ferments is the production of acetic acid

1 Buchner und Meisenheimer, Ber. d Deut. chem. Gesellsch., xxxvi, 1903. 
from weak solutions of ethyl alcohol. This process, which is the basis of vinegar production, is universally carried out by bacterial ferments. While possessed to some extent by a considerable number of microorganisms, acetic acid formation is a function pre-eminently of the bacterial groups described by Hansen, including "Bacterium aceti" and "Bacterium pasteurianum." To these two original groups a number of others have since been added.

The organisms are short, plump bacilli, with a tendency to chainformation, and occasionally showing characteristically swollen centers and many irregular involution forms. In the production of vinegar, as generally practiced by the farmer with cider or wine, these bacteria accumulate on the surface of the fluid as a pellicle or scum which is popularly known as the "mother of vinegar." Destruction of these bacteria by disinfectants or by sterilization with heat promptly arrests the process of vinegar formation. Chemically, the conversion of the alcohol consists in a double oxidation through ethyl aldehyde into acetic acid as shown in the following formulæ:

$$
\begin{aligned}
& \text { 1. } \mathrm{C}_{2} \mathrm{H}_{5}(\mathrm{OH})+\mathrm{O}=\mathrm{CH}_{3}(\mathrm{COH}) \\
& \text { - Alcohol Ethyl aldehyde }
\end{aligned}
$$

2. $\mathrm{CH}_{3}(\mathrm{COH})+\mathrm{O}=\mathrm{CH}_{3}(\mathrm{COOH})$ Acetic acid

Alcoholic Fermentation (Zymase). - The formation of alcohol as an end product of fermentation is of great importance in a number of the industries, primarily in the production of wine and beer. While accomplished by a number of bacteria, this form of fermentation is carried out chiefly by the yeasts.

Expressed in formulæ the simplest varieties of alcoholic fermentation, from mono- and disaccharids, may be represented as follows:

$$
\begin{aligned}
& \mathrm{C}_{6} \mathrm{H}_{12} \mathrm{O}_{6}=2 \mathrm{C}_{2} \mathrm{H}_{5}(\mathrm{OH})+2 \mathrm{CO}_{2} \\
& \text { Dextrose } \\
& \text { Ethyl alcohol }
\end{aligned}
$$

OI

$$
\begin{aligned}
& \mathrm{C}_{12} \mathrm{H}_{22} \mathrm{O}_{11}+\mathrm{H}_{2} \mathrm{O}=4 \mathrm{C}_{2} \mathrm{H}_{5}(\mathrm{OH})+4 \mathrm{CO}_{2} \\
& \text { Saccharose Ethyl alcohol }
\end{aligned}
$$

In all cases the process may not be so simple as indicated by the equations, since by-products, such as higher alcohols, glycerin, succinic and acetic acids, may often be found in small traces among the endproducts of such fermentations. The conditions which favor alcoholie 
fermentation by the yeasts are extremely important, since, upon obserservance of these, depends much of the uniformity of result which is so desirable in the industries mentioned above. The optimum concentration of sugar for the production of the highest quantity of alcohol is at or about 25 per cent. The temperature favoring the process ranges about $30^{\circ} \mathrm{C}$. Under such conditions fermentation may continue until the alcohol forms almost a 20-per-cent solution. Most of the fermentations important in the wine, beer, and spirit industries, take place under anaërobic conditions, since the carbon dioxide which is formed soon shuts out any excess of air.

In the industrial employment of yeasts for fermentative purposes, it is necessary to work with specific strains, and in scientifically conducted ${ }^{*}$ vineyards, breweries, and distilleries the study and pure cultivation of the yeasts form no unimportant part of the work. Certain races of yeasts are more uniform in their fermentative powers than others, and the byproducts formed by some races differ sufficiently from those of other races to cause material differences in the resulting substances. In the wine industries, the yeasts differ much from one another according to climatic and other environmental conditions. In vineyards, natural inoculation of the grapes occurs by transportation of the yeast from the soil to the surface of the grapes by wasps, bees, or other insects, through whose alimentary canals the microorganisms pass uninjured. In the autumn the yeast is returned to the soil by falling berries and remains alive in the upper layers of the ground throughout the winter months. In actual practice this natural yeast inoculation is not depended upon, but pure cultures of artificially cultivated yeasts are employed for inoculation. In some of the wine-growing countries these are supplied by special government experiment stations.

Denitrifying Bacteria.-Nitrogen is most readily absorbed by plants in the form of nitrates. These are furnished to the soil chiefly by the proteid decomposition induced by the proteolytic bacterial enzymes. It is self-evident, therefore, that any cleavage which reduces nitrogenous matter beyond the stage of nitrates, to nitrites and ammonia, detracts from the value of the nitrogen as a food stuff for plants, and the eventual setting free of nitrogen in the elementary state renders it entirely valueless for any but the leguminous plants.

Nevertheless, this process of nitrogen waste or denitrification is constantly going on in nature. In the course of ordinary decomposition, there is a constant reduction of nitrogenous matter to nitrites and salts of ammonia, actively taken part in by a host of bacteria, as many as 
85 out of 109 investigated by Maassen ${ }^{1}$ being found to possess this power. This, however, is not nearly so harmful a source of nitrogen waste as the process technically spoken of as true denitrification, in which nitrates are reduced, through nitric and nitrous oxides, to elementary nitrogen.

This phenomenon, more widely spread among bacteria than at first believed, depends essentially upon simple oxygen extraction from the nitrates by the bacteria, and for this reason goes on most actively when the supply of atmospheric oxygen is low. The first bacteria described as possessing this power of denitrification were the so-called B. denitrificans I and II, the first an obligatory anaërobe, the other a facultative aërobe. Since then numerous other bacteria, among them B. coli and B. pyocyaneus, have been shown to exhibit similar activities., It is important agriculturally, therefore, to know that many species which are able to utilize atmospheric oxygen when supplied with it, will get their oxygen by the reduction of nitrates and nitrites when free oxygen is withheld. It is thus clear that a loss of nitrogen is much more apt to proceed rapidly in manure heaps which are piled high and poorly aërated. There are other factors, however, in regard to the physiology of these microorganisms, which must be considered for practical purposes.

In order that these bacteria may develop their denitrifying powers to the best advantage, it is necessary to supply them with some carbon compound which is easily absorbed by them. This, in decomposing' material, is furnished by the products of the carbohydrate cleavage going on side by side with the proteolytic processes. It is still more or less an open question whether the facilitation of denitrification brought about in manure heaps by the presence of hay and straw is due to the carbon furnished by these materials, or whether it is due to the fact that bacilli of this group are apt to adhere to the straw which acts in that case as a means of inoculation.

The actual danger of nitrogen depletion of the soil by denitrifying processes is probably much less threatening than was formerly supposed; for, in the first place, the conditions for complete denitrification are much more perfect in the experiment than they ever can be in nature, and the nitrifying processes going on side by side with denitrification make up for much of the loss sustained.

${ }_{1}^{1}$ Maassen, Arb. a. d. kais. Gesundheitsamt, 1, xxviii, 1901. 


\section{ANABOLIC OR SYNTHETIC ACTIVITIES OF BACTERIA}

Nitrogen Fixation by Bacteria.-The constant withdrawal of nitrogenous substances from the soil by innumerable plants would soon lead to total depletion were it not for certain forces continually at work replenishing the supply out of the large store of free nitrogen in the atmosphere. This important function of returning nitrogen to the soil in suitable form for consumption by the plants is performed largely by bacteria.

It is well known that specimens of agricultural soil when allowed to stand for any length of time without further interference will increase in nitrogenous content, but that similar specimens, if sterilized, will show no such increase. ${ }^{1}$ The obvious conclusion to be drawn from this phenomenon is that some living factor in the unsterilized soil has aided in increasing the nitrogen supply. Light was thrown upon this problem when Winogradsky, ${ }^{2}$ in 1893, discovered a microorganism in soil which possessed the power of assimilating large quantities of nitrogen from the air. This bacterium, which he named "Clostridium Pasteurianum," is an obligatory anaërobe which in nature always occurs in symbiosis with two other facultatively anaërobic microorganisms. In symbiosis with these, it can be cultivated under aërobic conditions and thus grows readily in the upper well-aërated layers of the soil.

Although, until now, no other bacteria with equally well-developed nitrogen-fixing powers have been discovered, yet it is more than likely that Clostridium Pasteurianum is not the only microorganism endowed with this function. In fact, Penicillium glaucum and Aspergillus niger, two molds, and two other bacteria described by Winogradsky, have been shown to possess this power slightly, but in an incomparably less marked degree than Clostridium Pasteurianum. ${ }^{3}$ According to the calculations of Sachse, ${ }^{4}$ unsterilized soil may, under experimental conditions, gain as much as 25 milligrams of nitrogen in a season, a statement which permits the calculation of a gain of twelve kilograms of nitrogen per acre annually. ${ }^{5}$ It is very unlikely, however, that such gains actually occur in nature, where nitrogen-fixation and nitrogen-loss usually occur side by side.

\footnotetext{
${ }_{1}^{1}$ Berthelot, Compt. rend. de la soc. de biol., cxvi, 1893.

"Winogradsky, Compt. rend. de la soc. de biol., cxvi, 1893, ibid., t. cxviii, 1894.

3 Tacke, Land wirtsch. Jahresber., xviii, 1889.

"Sachse, "Agr. Chem.," 1883.

5 Pfeffer, Pflügers Physiologie, p. 395.
} 
Agriculturally of even greater importance than the free nitrogenfixing bacteria of the soil are the bacteria found in the root tubercles of a class of plants known as "leguminosæ." It has long been known that this class of plants, including clover, peas, beans, vetch, etc., not only does not withdraw nitrogen from the soil, but rather tends to enrich it. Upon this knowledge has depended the well-known method of alternation of crops employed by farmers the world over. The actual reason for the beneficial influence of the leguminosæ, however, was not known until 1887, when Hellriegel and Wilfarth ${ }^{1}$ succeeded in demonstrating that the nitrogen-accumulation was directly related to the root tubercles of the plants, and to the bacteria contained within them.

These tubercles, which are extremely numerous-as many as a thousand sometimes occurring upon one and the same plant-are formed by the infection of the roots with bacteria which probably enter through the delicate root-hairs. They vary in size, are usually situated near the main root-stem, and, in appearance, are not unlike fungus growths. Their development is in many respects comparable to the development of inflammatory granulations in animals after infection, inasmuch as the formation of the tubercle is largely due to a reactionary hyperplasia of the plant tissues themselves. They appear upon the seedlings within the first few weeks of their growth as small pink nodules, and enlarge rapidly as the plant grows. At the same time, later in the season, when the plants bear fruit, the root tubercles begin to shrink and crack. When the crops are harvested, the tubercles with the root remain, rot in the ground, and re-infect the soil.

Histologically the tubercles are seen to consist of large root cells which are densely crowded with microorganisms.

The microorganism itself, "Bacillus radicicola," was first observed within the tubercles by Woronin ${ }^{2}$ in 1866 . The bacilli are large, slender, and actively motile during the early development of the tubercles, but in the later stages assume a number of characteristic involution forms, commonly spoken of as "bacteroids." They become swollen, T and Y shaped, or branching and threadlike. Their isolation from the root tubercles usually presents little difficulty, since they grow readily upon gelatin and agar under strictly aërobic conditions. On the artificial media the bacillary form is usually well retained, involution forms appearing only upon old cultures.

${ }_{1}$ Hellriegel und Wilfarth, Cent. f. Bakt., 1887.

2 Woronin, Bot. Zeit., xxiv, 1866. 
The classical experiments of Hellriegel and Wilfarth conclusively demonstrated the important relation of these tubercle-bacteria to nitrogen assimilation by the leguminosæ.

These observers cultivated various members of this group of plants upon nitrogen-free soil-sand-and prevented the formation of root tubercles in some, by sterilization of the sand, while in others they encouraged tubercle formation by inoculation. An example of their results may be given as follows: ${ }^{1}$

Lupinus luteus was cultivated upon sterilized sand. Some of the pots were inoculated with B. radicicola, others were kept sterile. Comparative analyses were made of the plants grown in the different pots with the following striking result:

\begin{tabular}{|c|c|c|c|c|}
\hline \multirow{3}{*}{$\begin{array}{l}\text { Root tubercles } \\
\text { present ..... }\end{array}$} & $\begin{array}{l}\text { Harvested } \\
\text { dry weight }\end{array}$ & N. present & $\begin{array}{l}\text { soil, and soil- } \\
\text { extract }\end{array}$ & $\begin{array}{l}\text { Gain or } \\
\text { loss of } N .\end{array}$ \\
\hline & (a) 38.919 & .998 & .022 & +.975 \\
\hline & (b) 33.755 & .981 & .023 & $\div .958$ \\
\hline \multirow{2}{*}{ No root tubercles..... } & 0.989 & .016 & .020 & -.004 \\
\hline & 0.828 & .011 & .022 & -.009 \\
\hline
\end{tabular}

The great importance of this process in agriculture is demonstrated, furthermore, by a comparison made by the same observers between a legume, the pea, and one of the common nitrogen-consuming crops, oats. ${ }^{2}$

Nitrogen contents
of seed and soil.
Oats 0.027 grams
Peas 0.038 "6

Nitrogen contents of crop.

0.007 grams

0.459

Gain or loss.

$$
\begin{array}{r}
-.020 \\
+.421
\end{array}
$$

Exactly what the process is by which the bacteria supply nitrogen to the plant is as yet uncertain. Although the degenerating bacteroids in old nodules are bodily absorbed by the plant, this can not be conceived as the only method of supply, since the total nitrogen gain many times exceeds the total weight of bacteria in the nodules. It is probable that the microorganisms during life take up atmospheric nitrogen and secrete a nitrogenous substance which is absorbed by the plant cells.

Although formerly the relationship between plant and bacterium was regarded as one of symbiosis and of mutual benefit, the opinions as to this subject show wide divergence. While, according to some authors, the entrance of the bacteria into the plants is regarded as a true infection against which the plant offers at first a determined opposition as evidenced by tissue reactions, other observers, notably A. Fischer, regard

1 Pfeffer, "Planzenphysiologie," Leipzig, 1897.

${ }^{2}$ Hellriegel und Wilfarth, Zeit. d. Ver. f. d. Rübenzucker Industrie, 1888. Quoted from Fischer, "Vorles. über die Bakt.," Jena, 1903. 
the plant as a parasite upon the bacteria, in that it derives the sole benefit from the relationship and eventually bodily consumes its host.

Nitrifying Bacteria.-A process diametrically opposed in its chemistry to denitrification and reduction is that which brings about an oxidation of ammonia to nitrites and nitrates. The actual increase of nitrates in soil allowed to stand for any length of time and examined from time to time has been a well-established fact for many years; but it was believed until a comparatively short time ago that this increase was due to a simple chemical oxidation of ammonia by atmospheric oxygen. The dependence of nitrification upon the presence of living organisms was finally proved by Muntz and Schlossing ${ }^{1}$ in 1887, who demonstrated that nitrification was abruptly stopped when the soil was sterilized by heat or antiseptics. It remained, however, to isolate and identify the organisms which brought about this ammonia oxidation. This last step in our knowledge of nitrification was taken in 1890, by Winogradsky. Winogradsky ${ }^{2}$ found that the failures experienced by others who had attempted to isolate nitrifying bacteria were due to the fact that they had used the common culture media largely made up of organic substances. By using culture media containing no organic matter Winogradsky succeeded in isolating free from the soil, bacteria which have since that time been confirmed as being the causative factors in nitrification. During his first experiments this author observed that in some of his cultures the oxidation of ammonia went only as far as the stage of nitrite formation, while in others complete oxidation to nitrates took place. Following the clews indicated by this discrepancy, he finally succeeded in demonstrating that nitrification is a double process in which two entirely different varieties of microorganisms take part, the one capable of oxidizing ammonia to nitrites, the other continuing the process and converting the nitrites to nitrates. The nitrite-forming bacteria discovered by Winogradsky, and named Nitromonas or Nitrosomonas, are easily cultivated upon aqueous solutions containing ammonia, potassium sulphate, and magnesium carbonate. According to their discoverer they develop within a week in this medium as a gelatinous sediment. After further growth this sediment seems to break up and the bacteria appear as oval bodies, which swim actively about and develop flagella at one end. Upon the solid media in ordinary use they can not be cultivated. Special solid media suitable for their cul-

1 Muntz und Schlossing, Compt. rend. de l'acad. des sciences, 1887.

2 Winogradsky, Ann. Past. Inst., iv and v, 1890, 1891. 
tivation and composed of silicic acid and inorganic salts have been described by Winogradsky and by Omeliansky. ${ }^{1}$

Other nitrite-forming bacteria have since been described by various observers, all of them more or less limited to definite localities. Some of these are similar to nitrosomonas in that they exhibit the flagellated, actively motile stage. In others this stage is absent.

The nitrite-forming bacteria, apart from their great agricultural importance, claim our attention because of their unique position in relation to the animal and vegetable kingdoms. Extremely sensitive to the presence of organic compounds, they are able to grow and develop only upon media containing nothing but inorganic material; and this entirely without the aid of any substances comparable to the chlorophyll of the green plants. The source of energy from which this particular class of bacteria derive the power of building up organic compounds from simple substances is to some extent a mystery. The carbon which they unquestionably require for the building up of organic material may be, as Winogradsky believed, derived to a certain extent from ammonium carbonate. But it is also quite certain that they are capable of utilizing directly atmospheric $\mathrm{CO}_{2}$. In the absence of chlorophyll or of any highly organized chemical compound, it seems likely that the energy necessary for the utilization of the carbon obtained in this simple form is derived from the oxidation of ammonia during the process of nitrification.

The conversion of nitrites into nitrates is carried on by other species of bacteria also discovered by Winogradsky. These bacteria are much more generally distributed than nitrosomonas and probably include a number of varieties. The organism described by Winogradsky is an extremely small bacillus with pointed ends. Capsules have occasionally been made out. It may be cultivated upon aqueous solutions containing:

Sod. nitrite......................... per cent.

Potass. phosphate.......................... " "

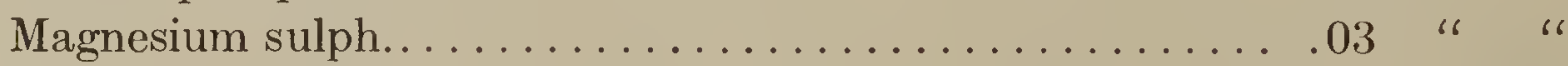

Sodium carbonate....................... " " "

Ferrous sulphate.................... "04 "

The development of the organism is slow and sparse, and is directly inhibited by the presence of organic matter. It is strongly inhibited by the presence of ammonia.

The Liberation of Energy by Bacteria.-Like all other living beings, 1 Omeliansky, Cent. f. Bakt., II, 5, 1899. 
bacteria in their metabolic processes liberate energy. It has been shown by several observers that slight quantities of heat are given off from actively growing cultures. The functions, furthermore, of reproduction, motility, and enzyme formation may be looked upon as forms of energy liberation. In addition to this, certain bacteria have been observed which may liberate energy in the form of light.

Light Production by Bacteria. - The production of light by bacteria is a power possessed chiefly by certain species inhabiting salt water. Thus, much of the phosphorescence observed at sea, though more frequently due to Medusa and other invertebrate animals, is caused by these bacteria. Numerous species which produce this phenomenon have been isolated, too many, and too unimportant, to be individually described. All of them are aërobes and require highly complex food stuffs. They are closely allied to the putrefactive bacteria, and in the sea are usually found upon rotting animal matter. ${ }^{1}$ The production of light seems directly dependent upon the free access of oxygen, since no light appears under anaërobic conditions. Their luminous quality, moreover, is not a true phosphorescence, in that it does not depend upon previous illumination and develops as well in cultures kept in the dark as in those which have been exposed to light. ${ }^{2}$

The Formation of Pigment by Bacteria (Chromobacteria).-A large number of bacteria, when cultivated upon suitable media, give rise to characteristic colors which are valuable as marks of differentiation. For each species, the color is usually constant, depending, to a certain extent, upon the conditions of cultivation. In only a few of the pigmented bacteria is the pigment contained within the cell body, and in only one variety, the sulphur bacteria, does the pigments appear to hold any distinct relationship to nutrition. In most cases, the coloring matter is found to be deposited in small intercellular gransles or globules. The absence of any relationship of the pigment to sunlight, as is the case with the chlorophyll of the green plants, is indicated by the fact that most of the chromobacteria thrive and produce pigment equally well in the dark as they do in the presence of light. Among the most common of the pigment bacteria met with in bacteriological work are Staphylococcus pyogenes aureus, Bacillus pyocyaneus, Bacillus prodigiosus, and some of the green fluorescent bacteria frequently found in feces.

The chemical nature of these pigments has been investigated quite thoroughly and it has been shown that they vary in composition.

1 Pflüger's Arch. f. Phys., xi, 1875.

${ }^{2}$ Fischer, Cent. f. Bakt., iii, 1888. 
Some of the pigments, like that of Staphylococcus aureus, are probably non-proteid and of a fatty nature. ${ }^{1}$ They are insoluble in water but soluble in alcohol, ether, and chloroform. Because of their probable composition, they have been spoken of as "lipochromes." Other pigments, like the pyocyanin, which lends the green color to cultures of Bacillus pyocyaneus, are water soluble and are probably of proteid composition. Pyocyanin may be crystallized out of aqueous solution in the form of fine needles. The crystals may be redissolved in chloroform. Aqueous solutions retain their color. Solutions in chloroform, however, are changed gradually to yellow.

The power of pigment production of various bacteria depends in each case upon cultural conditions. In most cases, this simply signifies that pigment is produced only when the microorganism, finding the most favorable environmental conditions, is enabled to develop all its functions to their fullest extent. Thus, a too high acidity or alkalinity of the culture medium may inhibit pigment formation. Oxygen is necessary for the production of color in some bacteria, since the bacteria themselves often produce the pigment only as a leuko-body which is then oxydized into the pigment proper. A notable example of this is the pigment of B. pyocyaneus. In other cases, temperature plays an important rôle in influencing color production. Thus, Bacillus prodigiosus refuses to produce its pigment when growing in the incubator. By persistent cultivation in an unfavorable environment, colored cultures may lose their power of pigment production.

Sulphur Bacteria.-Wherever the decomposition of organic matter gives rise to the formation of $\mathrm{H}_{2} \mathrm{~S}$, in cess-pools, in ditches, at the bottom of the sea, and in stagnant ponds, there is found a curiously interesting group of microorganisms, the so-called sulphur or thiobacteria. Red, purple, and colorless, these bacteria all possess the power of utilizing sulphuretted hydrogen and by its oxidation into free sulphur obtain the energy necessary for their metabolic processes. The colorless sulphur bacteria, the Beggiatoa and Thiothrices, usually appear as threads or chains which, in media containing sufficient $\mathrm{H}_{2} \mathrm{~S}$, are usually wellstccked with minute globules of sulphur. If found upon decomposing organic matter, they often cover this as a grayish mold-like layer. The red sulphur bacteria, of which numerous species have been described by Winogradsky, may appear as actively motile spirilla (Thiospirillum) or as short, thick bacillary forms.

${ }_{1}$ Schroeter, Cent. f. Bakt., xviii, 1895. 
The physiology of all the sulphur bacteria, and especially of the colored varieties, is of the greatest interest in that these microorganisms are among the few members of the bacterial group which behave metabolically like the green plants. The higher organic substances play little or no part in the nutrition of these microorganisms. Strictly aërobic, the colorless thiobacteria are independent of sunlight, while the red and purple varieties exhibit their physiological dependence upon light by accumulating under natural conditions in well-lighted spots. Both varieties possess equally the power of oxidizing sulphuretted hydrogen as a source of energy. The sulphur is then stored as elemental sulphur within the bacterial body and when a lack of food stuffs sets in, the store of sulphur can be further oxidized into sulphurous or sulphuric anhydrides. With this sole source of energy, these bacteria are capable of flourishing aërobically, while an absence of $\mathrm{H}_{2} \mathrm{~S}$, even in the presence of organic food stuffs, leads to a rapid disappearance of their sulphur contents and an inability to develop.

In the case of the colored thiobacteria, the red pigment appears to fulfil, to some extent, a function comparable to that of the chlorophyll of the green plants.

Engelmann, ${ }^{1}$ who has studied this pigment spectroscopically, has found that besides absorbing the red spectral rays there is an absorption of rays on the ultra-red end of the spectrum. The absorption of the red rays between the lines $\mathrm{B}$ and $\mathrm{C}$ of the spectrum, and of violet rays at the line $\mathrm{F}$, is the same as that of the absorption spectrum of chlorophyll, and it is in the zone of these rays that the physiological effects of chlorophyll are most active. In addition to these absorption bands, the bacteriopurpurin of the red sulphur bacteria shows absorption of the invisible ultra red rays of the spectrum.

Engelmann, with a microspectroscope, projected a spectrum into a miscroscopic field in which green algæ or, in the case under discussion, red sulphur bacteria had been placed. Other sources of light were, of course, excluded. By adding emulsions of strictly aërobic bacteria to such preparations, an accumulation of microorganisms was observed at those points in the spectrum at which most oxygen was liberated. In the case both of chlorophyll and of the red sulphur bacteria such areas of bacterial accumulation (in oxygen liberation) occurred in the zones of the absorption bands mentioned above. 


\section{CHAPTER V}

\section{THE DESTRUCTION OF" BACTERIA}

\section{GENERAL CONSIDERATIONS}

No branch of bacteriology has been more fruitful in practical application than that which deals with the factors which bring about the destruction of microorganisms. Upon the study of this branch has depended the growth and the development of modern surgery.

The agents which affect bacteria injuriously are many, and are both physical and chemical in nature.

When a procedure completely destroys bacterial life it is spoken of as sterilization or disinfection, the term disinfection being employed more especially to designate the use of chemical agents. When the procedure destroys vegetative forms only, leaving the more resistant spores uninjured, it is spoken of as "incomplete sterilization." When an agent, on the other hand, does not actually kill the microorganisms, but merely inhibits their growth and multiplication, it is spoken of as an antiseptic. The term deodorant is indiscriminately applied to substances which mask or destroy offensive odors, and may or may not possess disinfectant or antiseptic value. Some deodorants act chemically on the noxious gases, destroying them.

\section{PHYSICAL AGENTS INJURIOUS TO BACTERIA}

The principal physical agents which may exert deleterious action upon bacteria are: drying, light, electricity, and heat.

Drying.-Complete desiccation eventually destroys most of the pathogenic bacteria, yet great differences in resistance to this condition are shown by various microorganisms. Ficker, ${ }^{1}$ who has made a systematic study of the influence of complete drying upon bacteria, concludes that the resistance of bacteria to desiccation is influenced by the age of the culture investigated, the rapidity with which the withdrawal of moisture

I Ficker, Zeit, f. Hyg., xxix, 1896. 
is accomplished, and the temperature at which the process takes place. Microorganisms like the gonococcus and the Pfeiffer bacillus, are destroyed by drying within a few hours. The cholera vibrio dried upon a coverslip was found by Koch ${ }^{1}$ to be killed within four hours; by Burckholtz, ${ }^{2}$ to survive about twenty-four hours. The spore-forms of bacteria are infinitely more resistant to this influence than are the vegetative forms, though they may be destroyed by rapid and complete drying in a desiccator.

It is self-evident that many discrepancies in the experimental results of various authors may depend upon the technique of investigation, since the degree of drying attained depends intimately upon the thickness and consistence of the material investigated, and upon the methods employed for desiccation.

Light.-Direct sunlight is a powerful germicide for all bacteria except a limited number of species like the thio- or sulphur bacteria, which utilize sunlight for their metabolic processes as do the green plants.

Koch ${ }^{3}$ has shown that exposure to sunlight will destroy the tubercle bacillus within two hours or less, the time depending upon the thickness of the exposed layers and the material surrounding the bacilli. Confirmatory researches have been published by Mignesco ${ }^{4}$ and others. The powerful disinfecting influence of sunlight upon bacteria suspended in water has been shown by Buchner. ${ }^{5}$ Observations in regard to the influence of sunlight upon anthrax spores have been made by Arloing, ${ }^{6}$ and similar observations upon a number of other microorganisms have been carried out by Dieudonné, Janowski, v. Esmarch, and many others. All these observers, while differing somewhat as to the time necessary for bacterial destruction, agree in finding definite and powerful bactericidal action of sunlight. Diffuse light, of course, is less active than direct sunlight. According to Buchner, typhoid bacilli are inhibited by direct sunlight in one and one-half hours, by diffuse light in five hours. A remarkable statement is made by Arloing, who claims to have found that anthrax spores are more quickly destroyed by direct sunlight than are the vegetative cells. This fact would call for further confirmation.

1 Koch, Arb. a. d. kais. Gesundheitsamt, iii, 1887.

2 Burkholtz, Arb. a. d. kais. Gesundheitsamt, v, 1889.

${ }^{3}$ Koch, X Internat. Med. Congress, Berlin, 1890.

${ }^{4}$ Mignesco, Arch. f. Hyg., xxv, 1896.

${ }^{5}$ Buchner, Cent. f. Bakt., I, xi, 1892.

${ }^{6}$ Arloing, Compt. rend. de l'acad. d. sci., c, 1885. 
It has been shown by various authors that the influence of sunlight is not to be attributed in any way to temperature, nor always to a direct action of the light upon the bacteria, but depends largely upon photochemical changes produced by the light rays in the media. Richardson ${ }^{1}$ and Dieudonné ${ }^{2}$ conclude that under ordinary aërobic conditions in fluid environment peroxide of hydrogen is formed under the influence of light. Novy and Freer ${ }^{3}$ believe that the bactericidal effects in fluids noticed as a result of exposure to light are too strong to be explained by the formation of small quantities of peroxide of hydrogen, and attribute this action to organic peroxides formed under the described conditions, such as the peroxides of diacetyl, benzoylacetyl, and others. These views are somewhat strengthened by the fact that exclusion of oxygen from media markedly diminishes the bactericidal power of light. ${ }^{4}$ That the photochemical changes alone, however, do not explain this action follows from the fact that dried bacteria, not surrounded by media, are subject to a similar action. ${ }^{5}$

In analyzing sunlight in regard to its bactericidal power, it has been found by various observers that the most powerful action is exerted by the ultraviolet spectral rays, whereas the yellow, red, and ultra-red rays are practically innocuous. ${ }^{6}$

It is of importance to note that sunlight has been found also to have a strong attenuating influence ${ }^{7}$ upon some bacterial poisons, as shown by the experiments of Ferri and Celli upon tetanus toxin.

Electric light exerts a distinct bactericidal action when applied in strengths of 800 to 900 candle power for seven or eight hours. ${ }^{8}$

Röntgen or $x$-rays are said by Zeit, ${ }^{9}$ Blaise ${ }^{10}$ and Sambac, and others to be without appreciable germicidal power. Rieder, ${ }^{11}$ on the other hand, has reported definite inhibition of bacterial growth after exposures of half an hour to $x$-rays.

1 Richardson, Jour. Chem. Soc., i, 1893, Ref. Deut. chem. Gesells., xxvi.

2 Dieudonné, loc. cit.

${ }^{3}$ Novy and Freer, 3d Ann. Meeting Assn. Amer. Bacteriologists, Chicago, 1901.

${ }^{4}$ Roux, Ann. Inst. Past., ix, 1887.

5 Dieudonné, loc. cit.

${ }^{6}$ Ward, Proc. Royal Soc., 52, 1893.

7 Ferri and Celli, Cent. f. Bakt., I, xii, 1892.

${ }^{8}$ Dieudonné, loc. cit.

9 Zeit, Jour. Amer. Med. Assn., xxxvii, 1901.

10 Blaise and Sambac, Compt. rend. de la soc. de biol., 1896.

11 Rieder, Münch. med. Woch., 1898. 
Radium rays have a distinct inhibitory and even bactericidal power when applied at distances of a few centimeters for several hours. ${ }^{1}$

Electricity.-If we exclude the indirect actions of heat and electrolysis, it can hardly be said that the direct bactericidal action of electric currents has been satisfactorily demonstrated. Such action, however, has been claimed by d'Arsonville and Charrin, ${ }^{2}$ and by Spilker and Gottstein. ${ }^{3}$

Heat.-The most widely applicable and efficient physical agent for sterilization is heat.

The dependence of bacteria for growth and vitality upon the maintenance of a proper temperature in their environment, and the ranges of variation within which bacteria may thrive, have been discussed in a preceding section, in which a table of so-called "thermal death points" has been given. In the method of expressing these values it was seen that two elements entered into the destruction of bacteria by heat, namely, that of the degree of temperature which is applied, and that of the time of application.

The prolonged application of moderately high temperatures, in other words, may in certain instances, accomplish the same result as the brief use of extremely high ones. In general, the death of bacteria following proionged exposure to temperatures but slightly exceeding the optimum is due to the inability of the anabolic processes to keep pace with the accelerated katabolic processes, gradual attenuation resulting in death. At somewhat higher temperatures death results from coagulation of the bacterial protoplasm, and at still higher degrees of heat, applied in the dry form, direct burning of the bacteria may be the cause of their destruction.

Heat may be applied in the form of dry heat or as moist heat, these methods being of great practical value, but differently applicable according to the nature of the materials to be sterilized. The two methods, moreover, show a marked difference in efficiency, temperature for temperature. For the recognition of this fact we are largely indebted to the early researches of Koch and Wolffhügel, ${ }^{4}$ and of Koch, Gaffky, and Loeffler. ${ }^{5}$

1 Personal observations.

${ }_{2}$ D'Arsonville and Charrin, Compt. rend. de la soc. de biol.

${ }_{3}$ Spilker and Gottstein, Cent. f. Bakt., I, 9, 1891.

${ }^{4}$ Koch und Wolffhügel, Mitt. a. d. kais. Gesundheitsamt, 1, 1882.

${ }^{5}$ Koch, Gaffky and Loeffler, ibid. 
These observers were able to show that the spores of anthrax were destroyed by boiling water at $100^{\circ} \mathrm{C}$. in from one to twelve minutes, whereas dry hot air was efficient only after three hours' exposure to $140^{\circ} \mathrm{C}$. Extensive confirmation of these differences has been brought by many workers. An explanation of the phenomena observed is probably to be found in the changes in the coagulability of proteids brought about in them by the abstraction of water. Lewith, ${ }^{1}$ working with various proteids, found that these substances are coagulated by heat at lower temperatures when they contain abundant quantities of water, than when water has been abstracted from them. On the basis of actual experiment with egg albumin he obtained the following results, ${ }^{2}$ which illustrate the point in question:

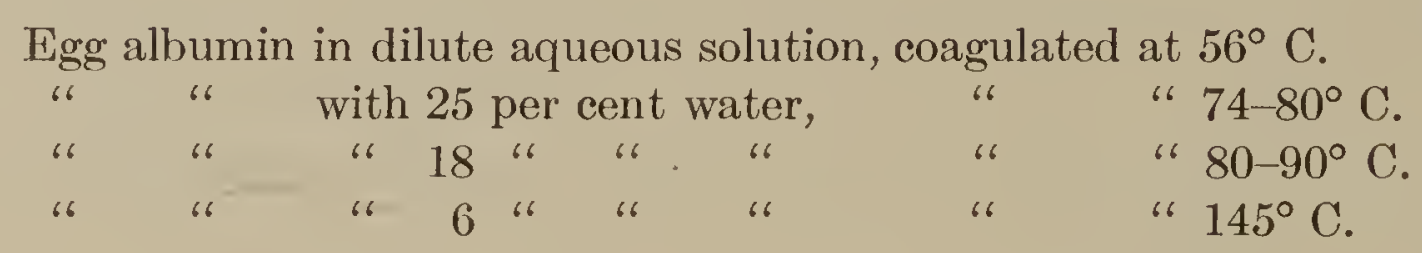

Absolutely anhydrous albumin, according to Haas, ${ }^{3}$ may be heated to $170^{\circ} \mathrm{C}$. without coagulation. It is thus clear that bacteria exposed to hot air may be considerably dehydrated before the temperature rises sufficiently to cause death by coagulation, complete dehydration necessitating their destruction possibly by actual burning.

Bacteria exposed to moist air or steam, on the other hand, may absorb water and become proportionately more coagulable.

The same principle, as Lewith points out, probably explains the great resistance to heat observed in the case of the highly concentrated protoplasm of spores.

Apart from the actually greater efficiency of moist heat when compared with dry heat of an equal temperature, an advantage of great practical significance possessed by moist heat lies in its greater powers of penetration. An experiment carried out by Koch and his associates illustrates this point clearly. Small packages of garden soil were surrounded by varying thicknesses of linen with thermometers so placed that the temperature under a definite number of layers could be deter-

${ }^{1}$ Lewith, Arch. f. exp. Path. u. Pharm., xxvi, 1890.

${ }^{2}$ Lewith, loc. cit., p. 351.

${ }^{3}$ Haas, Prag. med. Woch., 34-36, 1876. 
mined. Exposures to hot air and to steam were then made for comparison, and the results were as tabulated:1

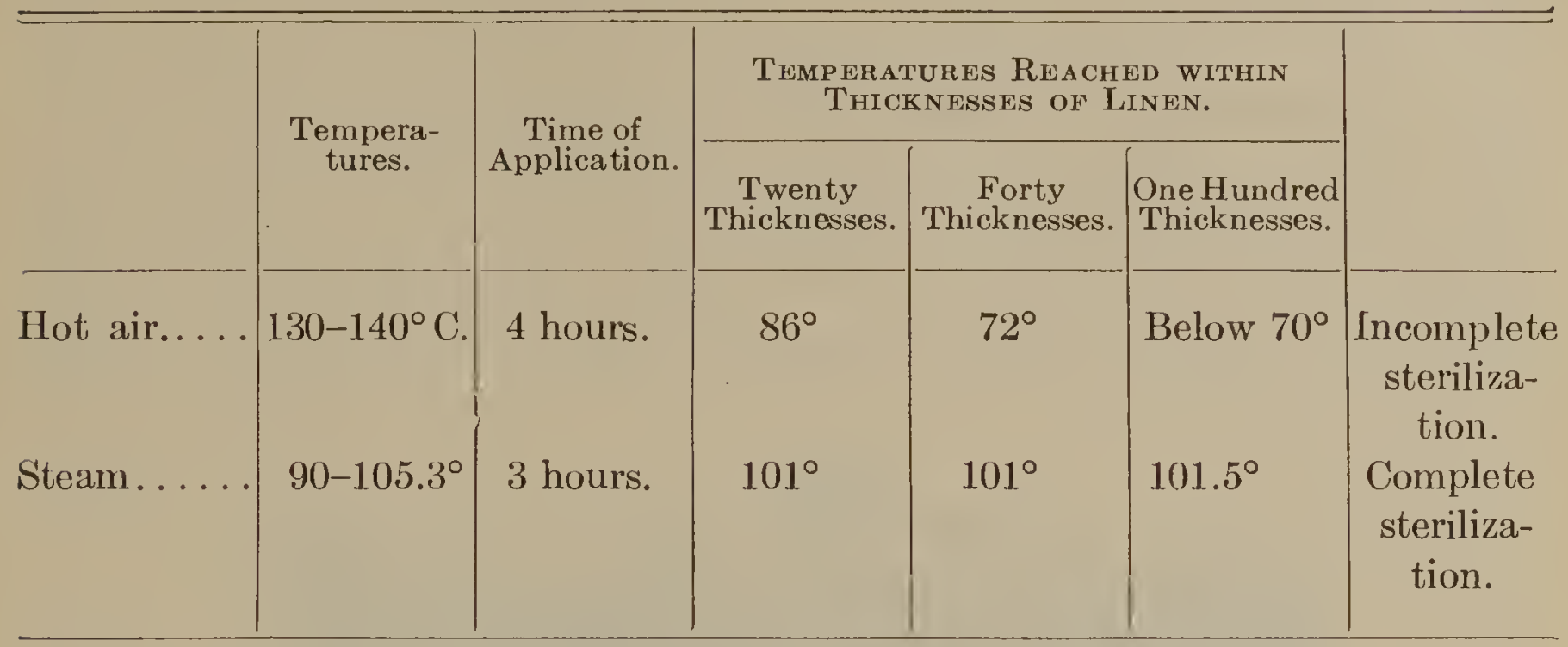

This great penetrating power of steam is due presumably to its comparatively low specific gravity which enables it to displace air from the interior of porous materials, and also to the fact that as the steam comes in contact with the objects to be disinfected a condensation takes place with the consequent liberation of heat. When a vapor passes into the liquid state it gives out a definite amount of heat, which in the case of water vapor, at $100^{\circ} \mathrm{C}$., amounts to about 537 calories. This brings about a rapid heating of the object in question. Following this process the further heating takes place by conduction, and it is, of course, well known that steam is a much better heat conductor than air. ${ }^{2}$

Moist heat may be applied as boiling water, in which, of course, the temperature varies little from $100^{\circ} \mathrm{C}$., or as steam. Steam may be used as live, flowing steam, without pressure, the temperature of which is more or less constant at $100^{\circ} \mathrm{C}$., or still higher efficiency may be attained by the use of steam under pressure, in which, of course, temperatures far exceeding $100^{\circ} \mathrm{C}$. may be produced, according to the amount of pressure which is used.

The spores of certain bacteria of the soil which can not be killed in live steam in less than several hours may be destroyed in a few minutes, or even instantaneously, in compressed steam at temperatures ranging from $120^{\circ}$ to $140^{\circ} \mathrm{C} .^{3}$

In all methods of steam sterilization, it is of great practical impor-

${ }^{1}$ Koch, Gaffky und Loeffler, loc. cit., p. 339.

${ }^{2}$ Gruber, Cent. f. Bakt., iii, 1888.

${ }^{3}$ Christen, Ref. Cent. f. Bakt., V, xiii, 1893. 
tance, as v. Esmarch ${ }^{1}$ has pointed out, that the steam shall be saturated, that is, shall contain as much vaporized water as its temperature permits. Unsaturated, or so-called "super-heated steam" is formed when heat is applied to steam, either by passage through heated piping or over heated metal plates. In such cases the temperature of the steam is raised, but no further water-vapor being supplied, the steam exerts less pressure and contains less water in proportion to its volume than saturated steam of an equal temperature. The super-heated steam, therefore, is heated considerably over its condensation temperature and becomes literally dried. In consequence, its action is more comparable to hot air than to saturated steam, and up to a certain temperature its disinfecting power is actually less than that of live steam at $100^{\circ} \mathrm{C}$. v. Esmarch, who has made a thorough study of these conditions, concludes that up to $125^{\circ} \mathrm{C}$., the efficiency of superheated steam is lower than that of live steam at $100^{\circ} \mathrm{C}$. Above this temperature, of course, it is again active as in the case of ordinary dry heat.

Practical Methods of Heat Sterilization.-Burning.-For objects without value, actual burning in a furnace is a certain and easily applicable method of sterilization. Flaming, by passage through a Bunsen or an alcohol flame, is the method in use for the sterilization of platinum needles, coverslips, or other small objects which are used in handling bacteria in the laboratory.

Hot air sterilization is carried out in the so-called "hot air chambers," simple devices of varied construction. The apparatus most commonly used (Fig. 8) consists of a sheet-iron, double-walled chamber, the joints of which, instead of being soldered, are closed by rivets. The inner case of this chamber is entirely closed except for an opening in the top through which a thermometer may be introduced, while the outer has a large opening at the bottom and two smaller ones at the top. A gasburner is adjusted under this so as to play directly upon the bottom of the inner case. A thermometer is fitted in the top in such a way that it penetrates into the inner chamber. The air in the chamber is heated directly by the flame and by the hot air, which, rising from the flame, courses upward within the jacket between the two cases and escapes at the top. To insure absolute sterilization of objects in such a chamber, the temperature should be kept between $150^{\circ}$ and $160^{\circ} \mathrm{C}$. for at least an hour. In sterilizing combustible articles in such a chamber, it should be remembered that cotton is browned at a temperature of $200^{\circ} \mathrm{C}$. and

3 v. Esmarch, Zeit, f, Hyg., iv. 1888, 
over. This method is used in laboratories for the sterilization of Petri dishes, flasks, test tubes, and pipettes, and for articles which may be injured by moisture. Both heating and subsequent cooling should be done gradually to avoid cracking of the glassware.

Moist Heat.-Instruments, syringes, and other suitable objects may be sterilized by boiling in water. Boiling for about five minutes is amply sufficient to destroy the vegetative forms of all bacteria. For the destruction of spores, boiling for one or two hours is usually sufficient, though the spores of certain saprophytes of the soil have been found

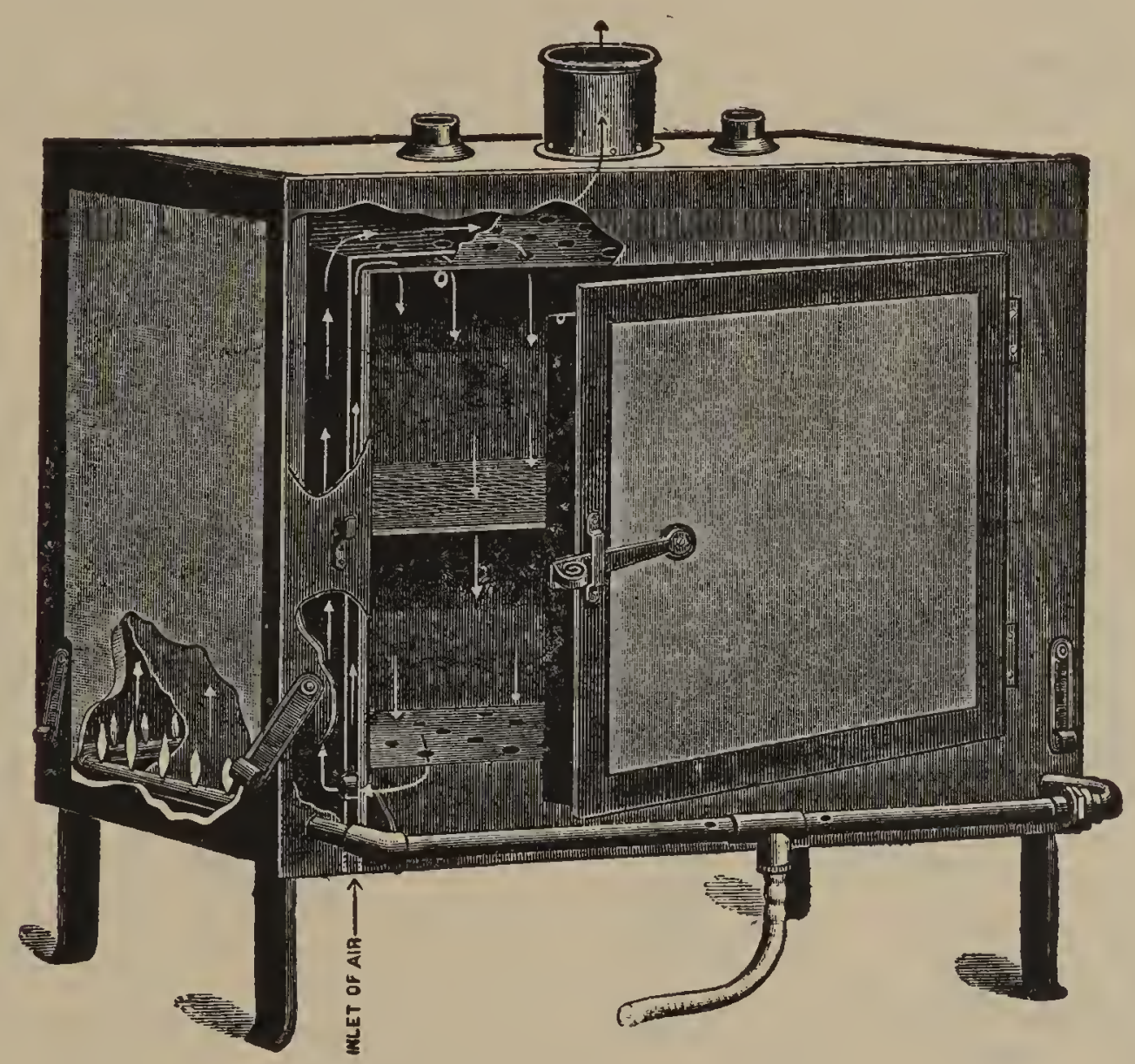

Fig. 8.-Hot Air STterilizer.

occasionally to withstand moist heat at a temperature of $100^{\circ} \mathrm{C}$. for as long as sixteen hours. ${ }^{1}$ The addition of 1 per cent of sodium carbonate to boiling water hastens the destruction of spores and prevents the rusting of metal objects sterilized in this way. The addition of carbolic acid to boiling water in from 2 to 5 per cent usually insures the destruction of anthrax spores, at least, within ten to fifteen minutes.

Exposure to live steam is probably the most practical of the methods of heat sterilization. It may be carried out by simple makeshifts of the kitchen, such as the use of potato-steamers or of wash-boilers. For 
laboratory purposes, the original steaming device introduced by Koch has been almost completely displaced by devices constructed on the plan of the so-called "Arnold" sterilizer (Fig. 9). In such an apparatus, water is poured into the reservoir $A$ and flows from there into the shallow receptacle $B$, formed by the double bottom. The flame underneath rapidly vaporizes the thin layer of water contained in $B$, and the steam rises rapidly, coursing through the main chamber $C$. Steam which escapes through the joints of the lid of this chamber is condensed under the hood and drops back into the reservoir. Exposure

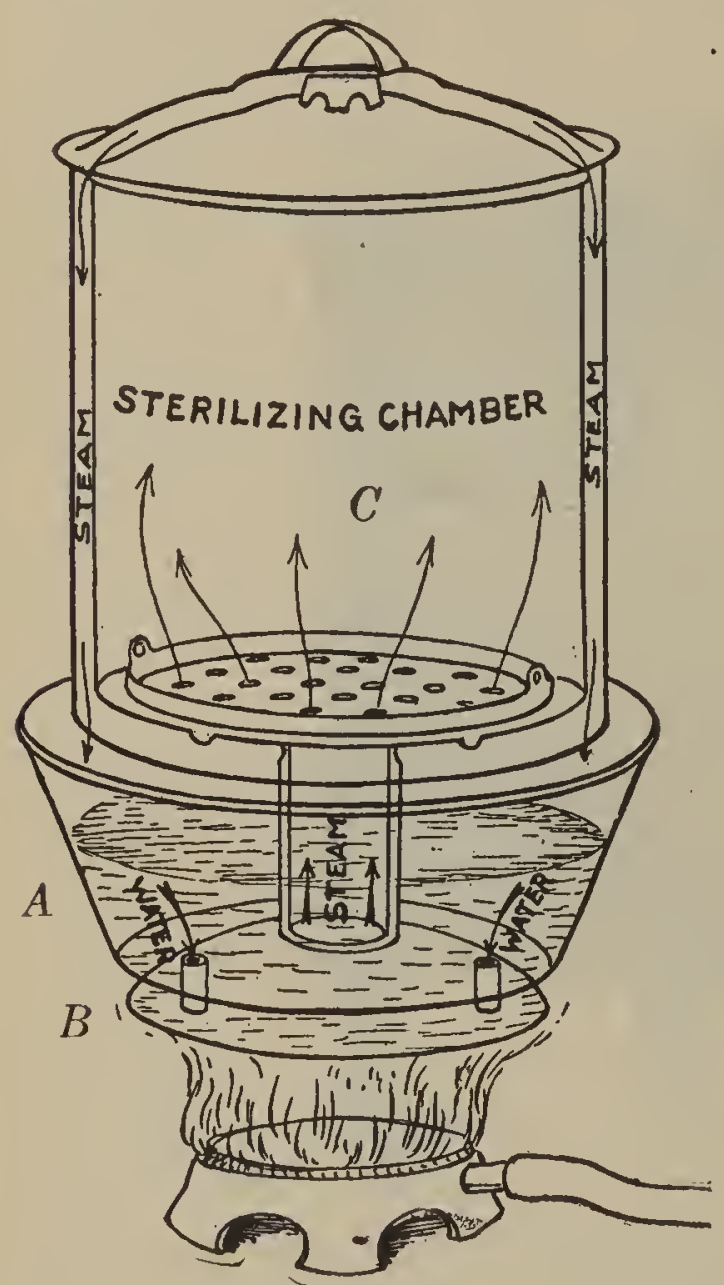

Fig. 9.-Arnold Sterilizer. to steam in such an apparatus for fifteen to thirty minutes insures the death of the vegetative forms of bacteria.

In the sterilization of media by such a device, the method of fractional steritization at $100^{\circ} \mathrm{C}$. is employed. The principle of this method depends upon repeated exposure of the media for fifteen minutes to one-half hour on three succeeding days. By the first exposure all vegetative forms are destroyed. The media may then be left at room temperature, or at incubator temperature $\left(37.5^{\circ} \mathrm{C}\right.$.) until the following day, when any spores which may be present will have developed into the vegetative stage. These are then killed by the second exposure. A repetition of this procedure on a third day insures sterility. It must always be remembered, however, that this method is applicable only in cases in which the substance to be sterilized is a favorable medium for bacterial growth in which it is likely that spores will develop into vegetative forms.

Exceptionally the method may fail even in favorable media when anaërobic spore-forming bacteria are present. Thus, it has been observed that anaërobic spores, failing to develop under the aërobic conditions prevailing during the intervals of fractional sterilization, have developed after inoculation of the media with other bacteria, when symbiosis had made their growth possible. Tetanus bacilli have, in this way, occurred in cultures of diphtheria bacilli employed for toxin production. 
In noting the time of an exposure in an Arnold sterilizer, it is important to time the process from the time when the temperature has reached $100^{\circ} \mathrm{C}$. and not from the time of lighting the flame.

The principle of fractional sterilization at low temperatures is applied also to the sterilization of substances which can not be subjected to temperatures as high as $100^{\circ} \mathrm{C}$. This is especially the case in the sterilization of media containing albuminous materials, when coagulation is to be avoided, or when both coagulation of the medium and sterilization are desired.

In such cases fractional sterilization may be practiced in simply constructed sterilizers, such as a Koch inspissator or, in the case of fluids, such as blood serum, by immersion in a water-bath at a temperature

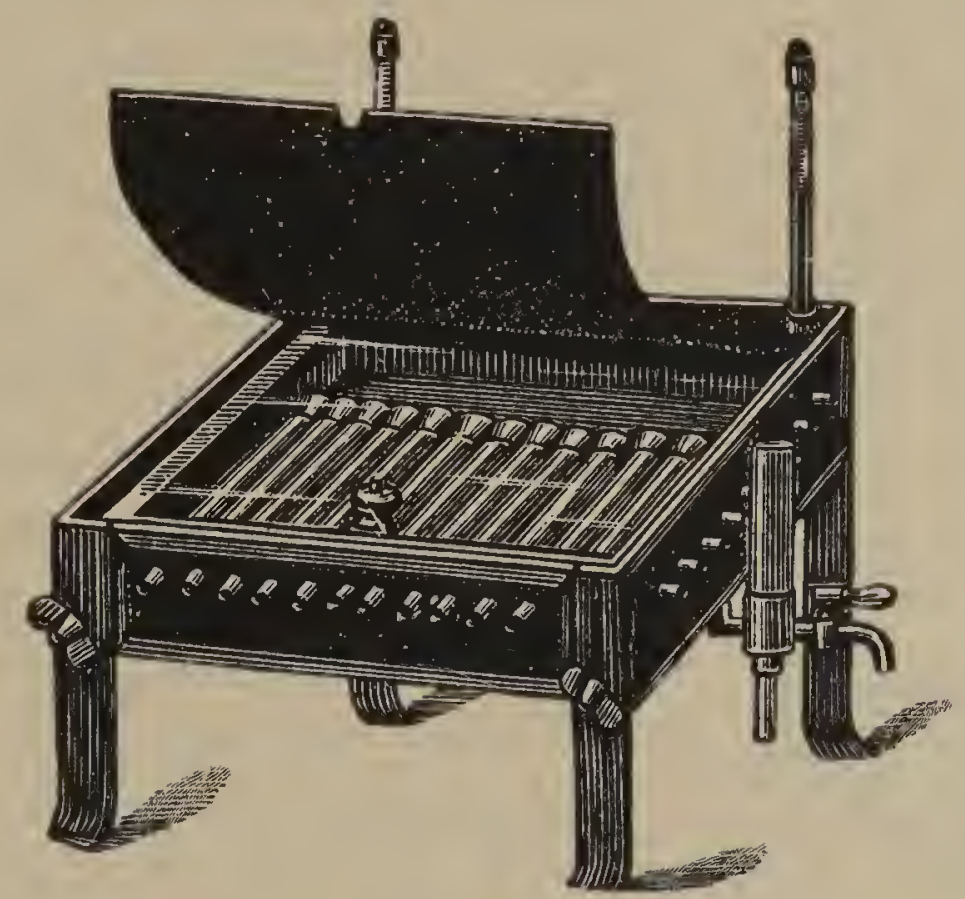

Fig. 10.-Low Temperature Sterilizer (Inspissator).

varying above $55^{\circ} \mathrm{C}$, according to circumstances. Exposures at such low temperatures may be repeated on five or six consecutive days, usually for an hour each day.

The use of steam under pressure is the most powerful method of heatdisinfection which we possess. It is applicable to the sterilization of fomites, clothing, or any objects of a size suitable to be contained in the apparatus at hand, and which are not injured by moisture. In laboratories this method is employed for the sterilization of infected apparatus, such as flasks, test tubes, Petri plates, etc., containing cultures. The device most commonly used in laboratories is the so-called autoclave, of which a variety of models may be obtained, both stationary and portable. The principle governing the construction of all of these 
is the same. The apparatus usually consists of a gun-metal cylinder supplied with a lid, which can be tightly closed by screws or nuts, and supplied with a thermometer, a safety-valve, and a steam pressure gauge. In the simpler autoclaves, water may be directly filled into the lower part of the cylinder, and the objects to be sterilized supported

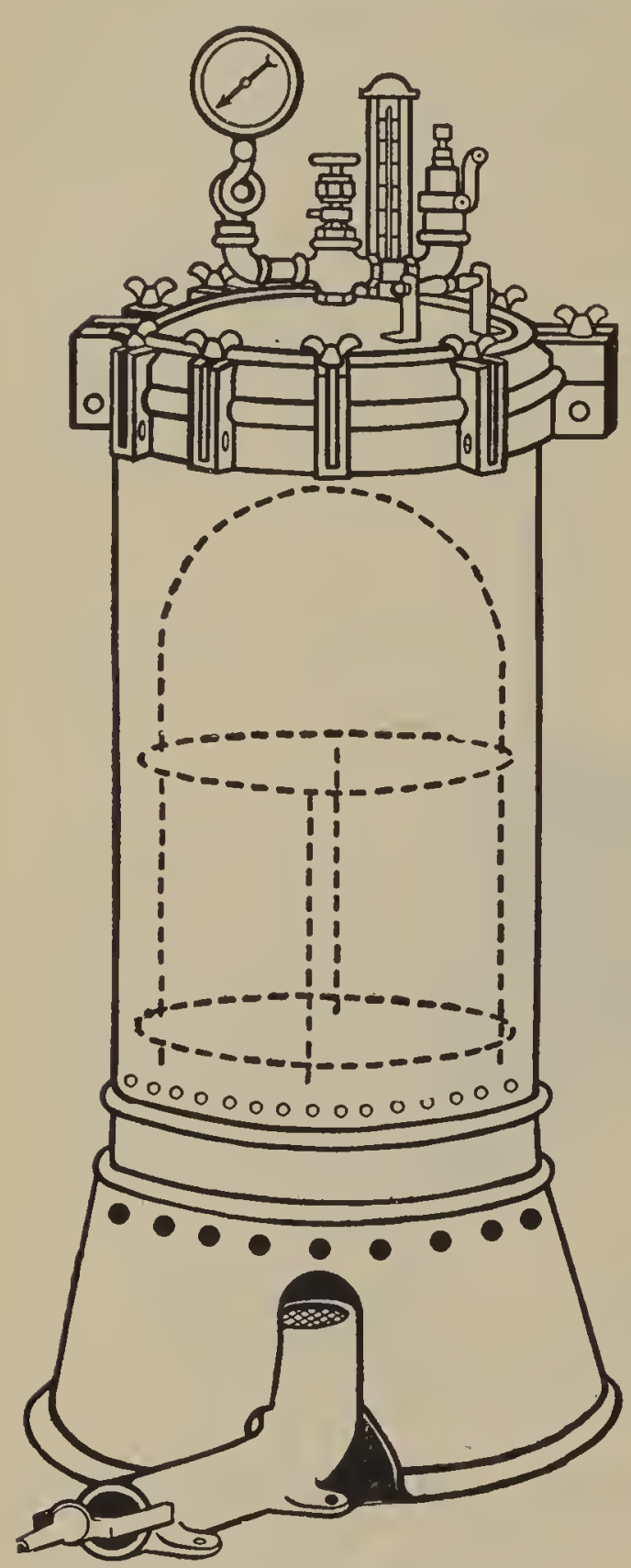

Fig. 11.-Autoclave. upon a perforated diaphragm. In this case the heat is directly applied by means of a gas flame. In the more elaborate stationary devices, steam may be let in by piping it from the regular supply used for heating purposes. Exposure to steam under fifteen pounds pressure (fifteen in addition to the usual atmospheric pressure of fifteen pounds to the square inch) for fifteen to twenty minutes, is sufficient to kill all forms of bacterial life, including: spores.

In applying autoclave sterilization practically, attention must be paid to certain technical details, neglect of which would result in failure of sterilization. It is necessary always to permit all air to escape from the autoclave before closing the vent. If this is not done, a poorly conducting air-jacket may be left about the objects to be sterilized, and these may not be heated to the temperature indicated by the pressure. It is also necessary to allow the reduction of pressure, after sterilization, to take place slowly. Any sudden relief of pressure, such as would be produced by opening the airvent while the pressure gauge is still above zero, will usually result in a sudden ebullition of fluid and a removal of stoppers from flasks.

The temperature attained by the application of various degrees of pressure is expressed in the following table:

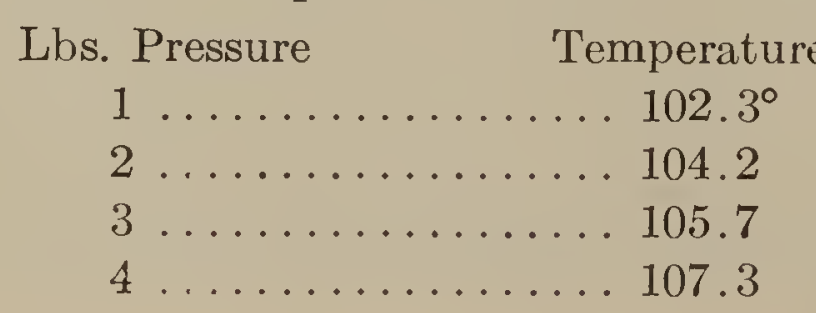

Lbs. Pressure

Temperature

$5 \ldots \ldots \ldots \ldots 108.8^{\circ}$

$6 \ldots \ldots \ldots \ldots \ldots 110.3$

$7 \ldots \ldots \ldots \ldots \ldots 111.7$

$8 \ldots \ldots \ldots \ldots \ldots 113$ 


\begin{tabular}{|c|c|c|c|}
\hline Lbs. Pressure & Temperature & Lbs. Pressure & Temperature \\
\hline 9 & $114.3^{\circ}$ & 17 & $123.3^{\circ}$ \\
\hline 10 & 115.6 & 18 & 124.3 \\
\hline 11 & 116.8 & 20 & 126.2 \\
\hline 12 & 118 & 22 & 128.1 \\
\hline 13 & 119.1 & 24 & 129.3 \\
\hline 14 & . 120.2 & 26 & 131.5 \\
\hline 15 & .121 .3 & 28 & 133.1 \\
\hline $16 \ldots$ & . . 122.4 & 30 & 134.6 \\
\hline
\end{tabular}

\section{CHEMICAL AGENTS INJURIOUS TO BACTERIA}

Since the time of Koch's ${ }^{1}$ fundamental rescarches upon chemical disinfectants, the known number of these substances has been enormously increased, and now embraces chemical agents of the most varied constitution. It is thus manifestly impossible to refer the injurious influence which these substances exert upon bacteria to any uniform law of action. The efficiency of a disinfecting agent, furthermore, is not alone dependent upon the nature and concentrations of the substance itself, but depends complexly upon the nature of the solvent in which it is employed, the temperature prevailing during its application, the numbers and biological characteristics of the bacteria in question, and the time of exposure. All these factors, therefore, must be considered in testing the efficiency of any given disinfectant. While it is true, furthermore, that all substances which in a given concentration exert bactericidal or disinfecting action upon a microorganism, will in greater dilution act antiseptically or inhibitively, no definite rules of proportion exist between the two values, which in each case must be determined by experiment.

Disinfectants Used in Solution.-The actual processes which take place in the injury of bacteria by disinfectants are to a large extent unknown. In the case of strong acids, or strongly oxidizing substances, there may be destruction of the bacterial body as a whole by rapid oxidation. Other substances may act by coagulation of the bacterial protoplasm; others again by diffusion through the cell membrane are able to enter into chemical combination with the protoplasm and exert a toxic action. Again, in other cases, a difference in tonicity between cell protoplasm and disinfectant may tend to withdrawal of water from the bacterial cell and consequent injury of the microorganism.

Among the inorganic disinfectants the most important are the metallic

${ }^{1}$ Koch, Arb. a. d. kais. Gesundheitsamt, i, 1881. 
salts, acids, and bases, the halogens and their derivatives, and certain oxidizing agents like peroxide of hydrogen and permanganate of potassium.

It has been shown by Scheuerlen and Spiro, ${ }^{1}$ Krönig and Paul, ${ }^{2}$ and others, that in the case of the salts, acids, and bases, there is a distinct and demonstrable relationship between the disinfecting power of these substances and their dissociation in solution.

According to the theory of electrolytic dissociation, when bodies of this class go into solution they are broken up or dissociated into an electro-positive and an electro-negative ion. Thus, metallic salts are broken up into the kation, or positive metal, and into the anion, or negative acid radicle $\left(\mathrm{AgNO}_{3}=\mathrm{Ag}\right.$, + ion and $\mathrm{NO}_{3},-$ ion $)$. In the case of the acids, ionization takes place into the hydrogen ions and the acid radicles, while in the case of the bases the dissociation occurs into the metal, on the one hand, and the OH group on the other. The degree of dissociation taking place depends upon the nature of the substance in solution, its concentration, and the nature of the solvent. Thus, in any such solution there appear three substances, the undissociated compound as such, its electro-negative ion, and its electropositive ion, their relative concentrations depending upon an interrelationship calculable by definite laws. It goes without saying, therefore, that any chemical or physical reaction, taken part in by such a solution, may be participated in, not only by the dissolved undissociated residue as a whole, but by its separate ions individually as well. In the case of many disinfectants, the writers referred to above have been able to demonstrate a relationship between the degree of dissociation and the bactericidal powers. According to Krönig and Paul, double metallic salts, in which the metal is a constituent of a complex ion and in which the concentration of the dissociated metal-ions is consequently low, have very little disinfecting power. Thus potassium-silver-cyanide, which is a comparatively weak disinfectant, dissociates into the kation IK and the complex anion $\mathrm{Ag}(\mathrm{CN})_{2}$, this latter further dissociating to a very slight degree only. The same writers conclude that the bactericidal action of mercuric chloride and of halogen combinations with metals is directly proportionate to the degree of dissociation. This consideration, moreover, explains why aqueous solutions of such substances are more active than are solutions in the alcohols or in ether, since it is well

${ }_{1}^{1}$ Scheuerlen und Spiro, Münch. med. Woch., 44, 1897.

${ }^{2}$ Krönig und Paul, Zeit. f. Hyg., xxv, 1897. 
known that metallic salts are ionized in these substances to a much slighter degree than they are in water. ${ }^{1}$

On the other hand, the addition of moderate quantities of ethyl and methyl alcohol or acetones to aqueous solutions of silver nitrate or mercuric chloride, definitely increases the disinfecting action of such solutions. In the case of mercuric chloride, Krönig and Paul obtained the most powerful effects in solutions to which alcohol had been added in a concentration of 25 per cent. For this empirical fact a satisfactory explanation has not yet been found. Krönig and Paul suggest that low percentages of alcohol may facilitate the penetration of the disinfectant through the cell membrane and thus increase its efficiency, while high percentages of alcohol have the opposite effect, by decreasing the degree of dissociation. In this connection it has been suggested, however, that absolute and strong alcohols possibly act as desiccating agents, thus actually rendering the bacteria dry and less susceptible to deleterious chemical influences.

In the case of acids and bases the same authors have determined that the powers of disinfection of these substances are again directly proportionate to the degree of their dissociation: that is, to the concentration of the hydrogen or hydroxyl ions, respectively. The hydrogen ions are more powerfully active than the hydroxyl ions in equal concentration; acids, therefore, are more efficient disinfectants than bases.

A fact which appears to strengthen the opinion as to the relationship between bactericidal powers and dissociation, is that brought forward by Scheuerlen and Spiro, that the addition of $\mathrm{NaCl}$ to bichloride of mercury solutions reduces the disinfecting power of such solutions, inasmuch as it diminishes the concentration of free ions. In practice, however, $\mathrm{NaCl}$ or $\mathrm{NH}_{4} \mathrm{Cl}$ is added to bichloride of mercury solutions, since these substances aid in holding in solution mercury compounds formed in the presence of alkaline albuminous material, blood serum, pus, etc.

In regard to the halogens, Krönig and Paul have shown that the germicidal power of this class of elements is inversely proportionate to their atomic weights. Thus, chlorine with the lowest atomic weight is the strongest disinfectant of the group. Next, and almost equal to this, is

${ }^{1}$ Water is the strongest dissociant known. Methyl alcohol has about one-half to two-thirds the dissociating power of water (Zelinsky, Zeit. f. physiol. Chemie, xx, 1896). Ethyl alcohol allows dissociation much less than methyl alcohol; ammonia allows dissociation to about one-third to one-fourth the extent of water. See Jones, "Elements of Physical Chemistry," p. 371. Macmillan, New York, 1902. 
bromin. Iodine with a much heavier atomic weight than either of the former is distinctly less bactericidal.

Chloride of Lime.-Of the halogen compounds commonly used in practice, the most important is what is popularly known as chloride of lime or bleaching powder. As to the composition of this substance, there is some difference of opinion. It was formerly believed to be a mixture of calcium hypochlorite, $\mathrm{Ca}\left(\mathrm{ClO}_{2}\right)$, and of calcium chloride, $\mathrm{CaCl}_{2}$. The fact that the substance is not deliquescent, however, speaks against the presence of calcium chloride as such, and it is probable that it consists of a single compound with the formula $\mathrm{CaOCl}_{2}$. The action of acids or even of atmospheric $\mathrm{CO}_{2}$ upon this substance results in the liberation of chlorine. For instance,

$$
\begin{aligned}
& \mathrm{Ca}\left(\mathrm{Cl}_{2} \mathrm{O}\right)+2 \mathrm{HCl}=\mathrm{CaCl}_{2}+2 \mathrm{HClO} . \\
& 2 \mathrm{HClO}+2 \mathrm{HCl}=2 \mathrm{H}_{2}+2 \mathrm{Cl}_{2} .
\end{aligned}
$$

Bleaching powder is readily soluble in about twenty parts of water. According to Nissen, ${ }^{1}$ solutions of 2 in 1,000 of this substance, destroy vegetative forms of bacteria in five to ten minutes.

TERChLORIDE OF IODINE $\left(\mathrm{ICl}_{3}\right)$, another halogen derivative, is an extremely strong disinfectant, being efficient for vegetative forms in solutions of 0.1 per cent in one minute and a 1 per cent solution destroying spores within a few minutes. ${ }^{2}$

Surgeons have found that painting with tincture of iodine (10 per cent) is a simple and reliable method of sterilizing the skin. It is now used in many clinics as the sole disinfecting agent in sterilizing the field of operation.

The oxidizing agents most commonly employed are peroxide of hydrogen $\left(\mathrm{H}_{2} \mathrm{O}_{2}\right)$ and permanganate of potassium $\left(\mathrm{KMnO}_{4}\right)$.

Peroxide of Hydrogen is formed by the action of dilute sulphuric acid upon peroxide of barium. It readily gives up oxygen and acts upon bacteria probably by virtue of the liberation of nascent oxygen. In the presence of organic matter such as blood, pus, etc., associated with bacteria, $\mathrm{H}_{2} \mathrm{O}_{2}$ is quickly reduced and weakened. It is important that the $\mathrm{H}_{2} \mathrm{O}_{2}$ come in immediate contact with the bacteria. In practice, therefore, blood and pus should be removed from wounds when applying the $\mathrm{H}_{2} \mathrm{O}_{2}$ or a large excess of $\mathrm{H}_{2} \mathrm{O}_{2}$ should be used.

Permanganate of Potassium, acting probably in the same way, is a powerful germicide. It also is readily reduced by many organic substances often associated with bacteria, being rendered weaker thereby.

${ }^{1}$ Nissen, Zeit. f. Hyg., viii, 1890.

${ }^{2} v$. Behring, Zeit. f. Hyg., ix, 1891. 
Among organic disinfectants those of most practical importance are the alcohols, formaldehydes, iodoform, members of the phenol group and its derivatives, carbolic acid, cresol, lysol, creolin, salicylic acid, certain ethereal oils, and, more recently introduced, organic silver salts such as protargol, argyrol, argonin, and others.

The ALCOHOLS are but indifferent disinfectants. Koch ${ }^{1}$ in 1881 found that anthrax spores remained alive for as long as four months when immersed in absolute and in 50 per cent ethyl alcohol. On the other hand, while absolute alcohol possesses practically no germicidal powers, possibly because of the formation of a protecting envelope by the coagulation of the bacterial ectoplasm, or, as suggested above, by desiccation due to the abstraction of water, dilute alcohol in a concentration of about 50 per cent is distinctly germicidal, destroying the vegetative forms of bacteria in from ten to fifteen minutes or less. ${ }^{2}$ Attention has already been called to the fact that moderate additions of alcohol to aqueous solutions of mercuric chloride enhance the germicidal power of this disinfectant. Additions of ethyl and methyl alcohol to carbolic acid or formaldehyde solutions, on the other hand, progressively decrease the bactericidal activities of these substances. $^{3}$

The value of boiling alcohol for the destruction of spores-especially in the sterilization of catgut-has been investigated by Saul, ${ }^{4}$ who found that boiling in absolute ethyl, methyl, or propyl alcohol is practically without effect, while spores are destroyed readily in boiling dilute alcohol, the most effectual being propyl alcohol of a concentration of from $10-40$ per cent.

Iodoform $\left(\mathrm{CHI}_{3}\right)^{5}$ is weakly antiseptic in itself, but when introduced into wounds where active reducing processes are taking place-often as the result of bacterial growth-iodine is liberated from it and active bactericidal action results.

CARBolic ACID $\left(\mathrm{C}_{6} \mathrm{H}_{5} \mathrm{OH}\right)$, at room temperature, consists of colorless crystals which become completely liquefied by the addition of 10 per cent of water. In contradistinction to most inorganic disinfectants, the action of carbolic acid and other members of the phenol group is

1 Koch. Arb. a. d. kais. Gesundheitsamt, i, 1881.

${ }^{2}$ Epstein, Zeit. f. Hyg., xxiv, 1897.

${ }^{3}$ Krönig und Paul, loc. cit.

- Saul, Archiv f. klin. Chir., 56, 1898.

5 v. Behring, "Bekaempfung d. Infektions-Krankh.," Leipzig, 1894, 
not in any way dependent upon dissociation. ${ }^{1}$ According to Beckmann ${ }^{2}$ and others, carbolic acid acts as a molecule and not by individual ions. The proof of this is brought out by the fact that the addition of $\mathrm{NaCl}$ to carbolic acid solutions, an addition which would tend to decrease the concentration of free ions, markedly increases the bactericidal powers of such solutions. On the other hand, as stated above, additions of alcohol progressively diminish the efficiency of the phenols.

Other members of this group of disinfectants are onTHO-, META-, and PARACRESOL $\left(\mathrm{C}_{6} \mathrm{H}_{4} \mathrm{CH}_{3} \mathrm{OH}\right)$, isomeric compounds differing only in the position of the $\mathrm{OH}$ radicle. Tricresol is a mixture of these three. The cresols are relatively more powerfully germicidal than is carbolic acid, but are less soluble in water. Lysou is a substance obtained by the solution of coal-tar cresol in neutral potassium-soap. Dissolved in water it forms an opalescent easily flowing liquid. According to Gruber, ${ }^{3}$ its germicidal action is slightly greater than that of carbolic acid. Creolin, another combination of the cresols with potassic soap, forms with water a turbid emulsion. v. Behring ${ }^{4}$ expressed the relative germicidal powers of carbolic acid, cresol, and creolin for vegetative forms by the numbers $1: 4: 10$, in the order named.

Formaldehyde (H-COH), or methyl aldehyde, is a gas which is easily produced by the incomplete combustion of methyl alcohol. The methods of actually generating it for purposes of fumigation will be discussed in a subsequent paragraph. In aqueous solution this substance forms a colorless liquid with a characteristic acrid odor, and in this form is largely used as a preservative for animal tissues and as a germicide. It is marketed as "formalin," which is an aqueous solution containing from 35 to 40 per cent of the gas and which exerts distinctly bactericidal action on vegetative forms in further dilutions of from 1 to 10 to 1 to 20 (formaldehyde gas 1: 400 to 1 : 800). Anthrax spores are killed in 35 per cent formaldehyde in ten to thirty minutes. ${ }^{5}$ Unlike the phenols, the addition of salt to formaldehyde solutions does not increase its efficiency, but similar to them, additions of ethyl and methyl alcohol markedly reduce its germicidal powers.

The essential oils which are most commonly used in practicelargely as intestinal antiseptics-are those of cinnamon, thyme, eucalyp-

1 Scheuerlen und Spiro, Münch. med. Woch., 44, 1897.

2 Beckmann, Cent. f. Bakt., I, xx, 1896.

${ }^{3}$ Gruber, Cent. f. Bakt, I., xi, 1892.

* v. Behring, loc. cit., p. 111.

'Krönig und Paul, loc. cit. 
tus, and peppermint. Omeltschenko ${ }^{1}$ believes that the employment of these oils in emulsions is illogical, inasmuch as their bactericidal powers depend upon their vaporization. He classifies the oils in decreasing order of their efficiency as follows: Oil of cinnamon, prunol, oil of thyme, oil of peppermint, oil of camphor, and eucalyptol.

Methods of Testing the Efficiency of Disinfectants.-The efficiency of any given disinfectant depends, as we have seen, upon a number of factors, any one of which, if variable, may lead to considerable differences in the end result. Thus, as far as the bacteria themselves are concerned, it is necessary to remember that not only do separate species differ in their resistance to disinfectants, but that different strains within the same species may show such variations as well. This fact largely accounts for the widely varying reports made by different investigators as to the resistance of anthrax spores, and depends possibly upon temporary or permanent biological differences produced in bacteria by the conditions of their previous environment.

The numbers of bacteria exposed to the disinfectant, furthermore, is a factor which should be kept constant in comparative tests. The medium, moreover, in which bacteria are brought into contact with the disinfectant is a matter of great importance, inasnuch as either by entering into chemical combination with the disinfectant it may detract from its concentration or by coagulation it may form a purely mechanical protection for the microorganism. Thus bacteria which may be destroyed in distilled water or salt-solution emulsion with comparative ease, may evince an apparently higher resistance if acted upon in the presence of blood serum, mucus, or other albuminous substances. Temperature influences bactericidal processes in that most chemical disinfectants are more actively bactericidal at higher than at lower temperatures, a fact due most likely to the favorable influence of temperature upon all chemical reactions. ${ }^{2}$ As far as merely inhibitory or antiseptic values are concerned, however, the temperature least favorable for the reaction of the antiseptic is that which represents the optimum growth temperature for the microorganism in question and the inhibitory effects of any substance are less marked at this point than at temperatures above or below it.

The important influence exerted by the solvent in which the

1 Omeltschenko, Cent. f. Bakt., I, ix, 1891.

${ }^{2} v$. Behring, "Bekaempf. der Infektions-Krankh., Infektion u. Desinfection," Leipzig, 1894. 
disinfectant is employed has already been discussed. For ordinary work it is customary to express absolute and comparative antiseptic and bactericidal values in terms of percentages based upon weight, and this, beyond question, is both simple and practical. For strictly scientific comparisons, however, as Krönig and Paul ${ }^{1}$ have pointed out, it is by far more accurate to work with equimolecular solutions.

Rideal and Walker ${ }^{2}$ have devised a method of testing disinfectants, in which an attempt is made to establish a standard for comparisons. They choose, as the standard, carbolic acid, and establish what they call the "carbolic-acid coefficient." This coefficient they obtain in the following way: the particular dilution of the disinfectant under investigation which will kill in a given time, is divided by the strength of carbolic acid which, under the same conditions, will kill the same bacteria in the same time. We quote an example of such a test, given by Simpson and Hewlett, ${ }^{3}$ comparing formalin and carbolic acid.

BACILLUS PESTIS.

\begin{tabular}{|c|c|c|c|c|c|c|c|}
\hline \multirow[b]{2}{*}{ Sample. } & \multirow[b]{2}{*}{ Dilution. } & \multicolumn{6}{|c|}{ Time in Minutes. } \\
\hline & & 2.5 & 5 & 7.5 & 10 & 12.5 & 15 \\
\hline $\left.\begin{array}{l}\text { Formalin........ } \\
\text { Carbolic acid...... }\end{array}\right\}$ & $\begin{array}{l}1 \text { in } 30 \\
1 \text { in } 40 \\
1 \text { in } 100 \\
1 \text { in } 110\end{array}$ & $\begin{array}{l}\text { growth } \\
\text { growth } \\
\cdots \cdots \\
\text { growth }\end{array}$ & $\begin{array}{c}\text { growth } \\
\text { growth } \\
\cdots \cdots \\
\text { growth }\end{array}$ & $\begin{array}{l}\cdots \cdots \\
\text { growth } \\
\ldots \ldots \\
\ldots\end{array}$ & & & $\cdots$ \\
\hline
\end{tabular}

In the above table, formalin 1 in 30 killed in the same time as carbolic acid 1 in 110 . Thus the carbolic-acid coefficient of formalin in this test $={ }^{30 / 110}=.27$.

The Rideal-Walker method has been much used and is recommended by many workers. ${ }^{4}$

The most precise method of standardizing disinfectants is that now in use in the U. S. Public Health Service. It is a modification of the Rideal-Walker procedure devised by Anderson and McClintic. ${ }^{5}$

Stock 5 per cent solutions of the disinfectant in question and of the

\footnotetext{
${ }^{1}$ Krönig und Paul, loc. cit

${ }^{2}$ Rideal and Walker, Jour. of the Sanitary Ins. London, xxiv.

${ }^{3}$ Simpson and Hewlett, Lancet, ii, 1904.

${ }^{4}$ Sommerville, Brit. Med. Jour., 1904.

${ }^{5}$ Anderson and McClintic, Jour. of Inf. Dis., 1911, viii, 1.
} 
standard (phenol) are first prepared and a series of accurate dilutions made with distilled water using graduated pipettes. (To make 1:70 take 4 c.c. of stock and 10 c.c. distilled water; $1: 80=4$ c.c. of stock +12 c.c. distilled water; $1: 90=4$ c.c. stock +14 c.c. distilled water; $1: 500$ $=2$ c.c. of stock +48 c.c. of distilled water. Complete dilution tables are given in their original article.) The series should include dilutions strong enough to kill B. typhosus in two and a half minutes and weak enough to fail to do so in fifteen minutes. If dilutions greater than 1500 are required, a second 1 per cent stock solution is prepared. They adopted the following scale for their tests: Dilutions up to 1:70 should vary from the next in the series by a difference of 5 (i.e., 5 parts of water).

From $1: 70$ to $1: 160$ by a difference of 10
From $1: 160$ to $1: 200$ by a difference of 20
From $1: 200$ to $1: 400$ by a difference of 25
From $1: 400$ to $1: 900$ by a difference of 50
From $1: 900$ to $1: 1800$ by a difference of 100
From $1: 1800$ to $1: 3200$ by a difference of 200

and so on if higher dilutions are necessary.

Short wide test tubes 1 inch by 3 inches are used in making the test. These are placed in a rack in a water bath at $20^{\circ} \mathrm{C}$. Five c.c. of each dilution are measured into a series of these tubes beginning with the strongest specimen and rinsing the pipette once with each dilution before the 5 c.c. are measured out. For inoculation, a 24-hour broth culture of $\mathrm{B}$. typhosus is prepared which has been transferred daily for at least 3 days. Before use it is shaken and filtered through sterile filter paper. The wide test tubes containing diluted disinfectant are inoculated with $1 / 10$ c.c. of this culture with a graduated pipette. The tip of the pipette is held against the side of the tube to insure accurate measurement and the tube immediately shaken to mix the bacteria thoroughly with the disinfectant. Test inoculations are made from this mixture at proper intervals into tubes containing 10 c.c. of standard extract broth of +1.5 acidity, using loops $4 \mathrm{~mm}$. in diameter. At least four such loops should be at hand, supported on a rack or wooden block so that a fan-tail Bunsen burner may be placed under each wire in turn. Each one is sterilized after a plant is made and allowed to cool while the other three are being used in order.

The test is conducted as follows: A row of ten wide tubes containing dilutions of the antiseptic is placed in the water bath at $20^{\circ} \mathrm{C}$. and time allowed for them to reach the temperature of the bath. They are then inoculated in order at intervals of exactly 15 seconds. Fifteen seconds 
after the last tube has been inoculated a subculture is made from the first tube of the series (i.e., $21 / 2$ minutes after this first tube was inoculated) and from the other tubes in order at 15-second intervals. Fifteen seconds after this first series of subcultures is completed a second series of subcultures is begun which will give the result of a 5-minute exposure to the antiseptic and the subinoculations continued at 15-second intervals until all dilutions have been tested for fifteen minutes. If the strength of the antiseptic is known approximately subcultures of the lower dilutions for the longer periods may be omitted. It is convenient to have an assistant at hand to call time and to label the subcultures as soon as made. The tubes may, however, be placed in order in suitable racks

\section{DETERMINATION OF THE CARBOLIC-ACID COEFFICIENT OF A DISINFECTANT.}

(Anderson and McClintic)

NAME. "A"

Temperature of Medication.............................. Culture Used B. Typhosus....................... Extract Broth, Filtered Proportion of Culture and Disinfectant............... 0.1 c.c. +5 c.c. Organic Matter, None; Kind, None; Amount, None.

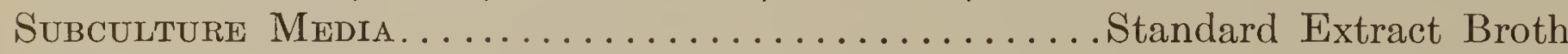

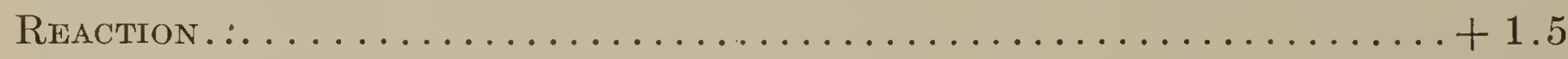

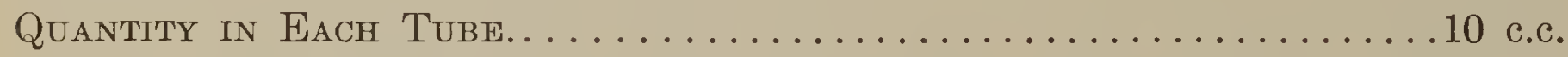

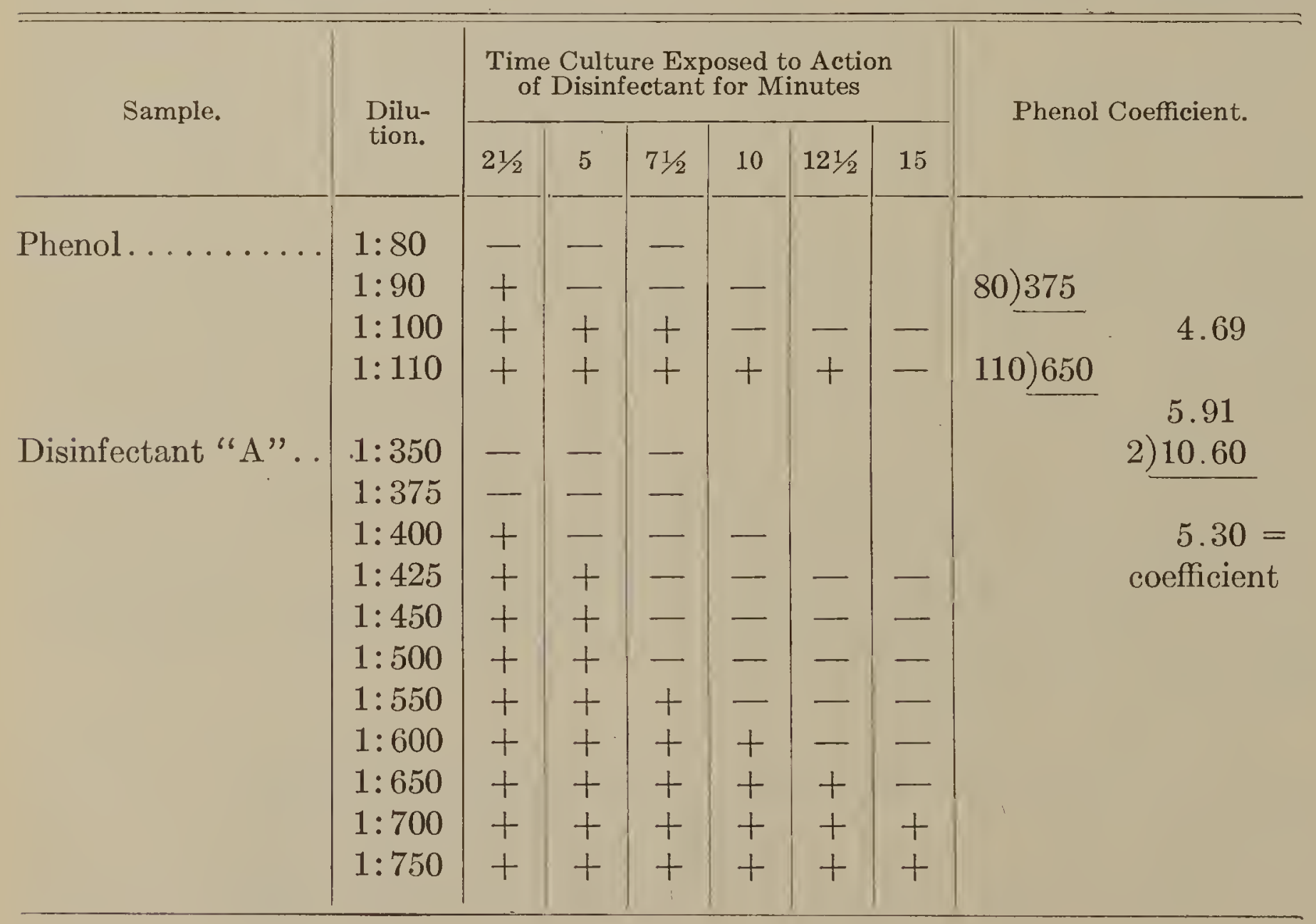


without labelling. The subculture tubes are incubated for 48 hours at $37^{\circ} \mathrm{C}$. and those in which growth is observed are recorded positive.

To obtain the coefficient the weakest dilution of the unknown antiseptic which kills in $2 \frac{1}{2}$ minutes is divided by the weakest dilution of phenol which kills in the same time. The same is done for the weakest strength that kills in 15 minutes and an average is taken. The results of such a test are shown in the table on page 82 .

As only the $21 / 2$-minute and 15 -minute intervals are used in determining this result it seems unnecessary to make plants at the intervening periods except in special cases where more detailed information is desired.

The procedure may be modified by adding some organic substance such as killed bacteria to the diluted antiseptic. For many substances, e.g., bichloride of mercury, the antispetic value in presence of organic matter is much lower than in watery solution. Anderson and McClintic insist that great care in making the dilutions and rigid adherence to a uniform technique are necessary to obtain consistent results in such tests.

Determination of Antiseptic Values.-The antiseptic or inhibitive strength of a chemical substance, sometimes spoken of as the "coefficient of inhibition," is determined by adding to definite quantities of a given culture medium, graded percentages of the chemical substance which is being investigated and planting in these mixtures equal quantities of the bacteria in question. The medium used for the tests may be nutrient broth or melted gelatin or agar. If broth is used, growth is estimated by turbidity of the medium and by morphological examination; if the agar or gelatin is employed, plates may be poured and actual growth observed.

Thus, in the case of carbolic acid, a 5 or 10 per cent solution is prepared and added to tubes of the medium, as follows:

Tube 1 contains $5 \%$ carbolic 2 c.c. + broth 8 c.c. $=1: 1,000$ carbolic acid.

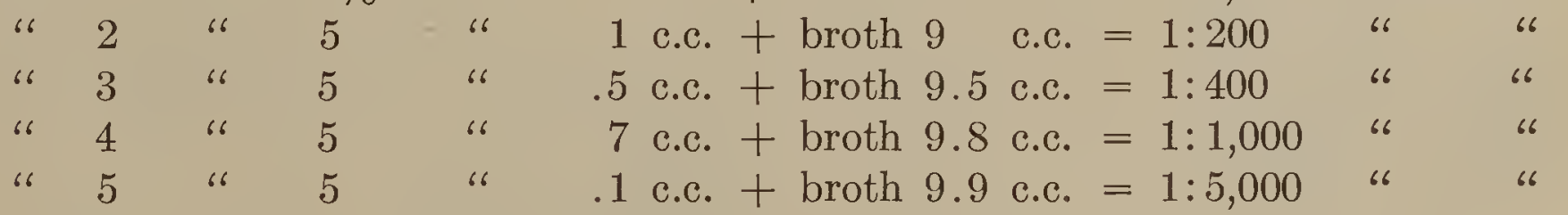

To each of these tubes a definite quantity of the bacteria is added either by means of a standard loopful of a fresh agar culture, or better by a measured volume of an even emulsion in sterile salt solution. The inoculated tubes are then incubated at a temperature corresponding to the optimum growth temperature for the microorganism in question. The tubes are examined for growth from day to day. From tubes containing higher dilutions, in which no growth is visible, transplants 
INHIBITION STRENGTHS OF VARIOUS ANTISEPTICS.

Adapted from FlügGe, Leipzig, 1902.

\begin{tabular}{|c|c|c|c|}
\hline & Anthrax Bacilli. & Other Bacteria. & $\begin{array}{l}\text { Putrefactive Bac } \\
\text { teria in Bouillon. }\end{array}$ \\
\hline \multicolumn{4}{|l|}{ AcIDs } \\
\hline Sulphuric. . & $1: 3,000$ & Chol. spir. $1: 6,000$ & \\
\hline Hydrochloric . & $1: 3,000$ & B. diph. $1: 3,000$ & \\
\hline & & $\begin{array}{l}\text { B. mallei } 1: 700 \\
\text { B. typh. } 1: 500\end{array}$ & \\
\hline Sulphurous & & Chol. spir. $1: 1,000$ & $1: 6,000$ \\
\hline Arsenous........... & ..... & $\ldots \ldots \ldots \ldots$ & $1: 200$ \\
\hline Boric & $1: 800$ & $\ldots \ldots \ldots$ & $1: 100$ \\
\hline \multicolumn{4}{|l|}{ Alkalies } \\
\hline \multirow[t]{3}{*}{ Potass. hydrox.......... } & $1: 700$ & B. diphth. $1: 600$ & \\
\hline & & Chol. spir. $1: 400$ & \\
\hline & & B. typh. $1: 400$ & \\
\hline \multirow[t]{2}{*}{ Ammon. hydrox. } & $1: 700$ & Chol. spir. $1: 500$ & \\
\hline & & B. typh. $1: 500$ & \\
\hline \multirow{2}{*}{ Calcium hydrox......... } & & Chol. spir. $1: 1,100$ & \\
\hline & & B. typh. $1: 1,100$ & \\
\hline \multicolumn{4}{|l|}{ SALTS } \\
\hline Copper sulphate & & & $1: 1,000$ \\
\hline Ferric sulphate & $\ldots \ldots$ & $\ldots \ldots \ldots \ldots \ldots$ & $1: 90$ \\
\hline Mercuric chlorid. ..... & $1: 100,000$ & B. typhosus $1: 60,000$ & $1: 20,000$ \\
\hline Silver nitrate ......... & $1: 60,000$ & $\begin{array}{l}\text { Chol. spir. } \\
\text { B. typhosus } 1: 50,000\end{array}$ & \\
\hline Potass. perman. & $1: 1,000$ & $\ldots \ldots \ldots \ldots \ldots$ & $1: 500$ \\
\hline \multicolumn{4}{|l|}{ HaLOGENS AND CoMpounds } \\
\hline Chlorin ... & $1: 1,500$ & & $1: 4,000$ \\
\hline Bromin ... & $1: 1,500$ & & ]$: 2,000$ \\
\hline Iodin . . . . . . . & $1: 5,000$ & $\ldots \ldots \ldots$ & $1: 5,000$ \\
\hline Potass. iodid & $\ldots \ldots \ldots$ & $\ldots \ldots \ldots \ldots$ & $1: 7$ \\
\hline Sodium chlor. . & $1: 60$ & & \\
\hline \multicolumn{4}{|l|}{ ORGaNIC COMPOUNDS } \\
\hline Ethyl alcohol....... & $1: 12$ & & $1: 10$ \\
\hline Acetic and oxalic acids & & B. diph. $1: 500$ & $1: 400$ \\
\hline Carbolic acid & 1. 800 & $\begin{array}{l}\text { B. typh. } 1: 400 \\
\text { Chol. spir. } 1: 600\end{array}$ & \\
\hline Benzoic acid & $1: 1,000$ & & \\
\hline Salicylic acid . ..... & $1: 1,500$ & & \\
\hline \multirow{2}{*}{$\begin{array}{c}\text { Formalin }(4 \% \text { formalde- } \\
\text { hyde }) \ldots \ldots \ldots \ldots \ldots\end{array}$} & & & \\
\hline & & $\begin{array}{l}\text { Chol. spir. } 1: 20,000 \\
\text { Staphylo. } 1: 5,000\end{array}$ & $1: 1,000$ \\
\hline Camphor............... & $1: 1,000$ & & \\
\hline Thymol ...... & $1: 10,000$ & . & $1: 3,500$ \\
\hline Oil mentha pip & $1: 3,000$ & & \\
\hline Oil of terebinth... & $1: 8,000$ & & \\
\hline Peroxide of hydrogen. . & & & $1: 2,000$ \\
\hline
\end{tabular}




\section{BACTERICIDAL STRENGTHS OF COMMON DISINFECTANTS.}

Adapted from Flügge, Leipzig, 1902.

\begin{tabular}{|c|c|c|c|c|}
\hline & \multirow{2}{*}{$\frac{\begin{array}{c}\text { Strepto-and } \\
\text { Staphylo- } \\
\text { cocci. }\end{array}}{5 \text { Minutes. }}$} & \multicolumn{2}{|c|}{$\begin{array}{l}\text { Anthrax and Typhoid Bacilli. } \\
\text { Cholera Spirillum. }\end{array}$} & \multirow{2}{*}{ Anthrax Spores. } \\
\hline & & 5 Minutes. & 2-24 Hours. & \\
\hline \multicolumn{5}{|l|}{ AcIDS } \\
\hline Sulphuric & $1: 10$ & $1: 100$ & $1: 1,500$ & $1: 50$ in 10 days \\
\hline Hydrochloric & $1: 10$ & $1: 100$ & $1: 1,500$ & $1: 50$ in 10 days \\
\hline Sulphurous . & .. & $\ldots \ldots$ & $\begin{array}{r}\text { (Typhoid } \\
1: 700)\end{array}$ & \\
\hline Sulphurous & & $\cdots$ & $\begin{array}{r}1: 300(\mathrm{Gas} \\
10 \text { vol. } \%)\end{array}$ & \\
\hline Boric. & & . & $1: 30$ & $\begin{array}{c}\text { Conc. sol. incomplete } \\
\text { disinfection }\end{array}$ \\
\hline \multicolumn{5}{|l|}{ AlKalies } \\
\hline Potass. hydrox. . & $1: 5$ & $1: 300$ & & \\
\hline Ammon. hydrox... & .. & $1: 300$ & & \\
\hline Calcium ........ & $\cdots$ & $1: 1,000$ & & \\
\hline \multicolumn{5}{|l|}{ SAlts } \\
\hline Copper sulphate .. & & $\ldots$ & & $1: 20$ (5 days $)$ \\
\hline Mercuric chlor. . & $\begin{array}{c}1: 10,000 \text { to } \\
1,000\end{array}$ & $1: 2,000$ & $1: 10,000$ & $1: 2,000$ (26 hours) \\
\hline Silver nitrate & $1: 1,000$ & . & $1: 4,000$ & \\
\hline $\begin{array}{l}\text { Potass. permang. . } \\
\text { "Calc. chlorid" . . }\end{array}$ & $\begin{array}{c}1: 200 \\
\ldots\end{array}$ & $1: 500$ & $\begin{array}{l}\cdots \\
\cdots \cdots\end{array}$ & $\begin{array}{l}1: 20 \text { ( } 1 \text { day }) \\
1: 20 \text { (1 hour })\end{array}$ \\
\hline \multicolumn{5}{|l|}{$\begin{array}{c}\text { HALOGENS AND COM- } \\
\text { POUNDS }\end{array}$} \\
\hline Chlorin .......... & 1 per cent. & .1 per cent. & & 2 per cent (in $1 \mathrm{hr}$.) \\
\hline Trichlorid of iodin.. & $1: 200$ & $1: 1,000$ & & $1: 1,000$ (in 12 hrs.) \\
\hline ORGANIC COMPOUNDS & & & & \\
\hline $\begin{array}{l}\text { Ethyl alcohol } \therefore . . . . \\
\text { Acetic and oxalic acids }\end{array}$ & $\begin{array}{l}70 \%-15 \\
\text { minutes }\end{array}$ & $70 \%-10 \mathrm{mins}$ & $1: 2-300$ & $\begin{array}{cc}\text { Alcol. } 50 \% & \text { for } 4 \\
\text { months } & \text { without } \\
\text { killing } & \text { spores. } \\
\text { Koch. } & \end{array}$ \\
\hline Carbolic acid & $1: 60$ & $\begin{array}{l}\text { Cholera 1:200 } \\
\text { Typh. 1 : } 50\end{array}$ & 1.300 & $\begin{array}{r}1: 20 \text { (4-45 days) } \\
\quad\left(\text { at } 40^{\circ} \text { in } 3 \text { hrs.) }\right.\end{array}$ \\
\hline Lysol & $1: 300$ & $1: 300$ & & \\
\hline Creolin ..... & $\ldots \ldots$ & $1: 100$ & $1: 3,000$ & $(10 \%$ in 5 hrs. $)$ \\
\hline Salicylic acid $\ldots \ldots$ & $1: 1,000$ & & & \\
\hline $\begin{array}{c}\text { Formalin }(40 \% \text { for- } \\
\text { maldehyde }) \quad \ldots \ldots\end{array}$ & $1: 10$ & $1: 20$ & $1: 1,000$ & $1: 20$ (in 6 hrs.) \\
\hline Peroxide of hydrogen & Conc. & $1: 200$ & $1: 500$ & $\begin{array}{l}1: 100 \text { (in } 1 \mathrm{hr} . \text { ) } \\
3: 100 \text { (in } 1 \mathrm{hr} . \text { ) }\end{array}$ \\
\hline
\end{tabular}

${ }^{1}$ Koch, Arb. a. d. kais. Gesundheitsamt, 1, 1881. 
are made to determine the presence of living bacteria and to distinguish between inhibition or antisepsis and bacterial death or disinfection.

The determination of the bactericidal or disinfectant value of a chemical substance upon spores may be carried out by a variety of methods. Koch, ${ }^{1}$ using anthrax spores as the indicator, dried the spores upon previously sterilized threads of silk. These were exposed to the disinfectant at a definite temperature for varying times, the disinfectant was then removed by washing in sterile water, and the threads planted upon gelatin or blood serum media and incubated. A serious objection to this method was pointed out by Geppert, ${ }^{2}$ who maintains that it is impossible by simple washing to remove completely the disinfectant in which the thread has been soaked. This author suggests that, whenever possible, the disinfectant, at the end of the time of exposure, should be removed by chemical means. In the case of bichloride of mercury Geppert exposes emulsions of the bacteria to aqueous solutions of the disinfectant, and at the end of exposure precipitates out the bichloride of mercury with ammonium sulphide. In the case of a large number of disinfectants, however, this is not possible, and, when the thread method is used, removal of the chemical agent by washing must be practised. Complete removal of the disinfectant is especially desirable, since spores previously exposed to these substances are more easily inhibited by dilute solutions than are normal spores. The spores may be dried upon the end of a glass rod, which, after exposure, is washed in distilled water or salt solution and then immersed in sterile broth. ${ }^{3}$

A simple method is that in which graded percentages of the disinfectant are added to the menstruum, blood, blood serum, broth, etc., in which the disinfectant is to be tested, and equal quantities of bacteria thoroughly emulsified in water or salt solution are added. Loopfuls of these mixtures are then planted from time to time in agar or gelatin plates upon which colony counts can afterward be made.

In all such tests it is important to remember that the presence of organic fluids, blood serum, mucus, etc., considerably alters the efficiency of germicides, and whenever practical deductions are made, experimental imitation of the actual conditions should be attempted.

Practical Disinfection.-In practical disinfection with chemical agents, the disinfectant must be chosen to a certain extent in accordance with the material to be disinfected.

1 Koch, Arb. a. d. kais. Gesundheitsamt, 1, 1881.

${ }^{2}$ Geppert, Berl. klin. Woch., xxvi, 1889.

${ }^{3}$ Hill, Rep. Am. Pub. Health Assn., xxiv, 1898. 
Sputum is a substance extremely difficult to disinfect because the bacteria present are surrounded by dense envelopes of mucus, through which disinfectants do not easily diffuse. For sputum disinfection, especially tuberculous sputum, carbolic acid-5 per cent solution-or any of the phenol derivatives in similar concentration, may be used. Bichloride of mercury is of very little use in sputum disinfection because of the dense protective layers of albuminated mercury which form about the microorganisms. Sputum should always be received into cups containing the disinfectant, and contaminated handkerchiefs should be soaked in the solution.

Feces from typhoid, dysentery, and cholera patients should be sterilized by burning, if possible, or by thoroughly mixing with large quantities of boiling water; but if chemical disinfectants are to be used, five per cent carbolic acid or dilute formalin are convenient. Milk of lime and chloride of lime are useful, though somewhat inconvenient. Bichloride of mercury is of little value in this case for the same reason that it is valueless in sputum disinfection. In all cases of feces disinfection it is extremely important that the chemical agent should be added in large quantities and thoroughly mixed with the discharge.

Linen, napkins, and other cloth materials which have come into contact with patients should be soaked for one or two hours in one per cent formaldehyde, five per cent carbolic acid, or $1: 5,000$ or $1: 10,000$ bichloride of mercury. After this, the material may be taken from the sick-room and boiled. It is extremely important that cloth material should never be removed from the sick-room in a dry state.

Urine may be easily disinfected by the addition in proper concentration of any of the disinfectants named above.

The methods for sterilization of surgical instruments and the preparation of the skin of the patient for operation are subject to so many local variations that it is hardly within the scope of a text-book on bacteriology to mention them. Metal instruments are usually sterilized by boiling in soda solution and may be subsequently immersed in five per cent carbolic acid solution. Catgut may be sterilized by boiling in alcohol or by subjecting it to temperatures of $140^{\circ} \mathrm{C}$. and over, for several hours in oils (albolin).

The disinfection of the hands is also a matter of much variation. Two methods frequently quoted are those of Welch and of Fürbringer.

In Welch's method the hands are brushed with green soap in water as hot as it can be borne for at least five minutes. They are then rinsed and immersed for two minutes in a warm saturated solution of perman- 
ganate of potash in which they are rubbed with a sponge or sterile cotton. They are then transferred to a saturated solution of oxalic acid, until the red color has entirely disappeared. Following this, they are rinsed in sterile water and then immersed in a 1:500 bichloride of mercury solution for one to two minutes.

According to Fürbringer's method, the finger nails are carefully cleaned with an orange-wood stick or nail file; the hands are then thoroughly brushed with a nail brush in green soap and hot water for five minutes. Following this they are immersed in 60 per cent alcohol for one minute, then in 3 per cent carbolic acid solution for one minute; after which they are rinsed in sterile water and dried.

Rooms, closets, and other closed spaces which are contaminated, must be disinfected largely by gaseous disinfectants. After such disinfection in the case of cellars, privies, and other places where feasible, walls and ceilings should be whitewashed.

Gaseous Disinfectants for Purposes of Fumigation.-There are a large number of gaseous agents which are harmful to bacteria. Only a few, however, are of sufficient bactericidal strength to be of practical importance.

Oxygen, especially in the nascent state, may exert distinct bactericidal action upon some bacteria. That strictly anaërobic strains are inhibited by its presence has already been mentioned.

Ozone was shown by Ransome and Fullerton ${ }^{1}$ to exert considerable germicidal power when passed through a liquid medium in which bacteria were suspended, but was absolutely without activity when employed in the dry state.

Chlorine because of its powerful germicidal action was once looked upon with favor, but has been found quite inadequate from a practical point of view because of its injurious action upon materials, and its irregularity of action. Chlorine, too, is but weakly efficient unless in the presence of moisture. ${ }^{2}$

Sulphur dioxide or sulphurous anhydrid $\left(\mathrm{SO}_{2}\right)$, which was formerly much used for room disinfection, is no longer regarded as uniformly efficient for general use. The gas is produced by burning ordinary roll sulphur. In order that it shall be at all effective, water should be vaporized at the same time, since the disinfectant action is dependent upon the formation of sulphurous acid. The concentration of the gas should be at least 8 per cent of the volume of air in the room. For this

${ }^{1}$ Ransome and Fullerton, Rep. Public Health, July, 1901.

${ }^{2}$ Fischer und Proskauer, Mitt. a. d. kais. Gesundheitsamt, x, 11, 1882. 
purpose about three pounds of sulphur should be burned for every thousand cubic feet of space. It should be allowed to act for not less than twenty-four hours. The researches both of Wolffhügel ${ }^{1}$ and of Koch ${ }^{2}$ have shown that the gas is not sufficient for the destruction of spores, under the best circumstances, probably because of its lack of penetrating power. Park ${ }^{3}$ believes that sulphur dioxide used in quantities of four pounds of sulphur to 1,000 cubic feet is of practical value for fumigating purposes in cases of diphtheria and the exanthemata.

Of all known gaseous disinfectants by far the most reliable is formaldehyde. There are many methods of generating this gas, and many devices for its practical use have been introduced. In all cases where formaldehyde fumigation is intended, clothing, bed-linen, and fabrics should be spread out, cupboards and drawers freely opened. The cracks of windows and doors should be hermetically sealed with paper strips or by calking with cotton. The generation of gas may be carried out in an apparatus left within the room or it may be generated outside and the gas introduced by a tube passed within the keyhole. In all cases moisture should be provided for, either in the generating apparatus or by a separate boiler.

The first of the methods of generating formaldehyde for fumigation purposes was that of Trillat, ${ }^{4}$ who devised a lamp in which formaldehyde was produced by the incomplete combustion of methyl alcohol. This method has proved expensive because of the complete oxidation of a large percentage of the alcohol.

Direct evaporation of formaldehyde from formalin solutions has been the principle underlying some other devices. If such evaporation is attempted from an open vessel, however, polymerization of formaldehyde to the solid trioxymethylene occurs. To prevent this, Trillat ${ }^{5}$ and others have constructed special autoclaves in which 20 per cent of calcium chloride is added to formalin which is then vaporized under pressure. By this means polymerization is practically eliminated.

The Trillat autoclave, as well as others constructed on the same principle, consists of a strong copper chamber of a capacity of about a gallon, fitted with a cover which can be tightly screwed into place. The cover is perforated by an outlet vent, a pressure gauge, and a

${ }^{1}$ Wolffhügel, Mitt. a. d. kais. Gesundheitsamt, i, 1881.

${ }^{2}$ Koch, Mitt. a. d. kais. Gesundheitsamt, i, 1881.

${ }^{3}$ Park, "Pathogen. Bact.," N. Y., 1908.

4 Trillat, Compt. rend. de l'acad. des sc., 1892.

5 Trillat, Compt. rend. de l'acad. des sc., 1896. 
thermometer. The whole apparatus is adjusted upon a stand and set over a kerosene lamp. Into the chamber is put about one-half to threcquarters its capacity of 40 per cent formaldehyde (commercial formalin) containing 15-20 per cent calcium chloride. The solution used should be free from methyl alcohol, since this leads to the formation, with formaldehyde, of methylal, which is absolutely without germicidal action. The flame is lighted and the exit tube kept closed until the pressure gauge indicates a pressure of three atmospheres. Then the vapor is allowed to escape through the tube. For a room of about 3,000 cubic feet Trillat advises the continuance of the gas flow for about an hour. The method is not uniformly reliable.

A method which has found much favor is that in which glycerinusually in a concentration of 10 per cent-is added to formalin. According to Schlossmann ${ }^{1}$ the presence of

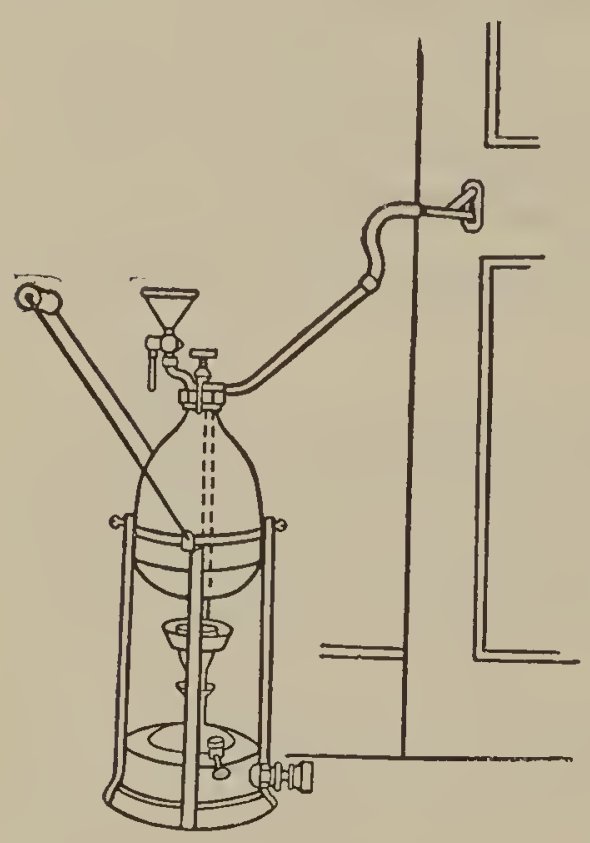

Fig. 12.-Lentz Formalin Apparatus. glycerin hinders polymerization. An apparatus in which this mixture is conveniently utilized is that of Lentz (see Fig. 12). Formalin with 10 per cent glycerin is placed in the copper tank and heated by a burner. Formaldehyde leaves the nozzle (which can be introduced through the keyhole) mixed in a fine spray with steam. This apparatus has been favorably endorsed by the War Department of the United States.

The so-called Breslau method of generating formaldehyde depends upon the evaporation of formaldehyde from dilute solutions. v. Brunn ${ }^{2}$ claims that where formalin in 30 to 40 per cent strength is evaporated, water vapor is generated more rapidly than formaldehyde is liberated, and a concentration leading to polymerization occurs. If, however, dilution is carried out until the formaldehyde in the solution is not more than 8 per cent, the generation of water vapor and formaldehyde takes place at about equal speed and no concentration occurs. Schlossmann ${ }^{1}$ furthermore claims that polymerization in the vaporized formaldehyde does not occur if sufficient water vapor is present-a principle which may also contribute to the efficiency of the Breslau method. In practice, the apparatus devised by v. Brunn (Fig. 13) consists of a

${ }^{1}$ Schlossmann, Münch. med. Woch., 45, 1898.

${ }^{2} v$. Brunn, Zeit. f. Hyg., xxx, 1899. 
strong copper kettle of about $34 \mathrm{~cm}$. diameter by $7.5 \mathrm{~cm}$. height. This is completely closed except for two openings in the slightly domed top, one of which is the exit vent, the other, laterally placed, is for purposes of filling and is closed by a screw stopper. The kettle is set up on a metal stand over an alcohol lamp, so arranged with a double circle of burners that heating may be carried out rapidly. The tank is filled with a solution of formalin of a strength of from 8 to 10 per cent (commercial formalin 1:4). The apparatus permits the evaporation of large quantities of fluid in a short time (3 liters in one hour). When the lamp is left in a closed room care should be taken to fill it with a quantity of alcohol proportionate to the amount of fluid to be evaporated. This, according to v. Brunn, is about one-quarter of the volume of formalin solution used. By using 1.5 liters of 8 per cent formalin for each 1,000 cubic feet of space, this apparatus is said to yield a concentration of formaldehyde of about 25 grams to the cubic meter, a strength sufficient to complete surface disinfection within seven hours.

To do away with the use of liquid formalin solutions, a meth-
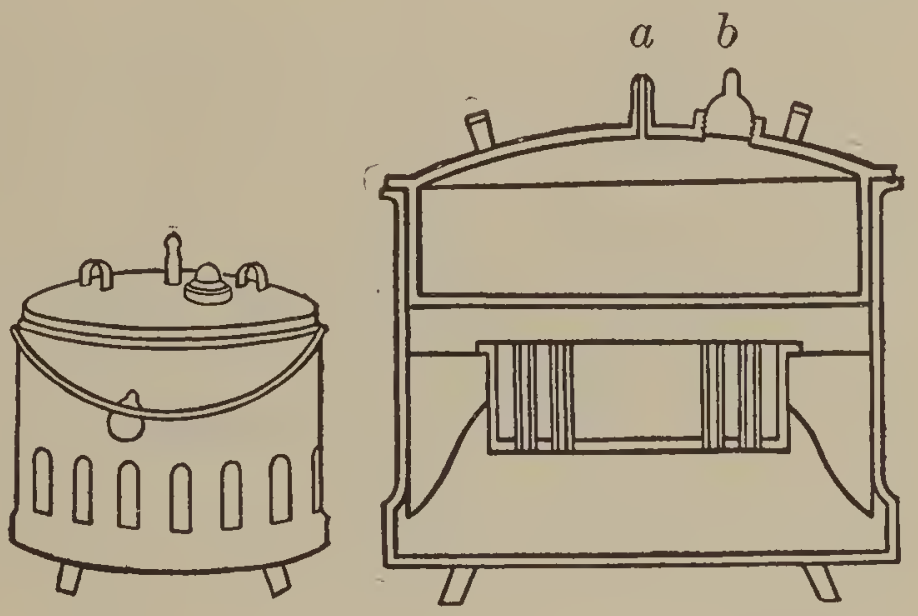

Fig. 13.-Breslau Formaldehyde Generator and Section of Same. (After v. Brunn.) $a$, Inlet; $b$, Exit vent. od has been devised which depends in principle upon the breaking up by heat of the solid polymer of formaldehyde (trioxymethylene). The apparatus (trade name, "Schering's Paraform Lamp") as described by Aronson ${ }^{1}$ consists of a cylindrical mantle of sheet-iron placed upon a stand and supplied below with an alcohol lamp. Set into the top of the mantle is a small chamber, into which 1 gram tablets of trioxymethylene are placed. The alcohol lamp, so placed that the wicks project but slightly-to avoid overheating-is lighted, and the formalin generated passes out through slits in the upper case, mingling with the water vapor and other gases liberated by the alcohol flame. The more modern devices have water-boiler attachments to insure sufficient moisture. Two tablets are sufficient for the fumigation of about thirty-five cubic feet, and 2 c.c. of alcohol are filled into the lamp for each tablet. One hundred to one hundred and fifty tablets are usually enough for the ordinary 
room. Modifications of this method are in common use, some wellknown firms preparing so-called "paraform candles," in which paraform, in the powdered state, is volatilized by heat.

A simple method of generating formaldehyde is that which is known as the "lime method." In a wide shallow pan 40 per cent formaldehyde solution (commercial formalin) is poured over quicklime $(\mathrm{CaO})$. According to Park, the previous addition of concentrated sulphuric acid to the formalin, in proportions of one to ten, increases the speed of formalin liberation, and aids in limiting polymerization. About one and one-half to two pounds (one-half to one kilogram) of quicklime are used for every 500 c.c. of the formalin solution. The heat generated in the slaking of the lime produces volatilization of the formalin.

A modification of this method is that of Schering ${ }^{1}$ in which tablets of paraform and unslaked lime are together laid into a pan and warm water is poured over them.

A highly efficient method, which has universal approval because of its simplicity, is the potassium permanganate method of Evans and Russell. ${ }^{2}$ This method depends upon the active reaction occurring when formalin and potassium permanganate are mixed. In practice, about 300 grams of small crystals of potassium permanganate are poured into a half liter of 40 per cent formalin. The mixture results in an active evolution of heat and the evaporation of formalin together with water vapor. Because of the active foaming which takes place, high cylindrical vessels should be used, about one foot in' height, preferably with a funnel-like flare at the top. The yield of gas by this method is said to be about 80 per cent of the amount present in the solution, and within the first five minutes most of this is liberated.

Harrington ${ }^{3}$ states that the equivalent of 110 c.c. for formalin suffices to produce sterility within two and a half hours in a space of one thousand cubic feet.

The room in which formaldehyde has been liberated is kept sealed, in the manner already described, for at least twelve hours, after which the windows and doors are opened and thorough airing practised. The odor which remains after formaldehyde fumigation may be removed by sprinkling with ammonia, or by the use of some one or another of the various sorts of apparatus devised for the liberation of ammonia.

${ }^{1}$ Schering, Hyg. Rundschau, 1900.

${ }^{2}$ Evans and Russell, Rep. State Bd. Health, Maine, 1904.

${ }^{3}$ Harrington, "Practical Hygiene." Phila., 1905. 


\section{CHAP'TER VI}

\section{METHODS USED IN THE MICROSCOPIC STUDY AND STAINING OF BACTERIA}

\section{MICROSCOPIC STUDY OF BACTERIA}

BACTERIA may be studied microscopically, in the living and unstained state, and, after the application of dyes, in colored preparations. For the manipulation of bacteria for such study, glass slides and coverslips of various design are used. These must be perfectly clean if the preparations are to be of any value. ${ }^{1}$

The Study of Bacteria in the Living State.-Living bacteria may be studied in what is spoken of as the "hanging-drop" preparation. For this purpose a so-called hollow slide is employed, in the center of which there is a circular concavity about three-quarters of a centimeter to one centimeter in diameter. The preparation is manipulated as follows: If the bacteria are growing in a fluid medium a drop of the culture fluid is transferred to the center of a cover-slip. If taken from solid media, an emulsion may be made in broth or in physiological salt solution, and a drop of this transferred to the cover-slip, or the bacteria may be emulsified in a drop of salt solution, or broth, directly upon the cover-slip. The concavity on the slide, having first been rimmed with vaseline, by means of a small camel's-hair brush, the cover-slip is inverted over the slide in such a way that the drop hangs freely within the hollow space. The preparation is then ready for examination under the microscope.

1 Although the silicates of which glass is composed are extremely stable, nevertheless alkaline silicates which are said to separate out on the surface, together with grease and dirt left upon the glass by handling, during blowing and cutting, necessitate cleansing before use. This may be accomplished by a variety of methods. A simple one suitable for general application is as follows: (1) The slides and coverslips are thrown singly into boiling water and left there for half an hour; (2) wash in twenty-five per cent sulphuric acid; (3) rinse in distilled water; (4) wash in alcohol; (5) wipe with a clean cloth and keep dry under a bell-jar. Another method convenient for routine use is to immerse, after thorough washing in soap-suds and acid, in ninety-five per cent alcohol and to leave in this until the time of use. 
Another method, known as the "hanging block method," devised by Hill, ${ }^{1}$ for the study of living bacteria in solid media is carried out as follows: Nutrient agar is poured into a Petri dish and allowed to solidify. Out of this layer a piece about a quarter of an inch square is cut. This is placed on a sterile slide. The upper surface of the agar block is then inoculated with bacteria by surface smearing, and the preparation covered with a sterile dish and allowed to dry for a few minutes in the incubator. A sterile cover-slip is then dropped upon the surface of the

Fig. 14.-Hanging Drop Preparation.

block and sealed about the edges with agar. Block and cover-slip are then taken from the slide and fastened over a moist chamber with paraffin. The entire preparation can be placed upon the stage of a microsocpe. This method is especially designed for the study of cell-division.

Living bacteria may also be studied in stained preparations by the so-called "intravital" method of Nakanishi. Thoroughly cleaned slides are covered with a saturated aqueous solution of methylene-blue. This is spread over the slide in an even film and allowed to dry. After drying the slide should appear of a transparent sky-blue color. The microorganisms which are to be examined are then emulsified in water, or are taken from a fluid medium and placed upon a cover-slip. This is dropped, face downward, upon the blue ground of the slide. In this way bacteria may be stained without being subjected to the often destructive processes of heat or chemical fixation. According to Nakanishi, cytoplasm is stained blue, while nuclear material assumes a reddish or purplish hue.

The Study of Bacteria in Fixed Preparations.-Stained preparations of bacteria are best prepared upon cover-slips, the process consisting of the following steps: (1) Spreading on cover-slip; (2) drying in air; (3) fixing; (4) staining; (5) washing in water; (6) blotting; (7) mounting.

(1) Sniearing.-Bacteria from a fluid medium are transferred in a small drop of the fluid, with a platinum loop, to a cover-slip and carefully spread over the surface in a thin film. If taken from a solid medium a small drop of sterile water is first placed upon the cover-slip and the bacteria are then in very small quantity carefully emulsified in this drop with the platinum needle or loop and spread in an extremely thin film.

+ Hill, Jour. of Med. Research, vii, 1902. 
(2) The film is allowed to dry in the air.

(3) When thoroughly dried, fixation is carried out by passing the preparation, film side up, three times through a Bunsen flame, at about the rate of a pendulum swing. Fixation by heat in this manner is most convenient for routine work, but is not the most delicate method, inasmuch as the degree of heat applied can not be accurately controlled. The other methods which may be employed are immersion in methyl alcohol, formalin, saturated aqueous bichloride of mercury, Zenker's fluid, or acetic acid. If chemical fixatives are used, they must be removed by washing in water before the stain is applied. If a preparation is made upon a slide instead of a cover-slip, passage through the flame should be repeated eight or nine times.

(4) Staining.--The dyes used for the staining of bacteria are, for the greater part, basic anilin dyes, such as methylene-blue, gentianviolet, and fuchsin. These may be applied for simple staining in 5 per cent aqueous solutions made up from filtered saturated alcoholic solutions, or directly by weight. They are conveniently kept in the laboratory as saturated alcoholic solutions. The strengths of some saturated solutions are as follows:

\section{Saturated Solutions ${ }^{1}$ (Stains Gruebler or Merck).}

Fuchsin (aqueous), 1.5 per cent.

Fuchsin (alcohol 96 per cent), 3 per cent.

Gentian-violet (aqueous), 1.5 per cent.

Gentian-violet (alcohol 96 per cent), 4.8 per cent.

Methylene-blue (aqueous), 6.7 per cent.

Methylene-blue (alcohol 96 per cent), 7 per cent.

The staining solution, in simple routine staining, is left upon the fixed bacterial film for from one-half to one and one-half minutes according to the efficiency of the stain used. Methylene-blue is the weakest of the three stains mentioned; gentian-violet the strongest.

(5) The excess stain is removed by washing with water.

(6) The preparation is thoroughly dried by a blotter or between layers of absorbent paper.

(7) A small drop of Canada balsam is placed upon the film side of the dry cover-slip, which is then inverted upon a slide. The preparation is now ready for microscopical examination.

1 After Wood, "Chemical and Microscopical Diagnosis," Appendix. N. Y., 1909. 
The chemical principles which underlie the staining process are still more or less in doubt. ${ }^{1}$ Suffice it to say here that most of the dyes in common use by bacteriologists and pathologists are coal-tar derivatives belonging to the aromatic series, all of them containing at least one "benzolring" combined with what Michaelis terms a "chromophore group," chief among which are the nitro-group $\left(\mathrm{NO}_{2}\right)$, the nitroso-group $(\mathrm{NO})$, and the azo-group $(\mathrm{N}=\mathrm{N})$. Just what the actual process of staining consists in, is a question about which various opinions are held, some believing that the phenomenon is purely chemical, in which a salt is formed by the combination of the dye and the protoplasm of the cells, others that there is no such salt formation, and that the process takes place by purely physical means. To support the latter view it is argued that certain substances like cellulose are stainable without possessing the property of salt formation, and that staining may often be accomplished without there being a chemical disruption of the dye itself. Michaelis sums up his views by stating that probably both processes actually take place. A dye stuff, as a whole, may enter into and be deposited upon a tissue or cell by a process which he speaks of as "insorption." In such a case the coloring matter may be subsequently extracted by any chemically indifferent solvent. On the other hand, a dye after being thus deposited upon or within a cell, may become chemically united to the protoplasm by the formation of a salt, and in such a case the color can be removed only by agents which are capable of decomposing salts, such as free acids.

The staining power of any solution may be intensified either by heating while staining, by prolonging the staining process, or by the addition of alkalies, acids, anilin oil, and other substances which will be mentioned in the detailed descriptions of special staining methods.

One of the most common examples of such an intensified stain is the so-called Loeffer's alkaline methylene-blue. This is made up in the following way:

Saturated alcoholic solution of methylene-blue, 30 c.c.

$1: 10,000$ solution potassium hydrate in water, 100 c.c.

Another solution designed with a similar purpose is the Koch-Ehrlich anilin-water solution. Anilin oil, one part, is shaken up with distilled water, nine parts; after thorough shaking, the mixture is filtered

${ }^{1}$ For comprehensive reviews of the subject, the reader is referred to dissertations such as those of Mann ("Physiol. Hist. Methods and Theory," Oxford, 1902) and of Michaelis ("Ein"ührung in dic Farbstoffchemie," etc., Berlin, 1902). 
through a moist filter paper until perfectly clear. A saturated alcoholic solution of either fuchsin or gentian-violet is added to this anilin water in proportions of about one to ten or until a slightly iridescent pellicle appears upon the surface of the solution.

An extremely useful and very strong staining solution is the Ziehl carbol-fuchsin solution, made up as follows: ${ }^{1}$

Fuchsin (basic) ........................ 1 gm.

Alcohol (absolute) ....................... 10 c.c.

Five per cent carbolic acid $\ldots \ldots \ldots \ldots \ldots \ldots \ldots$ c.c.

To make up this staining solution, $\operatorname{mix} 90$ c.c. of a five per cent aqueous solution of carbolic acid with 10 c.c. of saturated alcoholic basic fuchsin.

It may also be made up as follows:

Weigh out

Basic fuchsin ........................ 1 gram

Carbolic acid $\ldots \ldots \ldots \ldots \ldots \ldots \ldots \ldots \ldots \ldots \ldots$ grams

Dissolve in

Distilled water........................ 100 c.c.

Filter and add

Absolute alcohol ....................... 10 c.c.

\section{SPECIAL STAINING METHODS}

Spore Stains.-Aввотт's Meтнор. ${ }^{2}$-Cover-slips are smeared and fixed by heat in the usual manner.

Cover with Loeffler's alkaline methylene-blue and heat the stain until it boils, repeat the heating at intervals but do not boil continuously. Keep this up for one minute.

Rinse in water.

Decolorize with a mixture of alcohol eighty per cent 98 c.c. and nitric acid 2 c.c., until all blue has disappeared.

Rinse in water.

Dip from three to five seconds in saturated alcoholic solution of eosin 10 c.c., and water 90 c.c.

Rinse in water, blot, and mount in balsam.

By this method the spores are stained blue, the bodies of the bacilli are stained pink. 
Moeller's Method. ${ }^{1}$-Cover-slips are prepared as usual and fixed in the flame.

Wash in chloroform for two minutes.

Wash in water.

Cover with five per cent chromic acid one-half to two minutes:

Wash in water. Invert and float cover-slip on carbol-fuchsin solution in a small porcelain dish and heat gently with a flame until it steams; continue this for three to five minutes. (This step can also be done by covering the cover-glass with carbol-fuchsin and holding over flame.)

Decolorize with five per cent sulphuric acid five to ten seconds.

Wash in water.

Stain with aqueous methylene-blue one-half to one minute. By this method spores will be stained red, the body blue.

Capsule Stains.-Welch's Method. ${ }^{2}$ - Cover-slips are prepared as usual and fixed by heat.

Cover with glacial acetic acid for a few seconds. Pour off acetic acid and cover with anilin water gentian-violet, renewing stain repeatedly until all acid is removed. This is done by pouring the stain on and off three or four times and then finally leaving it on for about three minutes.

Wash in two per cent salt solution and examine in this solution.

Hiss' Methods. ${ }^{3}$ - (1) Copper Sulphate Method.-Cover-slip preparations are made by smearing the organisms in a drop of animal serum, preferably beef-blood serum.

Dry in air and fix by heat.

Stain for a few seconds with-

Saturated alcoholic solution of fuchsin or gentian-violet 5 c.c., in distilled water 95 c.c.

The cover-slip is flooded with the dye and the preparation held for a second over a free flame until it steams.

Wash off dye with twenty per cent aqueous copper sulphate solution.

Blot (do not wash).

Dry and mount.

By this method permanent preparations are obtained, the capsule appearing as a faint blue halo around a dark purple cell body.

(2) Potassium Carbonate Method.-This method consists in using as a dye a half-saturated solution of gentian-violet. Gentian-violet in

1 Moeller, Cent. f. Bakt., I, x, 1891.

2 Welch, Johns Hopkins Hosp. Bull., 1892.

${ }^{3}$ Hiss, Cent. f. Bakt., xxxi, 1902; Jour. Exper. Med., vi, 1905. 
substance is added in excess to distilled water and allowed to dissolve to its full extent. The solution is then filtered and diluted to twice its volume.

Cover-glass preparations are made by spreading the bacteria on a over-slip in a drop of animal serum as in preceding method. They are allowed to dry in the air and fixed by heat as usual. The dye is then poured upon the preparation and allowed to remain for a few seconds. It is then washed off with a twenty-five-hundredth per cent solution of potassium carbonate in water, and studied in this solution. The cover-slip inverted on a slide may be rimmed with vaseline to prevent evaporation.

Buerger's Method. ${ }^{1}$ - Cover-slip preparations are made by smearing in serum as in Hiss' method.

As the edges of the smear begin to dry, pour over it Zenker's fluid (without acetic acid) and warm in flame for three seconds.

(Zenker's fluid is composed of potassium bichromate $2.5 \mathrm{gm}$., sodium sulphate $1 \mathrm{gm}$., water 100 c.c., saturated with bichloride of mercury.)

Wash in water.

Flush with ninety-five per cent alcohol.

Cover with tincture of iodin, U. S. P., one to three minutes.

Wash with ninety-five per cent alcohol.

Dry in the air.

Stain with anilin water gentian-violet two to five seconds.

Wash with two per cent salt solution.

Mount and examine in salt solution.

Wadsworth's Method. ${ }^{2}$ - Wadsworth has devised a method of staining capsules which depends upon the fixation of smears with formalin. After such fixation capsules may be demonstrated both with simple stains and by Gram's method. The technique is as follows:

Smear preparations, made as usual, are treated as follows:

1. Formalin, 40 per cent, two to five minutes.

2. Wash in water, five secords.

Simple Stain.

Differential Stain (Gram's Method).

3. Ten per cent aqueous gentian-violet. 3. Anilin gentian-violet, two minutes.

4. Wash water, five seconds.

5. Dry, mount in balsam.

4. Iodin solution, two minutes.

5. Alcohol, 95 per cent, decolorize.

6. Fuchsin, dilute aqueous solution.

7. Wash water, two seconds.

8. Dry, mount in balsarn 
It is important that the formalin be fresh and the exposure to water momentary. When decolorizing in the Gram method, strong alcohol only should be used. Wadsworth also found that encapsulated pneumococci could be demonstrated in celloidin sections of pneumonic lesions hardened in strong formalin. The lungs should be distended with the formalin or the lesions cut in very thin bits, hardened, dehydrated, embedded, and cut in the usual way. The celloidin sections may be fixed on the slides by partially dissolving the celloidin in alcohol and ether and setting the celloidin quickly in water before staining. Failure to obtain pneumococci encapsulated in such sections is usually due to impioper or inadequate fixation in the formalin.

The differential method employed by Wadsworth for tissue staining is as follows:

1. Fix in formalin forty per cent, two to five minutes.

2. Wash in water.

3. Anilin gentian-violet, two minutes.

4. Iodin solution, two minutes.

5. Alcohol, ninety-five per cent, decolorize.

6. Eosin alcohol, counterstain.

7. Clear in oil of origanum.

8. Mount in balsam.

Flagella Stains.-All flagella stains, in order to be successful, necessitate particularly clean cover-slip preparations, best made from young agar cultures emulsified in sterile salt solution. Scrupulous care should be exercised in cleaning the glassware used.

LOEFFleR's Method. ${ }^{1-}$-The preparation is dried in the air and fixed by heat. It is then treated with the following mordant solution:

Twenty per cent aqueous tannic acid ..........10 parts.

Ferrous sulphate aq. sol. saturated at room temperature . 5 parts.

Saturated alcoholic fuchsin solution........... 1 part.

This solution, which should be freshly filtered before using, is poured over the cover-glass and allowed to remain there for one-half to one minute, during which time it should be gently heated, but not allowed to boil.

Wash thoroughly in water.

Stain with five per cent anilin water fuchsin or anilin water gen- 
tian-violet made slightly alkaline by the addition of one-tenth per cent sodium hydrate.

The stain should be filtered directly upon the cover-slip. Warm gently and leave on for one to two minutes. Wash in water. Mount in balsam.

Van Frmengem's Method. ${ }^{1}$ - This method requires the preparation of three solutions.

(1) Twenty per cent tannic acid solution .........60 c.c.

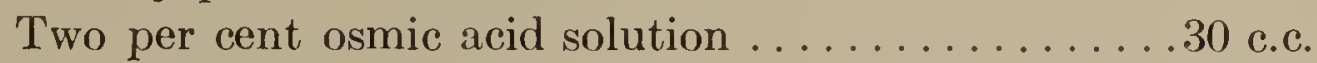

Glacial acetic acid . . . . . . . . . . . . . 4-5 drops.

The cover-slip carrying the fixed preparation is placed in this solution for one hour at room temperature, or for five minutes at $100^{\circ} \mathrm{C}$. (boiling).

Wash in water.

Wash in absolute alcohol.

Immerse the cover-slip for one to three seconds in

(2) Silver nitrate, $0.25-0.5$ per cent solution.

Without washing, transfer to

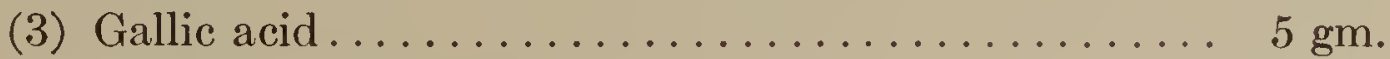

Tannic acid........................ 3 "

Fused potassium acetate ............... 10 "

Distilled water ........................ 350 c.c.

Immerse in this for a few minutes, moving the cover-slip about.

Return to the silver nitrate solution until the preparation turns black.

Wash thoroughly in water.

Blot and mount.

Smith's Modification of Pitfield's Methon. ${ }^{2}-\mathrm{A}$ saturated solution of bichloride of mercury is boiled and is poured while still hot into a bottle in which crystals of ammonia alum have been placed in quantity more than sufficient to saturate the fluid. The bottle is then shaken and allowed to cool. Ten c.c. of this solution are added to 10 c.c. of freshly prepared ten per cent tannic acid solution. To this add 5 c.c. carbolfuchsin solution. Mix and filter.

To stain, filter the above mordant directly upon the fixed cover-slip

1 Van Ermengem, Cent. f. Bakt., I, xv, 1894.

2 Smith, Brit. Med. Jour., I, 1901, p. 205. 
preparation. Heat gently for three minutes, but do not allow to boil. Wash in water and stain with the following solution:

Saturated alcoholic solution gentian-violet ......... 1 c.c. Saturated solution ammonia alum .............. 10 c.c.

Filter the stain directly upon the preparation and heat for three or four minutes. Wash in water, dry, and mount in balsam.

Differential Stains.-GRAm's METHOD. ${ }^{1}$-By this method of staining, which is extremely important in bacterial differentiation, bacteria are divided into those which retain the initial stain and those which are subsequently decolorized and take the counterstain. The former are often spoken of as the Gram-positive, the latter as Gram-negative bacteria.

Preparations are made on cover-slips or slides in the usual way.

The preparation is then covered with an anilin gentian-violet solution which is best made up freshly before use.

The staining fluid is made up, according to Gram's original directions, ${ }^{2}$ as follows:

Five c.c. of anilin oil are shaken up thoroughly with 125 c.c. of distilled water. This solution is then filtered through a moist filter paper.

To 108 c.c. of this anilin water, add 12 c.c. of a saturated alcoholic solution of gentian-violet. The stain acts best when twelve to twentyfour hours old, but may be used at once. It lasts, if well stoppered, for three to five days. A more convenient and simple method of making up the stain is as follows:

To 10 c.c. of distilled water in a test tube add anilin oil until on shaking the emulsion is opaque; roughly, one to ten. Filter this through a wet paper until the filtrate is clear. To this add saturated alcoholic solution of gentian-violet until the mixture is no longer transparent, and a metallic film on the surface indicates saturation. One part of alcoholic saturated gentian-violet to nine parts of the anilin water will give this result. This mixture may be used immediately and lasts two to five days if kept in a stoppered bottle.

Cover the preparation with this; leave on for 5 minutes. Pour off excess stain and cover with.Gram's iodin solution for 2 to 3 minutes.

Iodin ............................ $1 \mathrm{gm}$.

Potassium iodid...................... 2 gm.

Distilled water.............................

${ }^{1}$ Gram, Fortschr. d. Med., ii, $1884 . \quad{ }^{2}$ Gram, loc. cit. 
Decolorize with ninety-seven per cent alcohol until no further traces of the stain can be washed out of the preparation. This takes usually thirty seconds to two minutes, according to thinness of preparation.

Wash in water.

Counterstain with an aqueous contrast stain, preferably Bismarck brown. ${ }^{1}$

Paltauf's Modification of Gram's Stain. ${ }^{2}$ - The staining fluid as prepared by this modification possesses the advantage of retaining its staining power for a longer period than does the anilin-water-gentianviolet described in the original method.

The staining fluid is prepared as follows:

3-5 c.c. anilin oil are added to

90 c.c. distilled water and

7 c.c. absolute alcohol.

This mixture is thoroughly shaken and filtered through a moist filter paper until clear. Then add:

Gruebler's gentian-violet $2 \mathrm{gm}$.

The fluid should stand twenty-four hours, during which a precipitate forms. This is filtered before use.

This gentian-violet solution retains its staining power for from four to six weeks. It is good only when a metallic luster develops on the surface.

It is used in the following way: Spreads on cover-slips or slides are dried and fixed as usual.

Then apply:-

Anilin water gentian-violet (as above), three minutes.

Gram's iodin solution, two minutes.

Absolute alcohol (with stirring), thirty seconds.

Counterstain, without washing in water, in aqueous fuchsin or in weak carbol-fuchsin.

1 To make up Bismarck brown solution, prepare a saturated aqueous solution of the powdered dye by heating. Allow it to cool, and filter. Dilute one to ten with distilled water.

${ }^{2}$ Sharnosky, Proc. N. Y. Pathol. Soc., Oct., 1909, n. s., ix, 5. 
Classification of the Most Important Pathogenic Bacteria According to Gram's Stain.

Gram-positive.

(Retain the Gentian-violet.)

Micrococcus pyogenes aureus

Micrococcus pyogenes albus

Streptococcus pyogenes

Micrococcus tetragenus

Pneumococcus

Bacillus subtilis

Bacillus anthracis

Bacillus diphtheriæ

Bacillus tetanus

Bacillus tuberculosis and other acid-fast bacilli

Bacillus aërogenes capsulatus

Bacillus botulinus
Gram-negative.

(Take Counterstain.)

Meningococcus

Gonococcus

Micrococcus catarrhalis

Bacillus coli

Bacillus dysenteriæ

Bacillus typhosus

Bacillus paratyphosus

Bacillus fecalis alkaligenes

Bacillus enteritidis

Bacillus proteus (proteus)

Bacillus mallei

Bacillus pyocyaneus

Bacillus influenzæ

Bacillus mucosus capsulatus

Bacillus pestis

Bacillus maligni øedematis

Spirillum choleræ

Bacillus Koch-Weeks

Bacillus Morax-Axenfeld

Stains for Acid-Fast Bacteria.--These methods of staining are chiefly useful in the demonstration of tubercle bacilli. These bacteria because of their waxy cell membranes are not easily stained by any but the most intensified dyes, but when once stained, retain the color in spite of energetic decolorization with acid. For this reason they are known as acidfast bacilli. The first method devised for the staining of tubercle and allied bacilli was that of Ehrlich.

Ehrlich Method. ${ }^{1}$-This method is now rarely used. Cover-slip preparations are prepared as usual and fixed by heat.

Stain with anilin water gentian-violet, hot, three to five minutes, or twenty-four hours at room temperature.

1 Ehrlich, Deut. med. Woch. 1882. 
Decolorize with thirty-three per cent nitric acid one-half to one minute. off.

Treat with sixty per cent alcohol, until no color can be seen to come

Counterstain with aqueous methylene-blue.

Rinse in water, dry, and mount.

Zienl-Neelson Method. ${ }^{1}$-Thin smears are made upon coverslips or slides.

Fix by heat.

Stain in carbol-fuchsin solution as given on page 97 . The slide or cover-slip may be flooded with the stain, and this gently heated with the flame until it steams, or else the cover-slip may be inverted upon the surface of the staining fluid, in a porcelain dish or watch-glass, and this heated until it steams. This is continued for three to five minutes. Decolorize with either five per cent nitric acid, five per cent sulphuric acid, or one per cent hydrochloric acid for three to five seconds. The treatment with the acid is continued until subsequent washing with water will give only a faint pirk color to the preparation.

Wash with ninety per cent alcohol until no further color can be removed. If, after prolonged washing with alcohol, a red color still remains in very thick places upon the smear, while the thin areas appear entirely decolorized, this may be disregarded.

Wash in water and counterstain in aqueous methylene-blue for one-half to one minute.

Rinse $n$ water, dry, and mount.

By this method the tubercle bacilli are colored red, other bacteria and cellular elements which may be present are stained blue.

GabBe't's Method. ${ }^{2}$ - Gabbet has devised a rapid method in which the decolorization and counterstaining are accomplished by one solution. The specimen is prepared and stained with carbol-fuchsin as in the preceding method. It is then inmersed for one minute directly in the following solution:

Methylene-blue $2 \mathrm{gms}$.

Sulphuric acid 25 per cent (sp. gr. 1018) ............ 100 c.c.

Then rinse in water, dry, and mount.

This method, while rapid and very convenient, is not so reliable as the Ziehl-Neelson method.

1 Ziehl, Deut. med. Woch., 1882; Neelson, Deut. med. Woch., 1883.

${ }^{2}$ Gabbet, Lancet, 1887. 
Pappenheim's Method. ${ }^{1}$ - The method of Pappenheim is devised for the purpose of differentiating between the tubercle bacillus and the smegma bacillus. Confusion may occasionally arise between these two microorganisms, especially in the examination of urine where smegma bacilli are derived from the genitals, and less frequently in the examination of sputum where smegma bacilli may occasionally be mixed with the secretions of the pharynx and throat.

Preparations are smeared and fixed by heat in the usual way.

Stain with hot carbol-fuchsin solution for two minutes.

Pour off dye without washing and cover with the following mixture:

Corallin (rosolic acid) $\ldots \ldots \ldots \ldots \ldots \ldots \ldots \ldots, 1 \mathrm{gm}$.

Absolute alcohol........................100 c.c.

Methylene-blue added to saturation

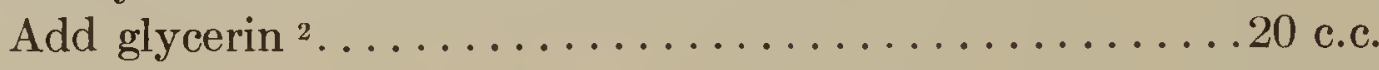

This mixture is poured on and drained off slowly, the procedure being repeated four or five times, and finally the preparation is washed in water. The combination of alcohol and rosolic acid decolorizes the smegma bacilli, but leaves the tubercle bacilli stained bright red.

Bunge and Trautenroth Method. ${ }^{3}$ - This method is designed to differentiate between the tubercle and smegma bacilli.

Smear and fix by heat in the usual way.

Wash with absolute alcohol to remove fat.

Treat with five per cent chromic acid for fifteen minutes.

Wash in several changes of water.

Stain with hot carbol-fuchsin for five minutes.

Decolorize with sixteen per cent sulphuric acid for three minutes.

Counterstain with alcoholic methylene-blue for five minutes.

Wash in water, dry, and mount.

By this method the tubercle bacillus remains red, the smegma bacil- . lus is decolorized.

Baumgarten's Method. "-This method is recommended by the author for differentiation between the bacillus of tuberculosis and the bacillus of leprosy and depends upon the fact that the tubercle bacillus is less easily stained than Bacillus lepræ.

Smears are prepared and fixed by heat in the usual way.

${ }^{1}$ Pappenheim, Berl. klin. Woch., 1898.

2 The glycerin is added after the other constituents have been mixed.

${ }^{3}$ Bunge und Trautenroth, Fortschr. d. Med., xiv, 1896.

${ }^{5}$ Baumgarten, Zeit. f. wissensch. Mikrosk., 1, 1884. 
Stain in dilute alcoholic fuchsin for five minutes.

Decolorize for twenty seconds in alcohol, ninety-five per cent, ten parts, nitric acid one part.

Wash in water.

Counterstain in methylene-blue.

Wash in water, dry, and mount.

The tubercle bacillus should be blue and the bacillus of leprosy red.

Special Stains for Polar Bodies.-These staining methods are designed to bring into view polar bodies as found, for instance, in the bacilli of diphtheria and plague.

Neisser's Method. ${ }^{1}$ - Smear and fix in the usual manner.

Stain for two to five seconds in the following solution:

Methylene-blue....................... $1 \mathrm{gm}$.

Absolute alcohol....................... 20 c.c.

Glacial acetic acid $\ldots \ldots \ldots \ldots \ldots \ldots \ldots \ldots \ldots$ c.c.

Distilled water ....................... 1,000 c.c.

Wash in water.

Counterstain in two per cent aqueous Bismarck brown solution for five seconds.

By this method polar bodies are stained blue, while the bacillary bodies are stained brown.

Roux's Method. ${ }^{2}-$ Two solutions are necessary.

(1) Dahlia violet................... $1 \mathrm{gm}$.

Alcohol 90 per cent................. 10 c.c.

Aqua destillata ad.................... 100 c.c.

(2) Methyl-green .................... $1 \mathrm{gm}$.

Alcohol 90 per cent. ................. 10 c.c.

Aqua destillata ad .................... 100 c.c.

Before use, one part of solution No. 1 is mixed with three parts of solution No. 2. The preparation is stained with the mixture for two minutes in the cold.

Polychrome Stains.--The various polychrome stains are of value to the bacteriologist chiefly for the staining of pus and exudates where the relation of bacteria to cellular elements is to be demonstrated. They are also extremely useful in the study of fixed specimens of protozoan parasites. There is a large number of these stains in use; a few only,

\footnotetext{
1 Neisser, Zeit. f. Hyg., xxiv, 1897.

2 Roux and Yersin, Annal. de l'inst. Past., 1890.
} 
however, can be given here. In principle, all these stains depend upon a combination of eosin and methylene-blue, these elements staining not only as units, but acting together in combination. One and the same solution, therefore, contains at least three elements which color the various structures of the preparation selectively.

JenneR's MeThon. ${ }^{1}$ - This stain, because of its simplicity, is useful for routine use. It is made up as follows: Equal parts of eosin (Gruebler, "W. G.") one and two-tenths per cent aqueous solution, and methyleneblue (medicinal, Gruebler) one per cent aqueous solution, are mixed and allowed to stand for twenty-four hours. A coarse granular precipitate is formed which appears dark, with a metallic luster on its surface. This is separated by filtration and washed with distilled water until the filtrate appears almost clear.

To make up the stain 0.5 gram of the dry precipitate is dissolved in 100 c.c. of methyl alcohol.

In using the stain, preparations are not fixed, but simply dried in the air and immersed in the stain for one to two minutes. After this, wash in distilled water and examine.

Wright's Modification of Leishman's Method. ${ }^{2}$ - A one per cent solution of methylene-blue (Gruebler) in five-tenths per cent solution of sodium bicarbonate in distilled water is steamed in a sterilizer at $100^{\circ}$ C. for one hour. After this has cooled, a one-tenth per cent aqueous solution of eosin (Gruebler, W. G.) is added until a metallic scum appears on the surface of the mixture. (About five parts of eosin solution to one of methylene-blue is necessary.) The precipitate which forms is collected by filtration, dried, and a saturated solution then made in methyl alcohol. This is filtered and diluted with one-quarter its bulk of methyl alcohol.

To stain, cover the dried preparation with the stain for one to one and one-half minutes. Dilute by dropping upon the stain distilled water from a pipette until a metallic film appears upon the top. Leave this on for three to fifteen minutes. Wash in distilled water.

Giemsa's Method. ${ }^{3}$ - The method of Giemsa is really a modification of the Romanowsky method. It is widely applicable, being of great value in the staining of the Spirochæte pallida, Vincent's spirilla, protozoa, and Negri bodies. The stain has been modified several times by

1 Jenner, Lancet, i, 1889.

${ }^{2}$ Wright, Jour. Med. Research, ii, 1902.

${ }^{3}$ Giemsa, Cent. f. Bakt., I, xxxvii, 1904. 
its originator, the following being the formula given by him in 1904: The substance referred to as azur II and purchasable under that name, consists of pure methylenazur chloralhydrate combined with an equal quantity of methylene-blue chloralhydrate. The substance referred to as azur II-eosin is a combination of this substance with eosin.

The staining fluid is made up as follows: ${ }^{1}$

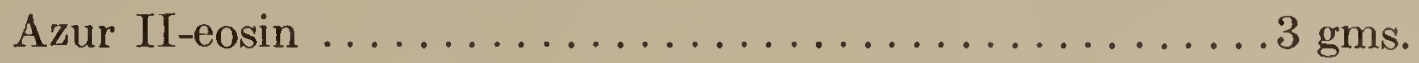

Azur II $\ldots \ldots \ldots \ldots \ldots \ldots \ldots \ldots \ldots \ldots \ldots \ldots \ldots \ldots$ gms.

This mixture is thoroughly dried over sulphuric acid in a desiccator, finely powdered, and rubbed through a fine sieve. It is then dissolved in 250 gms. of C. P. glycerin (Merck), at $60^{\circ} \mathrm{C}$. To this is added methyl alcohol (Kahlbaum) 250 c.c., previously warmed to $60^{\circ} \mathrm{C}$. This mixture is well shaken and allowed to stand at room temperature for twenty-four hours. The mixture is now ready for use.

For use 10 c.c. of distilled water are poured into a test tube and one to two drops of a one per cent potassium carbonate solution are added. Ten drops of the staining solution described above (one drop to the c.c.) are mixed with this slightly alkaline water. The preparation which is to be stained is fixed in methyl alcohol, dried, and covered with the diluted staining solution. For the staining of protozoa and exudates containing bacteria, ten to fifteen minutes are sufficient. For the staining of Negri bodies or Spirochæte pallida, one or more hours of staining should be employed. After staining, wash in running tap water and blot.

Wood's Method. ${ }^{2}$-Wood has devised a simple staining method based on the principles of the Giemsa stain, in which azur II and eosin may be used in separate solutions. Preparations are fixed in strong methyl alcohol for five minutes and are then stained in a 0.1 per cent aqueous solution of eosin until the preparation is pink. The eosin is then poured off and the preparation is covered with a 0.25 per cent aqueous solution of azur II for one-half to two minutes. Following this, it is washed in tap water and dried by blotting.

When an intense stain is desired, the solution of eosin and azur II may be flooded over the preparation together, using an excess of azur II. They are then left on from five to ten minutes. At the end of this time washing and drying as before completes the process.

${ }^{1}$ It is best not to attempt to make up the undiluted staining fluid, since this is purchasable under the name of "Giemsa Lösung für Romanowsky Färbung."

2 Wood, Med. News, 83, 1903. 
The Staining of Bacteria in Tissues.-The preparation of tissue for bacterial staining is, in general, the same as that employed for purposes of cellular studies, in histology. For bacteriological studies the most useful fixative is alcohol; other fixations, such as that by formalin, Zenker's fluid, or Mueller's fluid, give less satisfaction. In other respects the details of dehydration and embedding are the same as those used in histological studies, except that it is desirable that the tissues should be handled rather more carefully than is necessary for ordinary pathological work, and the changes from the weaker to the stronger alcohols should be made less abruptly. ${ }^{\text {- }}$

Embedding in paraffin is preferable to celloidin, although the latter method is not unsuccessful if carefully carried out. 'The chief disadvantages of celloidin are the retention of color by the celloidin itself and the consequent unclearness of differentiation. It is also easier to cut thin sections from paraffin blocks than from those prepared with celloidin.

When staining tissue sections for bacteria, it is most convenient to carry out the process with the section attached to a slide. For celloidin sections this may be accomplished by means of ether vapor. For paraffin sections it is necessary to cover the slide with an extremely thin layer of a filtered mixture of equal quantities of egg albumin and glycerin, to which a small crystal of camphor or a drop or two of carbolic acid has been added. The sections are then floated upon a slide so prepared, and set away in the thermostat for four or five hours.

LoefFler's Method. ${ }^{2}$-Stain in alcoholic methylene-blue solution five to fifteen minutes, or in Loeffler's alkaline methylene-blue solution one to twenty-four hours.

Wash in one to one-thousand acetic acid solution for about ten seconds.

Treat with absolute alcohol by pouring the alcohol over the preparation for ten to twenty seconds.

Clear with xylol.

Mount in balsam.

When celloidin sections are stained in this way ninety-five per cent alcohol should be substituted for the absolute. A number of other staining solutions may be used in the same way, aqueous fuchsin or aqueous gentian-violet yielding good result.

${ }^{1}$ For details of such work reference should be had to the standard textbooks on pathological technique, notably the very excellent one of Mallory and Wright.

${ }^{2}$ Loeffler, Mitt. a. d. kais. Gesundheitsamt, ii, 1884. 
Nicolle advises the use of a ten per cent aqueous solution of tannic acid for a few seconds after washing with the acetic acid. This fixes the stain and prevents a too vigorous decolorization during the process of dehydration.

Method of Staining Gram-Positive Bacteria in Tissue Sections. -Celloidin Sections.-After fixing section to the slide by pressure with a filter paper or by ether vapor, cover with anilin-water gentian-violet five minutes.

Pour off excess of stain and cover with Gram's iodin solution for two minutes.

Decolorize with ninety-five per cent alcohol until no more color comes out.

Stain quickly with eosin-alcohol (ninety-five per cent alcohol to which enough eosin has been added to give a transparent pink color; about $1: 15$ ). Clear in eosin-oil of origanum (oil of origanum, 25 c.c. and eosin alcohol, as above, about 3 c.c.).

Blot and mount in balsam.

Paraffin Sections. - Stain with anilin-water gentian-violet five to tea minutes.

Wash in water.

Cover with Gram's iodin solution one minute.

Wash in water.

Decolorize with absolute alcohol until no more color comes out.

Clear in xylol.

Mount in balsam.

Gram-Weigert Method. ${ }^{1}$-(For celloidin sections.) - Stain for one-half hour in the following freshly filtered solution:

Carmine . 3-5 grams.

Saturated aqueous solution of lithium carbonate... 100 c.c.

Dehydrate in ninety-five per cent alcohol.

Stick section to slide with ether vapor.

Stain in anilin-water gentian-violet for five to fifteen minutes (or $n$ a saturated solution of aqueous crystal violet diluted with water one to ten, five to fifteen minutes).

Wash in physiological salt solution.

Cover with Gram's iodin solution one to two minutes.

Wash in water and blot.

${ }^{1}$ Weigert, Fortschr. d. Med., v, 1887. 
Decolorize with anilin oil until no more color comes off.

This both decolorizes and dehydrates.

Treat with xylol. Mount in balsam.

Method of Staining For Tubercle Bacildi lis Sections. ${ }^{1-}$ Paraffin Sections.- Stain in carbol-fuchsin solution hot for five minutes (or better cold, for twenty-four hours).

Wash in water.

Decolorize and counterstain in Gabbet's methylene-blue sulphuric acid mixture for one minute.

Wash in water.

Dehydrate in absolute alcohol.

Clear in xylol.

Mount in balsam.

Celloidin Sections. ${ }^{2}$-Stain lightly in alum hematoxylin.

Wash in water.

Dehydrate in ninety-five per cent alcohol.

Attach the slide by ether vapor.

Stain with steaming carbol-fuchsin two to five minutes.

Wash in water.

Wash with Orth's acid alcohol (alcohol ninety per cent., 99 c.c.; conc. $\mathrm{HCl}, 1$ c.c.) one-half to one minute.

Wash in water several changes.

Treat with ninety-five per cent alcohol until red color is entirely gone.

Blot and cover with xylol until clear. Mount in balsam.

Method of Staining Actinomyces in Sections.-Mallory's Method ${ }^{3}$. -1. Stain deeply in saturated aqueous eosin ten minutes.

2. Wash in water.

3. Anilin gentian-violet two to five minutes.

4. Wash in normal saline solution.

5. Weigert's iodin solution (iodin $1, \mathrm{KI} 2$, and water 100 parts) one minute.

6. Wash in water and blot.

7. Clear in anilin oil.

8. Xylol several changes.

9. Mount in balsam.

1 Mallory and Wright, "Pathol. Tech.," p. 413.

2 After Mallory and Wright.

${ }^{3}$ Mallory and Wright, "Pathol. Tech.," 1904. 


\section{CHAPTER VII}

\section{THE PREPARATION OF CULTURE MEDIA}

\section{GENERAL TECHNIQUE}

THE successful cultivation of bacteria upon artificial media requires the establishment of an environment which shall be suitable in regard to the presence of assimilable nutritive material, moisture, and osmotic relations. These requirements are fulfilled in the composition of the nutrient media described in another section, media which are to somo extent varied according to the special requirements of the bacteria which are to be cultivated. If cultivation, furthermore, is to have any value for scientific study of individual species, it is necessary to obtain these species free from other varieties of microorganisms, that is, in pure culture, and to protect such cultures continuously from contamination with the other innumerable species which are everywhere present.

The technique which is employed for these purposes has been gradually evolved from the methods originally devised by Pasteur, Koch. Cohn, and others.

Bacterial cultivation is carried out in glassware of varied construction, the forms most commonly employed being test tubes of various sizes, Erlenmeyer flasks, the common Florence flasks, and Petri dishes. All glassware, of course, must be thoroughly cleansed before being used.

Preparation of Glassware.-The cleansing of glassware may be accomplished by any one of a number of methods. New glassware may be immersed in a one per cent solution of hydrochloric or nitric acid in order to remove the free alkali which is occasionally present on such glass. It is then transferred to a one per cent sodium hydrate solution for a few hours, and following this is washed in hot running water.

In the case of old glassware which has contained culture media, sterilization in the autoclave is first carried out, then the glassware is boiled in five per cent soda solution or in soapsuds. After this, thorough mechanical cleansing is practiced, and the glassware may be treated by acid and alkali followed by running water, as given above. These last 
steps, however, are not essential, thorough washing in hot water after the soapsuds or soda solution being usually sufficient to yield good results. Other workers have recommended immersion of the glassware after mechanical cleansing in five per cent to ten per cent potassium bichromate solution in twenty-five per cent sulphuric acid. This is followed by thorough washing in hot running water, and drying.

Clean flasks and test tubes are then stoppered with cotton, which has been found to be a convenient and efficient seal against the bacteria of the air, catching them in the meshes of the fibers as in a filter. The technique of the stoppering or plugging of glass receptacles is important,

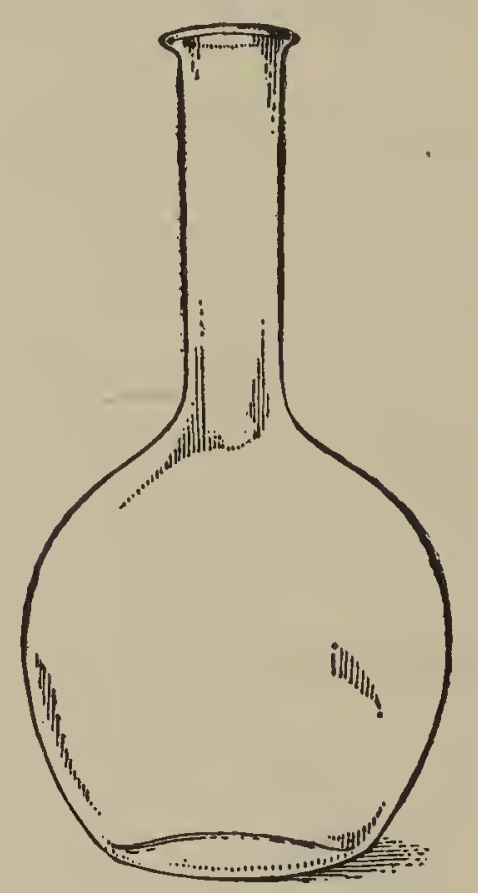

Fig. 15.-Florence Flask.

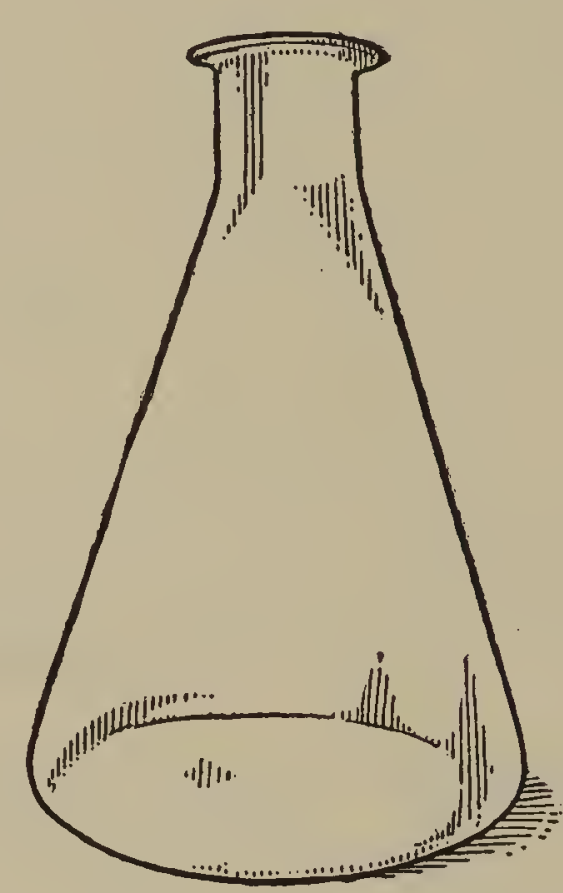

Fig. 16.-Erlenmeyer Flask.

in that, when poorly plugged, sterility is not safeguarded, and the purpose of culture study is defeated.

In almost all laboratories in this country non-absorbent cotton or "cotton batting" is used for the plug. In a few of the German laboratories the absorbent variety is employed. The disadvantages of the latter, especially in the case of fluid media, are obvious. The plugs should fit snugly, but not so tightly that force is necessary to remove them. Care should be taken, furthermore, that no creases are left between the surface of the glass and the periphery of the plug; for these, if present, may serve as channels for the entrance of bacteria. Fig. 18 , accompanying, will illustrate some of the more common and undesirable defects in poorly made plugs. The plugging itself is carried out by tearing a small piece of cotton, about $2 \times 2$ inches, from the roll, 
folding over one of its corners, and, applying the smooth end of a glass rod to the folded portion, gently pushing it into the mouth of the tube.

After plugging and before media are introduced into the tubes and flasks, these should be sterilized. This is best done in one of the "hotair sterilizers" (see Fig. 8, p. 69), by exposing the tubes for one hour to a temperature of $150^{\circ} \mathrm{C}$. If greater speed is desired exposure to $180^{\circ}$ to $190^{\circ} \mathrm{C}$. for half an hour is usually safe. If by mistake, however, the temperature is allowed to rise above $200^{\circ} \mathrm{C}$., a browning of the cotton plugs occurs and the glassware is apt to be stained by the burning of the fat and other organic material derived from the cotton. Petri dishes

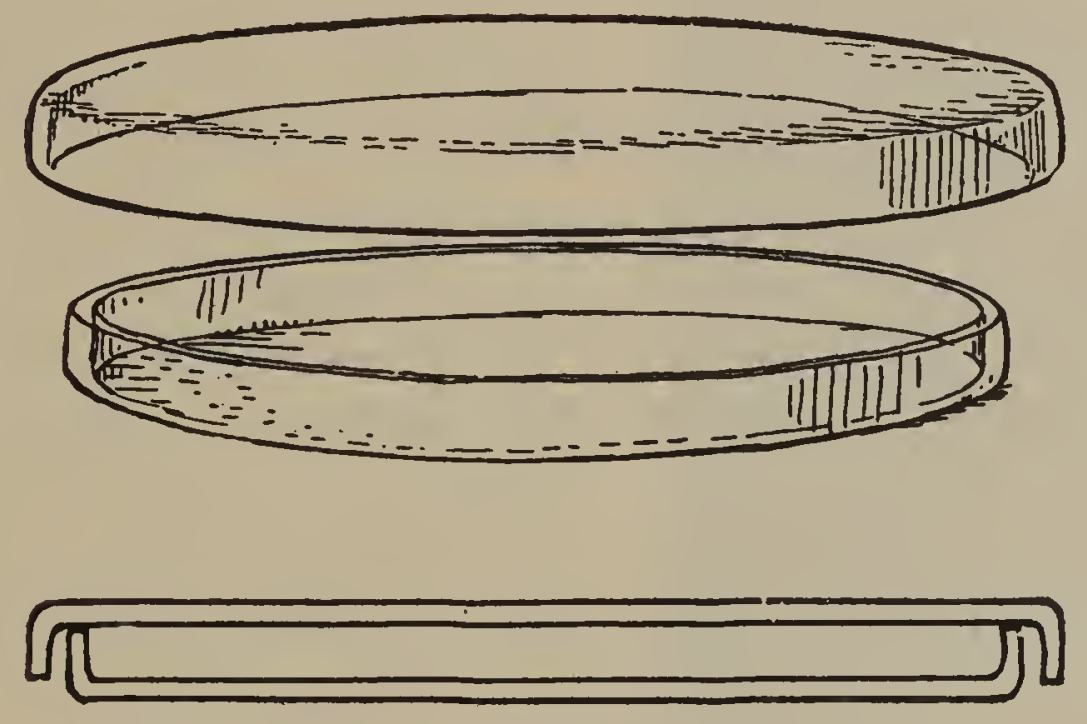

Fig. 17.-Petri Dish.

after cleansing are fitted together in the manner shown in Fig. 17, and are sterilized in the hot-air chamber at $150^{\circ} \mathrm{C}$. for one hour.

Glassware so prepared is ready for the reception of media.

Ingredients of Culture Media.-The food requirements of bacteria have been discussed in another section. From what has there been said, it is apparent that artificial culture media must, to a certain extent, be adjusted to the peculiarities of individual bacteria. In the cases of the more strictly parasitic microorganisms growth can be obtained only by the most rigid observance of special requirements. For the large majority of pathogenic bacteria, however, routine or standard media may be employed, which, while slightly more favorable for one species than for another, are sufficiently general in their composition to permit the growth of all but the most fastidious varieties.

The basis of many of our common media is formed by the soluble constituents of meat. These substances are best obtained by macerating 500 grams of lean beef in 1,000 c.c. of distilled water. The mixture is 
allowed to infuse in the ice chest over night, and then strained through cheese-cloth. To this infusion are added the other required constituents in the manner given in the detailed instructions below. The soluble constituents of meat, however, may also be procured in a simpler way by the use of the commercial meat extracts,

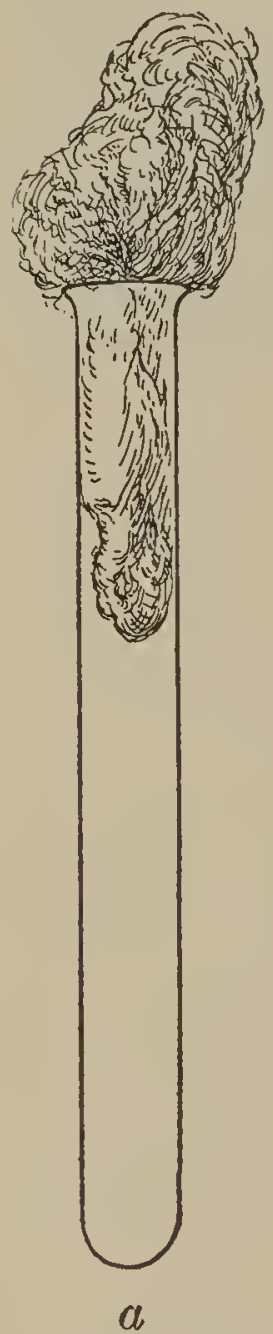

Fig. 18.-Test Tube $(a)$ incorrectly stoppered; (b) correctly stoppered. such as that of Liebig. These extracts are dissolved in quantities of five grams to the liter, and other constituents are added to this nutrient basis.

Though simpler to make, the meat-extract media are less favorable for the cultivation of the more delicate organisms than are the media made directly from fresh meat. Nevertheless, they suffice for the cultivation of the large majority of the more saprophytic pathogenic microorganisms and hold an important place in laboratory technique.

The ingredients and methods used in various laboratories in the preparation of such standard media should be, as much as possible, uniform, in order that confusion in results may be avoided; for, as is well known, the biological characteristics of one and the same bacterial species may vary considerably if grown on media differing in their composition.

A committee of the American Public Health Association, ${ }^{1}$ appointed in 1897 for the sake of standardizing the methods of preparation of media, recommended that the following rules should govern the choice of ingredients:

1. Distilled water should be used in all cases.

2. The meat used should be fresh, lean beef (when veal or chicken is substituted the change should be stated).

3. The pepton used should be Witte's pepton, dry, made from meat.

4. Only C. P. NaCl should be used.

5. For alkalinizing C. P. sodium hydrate should be used in normal solutions.

${ }^{1}$ Rep. Com. of Amer. Bact. to Com. of Amer. Pub. Health Assn. Meeting, Philadelphia, Sept., 1897. 
6. For acidification 'C. P. hydrochloric acid in normal solution should be used.

7. When glycerin is used, this should be of the redistilled variety.

8. The agar-agar employed should be of the finest grade of commercial thread agar.

9. The gelatin should be the commercial sheet gelatin washed as free as possible of acid and impurities.

10. Chemicals and carbohydrates which are used should be as nearly chemically pure as possible.

Titration of Media.-Next in importance to the actual composition of media is the adjustment of their reaction. Bacteria are highly susceptible to variations in the acidity and alkalinity of media, excessive degress of either may completely inhibit development or moderate variations may lead to marked modifications of cultural characteristics. It is necessary, therefore, to adjust the reaction both for the sake of favoring growth and in order to insure uniformity of growth characters. This is accomplished by titration which is best carried out according to the recommendations of the committee mentioned above.

The color indicator employed for the titration is a five-tenths per cent solution of phenolphthalein in fifty per cent alcohol. The chief advan= tage of this indicator over others is due to the fact that it indicates the presence of organic acid and acid compounds in its reaction. For

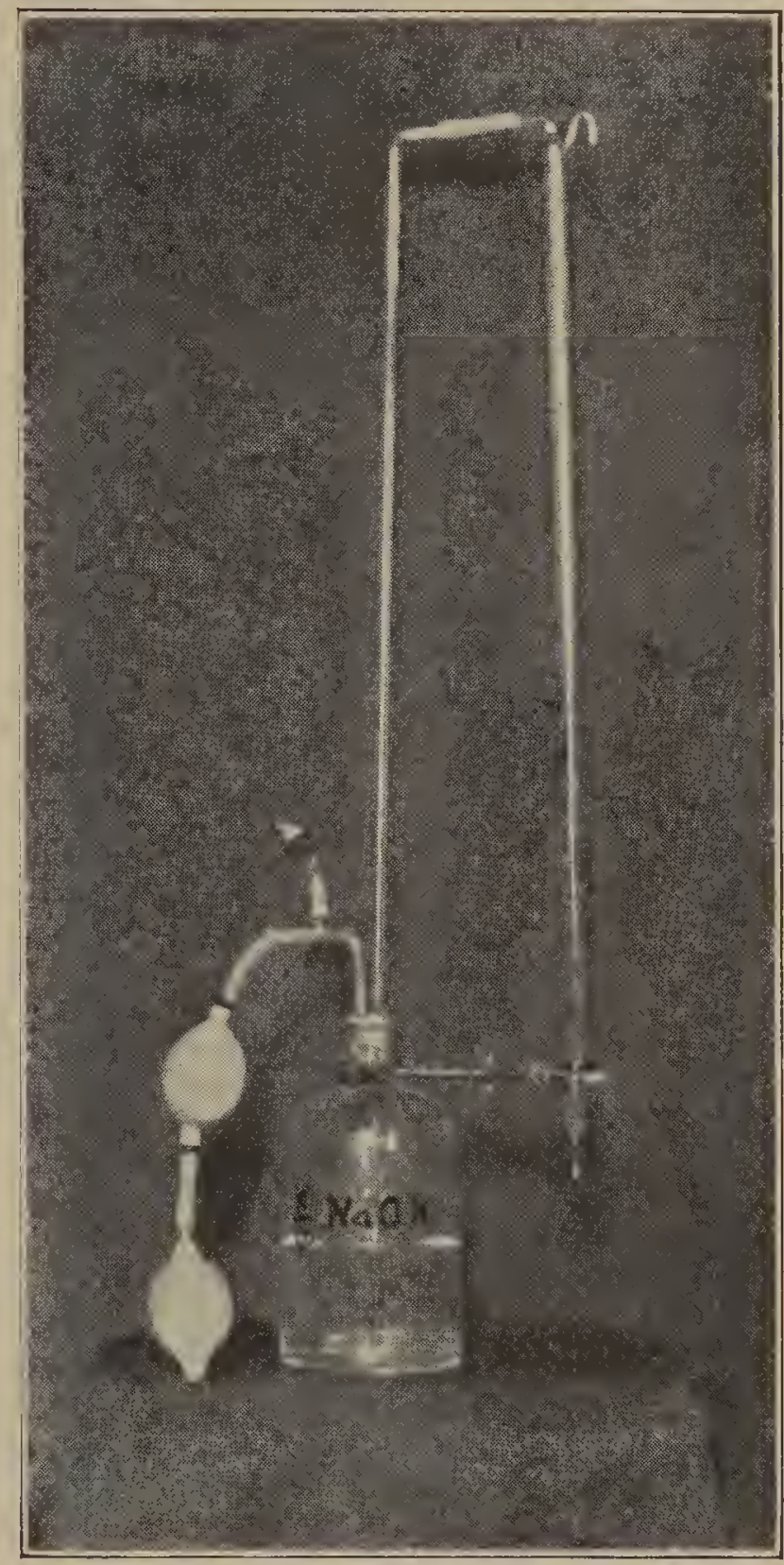

Fig. 19.-Burette For Titrating Media. actual titration $\frac{\mathrm{N}}{20}\left(\frac{1}{20}\right.$ normal $)$ solutions of sodium hydrate or of hydrochloric acid are used. Since media in the process of preparation are usually acid, the $\mathrm{NaOH}$ solution is the one most frequently needed. Five c.c. of the medium to be tested is measured accurately in a care- 
fully washed pipette and transferred into a porcelain evaporating dish. To this are added 45 c.c. of distilled water. The mixture is thoroughly boiled for three minutes over a free flame. The boiling drives off $\mathrm{CO}_{2}$, giving the true neutral point, and approximates the conditions prevailing during the further sterilization of

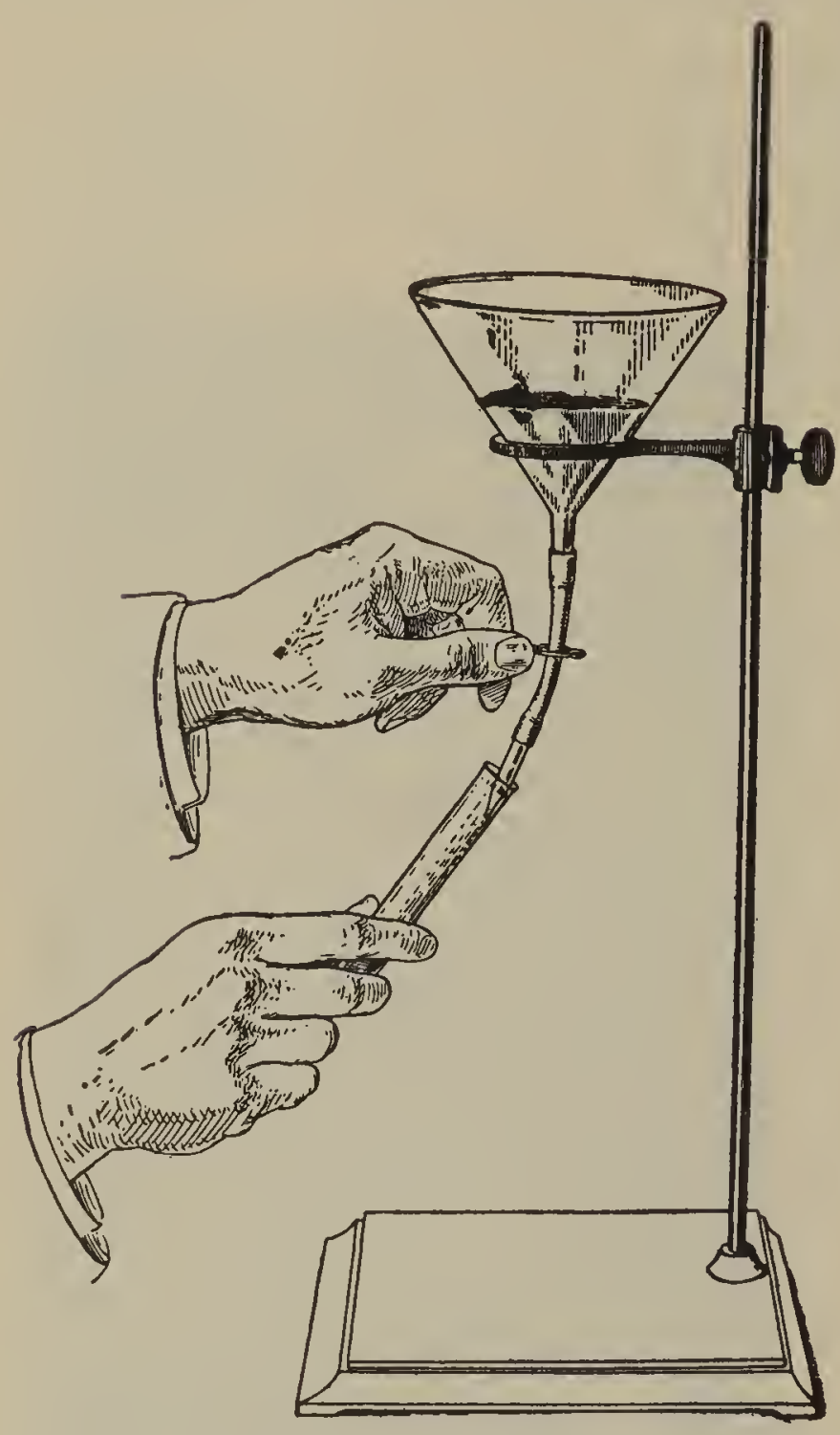

Fig. 20.-Tubing Media. the medium from which the 5 c.c. have been taken. After boiling, 1 c.c. of the phenolphthalein is added. If the medium is acid, no color is present; if alkaline, a pink or red color appears. The $\frac{\mathrm{N}}{20}$ alkali or acid solution is allowed to drop into the dish from a graduated burette. When the neutral point is approached in an acid solution, each drop of sodium hydrate added brings forth at first a deep red, which, however, upon slight. stirring with a clean rod, completely disappears. ${ }^{1}$ The end reaction is reached when a faint but clear and distinct pink color remains in the fluid after stirring.

When titrating alkaline media, the addition of the phenolphthalein produces a red color in the hot medium which gradually fades upon the addition of $\frac{\mathrm{N}}{20} \mathrm{HCl}$, becoming colorless at the end point of titration. Titration should be done quickly and in a hot solution. From the result of the titration the computation for the neutralization of the entire bulk of the medium can be made by a simple arithmetical process as illustrated in the following example:

Let us suppose that we have used:

2.5 c.c. of $\frac{\mathrm{N}}{20} \mathrm{NaOH}$ to neutralize 5 c.c. of the medium, $\begin{array}{ll}\text { then } 2.5 \text { c.c. of } & \frac{\mathrm{N}}{1} \mathrm{NaOH} \text { will neutralize } 100 \text { c.c. " " } \\ \text { and } 25 \text { c.c. of } & \frac{\mathrm{N}}{1} \mathrm{NaOH} \text { will neutralize } 1,000 \text { c.c., or one liter. }\end{array}$

${ }_{1}$ See standard textbooks on volumetric analysis. 
The adjustment of the reaction of media is largely determined by the particular uses for which the media are designed. For examinations in the practice of sanitation, such as analyses of water, ice, and milk, etc., the American Public Health Association recommends a standard reaction of +1 per cent (the plus sign is used to indicate acidity, the minus alkalinity; +1 per cent is the expression used to indicate that one per per cent of $\frac{\mathrm{N}}{1}$ sodium hydrate solution would be required to neutralize the medium or 10 c.c. to the liter). For general work with pathogenic bacteria, the most favorable reaction for routine media is slight alkalinity, neutrality, or an acidity not exceeding +1 per cent.

Methods of Clearing Media.-Clearing with Eggs.-When culture media are prepared from substances containing no coagulable proteid, it is often necessary, for purposes of clearing, to add the whites of eggs, and then to. heat for forty-five minutes in the Arnold sterilizer. In the following detailed descriptions, the direction "clear with egg" has been given whenever such a step is deemed necessary. The exact technique of such a procedure is as follows:

In a small pot or pan, the whites of several eggs (one or two eggs to each liter of medium) are beaten up thoroughly with a little water $(20$ c.c.). This egg white is then poured into the medium, which, if hot, as in the case of melted agar or gelatin, must first be cooled to about $50^{\circ}$ to $55^{\circ} \mathrm{C}$. The mixture is then thoroughly shaken and steamed in the Arnold sterilizer for thirty minutes. At the end of this time the flask containing the medium is removed from
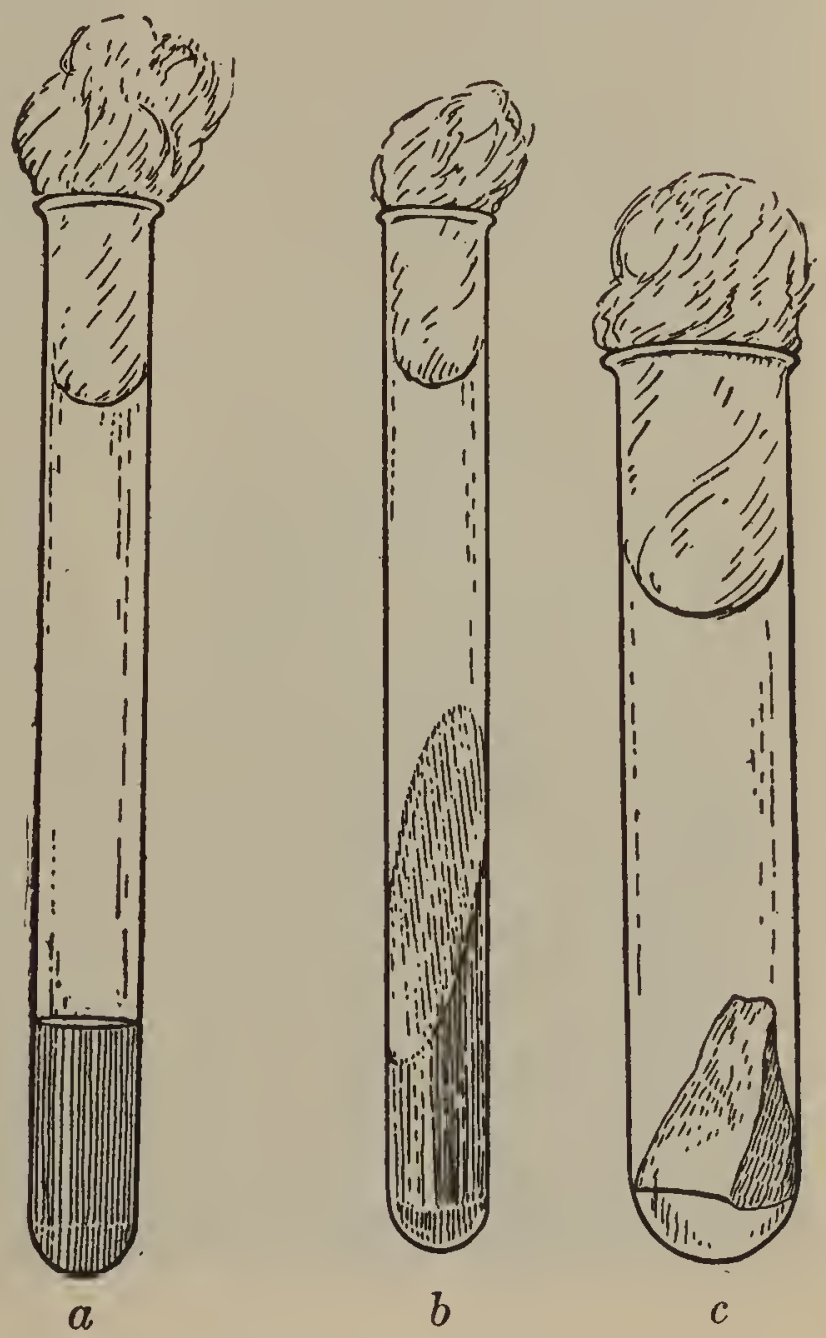

Fig. 21.-M HDiA in Tubes: $a$, broth; $b$, agar slant; $c$, potato. the sterilizer and thoroughly shaken so as completely to break up the coagulum which has formed. It is then replaced and allowed to steam for another fifteen minutes. At the end of this time the medium between the coagula should be clear. It is now ready for filtration through cotton. 
Filtering Media through Cotton.- The filtration of media after clearing, either by the addition of eggs or by the coagulation of the proteids originally contained in it, is best done through absorbent cotton. A small spiral, improvised of copper wire, is placed as a support in the bottom of a large glass funnel. A square piece of absorbent cotton is

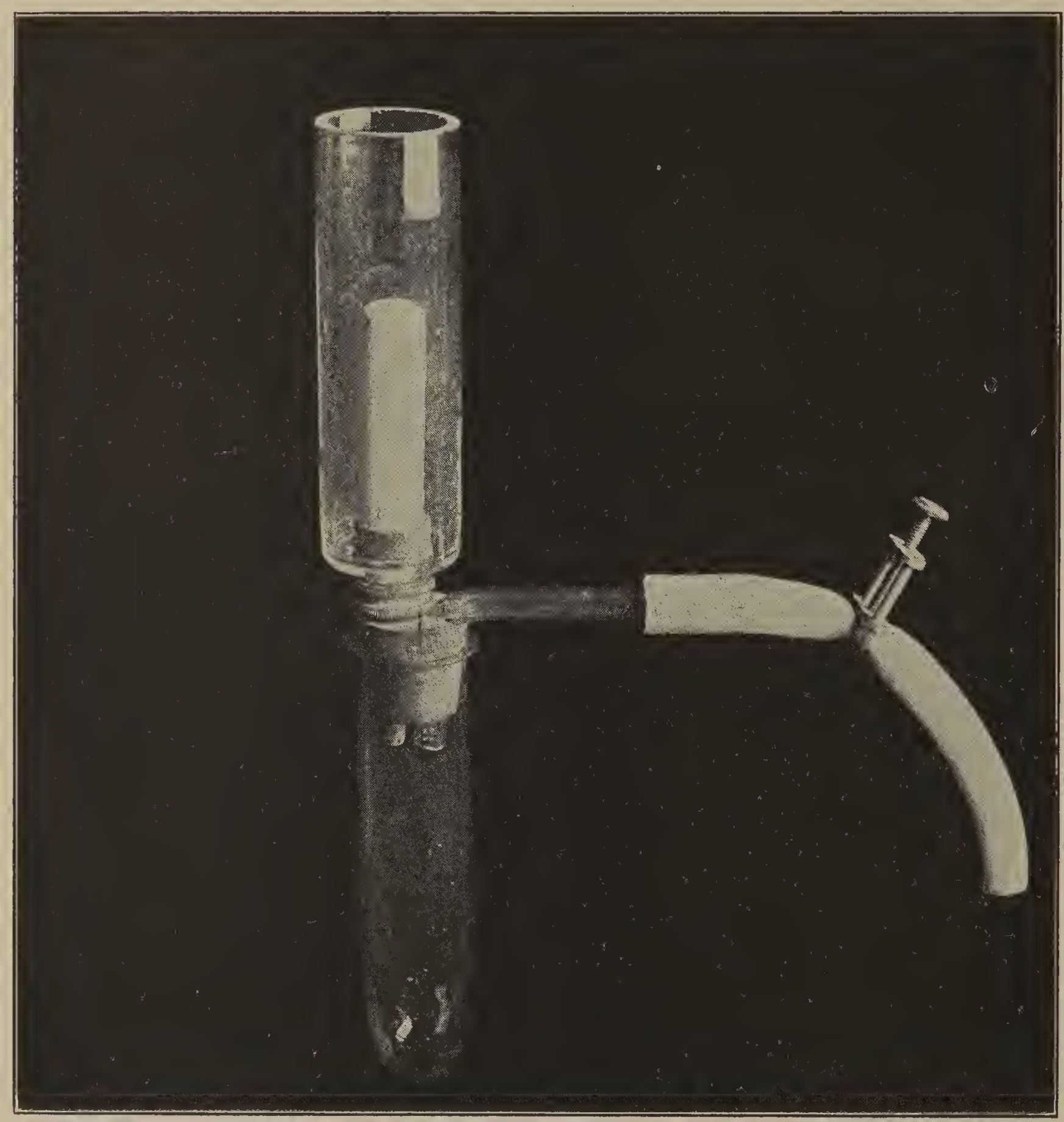

Fig. 22.-Berkefeld Filtirr.

then split horizonta ' $y$. giving two squares of equal size. Ragged edges and incisures shourd be avoided. These two layers of cotton are then placed in the funnel, one piece above the other in such a way that the direction of the fibers of the two layers is at right angles one to the other. They are then gently depressed into the filter with the closed fist. The 
edges of the cotton are made to adhere to the sides of the funnel by allowing a thin stream of tap water to run over them, while smoothing them against the glass with the hand.

The medium, when poured into such a filter, should be poured along a glass rod at first, to avoid running down the sides or bursting the filter. After filtration has begun, the filter should be kept as full as possible. The first liter or so which comes through may not be clear, but the filter gains in efficiency as the coagulum settles into the fibers of the cotton, and the first yield may be sent through a second time. Filtration of agar or gelatin is best done in a warm room with windows and doors closed, and the filter covered with a lid, to avoid too rapid cooling. The funnel and filter should be warmed just before use.

Filtering through Paper.-Many media may be efficiently cleared by filtration through close filter paper without the aid of coagula.

The Tubing of Media.-Most of the media described in the foregoing section are used in test tubes. In order to fill these tubes, the media are best poured into a large glass funnel to which a glass discharging tube has been fitted by means of a short piece of rubber tubing (see Fig. 20). Upon this is placed a thumb cock. The plug is then removed from the test tube by catching it between the small and ring fingers of the right hand and the glass outlet is thrust deeply into the test tube, in order to prevent the medium from touching the upper portion

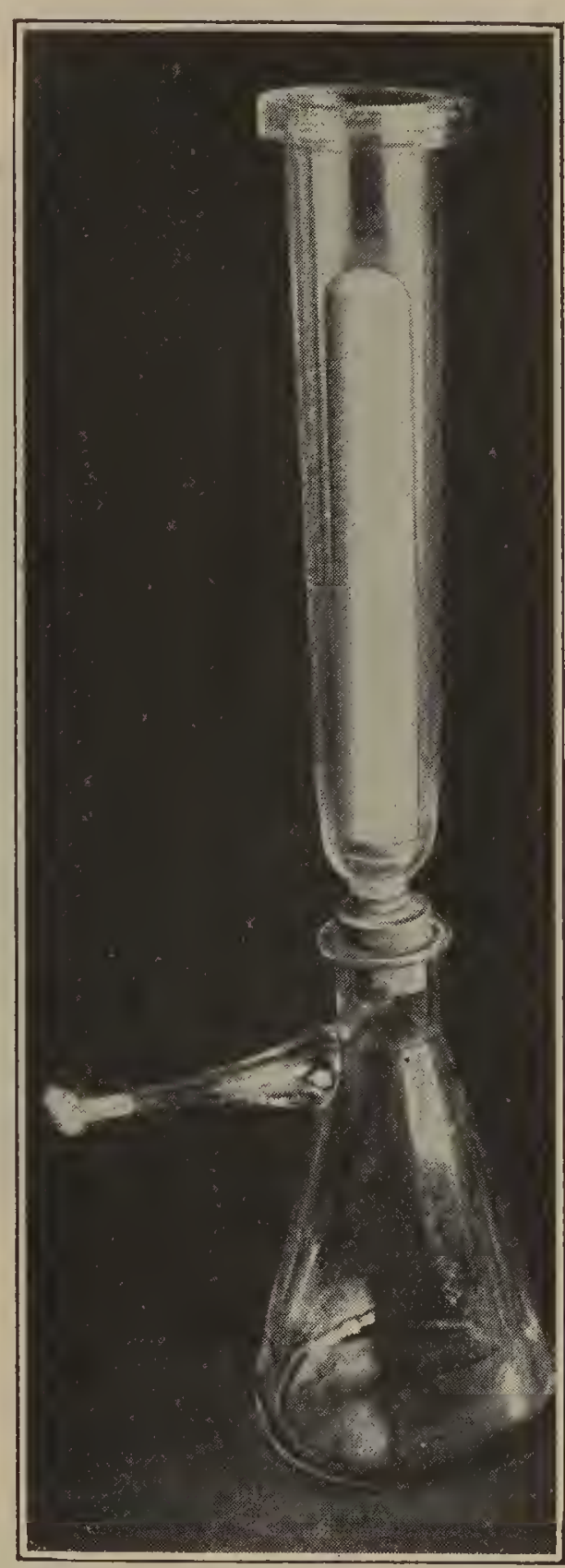

Fig. 23.-BERKEFELD Filter. of the test tube where the cotton plug will be lodged. About 7 to 8 c.c. is put in each test tube.

Sterilization of Media.-By Heat.-Media which contain neither sugars, gelatin, glycerin, nor animal serum may be sterilized in the autoclave at fifteen pounds pressure for fifteen minutes to half an hour. Media which contain these or other substances subject to injury from the high temperature, must be sterilized by the fractional method, 
i.e., by twenty minutes' exposure in the live steam sterilizer (Arnold, Fig. 9, p. 70) on each of three consecutive days. During the intervals between sterilizations, they should be kept at room temperature or in the incubator, to permit the germination of spores which may be present. Media containing animal serum or other albuminous solutions which are to be sterilized without coagulation, may be sterilized in wate baths, or in hot-air chambers (Fig. 10, p. 71), at temperatures varying

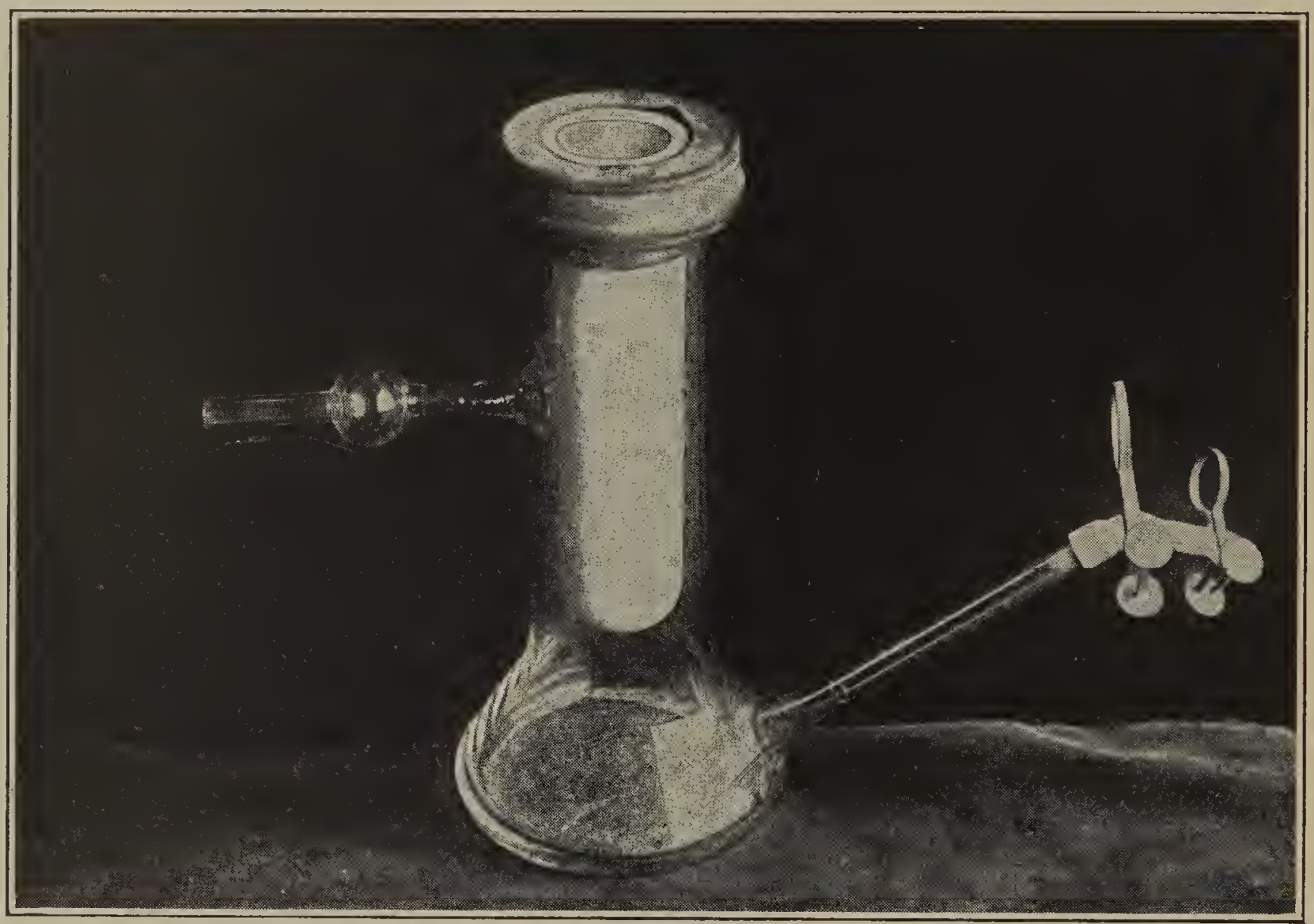

Fig. 24.-Reichel Filiter.

from $60^{\circ}$ to $70^{\circ} \mathrm{C}$, by the fractional method. In such cases five or six exposures of one hour on succeeding days should be employed.

By Filtration.-It is often desirable in bacteriological work to free fluid from bacteria. This is frequently necessary for the sterilization of blood-serum or exudate fluids, or for obtaining toxins free from bacteria. For these purposes a large variety of filters are in use. Those most commonly employed are of the Chamberland ${ }^{1}$ or Berkefeld type, which consist of hollow candles made of unglazed porcelain or diatomaceous earth. Both these types are made in various grades of fineness, upon which depend both the speed of filtration and the efficiency. They are made in various forms and models, some of which are shown

1 Pasteur and Chamberland, Compt. rend. de l'acad. des sci., 1884. 
in the accompanying figures. In most of the methods of filtration commonly employed the fluid which is to be filtered is sucked through the walls of the filter, either by a hand suction-pump or by some form of vacuum-pump attached to an ordinary water-tap.

The hollow candle-filter may either be firmly fitted into a cylindrical glass chimney and surrounded by the fluid which is to be filtered, or else the candle may be connected to the collecting flask with sterile rubber tubing and suspended freely in the fluid. Perfect filters of these types will hold back any of the bacteria known to us at present.

Filters before use must be sterilized. The candles themselves are subjected to $150^{\circ} \mathrm{C}$. in the hot-air sterilizer for one hour. The glassware and washers necessary for setting up the apparatus may be sterilized by boiling. In order that filters may be repeatedly used with good result, it is necessary that they should be carefully cleaned from time to time. This is best done in the following way:

Filters through which fluids from living cultures have passed are first sterilized in the Arnold steam sterilizer. Their exterior is then carefully cleaned with a fine brush. Following this a five-tenths per cent solution of potassium permanganate is passed through them and this again removed by sucking through a five per cent solution of bisulphite of soda. This last is washed out by sending a considerable quantity of distilled water through the filter, which is then dried and sterilized by heat.

The suction necessary for filtration through these filters is usually applied by means of the ordinary suction-pump attached to a running faucet.

Slanting of Media.-Dolid media which are to be used in slanted form in test tubes should be inclined on a ledge (easily improvised of glass tubing) at the proper slant, after the last sterilization. Agar, the medium 
most frequently cmployed in this way, should be left in this position for two or three days. (See Fig. 21, b.)

\section{ACTUAL STEPS IN THE PREPARATION OF NUTRIENT MEDIA}

Broth.-Meat Extract Broth.-1. To 1,000 c.c. of distilled or clear tap water add 5 gms. Liebig's meat extract, 10 gms. Witte's pepton, and 5 gms. common salt $(\mathrm{NaCl})$.

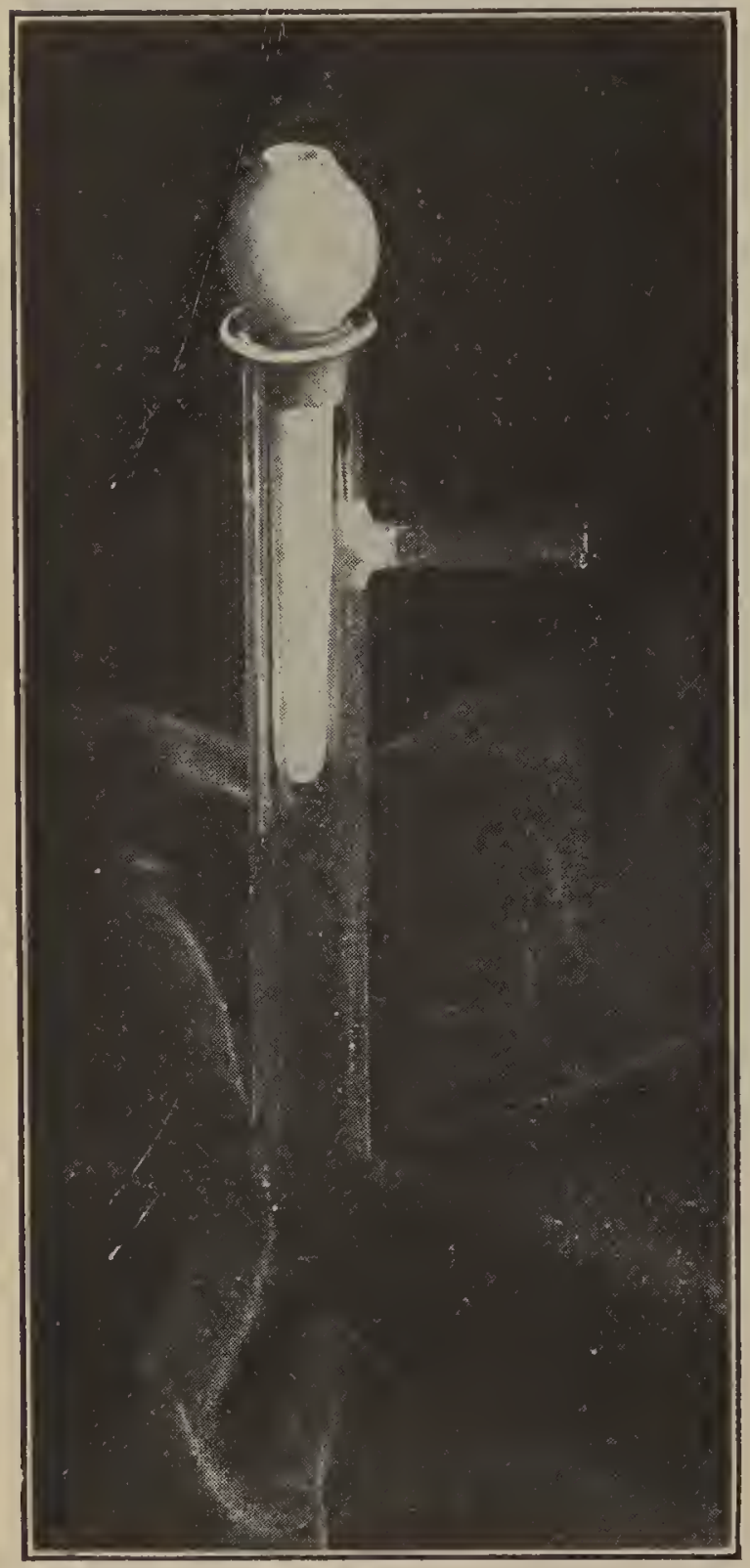

Fig. 26.-MAassen Filter, For small, Quantities of Fluid.

2. Weigh solution with containing vessel (any suitable agate-ware vessel or glass flask will do).

3. Heat over free flame until thoroughly dissolved, stirring constantly.

4. Weigh again and make up loss by evaporation.

5. Determine volume.

6. Titrate and adjust to required reaction, heating over free flame for five minutes.

7. Filter through paper until clear.

8. Sterilize.

If medium can not be cleared by filtering through paper, clearing by white of egg may be resorted to and the medium filtered through cotton.

Meat Infusion Broth.-1. Infuse 500 gms. $^{1}$ of lean meat, twelve to twenty-four hours, with 1,000 c.c. of distilled water in refrigerator.

2. Strain through wet cotton flannel or wet cheese-cloth and make up volume to 1,000 c.c.

3. Add 5 gms. common salt and 10 gms. Witte's pepton.

4. Weigh with containing vessel.

5. Warm over flame or water bath, stirring until pepton is dissolved, not allowing temperature to rise above $50^{\circ} \mathrm{C}$.

6. Determine volume.

${ }^{1}$ Roughly, 1 pound ( $1 \frac{1}{4} \mathrm{lb}$.). 
7. Titrate and adjust reaction to neutral.

8. Heat in Arnold sterilizer for thirty minutes; shake or stir well and heat again for fifteen minutes.

9. Determine weight and restore loss by evaporation.

10. Determine volume, titrate, and adjust reaction to desired point (usually one per cent acid).

11. Heat again for five minutes if adjustment of reaction has been necessary. ${ }^{1}$

12. Filter through absorbent cotton, passing the filtrate through the same filter until clear.

13. Titrate and record the final reaction.

Place in cotton-plugged sterile flasks or plugged sterile test tubes, and sterilize for thirty minutes in the Arnold sterilizer on three successive days, leaving at room temperature in the intervals.

Sugar-Free Broth.-1. Make 1 liter of meat infusion broth, following steps $1,2,3,4,5,6,7$, and $8^{2}$; then filter through thin cotton filter to remove gross particles-total clearing is not necessary.

2. Put the broth in a flask and cool. Then add 10 c.c. of a twentyfour-hour broth culture of B. coli communis.

3. Place the flask, stoppered with cotton, in the incubator at $37^{\circ} \mathrm{C}$. for eighteen hours. (The bacteria will ferment and thus destroy any sugar [monosaccharid] which may be present in the broth, and thus render the broth sugar-free and acid.)

4. Heat thoroughly to kill the bacteria.

5. Determine weight and bring to $1,015 \mathrm{gms}$. Then determine volume and titrate, and adjust to neutral. Heat thoroughly again.

6. Filter through filter paper until clear.

7. The pure sugars, dextrose, lactose, saccharose, etc., are then added to separate portions $(250$ c.c.) of the broth in the proportion of one per cent.

8. When the sugars are dissolved, tube the broth immediately in fermentation tubes, and sterilize by discontinuous sterilization, never heating over twenty minutes at a time, as heat tends to destroy or change the sugars.

Glycerin Broth.-To ordinary, slightly acid or neutral meat infusion broth, add six per cent of C. P. glycerin. Sterilize by fractional method.

${ }^{1}$ Media become more acid on boiling, probably because of a driving out of $\mathrm{CO}_{2}$, and a second titration therefore becomes necessary.

2 These steps refer to the regular directions for making infusion broth. One liter of previously made infusion broth may be used instead. 
Calcium Carbonate Broth.-This medium is designed for obtaining mass cultures of pneumococcus or streptococcus for purposes of immunization or agglutination.

To 100 c.c. of meat infusion broth in small flasks, add one per cent of powdered calcium carbonate, and one per cent of glucose. It is a wise precaution to sterilize the dried calcium carbonate in the hot-air chamber before using. Small pieces of marble may be used as suggested by Bolduan.

Pepton-Salt Solution (Dunham's solution):

1. Distilled water .....................

Pepton (Witte) .................... 10 gms.

$\mathrm{NaCl} \ldots \ldots \ldots \ldots \ldots \ldots \ldots \ldots \ldots \ldots, \quad 5$ ".

«. Heat until ingredients are thoroughly dissolved.

3. Filter through filter paper until perfectly clear.

4. Tube twenty-five tubes, and store remainder in 250 c.c. flasks.

Sterilize by discontinuous method.

Nitrate Solution.-

1. Distilled water................... 1,000 c.c.

Pepton................... 10 gms.

Potassium nitrate.$\ldots \ldots \ldots \ldots \ldots \ldots \ldots \ldots$. 0.2 "

2. Heat until ingredients are thoroughly dissolved.

3. Filter through filter paper until perfectly clear.

4. Tube twenty-five tubes, and store remainder in 250 c.c. flasks.

Sterilize by discontinuous sterilization.

Uschinsky's Proteid-Free Medium. ${ }^{1}$-To one liter of distilled water add:

Asparagin......................... 3.4 grams.

Ammonium lactate.................... 10 "

Sodium chloride ...................... 5 "

Magnesium sulphate $\ldots \ldots \ldots \ldots \ldots \ldots \ldots \ldots, 0.2$ "

Calcium chloride...................... 0.1 "

Potassium phosphate................. 1.0 "

When these substances are thoroughly dissolved, add 40 c.c. of glycerin Tube and sterilize.

Gelatin.-Meat-Extract Gelatin.-1. To 1,000 c.c. of distilled water add Liebig's extract 5 gms., pepton 10 gms., $\mathrm{NaCl} 5$ gms., and 120 gms. of the finest French sheet gelatin. ${ }^{2}$

${ }_{1}$ Uschinsky, Cent. f. Bakt., 1, xiv, 1893.

${ }_{2}$ The acidity and consistence of the different commercial gelatins vary considerably and care should be taken in selecting a uniform and suitable brand, such as Hesterberg's gold label gelatin. It is advisable, when working during the summer or in hot climates, to add 130 instead of 120 grams. 
2. Weigh with containing vessel.

3. Dissolve by warming.

4. Adjust weight, determine volume, titrate, and adjust reaction.

5. Cool to $60^{\circ} \mathrm{C}$., add whites of two eggs, and stir thoroughly.

6. Heat for thirty minutes, stir thoroughly, and heat for fifteen minutes.

7. Adjust weight.

8. Filter through cotton.

9. Sterilize.

Meat-Infusion Gelatin.-1. Infuse 500 gms. lean meat twelve to twenty-four hours with 1,000 c.c. of distilled water in refrigerator.

2. Strain through wet cotton flannel or wet cheese-cloth and make up volume to 1,000 c.c.

3. Add 5 gms. common salt, 10 gms. Witte's pepton, and 120 gms. of the finest French sheet gelatin.

4. Weigh with containing vessel.

5. Warm over flame or water bath, stirring till pepton and gelatin are dissolved and not allowing temperature to rise above $50^{\circ} \mathrm{C}$.

6. Determine volume.

7. Titrate and adjust reaction to neutral.

8. Heat in Arnold sterilizer for thirty minutes; shake or stir well and heat again for fifteen minutes.

9. Determine weight and restore loss by evaporation.

10. Determine volume, titrate, and adjust reaction to desired point, if necessary (one per cent acid).

11. Heat five minutes over free flame, constantly stirring, if adjustment of reaction has been necessary.

12. Filter through absorbent cotton, passing the filtrate through the same filter until clear.

13. Titrate and record the final reaction.

Place gelatin in cotton-plugged sterile 250 c.c. flasks or about 8 c.c. in plugged sterile test tubes and sterilize for thirty minutes in the Arnold sterilizer on three successive days, leaving at room temperature in the intervals. Never heat the gelatin for longer than is necessary to comply with directions, or it may not be solid enough for use. With some brands of gelatin it may be necessary to add thirteen per cent in order to obtain sufficient stiffness.

Agar.-Meat-Extract Agar.-1. To 1,000 c.c. of distilled water (or tap water) add $15 \mathrm{gms}$. of thread agar, 10 gms. of Witte's pepton, and $5 \mathrm{gms}$. of Liebig's meat extract, and $5 \mathrm{gms}$. of common salt. 
2. Weigh with containing vessel.

3. Heat over free flame until agar is dissolved, thirty to forty-five minutes. (Great care should be exercised in determining that agar is completely in solution.)

4. Determine weight and make up loss by evaporation.

5. Determine volume, titrate, and adjust to desired reaction.

6. Cool to $60^{\circ} \mathrm{C}$.

7. Add whites of two eggs and stir thoroughly.

8. Heat in Arnold sterilizer thirty minutes, stir, and reheat fifteen minutes.

9. Weigh and make up loss by evaporation.

10. Determine volume, titrate, and correct reaction if necessary. ${ }^{1}$

11. Heat for five minutes, if reaction is corrected.

12. Filter through cotton, tube, and sterilize.

Meat-Infusion Agar. ${ }^{2}$ - (A) 1. Infuse $500 \mathrm{gms}$. lean meat twelve to twenty-four hours in 500 c.c. of distilled water in refrigerator.

2. Strain through wet cotton flannel or wet cheese-cloth, and make up voiume to 500 c.c.

3. Add 10 gms. of Witte's pepton and 5 gms. of common salt.

4. Weigh solution and containing vessel.

5. Warm over free flame or water bath till pepton and salt are dissolved, not allowing temperature to rise above $50^{\circ} \mathrm{C}$.

6. Determine volume, titrate, and neutralize.

(B) 7 . Add $15 \mathrm{gms}$. of thread agar to 600 c.c. of distilled water and boil over free flame for thirty to forty-five minutes, watching and stirring constantly till agar is completely dissolved. This will lose weight by evaporation; final weight should be 515 gms.

8. Cool this to about $60^{\circ} \mathrm{C}$.

(C) 9. Then to the solution $\mathrm{A}$ of meat infusion (at $50^{\circ} \mathrm{C}$.) add the solution $\mathrm{B}$ of agar (at $60^{\circ} \mathrm{C}$.):

10. Heat for thirty minutes in Arnold sterilizer. Shake or stir thoroughly, and heat fifteen minutes more. Adjust weight by adding water.

11. Determine volume, titrate, and adjust reaction to plus one per cent acid or any desired reaction.

12. Boil for two minutes over free flame, constantly stirring.

1 While titrating, care should be taken that medium does not solidify along sides of vessel. Agar may be made more quickly and successfully in autoclave.

${ }^{2}$ Glycerin agar is made by adding 6 per cent of C. P. glycerin to meat-extract or meat-infusion agar. 
13. Filter through absorbent cotton, passing the filtrate through the same filter until clear.

14. Titrate and record final reaction.

Place agar in cotton-plugged sterile flasks or plugged sterile test tubes and sterilize for thirty minutes on three successive days.

Lactose-Litmus-Agar (Wurtz).-1. Put 1,500 c.c. distilled water in previously weighed agate-ware vessel.

2. Add $15 \mathrm{gms}$. thread agar and boil over free flame for thirty to forty-five minutes, watching and stirring constantly till the agar is completely dissolved.

3. Add 5 gms. Liebig:s extract of meat, $5 \mathrm{gms}$. NaCl, $10 \mathrm{gms}$. Witte's pepton, and dissolve completely.

4. Restore loss by evaporation to $1,035 \mathrm{gms}$.

5. Determine volume, titrate, and adjust reaction to one per cent acid.

6. Place in a flask and cool to $60^{\circ} \mathrm{C}$.

7. Add the whites of two eggs beaten up in 50 c.c. of water and mix thoroughly.

8. Heat for thirty minutes in Arnold sterilizer, shake thoroughly, and heat again for fifteen minutes.

9. Adjust weight.

10. Filter through absorbent cotton to clear.

11. Add two per cent pure lactose (milk sugar). ${ }^{1}$

12. Add enough pure five per cent litmus solution ${ }^{2}$ to bring to purple color when cold.

13. Tube and sterilize.

Welch's Modification of Guarnieri's Medium. ${ }^{3}$-This medium is made on a meat-infusion basis, according to the directions given for the preparation of meat-infusion agar. It contains 5 grams of agar, 80 grams of gelatin, 5 grams of $\mathrm{NaCl}$, and 10 grams of pepton to one liter. It should

1 Add lactose and litmus to 250 c.c. for 25 tubes; keep the remainder, with. out lactose, stored in small sterile flasks for further use.

2 The litmus solutions used in the preparation. of media are best made up as follows: Litmus in substance-Merck's purified, or Kaulbaum's--is dissolved in water to the extent of 5 per cent. The solution is made by heating in an Arnold sterilizer for about one to two hours, shaking occasionally. The solution is then filtered through paper and sterilized. It should be kept sterile, as molds will grow in it otherwise.

A standard litmus solution; which is marketed for laboratory purposes, known as "Kubel and Tiemann's" solution, may be used.

${ }^{3}$ Welch, Bull. Johns Hopkins Hosp. 
be adjusted to a neutral reaction. It is used for stab cultures and is designed chiefly for pneumococcus cultivation and storage.

Dorsett Egg Medium.-This medium is chiefly useful for the cultivation of tubercle bacilli.

1. Carefully break eggs and drop the contents into a wide-mouthecis flask. Break up the yolk with a sterile platinum wire, and shake up the flask until the whites and yolks are thoroughly mixed.

2. Add 25 c.c. of distilled water to every four eggs; strain through sterile cloth.

3. Pour 10 c.c. each into sterile test tubes and slant in an inspissator and expose to $73^{\circ} \mathrm{C}$. for four to five hours on two days.

4. On the third day, raise the temperature to $76^{\circ} \mathrm{C}$.

5. The sterilization may be finished by a single exposure to $100^{\circ}$ C. in the Arnold sterilizer for fifteen minutes. Before inoculation, add two or three drops of sterile water to each tube.

Potato Media.-Large potatoes are selected, carefully washed in hot water, and scrubbed with a nail brush. They are then peeled, considerably more than the cuticle being removed. The peeled potatoes are again washed in running water for a short time, following which cylindrical pieces are removed from them with a large apple corer. The cylinders are cut into wedges by oblique cuts.

Since the reaction of the potato is normally acid, this should be corrected by washing the pieces in running water over night, or, better, by immersing them in a one per cent solution of sodium carbonate for half an hour.

The pieces are then inserted into the large variety of test tubes known as "potato tubes." (See Fig. 21, c.) In the bottom of the tubes a small amout of water (about 1 c.c.) or a small quantity of moist absorbent cotton should be placed in order to retard drying out of the potato. The tubes are sterilized by fractional sterilization, twenty minutes to half an hour in the Arnold sterilizer on three successive days.

Glycerin Potato.-In preparing glycerin potato the potato wedges are treated as above, and are then soaked in a ten to twenty-five per cent aqueous glycerin solution for one to three hours. A small quantity of a ten per cent glycerin solution should be left in the tubes. In sterilizing these tubes, thirty minutes a day in the Arnold after heating of the sterilizer should be regarded as sufficient, to avoid changes in the; glycerin.

Milk Media.-Fresh milk is procured and is heated in a flask for 
fifteen minutes in an Arnold sterilizer. It is then set away in the ice chest for about twelve hours in order to allow the cream to rise. Milk and cream are then separated by siphoning the milk into another flask. It is rarely necessary to adjust the reaction of milk prepared in this way, since, if acid at all, it is usually but slightly so. If, however, it should prove more than 1.5 per cent acid, it should be discarded or neutralized with sodium hydrate. The milk may then be poured into test tubes without further additions, or litmus solution may be added in a quantity sufficient to give a purplish blue color. The tubes are sterilized by fractional sterilization in the Arnold sterilizer for thirty minutes on three successive days.

Serum Media.-Loeffler's Medium.-Beef blood is collected at the slaughter house in high cylindrical jars holding two quarts or more. It is desirable that attempts should be made to avoid contamination as much as is feasible by previously sterilizing the jars, keeping them covered, and exercising care in the collection of the blood.

The blood is allowed to coagulate in the jars, and should not be moved from the slaughter house until coagulated. All unnecessary shaking of jars should be avoided. As soon as the coagulum is fully formed, adhesions between the clot and the sides of the jar should be carefully separated with a sterile glass rod or wire. The jars are then set away in the ice chest for 24 to 36 hours. At the end of this time clear serum will be found over the top of the clot, and between the clot and the jar. This should be pipetted off, preferably with a large pipette of 50 to 100 c.c. capacity, or siphoned off with sterile glass tubing, and transferred to sterile flasks.

To three parts of the clear serum is then added one part of a one per cent glucose beef infusion or veal infusion bouillon. The mixture is filled into tubes, perferably the short test tubes commonly used for diagnostic diphtheria cultures. The tubes are then placed in a slanting position in the apparatus known as an inspissator (see p. 71). This is a double-walled copper box covered by a glass lid, cased in asbestos, and surrounded by a water jacket. It is heated below by a Bunsen flame. Together with the tubes a small open vessel containing water should be placed in the inspissator to insure sufficient moisture. The temperature of the inspissator is now raised to $70^{\circ}-75^{\circ} \mathrm{C}$., care being taken that the rise of temperature takes place slowly. The temperature is maintained at this point for two hours, and the process is repeated, for the same length of time, at the same temperature, on six successive days, preferably without removing the tubes from the inspissator at 
any time. It is also possible, though less regularly yielding good results, to sterilize in the inspissator for one day, following this on the second and third days by exposure for thirty minutes to $100^{\circ} \mathrm{C}$. in the Arnold steam sterilizer. In doing this, the Arnold should be very gradually heated, at first without outer jacket, this being lowered only after thorough heating has taken place.

Serum-Water Media for Fermentation Tests.-For the determination of the fermentative powers of various microorganisms for purposes of differentiation, Hiss has devised the following media in which the cleavage of any given carbohydrate is indicated, not only by the production of an acid reaction, but by the coagulation of the serum proteids.

Obtain clear beef serum by pipetting from clotted blood in the same way as this is obtained for the preparation of Loeffler's blood-serum medium. Add to this two or three times its bulk of distilled water, making a mixture of serum and water in proportions of one to two or three. Heat the mixture for fifteen minutes in an Arnold sterilizer at $100^{\circ} \mathrm{C}$. to destroy any diastatic ferments present in the serum. Add one per cent of a five per cent aqueous litmus solution (the variation in the different litmus preparations as obtained in laboratories necessitates a careful addition of an aqueous litmus solution until the proper color, a deep transparent blue, is obtained, rather than rigid adherence to any quantitative directions). Add to the various fractions of the medium thus made one per cent respectively of the sugars which are to be used for the tests.

For the preparation of inulin medium, made in this way for pneumococcus-streptococcus differentiation, it is necessary to sterilize the inulin dissolved in the water to be added to the serum in an autoclave at high temperature (15 pounds for 15 minutes) in order to kill spores before mixing with the serum. The serum-water media are sterilized by the fractional method at $100^{\circ} \mathrm{C}$, at which temperature they remain fluid.

Special Media for Colon-Typhoid Differentiation. ${ }^{1}$ - Hiss' Plating Medium. ${ }^{2}$ - The composition of this medium is as follows:

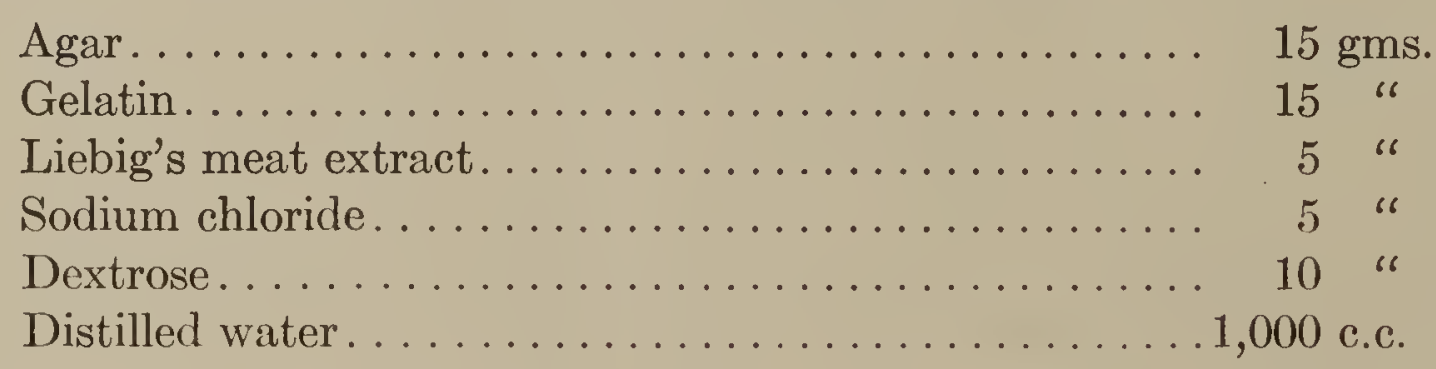

${ }^{1}$ For details of use of these special media see also chapter on Bacillus typhosus.

${ }^{2}$ Hiss, Jour. Exp. Med., ii, 1897; Jour. Med. Research, viii, 1902. 
The agar is thoroughly dissolved in 1,000 c.c. of distilled water. When the agar is melted, the gelatin, meat extract, and salt are added and dissolved by further heating. Any loss in weight is then adjusted by the addition of water. No titration or adjustment of reaction is necessary. The medium should be cleared with the whites of two eggs, and filtered through cotton. To the cleared medium is added one per cent of dextrose, and the medium tubed, about 8 c.c. to each tube, and sterilized.

Hiss' Tube Medium.-The composition is as follows:

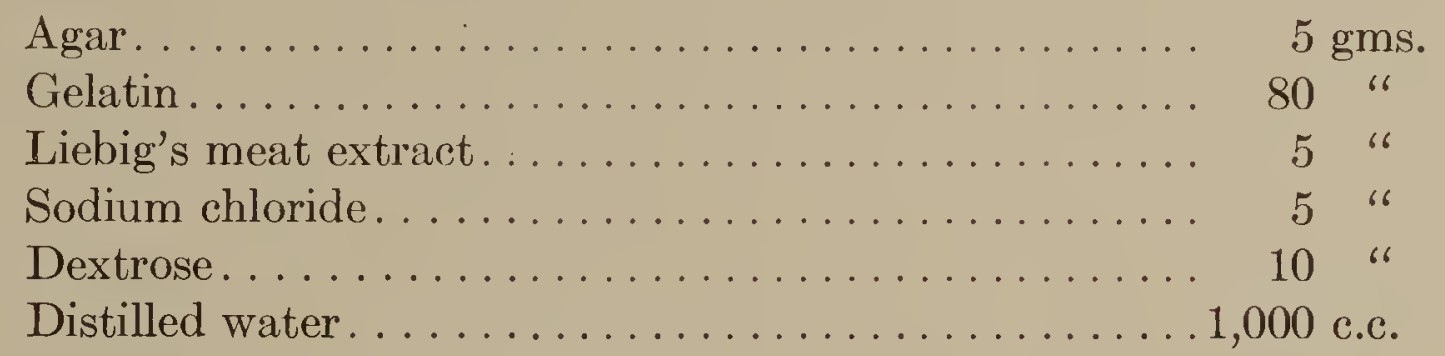

The method of preparation is the same as for the plating medium. The agar is thoroughly dissolved, and then the gelatin, meat extract, and salt are added and dissolved. After adjusting the loss in weight, the volume should be determined, a careful titration made, and the reaction adjusted to one and five-tenths per cent acid by the addition of $\frac{N}{1} \mathrm{HCl}$ solution. The medium is then cleared with the whites of eggs, filtered, and one per cent dextrose added. It is then tubed and sterilized.

Hesse's Medium. - - The medium devised by Hesse for typhoid-colon differentiation depends for its usefulness, as does the Hiss tube medium, upon the great motility of the typhoid bacillus. It may be used directly for the examination of feces or, as suggested by Jackson and Melia, ${ }^{2}$ after preliminary enrichment of the material to be examined by the use of the lactose-bile medium of Jackson. (See p. 138.)

The Hesse medium is made up as follows:

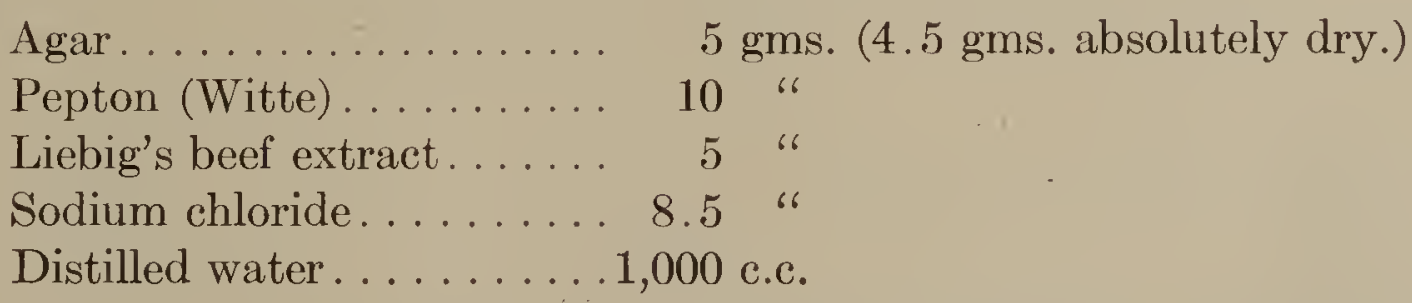

Jackson and Melia, in studying this medium, have found that complete drying of the agar and the use of $4.5 \mathrm{gms}$. of this dried preparation give more uniform results, since the amount of moisture in com-

\footnotetext{
${ }^{1}$ Hesse, Zeit. f. Hyg., Iviii, 1908.

${ }^{2}$ Jackson and Melia, Jour. of Inf. Dis., vi, 1909.
} 
mercial agar varies considerably. The preparation of the medium is as follows:

Dissolve 4.5 gms. of dry agar in 500 c.c. of distilled water over a free flame, making up for loss by evaporation. In another vessel 10 gms. of pepton, 5 gms. of beef extract, and $8.5 \mathrm{gms}$. of salt are dissolved in 500 c.c. of distilled water. This may be heated until complete solution has taken place and the loss by evaporation made up.

The two solutions are then mixed and heated for thirty minutes; loss by evaporation is then made up with distilled water and the solution is filtered through cotton until clear. The reaction is then adjusted to one per cent acidity and the medium tubed-10 c.c. to each tube. Sterilize in autoclave, cool, and store in ice chest.

The typhoid bacillus is characteristic on the Hesse medium only when the dilution poured in the plates is so high that only a few colonies appear. The typhoid colonies are much larger than are the colon colonies and may often be several centimeters in diameter.

Piorkowski's Urine Gelatin. 1 - Normal urine of a specific gravity of about 1.020 is collected for several days. At the end of this time, when its reaction has become alkaline, pepton 5 per cent and gelatin 3.3 per cent are added. This mixture is heated upon a water bath for one hour, filtered, and tubed. The tubes are sterilized by the fractional method.

In using this medium for the isolation of typhoid bacilli from the feces, two loopfuls of feces are placed into a tube of the melted urine gelatin, and from this dilutions are made into other tubes, taking four loopfuls from the first into the second and six to eight from the second to the third. Plates are then poured and kept at about $20^{\circ} \mathrm{C}$. The typhoid colonies show fine processes or filaments, while the colon colonies are quite compact.

Capaldi's Medium. ${ }^{2}$-The composition of this medium is as follows:

Distilled water. . . . . . . . . . . . . . . . . 1,000 c.c.

Pepton (Witte).................... 20 gms.

Gelatin.............................. 10 "

Agar............................. 20 "

Dextrose (or preferably mannit)............. 10 "

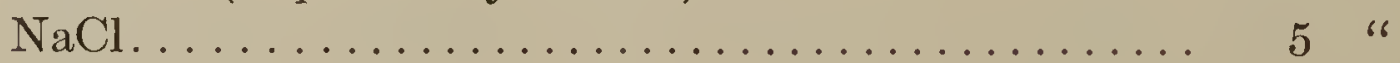

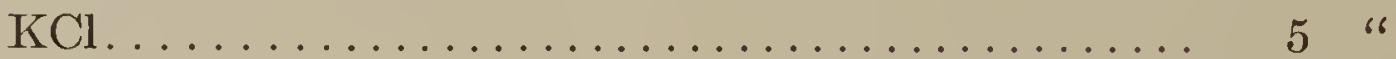

It is advisable to dissolve the agar in 500 c.c. of water, making up

${ }^{1}$ Piorkowski, Deut. med. Woch., vol. 25, 1899.

${ }^{2}$ Capaldi, Zeit. f. Hyg., xxiii, 1896. 
the loss by evaporation with distilled water, and to dissolve the other ingredients in a similar quantity of water, finally mixing the solutions. The mixture is rendered alkaline by the addition of 10 c.c. of normal $\mathrm{NaOH}$, and is cleared, filtered, and filled into test tubes.

Plates are made with this medium and surface smears made of the suspected material. B. typhosus grows in small, glistening, bluish translucent colonies. Colonies of $\mathrm{B}$. coli are larger, more opaque, and show a brownish tinge.

Conradi-Drigalski Medium. ${ }^{1}$ - The following are the directions given by the originators of this medium for its preparation. (a) Three pounds of meat are infused in two liters of water for twelve hours or more. After straining, boil for one hour and add 20 gms. of Witte's pepton, 20 gms. of nutrose, $10 \mathrm{gms}$. of $\mathrm{NaCl}$; boil one hour and filter. To the filtrate add 60 gms. of agar. Boil for three hours (or one hour in an autoclave) until agar is dissolved. Render weakly alkaline to litmus paper, filter, and boil for half an hour more.

(b) Litmus solution: Two hundred and sixty c.c. of litmus solution are boiled for ten minutes. (The litmus solution used by Conradi and Drigalski is the very sensitive aqueous litmus recommended by Kubel and Tiemann, and purchasable under the name.) After boiling, 30 grams of chemically pure lactose are added to the litmus solution. The mixture is then boiled for fifteen minutes, and, if a sediment has formed, is carefully decanted.

(c) Add the hot lactose mixture to the hot fluid agar solution; mix well and, if necessary, again adjust to a weakly alkaline reaction, litmus paper being used as an indicator. To this mixture add 4 c.c. of a hot, sterile, ten per cent solution of sodium carbonate, in order to render it alkaline, and 20 c.c. of a freshly made solution of crystal violet (c. p. Höchst), 0.1 gram in 100 c.c of sterile distilled water.

The medium contains thirteen per cent of litmus solution and one one-thousandth per cent of crystal violet.

(The plates used by Conradi and Drigalski are large plates 15 to $20 \mathrm{~cm}$. in diameter.) Surface smears are made upon the medium after solidification. These are incubated twenty-four hours. Typhoid colonies are small, blue, and transparent. Colon colonies are large, red, and opaque.

Endo's Medium. ${ }^{2}$ - 1 . Prepare one liter of meat infusion three per cent agar, containing 10 grams of pepton and 5 grams of $\mathrm{NaCl}$.

${ }^{1}$ Conradi-Drigalski, Zeit. f. Hyg., xxxix, 1902.

${ }^{2}$ Endo, Cent. f. Bakt., xxxv, 1904. 
2. Neutralize and clear by filtration.

3. Add 10 c.c. of ten per cent sodium carbonate solution in order to render it alkaline.

4. Add 10 grams of chemically pure lactose.

5. Add 5 c.c. of alcoholic fuchsin solution, filtered before using. (Endo in his original contribution does not mention the strength of this fuchsin solution, which, however, should be saturated.)

This colors the medium red.

6. Add 25 c.c. of a ten per cent sodium sulphite solution. This again decolorizes the medium, the color not entirely disappearing, however, until the agar is cooled.

7. Put into test tubes, 15 c.c. each, and sterilize.

The medium should be kept in the dark. For use, plates are poured and surface smears of stools made. Endo claims that upon this medium the typhoid bacillus outgrows the colon bacillus and its colonies remain colorless, while those of bacilli coli become red.

The preparation of Endo's medium presents certain difficulties which arise largely from the varying purity of the sodium sulphite. Kastle and Elvove ${ }^{1}$ accordingly recommend the use of anhydrous sodium sulphite instead of the crystallized variety which is hydrated. Harding and Ostenberg ${ }^{2}$ recommended the following method of preparing Endo's medium which we believe to be excellent. They adopted the method largely because $\mathrm{Na}_{2} \mathrm{SO}_{3}$ is easily oxidized and therefore varies in $\mathrm{SO}_{2}$ content. They add sodium sulphite solution to a measured amount of .5 per cent fuchsin solution until they determine the proportions which give the greatest delicacy of reaction as tested with formaldehyde. The proportions so determined are then added to the hot 3 per cent agar. This insures a delicate medium.

Although Endo described his medium as dependent upon the formation of acid by the bacteria, this is not so. Acids give no coloration of the sulphite-fuchsin mixture. Indeed this mixture is used by chemists under the name of Schiff's reagent as a test for aldehydes. Acids decolorize the red caused by aldehydes, and this accounts for the frequent late discoloration of red colon colonies on prolonged cultivation. The medium is red when hot, and colorless when cold, because the compound between sulphite and fuchsin dissociates in the hot solution.

Malachite-Green Media. ${ }^{3}$-The principle of these media is that mal-

${ }^{1}$ Kastle and Elvove, Jour. of Inf. Dis., xvi, 1909.

${ }^{2}$ Harding and Ostenberg, Jour. of Inf. Dis., xi, 1, 1909.

${ }^{3}$ Loeffler, Deut. med. Woch., 32, 1906. 
achite green inhibits the growth of the colon bacillus without exerting any such influence upon the typhoid bacillus. To make one liter:

1. Prepare a neutral, one-half strength, meat-infusion bouillon (500 grams of meat to 2 liters of water) by the usual technique.

2. Acidify this with 7.5 c.c. of normal hydrochloric acid (to facilitate the solution of agar).

3. Dissolve in this 30 grams of agar (three per cent) by boiling.

4. Neutralize with 7 c.c. normal $\mathrm{KOH}$ or $\mathrm{NaOH}$ (until neutral to litmus).

5. Add 5 c.c. of normal sodium carbonate solution to make it alkaline and heat in Arnold sterilizer for several hours.

6. Add 100 c.c. of a ten per cent nutrose solution (one per cent). This agar may be sterilized and stored in quantities of 100 c.c. without further manipulation.

7. Before use, redissolve, and to 100 c.c. add 2 to 2.9 c.c. of a two per cent solution of malachite green (trade mark, "Höchst 120"). This solution is made in sterilized water, but is not boiled.

8. Fifteen to twenty c.c. of this medium are poured into Petri dishes, allowed to cool, and inoculated by surface smears.

Malachite-Green Bouillon (Peabody and Pratt). ${ }^{1}$-To 100 c.c. of beef infusion broth add 10 c.c. of one per cent solution of malachite green, Höchst 120, made with sterile water. This is tubed, 10 to 15 c.c. being put in each tube.

This medium is used merely as an enriching fluid. One drop of the suspected material (emulsified stool) is added to each tube and after incubation for eighteen to twenty-four hours inoculations may be made upon Conradi-Drigalski plates or other media.

Peabody and Pratt found a reaction of .5 per cent acidity to phenolphthalein most favorable.

Bile Medium. ${ }^{2}$ - (Recommended for blood cultures by Buxton and Coleman.) The medium is prepared as follows:

Ox-bile ............................ 900 c.c.

Glycerin............................ 100 c.c.

Pepton ......................... 20 grams

Put into small flasks containing quantities of about 100 c.c. each and sterilized by fractional sterilization.

1 Peabody and Pratt, Boston Med.' and Surg. Jour., clviii, 7, 1908.

${ }^{2}$ Conradi, Deut. med. Woch., 32, 1906. 
Jackson's Lactose-Bile Medium. ${ }^{1}$-Jackson has devised a medium now in general use by water-analysts, which is of great use in isolating B. typhosus and B. coli from water, and serves as a valuable enriching medium in isolating these organisms from other sources, such as feces. Jackson and Melia ${ }^{2}$ have found that in this medium B. typhosus and B. coli outgrow all other microorganisms and that eventually B. typhosus will even outgrow B. coli.

It consists of sterilized undiluted ox-bile (or an eleven per cent solution of dry, fresh ox-bile) to which have been added one per cent pepton and one per cent lactose. It is filled into fermentation tubes holding 40 c.c., and sterilized by the fractional method.

MacConkey's Bile-Salt Agar.-

Sodium glycocholate............... 5 per cent.

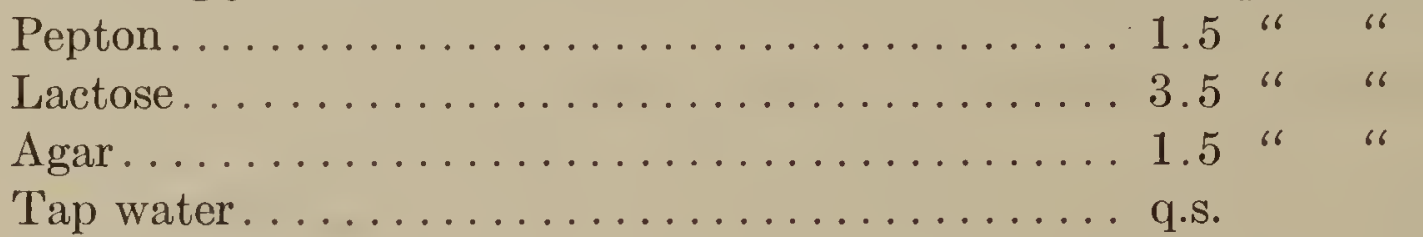

The agar and pepton are dissolved and cleared and the lactose and sodium glycocholate added before tubing. In this medium the B. typhosus produces no change; $B$. coli, by producing acid from the lactose, causes precipitation of the bile salts.

Neutral-Red Medium.-To 100 c.c. of a one or two per cent glucose agar add 1 c.c. of a saturated aqueous solution of a neutral-red.

The medium is used in tubes, stab or shake cultures. The typhoid bacillus produces no change, while members of the colon group render the medium colorless by reduction of the neutral-red and produce gas by fermentation of the sugar.

Barsiekow's Medium. ${ }^{3}$-To 200 c.c. of cold water, add 10 grams of nutrose and allow to soak for one-half to one hour. Pour this into 800 c.c. of boiling water, and heat for twenty minutes in an Arnold sterilizer at $100^{\circ} \mathrm{C}$. Filter through cotton and to the opalescent solution of nutrose add 5 grams of $\mathrm{NaCl}, 10$ grams of lactose, and sufficient aqueous litmus solution to give a pale blue color. ${ }^{4}$

Dieudonnés Selective Medium for cholera spirillum. See page 584.

Enriching Substances Used in Media.-For the cultivation of micro-

${ }^{1}$ Jackson, "Biol. Studies of Pupils of W. T. Sedgwick," 1906, Univ. Chicago Press.

${ }^{2}$ Jackson and Melia, Jour. Inf. Dis., vi, 1909.

${ }^{3}$ Barsiekow, Wien. klin. Rund., xliv, 1901.

${ }^{4}$ Filtration may be done through paper, but takes a long time. 
organisms which are sensitive to their food environment, it is often necessary or advisable to add to the ordinary media enriching substances, which empirical study has shown to favor the growth of the organism in question. The substances most commonly used for such enrichment are glucose, nutrose (sodium caseinate), glycerin, sodium formate, and unsolidified animal proteids. As animal and blood serum and whole blood must frequently be used in this way, an understanding of the methods employed in obtaining these substances is necessary.

Method of Obtaining Blood and Blood Media.-Blood serum from beef and sheep may be collected in the manner recommended for the collection of such serum in the preparation of Loeffler's medium, pipetted into test tubes, and sterilized in the fluid state by exposure to temperatures ranging from $60^{\circ}$ to $65^{\circ} \mathrm{C}$., for one hour upon six consecutive days. It is not a simple matter to sterilize serum in this way and requires much time and care.

The method most commonly employed, in laboratories which have access to hospitals, for obtaining clear serum depends upon the collection of exudate or transudate fluids by sterile methods directly from the pleural cavity, the abdominal cavity, or the hydrocele cavity. Sterile flasks or test tubes are prepared and the fluid is allowed to flow directly out of the cannula into these. It is necessary to avoid carbolic acid or other disinfectants in sterilizing instruments and rubber tubing used during the operation. These should be brought into the ward in the water in which they have been boiled and not in strong antiseptic solutions, as is frequently done. The fluid so obtained may be incubated and the contaminated tubes discarded. The serum may then be added, in proportions of one to three, to sterile broth or melted agar.

Agar thus used is melted and cooled to $60^{\circ} \mathrm{C}$, , or below. One-third of its volume of warmed exudate fluid is added, and the plates are poured.

Serum may be rendered free of bacteria by filtration through a Berkefeld or Pasteur-Chamberland filter. This is an effectual method, but requires much time and care.

Whole blood may be obtained for cultural purposes by bleeding rabbits or dogs or other animals directly from a blood-vessel into tubes of melted agar. In the case of a rabbit, after the administration of an anesthetic (ether), an incision is made directly over the trachea, and, by careful section, the carotid artery is isolated, lying close to the side of the trachea. 


\section{THE INFLUENCE OF DYE STUFFS UPON BACTERIAL GROWTH, AND AS ADDITIONS TO SELECTIVE MEDIA}

In describing the selective media for typhoid bacilli we have seen that malachite green and crystal violet have been found to exert a certain amount of selective action upon the typhoid and colon groups. The selective influence of various dyes has been recently again studied by Churchman. Churchman ${ }^{1}$ found that the addition of gentian violet in dilutions of $1: 100,000$, to media, inhibited some bacteria, while others grew luxuriantly in its presence. Extremely interesting, both practically and theoretically, is his observation that upon such gentian violet media bacteria fall into two groups. Those which grow on gentian violet correspond in a general way to the Gram-negative bacteria; those which fail to develop on these media correspond roughly with the Gram-positive species. One strain of the enteritidis group could not be cultivated on gentian violet, and this was found to differ from the others also in its agglutination tests.

Signorelli ${ }^{2}$ claims that dahlia is useful in differentiating true cholera strains from similar spirilla. The true cholera strains grew with colored colonies, while others remain colorless, in his experiments.

Krumwiede and Pratt $^{3}$ were unable recently to confirm the claims of Signorelli. However they fully confirm the findings of Churchman both as to the selective action of gentian violet and in regard to the classification of bacteria into two groups corresponding to their reaction to the Gram stain. They state that among Gram-negative bacteria a strain is occasionally found which will not grow on the gentian violet media, differing in this respect from other members of the same species. They find also that the reaction is quantitative.

The streptococcus-pneumococcus group, according to their investigations, differs from other bacteria in being able to grow in the presence of quantities of violet which inhibit other Gram-positive species. Dysentery bacilli show variations. Other dyes which they investigated showed no specific inhibitory properties which could be utilized for classification.

${ }^{1}$ Churchman, Jour. Exp. Med., 16, 1912; also Churchman and Michael, ibid.

${ }^{2}$ Signorelli, Centralbl. f. Bakt., Orig. 56, 1912.

${ }^{3}$ Krumwiede and Pratt, Centralbl. f. Bakt., Orig. 68, 1913; and Proc. N. Y. Path. Soc., xiii, 1913. 


\section{CHAPTER VIII}

METHODS USED IN THE CULTIVATION OF' BACTERIA

\section{INOCULATION OF MEDIA}

THE transference of bacteria from pathological material to media, or from medium to medium, for purposes of cultivation, is usually accomplished by means of a platinum wire or loop. The platinum wire used should be thin and yet possess a certain amount of stiffness and be about two to three inches in length. This is fused into the end of a glass rod six to eight inches long. It is an advantage, though not necessary, to use rods of so-called "sealing-in" glass which, having the same coefficient of expansion as platinum, does not crack during sterilization. For work with fluid media, the wire should be bent at its free end so as to form a small loop which will pick up a drop of the liquid. For the inoculation of solid media and the making of stab cultures, a straight "needle" or wire should be used. Other shapes of these wires and spatulæ from heavy wire have been devised for various purposes and are easily improvised as occasion demands. (See Fig. 27.)

When making a transfer from one test tube to another, the tubes should be held between the thumb and first and second fingers of the left hand, as shown in Fig. 28. The plugs are then removed by grasping them between the small and ring fingers and ring and middle fingers of the right hand, first loosening any possible adhesions between glass and plugs by a slight twisting motion. The platinum wire is held meanwhile by the thumb and index fingers of the right hand in the manner of a pen. The wire is heated red hot in a Bunsen flame, and is then passed into the culture tube without being allowed to touch the glass. It is held suspended within the tube for a few seconds to permit of cooling before touching the bacterial growth. The wire is then allowed to touch lightly the surface of the growth and a small amount is picked up. (See Fig. 29.) It is then removed from the tube without allowing it to touch the sides of the glass, and is passed into the tube which is to be inoculated. If the tube contains a slanted medium, such as agar, a light stroking motion from the bottom of the slant to its apex will deposit the bacteria 
upon the medium evenly along a central line. The needle may also be plunged downward into the substance of the nutritive material so that in the same tube both surface growth and deep growth may be observed. If a stab culture is to be made in unslanted agar or in gelatin,

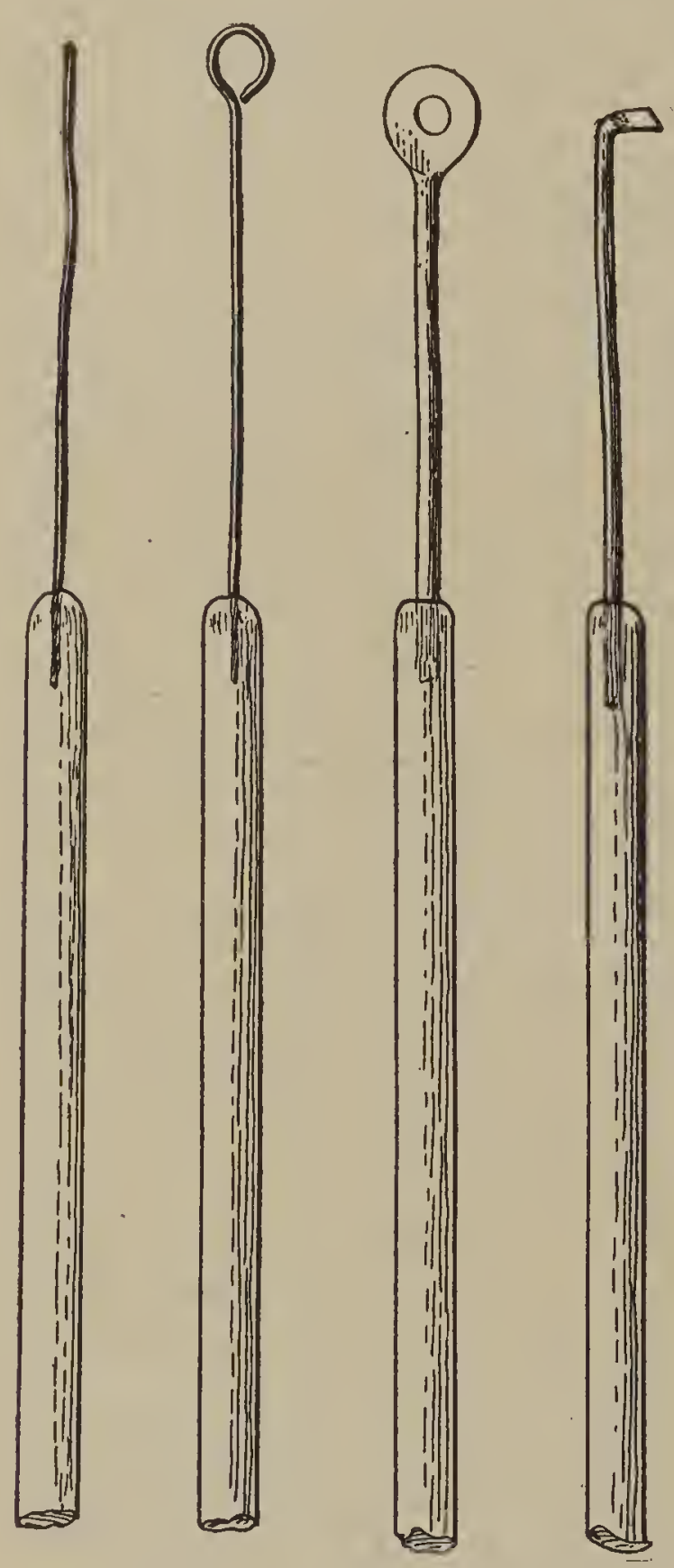

Fig. 27.-Platinum Wires. the needle is simply plunged straight downward as nearly as possible along the axis of the medium. If a fluid medium is being inoculated, the wire should be introduced only into the upper part of the liquid and the bacteria gently rubbed into emulsion against the side of the glass. The needle is then removed from the tube, the stopper carefully replaced, and the platinum wire immediately sterilized in the flame. This sterilization of platinum needles after they have been in contact with bacteria should become second nature to those working with bacteria, since an infraction against this rule may give rise to serious and widespread consequences. In burning off platinum needles it is well to remember that a part of the glass rod, as well as the wire itself, is introduced into the tubes and may become contaminated, and for this reason the rod itself, at least in its lower two or three inches, should be passed through the flame as well as the wire. As an extra precaution against contamination, the lips of test tubes and flasks and the protruding edges of cotton plugs may be passed through the flame and singed.

\section{THE ISOLATION OF BACTERIA IN PURE CULTURE}

It is obvious that in many cases where bacteria are cultivated from water, milk, pathological material, or other sources, many species may be present in the same specimen. It is likewise obvious that scientific bacteriological study of any bacterium can be made only if we obtain 
this particular species entirely apart from others, in what is known as "pure culture." The earliest methods for accomplishing this were the methods of Pasteur and of Cohn who depended upon the power of one species to outgrow all others, if cultivated for a sufficient length of time in fluid media. This method, of course, was inadequate in that it was often purely a matter of chance which one of the mixture of species was finally obtained by itself. A later method, by Klebs, depends upon serial dilution, in test tubes of fluid media, by which the eventual transference of one germ only, to the last tube was attempted. Such methods, none of them of great practical value, have been entirely dis-

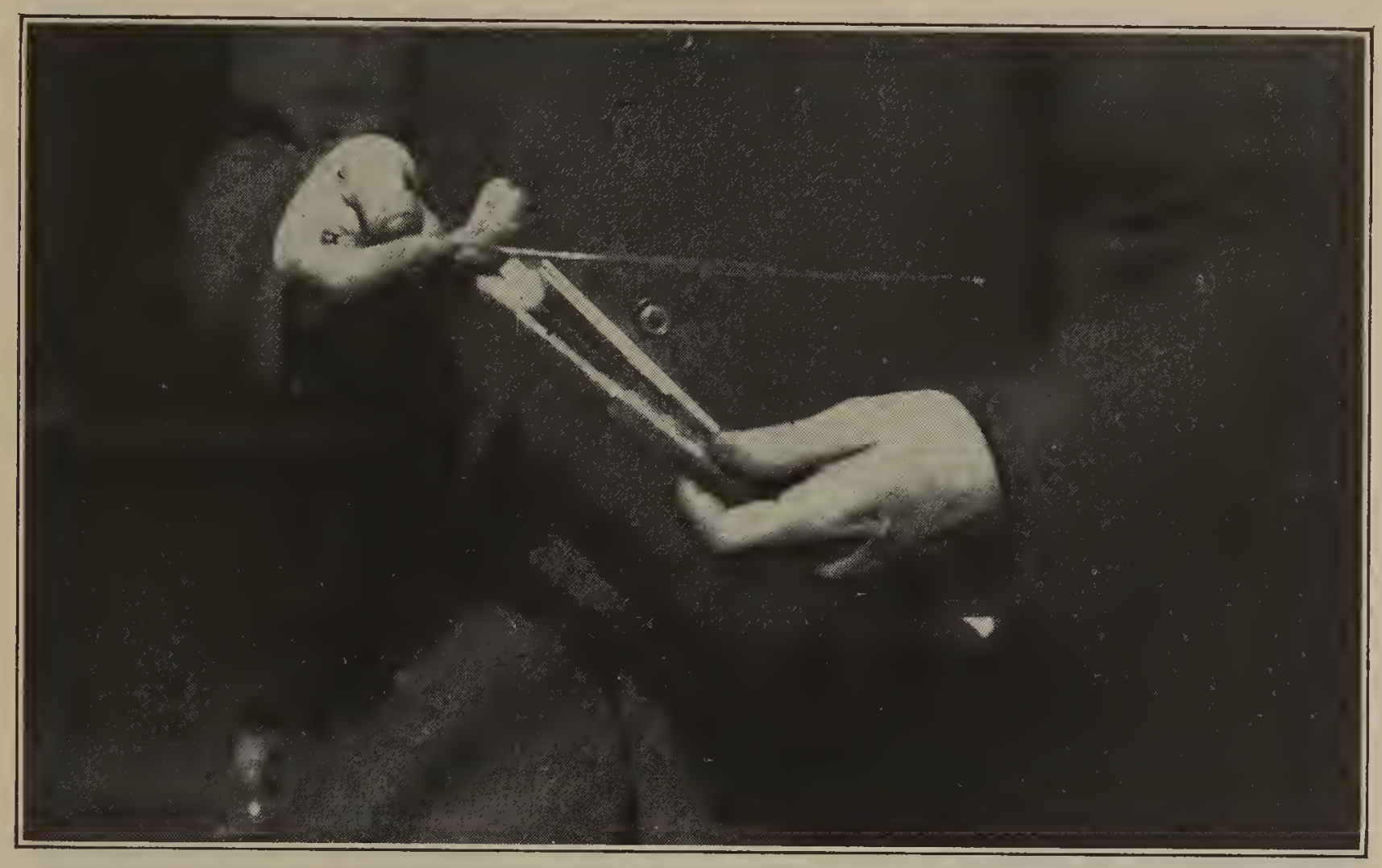

Fig. 28.-Taking Plugs from Tubes before Inoculation.

placed by those made possible by Koch's introduction of solid media which may be rendered fluid by heat.

The methods now employed for the isolation of bacteria depend upon the inoculation of gelatin or agar, when in the melted state, the thorough distribution of the bacteria in these liquids by mixing, and the subsequent congealing of these media in thin layers. By this means the individual bacteria, distributed in the medium when liquid, are held apart and separate when the medium becomes stiff. The masses of growth or "colonies" which develop from these single isolated microorganisms are discrete and are descendants of a single organism, and can be transferred, by means of a process known as "colony-fishing," to fresh sterile culture media. 
Plaitng.-The first method' employed by Koch for bacterial isolations was one that consisted in the use of simple plates of glass, about $3 \times 4$ inches in size, mounted upon a leveling stand. A wooden triangle, supported upon three adjustable screw-feet, formed the base of this apparatus. Upon this was set a covered crystallizing dish which could be filled with ice water. Upon the top of this rested the sterilized plates under a bell jar. By screwing up or down upon the supports of the triangle, leveling of the plate could be achieved and controlled by a spirit. level placed at its side. The inoculated gelatin was poured upon the

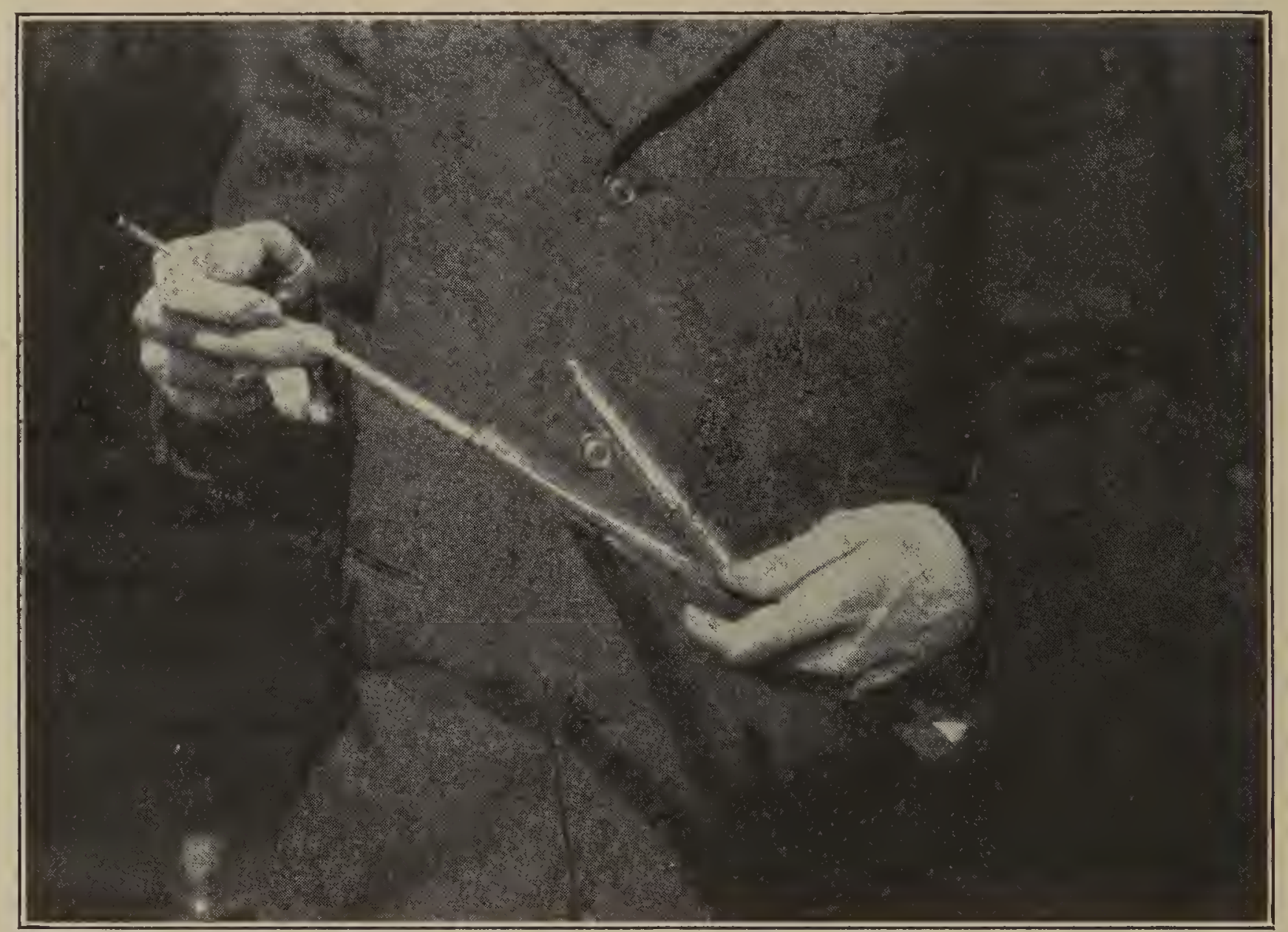

Fig. 29.-Inoculating.

plate and spread and rapidly cooled and hardened by the cold water contained in the crystallizing dish.

The original method of Koch has been modified considerably and the method universally employed at present depends upon the use of circular covered dishes, the so-called Petri dishes. These obviate the necessity of a leveling stand and prevent contamination of the plate when once poured. Each Petri dish plate consists of two circular glass dishes; the smaller and bottom dish has an area of 63.6 square centimeters; the larger is used as a cover for the smaller, and forms a loosely fitting lid. 
The plates when fitted together are sterilized and thus form a closed cell which, if properly handled, may remain sterile indefinitely.

The technique for making a pour plate for the purpose of isolating bacteria from mixed culture is as follows:

The actual "pouring" of plates is preceded by the preparation of usually three graded dilutions of the material to be examined. For this purpose three agar or gelatin tubes are melted and, in the case of the agar, are cooled to a temperature of about $42^{\circ} \mathrm{C}$. in a water bath. A platinum loopful of the material to be examined is transferred to one of these tubes. The bacteria are then thoroughly distributed throughout the melted

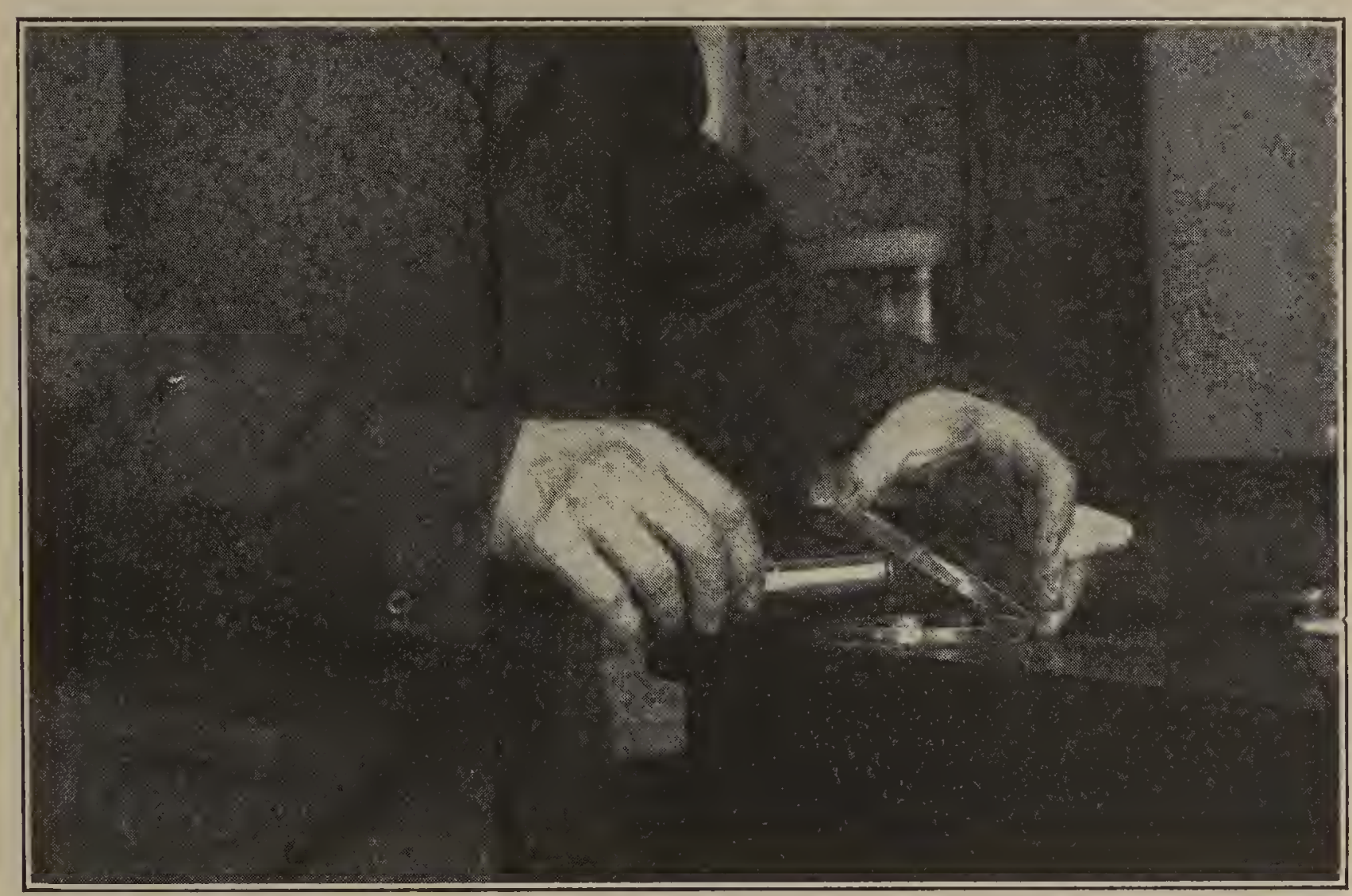

Fig. 30.--Pouring Inoculated Medium into Petri Plate.

gelatin or agar by alternately depressing and raising the plugged end of the tube, giving it a rotary motion at the same time. This thoroughly distributes the bacteria throughout the medium without allowing the formation of air-bubbles. Two loopfuls of this mixture are then transferred to the second tube and a similar mixing process is repeated. This second tube contains the bacteria in much greater dilution than the first and the colonies which will form in the plate poured from this tube will be farther apart. A third dilution is then made by transferring five loopfuls of the mixture in the second tube to the third. This again is mixed as before. The contents of the tubes are then poured into three 
sterile Petri dishes. The pouring should be done with great care. The cover of the dish is raised along one margin simply far enough to permit the insertion of the end of the test tube, the plug of which has been removed and the lips passed, with a rotary movement, through the flame. The medium is poured into the dish without the lips of the tube being allowed to touch either the bottom or the cover of the dish. The cover is then replaced and the medium allowed to harden.

When agar has been used, the dishes may be placed in an incubator at $37^{\circ} \mathrm{C}$. It is well to place the plates upside down in the incubator. This prevents the condensation water, squeezed out of the agar during hardening, from collecting on its surface, and forming channels for the diffuse spreading of bacteria. The same end may be attained by the use of Petri plates provided with porous earthenware lids, as suggested by Hill. Simple inversion of the plates, however, usually suffices. When gelatin has been used, the plates are allowed to remain in a dark place at room temperature or in a special thermostat kept at $22^{\circ}-25^{\circ} \mathrm{C}$.

Colonies in agar, kept at $37.5^{\circ} \mathrm{C}$., usually develop in eighteen to twentyfour hours; those in gelatin or agar at room temperature in from twentyfour to forty-eight hours, depending upon the species of bacteria which are being studied. Often in the second dilution, more frequently in the third, the colonies will be found well apart and can then be "fished." The process of "colony-fishing" is one which requires practice and should always be done with care, for upon its success depends the purity of the sub-culture obtained. Colonies should never be fished under the naked eye, no matter how far apart and discrete they may appear, since not infrequently close to the edge of or just beneath a larger colony there may be a minute colony of another species which may be too small to be visible to the naked eye, but which, nevertheless, if touched by accident will contaminate the sub-culture.

For proper "fishing," the Petri plate with cover removed, should be placed upon the stage of the microscope and examined with a low power objective, such as Leitz No. 2 or Zeiss AA. The sterilized platinum ncedle, held in the right hand, is then carefully directed into the line of focus of the lens, while the small finger of the hand is steadicd upon the edge of the microscope stage. When the point of the needle is clearly visible through the microscope, it is gently depressed until it is seen to touch the colony and to carry away a portion of it. The needle is then withdrawn without again touching the nutrient medium or the edges of the glass or the lens, and transferred to a tube of whatever medium is desired. In this way, individuals of one colony, de- 
scendants of a single bacterium of the original mixture,-are carried over to the fresh medium.

Esmarch Roll Tubes. ${ }^{1}$-A simple method of obtaining separate colonies is that devised by von Esmarch and known as "roll-tube" cultivation. Tubes of melted gelatin are inoculated with various dilutions of the bacterial mixture and, while still liquid, are laid in an almost horizon-

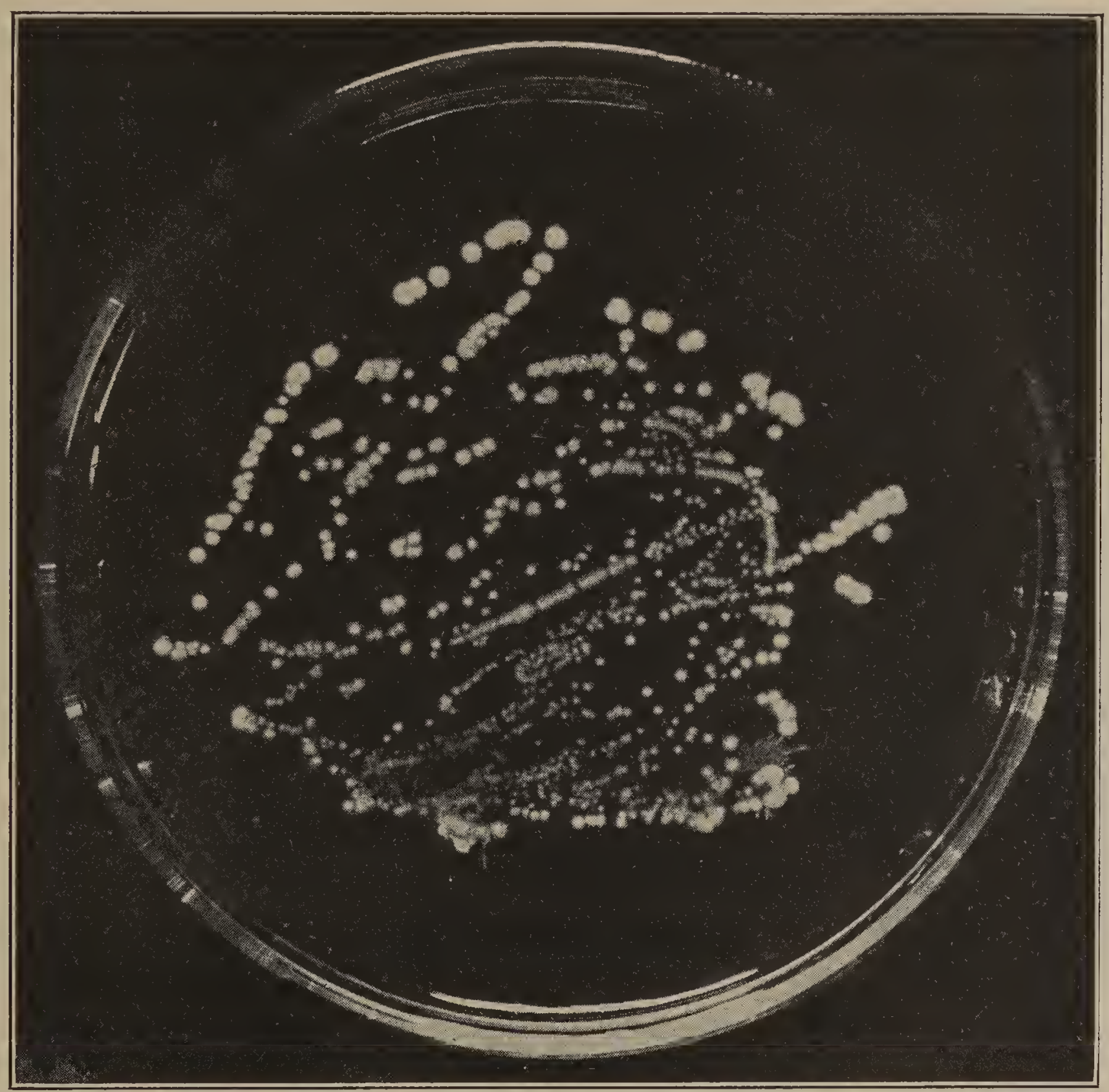

Fig. 31.-STtreak Plate.

tal position upon a block of ice, which has been grooved slightly by means of a test tube filled with hot water. The test tube containing the gelatin, after being placed in this groove, is rapidly revolved by passing the fingers of the right hand across it, while its base is carefully steadied with the left hand. If the revolving is carried out with

1 Esmarch, Zeit. f. Hyg., i, 1886. 
sufficient speed, the gelatin will harden in a thin layer on the inner surface of the tube. The colonies will develop in this layer and may be "fished" by means of a platinum wire with bent point introduced into the tube. This method is useful for certain purposes, but is too inconvenient for routine work. It is now rarely used.

Separation of Bacteria by Surface Streaking.-When it is necessary to

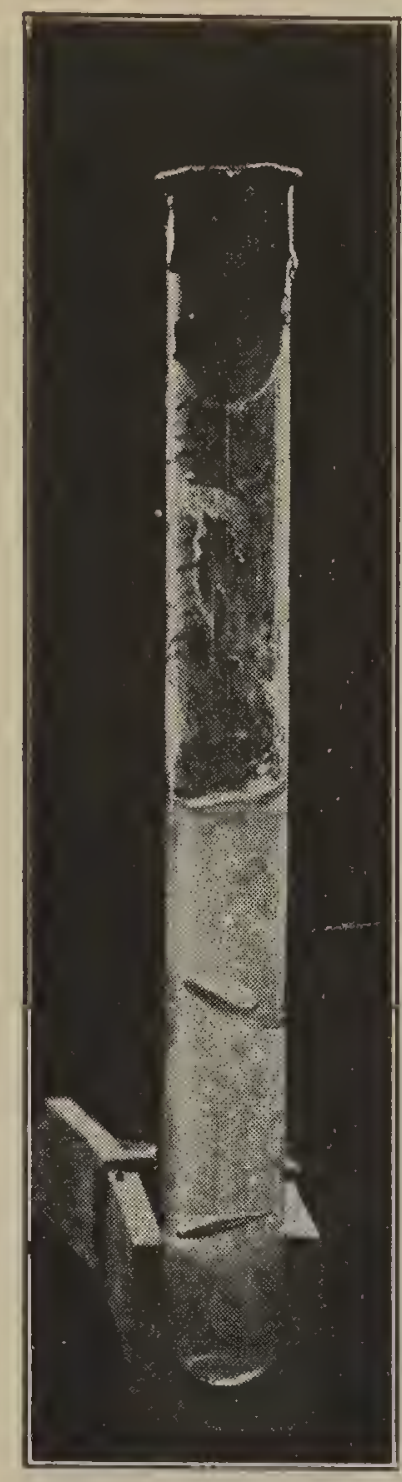

Fig. 32.- Deep Stae Cultivation OF ANÄ̈̈ROBIC BaCteria. isolate bacteria like the gonococcus, Bacillus influenzæ, the pneumococcus, and others, which, because of great sensitiveness to environment and possibly a preference for free oxygen, are not readily grown in pour plates, it is often advantageous first to pour plates of suitable media, allow them to harden, and then gently smear over their surfaces dilutions of the infectious material, usually in three or four parallel streaks. (See Fig. 31.)

Upon such plates, if dilutions have been properly made, and this is only a question of judgment based upon an estimation of the numbers of bacteria in the original material, discrete colonies of the microorganisms sought for may develop, and can be "fished" in the usual manner.

The media most favorable for the cultivation of various microorganisms will be discussed in the sections dealing with the individual species.

\section{ANAËROBIC METHODS}

We have seen in a preceding chapter ( $p .26)$ that many bacteria, the so-called anaërobes, will develop only in an environment from which free oxygen has been excluded.

The exclusion of oxygen for purposes of anaërobic cultivation may be accomplished by a variety of methods, depending upon a few simple principles which have been applied, either separately or in combination, by many workers.

The earliest methords depended upon the simple exclusion of air by mechanical devices. In other methods, the oxygen of the air is displaced by inert gases (hydrogen), and others again depend upon the oxygenabsorbing qualities of alkaline solutions of pyrogallol.

Cultivation by the Mechanical Exclusion of Air.-Koch succeeded in 
growing anaërobic bacteria upon plates by simply dropping upon the surface of the inoculated agar or gelatin a flat piece of sterile mica. This method, however, rarely succeeds in sufficiently excluding the air.

Liborius' Method. '- This method consists in the use of deeply filled tubes of agar or gelatin, from which all oxygen has been removed by boiling for fifteen minutes or more. It is advantageous, as has been pointed out in the section on anaërobiosis, that media used for this purpose should contain carbohydrates in some form, perferably glucose. After boiling, the tubes are rapidly transferred to ice water so that as little oxygen as possible may be absorbed during the hardening of the medium. The tubes are then inoculated by deep stabs. After inoculation, the medium may be covered with a thin layer of agar, gelatin, or oil (albolin), and further sealed with sealing-wax to prevent oxygenabsorption.

This method may be utilized for the isolation of anaërobes (as in the original method of Liborius) by inoculating the medium just before it solidifies. The tubes may be gently shaken in order to distribute the bacteria throughout the medium and then rapidly cooled. In this case colonies which develop may be scattered throughout the deeper layers of the agar or gelatin, and may be "fished" after breaking the tube.

Esmarch's Method. ${ }^{2}$-Von Esmarch has applied the principles of his roll-tube to the cultivation of anaërobic bacteria. Gelatin tubes are inoculated as above and roll-tubes prepared. The tubes are then set into cold water to prevent melting of the thin gelatin layer and the interior of the tube is filled with melted gelatin.

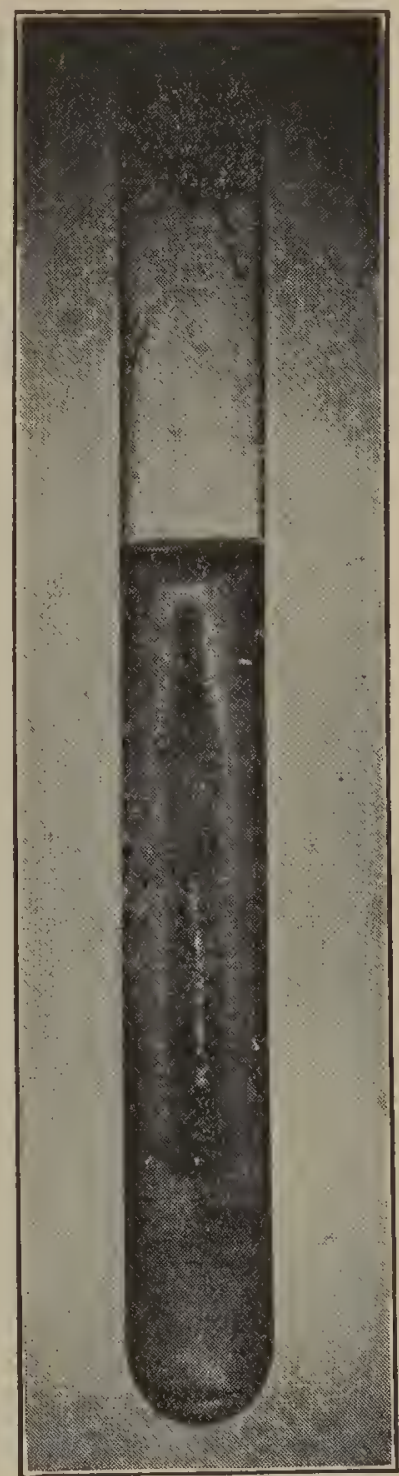

Fig。 33.-DeEP Stab CultivaTION OF ANÄ̈ROBIC BACTERIA.

Roux's METHOD. ${ }^{3}$-Anaërobic bacteria are cultivated by sucking the inoculated gelatin or agar into narrow tubes, which are then closed at both ends by fusing in the flame. After growth has taken place the tubes are broken and the organism recovered by "fishing." 
Fluid Media Covered with OrL.-Erlenmeyer flasks or other vessels are partially filled with glucose-bouillon over which a thin layer of albolin or other oil is allowed to flow. The oxygen is driven out of the liquid by vigorous boiling for fifteen minutes or more.

It should be remembered whenever using this or similar methods that a layer of fluid oil does not form an impermeable seal. By covering an alkaline pyrogallol solution with oil it

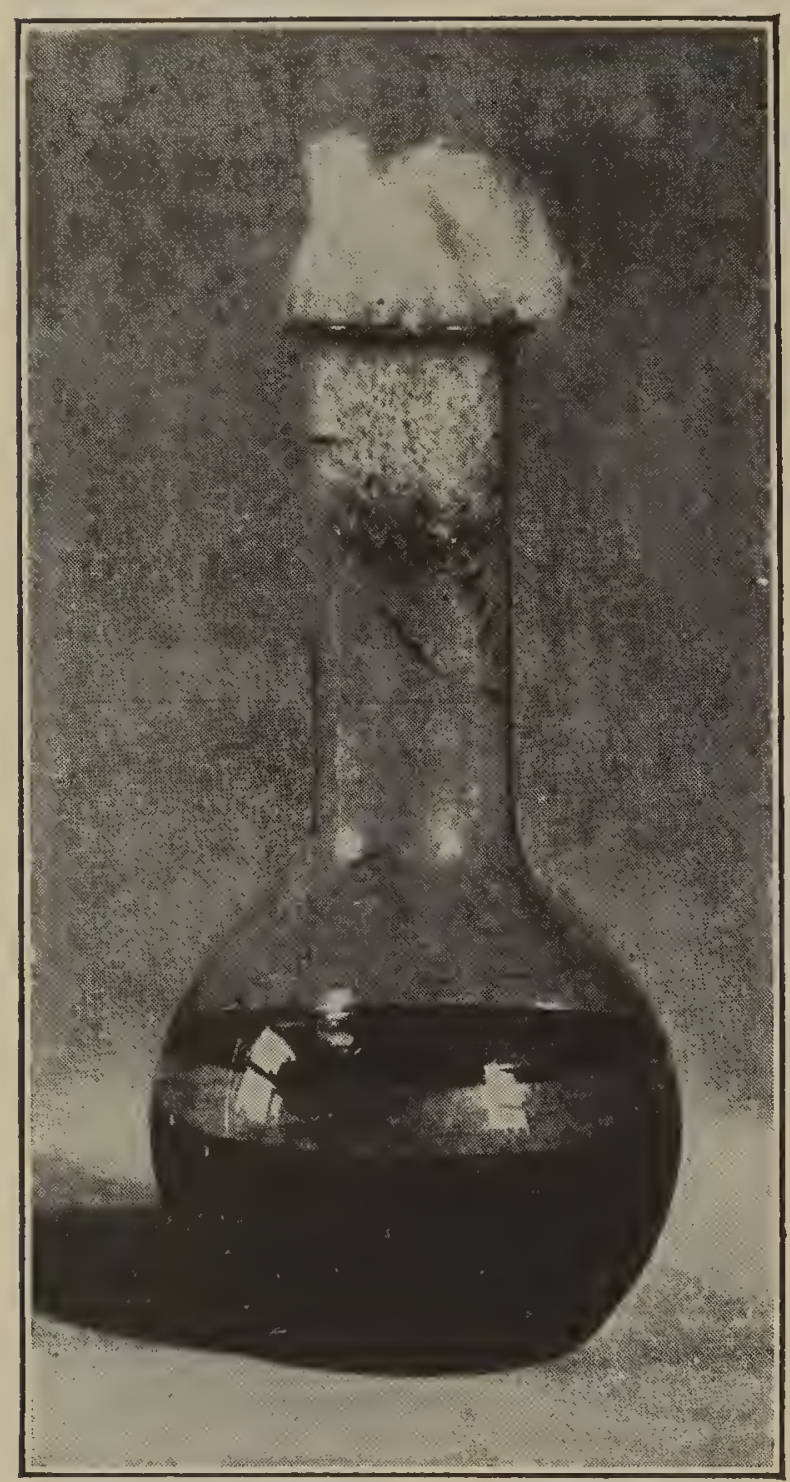

Fig. 34. - Cultivation of ANAËrobes in Fluid UNDER Albolin. can easily be shown that oxygen slowly diffuses through the oil into the medium below.

The simple exclusion of air, also, is the principle underlying the cultivation of anaërobic bacteria in the closed arm of a Smith fermentation tube.

Wright's Method. - Wright has described a simple and excellent method for the cultivation of anaërobic bacteria in fluid media. The apparatus necessary is easily improvised with the materials at hand in any laboratory. A short piece of glass tubing, constricted at both ends and fitted at each end with a small piece of soft-rubber tubing, is inserted into a test tube containing nutrient broth. The upper end of the inserted glass tubing is connected by the rubber with a pipette passed through the cotton plug in the tube. The entire apparatus, plus broth, may be sterilized after being put together. When a cultivation is made, the fluid in the test tube is inoculated as usual. The fluid is then sucked up into the glass tubing until this is completely filled. A downflow of the fluid is then prevented by placing the finger over the pipette through which the suction has been made or by constricting a small piece of rubber tubing attached to the upper end of the pipette. The entire system of tubes is then pushed downward in such a way that both pieces of rubber tubing, attached to the ends of the little glass

${ }^{1}$ Wright, J.H. Quoted from Mallory and Wright, "Path. Technique," Phila., 1904. 
chamber, are kinked. The entire apparatus may then be incubated. Growth of anaërobic bacteria takes place within the air-tight chamber formed by the short glass tubing within the test tube. The fluid in the test tube, outside of this chamber, usually remains clear.

When cultivation has been successful, the bacteria may be obtained either for morphological study or for further cultivation, by simply allowing the fluid to flow out of the little air-tight chamber back into the test tube. The method is simple and usually successful.

Methods Based upon the Displacement of Air by Hydrogen.-The principle of air-displacement by hydrogen, first utilized by Hauser, ${ }^{1}$ has been widely applied to the cultivation of anaërobic bacteria. In substance it consists of the conduction of a stream of hydrogen through an air-tight chamber in which plates or tubes containing inoculated media have been placed.

For the production of the hydrogen, the most convenient apparatus is the Kipp hydrogen generator or some modification of it. Hydrogen is generated from zine and sulphuric acid and this may be passed through a series of Woulfe-bottles, containing solutions of lead acetate and of pyrogallic acid, to remove traces of sulphuretted hydrogen and of oxygen, respectively. Some authors recommend also the interpolation of a bottle containing Lugol's solution to absorb traces of acid vapor, and of one with a silvernitrate solution to take up any hydrogen arsenide which may be derived from impurities contained in the zinc.
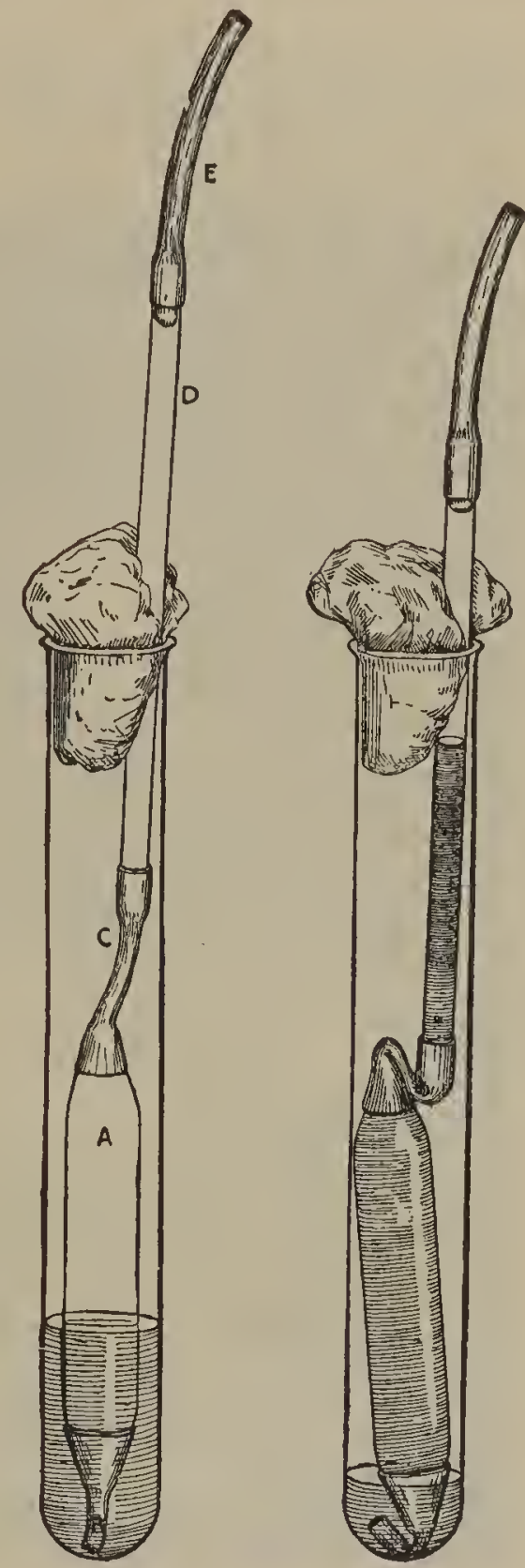

Fig. 35. - Wright's Method OF ANä̈robic Cultivation in Fluid Media.

For anaërobic cultivation upon solid media, the inoculated tubes or plates are placed in an apparatus such as the Novy jar. This is connected with the hydrogen apparatus and hydrogen allowed to flow through it for five or ten minutes, and the stop-cocks then closed. 
In applying the hydrogen method to fluid media, flasks containing the broth are fitted with sterile, tightly fitting rubber stoppers perforated by two holes, through which glass tubes are passed. One of these tubes, the inlet, passes below the surface of the liquid. The other one, the outlet, extends only a short distance below the stopper and is always kept above the surface of the medium. The flasks are inoculated and hydrogen is passed through the medium so that it enters the long tube, bubbles up through the fluid, and leaves by the short tube.

The broth may be covered with a

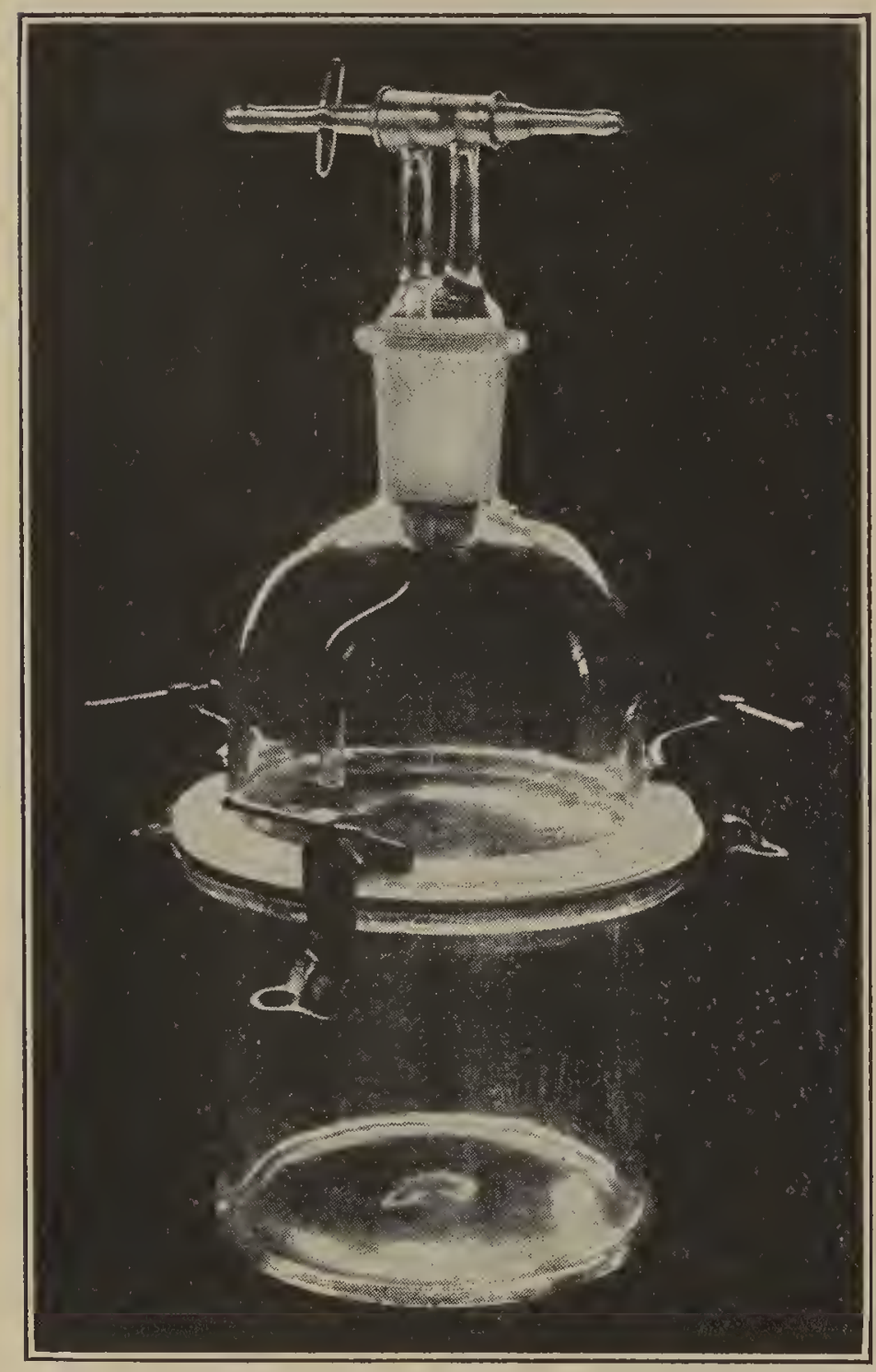

FIG $36 .-$ Novy JAR. thin layer of liquid paraffin or albolin.

The Use of Pyrogallic Acid Dissolved in Alkaline Solutions for Oxygen Absorption.-Buchner ${ }^{1}$ has applied the principle of chemical absorption for the removal of oxygen to the cultivation of anaërobic bacteria. This has been made use of in a number of different ways. The method is based upon the fact that alkaline solutions of pyrogallol possess the power of absorbing large quantities of free oxygen. At first such solutions are of a light straw-color, which becomes dark brown as oxygen is absorbed. The absorption of all the oxygen in the environment may be assumed when there is no further deepening of the brown color.

Buchner first utilized this principle by placing a small wire or glass holder within a large test tube, dropping dry pyrogallol (pyrogallic acid) into the bottom of this tube, then running a five per cent sodium hydrate solution into it, and inserting within this large tube a smaller test tube containing the inoculated culture medium. The large tube was then tightly closed 
with a rubber stopper. In this way, the air space surrounding the smaller tube was rendered oxygen free.

A simple modification of the preceding method of Buchner has been devised by Wright. ${ }^{1}$ Stab-cultures of gelatin or agar in test tubes are made in the usual way. The cotton stopper closing the tube is then thrust into the tube to such a depth that its upper end lies at least $1 \mathrm{~cm}$. below the mouth of the tube. A small quantity of sodium or potassium hydrate solution in which some pyrogallic acid has been dissolved, is then allowed to flow on to the cotton of the plug and the mouth of the tube is immediately sealed by a tightly fitting rubber stopper. The cotton stopper in these cases must be made of absorbent cotton; 1.5 to 2.5 c.c. of the pyrogallic acid solution is usually sufficient for test tubes of ordinary size.

For cultivation of anaërobic bacteria upon agar slants, a simple technique may be applied and easily improvised in the laboratory as follows: The tube of slant agar is inoculated with the infectious material in the usual way. It is then, with stopper removed, inverted into a tumbler or beaker containing about a gram of dry pyrogallic acid. A small quantity of a five per cent or three per cent sodium hydrate solution is then run into the tumbler and this is covered with a thin layer of liquid paraffin or albolin before the pyrogallic acid has been completely dissolved. In this way, completely anaërobic conditions are obtained in the tube and the growth of anaërobes takes place upon the surface of the slant.

For the cultivation of anaërobes in Petri

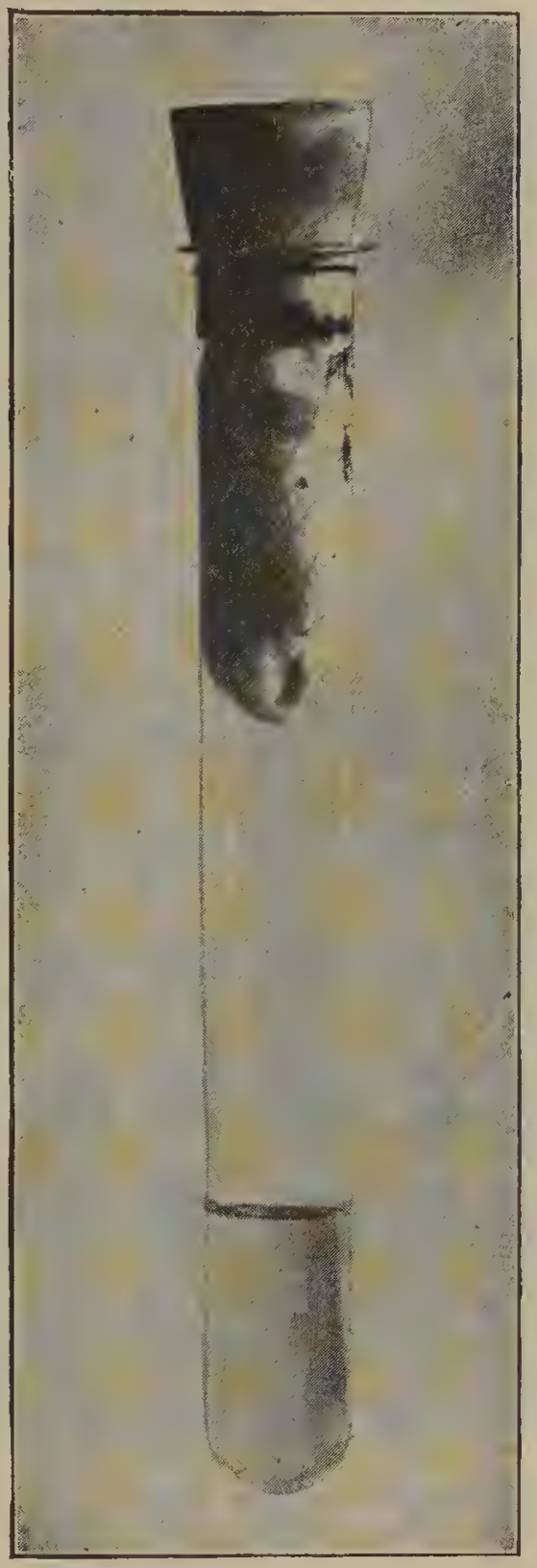

Fig. 37.-Wright's Method of Ana Ïrobic CultivaTION BY THE USE OF PyroGallic ACID Solution. dishes, for purposes of separation, a combination of the pyrogallic acid method and the hydrogen displacement methods is often employed. For this purpose the jars devised by Novy and by Bulloch are extremely convenient. 
In using the Novy jar, the inoculated plates are set upon a wire frame resting about an inch above the bottom of the jar. The cover is then tightly set in place and the air in the jar exhausted by means of a suction pump. The arrangement of the double stop-cock in the top renders it possible now, by simply turning this, to admit hydrogen from a Kipp generator into the jar. The process of alternate exhaustion and admission of hydrogen may be

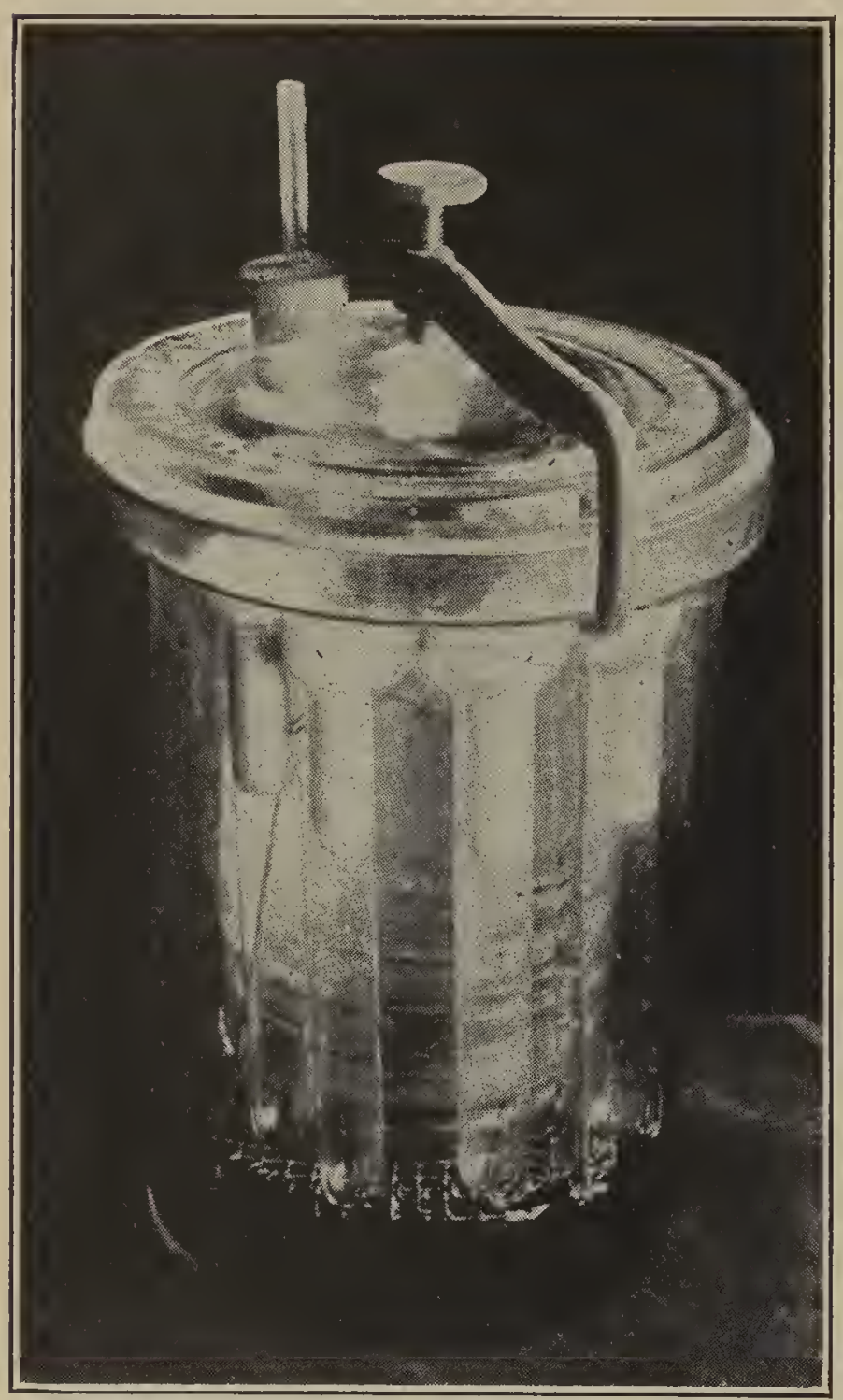

Fig. 38.-J JAR for ANAËROBIC CULTIVATION. several times repeated.

A combination of air exhaustion, oxygen absorption, and hydrogen replacement may be practiced in jars such as that shown in Fig. 39. Tubes or plates after inoculation are placed in this jar, on a raised wire frame. Dry pyrogallic acid is placed in the bottom of the jar and the cover tightly fitted. An opening in the side of the jar connects its interior with a bottle containing sodium or potassium hydrate solution. Through the stopper of this bottle pass two glass tubes, one of them of such length that it can be pushed down into the alkaline solution, or pulled upward above the level of the fluid. This tube connects the jar with the bottle. The other glass tube is short, passing just through the stopper and at the top made in the form of a $\mathrm{T}$, one arm of the $\mathrm{T}$

being connected with a Kipp hydrogen generator, the other with a suction-pump.

After the jar has been sealed, the glass tube connecting the jar and the bottle is raised above the level of the fluid in the bottle and, the connection to the hydrogen generator being closed, the air in the jar is exhausted with the suction-pump. Connection to the suction may then be closed, and the other arm of the $\mathrm{T}$ being open, hydrogen is allowed to flow into the jar. Alternate suction and hydrogen replacement may be 
carried out two or three times. After the last exhaustion, the glass tube in the bottle connecting it with the jar is pushed down into the fluid, and the vacuum will draw the sodium hydrate solution into the bottom of the jar, dissolving the pyrogallol, which will then absorb any traces of free oxygen remaining in the jar. Hydrogen is again introduced and the jar closed. If exhaustion of oxygen has been sufficiently thorough, the pyrogallic solution in the bottom of the jar will remain light brown.

A simple method for the separation of anaërobes in plates without the use of hydrogen or of specially constructed jars, may be carried out as follows ${ }^{1}$ : The apparatus used consists of two circular glass dishes, fitting one into the other as do the halves of a Petri dish, and similar to these in every respect except that they are higher, and that a slightly greater space is left between their sides when they are placed together. The

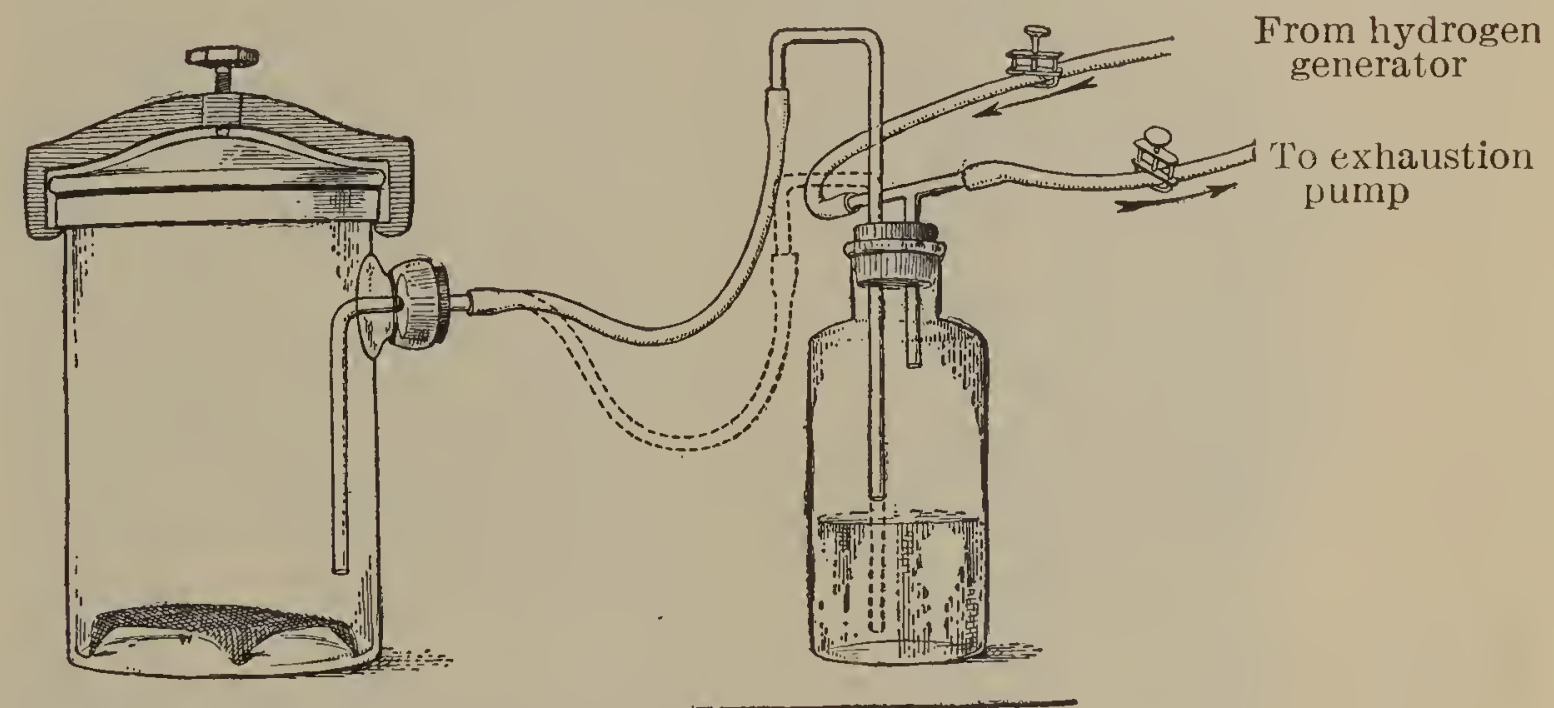

Fig. 30.-Apparatus for Combining the Methods of Exhaustion, Hydrogen, Replacement, and Oxygen Absorption.

dishes should be about three-fourths to one inch in height, they need be of no particular diameter, although those of about the same size as the usual Petri dish are most convenient. An important requirement necessary for the success of the method is that the trough left between the two plates, when put together, shall not be too broad, a quarter of an inch being the most favorable.

Into the smaller of these plates the inoculated agar is poured exactly as this is done into a Petri dish in the ordinary aërobic work. Prolonged boiling of the agar before plating is not essential. When the agar film has become sufficiently hard on the bottom of the smaller dish, the entire apparatus is inverted. The smaller dish is now lifted out of the 
larger, and placed, still inverted, over a moist surface-a towel or the wet surface of the table-to prevent contamination. Into the bottom of the larger dish, which now stands open, there is placed a quantity (about 3 grams) of dry pyrogallic acid. Into this, over the pyrogallic acid, the smaller dish, still inverted, is then placed. A five per cent solution of sodium hydrate is poured into the space left between the sides of the two dishes, in quantity sufficient to fill the receiving dish one-half full. While this is gradually dissolving the pyrogallic acid, albolin, or any other oil (and this is the only step that requires speed), is dropped from a pipette, previously filled and placed in readiness, into

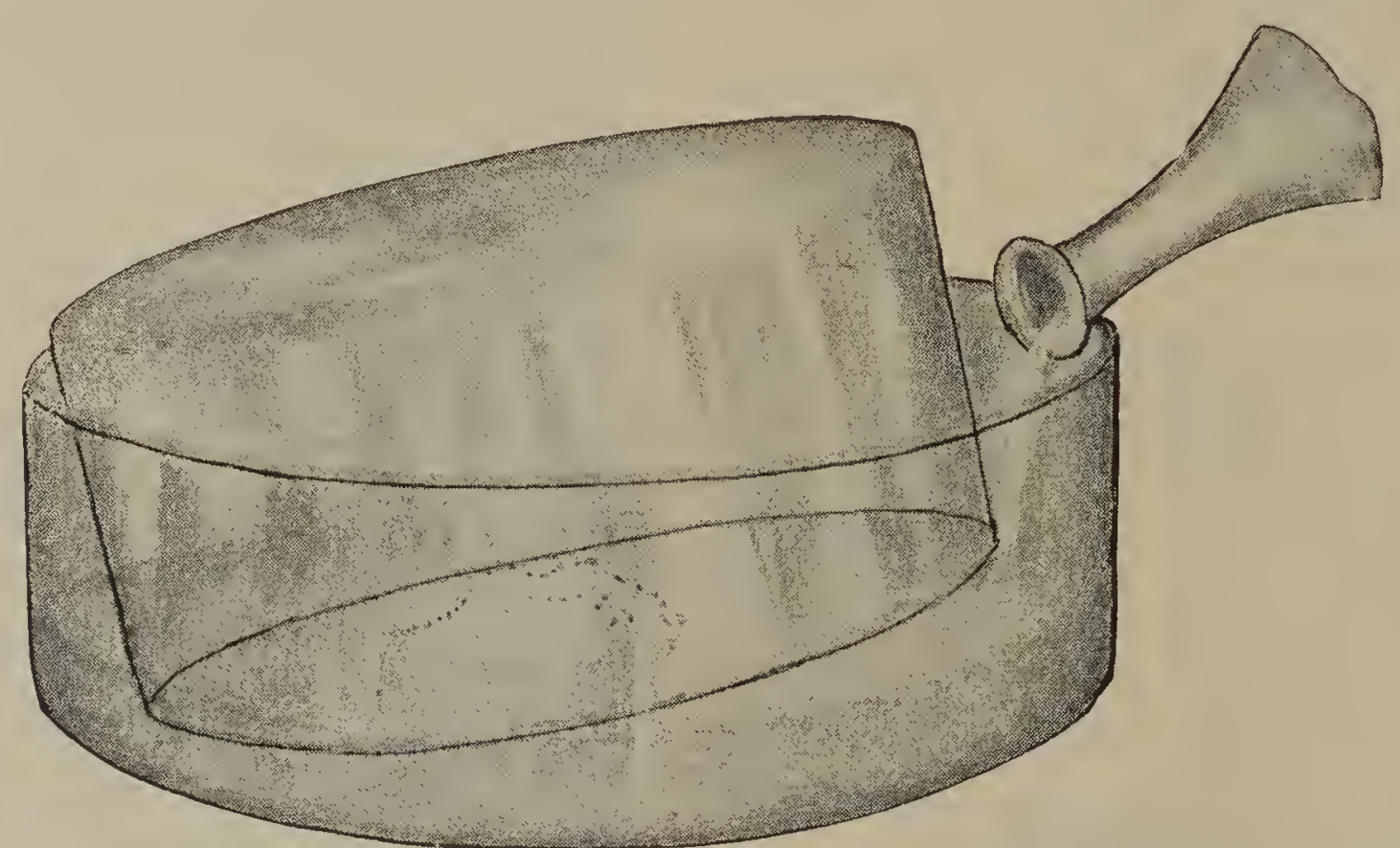

Fig. 40.-Simple Apparatus for Plate Cultitation of Anä̈robic Bacteria. (Zinsser.)

the same space, thus completely sealing the chamber formed by the two dishes.

If these steps have been performed successfully, the pyrogallic solution will at this time appear of a light brown color, and the smaller plate, with its agar film, will float unsteadily above the other. Very rapidly, as the pyrogallic acid absorbs the free oxygen in the chamber, this plate is drawn down close to the other, and the acid assumes a darker hue, which remains without further deepening even after three or four days' incubation.

The Use of Fresh Sterile Tissue as an Aid to Anaërobic Cultivation. - The addition of small pieces of fresh sterile tissue (rabbit or guineapig) to culture tubes, either solid or fluid, greatly favors the growth of anaërobic bacteria. By such a method anaërobes can be made to develop even when other precautions for the establishment of anaërobiosis 
are imperfectly observed. This was noticed first by Theobald Smith and by Tarozzi and has become an extremely useful reënforcement to other methods. It has been utilized most extensively by Noguchi of recent

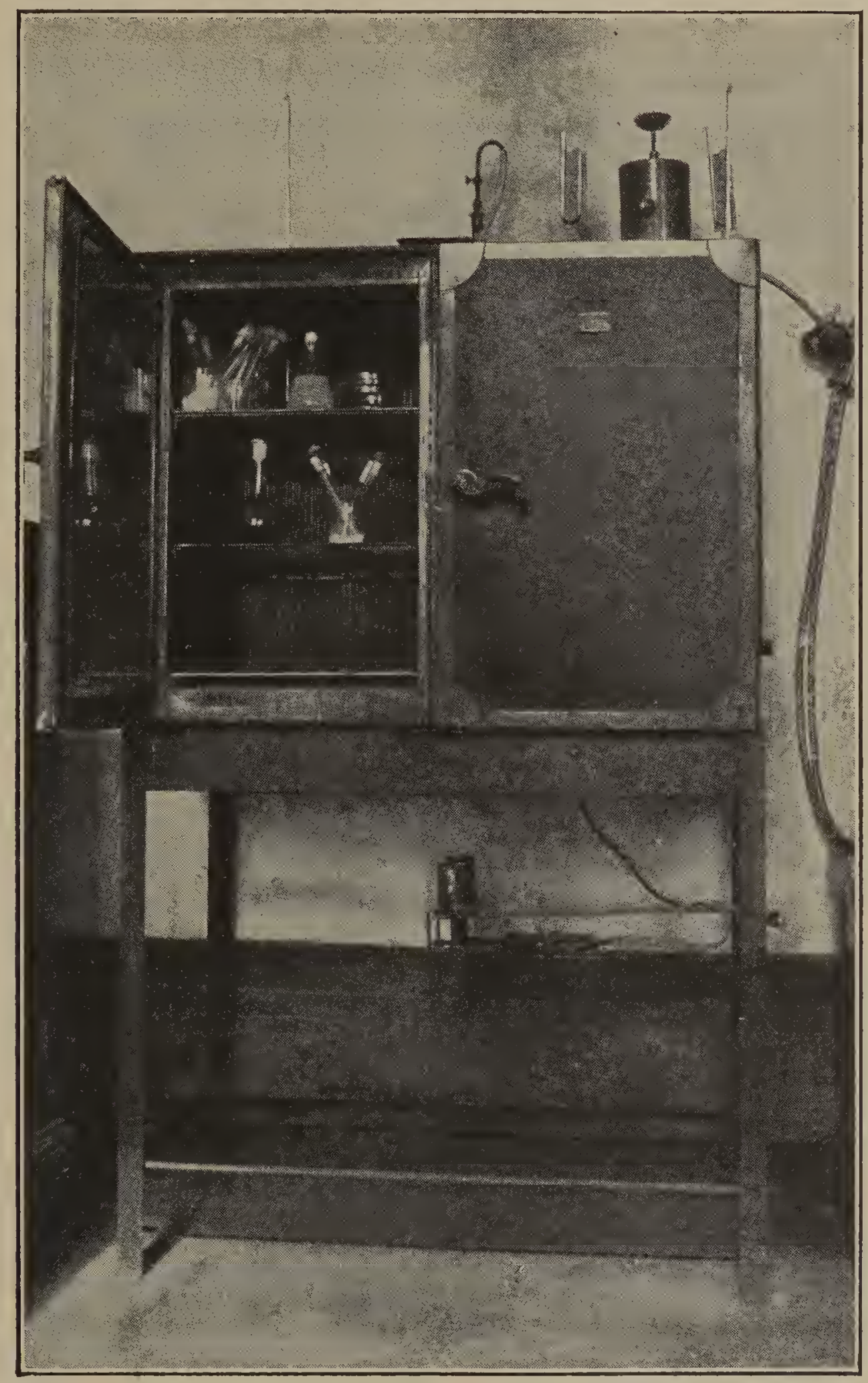

Fig. 41.-Incubator.

years in his technique for the cultivation of various treponemata. The simplest way to apply this method is to place a piece of freshly excised rabbit kidney, testicle, or spleen into the bottom of a high test tube $(20 \mathrm{~cm}$.) and then pouring over it the culture fluid. Kidney or 
other tissues are more suitable for this purpose than liver tissue since the latter is not easily obtained in a sterile condition, bacteria often getting into it during life through the portal circulation. The action of the tissues depends probably upon its great reducing power.

\section{THE INCUBATION OF CULTURES}

After bacteria have been transferred to suitable culture media, it is necessary to expose them to a temperature favorable to their develop-

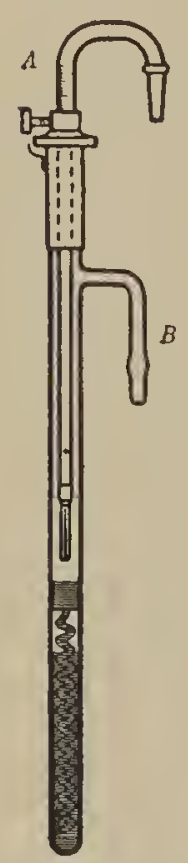

FIG. 42.

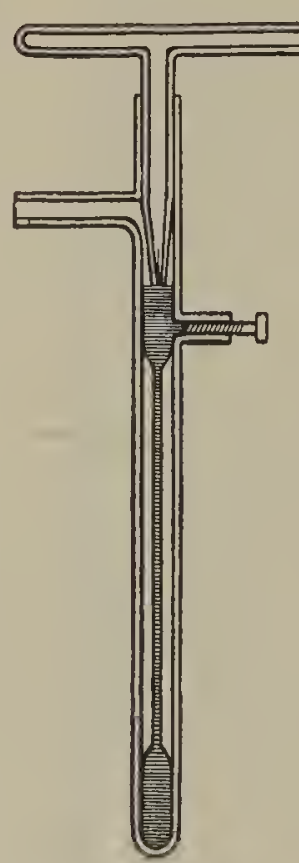

FIG. 43.
Fig. 42.-Thermo-Regulator.

(Lautenschläger.)

Fig. 43.-Thermo-regulator. (Reichert.)

ment. In the case of many saprophytes, the ordinary room temperature is sufficiently near the optimum to obviate the use of any special apparatus for maintaining a suitable temperature; in the case of most pathogenic bacteria, however, the body temperature of man, $37.5^{\circ}$ $\mathrm{C}$., is either a necessary requirement for their growth, or at any rate favors speedy and characteristic development.

For the purpose of obtaining a uniform temperature of any required degree, the apparatus in general use is the so-called incubator or thermostat. This may be adjusted for gelatin cultivation at 20 to $22^{\circ} \mathrm{C}$., or for agar, broth, or other media at $37.5^{\circ} \mathrm{C}$.

Incubators, while varying in detail, are all constructed upon the same principles. They consist of double-walled copper chambers, which are fitted with a set of double doors, the outer being made of asbestoscovered metal, the inner of glass. (See Fig. 41.) The space between the two walls is filled with water, which, being a poor heat conductor, tends to prevent rapid changes of temperature within the chamber as the result of changes in the external environment. Both walls are perforated above by openings to admit thermometers into the interior and one wall is perforated so that a thermo-regulator may be inserted into the water jacket, The under surface of the chamber is heated by a gas 
flame, the size of which is automatically regulated by the thermoregulator.

A number of thermo-regulators are on the market, all of them constructed upon modifications of the same principle. One of the most efficient of those in common use is that shown in Fig. 42. "This consists of a long tube of glass fitted with a metal cap through which an inlet tube $(A)$ projects into the interior. Slightly below the middle of the tube there is a glass diaphragm separating its interior into two compartments. In the middle of the diaphragm an aperture leads into a spiral of glass which projects into the lower compartment. The lower compartment is filled with ether and mercury. The lower end of the inlet tube $(A)$ has a wedge-shaped slit. The gas from the supply pipe passing through the tube $(A)$ is conducted through the slit-like opening in its lower end into the inner chamber and passes out to the burner through the elbow $(B)$. When the temperature is raised, the ether and mercury in the lower chamber expand and the mercury rises in the upper chamber, gradually restric ting the opening through the $V$-shaped slit in the inlet tube. Thus the gas supplied to the burner is diminished, the flame reduced, and the temperature again falls. The temperature can be arbitrarily adjusted by raising or lowering the inlet tube. A scale at the upper end of the inlet tube allows exact adjustment. Complete shutting off of the gas is prevented by a small circular opening placed in the inlet tube just above the slit.

Another cheaper and simpler therro-regulator is shown in Fig. 43. This consists of a long tube open at the top and fitted about $1 \frac{1}{2}$ inches from the top with two hollow glass elbows. One of these elbows remains open, the other, situated on a slightly lower level, is closed by a brass screw-cap. The tube is filled with mercury to a point slightly above the level of the elbow containing the screw-cap. The height of the mercury can thus be increased or decreased by screwing in or out upon the cap. Into the upper end of the tube there is fitted another device which consists of a $\mathrm{T}$-shaped system of glass tubes, one arm of the ' $\mathrm{T}$ being open and the other closed, the perpendicular leg of the $\mathrm{T}$ tapering to a minute opening at the bottom. The gas passes into one arm of the $\mathrm{T}$ down through the tapering leg and into the space immediately above the mercury. It then passes out through the open elbow of the main tube. As the mercury rises, it gradually diminishes the space between its surface and the small opening in the tapering tube above it, finally completely shutting off the gas from this source. Gas can now pass only through a minute hole perforating the vertical leg of the 
$\mathrm{T}$ an inch above its end. The flame decreases and the temperature again sinks.

Since gas pressure in laboratories is apt to vary, it is convenient to interpose between the gas supply and thermo-regulator some one of the various forms of gas-pressure regulators. The use of these is not absolutely necessary but aids considerably in the maintenance of a constant temperature. The one most commonly employed is the so-called Moitessier apparatus. This consists of a cylindrical metal chamber within which there is fitted an inverted metal bell. Glycerin is poured into the cylinder to the depth of about two inches. An inlet pipe con-

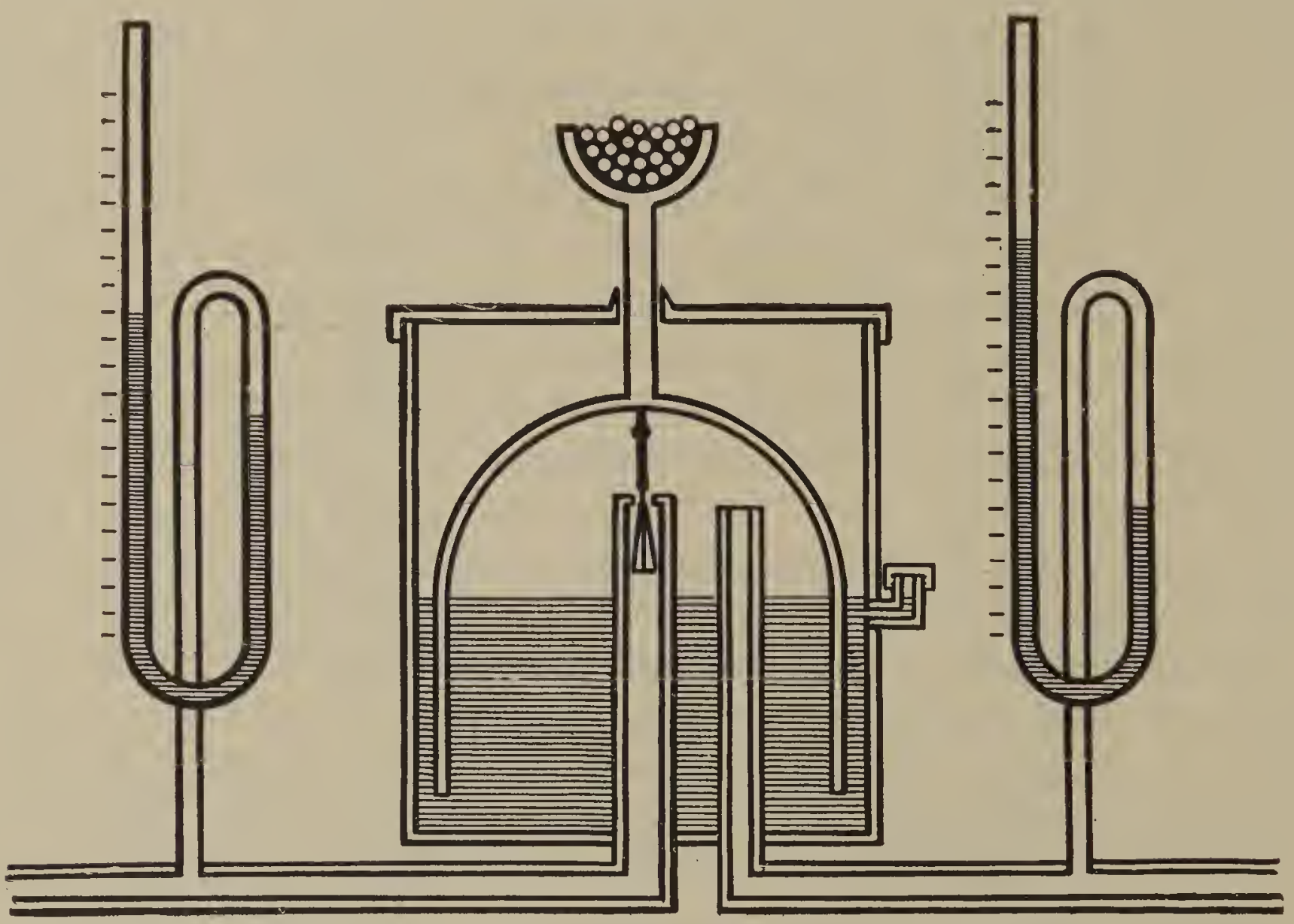

Fig. 44.-Moitessier Gas-Pressure Regulator.

ducts gas into the open space between the top of the glycerin and the bell. From the top of the bell is suspended a conical piece of metal which hangs free in the outlet pipe. As the gas pressure under the bell increases, this is raised and the opening of the outlet pipe is gradually diminished by the cone. Thus the relation between the pressure in the inlet pipe and the actual quantity of gas passing through is equalized. A cup connected to the top of the bell through the roof of the cylinder by a bar can be filled with birdshot and the pressure against the gas can thus be modified to conform with existing conditions. 
Colony Study.-Cultures are usually incubated for from twelve to forty-eight hours. Considerable aid to the recognition of species is derived from the observation of both the speed of growth and the appearance of the colonies. It is therefore necessary to proceed in the study of developed co onies in a systematic way. The development of colonies should be observed in all cases both upon gelatin and upon agar. In forming any judgment about colonies, the acidity or alkalinity, and the special constitution of the media should be taken into account. The colonies are carefully examined with a hand lens and with the low power (Leitz No. 2, Zeiss AA, Ocular No. 2) of the microscope. The colonies should be observed as to size, outline, transparency, texture, color, and elevation from the surface of the media. Much information,
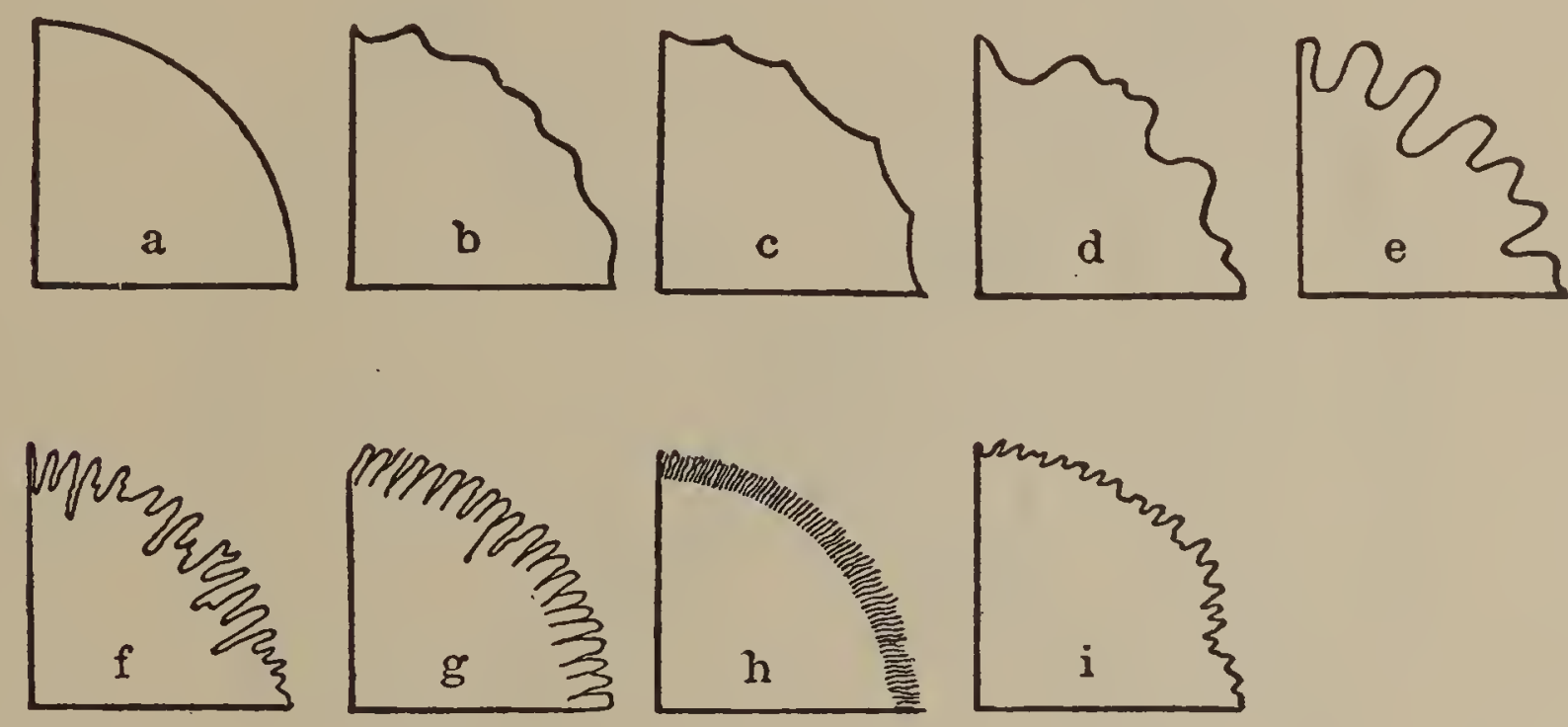

Fig. 45.-Variations in the Conformation of the Borders of Bacterial Colonies. (After Chester.)

also, can be obtained by observing whether a colony appears dry, mucoid, or glistening, like a drop of moisture. By a careful observation of these points, definite differentiation, of course, can not usually be made, but much corroborative evidence can be obtained which may guide us in the methods to be adopted for further identification and for a final summing up of species characteristic as a whole.

The Counting of Bacteria.-It is often necessary to determine the number of bacteria per c.c. contained in water, milk, or other substances. For this purpose definite quantities of the material to be analyzed are mixed with gelatin or agar and poured into Petri plates. The exact dilutions of the suspected material must largely depend upon the number of germs which one expects to find in it. The plates, if prepared with gelatin, are allowed to develop at room temperature for twenty-four to 
forty-eight hours. If agar has been used, they are usually placed in the incubator at $37.5^{\circ} \mathrm{C}$. At the end of this time, the colonies which have developed are enumerated. For this purpose, a Petri dish is placed upon a Wolffhügel plate. This plate consists of a disk or square of glass which is divided into small squares of one square centimeter each. Diagonal lines of these squares running at right angles to each other are subdivided into nine divisions each in order to facilitate counting when the colonies are unusually abundant. The Petri dish is placed upon the plate in such a way that the center of the dish corresponds to the center of the plate.

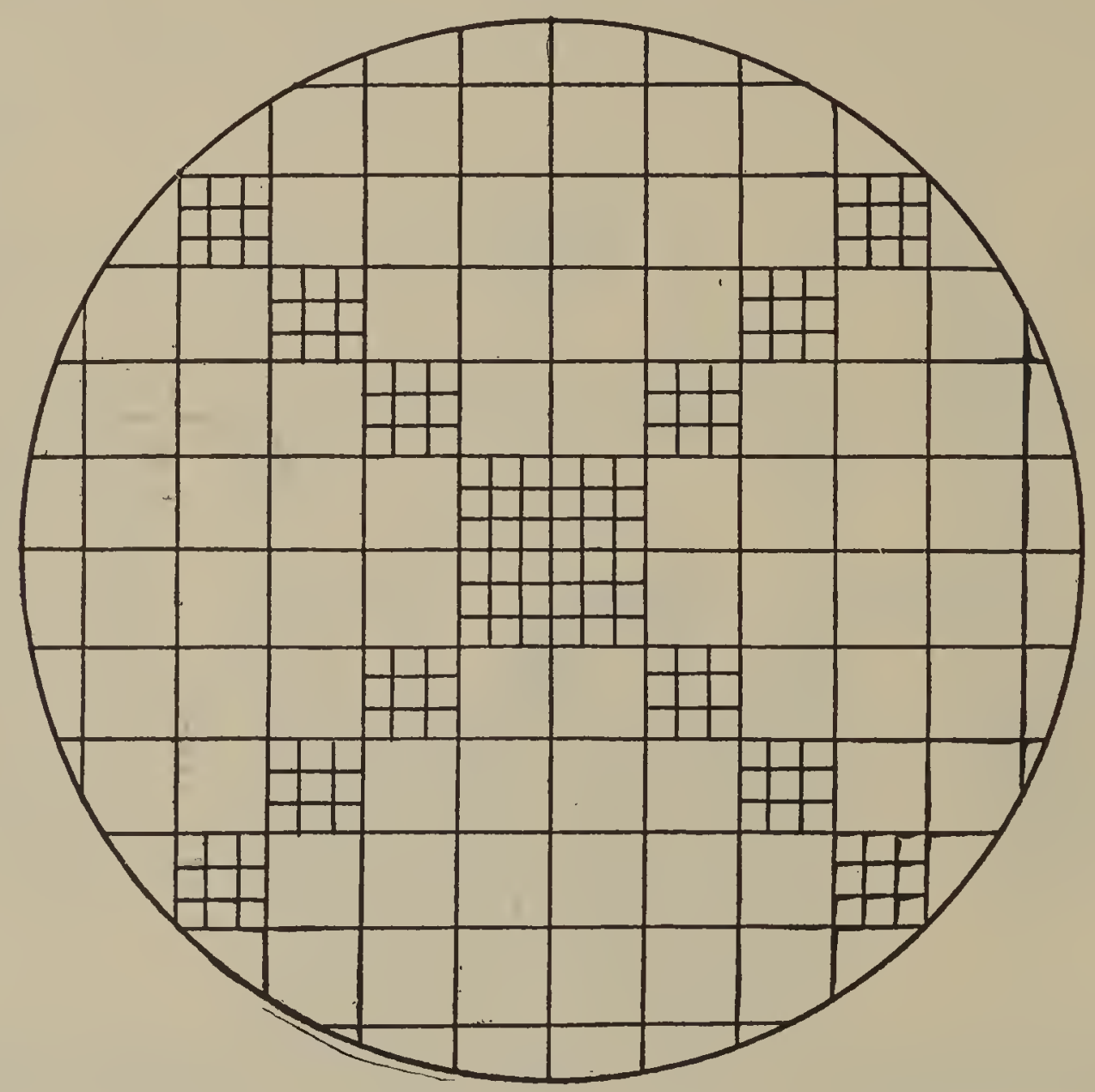

Fig. 46.-Wolffhü̈gel Counting Plate.

The colonies in a definite number of squares are then counted. The greater the number of squares that are counted the more accurate the estimation will be. When the growth is so abundant that only a limited number of squares can be counted, these should be chosen as much as possible from different parts of the plate, and in practice one counts usually six squares in one direction and six at right angles to these, so as to preclude errors arising from unequal distribution. The final calculation is then made by ascertaining the average number of colonies contained in each square centimeter. If standard Petri dishes have been 
used, this is multiplied by 63.6 , the number of squares in the area of the dish, and then by the dilution originally used.

Thus if twelve squares have been counted with a total number of one hundred and forty-four colonies - the average for each square is twelve. Twelve times 63.6 is 763.2 , which represents the total number of colonies in the plate. Now if 0.1 c.c. of the original material (water or milk) has been plated, this material may be assumed to have contained $10 \times 763.2$, or 7,632 bacteria to each cubic centimeter.

If dishes of an unusual size are employed, the square area must be ascertained by measuring the radius and multiplying its square by $\pi$ $\left(\pi \times \mathrm{R}^{2}=\right.$ area $)(\pi=3.141592)$. 


\section{CHAPTER IX}

METHODS OF DETERMINING BIOLOGICAL ACTIVITIES OF BACTERIA ANIMAL EXPERIMENTATION

Gas Formation.-Bacteria of many varieties produce gas from the proteid and the carbohydrate constituents of their environment.

Gas formation can be observed in a very simple manner by making stab cultures in gelatin or agar containing the fermentable nutrient substances. In such cultures bubbles of gas will form along the track of the inoculation, or, in the case of such semisolid media as the tube medium of Hiss, will spread throughout the tube. In the case of some anaërobes gas formation in stab cultures will occur to such an extent that the medium will split and break. It should be borne in mind in carrying out such methods that air is readily carried into the medium with the inoculating needle or loop by splitting of the medium, also that media which have been stored in the cold may absorb air. Expansion of the air in such tubes may simulate small amounts of gas formation and lead to error. It is advisable, therefore, whenever making stab inoculations with the above purpose, to heat the media and rapidly cool them before use.

A more accurate method of gas determination is by the use of fermentation tubes, such as those devised by Smith. The gas which is formed collects in the closed arm of the fermentation tube and may be quantitatively estimated. The fermentation, with gas production, of certain substances such as carbohydrates, may be determined by adding these materials in a pure state to the media before inoculation with organisms.

In the case of carbohydrates this method has proved of great differential value, since the power of splitting specific carbohydrates with gas production is a species characteristic of great constancy for many forms of bacteria.

Analysis of Gas Formed by BaCteria.-Carbon Dioxide.-For the estimation both qualitatively and roughly quantitatively of carbon dioxide produced by bacteria, cultures are grown in fermentation tubes containing sugar-free broth (see page 125) to which one per cent of pure, dextrose, lactose, saccharose, or other sugars has been added. The tubes are incubated until the column of gas formed in the closed arm no longer 
increases (twenty-four to forty-eight hours). The level of the fluid in the closed arm is then accurately marked and the column of gas measured.

The bulb of the fermentation tube is then completely filled with $\frac{\mathrm{N}}{5} \mathrm{NaOH}$ solution, the mouth closed with a clean rubber stopper, and the bulb inverted several times in order to mix the gas with the soda solution. The tube is then again placed in the upright position, allowing the gas remaining to collect in the closed arm. The gas lost may be roughly estimated as consisting of $\mathrm{CO}_{2}$.

Hydrogen.-The gas remaining, after removal of the $\mathrm{CO}_{2}$ in the preceding experiment, at least when working with carbohydrate solutions,
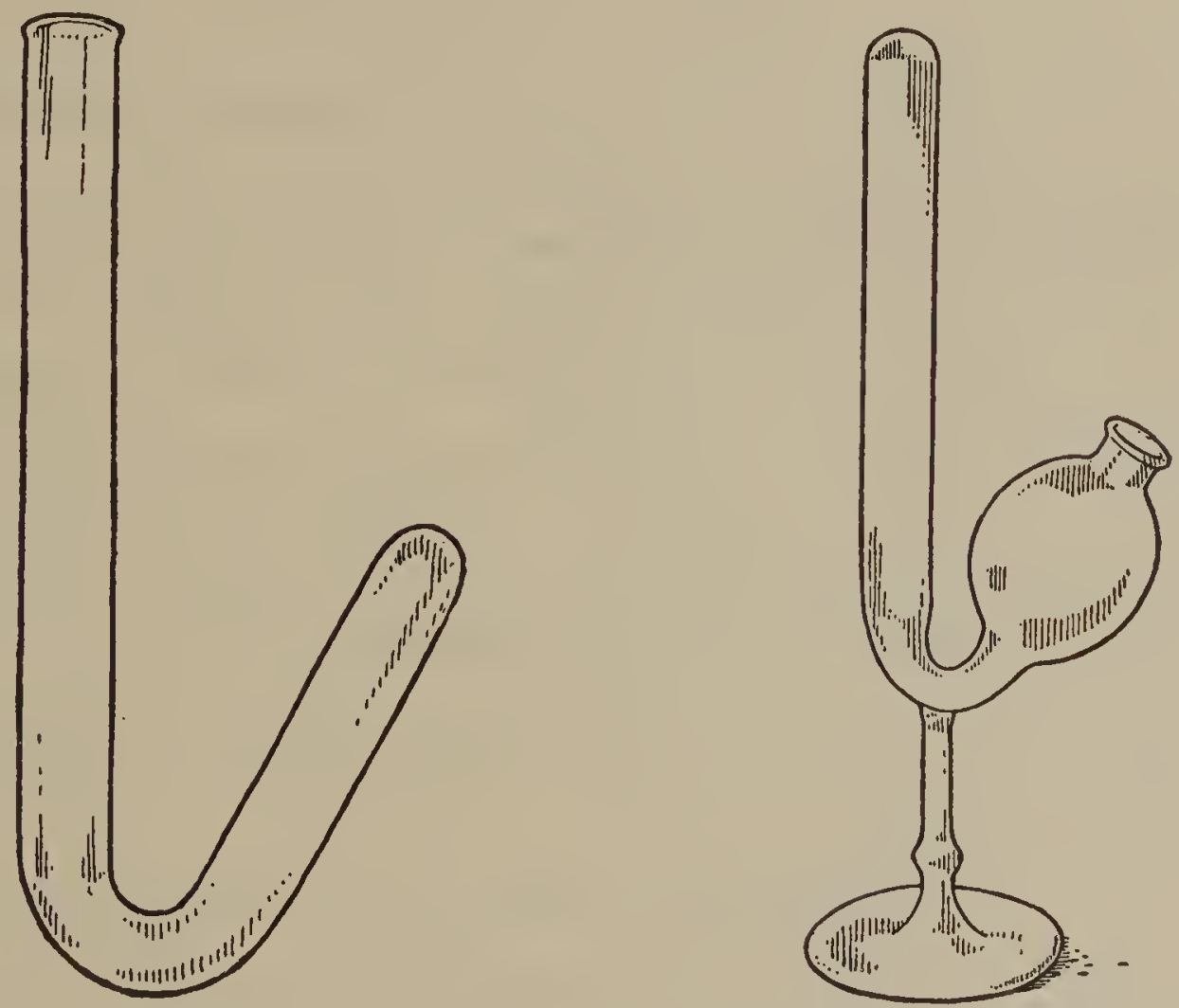

Fig. 47.-Types of Fermentation Tubes.

may be estimated as hydrogen. When allowed to collect near the mouth, further evidence of its being hydrogen may be gained by exploding it with a lighted match.

Hydrogen Sulphide ( $\mathrm{H}_{2} \mathrm{~S}$, Sulphuretted hydrogen).- - In alkaline media sulphuretted hydrogen, if formed, will not collect as gas, but will form a sulphide with any alkali in the solution. For the estimation of the formation of hydrogen sulphide, bacteria are cultivated in a strong pepton solution to which 0.1 c.c. of a one per cent solution of ferric tartrate or lead acetate has been added. The addition of these substances gives rise to a yellowish precipitate in the bottom of the tubes. If, on 
subsequent inoculation, the bacteria produce $\mathrm{H}_{2} \mathrm{~S}$, this precipitate will turn black. The solution recommended by Pake for this test is prepared as follows:

1. Weigh out 30 grams of pepton and emulsify in 200 c.c. of tap water at $60^{\circ} \mathrm{C}$.

2. Wash into a liter flask with 80 c.c. tap water.

3. Add sodium chloride 5 grams and sodium phosphate 3 grams.

4. Heat at $100^{\circ} \mathrm{C}$. for 30 minutes, to dissolve pepton.

5. Filter through paper.

6. Fill into tubes, 10 c.c. each, and to each tube add 0.1 c.c. of a one per cent solution of ferric tartrate or lead acetate. These solutions should be neutral.

7. Sterilize. ${ }^{1}$

ACCURATE QUANTITATIVE GaS ANALYSES of bacterial cultures can be made only by the more complicated methods used in chemical laboratories for quantitative gas analysis. The gas, in such cases, is collected in a bell jar mounted over mercury, and subjected to analysis by the usual method described in works on analytical chemistry.

Acid and Alkali Formation by Bacteria.-Many bacteria produce acid or alkaline reactions in culture media, their activity in this respect depending to a large extent upon the nature of the nutrient material. Many organisms which on carbohydrate media produce acid will give rise to alkali if cultivated upon media containing only proteids.

Information as to the production of acid or alkali can be obtained by the addition of one of a variety of indicators to neutral media. The indicators most often employed for this purpose are litmus or neutral red. Changes in the color of these indicators show whether acids or alkalis have been produced.

Great help in differentiation is obtained by adding chemically pure carbohydrates to media to which litmus has been added and then determining whether or not acid is formed from the substances by the microorganisms. These tests have been of special importance in the differentiation of the typhoid-colon groups of bacilli.

Quantitative estimation of the degree of acidity or alkalinity produced by bacteria may be made by careful titration of definite volumes of the medium before and after bacterial growth has taken place.

The variety of acid formed by bacteria depends largely upon the nature of the nutrient medium. The acids most commonly resulting from bacterial growth are lactic, acetic, oxalic, formic, and hippuric acids. Qualitative and quantitative estimation of these acids may be made by any of the methods employed by analytical chemists.

1 Quoted from Eyre, "Bact. Technique," Phila., 1903. 
Indol Production by Bacteria.-Many bacteria possess the power of producing indol. Though formerly regarded as a regular accompaniment of proteid decomposition, later researches have shown that indol production is not always coexistent with putrefaction processes and occurs only when pepton is present in the pabulum.

Indol formation by bacteria is determined by the so-called nitrosoindol reaction. Organisms are grown in sugar-free pepton broth or in the pepton-salt bouillon of Dunham. (See page 126.) Media containing fermentable substances are not favorable for indol production since acids interfere with its formation. The cultures are usually incubated for three or four days at $37^{\circ} \mathrm{C}$. At the end of this time, ten drops of concentrated sulphuric acid are run into each tube. If a pink color appears, indol is present, and we gather the additional information that the microorganism in question has been able to form nitrites by reduction (e.g., cholera spirillum). If the pink color does not appear after the addition of the sulphuric acid alone, nitrites must be supplied. This is done by adding to the fluid about 1 c.c. of a 0.01 per cent aqueous solution of sodium nitrite. The sodium nitrite solution does not keep for any length of time and should be freshly made up at short intervals.

Phenol Production by Bacteria.-Phenol is often a by-product in the course of proteid cleavage by bacteria. To determine its presence in cultures, bacteria are cultivated in flasks containing about 50-100 c.c. of nutrient broth. After three to four days' growth at $37^{\circ} \mathrm{C}$., 5 c.c. of concentrated $\mathrm{HCl}$ are added to the culture, the flask is connected with a condenser, and about 10-20 c.c. are distilled over.

To the distillate may be added 0.5 c.c. of Millon's reagent (solution of mercurous nitrate in nitric acid), when a red color will indicate phenol; or 0.5 c.c. of a ferric chloride solution, which will give a violet color if phenol is present.

Reducing Powers of Bacteria. - The power of reduction, possessed by many bacteria, is shown by their ability to form nitrites from nitrates. This is easily demonstrated by growing bacteria upon nitrate broth (see page 126). Bacteria are transferred to test tubes containing this solution and allowed to grow in the incubator for four or five days. The presence of nitrites is then chemically determined. ${ }^{1}$

${ }^{1}$ We are indebted to Dr. J. P. Mitchell, of Stanford University, for the following technique for nitrite tests:

I. Sulphanilic Acid.-Dissolve 0.5 g. in 150 c.c. of acetic acid of Sp. Gr. 1.04. 
In bacteriological work 4 c.c. of the culture fluid is poured into a clean test tube, and to it are gradually added 2 c.c. of the mixed test solutions. A pink color indicates the presence of nitrites, the intensity of the color being proportionate to the amount of nitrite present.

The reducing powers of bacteria may also be shown by their ability to decolorize litmus, methylene-blue, and some other anilin dyes, which on abstraction of oxygen form colorless leukobases.

Enzyme Action.-The action of the enzymes produced by bacteria may be demonstrated by bringing the bacteria, or their isolated ferments, into contact with the proper substances and observing both the physical and chemical changes produced. In obtaining enzymes free from living bacteria, it is convenient to kill the cultures by the addition either of toluol or of chloroform. Both of these substances will destroy the bacteria without injuring the enzymes. Enzymes may also be obtained separate from the bodies of the bacteria by filtration.

Proteolytic Enzymes. - The most common evidences of proteolytic enzyme action observed in bacteriology are the liquefaction of gelatin, fibrin or coagulated blood-serum, and the peptonization of milk. This may be observed both by allowing the proper bacteria to grow upon these media, or by mixing sterilized cultures with small quantities of these substances. ${ }^{1}$ The products of such a reaction may be separated from the bacteria by filtration and then tested for pepton by the biuret reaction.

Proteolytic ${ }^{2}$ enzymes may also be determined by growing the bacteria upon fluid media containing albumin solutions, blood serum, or milk serum, then precipitating the proteids by the addition of ammonium sulphate (about 30 grams to 20 c.c. of the culture fluid) and warming between 50 to $60^{\circ} \mathrm{C}$. for thirty minutes. The precipitate is then filtered off, the filtrate made strongly alkaline with $\mathrm{NaOH}$, and a few drops of copper sulphate solution added. A violet color indicates the presence of pepton-proving proteolysis of the original albumin.

(Acetic acid of 1.04 prepared by diluting 400 c.c. of conc. of Sp. Gr. 1.75 with $70 C$ c.c. of water.)

II. A-Naphthylamin.-Dissolve $0.1 \mathrm{~g}$. in 20 c.c. of water, boil, filter (if necessary), and to clear filtrate add 180 c.c. of acetic acid, Sp. Gr. 1.04 .

The solutions are kept separate and mixed in equal parts just before use.

In carrying out the test, put 2 c.c. of each reagent in a test tube and add substance to be tested. (In ordinary water analysis use 100 c.c.) Cover tube with watch glass and set in warm water for 20 minutes. Observe presence or absence of pink color promptly. Always run a blank on the distilled water used for rinsing to avoid errors due to nitrites in the water, or in the air of the laboratory.

${ }^{1}$ Bitter, Archiv f. Hyg., v. 1886.

${ }^{2}$ Hankin and Wesbrook, Ann. Past., vi., 1892. 
Diastatic Enzymes.- The presence of diastatic ferments may be determined by mixing broth cultures of the bacteria with thin starch paste. It is necessary that both the cultures and the starch paste be absolutely free from sugar. After remaining in the incubator for five or six hours, the fluid is filtered and the filtrate tested by methods used for determining the presence of sugars.

Inverting Ferments.- Inverting ferments are determined by a procedure similar to the above in principle. Dilute solutions of cane sugar are mixed with old cultures or culture filtrates of the respective bacteria and the mixture allowed to stand. It is then filtered, and the filtrate tested for glucose, preferably by Fehling's solution.

\section{ANIMAL EXPERIMENTATION}

In the study of pathogenic microorganisms, animal experimentation is essential in many instances. The virulence of any given organism for a definite animal species and the nature of the lesions produced are characteristics often of great value in differentiation. Isolation, moreover, of many bacteria is greatly facilitated by the inoculation of susceptible animals and recovery
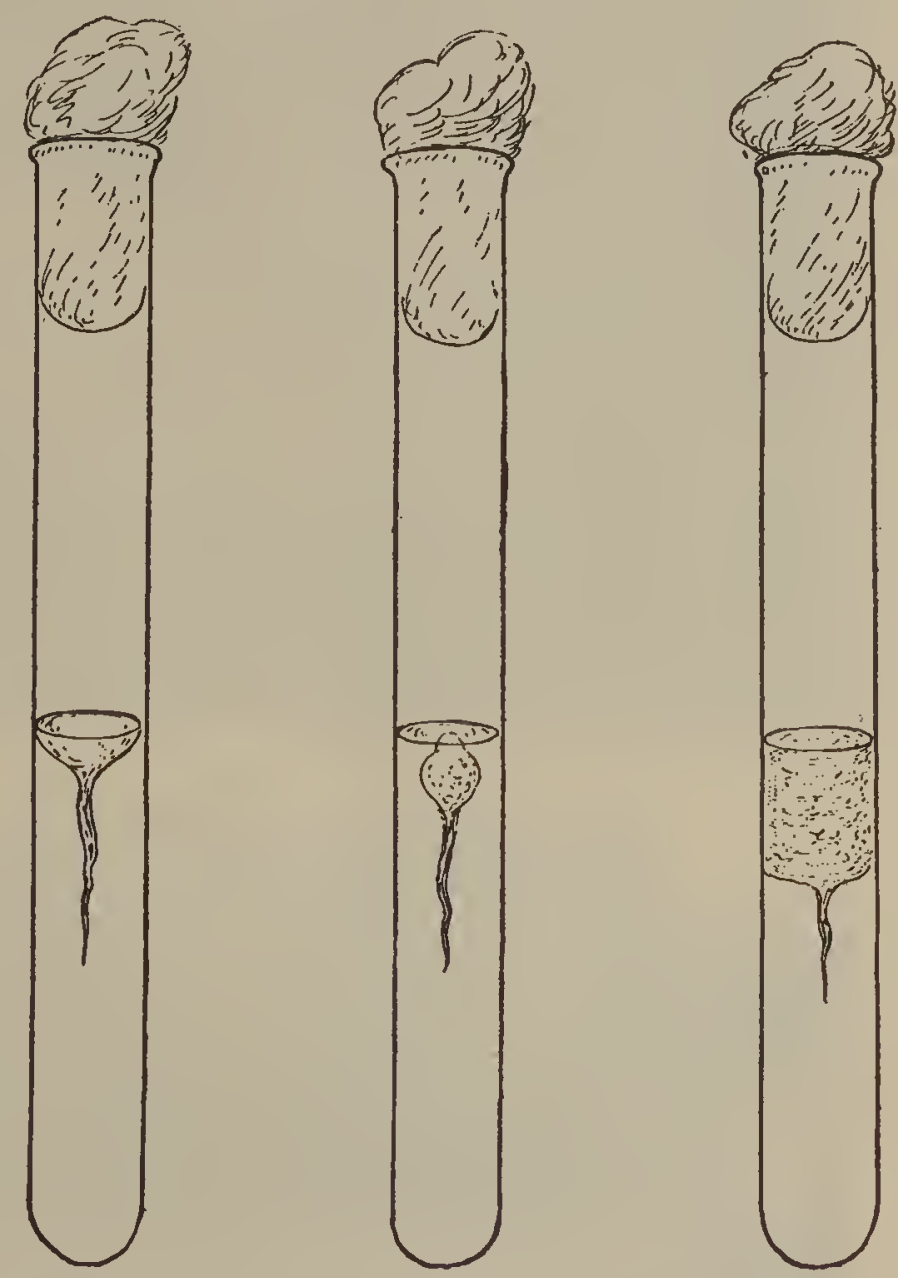

Frg. 48.-Types of Gelatin LiquefacTION BY BACTERIA. of the pathogenic organism from the heart's blood or from the lesions produced in various organs. That investigations into the phenomena of immunity would be absolutely impossible without the use of animal inoculation is, of course, selfevident, for by this method only can the action of bacteria in relation to living tissues, cells, and body-fluids be observed.

The animals most commonly employed for such observations are guinea-pigs, white mice, white rats, and rabbits. The method of inoculation may be either subcutaneous, intrapleural, intraperitoneal, intravenous, or subdural, etc. It must be borne in mind always that the mode of inoculation may influence the course of an 
infection no less than does the virulence of the microorganism or the size of the dose.

Inoculations are made with some form of hypodermic needle fitted to

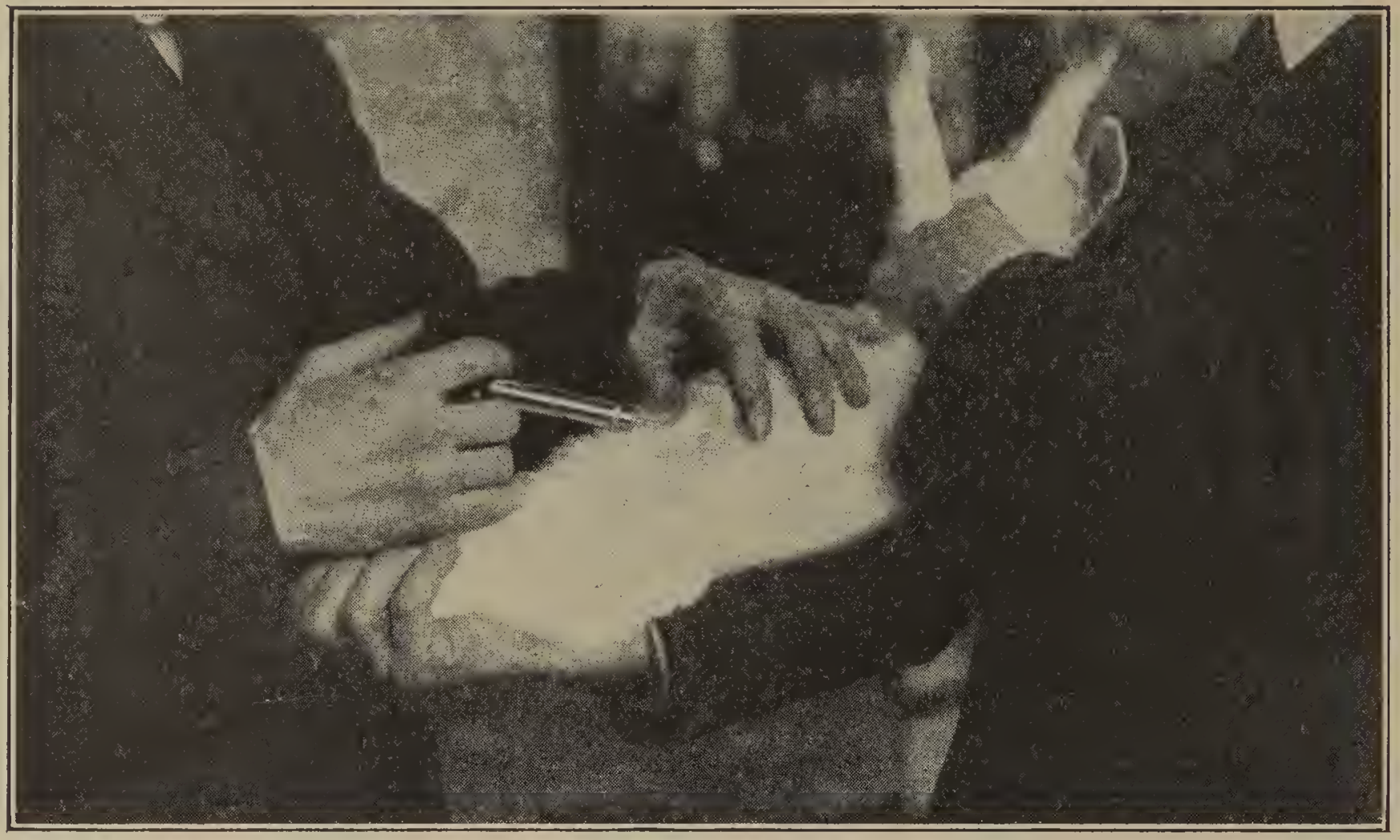

Fig. 49.-Intraperitoneat Inoculation of Rabbit.

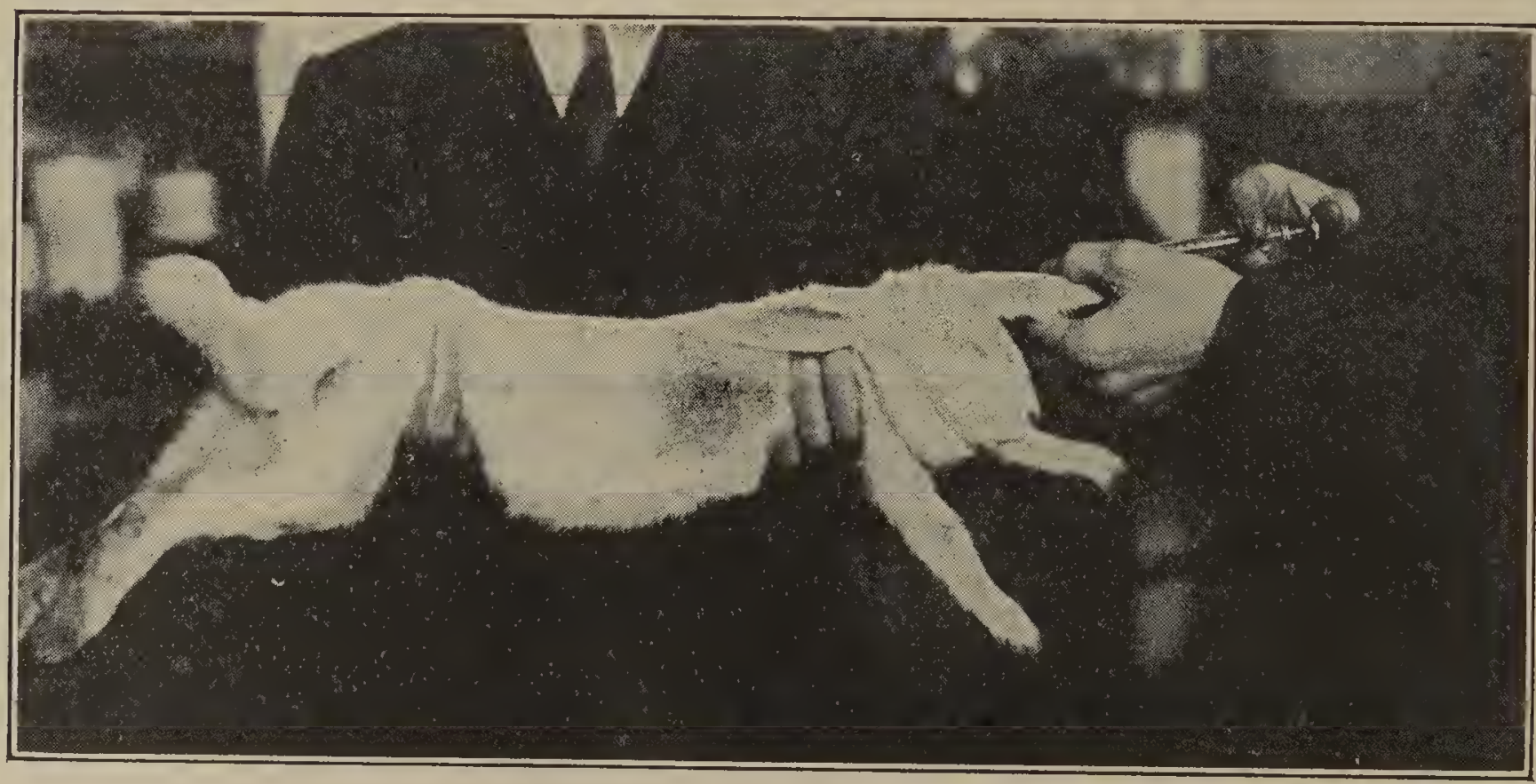

Fig. 50.-Intravenous Inoculation of Rabbit.

a syringe. The most convenient syringes are the all-glass Luer or the Debove syringes, which, however, are expensive. Any form of sterilizable syringe may be used. In making inoculations the hair of the 
animal should be clipped and the skin disinfected with carbolic acid or alcohol.

Subcutaneous inoculations are most conveniently made in the abdom..

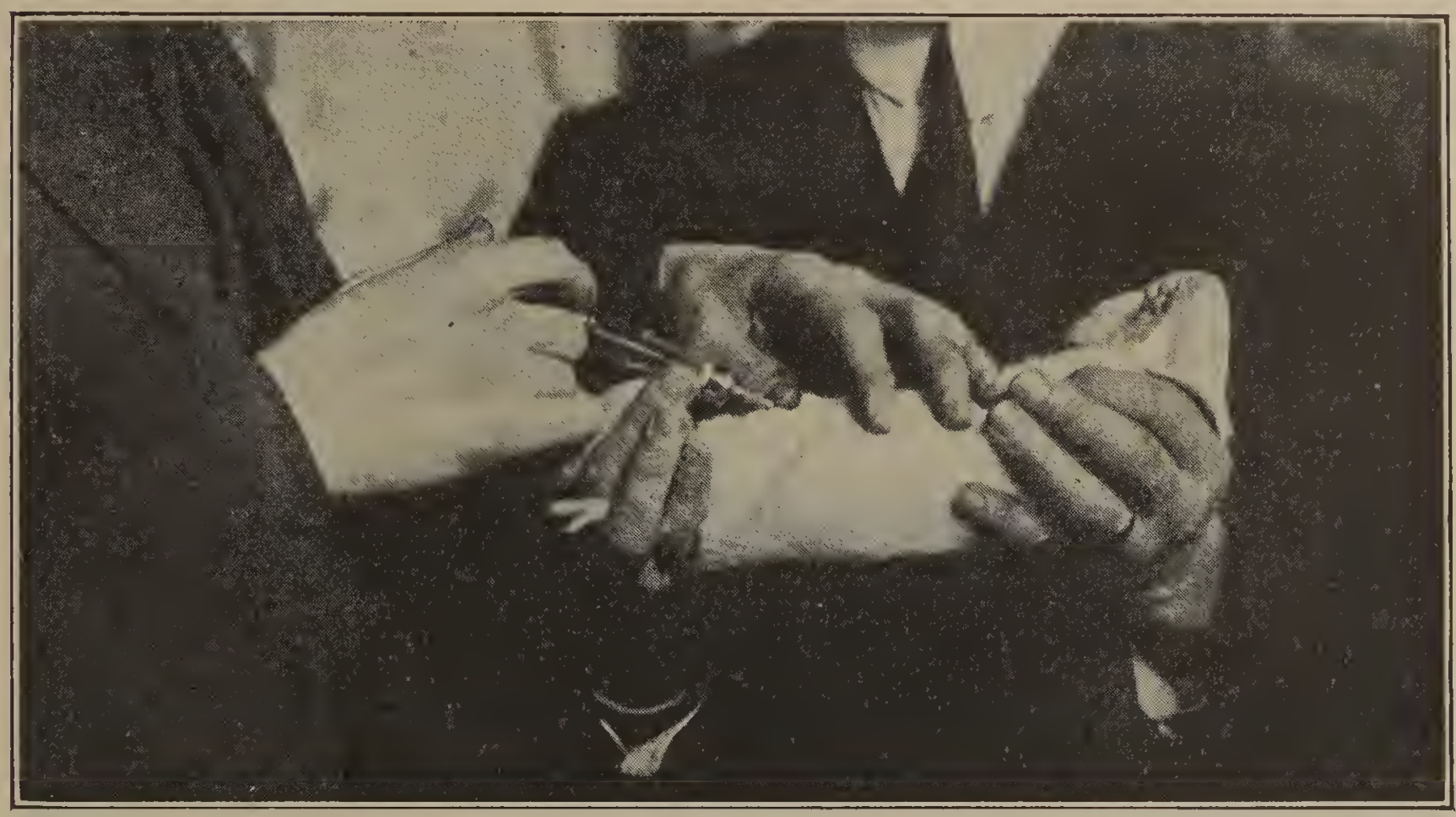

Fig. 51.-Intraperitoneal Inoculation of Guinea-Pig.

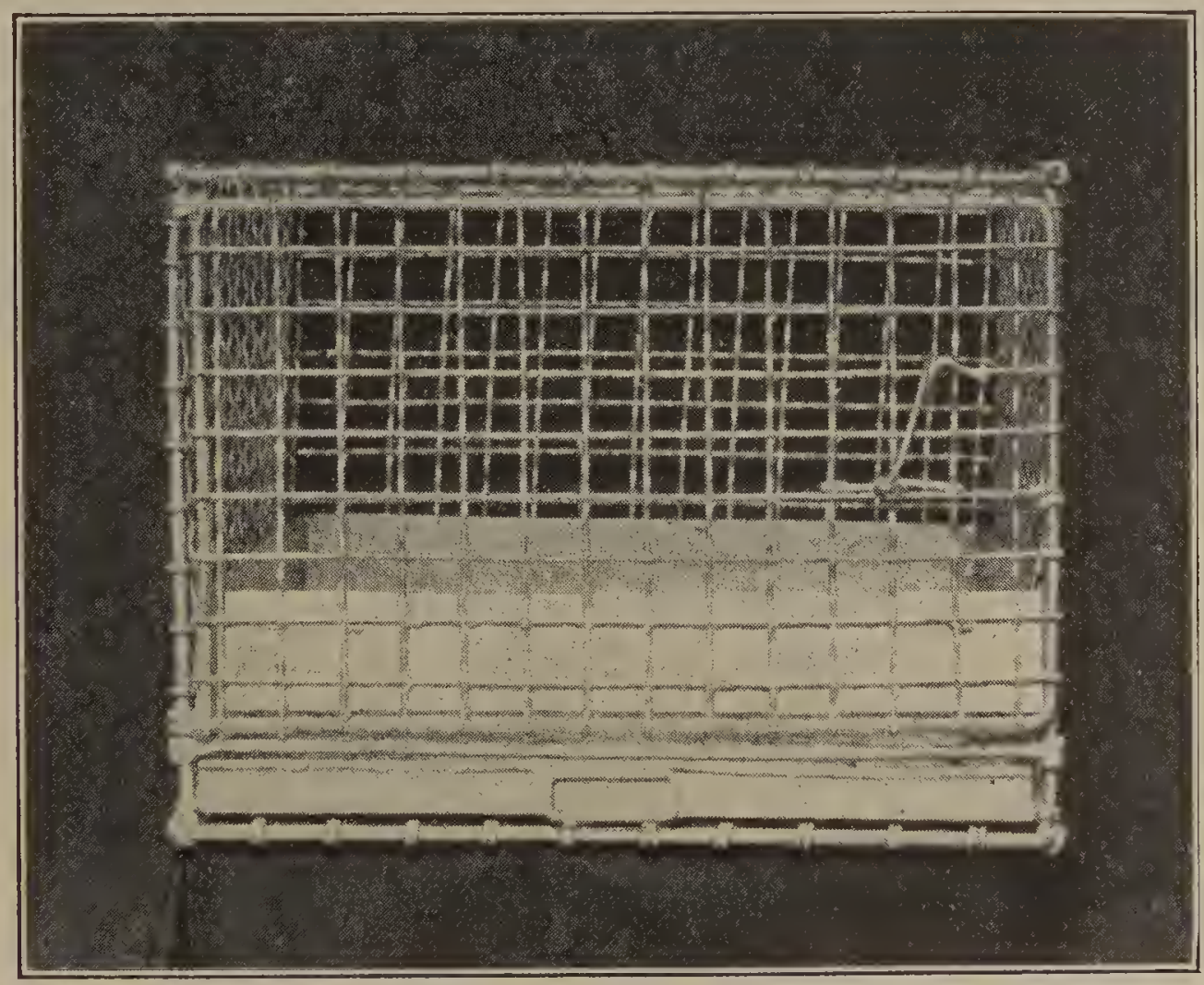

Fig. 52.-Guinea-Pig CAge.

inal wall, where the skin is thin. After clipping and sterilizing, the skiri is raised between the fingers of the left hand and the needle plunged 
in obliquely so as to avoid penetrating the abdominal wall and entering the peritoneum.

In making intraperitoneal inoculations, great care must be exercised not to puncture the gut. This can be avoided by passing the needle first through the skin in an oblique direction, then turning it into a position more vertical to the abdomen and perforating the muscles and peritoneum by a very short and carcfully executed stab.

Intravenous inoculations in rabbits are made into the veins running along the outer margins of the ears. The hair over the ear is clipped and the animal held for a short time head downward so that the vessels of the head may fill with blood. An assistant holds the animal firmly in

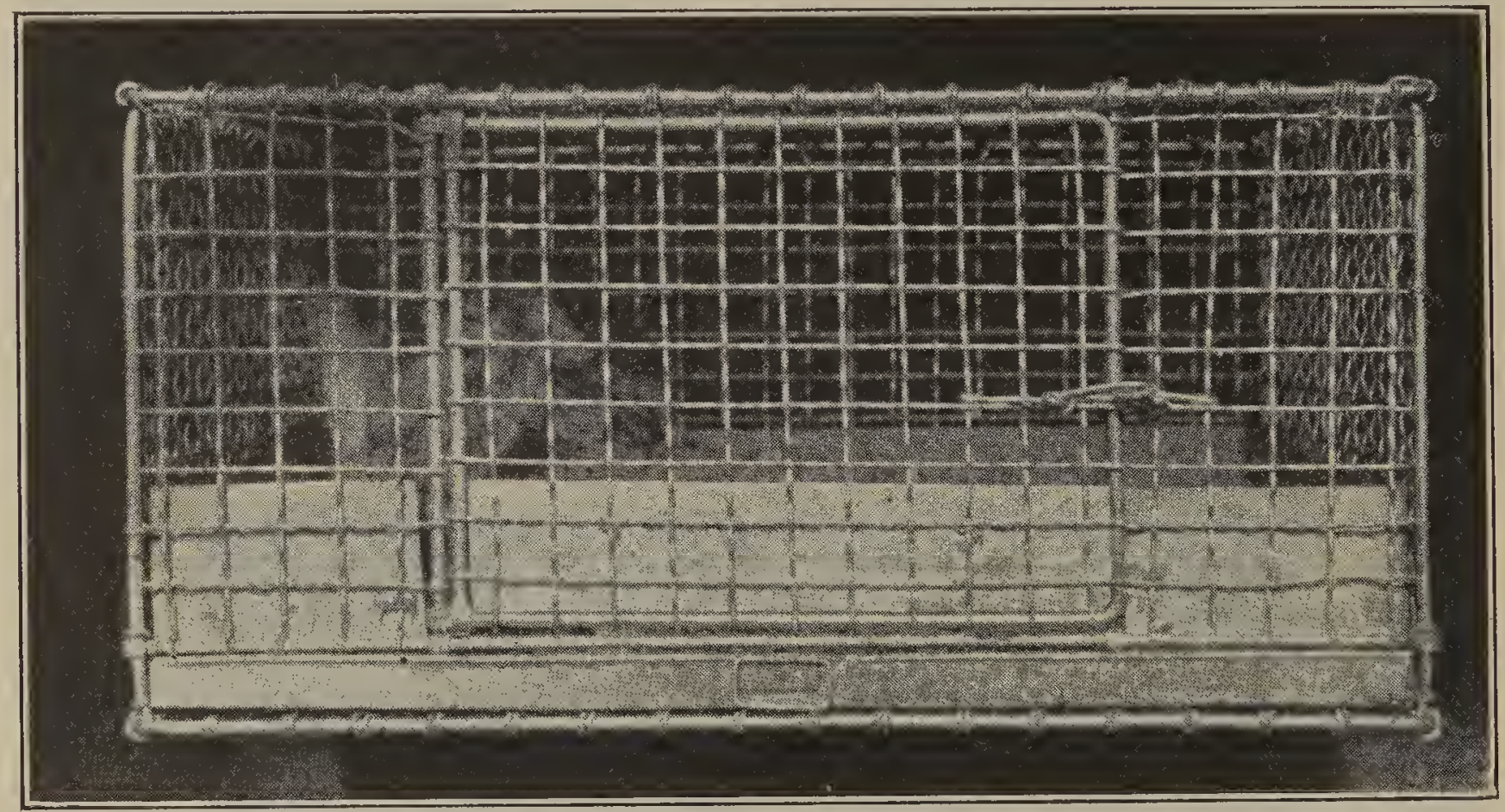

Fig. 53.-Rabrit Cage.

a horizontal position, the operator grasps the tip of the ears with the left hand, and carefully passes his needle into the vein in the direction as nearly as possible parallel to its course. (See Fig. 50.)

Mice are usually inoculated under the skin near the base of the tail. They may be placed in a jar over which a cover of stiff wire-gauze is held. They are then grasped by the tail, by which they are drawn up between the side of the jar and the edge of the wire cover, so that the lower end of the back shall be easily accessible. The skin is then wiped with a piece of cotton dipped in carbolic solution and the needle is inserted. Great care must be exercised to avoid passing the needle too close to the vertebral column. Mice are extremely delicate, and any injury to the spine usually causes immediate death. 
With proper care mice or rats may be easily injected intravenously if a sufficiently fine needle is used. There are four superficially placed veins running along the tail, which stand out prominently when rubbed with cotton moistened with xylol. Into these the injections are made.

When inoculating rats or guinea-pigs with bacillus pestis the Kolle vaccination method is used. The skin is merely shaved and a loopful of the culture vigorously rubbed into the shaven area.

The various forms of animal holders which have been devised are rarely necessary in bactcriological work unless working unassisted, immobilization of the animals being easily accomplished by the hands of a skilled assistant.

Autopsies upon infected animals must be carefully made. The animals are tied, back down, upon pans fitted in the corners with clamps for the strings. They are then moistened either with hot water or with a weak solution of carbolic acid, so that contamination by hair may be avoided. A median cut is made, the skin is carefully dissected back, and the body cavities are opened with sterile instruments. Cultures may then be taken from exudates, blood, or organs under precautions similar to those recommended below for similar procedures at autopsy upon man.

Inoculated animals should be, if possible, kept scparate from healthy animals. Rabbits and guinea-pigs are best kept in galvanized iron-wire cages, which are fitted with floor-pans that can be taken out and cleaned and sterilized. Mice may be kept in battery jars fitted with perforated metal covers. The mice should be supplied with large pieces of cotton upon batting since they are delicately susceptible to cold. 


\section{CHAP'T'ER X}

\section{THE BACTERIOLOGICAL EXAMINATION OF MATERIAL FROM PATIENTS}

IN making bacteriological examinations of material taken from living patients, or at autopsy, the validity of result is as fully dependent upon the technique by which the material is collected, as upon proper manipulation in the later stages of examination.

Material taken at autopsy should be, if possible, directly transferred from the cadaver to the proper culture media. If cultures are to be taken from the liver, spleen, or other organs, the surface of the organ should first be seared with a hot scalpel and an incision made through the capsule of the organ in the seared area, with the same instrument. The platinum needle can then be plunged through this incision and material for cultivation be taken with little chance of surface contamination. When blood is to be transferred from the heart, the heart muscle may be incised with a hot knife, or else the needle of a hypodermic syringe may be plunged through the previously seared heart muscle and the blood aspirated. The same end can be accomplished by means of a pointed, freshly prepared Pasteur pipette. In taking specimens of blood at autopsy it is safer to take them from the arm or leg, by allowing the blood to flow into a broad, deep cut made through the sterilized skin, than from the heart, since it has been found that post-mortem contamination of the hear't's blood takes place rapidly, probably through the large veins from the lungs. Exudates from the pleural cavities, the pericardium, or the peritoneum may be taken with a sterilized syringe or pipette.

Materials collected at the bedside or in the operating-room should be transferred directly to the proper media or else into sterile test tubes and so sent to the laboratory. When the material is scanty, it may be collected upon a sterile cotton swab, which should be immediately replaced in the sterilized containing tube and sent to the laboratory.

Syringes, when used for the collection of cxudates or blood, should be of some variety which is easily sterilizable by dry heat, or boiling. Most convenient of the forms in common use are the all-glass "Luer" syringe, or the cheaper "Sub-Q" model. Instruments which can be sterilized only by chemical disinfectants should not be used. When 
fluids are collected for bacteriological examination, such as spinal fluid, paracentesis fluid, or pleural exudate, it is convenient to have them taken directly into sterilized centrifuge tubes, since it is often necessary to concentrate cellular elements by centrifugalization. By immediate collection in these tubes, the danger of contamination is avoided.

Examination of Exudates.-Pus.-Pus should first be examined morphologically by some simple stain, such as gentian-violet, and by the Gram stain. It is convenient, also, to stain a specimen by Jenner's stain, in order to show clearly the relation of bacteria to the cells. Such morphological examination not only furnishes a guide to future manipulation, but supplies a control for the results obtained by cultural methods. Specimens of the pus are then transferred to the proper media, and pour-plates made or streaks made upon the surface of previously prepared agar or serum-agar plates.

A guide to the choice of media is often found in the result of the morphological examination. In most cases, it is well also to make anaërobic cultures by some simpler method. (See page 148 et seq.)

The colonies which develop upon the plates should be studied under the microscope, and specimens from the colonies transferred to cover-glasses and slides for morphological examination and to the various media for further growth and identification. Animal inoculation and agglutination tests must often also be resorted to. A knowledge of the source of the material may furnish considerable aid in making a bacteriological diagnosis, though great caution in depending upon such aid is recommended.

In the examination of peritoneal, pericardial, or pleural exudates it is often advantageous to use the sediment obtained by centrifugalization. A differential count of the cells present may be of aid in confirming the bacteriological findings. Morphological examination and cultural examination are made as in the case of pus. Specimens should also in these cases be stained for tubercle bacilli. Whenever morphological examinations of such fluids are negative, no bacteria being found, and especially when among the cellular elements the lymphocytes preponderate, the search for tubercle bacilli should be continued by means of animal inoculation. Guinea-pigs should be inoculated intraperitoneally from specimens of the fluid. The animals will usually die within six to eight weeks, but can be killed and examined at the end of about six weeks if they remain alive. The chances for a positive result are considerably increased if the fluid is set away in the ice-chest until a clot has formed and the animals are inoculated with the material from the broken-up clot 
The routine examination of spinal fluid is best made upon the sediment of centrifugalized specimens. The microorganisms with which we deal most frequently in this fluid are the meningococcus, the pneumococcus, the streptococcus, and the tubercle bacillus. If morphological examination reveals bacteria resembling the first three of these in appearance and staining-reaction, surface smears should preferably be made upon plates of serum agar, blood agar, or upon tubes of Loeffler's coagulated blood-serum. Failure to find organisms morphologically does not exclude their presence and careful cultivation should be done in all cases. When organisms are not found by simple morphological examination and the fluid and sediment are scanty, specimens should be stained by the Ziehl-Neelson method for tubercle bacilli. In such cases it is often of advantage to set away the specimen until a thin thread-like clot of fibrin has formed in the bottom of the tube. In smears of such a clot, tubercle bacilli are found with far greater ease than they are found in centrifugalized specimens. If these examinations are without result, inoculation of guinea-pigs should be resorted to.

Examination of Urine.-Bacteriological examination of the urine is of value only when specimens have been taken with sterile catheters, and care has been exercised in the disinfection of the external genitals. Many of the numerous finds of bacillus coli in urine are unquestionably due to defective methods of collecting material. Urine should be centrifugalized and the sediment examined morphologically and pourplates made and surface smears made upon the proper media. If necessary, animal inoculation may be done. In examining urine for tubercle bacilli, special care should be taken in staining methods so as to differentiate from Bacillus smegmatis.

Examination of Feces.-Human feces contain an enormous number of bacteria of many varieties. Klein, ${ }^{1}$ by special methods, estimated that there were about $75,000,000$ bacteria in one milligram of feces. It has been a noticeable result of all the investigations upon the feces, that although enormous numbers can be counted in morphological specimens, only a disproportionately smaller number can be cultivated from the same specimen. This is explicable upon the ground that special culture media are necessary for many of the species found in intestinal contents and upon the consideration that many of the bacteria which are present in the morphological specimen are dead, showing that there are bactericidal processes going on in some parts of the

${ }^{1}$ Klein, Ref. Cent. f. Bakt., I, xxx, 1901. 
intestinal tract, possibly through the agency of intestinal secretions, bile, and the action of the products of metabolism of the hardier species present. By far the greater part of the intestinal flora consists of members of the colon group, bacilli of the lactis aërogenes group, Bacillus fæcalis alkaligenes, Bacillus mesentericus, and relatively smaller numbers of streptococci, staphylococci, and Gram-positive anaërobes. Many other species, however, may be present without being necessarily considered of pathological significance. Certain writers have recently laid much stress upon a preponderance of Gram-positive bacteria in specimens of feces, claiming that such preponderance signifies some form of intestinal disturbance. Herter ${ }^{1}$ has recently advanced the opinion that the presence of Bacillus aërogenes capsulatus in the intestinal canal is definitely associated with pernicious anemia. The determination of these bacilli in the stools is made both by morphological examination by means of Gram stain and by isolation of the bacteria. Such isolation is easily done by the method of Welch and Nuttal. ${ }^{2}$ A suspension of small quantities of the feces in salt solution is made and 1 c.c. of the filtered suspension is injected into the ear vein of a rabbit. After a few minutes the rabbit is killed and placed in the incubator. After five hours of incubation, the rabbit is dissected, and if the Welch bacillus has been present in the feces, small bubbles of gas will have appeared in the liver from which the bacilli may be cultivated in anaërobic stabcultures.

Bacteriological examination of feces is most often undertaken for the isolation of Bacillus typhosus. This is accomplished with a great deal of difficulty because of the overwhelming numbers of colon bacilli which easily outgrow the typhoid germs, and because of the similarity of their colonies in most media. Many methods have been devised for this purpose, all of which depend upon the use of special media aimed at the inhibition of colon and other bacilli and the production of recognizable differences in the colonies of typhoid and colon bacilli. Such media are those of Elsner, Hiss, Conradi-Drigalski, Loeffler, Hesse, and others, which are described in the section upon special media. (See page 133.) The methods of using these media will be found described in the chapter on Bacillus typhosus (p. 399.)

Cholera spirilla may be recognized in and isolated from the stools of patients by morphological examination, and by cultivation. (See section on Sp. choleræ.)

${ }^{1}$ Herter, "Common Bacterial Infections of the Digestive Tract," N. Y., 1907.

${ }^{2}$ Welch and Nuttal. See ref. p. 469. 
For the isolation of dysentery bacilli from feces, no satisfactory special methods have as yet been devised, Here we can depend only upon careful plating upon agar and gelatin and extended colony "fishing," and the study of pure cultures. The complete absence of motility of these bacteria is of much aid in such identification.

The determination of tubercle bacilli in stools is difficult and of questionable significance, in that they may be present in people suffering from pulmonary tuberculosis as a consequence of swallowing sputum or infected food, and in that there may be other acid-fast bacilli, such as the timothy bacillus, present.

Blood Cultures.-The diagnosis of septicemia can be positively made during life only by the isolation of bacteria from the blood. Such examinations are of much value and are usually successful if the technique is properly carried out. A large number of methods are recommended, the writers giving, however, only the one which they have found successful and simple for general use.

The blood is taken by preference from the median basilic vein of the arm. Jf, for some reason (both forearms having been used for saline infusion), these veins are unavailable, blood may be taken from the internal saphenous vein as it turns over the internal malleolus of the ankle joint. The skin over the vein should be prepared, preferably an hour before the specimen is taken, with green soap, alcohol, and bichlorid of mercury, as for a surgical operation. The syringe which is used should be of some sterilizable variety (the most convenient the Luer model), which is easily manipulated and does not draw with a jerky, irregular motion. Its capacity should be at least 10 c.c. It may be sterilized by boiling for half an hour, or preferably, when all-glass syringes are used, they may be inserted into potato-tubes and sterilized at high temperature in the hot-air chamber. Before drawing the blood, a linen bandage is wound tightly about the upper arm of the patient in order to cause the veins to stand out prominently. When the veins are plainly in view, the needle may be plunged through the skin into the vein in a direction parallel to the vessel and in the direction of the blood-stream. After perforation of the skin, while the needle is groping for the vein, gentle suction may be exerted with the piston. Great care should be exercised, however, that the piston is not allowed to slip back, and air be, by accident, forced into the vessel. In most cases no suction is necessary, the pressure of the blood being sufficient to push up the piston. After the blood has been drawn, it should be immediately transferred to the proper media. Epstein has recently recommended the mixture of the blood 
with sterile two per cent ammonium oxalate solution in test tubes, by which means the clotting is prevented, and transfers can be made more leisurely to culture media. While this method is convenient in cases where blood must be taken at some distance from the laboratory, it is

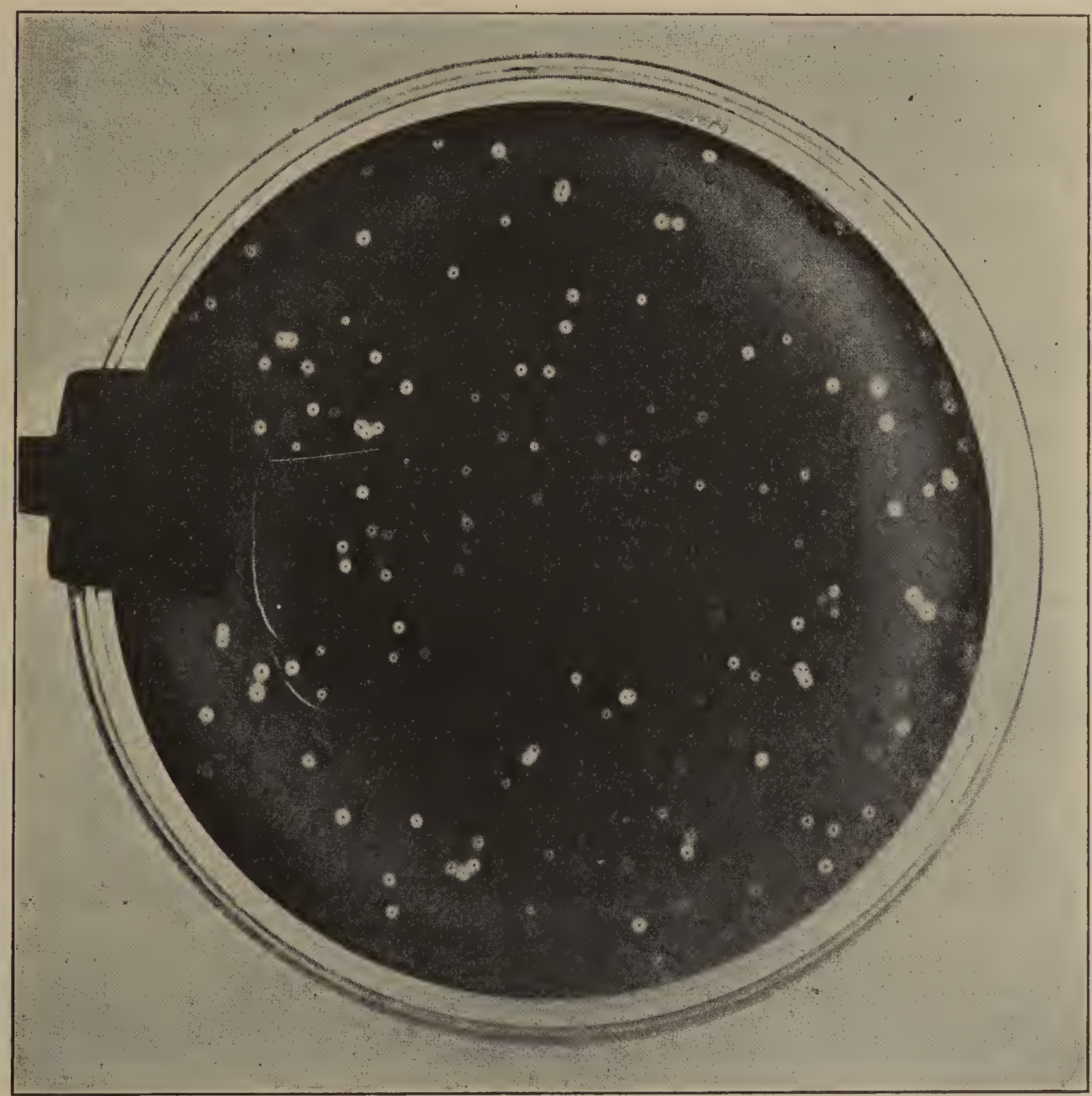

Fig. 54.-Blood-Culture Plate Showing Streptococcus Colonies. Note halo of hemolysis about each colony.

preferable, whenever possible, to make cultures from the blood immediately at the bedside.

The choice of culture media for blood cultures should, to a certain extent, be adapted to each individual case. For routine work, it is best to employ glucose-meat-infusion agar and glucose-meat-infusion broth. At least six glucose-agar tubes should be melted and immersed in water at $45^{\circ} \mathrm{C}$. Before the blood is mixed with the medium, the agar should be 
cooled to $41^{\circ}$ in order that bacteria, if present, may not be injured by the heat. The blood is added to the tubes in varying quantities, ranging from 0.25 to 1 c.c. each, in order that different degrees of concentration may be obtained. Mixing is accomplished by the usual dipping and rotary motion, the formation of air-bubbles being thus avoided. The mouth of each test tube should be passed through the flame before pouring the contents into the plates. Three flasks of glucose broth, containing 100 to 150 c.c. of fluid each, should be inoculated with varying quantities of blood-at least one of the flasks containing the blood in high dilution. The most stringent care in the withdrawal and replacement of the cotton stoppers should be exercised. ${ }^{1}$ The writers have found it convenient to use, in place of one of these flasks, one containing, in addition to the glucose, $1 \mathrm{gm}$. of powdered calcium carbonate. This insures neutrality, permitting pneumococci or streptococci, which are sensitive to acid, to develop and retain their vitality.

In making blood cultures from typhoid patients, various methods have been recommended. Buxton and Coleman ${ }^{2}$ have obtained excellent results by the use of pure ox-bile containing ten per cent of glycerin and two per cent of peptone in flasks. The writers have had no difficulty in obtaining typhoid cultures by the use of slightly acid meat-extract broth in flasks containing 200 or more c.c. to which comparatively little blood has been transferred in order to insure high dilution.

In estimating the results of a blood culture, the exclusion of contamination usually offers little difficulty. If the same microorganism appears in several of the plates and flasks, if colonies upon the plates are well distributed within the center and under the surface of the medium, and if the microorganisms themselver belong to species which commonly cause septicemia, such as streptococcus and pneumococcus, it is usually safe to assume that they have emanated from the patient's circulation. When colonies are present in one plate or in one flask only, when they are situated only near the edges of a plate or upon the surface of the medium, and when they belong to varieties which are often found saprophytic upon the skin or in the air, they must be looked upon with extreme suspicion. It is a good rule to look upon all staphylococcus blood cultures skeptically, discarding Staphylococcus albus as a contamination, and taking, if possible, another corroborative culture when the organism is Staphylococcus pyogenes aureus.

3 Small Florence flasks are preferable to the Erlenmeyer flasks usually employed.

${ }^{2}$ Buxton and Coleman, Am. Jour. of Med. Sci., 1907. 


\section{SECTION II}

\section{INFECTION AND IMMUNITY}

\section{CHAPTER XI}

\section{FUNDAMENTAL FACTORS OF PATHOGENICITY AND INFECTION}

When microorganisms gain entrance to the animal or human body: and give rise to disease, the process is spoken of as infection.

Bacteria are ever present in the environment of animals and human beings and some find constant lodgment on various parts of the body. The mouth, the nasal passages, the skin, the upper respiratory tract, the conjunctivæ, the ducts of the genital system, and the intestines are invairiably inhabited by numerous species of bacteria, which, while subject to no absolute constancy, conform to more or less definite characteristics of species distribution for each locality. Thus the colon organisms are invariably present in the normal bowel, Döderlein's bacillus in the vagina, Bacillus xerosis in many normal conjunctivæ, and staphylococcus, streptococcus, various spirilla, and pneumococcus in the mouth. In contact, therefore, with the bodies of animals and man, there is a large flora of microorganisms, some as constant parasites, others as transient invaders; some harmless saprophytes and others capable of becoming pathogenic. It is evident, therefore, that the production of an infection must depend upon other influences than the mere presence of the microcrganisms and their contact with the body, and that the occurrence of the reaction-for the phenomena of infection are in truth reactions between the germ and the body defenses-is governed by a number of important secondary factors.

In order to cause infection, it is necessary that the bacteria shall gain entrance to the body by a path adapted to their own respective cultural requirements, and shall be permitted to proliferate after gaining a foothold. Some of the bacteria then cause disease by rapid multiplication, progressively invading more and more extensive areas of the animal tissues, while others may remain localized at the point of invasion and 
exert their harmful action chiefly by local growth and the elaboration of specific poisons.

The inciting or inhibiting factors which permit or prohibit an infection are dependent in part upon the nature of the invading germ and in part upon the conditions of the defensive mechanism of the subject attacked.

Bacteria are roughly divided into two classes, saprophytes and parasites. The saprophytes are those bacteria which thrive best on dead organic matter and fulfill the enormously important function in nature of reducing by their physiological activities the excreta and dead bodies of more highly organized forms into those simple chemical substances which may again be utilized by the plants in their constructive processes. The saprophytes, thus, are of extreme importance in maintaining the chemical balance between the animal and plant kingdoms. Parasites, on the other hand, find the most favorable conditions for their development upon the living bodies of higher forms.

While a strict separation of the two divisions can not be made, numerous species forming transitions between the two, it may be said that the latter class comprises most of the so-called pathogenie or disease-producing bacteria. Strict saprophytes may cause disease, but only in cases where other factors have brought about the death of some part of the tissues, and the bacteria invade the necrotic areas and break down the proteids into poisonous chemical substances such as ptomains, or through their own destruction give rise to the liberation of toxic constituents of their bodies. It is necessary, therefore, that bacteria, in order to incite disease, should belong strictly or facultatively to the class known as parasitic. It must not be forgotten, however, that the terms are relative, and that bacteria ordinarily saprophytic may develop parasitic and pathogenic powers when the resisting forces of the invaded subject are reduced to a minimum by chronic constitutional disease or other causes.

Organisms that are parasitic, however, are not necessarily pathoge_ic, and there are certain more or less fundamental requirements which experience has taught us must be met by an organism in order that it may be infectious (or pathogenic) for any given animal; and by infectiousness is meant the ability of an organism to live and multiply in the animal fluids and tissues. For instance, an organism which is shown not to grow at the body temperature of warm-blooded animals may safely be assumed not to be infectious for such animals; and experience is 
gradually teaching us that strictly aërobic organisms, those thriving only in the presence of free oxygen and not able to obtain this gas in available combination from carbohydrates, can also be safely excluded from the infectious class. We have also learned that anaërobic organisms, although infectious when gaining entrance to tissues not abundantly supplied with blood, are practically unable to multiply in the blood stream and give rise to generalized infection.

The pathogenic microorganisms differ very much among themselves in the degree of their disease-inciting power. Such power is known as virulence. Variations in virulence occur, not only among different species of pathogenic bacteria, but may occur within the same species. Pneumococci, for instance, which have been kept upon artificial media or in other unfavorable environment for some time, exhibit less virulence than when freshly isolated from the bodies of man or animals. It is necessary, therefore, in order to produce infection, that the particular bacterium involved shall possess sufficient virulence.

Whether or not infection occurs depends also upon the number of bacteria which gain entrance to the animal tissues. A small number of bacteria, even though of proper species and of sufficient virulence, may easily be overcome by the first onslaught of the defensive forces of the body. Bacteria, therefore, must be in sufficient number to overcome local defenses and to gain a definite foothold and carry on their life processes, before they can give rise to an infection. The more virulent the germ, other conditions being equal, the smaller the number necessary for the production of disease. The introduction of a single individual of the anthrax species, it is claimed, is often sufficient to cause fatal infection; while forms less well adapted to the parasitic mode of life will gain a foothold in the animal body only after the introduction of large numbers.

The Path of Infection.-The portal by which bacteria gain entrance to the human body is of great importance in determining whether or not disease shall occur. Typhoid bacilli rubbed into the abraded skin may give rise to no reaction of importance, while the same microorganism, if swallowed, may cause fatal infection. Conversely, virulent streptococci, when swallowed, may cause no harmful effects, while the same bacteria rubbed into the skin may give rise to a severe reaction.

Animals and man are protected against invasion by bacteria in various ways. Externally the body is guarded by its coverings of skin and mucous membranes. When these are healthy and undisturbed, microorganisms are usually held at bay. While this is true in a general way bucteria may in occasional cases pass through uninjured 
skin and mucosa. Thus the Austrian Plague Commission found that guinea-pigs could be infected when plague bacilli were rubbed into the shaven skin, and there can hardly be much doubt of the fact that tubercle bacilli may occasionally pass through the intestinal mucosa into the lymphatics without causing local lesions.

Even after bacteria of a pathogenic species, in large numbers and of adequate virulence, have passed through a locally undefended area in the skin or mucosa of an animal or a human being by a path most favorably adapted to them, it is by no means certain that an infection will take place. The bodies of animals and of man have, as we shall see, at their disposal certain general, systemic weapons of defense, both in the blood serum and the cellular elements of blood and tissues which, if normally vigorous and active, will usually overcome a certain number of the invading bacteria. If these defenses are abnormally depressed, or the invading microorganisms are disproportionately virulent or plentiful, infection takes place.

Bacteria, after gaining an entrance to the body, may give rise merely to local inflammation, necrosis, and abscess formation. They may, on the other hand, from the local lesion, gain entrance into the lymphatics and blood-vessels and be carried freely into the circulation, where, if they survive, the resulting condition is known as bacteriemia or septicemia. Carried by the blood to other parts of the body, they may, under favorable circumstances, gain foothold in various organs and give rise to secondary foci of inflammation, necrosis, and abscess formation. Such a condition is known as pyemia. The disease processes arising as the result of bacterial invasion may depend wholly or in part upon the mechanical injury produced by the process of inflammation, the disturbance of function caused by the presence of the bacteria in the capillaries and tissue spaces, and the absorption of the necrotic products resulting from the reaction between the body cells and the microorganisms. To a large extent, however, infectious diseases are characterized by the symptoms resulting from the absorption or diffusion of the poisons produced by the bacteria themselves.

Bacterial Poisons.- It was plain, even to the earliest students of this subject, that mere mechanical capillary obstruction or the absorption of the products of a local inflammation were insufficient to explain the profound systemic disturbances which accompany many bacterial infections. The very nature of bacterial disease, therefore, suggested the presence of poisons.

It was in his investigations into the nature of these poisons that 
Brieger ${ }^{1}$ was led to the discovery of the ptomains. These bodies, first isolated by him from decomposing beef, fish, and human cadavers, have found more extended discussion in another section. Accurately classified, they are not true bacterial poisons in the sense in which the term is now employed. Although it is true that they are produced from proteid material by bacterial action, they are cleavage products derived from the culture medium upon the composition of which their nature intimately depends. The bacterial poisons proper, on the other hand, are specific products of the bacteria themselves, dependent upon the nature of the medium only as it favors or retards the full development of the physiological functions of the microorganisms. The poisons, produced to a greater or lesser extent by all pathogenic microorganisms, may be of several kinds. The true toxins, in the specialized meaning which the term has acquired, are soluble, truly secretory products of the bacterial cells, passing from them into the culture medium during their life. They may be obtained free from the bacteria by filtration and in a purer state from the filtrates by chemical precipitation and a variety of other methods. The most important examples of such poisons are those elaborated by Bacillus diphtheriæ and Bacillus tetani. If cultures of these bacteria or of others of this class are grown in fluid media for several days and the medium is then filtered through porcelain candles, the filtrate will be found toxic often to a high degree, while the residue will be either inactive or comparatively weak. Moreover, if the residue possesses any toxicity at all, the symptoms evidencing this will be different from those produced by the filtrate.

There are other microorganisms, however, notably the cholera spirillum and the typhoid bacillus, which act in an almost diametrically opposed manner. If these bacteria are cultivated and separated from the culture fluid by filtration in the method described above, the fluid filtrate will be toxic to only a very slight degree, whereas the residue may prove very poisonous. In these cases, we are dealing, evidently, with poisons not secreted into the medium by the bacteria, but rather attached more or less firmly to the bacterial body. Such poisons, separable from the bacteria only after death by some method of extraction, or by autolysis, are termed endotoxins. The greater number of the pathogenic bacteria seem to act chiefly by means of poisons of this class. The first to call attention to the existence of such intracellular poisons was Buchner, who formulated his conclusions from the results of ex- 
periments made with a number of microorganisms, notably the Friedländer bacillus and Staphylococcus pyogenes aureus, with dead cultures of which he induced the formation of sterile abcesses in animals and symptoms of toxemia. The conception of "endotoxins," subsequently, however, received its clearest and most definite expression in the work of Pfeiffer ${ }^{1}$ on cholera poison.

Some clarity of conception, based on visual perception, may possibly be gained by comparing some of the products of pathogenic bacteria with bacterial pigments and with insoluble interstitial or intercellular substance, which may be seen accompanying bacteria in cover-glass preparations. Soluble toxic secretions are to be compared to such pigments as the pyocyanin of Bacillus pyocyaneus, which is so readily soluble in culture media; endotoxins proper, to pigments confined to the bacterial cell, or at least, when secreted, being insoluble in culture media, such for instance as the well-known red pigment of Bacillus prodigiosus, which may often be seen free among the bacteria in irregular red granules like carmine powder. That bodies such as this latter might be extruded from pathogenic bacteria and not be soluble in the usual culture fluids, is not improbable, and the fact that more or less insoluble interstitial substances are not infrequent among bacteria is well known.

In all bacterial bodies, after removal of toxins and endotoxins, a certain proteid residue remains which, if injected into animals, may give rise to localized lesions such as abscesses or merely slight temporary inflammations. The nature of this residue has been carefully studied, especially by Buchner, who has named it bacterial protein and he believes the substance to be approximately the same in all bacteria, without specific toxic action, but with a general ability to exert a positive chemotactic effect on the white blood cells, thereby causing the formation of pus. The nature of the bacterial proteins is by no means clear, and it is still in doubt whether the separation of these substances from the endotoxins can be upheld.

A number of bacteria may give rise to both varieties of poisons. Thus, recently, Kraus has claimed the discovery of a soluble toxin for the cholera spirillum and Doerr for the dysentery bacillus, both of which microorganisms were regarded as being purely of the endotoxin-producing type.

It is plain, moreover, that occasionally it may be very difficult to distinguish between a soluble toxin and an endotoxin. In the filtration

${ }^{1}$ Pfeiffer, Zeit. f. Hyg., xl, 1892. 
experiment recorded above, it might well be claimed that the toxicity of the filtrate, when not very strong, may depend upon an extraction of endotoxins from the bodies of the bacteria by the medium. The final test, in such instances, lies in the power of true toxins to stimulate in animals the production of antitoxins; for, as we shall see later, the injection of true soluble toxins into animals gives rise to antitoxins, whereas the formation of such neutralizing bodies in the serum or plasma does not, it is claimed, follow the injection of endotoxins. This distinction will become clearer as we proceed in the discussion of immunity. It must not be forgotten, however, that our knowledge of bacterial poisons is by no means complete, and that sharp distinctions as those given above must be regarded to a certain extent as tentative.

In resistance to chemical action and heat, the various poisons show widely divergent properties. As a general rule, most true soluble toxins are delicately thermolabile, they are destroyed by moderate heating, and deteriorate easily on standing. Their chemical nature is by no means clear, but, on precipitation of toxic solutions with magnesium sulphate, these poisons come down together with the globulins. The nature of the endotoxins is still less clearly understood. Most of them, while less labile than the extracellular poisons, are, nevertheless, destroyed by exposure to $70^{\circ} \mathrm{C}$. On the other hand, certain specific and powerful intracellular poisons, like those of the Gärtner bacillus of meat poisoning, may undergo exposure to even $100^{\circ} \mathrm{C}$. and still retain their toxic properties. The nature of each individual poison will be discussed in connection with its microorganism.

The Mode of Action of Bacterial Poisons.-Close study of the toxic products of various microorganisms has shown that many of the bacterial poisons possess a more or less definite selective action upon special tissues and organs. Thus, certain soluble toxins of the tetanus bacillus and Bacillus botulinus attack specifically the nervous system. Again, certain poisons elaborated by the staphylococci, the tetanus bacillus, the streptococci, and other germs, the so-called "hemolysins," attack primarily the red blood corpuscles. Other poisons again act on the white blood corpuscles; in short, the characteristic affinity of specific bacterial poisons for certain organs is a widely recognized fact.

In explanation of this, behavior, much aid has been given by the researches of Meyer, ${ }^{1}$ Overton, ${ }^{2}$ Ehrlich, ${ }^{3}$ and others upon the causes for

1 Meyer, Arch. f. exper. Pathol., 1899, 1901.

2 Overton, "Studien üb. d. Narkose," Jena, 1901.

3 Ehrlich, "Sauerstoffs-Bed ürnniss des Organismus," Berlin, 1885. 
the analogous selective behavior of various narcotics and alkaloids. It seems probable, from the researches of these men, that the selective action of poisons depends upon the ability, chemical or physical or both, of the poisons to enter into combination with the specifically affected cells. From the nature of the combinations formed, it seems not unlikely that the physical factors, such as solubility in the cell plasma, may also play an important part.

Observations of a more purely bacteriological nature have tended to bear out these conclusions. Wassermann and Takaki, ${ }^{1}$ for instance, have shown that tetanus toxin, which specifically attacks the nervous system, may be removed from solution by the addition of brain substance. Removal of the brain tissue by centrifugation leaves the solution free from toxin. In the same way it has been shown that hemolytic poisons can be removed from solutions by contact with red blood cells, but only when the red blood cells of susceptible species are employed.

Similar observations have been made in the case of leukocidin, a bacterial poison acting upon the white blood cells specifically. ${ }^{2}$

That bacterial poisons injected into susceptible animals rapidly disappear from the circulation is a fact which bears out the view that a combination between affected tissie and toxin must take place. Dönitz, ${ }^{3}$ for instance, has shown that within four to eight minutes after the injections of certain toxins, considerable quantities will have disappeared from the sirculation. Conversely, Metchnikoff ${ }^{4}$ has observed that tetanus toxin injected into insusceptible animals (lizards) may be detected in the blood stream for as long as two months after administration.

${ }^{1}$ Wassermann und Takaki, Berl. klin. Woch., 1898.

2 Sachs, Hofmeister's Beiträge, 11, 1902.

3 Dönitz, Deut. med. Woch., 1897.

- Metchnikoff, "L'immunité dans les malad. infect." 


\section{CHAP'TER XII}

\section{DEFENSIVE FACTORS OF THE ANIMAL ORGANISM}

\section{GENERAL CONSIDERATIONS}

WE have seen that the mere entrance of a pathogenic microorganism into the human or animal body through a breach in the continuity of the mechanical defenses of skin or mucosa does not necessarily lead to the development of an infection. The opportunities for such an invasion are so numerous, and the contact of members of the animal kingdom with the germs of disease is so constant, that if this were the case, sooner or later all would succumb. It is plain, therefore, that the animal body must possess mor subtle means of defense, by virtue of which pathogenic germs are, even after their entrance into the tissues and fluids, disposed of, or at least prevented from proliferating and elaborating their poisons. The power which enables the body to accomplish this is spoken of as resistance. When this resistance, which in some degree is common to all members of the animal kingdom, is especially marked, it is spoken of as "immunity."

From this it follows naturally that the terms resistance and immunity, as well as their converse, susceptibility, are relative and not absolute terms. Degrees of resistance exist, which are determined to a certain extent by individual, racial, or species peculiarities; and persons or animals are spoken of as immune when they are unaffected by an exposure or an inoculation to which the normal average individual of the same species would ordinarily succumb. The word does not imply, however, that these individuals could not be infected with unusually virulent or large doses, or under particularly unfavorable circumstances. Thus, birds, while immune against the ordinary dangers of tetanus bacilli, may be killed by experimental inoculations with very large doses of tetanus toxin. ${ }^{1}$ Similarly, Pasteur rendered naturally immune hens susceptible to anthrax by cooling them to a subnormal temperature, and Canalis and Morpurgo did the same with doves by subjecting them to starvation.

1 Quoted from Abel, Kolle und Wassermann, "Handbuch," etc. 
Absolute immunity is exceedingly rare. The entire insusceptibility of cold-blooded animals (frogs and turtles) under normal conditions to inoculation with even the largest doses of many of the bacteria pathogenic for warm-blooded animals, and the immunity of all the lower animals against leprosy, are among the few instances of absolute immunity known. ${ }^{1}$ Apart from such exceptional cases, however, resistance, immunity, and susceptibility must be regarded as purely relative terms.

The power of resisting any specific infection may be the natural heritage of a race or species, and is then spoken of as natural immunity. It may, on the other hand, be acquired either accidentally or artificially by a member of an ordinarily susceptible species, and is then called acquired immunity.

Natural Immunity. - SPECIEs ImmUnITY. - It is well known that many of the infectious diseases which commonly affect man, do not, so far as we know, occur spontaneously in animals. Thus, infection with B. typhosus, the vibrio of cholera, or the meningococcus occurs in animals only after experimental inoculation. Gonorrheal and syphilitic infection, furthermore, not only does not occur spontaneously, but is produced experimentally in animals with the greatest difficulty-the consequent diseases being incomparably milder than those occurring in man. Other diseases, like leprosy, influenza, and the exanthemata, ${ }^{2}$ have never been successfully transmitted to animals.

Conversely, there are diseases among animals which do not spontaneously attack man. Thus, human beings enjoy immunity against Rinderpest, and, to a lesser degree, against chicken cholera.

Among animal species themselves great differences in susceptibility and resistance toward the various infections exist. Often-quoted examples of this are the remarkable resistance to anthrax of rats and dogs, and the immunity of the common fowl against tetanus.

The factors which determine these differences of susceptibility and resistance among the various species are not clearly understood. It has been suggested that diet in some instances may influence these relations, inasmuch as carnivorous animals are often highly resistant to glanders, anthrax, and even tuberculous infections, to which herbivorous animals are markedly susceptible. ${ }^{3}$ It is likely, too, that the great differences between animals of various species in their metabolism, temperature, etc., may call for special cultural adaptation on the part

Lubarsch, Zeit. f. klin. Mediz., xix.

With the possible exception of smallpox.

${ }^{3}$ Hahn, in Kolle und Wassermann, vol. iv. 
of the bacteria. The fact that the bacillus of avian tuberculosiswhose natural host has a normal body temperature of $40^{\circ} \mathrm{C}$. and above -will grow on culture media at 40 to $50^{\circ} \mathrm{C}$., whereas B. tuberculosis of man can not be cultivated at a temperature above $40^{\circ} \mathrm{C}$., would seem to lend some support to this view. The difference between warmand cold-blooded animals has already been noted. The necessity for cultural adaptation, too, would seem to be borne out by the great enhancement observed in the virulence of certain microorganisms for a given species after repeated passage through individuals of this species.

Racial Immunity.-Just as differences in susceptibility and immunity exist among the various animal species, so the separate races or varieties within the same species may display differences in their reactions toward pathogenic germs. Algerian sheep, for instance, show a much higher resistance to anthrax than do our own domestic sheep, and the various races of mice differ in their susceptibility to anthrax and to glanders.

Similar racial differences are common among human beings. As a general rule, it may be said that a race among whom a certain disease has been endemic for many ages is less susceptible to this disease than are other races among whom it has been more recently introduced. The appalling ravages of tuberculosis among negroes, American Indians, and Esquimaux, bear striking witness to this fact. Conversely, the comparative immunity of the negro from yellow fever, a disease of the greatest virulence for Caucasians, furnishes further evidence in favor of this opinion. It must not be forgotten, however, in judging of these relations, that the great differences in the customs of personal and social hygiene existing among the various races may considerably affect the transmission of disease and lead to false conclusions.

In so far as the statement made above is true, however, it seems to indicate that the endemic diseases have carried in their train a certain degree of inherited immunity.

In other cases ${ }^{1}$-as in the instance of the malaria-immunity of negroes-the resistance seems to be acquired rather than inherited, for, as Hirsch was first to note, death from this disease occurred frequently among the children, while adult negroes were rarely attacked.

Differences in Individual Resistance.-In bacteriological experimentation with smaller test animals, a direct ratio may often exist between body weight and dosage in determining the outcome of an

: Hahn, in Kolle und Wassermann, loc. cit. 
infection, provided the mode of inoculation has been the same and the virulence of the germ not excessive. It would seem, therefore, that among these animals the difference in resistance in the face of an artificial infection between individuals of the same race is very slight.

In higher animals, however, especially in the case of man, the existence of such apparent individual differences is a well-established fact, although in judging of them we must not forget that the conditions of infection are not subject to the uniformity and control which animal experimentation permits. Of a number of persons exposed to any given infection there are always some who are entirely unaffected and there are great variations in the severity of the disease in those who are attacked. In the absence of positive evidence in support of the direct inheritance of this individual immunity, the most reasonable explanation for such differences in resistance seems to lie in attributing them to individual variations in metabolism or body chemistry. Depressions, for instance, in the acidity of the gastric secretion would predispose to certain infections of gastro-intestinal origin. Anatomical differences, too, may possibly influence resistance. Thus, Birch-Hirschfeld believed that certain anomalous arrangements of the bronchial tubes predisposed to tuberculosis.

Instances of transient susceptibility induced by physical or mental overwork, starvation, etc., should hardly be classified under this heading, since the conditions in such cases correspond simply to experimental depression of natural species or race resistance.

Acquired Immunity.- It is a matter of common experience that many of the infectious diseases occur but once in the same individual. This is notably the case with typhoid fever, yellow fever, and most of the exanthemata, and is too general an observation to require extensive illustration. A single attack of any of the diseases of this class alters in some way the resistance of the individual so that further exposure to the infective agent is usually without menace, either for a limited period after the attack, or for life. Resistance acquired in this way is often spoken of as acquired immunity.

The protection conferred by certain diseases against further attack was recognized many centuries ago, and there are records which show that attempts were made in ancient China and India to inoculate healthy individuals with pus from small-pox pustules in the hope of producing by this process a mild form of the disease and its consequent immunity.

Pasteur, before all others, thought philosophically about the phenomena of acquired immunity, and, with adequate knowledge, realized the 
possibility of artifically bestowing immunity without inflicting the dangers of the fully potent infective agent. The first observation which, made by him purely accidentally, inspired the hope of the achievement of such a result, occuired during his experiments with chicken cholera. The failure of animals to die after inoculation with an old culture of the bacilli of chicken cholera, fully potent but a few weeks previously, pointed to the attenuation of these bacilli by their prolonged cultivation without transplantation. With this observation as a point of departure he carried out a series of investigations with the purpose of discovering a method of so weakening or attenuating various incitants of disease that they could be introduced into susceptible individuals without endangering life and yet without losing their property of conferring protection. The brilliant results achieved by Jenner, many years before, in protecting against smallpox by inoculating with the entirely innocuous products of the pustules of cowpox furnished an analogy which gave much encouraging support to this prospect.

The experimental work which Pasteur carried out to solve this problem not only reaped a rich harvest of facts, but gave to science the first and brilliant examples of the application of exact laboratory methods to problems of immunity.

\section{ACTIVE IM MUNITY}

Active Artificial Immunity.-The process of conferring protection by treatment with either an attenuated form or a sublethal quantity of the infectious agent of a disease, or its products, is spoken of as "active immunization."

Whatever the method employed, the immunized individuals gain their power of resistance by the unaided reactions of their own tissues. They themselves take an active physiological part in the acquisition of this new property of immunity. For this reason, Ehrlich has aptly termed these processes "active immunization."

There are various methods by which this can be accomplished, all of which were, in actual application or in principle, discovered by Pasteur and his associates, and can be best reviewed by a study of their work.

Active Immunization with Attenuated Cultures. - In the course of his experiments upon chicken cholera, as mentioned above, Pasteur ${ }^{1}$

${ }^{1}$ Pasteur, Compt. rend. de l'acad. des sci., 1880, t. xc. 
accidentally discovered that the virulence of the bacilli of this disease was greatly reduced by prolonged cultivation upon artificial media. This was especially noticeable in broth cultures which had been stored for long periods without transplantation. By repeated injections of such cultures into fowl, he succeeded in rendering the animals immune against subsequent inoculations with lethal doses of fully virulent strains.

During the same year, 1880, in which Pasteur published his observations on chicken cholera, 'Toussaint ${ }^{1}$ succeeded in immunizing sheep against anthrax by inoculating them with blood from infected animals, defibrinated and heated to $55^{\circ} \mathrm{C}$. for ten minutes. Toussaint wrongly believed, however, that the blood which had been used in his immunizations was free from living bacteria. In repeating this work Pasteur. showed that the protection in Toussaint's cases was conferred by living bacteria, the virulence of which had been reduced by their subjection to heat.

In following out the suggestions offered by these experiments, Pasteur ${ }^{2}$ discovered that he could reduce the virulence of anthrax bacilli much more reliably than by Toussaint's method, by cultivating the organisms at increased temperatures $\left(42^{\circ}\right.$ to $43^{\circ} \mathrm{C}$.). By this process of attenuation he was able to produce "vaccines" of roughly measurable strength, with which he succeeded in immunizing sheep and cattle. A successful demonstration of his discovery was made by him at Pouillyle-Fort, soon after, upon a large number of animals and before a commission of professional men.

It is a fact well known to bacteriologists that certain of the pathogenic microorganisms, when passed through several individuals of the same animal species, become gradually more virulent for this species. In his studies on the bacillus of hog cholera, Pasteur observed that when this microorganism was passed through the bodies of several rabbits it gained in virulence for rabbits, but became less potent against hogs. He succeeded, subsequently, in protecting hogs against fully virulent cultures by treating them with strains which had been attenuated by their passage through rabbits.

A further principle of attenuation for purposes of immunization was, at about this time, contributed by Chamberland and Roux, ${ }^{4}$ who re-

1 Toussaint, Compt. rend. de l'acad. des sci., 1880, t. xci.

${ }^{2}$ Pasteur, Chamberland et Roux, Compt. rend. de l'acad. des sci., 1881, t. xcii.

${ }^{3}$ Pasteur, Compt. rend. de l'acad. des sci., 1882, t. xcv.

${ }^{4}$ Chamberland et Roux, Compt. rend. de l'acad. des sci., 1882, t. xevi. 
duced the virulence of anthrax cultures by growing them in the presence of weak antiseptics (carbolic acid $1: 600$, potassium bichromate $1: 5,000$, or sulphuric acid $1: 200$ ). Cultivated under such conditions the bacilli lost their ability to form sporss and became entirely avirulent for sheen within ten days. A similar result was later obtained by Behring ${ }^{1}$ when attenuating $\mathrm{B}$. diphtherix cultures by the addition of terchlorid of iodin.

Active Immunization with Sublethal Doses of Fully Virulent BACTERIA.-The use of fully virulent microorganisms in minute quantities for purposes of immunization was first suggested by Chauveau, ${ }^{2}$ and is naturally inapplicable to extremely virulent organisms like B. anthracis. The principle, however, is perfectly valid, and has been experimentally applied by many observers, notably by Ferran ${ }^{3}$ in the case of cholera. A similar method proved of practical value in the hands of Theobald Smith and Kilborne ${ }^{4}$ in prophylaxis against the protozoan disease, Texas fever.

Active Immunization with Dead Bacteria.--Suggested by Chauveau, the method of active immunization with gradually increasing doses of dead microorganisms has been successfully employed by various observers, chief among whom are Pfeiffer, Brieger, Wright, and Wassermann. The method is especially useful against that class of bacteria in which the cell bodies (endotoxins) have been found to be incomparably more poisonous than their extracellular products (toxins). From a practical point of view, the method is of the greatest importance in routine laboratory immunization against B. typhosus, Vibrio choleræ asiaticx, B. pestis, and a number of other bacteria. In the therapy of human disease, this method has recently come into great prominence, chiefly through the work of Wright, whose investigations will be more fully discussed in a subsequent section.

Active Immunization with Bacterial Products.-Many bacteria when grown in fluid media produce extracellular, soluble poisons which remain in the medium after the microorganisms have been removed by filtration or centrifugalization. Since the diseases caused by such microorganisms are, to a large extent, due to the soluble poisons excreted by them, animals can be actively immunized against this class of bac-

1 Behring, Zeit. f. Hyg., xii, 1892.

${ }^{2}$ Chauveau, Compt. rend. de l'acad. des sci., 1881, t. xcii.

3 Ferran, Compt. rend. de l'acad. des sci., 1895, t. ci.

${ }^{4}$ Th. Smith and Kilborne, U. S. Dept. of Agri., Bureau of Ani. Indust., Wash., 1893. 
teria by the inoculation of gradually increasing doses of the specific poison or toxin. This method is naturally most successful against those microorganisms which possess the power of toxin formation to a highly developed degree. Most important among these are B. diphtheriæ and B. tetani. The first successful application of this principle of active immunization, however, was made by Salmon and Smith ${ }^{1}$ in the case of hog cholera.

\section{PASSIVE IMMUNITY}

In Pasteur's basic experiments, as in those of the other scientists who followed in his footsteps, the methods of immunization were based upon the development of a high resistance in the treated subject by virtue of its own physiological activities. This process we have spoken of as "active immunization" and it is self-evident that a method of this kind can, in the treatment of disease, be employed prophylactically only against possible infection, or in localized acute infections, or at the beginning of a long period of incubation before actual symptoms have appeared, as in rabies or in chronic conditions in which the infection is not of a severe or acute nature.

A new and therapeutically more hopeful direction was given to the study of immunity when, in 1890 and 1892, v. Behring and his collaborators discovered that the sera of animals immunized against the toxins of tetanus ${ }^{2}$ and of diphtheria ${ }^{3}$ bacilli would protect normal animals against the harmful action of these poisons. The animals thus protected obviously had taken no active part in their own defense, but were protected from the action of the poison by the substances transferred to them in the sera of the actively immunized animals. Such immunity or protection, therefore, is a purely passive phenomenon so far as the treated animal is concerned, and the process is for this reason spoken of as "passive immunization."

Passive immunization of this description is practically applicable chiefly against diseases caused by bacteria which produce powerful toxins, and the sera of animals actively immunized against such toxins are called antitoxic sera. In the treatment of the two diseases mentioned above, diphtheria and tetanus, the respective antitoxic sera have

1 Salmon and Smith, Rep. of Com. of Agri., Wash., 1885 and 1886.

${ }^{2} v$. Behring and Kitasato, Deut. med. Woch., 49, 1890.

s v. Behring and Wernicke, Zeit. f. Hyg., 1892. 
reached broad and beneficial therapeutic application, and innumerable lives have been saved by their use.

Passive immunization against microorganisms not char.cterized by marked toxin formation was attempted, even before Behring's discovery, by Richet and Hericourt, ${ }^{1}$ experimenting with cocci, and by Babes, ${ }^{2}$ in the case of rabies; and the underlying thought had been the basis of Toussaint's work upon anthrax. Microorganisms, however, which exert their harmful action rather by the contents of the bacterial cells than by secreted, soluble toxins, do not, so far as is known, produce antitoxins in the sera of immunized animals. The substances which they call forth in the process are directed against the invading organisms themselves in that they possess the power of destroying or of causing dissolution of the specific germs used in their production.

Such antibacterial sera are extensively used in the laboratory in the passive immunization of animals against a large number of germs, and are fairly effectual when used before, at the same time with, or soon after, infection. Their therapeutic use in human disease, however, has, up to the present time, been disappointing and their prophylactic and curative action has been almost invariably ineffectual or feeble at best, except when the antibacterial sera could be brought in direct contact with the germs, in closed cavities or localized lesions. Thus, in epidemic meningitis, such sera have proved extremely useful in the hands of Flexner, when injected directly into the spinal canal.

\section{ANTIBODIES AND THE SUBSTANCES GIVING RISE TO THEM}

In the foregoing sections we have seen that the process of active immunization so changes the animal body that it becomes highly resistant against an infection to which it had formerly in many instances been delicately susceptible. In the absence of visible anatomical or histological changes accompanying the acquisition of this new power, investigators, in order to account for it, were led to examine the physiological properties of the body cells and fluids of immunized subjects. While it was reasonable to suppose that all the cells and tissues were affected by, or might have taken part in, a physiological change so profoundly influencing the individual, the blood, because of its unquestionably close relation to inflammatory reactions, and because of the

1 Richet et Hericourt, Compt. rend. de l'acad. des sci., 1888.

2 Babes et Lepp, Ann. de l'inst. Pasteur, 1889. 
ease with which it could be obtained and studied, claimed the first and closest attention. The bactericidal properties of normal blood serum noted in 1886 by Nuttall, ${ }^{1}$ v. Fodor, ${ }^{2}$ and Flügge, moreover, aided in pointing to this tissue as primarily the seat of the immunizing agents. It is an interesting historical fact, that, long before this time, the English physician Hunter had noted that blood did not decompose so rapidly as other animal tissues.

The study of the blood serum of immunized animals as to simple changes in chemical composition or physical properties has shed little light upon the subject. Beljaeff ${ }^{3}$ in a recent investigation found little or no alteration from the normal in the blood sera of immunized animals as to index of refraction, specific gravity, and alkalinity. Joachim ${ }^{4}$ and Moll agree in stating that immune blood serum is comparatively richer in globulin than normal serum. Similar observations had been made by Hiss and Atkinson ${ }^{5}$ and others. Important and significant as these purely chemical observations are, they have helped little in explaining the nature of the processes going on in immune sera. The first actual light was thrown upon the mysterious phenomena of immunity by the investigations of Nuttall, ${ }^{6}$ v. Fodor, Buchner, and others, who not only demonstrated the power of normal blood serum to destroy bacteria, but also showed that this property of blood serum became diminished with age and was destroyed completely by heating to $56^{\circ} \mathrm{C}$. The thermolabile substance of the blood serum possessing this power was called by Buchner, alexin.

Soon after this work, Behring, in collaboration with Kitasato ${ }^{8}$ and Wernicke, ${ }^{9}$ in 1890 and 1892 , made further important advances in the elucidation of the immunizing processes by showing that the blood sera of animals actively immunized against the toxins of diphtheria and tetanus would protect normal animals against the poisons of these diseases. He believed, at the time of discovery, that such sera contained substances which had the power of destroying the specific toxins which had been

${ }^{1}$ Nuttall, Zeit. f. Hyg., i, 1886.

${ }^{2} v$. Fodor, Deut. med. Woch., 1886.

3 Beljaeff, Cent. f. Bakt., xxxiii.

4 Joachim, Pflügers Archiv, xciii.

${ }^{5}$ Hiss and Atkinson, Jour. Exper. Med., v, 1900.

${ }^{6}$ Nuttall, Zeit. f. Hyg., 1886.

7 Buchner, Cent. f. Bakt., i, 1889.

8 Behring und Kitasato, Deut. med. Woch., 1890, No. 49.

- Behring und Wernicke, Zeit. f. Hyg., 1892. 
used in the immunization. He called these bodies antitoxins. While Behring's first conception of actual toxin destruction soon proved to be erroneous, his discovery of the presence in immune sera of bodies specifically antagonistic to toxins was soon confirmed and extended, and stands to-day as an established fact.

Ehrlich, ${ }^{1}$ soon after Behring's announcement, showed that specific antitoxins could also be produced against the poisons of some of the higher plants (antiricin, antikrotin, antirobin), and Calmette ${ }^{2}$ produced similar antitoxins against snake poison (antivenin). Stimulated by these researches, other observers have, since then, added extensively to the list of poisons against which antitoxins can be produced. Kempner ${ }^{3}$ has produced antitoxin against the poison of Bacillus botulinus, and Wassermann, ${ }^{4}$ against that of Bacillus pyocyaneus. Antitoxin has been produced by Calmette ${ }^{5}$ against the poison of the scorpion, and by Sachs ${ }^{6}$ against that of the spider. Thus a large number of poisons of animal, plant, or bacterial origin have been found capable of causing the production of specific antibodies in the sera of animals into which they are injected.

The formation of antitoxins directed against soluble poisons, however, did not explain the immunity acquired by animals against bacteria like Bacillus anthracis, the cholera vibrio, and others which, unlike diphtheria and tetanus, produced little or no soluble toxin. It was evident that the antitoxic property of immune blood serum was by no means the sole expression of its protective powers. Much light was shed upon this phase of the subject by the discoveries of Pfeiffer in 1894, who worked along the lines suggested by the investigations of Nuttall and Buchner. Pfeiffer ${ }^{7}$ showed that when cholera spirilla were injected into the peritoneal cavity of cholera-immune guinea-pigs, the microorganisms rapidly swelled up, became granular, and often underwent complete solution. The same phenomenon could be observed when the bacteria were injected into a normal animal together with a sufficient quantity of cholera-immune ${ }^{8}$ serum.

1 Ehrlich, Deut. med. Woch., 1891.

${ }^{2}$ Calmette, Compt. rend. de la soc. de biol., 1894.

${ }^{3}$ Kempner, Zeit. f. Hyg., 1897.

${ }^{4}$ Wassermann, Zeit. f. Hyg., xxii.

${ }^{5}$ Calmette, Ann. de l'inst. Pasteur, 1898.

- Sachs, Hofm. Beit., 1902.

- Pfeiffer, Zeit. f. Hyg., xviii, 1894.

- Pfeiffer und Isaeff, ibid. 
This process he observed microscopically by abstracting, from time to time, a small quantity of the peritoneal exudate and studying it in hanging-drop preparations. The reaction was specific in that the destructive process took place to any marked extent only in the case of the bacteria employed in the immunization.

Metchnikoff, ${ }^{1}$ Bordet, and others not only confirmed Pfeiffer's observation, but were able to show that the lytic process would take place in vitro, as well as in the animal body. The existence of a specific destructive process in immune serum was thus established for the vibrio of cholera and soon extended to other microorganisms. The constituents of the blood serum which gave rise to this destructive phenomenon were spoken of as bacteriolysins.

Following closely upon the heels of Pfeiffer's observation came the discovery of another specific property of immune serum by Gruber and Durham. $^{2}$ These workers noticed that certain bacteria, when brought into contact with the serum of an animal immunized against them, were clumped together, deprived of motility, and firmly agglutinated. They spoke of the phenomenon as agglutination and of the substances in the serum giving rise to it as agglutinins.

The list of antibodies was again enlarged by Kraus, ${ }^{3}$ who in 1897 showed that precipitates were formed when filtrates of cultures of cholera, typhoid, and plague bacilli were mixed with their specific immune sera. He called the substances which bestowed this property upon the sera precipitins.

The treatment of the animal body, therefore, with bacteria or their products gives rise to a variety of reactions which result in the presence of the "antibodies" described above. Extensive investigation has shown, however, that the power of stimulating antibody production is a phenomenon not limited to bacteria and their products alone. Antitoxins, we have already seen, may be produced with a variety of poisons of plant and animal origin. Lysins, agglutinins, and precipitins, likewise: may be produced by the use of a large number of different substances. Chief among these, because of the great aid they have given to the theoretical investigation of the phenomena of immunity, are the red blood cells. Bordet ${ }^{4}$ and, independently of him, Belfanti and Carbone ${ }^{5}$ showed

${ }^{1}$ Metchnikoff, Ann. de l'inst. Pasteur, 1895.

${ }^{2}$ Gruber und Durham, Münch. med. Woch., 1896.

${ }^{3}$ Kraus, R., Wien. klin. Woch., 32, 1897.

4 Bordet, Ann. de l'inst. Pasteur, 1898.

5 Belfanti et Carbone, Giornale della R. Acad. di Torino, July, 1898. 
in 1898 that the serum of animals repeatedly injected with the defibrinated blood of another species exhibited the specific power of dissolving the red blood corpuscles of this species. This was the first demonstration of "hemolysis" - a phenomenon which, because of the ease with which it can be observed in vitro, has much facilitated investigation.

The knowledge that specific "cytotoxins" or cell-destroying antibodies could be produced by injection of red blood cells naturally suggested the possibility of analogous reactions for other tissue cells. It was not long, therefore, before Metchnikoff ${ }^{1}$ and, independently of him, Landsteiner ${ }^{2}$ succeeded, by repeated injections of spermatozoa, in producing a serum which would seriously injure these specialized cells. Von Dungern ${ }^{3}$ obtained similar results with the ciliated epithelium of the trachea. Since then a host of cytotoxins have been produced with the cells of various organs and tissues. Thus, Neisser and Wechsberg ${ }^{4}$ produced leucotoxin (leucocytes); Delezenne, ${ }^{5}$ neurotoxin and hepatotoxin; Surmont, ${ }^{6}$ pancreas cytotoxin; and Bogart and Bernard, ${ }^{7}$ suprarenal cytotoxin.

One of the most interesting of the cytotoxins, moreover, is nephrotoxin-produced by the treatment of animals with injections of emulsions of kidney tissue.

In all cases it was supposed by those first working with these bodies, that the injection of the sera of animals previously treated with any particular tissue substance would produce specific injury upon the organs homologous to the ones used in immunization. It need hardly be pointed out how very important such phenomena would be in throwing light upon the degenerative pathological lesions occurring in disease. As a matter of fact, however, sera so produced have been shown to be specific for certain organs in a limited sense only. The question of specific cytotoxins has been of especial importance in the case of nephritis, where Ascoli and Figari ${ }^{8}$ and others have suggested an autonephrotoxin as the basis of the pathology of this disease. In the hands of Pearce and others, however, the strict specificity of

${ }^{1}$ Metchnikoff, Ann. de l'inst. Pasteur, 1898.

${ }^{2}$ Landsteiner, Cent. f. Bakt., i, 25, 1899.

3 v. Dungern, Münch. med. Woch., 1899.

${ }^{4}$ Neisser und Wechsberg, Zeit. f.'Hyg., xxxvi, 1901.

${ }^{5}$ Delezenne, Ann. de l'inst. Past. 1900; Compt. rend. de l'acad. des sci. 1900.

${ }^{6}$ Surmont, Compt. rend. de la soc. de biol., 1901.

${ }^{7}$ Bogart et Bernard, ibid., 1891.

${ }^{8}$ Ascoli and Figari, Berl. klin. Woch., 1902. 
nephrotoxin could not be upheld and the subject is still in the experimental stage.

Recent experiments by Pearce ${ }^{1}$ suggest that at least a part of the local injury to organs exerted by such "cytotoxic" sera may not be due to a specific action upon the organ cells so much as upon the hemagglutinating action of the sera causing embolism and necrosis.

It is a fact also that most cytotoxic sera are usually hemolytic as well. It is not easy to decide, therefore, how much of the action upon the organs is due to their true cytotoxic properties and how much is attributable to the concomitant action upon blood cells. The extravagant hopes at first based upon cytotoxin investigation, especially in regard to the problem of malignant tumors, have been disappointed, and much is still obscure in regard to the cytotoxins which calls for further research.

The many points of similarity existing between bacterial toxins and digestive ferments, by animal inoculation, suggested to several observers the possibility of producing antibodies against the latter. As a result, a number of antiferments have been obtained, chief among which are antilab (Morgenroth ${ }^{2}$ ), antipepsin $\left(\right.$ Sachs $\left.^{3}\right)$, antisteapsin $\left(\right.$ Schütze $\left.^{4}\right)$, and antilactase (Schütze).

The stimulation of antibody formation in the sera of animals is a consequence, therefore, of the injection of a large variety of substancessome of them poisonous, some of them entirely innocuous. The substances possessing this power have been conveniently named antigens or antibody-producers by German writers. The term antigen-though etymologically wrong, nevertheless is convenient and has crept into general usage. It signifies simply a substance which can stimulate the production or formation of an antibody. Such substances, so far as is known, belong to the group of proteids and are derivatives of animal or plant tissues. Being proteids, all antigens are colloids. Recently, however, some crystalloidal substances have been described as possessing antigenic properties.

1 Pearce, Jour. Exper. Med., viii, 1906.

2 Morgenroth, Cent. f. Bakt., 1899.

${ }^{3}$ Sachs, Fort. d. Med., 1902.

'Schütze, Deut. med. Woch., 1904; Zeit. f. Hyg., 1905. 


\section{CHAPTER XIII}

\section{TOXINS AND ANTITOXINS}

The Toxin-Antitoxin Reaction.-Apart from the therapeutic possibilities disclosed by the discovery of antitoxins, new light of inestimable value was thrown by these observations upon the biological processes involved in immunization. The most vital problem, of course, which immediately thrust itself upon all workers in this field was the question as to the nature of the reaction in which toxin was rendered innocuous by antitoxin.

The simplest conception of this process would be an actual destruction of the toxin by its specific antitoxin, and it is not unnatural, therefore, that this was the view which, for a short time, found favor with some observers. Roux, and more particularly Buchner, ${ }^{1}$ however, under the sway of cellular pathology, advanced the opinion that the antitoxins in some way influenced the tissue cells, rendering them more resistant against the toxins. Antitoxin, according to this theory, did not act directly upon toxin, but affected it indirectly through the mediation of tissue cells. Ehrlich, ${ }^{2}$ on the other hand, conceived that the reaction of toxin and antitoxin was a direct union, analogous to the chemical neutralization of an acid by a base-an opinion in which Behring soon joined him.

The conception of toxin destruction received unanswerable refutation by the experiments of Calmette. ${ }^{3}$ This observer, working with snake poison, found that the poison itself (unlike most other toxins) possessed the property of resisting heat even to $100^{\circ} \mathrm{C}$., while its specific antitoxin, like other antitoxins, was delicately thermolabile. He noted, furthermore, that non-toxic mixtures of the two substances, when subjected to heat, regained their toxic properties. The natural inference from these observations could only be that the toxin in the original mixture had not been destroyed, but had been merely inactivated by the

${ }^{1}$ Buchner, "Schutzimpfung," etc., in Penzoldt u. Stinzing, "Handbuch d. spez. Therap. d. Infektkrank.," 1894.

${ }^{2}$ Ehrlich, Deut. med. Woch., 1891. $\quad{ }^{3}$ Calmette, Ann. de l'inst. Past., 1895. 
presence of the antitoxin, and again set free after destruction of the antitoxin by heat. A similar observation, made soon after by Wassermann ${ }^{1}$ in the case of pyocyaneus toxin and antitoxin, fully supported the results of Calmette.

An ingenious proof of the direct action of antitoxin upon toxin was obtained by Martin and Cherry. ${ }^{2}$ It was found by them that very dense filters, the pores of which had been filled with gelatin, permitted toxin to pass through under high pressure, while the presumably larger antitoxin molecule was held back. Through such filters they forced toxin-antitoxin mixtures, under a pressure of fifty atmospheres, at varying intervals after mixing. They found that, if filtered immediately, all the toxin in the mixtures came through, but that, as the interval elapsing between mixing and filtration was prolonged, less and less toxin appeared in the filtrate, until, finally, two hours after mixing, no toxin whatever passed through the filter. Besides demonstrating the direct action of antitoxin upon toxin, this work of Martin and Cherry showed that the element of time entered into the toxin-antitoxin reaction, just as it enters into reactions of known chemical nature. The absolute nonparticipation of the living tissue cells in these reactions was demonstrated by Ehrlich himself. Kobert and Stillmarck ${ }^{3}$ had shown that ricin possessed the power of causing the red blood cells of defibrinated blood to agglutinate in solid clumps, a reaction which could easily be observed in vitro. Ehrlich, ${ }^{4}$ who had obtained antiricin in 1891 by injecting rabbits with increasing doses of ricin, found that this antibody possessed the power of preventing the hemagglutinating action of ricin in the test tube. By a series of quantitatively graded mixtures of ricin and antiricin, with red blood cells as the indicator for the reaction, he succeeded in proving not only that the toxin-antitoxin neutralization was in no way dependent upon the living animal body, but that definite quantitative relations existed between the two substances entirely analogous to those which, according to the law of multiple proportions, govern reactions between different substances of known chemical nature. Similar quantitative results were subsequently obtained by Stephens and Myers ${ }^{5}$ for cobra poison and its antitoxin, by Kossel ${ }^{\theta}$

1 Wassermann, Zeit. f. Hyg., xxii, 1896.

${ }^{2}$ Martin and Cherry, Proc. Royal Soc., London, lxiii, 1898.

${ }^{3}$ Kobert und Stillmarck, Arb. d. phar. Inst. Dorpat, 1889.

- Ehrlich, Fort. d. Med., 1897.

${ }^{5}$ Stephens and Myers, Jour. of Path. and Bact., 1898.

- Kossel, Berl. klin. Woch., 1898. 
for the toxic eel blood serum, and by Ehrlich ${ }^{1}$ for the hemolytic tetanus poison known as tetanolysin.

The introduction of the test-tube experiment into the investigation of these reactions permitted of much more exact observations, and by this means, as well as by careful, quantitatively graded, animal experiments, the further facts were ascertained that toxin and antitoxin combined more speedily in concentrated than in dilute solutions, and that warmth hastened, while cold retarded, the reaction-observations ${ }^{2}$ which in every way seem to bear out Ehrlich's conception of the chemical nature of the process.

Ehrlich's Analysis of Diphtheria Toxin.-Shortly after the discovery and therapeutic application of diphtheria antitoxin, it became apparent that no two sera, though similarly produced, could have exactly the same protective value. It was necessary, therefore, to establish some measure or standard by which the approximate strength of a given antitoxin could be estimated. Von Behring ${ }^{3}$ attempted to do this for both tetanus and diphtheria antitoxins by determining the quantity of immune sera which, in each case, was needed to protect a guinea-pig of known weight against a definite dose of a standard poison. He ascertained the quantity of standard toxin-bouillon which would suffice to kill a guinea-pig of 250 grams, and called this quantity the "toxin unit." This unit was later more exactly limited by Ehrlich, who, considering the element of time, stated it as the quantity sufficient to kill a guineapig of the given weight in from four to five days.

Appropriating the terminology of chemical titration, v. Behring spoke of a toxin-bouillon which contained one hundred such toxin units in a cubic centimeter, as a "normal toxin solution" (" $\mathrm{DTN}^{1} \mathrm{M}^{250}$ "), and designated as "normal antitoxin" a serum capable of neutralizing, cubic centimeter for cubic centimeter, the normal poison." A cubic centimeter of such an antitoxic serum was sufficient, therefore, to neutralize one hundred toxin units, and was spoken of as an "antitoxin unit." In the experiments of v. Behring, toxin and antitoxin had been separately injected. Ehrlich ${ }^{5}$ improved upon this method by mixing. toxin and antitoxin before injection, thereby obviating errors arising

Ehrlich, Berl. klin. Woch., 1898.

Knorr, Fort. d. Med., 1897.

s $v$. Behring, Deut. med. Woch., 1893.

$4 \mathrm{DTN}^{1}$ M 250 signifies: D, Diphtheria; TN¹, Normal Toxin solution; M250, Meerschweinchen or guinea-pig weighing 250 grams.

s Ehrlich, Kossel und Wassermann, Deut. med. Woch., 1894. 
from differences which may have existed in the depth of injection or rapidity of absorption.

In order, however, that any such method of standardization of antitoxin may be practically applicable, it is necessary to produce either a stable toxin or an unchangeable antitoxin. This Ehrlich achicved for antitoxin by drying antitoxic scrum in vacuo and prescrving it in the dark, at a low tempcrature and in the presence of anhydrous phosphoric acid. By the use of such a stable antitoxin, various toxins may be measured and other antitoxic sera estimated against these.

Given thus a constant antitoxin, the standardization of toxins would be a comparatively simple matter were the poison obtainable in a perfectly pure state. Unfortunatcly for the ease of measurement, however, this is not the case. The problem is rendered difficult by a number of complicating factors, many of which have been brought to light by Ehrlich ${ }^{1}$ in his laborious researches into the quantitative relationship between the two reacting bodies.

As previously stated, it had been noted by Ehrlich and others that toxin solutions would deteriorate with time; that is, a toxin-bouillon

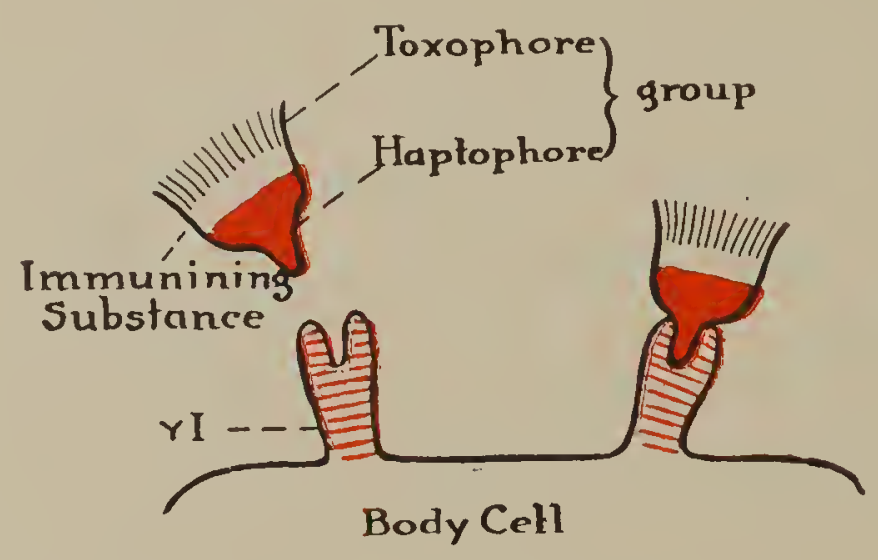

Fig. 55.-Toxin and Body Celit.

which was found soon after production to contain, say, cighty toxin units in each cubic centimeter, would, after four or five months, be found to contain but forty units in the same gross quantity. It had lost, therefore, in this case, just one-half of its toxic power. In spite of this loss, however, Ehrlich found that such bouillon had retained its full originai power of neutralizing antitoxin. If the reaction was purcly one of chemical neutralization, there scemed to be but one explanation of this. The toxin molecule must contain two separate atom groups. One of these must possess the powcr of binding antitoxin and be stable; this

${ }^{1}$ Ehrlich, Klin. Jahrbuch, vi, 1897; Deut. med. Woch., 1898. 
he designates as the "haptophore" or "anchoring" group. The other, the one by which the ioxin molecule exerts its deleterious action, must be more easily changed or destroyed; this he calls the "toxophore" or "poison" group. In the altered toxin-bouillon in which a part of the poisonous action has been lost while the antitoxin-neutralizing power is intact, the toxophore group of some of the toxin must have been changed or destroyed. Such altered toxin he spcaks of as "toxoid."

In support of this hypothesis and for the purpose of perfecting the methods of standardization, Ehrlich was led to determine, for a large valiety of specimens of diphtheria toxin, the precise quantity, in cubic centimeters, which was necessary to neutralize exactly one unit of his standard antitoxin. This he accomplished by making a series of toxin-antitoxin mixtures, in each of which the quantity of antitoxin was exactly one unit, while the amount of toxin was gradually increased. These mixtures were injected into guinea-pigs of 2.50 grams weight. It is self-evident that in such an experiment the mixtures containins the smaller quantities of toxin would have no effect upon the guineapigs. Soon, however, a mixture would be reached in which toxin would be sufficiently in excess of antitoxin to produce the symptoms of slight poisoning, as evidenced in local edema, rise of temperature, ete. The largest quantity of toxin which could be added without producing such symptoms was then regarded as exactly neutralizing one antitoxin unit. This quantity of toxin Ehrlich speaks of as "Limes zero" (Limes= threshold) or, briefly, " $\mathrm{L}_{0}$."

For instance:

One antitoxin unit +0.6 c.c. toxin........ No symptoms of poisoning.

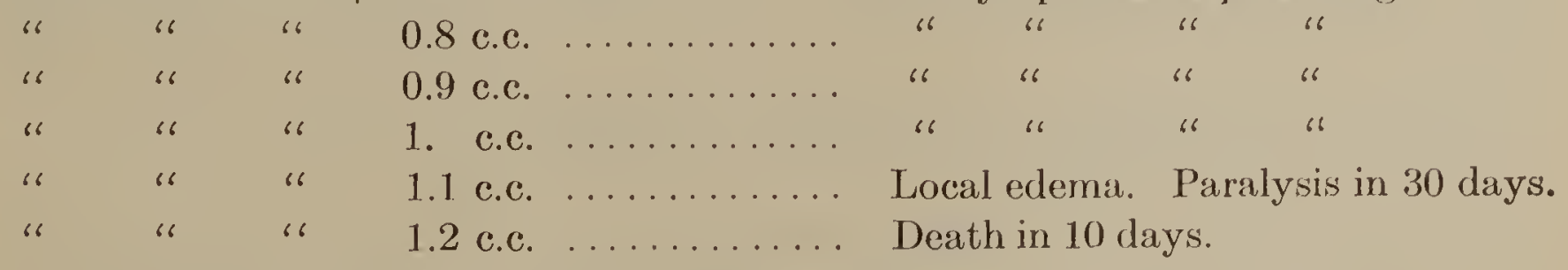

In this example, $\mathrm{L}_{0}$, therefore, equals 1 c.c.

It is obvious, however, that because of the great difficulty in estimating the very slightest evidences of toxic action in guinea-pigs, a more exact method of standardizing the poisons against antitoxin would be to determine how much toxin would be required to neutralize one antitoxin unit and still be sufficiently in excess to cause the death of a guinea-pig of 250 grams in four to five days. This would then correspond to the action of one toxin linit, unmixed with antitoxin. A priori it would seem that this value (expressed by Ehrlich as "Iimes 
death" or " $\mathrm{L}_{+}$) must simply be $\mathrm{L}_{\mathrm{o}}$ plus one toxin unit. This, however, was found not to be the case. Thus, in the example given, in which $\mathrm{T}$ (the toxin unit-the quantity of the bouillon killing a guinea-pig of 250 grams in four to five days) was equal to 0.01c.c., $\mathrm{L}_{0}$ (the quantity of toxin completely neutralizing one antitoxin unit) was found to be 1 c.c. or $100 \mathrm{~T}$. In this same poison, however, $\mathrm{L}_{+}$(the quantity of toxin necessary both to neutralize one antitoxin unit and yet to be sufficiently in excess of neutralization to kill a guinea-pig of 250 grams in four or five days) was not found to be merely $\mathrm{L}_{0}+1 \mathrm{~T}$; but on actual experiment proved to be $\mathrm{L}_{0}+101 \mathrm{~T}$.

Expressed graphically, the conditions may be stated as follows:

$T=.01$ c.c. of the toxin bouillon.

$L_{+}$(neutral. of 1 antitox. unit yet killing 1 pig) $=2.01$ c.c. or $201 \mathrm{~T}$.

$L_{0}$ (complete neutral. of 1 antitox. unit) $=1$. c.c. or $100 T$.

Difference $=1.01$ c.c. or $101 T$.

Ehrlich, at first, endeavored to explain this surprising phenomenon on the basis of toxoids. He argued that the toxoids formed by deterioration of toxin might be conceived as possessing three different degrees of affinity for antitoxin. If their affinity for antitoxin were equal to, or more marked than, that of the toxin itself, they could have no influence upon the dose $\mathrm{L}_{+}$. If, however, their affinity for antitoxin were weaker than that of toxin, each fresh toxin unit added to the dose $\mathrm{L}_{\mathrm{o}}$ would, first uniting with antitoxin, replace a corresponding quantity of these nontoxic substances of weaker affinity, and $\mathrm{L}_{+}$would be reached only after all of these "epitoxoids," as Ehrlich called them, had been replaced, and toxin became free in the mixture.

Thus, in analyzing our example, we have:

100 tox.-antitox. +100 epitox.-antitox. $=L_{\mathrm{o}}$;

add $1 T$, and we have 101 tox.-antitox. +99 epitoxoid-antitoxin +1 epitoxoid free; add $101 T$ and we have 200 toxin-antitoxin +100 epitoxoid free $+1 T$ free $=L_{+}$.

Two facts, however, led Ehrlich to abandon the opinion that epitoxoid was merely a variety of toxoid. He found, in the first place, that the stated relations between $\mathrm{L}_{0}$ and $\mathrm{L}_{+}$were true for perfectly, fresh toxin-bouillon in which little or no deterioration had taken place. He observed, furthermore, that in old, altered toxin bouillon, while $\mathrm{T}$ was very much affected, the quantity needed to kill a pig constantly increasing, and the number of actual fatal doses in $\mathrm{L}_{0}$ con- 
stantly decreasing (by reason of toxoid formation), $\mathrm{L}_{+}$remained practically unchanged.

Simply stated, this means that the epitoxoids or substances which have weaker affinity for antitoxin than toxin itself are already present in fresh bouillon and are not increased with time. For this reason, Ehrlich has separated these substances from toxoids. He calls them "toxon" and believes them to be, like toxin, primary secretory products of the diphtheria bacilli. The toxoids themselves, Ehrlich believes, are of two kinds, those with a stronger affinity for antitoxin than toxin itself (protoxoids), and those whose affinity for antitoxin is equal to that of toxin. These latter he calls "syntoxoids."

The toxon (epitoxoid originally), as Ehrlich believes, has a haptophore or "binding" group similar to that of toxin, but a different toxophore or "poisoning" group. Qualitatively it has been shown to differ from toxin in that, lacking the power to produce acute symptoms, it causes gradual emaciation and paresis in animals.

That this difference in the poisonous action of toxin and toxon is not merely a quantitative difference, referable to small quantities of toxin, was proved by Dreyer and Madsen, ${ }^{1}$ who showed that if they made a toxin-antitoxin mixture in which after injection the only evidence of incomplete neutralization lay in the emaciation and final paralysis of the test animals, the quantity of such a mixture could be increased five- and tenfold, without producing the true toxin symptoms in animals. These authors, too, claim to have been able to immunize against toxin with such mixtures, thereby proving the identity of the haptophore groups of the two substances. The importance of this observation will become more evident in connection with the section on the "sidechain theory."

Method of Partial Absorption of Toxin.-Ehrlich ${ }^{2}$ has gathered more exact data in support of his views from what he terms the "Method of Partial Absorption" of toxin by antitoxin.

In order to understand this method clearly, it is necessary to remember that Ehrlich ${ }^{3}$ believes the union of toxin with antitoxin to take place according to the chemical laws of valency. Just as in $\mathrm{H}_{2} \mathrm{O}$ oxygen has an atomic valency of 2 for hydrogen, so, in the fully neutralized toxin-antitoxin compound, he believes antitoxin to have a

${ }_{1}^{1}$ Dreyer und Madsen, Zeit. f. Hyg., xxxvii, 1901.

2 Ehrlich, "Gesammelte Arbeiten zur Immunitätsforsch.," Berlin, 1904.

3 Ehrlich, Deut. med. Woch., 1898. 
valency of 200 for toxin. It would require, according to this, $200 \mathrm{~T}$ or toxin molecules to satisfy the affinities of one antitoxin molecule. ${ }^{1}$

This belief is based upon the following consideration: In determining the $\mathrm{I}_{\mathrm{o}}$ dose, or fully neutralized toxin-antitoxin union, Ehrlich, as well as Madsen, found that the number of $\mathrm{T}$ units contained in such a dose was almost regularly a factor of one hundred, recurring again and again as $25,33,50,75$, etc. This pointed to more or less regularity in the deterioration of toxin into toxoid, and to a more or less regular relation of toxin to toxon. Now, as we have seen before, if we could procure a perfectly pure toxin, the $\mathrm{L}_{0}$ dose plus one toxin unit would give us the $\mathrm{L}_{+}$dose; that is, one toxin unit in excess of full neutralization would suffice to kill a guinea-pig of 250 grams in four to five days. Since a perfectly pure toxin, however, has not been obtainable up to the present time, it is clear that the number of pure toxin bonds contained in $\mathrm{L}_{+}$must be less than the actual number of neutralizing units in the combination, a part of the antitoxin being bound by toxon and toxoid. The actual values obtained for the number of $\mathrm{T}$ units in $\mathrm{L}_{+}$has never exceeded 200, and has usually been more than 100, the highest value ascertained by Madsen being 160. Given, therefore, a combining value which, being a multiple of one hundred, is often more than one hundred, but in an obviously impure state has never reached 200, it is most likely that 200 represents the actual value sought for.

Assuming, therefore, upon the foregoing considerations, that the valency of antitoxin for toxin is 200 , Ehrlich carries out his experiments in the following way:

Given a toxin, the unit $(\mathrm{T})$ of which is 0.024 c.c., he first determines the $\mathrm{L}_{+}$dose which, tested against the standard antitoxin unit, in this case is 2.05 c.c. But 2.05 c.c. $=85 \mathrm{~T}$. (or $2.05 \div .024$ ) units. By mixing the $\mathrm{L}_{+}$dose of toxin and antitoxin in such a way that the quantity of antitoxin is gradually increased, while the toxin remains always $\mathrm{L}_{+}$, and determining upon animals the amount of free toxin contained in each mixture, the following table may be constructed: ${ }^{2}$

0 antitox. unit representing

.1 " " " "

.25 " " " "

.8 " " " " "

.9 "

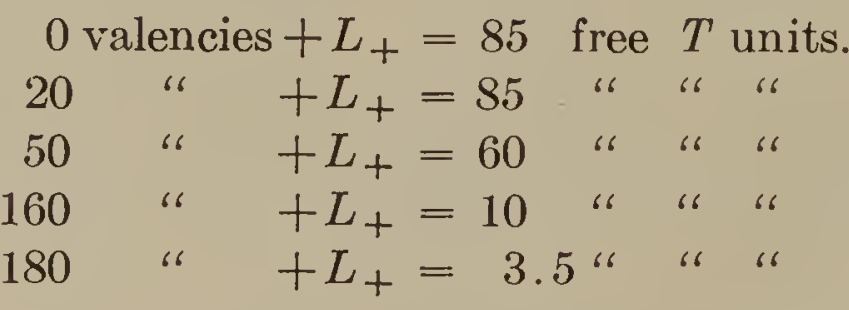

1 Ehrlich, "Schlussbetrachtungen," Nothnagel's System.

${ }^{2}$ Example taken from Ehrlich, Deut. med. Woch., 1898. 
It is plain that the substances with the strongest affinity for antitoxin must be bound first by the antitoxin. This does not diminish the toxic value of the mixture; and these are the protoxoids. Next are bound syntoxoids and toxins, and, finally, the toxons. It is plain that, by this method, the constitution of any given toxin may be ascertained, and Ehrlich has constructed, on the basis of these observations, what he terms his toxin spectrum. Minor differences of toxicity and affinity for the antibody have caused him, by the partial saturation method described, still further to divide toxin into proto-, deutero-, and trito-toxin.

His spectra graphically describe the constitution of any given toxic bouillon and trace its deterioration as follows:

Fig. 1.

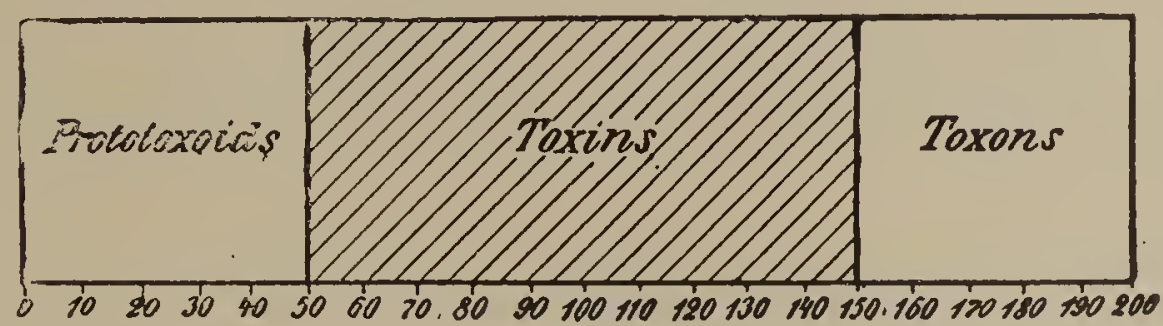

Fig.2.
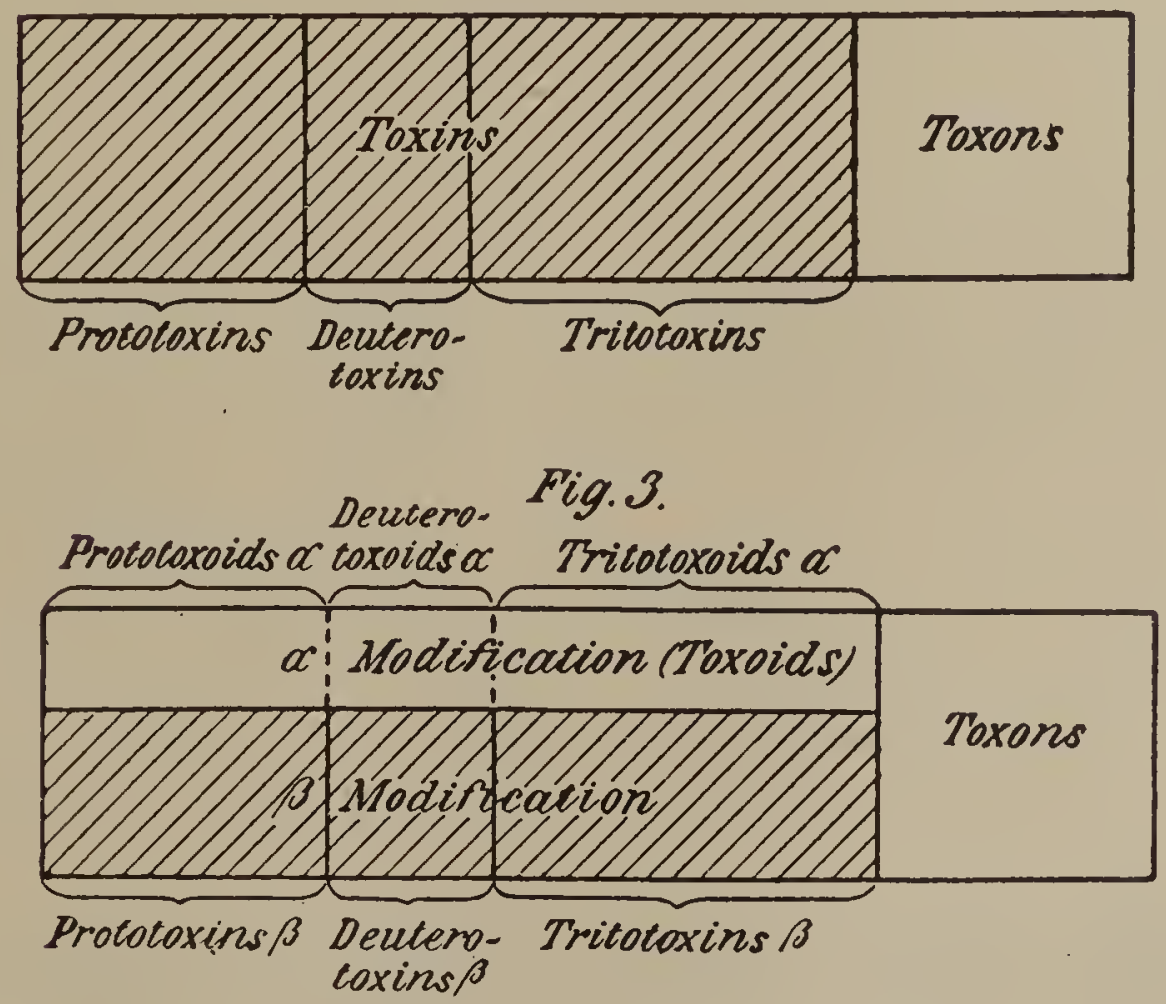

Ehrlich's opinion as to the constitution of toxin is by no means fully accepted. Arrhenius, ${ }^{1}$ the great physical chemist, and Madsen,

${ }^{1}$ Arrhenius und Madsen, Zeit. f. physik. Chem., 1902; Festschrift, Kopenhagen, 1902. 15 
a bacteriologist who was once a pupil of Ehrlich, have recently opposed Ehrlich's theory on grounds of physical chemistry.

Modern theories of solution maintain that substances in solution are broken up into their atoms or atom-groups, known as ions. Thus, $\mathrm{NaCl}$ in solution would be "dissociated" into its $\mathrm{Na}$ ion and its $\mathrm{Cl}$ ion, the completeness of the dissociation depending upon the concentration of the solution. A solution of $\mathrm{NaCl}$, therefore, contains, according to this view, three substances, $\mathrm{NaCl}$ undissociated and free ions of $\mathrm{Na}$ and $\mathrm{Cl}$, the relative quantities of the three present in any given solution being calculable, and depend upon a law known as the law of massaction of Guldberg and Waage. These free ions are the elements, therefore, which are active in the formation of further chemical combination. When a strong acid, in solution, acts upon a base, say $\mathrm{HCl}$ upon ammonia $\left(\mathrm{NH}_{3}\right)$, strong acid having the property of quite complete dissociation in relatively concentrated solutions, little or no ammonia would remain unbound. A weak acid, like boric acid, however, not being as completely dissociated, would leave some ammonia uncombined even after more than quantitatively sufficient boric acid had been added. Arrhenius and Madsen, on the basis of careful researches into the reaction between tetanolysin and its antibody, believe that toxin and antitoxin possess weak chemical avidity for each other, their interaction being comparable to that taking place between a weak acid and a base. Toxin-antitoxin solutions, therefore, would contain the neutral compound, but at the same time uncombined toxin and antitoxin. The qualities which Ehrlich ascribes to toxon, they believe, are due to the unbound toxin present in such mixtures. In careful studies in which they inhibited the hemolytic action of ammonia by gradual addition of boric acid, they were able to show complete parallelism between the conditions governing this neutralization and those concerned in their tetanus experiments. Their explanation has the advantage of extreme simplicity over that of Ehrlich, but since the differences of opinion are now the subject of active experimental controversy, a critical discussion must rest until further facts are revealed.

The Side-Chain Theory.-We have seen that the extensive researches of Ehrlich into the nature of the toxin-antitoxin reaction led him to believe that the two bodies underwent chemical union, forming a neutral compound. The strictly specific character of such reactions, furthermore, diphtheria antitoxin binding only diphtheria toxin, tetanus antitoxin only tetanus toxin, etc., led him to assume that the chemical 
affinity between each antibody and its respective antigen depended upon definite atom groups contained in each.

Ehrlich ${ }^{1}$ had, in 1885, published a treatise in which he discussed the manner of cell-nutrition and advanced the opinion that in order to nourish a cell, the nutritive substance must enter directly into chemical combination with some elements of the cell protoplasm. The great number and variety of chemical substances which act as nutriment led him to believe that the highly complex protoplasmic molecules of cells were made up of a central atom-group (Leistungs-Kern) upon which depended the specialized activities of the cell, and a multiplicity of side chains (a term borrowed from the chemistry of the benzol group), by means of which the cell entered into chemical relation with food and other substances brought to it by the circulation. If we illustrate graphically by the chemical conception from which the term side chain was borrowed, in salicylic acid, the formula given, the<smiles>O=C(O)C1CCC=CC1O</smiles>

benzol ring represents the "Leistungs-Kern," or radicle, while $\mathrm{COOH}$ and $\mathrm{OH}$ are side chains by means of which a variety of other substances may be brought into relation with the "radicle," for instance, as in methyl salicylate.<smiles>CCOC(=O)C1CCCC1O</smiles>

Just as nutritious substances are thus brought into workable relation with the cell by means of the atom-groups corresponding to side chains, so Ehrlich believes toxins exert their deleterious action only because the cells possess side chains by means of which the toxin can be chemically bound. These side chains, Ehrlich in his later work calls "receptors." The receptors or side chains present in the cells and

${ }^{1}$ Ehrlich, "Das Sauerstoffbedürfniss des Organismus," Berlin, 1885. 
possessing by chance specific affinity for a given toxin, are, by their union with toxin, rendered useless for their normal physiological function. By the normal reparative mechanism of the body these receptors are probably cast off and regenerated. Regenerative processes of the body, however, do not, as a rule, stop at simple replacement of lost elements, but, according to the hypothesis of Weigert, ${ }^{1}$ usually tend to over'compensation. The receptors eliminated

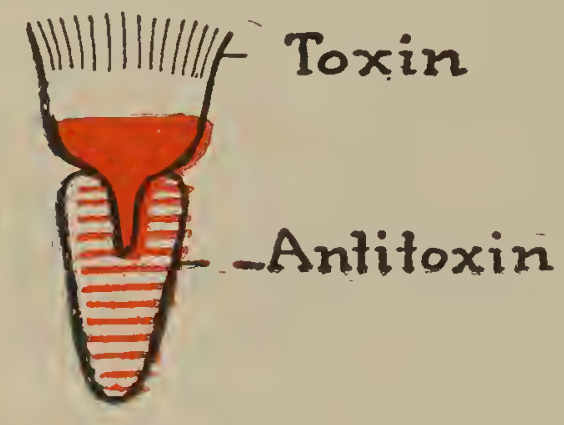

Fig. 56.-Toxin AND ANTitoxin. by toxin absorption are not, therefore, simply reproduced in the same quantity in which they are lost, but are reproduced in excess of the simple physiological needs of the cell. Continuous and increasing dosage with the poison, consequently, soon leads to such excessive production of the particular receptive atom-groups that the cells involved in the process become overstocked and cast them off to circulate freely in the blood. These freely circulating receptors-atom-groups with specific affinity for the toxins used in their production-represent the antitoxins. These, by uniting with the poison before it can reach the sensitive cells, prevent its deleterious action. (Fig. 56.)

The theory of Ehrlich, in brief, then, depends upon the assumptions that toxin and antitoxin enter into chemical union, that each toxin possesses a specific atom-group by means of which it is bound to a preexisting side chain of the affected cell, and that these side chains, in accordance with Weigert's law, under the influence of repeated toxin stimulation, are eventually overproduced and cast off by the cell into the circulation.

It stands to reason that this theoretical conception would be vastly strengthened were it possible to show that such receptors or toxinbinding atom-groups actually pre-existed in the animal body, and such support was indeed given by the experiments of Wassermann and Takaki. $^{2} \quad$ These observers succeeded in showing that tetanus toxin could be rendered innocuous if, before injection into animals, it was thoroughly mixed with a sufficient quantity of the fresh brain substance of guineapigs. Similar observations were independently made by Asakawa, ${ }^{3}$ and

${ }^{1}$ Weigert, Verhandl. d. Ges. Deutsch. Naturf. u. Aerzte, Frankfurt, 1896.

${ }^{2}$ Wassermann und Takaki, Berl. klin. Woch., 1898.

3 Asakawa, Cent. f. Bakt., 1898. 
variously confirmed. Kempner and Schepilewsky ${ }^{1}$ showed a similar relation to exist between brain tissue and botulismus toxin, and Myers ${ }^{2}$ brought proof of analogous conditions in the case of suprarenal tissue and cobra poison.

In the discussion of Ehrlich's toxin analysis, we have seen that he accounted for variations in the quantitative relations by the existence of toxoids and toxons. He explained the striking fact that toxoids had lost their poisonous nature and yet retained full powers to neutralize antitoxin by the assumption that toxin was made up of two separate atom-groups; one, the haptophore group which possessed the specific affinity for the antitoxin or cell receptor; the other, the toxophore group by means of which the actually harmful effects were produced. The haptophore groups of all three of these substances, toxin, toxoid, and toxon, by virtue of their antitoxin-binding power, he assumed to be alike; in toxoid, the toxophore group has been destroyed or altered; in toxon, the toxophore group is qualitatively different from that of toxin. The haptophore group, however, being alone concerned in neutralizing receptors, all three of these substances should, if Ehrlich's theory is to be tenable, produce antitoxin. Dreyer and Madsen, ${ }^{3}$ accordingly, actually succeeded in producing diphtheria antitoxin by immunization with toxon. Attempts to produce antitoxin with toxoids have succeeded in the hands of Ehrlich and others. Such experiments have not, however, been always successful, a notable failure being that of Bruck. ${ }^{4}$ On the basis of such negative results the theory was advanced by Wassermann that overproduction of receptors was stimulated by the irritation (Zellreiz) produced by the toxophore group-a stimulation not present in the case of toxoids.

${ }_{1}$ Kempner und Schepilewsky, Zeit. f. Hyg., 1898.

2 Myers, Cent. f. Bakt., i, 1899.

${ }^{3}$ Dreyer und Madsen, Zeit. f. Hyg., 1901.

${ }^{4}$ Bruck, Zeit. f. Hyg., 1904. 


\section{CHAPTER XIV}

\section{PRODUCTION AND TESTING OF ANTITOXINS}

\section{DIPHTHERIA ANTITOXIN}

In spite of the great advances in our theoretical knowledge of antibodies, gained during the last three decades, extensive therapeutic application has been made of the antitoxins only. Pre-eminent among these from a practical point of view are the antitoxins against diphtheria and tetanus toxin. For diphtheria, careful statistical studies have demonstrated, beyond doubt, the therapeutic value of the serum treatment. Biggs and Guerard, in a general statistical review, arrived at the conclusion that the death rate of diphtheria had been reduced fifty per cent by the use of antitoxin. Approximately the same estimate is made by Dieudonné ${ }^{1}$ who studied almost 10,000 treated cases.

Production of Diphtheria Antitoxin.-The methods for producing diphtheria antitoxin vary only in minor technical details. The first requisite for successful antitoxin production is the possession of a strong toxin. The various means of obtaining this are outlined in the section on diphtheria toxin. The toxin used should be of such potency that less than 0.01. cc. will kill a guinea-pig of 250 grams weight in four to five days. ${ }^{2}$

For experimental purposes, goats or sheep may be used for immunization; for antitoxin production on a large scale, horses have been found to be the most useful animals. The horses should be young, four to six years old, vigorous, and healthy. It is advisable that they be subjected to the mallein test to exclude possible infection with glanders.

The toxin injections are made subcutaneously. Because of the differences in susceptibility noted in various hor'ses, it is advisable that the first doses of toxin should be either very small or weakened by chemicals or heat, or cornbined with antitoxin. In the Pasteur Institute in Paris, the small initial dose of toxin (0.5 c.c.) is mixed before injection with an equal quantity of Lugol's solution (iodin-potassium iodid solution).

"Dieudonné, Arb. a. d. kais. Gesundheitsamt, 1895 and 1897.

${ }^{2}$ Park, "Pathog. Bacteria and Protozoa," N. Y., 1908. 
Park ${ }^{1}$ advises an initial dose of 5,000 toxin units (about 20 c.c. of a strong toxin) combined with 100 units of antitoxin. The same amount is given with the second and third doses of toxin. The intervals between injections are from five days to a week, depending upon the time necessary for complete subsidence of the reaction (temperature). The doses of toxin are gradually increased until, at the end of two or three months, more than ten times the original dose is given $(50,000$ units).

Horses vary greatly in the strength of antitoxin which they will produce. At the end of three or four months in favorable animals one cubic centimeter of serum may contain 250 to 800 antitoxin units. Further immunization will often increase the antitoxin output to 1,000 and more units to the cubic centimeter of serum. Park states that none of the horses used by him has ever yielded 2,000 units to the cubic centimeter. The same writer advises a three months' period of rest from immunization at the end of every nine months. Given such resting periods, some horses have continued to furnish high-grade antitoxin for from two to four years.

In order to obtain serum from horses, a sharp cannula is introduced into the jugular vein. After leading the horse into a specially constructed stall, its head is slightly deflected and pressure is made upon the jugular vein below the point into which the needle is to be plunged. Compression can also be made by surrounding the neck of the horse close to the shoulders with a leather strap over a pad laid directly upon the vein. The vein becomes visible along the lower margin of the neck in a line running from the angle of the jaw to the edge of the scapula. The skin, of course, is previously shaved and sterilized. The cannula is then plunged into the vein, either with or without previous incision through the skin, and, through a sterile rubber tube, the blood is allowed to flow into high glass cylinders or slanted Erlenmeyer flasks. In this way, large quantities of blood may be obtained and, according to Kretz, ${ }^{2}$ as much as six liters may be taken at a time at intervals of a month, without injuring the animal. Ligature of the vein after bleeding is unnecessary.

The cylinders and flasks are allowed to remain standing for two or three days in a cool place, preferably at or below $10^{\circ} \mathrm{C}$. At the end of this time, the serum may be pipetted or siphoned away from the

${ }^{1}$ Park, loc. cit., p. 212.

${ }^{2}$ Kretz, in "Handb. der Techn. u. Meth. d. Immun.," Kraus and Levaditi, vol. ii, 1908. 
clot and stored in the refrigerator. In order to diminish the chances of contamination, five-tenths per cent of carbolic acid or four-tenths per cent of tri-cresol may be added.

Antitoxin is fairly stable and if kept in a cool, dark place, may remain active, with but slight deterioration, for as long as a year. Kept in a dry state, in vacuo, over anhydrous phosphoric acid, by the method of Ehrlich, it retains its strength indefinitely.

Standardization.-Since antitoxin units are measured in terms of toxin, it is obvious that uniformity of measurement necessitates the possession by the various laboratories of a uniform toxin. Antitoxin being more stable than toxin, uniformity of toxin is obtained by means of a standard antitoxin distributed from a central laboratory. This was first done by Ehrlich in Germany, and is now done for the United States by the Public Health and Marine Hospital Service laboratories. Bottles of the distributed antitoxin are marked with the number of units contained in each cubic centimeter. Dilutions of this antitoxin are mixed with varying quantities of the toxin to be tested, the mixtures are allowed to stand for fifteen minutes to permit union of the two elements, and injections into guinea-pigs of 250 grams weight are made. In this way, the $\mathrm{L}_{+}$dose of the toxin is determined. (The $\mathrm{L}_{+}$dose, as we have seen in a previous section [p. 208], is the quantity of poison not only sufficient to neutralize one antitoxin unit, ${ }^{1}$ but to contain an excess beyond this sufficient to kill a guinea-pig of 250 grams in four to five days. $\mathrm{L}_{+}$is chosen rather than $\mathrm{L}_{0}$, the simple neutralizing dose, because of the difference between toxins in their contents of toxoid and toxon. ${ }^{2}$ )

The $\mathrm{L}_{+}$dose of the toxin having thus been determined, this quantity is mixed with varying dilutions of the unknown antitoxin. ${ }^{3}$ Thus, given an antitoxin in which 300 to 400 units to the cubic centimeter are suspected, dilutions of $1: 200,1: 250,1: 300$, etc., are made. One cubic centimeter of each of these is mixed with the $\mathrm{L}_{+}$dose of the toxin, and the mixtures are injected into guinea-pigs of about 250 grams. If the guinea-pig receiving $\mathrm{L}_{+}$plus the $1: 250$ dilution lives and the one receiving $\mathrm{L}_{+}$plus the $1: 300$ dilution dies in the given time, we know that the unit sought must lie between these two values, and further similar experiments will easily limit it more exactly. The possibility of error in

1 A unit of diphtheria antitoxin is a quantity of antitoxin sufficient to protect a guinea-pig of 250 grams against 100 times the fatal dose of diphtheria toxin.

2 Madsen, in Kraus u. Levaditi, "Handbuch," etc., 1907.

3 Dönitz, "Die Werthbem. der Heilsera," in Kolle u. Wassermann, "Handbuch," etc. 
measurement is much diminished by the use of larger quantities of dilutions higher than those given. 'Four c.c. is the volume usually injected.

Since 1902, the production and sale of diphtheria antitoxin has been regulated by law in the United States. From time to time, antitoxin is bought in the open market and examined at the hygienic laboratories of the United States Public Health and Marine Hospital Service. Antitoxic serum which contains less than two hundred units to each cubic centimeter is not permitted upon the market.

In a previous section we have seen that Hiss and Atkinson ${ }^{1}$ and others have shown an increase in the globulin contents of blood serum of immunized animals. It has been shown, furthermore, that the precipitation of such serum with ammonium sulphate carried down in the globulin precipitate all the antitoxic substances contained in the serum. Upon a basis of globulin precipitation, Gibson ${ }^{2}$ has recently perfected a method of concentrating and purifying diphtheria antitoxin for therapeutic use. This procedure, as carried out at the New York Department of Health, is, in principle, as follows:

The serum, as taken from the horse, is heated to $56^{\circ} \mathrm{C}$. for twelve hours. This converts about half of the pseudoglobulin into euglobulin, the antitoxin remaining in the pseudoglobulin fraction. ${ }^{3}$ It is then ${ }^{4}$ precipitated with an equal volume of a saturated ammonium sulphate solution. After two hours, the precipitate is caught in a filter and redissolved in a quantity of water corresponding to the original quantity of serum. After filtration, this solution is again precipitated with saturated ammonium sulphate solution and the precipitate again filtered off. The precipitate is then treated with a saturated solution of sodium chloride of double the volume of the original serum. This is allowed to stand for about twelve hours. At the end of this time the antitoxin-containing globulin is in solution and is pipetted away from the precipitate and filtered. This salt-solution extract is then precipitated with twenty-five hundredths per cent acetic acid. The resulting precipitate of globulin is thoroughly dried by pressure between filter papers and placed in a parchment dialyzer. Dialysis with running water is continued for seven to eight days, after neutralization with sodium carbonate, in order to remove the sodium chloride. At the end of this time, the globulin solution remaining in the dialyzer is fil-

${ }^{1}$ Hiss and Atkinson, Jour. Exper. Med., v, 1900.

${ }^{2}$ Gibson, Jour. of Biol. Chem., i, 1906.

3 Dr. Banzhaf, personal communication.

${ }^{4}$ Gibson and Collins, Jour. of Biol. Chem., iii, 1907. 
tered through a Berkefeld candle for the purpose of sterilization, after the addition of 0.8 per cent sodium chlorid. According to Gibson, this method produces a yield of antitoxin which equals about four-fifths of the original quantity but is concentrated five- to seven-fold. The method has more recently been modified as follows:

After heating to $56^{\circ} \mathrm{C}$, as above, and cooling, ammonium sulphate is added to the serum to thirty per cent saturation. This brings down all the euglobulins. This is then filtered and the filtrate, which contains the pseudoglobulins with the antitoxin, is again precipitated with ammonium sulphate in a concentration of fifty-four per cent of saturation. The precipitate is then separated on a paper, pressed to dryness, and directly dialyzed. ${ }^{1}$

Park and Thorne ${ }^{2}$ have found that the use of such concentrated antitoxin is, therapeutically, equally efficient as the unconcentrated, and possesses the advantage of less frequently giving rise to the secondary reactions in skin and mucous membranes occasionally noticed after the use of ordinary antitoxin, and referable, probably, to some other constituent of the horse serum.

Diphtheria antitoxin is therapeutically used in doses ranging from 3,000 to 20,000 units. For prophylactic immunization of healthy individuals, about 500 units should be used.

\section{TETANUS ANTITOXIN}

Production of Tetanus Antitoxin.-The production of tetanus antitoxin is, in every way, analogous to that of diphtheria antitoxin. It is necessary in the first place to produce a powerful tetanus toxin. The methods of procuring this will be discussed in the section upon tetanus toxin, page 458. Suffice it to say here that the most satisfactory method of obtaining toxins consists in cultivating the bacilli upon veal broth containing five-tenths per cent to two per cent sodium chlorid and one per cent pepton. It has been advised, also, that the broth should be neutralized by means of magnesium carbonate rather than with sodium hydrate. The bacilli are cultivated for eight to ten days at incubator temperature and the broth filtered rapidly through Berkefeld filters. The toxin may be preserved in the liquid form with the addition of five-tenths per cent carbolic acid, or may be preserved in the dry state after precipitation with ammonium sulphate.

${ }^{1}$ Dr. Banzhaf', personal communication.

${ }^{2}$ Park and Throne, Amer. Jour. Med. Sci., Nov., 1906. 
It is necessary to determine the strength of the poison. This is done according to Behring ${ }^{1}$ by determining the smallest amount of toxin which will kill a white mouse of twenty grams weight within four days. This is most easily done by making dilutions of the toxin ranging from $1: 100$ to $1: 1,000$, and then injecting quantities of 0.1 c.c. of each of these dilutions subcutaneously into white mice. In this way, the minimal lethal dose is ascertained.

For the actual production of antitoxin, horses have been generally found to be the most favorable animals. The horses should be healthy and from five to seven years old. The first injection of toxin administered to these animals should be attenuated in some way. Various methods for accomplishing this have been in use. In America, the first injection of about ten to twenty thousand minimal lethal doses ${ }^{2}$ (for mice of twenty grams weight) is usually made subcutaneously together with sufficient antitoxin to neutralize this quantity. In Germany, v. Behring uses, for his first injection, a much larger dose of toxin to which about 0.25 per cent of terchlorid of iodin has been added. Inmediately after an injection, the animals will usually show a reaction expressed by a rise of temperature, refusal of food, and sometimes muscular twitching. A second injection should never be given until all such symptoms have completely subsided. This being the case, after five to eight days double the original dose is given together with a neutralizing amount of antitoxin or with the addition of terchlorid of iodin. Again after five to eight days, a larger dose is given and thereafter, at similar intervals, the quantity of toxin is rapidly increased. In America the neutralizing antitoxin is omitted after the third or fourth injection; in v. Behring's laboratory the quantity of terchlorid of iodin is gradually diminished. The increase of dosage is often controlled by the determination of the antitoxin contents of the animal's blood serum. The immunization is increased until enormous doses (500 c.c.) of a toxin in which the minimal lethal dose for mice is represented by 0.0001 c.c., or less, is borne by the horse without apparent harm.

The antitoxic serum is then obtained by bleeding from the jugular vein, as in the case of diphtheria antitoxin. It may be preserved in the liquid state by the addition of five-tenths per cent of carbolic acid or four-tenths per cent of tricresol.

${ }^{1} v$. Behring, Zeit. f. Hyg., xii, 1892 ; Deut. med. Woch., 1900.

${ }^{2}$ According to Park the "horses receive 5 c.c. as the initial dose of a toxin of which 1 c.c. kills 250,000 grams of guinea-pig, and along with this a sufficient amount of antitoxin to neutralize it." 
Standardization.-The universal prophylactic use of tetanus antitoxin has, as in the case of diphtheria antitoxin, necessitated its standardization. A variety of methods are in use in different parts of the world. In the following description the American method only will be considered as laid down under the law of July, 1908, and based upon the work of Rosenau and Anderson ${ }^{1}$ at the United States Hygienic Laboratories at Washington.

In conjunction with a committee of the Society of American Bacteriologists, these authors have defined the unit of tetanus antitoxin as follows:

The unit shall be ten times the least amount of serum necessary to save the life of a 350 gram guinea-pig for ninety-six hours against the official test dose of standard toxin. The test dose consists of 100 minimal lethal doses of a precipitated toxin preserved under special conditions at the hygienic laboratory of the Public Health and Marine Hospital Service. (The minimal lethal dose is in this case, unlike Behring's minimal lethal dose, measured not against 20 gram mice, but against 350 gram guinea-pigs.)

In the actual standardization of tetanus antitoxin, as in that of diphtheria antitoxin, the $\mathrm{L}_{+}$dose of toxin is employed. The $\mathrm{L}_{+}$dose is, however, in this case, defined as the smallest quantity of tetanus toxin that will neutralize one-tenth of an immunity unit, plus a quantity of toxin sufficient to kill a 350 gram guinea-pig in just four days. At the Hygienic Laboratory at Washington, a standard toxin and antitoxin are preserved under special conditions, and standard toxin and antitoxin, arbitrary in their first establishment, are kept constant by being measured against each other from time to time. In measuring the antitoxic serum thus preserved, at the Hygienic Laboratory, a mixture of one-tenth of a unit of antitoxin and 100 minimal lethal doses of the standard toxin must contain just enough free poison to kill the guineapig in four days. This $\mathrm{L}_{+}$dose of the standard toxin is given out to those interested commercially orotherwise in the production of antitoxin.

In measuring an unknown antitoxic serum against this $\mathrm{L}_{+}$dose of toxin, a large number of mixtures are made, each containing the $\mathrm{L}_{+}$dose of the toxin and varying quantities of the antitoxin. Dilutions must always be made with 0.85 per cent salt solution and the total quantity injected into the animals should always be brought up to

${ }^{1}$ Rosenau and Anderson, Pub. Health and Mar. Hosp. Serv. U. S., Hyg. Lab. Bull. 43, 1908. 
4 c.c. with salt solution in order to equalize the conditions of concentration and pressure. The mixtures are then kept for one hour at room temperature in diffused light. After this they are subcutaneously injected into a series of guinea-pigs weighing from 300 to 400 grams. The following example of a test is taken from the article by Rosenau and Anderson quoted above.

\begin{tabular}{|c|c|c|c|c|}
\hline \multirow{2}{*}{$\begin{array}{l}\text { No. of } \\
\text { Guinea- } \\
\text { pig. }\end{array}$} & \multirow{2}{*}{$\begin{array}{l}\text { Weight of } \\
\text { Guinea-pig } \\
\text { (Grams.) }\end{array}$} & \multicolumn{2}{|c|}{$\begin{array}{l}\text { Subcutaneous Injection of a } \\
\text { Mixture of }\end{array}$} & \multirow{2}{*}{ Time of Death. } \\
\hline & & $\begin{array}{l}\text { Toxin } \\
\text { (Test Dose). }\end{array}$ & Antitoxin. & \\
\hline $\begin{array}{l}1 \\
2 \\
3 \\
4 \\
5\end{array}$ & $\begin{array}{l}360 \\
350 \\
350 \\
360 \\
350\end{array}$ & $\begin{array}{r}\text { Gram. } \\
0.0006 \\
.0006 \\
.0006 \\
.0006 \\
.0006\end{array}$ & $\begin{array}{l}\text { c.c. } \\
0.001 \\
.0015 \\
.002 \\
.0025 \\
.003\end{array}$ & $\begin{array}{l}2 \text { days } 4 \text { hours } \\
4 \text { days } 1 \text { hour } \\
\text { Symptoms } \\
\text { Slight symptoms } \\
\text { No symptoms }\end{array}$ \\
\hline
\end{tabular}

In this series the guinea-pig, receiving 0.0015 c.c. of the antitoxin, died in approximately four days; 0.0015 c.c. therefore represents one-tenth of an immunity unit.

In therapeutically employing antitoxin for prophylactic purposes, about 1,500 units should be employed. 


\section{CHAPTER XV}

\section{LYSINS, AGGLUTININS, PRECIPITINS, AND OTHER ANTIBODIES}

\section{LYSINS}

IN the immediately preceding sections, we have dealt solely with immunity as it occurs where soluble toxins play an important part and in which antitoxins are developed in the immunized subject. There are many species of pathogenic bacteria, however, which stimulate the production of little or no antitoxic substance when introduced into animals, and the resistance of the immunized animal can not, therefore, be explained by the presence of antitoxin in the blood.

v. Fodor, ${ }^{1}$ Nuttall, ${ }^{2}$ Buchner,${ }^{3}$ and others had in 1886 and the years following carried on investigations which showed that normal blood serum possessed the power of killing certain of the pathogenic bacteria. Nuttall, working under the direction of Flügge, made the important discovery that this bactericidal power became gradually diminished with time, and could be experimentally destroyed by exposure of the serum to a temperature of $56^{\circ} \mathrm{C}$. for one-half hour. Buchner, who confirmed and extended the observations of Nuttall, called this thermolabile substance upon which the bactericidal character of the serum seemed to depend "alexin."

Our knowledge of the bactericidal action of serum was, soon thereafter, extensively increased by the discovery, by Pfeiffer and Isaeff, ${ }^{4}$ that cholera spirilla injected into the peritoneal cavity of a choleraimmune guinea-pig were promptly killed and almost completely dissolved. The same phenomenon could be observed when the spirilla, mixed with fresh immune serum, were injected into the peritoneum of a normal guinea-pig.

The processes observed by Pfeiffer as taking place intraperitoneally were soon shown by Metchnikoff, ${ }^{5}$ Bordet, ${ }^{6}$ and others to take place, though to a lesser extent, in vitro. Bordet, furthermore, observed that

$1 v$. Fodor, Deut. med. Woch., 1886.

${ }^{3}$ Buchner, Cent. f. Bakt., 1889.

5 Metchnikoff, Ann. de l'inst. Pasteur, 1895.
${ }^{2}$ Nuttall, Zeit. f. Hyg., 1886.

${ }^{4}$ Pfeiffer und Isaeff, Zeit. f. Hyg., 1894.

${ }^{6}$ Bordet, ibid., 1895. 
the bacteriolytic digestive power of such immune serum, when destroyed by heating, or after being attenuated by time, could be restored by the addition to it of small quantities of normal blood serum. It could, in other words, be "reactivated" by normal serum. From this observation Bordet drew the conclusion that the bactericidal or bacteriolytic action of the serum depended upon two distinct substances. The one present in normal serum and thermolabile, he conceived to be identical with Buchner's alexin. The other, more stable, produced or at least increased in the serum by the process of immunization, he called the "sensitizing substance." This substance, he believed, acting upon the bacterial cells, rendered them vulnerable to the action of the alexin. Without the previous preparatory action of the "sensitizing substance" the alexin was unable to act. Without the co-operation of alexin, the "sensitizing substance" produced no visible effects.

Bordet's interpretation of the phenomenon of lysis differs essentially from that of Ehrlich, in that both active serum components are conceived by him, though independent, to act directly upon the bacterial cell. A few years later, Bordet was able to show that exactly analogous conditions governed the phenomenon known as "hemolysis" or disintegration of red blood cells.

It had been known for many years that in the transfusion of blood from an animal of one species into an animal of another species, injury was done to the red corpuscles which were introduced. Observed in the test tube, the red cells in the heterologous serum were seen to give up their hemoglobin in the fluid, the mixture taking on the red transparency characteristic of what is known as "laked" blood. Buchner, ${ }^{1}$ in his alexin studies, had shown that the blood-cell destroying action of the normal serum was subject to the same laws as the bactericidal power of similar serum, in that it was destroyed by heating, and he assumed that both the bacteriolytic and the hemolytic action of normal serum were due to the same "alexin." Metchnikoff, ${ }^{2}$ moreover, had pointed out the striking analogy between the two phenomena as early as 1889 .

Bordet ${ }^{3}$ now observed that the blood serum of guinea-pigs previously treated with the defibrinated blood of rabbits developed marked powers of dissolving rabbits' corpuscles, and that this hemolytic action could

1 Buchner, Arch. f. Hyg., xvii, 1893; Waremberg, Arch. d. méd. exper., 1891.

, 2 Metchnikoff, Ann. de l'inst. Pasteur, 1889.

3 Bordet, Ann, de l'inst. Pasteur, t. xii, 1898. 
be destroyed by heating to $56^{\circ} \mathrm{C}$., but "reactivated" by the addition of fresh normal serum. He had thus produced an immune hemolysin, just as Pfeiffer had produced immune bacteriolysin, and had demonstrated the complete parallelism which existed between the two phenomena.

A practical test-tube method was thus given for the investigation of the lysins, just as a practical test-tube method for antitoxin researches had been developed by Ehrlich in his ricin-antiricin experiments.

The path of investigation thus pointed out by Bordet was soon explored in greater detail by Ehrlich and Morgenroth. ${ }^{1}$ The reasoning which Ehrlich had applied in explaining the production of antitoxins was thought, by these observers, to be equally applicable to the phenomena of bacteriolysis and hemolysis.

Since the thermolabile substance or alexin, renamed by Ehrlich "complement," was already present in normal serum and had been shown to be little, if at all, increased during the process of immunization, this substance could have but little relation to the changes taking place in the animal body as immunity was acquired. 'The more stable serumcomponent, however, the "substance sensibilisatrice" of Bordet, or, as Ehrlich now called it, the "immune body," was the one which seened specifically called forth by the process of active immunization. Ehrlich argued, therefore, that when bacteria or blood cells were injected into the animal, certain atom-groups or chemical components of the injected substances were united to other atom-groups or "side chains" of the protoplasm of the tissue cells. These "side chains" or receptors, then reproduced in excess and finally thrown free into the circulation, constituted the "immune body." The immune body, therefore, he concluded, must possess atom complexes which endow it with specific chemical affinity for the bacteria or red blood cells used in its production. This contention was supported by Ehrlich and Morgenroth by an ingenious series of experiments.

Having in their possession, at that time, the blood serum of a goat immunized against the red blood cells of a sheep, they inactivated it (destroyed the complement or alexin) by heating to $56^{\circ} \mathrm{C}$. The serum then contained only the "substance sensibilisatrice" or immune body. To this inactivated serum they added sheep's red corpuscles, without obtaining hemolysis. Having left the inactive serum and the sheep's corpuscles in contact with each other for some time, they separated 
them by centrifugalization. To the supernatant fluid, they now added sheep-blood corpuscles and normal goat serum (complement) and found that no hemolysis took place. The immune body had apparently gone out of the serum. The red cells which had been in contact with the serum and separated by the centrifuge were then washed in salt solution and to them complement was added in the form of fresh normal serum.

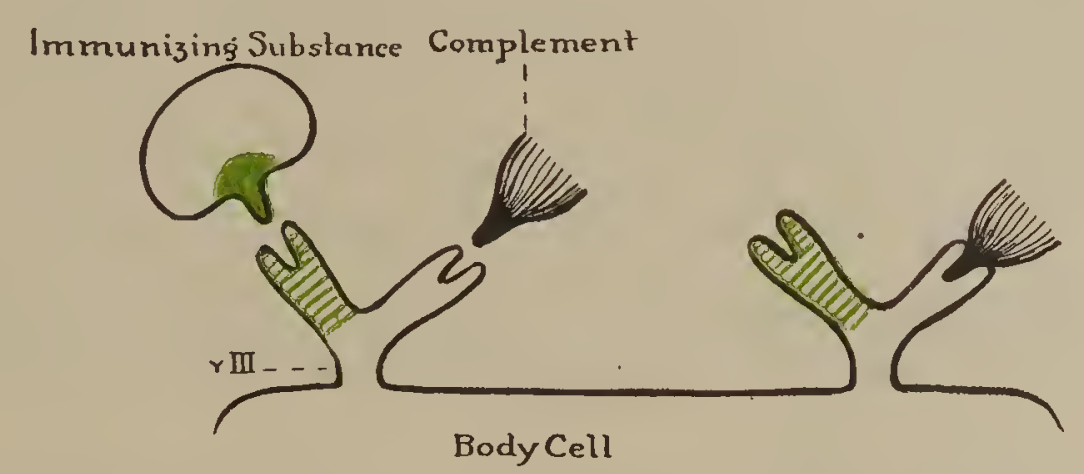

Fig. 57.-Ehrlich's Conception of Cell-Receptors, Giving Rise to Lytic Immune Bodies (Haptines of the Third Order).

Hemolysis occurred. It was plain, therefore, that the immune body of the inactivated serum had gone out of solution and had become attached to the red blood cells, or, as Fhrlich expressed it, the immune body by means of its "haptophore" atom-group had become united to the corpuscles. In contrast to this, if normal goat serum (containing

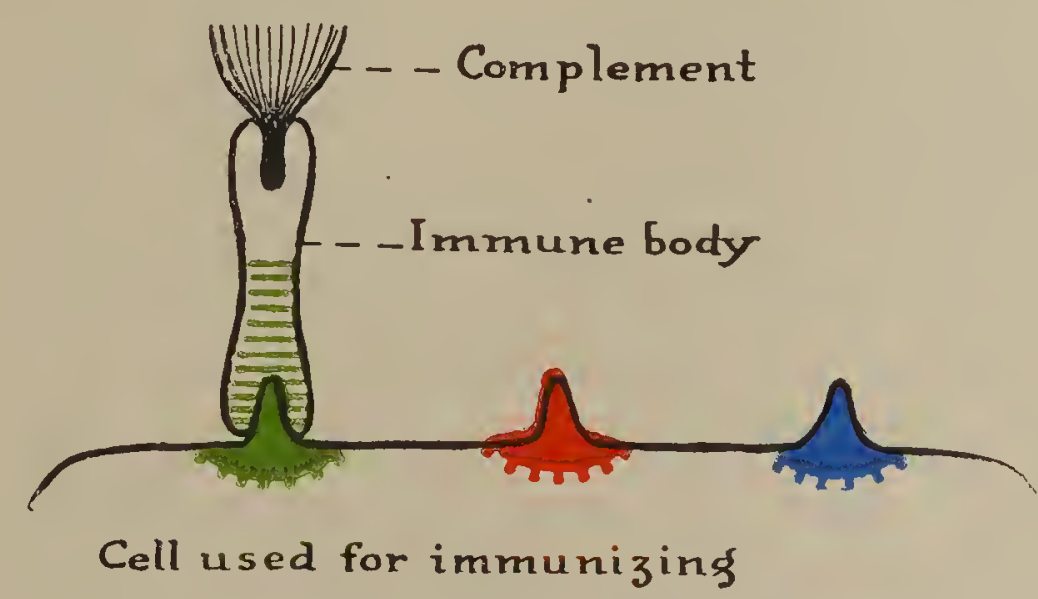

Fig. 58.-Complement, Amboceptor or Immune Body, and Antigein on Immunizing Substance.

complement only) was added to sheep corpuscles and separated again by centrifugalization, the supernatant fluid was found to be still capable of reactivating inactivated serum (immune body). This he interpreted as proving that the complement was not bound to the corpuscles directly.

If the three factors concerned-corpuscles, immune body, and complement-were mixed and the mixture kept at $0^{\circ} \mathrm{C}$., no hemolysis 
occurred; yet, centrifugalized at this temperature, immune body was found to have become bound to the corpuscles, the complement remaining free in the supernatant fluid. If the same mixture, however, was exposed to $37^{\circ} \mathrm{C}$., hemolysis promptly occurred.

From these facts, Ehrlich concluded that complement did not directly combine with the corpuscles, but did so through the intervention of the immune body. This immune body, he reasoned, possessed two distinct atom-groups or haptophores; one, the cytophile haptophore group, possessing strong chemical affinity for the red blood cell; the other, or complementophile haptophore group, with weaker avidity for the complement. Because of this double combining power, Ehrlich speaks of the immune body as " amboceptor." His views as to the nature and action of immune body and complement are graphically represented in Figs. 57 and 58 (p. 227).

From what has been said before, it will be seen that the fundamental difference between the conceptions of the mechanism of the lytic processes as held by Bordet and by Ehrlich lies in the ability of the alexin or complement to act directly upon the antigen, as claimed by Bordet, or, as Ehrlich holds, only through the intermediation of the immune body. Bordet's views, ${ }^{1}$ by no means disproved and still held by many bacteriologists, may be summed up in his own words as follows: "Neither immune body nor antigen (bacterium, blood cell, etc.) alone has any manifest affinity for alexin (complement); but, united, they form a complex which can absorb alexin." The absorption of complement is thus conceived as a property of the immune body or amboceptor (or, in Bordet's language, sensitizẹr) plus its specific antigen-acting as a complex and not through a complementophile group of the immune body.

\section{AGGLUTININS}

Although Metchnikoff ${ }^{2}$ and Charrin and Roger ${ }^{3}$ had noticed peculiarities in the growth of bacteria when cultivated in immune sera, which were unquestionably due to agglutination, the first recognition of the agglutination reaction as a separate function of immune sera was the achievement of Gruber and Durham. While investigating the Pfeiffer reaction with B. coli and the cholera vibrio, Gruber and Durham ${ }^{4}$

${ }^{1}$ Bordet, A Résumé of Immunity in "Studies in Immunity." Trarısl. by Gay, Wiley \& Son, 1909.

2 Metchnikoff, "Etudes sur l'immunité," IV Memoir, 1891.

${ }^{3}$ Charrin et Roger, Compt. rend. de la soc. de biol., 1889.

${ }^{4}$ Gruber und Durham. Münch. med. Woch., 1896. 
noticed that if the respective immune sera were added to bouillon cul. tures of these two species, the cultures would lose their turbidity and flake-like clumps would sink to the bottom of the tube, the supernatant fluid becoming clear. Gruber, at the same time, called attention to the fact that immune sera would affect in this way not only the microorganism used in their production, but, to a less energetic extent, other closely related bacteria as well.

Widal, very soon after Gruber and Durham's announcement, applied the agglutination reaction to the practical diagnosis of typhoid fever, finding that the serum of patients afflicted with this disease showed agglutinating power over the typhoid bacillus at early stages in the course of the fever. The reaction, thus practically applied to clinical diagnosis, was soon shown to be of great importance in its
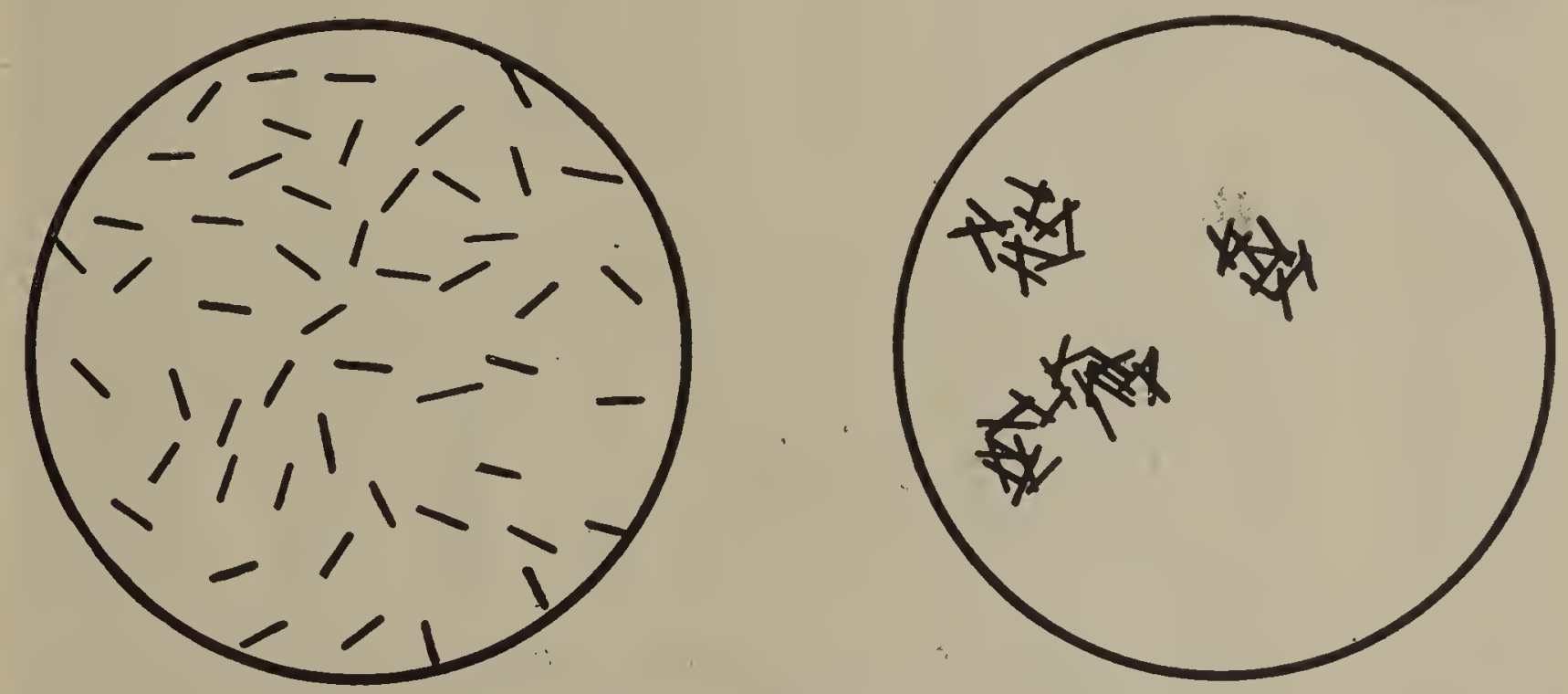

Fig. 59.-Mrcroscopic Agglutination Reaction.

bearing on bacteriological' species differentiation. Since animals immunized against a definite species of bacteria acquire in their sera specific agglutinating powers for these bacteria and at best only slight agglutinating powers for other species, immune sera can be used extensively in differentiating between bacterial varieties.

Agglutination may be observed microscopically or macroscopically. Bacteria brought into contact with agglutinating serum in the hanging drop rapidly lose their motility, if motile, as in the case of typhoid bacilli, and gather together in small clumps or masses. The microscopic picture is striking and easily recognized and the reaction takes place with varying speed and completeness, according to the strength of the agglutinating serum.

As the reaction approaches completeness, the clumps grow larger, 
individual microorganisms become more and more scarce, finally leaving the medium between clumps entirely clear. While the clumping of a motile organism suggests that motility has something to do with the coming together in clumps, it nevertheless has no relation whatever to agglutination, motile and non-motile organisms alike being subject to the reaction.

Macroscopically observed, in small test tubes or capillary tubes, agglutination evidences itself by the formation of flake-like masses which

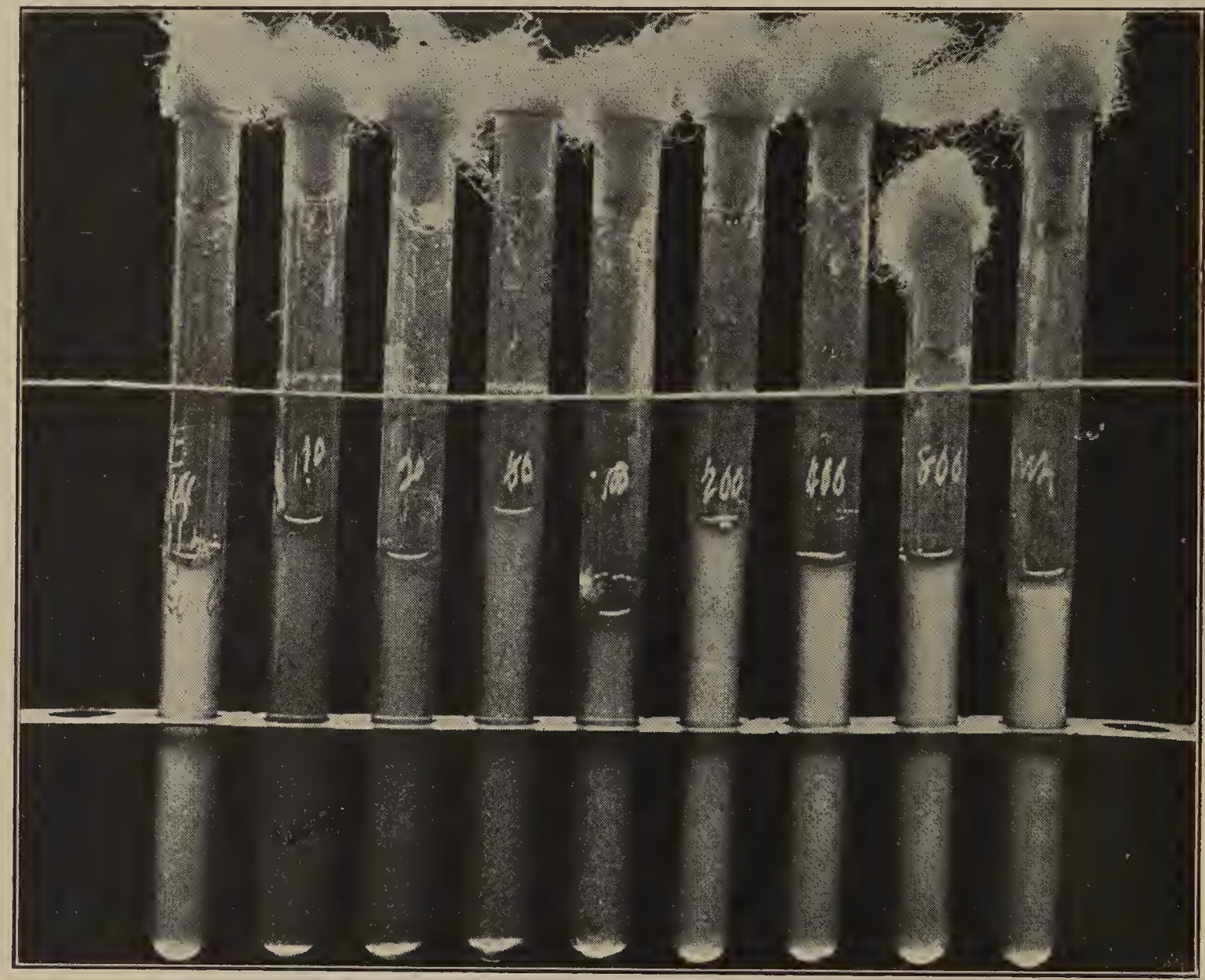

Fig. 60.-Macroscopic Agglutination. Dilutions from 1 in 10 to 1 in 1,000. The first tube contains a $1: 20$ control with the bacteria and normal serum. Agglntination complete in the tubes marked 10, 20, 50, 100.

settle into irregular heaps at the bottom, leaving the supernatant fluid clear, in distinct contrast to the even flat sediment and the clouded supernatant fluid of the control. Macroscopically, too, agglutination is evidenced when bacteria are grown in broth to which immune serum has been added. Instead of evenly clouding the broth, the microorganisms develop in clumps or chains.

Another phenomenon probably produced by agglutinins is the so- 
called "thread-reaction", of Pfaundler." This consists in the formation of long convoluted threads of bacterial growth in the hanging drop of dilute immune serum after twenty-four hours. Very strict specificity is attributed to this reaction by Pfaundler.

Agglutinins act upon dead as well as upon living bacteria. For the microscopic tests bacterial emulsions killed by formalin were introduced by Neisser.

Ficker ${ }^{2}$ has recently succeeded in preparing an emulsion of typhoid bacilli, which is permanent and may be kept indefinitely, and may be employed for macroscopic agglutinations. ${ }^{3}$

Attention has been called by various workers to a source of error in all these methods, known as pseudo-clumping. ${ }^{4}$ The causes for such clumping not due to agglutinins seem to lie in the presence of blood cells in the serum or excessive alkalinity of the culture medium. ${ }^{5}$

While the microscopic methods are more suitable for clinical-diagnostic purposes, because of the smaller amounts of blood required, the macroscopic tests are far preferable for the purposes of bacterial differentiation and research. Greater exactitude of dilution is possible when dealing with larger quantities; microscopic unevenness in the bacterial emulsion does not become a source of error; and positive and negative reactions are more sharply defined.

Nature of Agglutinins.- Gruber and Durham, ${ }^{6}$ the discoverers of agglutinins, at first advanced the opinion that the agglutinins were identical with the immune body concerned in the Pfeiffer reaction, which by injuring the bacteria rendered them susceptible to the alexins. Pfeiffer ${ }^{7}$ and Kolle ${ }^{8}$ soon showed, however, that by the addition of cholera vibrio to immune serum, the agglutinins could be completely absorbed, or used up, while bacteriolytic substances still remained. The same authors demonstrated that immune serum, preserved for several months, would lose its agglutinins without a corresponding loss of bacteriolytic power. It has been variously shown since then, by these and other author's, that the agglutinins and the bactericidal substances are in no way parallel

1 Pfaundler, Cent. f. Bakt., xxiii, 1898.

${ }^{2}$ Ficker, Berl. klin. Woch., 1903.

3 The exact method of production of "Ficker's Diagnosticum" is a proprietary secret.

- Savage, Jour. of Path. and Bact., 1901.

${ }^{5}$ Biggs and Park, Amer. Jour. of Med. Sci., 1897; Block, Brit. Med. Jour., 1897.

${ }^{6}$ Loc. cit.

7 Pfeiffer, Deut. med. Woch., 1896.

${ }^{8}$ Pfeiffer und Kolle, Cent. f. Bakt., xx, 1896. 
in their development, and that strongly agglutinating sera may be extremely weak in bactericidal substances and vice versa, the relative quantity of either of these substances depending to a certain extent upon the method of immunization. Whether or not agglutinins possess any direct protective function can not at present be stated with certainty. Metchnikoff ${ }^{1}$ assigns to them a purely secondary rôle. As a matter of fact, agglutinated bacteria ${ }^{2}$ are not killed by the act of agglutination and are often as virulent as non-agglutinated cultures.

The agglutinins, furthermore, unlike the bactericidal substances in sera, remain active after exposure to temperatures of over $55^{\circ} \mathrm{C}$, , some of them withstanding even $65^{\circ}$ to $70^{\circ}$, and can not be reactivated by the subsequent addition of normal serum. These facts definitely preclude the participation in the reaction of the alexin or complement and have an important bearing upon Ehrlich's views of their structure ${ }^{3}$ (see page 238 ).

As a result of these and a multitude of other studies, the agglutinins have come to be regarded as separate antibodies, closely related to the precipitins.

The agglutinins may be chemically precipitated out of serum together with the globulins. They do not dialyze. Bordet ${ }^{4}$ made the observation that agglutinins do not act in the absence of $\mathrm{NaCl}$. Whether the presence of the salt aids the reaction in a chemical or purely physical way, as Bordet supposed, is uncertain.

Production of Agglutinins.- Just as normal sera contain small quantities of bactericidal substances, so do they contain agglutinins in small amount. In a general way these "normal agglutinins" have the same nature as the immune agglutinins, and it is probable that their presence is traceable to the various microorganisms parasitic upon the human and animal body.

As a matter of fact, the blood serum of new-born guinea-pigs hardly ever contains agglutinin for $\mathrm{B}$. coli, while that of adults acts upon these bacilli in dilutions of $1: 20 .^{5}$ Similarly, infants show lower normal agglutinating values than adults. ${ }^{6}$

: Metchnikoff, “L'immunité," etc., 1901, p. 214.

${ }^{2}$ Mesnil, Ann. de l'inst. Pasteur, 1898.

${ }_{3}$ Pane, Cent. f. Bakt., 1897; Trumpp, Arch. f. Hyg., 1898; F örster, Zeit. f. Hyg., xxiv.

${ }^{4}$ Bordet, Ann. de l'inst. Pasteur, 1899.

${ }^{5}$ Kraus und Löw, Gesell. d. Aerzte, Wien, 1899.

${ }^{6}$ Pfaundler, Jahrb. f. Kinderheilk., Bd. 50. 
Agglutinins may be produced in the sera of animals by the introduction of microorganisms subcutaneously, intravenously, or intraperitoneally. The intravenous method seems to give the most abundant and speedy results. ${ }^{1}$ The formation of agglutinins is a reaction to the bodysubstances of the bacteria themselves, rather than to their toxic products. Thus agglutinins are produced in response to the introduction of dead bacteria and soluble extracts of cultures. Pathogenicity ${ }^{2}$ does not influence agglutinin formation to any great extent, non-pathogenic as well as pathogenic giving rise to these substances in serum. As a rule, however, agglutinins are more easily produced against avirulent than against fully virulent strains of bacteria of the same species.

While agglutinins can be produced with almost all the known bacteria, there are great differences between various species in the quantity and speed of production, and Nicolle and Thenel ${ }^{3}$ have classified bacteria in three groups according to their power of stimulating the production of agglutinins in immunized animals. As a rule, the agglutinins appear in the blood of animals three to six days after the introduction of bacteria. From the third to the sixth day they rapidly increase to a maximum at the seventh to thirteenth day. They then fall off rapidly until they reach a level at which they remain for a long period without very considerable change. Curves to illustrate these phases have been constructed by Jorgensen and Madsen. ${ }^{4}$

The Reaction between Agglutinin and Agglutinin-Stimulating Substances (Agglutinogen). - The fact that agglutinin can be removed from, or absorbed out of, serum by the specific bacilli which have led to its formation indicates that there is in the act of agglutination a combination between the agglutinin and the agglutinin-stimulating substance (agglutinogen). It is likely that this combination is of a chemical nature, since; as we have mentioned, agglutinins result from the injection of bacterial extracts as well as from the introduction of living bacteria. The probability that the process follows chemical laws of combination is furthermore strengthened by the work of $\mathrm{Joos}^{5}$ and others, who have demonstrated that definite quantitative relations exist between the agglutinin-stimulating substances and the agglutinins. Every agglutination reaction, therefore, will vary in its degree of com-

1 Hoffmann, Hyg. Rundschau, 1903.

2 Nicolle, Ann. de l'inst. Pasteur, 1898.

3 Nicolle et Thenel, Ann. de l'inst. Pasteur, 1902.

${ }^{4}$ Jorgensen and Madsen, Festschrift, Kopenhagen, 1902.

5 Joos, Zeits, f, Hyg., xxxvi, 1901. 
pleteness with the quantities of agglutinin and agglutinogen, a fact which makes it necessary, especially for clinical tests, to preserve a certain uniformity in the quantity and density of the bacterial culture or emulsion employed.

Specificity.-From the very beginning, Gruber and Durham ${ }^{1}$ had claimed specificity for the agglutination reaction, and in this sense it was clinically utilized by Widal for the diagnosis of typhoid fever. It was noticed, however, even by these earliest workers, that the serum of an animal immunized against one microorganism would often agglutinate, to a less potent degree, other closely related species. Thus, the serum of a typhoid-immune animal may agglutinate the typhoid bacillus in dilutions of $1: 1,000$, and the colon bacillus in dilutions as high as $1: 200$; while the agglutinating power of normal serum for the colon bacillus rarely exceeds $1: 20$. The specificity of the reaction for practical purposes, thus, is not destroyed if proper dilution is carried out, the degree of agglutinin formation being always far higher for the specific organism used in immunization than it is for allied organisms. The specific immune-agglutinin in such experiments is spoken of as the chief agglutinin (hauptagglutinin), and the agglutinins formed parallel with it, as the partial agglutinin (metagglutinin), terms introduced by Wassermann. Hiss has spoken of these as major and minor agglutinins. The relative quantities of the specific chief agglutinin and partial agglutinins present in any immune serum depend upon the individual cultures used for immunization, and the phenomenon is probably dependent upon the fact that certain elements in the complicated bacterial cell-body may be common to several species and find common receptors in the animal body. Whenever an immune serum agglutinates a number of members of the group related to the specific organism used for its production, the reaction is spoken of "group agglutination."

The partial agglutinins (metagglutinins) have been extensively studied by Castellani ${ }^{2}$ and others, by a method spoken of as the "absorption method." This consists in the separate addition of bacterial emulsions (agglutinogens) of the various species concerned in a group agglutination, to the agglutinating serum. In this way, specific and partial agglutinins can be separately removed from the immune serum by absorption-each by its corresponding agglutinogen. In such experiments all agglutinins will bo removed by the organisms used for im-

${ }^{1}$ Gruber und Durham, loc. cit.

${ }^{2}$ Cast llani, Zeits. f. Hyg., xl, 1902. 
munization, a partial removal only resulting from the addition of allied strains. This method has thrown much light upon the intimate relations existing between members of various bacterial species, and has been particularly valuable in the study of the typhoid-colon-dysentery group. It is important to mention, however, that "groups" as determined by agglutination tests do not always correspond to classifications depending upon morphological and cultural characteristics.

An interesting phenomenon of great practical importance, which has been noticed by a number of observers, and which may often be encountered in routine agglutination tests, is the frequent failure of a strongly agglutinating serum to produce agglutination if used in concentration, while in dilutions it produces a characteristic reaction. This has been explained theoretically by what is known as the "proagglutinoid zone." It is assumed that agglutinins may deteriorate as do toxins and be converted into substances which are capable of combining with agglutinogen without causing agglutination. Such substances, as we will see in discussing Ehrlich's views on the structure of agglutinins, may have a stronger affinity for agglutinogen than the agglutinins themselves, and are, therefore, termed "proagglutinoids." In strongly agglutinating sera these proagglutinoids may be present in considerable quantities and prevent the combination of agglutinin with agglutinogen. In dilution, this proagglutinoid action would naturally become weaker and of no actual significance in obscuring the reaction.

Agglutination, like other immune phenomena, is a manifestation of broad biological laws and not limited to bacteria. Thus, as hemolysins are produced by the injection of red blood cells, so hemagglutinins, or substances which clump together red blood cells, are similarly formed.

\section{PRECIPITINS}

\section{(Coagulins)}

In the year 1897, R. Kraus, ${ }^{1}$ of Vienna, demonstrated that the sera of animals immunized against B. pestis, B. typhosus, and Vibrio choleræ, when mixed with the clear filtrate of bouillon cultures of the respective organisms, gave rise to macroscopically visible precipitates. The precipitates occurred only when filtrate and immune serum were homologous, that is to say, the animal from which the serum was obtained was immunized by the same species of microorganism as that used in the test; and for this reason Kraus spoke of them as "specific

\footnotetext{
${ }^{1}$ Kraus, Wien. klin. Woch., 1897.
} 
precipitates." It was evident, therefore, that during the process of active immunization with these organisms, a specific antibody had been produced in the serum of the treated animal, which, because of its precipitating quality, was námed "precipitin." This peculiar reaction was soon found to hold good, not only for the bacteria used by Kraus, but also for other bacteria, few failing to stimulate the production of specific precipitins in the sera of immunized animals. The phenomenon of precipitation, however, is not limited to bacterial immunization, but has been found, like the phenomena of agglutination and lysis, to depend upon biological laws of broad application. Thus, Bordet ${ }^{1}$ found that the blood serum of rabbits treated with the serum of the chicken gave a specific precipitate when mixed with chicken serum. Tchistovitch ${ }^{2}$ demonstrated a similar reaction with the sera of rabbits treated with horse and eel sera. By the injection of milk, Wassermann, ${ }^{3}$ Schütze, ${ }^{4}$ and others produced an antibody which precipitated the casein of the particular variety of milk employed for immunization. The reaction was thus applicable to many albuminous substances. These substances, because of their precipitin-stimulating quality, are called "precipitinogens."

Nature of Precipitins. - The precipitins, like the agglutinins, may be inactivated by heating to from $60^{\circ}$ to $70^{\circ} \mathrm{C}$., and can not be reactivated by the addition of normal serum or by any other known method. Such inactivated precipitin, however, while unable to produce precipitates, has not lost its power of binding the precipitinogen. This is shown by the fact that the inactivated precipitin, when mixed with precipitinogen, will prevent subsequently added fresh precipitin from causing a reaction. From these facts the conclusion has been drawn that precipitin, like toxin, is built up of two atom groups, ${ }^{5}$ a stable haptophore and a labile precipitophore group. By the destruction of the latter, an inactive, yet neutralizing substance is produced which is spoken of as "precipitoid." The precipitoids, like protoxoids, have a higher affinity for precipitinogen than the unchanged precipitin, and thus are able to prevent the action of these.

Our own opinion would rather incline toward regarding the precipitins as identical in structure with sensitizer or amboceptor-being in fact "albuminolysins" in the sense of Gengou. This problem is too

1 Bordet, Ann. de l'inst. Pasteur, 1899.

2 Tchistovitch, Ann. de l'inst. Pasteur, 1899.

${ }^{3}$ Wassermann, Deut. med. Woch., 29, 1900.

${ }^{4}$ Schütze, Zeit. f. Hyg., 1901.

${ }^{5}$ Kraus und v. Pirquet, Cent. f. Bakt., Orig. Bd. xxxii. 
complex to be discussed in detail in a summary of immunity as brief as the one here presented.

Specificity.-The specificity of precipitins is a question of the greatest importance, since, as we shall see, these bodies have been used extensively for the differentiation of animal proteids. In regard to bacterial precipitins it may be said that, just as in agglutination, there is in precipitation a certain degree of "group reaction." The precipitin obtained with a colon bacillus, for instance, will cause precipitation with culture-filtrates of closely allied organisms, though in a less marked degree. According to Kraus, such confusion may be easily overcome by the proper use of dilution and quantitative adjustment, similar to that used in agglutination tests. Norris ${ }^{1}$ found that the precipitates given by immune sera with the filtrates of the homologous bacteria were invariably heavier than those given with allied strains and that the latter could be eliminated entirely by sufficient dilution.

Specificity becomes of still greater importance in the forensic use of the precipitin reaction introduced by Uhlenhuth, ${ }^{2}$ Wassermann and Schütze, ${ }^{3}$ and Stern. ${ }^{4}$ These authors found that the precipitin reaction furnished a means of distinguishing the blood of one species from that of another. Thus, blood spots, dissolved out in normal salt solution, could be recognized by this reaction as originating from man or from an animal, even after months of drying and in dilutions as high as 1:50,000. Since the value of this test depends entirely upon the strict specificity of the reaction, this question has been studied with especial care, notably by Nuttall. ${ }^{5}$ All who have investigated the subject find the only important source of confusion in the blood of the anthropoid apes. The specificity of the reaction, too, has been found to depend very closely upon the amount of precipitin in the serum employed. If a highly immune serum is insufficiently diluted, the reaction loses much of its specific value. ${ }^{6}$ This source of error is easily eliminated in practice by careful control and titration of the sera used for the tests.

Unlike agglutinins, precipitins have, so far, not been demonstrated in normal sera. ${ }^{7}$

${ }^{1}$ Norris, Jour. Inf. Dis., i, 3, 1904.

2 Uhlenhuth, Deut. med. Woch., xlvi, 1900; vi and xvii, 1901.

${ }^{3}$ Wassermann und Schütze, Berl. klin. Woch., vi, 1901.

${ }^{4}$ Stern, Deut. med. Woch., 1901.

${ }^{5}$ Nuttall, Brit. Med. Jour., i, 1901; ii, 1902.

(5ister und Wolf, Zeit. f. Medizinal-Beamte, 1902.

${ }^{7}$ Kraus, loc. cit., and Norris, loc. cit. 


\section{Theoretical Considerations Concerning Agglutinins and Precipitins.-} We have seen that Ehrlich evolved his theories of antibody formation from his early views upon the absorption of nutritive substances by the body cells, and we have followed, in more or less detail, the steps of his reasoning as he developed his hypothesis in its application to the antitoxic and the lytic substances. There still remained the agglutinins and precipitins, bodies which because of their individual characteristics can be classed neither with the group of antitoxic, nor with that of the lytic substances. These two antibodies, while by no means identical, possess the common characteristics of being more thermostable than the bacteriolytic substances, and of being insusceptible to reactivation by normal serum. It is plain, therefore, that both agglutinating and precipitating reactions take place without the co-operation of complement. The substances which give rise to precipitins and agglutinins,

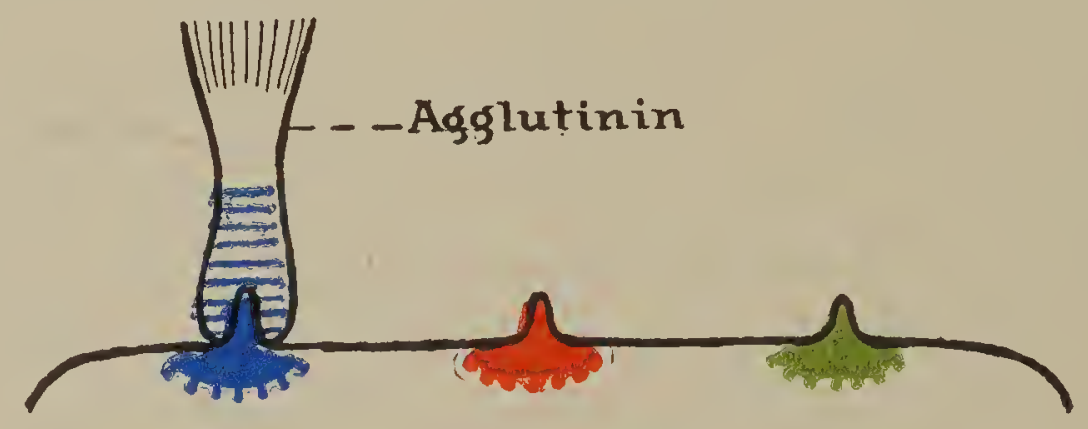

\section{Cell used for immunizing}

Fig. 61.-Ehrlich's Conception of the Structure of Agglutinins and Precipitins.

moreover, are not of the relatively simple soluble character of the toxins, but are intrinsic portions of complex albuminous molecules, comparable to and often identical with the true nutritive substances. For these reasons Ehrlich believes that the cell-receptors for the various substances which give rise to agglutinins and precipitins are neither of the simple structure of the toxin receptor, nor of the double-haptophore nature of the bacteriolytic receptors, but contain a single haptophore group for the anchorage of the ingested material and at the same time a constantly attached zymophore group or ferment by means of which the anchored substance is transformed preparatory to its absorption by the cell protoplasm. For the sake of clearness, this form of receptor may be compared to a bacteriolytic or hemolytic amboceptor with a permanently attached and inseparable complement.

Three forms of receptors, then, are proposed by Ehrlich in explanation of all known varieties of antibodies. The first, the simplest side 


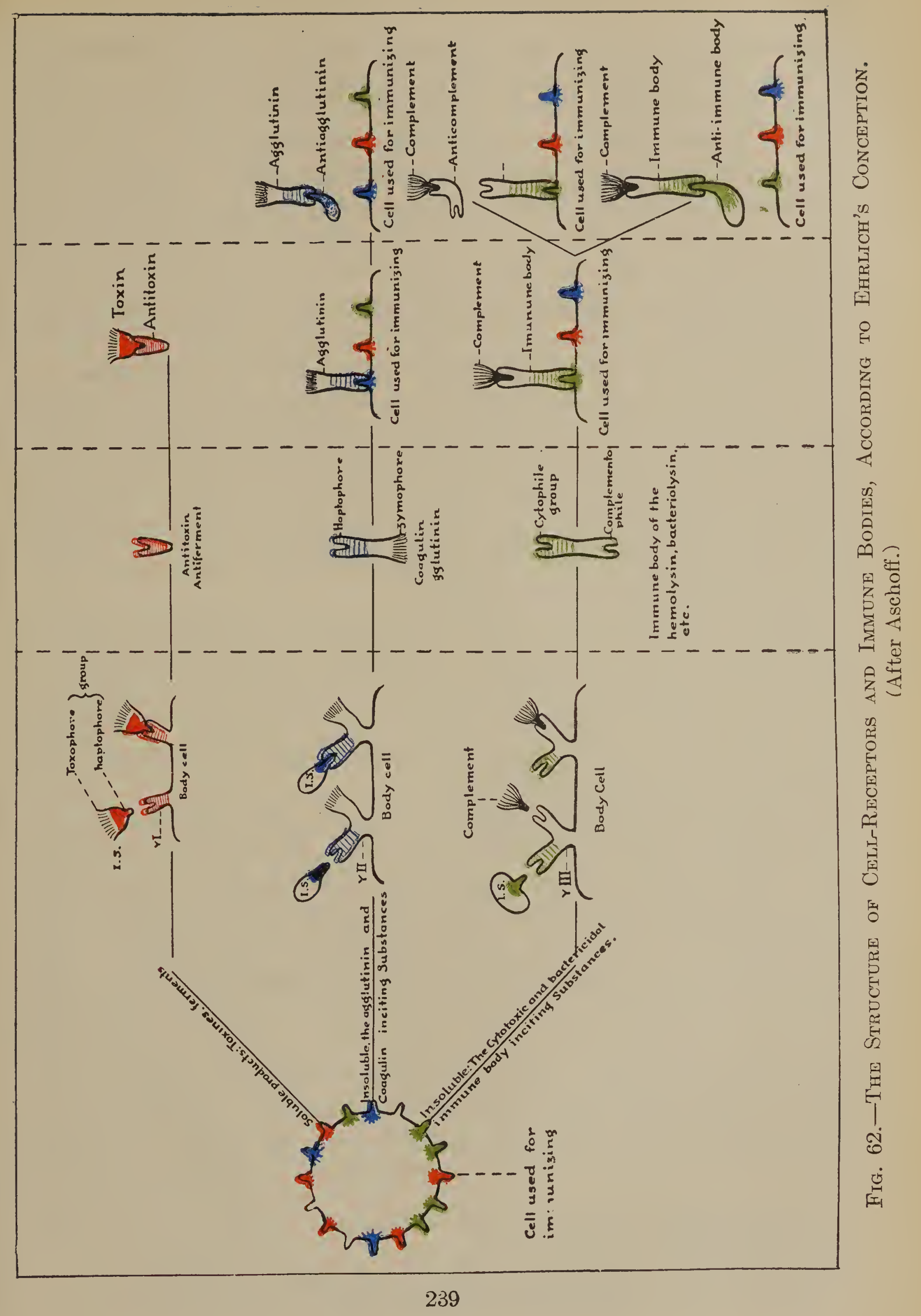


chains of the body cells, he calls "receptors or haptines of the first order." These, overproduced and cast off, constitute the antitoxin and antiferments. Next "haptines of the second order" are the receptors planned both for the anchorage and further digestion of antigens. These, free in the circulation, are the precipitins and agglutinins. "Faptines or receptors of the third order" are merely able to anchor a suitable substance, but exert no further action upon it until re-enforced by the complement normally present in the serum. These, free in the circulation, with a chemical group having avidity for the antigen, and another complementophile group, are the amboceptors or immune bodies of bacteriolytic, cytolytic, and hemolytic sera. (See Fig. 62.)

It is plain that all these receptors while still parts of their respective cells, serve by their chemical affinity to attract and hold the foreign substances injected; freely circulating, on the other hand, they serve in preventing these substances from reaching the cells. As Behring has aptly expressed it, the very elements which situated in the animal cells render the body susceptible to toxic substances serve to protect when circulating freely in the blood.

Bordet, ${ }^{1}$ at present the strongest antagonist of Ehrlich's point of view, claims that the conception of Ehrlich rests upon the basis of a number of undemonstrated hypotheses. He asserts, and with justice, that it has never been shown beyond question that the antibodies, free in the serum, are identical with the receptors of the body cells upon which the antigen originally acts.

In regard to agglutinins, Ehrlich, as we have seen, believes that it is the agglutinin itself which, first uniting with its antigen by its haptophore group, then causes clumping by its zymophore group. Now, as a matter of fact, Bordet ${ }^{2}$ has shown that it is not the agglutinin itself which agglutinates, but that agglutinin with its antigen forms a complex which is then agglutinable by the salt present in the solution. This conclusion seems borne out by the later work of Gengou, ${ }^{3}$ Landsteiner and Jagic, ${ }^{4}$ and others, who have shown that bacteria which have absorbed other substances, such as uranium compounds, colloidal silicic acid, etc., are subsequently agglutinable by salts. In consequence, from these and other observations, Bordet concludes that it is neither necessary nor accurate for the explanation of these phenomena, to

1 Bordet, Résumé of Immunity in Bordet's "Studies in Immunity," transl. by Gay, Wiley \& Sons, 1909.

2 Bordet. Ann. de l'inst. Pasteur, 1899.

${ }^{4}$ Landsteiner und Jagic. Wien. klin. Woch., iii, 1904.

${ }^{3}$ Gengou, Annal. Past., 1904. 
assume the conditions conceived by Ehrlich, but that the phenomenon of agglutination consists primarily of the union of the antibody with its antigen in a colloidal solution, and that the actual subsequent agglutination is a purely secondary phenomenon which depends possibly upon a change in the physical properties of the emulsion-upon, as he expresses it, its colloidal stability. A similar condition he assumes for precipitins.

\section{FURTHER FACTS AND THEORIES CONCERNING ANTIBODIES}

Multiplicity of Amboceptors.-Fresh normal serum, as Nuttall ${ }^{1}$ was first to show, possesses moderate bactericidal powers which are lost when the serum is subjected to heat. Since such inactivated normal serum can be reactivated by the addition of fresh peritoneal exudates, as the experiments of Moxter ${ }^{2}$ have demonstrated, it is plain that the bactericidal power of normal serum must depend, like that of immune serum, upon amboceptor and complement. But normal serum often exerts lytic powers upon several species of bacteria, or, in the case of hemolytic tests, upon the red blood cells of several species of animals. It is supposed that this multiplicity of action is due to the presence in the normal serum of a variety of different amboceptors or immune bodies. The method for proving this was devised by Ehrlich and Morgenroth. $^{3}$ They worked with normal goat's serum, which has the power of hemolyzing the red blood cells of guinea-pigs as well as those of rabbits. Goat serum, inactivated by heat, was mixed with rabbits' corpuscles. After the mixture had been allowed to stand for a short time, the corpuscles were removed by centrifugalization. The serum was then reactivated and found still to possess its hemolytic power for guinea-pigs' blood, but to have lost this power for rabbits' blood. By a similar technique, Pfeiffer and Friedberger ${ }^{4}$ were able to demonstrate the multiplicity of bastericidal immune bodies in normal sera.

The immunity acquired by an animal as the result of treatment with any of the various antigens is specific. An animal immunized against the cholera vibrio, for instance, possesses marked bactericidal powers for the cholera vibrio only.

1 Nuttall, loc. cit.

2 Moxter, Cent. f. Bakt., xxvi, 1896.

3 Ehrlich und Morgenroth, Berl. klin. Woch., 1901.

- Pfeiffer und Friedberger, Deut. med. Woch., 1901. 
According to Ehrlich's views, the amboceptor or immune body alone enters into direct relation with the substance used for immunization, and it would seem natural therefore that the specificity of immune sera depends entirely upon the increase of amboceptor or immune body.

Von Dungern, ${ }^{1}$ indeed, was able to show that the specific amboceptor was increased as immunity was acquired, without there being a corresponding enhancement of the complement. The chief difference between a normal and an immune serum in this respect, therefore, consists in an enormous increase, in the latter, of the specific amboceptor.

Multiplicity of the Complement.-A number of very complicated experiments have been carried out by Ehrlich, Morgenroth, ${ }^{2}$ Sachs, ${ }^{3}$ and others, which seem to show that the same serum may contain a variety of complements. Similar conclusions have been drawn by Wechsberg ${ }^{4}$ and by Wassermann, ${ }^{5}$ who demonstrated separate complements for bactericidal and hemolytic amboceptors in the same serum. Bordet ${ }^{6}$ and his school, on the other hand, deny the multiplicity of the complement, and, basing their views upon numerous experimental data, contend that any given serum contains but one alexin or complement. Buchner and Gruber share the views of Bordet, and, in the light of recent work, especially with complement fixation (see below), it seems more likely that one and only one alexin exists in any given serum.

Anticomplements and Antiamboceptors.-Hemolytic sera, having the power of destroying red blood cells, must necessarily prove in the presence of sufficient complement to be powerful poisons when introduced into animals whose corpuscles they are able to injure. By careful and gradual dosage with such hemolytic sera, Ehrlich and Morgenroth, ${ }^{7}$ as well as Bordet, ${ }^{8}$ have been able to produce immunity against the hemolytic action. Thus antihemolytic sera have been produced, the action of which may depend either upon the presence of anticomplement or of antiamboceptor. The presence of anticomplement in such sera, it is believed, has been demonstrated by mixing inactivated hemolytic serum with its respective red blood cells, then adding the

I v. Dungern, Münch. med. Woch., xx, 1900.

${ }^{2}$ Ehrlich und Morgenroth, Berl. klin. Woch., 1900.

3 Ehrlich und Sachs, Berl. klin. Woch., 1902.

${ }^{4}$ Wechsberg, Zeit. f. Hyg., 1902.

${ }^{5}$ Wassermann, Zeit. f. Hyg., 1901.

${ }^{6}$ Bordet, Ann. de l'inst. Pasteur, 1900 and 1901.

7 Ehrlich und Morgenroth, Berl. klin. Woch., xxxi, 1900.

8 Bordet, Ann. de l'inst. Pasteur. t. 14, 1900. 
antiserum and later complement. After centrifugalization and separation of the corpuscles, these may be dissolved by the addition of fresh complement. This proves conclusively that there was no obstacle in the original mixture to the absorption of the immune body by the red blood cells, and that the antihemolytic properties of the scrum must be attributed to an anticomplement. This was the method of experimentation employed by Ehrlich and Morgenroth. ${ }^{1}$ Antiamboceptors have been produced by the same authors as well as by Bordet ${ }^{2}$ and Müller, ${ }^{3}$ against hemolytic amboceptors.

Complementoids.-Ehrlich and Morgenroth and Müller have succeeded in producing anticomplements by the treatment of animals with normal heated serum. They explain this by assuming that the heating has not entirely destroyed the complement in the normal serum, but that this, analogous to toxin, possesses two groups, a haptophore and a zymophore group. Heating destroys the zymophore without affecting the haptophore group. The resulting body, which corresponds to toxoid, they call "complementoid."

Further evidence for the existence of such complementoids has been claimed by Ehrlich and Sachs ${ }^{4}$ in working with dog serum. Unheated dog scrum hemolyzes guinea-pig corpuscles. Heated to $52^{\circ}$ C. for thirty minutes, however, it no longer hemolyzes these corpuscles owing to complement destruction. Such heated dog serum can be reactivated by fresh guinea-pig serum (fresh complement). If, however, the corpuscles are left in contact with the heated dog blood for two hours, reactivation by the guinea-pig serum no longer occurs-that is, the addition of guinea-pig serum no longer causes hemolysis. They conclude from this that the hemolytic amboceptor of the dog scrum has been attached by its complementophile group to complementoids produced in the heating-leaving no point of attachment for the complement added later. These experiments have failed of confirmation by Gay ${ }^{5}$ - who with Bordet denies the existence of complementoids.

Muir, on the other hand, claims to have demonstrated the existence of complementoids by experiments too complicated to be detailed in this place. The question of complementoids must be left undecided until further work has been done.

\footnotetext{
1 Ehrlich und Morgenroth, loc. cit.

${ }^{2}$ Bordet, loc. cit.

${ }^{3}$ P. Th. Müller, Cent. f. Bakt., 1901.

"Ehrlich and Sachs, "Ehrlich Collected Studies on Immunity," trans. by Boldnau.

5 Gay, Cent. f. Bakt., I, xxxix, 1905.
} 
Filtration of Immune Body and Complement.-Muir and Browning have recently shown that, on the filtration of serum, amboceptor or immune body will pass through the filter, whereas alexin or complement is held back. The amboceptor filters equally well, whether or not mixed with the complement.

The Fixation of Complement by Precipitates.-It has been found by Gengou ${ }^{1}$ and confirmed by Gay ${ }^{2}$ and others, that when the serum of an animal immunized with the serum of another species or with a foreign albumin is mixed with a solution of the substance used in the immunization, the precipitate formed will remove complement from the mixture. In other words, precipitates formed by the reaction of precipitin with its antigen will fix complement. This phenomenon is of great importance in complement-fixation tests such as those of Wassermann or Noguchi (see below); for because of insufficient washing, the blood cells used in producing the hemolytic amboceptor, may, from the presence of serum, give rise to a precipitin as well as a hemolysin. In the test done subsequently, a precipitin reacion may take place and by thus removing complement may give a false result. The absorption of complement by such precipitates takes place when the two reacting factors, the precipitin and its antigen, are in extremely high dilution-in fact, when a visible precipitate can not be observed.

\section{Quantitative Relationship Between Amboceptor and Complement.-} Morgenroth and Sachs, ${ }^{3}$ in studying the quantitative relationship existing between hemolytic amboceptor and its complement, have succeeded in showing that within certain limits an inverse relationship exists between these two bodies. If for a given quantity of red blood cells a certain quantity of amboceptor and complement suffices to produce complete hemolysis, reduction of either the complement or the amboceptor necessitates an increase of the other factor. As amboceptor is increased, in other words, complement may be reduced and vice versa. This result is of great importance in arguing against the original conception of Ehrlich in supposing these substances to act together multiple for multiple as do compounds in chemical reactions.

Deviation of the Complement (Complement-Ablenkung).--It was noticed by Neisser and Wechsberg ${ }^{4}$ that in mixing together bacteria,

1 Gengou, Ann. Past., 1902.

${ }^{2}$ Gay, Cent. f. Bakt., I, xxix, 1905.

3 Morgenroth und Sachs, "Gesammel. Arb. für Immunitätsforschung." Berlin, Hirschwald, 1904.

${ }^{4}$ Neisser und Wechsberg, Münch. med. Woch., xviii, 1901. 
inactivated bactericidal immune serum (immune body), and complement in the test tube, a great excess of immune body hindered rather than helped bactericidal action. As the amount of immune body in the mixture was carried beyond the experimental optimum, bactericidal action became less and less pronounced, and was finally completely suspended. They explain this by assuming that free immune body, uncombined with complement, has a greater affinity for the bacterial receptor than the immune body combined with complement. The complement is consequently diverted and prevented from activating the amboceptor attached to the bacterial cell. Graphically, the conditions may be illustrated as follows:
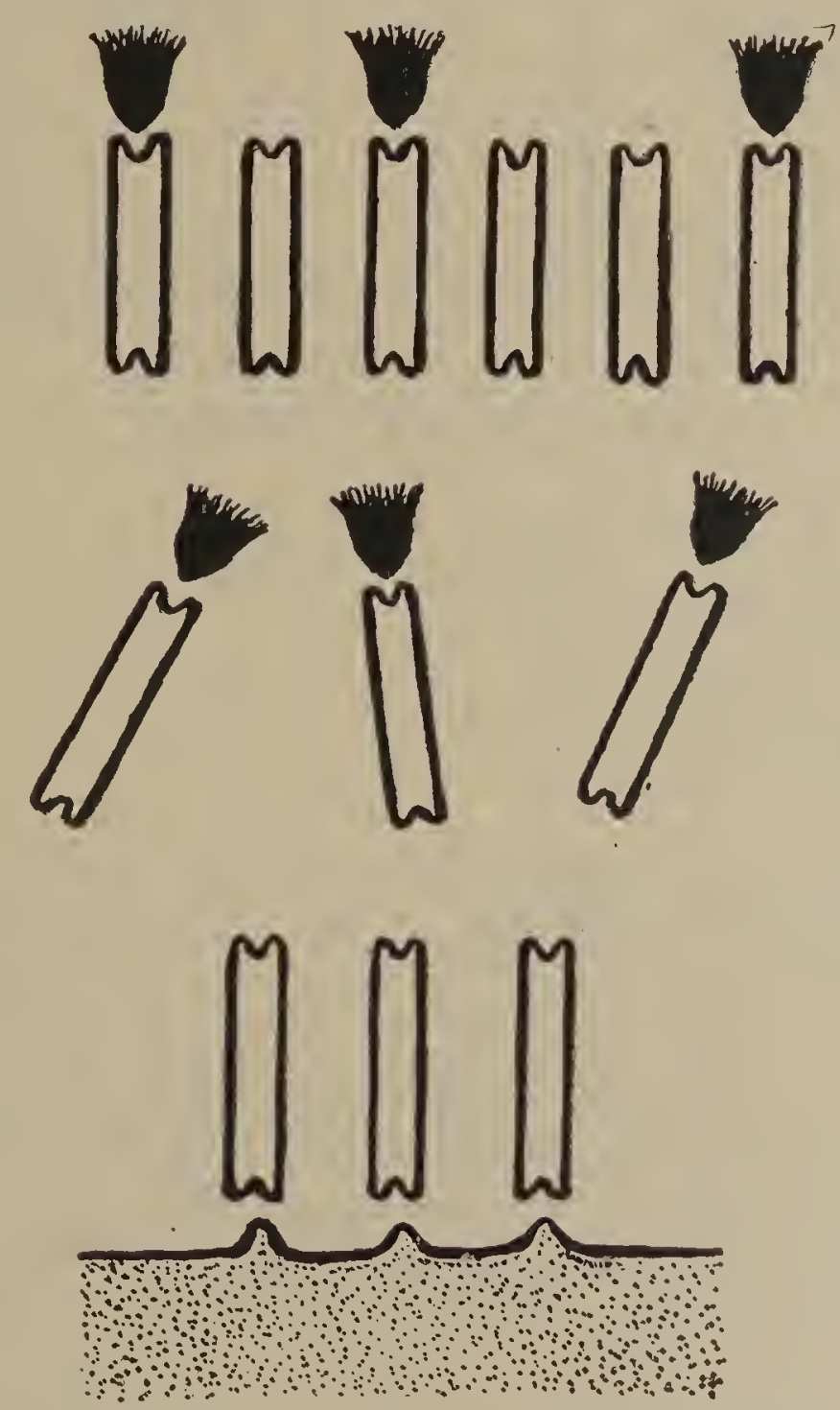

Fig. 63. - Neisser and Wechsberg's Conception of Complement Deviation.

The above theory of Neisser and Wechsberg is here stated simply because of the wide discussion it has aroused. In the light of our present knowledge concerning the relations between antigen, amboceptor, and complement, their conception is obviously erroneous. 
Fixation of the Complement.-Bordet and Gengou ${ }^{1}$ in 1901, devised an ingenious method of experimentation by which even very small quantities of any given immune body (amboceptor) can be demonstrated in serum. The term "fixation of complement," by which their method of investigation is now generally known, explains itself, as the steps of experimentation are followed. They prepared the following mixtures:

(a)

Bacteriolytic amboceptor

(Plague immune serum, heated)

$+$

Plague emulsion

$+$

Complement

(Fresh normal serum)
Normal serum, heated

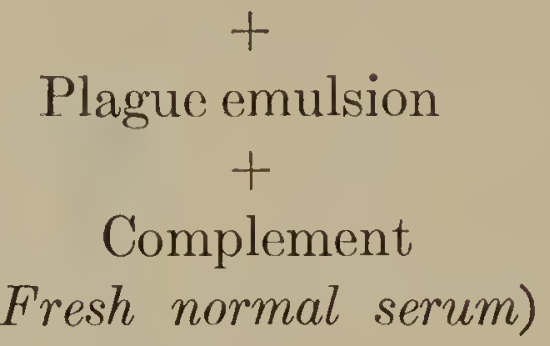

To both of these after five hours was added

Hemolytic amboceptor

(Heated hemolytic serum)

\section{Red blood cells}

Results:

(a) showed no hemolysis.

(b) showed hemolysis + .

The conclusion to be drawn from this was that in (a) the presence of immune body had led to absorption of all the complement. In (b), there being no bacteriolytic immune body to sensitize the bacteria and enable them to absorb complement, the latter substance was left free to activate the subsequently added hemolytic amboceptors. The Bordet-Gengou phenomenon has been extensively used by Wassermann and Bruck, ${ }^{1}$ Neisser and Sachs, ${ }^{2}$ and others to demonstrate the presence of immune bodies in various sera. (See p.262.)

It should be noted that this method, if valid, must presuppose the identity of the hemolytic and bactericidal complement in the activating serum.

Complement fixation will be more extensively discussed in the section dealing with the Wassermann reaction.

1 Bordet et Gengou, Ann. de l'inst. Pasteur, 1901.

1 Wassermann und Bruck, Med. Klin., 1905.

${ }^{2}$ Neisser und Sachs, Berl. klin. Woch., xliv, 1905, and i, 1906. 
The Specificity of Hemolysins.-In the sections preceding we have scen that the blood cells of one animal, injected into an animal of another species, give rise to a hemolytic substance in the blood serum of the second animal, which is strictly specific for the variety of cells injected. Such hemolysins, when produced in one animal
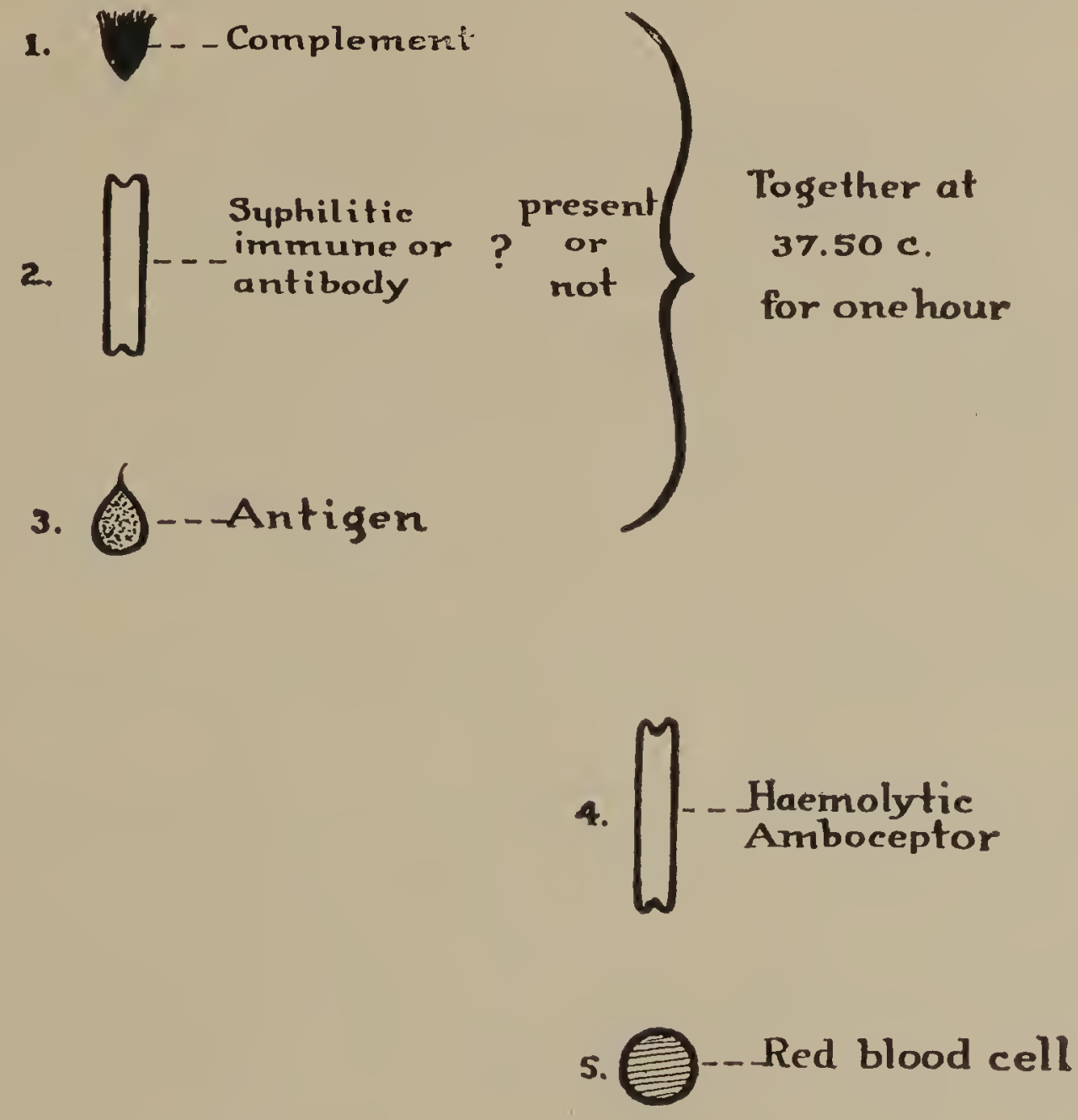

\section{If (2) present, nohaemolysis. \\ If $(z)$ not present, + haemolysis.}

Fig. 64.-Schematic Representation of Complement Fixation in the Bordet-Gengou Reaction.

against blood cells of another species, are spoken of as heterolysins. In studying the nature of hemolysis, Ehrlich and Morgenroth ${ }^{3}$ now discovered that hemolysins could also be produced if an animal was injected with red blood cells of a member of its own species. Such hemolytic substances they called isolysins. In their experiments they injected goats with the washed red blood corpuscles of other goats and found that the serum of the recipient developed the power of

${ }^{3}$ Ehrlich und Morgenroth, Berliner klin. Woch., xxi, 1900. 
causing hemolysis of the red blood cells of the particular goat whose blood had been used for injection. It did not, however, possess the power of producing hemolysis in the blood of all goats, nor did it produce hemolysis with the red corpuscles of its own blood. It is thus shown that the specificity of the hemolysins extends even within the limits of species, and is, to a certain extent at least, an individual property.

The production of autolysins, that is, of substances in the blood serum which will produce hemolysis of the individual's own corpuscles, has, so far, been unsuccessful.

Ehrlich and Morgenroth, in the course of these experiments, furthermore succeeded in showing that the injection of isolysins into animals produced antiisolysins, and that these again were strictly specific.

The almost universal failure of autolysin production has found no satisfactory explanation. It is supposed by Ehrlich and Morgenroth that autolysins may be formed, but are probably speedily neutralized by the production of antiautolysins.

The clinical significance of the presence of isolysins and possibly of autolysins in human beings, is too evident to require much discussion. A practical and extremely interesting result which these investigations have yielded is that of Donath and Landsteiner, ${ }^{1}$ who discovered an autolysin in the blood serum of patients suffering from paroxysmal hemoglobinuria. In these cases the sensitizing substance or amboceptor appeared to be absorbed by the red blood cells only at low temperatures-probably in the capillaries during exposure to the cold, and hemolysis subsequently resulted in the blood stream by the action of complement. These observations have been confirmed by other writers, but the phenomenon is surely not present in all cases of paroxysmal hemoglobinuria. 'The writers have had occasion to examine carefully several clinically typical cases with negative results.

I Donath und Landsteiner, Münch. med. Woch., xxxvi, 1904. 


\section{HAPTER XVI \\ THE TECHNIQUE OF SERUM REACTIONS}

Obtaining Serum from Animals and Man.-To obtain blood serum from man, the blood may be taken from the finger or the ear, either into a sterile centrifuge tube or into a Wright capsule. (See section on Opsonins, page 284.) When taken into a centrifuge tube, the blood is allowed to clot and the serum separated from the coagulum by a few revolutions of the centrifuge. When larger quantities of blood are desired, it may be taken with a syringe from the median basilic vein and either slanted in sterile test tubes in the ice chest or put into centrifuge tubes and centrifugalized.

In bleeding small laboratory animals, a number of methods may be employed, depending upon whether a large or small amount of serum is required.

The animals most frequently used for laboratory purposes are rabbits. To obtain small quantities of serum from rabbits, the animals may be bled from the marginal vein of the ear. In doing this, a satisfactory yield of blood may be obtained by following a simple method devised by Wadsworth. The animal is strapped upon a tray and underneath it is placed a rubber bag filled with warm water. This keeps the body temperature of the rabbit somewhat higher than normal, causes dilatation of the vessels, and thus facilitates the flow of blood. The tray is then placed upon a simply constructed easel so that the animal's head hangs downward. The skin over the ear vein is shaved and sterilized, and an incision is made into the vein in its long axis with a sharp knife. The blood is caught in test tubes or centrifuge tubes.

When larger quantities of blood are desired, the animal is strapped down, anesthetized, and the neck shaved and sterilized. The carotid artery is then isolated by dissection. In rabbits, the carotid artery may be found lying just lateral to the trachea and deeply placed, and must be carefully separated from the pneumogastric nerve by blunt dissection. The distal end of the artery is then tied off and the proximal end temporarily closed with a small clamp. (This clamp should be rather weak and not exert sufficient pressure to injury the artery and cause throm. 
bosis.) The artery is then raised out of the wound on a knife or forceps handle and, with sharp-pointed scissors, a small incision is made into but not through the vessel. A small glass cannula is now introduced into the proximal end of the artery through the incision and tied into place by a thread. To this cannula a small rubber tube fitted with a pinchcock should have been attached, the whole being sterilized. The clamp on the artery is then released and the blood allowed to flow into sterile test tubes which are slanted and placed in the cold for separation of the serum. A larger yield of serum will be obtained if, after coagulation, the clot is separated from the glass with a sterile platinum wire.

In obtaining blood from larger animals, horses, sheep, etc., a cannula may be introduced into the jugular or internal saphenous veins. The skin is shaved and sterilized and a rubber tourniquet placed about the neck or thigh, as the case may be, in order to cause the vein to stand out. A small incision may be made through the skin over the vein, but is not necessary. A cannula, with rubber tubing attached, is then plunged into the vein and the blood caught in sterile high cylindrical jars, allowed to clot, and placed in the refrigerator. The serum is taken off after twenty-four to forty-eight hours with sterile pipettes.

Agglutination Tests.-For the determination of the agglutinating power of serum it is necessary to make suitable dilutions of the serum, and to prepare an even emulsion of the microorganisms to be tested. The test may be made microscopically or macroscopically. The microscopic test is the one in general use in the diagnosis of typhoid fever, and is occasionally applied to some other diseases. In its application to typhoid fever it is usually spoken of as the Gruber-Widal reaction.

Twelve- to eighteen-hour broth cultures of the typhoid bacillus, grown at incubator temperature, may be used. It is preferable, bowever, to use an emulsion of a twelve to twenty-four hour old agar culture in physiological salt solution (0.85 per cent). The salt-solution emulsion is made by adding about 10 c.c. of normal salt solution to the fresh agar slant culture, carefully detaching the culture from the surface of the agar with a flexible platinum wire, and pipetting off the emulsion thus made. With some microorganisms it is sufficient simply to allow the larger clumps to settle and to pipette off the supernatant turbid emulsion. With other microorganisms, the tendency to form clumps makes it necessary to resort to further methods of securing an even distribution of the bacteria. This may be done either by sucking the emulsion in and out through a narrow pipette held perpendicularly against the bottom of a watch glass, as in Wright's tachnique for the opsonic test (see section 
on Opsonins, p. 285), or by carefully rubbing the clumps against the watch glass with a stiff platinum wire. In the case of the tubercle bacillus not even this suffices, but it becomes necessary to grind the moist bacillary masses in a mortar before emulsifying. With the tubercle bacillus, too, it is preferable to use salt solution at 1.5 per cent concentration.

In preparing cultures of streptococcus and pneumococcus for agglutination tests, it has been found convenient by Hiss to grow microorganisms for about four days in flasks of a one per cent glucose, two per cent pepton meat-infusion broth, to which has been added one per cent of calcium carbonate. (See page 126.) The insoluble calcium carbonate sinks to the bottom, but by neutralizing the inhibiting acid formed in the broth by the microorganisms, permits the development of a mass culture. The flasks should be shaken thoroughly at least once a day. The broth may be pipetted off and the clumps may be removed by a few revolutions of a centrifuge. Without this technique it is sometimes difficult to get sufficient growths of these bacteria for any quantity of emulsion unless large surfaces of agar are employed in special receptacles or by making many slant cultures.

The serum dilutions are obtained by first making a one to ten dilution of serum with normal salt solution. The serum used for this purpose may be cleared of red blood corpuscles by centrifugalization. From the one to ten dilution any number of higher dilutions may be made, simply by mixing given parts of the one to ten dilution with normal salt solution; thus one part of a one to ten dilution plus an equal quantity of salt solution gives a dilution of one to twenty. One part of one to ten dilution plus two parts of normal salt solution gives one to thirty, and one part of one to twenty dilution plus one part normal salt solution gives one to forty, etc. It must not be forgotten that, when equal parts of the serum and bacillary emulsion have been mixed, each one of these dilutions is doubled.

In making the microscopic agglutination test, minute equal quantities of serum dilution and bacterial emulsion are mixed upon the surface of a cover-slip. The mixture may be made either by measuring out a drop of each substance with a standard platinum loop, depositing them close together on the cover-slip, and mixing; or, more exactly, equal quantities may be sucked up, each to a given mark, in a capillary pipette, mixed by suction in and out, and then deposited upon the cover-slip. The cover-slip is inverted over a hollow glass slide, the rim of which has been greased with vaseline. The drop is then observed, preferably through a (Leitz) No. 7 lens, ocular No. 3. 
The macroscopic agglutination test, always preferable for exact laboratory research, is made in narrow test tubes especially designed for the purpose, measuring about $0.5 \mathrm{~cm}$. in diameter and about $5 \mathrm{~cm}$. in length (Fig. 60, p. 230).

In these test tubes equal quantities, usually 1 c.c. each, of serum dilution and emulsion are mixed. A series of tubes is prepared, in each subsequent one of which the dilution is higher. These mixtures may be placed in the incubator for a few hours and then lept at room temperature. It has been observed by Hiss that after removal from the incubator agglutination is in some instances hastened by transference to the ice chest. When agglutination takes place in these tubes, clumps of bacteria may be seen to form, which settle to the bottom of the tube, very much like snow-flakes. The surface of the sediment is heaped up and irregular. The supernatant fluid becomes entirely clear. When the reaction does not occur the sediment is an even, granular one with a flat surface, and the emulsion remains turbid.

Instead of using test tubes as described above, Wright has suggested the use of throttle pipettes of comparatively large diameter into each of which at least three or four different dilutions can be sucked with a nipple, a small air bubble being left between the mixtures. By sealing the distal end of these pipettes in a flame the various dilutions are kept at a distance from each other, and the pipettes may be set on end in a tumbler and observed just as are the test tubes (Fig. 68, p. 285).

Precipitin Tests.-In an earlier section on precipitins we have seen that precipitates are formed when clear filtrates of bacterial extracts or of broth cultures are mixed with their specific immune sera. Such precipitin reactions are not limited to the realm of bacteria, but have a broad biological significance, in that specific precipitating sera may be produced with proteids of varied source.

For actually carrying out a precipitin test, the following reagents are required:

1. A specific precipitating antiserum (antibacterial or antiproteid);

2. A bacterial filtrate or proteid solution.

The Production of Precipitating Antisera. ${ }^{1}$ - Antibacterial precipitins may be produced in animals by any one of a variety of methods. Animals, preferably rabbits, are injected either with broth cultures or with salt solution emulsions of agar cultures of the bacteria, in gradually increasing quantities. Five or six injections are given in-

${ }^{1}$ R. Kraus, Wien. klin. Woch., 1897; Norris, Jour. Inf. Dis., 1 and 3, 1904. 
traperitoneally or intravenously, at intervals of from five to six days, the dosage and mode of administration being adapted in each case to the pathogenic properties of the microorganisms in question. It has been asserted by Myers ${ }^{1}$ that when pepton-broth cultures are used for immunization a specific precipitin for pepton may be formed which by giving a precipitate with a culture filtrate containing pepton may lead to error. This observation could not be confirmed by Norris. ${ }^{2}$

The immunized animals should be bled about seven to twelve days after the last injection of bacteria.

Specific precipitating antisera against proteid solutions are prepared by methods analogous to those employed for the production of antibacterial sera. A variety of methods have been described. The sera or proteid solutions used should be sterile. This may be accomplished by filtration through small porcelain filters. The injections into animals may be made subcutaneously, intraperitoneally, or intravenously. The subcutaneous route has no advantages unless the substances to be used are contaminated.

Nuttall advises the use of rabbits. The animals should be weighed from time to time, and if considerable loss of weight ensues during immunization, the intervals between injections should be increased. Dosage should be carefully graded, beginning, in the case of an animal serum, for instance, with 2 c.c. and increasing gradually through 3,5 , and 8 c.c. to possibly 15 c.c. at the last injection. A single injection of a large quantity has occasionally yielded a precipitating serum of considerable strength, ${ }^{3}$ but this method is not usually successful. Injections are made at intervals of from five to seven days. Seven to twelve days after the last injection the animals may be bled, and a preliminary test made to ascertain the precipitating value of the serum. If this is insufficient for the desired purposes, more injections may be made before the animal is finally bled. Bleeding should be done seven to twelve days after the last injection. Such sera may be preserved by sealing in glass bulbs and keeping in the dark and at a low temperature. If a preservative is to be added, Nuttall recommends chloroform, but disapproves of the phenols, because of occasional turbidity produced by these.

The precipitating antisera used for the tests should be absolutely

1 Myers, Lancet, ii, 1900.

${ }^{2}$ Norris, loc. cit.

${ }^{3}$ Michaelis, Deut. med. Woch., 1902. 
clear. If turbidity is present, the sera should be filtered through small Berkefeld or porcelain candles.

Preparation of Bacterial Filtrates and Proteid Solutions For Precipitin Tests.-Bacteria may be grown in broth made of Liebig's beef extract five-tenths per cent, pepton one per cent, $\mathrm{NaCl}$ fivetenths per cent, and having an initial reaction of neutrality or five-tenths per cent acidity to phenolphthalein. The cultures are incubated for times varying from a week to several months, and are then filtered through porcelain or Berkefeld candles until perfectly clear. Bacterial extracts may also be made by emulsifying agar cultures in salt solution, placing at $37.5^{\circ} \mathrm{C}$. in the incubator for a week or longer, and filtering. More rapid extraction of bacteria may be accomplished by repeated, rapid freezing and thawing of salt-solution emulsions. This is easily and simply done by placing the test tubes in battery jars filled with brine and cracked ice.

Proteid solutions to be tested should be made in salt solution. When dealing with blood stains, as is frequently the case in doing the test for forensic purposes, the stains should be dissolved out in salt solution, an approximate dilution of one in five hundred being aimed at. This solution if turbid should be filtered through a small porcelain filter. Before use it should be perfectly clear and colorless, should show a faint cloud on boiling with dilute acetic acid, and, according to Müller, should show distinct frothing when shaken.

When the reaction is to be done with the purpose of determining the nature of meat (detection of horse-meat substitution for beef, etc.), about 20 to 40 grams of the suspected meat are macerated by being placed in a flask, and covered with 100 c.c. of physiological salt solution. This mixture is allowed to infuse at room temperature for three to four hours, and is then placed in the refrigerator for twelve hours or more. At the end of this time 2 c.c. may be poured into a test tube and shaken. If frothing ${ }^{1}$ appears easily and profusely, the extract is ready for use. It is then filtered clear, either through paper, or, if this is unsuccessful, through infusorial earth in a Buchner or Nutsche filter. Berkefeld filters may also be used, but their use is less simple. The clear solution is then further diluted until the addition of concentrated $\mathrm{HNO}_{3}$ produces only a slight even turbidity. Before use, furthermore, the reaction of the meat extract should be tested, and if necessary adjusted to neutrality or slight acidity or alkalinity. 
In the actual test with bacterial filtrate, the procedure is as follows: in a series of narrow test tubes, the following mixtures are made:

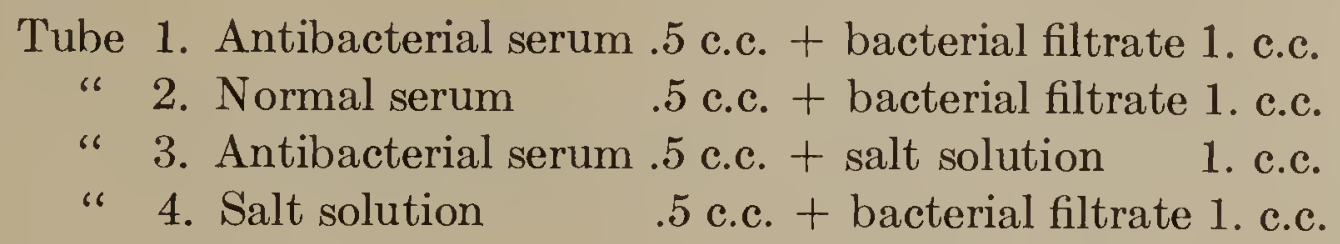

Place the tubes in the incubator at $37.5^{\circ} \mathrm{C}$. In a positive test, tube 1 only should show a haziness which develops into a distinct cloudiness or even a flocculent precipitate within one hour. Tubes 2,3 , and 4 should remain clear.

For the testing of an unknown proteid with the serum of an animal immunized with the proteid sought for, the technique of the test is as follows:

1. 0.1 c.c. immune serum +2 c.c. unknown proteid solution.

2. 0.1 c.c. immune serum +2 c.c. known proteid solution of variety suspected (similarly diluted).

3. 0.1 c.c. immune serum +2 c.c. proteid solution of different nature (similarly diluted).

4. 0.1 c.c. immune serum +2 c.c. salt solution.

5. 2 c.c. unknown proteid solution.

The test is positive when a precipitate appears in tube 1 and in tube 2 , but not in any of the others. The precipitate should appear definitely within fifteen to twenty minutes.

Bactericidal and Bacteriolytic Tests.--The bactericidal and bacteriolytic powers of serum may be tested either in the animal body or in the test tube. The most common bacteriolytic test, in vivo, is that which is known as Pfeiffer's test. This test depends upon the fact considered in a previous section, that bacteria, when injected into the peritoneal cavity of a guinea-pig, together with a homologous immune serum, undergo dissolution.

As practiced in bacteriological work, the test finds a double application. It may be carried out either for the determination of the specific bacteriolytic power of a given serum against a known microorganism, or for the identification of a particular microorganism by means of its susceptibility to lysis in a known immune serum.

1. Determination of the bacteriolytic power of serum against a known microorganism in vivo: ${ }^{1}$

1 P. Th. Müller, "Technik d. serodiagnos. Methoden," Jena, 1909. 
A number of dilutions of the serum are made with sterile neutral bouillon or salt solution, ranging from 1 in 20 to 1 in 500, or higher. It is convenient to make a first solution of 1 in 20. One c.c. of this mixed with 4 c.c. of broth will give 1 in 100 . One c.c. of the 1 in 100 dilution with 1 c.c. of broth, 2 c.c. of broth and 4 c.c. of broth will give 1 in 200 , 1 in 300 , and 1 in 500, respectively. Into one cubic centimeter of each of these dilutions there is placed one platinum loopful of a twenty-fourhour agar culture of the microorganism against which the serum is to be tested. Into another test tube is placed 4 c.c. of broth, without serum, and with one loopful of the microorganisms. The mixtures are thoroughly emulsified in each case by rubbing the bacteria against the sides of the tube with the platinum loop.

Intraperitoneal injections into guinea-pigs are then made of 1 c.c. of each of the serum-dilution-bacterial-emulsions. A control guinea-pig (better two or three) receives 1c. c. of the broth emulsion-one-fourth as many bacteria, therefore, as the animals receiving the serum dilutions.

Before making the injections, areas on the lateral abdominal walls of the guinea-pigs are shaved, and small incisions made through the

Fig. 65.-Capillary Pipette for Removal of Exudate in doing the Pfeiffer 'Test.

skin, down to the muscular layers. The needle of the syringe is then introduced perpendicular to the skin until it has penetrated the peritoneum, and then carefully slanted to avoid puncturing the gut. The animals need not be strapped down during this procedure and afterward may be allowed to run about.

After one-half hour, and again after one hour has elapsed, a drop of peritoneal exudate is removed from each guinea-pig and examined in the hanging drop for granulation and swelling of the bacteria. The method of obtaining the peritoneal exudate is as follows: Small glass tubing is drawn out into capillary pipettes, the ends of the capillaries being again drawn to fine points in a small yellow flame. A number of such pipettes should be prepared before the test is begun. The guineapig is then held down upon a table, either by an assistant or by the left han.l of the operator, and the point of the pipette pushed through the cut in the abdominal wall into the peritoneum by a sharp, quick thrust- 
ing motion. A column of peritoneal fluid will run into the glass tubing by capillary attraction; this can then be blown out upon a cover-slip for hanging-drop examination or may be blown upon a slide, smeared, and examined after staining. The reaction is regarded as positive if within thirty minutes to an hour the peritoneal exudates of the animals receiving immune sera contain only swollen or disintegrated microorganisms, while in that of the control animals only well-preserved and undegenerated bacteria are found. In dealing with typhoid bacilli and cholera spirilla, in connection with which the test is most often used, active motility in the controls is of much help. Should there be extensive degeneration of the bacteria in the exudate of the control animals the test is of no value.

2. Identification of a microorganism by observing its a susceptibility to lysis in a known immune serum in vivo:

The technique for this test is practically the same as that of the preceding except that in this case we require a potent known immune serum and normal serum for control. It is necessary, furthermore, that by previous tests we should know the degree of dilution in which the immune serum will cause complete bacteriolysis of the microorganism used in its production. Thus, if we are employing a typhoid immune serum and are about to test by this method an unknown Gram-negative bacillus, we must know the titer of the serum for the typhoid bacillus itself.

Mixtures are then made of dilutions of this serum and definite quantities of the microorganism to be tested. It is best, always, to employ from ten to one hundred times the amount of immune serum which suffices to produce lysis with its homologous microorganism. Thus, if the serum has been found to be active in dilutions of $1: 1,000$, it is employed in the test in dilutions of $1: 1,000,1: 100$, and $1: 10$. These dilutions are then injected into guinea-pigs in quantities of 1 c.c. together with the bacteria to be tested, and control guinea-pigs are injected with undiluted normal serum mixed with the bacteria and with salt solution and the bacteria. The exudates are then observed in the same way as in the preceding experiment.

Bactericidal Reactions in the Test Tube-Bactericidal reactions in the test tubes may be made by mixing in small sterile test tubes, definite quantities of the bacteria with inactivated serum and complement, the latter in the form of unheated normal serum. The mixtures, diluted with equal volumes of neutral broth or salt solution, are set away for a definite time three to four hours in an incubator at 
$37.5^{\circ} \mathrm{C}$, and equal quantities from all the tubes are then inoculated into melted agar at $40^{\circ} \mathrm{C}$., and plates are poured. Control plates must be made in each case with mixtures of similar quantities of bacteria in salt solution, and similar quantities of bacteria in normal serum. By colony counting after the plates have developed, it is then possible to estimate the degree of bacterial destruction in any of the given dilutions.

In actually carrying out the test, dilutions of the inactivated serum are first made, ranging from $1: 10$ to $1: 1,000$ and over. An emulsion of bacteria from a twenty-four-hour agar slant is then made in salt solution, or a twenty-four-hour broth culture properly diluted may be used. Complement is obtained by taking fresh normal rabbit serum and diluting it with salt solution $1: 10$ or $1: 15$. Into a series of test tubes, then, 1 c.c. of each of the serum dilutions is placed, and to each tube is added 0.5 c.c. of the diluted fresh normal rabbit serum (complement). To these mixtures the bacteria are then added. In adding the bacterial emulsion to these tubes, the writers have found it more accurate to discard the use of the platinum loop and to measure the bacterial emulsion in a marked capillary pipette such as that used in the opsonin test. (See page 285, Fig. 68.) The controls are set up in a similar way, all of them containing a similar quantity of bacterial emulsion, one control containing 1.5 c.c. of salt solution, another control containing 1 c.c. of salt solution + 0.5 c.c. of the diluted complement, and the third control containing inactivated normal serum 1 c.c. +0.5 c.c. of diluted complement. Definite quantities of these mixtures, taken with a standard loop, or preferably with a capillary pipette, are plated in agar immediately after mixing.

\section{BACTERICIDAL TEST IN VITRO}

(To Determine the BaCtericidal Power of a Typhoid Immune Serum against Typhoid Bacilli).

Plates
Poured
After $3 \mathrm{Hrs}$.
at $37^{\circ} \mathrm{C}$.
$5\}$
Colonies.
$100-1,000$
Colonies
More than
10,000
Colonies

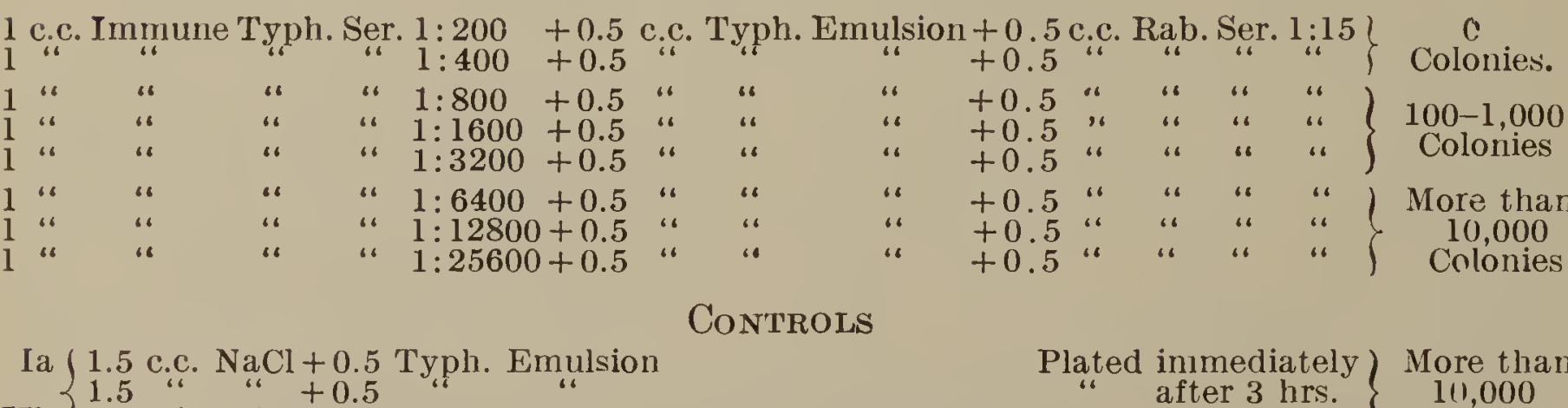

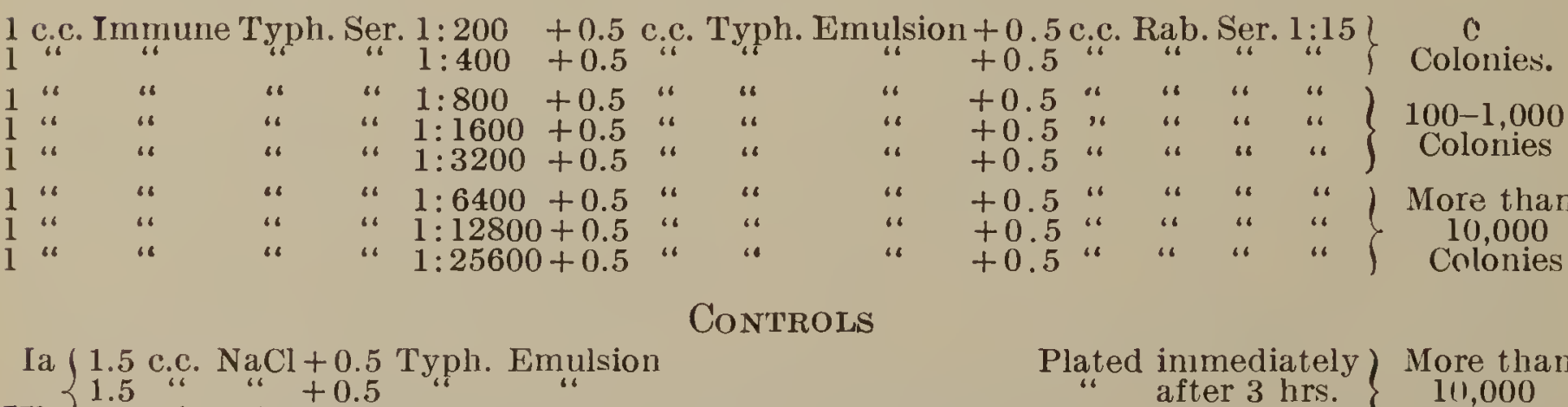

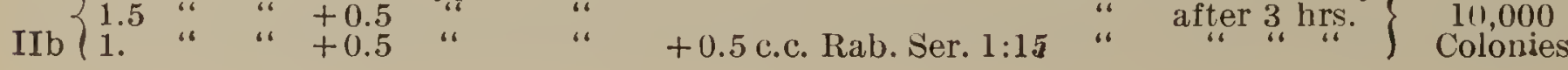

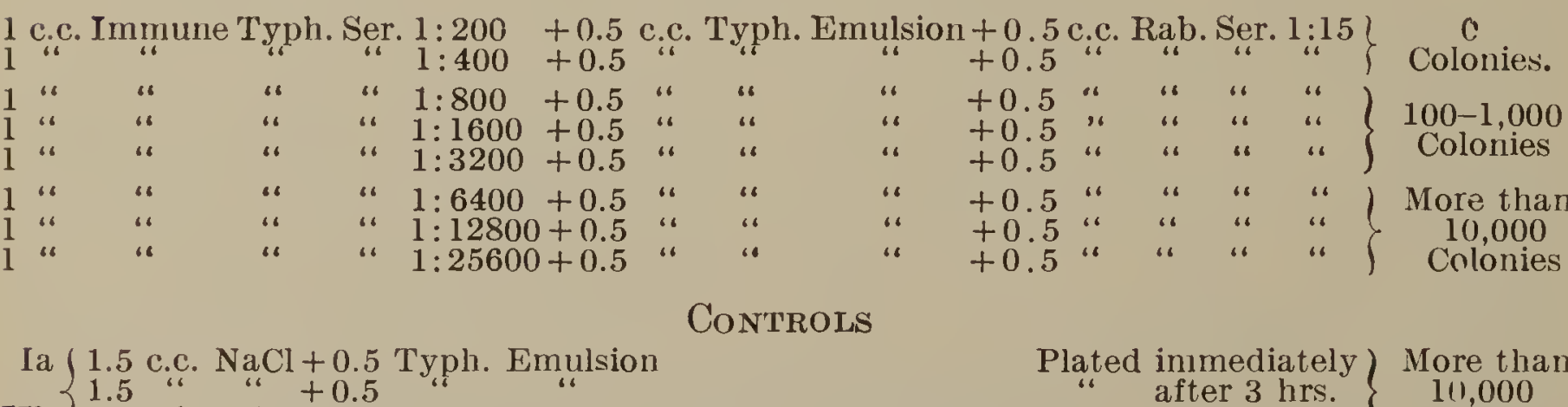


After incubation for two or three hours similar quantities are again measured into tubes of melted agar with the capillary pipette. With a little practice, great accuracy in these measurements can be acquired. The inoculated agar tubes are very thoroughly mixed, and plates are poured. At the end of twenty-four hours' incubation, an enumeration of the colonies in the various plates is made and the results are compared.

The in vitro bactericidal tests have been employed, practically, chiefly in the diagnosis of typhoid fever by Stern and Korte. ${ }^{1}$ While the serum of normal individuals shows practically no bactericidal power for typhoid bacilli, the sera of typhoid patients may be actively bactericidal in dilutions as high as $1: 50,000$.

Hemolytic Tests.-Determination of the hemolytic action of blood serum, bacterial filtrates, and of a variety of other substances, such as tissue extracts and animal and plant poisons, is frequently made in bacteriological laboratories. Familiarity with the methods of carrying out such tests is especially essential since hemolytic tests are also employed in determining other serum reactions, such as the "complementfixation tests" discussed in another section.

For these tests it is necessary to prepare washed red corpuscles of the species of animal against which the hemolysins are to be tested, and to obtain these, blood may be taken in one of the following ways:

$A$. If small quantities of blood corpuscles are desired, the blood may be received into a sterile test tube into which a copper or other wire bent into a loop at the lower end has been introduced. This is used to prevent clotting and to remove the fibrin. Immediately after receiving the blood into this tube, the wire is twirled between the fingers so that the blood is beaten by the wire as by an egg-beater. At the end of five minutes of continuous agitation, the fibrin adhering in a mass to the wire may be lifted out. The corpuscles are then washed and centrifugalized in several changes of salt solution to remove all traces of serum, and are finally emulsified in salt solution.

$B$. The blood may be taken into a centrifuge tube and immediately centrifugalized before clotting has taken place. The plasma is then poured off and the corpuscles are washed with salt solution, as before, to remove the serum.

$C$. The blood may be taken directly into a solution containing five-tenths per cent sodium chlorid and one per cent sodium citrate. 
The corpuscles are concentrated by centrifugalization, the citrate solution is decanted, and corpuscles are washed with salt solution, as before, to remove the serum.

$D$. When large quantities of blood are desired, either from man or from an animal, the blood may be received directly into a flask into which a dozen or more glass beads or short pieces of glass tubing have been placed. The flask is shaken for five or ten minutes, immediately after the blood has been taken and, in this way, defibrination is accomplished.

Since, for comparative tests, it is necessary to establish some standard concentration of red blood cells, it is customary in these tests to employ a five per cent emulsion of corpuscles in salt solution. To obtain this, the thoroughly washed corpuscles from one volume of the original blood are mixed with ninèteen parts of 0.85 per cent salt solution. ${ }^{1}$ Such an emulsion, if kept sterile and in the refrigerator, will serve for hemolytic tests for from one to three days. An cmulsion should not be used if the supernatant salt solution shows any transparent redness, as this indicates hemolysis.

If the substance in which hemolysins are to be determined is serum, this may be used either as such or it may be inactivated by exposure to $56^{\circ} \mathrm{C}$. in a water bath, and to each test, complement may be added in the form of fresh guinea-pig or rabbit's serum. No absolute rule for the quantity of complement to be used in these tests can be given. As a starting-point, however, when 1 c.c. of a 5 per cent emulsion of red corpuscles is used, it is best to use about 0.1 to 0.2 c.c. of fresh guineapig serum as complement.

In the actual test, mixtures are made of the corpuscle emulsion, the inactivated immune serum, and complement in small test tubes and the volumes of the various tubes made equal by the addition of definite quantities of salt solution. The contents of the tubes are thoroughly mixed and the tubes put in the incubator or in a water bath at $37.5^{\circ} \mathrm{C}$. If complete hemolysis occurs, the fluid in the tube will assume a deep Burgundy red. If no hemolysis occurs, the fluid will remain uncolored and the corpuscles will settle out. Incomplete hemolysis will be evidenced by a lighter tinge of red in the tube and by the settling out of a varying quantity of blood corpuscles.

1 The method here given was formerly much employed. It is now the general practice, however, to use one volume of the actual sediment to nineteen volumes of salt solution. 
In all hemolytic tests the time element is important. No hemolysis should be adjudged as incomplete unless at least one hour has elapsed.

It is often necessary to carry out hemolytic tests on the blood corpuscles of one human being with the serum of another in order to determine the advisability of performing transfusion. In this case, the serum of the recipient is mixed with a corpuscle emulsion of the cells of the donor, and vice versa. Since it is often difficult to obtain much blood for these tests, the writers have found it convenient to make the test in throttle pipettes, instead of in test tubes. By this technique, ten or fifteen drops of blood and a very small amount of serum will suffice. It should be stated, however, that whenever sufficient quantities of serum can be obtained this technique should not be employed.

The Determination of Antibodies in Sera by Complement Fixation.The principle of complement fixation, discovered by Bordet and Gengou ${ }^{1}$ in 1901, has been utilized both in bacteriological investigations, and in practical diagnosis for the determination in serum of the presence of specific antibodies. Although spoken of in another section of this book, it may be well to review, briefly, the principles of the Bordet-Gengou phenomenon. The reaction depends upon the fact that when an antigen, i.e., a substance capable of stimulating the formation of antibodies in animals or man, is mixed with its inactivated antiserum, in the presence of complement, the complement is firmly fixed by the combined immune body and antigen in such a way that it can no longer be found free in the mixture. If such a mixture is allowed to stand at a suitable temperature for an hour or more, and to it is then added an emulsion of red blood cells together with a suitable quantity of inactivated hemolytic serum, no hemolysis will take place, since there is no free complement available to complete the hemolytic system. If, on the other hand, the original mixture contains no antibody for the antigen used, the complement present is not fixed and is available for the activation of the hemolytic serum later added.

The reaction thus depends, in principle, intimately upon the fact that neither antigen ${ }^{2}$ alone, nor amboceptor (antibody) alone, can fix complement, but that this fixation is carried out only by the combination of antigen plus amboceptor. Any specific amboceptor can be determined by this method, provided the homologous or stimulating antigen

1 Bordet and Gengou, Ann. de l'inst. Pasteur, xv, 1901.

2 Bordet and Gay, Ann. de l'inst. Pasteur, xx, 1906. 
is used; and vice versa, by the use of a known antibody a suspected antigen may be determined.

When testing immune sera for amboceptors given rise to in man or animals by microorganisms which can be cultivated, either the whole bacteria or extracts of the bacteria may be used as an antigen.

For the diagnosis of syphilis by this method, in the so-called "Wassermann reaction," the antigen employed was originally obtained by the extraction of syphilitic organs, in which free syphilitic antigens, i.e., uncombined products of Spirochæte pallida, were assumed to be present. As this reaction has recently become prominent and has proven of no inconsiderable diagnostic value, the technique given below for immunebody determination by complement fixation will be that utilized in the Wassermann test for syphilis.

The reader will, however, bear in mind that the test may be applied to other diseases simply by the substitution of the suitable, specific antigen. Thus, when cultivatable bacteria are used as antigens, Bordet and Gengou make use of a thick salt-solution emulsion of a twenty-four-hour agar-slant culture of the microorganisms. In the case of the tubercle bacilli, these authors emulsify 80 milligrams of the bacilli in 1 c.c. of the salt solution. Wassermann and Bruck, ${ }^{1}$ on the other hand, prepare their bacterial antigen in the following way: The growths of about ten agar slant cultures are emulsified in 10 c.c. of sterile, distilled water. This emulsion is shaken for twenty-four hours in a shaking apparatus. At the end of this time 0.5 per cent of carbolic acid is added and the fluid cleared by centrifugalization. These antigens become slightly weaker during the first ten or fourteen days, but after that remain fairly constant. For the determination of tuberculosis antibody, these authors make use of either old tuberculin or the new tuberculins "TR" or "Bazillen Emulsion."

The Wassermann Test for the Diagnosis of Syphilis. ${ }^{2}$-The substances for the test are the following:

I. The Antigen.-In their original experiments, Wassermann and his collaborators made use of salt-solution extracts of the organs (chiefly of the spleen) of a syphilitic fetus. The tissue substance was cut into small pieces and to one part by weight of this substance, four parts of normal salt solution and 0.5 per cent of carbolic acid were added. This

1 Wassermann und Bruck, Med. Klinik, 55, 1905, and Deut. med. Woch., xii, 1906.

${ }^{2}$ Wassermann, Neisser und Bruck, Deut. med. Woch., xix, 1906; Wassermann, Neisser, Bruck und Schucht. Zeit. f. Hyg., lv, 1906. 
mixture was shaken in a shaking apparatus for twenty-four hours, and after this the coarser particles were removed by centrifugalization. The reddish supernatant fluid was used as the antigen and could be preserved for a long time in dark bottles in the ice chest.

Michaelis ${ }^{1}$ obtained the antigen in the following way: The liver of a syphilitic fetus was preserved in a frozen state and from time to time small quantities of extract were prepared for the purpose of obtaining antigen. This was obtained by thoroughly grinding up a small piece of the liver in a mortar and adding five parts of salt solution and about 0.5 per cent of carbolic acid. This mixture was shaken in a shaking apparatus for several hours and was then allowed to stand at a temperature slightly above $0^{\circ} \mathrm{C}$. for several days. Finally it was cleared by filtration or centrifugalization.

Alcoholic extracts of syphilitic organs have been used by a number of authors. Porges and Meier ${ }^{2}$ extract the chopped-up syphilitic liver for twenty-four hours with five times the volume of absolute alcohol. This is then filtered through paper and the alcohol evaporated in vacuo at a temperature not above $40^{\circ} \mathrm{C}$. The greenish sticky residue should have an alkaline or neutral reaction. About 1 gram of this material is then emulsified in 100 c.c. of salt solution to which 0.5 per cent of carbolic acid has been added. The fine emulsion which results is filtered through thin paper and the filtrate used as the antigen.

Porges and Meier, as well as a number of others, have discovered that in actual practice it is not necessary to make use of syphilitic organs in order to obtain an antigen which will combine with syphilitic immune body. This fact, of course, has thrown much suspicion upon the specificity of the phenomenon. In practice, however, it appears as a purely empirical fact that many of the non-specific antigens, nevertheless, give reasonably reliable results. The authors mentioned above have found that a 1 per cent emulsion of commercial lecithin (Kahlbaum) in carbolized salt solution furnishes a suitable antigen. This has not been universally confirmed. The same authors have obtained good results by extracting a normal fetal liver by alcohol in the same way as they extracted the syphilitic organ. Landsteiner, Müller, and Poetzl ${ }^{3}$ have successfully employed an alcoholic extract of the heart substance of a guinea-pig.

1 Michaelis, Berl. klin. Woch., 1907.

${ }^{2}$ Porges und Meier, Berl. klin. Woch., xv, 1908.

${ }^{3}$ Landsteiner, Mïller und Poelzl, Wien, klin. Woch, 50, 1907. 
Similar alcoholic extracts of normal human spleen or of normal rabbit's liver may be employed. Although often claimed that the antigen in such extracts is furnished by the lipoids, as a matter of fact it is at the present day unknown to which ingredient the immune-body binding power is to be attributed.

The antigen used in several hundred reactions by the writers with satisfactory result is one prepared according to the method of Noguchi, ${ }^{1}$ as follows:

Fresh normal liver or spleen is covered and thoroughly macerated with five times its volume of absolute alcohol. This is allowed to extract in the incubator for six to eight days, being thoroughly stirred up at least once a day. It is then pressed through cheese-cloth and filtered through paper. This alcoholic extract is evaporated to dryness at room temperature with the aid of a wind fan. The sticky, brownish residue resulting is taken up in a small quantity of ether and the solution poured into four times its volume of C. P. acetone. A heavy flocculent precipitate forms which settles to the bottom as a sticky brown mass. This is retained as antigen and may be preserved under acetone. The acetone-soluble fraction is thrown away. For use, about 0.2 gram of the sticky paste is dissolved in about 5 c.c. of ether and 100 c.c. of salt solution added. This is shaken until the ether has evaporated. The resultant antigen, ready for use, is a slightly opalescent greenish fluid from which nothing settles out on standing.

Before an antigen can be used for the actual test, it is necessary to determine the quantity which will furnish a valid result. The substances which are used as antigens often have the power, if used in too large quantity, of themselves binding complement. It is necessary, therefore, to determine the largest quantity of each given antigen which may be used without exerting an anti-complementary action, i.e., which will not inhibit in the presence of normal serum but which will at the same time inhibit hemolysis when syphilitic serum is used. This is done by mixing graded quantities of the antigen with a constant quantity of complement ( 0.1 c.c. of fresh guinea-pig serum), in duplicate sets, adding to each tube of one set 0.2 c.c. of a normal serum, and to the other 0.2 c.c. of a known syphilitic serum. These substances are allowed to remain together for one hour and then red blood corpuscles and inactivated hemolytic serum are added. The quantity which has given. complete inhibition with the syphilitic serum, but absolutely no inhibi-

1. Noguchi, Personal communication. 
tion with normal serum, is the one to be employed in subsequent reactions. Before actual use, it is convenient to make a dilution of antigen in salt solution in such a way that 1 c.c. shall contain the amount required. Thus if 0.05 c.c. is wanted, mix 0.5 c.c. with 9.5 c.c. salt solution. Then 1 c.c.of this can be added to each tube in the test.

II. The Hemolytic Serum.-The hemolytic amboceptor, for the reaction, is obtained by injecting into rabbits the washed red blood corpuscles of a sheep. A 5 per cent emulsion of the corpuscles is made and of this 5 c.c., 10 c.c., 15 c.c., etc., are injected at intervals of five or six days. Three or four graded injections of this kind are usually sufficient to furnish a serum of adequate hemolytic power. The injections may be made intraperitoneally or intravenously. About nine or ten days after the last injection of corpuscles, the rabbit is bled from the carotid artery and the serum obtained by pipetting it from the clot.

It is best to have a hemolytic serum of high potency in order that the quantities used for the reaction may be as small as possible. This is desirable because of the fact that the serum may contain small amounts of precipitins for sheep's serum, due to insufficient washing of the corpuscles employed in the immunization. If such precipitins should be present in any quantity in the serum used for the reaction, precipitates might be formed, and these, as we know, have a tendency to carry down complement from a mixture.

While the quantitative relations of the complement and antigen in the Wassermann reaction are important, they are vastly more so in the case of the hemolytic amboceptor. For the actual reaction most observers make use of two hemolytic units. A hemolytic unit is the quantity of inactivated immune serum which, in the presence of complement, suffices to cause complete hemolysis in 1 c.c. of a 5 per cent emulsion of washed blood corpuscles. Noguchi ${ }^{1}$ has pointed out very clearly the dangers of not delicately adjusting the quantity of amboceptor used in the reaction. In a recent communication upon the subject, he has called attention to the experiments of Morgenroth and Sachs ${ }^{2}$ who have shown that the relationship between complement and amboceptor necessary for hemolytic reactions is one of inverse proportions. To state it more clearly, in their own words, "in the presence of larger quantities of amboceptor, smaller quantities, of complement suffice," and vice versa. Noguchi, in his work, has found that, while, in the

1 Noguchi, Proc. Soc. for Exper. Biol. and Med., VI., 3, 1909.

2 Morgenroth und Sachs, in Ehrlich's “ Gesammelte Arbeiten," etc., Berlin, 1904. 
presence of one unit of amboceptor, 0.1 c.c. of guinea-pig's complement is required to produce hemolysis, by using four, eight, and twenty units of amboceptor, complete hemolysis is obtainable with one-third, onefifth, and one-tenth of the 0.1 c.c. of complement, respectively. For this reason an excess of amboceptor might result in complete hemolysis in a test, if a small fraction of the complement were left unfixed by the syphilitic antibody. Another result of an excess of amboceptor would consist in a partial dissociation of the complement from its combination with the antigen-antibody compound. As Noguchi puts it, "a quantity of syphilitic antibody just sufficient to fix 0.1 c.c. of the complement against two units of the amboceptor is no longer efficient in holding back the complement from partial liberation against the influence exerted by more than four units of the amboceptor."

From these considerations it follows that the serum from rabbits immunized against sheep corpuscles must, in each case, be titrated in order to determine the hemolytic unit. For this purpose a number of mixtures are made in test tubes, containing each 0.1 c.c. of complement (fresh guinea-pig serum), 1 c.c. of a 5 per cent emulsion of sheep's corpuscles, and diminishing quantities of the inactivated hemolytic serum, thus:

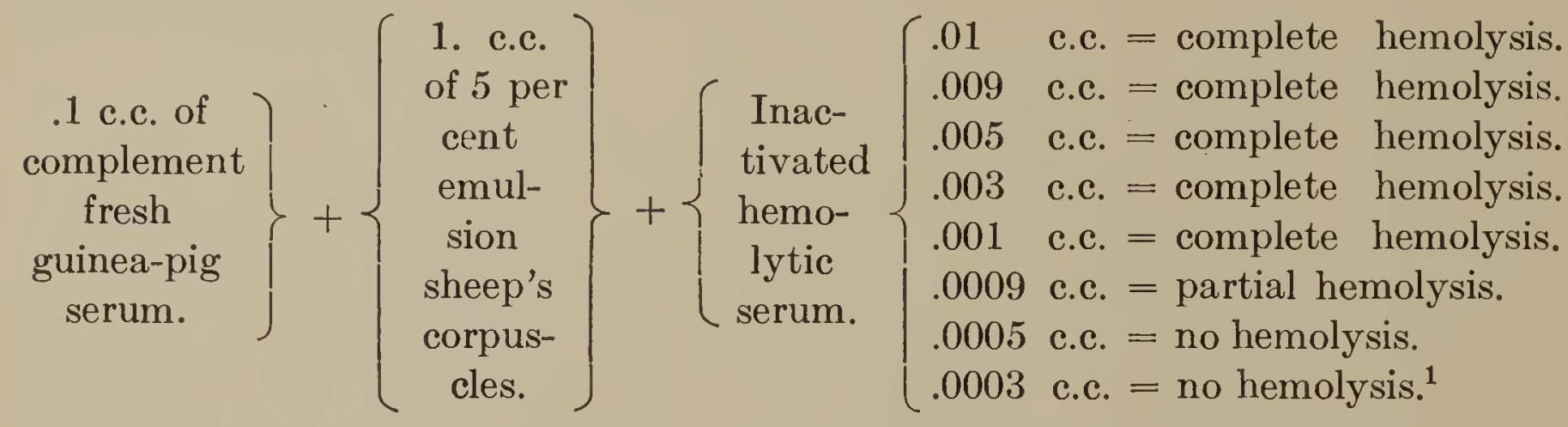

In the given case, 0.001 c.c. of the serum represents one unit, and 0.002 c.c., two units, is the quantity to be used for each test.

III. The Complement.-The complement for the Wassermann reaction is used in the form of fresh guinea-pig serum. This may be obtained in one of the following ways: A guinea-pig may be killed by an incision in the throat and the blood allowed to flow into a large Petri dish. This is set away in the ice chest until clear beads of serum have formed upon the surface, and these are then carefully removed with a pipette. The writers have found it convenient, however, to anesthetize the guinea-pigs, then, by a longitudinal incision into the

${ }^{1}$ In each tube the volume of the mixture should be made up to 5 c.c. with 0.85 per cent salt solution. 
neck to lay bare the carotid artery and, severing this, to allow the blood to flow into a sterile centrifuge tube. When clotting has occurred, the clot is loosened from the glass with a platinum needle and the serum separated by centrifugalization. Such serum should be used for no longer than three days after being taken and should be kept, except when in actual use, at a low temperature. The complement in guinea-pig serum is sufficiently constant in quantity for practical purposes.

IV. The Sheep Corpuscles.-The sheep corpuscles for the actual reaction are obtained by receiving the blood of a sheep in a small flask containing a sterile solution of a 0.5 per cent sodium citrate and 0.85 per cent sodium chloride, or into one containing glass beads or short pieces of glass tubing. In the former case, the citrate solution prevents clotting and the corpuscles may be washed free from the citrate solution and emulsified in salt solution before use in the test. In the latter case, it is necessary to shake the blood in the flask immediately after taking, and to continue the shaking motion for about ten minutes. At the end of this time, the blood will be defibrinated and the corpuscles are washed free from serum by centrifugalization in salt solution. A 5 per cent emulsion of the corpuscles in salt solution is employed for the test, made by measuring the bulk of centrifugalized corpuscles and adding nineteen parts of sterile salt solution. Thorough washing of the corpuscles is essential both in order to preclude the occurrence of precipitates and to remove any traces of complement present in the serum.

V. The Serum to be Tested for Syphilitic Antibody.-The serum of the patient upon whom the test is to be made is best obtained in the same way that blood is obtained for blood cultures. After surgical precautions as to sterilization, a needle is plunged into the median basilic vein and 3 or 4 c.c. of blood are removed. Whenever circumstances do not permit such procedure, blood may be obtained from the finger or the ear, always in sufficient quantity to furnish at least 1 c.c. of clear serum. Before use for the test, the patient's serum must be inactivated by heating in a water bath to $56^{\circ} \mathrm{C}$. for twenty minutes to half an hour. As, according to some observers, $56^{\circ} \mathrm{C}$. destroys the syphilitic antibody in part, Noguchi advises inactivation at $54^{\circ} \mathrm{C}$.

The TesT.-The actual test for antibody in a suspected serum is carried out in the following way: In a test-tube of suitable size, 0.1 c.c. of complement, 0.2 c.c. of the inactivated suspected serum, and the antigen, in quantity determined by titration, are mixed, and the total 
volume brought up to 3 c.c. with normal salt solution. This mixture is thoroughly shaken, and placed for one hour in a water bath or in the incubator at $37.5^{\circ} \mathrm{C}$. At the end of this time, there is added 1 c.c. of a 5 per cent emulsion of sheep's corpuscles, and two units of hemolytic amboceptor, determined by a titration of the inactivated hemolytic rabbit serum, as described above. This mixture is again placed at $37.5^{\circ}$ C. for one to two hours. If the antibody is present in the suspected serum, no hemolysis takes place. If absent, hæmolysis is complete.

No test is of use unless suitable controls are made. The controls set up should be as follows:

Control 1. For each serum tested the mixture described above, omitting antigen.

Controls 2 and 3. The mixture made as in the test but with known syphilitic serum (2) with and (3) without antigen.

Controls 4 and 5 . The mixture made as in the test, but with normal serum (4) with and (5) without antigen.

Control 6. Antigen and complement alone, left together for an hour before the addition of blood cells and amboceptor in order to preclude the possibility of the antigen itseif fixing complement. When working with a well-controlled antigen this control may be omitted.

Controls 7 and 8. The hemolytic system, complement, blood cells and amboceptor, set up in order to show that the system is in working order (7) with and (8) without antigen. It is convenient to set the tubes in two rows in a rack, the front row containing antigen, the back row containing the same mixture without antigen.

In a positive test, the test itself, and Control 2, alone, should show inhibited hemolysis. The other tubes should show complete solution of the hemoglobin. (See scheme, p. 259.)

Modifications of the Wassermann Test.-Bauer's Modification.Bauer ${ }^{1}$ utilizes the fact that normal human serum contains a certain amount of hemolytic amboceptor for sheep's corpuscles. In consequence he omits in his reaction the use of specifically immunized hemolytic rabbit serum. In carrying out the test he uses but four tubes:

1. Contains 0.1 c.c. of complement, the titrated amount of antigen, and 0.2 c.c. of the inactivated serum to be tested.

2. Contains the same mixture without antigen.

3. Is like one, except that normal serum is substituted for that of the patient.

1 Bauer, Deut. med. Woch., xii, 1908, and Berl. klin. Woch., xvii, 1908. 
4. Is like three, except that antigen is omitted.

If this modification is used at all (and its value is by no means established), a fifth control should be added in which known syphilitic serum is used.

These tubes are exposed to $37.5^{\circ} \mathrm{C}$. for one hour, at the end of which time 1 c.c. of a 5 per cent emulsion of sheep's corpuscles are added.

If the test is positive, tube one should be without hemolysis, as well as the fifth control with known syphilitic serum. Tubes two, three,

\section{SCHEME FOR WASSERMANN TEST.}

ADAPTED TO ORIGINAL WASSERMANN SYSTEM AFTER SCHEME OF NOGUCHI.

\begin{tabular}{|c|c|c|c|c|c|}
\hline & \multicolumn{2}{|l|}{$\begin{array}{l}\text { Test with Unknown } \\
\text { Serum. }\end{array}$} & $\begin{array}{l}\text { Test with Known } \\
\text { Positive Syphilic } \\
\text { Serum. }\end{array}$ & $\begin{array}{l}\text { Test with Known } \\
\text { Negative Normal } \\
\text { Serum. }\end{array}$ & $\begin{array}{c}\text { Test without Serum } \\
\text { to Control Efficien cy } \\
\text { of Hemolytic } \\
\text { System. }\end{array}$ \\
\hline 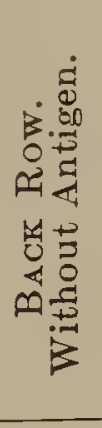 & 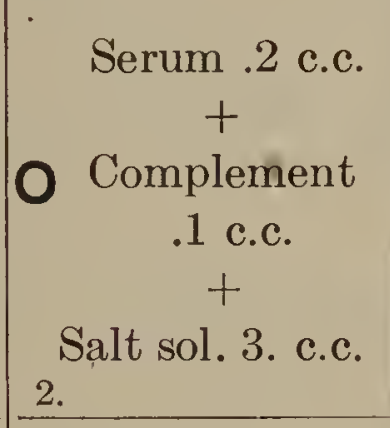 & 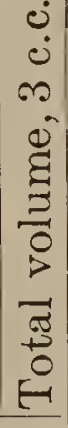 & 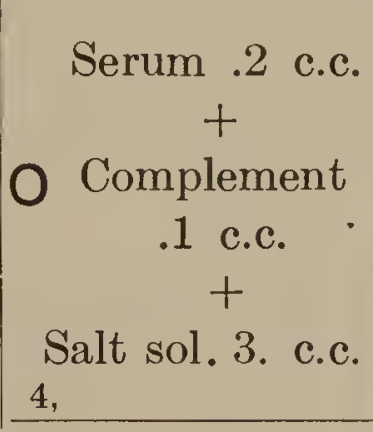 & 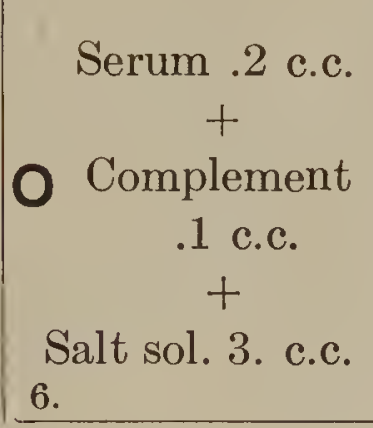 & $\begin{array}{c}\text { Complement } \\
.1 \text { c.c. } \\
+ \\
\text { Salt sol. 3. c.c. } \\
8 .\end{array}$ \\
\hline 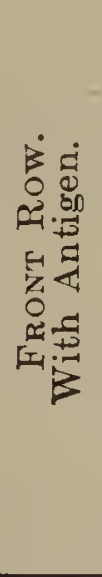 & $\begin{array}{c}\text { Serum .2 c.c. } \\
+ \\
\text { Complement } \\
.1 \text { c.c. } \\
+ \\
\text { Antigen } \\
\text { (required amount } \\
\text { in 1 c.c. salt sol.). } \\
+ \\
\text { Salt sol. 2. c.c. } \\
\text { 1. }\end{array}$ & 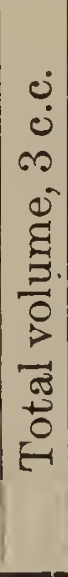 & $\begin{array}{c}\text { Serum .2 c.c. } \\
+ \\
\text { Complement } \\
.1 \text { c.c. } \\
+ \\
\text { Antigen } \\
+ \\
\text { Salt sol. } 2 \text { c.c. } \\
\text { 3. }\end{array}$ & $\begin{array}{c}\text { Serum .2 c.c. } \\
+ \\
\text { Complement } \\
.1 \text { c.c. } \\
+ \\
\text { Antigen } \\
+ \\
\text { Salt sol. 2. c.c. } \\
5 .\end{array}$ & $\begin{array}{l}\text { Complement } \\
.1 \text { c.c } \\
+ \\
\text { Antigen } \\
+ \\
\text { Salt. sol. 2. c.c. } \\
7 .\end{array}$ \\
\hline
\end{tabular}

$\mathbf{O}=$ test tube.

Place in water bath at $40^{\circ} \mathrm{C}$. for one hour, then add to all tubes red blood cells and amboceptor. These are previously mixed so that 2 c.c. contains the equivalents of 1 c.c. of a 5 per cent emulsion of sheep corpuscles and 2 units of amboceptor. Again expose to $40^{\circ} \mathrm{C}$. If the serum tested is positive, tubes 1 and 3 should show no hemolysis, all the other tubes showing complete hemolysis in one hour.

and four, on the other hand, should show complete hemolysis. This modification of the Wassermann test has not found universal adoption and is little used at present. 
Noguchi's Modification.-Noguchi ${ }^{1}$ has much simplified the test by making use of an anti-human hemolytic amboceptor instead of an antisheep amboceptor. In this way, he avoids the necessity of procuring fresh sheep corpuscles for each test by using the corpuscles of the patient himself. He has determined empirically that human serum contains, normally, no amboceptor active against the human red corpuscles. This fact is extremely important and has a decided advantage over the original Wassermann test, in that in any reaction in which sheep corpuscles are used as an indicator with human serum, the actual amount of hemolytic amboceptor used in the test is uncertain. For, as we have mentioned above, human serum, normally, may contain a variable quantity of amboceptor for sheep corpuscles. In Noguchi's test, therefore, the actual quantity of amboceptor is exactly known by previous titration. The hemolytic amboceptor for Noguchi's test is obtained by four or five injections of washed human corpuscles into rabbits. These corpuscles may be obtained from the heart's blood at autopsies, or better, if possible, from placentæ at childbirth. The unit for this amboceptor is obtained by titration as in the case of the sheep-blood amboceptor for the original Wassermann test. In setting up Noguchi's test, the following substances are used:-

(a) Patient's serum. Obtained in small glass capsule. About 2 c.c. should be taken.

(b) Complement. Fresh guinea-pig serum: 0.1 c.c. of a forty per cent, fresh, guinea-pig serum in salt solution is used in the test. Obtain by adding 1 part of guinea-pig serum to one and one-half parts of salt solution.

(c) Antigen. Substance prepared as in the Wassermann test by extraction of syphilitic or normal organ.

(d) Human corpuscles. Normal corpuscles or those of the patient himself may be employed. If the patient's red cells are chosen, these should not be used for other tests than that on the patient's own serum; 1 c.c. of a one per cent emulsion of washed corpuscles is used for the test.

(e) Anti-human amboceptor prepared by the injection of washed human corpuscles into rabbits and titrated against human corpuscles. Two units are used in the test.

The test itself is set up as follows: 
Tube 1. 1 drop patient's serum + complement (.1 c.c. of 40 per cent guineapig serum + antigen.

Tube 2. 1 drop patient's serum + complement (no antigen).

Tube 3. 1 drop known syphilitic serum + complement + antigen.

Tube 4. 1 drop known syphilitic serum + complement (no antigen).

Tube 5. 1 drop known normal serum + complement + antigen.

Tube 6. 1 drop known normal serum + complement (no antigen).

Tube 7. Complement alone (for hemolytic system control).

To each tube then add 1 c.c. of the one per cent emulsion of human corpuscles. Shake mixtures thoroughly and incubate or place in water bath at $38-40^{\circ} \mathrm{C}$. for one hour. Then add to each tube two units of amboceptor and replace in water bath for one hour. At the end of this time in a positive test there will be no hemolysis in tubes one and three while all the other tubes will show hemolysis.

Noguchi has simplified the technique of complement fixation further by drying measured amounts of antigen and amboceptor upon small squares of blotting paper. These may be dropped into the tubes directly, obviating the necessity of preparing fresh dilutions of the concentrated substances for each test. The substances in the dried state, moreover, may be preserved for longer periods than when kept in the liquid form.

The Determination of Antigen by Complement Fixation.-The principles underlying the preceding tests for the determination of suspected antibodies may be equally applied to the determination of suspected antigen. In the former case it was necessary to bring the serum to be tested into contact with the antigen specific for the suspected antibody, in the presence of complement, and at a suitable temperature. At the end of an hour the mixture was tested for free comple ment by the addition of hemolytic amboceptor and red blood cells. In testing for antigen, the procedure is reversed, in that the serum or other substance (bacterial extract) to be tested is brought into contact with an antibody specific for the antigen, in the presence of complement; and at the end of an hour at suitable temperature, free complement is again determined by hemolytic reaction as before.

When dealing with bacterial antigen, it is necessary, therefore, to prepare a highly potent immune serum against the bacteria which contain the specific antigen which is sought.

Thus in testing for typhoid-bacillus antigen in the serum of a patient, the substances required are as follows:

1. Complement: obtained from fresh guinea-pig serum. It is best to titrate the complement when possible, using for the test double the 
quantity necessary to produce complete hemolysis of 1 c.c. of a five per cent emulsion of blood cells, in the presence of two units of amboceptor. When titration is omitted 0.1 c.c. may be used in routine work, and is sufficiently accurate.

2. Hemolytic amboceptor: rabbit serum hemolytic for sheep corpuscles. Inactivated and titrated as for Wassermann test. Two units are used in the test.

3. A five-per-cent emulsion of sheep corpuscles in salt solution, prepared as for Wassermann test.

4. A highly potent typhoid antiserum obtained from an immunized rabbit. In this case the smallest quantity of the immune serum which will cause the fixation of complement in the presence of an emulsion or extract of typhoid bacilli is determined by experiment. The bacillary emul sion is prepared by scraping the growth from twenty-four-hour agar slant cultures, drying it, and macerating in a mortar with salt solution until a slightly opalescent emulsion is formed. A series of tubes is prepared into each of which is placed 0.1 c.c. of the emulsion of bacteria, 0.1 c.c. of fresh guinea-pig serum, as complement, and gradually diminishing quantities of the inactivated specific immune serum, ranging from 0.1 c.c. downward. These tubes are left for one hour at $38^{\circ}$ to $40^{\circ} \mathrm{C}$., and, following this, there are added the required quantities of red blood cells and hemolytic immune serum. The smallest quantity of immune serum which has completely inhibited hemolysis is the unit and a quantity slightly greater than this should be used in the actual test. ${ }^{1}$

5. Serum from the patient, inactivated at $56^{\circ} \mathrm{C}$. for twenty minutes.

In the actual test a series of tubes are prepared each of which contains:

1. Complement, the determined quantity or 0.1 c.c.

2. Antiserum, the determined quantity.

3. Diminishing quantities of the serum to be tested for antigen beginning with 1 c.c.

Salt solution is added for dilution to 3 c.c.

These substances are left together at $40^{\circ} \mathrm{C}$. for one hour and then the required quantities of amboceptor and red cells are added. The reaction is controlled by tubes containing the same ingredients without

1 Müller, "Technik d. serodiagnos. Methoden," Jena, 1909; Leidke, "Zur Kenntniss d. Komplemente," Würzburg, 1908. 
the typhoid antiserum. In a positive test there will be no hemolysis in the tubes containing the patient's serum.

Proteid Differentiation by Complement Fixation.-That the technique of complement fixation was applicable to the determination of specific proteid antigen - such as human or animal blood-was shown by Gengou ${ }^{1}$ in 1902 . The principles worked out by him have been practically applied by Neisser and Sachs ${ }^{2}$ and others to the forensic differentiation of animal proteids and these tests are said to be more delicate and reliable than precipitation tests made for the same purpose.

The substances necessary for the reaction are as follows:

1. Complement, titrated as above.

2. Hemolytic amboceptor as above.

3. A five-per-cent emulsion of sheep corpuscles as above.

4. Specific antiserum.

This is obtained from a rabbit immunized with the proteid for which the test is to be made; viz.: human or animal blood serum. This must be titrated. In order to do this, diminishing quantities of the antiserum are mixed in a series of tubes with the determined quantity of complement, and the antigen which is to be tested for, i.e., the homologous serum with which the antiserum has been produced. Since the test should be sufficiently delicate to determine 0.0001 c.c. of the antigen, this quantity is added to each tube. The actual titration is as follows: ${ }^{3}$

1. Antiserum, undiluted $.1+$ homologous serum $.0001+$ complement.

2. Antiserum diluted by $10.75+$ homologous serum $.0001+$ complement.

3. Antiserum diluted by $10.75+$ homologous serum $.0001+$ complement.

4. Antiserum diluted by $10.3+$ homologous serum $.0001+$ complement. etc., down to .1

These tubes are incubated for one hour and hemolytic amboceptor and red blood cells are added. The smallest quantity of antiserum which has completely inhibited hemolysis is the "unit" and one and a half to two times this quantity is used for the test.

5. A solution of the blood spot or other material to be tested prepared as for precipitin test. (See page 254.)

1 Gengou, Ann. de l'inst. Pasteur, 1902.

${ }^{2}$ Neisser und Sachs, Berl. klin. Woch., 1905 and 1906. See also Citron, in Kraus and Levaditi " Handbuch," etc.

${ }^{3}$ Citron, loc. cit. 
For the actual test the following mixtures are made in a series of tubes, each of which contains:

1. Complement

2. Antiserum $\}$ quantity determined by titration.

3. Diminishing quantities of the substance in which the antigen is suspected, ranging from 0.1 c.c. downward to 0.0001 c.c.

Salt solution is added as a diluent up to 3 c.c. and the tubes are placed in the incubator or water-bath at $37.5^{\circ}$ to $40^{\circ} \mathrm{C}$. At the end of this time red blood cells and amboceptor are added as before.

The tubes are controlled by a series containing all the above ingredi. ents except the antiserum. 


\section{CHAPTER XVII}

\section{PHAGOCYTOSIS}

THE studies on immunity which we have outlined in the preceding sections have dealt entirely with the phenomena occurring in the reaction between bacteria or bacterial products and the body fluids. These studies, we have seen, have formed the basis of a theoretical conception of immunity formulated chiefly by the German school of bacteriologists under the leadership of Ehrlich, Pfeiffer, Kruse, and others. Parallel with these developments, however, investigations on immunity have been carried on which have brought to light many important facts concerning the participation of the cellular elements of the body in its resistance to infectious germs.

The inspiration for this work and the greater part of the theoretical considerations which have been based upon it have emanated from Metchnikoff ${ }^{1}$ and his numerous pupils at the Pasteur Institute in Paris. The phenomenon which these observers have studied in great detail and upon the occurrence of which they have based their conceptions of immunity, is known as phagocytosis.

It is well known that among the lowest unicellular animals the nutritive process consists in the ingestion of minute particles of organic matter by the cell. The rhizopods, which may be found and studied in water from stagnant pools or infusions, when observed under the microscope, may be seen to send out short protoplasmic processes, the pseudopodia, by means of which they gradually flow about any foreign particle with which they come in contact. If the ingested particle is of an inorganic nature and indigestible, it will be again extruded after a varying period. If, however, the ingested substance is of a nature which can be utilized in the nutrition of the protozoon, it is rapidly surrounded by a small vacuole within which it is gradually dissolved and becomes a part of the cellular protoplasm. This digestion within the unicellular organism is probably due to a proteolytic enzyme ${ }^{2}$ which acts in the

Metchnikoff, "L'Immunité dans les maladies infectueuses." 2 Mouton, Ann. de l'inst. Pasteur, xvi, 1902. 
presence of a weakly alkaline reaction. This has been shown by the actual extraction, from amebæ, of a trypsin-like ferment.

As we proceed higher in the scale of the animal kingdom, we find that this power of intracellular digestion, while not uniformly an attribute of all the body cells, is still well developed and a necessary physiological function of certain cells which have retained primitive characters. In animals like the cœlenterata, in which there are two cell layers, an entoderm and an ectoderm, the ectodermal cells have lost the power of intracellular digestion, while the entodermal cells are still able to ingest and digest suitable foreign particles. It is only as we proceed to animals of a much higher organization that the function of cell ingestion of crude food is entirely removed from the process of general nutrition. Nevertheless, in these animals also, the actual cell ingestion of foreign particles occurs, but it is now limited entirely to a definite group of cells. In the higher animals and in man, this function of phagocytosis is limited to the white blood cells of the circulation, or leucocytes, to certain large endothelial cells lining the serous cavities and blood-vessels, and to cells of a rather obscure origin which contribute to the formation of giant cells within the tissues. A convenient division of these phagocytic cells is that into "wandering cells" and "fixed cells." The wandering cells are the polymorphonuclear leucocytes, called "microphages" by Metchnikoff, and certain large mononuclear elements or "macrophages." Fixed cells, also called macrophages by Metchnikoff and possessing the power of ameboid motion, include the cells lining the serous cavities, and the blood and lymph spaces. The small lymphocytes, so far as we know, have no phagocytic functions.

In studying the cellular activities which come into play whenever foreign material of any description gains entrance into the animal body, a definite reaction on the part of the phagocytic cells may be observed. When we inject into the peritoneal cavity of a guinea-pig a small quantity of nutrient broth, and examine the exudate within the cavity from time to time, we can observe at first a diminution from the normal of the cells present in the peritoneal fluid. This may be due either to an injury of the leucocytes by the injected substance, or to an actual repellent influence which the injected foreign material exerts upon the wandering cells. ${ }^{1}$ Very soon after this, however, the exudate becomes extremely rich in leucocytes, chiefly of the polymorphonuclear variety, the maximum of the reaction being reached about eighteen to twenty-four 
hours after the injection. After this, there is a gradual diminution in the leucocytic elements until the fluid in the peritoneal cavity again reaches its normal condition. It is plain, therefore, that the presence of the foreign material in the peritoneal cavity has, after a primary repellent action upon the phagocytes, attracted them in large numbers to the site of the foreign substance. Such repelling or attracting influences upon the leucocytes are spoken of as negative or positive chemotaxis. The reasons for chemotaxis are not well understood. In the case of bacteria, which chiefly interest us in the present connection, chemotactic attraction or repulsion is intimately dependent upon the nature of the microorganism, and very probably has a definite relationship to its virulence. Whether or not the principles of chemotaxis may serve to explain the hypo- and hyper-leucocytoses, observed and diagnostically utilized in clinical medicine, is by no means positive. It is likely, however, that the two phenomena are closely associated. Levaditi ${ }^{1}$ believed that he obtained some evidence that negative chemotaxis may take place within the blood-vessels when he noticed that the intravenous injection of cholera spirilla into immunized guinea-pigs resulted in an immediate disappearance of leucocytes from the circulating blood, and their accumulation in the internal organs. On the other hand, this may possibly be more logically explained by a concentration of both bacteria and leucocytes in the capillary system of such an organ as the liver, as it is known that injected bacteria rapidly disappear from the general circulation, but may be demonstrated in the various organs for some time after injection.

We have seen, therefore, that the invasion of the animal body by foreign material, living or dead, is followed by a prompt response on the part of the phagocytic cells. In the case of bacteria, when these are deposited in the subcutaneous areolar tissues, the inflammatory reaction which follows brings with it an emigration of microphages (polynuclear leucocytes) from the blood-vessels-and these are the so-called pus cells. When the injection of bacteria is intraperitoneal, after a primary diminution, there is an increase of leucocytes in the peritoneal cavity which soon results in the formation of a copious turbid exudate. If the pus of an abscess or the exudate from an infected peritoneum is examined microscopically, it will be seen that many of the microphages have taken bacteria into their cytoplasm. That fully virulent living bacteria can be so taken up has been variously proven. The phago-

1 Levaditi, Presse méd., 1900. 
cytosis is, therefore, not simply a removal of the dead bodies of bacteria previously killed by the body-fluids, but represents an actual attack upon living and fully virulent microorganisms. That the ingested bacteria are often alive after ingestion is proved by the fact that the injection of exudate containing, so far as can be determined, only intracellular bacteria, has, in several instances, been found to give rise to infection.

After the bacteria have remained for some time within the cytoplasm of the leucocyte, vacuoles may be seen to form about them, similar to those mentioned in discussing the digestive processes of amebæ. If the preparations are, at this stage or later, stained with a one-per-cent solution of neutral red, it will be found that the bacteria, colorless under normal conditions, will be stained pink, an evidence of their beginning disintegration. At a later stage in the process of intracellular digestion, the bacteria will lose their form, and appear swollen, granular, and vacuolated, and finally will be no longer distinguishable. If, on the other hand, the ingestion of bacteria brings about the death of a leucocyte, the neutral red will not stain the bacteria, the digestive vacuoles will not form, and the leucocyte itself will disintegrate.

It must not be forgotten, however, that not all microorganisms are equally susceptible to phagocytosis. Some may resist ingestion more energetically than others by agencies not fully understood. Others again, like the tubercle bacillus and the anthrax bacillus for instance, may, after ingestion, oppose great difficulties to intracellular digestion.

To a certain extent, moreover, the variety of the bacterium determines the variety of phagocyte attracted to the point of invasion. In the cases of most of the bacteria of acute diseases, the microphages or polymorphonuclear leucocytes are the ones upon which the brunt of the battle devolves. Other invaders, like the Bacillus tuberculosis, blastomyces, and others, find themselves opposed chiefly by the macrophages. Cells of animal origin, such as the dead or injured cells of the animals' own body or the cells of other animals artificially introduced, are ingested by macrophages. This is true also of many parasites of animal nature.

It is clear, thus, that the process of phagocytosis is a universal response on the part of the body to the invasion of foreign particles of dead material, of alien cells, and of living microorganisms. It remains to be shown upon what basis this process may be regarded as an essential feature in protecting the body against infection.

The numerous researches of Metchnikoff have brought out the important fact that phagocytosis is regularly more active in cases in 
which the infected animal or human being eventually recovers. In animals, furthermore, which show a high natural resistance against any given microorganism, phagocytosis is decidedly more energetic than it is in animals more susceptible to the same incitant. Thus, experimenting with anthrax infection in rats, Metchnikoff was able to show that, in these animals, a decidedly more rapid and extensive phagocytosis of anthrax bacilli takes place than in rabbits and guinea-pigs and other animals which are delicately susceptible to this infection. While different interpretations have been attached to this phenomenon, its actual occurrence may be accepted as a proven fact.

In his later investigations, furthermore, Metchnikoff was able to show that a direct parallelism existed between the development of immunity in an artificially immunized animal and the phagocytic powers of its white cells. He showed that rabbits artificially immunized to anthrax, responded to anthrax infection by a far more active phagocytosis than did normal, fully susceptible animals of the same species.

It is quite impossible, in the space allotted, to recount the many similar experiments by which the accuracy of these observations has been confirmed. While few bacteriologists at the presentu day harbor any doubt as to the truth of these contentions, the fundamental differences between the conclusions drawn from these various phenomena by the school of Metchnikoff and by that of the German workers may be clearly stated as follows: Metchnikoff believes that phagocytosis is the cardinal factor which determines immunity, while Pfeiffer and others maintain that the determining factors upon which recovery or lethal outcome depends, lie in the fluids of the body, the serous exudate and its contents of immune body and complement, while the phagocytosis occurring coincidently, is merely a means of removal of the bacteria after the outcome has already been decided.

In the further developments of his theory, Metchnikoff has claimed that the immune body and complement-the presence of which in blood serum and exudates he by no means overlooks-are derivatives of the leucocytes.

The immune body or "fixator," as Metchnikoff has named it, has been shown by Wassermann and Takaki ${ }^{1}$ to be most plentiful in the spleen, lymph nodes, and bone marrow of animals-all of them organs in which large collections of leucocytic elcments are found. Metchnikoff's opinions as to the leucocytic origin of the complement, or "cytase,"

${ }^{1}$ Wassermann und Takaki, Berl. klin. Woch., 1898. 
have found support in the experiments of Levaditi, ${ }^{1}$ who was able to demonstrate the absence of complement in blood plasma, -i.e., where no destruction of leucocytes had taken place-and in those of Cantacuzène, ${ }^{2}$ who showed that cholera-immune guinea-pigs would succumb to intraperitoneal injection of these bacteria when the diapedesis of leucocytes had been prevented by the administration of opium.

The chapter of phagocytosis in its relation to bacterial immunity is by no means closed. The problems involved in it are intricate and will require much further study. The subsequent sections upon opsonins, aggressins, and upon leucocyte extract, incorporate the more recent studies which may be said to have followed logically in the footsteps of Metchnikoff's work.

1 Levaditi, Presse méd., 1900.

2 Cantacuzène, Ann. de l'inst. Pasteur, 1897. 


\section{CHAPTER XVIII}

\section{OPSONINS, LEUCOCYTE EXTRACT, AND AGGRESSINS}

\section{OPSONINS}

Although the theories of immunity are, as we have stated, generally classified as the humoral and the cellular or phagocytic theories, the separation has never, even in the minds of the warmest partisans, been an absolute one. Thus, Buchner and his successors looked for the origin, first, of alexin, then of complement, in the leucocytes, and Metchnikoff attributed to immune serum the quality of stimulating the leucocytes (stimulins) to increased phagocytosis. The serum, according to Metchnikoff, acted, not directly upon the bacteria, in the nature of bactericidal or lytic substances, but rather upon the leucocytes, preparing or arming these for the fray. Denys and Leclef ${ }^{1}$ were the first definitely to oppose this view. These authors, on the basis of ex. periments done upon streptococcus immunity in rabbits, came to the conclusion that the serum aided phagocytosis rather by its action upon the bacteria than by its influence upon the leucocytes.

Wright ${ }^{2}$ in 1903 and 1904 undertook a systematic study of the relation of the blood serum to phagocytosis, in a series of careful experiments. Using his own modifications of the technique of Leishman, ${ }^{3}$ he first determined the direct dependence of phagocytosis upon some substance contained in the blood serum. He further proved conclusively that this serum component acts upon the bacteria directly and not upon the leucocytes, is bound by the bacteria, and renders them subject to phagocytosis. The presence of these substances in sera, furthermore, which appear entirely free from bactericidal or lytic bodies, and the thermolabile character of the substances $\left(60^{\circ}\right.$ for ten or fifteen minutes destroys them) seemed to exclude their identity with the immune bodies of other authors.

1 Denys et Leclef, La cellule, xi, 1895.

${ }^{2}$ Wright and Douglas, Proc. Royal Soc. London, Ixxii, 1904.

${ }^{3}$ Leishman, Brit. Med. Jour., i, 1902. 
Because of their action in preparing the bacteria for ingestion by the leucocytes, he named these bodies "opsonins" ( $\dot{\psi} \psi \omega \nu \hat{\varepsilon} \omega$, to prepare food).

Neufeld and Rimpau ${ }^{1}$ soon after, and independently of Wright, described similar substances in the blood serum of streptococcus and pneumococcus immune animals, which they called bacteriotropins. Because of their greater thermostability it is not yet possible to identify these bacteriotropins absolutely with the opsonins.

The importance of these opsonic substances in immunity was shown by Wright ${ }^{2}$ in a series of experiments in which he determined that in persons ill with staphylococcus or tubercle-bacillus infections, the phagocytic powers were relatively diminished toward these microorganisms, but could be specifically increased upon active immunization with dead bacteria or bacterial products.

The results of Wright have been confirmed and elaborated by numerous workers.

The diminished power of leucocytes to take up bacteria without the co-operation of serum was demonstrated, after Wright, by Hektoen and Ruediger, ${ }^{3}$ who worked with gradually increasing dilutions of serum. The contention of the Wright school, however, that leucocytes are entirely impotent for phagocytosis without the aid of serum, can not be regarded as proven, in face of the work of Löhlein ${ }^{4}$ and others who have observed phagocytosis on the part of washed leucocytes.

The specificity of opsonins and their multiplicity in a given serum were shown mainly by the work of Bullock and Atkin, ${ }^{5}$ Hektoen and Ruediger, ${ }^{6}$ and Bullock and Western. ${ }^{7}$ These authors showed that the opsonic substances in sera could be absorbed out of the sera, one by one, by treatment with various species of bacteria, a procedure analogous to the method of absorption used in the study of agglutinins.

The increase of phagocytic power demonstrated by Wright in immune sera naturally led to the question whether this depended merely upon an increase of the normal opsonins or whether the newly formed immune opsonins were entirely different substances. The greater thermostability of the opsonins in immune sera seemed, at first, to support the

${ }^{1}$ Neufeld und Rimpau, Deut. med. Woch., xl, 1904.

${ }^{2}$ Wright and Douglas, Proc. Roy. Soc., London, Ixxiv, 1905

3 Hektoen and Ruediger, Jour. Inf. Dis., ii, 1905.

- Löhlein, Ann. de l'inst. Pasteur, 1905 and 1906.

s Bullock and Atkin, Proc. Roy. Soc., London, lxxiv, 1905.

- Hektoen and Ruediger, loc. cit.

- Bullock and Western. Proc. Roy. Soc., loc. cit. 
latter view. Dean, ${ }^{1}$ however, showed that not all of the normal opsonins are thermolabile and that, by absorption experiments, bacteria treated with normal sera could be prevented from taking up opsonins from immune sera. These facts seem to point strongly toward the identity of normal and immune opsonic substances.

Further study of the opsonins has led to numerous other questions regarding their structure, their relation to other immune bodies, etc., which are largely still in the stage of controversy, and for which the original monographs must be consulted.

The controversial questions may be briefly reviewed as follows:

As stated above, Wright believed originally that the bodies discovered by him in normal sera, the "normal opsonins," in other words, were distinct bodies that could not be identified with either the complement or antibodies present in serum. Neufeld and Hüne, ${ }^{2}$ Levaditi and Inmann, ${ }^{3}$ and others, on the other hand, maintain that the opsonic action of normal serum, at least, is intimately related to the complement contents of such serum.

They base this contention not only upon the thermolability of normal opsonins, but also upon the fact that opsonin may be removed from normal serum at the same time as complement by the method of complement fixation, detailed in another section (see pp. 245 and 261). ${ }^{4}$

The contention of Wright that the thermostable opsonic substances of immune serum are distinct bodies, not identical with the amboceptors, is supported by the work of Hektoen, ${ }^{5}$ Neufeld and Töpfer, ${ }^{6}$ and others. The problem, however, can by no means be regarded as finally settled, since other workers, notably Levaditi, are inclined to identify the immune opsonins with lytic amboceptors.

As to the structure of the opsonic substances, moreover, differences of opinion still exist. Hektoen and Ruediger ${ }^{7}$ who have investigated the question attribute to opsonins a complex constitution. They believe them to possess a thermostable haptoohore group and a thermolabile "opsonophore" group and that heating beyond a definite temperature converts the opsonins into opsonoids by

1 Dean, Proc. Roy. Soc., London, lxxvi, 1905.

2 Neufeld and Hüne, Arb. a. d. kais. Gesundheitsamt, xxv.

3 Levaditi and Inmann, Compt. rend. de la soc. de biol., 62, 1907.

4 Levaditi, Presse médicale, 70, 1907.

5 Hektoen, Jour. of Inf. Dis., iii, 1906.

- Neufeld und Töpfer, Cent: f. Bakt., xxxviii, 1905.

- Hektoen and Ruediger, Jour. of Inf. Dis., ii, 1905. 
destruction or alteration of the "opsonophore" group. This view is not shared by all workers and has been disputed by Bullock and Atkin."

The Technique of Wright.-The three factors necessary for the performance of an opsonic test are (1) the blood serum to be tested; (2) an even emulsion of bacteria, and (3) leucocytes.

(1) Blood serum is obtained by bleeding from the finger and receiving the blood into glass capsules (Fig. 66). These are sealed at both ends; the blood is allowed to clot; and the separation of serum is hastened by a few revolutions of a centrifuge.

(2) The bacterial emulsion is obtained by rubbing up a few loopfuls of a twenty-four-hour slant agar culture with a little physiological salt solution ( 0.85 per cent) in a watch glass. A very small

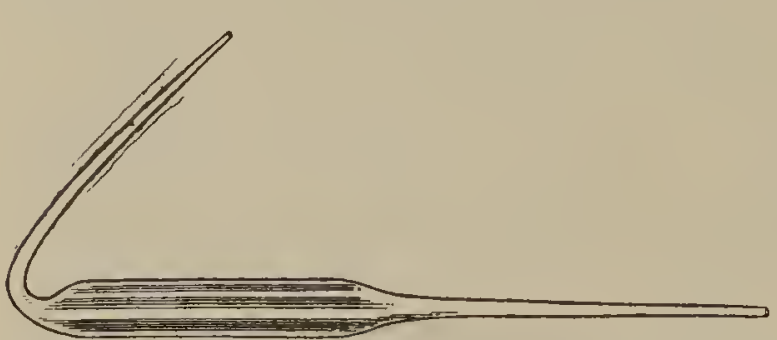

Fig. 66.-Wright's Capsule for Collecting Blood. amount of salt solution is used at first and more is gradually added, drop by drop, as the emulsion becomes more even. The final breaking up of the smaller clumps is best accomplished by cutting off very squarely the end of a capillary pipette, placing it perpendicularly against the bottom of the watch glass, and sucking the emulsion in and out through the narrow chink thus formed. (Fig. 67.)

Emulsions of tubercle bacilli are more difficult to make. The bacilli filtered off in the manufacture of old tuberculin are commonly used. These are washed in salt solution on the filter, and are then scraped off and sterilized. They are then, in a moist condition, placed in a mortar and thoroughly ground into a paste. While grinding, salt solution 1.5 cent) is gradually added until a thick emulsion appears. This emulsion may be diluted and larger clumps separated by centrifugalization.

(3) The leucocytes are obtained by bleeding from the ear or finger directly into a solution containing eighty-five hundredths per cent to one per cent of sodium chlorid and five-tenths to one and five-tenths per cent of sodium citrate. Ten or fifteen drops of blood to 5 or 6 c.c. of the solution will furnish sufficient leucocytes for a dozen tests. This mixture is then centrifugalized at moderate speed for five to six minutes. At the end of this time, the corpuscles at the bottom of the tube will be covered by a thin grayish pellicle, the buffy coat, consisting 
chiefly of leucocytes. These are pipetted off with a capillary pipette (by careful superficial scratching movements over the surface of the buffy coat).

There being, of course, no absolute scale for phagocytosis, whenever an opsonin determination is made upon an unknown serum, a parallel control test must be made upon a normal serum. This normal is best obtained by a "pool" or mixture of the sera of five or six supposedly normal individuals.

The three ingredients - serum, bacterial emulsion, and leucocyteshaving thus been prepared, the actual test is carried out as follows:

Fig. 67.-Pipette for Opsonic Work.

Capillary pipettes of about six or seven inches in length and of nearly even diameter throughout, are made. These are fitted with a nipple and a mark is made upon them with a grease-pencil about 2 to $3 \mathrm{~cm}$. from the end (Fig. 68). Corpuscles, bacteria, and serum are then successively, in the order named, sucked into the pipette up to the mark, being separated from each other by small air-bubbles. Equal quantities of each having thus been secured, they are mixed thoroughly by repeatedly drawing them in and out of the pipette upon a slide. The mixture is then drawn into the pipette; the end is sealed; and incubation at $37.5^{\circ}$ is carried on for an arbitrary time, usually fifteen to thirty minutes. ${ }^{1} \quad$ The control with normal serum is treated in exactly

Fig. 68.-Pipette with three Substances, Corpuscles, Bacteria, and Serum, AS FIRST TAKEN UP.

the same way. After incubation the end of the pipette is broken off, the contents are again mixed, and smears are made upon glass slides in the ordinary manner of blood smearing. Staining may be done by Wright's modification of Leishman's stain, by Jenner's, or by any other of the usual blood stains. In these smears, then, the number of bacteria contained in each leucocyte is counted. The contents of about eighty

${ }^{1}$ For the purpose of incubation, specially constructed water baths, marketed under the name of "opsonizers," may be used. 
to one hundred cells are usually counted and an average is taken. This average number of bacteria in such leucocytes is spoken of as the "phagocytic index." The phagocytic index of the tested serum, divided by that of the "normal pool" (control) serum, gives the "opsonic index."

Another method of estimating the opsonic content of a given blood serum has been contributed by Simon, Lamar, and Bispham. ${ }^{1}$ These authors employed dilutions both of the patient's serum and of normal serum ranging from one in ten to one in one hundred. With these dilutions, they carry out opsonic experiments with bacterial emulsions and washed leucocytes in the same way as this is done in the Wright method, except that they recommend the employment of thinner bacterial emulsions than are usually employed in the former method. In examining their slides, they do not estimate the number of bacteria found within the leucocytes, but rather the percentage of leucocytes which actually take part in the phagocytic process, ${ }^{2}$ i.e., which contain bacteria.

By the same method of dilution, they determine what they have called "the opsonic coefficient of extinction," a phrase which is used to express the degree of dilution of the serum at which no further phagocytosis takes place. They claim for their methods the more delicate determination of variations in opsonic power. The method has not been sufficiently used to permit the expression of an opinion as to its value.

The Vaccine Therapy of Wright.-In connection with his more theoretical work upon opsonins, Wright has laid much stress upon the value of active immunization in the treatment of infectious diseases. Beginning his work with staphylococcus and tubercle-bacillus infections, he has extended his methods, with the aid of many collaborators, to gonococcus, streptococcus, pneumococcus, and a number of other bacterial infections. In all these cases, when possible, he uses for therapeutic purposes a so-called "autogenous vaccine" which is made with the bacteria isolated from the patient himself. In the case of tubercle-bacillus infections, he uses for treatment the new-tuberculinbacillary-emulsion of Koch. The production of vaccme is, according to Wright, as follows:

Production of Vaccines.-After isolation of the organisms from the patient, cultures are made with a view of obtaining considerable amounts

'Simon, Lamar, and Bispham, Jour. Exp. Med., viii, 1906.

2 Simon and Lamar, Johns Hopkins Hosp. Bull., xvii, 1906. 
of bacterial growth. In making vaccines with poorly growing organisms, large surfaces must be inoculated. Organisms are best grown for this purpose upon the surface of agar or glucose agar (the enrichment of the agar with sugar or acetic fluid, etc., depending upon the cultural requirements of the urganism in question), in square eight-ounce medicine bottles laid upon their sides. This furnishes a large area for inoculation. After sufficient growth has taken place upon the agar, two or three cubic centimeters of sterile normal salt solution are introduced into the bottles with a sterile pipette. With this the growth is gently washed off the surface of the agar, more salt solution gradually being added as necessary. The emulsification may be facilitated by gently scraping the growth off the medium by means of a flexible platinum loop. This thick bacterial emulsion is then pipetted out of the bottles, during which process an equalization of the emulsion can be attained by repeated sucking in and out with the pipette. The emulsion is then placed in a sterile test tube which may then be drawn out at its open end into a capillary opening. It is a point of practical importance that, in preparing such capsules out of a test tube, a few inches of air space should be left above the surface of the emulsion, so that expansion during heating may not blow out the top of the glass tube. A dozen or so of sterile glass beads may be put into these tubes in order to aid in emulsification. Shaking the beads in such a tube will help in breaking up small clumps of bacteria.

The emulsion is then standardized; that is, a numerical estimation of bacteria per cubic centimeter must be made. This standardization is best done before sterilization, because during the latter process a number of bacteria may be broken up, and, while unrecognizable morphologically, are, nevertheless, represented in the emulsion by their products. The standardization may be accomplished by highly diluting a definite volume of the emulsion, planting plates with definite quantities of the dilution, and counting colonies. Wright prefer's, as more exact, an enumeration of the bacteria against red blood cells. This is done in the following way:

A little of the emulsion is placed in a watch glass and from it, with a pipette as used in the estimation of the opsonic index, one volume is taken and is mixed with an equal volume of blood from the finger and two or three volumes of salt solution. The salt solution is added in order to dilute the red cells so that they can be conveniently counted and to prevent clotting. These substances are thoroughly mixed in a pipette and spread upon a slide as in making a blood smear, and as even 
and uniform a smear as possible should be made. They are then stained either by Jenner's or Wright's blood stain.

The preparations are examined with an oil-immersion lens. In order to limit a definite microscopic field, it is convenient. to use an Ehrlich diaphragm, or else, in lieu of this, to mark a circle with a blue pencil upon the lens of the eye-piece. The red blood cells and bacteria, in a number of these fields, are counted and the ratio between them is estimated. Knowing the number of red blood cells to the cubic millimeter in the particular blood employed, by previous blood count, and knowing that equal volumes of blood and of bacterial emulsion have been used in the mixture, it is easy from this ratio to ascertain the number of bacteria contained in a cubic millimeter of the original emulsion. Thus, for instance, if in an average of twenty fields bacteria are to red blood cells as two is to one, and the blood employed contains five million red blood cells to each cubic millimeter, then a cubic millimeter of our emulsion contained ten million bacteria, and a cubic centimeter one thousand times as many.

The vaccine, thus produced and standardized, is sterilized by suspension in a water bath at $60^{\circ} \mathrm{C}$. for one hour on each of five or six consecutive days. Its sterility is then controlled by culture.

From this stock emulsion small quantities may be drawn off and diluted for therapeutic use.

The initial dose given by Wright in staphylococcus infections, in which the method has been most frequently employed, varies from fifty to one hundred millions of bacteria. In working with the tubercle bacillus, the ordinary tuberculin dosage is adhered to.

Wright, in his work, makes use of the opsonic index in order to estimate changes in the resistance of the patient against the given infection. In other words, he bases his judgment as to whether the patient is improving or not, upon the opsonic power of the patient's serum. In following the opsonic index of a patient during systematic treatment with vaccine, Wright has found definite changes upon the basis of which he constructs a curve of opsonic power. Immediately after the injection of vaccine, he finds that there is a brief period during which the opsonic power of the patient is depressed below its original state. This he calls the negative phase. The length of time occupied by this negative phase depends both upon the condition of the patient and upon the size of the dose given. It is usually completed within twenty-four hours. After this, there is a gradual rise in the opsonic power, at first rapid, later more slow, until a maximum is reached after a vary- 
ing number of days. This period of rise represents the positive phase. The second inoculation with vaccine should, according to Wright, be made when the opsonic power is again beginning to sink after the highest point of the positive phase.

The facts of Wright's investigations have been given in the preceding pages, purposely without critical considerations. The existence of opsonic or phagocytosis stimulating substances in blood serum may be accepted as fact. It is also of unquestionable value to the science of immunity that renewed vigor has been infused into the investigation of active therapeutic immunization. The far-reaching claims of therapeutic benefit, which have been made by Wright and his school, however, have not yet received sufficient support by clinical observation to be fully accepted, although intelligent application of this treatment in suitable cases has undoubtedly proved its therapeutic value.

\section{LEUCOCYTE EXTRACT}

In the foregoing sections upon Phagocytosis and Opsonins, we have discussed the protective action exerted by the living leucocytes against bacterial infection and the relation of these cells to the blood serum. We have seen, furthermore, that, while our knowledge of the blood serum, as developed at present, shows that puagocytes may be aided by this in the ingestion of bacteria, the subsequent digestion of the germs, and possibly the neutralization or destruction of their intracellular poisons, is, as far as we know, largely accomplished by the unaided phagocytic cell. It is an obvious thought, therefore, that, in the struggle with bacterial invaders, the leucocytic defenders might be considerably re-enforced if they were furnished, as directly as possible, with a further supply of the very weapons which they were using in the fight with the microorganisms. With this thought as a point of departure, Hiss ${ }^{1}$ conceived the plan of injecting into infected subjects the substances composing the chief cells or all the cells usually found in exudates, in the most diffusible form and as little changed by manipulation as possible; and he also assumed that extracts would be more efficacious than living leucocytes themselves, since if diffusible they would be distributed impartially to all parts of the body by the circulatory mechanism. They would then, as quickly as absorption would permit, relieve the fatigued leucocyte and also protect by any toxin-neutralizing or other power they might possess, the cells of highly specialized functions.

${ }^{1}$ Hiss, Jour. Med. Res., N. S., xiv, 3, 1908. 
The method of obtaining these substances as used both in animal experiments and in the treatment of human subjects is at present as follows:

Rabbits, preferably of 1,500 grams weight or heavier, receive intrapleural injections of aleuronat. This is prepared by making a three per cent solution of starch in meat-extract broth, without heating, and adding to this, after the starch has gone into thorough emulsion, five per cent of powdered aleuronat. This is thoroughly mixed, boiled for five minutes, and filled into sterile potato tubes, 20 c.c. into each tube. Final sterilization is done preferably in an autoclave. The rabbit injections are carried out by injecting 10 c.c. into each pleural cavity in the intercostal spaces at the level of the end of the sternum, in the anterior axillary line, great care being exerted to avoid puncturing of the lungs. The rabbits are left for twenty-four hours, at the end of which time a copious and very cellular exudate will have accumulated in the pleural cavities. This is removed, after killing the animals with chloroform, by opening the anterior chest wall under rigid precautions of sterility, and pipetting the exudate into sterile centrifuge tubes. Immediate centrifugalization before clotting can take place then permits the decanting of the supernatant exudate fluid. To the leucocytic sediment is then added about 2 c.c. of sterile distilled water, and the emulsion is thoroughly beaten up with a stiff bent platinum spatula. Smears are now made on slides, stained by Jenner's blood stain, and examined for possible bacterial contamination. It is well also to take cultures. Sterile distilled water is then added to each tube, about twenty volumes to one volume of sediment, and the tubes are set away in the incubator for eight hours. At the end of this time the sterility is again controlled as above, and further extraction in the refrigerator continued until the extract is used.

In experimenting upon animals, Hiss ${ }^{1}$ observed that pneumococcus, staphylococcus, streptococcus, meningococcus, and typhoid, dysentery, and cholera infections in rabbits and guinea-pigs were profoundly modified when injections of leucocyte extracts, prepared as above, were administered intraperitoneally or subcutaneously during the course of the infection. In many cases animals were saved by these substances from infections which proved rapidly fatal in untreated control animals, even when the protective injections were made as late as twenty-four hours after intravenous infection.

${ }^{1}$ Hiss, Jour. Med. Res., N. S., xiv, 3, 1908. 
In applying this method of treatment, by subcutaneous injections, to infections in man, Hiss and Zinsser observed distinctly beneficial results in cases of epidemic cerebrospinal meningitis, in lobar pneumonia, in staphylococcus infections, and in erysipelas. ${ }^{1}$

In experimenting with the leucocyte extracts in vitro the same authors were able to show that precipitates occurred when clear leucocyte extract and the clear extract of various bacteria were mixed. ${ }^{2}$

Further experiments, carried out both in animals and in the test tube, showed that while the leucocytic extracts possessed slight bactericidal powers for a variety of microorganisms, these attributes did not seem sufficient to explain the profound, modifying influences exerted upon bacterial infections by these extracts. Experiments have also shown that the leucocyte extracts possess some distinct power of neutralizing or destroying the poisonous products of typhoid and dysentery bacilli, Whether or not the final explanation of the action of these extracts will be fourd to lie in these endotoxin-neutralizing properties of the leucocytic substances, can not as yet be determined, and this problem must be leit for further research to decide.

That bactericidal substances can be extracted from leucocytes by various methods has been repeatedly shown by Schattenfroh, Petterson, Korschun, and others. ${ }^{3}$ The researches of Petterson as well as, more recently, the work of Zinsser, have shown that these "endolysins," as Petterson has called them, have a structure quite different from that of the serum bacteriolysins in that they are not rendered inactive by temperatures under $80^{\circ} \mathrm{C}$, , but, when once destroyed by higher temperatures, can not be reactivated either by the addition of fresh serum or of unheated leucocyte extracts. The last-named authors, moreover, have shown that these endocellular bactericidal substances are not increased by immunization, the quantity present in each leucocyte being probably at all times simply sufficient for the digestion of the limited number of bacteria which can be taken up by the individual leucocyte.

\section{AGGRESSINS}

An extremely obscure chapter in our knowledge of the reaction of animals and man against infection is the one dealing with the questions

${ }^{1}$ Hiss and Zinsser, Jour. Med. Res., N. S., xiv, 3, 1908; ibid., xv, 3, 1909.

${ }^{2}$ Hiss and Zinsser, ibid., xiv, 3, 1908.

${ }^{3}$ Schattenfroh, Arch. f. Hyg., 1897; Petterson, Cent. f. Bakt., I, xxxix, 1905, and ibid., xlvi, 1908; Korschun, Ann. de l'inst. Pasteur, xxii, 1908; Zinsser, Jour. Med. Res., xxii, 3, 1910. 
of varying pathogenicity between different bacterial species and between different races of the same microorganism. We know that certain bacteria may be injected into an animal or human being in considerable quantities, without producing anything more than the temporary local disturbance following the subcutaneous administration of any innocuous material. Other bacteria, on the other hand, such as the bacillus of anthrax or the bacillus of chicken cholera, injected in the most minute dosage, may give rise to a rapidly fatal septicemia. Within the same species, furthermore, fluctuations in virulence may take place which may depend upon a variety of influences which have been discussed in another section and need not be recapitulated. Suffice it to say that variations in the susceptibility of inoculated subjects do not, in any way, furnish a sufficient explanation for these phenomena and we are forced to seek for the key to the problem in the activities of the bacteria themselves.

In an effort to cast light upon this subject, Bail, following in the footsteps of his predecessors, Kruse, ${ }^{1}$ Deutsch and Feistmantel, ${ }^{2}$ has formulated his so-called "aggressin-theory." In its reasoning, this theory is indirectly an offspring of Metchnikoff's phagocytic theory and is, in many of its phases, antagonistic to the purely humoral conception of immunity.

Bail ${ }^{3}$ was first led to the formulation of his theory by extensive researches which he had made in conjunction with Petterson ${ }^{4}$ into anthrax immunity. He had noted, as others before him had, that animals, highly susceptible to anthrax, often possessed marked bactericidal powers against this bacillus. When such animals, whose serum should surely be capable of bringing about the death of, at least, a few hundred anthrax bacilli, were injected with doses far less than this number they nevertheless succumbed rapidly and the bacilli multiplied enormously in their bodies. He argued from this that the injected microorganisms must possess some weapon whereby they were enabled to counteract the protective forces of the animal organism. In an anthrax-immune animal, as a matter of fact, no proliferation of bacteria took place and the injected germs were rapidly disposed of by the protective forces, foremost of which was phagocytosis.

1 Kruse, Ziegler's Beiträge, xii, 1893.

2 Deutsch und Feistmantel, "Die Impfstoffe in Sera," Leipzig, 1903.

${ }^{3}$ Bail, Cent. f. Bakt. I, xxvii, 1900, and xxxiii, 1902.

4 Bail und Petterson, Cent. f. Bakt., I, xxxiv, 1903; xxxv, 1904; xxxvi, 1904. 
The theory of Bail ${ }^{1}$ as eventually formulated, after extended investigations which need not be outlined, contains the following basic principles: ${ }^{2}$

Pathogenic bacteria differ fundamentally from non-pathogenic bacteria in their power to overcome the protective mechanism of the animal body, and to proliferate within it. They accomplish this by virtue of definite substances given off by them, probably in the nature of a secretion, which acts primarily by protecting them against phagocytosis. These substances (referred to by Kruse as "Lysins") were named by Bail, "Aggressins." The production of aggressins by pathogenic germs is probably absent in test-tube cultures, or, at any rate, is greatly depressed under such conditions, but is called forth in the animal body by the onslaught of the germicidal or other influences encountered after inoculation.

These aggressins can be found, according to Bail, in the exudates occurring about the site of inoculation in rapidly fatal infections. $\mathrm{He}$ obtained them, separate from the bacteria themselves, by the prolonged centrifugation and subsequent decanting of edema fluid, and pleural and peritoneal exudates.

Two fundamental experimental observations are brought forward by Bail in support of the truth of his contentions. In the first place, he was able to show that fatal infection could be produced in animals by the injection of sublethal doses of bacteria, when, together with the germs, there was administered a small quantity of "aggressin." $\mathrm{He}$ inferred from this experiment that the injected aggressin had served in paralyzing the onslaught of phagocytic and other protective agencies, and had thus made it possible for the bacteria to gain a foothold and to proliferate.

The second experimental support upon which Bail's theory is founded consists in the successful immunization of animals with aggressin. Animals were treated with aggressive exudates, from which all bacteria had been removed by prolonged centrifugalization and which had been rendered entirely sterile by three hours' heating to $60^{\circ} \mathrm{C}$. and by the addition of five-tenths per cent of phenol. Animals so treated were not only immune themselves, but contained a substance in their serum which permitted the passive immunization of other untreated animals. Bail explained this by assuming the production of antiaggressins in the

${ }_{1}^{1}$ Bail, Arch. f. Hyg., lii, 1905; liii, 1905; Wien. klin. Woch., xvii, 1905.

${ }^{2}$ Bail und Weil, Wien. klin. Woch., ix, 1906; Cent. f. Bakt., I, xl, 1906; xlii, 1906. 
treated subjects. His cxperiments and those of his pupils were conducted with a large variety of microorganisms, among which were the typhoid and dysentery bacilli, the bacilli of chicken cholera and of plague, the cholera spirillum, and various micrococci. According to whether a microorganism is capable of producing an aggressin and consequently of invading the animal body, he divides bacteria into "pure parasites," "half parasites," and "saprophytes."

The theory of Bail has been extensively attacked by a number of authors, chief among whom are Wassermann and Citron, ${ }^{1}$ Wolff, ${ }^{2}$ and Sauerbeck. ${ }^{3}$ The criticism which these investigators make of Bail's views is based upon laborious experimentation and has succeeded in placing the "aggressin" theory upon a very precarious footing. It is claimed by them, in the first place, that much of the "aggressive" character of Bail's exudates is due to their containing liberated bacterial poisons (endotoxins). This they have nuaintained both because the sterile "aggressin" exudates could be shown to possess a considerable degree of independent toxicity and because the aggressive action of such an exudate could be duplicated by aqueous extracts of bacteria. Citron, ${ }^{4}$ furthermore, was able to show, by the Bordet-Gengou method of complement fixation, that the exudates of Bail contained considerable quantities of free bacterial receptors, which, in taking up bacteriolytic immune body, would neutralize any lytic power on the part of the infected animal. By this antilytic action, he believes, Bail's first contention, the virulence-enhancing action of the exudates, can be explained. The nature of the immunity produced in animals by Bail's method of treatment is less easily explained and less exposed to adverse criticism. Bacteriolytic immunity alone probably can not account for the high degree of resistance imparted by a few injections of the aggressins. On the other hand, the establishment of an antiaggressive immunity has not been sufficiently supported to stand as a proven fact. Final judgment must be postponed until further investigation shall have brought a better understanding of the phenomenon.

${ }_{1}$ Wassermann and Citron, Deut. med. Woch., xxviii, 1905.

${ }^{2}$ Wolff, Cent. f. Bakt., I, xxx viii, 1906.

3 Sauerbeck, Zeit. f. Hyg., Ivi, 1907.

Citron, Cent. f. Bakt., I, xl, 1905; xli, 1906; and Zeit. f. Hyg., lii, 1905. 


\section{CHAPTER XIX}

\section{ANAPHYLAXIS OR HYPERSUSCEPTIBILITY}

\section{PHENOMENA OF ANAPHYLAXIS}

THE phenomena now grouped together under the heading of anaphylaxis and hypersusceptibility have but recently become the subject of systematic experimentation. Nevertheless, manifestations now recognized as belonging to this category, had not escaped the attention of a number of the earlier workers in immunity.

By anaphylaxis is meant the following train of phenomena: When a foreign proteid is introduced by subcutaneous, intraperitoneal, intravenous, or subdural injection (or in some cases by feeding) into the animal body, after a time there will appear a specific hypersusceptibility of the animal for this proteid. After a definite interval, a second injection of the same substance, harmless in itself, will produce violent symptoms of illness and often rapid death in an animal so prepared. The phenomena are not limited to any given class of proteids, but are manifest in the case of animal, vegetable, and bacterial proteids, and within certain limits are specific.

As early as 1893 , Behring ${ }^{1}$ and his pupils ${ }^{2}$ had noticed that animals, highly immunized against diphtheria toxin, with high antitoxin content of the blood, would occasionally show marked susceptibility to injections of small doses of the toxin.

The phenomena observed by them was interpreted as an increased tissue susceptibility to the toxin, and Wassermann, reasoning on the basis of Ehrlich's side-chain theory, formulated the conception that the increased susceptibility was due to toxin receptors, increased in number by immunization, but not yet separated from the cells that had produced them; the cells thereby becoming more vulnerable to the poison. In the same category belongs the observation of Kretz, who noticed that normal guinea-pigs did not show any reaction after injections of innocuous

Behring, Deut. med. Woch., 1893.

${ }^{2}$ Knorr, Dissert., Marburg, 1895; Behring und Kitashina, Berl. klin. Woch., 1901. 
toxin-antitoxin mixtures, but that marked symptoms of illness often followed such injections when made into immunized guinea-pigs. Other phenomena which are now regarded, a posteriori, as probably depending upon the principles involved in anaphylaxis, are the tuberculin and mallein reactions, fully described in another place, and the adverse effects often following the injections of antitoxins in human beings, conditions spoken of under the heading of "serum sickness." The lastnamed condition has been made the subject of an exhaustive study by v. Pirquet and Schick. ${ }^{1}$

That the injection of diphtheria antitoxin in human beings is of ten followed, after an incubation time of from three to ten days, by exanthematous eruptions, urticaria, swelling of the lymph glands, and often albuminuria and mild pulmonary inflammations, has been noticed by many clinicians, who have made extensive therapeutic use of antitoxin. It was recognized early that such symptoms were entirely independent of the antitoxic nature of the serum, but depended upon other constituents or properties peculiar to the antitoxic serum. Moreover, symptoms of this description were by no means regular in patients injected for the first time, but seemed to depend upon an individual predisposition, or idiosyncrasy. v. Pirquet and Schick, however, noticed that in those injected a second time, after intervals of weeks or months, the consequent evil effects were rapid in development, severe, and occurred with greater regularity. Many of the phases of such "serum sickness" are still obscure, since experimental conditions can not be controlled, and many modifying factors can not be excluded in observations made upon human beings, and the grouping of the above conditions with the phenomena of anaphylaxis is still tentative.

The fundamental observations from which our present knowledge of anaphylaxis takes its origin are those made in 1898 by Hericourt and Richet, ${ }^{2}$ who observed that repeated injections of eel serum into dogs gave rise to an increased susceptibility toward this substance instead of immunizing the dogs against it. Following up the lines of thought suggested by this phenomenon, Portier and Richet ${ }^{3}$ later made an interesting observation while working with actino-congestin-a toxic substance which they extracted from the tentacles of Actinia. This

1v. Pirquet and Schick, "Die Serum Krankheit," monograph, Leipzig and Wien, 1905.

${ }_{2}^{2}$ Hericourt and Richet, Compt. rend. de la soc. de biol., 53, 1898.

3 Portier and Richet, Compt. rend. de la soc. de biol., 1902; Richet, Ann. de l'inst. Pasteur, 1907 and 1908. 
substance in doses of 0.042 grams per kilogram produced vomiting, diarrhea, collapse, and death in dogs. If doses considerably smaller than this were given in quantities sufficient to cause only temporary illness, and several days allowed to elapse, a second injection of a quantity less than one-quarter or one-fifth of the ordinary lethal dose would cause rapid and severe symptoms and often death. Similar observations were made soon after this by Richet with mytilo-congestin, a toxic substance isolated from mussels. In these experiments there remained little doubt as to the fact that the first injection had given rise to a well-marked increased susceptibility of the dogs for the poison used.

It was Richet who first applied to this phenomenon the term "ana-

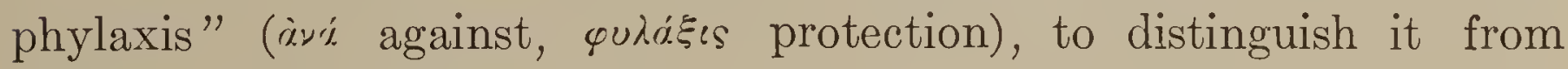
immunization or prophylaxis.

Soon after Richet's earlier experiments, and simultaneously with his later work, Arthus ${ }^{1}$ made an observation which plainly confirmed Richet's observations, though in a somewhat different field. The observation of Arthus is universally spoken of as the "phenomenon of Arthus."

He noticed that the injection of rabbits with horse serum (a substance in itself without toxic properties for normal rabbits) rendered the rabbits delicately susceptible to a second injection made after an interval of six or seven days. The second injection-even of small doses -regularly produced severe symptoms and often death in these animals:

An observation very similar to that of Arthus was made by Theobald Smith ${ }^{2}$ in 1904. Smith observed that guinea-pigs injected with diphtheria toxin-antitoxin mixtures in the course of antitoxin standardization, would be killed if after a short interval they were given a subcutaneous injection of normal horse serum.

The fundamental facts of hypersusceptibility had thus been observed, and Otto ${ }^{3}$ working directly upon the basis of Smith's observation, carried on an elaborate inquiry into the phenomenon. Almost simultaneously with Otto's publication there appeared a thorough study of the condition by Rosenau and Anderson. ${ }^{4}$

The researches of Otto, and Rosenau and Anderson, besides confirming the observations of previous workers, brought out a large number

1 Arthus, Compt. rend. de la soc. de biol., 55, 1903.

2 Th. Smith, Jour. Med. Res., 1904.

3 Otto, "Leuthold Gedenkschrift," 1905.

"Rosenau and Anderson, Hyg. Lab. U. S. Pub. Health and Marine Hosp. Serv. Bull., 29, 36, 1906, 1907. 
of new facts. They showed conclusively that the action of the horse serum had no relationship to its toxin or to its antitoxin constituents, that the "sensitization" of the guinea-pigs by the first injection became most marked after a definite incubation time of about ten days. Sensitization was accomplished by extremely small doses (one one-millionth in one case, usual doses $\frac{1}{2} \frac{1}{50}$ to 1 c.c.). Rosenau and Anderson, furthermore, excluded hemolysin or precipitin action as explanations of the phenomena, and proved that hypersusceptibility was transmissible from mother to offspring; and that it was specific-animals sensitized with horse serum not being sensitive to subsequent injections of other proteids. These authors, Vaughan ${ }^{1}$ and Wheeler, Nicolle, ${ }^{2}$ and others, furthermore, showed that the reaction was by no means limited to animal sera, but was elicited by proteids in general, pepton, egg albumin, milk, the extract of peas, and bacterial extracts.

The typical anaphylactic reaction, then, is obtained when animals, preferably guinea-pigs, are injected with a small quantity of a given proteid, and ten or fifteen days subsequently given a second injection of the same substance employed for the first or sensitizing inoculation. The quantity used for the second injection should be considerably larger than that used for sensitization when the injection is made intraperitoneally or subcutaneously. When given intravenously, intracranially, or intracardially; amounts as small as 0.25 to 0.008 c.c. may suffice. The time at which a second injection gives rise to the most violent symptoms, moreover, is to a large extent dependent upon the size of the sensitizing dose. ${ }^{3}$ After extremely small initial quantities (0.005-0.002 c.c.), the anaphylactic state is usually well developed, according to Rosenau and Anderson, ${ }^{4}$ after twelve or fourteen days. After larger doses ${ }^{5}$ the time required for the development of anaphylaxis is usually longer-extending of ten over weeks, or even months.

While the sensitizing or first dose may be given subcutaneously, intravenously, intraperitoneally, or intracardially with equal success, Besredka and Steinhardt maintain that no anaphylaxis results if the first dose is given intracranially. This statement, however, has found contradiction in the work of Rosenau and Anderson. The time required for full sensitization, furthermore, depends, according to the last-named

1 Vaughan, Assn. Am. Phys., May, 1907.

2 Nicolle, Ann. de l'inst. Pasteur, 2, 1903.

3 Besredka, Ann. de l'inst. Pasteur, 1907.

* Rosenau and Anderson, loc. cit.

s Otto, Münch. med. Woch., 1907. 
authors, also upon the mode of injection of the first dose; on this point, however, no conclusions are, at present, justified.

At reinjection, the symptoms are more prompt in developing and more severe when the injection is made intraperitoneally, intracardially, or intracerebrally than when the subcutaneous route is chosen.

The symptoms occurring in sensitized animals after the second or anaphylactic injection are usually well-marked and unmistakable. The animals move about restlessly, breathe rapidly, and may cough. Often they stagger about or fall upon one side, and die frequently in convulsions within a time ranging from five minutes to one hour after the injection. During this time there is a rapid fall of temperature and frequently defecation and urination. Animals that recover from the condition after such symptoms, return to normal within a remarkably short time-twelve to twenty-four hours.

Animals dead of anaphylaxis, according to Gay and Southard, ${ }^{1}$ show congestion of the serous membranes of peritoneum, pleura, and pericardium, with small hemorrhagic spots on the heart and lungs and the pleura. In some cases there is fatty degeneration in the parenchyma cells of the heart, the muscles, and in the nervous system. Such lesions, however, could not be found by Otto, but were found in some cases by Doerr. $^{2}$

When sensitized animals recover from the second injections, they are thereafter immune-that is, they do not react to subsequent injections of the same substance.

This immunity or "antianaphylaxis" as Besredka ${ }^{3}$ and Steinhardt have called it, appears immediately after recovery from the second injection. Antianaphylaxis may also be produced if animals which have received the first or sensitizing dose are injected with comparatively large quantities of the same substance during the preanaphylactic period -or, as it is sometimes spoken of, during the anaphylactic incubation. time. This injection should not be done too soon after the first dose, but rather toward the middle or end of the preanaphylactic period.

If given within one or two days after the sensitizing injection, anaphylaxis will develop, nevertheless. Whether or not the antianaphylactic condition is transitory or permanent is not yet fully shown. Besredka and Steinhardt believe that it lasts a long time, while Otto found guinea-

${ }^{1}$ Gay and Southard, Jour. Med. Res., May, 1907.

2 Doerr, in Krauss and Levaditi, "Handbuch," etc.; 2, Die Antikörper.

"Besredka and Steinhardt, Ann. de l'inst. Pasteur, 1907. 
pigs immunized in the above manner to lose their antianaphylaxis within three weeks.

An important development of our knowledge of the phenomena of anaphylaxis was achieved when Nicolle, Otto, ${ }^{1}$ Gay and Southard, ${ }^{2}$ and others ${ }^{3}$ succeeded in showing that the hypersusceptible state could be passively transferred to normal animals by injecting them with the serum of anaphylactic animals. In such experiments the serum of the anaphylactic animal is first injected in quantities of 0.5 c.c. or preferably more, and twenty-four hours later an injection of the specific antigenthat is, the proteid used for sensitization-is given. The animals so treated show typical symptoms of hypersusceptibility and often die.

Simultaneous inoculation of the two substances, either mixed or injected separately, does not, according to Rosenau and Anderson, produce the same effect. On this point, however, there is not complete unanimity, since Weill-Hallé and Jemaire ${ }^{4}$ report aphylactic syn!ptoms in guinea-pigs injected simultaneously with horse serum, and the serum of guinea-pigs hypersusceptible to horse serum. Reversal of the procedure originally described, however, may be successfully practiced. Thus Pick and Yamanouchi ${ }^{5}$ have recently succeeded in producing anaphylactic symptoms by injecting rabbits first with beef serum, and some time later with anti-beef serum from rabbits. Their experiments, however, are not entirely analogous to those given above, since the antiserum used by them for reinjection was actually a precipitating immune serum. A remarkable fact, observed by Otto, is that the serum of guinea-pigs who have been given the sensitizing or first injection will confer passive anaphylaxis on the eighth or tenth day after injection, before the animals themselves show evidences of being actively. hypersensitized. It is also true that occasionally the serum of antianaphylactic animals will possess the power of conferring passive anaphylaxis upon other normal animals.

Anaphylaxis may also be passively transmitted by inheritance. Thus, according to Rosenau and Anderson, the young of anaphylactic guinea-pigs show hypersusceptibility, irrespective of whether the mother became hypersusceptible before or after the beginning of pregnancy.

1 Otto, loc. cit.

${ }^{2}$ Gay and Southard, loc. cit.

3 Nicolle, Ann. de l'inst. Pasteur, 1907, 1908.

4 Weill-Hallé and Lemaire, Compt. rend. de la soc. de biol., 1907.

${ }_{5}^{5}$ Pick and Yamanouchi, Wien. klin. Woch., xliv, 1908. 
Such anaphylaxis has no reference whatever to the condition of the father, and is not transmitted by the milk.

\section{THEORIES CONCERNING ANAPHYLAXIS}

Our present knowledge of anaphylaxis is largely empirical. We are not yet in a position to correlate the many data gained experimentally into a theory which offers anything like a satisfactory explanation for all the phenomena observed. Nevertheless, a number of hypotheses have been advanced which deserve serious consideration, since they are experimentally supported and may serve as points of departure for future research.

One of the earliest ideas advanced was based upon the Ehrlich theory of receptor overproduction by tissue cells during immunization. It was suggested that hypersusceptibility might well be due to the stimulation of new specific receptors which as yet remained sessile upon the body cells instead of having been thrown off into the blood stream. As a consequence, the cells, having an affinity for more of the toxic substance of the antigen than they possessed normally, had become more vulnerable. This opinion, however, is hardly tenable, in face of the facts of passive anaphylaxis, in which, we have seen, the hypersusceptibility may be transmitted with the serum of the sensitized individual.

v. Pirquet and Schick, ${ }^{1}$ as well as many other observers, have regarded the anaphylactic process as analogous to other inmune reactions, and believe that an antigen in the serum first injected produces a specific antibody. The reaction between these two substances following the second injection gives rise to the anaphylactic symptoms. The essential fcature of this opinion is the assumption that the substance which sensitizes after the first injection is identical with that which exerts the anaphylactic injury after reinjection.

Wolff-Eisner ${ }^{2}$ has expressed a belief which has found much experimental support in the hands of Vaughan and Wheeler. ${ }^{2}$ Wolff-Eisner holds that all cells and proteids contain a toxic substance which is characterized by its inability to produce a neutralizing antibody when injected into animals. The first injection produces a lysin for the proteid injected, which possesses the power of liberating such poisons from the complex molecule. A second injection is followed, conse- 
quently, by a rapid liberation of the toxic fraction, and injury to the animal results. This view has been expressed in slightly different form by Richet ${ }^{1}$ and has been more clearly formulated and experimentally supported by Vaughan and Wheeler ${ }^{2}$ who were actually able to extract from various proteids toxic substances which gave rise in animals to a symptom complex not unlike that of typical anaphylaxis. (Extraction with alkalinized seventy-per-cent alcohol.)

Not entirely unlike these views is the hypothesis advanced by Gay and Southard, ${ }^{3}$ who assume that a part of the proteid introduced on first injection is assimilated and removed, but that another part, unassimilable, remains in the circulation and exerts a constant irritation upon the tissue cells, rendering them abnormally susceptible to reinjections of the same substance. They speak of this toxic or irritating, nonassimilable substance as "anaphylactin," and believe that in passive sensitization it is the transference of this element which renders the recipient anaphylactic.

Opposed to the opinions of most other worker's is that expressed by Besredka. ${ }^{4}$ Besredka believes that the substance which produces sensitization is not identical with that which gives rise to the symptoms on reinjection. According to his conception of the process, the sensitizing injection contains an active element (called by him "sensibilisinogen"), which gives rise in the injected animal to a specific antibody ("sensibilisin"). This sensibilisin circulates in the blood and is stored by the cells of the central nervous system. On reinjection of the same proteid, a reaction takes place between the anchored sensibilisin and a third substance, present in the proteid and not identical with sensibilisinogen, which acts typically upon the nerve cells and gives rise to the symptoms. This third substance he speaks of as "antisensibilisin." That the process of anaphylaxis takes place probably in the nervous system is rendered plausible by the fact that ether narcosis seems to prevent its occurrence (a fact maintained by Besredka but contradicted by Rosenau and Anderson). Furthermore, direct introduction of the second dose into the brain gives rise to more rapid and violent anaphylaxis than when any other route is chosen. Besredka calls attention to the fact, furthermore, that no anaphylactic symptoms occur when the serum of a sensitive animal is introduced into another animal simul-

1 Richet, Ann. de l'inst. Pasteur, xxi, 1907.

${ }^{2}$ Vaughan and Wheeler, Jour. Infect. Dis., iv, 1907.

${ }^{3}$ Gay and Southard, loc. cit.

- Besredka, Ann. de l'inst. Pasteur, 1907 and 1908. 
taneously with its specific proteid, but only when the serum of the sensitized animal precedes the injection of its antigen. This, he argues, points to an anchorage of the sensitizing elements to the body cells before an anaphylactic injury can occur. If the two substancse, sensibilisin and antisensibilisin, meet in the blood stream, no harm results, the elements neutralize each other and the animal is anti-anaphylactic. His contention that there are two separate elements, sensibilisinogen and antisensibilisin, in the original proteid, is based on the fact that sensitization can be accomplished by sera heated to from $100^{\circ}$ to $120^{\circ} \mathrm{C}$, but that, after sensitization, no anaphylaxis results if the injected serum be exposed previously to temperatures of $100^{\circ} \mathrm{C}$., and the reaction is rendered much less violent even by exposure of sera to temperature of 50 to $60^{\circ} \mathrm{C}$.

Recently an attempt has been made to associate the phenomena of anaphylaxis with the formation of precipitates. Hamburger ${ }^{1}$ early expressed the opinion that anaphylaxis may be nothing more than the formation of emboli by serum precipitates. This view, however, has found few adherents in face of the facts that we have no positive evidence of the actual occurrence of precipitates in the blood streams of living animals, and that it has been shown by Friedemann ${ }^{2}$ that the precipitates produced in vitro will, when injected intravenously in animals, pass through the capillaries without harmful effects.

Doerr and Russ, ${ }^{3}$ on the other hand, have recently studied carefully the relationship between anaphylaxis and the precipitin reaction and have shown a close parallelism between the two. These observers injected rabbits with precipitating antisera and twenty-four hours later treated the same animals with the antigen employed for the production of these precipitating sera. They found that, in such experiments, the regularity of occurrence and degree of anaphylaxis which ensued, were directly proportionate to the precipitating power's of the serum first injected. They claim, from such results, that precipitable antigen and anaphylactic antigen are identical substances. They conceive the phenomenon of anaphylaxis as a reaction between precipitins, attached to the tissue cells, and the precipitable antigen. In other words, the anaphylactic shock is looked upon as an intracellular precipitin reaction.

Friedberger and Hartoch ${ }^{4}$ have recently called attention to another

${ }^{1}$ Hamburger, quoted by U. Friedemann, Zeit. f. Immunitätsforschung, ii, $19(09$.

2 Friedemann, loc. cit.

3 Doerr and Russ, Zeit. f. Immunitätsforschung, iii, 1909.

- Friedberger und Hartoch, Zeit. f. Immunitätsforschung, iii, 1909. 
factor which may possibly lead to further elucidation of phenomena of hypersusceptibility. They have shown that, in passive anaphylaxis at least, simultaneously with the occurrence of symptoms, there is a marked diminution of complement in the serum of the treated animal. Intravenous injection of substances which prevent complement absorption in vitro-concentrated salt solution, for instance-prevented anaphylaxis in both actively and passively sensitized animals. They suggest that sudden removal of complement from the circulation has a definite causal relationship to anaphylaxis.

Friedberger's more recent work has shown that the action of complement in vitro, both upon bacteria and upon the precipitates formed when a dissolved antigen is mixed with its antiserum, will produce poisons which kill guinea-pigs in typical anaphylactic shock. His results, much confirmed by his own work and that of others, seem to indicate that anaphylactic shock may be due to a poison, "anaphylatoxin," which is formed by the proteolytic action of the complement upon the foreign protein which is injected into the animal, and which is sensitized to the action of the complement by the antibody formed in response to the first injection.

The toxic effects of Friedberger's "anaphylatoxins" are in many ways similar to those of Vaughan's toxic-protein-split products, and the subsequent development of his theory of anaphylaxis and infectious disease may be logically regarded as a further elaboration of Vaughan's views, though approached from a different point of departure. 


\section{GHAPTER XX}

\section{FACTS AND PROBLEMS OF IMMUNITY IN THEIR BEARING UPON THE TREATMENT OF INFECTIOUS DISEASES}

WHILE the various facts and theories of immunity and infection have been given in the preceding sections, no systematic attempts have been made to correlate the facts presented, or to determine their bearing on the most vital problem of all-the treatment of infectious diseases.

To understand more fully this point of view, it is necessary briefly to recall certain of the facts which are known about the physiology, metabolism, and composition of the bacteria, and of their ability to neutralize directly or to respond adaptively to the agents directed against them by the invaded animal. Some of these facts are so well understood that passing mention here is sufficient: such, for instance, is the fact that certain microorganisms, especially the bacilli of diphtheria and tetanus, secrete soluble poisons both during artificial cultivation and during their life in the animal body, which poisons are eminently toxic. These poisons are true secretions and are largely independent of the composition of the surrounding medium so long as this favors the physiologic activities and growth of the germs. Such germs, then, once having gained even an insecure foothold in the animal body, by no matter what favoring circumstances, are possessed of a powerful weapon of offense against the sensitive physiologic bases of the host and, possibly, of defense against its more immediate and mobile means of combating the germs themselves. In the case, however, of most other pathogenic bacteria, the secretion, at least in artificial media, of such highly soluble and potent poisons has not been demonstrated satisfactorily, although certain investigations point fairly conclusively to the production of some minor bodies which have been shown to act deleteriously on the red blood cells and on the leucocytes-the hemolytic, leucocidic, and leucolytic substances which are looked on as probably true soluble toxins, like the toxins of diphtheria and tetanus, which give rise in the animal body to the production of true antitoxins: i.e., are neutralized by their antisera, unit for unit, according to the law of multiples. 
Other minor poisons may in some instances be demonstrated in culture media, and also may possibly be formed in the animal body by the metabolic activities of the germs. These are either simply waste products of metabolism or bodies due to the decomposition of the nutrient media in which the germs are gowing. These bodies are usually referred to as ptomains, and differ entirely from the true secreted toxins, both in their chemical composition and in their physiologic action, resembling in both of these the alkaloids. They are not known to give rise to antibodies of any kind in animals.

Apart from all the poisons just mentioned; i.e., the toxins, hemolysins, leucocidins, and ptomains, there is supposed to exist a most vit lly important and interesting group of poisonous substances, the so-called endotoxins. These, so far as our knowledge goes, are poisons rather firmly seated in the bacterial cell, which are not secreted in our ordinary culture! media, and are supposed by most observers not to be separable in the animal fluids and tissues from the intact bacterial cell. These poisons may be demonstrated in old cultures, in which the bacteria are dead and disintegrating or undergoing autolysis-although Pfeiffer does not consider autolytic products necessarily similar to endotoxins —or they may be obtained by destroying the bacteria mechanically by pressure and grinding, or by breaking them while frozen. In the animal body they are said to become free when the bacteria die and decompose or are disintegrated by the digestive bodies by which they have been attacked. These endotoxins are recognized by the fact that they do not call out true antitoxins which become free in the plasma and serum, but do, nevertheless, lead to the formation of digestive antibodies, these not following, however, the "law of multiples" in protecting infected animals from the poisons. The liberation of these poisons by the destruction of bacteria in the animal body is best illustrated by the socalled phenomenon of Pfeiffer which takes place when cholera vibrios and immune cholera serum are introduced into the peritoneal cavity of a guinea-pig. If specimens are withdrawn from time to time from the peritoneal cavity of an animal so treated, a rapid swelling up, disintegration, and disappearance of the vibrios can readily be demonstrated. The organisms apparently do not multiply in the animal body under these conditions and are almost immediately destroyed. This disintegrating power is also claimed for the body fluids of normal animals and is supposed to be demonstrated by the following experiment. When graded quantities of a fresh cholera culture are introduced into the peritoneal cavity of normal guinea-pigs of equal weight, the 
following phenomena can be regularly observed: Minimal doses of the culture produce a febrile condition which continues for a few hours with no serious symptoms. Slightly larger doses give rise, after a short interval, during which there is fever, to a marked drop in temperature and definite symptoms of cholera poisoning--muscular weakness, twitching, and general prostration. These symptoms of poisoning then gradually disappear, and after twenty-four hours the guinea-pigs are again normal. If the quantity of cholera culture injected is carefully increased up to the minimal lethal dose, the animal dies with all the symptoms of cholera intoxication, but on autopsy the peritoneum is found to be entirely sterile, or only a few isolated cholera spirilla are found, usually inclosed in pus cells. Finally, if larger quantities of living cholera spirilla are injected, the peritoneal cavity shows a profuse, serous, sometimes hemorrhagic exudate, which contains innumerable actively motile microorganisms. The point of interest in this experiment is the demonstration of the fact that the normal guinea-pigs which receive enough of the cholera vibrios to prove fatal have destroyed the vibrios and presumably died from the poison thus liberated, and not from poisons secreted by living vibrios, or from an overcoming of their systems by the rapid multiplication of the organisnı. It is only when the animal system is previously flooded with an overwhelming dose that the vibrios are found alive and multiplying even locally in the peritoneum after death. This does not mean, however, that no multiplicacation ever goes on hand in hand with the destruction of the germs in the infected animal; on the contrary, such a multiplication is probably the rule rather than the exception, as has been shown fairly conclusively by the experiments of Radziewsky, and was beautifully illustrated by an experiment of Pfeiffer and Wassermann, who after having shown that the blood serum of human beings who have recovered from Asiatic cholera has the power to protect guinea-pigs from ordinarily fatal doses of cholera spirilla, even when used in high dilutions, then proved that this protective power is not an antitoxic one, but depends largely, if not entirely, on the ability of the serum to aid in the immediate dissolution of the vibrios. Thus animals which received only a fraction of a milligram of such a serum were able to bear the injection of a loopful of virulent cholera vibrios, practically without reaction, while control animals succumbed to one-fourth of the dose with typical symptoms. Now, however, if the dose was increased to three or five loopfuls, not even ten thousand times the original amount of the serum would protect the animals against the inoculation. The toxic effects may, in fact, as 
shown by Pfeiffer, appear with extraordinary rapidity, so that in these animals the temperature may show the lethal drop within two hours after inoculation, while control animals which have received the same quantity of cholera germs without the serum may not show a similar lethal drop in temperature for four to five hours.

An explanation of the results of this experiment is found, probably, in the fact that guinea-pigs are able to withstand a certain quantity of the intracellular cholera poison (endotoxin) which may be represented by one loopful of a fresh culture. If the animals are given smaller quantities without the serum, say one-fourth to one-half loopful, the bacteria may increase for a time without producing marked symptoms. Parallel with the increase, however, the phenomenon of germ destruction is going on and characteristic symptoms of intoxication appear at the moment when the number of vibrios destroyed has become so large that it corresponds to more than one loopful of the cholera culture. An animal will thus withstand a culture of any size when mixed with immune serum, if the dose does not exceed the limit of intoxication before it is entirely destroyed. On the other hand, when guinea-pigs receive the larger dose of three to five loopfuls, the serum, not being antitoxic, is not able to counteract the fatal effects of the liberated cholera poisons, but, on the other hand, enormously increases the rate of destruction of the vibrios, and hence intoxication appears earlier in such treated animals than in the controls receiving the organisms alone.

This classic cholera experiment has been selected because it illustrates the most extreme limit of the endotoxin point of view, and, further, because the cholera organism, standing at one end of the scale, is the most extreme example of pathogenicity by virtue of its own destruction, while the diphtheria bacillus at the other end, as we have seen, is one of the classic examples of pathogenicity by virtue of secreted toxins. Neither of these organisms is truly invasive or highly parasitic, and both are harmful usually by the action of their poisons alone and acting, as it were, from a base of supply on the periphery of the animal system. Between these two extremes stand all grades of pathogenic and infective germs.

These two organisms are typical examples of their kind, but there are few organisms which secrete such highly toxic soluble bodies as do diphtheria bacilli, and there are few so susceptible as the cholera organism to disintegration within the animal body; and yet there are many germs which are extremely pathogenic, and in many cases capable of 
severely and detrimentally infeeting the animal body. In view of this unquestioned fact, the teaching which considers all poisonings as due either to true soluble secreted poisons, or to true endotoxins liberated only on disintegration of the bacterial cell, is probably too narrow; and it would seem not unlikely that many organisms, possibly all, secrete bodies which are not soluble in their condition at secretion in culture media or in the body fluids, but which are susceptible to digestion in the animal body, and may thus become soluble and assimilable, and when toxic act harmfully on the body cells. This question is an important one and will be considered later. Besides these actively poisonous bodies which we have been considering, there are probably bodies such as some at least of the substances called aggressins by Bail, which, while not being toxic in themselves for the animal body, nevertheless are active defensive agents of the bacteria, probably neutralizing certain bodies of the animal economy, which are indirectly injurious to the bacteria. Further than this, certain bacteria may be furnished with envelopes, capable possibly of protecting them either chemically or physically from harmful influences.

Some clarity of conception may, as we have suggested, be gained by comparing some of the products of pathogenic bacteria with bacterial pigments and with insoluble interstitial or intercellular substance, which may be seen accompanying bacteria in cover-glass preparations. Soluble toxic secretions are to be compared to such pigments as the pyocyanin of Bacillus pyocyaneus, which is so readily soluble in culture media; endotoxins proper, to pigments confined to the bacterial cell or, at least when secreted, being insoluble in culture media, such, for instance, as the well-known red pigment of Bacillus prodigiosus, which may often be seen free among the bacteria in irregular red granules like carmine powder. That bodies such as this latter might be extruded from pathogenic bacteria, and not be soluble in the usual culture fluids, is not improbable, and the fact that more or less insoluble interstitial substances are not infrequent among bacteria is well known. Among pathogenic germs these characters are often more marked in freshly isolated cultures. The sticky, almost slimy character of cultures of meningococcus may be recalled, a character which tends to disappear after a few generations of artificial cultivation, and the highly mucinous capsule of the Streptococcus mucosus which tends to decrease under artificial cultivation, as do also the capsules of pneumococci and streptococci.

Now, it seems-and this view has been supported by Walker, Deutsch, 
Welch, and Eisenberg, and is, in fact, but an axiom which would be recognized immediately by any trained biologist-that all microorganisms will adapt themselves so far as is permitted by their physiologic peculiarities to the stress of the environment, the exact direction which this adaptation will take being determined by the character of the environment, chenical and physical, and the physical, chemical, and physiologic characteristics of the germ involved.

Thus far, in considering the means of offense and defense at the command of the bacteria, we have largely left out of consideration the animal organism against which these are directed, or by the changes in whose functions, metabolism, tissues, cells, and fluids, we are largely made aware of their existence.

The internal defenses of the animal body-and with these alone we are concerned-have largely been clucidated, as we have seen, through morphologic investigation of cellular activities taking place in the animal body or under controlled conditions in the test tube, and by visible reactions taking place in test tubes between the fluids of normal or immunized animals and the bacteria and their products, and, finally, by the more purely physiologic tests of the protecting power and mechanism of action of animal fluids or extracts when introduced into another animal of the same or different species, along with the bacteria or their products.

Such studies have, as is well known, afforded a vast amount of information. Through them the soluble secreted bacterial poisons have been demonstrated and have been found to stimulate the production of neutralizing bodies, the antitoxins; bacteria and their culture filtrates have been shown to eall forth bodies which are present in the serum of animals treated with them, and which cause a precipitation of certain bacterial constituents of the filtrate-the precipitins; and injections of animals with bacteria or their products have been found to cause the production of bodies which are present in the serum and which have the power of agglutinating the bacteria when brought into contact with them-the agglutinins; and other bodies are likewise produced which are capable under proper conditions of killing the bacteria-the bactericidal substances-or even of dissolving them as we have seen in some instances - the bacteriolytic substances. All of these bodies may be demonstrated in the serum of certain normal animals and may be shown to be increased during the immunization of these animals with bacteria or their products. The complementing body, however, which is necessary for the activation of the bactericidal and bacteriolytic 
bodies is not known to be increased during immunization, at least so far as its presence in the serum is concerned.

These facts we have learned from the study of the serum; on the other hand, the morphologic investigations instigated and carried on largely by Metchnikoff and his followers have taught us the great part which the formed elements of the blood and lymph play in the protection against and cure of germ diseases, and the importance of the polymorphonuclear and large mononuclear leucocytes as phagocytes is now widely recognized.

Of these cells, the polymorphonuclear leucocytes take a very active part in the ingestion and destruction of bacteria, while the large mononuclear leucocytes and endothelial cells, especially those lining the blood vessels and body cavities, although also able to ingest bacteria directly, are chiefly active in taking up cells of animal origin, principally those which necessarily, in the normal course of events, belong to the same animal and have probably become injured or have suffered death.

It does not seem, in this connection, a far-fetched idea to suppose that phagocytic cells may use naturally other cells and bacteria as a part of their regular food supply. The polymorphonuclear leucocytes may thus depend to some extent on the ever-entering bacteria and their remains; for, as we know, bacteria are constantly entering along the regular channels of absorption; and it is just as obvious that numbers of blood and tissue cells are constantly dying out and must be disposed of, for such processes are always in evidence in the spleen, and the ingestion of polymorphonuclear leucocytes by the large mononuclears can be observed wherever leucocytes are collected in exudates, due either to infections, poisons, or supposedly benign irritants. The simple fact that these cells retain the basic physiologic activities and an ability to ingest and digest food in its crudest form, which ability was the heritage of their free-swimming ancestors, and that they have not suffered the total specialization and physiologic degeneracy of the fixed tissue cells, seems sufficient evidence to warrant the conclusion that they are most active factors in the protection of the specialized internal tissue cells, which control the general metabolism and higher functions of the animal body. It seems worth mentioning that the leucocytes, alone probably of all the true cells of the body, are entirely independent of the nerve control, and are subject only to the stimulation of their chemical and physical environment, and are thus susceptible of adaptation to and capable of subserving various purposes which would be fatal to the duties of cells controlled by the nerve mechanism for the special func- 
tions of the organism at large. Further than this the death of leucocytes does not matter, as would the death of specialized and nervecontrolled cells, for no special metabolic or functional derangement occurs from their destruction.

In considering this independence of the leucocytes it must not be assumed that they have not varied from primitive ameboid cells, for undoubtedly their life and proper functioning are largely determined by the special plasma in which they live, and it may be that their food, although at times crude compared with that of the other body cells, is nevertheless usually prepared for them by processes going on in the plasma.

Questions relating to the independence and to the interrelation of the plasma and leucocytes in their action on invading microorganisms and the action of plasma as compared with serum have been ground for scientific strife for many years, one side contending for the activity of the plasma, the other for the activity of the phagocytes; the humoralist at first neglecting, if not absolutely forgetting, that a fluid can not be self-replenishing, while the supporters of phagocytosis largely overlooked the fact that plasma is not necessarily an inert menstruum such as salt solution.

While these differences have been to some extent adjusted by the theory and work of Ehrlich, an immediate point of contention is still the question of the similarity of action of plasma and serum. The humoral school contends that the alexin of Buchner-complement of later writers-is secreted into the plasma, while the Metchnikoff school claims that it is only given up from injured leucocytes in the body, and to the serum by destruction of leucocytes during coagulation. The Metchnikoff school admits, however, that the amboceptors necessary for bactericidal and bacteriolytic action are formed. in excess in the phagocytes, and given off from these to the plasma, yet asserts that they are inactive for lack of the complement which is normally retained in the leucocytes, and that they simply prepare the bacteria for complete digestion in the leucocytes. The relation of the bacteriolytic amboceptors to intracellular digestion is not settled, although it seems illogical for a digestive body to be produced in excess that has not arisen from cells by the stimulation of its use, and, as the leucocytes take up the bacteria, they are the most likely producers and users of this body.

In 1894 a further adjustment of differences took place, when certain phenomena observed by Denys and his pupil Leclef demonstrated that the act of phagocytosis when performed in serum, in some instances at least, was dependent on the presence of certain substances in the 
serum. Thus, they were able to show that leucocytes removed from normal blood and placed with bacteria in immune serum enulfed the bacteria actively, while leucocytes from immunized animals mixed with bacteria in normal serum took up the organisms no more actively than the normal leucocytes. The bodies inciting the phagocytosis must obviously, then, they concluded, be in the serum. Whether these bodies acted on the leucocyte or on the bacteria was not then determined, but Denys concluded, in 1898, that the bacteria were directly affected. The fact that the action is exerted on the bacteria was recently determined positively by Wright for normal serum, and by Neufeld and Rimpau, independently of Wright, for immune serum. These bodies have been called opsonins by Wright, and bacteriotropins by Neufeld, and have been shown to attach themselves to the bacteria and thus prepare them for ingestion by the phagocytes. It has also been shown by various observers that the more virulent the germ, the less susceptible it is to phagocytosis and the more potent the antisera must be to permit of the ingestion by the cells.

If now, for clarity of conception, we summarize briefly the diseaseproducing agents possessed by the bacteria and the opposing substances of the serum and processes of the animal body, we find the true toxins, including probably leucocidins and hemolysins, opposed by antitoxins which become free in the plasma; the bacterial bodies and probably the endotoxins opposed by leucocytes, and possibly directly in the plasma by lytic substances formed of amboceptor and complement, which either kill or dissolve the bacteria and free the endotoxins, but do not neutralize them; and, third, we have probably certain secretions which oppose the opsonins, and thus prevent phagocytosis-antiopsonins -bodies which may possibly be the so-called aggressins of Bail, and which are present in exudates and, although not toxic in themselves, increase the infectiousness of the bacteria with which they are injected; and, finally, opposed to bacteria and their broth filtrates we have the agglutinins and precipitins, the activities of which are manifest in serum, but whose relation to immunity is not altogether obvious, as they have not been shown satisfactorily to bring about agglutination or precipitation in the animal body.

While all of these different functions and chemical substances are possessed by animals as a class, it is becoming more and more obvious that these are not always present or active in the same degree, and that there are recognizable differences in the protective mechanism of different animal species-in species, in fact, not far removed from each 
other in the natural classification. An explanation of reactions to a given infection which applies in the case of one species is not, therefore, obviously applicable in the case of another species. This is true not only of the mechanism of protection as it takes place in the serum of different animals and in their plasma, but also of phagocytosis and phagocytic digestion and the factors which contribute to the perfection of these processes. The constant stumbling-block in the way of a correct interpretation of processes going on in the animal body is our inability, as we have seen, to argue from serum phenomena to phenomena occurring in the plasma. A failure to keep this in mind, although it is fully recognizen, has undoubtedly led to many hasty conclusions, particularly connected with the theory of lytic immunity. This may be illustrated by a well-known example: Fresh rabbit serum is actively germicidal for anthrax bacilli, dog serum is not; yet rabbits are extremely sensitive to a true anthrax infection, while dogs are very resistant. Experiment has shown that there are lytic amboceptors in the sera of both these animals, but that the dog's serum does not contain the complement necessary for their action on the bacilli; the complement presumably has remained in the body cells, whereas in the case of the rabbit it has possibly been liberated from the leucocytes during clotting. The reason the $\operatorname{dog}$ is insusceptible is, then, not because of a more active plasma destruction of the invading anthrax germs, but probably because of a more perfect adjustment of the cellular mechanism to the infection, although if we simply followed the theory of the bactericidal action of serum and plasma as being coextensive, and the active protective mechanism, the rabbit should have been protected, while the dog should have succumbed. The difference here probably depends upon the possession of all requisites for the perfect performance of phagocytosis, and the complete digestion of the bacteria by the phagocytes of of the $\operatorname{dog}$, while in the rabbit either the mechanism of ingestion is incomplete or the cells fail to cope successfully with their contents after ingestion. This example has been selected because anthrax bacilli have been shown to contain less toxic intracellular bodies-endotoxinsthan many other infectious germs, and the likelihood of the rabbit being poisoned by any primary plasma disintegration of the bacilli is not very great, so that if the plasma mechanism had corresponded to that of the serum the animal should have been saved. The validity of such an argument would not have been so apparent if we had substituted cholera vibrios for anthrax bacilli in rabbits, for the bodies liberated from cholera bacilli at their disintegration are very toxic. 
Even if the evidence so far in our possession warranted the conclusion that the bactericidal and bacteriolytic bodies which are present in the sera of various animals are present and active against certain microorganisms in the same manner in their plasma, we should, nevertheless, still have a number of microorganisms which are singularly insusceptible to such action of the serum or plasma, even of animals highly immunized against them. The method of resistance against these would have to be explained by a different mechanisn, and if this death and destruction are not accomplished in the plasma, then we must look largely to the activities of the leucocytes for their accomplishment.

Now, not only the serum substances which further leucocytosis have, as we have seen, received much attention of late, but the bodies antagonistic to the bacteria which are supposed to be contained in the leucocytes have also been extensively investigated.

Experiments bearing on these questions make it appear extremely probable that bactericidal and bacteriolytic action depend on two processes; one of these is the bacteriolytic action of the serum and plasma, the other the bactericidal action of substances retained in the leucocytes. As an example of the type supposed to depend solely on the bactericidal substances of the serum or plasma, the mechanism of the natural and artificial immunity of guinea-pigs to typhoid and cholera may be cited, since in these animals no one has as yet succeeded in demonstrating that substances derived from the leucocytes by extraction have any bactericidal action on the organisms of these two diseases. This does not mean, however, that the bactericidal action takes place naturally outside of the leucocytes, for the bacteria loaded with amboceptors are probably taken into the leucocyte and there digested. As examples of immunity depending on substances within the leucocytes, the natural and artificial inmunity of dogs and cats to anthrax, and the immunity of guinea-pigs to certain strains of proteus, may be cited, for in these cases the leucocyte extract is germicidal, while the serum is not.

Stated impartially, then, our knowledge of immune bodies and processes stands somewhat thus: Bodies which are bactericidal and bacteriolytic may be present in the plasma, and certainly in the serum, wherever this is formed in a pathologic process, which when supplied with complement, either normally present in the plasma or derived from injured leucocytes or other sources, may be active against microorganisms, either killing them or actually breaking them up in some cases and liberating bodies which are then directly poisonous or become so by fur21 
ther digestion. Besides these germicidal bodies, there are other bodies which, while not directly harmful to the bacteria, render them powerless against the phagocyting power of the leucocytes. These bodies are probably present in the plasma, certainly in the serum. They are the opsonins or bacteriotropins.

After phagocytosis has taken place, the germs may be killed and digested. Some of the bactericidal bodies of the phagocytes are bodies differing in character from the lytic bodies of the serum, and are either not given off to the serum or are not active in it; but there is no proof that the lytic amboceptors present in the serum are not normally, in part at least, derived from the leucocytes, and active in intracellular digestion when activated by complement. This is supported by the supposition that guinea-pig leucocyte extracts are not germicidal for cholera and typhoid organisms. Nevertheless, intracellular digestion of these germs does go on; it is possible, therefore, that the amboceptors present in the plasma, whatever their source, attach themselves to the germs and aid in intracellular digestion.

None of the processes just mentioned leads to the formation of antitoxins which become free in the plasma or serum. Now, in view of these facts and suppositions, it may possibly be logical to conceive that nearly all pathogenic germs secrete bodies which are not readily soluble in culture fluids or in the fluids of the animal body; that these bodies are not readily, if at all, assimilable by non-phagocyting cells. These bodies may, however, be broken up by digestive bodies present in the serum, and from them may thus be liberated a poisonous substance, which may then be assimilated by the higher cells of the body, and, when in sufficient quantity, cause death. The more rapid the process of liberation the more quickly death ensues. This plasma digestion is, then, according to this conception, a mechanism which is faulty when applied to bacteria and their products, and if this conception is correct the fault may occur somewhat as follows: Bacteria and their insoluble or non-assimilable products when taken into the phagocyte are subjected to two processes, a primary bactericidal and coagulating one, and then a more leisurely lytic or disintegrating action, during which poisonous products are probably liberated, but slowly enough to be taken care of by destroying or neutralizing bodies. Even if the leucocyte dies, it is usually taken up by a mononuclear cell, and the poisons do not become free in the fluids. Now, in this process the only bodies which are produced in excess and at the same time are capable of escaping from the leucocytes are the lytic bodies; neither the toxin-neutralizing body nor the coagulating 
body are secreted or given off from the cell. Such lytic amboceptors, then, when present in the plasma and activated by complement, may thus become an active agent for harm by liberating poisonous substances from the bodies of germs which are susceptible to such action, or from the insoluble or non-assimilable products of these or more lysisresisting members of the invasive organisms; and by the action of these poisons, phagocytosis may be hindered and the specialized cells poisoned. Since the neutralizing or poison-destroying bodies are not present in the plasma, the leucocytes are then poisoned from without, just as are the specialized cells, and the more active the plasma digestion, the more deranged the true protective mechanism becomes.

These are some of the problems of immunity, particularly those relating to the microorganisms which are harmful to the animal body, not so much through their ability to secrete harmful soluble poisons, as through their insistently invasive character, or by the liberation of the toxic products resulting from the destruction of their secretions or of their own bodies. It is the diseases caused by these organisms on which the attention of bacteriologists is now chiefly centered.

The organisms of these diseases undoubtedly belong to two or more classes, in one of which may be placed the typical septicemia producers -anthrax, pneumococcus, streptococcus, ete.-in the other the less invasive organisms, typified by cholera and to some degree by typhoid. Between these two extremes there are all grades.

If the data amassed in the study of these types of microorganisms, and of the processes supposed to be involved in meeting infection and establishing cure and immunity from them, have been made clear, it may be easier to comprehend some of the problems which daily face investigators in their struggle to arrive at a rational method of biologic treatment, and to realize more fully, in the light of this knowledge, why disappointment has so persistently followed in the wake of serum therapy as applied to these infectious diseases. For, in spite of the most persistent attempts to produce curative sera, the results have not been satisfactory and have not led, except in rare instances, to the practical use of such sera in the treatment of disease in man.

The sera, thus produced, have not, except in a very minor way, been antitoxic in the usually accepted sense, and depend, as we have seen, probably, for any protective value they may possess, on their germicidal and bacteriolytic power and on the opsonins they may carry, and thus facilitate phagocytosis. These sera are capable of protecting an animal from an infecting organism, when mixed with it ị surprisingly 
minute quantities; but that consistent curative effects, other than nierely local, have been definitely determined as due to their action, after an infection has once been established, is open to serious doubt.

On the other hand, indeed, test and experiment have shown that animals and man suffering from a true infection may and often do theniselves furnish sera capable of strong bactericidal and bacteriolytic action (when combined with normal sera containing complement), and yet in spite of this, they succumb or may be subject to severe relapses.

In the light of these and other facts which have been cited, it seems that one might well refrain from attempts to produce beneficial effects by injecting still further amounts of bacteriolytic or similar bodies, and seek further for an explanation of the exact methods and processes of the cure effected in those animals and man who do survive an infection.

Failure to solve these problems on lines hisherto followed should not discourage us, however, while we know that animals and man do recover naturally from such infections. The conclusion that this power must reside in increased digestive and neutralizing or poison-destroying powers of the animal organisms can not well be avoided, and these functions of the animal mechanism will probably be found to take place largely in some group of cells.

The animal body, then, ideally protected in the time of bacterial invasion, may well be one in which some set of cells-phagocytes-are immediately ready and able to take up the bacterial invaders and destroy them, and within their own bodies to neutralize any poisons secreted by such invaders or arising from their destruction by digestion, and this without serious harm to the ingesting cells; or-failing this full immunity from serious harm - it may be that these ingesting cells are, in their turn, taken up and, with their noxious contents, digested by other scavenging cells, with a minimum liberation of the substances which could injure the body cells dedicated to specialized functions. The whole struggle of the infected organism may be summed up as a conflict between the leucocytes and the germs, and that it is an attempt to bring the invading germs within the leucocytes, and is a process with which the system at large often has little or nothing to do, except as an innocent and injured bystander, and that extracellular destruction of bacteria and toxicogenic bodies is an untoward event after the thorough establishment of infection often leading to dire consequences, and depending on the chance occurrence of suitable digestive bodies in the serum which have been thrown off in excess from the cells, and which 
may thus become a menace to the system at large by liberating woisonous bodies from comparatively harmless compounds.

Thus, in many instances, it seems we are probably dealing with an immunity, a large part of the mechanism of which is intracellular, not only in the sense of phagocytosis and digestion, but in the neutralization or destruction of poisons which arise from the disintegration of the bacteria and their products - a mechanism in which the protecting cells must intervene and, largely unaided by antitoxic bodies in the plasma, neutralize or destroy within themselves the poisonous products of the invading microorganisms.

It was this thought which suggested the idea of treating infections with the extract of leucocytes, and thus aiding the phagocytes by furnishing them as directly as possible with the weapons which they use in their fight with invading microorganisms, and also to protect them and the cells of specialized function from destruction and give them an opportunity to recuperate and carry on successfully their struggle against the invading germs.

The treatment of infections with vaccines also is based upon the recognition of the necessity of the direct intervention of phagocytes in the cure of certain bacterial diseases and is, as we have seen, an endeavor to stimulate the production of substances facilitating the ingestion of the organisms by the phagocytes.

Finally, it must be remembered that while animal experiments are necessary, and often extremely instructive, one can, nevertheless, not always argue directly from these to occurrences in man. An injection disease is not an infectious disease, and we are dealing usually with conditions in man which are at least not entirely analogous to artificial infections in animals. Artificial infections are usually accomplished by an abrupt introduction of a large quantity of infecting germs and their products; the animal powers of resistance are often immediately and severely taxed; the incubation period thus artificially shortened; and the germs themselves, being present in large numbers, are not subjected to such a searching elimination as is usually the case with the few organisms gaining a foothold by the natural channels of infection. This difference is most marked in septicemias, in which, in animal experiments, the organisms have been introduced directly into the circulation in quantities sufficient to bring about a very rapid poisoning and overwhelming of the animal, with probably only a very partial adaptation of the bacteria to the animal agents of resistance. On the other hand, the septicemic invasion in man most often follows the adaptation of the germs in some 
more favorable nidus, and probably has to do with an evolution in the bacterial resistance to the protective powers, rather than a decrease in protective strength on the part of man. Indeed, both of these processes may increase hand in hand, and we may have septicemias extending over weeks, months, and even years. We may have, in fact, an "armed peace" and the prepared bacterial army is not to be routed by the application of means which under other circumstances might prove efficacious, for we have seen how the bacteria may possibly become resistant to the protective agents of the animal body, and may continue to survive attacks which might well prove fatal to less well-adapted members of their species.

Theoretically, then, we are safe in assuming that the infections in man which most closely simulate the usual artificial infection in animals are fresh local infections, and infections of any character, in their earliest stages, before the bacteria have been adapted to carry on their fight with the powers of the infected body.

The point which should be made clear is that the outcome of our attempts to treat infectious diseases is, if we have the real means in our hands, probably more dependent on the degree of adaptation of the germs than the actual powers of resistance of the patient. These latter, of course, determine largely the picture of the disease, but give little information as to the power of the invader. This has been forced on us by the fact that, although we are able to cure positively acute septicemias in animals, the more subacute septicemias of man do not yield readily to our present modes of treatment, whereas even extremely severe acute and chronic localized diseases, due to the same organisms, respond to treatment. 


\section{SECTION III}

\section{PATHOGENIC MICROORGANISMS}

\section{CHAPTER XXI}

\section{THE STAPHYLOCOCCI (MICROCOCCI)}

THE power to incite purulent and sero-purulent inflammations and localized abscesses in man and animals is possessed by a large variety of pathogenic bacteria. Most infections, in fact, in which the relative virulence of the incitant and the resistance of the infected subject are so balanced that temporary or permanent localization of the infectious process takes place are apt to be accompanied by the formation of pus. The large majority of acute and subacute purulent processes, however, are caused by the members of a well-defined group of bacteria spoken of as the pyogenic cocci. Among these, pre-eminent in importance, are the "staphylococci" or "micrococci."

Many of the earlier investigators of surgical infections had seen small round bodies in the pus discharged from abscesses and sinuses and had given them a variety of names. Careful bacteriological studies, however, were not made until 1879 and the years immediately following, when Koch, Pasteur, Ogston, ${ }^{1}$ and others not only described morphologically, but cultivated the cocci from surgical lesions of animals and man. Of fundamental importance are the studies published by Rosenbach ${ }^{2}$ in 1884, in which the technical methods of modern bacteriology were brought to. bear upon this subject for the first time. The group of staphylococci-so named from their growth in irregular, grape-like clusters -is made up of several members, by far the most important of which, pathologically, is the Staphylococcus pyogenes aureus.

1 Ogston, Brit. Med. Jour., 1881.

${ }^{2}$ Rosenbach, "Microorganismen bei Wundinfektion," 1884. 


\section{STAPHYLOCOCCUS PYOGENES AUREUS}

Morphology and Staining.-This microorganism, the most frequent cause of abscesses, boils, and many surgical suppurations, is a spherical coccus having an average diameter of about 0.8 micra, but varying within the extreme limits of 0.4 to 1.2 micra. Any considerable variation from the average size, however, is rare. The perfectly spherical character may not develop, whenever, as is usually the case, two or more are

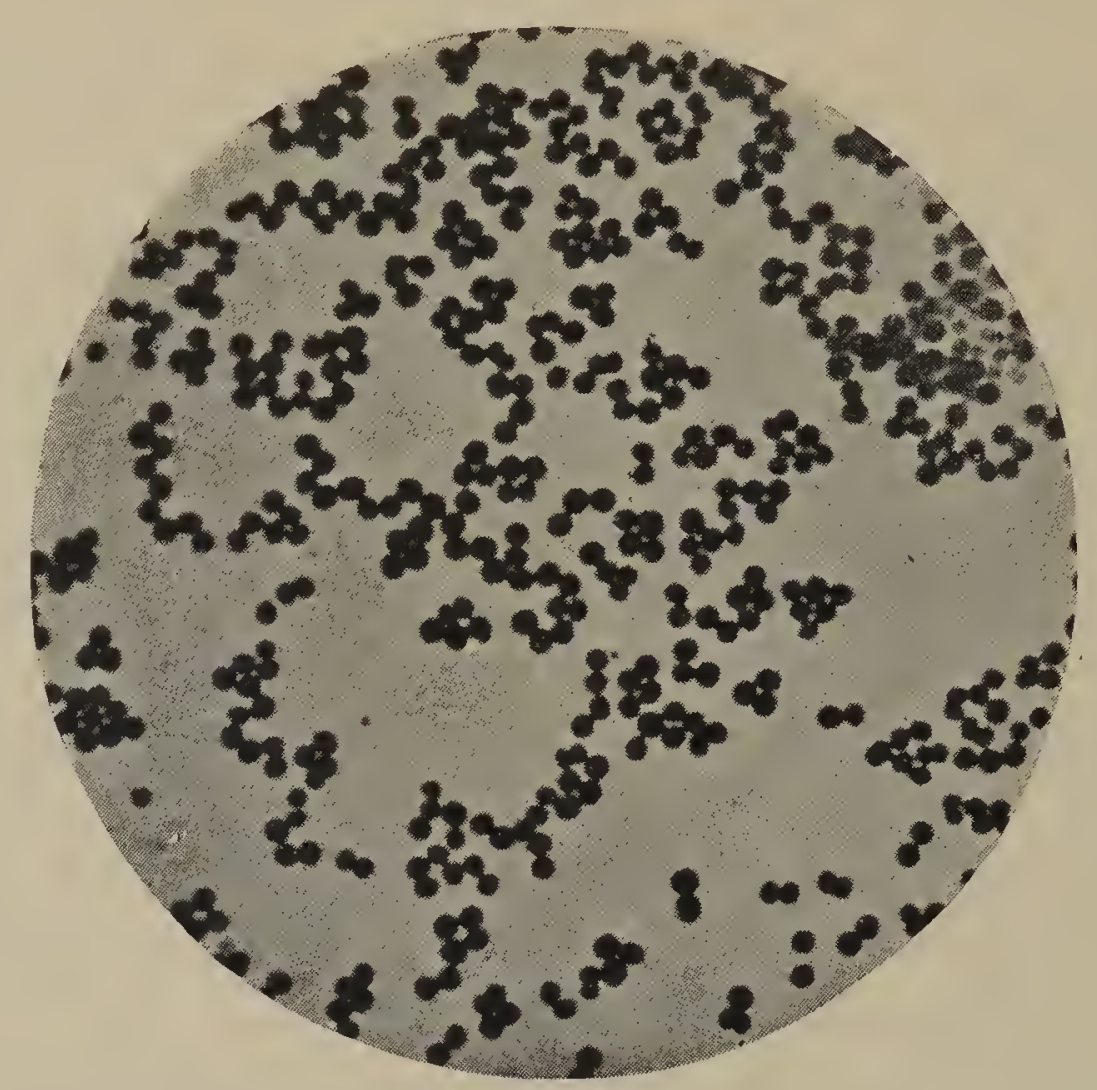

Fig. 69.--Staphylococcus Pyogenes aUReus. (After Günther.)

grouped together, unseparated after cell cleavage. In this case, adjacent cocci are slightly flattened along their contiguous surfaces.

Examined in smears from cultures or pus, the staphylococci may appear as single individuals, in pairs, or, most frequently, in irregular grape-like clusters. Occasionally, short chains of three or four may be seen. In very young cultures in fluid media, the diplococcus form may predominate.

The staphylococci stain with all the usual basic aqueous anilin dyes, and, less intensely, with some of the acid dyes. Stained by the method of Gram, they retain the anilin-gentian-violet. Gram's method of staining is excellently adapted for demonstration of these cocci in tissue sections. 
Although exhibiting marked Brownian movements in the hanging drop, staphylococci are non-motile and possess no flagella. They are non-sporogenous and form no capsules.

Cultural Characters.-Staphylococci grow readily upon the usual laboratory media. The simpler media, made of meat extract, are quite as efficient for their cultivation as are the freshly made meat-infusion

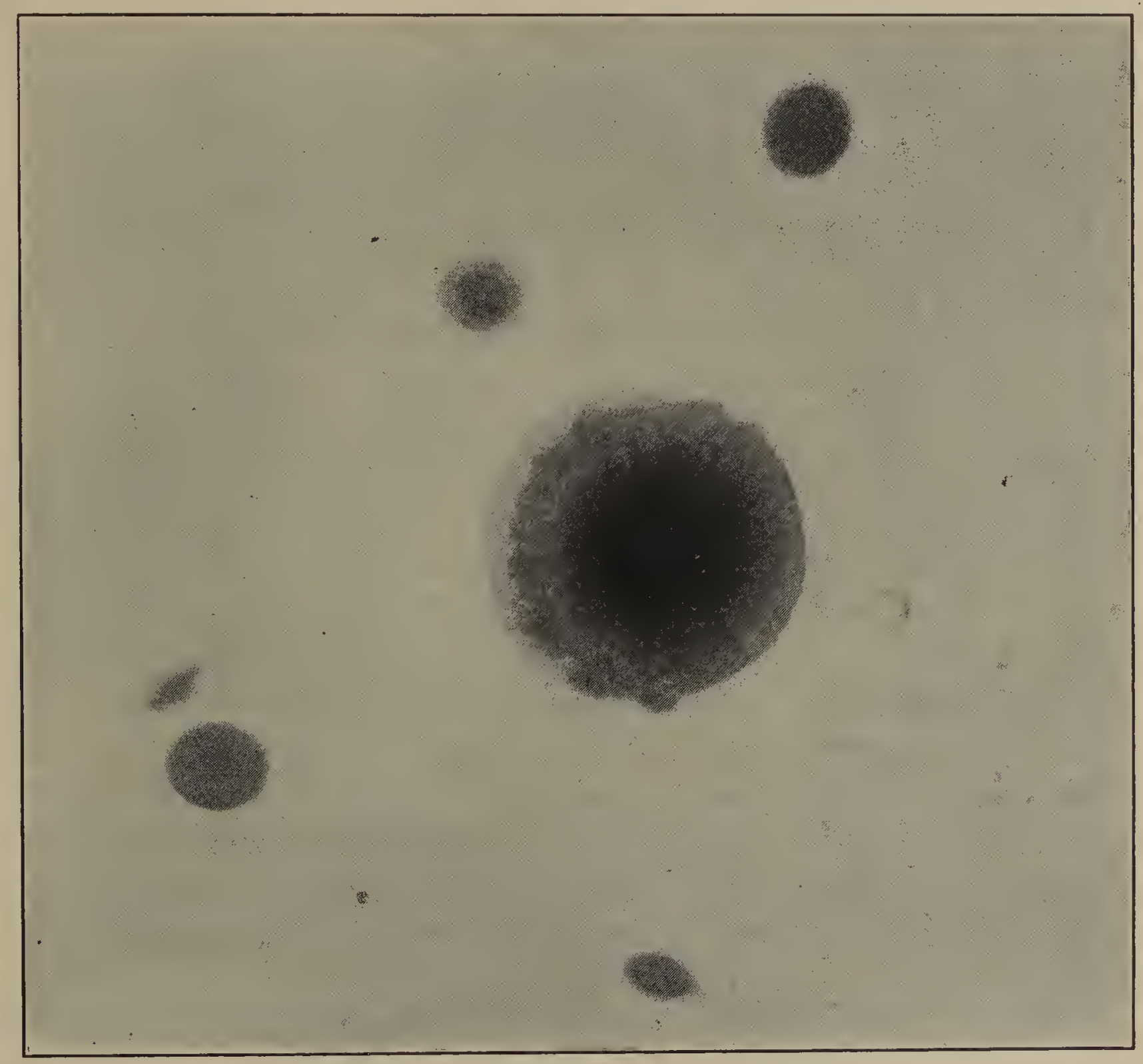

Fig. 70.-Staphylococcus Colonies.

products. The optimum temperature for staphylococcus cultivation lies at or about $30^{\circ} \mathrm{C}$., though growth readily takes place at temperatures as low as $15^{\circ} \mathrm{C}$, , and as high as $40^{\circ} \mathrm{C}$. Slow but definite growth has been observed at a temperature as low as $10^{\circ} \mathrm{C}$.

While development is most characteristic and luxuriant under aërobic conditions, staphylococci are facultatively anaërobic on suitable media. They grow readily in an atmosphere of hydrogen. 
As to the reaction of media, staphylococcus develops most favorably upon those having a slightly alkaline titer. Moderately increased alkalinity or even moderate acidity of media does not inhibit growth.

On gelatin plates, growth occurs readily at room temperature, forming within thirty-six to forty-eight hours, small, shining, pin-head shaped colonies, appearing, at first, grayish-white, and later assuming a yellowish hue, which intensifies into a light brown and often a bronze color as the colony grows older. The intensity of the color differs considerably in different races of staphylococci. Liquefaction of the gelatin occurs, and, shallow, saucer-shaped depressions are formed about the colonies after forty-eight hours or more. These zones of fluidification grow larger as the colonies grow, finally becoming confluent. Microscopically, the colonies themselves, before liquefaction has destroyed their outline, are round, rather finely granular, with smooth edges. They are not flat, but rise from the surface of the medium as the segment of a sphere. In gelatin stab cultures in tubes, fluidification leads to the formation of a funnel-shaped depression, with, finally, complete liquefaction of the medium and sedimentation of the bacteria. Liquefaction of gelatin by the staphylococcus is due to a ferment-like body elaborated by it, which is spoken of as "gelatinase." This substance can be obtained apart from the cocci by the filtration of cultures. ${ }^{1}$ It is an extremely thermolabile body.

On agar plates the characteristics of the growth, barring liquefaction, are much like those on gelatin. Colonies do not show a tendency toward confluence, remaining discrete, and show a rather remarkable difference in the size of the colonies occurring upon the same plate. Upon slanted agar in tubes, rapid growth occurs, at first grayish-white, but soon covering the surface of the slant as a glistening, golden-brown layer.

In broth, growth is rapid, leading to a general, even clouding of the medium, and giving rise, after forty-eight or more hours, to the formation of a thin surface pellicle. As growth increases, the bacteria sink to the bottom, forming a heavy, mucoid sediment. The odor of old cultures is often peculiarly acrid, not unlike weak butyric acid.

In milk, staphylococcus causes coagulation usually within three or four days, with the formation of lactic and butyric acids.

On potato, growth is abundant, rather dry and usually deeply pigmented.

${ }^{1}$ Loeb, Cent. f. Bakt., xxxii, 1902. 
Upon coagulated animal seru, rapid grow th takesplace and eventually slight liquefaction of the medium is said to occur.

In nitrate solutions, reduction of the nitrates to nitrites is caused.

In Dunham's broth, indol is formed.

In media containing the carbohydrates - dextrose, lactose, or saccharose-acidification takes place with the formation chiefly of lactic, butyric, and formic acids. There is no gas formation, however. In proteid media free from sugars, the staphylococcus produces alkali.

The reducing action of staphylococcus is shown by decolorization in cultures of litmus, methylene-blue, and rosanilin. ${ }^{1}$

Pigment Formation. - Differentiation between the various members of the staphylococcus group is based largely upon the formation of pigments. These pigments, so far as we know, seem to be species characteristics. Thus, Staphylococcus pyogenes aureus is recognized primarily by its production of a yellowish-brown pigment, varying in different strains from a pale brown hue to a deep golden yellow. Prolonged cultivation upon artificial media may lead to a diminution in the depth of color produced. ${ }^{2}$ It appears only when cultivation is carried on under freely aërobic conditions, anaërobic cultivation resulting in unpigmented colonies. The coloring matter is insoluble in water but soluble in alcohol, chloroform, ether, and benzol. ${ }^{3}$ According to Schneider, the pigment belongs to the class of "lipochromes" or fatty pigments, and is probably composed of carbon, oxygen, and hydrogen, without nitrogen. Treatment with concentrated sulphuric acid changes it to a green or greenish-blue. ${ }^{5}$

Resistance.-Although not spore formers, staphylococci are luore resistant to heat than many other purely vegetative forms. The thermal death point given for Staphylococcus pyogenes aureus by Sternberg ${ }^{6}$ lies between $56^{\circ}$ and $58^{\circ} \mathrm{C}$., the time of exposure being ten minutes. The same author states that, when in a completely dried state, the coccus is still more resistant, a temperature of from $90^{\circ}$ to $100^{\circ} \mathrm{C}$. being required for its destruction. Against low temperatures, staphylococci are extremely resistant, repeated freezing often failing to sterilize cultures.

\footnotetext{
1 Fr. Müller, Cent. f. Bakt., xxvi, 1899.

2 Flügge, "Die Microorg.," etc.

3 Migula, "System d. Bakt.," Jena, 1897.

4 Schneider, Arb. a. d. bakt. Inst., Karlsruhe, 1, vol. i, 1894.

${ }^{5}$ Fischer, "Vorles. über die Bakt.," Jena, 1903.

6 Sternberg, "Textbook," etc., N. Y., 1901, p. 375.
} 
Desiccation is usually well borne, staphylococci remaining alive for six to fourteen weeks when dried upon paper or cloth. ${ }^{1}$ On slant agar, staphylococci may be safely left for three or four months without transplantation, and remain alive. ${ }^{2}$

The resistance of staphylococci to chemicals, a question of great surgical importance, has been made the subject of extensive researches, notably by Lübbert, ${ }^{3}$ Abbott, ${ }^{4}$ Franzott, ${ }^{5}$ and many others. According to Lübbert, inhibition of staphylococcus growth is attained by the use of boric acid 1 in 327, salicylic acid 1 in 650, corrosive sublimate 1 in 80,000 , carbolic acid 1 in 800, thymol 1 in 11,000. Staphylococci are killed by corrosive sublimate 1 in 1,000 in ten minutes, by carbolic acid 1 per cent in 35 minutes, 3 per cent in 2 minutes (Franzott). Ethyl alcohol, ${ }^{6}$ even when absolute, is not very efficient as a disinfectant. Nascent iodin, as split off from iodoform in wounds, is extremely powerful in destroying staphylococci.

Pathogenicity.-Separate strains of Staphylococcus pyogenes aureus show wide variations in relative virulence. The most highly virulent are usually those recently isolated from human suppurative lesions, but no definite rule can be formulated in this respect. The virulence of a given strain, furthermore, may be occasionally enhanced by repeated passages through the body of a susceptible animal. Prolonged cultivation upon artificial media is liable to decrease the virulence of any given strain, though this is not regularly the case. There are, moreover, unquestionably, many staphylococci constantly present in the air, dust, and water, which although morphologically and culturally not unlike the pathogenically important species, may be regarded as harmless saprophytes.

The susceptibility of animals to staphylococcus infection is, likewise, subject to extreme variations, depending both upon differences between species and upon fortuitous individual differences in susceptibility among animals within the same species. Animals on the whole are less susceptible to staphylococcus than is man. Among the ordinary laboratory animals, rabbits are most susceptible to this microorganism. Mice, and especially the white

${ }_{1}$ Deslongchamps, Paris, 1897.

${ }^{2}$ Passet, Fort. d. Med., 2 and 3, 1885.

${ }^{3}$ Lübbert, "Biol. Untersuch.," Würzburg, 1886.

* Abbott, Medical News, Phila., 1886.

5 Franzott, Zeit. f. Hyg., 1893.

${ }^{6}$ Hanel, Beit. z. klin. Chir., xxvi. 
Japanese mice, show considerable susceptibility. Guinea-pigs possess a relatively higher resistance. ${ }^{1}$

Subcutaneous or intramuscular inoculation of a susceptible animal usually results in the formation of a localized abscess with much pus formation and eventual recovery. Intraperitoneal inoculation is more often fatal. Intravenous inoculation of doses of 0.5 c.c., or more, of fresh broth cultures of virulent staphylococci usually leads to pyemia with the production of secondary abscesses, located chiefly in the kidneys and the heart and voluntary muscles, but not infrequently in other organs as well. In the kidney they occur as small foci, situated most often in the cortex, composed of a central, necrotic pus cavity, surrounded by a zone of acute inflammatory exudation. Staphylococcus lesions form histologically the typical "acute abscess." Not infrequently the pyemic condition is accompanied by suppurative lesions in the joints. Intravenous injections of virulent staphylococci preceded by injury to a bone is often followed by the development of osteomyelitis. Mechanical or chemical injury of the heart valves preceding intravascular staphylococcus inoculation may result in localization of the infection on or about the heart valves, leading to "malignant endocarditis." The pyemic conditions following staphylococcus inoculation usually lead to chronic emaciation and death after an interval dependent upon the relative virulence of the microorganism, the amount injected, and the resistance of the infected subject. Large doses of unusually virulent cultures cause death within twenty-four hours, or even less, without abscess formation.

As above stated, the susceptibility of man to spontaneous staphylococcus infection is decidedly more marked than is that of animals. The form of infection most frequently observed is the common boil or furuncle. As Garré, ${ }^{2}$ Büdinger, ${ }^{3}$ Schimmelbusch, ${ }^{4}$ and others have demonstrated by experiments upon their own bodies, energetic rubbing of the skin with virulent staphylococcus cultures may often be followed by the development of a furuncle. Subcutaneous inoculation of the human subject invariably gives rise to an abscess. The pathological lesions which may be produced in man by virulent staphylococci are naturally of great variety, depending upon the mode of inoculation, and

${ }_{1}^{1}$ Terin, Ref. in Lubarsch und Ostertag, Ergebnisse, 1896; Lingelsheim, "Aetiol. d. Staph. Inf.," etc., Wien, 1900.

${ }^{2}$ Garré, Beit. z. klin. Chir.; x, 1893.

${ }^{3}$ Büdinger, Lubarsch und Ostertag, Ergebnisse, etc., 1896.

${ }^{4}$ Schimmelbusch, Ref. by Büdinger. 
upon the relation between the virulence of the incitant and the resistance of the subject. Apart from the formation of localized abscesses, staphylococci are common as the incitants of surgical suppurations and wound infections. The large majority of acute suppurative inflammations of bone (osteomyelitis) are caused by staphylococci. Abscesses of the brain, of the liver, and of the lung may be due to this microorganism. It may give rise to ascending infections of the genitourinary tract, leading to pyelonephritis. Empyema or peritonitis may be caused by its entrance into the serous cavities from the lung or bowel. When gaining access to the circulation from some localized focus, it gives rise to septicemia and may lead to malignant endocarditis and, by secondary localization in the viscera, to general pyemia. As the incitant of septicemia it can frequently be found by blood culture during the life of the patient. Puerperal sepsis is not infrequently a staphylococcus disease. Of recent years several authors have claimed direct etiological relationship for the Staphylococcus pyogenes aureus with acute articular rheumatism. ${ }^{1}$ While not unlikely, this claim is not, at present, substantiated by sufficiently exact evidence.

Apart from the local inflamratory reactions called forth by staphylococcus invasion, all such infections, if severe or prolonged, give rise to profound toxic marifestations evidenced by characteristically irregular temperature (the so-called "septic type"), by headache, nausea, and general malaise, and not infrequently by chills. Prolonged chronic infection with staphylococci may give rise to the so-called amyloid changes in liver, spleen, and kidneys.

Toxic Products.-Endotoxins. - The dead bodies of staphylococci injected into animals may occasionally give rise to abscess formation, and, ${ }^{2}$ if in sufficient quantity, may cause death. To obtain the latter result, however, large quantities are necessary, the endotoxic substances within the dead cell body of these microorganisms being probably neither very poisonous nor abundant. ${ }^{3}$

That dead cultures of Staphylococcus aureus exert a strong positive chemotaxis for leucocytes was shown beyond question by the experiments of Borissow. ${ }^{4}$

Hemolysins.-In 1900 Kraus $^{5}$ noticed the hemolytic action of

${ }^{1}$ A. H. Weis, Inaug.-Diss., Berlin, 1901.

2 Schattenfroh, Arch. f. Hyg., xxxi, 1887.

3 v. Lingelsheim, "Aetiol. u. Therap. d. Staph. Krank.," Wien, 1900.

"Borissow Zieglers Beitr., xvi, 1894.

5 Kraus, Wien. klin. Woch., iii, 1900. 
staphylococci growing upon blood-agar plate cultures. Neisser and Wechsberg ${ }^{1}$ then showed that this hemolytic substance, secreted by the staphylococcus, could be demonstrated in filtrates of bouillon cultures. Such hemolysins are produced by Staphylococcus aureus, and, to a lesser degree, by Staphylococcus albus. The quantity produced varies enormously with different strains and seems to be roughly proportionate to the virulence of the particular microorganism, though exceptions to this rule are not uncommon. Absolutely avirulent races do not, so far as we know, produce hemolysins. The culture medium most favorable to the formation of these substances is, according to Neisser and Wechsberg, a moderately alkaline beef bouillon. Cultivated at $37.5^{\circ} \mathrm{C}$., the bouillon contains the maximum amount of hemolytic substance between the eighth and fourteenth day, and this may be separated from the bacteria by filtration through Berkefeld or Chamberland filters.

The hemolytic action may be observed by the general technique for determining hemolysis (given on page 259). It is important to wash the red blood corpuscles used for the experiments, since many animals normally possess small quantities of antihemolysin in their blood-sera (man and horse especially). ${ }^{2}$ The red blood corpuscles of rabbits, dogs, and guinea-pigs are extremely susceptible to the action of the staphylohemolysin. Those of man are less easily injured by it. The hemolytic action takes place, as Todd $^{3}$ and others ${ }^{4}$ have shown, not only in vitro, but in the living animal as well.

The staphylo-hemolysin is comparatively thermolabile. According to Neisser and Wechsberg, heating it to $56^{\circ} \mathrm{C}$. for twenty minutes destroys it. According to some other authors, however, higher temperatures $\left(60^{\circ}\right.$ to $80^{\circ} \mathrm{C}$.) are required. Reactivation of a destroyed staphylohemolysin has so far been unsuccessful. The fact that antistaphylolysin is occasionally present in normal sera has been mentioned above. This antibody is most abundant in the blood of horses and of man. Artificially antistaphylolysin formation is easily induced by subcutaneous inoculation of staphylolysin into rabbits.

Leucocidin.-In 1894, Van de Velde ${ }^{5}$ discovered that the pleural exudate of rabbits following the injection of virulent staphylococci showed marked evidences of leucocyte destruction. He was subse-

${ }_{1}$ Neisser und Wechsberg, Zeit. f. Hyg., xxxvi, 1901.

2 Neisser, Deut. med. Woch., 1900.

3 Todd, Trans. London Path. Soc., 1902.

Kraus, Wien. klin. Woch., 1902.

5 Van de Velde, La Cellule, $\mathrm{x}, 1894$. 
quently able to show that the substance causing the death and partial solution of the leucocytes was a soluble toxin formed by the staphylococcus, not only in vivo, but in vitro as well; for cultures of Staphylococcus pyogenes aureus, grown in mixtures of bouillon and blood serum, contained, within forty-eight hours, marked quantities of this "leucocidin."

Other workers since Van de Velde have evolved various methods for obtaining potent leucocidin. Bail ${ }^{1}$ obtained it by growing virulent staphylococcus in mixtures of one-per-cent glycerin solutions and rabbit serum. Neisser and Wechsberg ${ }^{2}$ advise the use of a carefully titrated alkaline bouillon. To obtain the leucocidin free from bacteria, the cultures are passed through Chamberland or Berkefeld filters, after about eight to eleven days' growth at $37^{\circ} \mathrm{C}$., at which time the contents in leucocidin are usually at their highest point.

The action of leucocidin upon leucocytes may be observed in vivo by the simple method of Van de Velde, of injecting virulent staphylococci intrapleurally into rabbits and examining the exudate. Bail advises the production of leucocytic intrapleural exudates by the use of aleuronat and following this after twenty-four hours by an injection of leucocidin-filtrate. In vitro the phenomenon may be observed by direct examination of mixtures of leucocytes and leucocidin in the hanging drop on a warmed stage, or by the "methylene-blue method" of Neisser and Wechsberg. This method is based upon the fact that living leucocytes will reduce methylene-blue solutions and render them colorless, while dead leucocytes have lost this power. Leucocidin and leucocytes are allowed to remain in contact for a given time and to them is then added an extremely dilute solution of methylene-blue. If the leucocytes have been actively attacked by leucocidin, no reduction takes place. This method is particularly adapted for quantitative tests.

All staphylococcus strains do not produce leucocidin to the same degree. Almost all true Staphylococcus pyogenes aureus cultures produce some of this toxin, but one strain may produce fifty- and a hundred-fold the quantity produced by another. Staphylococcus pyogenes albus gives rise to this substance but rarely, and then in small quantity.

Leucocidin seems to be similar to the soluble toxins of other bacteria. It is rapidly destroyed by heat at $58^{\circ} \mathrm{C}$, and deteriorates quickly in

1 Bail, Arch. f. Hyg., xxxii, 1898.

${ }^{2}$ Neisser und Wechsberg, Zeit. f. Hyg., xxxvi, 1901. 
culture fluids at incubator temperatures. It is distinct from staphylohemolysin as shown by differences in thermostability.

Soon after Van de Velde's discovery of leucocidin, Denys and Van de Velde ${ }^{1}$ produced an antileucocidin by treating rabbits with pleural exudate containing leucocidin. Neisser and Wechsberg ${ }^{2}$ later confirmed these results and showed that among staphylococci, leucocidin is not specific, the toxin of all strains of Staphylococcus aureus and albus examined being neutralizable by the same antileucocidin. Antileucocidin is often found in the normal sera of horses and man. ${ }^{3}$

Leucocidin should not be confounded with "leucotoxin," a substance obtained in serum by treatment of animals with leucocytes, a true "cytotoxin," having no connection whatever with the staphylococcus.

Staphylococci, besides the toxic substances already mentioned, give rise to gelatinase, spoken of in the section upon cultivation, and to a proteolytic ferment by means of which albuminous media (Loeffler's serum) may be slightly digested.

Immunization.-Animals can be rendered actively immune by repeated inoculations with carefully graded doses of living or dead staphylococcus cultures. ${ }^{4}$ The production of antistaphylolysin and of antileucocidin in the sera of animals so treated, has been alluded to in the preceding sections. The sera of such actively immunized animals possess distinct protective power when administered to other animals, slightly before or at the same time with an inoculation of staphylococci. They do not, however, exhibit very high bactericidal value in vitro. The use of immune sera to combat staphylococcus infection has not so far given very encouraging results. ${ }^{5}$

Agglutinins have been demonstrated in staphylococcus immune sera by a number of authors, and have been shown to be of value in differentiating between the several groups of staphylococci. ${ }^{6}$ A rather surprising result of these researches has been the recognition that immune sera obtained with pathogenic staphylococci will agglutinate other pathogenic staphylococci, whether belonging to the group of Staphylococcus pyogenes aureus or that of Staphylococcus pyogenes albus,

1 Denys et Van de Velde, La Cellule, xi, 1895.

2 Loc. cit.

3 Van de Velde, Presse médicale, i, 1900.

4 Richet et Hericourt, Compt. rend. de l'acad. des sci., cvii, 1888.

5 Kolle und Otto, Zeit. f. Hyg., xli, 1902.

"Proscher, Cent. f. Bakt., xxxiv, 1903; v. Lingelsheim, "Aetiol. u. Therap. d Staphyl.," etc., Wien, 1900. 
but will not agglutinate any of the non-pathogenic members of either group. ${ }^{1}$

Active immunization of human beings suffering from staphylococcus infections has been extensively practiced by Wright, in connection with his work on opsonins. There can be no question about the fact that the opsonic substances in the blood are increased by the injection of dead staphylococci. The procedure is of therapeutic value in subacute and chronic cases. The work of Hiss on the use of leucocyte extracts in animals infected with Staphylococcus pyogenes aureus has given encouragement for such treatment in human beings. A number of staphylococcus infections in man have been successfully treated with leucocyte extract by Hiss and Zinsser.

\section{STAPHYLOCOCCUS PYOGENES ALBUS}

This coccus differs from Staphylococcus pyogenes aureus simply in the absence of the golden yellow coloration of its cultures. Morphologically, culturally, and pathogenically, it is in every way identical with the staphylococcus described in the preceding section, but its toxin- and enzyme-producing powers in general are less developed than those of the aureus variety. Its close biological relationship to aureus is furthermore demonstrated by its agglutination in Staphylococcus pyogenes aureus immune sera.

\section{STAPHYLOCOCCUS EPIDER MIDIS ALBUS}

The Staphylococcus epidermidis albus described by Welch is merely. one of the non-pathogenic varieties of Staphylococcus pyogenes albus and possibly does not deserve separate classification. It may give rise to unimportant stitch abscesses.

\section{STAPHYLOCOCCUS PYOGENES CITREUS}

Staphylococcus pyogenes citreus produces a bright yellow or lemoncolored pigment of distinctly different hue from that of Staphylococcus pyogenes aureus. It may be pyogenic and in every way similar to Staphylococcus pyogenes aureus, but is less often found in connection with pathological lesions than either of the preceding staphylococci.

1 Proscher, Deut. med. Woch., xi, 1903. 
A large number of staphylococci, differing from those described above in one or another detail, have been observed. They are of common occurrence and are met with chiefly as contaminations in the course of bacteriological work. Few of these have any pathological significance and none of them are toxin-producers, so far as we know. Many of them differ, furthermore, in their inability to liquefy gelatin. All of them belong more strictly to the field of botany than to that of pathological bacteriology.

Atypical pathogenic staphylococci have been described by a number of observers. Thus Weichselbaum ${ }^{1}$ isolated a staphylococcus from a case of malignant endocarditis which could not be cultivated at room temperature, and grew only in very delicate colonies. Veillon, ${ }^{2}$ moreover, has described a strictly anaërobic staphylococcus cultivated from the pus of an intra-abdominal abscess.

\section{MICROCOCCUS TETRAGENUS}

In 1881, Gaffky ${ }^{3}$ discovered a micrococcus which occur's regularly in groups of four or tetrads. He first isolated it from the pus discharged by tuberculous patients with pulmonary lesions. Observed in smear preparations from pus, the tetrads are slightly larger in size than the ordinary staphylococcus, flattened along their adjacent surfaces, and surrounded by a thick halo-like capsule. Preparations from cultures often lack these capsules. The micrococcus is easily stained by the usual basic anilin dyes. Stained by Gram's method, it is not decolorized, retaining the gentian-violet.

Cultivation.-Micrococcus tetragenus grows on the ordinary laboratory media, showing a rather more delicate growth than do the staphylococci.

On agar, the colonies are at first transparent, later they become grayish-white, but are always more transparent than are staphylococcus cultures.

On gelatin, growth is rather slow and no liquefaction takes place.

Broth is evenly clouded.

On potato there is a white, moist growth which shows a tendency to confluence.

1 Weichselbaum, Baumgarten Jahresb., 1899, Ref.

2 Veillon, Compt. rend. soc. de biol., 1893.

${ }^{3}$ Gaffky, Mitteil. a. d. kais. Gesundheitsamt, i, 1881. 
Milk is coagulated and litmus milk indicates acid formation.

Pathogenicity.-Micrococcus tetragenus is especially pathogenic for Japanese mice, which succumb within three or four days to subcutaneous inoculation. ${ }^{1}$ Gray mice, rats, guinea-pigs, and rabbits are less susceptible, showing only a localized reaction at the point of inoculation.

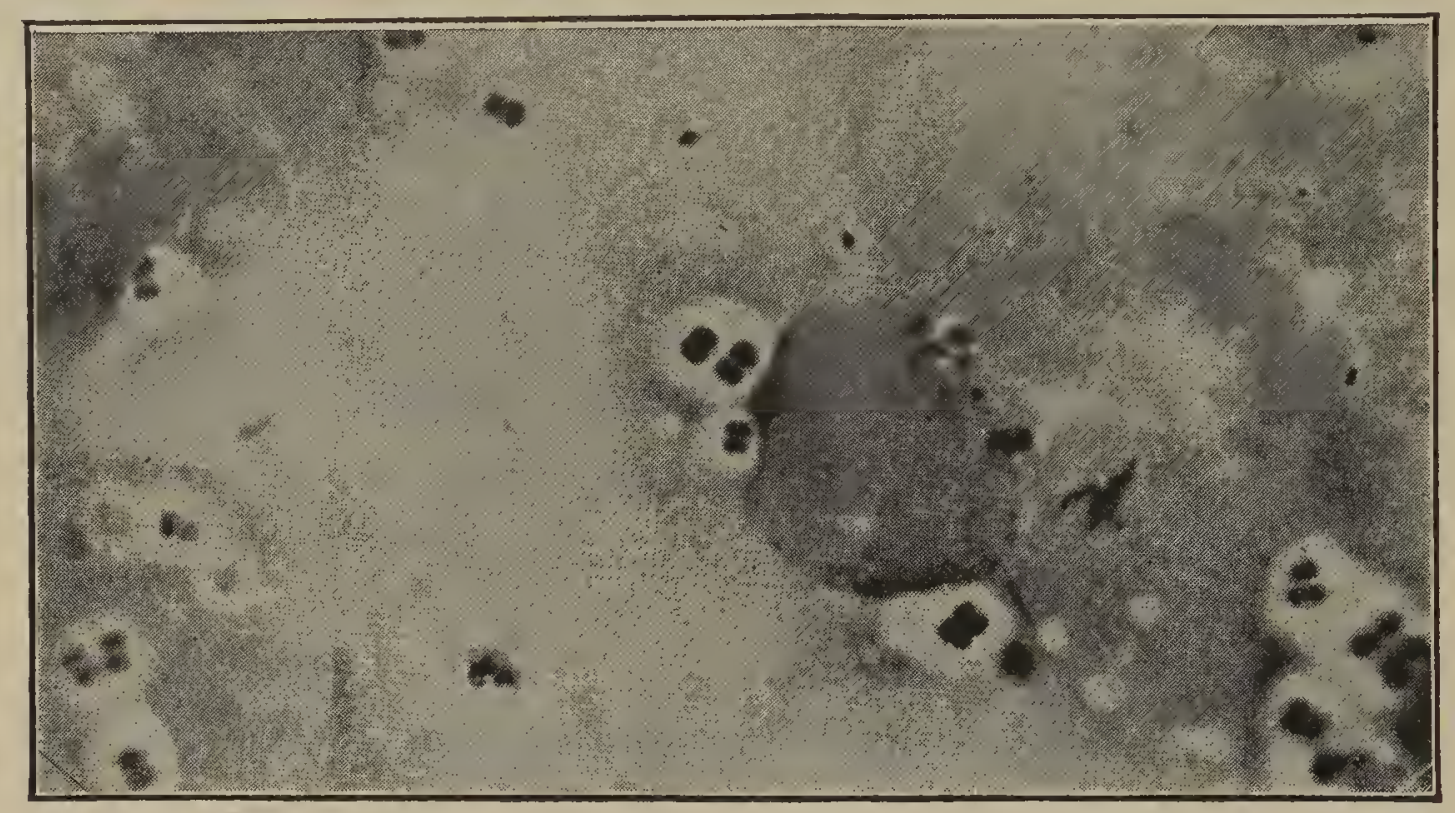

Fig. 71.-Micrococcus Tetragenus. (In spleen of infected mouse.)

The organism has occasionally been isolated from spontaneous abscesses observed in domestic animals.

In man, this microorganism is usually found without any particular pathogenic significance, in sputum or saliva. In isolated cases, however, it has been described as the sole incitant of abscesses.

Bezançon ${ }^{2}$ has isolated Micrococcus tetragenus from a case of meningitis. A single case of tetragenus septicemia is on record, reported in 1905 by Forneaca. ${ }^{3}$

In America, this microorganism has not been frequently observed in connection with disease. It is often found, however, in considerable numbers, in smears of sputum which are being examined for pneumococci or tubercle bacilli.

\footnotetext{
1 Müller, Wien. klin. Woch., 17, 1904.

2 Bezançon, Semaine méd., 1898.

${ }^{3}$ Forneaca, Rif. med., 1903.
} 


\section{CHAPTER XXII}

\section{THE STREPTOCOCCI}

Among the pyogenic cocci, there is a large and important group of organisms which multiply by division in one plane of space only, and thus give rise to appearances not unlike chains or strings of beads. The term streptococcus or chain-coccus is, therefore, a purely morphological one which includes within its limits microorganisms which may differ from each other considerably, both as to cultural and pathogenic properties. Thus, cocci which form chains may be isolated from water, milk, dust, and the feces of animals and man. These may have little but their morphological appearance in common with the pyogenic streptococci which are so important as the incitants of disease. The interrelationship between streptococci from different sources, however, is by no means fully understood, and we are forced at present to content ourselves with the recognition of a large morphological group, in no individual case taking the pathogenic or more special cultural characteristics for granted.

\section{STREPTOCOCCUS PYOGENES}

Of paramount importance among the streptococci are those which possess the power of giving lise to disease processes in animals and in man, and which, because of their frequent association with suppurative inflammations, are roughly grouped under the heading of Streptococcus pyogenes.

The same researches upon surgical infections which led to the discovery of the staphylococci, laid the basis for our knowledge of the streptococci. The fundamental studies of Pasteur and Koch ${ }^{1}$ were followed, in 1881, by the work of Ogston, ${ }^{2}$ who was the first to differentiate between the irregularly grouped staphylococci and the chain-cocci.

1 Koch, "Untersuch. über Wundinfektion," etc., 1878.

2 Ogston, Brit. Med. Jour., 1881. 
Pure cultures of streptococei were first obtained by Fehleisen ${ }^{1}$ in 1883 and by Rosenbach ${ }^{2}$ in 1884 . The thorough and systematic researches of the last-named authors, together with those of Passet, ${ }^{3}$ were of special

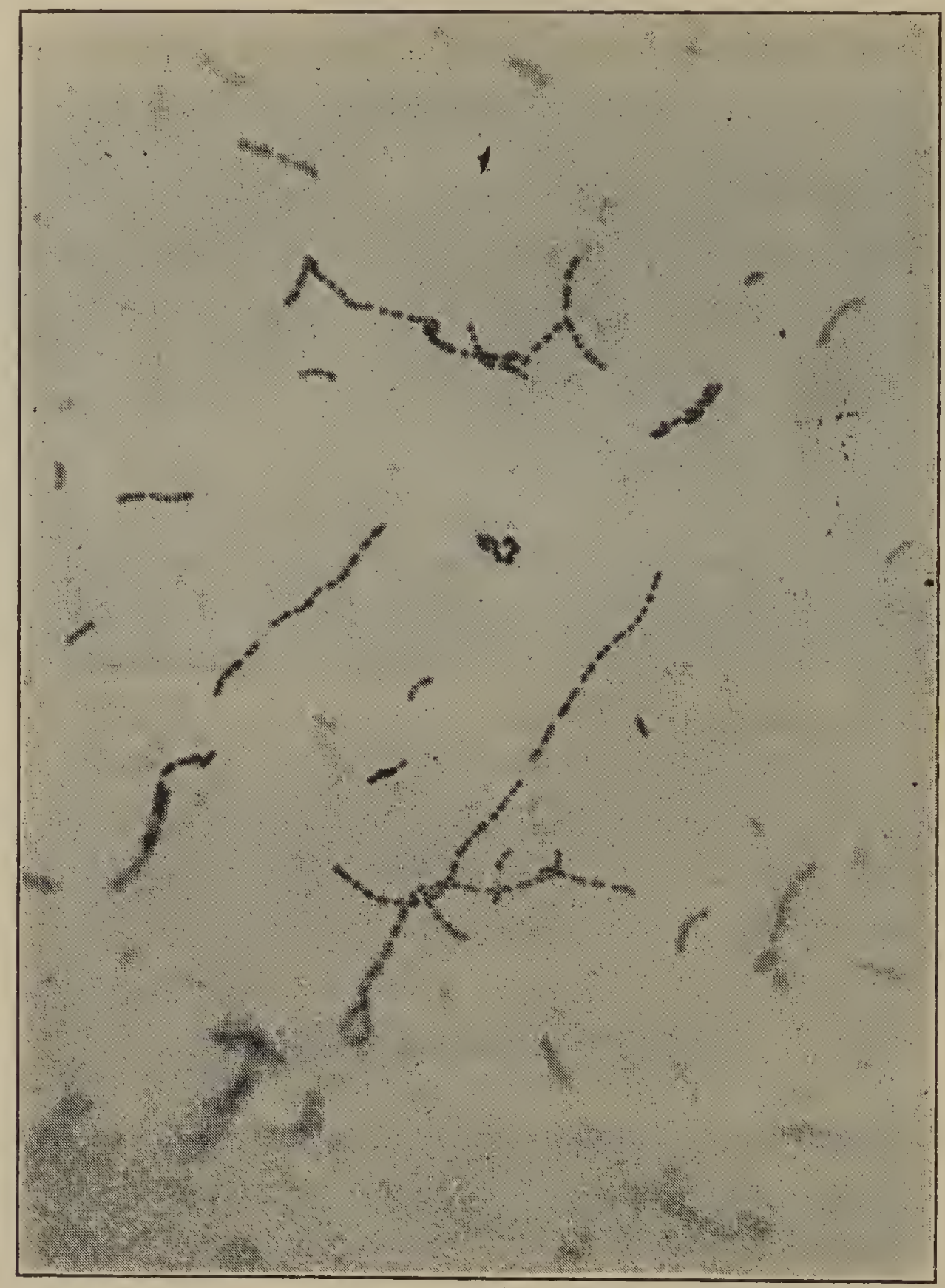

Fig. 72.-Streptococcus Pyogenes.

influence in placing our knowledge of the pathogenic properties of streptococci upon a scientific basis.

Morphology and Staining.-The individual streptococcus is a spherical microorganism measuring from 0.5 micron to 1 micron in diameter. Since the line of cleavage of cocci, when in chains, is perpendicular to the

"Fehleisen, "Aetiol. d. Erysipelas," Berlin, 1883.

2 Rosenbach, "Mikroorg. bei Wundinfektion," etc., Wiesbaden, 1884.

3 Passet, "Untersuch. über die eitrigen Phlegm.," etc., Berlin, 1885. 
long axis of the chain, adjacent cocci often show slight flattening of the contiguous surfaces, forming, as it were, a series of diplococci arranged end to end. As a general rule the streptococci pathogenic for man, when grown upon favorable media, have a tendency to form chains made up of at least eight or more individuals, while the more saprophytic, less pathogenic varieties are apt to be united in shorter groups. Upon this basis a rough morphological distinction has been made by $\mathrm{v}$. Lingelsheim, " who first employed the terms Streptococcus "longus" and "brevis." A differentiation of this kind can hardly be relied upon, however, since the length of chains is to some degree dependent upon cultural and other environmental conditions. Species which exhibit long and tortuous chains, when grown upon suitably alkaline bouillon, or ascitic broth, may appear in short groups of three or four, or even in the diplo form, when cultivated upon solid media or unfavorable fluid media. Stained specimens often show swelling and enlargement of individual cocci, giving the chains an irregularly beaded appearance. These swollen individuals are probably to be interpreted as involution forms and are seen with especial frequency in old cultures. Streptococci do not form spores, are non-motile, and do not possess flagella. There can be no doubt that certain species of true streptococci may possess capsules, though these are not so regularly demonstrable and are more delicately dependent upon cultural conditions than are the capsules of the pneumococci. ${ }^{2}$ The capsulated streptococci will be discussed more comprehensively in the section upon the differentiation of pneumococcus from streptococcus (page 367).

Streptococci are easily stained by the usual anilin dyes. Stained by the method of Gram, the pyogenic streptococci are not decolorized and invariably retain the gentian-violet. Certain species found in stools and described as Gram-negative, are rare and are non-pathogenic. Others of the "Streptococcus brevis" variety, and purely saprophytic, may stain irregularly by the Gram method.

Cultivation.-The pyogenic streptococci are easily cultivated upon all the richer artificial media. While meat extract-pepton media may suffice for certain strains, it is usually better to employ those media which have the beef or veal infusion for a basis. For the cultivation of more delicate strains of streptococci, especially when

1 v. Lingelsheim, "Aetiol. u. Therap. d. Streptok. Infek." Beit. z. Exp. Ther., Hft. 1, 1899.

2 Pasquale, Zieglers Beit., xii; Bordet, Ann. de l'inst. Pasteur, 1887; Schottmüller, Münch. med. Woch., xx, 1903; Hiss, Jour. Exp. Med., vi, 1905. 
taken directly from the animal or human body, it is well to add to the media animal albumin in the form of whole blood, blood serum, or ascitic or pleural transudates. Glucose, added in proportions of one to two per cent, likewise renders media more favorable for streptococcus cultivation. Prolonged cultivation of all races upon artificial media renders them less fastidious as to cultural requirements. The most favorable reaction of media for streptococcus cultivation is moderate alkalinity (two-tenths to five-tenths per cent alkalinity to phenolphthalein). Growth may be readily obtained, however, in neutral media or even in those slightly acid. The optimum temperature for growth is at or about $37.5^{\circ} \mathrm{C}$. Above $43^{\circ}$ to $45^{\circ} \mathrm{C}$, development ceases. At from $15^{\circ}$ to $20^{\circ} \mathrm{C}$, growth, while not energetic, still takes place, an important point in the differentiation of these microorganisms from pneumococci. While the free access of oxygen furnishes the most suitable environment for most races of streptococci, complete anaërobiosis does not prevent development in favorable media. Strictly anaërobic streptococci have been cultivated from the human intestinal tract by Perrone ${ }^{1}$ and others.

In alkaline bouillon at $37.5^{\circ} \mathrm{C}$, pyogenic streptococci grow rapidly, form long and tortuous chains, and have a tendency to form flakes which rapidly sink to the bottom. Diffuse clouding occurs rarely and is a characteristic rather of the shorter so-called Streptococcus brevis. When sugar has been added to the broth the rapid formation of lactic acid soon interferes with extensive development. This may be obviated, especially when mass cultures are desired, without sacrifice of the growthincreasing influence of the glucose, by adding to the sugar-broth one per cent of sterile powdered $\mathrm{CaCO}_{3}{ }^{2}{ }^{2}$

In milk, Streptococcus pyogenes grows readily with the formation of acid, followed, in most cases, by coagulation of the medium.

On agar-plates at $37.5^{\circ} \mathrm{C}$., growth appears within eighteen to twenty-. four hours. The colonies are small, grayish, and delicately opalescent. They are round with smooth or very slightly corrugated or lace-like edges, and rise from the surface of the medium in regular arcs, like small droplets of fluid. Microscopically they appear finely granular and occasionally, under high magnification, may be seen to be composed of long intertwining loops of streptococcus chains, which form the lace-like edges. When ascitic fluid or blood serum has been added to agar, growth is more energetic and the colonies correspondingly more rapid in

1 Perrone, Ann. de l'inst. Pasteur, xix, 1905.

${ }^{2}$ Hiss, Jour. Exp. Med., vi, 1905. 
appearance and luxuriant in development. In glucose-ascitic-agar, acid formation from the sugar causes coagulation of albumin with the consequent formation of flaky white precipitates throughout the medium. ${ }^{1}$

In gelatin stab-cultures growth takes place slowly, appearing after twenty-four to thirty-six hours as a very thin white line, or as disconnected little spheres along the line of the stab. The colonies on gelatin plates are similar in form to those on agar, but are usually more opaque and more distinctly white. The gelatin is not liquefied by the pyogenic streptococci, though certain of the more saprophytic forms may occasionally bring about slow fluidification.

On Loeffler's coagulated blood serum, growth is rapid and luxuriant, and may show a slight tendency to confluence if the medium is very moist. Good chain formation takes place on this medium.

Upon potatoes, growth is said not to take place. $^{2}$

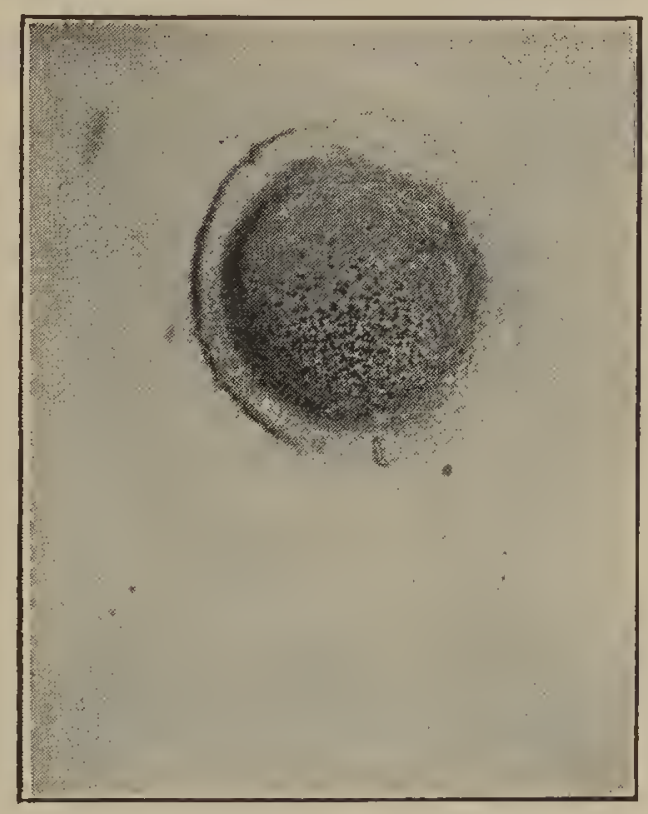

Fig. 73.-STreptococcus Colonies, on Serum Agar.

On media containing red blood cells, most pathogenic streptococci cause hemolysis and decolorization (see Fig. 74, p. 345). It is useful to remember this when examining blood-culture plates, for here the yellow transparent halo of hemolysis and decolorization surrounding the colonies may aid in differentiating these bacteria from pneumococci. This is of especial importance, since many streptococci, when cultivated directly out of the human blood, do not exhibit chain formation, but appear as diplococci.

In the inulin-serum media of Hiss, ${ }^{3}$ streptococci do not produce acid and coagulation. The so-called Streptococcus mucosus, a capsulebearing, inulin-fermenting microorganism, is very probably a sub-species of the pneumococcus (see later section).

Resistance.-Streptococci on the ordinary culture media, without transplantation and kept at room temperature, usually die out within ten days or two weeks. They may be kept alive for much longer periods by the use of the calcium-carbonate-glucose bouillon, if the cultures are

${ }^{1}$ Libman, Medical Record, lvii, 1900.

${ }^{2}$ Frosch und Kolle, in Flügge, "Die Mikroorganismen," 1891.

${ }^{3}$ Hiss, Jour. Exp. Med., vi, 1905. 
thoroughly shaken and the powdered marble thoroughly mixed with the bouillon from time to time. ${ }^{1}$ Preservation at low temperatures $\left(1^{\circ}\right.$ to $2^{\circ} \mathrm{C}$.), in the ice chest, considerably prolongs the life of cultures. Virulence is preserved longest by frequent transplantation upon albuminous media. In sputum or animal excreta, streptococci may remain alive for several weeks.

Streptococci are killed by exposure to a temperature of $54^{\circ} \mathrm{C}$. for ten minutes. ${ }^{2}$ Low temperatures, and even freezing, do not destroy some races.

The action of various chemical disinfectants has been thoroughly investigated by $\mathrm{v}$. Lingelsheim, ${ }^{3}$ who reports among others the following results: Carbolic acid $1: 200$ kills streptococci in fifteen minutes. In the same time, bichloride of mercury is efficient in a dilution of $1: 1,500$, lysol in a dilution of $1: 200$, peroxide of hydrogen $1: 35$, sulphuric acid $1: 150$, and hydrochloric acid $1: 150$. Inhibition is exerted by carbolic acid $1: 550$, and by bichloride of mercury $1: 65,000$. Exposure to direct sunlight kills streptococci in a few hours.

Virulence and Pathogenicity.-Different races of pyogenic streptococci show considerable variations in virulence, and there are few organisms, pathogenic both for animale and man, which show such peculiarities in virulence. The character or severity of the lesion in man gives little evidence as to the virulence of the organism for animals. Such differences are, to a certain extent, dependent upon inherent individual characteristics, but are rather more likely to be the consequences of previous environment or habitat. Prolonged cultivation upon artificial media usually results in the reduction of the virulence of a streptococcus, while an originally low or reduced virulence may often be much enhanced by repeated passage of the streptococci through animals. It is noteworthy, however, that while the passage of a streptococcus through rabbits will usually enhance its virulence for susceptible animals in general, repeated passages through mice may increase the virulence for these animals only, even occasionally depressing the virulence for rabbits. ${ }^{4}$

Among the domestic animals, those most susceptible to experimental streptococcus infection are white mice and rabbits. Guinea-pigs and

${ }^{1}$ Hiss, loc. cit.

${ }^{2}$ Sternberg, "Textbook of Bact.," 2d ed., 1901; Hartmann, Arch. f. Hyg., vii.

${ }^{3} v$. Lingelsheim, "Aetiol. u. Therap. d. Streptoc. Inf.," etc., Beit. z. Exper. Therap., Hft. 1, 1899.

${ }^{4}$ Knorr, Zeit. f. Hyg., xiii. 
rats are less easily infected, and the larger domestic animals, cattle, horses, goats, cats, and dogs, are extremely refractory. Almost complete immunity toward streptococcus infections prevails among birds.

The nature of the lesions following animal inoculation depends upon the manner of inoculation, the size of the dose given, and most of all upon the grade of virulence of the inoculated germ. Subcutaneous inoculations, according to the virulence of the inoculated material, may result in a simple localized abscess, differing from a staphylococcus abscess only in the more serous nature of the exudate and the frequent occurrence of edema, or in a severe general septicemia with a hardly noticeable local lesion. Subcutaneous inoculation of mice results almost invariably in general sepsis followed by death within thirty-six to fortyeight hours, or less, and the presence of streptococci in the heart's blood and the viscera. Intrapleural or intraperitoneal inoculation of susceptible animals with virulent streptococci leads usually to a peculiarly hemorrhagic form of exudate, due both to the diapedesis caused by the violent inflammatory process, and to the hemolysis of the red cells by the streptococcic hemolysins. Inoculation of rabbits at the base of the ear with virulent streptococci may result in the formation of a lesion indistinguishable histologically from erysipelas in man. ${ }^{1}$ Marbaix ${ }^{2}$ has shown that such erysipeloid lesions could be produced in rabbits by streptococci from various and indifferent sources, provided that the virulence of each strain could be sufficiently enhanced. This marked variability of the resulting lesion as determined by the degree of virulence of the incitant, whatever its original source, forms a strong argument in favor of the opinion that all the pyogenic streptococci are members of a single species.

Intravenous inoculation of rabbits with virulent cultures usually results in a rapidly fatal septicemia. An animal which has died of a streptococcus infection usually shows serosanguineous edema about the point of inoculation, multiple hemorrhagic spots upon the serous membranes, and congestion of the viscera. The microorganisms can almost invariably be found in the heart's blood, in the spleen, and in the exudate about the inoculated area. Microscopically, when the process has lasted sufficiently long, parenchymatous degeneration of all the organs may be observed. In the more chronic infections articular and periarticular lesions may occur. ${ }^{3}$

1 Fehleisen, loc. cit.; Frankel, Cent. f. Bakt., vi.

${ }^{2}$ Marbaix, La Cellule, 1892.

${ }^{3}$ Schütz, Zeit. f. Hyg., iii; Hiss, Jour. Med. Res', xix, 1908. 
Spontaneous streptococcus disease seems to occur among some of the larger domestic animals. Thus, a contagious form of inflammation of the respiratory passages of horses has been attributed to streptococcus infection. ${ }^{1}$ Among cattle these microorganisms have been found to produce purulent inflammation of the udder and occasionally postpartum uterine inflammation in cows. Among the smaller laboratory animals, occasional streptococcus infections may be observed in rabbits. Recently an epidemic disease among white mice due to streptococcus was studied by Kutscher. ${ }^{2}$ As a rule, however, streptococcus disease is by far more rare among animals than it is among human beings.

In man, a large variety of pathological processes may be caused by streptococci and here again the nature of the infection, whether definitely localized or generally distributed, depends upon the relationship existing between the virulence of the incitant and the resistance of the subject.

The first cultivation of streptococcus from human lesions was made by Fehleisen, ${ }^{3}$ who obtained them from cases of erysipelas. It was long believed that the so-called Streptococcus erysipelatis was a similar but essentially different species from the common Streptococcus pyogenes. The production of erysipelas in animals with streptococci from other sources, however, has shown definitely that the two groups can not be separated. ${ }^{4}$ Superficial cutaneous infections are frequently caused by streptococci and these in the milder cases may be similar to the localized abscesses caused by staphylococci. In severe cases, however, infection is followed by rapidly spreading edema, lymphangitis, and severe systemic manifestations with the development of a grave cellulitis, often threatening life and requiring energetic surgical interference. Invasion of the respiratory organs by streptococci is not rare, and may lead to bronchitis, pneumonia, or empyema. They are frequently present also as secondary invaders in pulmonary tuberculosis. ${ }^{5}$ Streptococcus infections of the lungs and pleura not infrequently lead to pericardial involvement.

Suppurations of bone may be caused by streptococci, and constitute a severe form of osteomyelitis. Such lesions when occurring in the mastoid bone are not infrequently secondary to streptococcus otitis and may lead to a form of meningitis which is in most cases fatal. In the

1 Van de Velde, Monatsheft Bakt., Thierheilk., ii.

${ }^{2}$ Kutscher, Cent. f. Bakt., xlvi.

${ }^{3}$ Fehleisen, loc. cit.

${ }^{4}$ Marbaix, La Cellule, 1892; Petruschky, Zeit. f. Hyg., xxiii.

${ }^{5}$ Cornet, "Die Tuberkulose," Wien, 1899. 
mouth and throat streptococci may give rise to pharyngitis and are one of the most frequent causes of a form of tonsillitis often clinically indistinguishable from diphtheria. The throat inflammation accompanying scarlatina is, almost without exception, referable to streptococcus infection. ${ }^{1}$ The occasional presence of the streptococcus in the blood of scarlatina patients, moreover, has led some authors to suggest a possible etiological connection between this microorganism and the disease. ${ }^{2}$ This, however, is at present merely conjectural.

In diphtheric inflammations of the throat, a secondary streptococcus infection is a frequent and serious complication. As incitants of disease of the intestines, streptococci have been found in appendicular abscesses ${ }^{3}$ and have been described as the cause of some forms of infantile diarrhea. ${ }^{4}$ From any of the local processes streptococci may pass into the circulation, causing sepsis. The septicemia occurring during the puerperium is most often caused by this microorganism.

Secondary foci in the viscera may be established, leading to pyemia, ${ }^{5}$ or, if these localizations occur upon the heart valves, septic endocarditis may ensue. All such forms of general streptococcus infection, whether running acute or chronic courses, present a high rate of mortality. The diagnosis in these cases is usually easy if blood cultures are taken upon suitable media.

Streptococcus throat infections have recently appeared in fulminating epidemics. Several small epidemics were described in England, and three extensive outbreaks have occurred in this country; one in Boston of 1,400 cases; a second in Baltimore of about 1,000 cases, and a third in Chicago of about 10,000 cases. These outbreaks were studied by Winslow, Stokes, Davis, ${ }^{6}$ and by Rosenow. ${ }^{7}$ In each case the majority of infections were traced to a single milk supply, though secondary cases doubtless occurred by contact. Severe complications such as suppurative adenitis, otitis, erysipelas, peritonitis, and septicemia were not uncommon. A similar organism-a capsulated, hemolytic streptococcus - was found in each epidemic.

${ }_{1}^{1}$ Baginsky, Deut. med. Zeit., 1900.

${ }^{2}$ Baginsky und Sommerfeld, Berl. klin. Woch., xxvii, 1900.

${ }^{3}$ Kelly, "Pathogenesis of Appendicitis."

${ }^{4}$ Lanz and Tavel, Rev. de Chir., 1904; Perrone, Ann. de l'inst. Pasteur, 1905; Escherich, Jahrb. f. Kinderheilkunde, 1899.

${ }^{5}$ Libman, Cent. f. Bakt., xxii.

${ }^{6}$ Cited from Capps, Jour. A. M. A., 1912, p. 1848.

${ }^{7}$ Rosenow, Jour. of Inf. Dis., 1912. 
Toxic Products. - In spite of extensive researches by many investigators upon the nature of the poisons produced by streptococci, our understanding of these substances is still very incomplete. The grave systemic symptoms so often accompanying comparatively slight streptococcus lesions argue strongly for the production by these microorganisms of a powerful diffusible poison. Toxic filtrates of streptococcus cultures have indeed been obtained by Roger, ${ }^{1}$ Marmier, ${ }^{2}$ Baginsky and Sommerfeld, ${ }^{3}$ Marmorek, ${ }^{4}$ and many others; but these have in no case been comparable in potency to the soluble toxins of diphtheria or of tetanus. When injected into young guinea-pigs in sufficient quantity, these filtrates produce rapid collapse and death. The inability to produce strong toxins is generally attributed to the difficulty of obtaining very abundant growth of these bacteria upon fluid media, development being self-limiting, either because of the exhaustion of specific nutritive material (Marmorek ${ }^{5}$ ), or, more probably, because of the inhibitory effects of the products of growth, chiefly acid formation. This last factor can be partially overcome by the use of the glucose-calcium-carbonate broth mentioned above, in which acid neutralization is constantly taking place. For toxin production, Baginsky and Sommerfeld ${ }^{6}$ advise a strongly alkaline reaction of the media; Marmorek $^{7}$ has used human blood-serum-bouillon with success. The toxins so produced are relatively thermostable. According to v. Lingelsheim, heating to $60^{\circ}$ or $70^{\circ} \mathrm{C}$. destroys them in part only. The endotoxins contained within the cell-bodies of streptococci themselves have been found to possess but slight toxic qualities.

Apart from these substances, some streptococci produce a hemolysin which has the power of bringing about destruction of red blood corpuscles. The observation of this phenomenon for streptococci was first made by Marmorek ${ }^{8}$ in 1895. According to this author, there is a direct relationship between virulence and hemolytic power. Other investigators, however, notably Schottmüller, ${ }^{9}$ believe the hemolytic power to be a constant characteristic of certain strains unchangeable by

\footnotetext{
${ }^{1}$ Roger, Rev. de méd., 1892.

${ }^{2}$ Marmier, Ann. de l'inst. Pasteur, ix, 1895, p. 533.

${ }^{3}$ Baginsky und Sommerfeld, Berl. klin. Woch., 1900.

${ }^{4}$ Marmorek, Berl. klin. Woch., 1902.

5 Marmorek, Berl. klin. Woch., xiv, 1902.

${ }^{6}$ Loc. cit.

7 Marmorek, Ann. de l'inst. Pasteur, 1895.

${ }^{8}$ Marmorek, Ann. de l'inst. Pasteur, 1895.

${ }^{9}$ Schottmüller, Münch. med. Woch., 1903.
} 
experimental enhancement or reduction of the virulence. Streptococcus hemolysins may be conveniently observed by cultivation of the organisms upon blood-agar plates. They may be produced in alkaline peptonbroth and obtained separate from the bacteria by filtration-a procedure, however, in which the quantities obtained are never large. Besredka ${ }^{1}$ and Schlesinger ${ }^{2}$ believe, for this reason, that the hemolytic substances are closely attached to the bacterial bodies. The last-named author, furthermore, has determined that, in contradistinction to the other toxic substances, streptococcus hemolysins are extremely labile, disappearing from culture fluids after standing for from five to seven days at ordinary room temperature.

Immunization.-For reasons not wholly understood at present, recovery from streptococcus infection does not to any marked degree produce immunity against these

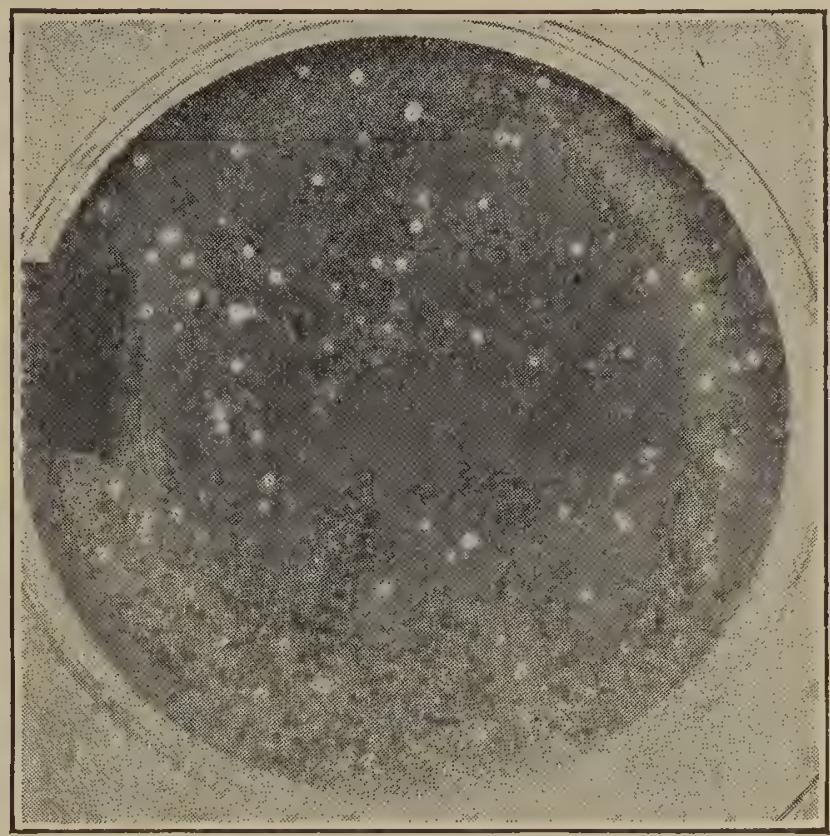

Fig. 74.-Streptococcus Colonies From Blood Culture on BloodAgar Plate. Showing areas of hemolysis about colonies. bacteria. Active immunity may, however, be produced in rabbits, goats, horses, and other domestic animals by treatment with gradually increasing doses of streptococcus cultures. ${ }^{3}$

In carrying out such immunizations it is necessary to use for the first injection attenuated or dead bacteria. Attenuation may be accomplished by moderate heating or by the addition of chemicals (terchloride of iodin). Neufeld ${ }^{4}$ advises, for the first injection in immunizing rabbits, the use of ascitic-broth cultures killed by heating to $70^{\circ} \mathrm{C}$. This is followed, after ten days, by a second injection of a small quantity of fully virulent cocci. Following this, injections are made at intervals of ten days with constantly increasing doses. Modifications of these general principles are employed in most laboratories.

The sera of animals so treated contain no demonstrable antitoxic or

${ }^{1}$ Besredka, Ann. de l'inst. Pasteur, xv, 1901, p. 880.

${ }^{2}$ Schlesinger, Zeit. f. Hyg., xxiv, 1903.

${ }^{3}$ Koch und Petruschky, Zeit. f. Hyg., xxiii, 1896.

${ }^{4}$ Neufeld, Zeit. f. Hyg., xliv, 1903. 
antihemolytic substances. ${ }^{1}$ They exert, however, demonstrable bactericidal power both in vivo and in vitro and distinctly enhance phagocytosis when brought into contact with leucocytes and streptococci. This "opsonic" power has been noticed both intraperitoneally (Bordet ${ }^{2}$ ) and in vitro (Denys and Leclef ${ }^{3}$ ).

The protective value of streptococcus immune sera for infected animals is considerable, reaching often a potency hardly explicable by the demonstrable bactericidal or opsonic power, and thereby suggesting some other active factor not understood as yet. ${ }^{4}$ Aronson ${ }^{5}$ has produced immune sera by the treatment of horses with a streptococcus derived from a case of scarlatina, 0.0004 c.c. of which sufficed to protect mice from ten times the fatal dose of a streptococcus culture. These high protective values, however, are obtained only when the serum injections are given simultaneously with the bacteria. Given four or six hours after infection, much higher dosage must be employed and protective results are much less regular in occurrence. ${ }^{6}$ Other antistreptococcic sera have been produced by Denys, Menger, Tavel, and others, all showing more or less marked potency in protecting animals. ${ }^{7}$

Since these sera, while in a general way potent against all streptococci, have been found protective chiefly against the specific microorganism employed for their production, Van de Velde, ${ }^{8}$ Denys, Aronson, and others have advised the immunization of the animal with a large variety of streptococcus races, derived from many different human sources. The resulting "polyvalent" serum is more apt to exert equally high protective powers against all streptococcus infections. The therapeutic value of such sera in the treatment of human infections is still sub judice. Undeniably favorable reports are published each year in increasing number, but are by no means regular or comparable to results such as those obtained in diphtheria with diphtheria antitoxin. Nevertheless, in mild cases or in those in which the lesions have been distinctly localized, the

${ }^{1}$ Lingelsheim, Zeit. f. Hyg., x, 1891.

${ }^{2}$ Bordet, Ann. de l'inst. Pasteur, 1897.

${ }^{3}$ Denys et Leclef, Cellule, t. ix.

"Denys et Marchand, "Mécanisme de l'immunité," etc., Brussels, 1896.

${ }^{5}$ Aronson, Berl. klin. Woch., xxxii, 1896; ibid., xlii and xliii, 1902; ibid., viii and ix, 1905 .

${ }^{6}$ Denys, "Le Sérum antistreptoc.," Louvain, 1896; Van de Velde, Ann. de l'inst. Pasteur, 1896.

${ }^{7}$ Denys et Marchand, Bull. de l'acad. roy. de méd. de Belgique, 1898; Menger, Berl. klin. Woch., 1902; Tavel, Corr.-Bl. f. Schw. Aerzte.

${ }^{8} V$ an de Velde, Arch. de méd. expér., 1897. 
sera have seemed to be sufficiently useful to justify their use and to necessitate their standardization.

Standardization is accomplished by the methods first devised by Marx ${ }^{2}$ for the standardization of swine-plague serum, and depends upon the ability of the serum to protect animals against a measured dose of virulent streptococci. Aronson ${ }^{2}$ designates as a "normal serum" one of which 0.01 c.c. will protect a mouse against ten to one hundred times the fatal dose of virulent streptococci. One cubic centimeter of this serum equals one serum unit. Comparisons by animal experiment with this standard serum approximately determine the value of other sera.

Leucocyte extracts have been employed by the writers and others, as advised by Hiss, ${ }^{3}$ in various forms of streptococcus infections of man, with success in many cases. Very uniformly favorable results have been obtained with these extracts in cases of erysipelas.

The agglutinins found in streptococcus immune sera are usually most active toward the race of bacteria employed in the immunization. Other streptococci, however, are also agglutinated, but in relatively higher concentration of the serum. Thus, while a specific group reaction is extremely useful in differentiating streptococci from other species, such as pneumococci, agglutination can not be relied upon to differentiate individual streptococci from one another (Hiss). It has even been found that a serum produced with a streptococcus from one source contained a higher agglutinating value for some other streptococcus than for the one employed in its production. Agglutinins may be produced by treating animals with dead as well as with the living virulent streptococci. While the technique of the streptococcus agglutination tests is not difficult when we are dealing with strains which grow diffusely and with even clouding in fluid media, the frequency with which these microorganisms clump spontaneously in broth cultures necessitates the use of a special technique. The most simple of these methods, and possibly the best, is the one in which calcium-carbonate-glucose broth is used for cultivation. ${ }^{4}$ Growing in this medium and thoroughly shaken once a day, the streptococci are usually found evenly divided in the supernatant fluid after the settling out of the heavier calcium-carbonate powder.

1 Marx, Deutsche thierärzt. Woch., vi, 1901.

${ }^{2}$ Aronson, Berl. klin. Woch., xliii, 1902; Otto, Arb. a. d. königl. Inst., etc., Frankfurt a. M., Heft 2, 1906.

${ }^{3}$ Hiss, Jour. Med. Res., xix, 1908.

${ }^{4}$ Hiss, Jour. Exp. Med., vii, 1905. 
Precipitins have been found by Aronson ${ }^{1}$ in streptococcus immune horse serum. Special methods of extracting the bacteria were employed.

Classification.-Frequently observed differences in the minor cultural characteristics and in the virulence of streptococci obtained from various sources have given rise to much discussion as to the identity of all races of streptococci. The earliest observers were forced to abandon their separation of the streptococci of erysipelas from other streptococci because of the work of Marbaix ${ }^{2}$ and others, who produced erysipelas in rabbits with streptococci from non-erysipelatous lesions, after enhancement of their virulence. V. Lingelsheim ${ }^{3}$ has proposed a purely morphological differentiation of "longus" and "brevis"; the former class including the streptococci most usually found in pyogenic lesions and having a tendency to form chains of six or more links, the latter designating the short-chained varieties, including, as a rule, the less virulent streptococci. This classification, however, is not scientifically tenable because of the considerable dependence of chain formation upon reaction, consistency, and nutritive qualities of the media employed for cultivation, and upon the influence of animal fluids if the microorganisms are taken direct from lesions. Schottmüller, ${ }^{4}$ who has made a careful study of streptococci, in 1903 proposed a classification based both upon morphology and the appearance of cultures upon human blood agar. By this method he divided streptococci into two main groups as follows: I. Streptococcus longus seu erysipelatos, consisting of the most virulent varieties, having a tendency to form long chains, and regularly producing hemolysis upon blood media. II. Streptococcus mitior seu viridans, including less virulent strains, with usually shorter chain-formation, and producing green, non-hemolyzing colonies upon blood media. These are the streptococci which he usually obtained from milder or more chronic lesions. A third group which he adds, Streptococcus mucosus, will receive special consideration in a separate section, and is probably more closely related to the pneumococci than to the streptococcus groups.

Attempts to separate the streptococci into subdivisions by their powers to ferment various carbohydrates have been made by Hiss, Gordon, and others. These attempts have, so far, been without practical

\footnotetext{
${ }^{1}$ Aronson, Deut. med. Woch., 25, 1903.

${ }^{2}$ Marbaix, loc. cit.

${ }^{3} v$. Lingelsheim, "Aetiol. u. Therap. d. Streptokok. Krankh.," etc., Berlin, 1899.

${ }^{4}$ Schottmüller, Münch. med. Woch., 1903.
} 
result. Hiss ${ }^{1}$ indicated a tentative division of streptococci into those which fermented monosaccharids alone, those which were also able to ferment disaccharids, and those in which the fermentative powers were extended to the polysaccharids, starch, dextrin, and glycogen.

Gordon, ${ }^{2}$ after a thorough study of many strains upon seven carbohydrates, found ten different fermentation reactions among twenty pyogenic streptococci examined, and forty-eight different fermentation reactions among two hundred streptococci isolated from saliva. Other work by Andrewes and Horder and by Buerger ${ }^{3}$ confirms the irregularity of the fermentation reactions within this group.

Andrewes and Horder suggest the following classification:

(1) Streptococcus pyogenes. A type which grows in long chains and which ferments lactose, saccharose, and salicin, but does not coagulate milk. Most of the streptococci which cause suppurative lesions or severe systemic infections belong to this group.

(2) Streptococcus mitis. A saprophytic type found frequently in the mouth which shows the same cultural characteristics as the streptococcus pyogenes, but grows in short chains.

(3) Streptococcus anginosus. A type found frequently in the throats of scarlet-fever patients which differs from the pyogenes only in coagulating milk.

(4) Streptococcus salivarius. A short-chain type which ferments lactose, saccharose, and raffinose, and coagulates milk. Streptococci of this type are found frequently in the mouth, but are rarely pathogenic.

(5) Streptococcus fecalis. A short-chain type which ferments lactose, saccharose, and mannite. This type is found normally in the intestine, and is occasionally pathogenic.

(6) Streptococcus equinus. A short-chain type which does not ferment lactose. It is found normally in horse dung and is never pathogenic.

Quantitative determinations of the amount of acid formed in various sugars by different races have also been made by Winslow and Palmer ${ }^{4}$ and others, but have led to no satisfactory classification.

Studies by Hopkins and Lang seem to show that the streptococci found in most human infections may be differentiated from the ordinary

\footnotetext{
${ }^{1}$ Hiss, Cent. f. Bakt., xxxi, 1902; Jour. Exp. Med., vi, 1905.

${ }^{2}$ Gordon, Annual Report, Local Govern. Board, 33, London, 1903.

${ }^{3}$ Andrewes and Horder, Lancet, 1906; Buerger, Jour, Exp. Med., ix, 1907.

${ }^{4}$ Jour. of Inf. Dis., No. vịi, 1910, 1 ,
} 
saprophytic types by the fact that they ferment lactose and salicin, but fail to ferment raffinose, inulin, or mannite. According to their results, the usual saprophytic types found in the mouth either fail to ferment salicin or ferment raffinose or inulin, whereas the usual fecal types ferment mannite. They also found in infection mannite fermenters which were apparently of fecal origin. Streptococci which gave the same fermentative reaction as the mouth saprophytes were, however, frequently found in malignant endocarditis.

Probably the most reliable method of determining the interrelationships existing between bacteria, not only within this group, but in all bacterial classes, is that depending upon their reactions to immune sera. The work of Aronson, ${ }^{1}$ Marmorek, ${ }^{2}$ and others has shown that streptococcus immune sera produced with any one race of pyogenic streptococci exerted considerable, though variable, protective action against many other strains of streptococci. The same authors, as well as many others, working with the agglutination reaction, have shown that the agglutinins produced with one streptococcus strain were active against many other streptococci. While most active usually against the particular microorganism with which they were produced, this was by no means the rule, a serum produced with a streptococcus from a case of sepsis, in one case, agglutinating a streptococcus from a case of scarlatina more highly than its own microorganism. As with other "group agglutinations," the more highly immune the serum is, the more general is the agglutinating power over the whole group. Thus, while agglutination is practically useless in separating streptococci from one another, it is highly useful in differentiating these organisms from allied groups, such as the pneumococci. The immune reactions, therefore, seem to indicate a very close relationship between streptococci as a class.

Streptococcus mucosus.-This microorganism was first definitely described by Howard and Perkins ${ }^{3}$ in 1901, and was subsequently carefully studied by Schottmüller, ${ }^{4}$ who isolated it from cases of parametritis, peritonitis, meningitis, and phlebitis. The organism has since been described by many observers as the incitant of a variety of lesions and as an apparently harmless inhabitant of the normal mouth.

1 Aronson, Berl. klin. Woch., 1902; ibid., 1903.

2 Marmorek, Berl. klin. Woch., 1902.

${ }^{3}$ Howard and Perkins, Jour. Med. Res., 1901, N. S., i.

${ }^{4}$ Schottmüller, Münch. med. Woch., xxi, 1903. 
Morphologically, though showing a marked tendency to form chains, on solid media it often appears in the diplococcus form. It is enclosed in an extensive capsule, which appears with much regularity and persistence. Though very similar in appearance, therefore, to pneumococci, these bacteria do not appear in the typical lancet shape. Upon solid media they show a tendency to grow in transparent moist masses. The regularity with which this microorganism ferments inulin medium, and its agglutinative characters, make it probable that it is more accurate to place it with the group of pneumococci than with that of streptococci. ${ }^{1}$ (For agglutinations see section on pneumococcus agglutination, p. 364.)

\section{STREPTOCOCCI AND RHEUMATISM}

In 1910 Poynton and Paine ${ }^{2}$ described a diplococcus which they obtained from eight cases of acute rheumatic fever and with which they were able to produce lesions in rabbits which they considered typical of rheumatism. The organism was recovered from the blood, the pericardial fluid, or the tonsil of their patients. They described a minute Gram-negative diplococcus growing best in acid media and under anaërobic conditions, but capable of growth on the surface of ordinary media. Many investigators have attempted to confirm their work, but with negative results for the most part, though some have found streptococci and diplococci from rheumatic lesions. Recently Rosenow ${ }^{3}$ has reported the isolation of a streptococcus from the joints of seven cases of articular rheumatism. He was also able to produce nonsuppurative arthritis, endocarditis, and pericarditis in rabbits with these cultures. He describes them as intermediate in character between the streptococcus viridans and streptococcus hemolyticus.

More recently Rosenow ${ }^{4}$ has reported the production of gastric ulcers in rabbits and dogs with streptococci of a certain grade of virulence. He has also obtained streptococci from human peptic ulcers which showed a remarkable "affinity" for the gastric mucous membranes of experimental animals.

${ }^{1}$ Hiss, Jour. Exp. Med., 1905; Buerger, Cent. f. Bakt., I, xli, 1906.

${ }^{2}$ Poynton and Paine, Lancet, 1900, ii, 861, 932.

${ }^{3}$ Rosenow, Jour. A. M. A., 1913, lx, 1223.

${ }^{4}$ Rosenow, Jour. A. M. A., 1913, lxi, 1947, 2007. 


\section{CHAPTER XXIII}

\section{DIPLOCOCCUS PNEUMONIÆ}

\section{(Pneumococcus, Diplococcus lancenlatus)}

THE opinion that lobar pneumonia is an infectious disease was held by many far-sighted clinicians long before the actual bacteriological facts had been ascertained. This idea, so well founded upon the nature of the clinical course of the disease, with its violent onset and equally rapid defervescence, led many of the earlier bacteriologists to make it the subject of their investigations-a subject made doubly difficult by the abundant bacterial flora found normally in the upper respiratory passages, and by the fact, which is now recognized, that lobar and other pneumonias are by no means always caused by one and the same microorganisms.

Cocci of various descriptions and cultural characteristics were isolated from pneumonia cases by Klebs, ${ }^{1}$ Koch, ${ }^{2}$ Günther, ${ }^{3}$ Talamon, ${ }^{4}$ and many others, which, however, owing to the insufficient differential methods at the command of these investigators, can not positively be identified with the microorganism now known to us as Diplococcus pneumonix or the pneumococcus. Although thus unsuccessful as to their initial object, these early investigations were by no means futile, in that they gave valuable information regarding the manifold bacterial factors involved in acute pulmonary disease and incidentally led to the discovery by Friedländer ${ }^{5}$ of B. mucosus capsulatus.

Communications upon lance-shaped cocci found in saliva, and capable of producing septicemia in rabbits, were published almost simultaneously by Sternberg ${ }^{6}$ and by Pasteur ${ }^{7}$ in 1880 . These workers

${ }_{1}$ Klebs, Arch. f. exp. Path., 1873.

${ }^{2}$ Koch, Mitt. a. d. kais. Gesundheitsamt, Bd. 1.

${ }^{3}$ Günther, Deut. med. Woch., 1882.

4 Talamon, Progr. méd., 1883.

5 Friedländer, Virchow's Arch., Ixxxvii.

- Sternberg, Nat. Board of Health Bull., 1881.

- Pasteur, Bull. de l'acad. de méd., 1881. 
beyond reasonable doubt were dealing with the true pneumococcus, but did not in any way associate the microorganisms they described with lobar pneumonia. The solution of this problem was reserved for the labors of A. Fränkel ${ }^{1}$ and Weichselbaum ${ }^{2}$ who published their results, independently of each other, in 1886, demonstrating beyond question that the pneumococcus is the etiological factor in a large majority of cases of lobar pneumonia.

Morphology and Staining.-The morphology of the pneumococcus is, in general, one of the most valuable guides to its identity.

When typical, the pneumococcus is a rather large, lancet-shaped coccus, occurring in pairs, and surrounded by a definite and often wide capsule, which usually includes the two approximated cocci without a definite indentation opposite their lines of division. The pneumococci may, however, occur singly or in short chains, and even fairly long chains are not infrequently met with under artificial cultural conditions. This may be chiefly due to the cultural conditions or may be a prominent characteristic of certain strains. Apparently the capsules of organisms making up the chains are continuous; wavy indentations are usually present, however, in the capsule of chains, and at times distinct divisions are observed.

The chief variations from the typical morphology consist either in the assumption of a more distinctly spherical coccus type, or in an elongation approximating the bacillary form. Under certain conditions of artificial cultivation a distinct flattening of the organisms, particularly of those making up chains, may be seen, and even the impression of a longitudinal line of division, characteristic of many streptococcus cultures, is not infrequently gained.

The capsules under certain conditions, especially in artificial media, may be absent or not demonstrable, and in certain strains capsules apparently may not be present under any conditions. Practically any of the described variations may dominate one and the same culture under different or even apparently the same conditions of cultivation, and all grades may occur in capsule development, from its typical formation through all variations, to its total and apparently permanent absence.

The presence or absence of capsules depends, to a large extent, upon the previous environment of the pneumococci under observation. The most favorable conditions for the development or preservation of the pneumococcus capsule are found in the body fluids of man and animals

${ }^{1}$ A. Fränkel, Zeit. f. klin. Med., x, 1886.

${ }^{2}$ Weichselbaum, Med. Jahrbücher, Wien, 1886. 
suffering from pneumococcus infection. For instance, capsules may be demonstrated with ease by the usual capsule-staining methods in the blood, serum, and inflammatory exudate of the infected rabbit and white mouse. Capsules may be equally well marked in the fresh sputum of pneumonia patients, especially in the early stages of the disease and in the exudate accompanying such pneumococcus infections as meningitis, otitis media, and empyema. In sputum and the exudates of various localized infections, the organisms are, however, frequently degenerated or under chemical conditions unfavorable for capsule staining, and satisfactory results are not then easily obtained. The

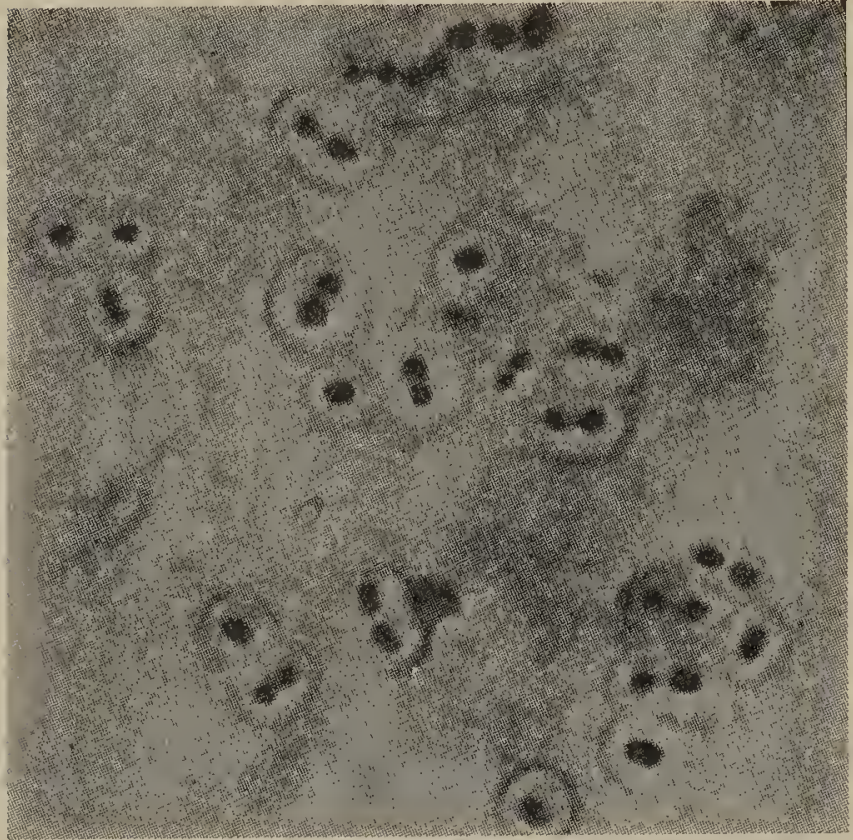

Fig. 75.-Pneumococci, Grown on Loeriler's Serum. (Capsule stain by gentian-violet-potassium-carbonate method.)

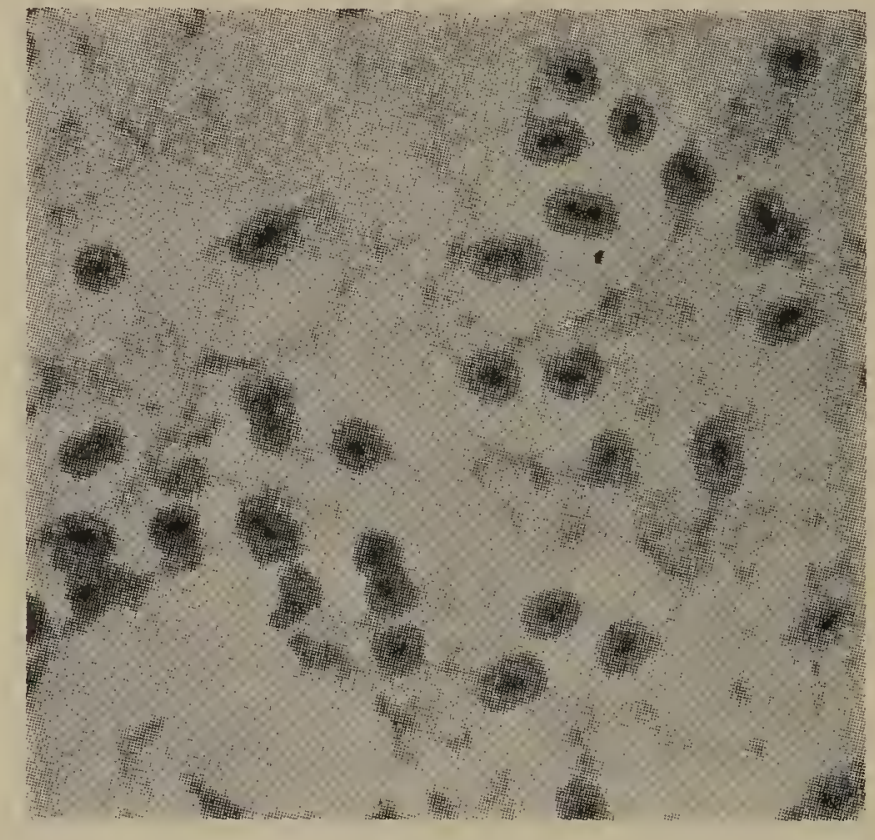

Fig. 76.-PNeUmococCI, FROM RABbit's Heart Blood. (Capsule stain by copper-sulphate method.)

same is often true of the scrapings from lungs of patients dead of pneumonia, even in the stage of red hepatization.

In artificial cultivation, if the nutrient medium is not milk or does not contain serum, capsules can not usually be demonstrated by the ordinary methods of preparing and staining. Capsules may, however, with much regularity be demonstrated on pneumococci, in agar, broth, or on almost all, if not all, artificial media, irrespective of the length of time the organisms have been under artificial cultivation if beef or rabbit serum is used as the diluent, when they are spread on the cover-glass for staining. ${ }^{1}$

The pneumococcus is non-motile and possesses no flagella. Spores are not formed. Swollen and irregular involution forms are common in cultures more than a day old.

${ }^{1}$ Hiss, Cent. f. Bakt., xxxi, 1902; Jour. Exp. Med., vi, 1905. 
The pneumococcus is stained readily with all the usual aqueous anilin dyes. Stained by the method of Gram, it is not decolorized. Special methods of staining have been devised for demonstration of the capsule. The ones most generally used are the glacial acetic-acid method of Welch ${ }^{1}$ and the copper-sulphate method of Hiss. ${ }^{2}$ More recently Buerger ${ }^{3}$ has devised a more complicated method for staining capsules, for which he claims differential value. (For methods see section on Technique, p. 98.)

For simple staining of pneumococci in tissue sections, the GramWeigert technique is excellent. For demonstration of the capsules in tissue sections, Wadsworth ${ }^{4}$ has described a simple method.

Cultivation and Isolation.-The pneumococcus being more strictly parasitic than many other bacteria, presents greater difficulties in its cultivation. On meat-extract media growth does not take place with regularity. On those media, however, which have beef or veal infusion for their basis, growth can be obtained with considerable regularity, although such growth may be sparse and delicate.

Growth takes place most regularly at a temperature of $37.5^{\circ} \mathrm{C}$. Development does not usually occur below $25^{\circ}$ nor above $41^{\circ} \mathrm{C} .^{5}$ At crdinary room temperature, $18-22^{\circ} \mathrm{C}$., the temperature used for gelatin cultivation, growth either does not take place at all or is exceedingly slow and unenergetic. Aërobic and anaërobic conditions are equally favorable for pneumococcus cultivation, there being very little difference in speed or extent of growth along the course of deep stab cultures in favorable media. The most favorable reaction of media for the cultivation of this microorganism is neutrality or moderate alkalinity (twotenths to eight-tenths per cent alkalinity to phenolphthalein). Slight acidity, however, if not exceeding eight-tenths per cent, does not materially hamper development.

The growth of pneumococci on all media may be considerably enhanced by the addition to these media of animal or human serum or whole blood. Additional substances which, among others, unquestionably have a favorable influence upon pneumococcus growth, are glucose, nutrose, and glycerin. The addition of the latter substances to the media, however, probably because of acid formation, hastens the death

1 Welch, Johns Hopk. Hosp. Bull., xiii, 1892.

${ }^{2}$ Hiss, Cent. f. Bakt., xxxi, 1902; Jour. Exp. Med., vi, 1905.

3 Buerger, Medical News, lxxxviii, 1904.

4 Wadsworth, "Studies by the Pupils of W. T. Sedgwick," Chicago, 1896.

5 A. Frankel, Dent. med. Woch., xiii, 1886. 
of pneumococcus cultures. An increase of the amount of pepton used for the preparation of media is desirable for the cultivation of this microorganism; two to four per cent of pepton may be found advantageous.

In suitably alkaline, nutrient broth, growth is rapid, and within twenty-four hours leads to slight clouding of the fluid. 'This clouding, as a rule, eventually disappears as the microorganisms, sinking to the bottom of the tube or disintegrating, leave the fluid more or less clear. In broth, pneumococci have a tendency to form short chains. When glucose has been added to the broth, growth is more rapid and profuse, but considerable acid formation causes the cultures to die out rapidly. It is possible, however, to employ glucose as a growth-enhancing element in broth cultures without interfering with the viability of the cultures by adding small quantities (one per cent) of sterile, powdered calcium carbonate. This method of cultivation in broth is especially adapted to the production of mass cultures for purposes of immunization or agglutination. ${ }^{1}$ The addition of ascitic fluid or blood serum to broth, in the proportion of one to three, makes an extremely favorable medium in which growth is rapid and profuse.

Upon agar plates, pneumococcus growth is not unlike that of streptococcus. The colonies are small, round, and slightly more transparent than those of the streptococci. They appear more moist than streptococcus colonies and often are more flat. Microscopically examined, the colonies are finely granular, with dark centers and slightly corrugated lighter-colored peripheral areas. Under high magnification no such intertwining convolutions can be seen as those noticed under similar magnification in streptococcus cultures. The addition of animal albumin to agar results in the more rapid development, larger size, and deeper opacity of the colonies.

Agar stab cultures show growth within twenty-four to thirty-six hours, which takes place with equal thickness along the entire course of the stab. There is nothing distinctive in these cultures to differentiate them from similar streptococcus cultures.

In gelatin plate and stab cultures at $22^{\circ} \mathrm{C}$., growth, as a rule, does not take place. This, however, is not true of all races of pneumococci. Occasionally strains are met with which will grow fairly abundantly in gelatin at a temperature of $22^{\circ} \mathrm{C}$. When the gelatin is rendered sufficiently firm to bear $25^{\circ}$ to $26^{\circ} \mathrm{C}$. without melting, growth appears 
slowly and sparsely as minute, grayish-white, transparent colonies. The gelatin is not liquefied by the organisms.

Growth upon milk is rapid and profuse, resulting usually in the production of acid and consequent, coagulation of the medium. Exceptionally, races are encountered in which this function is suppressed and coagulation in milk is absent or long delayed.

Upon potato, a thin, grayish, moist growth occurs, hardly visible to the naked eye, and often indistinguishable from an increased moisture on the surface of the medium.

Upon Loeffler's coagulated blood serum, the pneumococcus develops into moist, watery, discrete colonies which tend to disappear by a drying out of the colonies after some days, differing in this from streptococcus colonics, which, though also discrete, are usually more opaque and whiter in appearance than those of the pneumococcus and remain unchanged for a longer time. This medium, as, will be seen, is useful in differentiating pneumococci from the so-called Streptococcus mucosus.

Upon a medium made up of mixtures of whole rabbit's blood and agar, the pneumococcus grows with considerable luxuriance, and forms, after four or five days or longer, thick black surface colonies, not unlike small sun blisters on red paint. These colonies are easily distinguished from the hemolyzing colonies of most streptococci, and are in this respect of considerable differential value. ${ }^{1}$

Special media of various descriptions have been devised for pneumococcus cultivation. Thus, Guarnieri ${ }^{2}$ has recommended a medium with a pepton-beef-infusion basis rendered semisolid by mixtures of agar and high percentages of gelatin. A modification of this medium has been described by Welch ${ }^{3}$ and has been much employed in work with the pneumococcus. Cultivation within eggs and upon egg media ${ }^{4}$ has been advised and used by various observer's. Wadsworth ${ }^{5}$ has recommended a medium composed of ascitic fluid to which three-tenths per cent agar has been added-sufficient to give a soft jelly-like consistency to the medium. He observed prolonged viability and the preservation of the virulence in this medium.

For the purpose of differentiating pneumococci from streptococci,

${ }^{1}$ Hiss, loc. cit.

${ }_{2}$ Guarnieri, Att. dell' Acad. di Roma, 1883.

3 Welch, Johns Hopk. Hosp. Bull., iii, 1892.

4 Sclavo, Riv. d'Igiene, 1894.

5 Wadsworth, Proc. N. Y. Path. Soc., 1903. 
Hiss ' devised a medium composed of beef serum one part, and distilled water two parts, to which is added one per cent of inulin (c. p.), and enough litmus to render the medium a clear, transparent blue. By fermentation of the inulin the pneumococcus acidifies this mixture, rendering the litmus red and causing coagulation of the serum. Streptococei do not ferment inulin and the medium remains blue and fluid. (For the preparation of special media, see section on Media, p. 132.)

For the isolation of pneumococci from mixed cultures or from material containing other species, such as sputum, the most reliable method is to make surface smears of the material containing the bacteria upon plates of neutral glucose-agar or preferably of glucose-serumagar. According to the number of bacteria present in the infected material from which the isolation is to be made, it may be smeared directly upon the plate, or diluted with sterile broth or salt solution before planting. After incubation for twenty-four hours, the pneumococcus colonies are easily differentiated from all but those of streptococcus. With practice, however, they may be distinguished from these also, by their smoother edges and greater transparency and flatness. Pour-plates, prepared in the usual way, can also be made but are less useful since deep colonies of pneumococei show no distinctive features.

Another method for pneumococcus isolation, useful to eliminate other bacteria, is that of animal inoculation. White mice are inoculated with 0.5 to 1 c.c. of the infectious material by subcutaneous injection, made most easily at the base of the tail. If virulent pneumococci are present in the inoculated material, death from septicemia usually occurs within twenty-four to forty-eight hours. Surface smears should be made on glucose-agar plates with the heart's blood. By this method pure cultures may usually be obtained directly from the mouse blood.

Resistance.-Kept upon artificial media, the viability of the pneumococcus is not great. Cultures upon agar or bouillon should, to be kept alive, be transplanted every third or fourth day, if the cultures are kept at incubator temperatures. In all media in which rapid acid formation takes place, such as glucose media, the death of cultures may occur even more rapidly. In media containing albumin and of a proper degree of alkalinity, preservation for one or even two weeks is possible. The longer the particular race has been kept upon artificial media, the

Hiss, Jour. Exp. Med., vi, 1905. 
more profuse is its growth, and the greater its viability, both qualities going hand in hand with its diminishing parasitism. The length of life of these bacteria may be much increased by their preservation at a low temperature, in the dark, and by the exclusion of air. By far the best medium for keeping pneumococci alive is the previously mentioned calcium-carbonate-infusion broth. Grown in this medium and kept in the ice-chest, cultures may often remain alive for months.

In sputum the viability of pneumococci seems far to exceed that observed upon culture media. The studies of Guarnieri, ${ }^{1}$ BordoniUffreduzzi, ${ }^{2}$ and others have shown that pneumococci slowly dried in sputum may remain not only alive but virulent, after from one to four months, when protected from light; and as long as nineteen days when exposed to diffused light at room temperature. Experiments by Ottolenghi ${ }^{3}$ have, in the main, confirmed these results; the virulence seems, in Ottolenghi's experiments, to have become considerably attenuated before the death of the cocci occurred. More recent studies by Wood, ${ }^{4}$ whose attention was focused chiefly upon pneumococcus viability in finely divided sputum-in a condition, in other words, in which inhalation transmission would be possible-have shown that pneumococci in finely sprayed sputum survive for only about one and one-half hours, under ordinary conditions of light and temperature. Exposed to strong sunlight pneumococci die off within an hour, often within a few minutes.

Low temperatures are well borne by pneumococci, temperatures slightly above zero being even conducive to the prolongation of life and the preservation of virulence.

The resistance of the pneumococcus to heat, on the other hand, is low, $52^{\circ} \mathrm{C}$. destroying it within ten minutes. ${ }^{5}$ To germicidal agents, carbolic acid, bichlorid of mercury, permanganate of potassium, etc., the pneumococcus is extremely sensitive, being destroyed by weak solutions after short exposures.

The disinfection of sputum, offering considerable difficulties because of the protective coating of the secretions about the bacteria, has been recently made the subject of a spec al study by Wadsworth. ${ }^{6}$ The conclusions reached by this writer indicate that pneumococci in exudates

${ }^{1}$ Guarnieri, Att. della R. Acad. Med. di Roma, iv, 1888.

${ }_{2}$ Bordoni-Uffreduzzi, Arch. p. 1. sc. med., xv, 1891.

${ }^{3}$ Ottolenghi, Cent. f. Bakt., xxv, 1889.

4 Wood, Jour. Exp. Med., vii, 1905.

5 Sternberg, Cent. f. Bakt., xii, 1891.

- Wadsworth, Jour. Inf. Diseases, iii, 1906. 
are most rapidly destroyed by twenty per cent alcohol, other and stronger disinfectants being less efficient, probably because of slighter powers of diffusion.

Virulence and Pathogenicity.-The virulence of pneumococci is subject to much variation, depending largely upon the length of time during which the microorganism has been cultivated under artificial conditions. It has been mentioned above that under certain conditions - such as those prevailing in dried sputum or blood ${ }^{1}$ - the virulence of pneumococci may be preserved for several weeks. Ordinarily, however, the virulence diminishes gradually as the cocci adapt themselves more saprophytically to life upon artificial media. Upon media containing animal albumin, such as ascitic fluid or blood agar, this attenuation is less rapid than upon the simple meat-infusion preparations.

In the blood of rabbits dead of a pneumococcus infection, taken directly into sterilized tubes, sealed and kept in the dark, Foa has been able to preserve the virulence of pneumococci for as long as forty-five days. Whether or not the virulence of pneumococci is attenuated by sojourn within the human body during disease is a question much discussed but hardly settled. It is a matter of fact, however, that many pneumococci obtained by blood culture from more or less chronic cases of pneumococcus septicemia fail to kill susceptible test animals, even when injected in considerable doses. The attenuation of virulent pneumococci on artificial media may be hastened, according to Fränkel, ${ }^{3}$ by cultivation of the organism at or above a temperature of $41^{\circ} \mathrm{C}$.

Freshly isolated from the human saliva or pneumonic lesions, the differences in virulence between various strains of pneumococci are not very marked, almost all such strains showing considerable pathogenic powers toward the usual test animals.

The virulence of attenuated cultures may be rapidly enhanced by the passage of the organisms through the bodies of susceptible animals. The extreme virulence of some of these pneumococcus strains may be illustrated by citing the experiments of Eyre and Washburn ${ }^{4}$ who possessed cultures of which one millionth of a loopful would kill a mouse within four days.

Among the domestic animals those most susceptible to pneumococcus infection are white mice and rabbits. Guinea-pigs, dogs, rats, and cats

${ }^{1}$ Guarnieri, loc. cit.

${ }^{2}$ Foa, Zeit. f. Hyg., iv, 1888.

3 Fränkel, Deut. med. Woch., 13, 1886.

${ }^{4}$ Eyre and Washburn, Jour, of Path, and Bac., v. 
are more resistant, but still may be infected with large doses. Young animals are usually more susceptible than adults. Birds are practically immune.

The results of pneumococcus inoculation into susceptible animals vary according to the size of the dose, the virulence of the introduced bacteria, the mode of administration, and the susceptibility of the subject of the inoculation. Subcutaneous inoculation of virulent pneumococci into mice and rabbits usually results in an edematous, often fibrinous exudation at the point of inoculation, which, in all cases $\mathrm{n}$ which the dose given has not been extremely small, leads to septicemia and death within twenty-four to seventy-two or more hours. When the dose has been extremely small or the culture unusually attenuated, a localized abscess may be the only result. Intravenous inoculation is usually more rapidly fatal in these animals than the subcutaneous method. Intraperitoneal inoculation in rabbits results in the formation of a rapidly spreading peritonitis in which the inflammatory exudate in many cases exhibits differences from similar exudates produced by the streptococcus. Pneumococcus exudates are apt to be thicker, to be accompanied by a deposit of fibrin, and to lack the transparent red color so often caused by the hemolyzing streptococci. With very virulent strains, these differences are less marked. In almost all of these infections death is preceded by septicemia and the microorganisms can be recovered from the heart's blood of the victims.

After such infections, the animals exhibit a rise of temperature, at times visible depression, and, rarely, diarrhea. General hyperemia of the organs with secondary effusions in the pleural cavities and often hemorrhages upon the serous surfaces may be found at autopsy.

The production in animals of lesions comparable to the lobar pneumonia of human subjects has been the aim of many investigators. Wadsworth, ${ }^{1}$ recognizing that such lesions probably depended upon the partial immunity which enabled the infected subjects to localize the pneumococcus processes in the lungs after infection by way of the respiratory passages, succeeded in producing typical lobar pneumonia in rabbits by partially immunizing these animals and inoculating them intratracheally with pneumococci of varying virulence. By this method he actually carried out, for the first time, Koch's postulates in regard to lobar pneumonia.

In man, the most frequent lesion produced by the pneumococcus is

${ }^{1}$ Warlsworth, Amer. Jour. Med. Sci., May, 1904. 
acute lobar pneumonia. About ninety per cent of all cases of this disease are caused by the pneumococcus, ${ }^{1}$ the remainder being due to streptococci, influenza bacilli, Friedländer's bacilli, and exceptionally to other microorganisms. Lobular pneumonia is caused by the pneumococcus with almost equal regularity. During the course of these diseases the cocci are found in large numbers within the pulmonary alveoli, and in the capillaries and lymph vessels of the lung. Whether or not the pneumococci enter the blood stream in all these cases is a question not yet definitely settled. Fränkel ${ }^{2}$ states it as his belief that in most, if not in all, cases, the diplococci at some time during the disease could be found in the circulating blood. Prochaska in a study of ten unselected cases obtained positive blood cultures in every one of them. A review of the literature upon the question indicates positive bloodculture findings in certainly over twenty-five per cent of the cases.

In complications of pneumonia, pneumococci are found usually in the pleura where they may cause a simple dry pleurisy or even empyema. Less frequently they may cause pericarditis and endocarditis. Meningitis may be caused by pneumococci, either secondarily to preumonia or independently. Such cases are extremely grave, almost invariably ending in death. Other lesions which may be caused by pneumococci, either as post-pneumonic processes or without previous pneumonia, are otitis media, osteomyelitis, and arthritis. Cases of pneumococcus peritonitis occur sometimes secondary to appendicular inflammations, occasionally without traceable portal of entry. Severe catarrhal conjunctivitis may be caused by these diplococci, usually during the course of a pneumonia. Ulcerative endocarditis with pneumococcus septicemia, apparently independent of a pulmonary lesion, is not infrequent.

Toxic Products of the Pneumococcus.-Our knowledge of pneumococcus poisons is still very imperfect. Attempts to obtain soluble toxins by the filtration of cultures have been practically unsuccessful in the hands of many careful workers. G. and F. Klemperer, ${ }^{3}$ Mennes, ${ }^{4}$ Pane, ${ }^{5}$ Foa and Carbone, ${ }^{6}$ and others failed to obtain pneumococcus filtrates of any marked degree of toxicity, though working with highly virulent

1 Netter, Compt. rend. de la soc. de biol., 1890.

2 Fränkel, "v. Leyden Festschr.," 1902.

${ }^{3} G$. and $F$. Klemperer, Berl. klin. Woch., xxxiv and $\mathrm{xxxv}, 1891$.

${ }^{4}$ Mennes, Zeit. f. Hyg., xxv, 1897.

- Pane, Rif. med., xxi, 1898.

- Foa und Carbone, Cent. f. Bakt., x, 1899. 
strains. Attempts to demonstrate by the production of antitoxin the specific nature of the feeble poisons obtained have also met with failure. Isaeff, ${ }^{1}$ though confirming the feeble toxicity of fluid cultures, made the interesting observation that a filtrate of the blood of pneu:nococcus-infected rabbits contained a poison often more potent than that obtained in culture filtrates. Carnot and Fournier ${ }^{2}$ obtained a poison of distinct though feeble potency by dialysis of pneumococcus cultures.

The general failure, however, to procure strong soluble poisons from cultures, gives weight to the assumption that the most potent toxic products of pneumococci are in the nature of endotoxins and closely bound to the cell-bodies themselves. This assumption is borne out by the more recent experiments of Macfadyen. ${ }^{3}$ This author obtained acutely poisonous substances from pneumococci by trituration of the organisms after freezing, and extracting them with a one $1: 1,000$ caustic potash solution. With the filtrates of these extracts he was able to cause rapid death in rabbits and guinea-pigs by the use of doses not exceeding 0.5 to 1 c.c. He found, furthermore, a striking parallelism between the degree of toxicity and the virulence of the extracted culture.

Immunization.--Recovery from a spontaneous pneumococcus infection confers immunity for only a short period. Two and three attacks of lobar pneumonia in the same individual are not unusual, and it is uncertain whether even a temporary immunity is acquired in such infections. Active immunization of laboratory animals may be carried out by various methods. The method usually followed is to begin by injecting attenuated ${ }^{4}$ or dead bacteria or bacterial extracts. Subsequent injections are then made with gradually increasing doses of living, virulent microorganisms. Great care in increasing the dosage should be exercised since the loss of an animal after two or three weeks' treatment by a carelessly high dose of pneumococci is not unusual. Wadsworth has recommended the following method for preparing pneumococci for the first injections in immunizing rabbits. Freshly grown pneumococcus cultures are centrifugalized, and the supernatant bouillon is thoroughly decanted. To the pneumococcic sediment a definite quantity of concentrated salt solution is added, and the mixture is allowed to stand over night. At the end of this time, the pneumococci are dead and considerable destruction of the cell-bodies

1 Isaeff, Ann. de l'inst. Pasteur, vii, 1893.

${ }^{2}$ Carnot et Fournier, Arch. de méd. expér., 1900.

3 Macfadyen, Brit. Med. Jour., ii, 1906.

- Radziewsky, Zeit. f. Hyg., xxxvii, 1901; Neufeld, Zeit. f. Hyg., xi, 1902. 
has taken place. Dilution with water until the solution equals 0.85 per cent $\mathrm{NaCl}$ now prepares the emulsion for inoculation. Whichever of the various methods is adopted, the intervals of injection should not be shorter than a week, preferably ten days. The animals so immunized will at the end of six or more weeks withstand an inoculation with many times the fatal dose of virulent pneumococci. The sera of animals immunized with pneumococei contain active bacteriolytic and bactericidal substances, easily demonstrable in vivo and in vitro.

Specific agglutinins in pneumococcus immune sera were first thoroughly studied by Neufeld ${ }^{1}$ and since then have been made the subject of extensive studies by Wadsworth, ${ }^{2}$ Hiss, ${ }^{3}$ and many others. In the sera of normal animals and man, pneumococci are rarely agglutinated in dilutions higher than one in ten. In the serum of patients suffering from lobar pneumonia, pneumococci agglutinate in dilutions ranging anywhere from one in ten to one in fifty. In the sera of immunized rabbits, readings up to one in 800 are not rare. Such specific agglutinating sera are most reliable in differentiating between pneumococci and closely allied bacteria and in identifying all pneumococci.

The table on page 365 illustrates this uniformly high agglutinative power of various pneumococcus-immune sera upon several races of this microorganism, and shows the value of such sera for biological differentiation. The table, furthermore, records the peculiar fact that pneumococci are agglutinated in high dilution by sera obtained by immunization with Streptococcus mucosus, a fact which argues strongly in favor of classifying Streptococcus mucosus more intimately with the pneumococci than with the Streptococci of the pyogenes group.

To overcome the difficuities often attending agglutination tests with pneumococci, Wadsworth ${ }^{4}$ has proposed centrifugalizing young broth cultures and shaking up the sediment with small quantities of isotonic salt solution. Hiss recommends ${ }^{5}$ cultivation in glucosecalcium-carbonate broth in small flasks containing 100 to 150 c.c each. After three or four days at $37^{\circ} \mathrm{C}$., the growth is usually at its optimum for agglutination work. The flasks should be thoroughly shaken at least once in twenty-four hours. About one hour before use the flasks are again shaken and the calcium carbonate and larger clumps are allowed to settle.

1 Neufeld, loc. cit.

${ }^{2}$ Wadsworth, loc. cit.

${ }^{3}$ Hiss, Jour. Exp. Med., vii, 1905.

${ }^{4}$ Wadsworth, Jour. Med. Res., x, 1905.

${ }^{5} \mathrm{Hiss}$, loc. cit. 
Precipitins have been demonstrated in pneumococcus immune sera by Neufeld, ${ }^{1}$ Wadsworth, ${ }^{2}$ and others. Neufeld obtained precipitates with pneumococcus cultures in which lysis had been produced by the addition of bile. He found that normal rabbit's bile added to pneu-

\begin{tabular}{|c|c|c|c|c|c|c|}
\hline \multirow[b]{2}{*}{ Organism. } & \multicolumn{6}{|c|}{ IMMUNE SERA 3} \\
\hline & $\begin{array}{l}\text { Pneum. } \\
\quad 1 .\end{array}$ & $\begin{array}{l}\text { Pneum. } \\
3 .\end{array}$ & $\begin{array}{l}\text { Pneum. } \\
\text { 23. }\end{array}$ & $\begin{array}{l}\text { Strepto- } \\
\text { coccus } \\
\text { mucosus } \\
\quad 7 .\end{array}$ & $\begin{array}{l}\text { Strepto- } \\
\text { coccus } \\
\text { mucosus } \\
7 \text { a. }\end{array}$ & $\begin{array}{c}\text { Strepto- } \\
\text { coccus } \\
\text { pyo- } \\
\text { genes. }\end{array}$ \\
\hline Pneumo. 1.... & $400-800$ & $200-800$ & $400-800$ & $200-800$ & 400 & $0-100$ \\
\hline Pneumo. 3.... & & $400-800$ & & & $200-800$ & $0-100$ \\
\hline Pneumo. 23... & & $100-800$ & $200-800$ & $100-200$ & & \\
\hline Pneumo. 45... & & $400-800$ & 600-200 & $200-800$ & $100-400$ & \\
\hline Pneum. E 1.. & $\ldots$ & $100-800$ & & $\ldots$ & $100-200$ & \\
\hline Pneum. E 32. & & $200-800$ & & $100+$ & & \\
\hline Pneum. E 55.. & $100-400$ & $\ldots \ldots \ldots$ & $400-800$ & $200-400$ & & \\
\hline Pneum. N 7. & $200-800$ & & 800 & $200-800$ & $200-800$ & \\
\hline Pneum. N 17. & $200-800$ & $\ldots \ldots \ldots$ & 800 & $200-800$ & $200-800$ & \\
\hline $\begin{array}{r}\text { Streptococcus } \\
\text { pyog. } 1 \ldots\end{array}$ & & $200-800$ & & & & $800-6400$ \\
\hline $\begin{array}{l}\text { Streptococcus } \\
\text { mucos. } 7 \ldots\end{array}$ & $0-10$ & $0-10$ & & $10-100$ & $10-200$ & $0-50$ \\
\hline $\begin{array}{l}\text { Streptococcus } \\
\text { mucos. } 22 .\end{array}$ & $0-10$ & $0-10$ & & $10-50$ & $0-50$ & \\
\hline
\end{tabular}

mococcus cultures (one drop to 1 c.c. of culture) caused the cultures to become perfectly clear and transparent, and no longer contain demonstrable pneumococcus cell bodies. The addition of pneumococcus immune sera to such cultures produced precipitates. Wadsworth ob- 
taned similar precipitates with pneumococcus cultures treated with concentrated salt solution as described above (see p. 363).

Pneumococcus immune sera also contain specific phagocytosisstimulating substances. The first investigators to describe these substances for pneumococcus sera, Neufeld and Rimpau, ${ }^{1}$ separated them from the opsonins on the basis of their greater thermo-stability and named them bacteriotropins. It is doubtful whether such differentiation is tenable. Great importance for pneumococcus immunity is attributed to these bodies by some authors. This question has been studied more recently by Park and Williams, ${ }^{2}$ however, who were unable to find distinct parallelism between opsonic power and the protective value of a serum.

Passive immunization with pneumococcus immune sera has been extensively attempted. Washburn, ${ }^{3}$ Mennes, ${ }^{4}$ Pane, ${ }^{5}$ and many others have succeeded in protecting subsequently infected animals by treatment with such sera. Neufeld and Haendel in Germany, and in this country Cole, have recently used pneumococcus immune sera extensively in the treatment of man. The serum, given intravenously, seems to exert a favorable influence, and while unfinished, the work is exceedingly encouraging. Encouraging results were obtained by Hiss in treatment of pneumococcus infection in animals and by Hiss and Zinsser ${ }^{6}$ in treatment of pneumonia in man with aqueous leucocyte extracts.

Experiments both with passive immunization and with agglutination show that all pneumococci do not react alike. Sera which will protect white mice against the homologous strain react similarly to some other strains, but not to all. Neufeld and Haendel ${ }^{7}$ as a result of protection experiments concluded that the majority of pneumococci belonged to one type, but that a number of other types could be recognized. On the basis of agglutination and protection tests Dochez and Gillespie $^{8}$ describe three distinct races of pneumococci and a fourth group of heterogeneous strains. One of these definite types occurred in nearly half of their sixty-two cases of lobar pneumonia.

${ }^{1}$ Neufeld und Rimpau, Deut. med. Woch., 1904.

${ }^{2}$ Park and Williams, Jour. Exp. Med., vii, 1905.

${ }^{3}$ Washburn, Brit. Med. Jour., 1897.

"Mennes, Zeit. f. Hyg., 1897.

${ }^{5}$ Pane, Rif. med., 1897.

${ }^{6}$ Hiss and Zinsser, Jour. Med. Res., xix, 1908.

${ }^{7}$ Neufeld and Haendel, Arb. aus dem Kais. Gesunds., 1910, xxxiv, 293.

${ }^{8}$ Dochez and Gillespie, J. A. M. A., 1913, lxi, 727. 
Differentiation of Pneumococcus from Streptococcus.-Pneumococci and streptococci which do not differ in morphology from their classic types can usually be differentiated from each other and identified by their morphological characters without difficulty; but it is equally true that certain cultures of these organisms, either at the time of their isolation or after cultivation on artificial media, approach the type of the other so closely that it may be impossible to identify them by their molphology alone. When such morphological variations occur there are no constant and distinctive cultural or pathogenic characters as yet demonstrated which can with certainty be depended upon as distinguishing marks between these organisms.

This lack of distinct cultural differences between pneumococci and streptococci has not infrequently led to confusion, and that uncertainty should exist and mistakes be made in identification is not surprising when one considers the characters usually depended upon to distinguish pneumococci from streptococci. Chief among these, as has just been implied, are the morphological features which are, in the case of pneumococci, a slightly lancet or elongated form rather than the more typical coccus form characteristic of the streptococci, and an arrangement of such cocci in pairs rather than in chains; added to these features is the possession of a more or less well-defined capsule. All of these characters are subject to variation or may be absent. Compared with the morphological, the cultural characters are of minor importance and are variable. They consist in a more moist and flatter appearance of the pneumococcus colonies on coagulated blood serum and on agar, and in the usual inability of the freshly isolated pneumococcus to develop readily or at all on gelatin at temperatures below $22^{\circ} \mathrm{C}$.

The distinctness of the capsule of the pneumococcus in the body fluids of man and animals, and at times when this organism is cultivated artificially on blood serum, milk, or serum agar, has really been depended upon as the chief distinguishing and diagnostic character.

Nevertheless, from time to time, instances have been reported of distinct capsule formation by organisms which had either been previously identified as Streptococcus pyogenes, or at the time of their isolation could not be definitely identified by their discoverers as belonging to either this group or to the pneumococci, but were considered intermediate in their character. ${ }^{1}$

${ }^{1}$ Brief Description of Organisms Reported as Capsulated Streptococei.-Bordet (Bordet, Ann. de l'inst. Pasteur, 1897, xi, p. 177), working with an organism previously 
There are occasions, then, both within the animal body and in artificial cultivations, when it is practically impossible to distinguish definitely between some races of pneumococci and races of streptococci. This difficulty is especially heightened when the pneumococcus has become non-virulent, and at the same time no very typical morphology or capsule formation is to be determined and a tendency to chain-formation is marked. Cultures of pneumococci in such condition can not readily be distinguished morphologically from streptococcus cultures.

Under these circumstances recourse must be had to a careful biological study of the organism in question. The following are the criteria mainly relied upon at present for the differentiation of these two groups.

identified as Streptococcus pyogenes, described such capsule formation occurring in the peritoneal exudate of infected rabbits.

Schuetz' (Schuetz, Cent.f. Bakt., Ref. 1, 1887, p. 393) Diplokokkus der Brustseuche der Pferde, Poels and Nolen's (Poels und Nolen, Fort. d. Med., iv, 1886, p. 217) streptococcus of contagious pneumonia of cattle, and especially the organism described by Bonome (Bonome, Ziegler's Beit., viii, 1890, p. 377) as Streptococcus der meningitis cerebrospinalis epidemica, may all be looked upon as organisms differentiated on insecure grounds from either pneumococcus or streptococcus. The first two of these organisms, however, are said to be decolorized by Gram's method, and as suggested by Frosch and Kolle (F'rosch und Kolle, Flügge's "Mikroorganis.," ii, 1896, p. 161), in the case of Schuetz' organism may belong to a group intermediate between Fraenkel's diplococcus and the chicken-cholera group.

Tavel and Krumbein (Tavel und Krumbein, Cent. f. Bakt., xviii, 1895, p. 547) describe a streptococcus with a capsule, which was isolated from a small abscess on the finger of a child. Capsules were also present in the artificial cultures, and although ordinarily remaining uncolored, could be stained by Loeffler's flagella stain. This organism was said to be differentiated from Fraenkel's diplococcus and also in general from streptococcus (pyogenes) by a rapid and rich growth on gelatin, agar, and potato. A pellicle was formed on broth. The organisms forming this pellicle possessed capsules, but those in the deeper portions of the broth generally lacked the capsule.

In 1897, Binaghi (Binaghi, Cent. f. Bakt., xxii, 1897, p. 273) described a capsulated streptococcus isolated from a guinea-pig dead of a spontaneous peribronchitis and multiple pulmonary abscesses. In the pus were found some diplococci and short chains (four to six) surrounded by a capsule, which could be made evident by staining with carbol fuchsin. This organism he proposes to call Streptococcus capsulatus.

Le Roy des Barres and Weinberg in 1899 (Le Roy des Barres et Weinberg, Arch. d. méd. expér., xi, 1899, p. 399) published an account of a streptococcus with a capsule. This was isolated from a man who had apparently been infected from a horse which had died of an acute intestinal disorder. The patient neglected the infection and died. Diplococci and short chains furnished with a capsule were found in the subcutaneous tissue at the area of infection. The blood, liver, and 
Pneumococci ferment inulin, if cultivated in inulin-serum-water medium. Acid formation from the inulin results within two days or more in coagulation of the serum and reddening of the litmus. Streptococci because of their inability to attack the inulin leave the medium unchanged. ${ }^{1}$

Cultivated on whole-blood-agar, streptococci usually cause hemolysis, pneumococci usually do not. ${ }^{2}$ In contradistinction to Streptococcus viridans which does not hemolyze, pneumococci have a tendency on these media to form the black, dry, paint-blister colonies. ${ }^{3}$

Neufeld, ${ }^{4}$ in 1900 , noticed that normal rabbits' bile added in quan-

spleen also contained these organisms. The capsule in all the preparations remained uncolored, but the authors say that its existence was not to be doubted. Ascitic broth inoculated from the peritoneal exudate of a rabbit dying from the infection gave streptococci in extremely long chains and surrounded by capsules. These were not so distinct as in the case of the organisms in the original smear preparations. All fluid media (bouillon, milk, and ascitic broth) were said to be strongly acid after twenty-four hours. These authors report that Achard and Marmorek have assured them that they have seen capsulated streptococci, and that Marmorek showed them some preparations in which one of his streptococci presented the same characters as that isolated by them.

Although Le Roy des Barres and Weinberg have used the term encapsulated, they believe that it would perhaps be more prudent to call their organism streptocoque aureole, since they were not able to define this capsule by staining it.

Howard and Perkins (Howard and Perkins, Jour. Med. Res., 1901, iv, p. 163) have lately described an organism, probably of the foregoing type, which was present in a tubo-ovarian abscess and in the peritoneal exudate, the blood, and some of the organs of a woman dying in the Lakeside Hospital, Cleveland, Ohio. The organisms were biscuit-shaped cocci in pairs, usually arranged in chains of four, six, eight, or twenty elements, and surrounded by a wide and sharply staining capsule. In the artificial cultures special capsule stains, it was noted, failed to stain any definite area, but numerous small deeply stained granules were to be seen within the halo, especially near its outer border. Howard and Perkins propose for the group composed of the streptococci of Bonome, Binaghi, and their own organism, the name Streptococcus mucosus. Streptococci isolated from cases of epidemic sore-throat have also shown capsules (p. 343).

Reference to the original descriptions of these various capsulated streptococci will show that, with the exception of a rather poorly staining capsule, the majority of these organisms are separated from the typical Streptococcus pyogenes or from the pneumococcus by exceedingly slight and unstable morphological and cultural characters. The same is true of the difference observed in their pathogenic action in animals.

${ }^{1}$ Hiss, Cent. f. Bakt., xxxi, 1902; Jour. Exp. Med., vi, 1905.

2 Schottmüller, Münch. med. Woch.

${ }^{3}$ Hiss, Jour. Exp. Med., vii, 1905.

${ }^{4}$ Neufeld, Zeit. f. Hyg., 1901. 
tities of 0.1 c.c. to each one or two cubic centimeters of a pneumococcus broth culture caused lysis of the bacteria, rendering the culture fluid transparent and clear. This phenomenon does not occur with streptococci, and has been used to differentiate the two species. According to the recent studies of Libman and Rosenthal, ${ }^{1}$ great reliance may be placed upon this method.

The most convenient reagent for use in the Neufeld bile test is a 10 per cent solution of sodium taurocholate in physiological salt solution. This should be sterilized or kept on ice. One-tenth volume of such a solution produces prompt lysis in a broth culture of pneumococci.

Decisive differential importance may be attached to the agglutinations of these microorganisms in immune sera (see p. 364).

The permanency of the various types in the pneumococcus-streptococcus group is still open to question. E. C. Rosenow ${ }^{2}$ has recently reported that he has transmuted typical pneumococci into typical hemolytic streptococci by methods which he has not as yet fully described, but among which were animal passage, growth in symbiosis with bacillus subtilis, and growth in an atmosphere of oxygen. The pneumococci when first altered took on the characteristics of the streptococcus viridans, later of the so-called streptococcus rheumaticus, and finally of streptococcus hemolyticus. Together with cultural characteristics the pathogenicity of these various strains for rabbits changed. The pneumococcus produced acute sepsis, the streptococcus viridans caused endocarditis, the streptococcus rheumaticus periarticular or serous arthritis, and hemolyticus suppurative arthritis. An intermediate stage was found in which the organisms quite regularly produced myositis. Although he was able to transmute these types one into the other in both directions, Rosenow believes that the cultural characteristics of each type correspond to a fairly definite type of pathogenicity both in animals and man. This work has not as yet appeared in detail and has not been confirmed.

${ }_{1}$ Libman and Rosenthal, Proc. N. Y. Path. Soc., March, 1908.

${ }^{2}$ Rosenow, J. A. M. A., 1913, lxi, 2007. 


\section{CHAPTER XXIV}

\section{MICROCOCCUS INTRACELLULARIS MENINGITIDIS (MENINGOCOCCUS)}

Infectious processes in the meninges may be caused by many different microorganisms.

Meningitis may be primary or secondary. Secondary meningitis may often occur during the course of pneumonia, when pneumococci, carried to the meninges by the blood stream, give rise to a usually fatal form of the disease. More rarely a similar process may occur as a secondary manifestation of typhoid fever or influenza. Meningitis may also result secondarily by direct extension from suppurative lesions about the skull, such as those occurring in diseases of the middle ear or frontal sinuses or after compound fractures. In such cases the invading organisms are usually staphylococci, streptococci, or pneumococci.

Isolated cases of meningeal infection with B. coli, B. paratyphosus, Bacillus pestis, and Bacillus mallei have been reported. A frequent, more chronic form of the disease is caused by Bacillus tuberculosis.

Primary acute meningeal infection, however, is due chiefly to two microorganisms, Micrococcus intracellularis meningitidis, and the pneumococcus.

A tabulation of the comparative frequency with which the various microorganisms are found in the meninges has been attempted by Marschal. ${ }^{1}$ This author estimates that about 69.2 per cent of all acute cases are due to the meningococcus, 20.8 per cent to Diplococcus pneumoniæ, and the remaining 10 per cent to the other bacteria mentioned.

The cases caused by the pneumococcus and the other less frequent incitants usually occur sporadically. When the disease occurs in epidemic form, it is almost always due to the meningococcus.

Diplococcus intracellularis meningitidis was first seen in meningeal exudates by Marchiafava and Celli ${ }^{2}$ in 1884 . These authors not only described accurately the morphological characteristics now recog-

${ }^{1}$ Marschal, Diss. Strassburg, 1901, Quoted from Weichselbaum, in Kolle und Wassermann, "Handbuch."

${ }^{2}$ Marchiafava and Celli, Gaz. degli ospedali, 8, 1884. 
nized, but also called attention to the intracellular position of the microorganism and to its gonococcus-like appearance. They failed, however, to cultivate it.

Observations confirmatory of the Italian authors were, soon after, made by Leichtenstern. ${ }^{1}$ Cultivation and positive identification as a separate species was not accomplished, however, until Weichselbaum, ${ }^{2}$ in 1887, reported his observations upon six cases of epidemic cerebro-

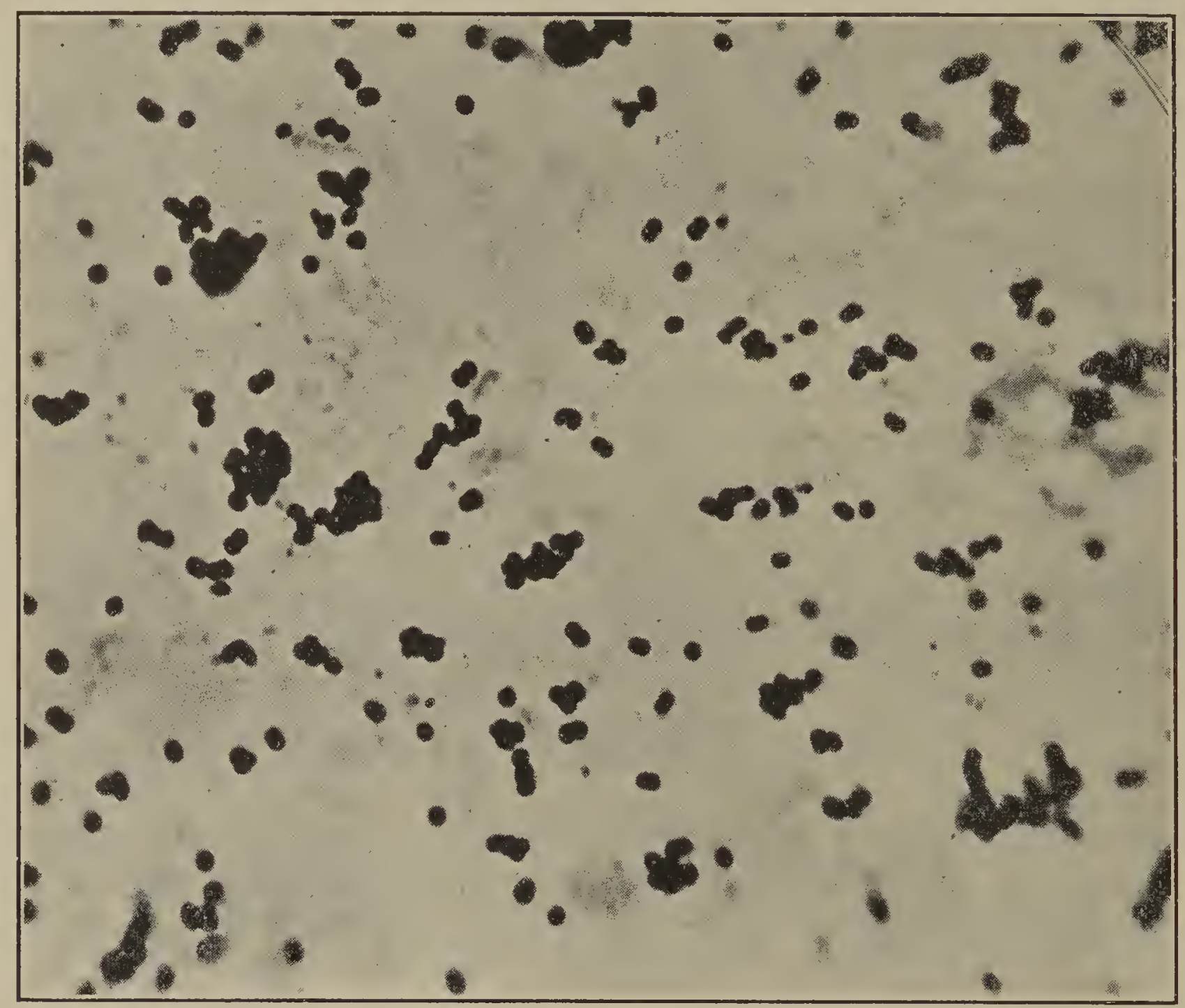

Fig. 77.-Meningococcus, Pure Culture. (Very highly magnified.)

spinal meningitis in which he not only found the cocci morphologically, but was able to study their biological characteristics in pure culture. The researches of Weichselbaum were soon confirmed and extended by elaborate studies ${ }^{3}$ which left no doubt as to the specific relationship between the microorganism cultivated by him and the clinical condition.

${ }^{1}$ Leichtenstern, Deut. med. Woch., 1885.

${ }^{2}$ Weichselbaum, Fort. d. Med., 1887.

${ }^{3}$ Councilman, Mallory, and Wright, Special Rep. Mass. Board of Health, 1898; Albrecht und Ghon, Wien. klin. Woch., 1901. 
Morphology and Staining.-Stained in the spinal fluid from an infected patient, the meningococcus bears a striking similarity to the gonococcus. The microorganisms appear intra- and extracellularly, usually in diplococcus groups, sometimes as tetrads, or even in larger agglomerations. The individual diplo-forms are flattened on the sides facing each other, presenting somewhat the biscuit-form of the gonococcus. The variation in size of the cocci in the same smear is a noticeable feature

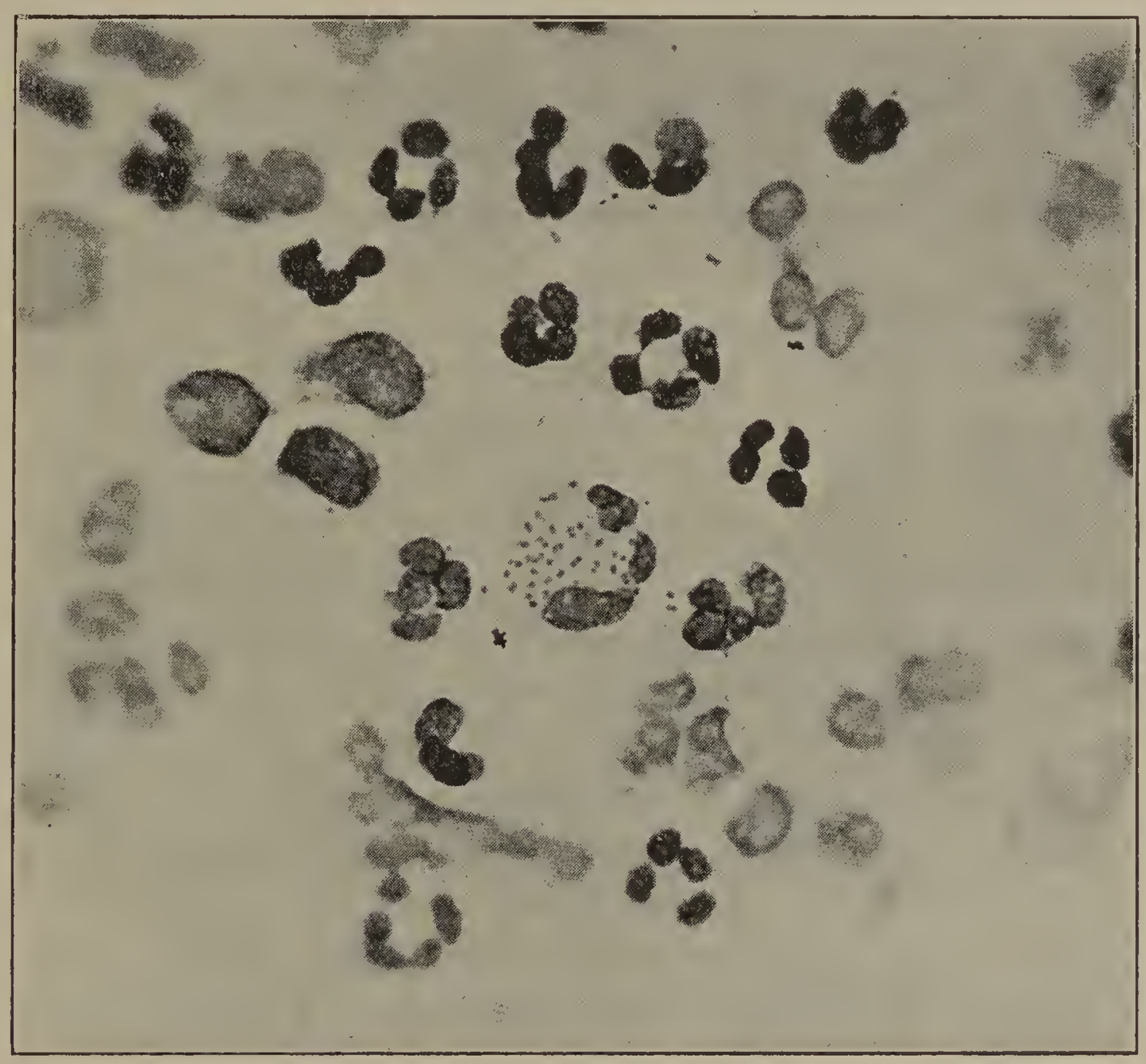

Fig. 78.-Meningococcus in Spinal Fluid.

and of some diagnostic importance. This dissimilarity in size is noticeable also in cultures, which, especially when older than twenty-four hours, contain forms double or even triple the size of the average coccus. These may possibly be involution forms.

The meningococcus is non-motile and non-spore forming. It stains easily with all the usual aqueous aniiin dyes. Its behavior toward Gram's stain was long a subject of controversy, owing to the error of Jaeger, ${ }^{1}$ who claimed to have found it Gram-positive. There

${ }^{1}$ Jaeger, Zeit. f. Hyg., xix, 1895. 
is no question now, however, that the cocci decolorize by Gram's method when this is carefully carried out.

In spinal fluid very beautiful preparations may be obtained by staining in Jenner's blood stain. Councilman, Mallory, and Wright ${ }^{1}$ were the first to notice that, when stained with Loeffler's methylene-blue, meningococcus stains irregularly, showing metachromatic granules in the center of the cell bodies. These granules can be demonstrated more clearly with the Neisser stain employed for similar demonstration in the case of B. diphtheriæ (see p. 107) and have some value in differentiating meningococcus from gonococcus.

Cultivation.--Micrococcus intracellularis meningitidis grows readily upon all the meat-infusion culture media. It may even be cultivated upon meat-extract media, but growth upon these is not profuse. Upon agar, colonies appear within eighteen to twenty-four hours as grayish, glistening spots with smooth edges and raised granular centers. These show a tendency to enlargement and eventual confluence.

Growth is more luxuriant and rapid upon media to which animal proteid in the form of blood serum or ascitic fluid has been added. Coagulated serum is not liquefied. For cultivation of the meningococcus directly from the human body it is wise to use the richer serum or blood media, ability to grow easily upon simple agar being occasionally acquired only after previous cultivation upon richer media. Agar to which whole rabbit's blood has been added forms an excellent medium, both for cultivation and for keeping the organism alive. Loeffler's blood serum is also very favorable. It is advisable, too, when cultivating directly from spinal fluid, to plant rather large quantities (1 to 2 c.c.), since many of the cocci in the exudate will fail to develop colonies, possibly because of their prolonged exposure either to the body fluids or to their own products in a closed space.

Upon broth, growth is slow and takes place chiefly upon the surface, the sediment consisting mainly of dead bacteria. Glucose added to agar or to broth renders the medium more favorable for rapid growth, but, owing to acid formation, tends to cause a more rapid death of the culture. In flasks of broth containing glucose one per cent, and $\mathrm{CaCO}_{3}$ one per cent, however, cultures have been kept alive for as long as fourteen months (Hiss). On milk, growth takes place without coagulation of the casein. Potatoes are not a favorable medium, though growth occasionally takes place.

${ }^{1}$ Councilman, Mallory, and Wright, Rep. Mass. State Bd. of Health, 1898. 
While slight alkalinity or acidity does not inhibit, the most favorable reaction of media is neutrality.

Oxygen is necessary for development. Complete anaërobiosis, while not absolutely inhibitory, is extremely unfavorable, unless proper carbohydrates be present.

While growth may take place at temperatures ranging from $25^{\circ}$

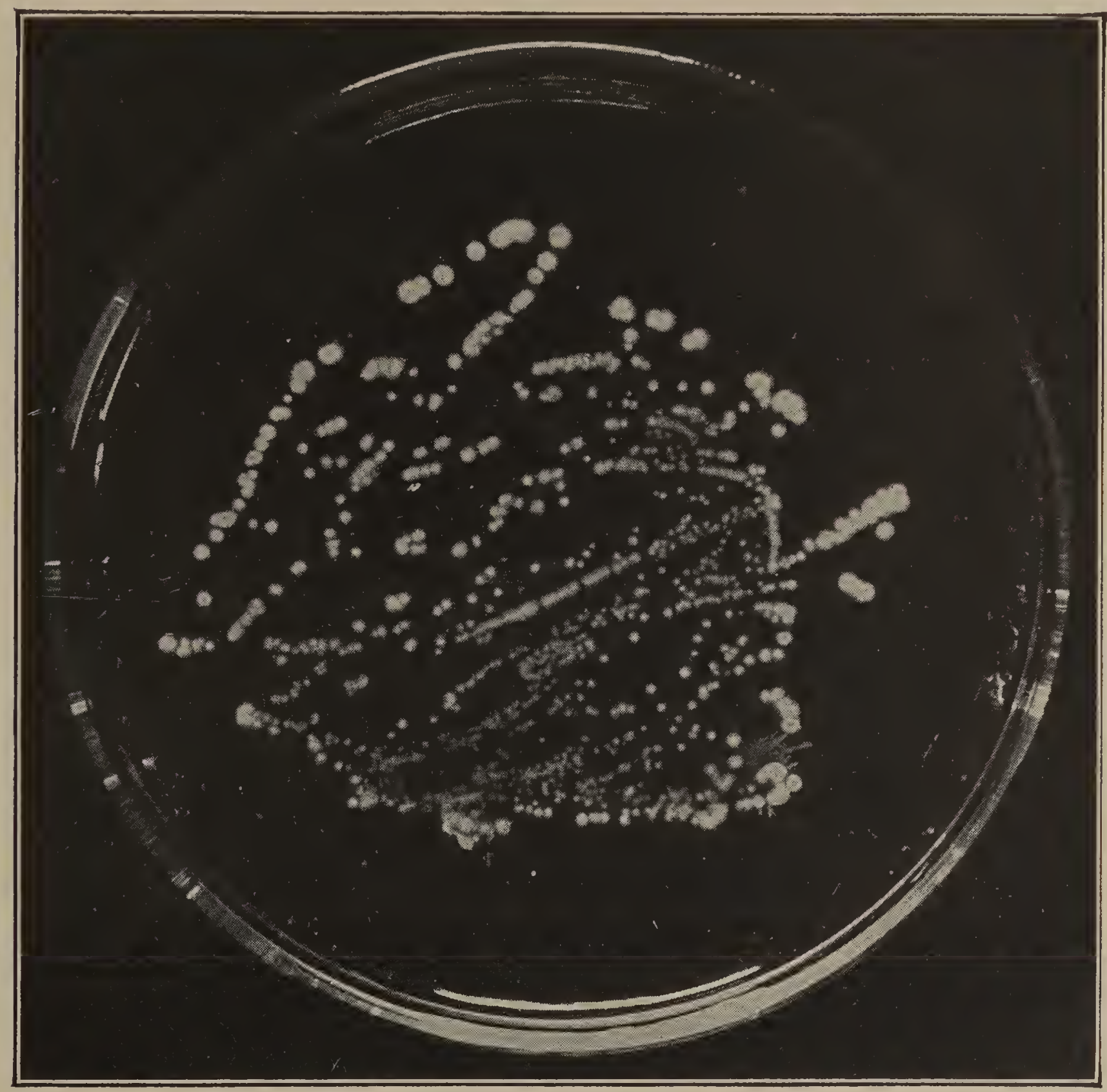

Fig. 79.-Meningococcus Culture. Streak culture from spinal fluid on serum agar-plate.

to $42^{\circ} \mathrm{C}$, the optimum is $37.5^{\circ} \mathrm{C}$. Apart from the remarkable viability displayed upon calcium-carbonate broth, the average length of time during which the meningooocus will remain alive without transplantation is rather short. Recently isolated cultures grown on agar or serumagar may die within two or three days. Accustomed to artificial cultivation through a number of generations, however, the cultures become 
more hardy and transplantation may safely be delayed for a week or even longer. Albrecht and Ghon ${ }^{1}$ have kept a culture alive on agar for one hundred and eighty-five days. It is a strange fact that after prolonged artificial cultivation some strains of meningococcus may gradually lose their growth energy and finally be lost because of their refusal to develop in fresh transplants. Storage is best carried out at incubator temperatures. At room temperatures or in the ice chest, the diplococcus dies rapidly. ${ }^{2}$

Resistance.-The meningococcus is killed by exposure to sunlight or to drying within twenty-four hours. ${ }^{3}$ It is extremely sensitive to heat and cold and by the common disinfectants is killed in high dilutions and by short exposures. At $0^{\circ} \mathrm{C}$. it usually dies within two or three days.

Pathogenicity.-As stated above, the form of meningitis caused by the diplococcus of Weichselbaum occurs usually in epidemics, though isolated sporadic cases are seen from time to time in all crowded communities. Epidemics have been numerous and widespread, and their records far antedate the discovery of their causative agent. As a rule, these epidemics have occurred during the winter and spring months, and have attacked chiefly that part of the population which is forced by poverty to live in crowded unhygienic surroundings. The manner in which the microorganism enters the human body is still a subject for investigation. Weichselbaum, ${ }^{4}$ Ghon and Pfeiffer, ${ }^{5}$ and, more recently, Goodwin and v. Sholly ${ }^{6}$ of the New York Department of Health, have succeeded in demonstrating culturally the presence of the meningococcus in the nasal cavities, not only of patients suffering from the disease, but occasionally in those of healthy subjects as well. Similar findings have been reported by many others; but in many cases morphological examination only was made, which, owing to the danger of confusion with Micrococcus catarrhalis, a frequent inhabitant of the nose, renders such reports valueless. The careful work of the writers inentioned, however, has given ground for the theory that meningeal infection, which is

1 Albrecht und Ghon, Wien. klin. Woch., 1901.

${ }^{2}$ A very thorough biological study of meningococcus and related organisms has recently been made by Elser and Huntoon (Jour. Med. Res., N. S. vol. xv, 1999), which may be consulted for a more detailed description of cultural characteristics.

${ }^{3}$ Councilman, Mallory, and Wright, Boston, 1898; Albrecht and Ghon, loc. cit.

${ }^{4}$ Weichselbaum, Fort. d. Med., 1887.

${ }^{5}$ Ghon und Pfeiffer, Zeit. f. klin. Med., xliv, 1901.

'Goodwin und v. Sholly, Jour. Inf. Dis., Suppl. 2, Feb., 1906. 
often preceded by nasal catarrh, may take place along the paths of the lymphatics, passing out of the nose and its accessory cavities toward the base of the skull. These facts, together with the low resistance shown by the meningococcus against drying, and the general failure so far to demonstrate it in air, dust, or fomites, would seem to indicate that transmission usually occurs directly from one human being to another.

The disease produced in man consists anatomically in a suppurative lesion of the meninges, involving the base and cortex of the brain and the surface of the spinal cord. The nature of the exudate may vary from a slightly turbid serous fluid to that of a thick fibrinous exudate. In chronic cases encephalitis and dilatation of the ventricles may take place. Apart from their presence in the meninges and in the nasopharynx, meningococci have not been satisfactorily demonstrated in any of the complicating lesions of the disease. Reports of their presence in the conjunctivæ, in the bronchial secretions from broncho- or lobar pneumonia, and in otitis media, have usually been based upon insufficient bacteriological evidence.

The occurrence of this microorganism in the circulating blood of meningitis cases has been definitely proved by Elser, ${ }^{1}$ who found it in ten cases.

Animals are not very susceptible to infection with Diplococcus meningitidis. Subcutaneous inoculation is rarely followed by more than a local reaction unless large quantities are used. White mice are rather more susceptible than other species. Intraperitoneal and intravenous inoculation of sufficient quantities usually results in the death of mice, rabbits, guinea-pigs, and dogs. Occasional strains have been found to possess a not inconsiderable degree of toxicity for rabbits, grave symptoms or even death following intravenous injection of but moderate quantities without any traceable development of the microorganisms in the organs of the animals.

Similar observations have been made by Albrecht and Ghon, ${ }^{2}$ who succeeded in killing white mice with dead cultures. It would seem therefore that the effect of this coccus upon animals depends chiefly upon the poisonous substances contained in the bacterial bodies (endotoxins). Lepierre ${ }^{3}$ has obtained the meningococcus toxin by alcohol precipitation of broth cultures.

Weichselbaum himself succeeded in producing meningeal suppura-

1 Elser, Jour. Med. Res., xiv, 1906.

2 Albrecht und Ghon., loc. cit.

${ }^{3}$ Lepierre, Jour. de phys. et de path. gén., v, No. 3. 
tion and, in one case, brain abscess, by subdural inoculation of dogs. Councilman, Mallory, and Wright ${ }^{1}$ produced a disease in many respects similar to the human disease by intraspinous inoculation of a goat. Recently, Flexner ${ }^{2}$ has succeeded in producing in monkeys a condition entirely analogous to that occurring in human beings.

Agglutination. - Immunization of animals by repeated inoculations of meningococcus ${ }^{3}$ results in the formation in the blood serum of agglutinins. Kolle and Wassermann ${ }^{4}$ obtained from horses a serum which had an agglutinating value of $1: 3,000$ for the homologous strain, and of as much as $1: 500$ for other true meningococcus strains. Similar experiments by Dunham ${ }^{5}$ and others have proved the unquestionable value of agglutination for species identification of this group. Great differences may, however, exist between individual races in their agglutinability in the same immune serum.

Kutscher has recently called attention to the fact that strains which can not be agglutinated in specific sera at $37^{\circ} \mathrm{C}$. will often yield positive results when subjected to $55^{\circ} \mathrm{C}$, a fact of some practical importance if confirmed.

Elser and Huntoon ${ }^{6}$ have shown that in the serum of infected human subjects agglutination of some strains takes place in dilutions as high as $1: 400$.

Serum Therapy of Meningitis.-During recent years, many attempts have been made to treat epidemic cerebrospinal meningitis by injections, subcutaneous and intraspinous, of meningococcus-immune serum. Wassermann, ${ }^{7}$ in 1907 , reported the results obtained by such treatment in one hundred and two patients, with a recovery of 32.7 per cent. The serum, as manufactured by Wassermann and his associates, was obtained from horses immunized with pure cultures of meningococcus and with toxic meningococcus extracts. More recently Flexner and Jobling ${ }^{8}$ have used a similar serum in the United States with apparently excellent results. The serum, in Flexner's cases, is injected intraspinously after a quantity of spinal fluid had been withdrawn. The cases treated by Flexner and Jobling's method have now reached

${ }^{1}$ Councilman, Mallory, and Wright, loc. cit.

2 Flexner, Jour. Exp. Med., 1906.

3 Albrecht and Ghon, Wien. klin. Woch., 1901.

Kolle und Wassermann, Deut. med. Woch., 15, 1906.

- Dunham, Jour. Inf. Dis., 11, 1907.

- Elser and Huntoon, loc. cit.

7 Wassermann, Deut. med. Woch., 39, 1907.

8 Flexner and Jobling, Jour. Exper. Med., x, 1908. 
large numbers, both in this and foreign countries and the value of the serum as a therapeutic agent seems firmly established.

Hiss and Zinsser ${ }^{1}$ have treated a number of meningitis patients with subcutaneous injections of leucocyte extracts and believe that they have favorably influenced the course of the disease.

Pseudomeningococcus.-Elser and Huntoon ${ }^{2}$ have described a diplococcus very similar to the meningococcus which they differentiated from it only by serum reactions. This diplococcus could be identified only by agglutinin absorption tests. They named it pseudomeningococcus.

1 Hiss and Zinsser, Jour. Med. Res., Nov., 1908.

2 Elser and Huntoon, Jour. Med. Res., xxi, 1909. 


\section{CHAPTER XXV}

\section{DIPLOCOCCUS GONORRHEE (GONOCOCCUS), MICROCOCCUS CATARRHALIS, AND OTHER GRAM-NEGATIVE COCCI}

\section{DIPLOCOCCUS GONORRHEA}

Neisser, ${ }^{1}$ in 1879 , described diplococci which he had found regularly in the purulent secretions of acute cases of urethritis and vaginitis and in the acute conjunctivitis of the new-born. His researches were purely morphological, as were the numerous confirmatory investigations which rapidly followed his announcement.

Cultivation of this diplococeus, now usually spoken of as gonococcus, was not definitely successful until 1885, when Bumm ${ }^{2}$ obtained growth upon tubes of coagulated human blood serum. Bumm was not only able to keep the organisms alive by transplantation in pure culture, but produced the disease by inoculation of his cultures upon the healthy urethra.

Morphology and Staining.-The gonococcus is usually seen in the diplococcus form, the pairs being characteristically flattened along the surfaces facing each other. This gives the cocci a peculiar coffee-bean or biscuit shape. The size of the diploforms is about 1.6 micra in the long diameter, about 0.8 micron in width. Stained directly in gonorrheal pus from acute cases, the microorganisms are found both intra- and extracellularly, a large number of them crowded characteristically within the leucocytes. They are never found within the nucleus. The phagocytosis which produces this picture has been shown by Scholtz ${ }^{3}$ and others to take place in the free secretions, not in the depth of the tissues. The intracellular position, which is of considerable diagnostic importance, is lost to a great extent in secretions from chronic cases. In smears made from pure cultures the arrangement in groups of two may often be less marked than in pus, clusters of eight or more being common.

1 Neisser, Cent. f. d. med. Wiss., 1879.

2 Bumm, "Beitr. z. Kenntniss des Gonococcus," Wiesbaden, 1885.

3 Scholtz, Arch. f. Dermat., 1899. 
The gonococcus is non-motile and does not form spores. It is easily stained with the usual aqueous anilin dyes. Methylene-blue alone, or eosin followed by methylene-blue, or the neutral red stain of Plato, ${ }^{1}$ gives good results. Gram's method of staining, however, is the only one of differential value. With this method the gonococcus is rapidly decolorized and can be counterstained with fuchsin or Bismarck brown. The Gram stain applied to pus from the male urethra, while not absolutely reliable, is, for practical purposes, sufficiently so to make a diagnosis. In exudates from the vagina or from the eye the morphological picture is not so reliable, owing to the frequent presence

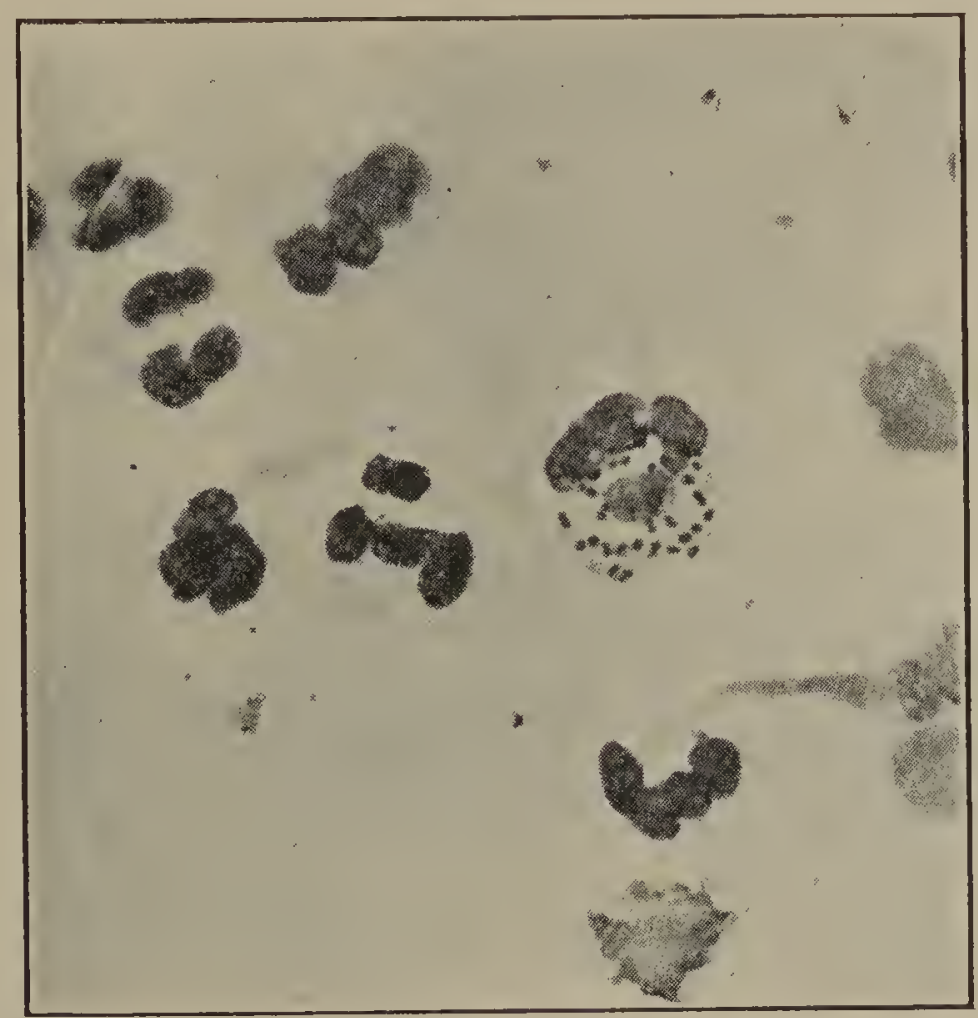

Fig. 80.-Gonorrheal Pus from Urethra, showing the Cocci within $\Lambda$ LEUCOCYTE.

in these regions of other Gram-negative cocci. The great scarcity of gonococci in very chronic discharges necessitates thorough cultural investigation; negative morphological examination in such cases can not be regarded as conclusive. ${ }^{2}$

Cultivation.-The gonococcus is extremely delicate and is difficult to cultivate. After many failures to grow it upon the ordinary media, Bumm ${ }^{3}$ obtained his first growths upon human blood serum which had been heated to partial coagulation.

The medium most commonly used at the present day was introduced

1 Plato, Berl, klin. Woch., 1894.

${ }^{2}$ Heiman, Medical Record, 1896.

3 Bumm, Deut. med. Woch., 1885. 
by Wertheim, ${ }^{1}$ and consists of a mixture of two or three parts of meat infusion-agar with one part of uncoagulated human ascitic fluid, hydrocele fluid, or blood serum. The agar is melted and cooled to $45^{\circ}$ before the serum is added. The mixture may then be slanted in the test tube or poured into a Petri plate. This medium may be improved by the addition to the agar of six per cent of glycerin or one per cent of

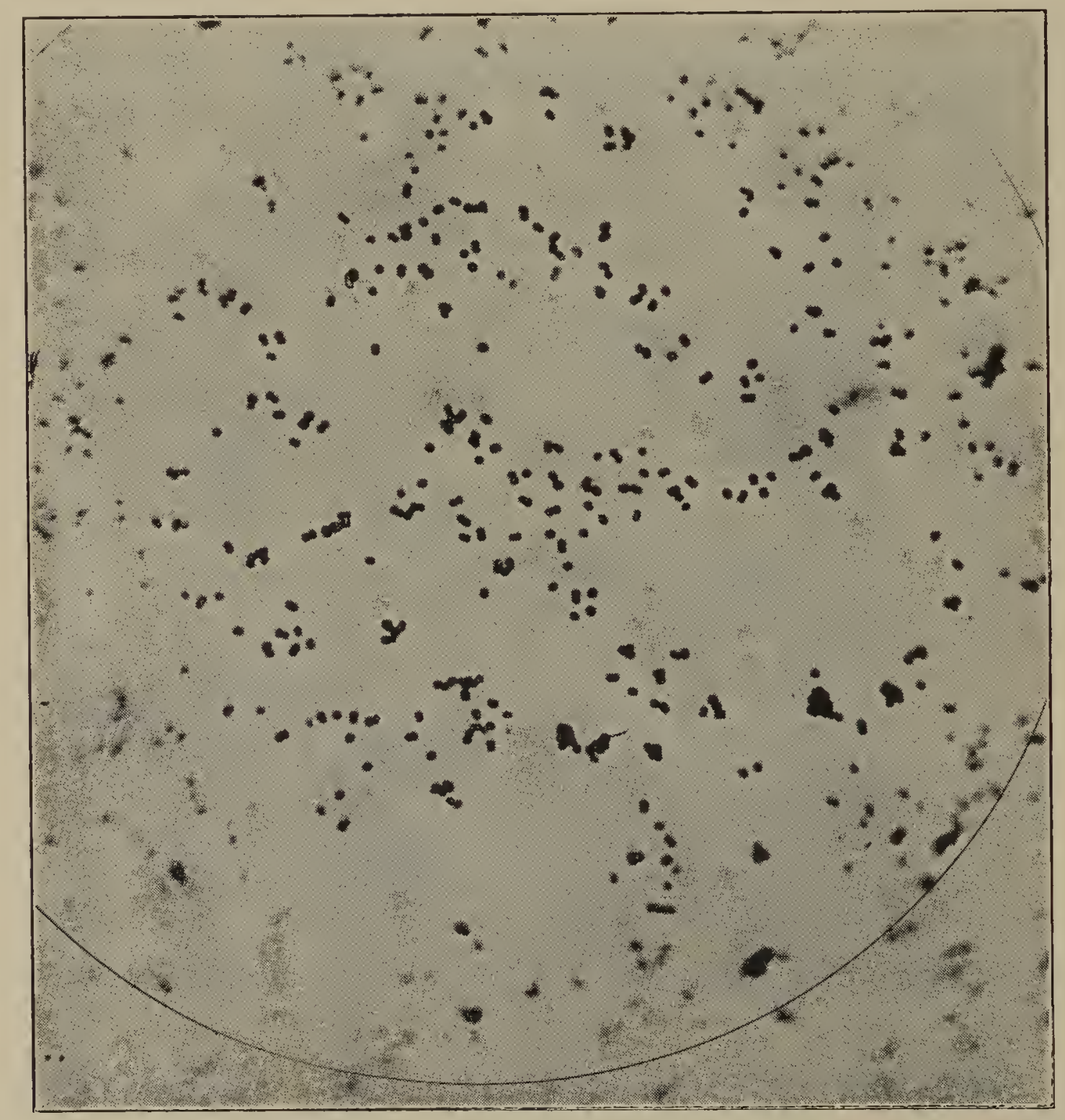

Fig. 81.-Gonococcus. Smear from pure culture.

glucose. Cultures in fluid media may be obtained by similar additions of serum to meat-infusion-pepton-broth (pepton, one to two per cent). While human sera may be replaced by animal sera, these in general are not so favorable for growth of the gonococcus. They are useful chiefly in cases where it is difficult to obtain the human serum. Whole rabbit's blood added to agar, or the swine- 
serum-nutrose medium of Wassermann ${ }^{1}$ may occasionally be used with success.

Plates may also be made by smearing for enrichment a drop of blood from the finger over the surface of agar in the manner of Pfeiffer's method for influenza-bacillus cultivation. Inoculations from gonorrheal material are best made by surface smearing upon plates, since the gonococcus grows best in the presence of free oxygen. Growth is more easily obtained and becomes more luxuriant after prolonged culti-

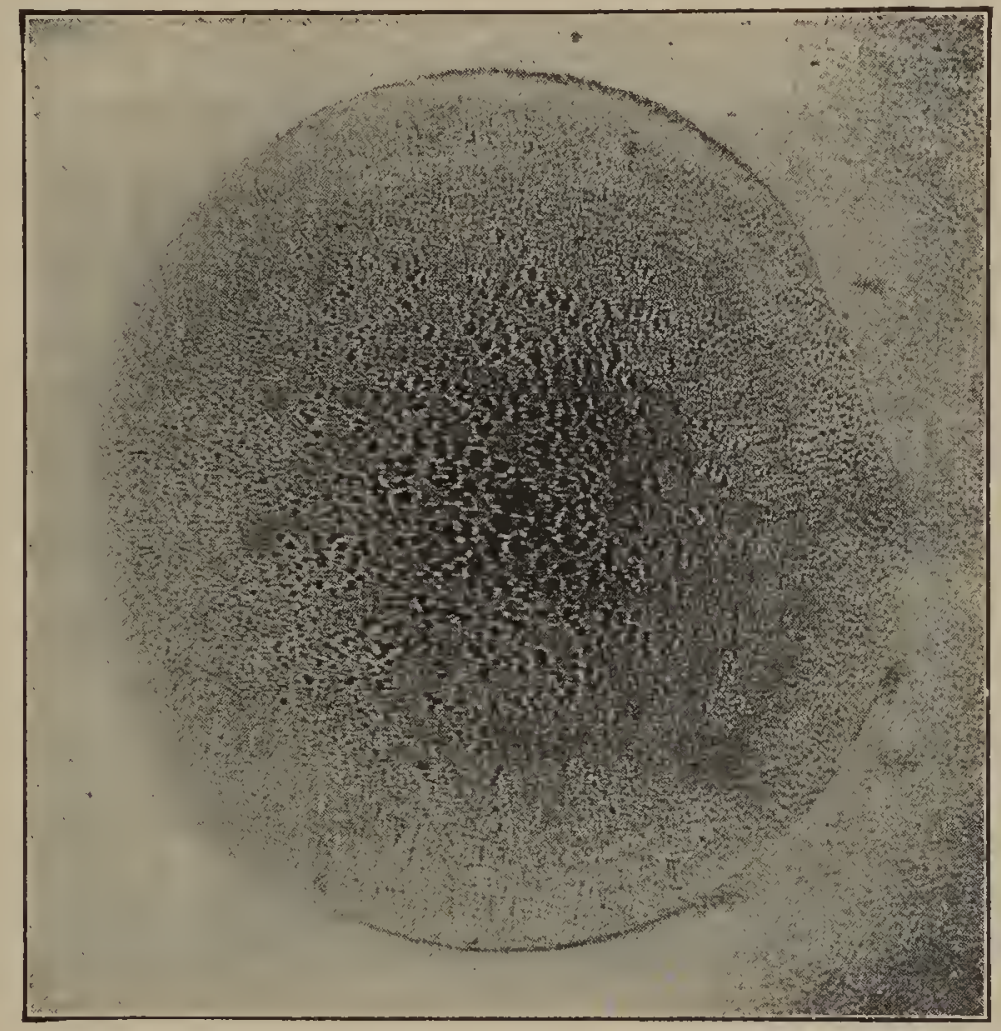

Fig. 82.-Gonococcus Colony. Low power of magnification. (After Mallory and Wright.)

vation upon artificial media. The most favorable reaction of media is neutrality or slight acidity.

Whenever the gonococcus has been successfully cultivated from pus upon media without serum additions, the success has probably been due to the substances carried over in the pus.

The gonococcus will develop a sparse growth under anaërobic conditions, but displays a very marked preference for aërobiosis. The optimum temperature for growth is $37.5^{\circ} \mathrm{C}$. Growth ceases above $38.5^{\circ}$ and below $30^{\circ}$.

Wassermann, Berl. klin. Woch., 1897.

(Fifteen c.c. swine-serum, 35 c.c. of water, 3 c.c. glycerin, with two per cent nutrose. The nutrose is dissolved by boiling and the solution sterilized. This is then added to agar, in equal parts, and used in plates.) 
Upon suitable media colonies appear as extremely delicate, grayish, opalescent spots, at the end of twenty to twenty-four hours. The separate colonies do not tend to confluence and have slightly undulated margins. Touched with a platinum loop their consistency is found to be slimy or sticky. In fluid media, growth takes place chiefly at the surface and there is no general clouding.

Resistance.-Cultures of gonococcus, if not transplanted, usually die out within five or six days at incubator temperature. At room temperature they die more rapidly. In the ice chest they may be kept alive for somewhat longer periods. In this they differ from meningococci, which are always killed by temperatures approximating zero C. In these respects, however, individual strains show much variation, some cultures dying out after but a few hours' removal from the incubator.

The resistance of the gonococcus to light and heat is very slight. A temperature of $41^{\circ}$ to $42^{\circ}$ kills it after a brief exposure. Complete drying destroys it in a short time. Incompletely dried, however, and protected from light (gonorrheal pus) it may live, on sheets and clothing, for as long as eighteen to twenty-four hours. ${ }^{1}$

It is easily killed by most disinfectant solutions ${ }^{2}$ even when these are highly diluted and seems to be almost specifically sensitive to the various silver salts, a fact of therapeutic importance.

Pathogenicity.-Gonorrheal infection occurs spontaneously only in man. True gonorrheal urethritis has never been experimentally produced in animals. In human beings, apart from the common seats of the infection in the male and female genital tracts, and in the conjunctivæ, the gonococcus may produce cystitis, proctitis, and stomatitis. It may enter the general circulation, giving rise to septicemia ${ }^{3}$ and, secondarily, to endocarditis and arthritis. Isolated cases of gonorrheal periostitis and osteomyelitis have been reported. ${ }^{4}$

The common acute infections of the genito-urinary passages in man are often followed by an indefinitely prolonged chronic infection, which, though quiescent, may for many years be a source of social danger. In children, especially females, the infection is not rare, and may assume epidemic characters, traveling from bed to bed in institutions. Such hospital epidemics can be stopped only by the most rigid isolation. It is advisable, in view of this danger, to examine all

1 Heiman, Medical Record, 1896.

${ }^{2}$ Schueffer und Steinschneider, Kong. Deut. Dermat. Gesells., Breslau, 1894.

3 Review of cases of Gon. Septicemia, Faure-Beaulieu, Thesis, Paris, 1906.

${ }_{4}^{4}$ Ullmann, Wien. med. Presse, 1900. 
female children applying for admission to a hospital, by vaginal smear and, if possible, to keep them in a receiving ward for twentyfour hours in order that the examination may be repeated before admission to the general wards. In the best-equipped institutions, furthermore, separate thermometers, bed linen, and diapers are set aside for each child in order to preclude any possibility of accidental transmission from cases which may have escaped detection by smear examination.

While inoculation of animals has never resulted in active proliferation of the gonococcus upon the new host, local necrosis, suppuration, and temporary systemic reactions have been produced by subcutaneous and intraperitoneal inoculation. These are probably referable to the endotoxin contained in the bodies of the gonococci. This toxin has been isolated by Nikolaysen ${ }^{1}$ by extraction from the bacterial bodies with distilled water or sodium hydrate solutions. It was found to be resistarit to a temperature of $120^{\circ}$ and to remain potent after complete drying. The same author found that the isolated toxin and dead cultures were fully as toxic for animals as living cultures, 0.01 gram killing a white mouse.

Specific injury to the nervous system by injections of gonococcus toxin has been reported by Moltschanoff. ${ }^{2}$

The secretion of a true soluble toxin by the gonococcus, asserted by Christmas, ${ }^{3}$ is denied by Wassermann, ${ }^{4}$ Nikolaysen, ${ }^{5}$ and others. The two authors last named, furthermore, do not believe that a general immunity is developed in subjects infected with gonococcus. Christmas ${ }^{6}$ on the other hand, and, more recently, Torrey ${ }^{7}$ have reported successful active immunization of animals by repeated injections of whole bacteria. Torrey and others apparently have successfully treated human cases by injections of the serum of immunized animals.

\section{MICROCOCCUS CATARRHALIS}

Micrococcus catarrhalis is a diplococcus described first by R. Pfeiffer, ${ }^{8}$ who found it in the sputum of patients suffering from catarrhal in-

1 Nikolaysen, Cent. f. Bakt., 1897.

2 Moltschanoff, Münch. med. Woch., 1899.

${ }^{3}$ Christmas, Ann. de l'inst. Pasteur, 1897.

${ }^{4}$ Wassermann, Zeit. f. Hyg., xxvii, 1897.

${ }^{5}$ Nikolaysen, Fort. d. Med., xxi, 1897.

${ }^{6}$ Christmas, loc. cit.

7 Torrey, Jour. Amer. Med. Assn., xlvi, 1906.

\& Flügge, "Die Mikroorg.," 3d ed., 1896. 
flammations of the upper respiratory tract. It was subsequently carefully studied by Ghon and $\mathrm{H}$. Pfeiffer. ${ }^{1}$ According to these authors the pathogenic significance of the micrococcus is slight, though occasionally it may be regarded as the causative factor in catarrhal inflammations. Its chief claim to attention, however, lies in its similarity to the meningococcus and the gonococcus, from neither of which it can be morphologically distinguished. It is decolorized by Gram's stain, appears often in the diplococcus form, and has a tendency, in exudates, to be located intracellularly. Not unlike the two microorganisms mentioned, too, it shows but slight pathogenicity for animals.

Differentiation from gonococcus is extremely simple in that Micrococcus catarrhalis grows easily on simple culture media and shows none of the fastidious cultural requirements of the gonococcus.

From meningococcus the differentiation is less simple and, because of the presence of both microorganisms in the nose, is of great importance.

Distinction between the two is made entirely upon cultural characteristics and agglutination reactions. Culturally, Micrococcus catarrhalis grows more heavily than meningococcus upon the ordinary culture media. The colonies of Micrococcus catarrhalis are coarsely granular and distinctly white in contradistinction to the finely granular, grayish meningococcus colonies. ${ }^{2}$ Micrococcus catarrhalis will develop at temperatures below $20^{\circ} \mathrm{C}$, while meningococcus will not grow at temperatures below $25^{\circ} \mathrm{C} .^{3}$

Dunham, ${ }^{4}$ who has recently made a comparative study of meningococcus and other Gram-negative diplococci from the nose and throat, states that while some of the supposed Micrococcus catarrhalis cultures are easily distinguished from meningococcus simply by the characteristics of their growths upon two-per-cent glucose agar, others offer great difficulties to differentiation. He recommends as a differential medium a mixture of sheep serum and bouillon containing one per cent of glucose. Upon this medium all true meningococci produce acid, but no coagulation, with twenty-four hours. Cultures from the nose and throat, however, produce acid and coagulation, or else produce an alkaline reaction.

${ }^{1}$ Ghon und H. Pfeiffer, Zeit. f. klin. Med., 1902.

${ }^{2}$ Ghon und Pfeiffer, loc. cit.

${ }^{3}$ Weichselbaum, in Kolle und Wassermann, Bd. iii, p. 269.

- Dunham, Jour. Inf. Dis., 1907. 


\section{OTHER GRAM-NEGATIVE COCCI}

Micrococcus pharyngis siccus.-This organism was first described by von Lingelsheim ${ }^{1}$ in 1906. It is described by Elser and Huntoon as readily differentiable from meningococcus and other Gram-negative cocci by the firm adherence and dryness of its colonies. It is similar to Micrococcus catarrhalis from which it may, however, be differentiated by fermentation tests.

Diplococcus mucosus.-This organism was also described by von Lingelsheim together with the preceding one. Its colony formation is similar to that of meningococcus, but slightly more sticky and mucoid. Stained by the capsule methods, it is seen to possess a distinct capsule.

Chromogenic Gram-negative Cocci.-These microorganisms all produce a greerish-yellow pigment on the ordinary culture media. When pigment is absent, as is frequently the case when grown upon sugar-free media, these microorganisms can be distinguished from meningococcus only by sugar fermentation and serum reactions.

An exhaustive study of Gram-negative micrococci has recently been made by Elser and Huntoon. ${ }^{2}$ These authors, in studying the differential value of sugar fermentation in the diagnosis of these bacteria, have constructed the following table:

\begin{tabular}{|c|c|c|c|c|c|c|c|}
\hline Strains Tested. & 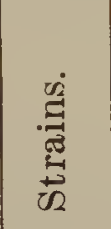 & 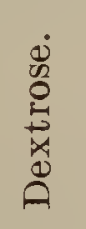 & 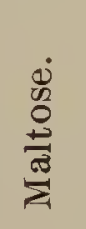 & 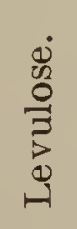 & 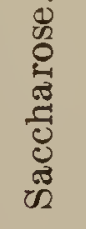 & 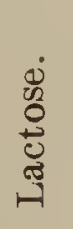 & 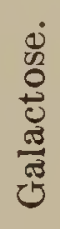 \\
\hline Meningococcus. . & 200 & + & + & 0 & 0 & 0 & 0 \\
\hline Pseudomeningococcus... & 6 & + & + & 0 & 0 & 0 & 0 \\
\hline Gonococcus........... & 15 & + & 0 & 0 & 0 & 0 & 0 \\
\hline Micrococcus catarrhalis.. & 64 & 0 & 0 & 0 & 0 & 0 & 0 \\
\hline Micrococcus pharyngis siccus.... & 2 & + & + & + & + & 0 & 0 \\
\hline Chromogenic group I & 28 & + & + & + & + & 0 & 0 \\
\hline Chromogenic group II. & 11 & + & + & + & 0 & 0 & 0 \\
\hline Chromogenic group III. & 9 & + & + & 0 & 0 & 0 & 0 \\
\hline Jaeger meningococcus, Kral. . . . . . . . . . . . & 1 & + & + & + & + & + & + \\
\hline Diplococcus crassus, Kral............... & 1 & + & + & + & + & + & + \\
\hline
\end{tabular}

${ }^{1} v$. Lingelsheim, Klin. Jahrb., 15, 1906.

${ }_{2}$ Elser and Huntoon, loc. cit. 


\section{CHAPTER XXVI}

\section{BACILLI OF THE COLON-TYPHOID-DYSENTERY GROUP}

THE bacilli belonging to this group of microorganisms, while presenting great differences in their pathogenic characteristics, possess many points of morphological and biological similarity which have made their differentiation extremely difficult. Among pathogenic bacilli, they are probably the ones most commonly encountered and because of the fact that some of them are specifically pathogenic, while others are essentially saprophytic and are pathogenic only under exceptional conditions, the necessity of accurate differentiation is a daily occurrence in bacteriological laboratories. It has been through the study of this group particularly that many of the modern differential methods of bacteriology have been developed.

The group includes the colon bacillus and its allies, the typhoid bacillus, the paratyphoid organisms, the several varieties of dysentery bacillus and numerous closely related species, and Bacillus fecalis alkaligenes. Closely related to the group though not properly within it, are Bacillus lactis aërogenes, bacilli of the Friedländer or mucosus capsulatus group, and a number of less important subdivisions of this last group.

All bacilli of the group possess morphological characteristics which, although exhibiting slight differences, are insufficient to permit accurate morphological diagnosis. They are none of them spore-bearing. Stained by Gram's method they are decolorized.

Cultivated upon artificial media, they grow readily both at room and at incubator temperatures. None of them liquefies gelatin. Though showing, often, distinct differences in the speed and luxuriance of growth upon ordinary media, these differences are, nevertheless, too slight to become the basis of differentiation.

In order to distinguish between the individual members of this group, therefore, we are forced to a careful biological and cultural study. This is carried out by the observation of the cultural characteristics upon special media and by the study of serum reactions in specific immune sera. Our mainstays in the accurate differentiation of these 
bacilli are the observation of their fermentative action upon carbohydrate media, and their agglutinating reactions in immune sera. These points will be alluded to in the description of the individual microorganisms, and will be again summarized in the differential tables given at the end of the chapters dealing with this group.

\section{BACILLUS COLI COMMUNIS AND MEMBERS OF THE COLON BACILLUS GROUP}

Under the name of "colon bacilli" are grouped a number of bacterial varieties differing from one another somewhat in minor characteristics, but corresponding in certain cardinal points which stamp them as close relatives and amply warrant their consideration under one heading. While usually living as harmless parasites upon the animal and human body, and capable of leading a purely saprophytic existence, they may, nevertheless, under certain circumstances become pathogenic and thus, both culturally and in their pathological significance, form a link between pure saprophytes like Bacillus lactis aërogenes, on the one hand, and the more strictly pathogenic Gram-negative bacilli of the paratyphoid, typhoid, and dysentery groups, on the other. As a type of the group we may consider in detail its most prominent and thoroughly studied member, Bacillus coli communis.

\section{BACILLUS COLI COMMUNIS}

This microorganism was seen and described by Buchner ${ }^{1}$ in 1885 . It was thoroughly studied in the years immediately following, especially by Escherich, ${ }^{2}$ in connection with the intestinal contents of infants.

Morphology.-Bacillus coli communis is a short, plump rod about 1-3 micra long, and varying in thickness from one-third to one-fifth of its length. Under varying conditions of cultivation, it may appear to be more slender than this or shorter and even coccoid in form. In stained preparations, it usually appears singly, but occasionally may be seen in short chains. It stains readily with the usual anilin dyes and decolorizes by Gram's method. Spores are not formed. It is motile, and fiagella staining reveals eight or more flagella peripherally arranged. Its motility is subject to wide variations. Young cultures, in the first gen-

${ }_{1}$ Buchner, Arch. f. Hyg., 3, 1885.

${ }^{2}$ Escherich, "Die Darmbakt. des Säuglings," Stuttgart, 1886; Cent. f. Bakt., 1, 1887. 
eration, after isolation from the body, may be extremely motile, while old laboratory strains may show almost no motility. Independent of these modifying conditions, however, separate races may show individual characteristics as to motility, varying in range between a motility hardly distinguishable from Brownian movement and one which is so active as to be but little less than that of the typhoid bacillus. Ordinarily, the colon bacillus possesses a motility intermediate between these two extremes.

Cultivation.--The bacillus is an aërobe capable of anaërobic growth under suitable cultural conditions. It grows well on the simplest media

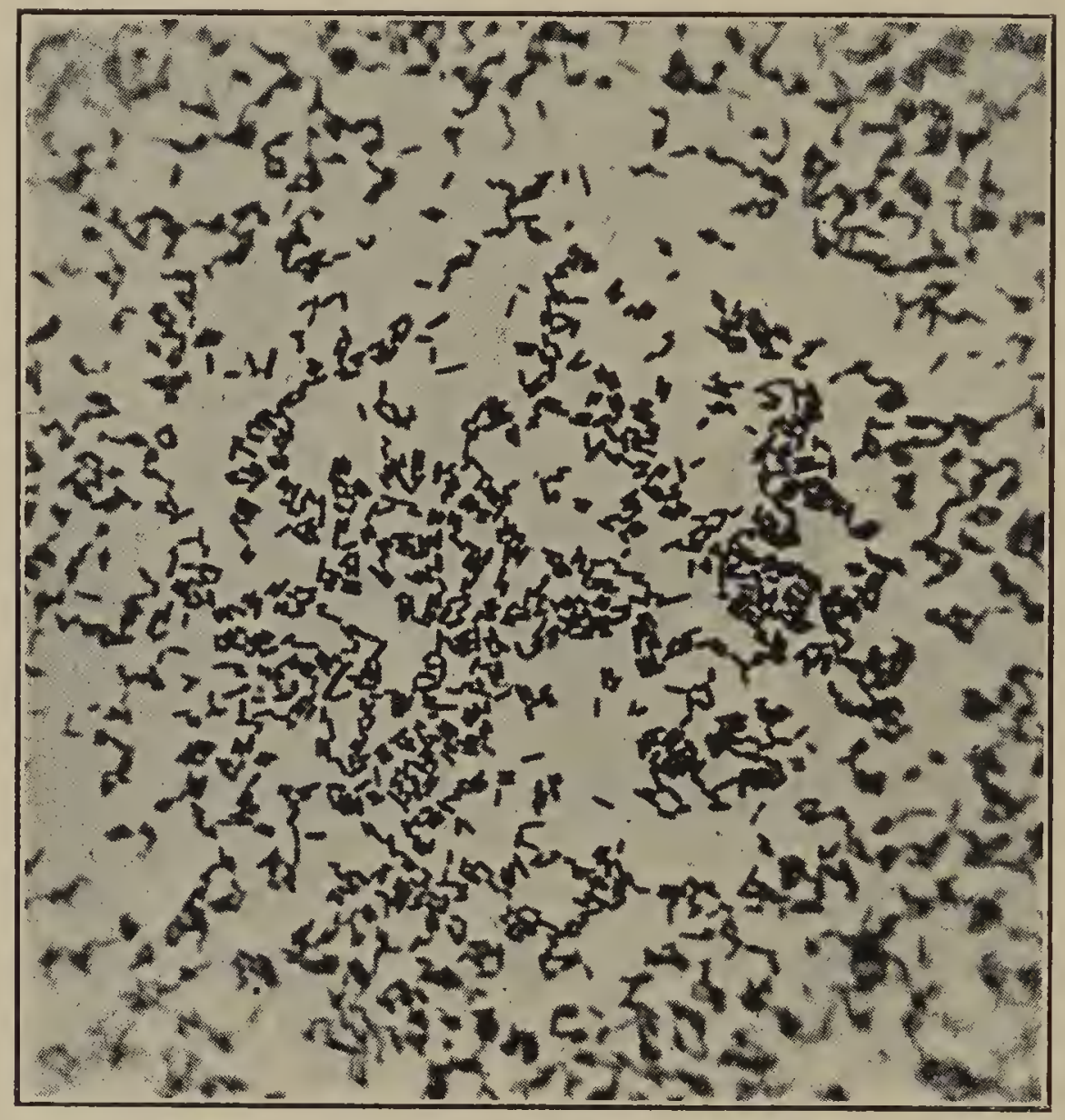

Fig. 83.-Bactllus COLI COMMUNis.

at temperatures ranging from $20^{\circ}$ to $40^{\circ} \mathrm{C}$., but finds its optimum growth at about $37.5^{\circ} \mathrm{C}$. Upon broth it grows rapidly, giving rise to general clouding; later to a pellicle and a light, slightly slimy sediment. Within moderate ranges, it is not delicately susceptible to reaction, growing equally well on media slightly acid and on those of a moderate alkalinity.

Upon agar, it forms grayish colonies which become visible within twelve to eighteen hours, gradually becoming more and more opaque as they grow older. The deep colonies are dense, evenly granular, oval, 
or round. Surface colonies often show a characteristic grape-leaf structure, or may be round and flat, and show a definitely raised, glistening surface. Upon agar slants, growth occurs in a uniform layer.

On gelatin the colon bacillus grows rapidly, causing no liquefaction. Surface colonies are apt to show the typical grape-leaf formation. Deep colonies are round, oblong, and glistening. In gelatin stabs growth takes place along the entire line of inoculation, spreading in a thin layer over the surface of the medium.

On potato, growth is abundant and easily visible within eighteen to twenty-four hours, as a grayish-white, glistening layer which later turns to a yellowish-brown, and in old cultures often to a dirty greenish-brown color.

In pepton solution indol is formed. In milk there is acidity and coagulation. In lactose-litmus-agar acid is formed, the medium becoming red, and gas-bubbles appear along the line of the stab inoculation.

In carbohydrate broth, gas is formed in dextrose, lactose, and mannit, but not in saccharose. Levulose, galactose, and maltose are also fermented with the formation of acid and gas.

Cultures of the colon bacillus are characterized by a peculiar fetid odor which is not unlike that of diluted feces. The acids formed by the colon bacillus from sugars are chiefly lactic, acetic, and formic acids. The gas it produces consists chiefly of $\mathrm{CO}_{2}$ and hydrogen. The bacillus grows well on media containing urine and on those containing bile. Upon the latter fact some methods for the isolation of the colon bacillus from water and feces have been based.

Isolation of the colon bacillus from mixed cultures is most easily accomplished by plating upon lactose-litmus-agar, the Conradi-Drigalski medium, or the Endo medium after preliminary enrichment of the specimen to be tested in bile or malachite-green broth. (In the case of feces such enrichment is superfluous.)

Distribution.-The colon bacillus is a constant inhabitant of the intestinal canal of human beings and animals. It is also found occasionally in soil, in air, in water, and in milk and is practically ubiquitous in all neighborhoods which are thickly inhabited. When found in nature its presence is generally taken to be an indication of contamination from human or animal sources. Thus, when found in water or milk, much hygienic importance is attached to it. Recently, Papasotiriu ${ }^{1}$ and, independently of him, Prescott, ${ }^{2}$ have reported finding bacilli apparently

${ }^{1}$ Papasotiriu, Arçh. f. Hyg., xli. $\quad 2$ Prescott, Cent. f. Bakt., Ref., xxxiii, 1903, 
identical with Bacillus coli upon rye, barley, and other grains. They believe, upon the basis of this discovery, that Bacillus coli is widely distributed in nature and that its presence, unless it appears in large numbers, does not necessarily indicate recent fecal contamination. These reports, however, have not found confirmation by the work of others, and can not, therefore, be as yet accepted.

In man, Bacillus coli appears in the intestine normally soon after birth, at about the time of taking the first nourishment. ${ }^{1}$ From this time on, throughout life, the bacillus is a constant intestinal inhabitant apparently without dependence upon the diet. Its distribution within the intestine, according to Cushing and Livingood, ${ }^{2}$ is not uniform, it being found in the greatest numbers at or about the ileocecal valve, diminishing from this point upward to the duodenum and downward as far as the rectum. Adami ${ }^{3}$ and others claim that, under normal conditions, the bacillus may invade the portal circulation, possibly by the intermediation of leucosytic emigration during digestion. After death, at autopsy, Bacillus coli is often found in the tissues and the blood without there being visible lesions of the intestinal mucous membrane. ${ }^{4}$ It is probable, also, that it may enter and live in the circulation a few hours before death during the agonal stages.

Extensive investigations have been carried out to determine whether or not the constant presence of this microorganism in the intestinal tract is an indication of its possessing a definite physiological function of advantage to its host. It has been argued that it may aid in the fermentation of carbohydrates. The question has been approached experimentally by a number of investigators. Nuttall and Thierfelder ${ }^{5}$ delivered guinea-pigs from the mother by Cesarean section and succeeded in keeping them without infection of the intestinal canal for thirteen days. Although no microorganisms of any kind were found in the feces of these animals, no harm seemed to accrue to them, and some of them even gained in weight. Schottelius, ${ }^{6}$ on the other hand, obtained contradictory results when working with chicks. Allowing eggs to hatch in an especially constructed glass compartment, he succeeded in keeping the

${ }_{1}^{1}$ Schild, Zeit. f. Hyg., xix, 1895; Lembke, Arch. f. Hyg., xxvi, 1896.

${ }^{2}$ Cushing and Livingood, "Contributions to Med. Sci. by Pupils of Wm. Welch," Johns Hopk. Press, 1900.

${ }^{3}$ Adami, Jour. of Amer. Med. Assn., Dec., 1899.

${ }^{4}$ Birch-Hirschfeld, Ziegler's Beitr., 24, 1898.

${ }^{5}$ Nuttall und Thierfelder, Zeit. f. Physiol. Chemie, xxi and xxii,

s Schottelius, Arch. f. Hyg, xxxiv, 1889. 
chicks and their entire environment sterile for seventeen days. During. this time they lost weight, did not thrive, and some of them were moribund at the end of the second week, in marked contrast to the healthy, well-nourished controls, fed in the same way, but under ordinary environmental conditions. Although insufficient work has been done upon this important question, and no definite statement can be made, it is more than likely that the function of the Bacillus coli in the intestine is not inconsiderable if only because of its possible antagonism to certain putrefactive bacteria, a fact which has been demonstrated in interesting studies by Bienstock ${ }^{1}$ and others. ${ }^{2}$

Pathogenicity. - The pathogenicity of the colon bacillus for animals is slight and varies greatly with different strains. Intraperitoneal injections of 1 c.c. or more of a broth culture will often cause death in guinea-pigs. Large doses intravenously administered to rabbits may frequently cause a rapid sinking of the temperature and death with symptoms of violent intoxication within twenty-four to forty-eight hours. Subcutaneous inoculation of moderate doses usually results in nothing more than a localized abscess from which the animals recover. It is likely that, even in fatal cases, death results chiefly from the action of poisons liberated from the disintegrating bacteria and not from the multiplication of the bacilli themselves, for often no living organism can be found unless large doses are given.

In man, a large variety of lesions produced by Bacillus coli have been described. It is a surprising fact that disease should be caused at all, in man, by a microorganism which is so constantly present in large numbers in the intestine and against which, therefore, it is to be expected that a certain amount of immunity should be developed. A number of explanations for this state of affairs have been advanced, none of them entirely satisfactory. It is probable that none of the poisonous products of the colon bacillus is absorbed unchanged by the healthy unbroken mucosa and that, therefore, the microorganisms are, strictly speaking, at all times, outside of the body proper. Under these circumstances, no process of immunization would be anticipated. It is also possible that, whenever an infection with Bacillus coli does occur, the infecting organism is one which has been recently acquired from another host, having no specific adaptation to the infected body. Virulence may possibly be enhanced by inflammatory processes caused by other organisms. Considering the subject from another point of view,

1 Bienstock, Arch. f. Hyg., xxxix, 1901.

${ }^{2}$ Tissier and Martelly, Ann. de l'inst. Pasteur, 1902. 
colon-bacillus infection may possibly take place simply because of unusual temporary reduction of the resistance of the host. Whether or not altered cultural conditions in the intestine may lead to marked enhancement in the virulence of the colon bacilli can not at present be decided. The opinion has been frequently advanced, however, without adequate experimental support.

Septicemia, due to the colon bacillus, has been described by a large number of observers. It is doubtful, however, whether many of these cases represent an actual primary invasion of the circulation by the bacilli, or whether their entrance was not simply a secondary phenomenon occurring during the agonal stages of another condition. A few unquestionable cases, however, have been reported, and there can be no doubt about the occurrence of the condition, although it is probably less frequent than formerly supposed. The writers have observed it on two occasions in cases during the lethal stages of severe systemic disease due to other causes. An extremely interesting group of such cases are those occurring in new-born infants, in which generalized colon-bacillus infection may lead to a fatal condition known as Winckel's disease or hemorrhagic septicemia. ${ }^{1}$ Prominent among disease processes attributed to these microorganisms are various diarrheal conditions, such as cholera nostras and cholera infantum. The relation of these maladies to the colon bacillus has been studied especially by Escherich, ${ }^{2}$ but satisfactory evidence that these bacilli may specifically cause such conditions has not been brought. While it is not unlikely that under conditions of an excessive carbohydrate diet, colon bacilli may aggravate morbid processes by a voluminous formation of gas, they do not, of themselves, take part in actual putrefactive processes. It is likely, therefore, that in most of the intestinal diseases formerly attributed purely to bacilli of the colon group, these microorganisms actually play but a secondary part. ${ }^{3}$

It is equally difficult to decide whether or not these bacilli may be regarded as the primary cause of peritonitis following perforation of the gut. Although regularly found in such conditions, they are hardly ever found in pure culture, being accompanied usually by staphylococci, streptococci, or other microorganisms, whose relationship to disease is far more definitely established. Isolated cases have been reported, however, one of them by Welch, in which Bacillus coli was present in

${ }^{1}$ Kamen, Ziegler's Beitr., 14, 1896.

${ }^{2}$ Escherich, loc. cit.

${ }^{3}$ Herter, "Bact. Infec. of Digest. Tract," N. Y., 1907. 
the peritoneum in pure culture without there having been any intestinal perforation. 'Granting that the bacillus is able to proliferate within the peritoneum, there is no reason for doubting its ability of giving rise to a mild suppurative process.

Inflammatory conditions in the liver and gall-bladder have frequently been attributed to the colon bacillus. It has been isolated from liver abscesses, from the bile, and from the center of gall-stones. Welch has reported a case of acute hemorrhagic pancreatitis in which the bacillus was isolated from the gall-bladder and from the pancreas.

In the bladder, Bacillus coli frequently gives rise to cystitis and occasionally to ascending pyonephrosis. No other microorganism, in fact, is found so frequently in the urine as this one. It may be present, often, in individuals in whom all morbid processes are absent. The condition is frequently observed during the convalescence from typhoid fever. It may disappear spontaneously, or cystitis, usually of a mild, chronic variety, may supervene.

Localized suppurations due to this bacillus may take place in all parts of the body. They are most frequently localized about the anus and the genitals. They are usually mild and easily amenable to the simplest surgical treatment.

Poisonous Products of the Colon Bacillus.-The colon bacillus belongs essentially to that group of bacteria whose toxic action is supposed to be due to the poisonous substances contained within the bacillary body. Culture filtrates of the colon bacillus show very little toxicity when injected into animals; whereas the injection of dead bacilli produces symptoms almost equal in severity to those induced by injection of the live microorganisms. Corroborative of the assumption of this endotoxic nature of the colon-bacillus poison is the fact that, so far, no antitoxic bodies have been demonstrated in serum as resulting from immunization.

Immunization with the Colon Bacillus.-The injection into animals of gradually increasing doses of living or dead colon bacilli gives rise to specific bacteriolytic, agglutinating, and precipitating substances.

The bacteriolytic substances may be easily demonstrated by the technique of the Pfeiffer reaction. In vitro bacteriolysis is less marked than in the case of some other microorganisms such as the cholera spirillum or the typhoid bacillus. Owing probably to the habitual presence of colon bacilli in the intestinal tracts of animals and man, considerable 
bacteriolysis may occasionally be demonstrated in the serum of normal individuals.

Agglutinins for the colon bacillus have often been produced in the sera of immunized animals in concentration sufficient to be active in dilutions of $1: 5,000$ and over. The agglutinins are produced equally well by the injection of live cultures and of those killed by heat, if the temperature used for sterilization does not exceed $100^{\circ} \mathrm{C}$. It is ${ }^{1}$ a notice-

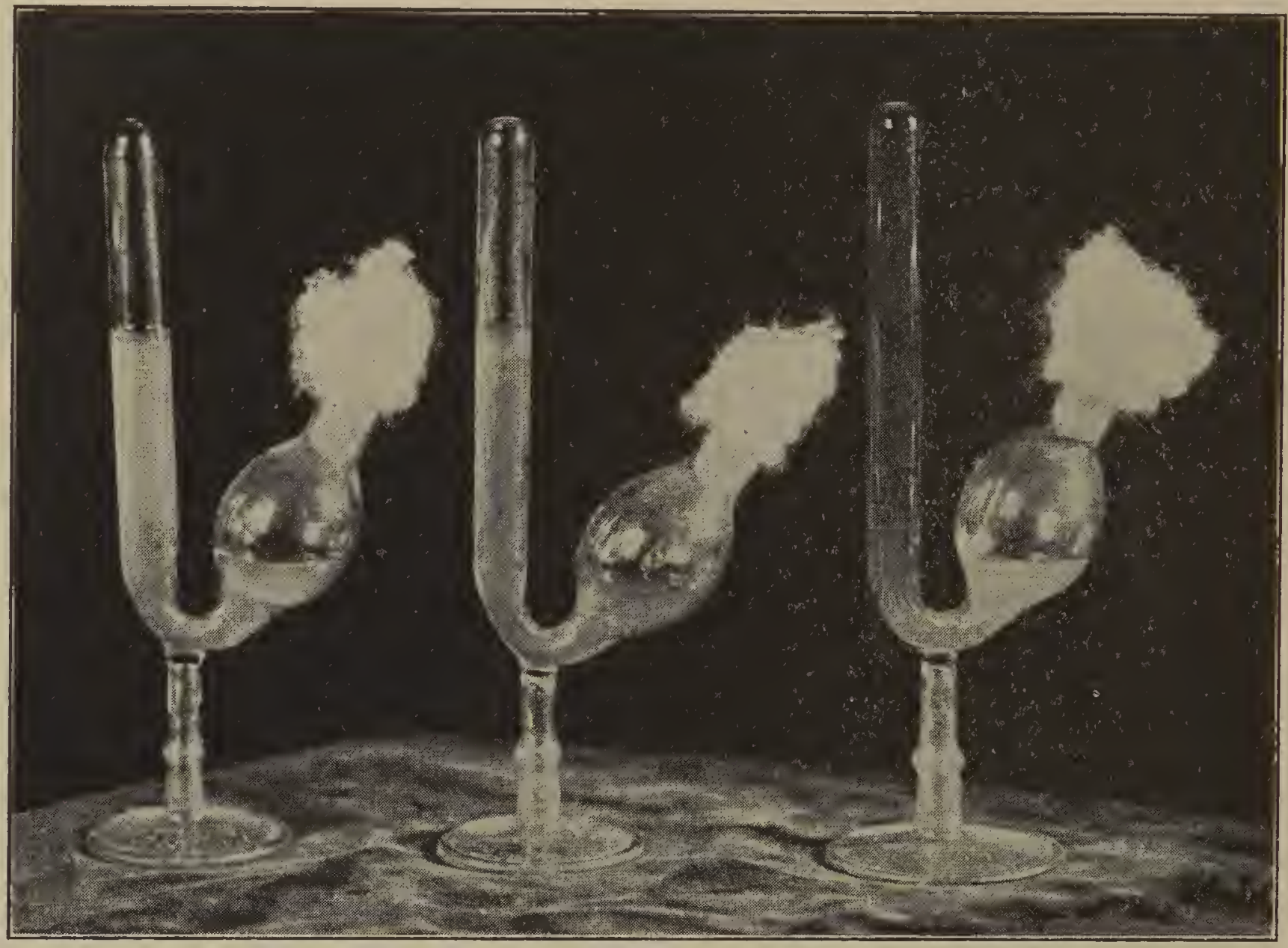

1

2

3

Fig. 84.-Bacillus coli communis. Grown in: 1. Dextrose, 2. Lactose, 3. Saccharose broth. The bacillus forms acid and gas from dextrose and lactose, not from saccharose. Note the absence of growth in the closed arm of the saccharose tube, in which no acid or gas is formed.

able fact that the injection of any specific race of colon bacilli produces, in the immunized animal, high agglutination values only for the individual culture used for immunization, while other strains of colon bacilli, although agglutinated by the serum in higher dilution than are paratyphoid or typhoid bacilli, require much higher concentration than does the original strain. The subject has been extensively studied by a number of observers and illustrates the extreme individual 
specificity of the agglutination reaction. Thus a serum which will agglutinate its homologous strains in dilutions of one 1:1,000 will often fail to agglutinate other races of Bacillus coli in dilutions of $1: 500$ or $1: 600$.

The normal serum of adult animals and man will often agglutinate this bacillus in dilutions as high as $1: 10$ or $1: 20$-a phenomenon possibly referable to its habitual presence within the body. Corroborating this assumption is the observation of Kraus and Löw, that the serum of new-born animals possesses no such agglutinating powers. The fact that agglutinins for the colon bacillus are increased

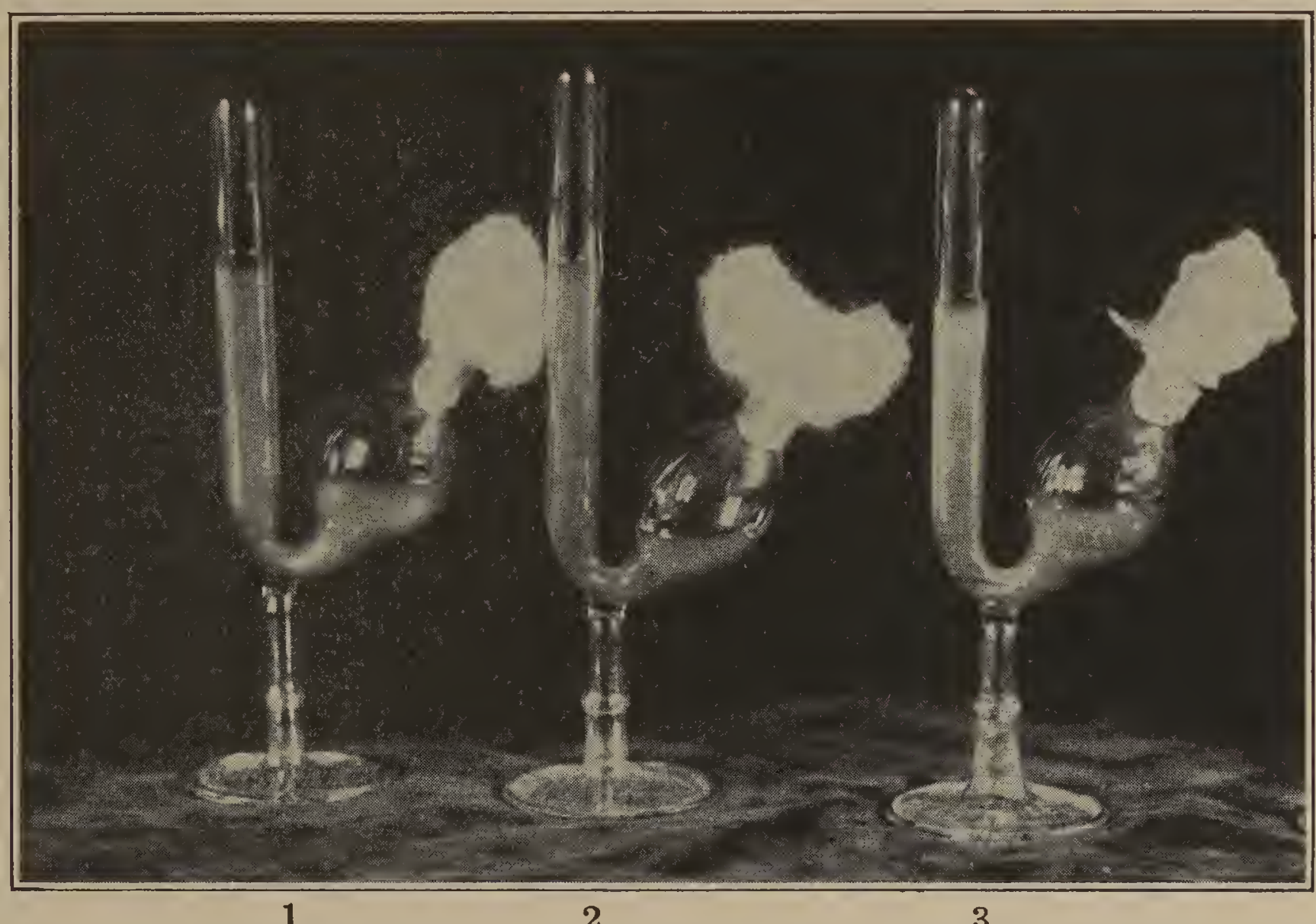

1

2

3

Fig. 85.-Bacillus coli communior. Grown in: 1. Dextrose, 2. Lactose, 3. Saccharose broth.

in the serum of patients convalescing from typhoid fever or dysentery is probably to be explained, partly by the increase of the group agglutinins produced by the specific infecting agent, and partly by the invasion of colon bacilli, or the absorption of its products induced by the diseased state of the intestinal mucous membrane.

Varieties of the Colon Bacillus.-During the earlier days of bacteriological investigations, a large number of distinct varieties of colon bacilli were described, many of which may now be dismissed as based simply 
upon a temporary depression of one or another cultural characteristic of Bacillus coli communis, while others can be definitely included within other closely related, but distinct groups.

That secondary features, such as dimensions, motility, and luxuriance of growth upon various media, may be markedly altered by artificial cultivation is a common observation. It has not, however, been satisfactorily shown that cardinal characteristics, such as the formation of indol from pepton, or the power to produce gas from dextrose and lactose, can be permanently suppressed without actual injury or inhibition of the normal vitality of the microolganism. Such alteration is, in fact, contrary to experience, which demonstrates that whenever such changes do occur, they are purely temporary and a few generations of cultivation under favorable environmental conditions will regularly restore the organism to its normal activity.

Bacillus coli communior.-Distinct and constant varieties of Bacillus coli, however, do occur. The most common of these is one which Dunham has named Bacillus coli communior, because of the fact that he believes it to be more abundant in the human and animal intestine than is coli communis itself. This bacillus possesses all the cardinal characteristies of the colon group. It is a Gram-negative bacillus, moderately motile, non-sporulating, and morphologically indistinguishable from the communis variety. It does not liquefy gelatin, it produces indol from pepton, coagulates and acidifies milk, and grows characteristically upon agar and potato. It differs from Bacillus coli communis in that it produces acid and gas from saccharose as well as from dextrose and lactose, whereas the former does not form acid or gas from saccharose. 


\section{CHAPTER XX'VIT}

\section{BACILLI OF THE COLON-TYPHOID-DYSENTERY GROUP (Continued)}

\section{THE BACILLUS OF TYPHOID FEVER}

(Bacillus typhosus, Bacillus typhi abdominalis)

TyPHoID fever, because of its wide distribution and almost constant presence in most communities, has from the earliest days been the subject of much etiological inquiry. A definite conception as to its infectiousness and transmission from case to case was formed as early as 1856 by Budd. ${ }^{1}$

But it was not until 1880 that Eberth ${ }^{2}$ discovered in the spleen and mesenteric glands of typhoid-fever patients who had come to autopsy, a bacillus which we now know to be the cause of the disease. Final proof of such an etiological connection was then brought by Gaffky, ${ }^{3}$ who not only saw the bacteria referred to by Eberth, but succeeded in obtaining them in pure culture and studying their growth characteristics.

Morphology and Staining.- The typhoid bacillus is a short rod from 1-3.5 $\mu$ in length with a varying width of from .5 to $.8 \mu$. In appearance it has nothing absolutely distinctive which could serve to differentiate it from other bacilli of the typhoid-colon group, except that it has a general tendency to greater slenderness. Its ends are rounded without ever being club-shaped. Contrary to the descriptions of the earliest observers, typhoid bacilli do not form spores. They are actively motile and have twelve or more flagella peripherally arranged.

- The bacilli stain readily with the usual anilin dyes. Stained by Gram's method, they are decolorized.

Cultivation.-Bacillus typhosus is easily cultivated upon the usual laboratory media. It is not delicately susceptible to reaction, but will grow well upon media moderately alkaline or acid. It is an aërobic and facultative anaërobic organism, when the proper nutriment is present. Upon agar plates growth appears within eighteen to twenty-four hours

${ }^{1}$ Budd, "Intestinal Fever," Lancet, 1856.

2 Eberth, Virch Archiv, 81, 1880, and 83, 1881.

${ }^{3}$ Gaffky, Mitt. a. d. kais. Gesundheitsamt, 2, 1884. 
as small grayish colonies at first transparent, later opaque. Upon agar slants growth takes place in a uniform layer. There is nothing characteristic about this growth to aid in differentiation.

In broth, the typhoid bacillus grows rapidly, giving rise to an even clouding, rarely to a pellicle.

Upon gelatin, the typhoid bacillus grows readily and does not liquefy the medium. In stabs, growth takes place along the entire extent of the stab and over the surface of the gelatin in a thin layer. In gelatin plates the growth may show some distinction from that of other members of this group, and this medium was formerly much used for isolation

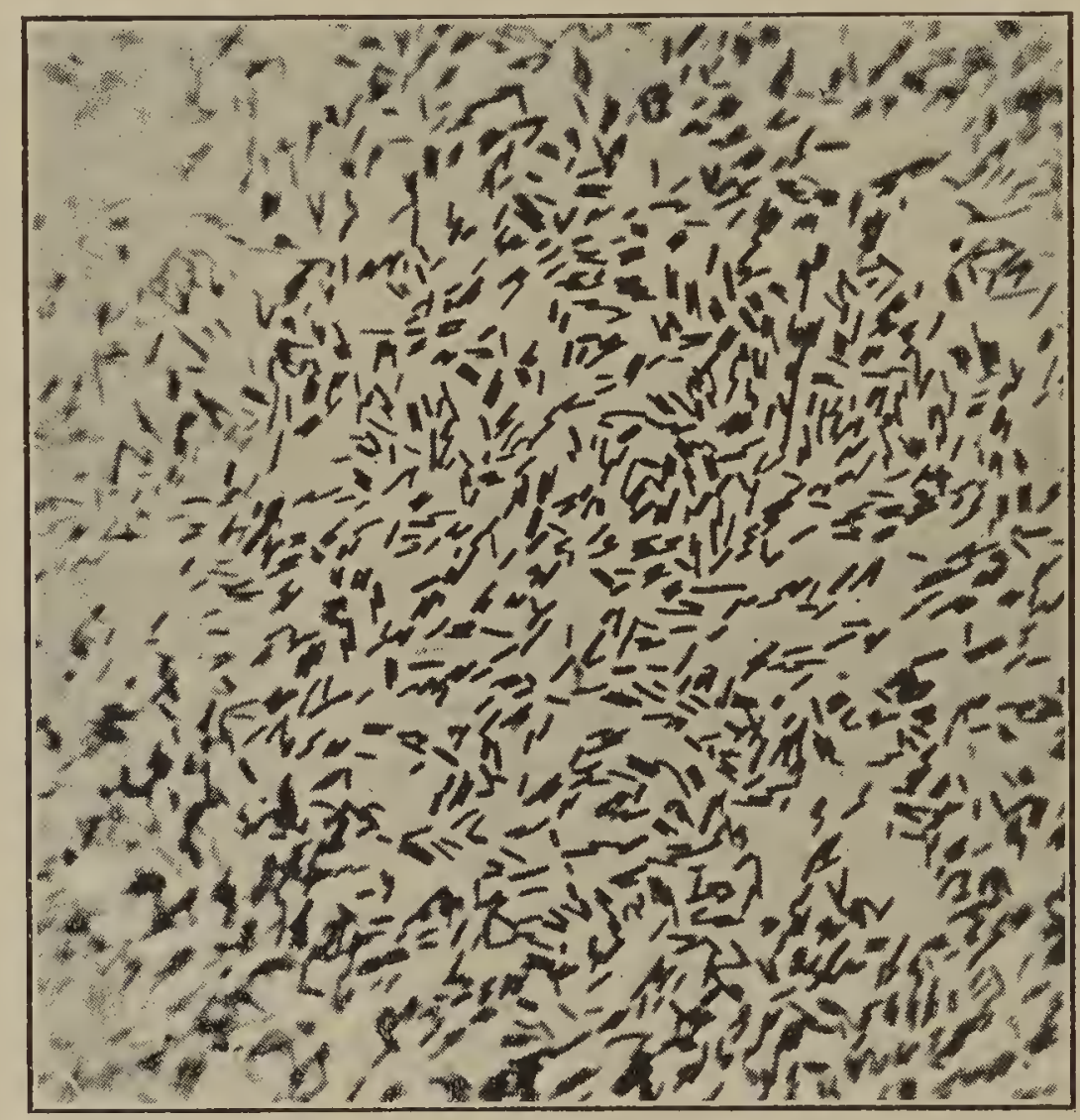

Fig. 86.-Bacillus typhosus, from twenty-four-hour culture on agar.

of the bacillus from mixed cultures. Growth appears within twentyfour hours as small, transparent, oval, round, or occasionally leaf-shaped colonies which are smaller, more delicate, and more transparent than contemporary colonies of the colon bacillus. They do not, however, show any reliable differential features from bacilli of the dysentery group. As the colonies grow older they grow heavier, more opaque, and lose much of their early differential value.

On potato the growth of typhoid bacilli is distinctive, and this medium was recommended by Gaffky ${ }^{1}$ in his early researches for purposes of 
identification. On it typhoid bacilli, after twenty-four to forty-eight hours, produce a hardly visible growth, evident to the naked eye only by a slight moist glistening, an appearance which is in marked contrast to the grayish-yellow or even brown and abundant growth of the colon bacilli. If the potato medium is rendered neutral or alkaline, this distinction disappears, the typhoid bacillus growing more abundantly.

In milk, typhoid bacilli do not produce coagulation. In litmus-milk, during the first twenty-four hours, the color is changed to a reddish or violet tinge by the formation of acid from the small quantities of mono-

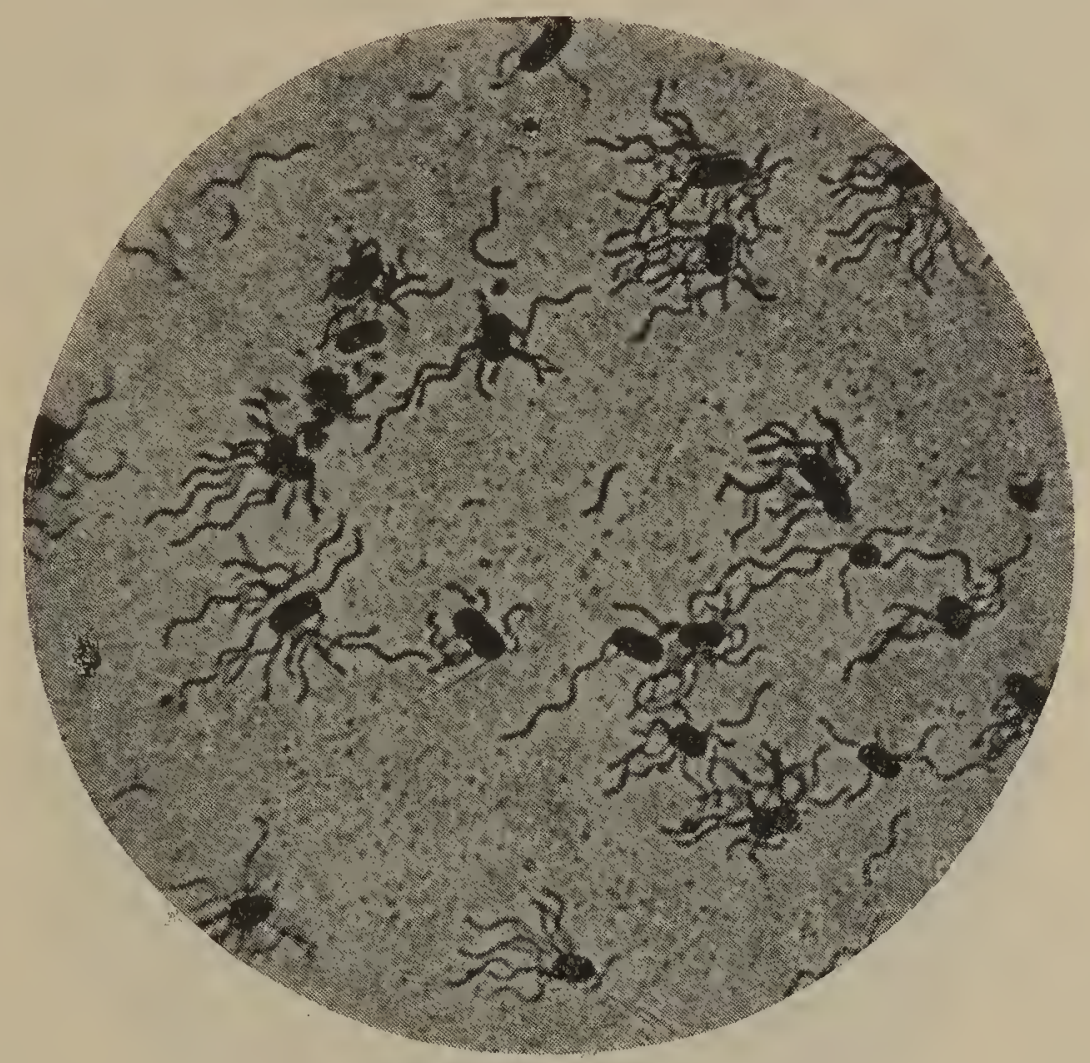

Fig. 87.-Bacillus typhosus, showing flagella. (After Fränkel and Pfeiffer.)

saccharid present. Later the color becomes deep blue from the formation of alkali.

In Dunham's pepton solution no indol is produced. According to Peckham, however, continuous cultivation in rich pepton media may lead to eventual indol formation by typhoid bacilli. This fact appears to have no bearing on the value of the indol test, as indol is never formed under the usual cultural conditions.

In dextrose, mannite, lactose, and saccharose broth, the typhoid bacillus produces no gas. A comparative summary of the action of other bacilli of this group in these sugar media will be given in the final differential table on page 443 . 
Tested for its power to form acid from sugars commonly used in differential tests, typhoid bacilli give the following reactions:

\begin{tabular}{lc||lc}
\multicolumn{2}{r}{ Acid formation } & \multicolumn{2}{r}{ Acid formation } \\
Dextrose & + & Maltose & + \\
Levulose & + & Lactose & - \\
Galactose & + & Saccharose & - \\
Mannit & + & Dextrin & +
\end{tabular}

In the Hiss tube medium (see section on Media, page 133), the typhoid bacillus within eighteen to twenty-four hours produces an even clouding by virtue of its motility, but does not form gas. In contradistinction to this, dysentery bacilli grow only along the line of inocula-

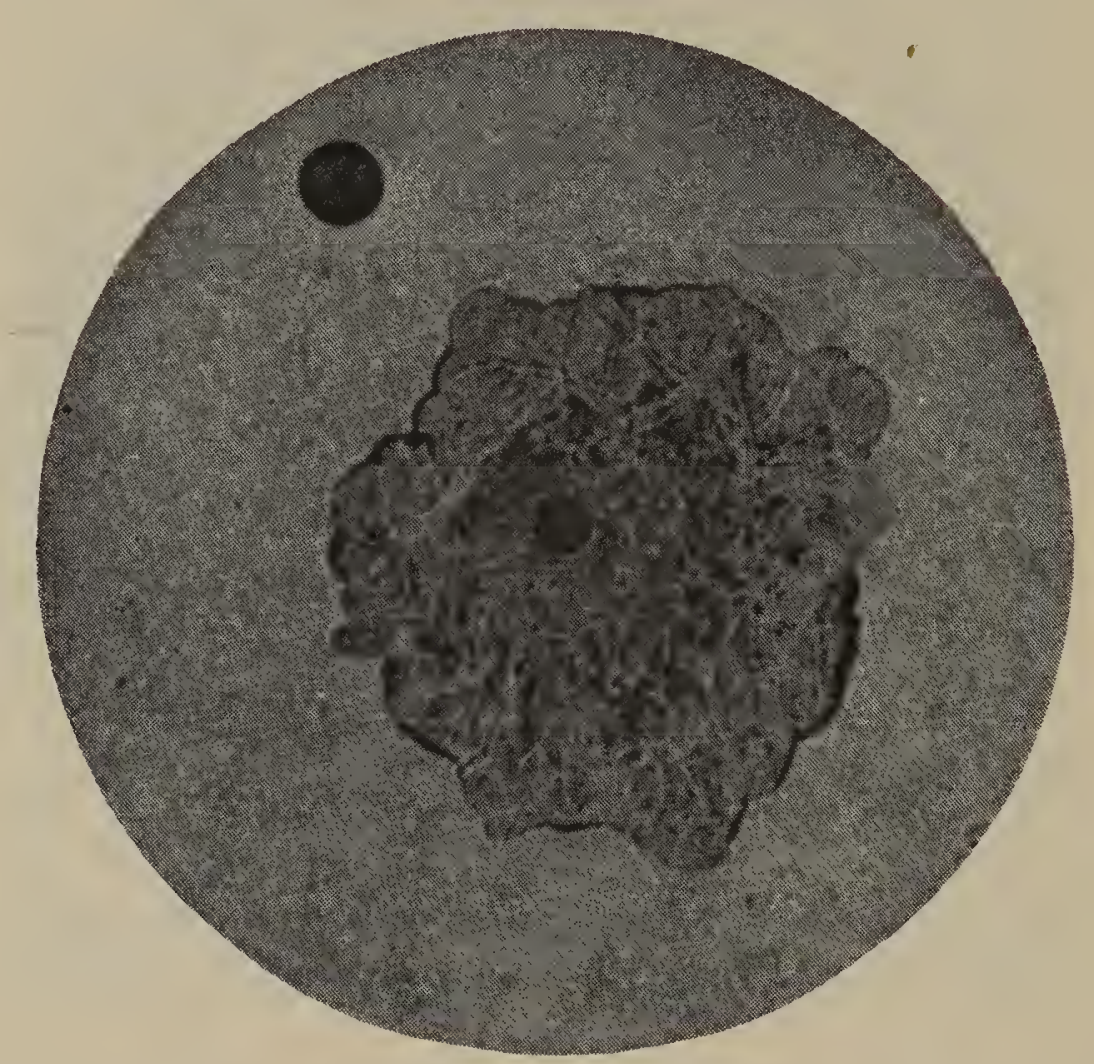

Fig. 88. Surface Colony of Bacillus typhosus on Gelatin. (After Heim.)

tion, while bacilli of the colon group move in irregular sky-rocket-like figures away from the stab, at the same time breaking up the medium by the formation of gas-bubbles. Some actively motile colon bacilli cloud the medium, but the ruptures caused by the gas are always evident.

The differentiation of the typhoid bacillus in pure culture from similar microorganisms by means of its growth upon media has been the subject of many investigations. It is neither practicable nor desirable to enumerate all the various media which have been devised and reported. 
The aim has been chiefly the differentiation of typhoid bacilli from the bacilli of the colon group, and most of the media have been devised with this end in view. (See section on Media.)

Rothberger ${ }^{1}$ has devised a mixture of glucose agar to which is added one per cent of a saturated aqueous solution of neutral-red. Shake-cultures or stab-cultures are made in tubes of this medium. The typhoid bacillus causes no changes in it, while members of the colon group, by reduction of the neutral-red, decolorize the medium and produce gas by fermentation of the sugar.

Utilizing the fact that bile-salts are precipitated in the presence of acids, Macconkey devised a medium composed of sodium glycocholate, pepton, lactose, and agar (the composition of this medium is given on page 138), in which Bacillus typhosus grows without causing much change, but distinct clouding results from the growth of the colon bacillus which, producing acid from the lactose, causes precipitation of the bilesalts.

On Wurtz's lactose-litmus-agar (see page 129) the typhoid bacillus produces no acid, but eventually deepens the purple color to blue; the colon bacillus produces acid and in stab-cultures gas bubbles and the color changes to red.

In Barsiekow's (see page 139) lactose-nutrose-litmus mixture the typhoid bacillus causes no change, while the colon bacillus produces coagulation and an acid reaction.

Especially designed for the isolation of typhoid bacilli from the feces, are the media of Drigalski and Conradi, the agar-gelatin media of Hiss, the medium of Hesse, the fuchsin medium of Endo, and the malachite-green media of Loeffler, and others. These media have all been described in detail in the section on the preparation of media, pages $133-138$.

Biological Considerations.-The typhoid bacillus is an aërobic and facultatively anaërobic organism growing well both in the presence and in the absence of oxygen when certain sugars are present, showing a slight preference, however, for well aërated conditions. It grows most luxuriantly at temperatures about $37.5^{\circ} \mathrm{C}$., but continues to grow within a range of temperature lying between $15^{\circ}$ and $41^{\circ} \mathrm{C}$. Its thermal death point, according to Sternberg, is $56^{\circ} \mathrm{C}$. in ten minutes. It remains alive in artificial cultures for several months or even years if moisture is supplied. In carefully sealed agar tubes Hiss has found the organisms

${ }^{1}$ Rothberger, Cent. f. Bakt., xxiv, 1898. 
alive after thirteen years. In natural waters it may remain alive as long as thirty-six days, according to Klein. ${ }^{1}$ In ice, according to Prudden, ${ }^{2}$ it may remain alive for three months or over. Against the ordinary disinfectants, the typhoid bacillus is comparatively more resistant than some other vegetative forms. It is killed, however, by $1: 500$ bichlorid or five-per-cent carbulic acid within five minutes.

Pathogenicity.--In animals, some early investigators to the contrary, typhoidal infection does not occur spontaneously and artificial inoculation with the typhoid bacillus does not produce a disease analogous to typhoid fever in the human being. Fränkel ${ }^{3}$ was able to produce intestinal lesions in guinea-pigs by injection of the bacilli into the duodenum, and recovered the bacteria from the spleens of the animals after death, but the disease produced was in no other respect analogous to typhoid fever in the human being. It is probable that typhoid bacilli injected into animals do not multiply extensively and that most of the symptoms produced are due to the endotoxins liberated from the dead bacteria. In corroboration of this view is the observation that inoculation with dead cultures is followed by essentially the same train of symptoms as inoculation with live cultures. ${ }^{4}$ The injection of large doses into rabbits or guinea-pigs intravenously or intraperitoneally is usually followed by a rapid drop in temperature, often by respiratory embarrassment and diarrhea. Occasionally blood may be present in the stools. According to the size of the dose or the weight of the animal, death may ensue within a few hours, or, with progressive emaciation, after a number of days, or the animal may gradually recover. Welch and Blachstein ${ }^{5}$ have shown that typhoid bacilli injected into the ear vein of a rabbit appear in the bile and may persist in the gallbladder for weeks. Typhoid bacilli isolated from different sources may show considerable variations in virulence and toxicity.

Doerr, ${ }^{6}$ Koch, ${ }^{7}$ Morgan, ${ }^{8}$ and more recently Johnston ${ }^{9}$ have all confirmed this, the last named showing that the typhoid bacillus could

${ }^{1}$ Klein, Med. Officers' Report, Local Govern. Bd., London, 1894.

${ }^{2}$ Prudden, Med. Rec., 1887.

${ }^{3}$ Fränkel, Cent. f. klin. Med., 10, 1886.

${ }^{4}$ Petruschky, Zeit. f. Hyg., xii, 1892.

${ }^{5}$ Welch and Blachstein, Bull. Johns Hop. Hosp., ii, 1891.

${ }^{6}$ Doerr, Centralbl. f. Bakt., 1905.

${ }^{7}$ Koch, Zeitschr. f. Hyg., 1909.

8 Morgan, Jour. of Hyg., 1911.

${ }^{9}$ Johnston, Jour. of Med. Res., xxvii, 1912. 
not only remain latent for a long time in the gall-bladder of rabbits, but would appear in the blood stream with considerable regularity after the seventh or ninth day, and persist for as long as 125 days. Gay and Claypole ${ }^{1}$ have been able to produce the carrier state in rabbits with great regularity by growing the typhoid cultures used for inoculation upon agar containing 10 per cent defibrinated rabbit's blood. Such cultures are not as readily agglutinated by immune serum as are those grown on plain agar, and it may well be that they have acquired a certain degree of resistance to the serum antibodies which renders them more competent to survive in the body of the rabbit. Gay has used rabbits inoculated with such cultures for the determination of the efficacy of his sensitized vaccines.

In man the overwhelming majority of typhoid infections take the form of the disease clinically known as typhoid fever. For a description of the clinical course and pathological lesions of the disease, the reader is referred to the standard text-books of medicine and pathology. During the course of the disease, and during convalescence, the bacilli may be cultivated from the circulating blood, the rose spots, the feces, the urine, and in exceptional cases from the sputum. At autopsy the bacilli may be obtained from these sources as well as from the lesions in the intestine, the spleen, and often from the liver, kidneys, and from the gall-bladder.

Though formerly regarded as primarily an intestinal disease, recent investigations have brought convincing proof that the disease is in its inception actually a bacteriemia. It is not unlikely that the intestinal lesions are largely the result of toxic products which are excreted through the intestinal wall.

Typhoid Bacilli in the Blood during the Disease.-The investigations of many workers have shown that typhoid bacilli are present in the circulating blood of practically all patienis during the early weeks of the disease. Series of cases have been studied by Castellani, ${ }^{2}$ Schottmüller, ${ }^{3}$ and many others. More recently Coleman and Buxton ${ }^{4}$ have reported their researches upon 123 cases, and have at the same time analyzed all cases previously reported. Their analysis of blood cultures taken at different stages in the disease is as follows:

${ }^{1}$ Gay and Claypole, Arch. of Inf. Med., Dec., 1913.

${ }^{2}$ Castellani, Riforma medica, 1900.

${ }^{3}$ Schottmueller, Deut. med. Woch., xxxii, 1900, and Zeit. f. Hyg., xxxvi, 1901.

${ }^{4}$ Coleman and Buxton, Am. Jour. of Med. Sci., 133, 1907. 
Of 224 cases during first week, 89 per cent were positive.

Of 484 cases during second week, 73 per cent were positive.

Of 268 cases during third week, 60 per cent were positive.

Of 103 cases during fourth week, 38 per cent were positive.

Of 58 cases after fourth week, 26 per cent were positive.

The technique recommended by Coleman and Buxton for obtaining blood cultures is that recommended by Conradi, ${ }^{1}$ slightly modified. The blood is taken into flasks each containing about 20 c.c. of the following mixture:

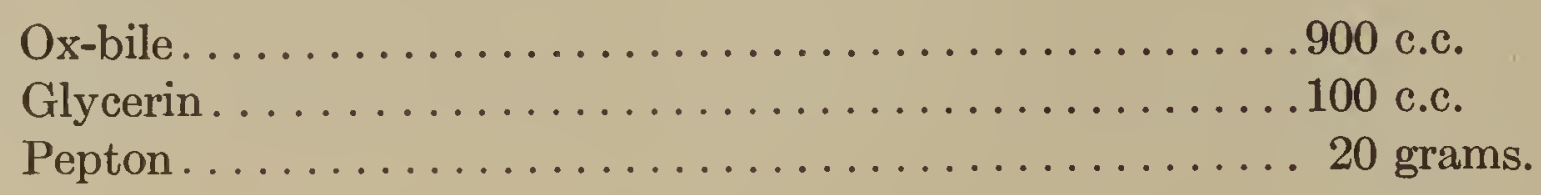

About 3 c.c. of blood are put into each flask. The ox-bile, besides preventing coagulation, may possibly neutralize the bactericidal substances present in the drawn blood. The flasks are incubated for eighteen to twenty-four hours, at the end of which time streaks are made upon plates of lactose-litmus-agar and the organisms identified by agglutination or by cultural tests.

European workers have generally preferred to make high dilution of the blood in flasks of bouillon, small quantities of blood, 1 to 2 c.c., being mixed with 100 to 150 c.c. of nutrient broth.

Epstein ${ }^{2}$ has reported excellent results from mixing the blood in considerable concentration with two-per-cent glucose agar and pouring plates.

The writers in hospital work have had equally good results with the bile medium and with broth in flasks, rather less uniform but still satisfactory results with the plating method. In general it may be said that any one of these methods carried out with reasonable accuracy may be satisfactorily employed.

Typhoid Bacilli in the Stools. - The examination of the stools for typhoid bacillus is performed for diagnostic purposes chiefly in obscure cases. It may, furthermore, furnish information of extreme hygienic importance. Thus Drigalski ${ }^{3}$ and Conradi have succeeded in isolating typhoid bacilli from the stools o ambulant cases so mild that they were not clinically suspected. It 1s py means of such examinations that the so-called typhoid-carriers are detected, cases which,

${ }^{1}$ Conradi, Deut. med. Woch., xxxii, 1906.

${ }^{2}$ Epstein, Proc. N. Y. Path. Soc., N. S., vi, 1906.

${ }^{3}$ Drigalski and Conradi, Zeit. f. Hyg., xxxix, 1902. 
though perfectly well themselves, may be a means of spreading the disease. Such cases have been known to harbor the bacilli for periods as long as several years.

The examination itself is fraught with great difficulties, owing to the preponderating numbers of colon bacilli found in all feces and the difficulty of isolating the typhoid bacilli from such mixtures.

Reviewing the data collected by a number of investigators, it seems probable that the bacilli do not appear in the stools, at least in numbers sufficient for recognition, much before the middle of the second week, or, in other words, as pointed out by Hiss, about the time that the intestinal lesions are well advanced and ulceration is occurring. Thus Wiltschour ${ }^{1}$ could not determine their presence before the tenth day; Redtenbacher, ${ }^{2}$ in reviewing the statistics, states that in a majority of cases the bacilli first appear toward the end of the second week, and Horton-Smith ${ }^{3}$ could not find the bacilli before the eleventh day. Hiss, ${ }^{4}$ in an investigation of the same subject, obtained the following results:

First to tenth day, inclusive, twentyeight cases examined; typhoid bacilli isolated from three; percentage of positive cases

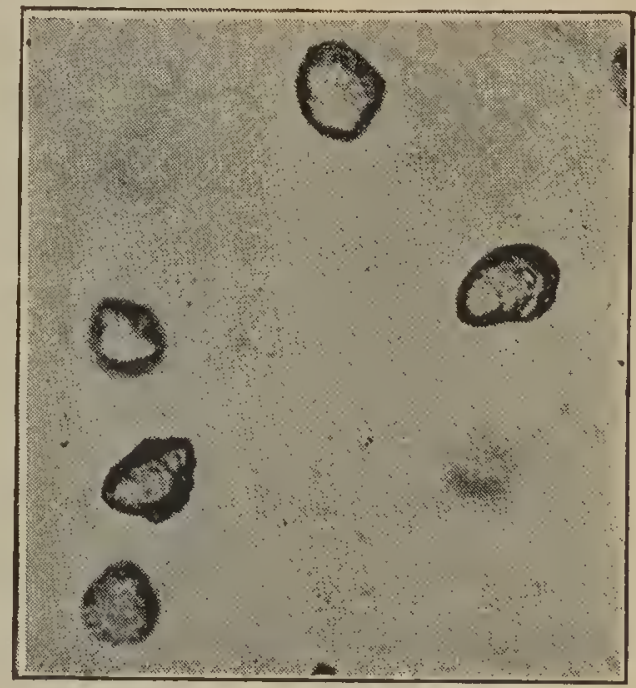

Fig. 89.-BAcillú Coli. Deep colonies on Hiss plate medium. 10.7 per cent.

Eleventh to twentieth day, inclusive, forty-four cases examined; typhoid bacilli from twenty-two; percentage of positive cases 50 per cent.

Twenty-first day to convalescence, sixteen cases examined; typhoid bacilli isolated from thirteen; percentage of positive cases 81.2 per cent.

The difficulties encountered in such examinations have led to the development of a large number of methods. The first method which yielded successful results was that of Elsner, ${ }^{5}$. who employed a potatoextract gelatin containing one per cent of potassium iodid, a medium which prevented the growth of many intestinal bacteria, allowing only

1 Wiltschour, Cent. f. Bakt., 1890.

${ }^{2}$ Redtenbacher, Zeit. f. klin. Med., xix, 1891.

${ }^{3}$ Horton-Smith, Lancet, May, 1899.

1 Hiss, Med. News, May, 1901.

5 Elsner, Zeit. f. Hyg., xxi, 1895. 
colon, typhoid, and a few others to develop. This medium is at present rarely used.

Hiss ${ }^{1}$ has employed with success an agar-gelatin mixture containing one per cent of glucose, the preparation of which has been described in detail in the section on media. The actual technique of the test is as follows: One to two loopfuls of feces are transferred to a tube of broth, making the broth fairly cloudy. From this emulsion five or six plates are made by transferring in series one to five loopfuls of the emulsion to tubes containing the melted plate medium, and then pouring the contents of these tubes into Petri dishes. These dishes, after the medium

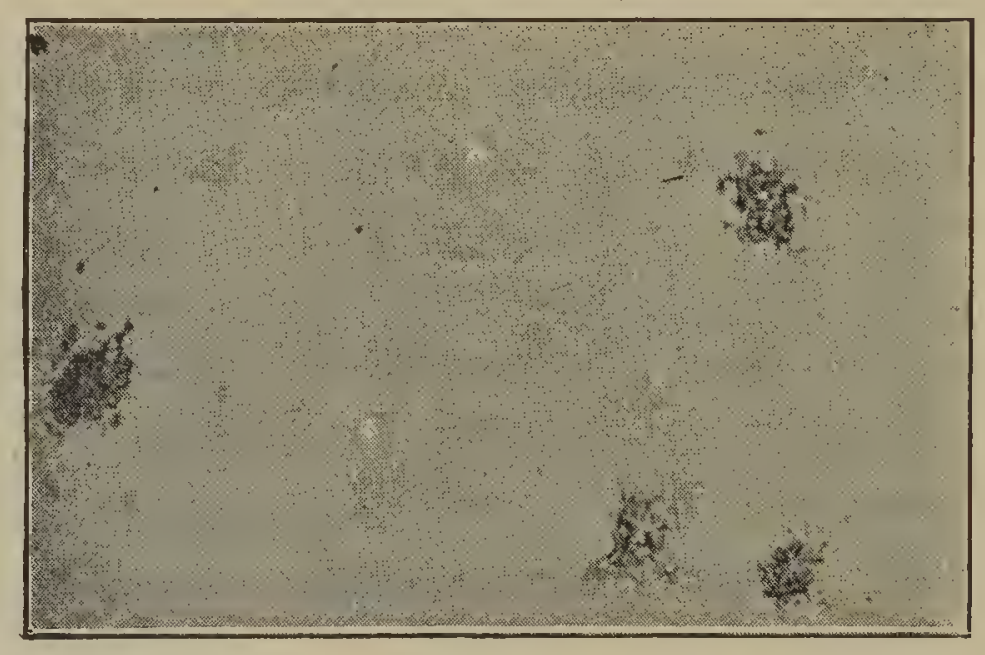

Fig. 90.-Bacillus typhosus. Deep colonies in Hiss plate medium.

has hardened, are placed in an incubator at $37^{\circ} \mathrm{C}$., and allowed to remain for eighteen to twenty-four hours, when they are ready for examination. If typhoid bacilli are present they will be found as small, usually glistening colonies with a fringe of threads growing out like flagella from their peripheries (see Figs. 90 and 91). These colonies are smaller and quite distinct from those of colon bacilli, which are heavier and darker and do not display the fringing threads. Suspicious colonies may be fished and transferred to the Hiss tube medium (see page 133) or identified by other reliable methods.

A method which has been found useful, especially in Europe, is that in which smears of diluted feces are made upon large plates of the Conradi-Drigalski medium. (For preparation see page 135.) The principles underlying the use of this medium are the formation of acid from the lactose by the colon bacilli and the inhibition of cocci and many other bacteria by the crystal-violet. In practice, an emul-

${ }^{1}$ Hiss, Jour. of Exp. Med., ii, 1897; Med. News, May, 1901; and Jour. Med. Res., N. S., iii, 1902. 
sion is made of a loopful of feces in a tube of broth. Into this is dipped a bent glass smearing rod, the excess of fluid is allowed to drip off, and smears are made upon plates of the medium, several plates being smeared without redipping the rod. Colonies of the colon bacillus on these plates will appear opaque, comparatively large, and will produce an acid reaction with consequent reddening of the medium. Typhoid colonies will be smaller, transparent, and without acid formation. These colonies are fished and the microorganisms may

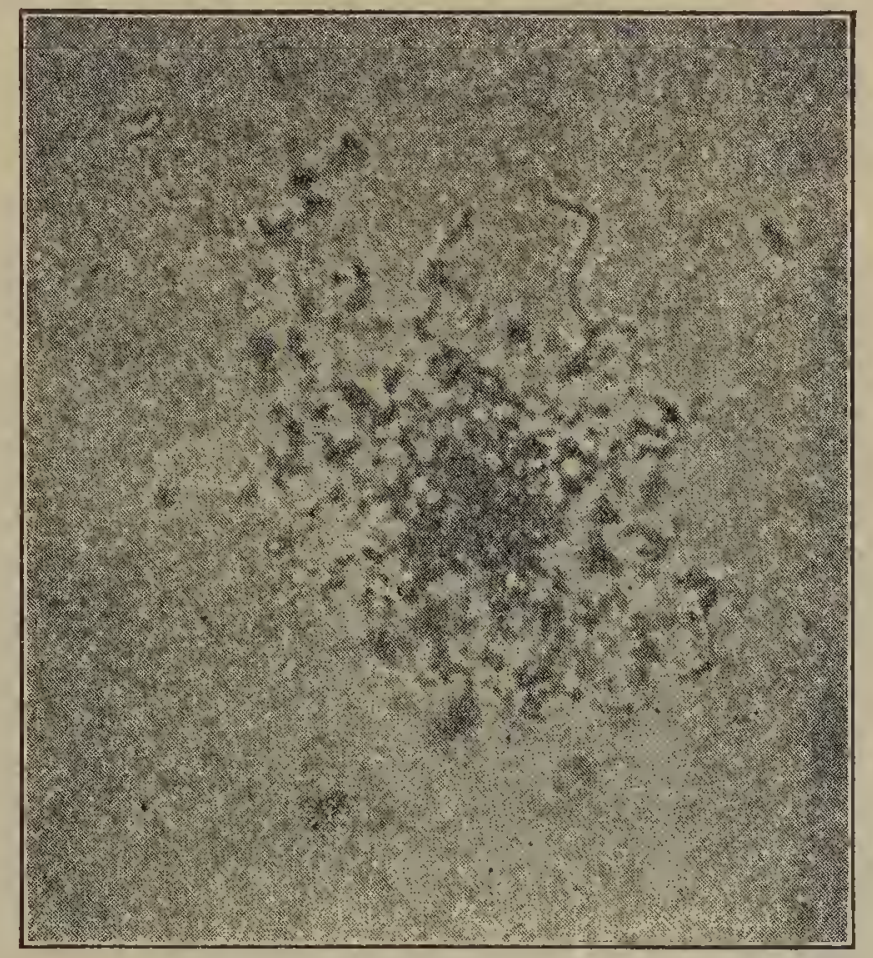

Fig. 91.-Bacillus typhosus. Colony in Hiss plate medium, highly magnified.

be identified by agglutination or by stab cultures in the Hiss tube medium.

The malachite-green media of Loeffler and others have found less general use than was originally expected, because of the difficulty in obtaining uniform preparations of malachite-green. Peabody and Pratt ${ }^{1}$ have applied the principle of colon-bacillus inhibition by malachite-green, by adding this dye to broth in the manner described in the section on media (page 137), planting the feces directly into this broth, and, after incubation for several hours, making smears from these tubes upon plates of the Conradi-Drigalski medium.

Marked success has been reported in the isolation of typhoid bacilli 
from the feces by the use of the Endo fuchsin-agar. Emulsions of feces are made in tubes of ordinary broth in the manner described in the Conradi-Drigalski method, and smears of this emulsion are made upon plates of the fuchsin-agar by means of a glass smearing rod. The colonies of Bacillus coli, after eighteen or more hours of incubation, will be found to have brought back a deep red color to the medium, whereas the typhoid colonies are smaller, more transparent, and have left the medium uncolored.

In all cases where plates are prepared from broth emulsions of feces, it is desirable to allow the emulsion to stand at incubator temperature for several hours, or, better, to centrifugalize the emulsion and then allow it to stand without agitation. Subsequent removal of fluid from the

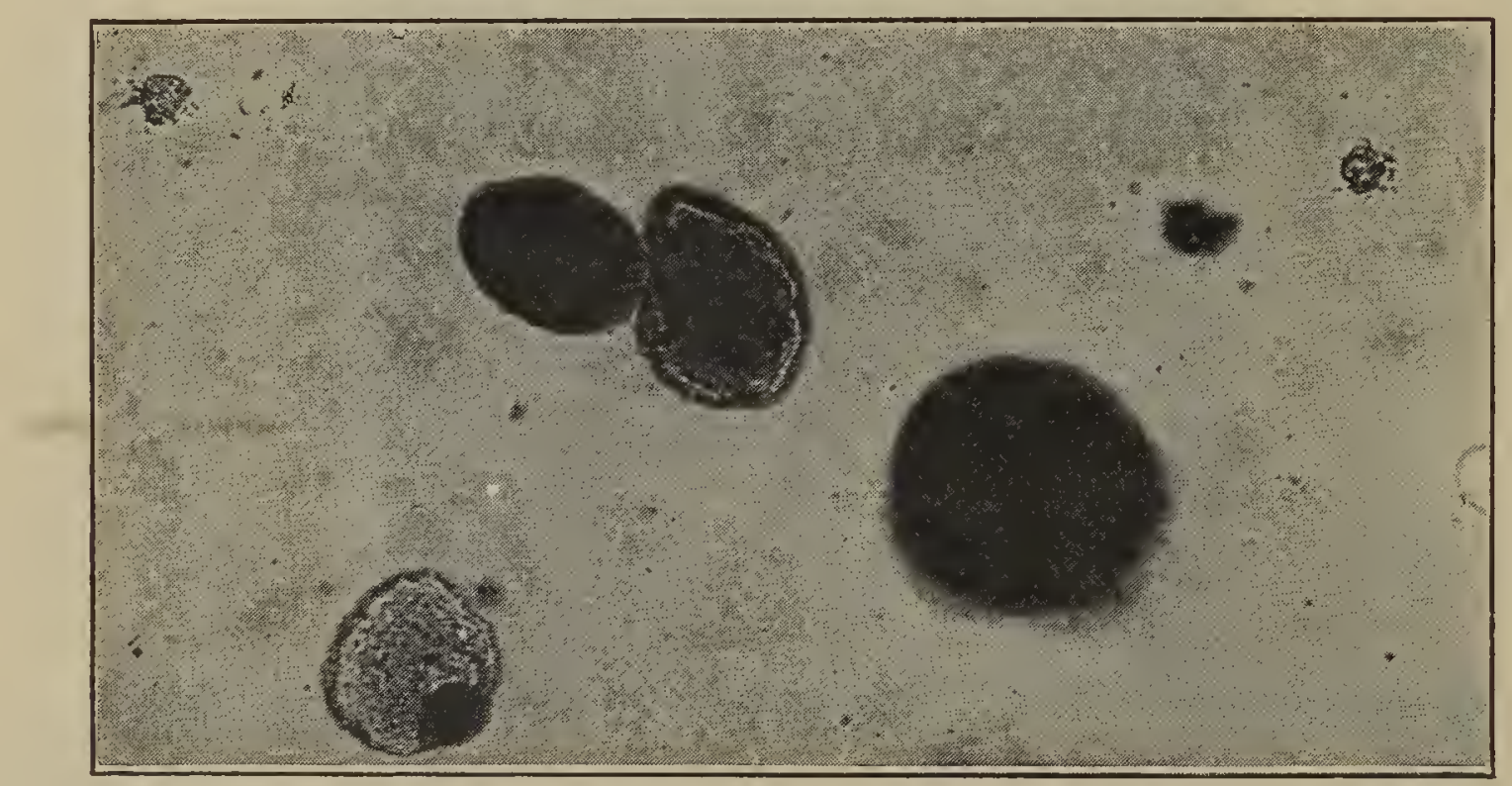

Fig. 92.-Colon and Typhoid Colonies in Hiss Plate Medium. (Planted from. stool. Note the small thread-forming typhoid colonies.)

upper layers of the medium is likely to bring away a comparatively larger number of the motile organisms.

The methods of isolating typhoid bacilli given above do not exhaust the records of work done upon this problem. Other methods have been devised, but those given are the ones most generally in use. It is not satisfactory to compare any two of these methods as to practical value, since all of them require a considerable amount of working familiarity with organisms and media. In fact, it may be said that all of the methods given are satisfactory if consistently employed by a worker who has become thoroughly accustomed to the peculiarities and variations of the typhoid colonies upon the medium with which be is working. 
Typhoid Bacilli in the Urine.-Careful investigation by a number of workers has revealed typhoid bacilli in the urine in about twenty-five per cent of all patients. Neumann ${ }^{1}$ discovered the bacilli in eleven out of forty-six cases and Karlinski ${ }^{2}$ in twenty-one out of forty-four cases. Investigations by Petruschy, ${ }^{3}$ Richardson, ${ }^{4}$ Horton-Smith, ${ }^{5}$ Hiss, ${ }^{6}$ and others have confirmed these results. In general the bacilli have not been found before the fifteenth day of the disease, and examination of the urine, therefore, can be of little early diagnostic value. A series of seventy-five cases examined by Hiss before the fourteenth day of the disease did not once reveal typhoid bacilli in the urine. On the other hand, they have been found to be present for weeks, months, and, in isolated cases, for years after convalescence, the examination thus having much hygienic importance. They are probably present in about twelve per cent of cases during the early days of convalescence. In most of these cases where typhoid bacilli are found, albumin is present in the urine in considerable quantities. The bacilli usually appear and disappear with the albuminuria.

It is not infrequent that an obstinate cystitis caused by typhoid bacilli may follow in the path of typhoid fever. Such cases have been reported by Blumer, ${ }^{7}$ Richardson, ${ }^{8}$ and others. Suppurative processes in the kidneys are less frequent. It is noteworthy, also, that in the course of, and following, typhoid fever there often occurs voiding of Bacillus coli with the urine. This may obstinately persist for considerabe periods after convalescence. The reasons for this are not entirely clear.

Typhoid Bacilli in the Gall-Bladder.-Typhoid bacilli have been frequently observed in the gall-bladder at autopsy. They have also been found present in this organ, at operations for cholecystitis, months and years after the occurrence of typhoid fever. Miller ${ }^{9}$ has reported a case in which typhoid bacilli were present in the gall-bladder seven years after the disease; $v$. Dungern ${ }^{10}$ has cultivated them from an inflamed gall-bladder fifteen years after the disease. Zinsser has had

1 Neumann, Berl. klin. Woch., xxvii, 1890.

${ }^{2}$ Karlinsky, Prag. med. Woch., xv, 1890.

${ }^{3}$ Petruschy, Cent. f. Hyg., xxiii, 1898.

4 Richardson, Jour. Exp. Med., 3, 1898.

${ }^{5}$ Horton-Smith, Lancet, May, 1899.

${ }^{6}$ Hiss, Med. News, May, 1901.

7 Blumer, Johns Hopkins Hosp. Reports, 5, 1895.

8 Richardson, loc. cit.

${ }^{9}$ Miller, Johns Hopkins Hosp. Bull., 1898.

$10 v$. Dungern, Münch. med. Woch., 1897. 
occasion ${ }^{1}$ to observe a case in which an operation for gall-stone seventeen years after the occurrence of typhoid fever revealed the presence of the bacilli in the gall-bladder. In such cases typhoid bacilli may be constantly discharged from the intestine with the feces and prove a menace to the health of the community. An extremely interesting example of such a typhoid carrier has been carefully studied and reported by Park. ${ }^{2}$

Typhoid Bacilli in the Rose Spots.-A number of observers have succeeded in isolating typhoid bacilli from the rose spots. Neufeld, ${ }^{3}$ who made an extensive investigation of this question, obtained positive results in thirteen out of fourteen cases. According to his researches and those of Fränkel, ${ }^{4}$ the bacilli are localized not in the blood, which is taken when the rose spots are incised, but are crowded in large numbers within the lymph spaces.

Typhoid Bacilli in the Sputum.-In rare cases typhoid bacilli have been found in the sputum of cases complicated by bronchitis, bronchopneumonia, and pleurisy. Such cases have been reported by Chantemesse and Widal, ${ }^{5}$ Fränkel, ${ }^{6}$ and a number of others. Empyema, when it occurs in connection with such cases, is usually accompanied by a mixed infection. From a hygienic point of view the spread of typhoid fever by means of the sputum must be considered, but is probably of rare occurrence.

Suppurative Lesions Due to Typhoid Bacillus.-In the course of typhoid convalescence or during the latter weeks of the disease, suppurative lesions may occur in various parts of the body. The most frequent localization of these is in the periosteum, especially of the long bones, and in the joints. A large number of such lesions have been described by Welch, Richardson, ${ }^{7}$ and others. They usually take the form of periosteal abscesses, often located upon the tibia, occurring either late in the disease or even months after convalescence, and are characterized by very severe pain. Osteomyelitis may also occur, but is comparatively rare. Subcutaneous abscesses and deep abscesses in the muscles, due to this bacillus, have been described by Pratt. ${ }^{8}$ Synovitis may also occur.

${ }_{1}$ Zinsser, Proc. N. Y. Pathol. Soc., 1908.

2 Park, "Pathogenic Bacteria," N. Y., 1908.

${ }^{3}$ Neufeld, Zeit. f. Hyg., xxx, 1899.

4 Fränkel, Zeit. f. Hyg., xxxiv, 1900.

${ }^{5}$ Chantemesse and Widal, Arch. de physiol. norm. et path., 1887.

6 Fränkel, Deut. med. Woch., xv and xvi, 1899.

7 Richardson, Jour. Boston Soc. Med. Sci., 5, 1900.

8 Pratt, Jour. Boston Soc. Med. Sci., 3, 1899. 
Meningitis, due to the typhoid bacillus, occurs not infrequently, usually during convalescence from typhoid fever. A case of primary typhoid meningitis has been reported by Farnet. ${ }^{1}$

Peritoneal abscesses, due to the typhoid bacillus, have been reported. Zinsser ${ }^{2}$ has reported a case in which typhoid bacilli were found free in the peritoneal cavity during typhoid fever without perforation of the gut.

Isolated instances of typhoid bacilli in abscesses of the thyroid and parotid glands and in brain abscesses have been observed.

Typhoid Fever without Intestinal Lesions.-A considerable number of cases have been reported in which typhoid bacilli have been isolated from the organs after death or from the secretions during life of patients in whom the characteristic lesions of typhoid fever have been lacking. Most of these cases must be regarded as true typhoid septicemias. In some cases the bacilli were isolated from the spleen, liver, or kidneys; in others, from the urine or the gall-bladder. In a case observed by Zinsser the bacilli were isolated from an infarct of the kidney removed by operation. In this case the clinical course of the disease had pointed only toward the existence of an indefinite fever accompanied by symptoms referable to the kidneys. The Widal test, however, was positive. An excellent summary of such cases, together with several personally observed, has been given by Flexner. ${ }^{3}$

Hygienic Considerations.-Although typhoid fever is frequently spoken of as an epidemic disease, it is, more truly, endemic in character in almost all parts of the world, but subject to occasional epidemic exacerbations. In the larger communities of the temperate zones these epidemic increases take place chiefly in the autumn and, unlike epidemics of diseases such as influenza, are usually distinctly circumscribedlimited usually by the distribution of a particular water-supply.

Since the disease never occurs except by transmission, directly or indirectly, from a previous case, it is amenable more than most other maladies to sanitary regulation, and it may be said without exaggeration, in the light of our present knowledge, that any extensive prevalence of typhoid fever in a large community is a direct consequence of some defect in the system of sanitation. The disease is acquired by ingestion of the specific bacteria. Infection by any other channel than that of the alimentary tract has not, so far, been satisfactorily demonstrated.

${ }_{1}$ Farnet, Bull. de la soc. méd. des hôp. de P., 3, 1891.

${ }^{2}$ Zinsser, Proc. N. Y. Path. Soc., 1907.

3 Flexner, Johns Hopkins, Rep., 5, 1896. 
Prophylactic measures in typhoid fever, therefore, should begin with the isolation of the patient and the disinfection of excreta, discharges, linen, and all utensils which have been in contact with the patient. The bacilli leave the body chiefly in the feces and the urine and the dangers of contamination, by these substances, of all objects in immediate contact with the patient are considerable. Excreta should therefore be either mixed with boiling water or chemically disinfected, preferably by means of thoroughly mixing with carbolic acid, lysol, or a solution of freshly slaked lime, and, if possible, destroyed by buriing. Linen, tableware, and eating utensils should be soaked in similar solutions and boiled. The observance of such measures, furthermore, should not be discontinued until bacteriological examination has demonstrated the absence of the bacilli from feces and urine. Disregard of this last precaution may well be one of the main causes of the endemic persistence of the disease in large cities-especially considered in the light of our recent knowledge of "typhoid carriers" in whom chronic infection of the gall-bladder leads to the discharge of the bacilli in the feces for months and even years after the cessation of symptoms.

It can hardly be doubted, at the present day, that typhoid fever, in the large majority of cases, is transmitted by the agency of water. In an analysis of six hundred and fifty typhoid epidemics Schüder ${ }^{1}$ found four hundred and sixty-two reported, upon reasonable evidence, as originating from water. The technical difficulties attending the isolation of typhoid bacilli from contaminated water have prevented actual bacteriological proof in most epidemics; nevertheless, indirect evidence of pollution of the suspected water-supply, correspondence of the distribution of this supply with that of the disease, and reduction of typhoid morbidity upon the substitution of an uncontaminated supply are sufficiently convincing to remove reasonable doubt. Added to this is our knowledge, from the experiments of Jordan, Russell, and Zeit ${ }^{2}$ and others, that typhoid bacilli may remain alive in natural waters for as long as five days. That the bacilli may survive freezing for as long as three months has been demonstrated by Prudden, and dangers of infection from this source are therefore considerable.

Next to water, the most important source of typhoid fever is found in contaminated milk. In the statistical summary by Schüder, ${ }^{3}$ quoted above, one hundred and ten of the four hundred and sixty epidemics

${ }_{1}^{1}$ Schüder, Zeit. f. Hyg., xxxviii, 1901.

${ }^{2}$ Jordan, Russell, and Zeit, Jour. of Inf. Dis., 1, 1904.

3 Schüder, loc. cit, 
recorded, were attributable to milk. Actual discovery of Bacillus typhosus in milk by Vaughan, Conradi, and others has been discussed in another section (see page 685). The fact that this bacillus causes no visible modifications in milk makes this source especially insidious. When contamination of milk has occurred, it has often been traceable to the water used in washing the cans or to attendants employed at the dairies, who had been in contact with typhoid cases, or who are convalescing from, or actually suffering from, the infection themselves.

Excluding water and milk, all remaining causes of typhoid dissemination constitute about twelve per cent and are found chiefly in the use of vegetables contaminated from infected soil, and other food products. Recently Conn has called attention to the fact that oysters grown in waters close to sewage discharges may be the means of typhoid transmission. An epidemic occurring at Wesleyan University was attributed by him to this cause. Experiments by Foote ${ }^{2}$ have actually demonstrated that typhoid bacilli may be found alive within oysters for three weeks or more after they have disappeared from the surrounding water.

Indirect contamination of food and water by the intermediation of flies and other insects has been emphasized by Veeder ${ }^{3}$ as one of the methods of typhoid transmission. This observer called attention to the fact that in camps during the Spanish-American War flies in large numbers traveled to and fro between the sinks and the cook-tents, and it is not unlikely that at least some of the typhoid fever occurring at that time may have been caused in this way.

Poisons of the Typhoid Bacillus.-The investigation of the toxic products of the typhoid bacillus has occupied the attention of a large number of workers. The first to do experimental work upon the subject was Brieger ${ }^{4}$ soon after the discovery and cultivation of the microorganism. That toxic substances can be obtained from typhoid cultures is beyond question. There is, however, a definite difference of opinion as to whether these poisons are so-called endotoxins only, or whether they are in part composed of soluble toxins comparable to those of diphtheria and tetanus, following the injection of which antitoxic substances may be formed.

The evidence so far seems to bear out the original contention of

1. Conn, Med. Record, Dec., 1894.

3 Veeder, Med. Record, 45, 1898.
2 Foote, Med. News, 1895.

4 Brieger, Deut. med. Woch., xxvii, 1902. 
Pfeiffer, ${ }^{1}$ who first advanced the opinion that the poisonous substances are products of the bacterial body set free by destruction of the bacteria by the lytic substances of the invaded animal or human being. These poisons, when injected into animals for purposes of immunization, in Pfeiffer's experiments, did not incite the production of neutralizing or antitoxic bodies, but of bactericidal and lytic substances. That these endotoxins constitute by far the greater part of the toxic products of the typhoid bacillus can be easily demonstrated in the laboratory, by the simple experiment of filtering a young typhoid culture (eight or nine days old) and injecting into separate animals the residue of bacilli and the clear filtrate respectively. In such an experiment there will be little question as to the overwhelmingly greater toxicity of the bacillary bodies as compared with that of the culture filtrate. On the other hand, if such cultures, especially in alkaline media, are allowed to stand for several months and the bacilli thus thoroughly extracted by the broth, the toxicity of the filtrate is found to be greatly increased.

Nevertheless, more recent experiments by Besredka, ${ }^{2}$ Macfadyen, ${ }^{3}$ Kraus and Stenitzer, ${ }^{4}$ and others have tended to show that, together with such endotoxic substances, typhoid bacilli may produce a true toxin which is not only obtainable by proper methods from comparatively young typhoid cultures, but which fulfils the necessary requirement of this class of poisons by producing in treated animals a true antitoxic neutralizing body.

The typhoid endotoxins may be obtained by a variety of methods. Hahn ${ }^{5}$ has obtained what he calls "typhoplasmin" by subjecting thern to a pressure of about four hundred atmospheres in a Buchner press. The cell juices so obtained are cleared by filtration. Macfadyen has obtained typhoid endotoxins by triturating the bacilli after freezing them with liquid air and extracting in $1: 1,000$ potassium hydrate. Besredka obtained toxic substances by emulsifying agar cultures of bacilli in salt solution, sterilizing them by heating to $60^{\circ} \mathrm{C}$. for about one hour, and drying in vacuo. The dried bacillary mass was then ground in a mortar and washed in sterile salt solution which was again heated to $60^{\circ} \mathrm{C}$. for two hours. The remnants of the bacterial

${ }^{1}$ Pfeiffer, Deut. med.Woch., xlviii, 1894; Pfeiffer und Kolle, Zeit. f. Hyg., xxi, 1896.

2 Besredka, Ann. de l'inst. Pasteur, 1895, 1896.

${ }^{3}$ Macfadyen and Rowland, Cent. f. Bakt., I, xxx, 1901; Macfadyen, Cent. $\mathrm{f}_{\mathrm{*}}$ Bakt., I, 1906.

${ }^{4}$ Kraus und Stenitzer, Quoted from "Handb.d. Tech.," etc., 1, Fischer, Jena, 1907.

${ }^{5}$ Hahn, Münch. med. Woch., xxiii, 1906. 
bodies settle out and the slightly turbid supernatant fluid contains the toxic substances.

Vaughan ${ }^{1}$ has obtained poisons from typhoid bacilli by extracting at $78^{\circ} \mathrm{C}$. with a two-per-cent solution of sodium hydrate in absolute alcohol. In this way he claims to separate by hydrolysis a poisonous and a non-poisonous fraction. He claims, moreover, that this poisonous fraction is similar to the poisons obtained in the same way from Bacillus coli and the tubercle bacillus, and other proteid substances, believing that the specific nature of such proteids depends upon the non-toxic fraction.

A simple method of obtaining toxins from typhoid bacilli is carried out by cultivating the microorganisms in meat-infusion broth, rendered alkaline with sodium hydrate to the extent of about one per cent. The cultures are allowed to grow for two or three weeks and then sterilized by heating to $60^{\circ} \mathrm{C}$. for one hour, and allowed to stand for three or four weeks at room temperature. At the end of this time the cultures may be filtered through a Berkefeld or Pasteur-Chamberland filter and will be found to contain strong toxic substances.

The accounts concerning the thermostability of the various toxins obtained are considerably at variance. In general, corresponding with other endotoxins, observers agree in considering them moderately resistant to heat, rarely being destroyed at temperatures below $70^{\circ} \mathrm{C}$.

Intravenous inoculation of rabbits with typhoid endotoxins, if in sufficient quantity, produces, usually within a few hours, a very marked drop in temperature, diarrhea, respiratory embarrassment, and death. If given in smaller doses or by other methods of inoculationsubcutaneous or intraperitoneal-rabbits are rendered extremely ill, with a primary drop in temperature, but may live for a week or ten days, and die with marked progressive emaciation, or may survive. Guineapigs and mice are susceptible to the endotoxins, though somewhat less so than rabbits.

Immunity in Typhoid Fever.-As a rule, one attack of typhoid fever protects against subsequent ones. Although exceptions to this rule may occur, they are so rare that the history of a previous attack of this disease practically excludes its consideration in the diagnosis of any obscure condition.

Animals may be actively immunized by the injection of typhoid bacilli in gradually increasing doses. In actual practice, this is best

${ }_{1}$ Vaughan, Am. Jour. of Med. Sci., 136, No. 3, 1908. 
accomplished by beginning with an injection of about 1 c.c. of broth culture heated for ten minutes at $60^{\circ}$ in order to kill the bacilli. After five or six days, a second injection of a larger dose of dead bacilli is administered; at similar intervals, gradually increasing doses of dead bacilli are given and finally considerable quantities of a living and fully virulent culture may be injected without serious consequences to the animal. While this method is convenient and usually successful, it is also possible to obtain satisfactory immunization by beginning with very small doses of living microorganisms, according to the early method of Chantemesse and Widal, ${ }^{1}$ and others.

Such active immunization, successfully carried out upon rabbits and guinea-pigs, within a short time after the discovery of the typhoid bacillus, was believed to depend upon the development of antitoxic substances in immunized animals. This point of view, however, was not long tenable, and was definitely disproven by the investigations of Pfeiffer and Kolle ${ }^{2}$ in 1896. These investigators, as well as a large number of others working subsequently, have shown satisfactorily that there are present in the blood serum of typhoid-immune animals and human beings, bacteriolytic, bactericidal, and agglutinating substances, and to a lesser extent, precipitating and opsonic bodies.

Bactericidal and Bacteriolytic Substances.-The bacteriolytic substances in typhoid-immune serum may be demonstrated either by the intraperitoneal technique of Pfeiffer or in vitro. In the former experiment a small quantity of a fresh culture of typhoid bacilli is mixed with the diluted immune serum and the emulsion injected into the peritoneal cavity of a guinea-pig. Removal of peritoneal exudate with a capillary pipette and examination in the hanging drop will reveal, within a short time, a swelling and granulation of the bacteria-the so-called Pfeiffer phenomenon. The test in vitro, as recommended by Stern and Korte, ${ }^{3}$ may be carried out by adding definite quantities of a fresh agar culture of typhoid bacilli to progressively increasing dilutions of inactivated immune serum together with definite quantities of complement in the form of fresh normal rabbit or guinea-pig serum. At the end of several hours' incubation at $37.5^{\circ} \mathrm{C}$. definite quantities of the fluid from the various tubes are inoculated into melted agar and plates are poured to determine the bactericidal action. Carefu? colony counting in these plates and comparison with proper controls

${ }^{1}$ Chantemesse and Widal, Ann. de l'inst. Pasteur, 1892.

${ }^{2}$ Pfeiffer und Kolle, Zeit. f. Hyg., xxi, 1896.

3 Stern und Korte, Berl. klin. Woch., x., 1904. 
will not only definitely demonstrate the presence of bactericidal substances in the immune serum, but will furnish a reasonably accurate quantitative estimation. (For these tests see p. 255.)

Although normal human serum contains in small quantity substances bactericidal to typhoid bacilli, moderate dilution, $1: 10$ or $1: 20$, of such serum will usually suffice to eliminate any appreciable bactericidal action. The bactericidal powers of immune serum, on the other hand, are often active, according to Stern and Korte, in dilutions of over $1: 4,000$ and in one case even of $1: 4,000,000$. The specificity of such reactions gives them a considerable degree of practical value, both in the biological identification of a suspected typhoid bacillus in known serum and in the diagnosis of typhoid fever in the human patient by the artion of the patient's serum on known typhoid bacilli. In the publication of Stern and Korte, quoted above, it was found that typhoid patients during the second week often possess a bactericidal power exceeding $1: 1,000$, whereas the blood of normal human beings was rarely active in dilutions exceeding $1: 50$ or $1: 100$. While scientifically accurate, the practical application of bactericidal determinations for diagnosis presents considerable technical difficulties, and gives way to the no less accurate method of agglutination.

Agglutinins.-Agglutinins are formed in animals and man inoculated with typhoid bacilli, and in the course of typhoid fever. It was, in fact, while studying the typhoid bacillus that the agglutinins were first discovered by Gruber and Durham.

In animals, by careful immunization, specific typhoid agglutinins may easily be produced in sufficient quantity to be active in dilution of $1: 10,000$, and occasionally even $1: 50,000$ or over. In the blood of typhoid patients, the agglutinins may often be found in dilutions of $1: 100$ and over. It is interesting to note that irrespective of the agglutinin contents of any given serum, there may occasionally be noted differences in the agglutinability of various typhoid cultures, a point which is practically important in the choice of a typhoid culture for routine diagnosis work. Weeny ${ }^{1}$ has called attention to the fact that bacilli which do not readily agglutinate when directly cultivated from the body, may often be rendered more sensitive to this reaction by several generations of cultivation upon artificial media. Walker has noted ${ }^{2}$ a loss of agglutinability if the bacilli

1 Weeny, Brit. Med. Jour., 1889.

${ }^{2}$ Walker, Jour. of Path. and Bact., 1892; Totsuka, Zeit. f. Hyg., xlv, 1903. 
are cultivated in immune serum. A similar alteration in the agglutinability of typhoid bacilli was noted by Eisenberg and Volk ${ }^{1}$ when they subjected the microorganism to moderate heat or to weak acids such as $\frac{\mathrm{N}}{4} \mathrm{HCl}$.

The practical application of agglutination to bacteriological work is found, as in the case of the bactericidal substances, in the identification of suspected typhoid bacilli, and in the diagnosis of typhoid fever.

When it is desired to determine by means of agglutination whether or not a given bacillus is a typhoid bacillus, mixtures may be made of young broth cultures, or preferably of emulsions of young agar cultures in salt solution, with dilutions of immune serum. The tests are made microscopically in the hanging-drop preparation or, preferably, macroscopically in small test tubes. In all cases it is desirable first to determine the agglutinating power of the serum when tested against a known typhoid culture. (For detailed technique, see chapter on Technique of Serum Reactions, p. 250.)

In seientific investigations, specific agglutinations in high dilutions of immune serum constitute very strong proof of the species of the microorganism and may often furnish much information as to the biological relationships between similar species. It is found in immunizing animals with any given strain of typhoid bacilli, that there are formed the "chief" or "major" agglutinins which are specific and active against the species used in immunization, and the "group" or " minor" agglutinins, active also against closely related microorganisms. The following extract from a table will serve to illustrate this point in the case of typhoid and allied bacilli.

\begin{tabular}{|c|c|c|c|c|c|}
\hline & \multicolumn{5}{|c|}{ Highly Immune Typhoid Serum. } \\
\hline & $1: 100$ & $1: 250$ & $1: 500$ & $1: 1,000$ & $1: 2,500$ \\
\hline B. typh......... & + & + & + & + & + \\
\hline B. paratyph. (Schottmüller). & + & + & + & - & - \\
\hline B. enteritidis........... & + & - & - & - & - \\
\hline B. coli communis.. & + & - & - & - & - \\
\hline
\end{tabular}

The sera of most adult normal animals and human beings usually contain a small amount of agglutinin for these bacilli. Immunization with the typhoid bacillus, while increasing chiefly the agglutinin

${ }_{1}$ Eisenberg und Volk, Zeit. f. Hyg., xlv, 1903. 
for this bacillus itself, also to a slighter extent increases the group agglutinins for other closely allied species. That these group agglutinins are separate substances and not merely a weaker manifestation of the action of the typhoid agglutinin itself upon these other microorganisms, may be demonstrated by the experiments of agglutinin absorption. (See section on Agglutinins, page 234.)

Immune serum obtained by immunization with one particular typhoid culture usually agglutinates this culture in higher dilutions than it will agglutinate other typhoid strains. This has been noticed in a large number of investigations, but is not always the case.

In the clinical diagnosis of typhoid fever, the phenomenon of agglutination was first utilized by Widal. ${ }^{1}$ This observer called attention to the fact that during the last part of the first or the earlier days of the second week of typhoid fever, as well as later in the disease and in convalescence, the blood serum of patients would cause agglutination of typhoid bacilli in dilutions of 1:10, or over, whereas the serum of normal individuals usually exerted no such influence. Upon this basis he recommended, for the diagnosis of the disease, the employment of a microscopic agglutination test carried out by the usual hanging-drop technique. The reaction of Widal is, at present, widely depended upon for diagnostic purposes and although not universally successful, owing to irregularities in agglutinin formation in some patients, and because of differences in agglutinability of the cultures employed, it is nevertheless of much value. The original conclusions as to the dilutions of the serum which must be employed, have, however, necessarily been modified. Owing to the fact that Gruber, ${ }^{2}$ Stern, ${ }^{3}$ Fränkel, ${ }^{4}$ and a number of others have found that occasionally normal serum will give rise to agglutination of typhoid bacilli in dilutions exceeding $1: 10$, it has been found necessary, whenever making a diagnostic test, to make several dilutions, the ones most commonly employed being $1: 20,1: 40,1: 60$, and $1: 80$. The wide application of the method has given rise to the development of a number of technical procedures, all of them devised with a view toward simplification. In ordinary hospital work, it is most convenient to keep on hand upon slant agar, a stock typhoid culture, the agglutinability of which is well known. From this stock culture, fresh

${ }_{1}$ Widal, Bull. de Ia soc. méd. des hôpit., vi, 1896; Widal et Sicard, Ann. de l'inst. Pasteur, xi, 1897.

${ }^{2}$ Gruber, Verhand. Congr. f. inn. Med., Wiesbaden, 1896.

3 Stern, Cent. f. inn. Med., xlix, 1896.

${ }^{4}$ Fränkel, Deut. med. Woch., ii, 1897. 
inoculations upon neutral bouillon should be made each day, so that a young broth culture may always be on hand to furnish actively motile, evenly distributed bacteria. These bouillon cultures may be grown for from six to eight hours at incubator temperature or for from twelve to eighteen hours at room temperature. The temperatures at which the broth cultures are kept must depend, to a certain extent, upon the peculiarities of the typhoid bacillus employed, since some strains are rather more actively motile and furnish a more suitable emulsion if kept at a temperature lower than, $37.5^{\circ} \mathrm{C}$. A false clumping in the broth cultures due to a too high acidity of the bouillon or a too prolonged incubation, must be carefully guarded against. It is also possible to use for this test an emulsion of typhoid bacilli prepared by rubbing up a small quantity of a young agar culture in salt solution. Uniformity in the preparation of broth cultures or of emulsions should be observed, since the quantitative relationship between typhoid bacilli and agglutinins will markedly affect the completeness or incompleteness of the reaction. In high dilutions an excess of typhoid bacilli may bring about complete absorption of all the agglutinins present, without agglutinating all the microorganisms.

The blood of the patient to be used for a Widal test may be obtained in a number of ways. The most convenient method is to bleed the patient from the ear or finger into a small glass capsule, in the form of that used in obtaining blood for the opsonin test, or into a small centrifuge tube. About 0.5 to 1 c.c. is amply sufficient. These capsules or tubes, after clotting of the blood, may be placed in the centrifuge which in a few revolutions will separate clear serum from clot. The dilutions of the serum are then made. It is best to use sterile physiological salt solution as a diluent, but neutral broth may be used. The dilutions may be made either by means of an ordinary blood-counting pipette or by means of a capillary pipette upon which a mark with a grease pencil, made about an inch from the tip, furnishes a unit of measure, and upon which suction is made by means of a rubber nipple. It is convenient to have at hand a small porcelain palette such as that used by painters, in which the various cup-like impressions may be utilized to contain the various dilutions. Dilutions of the serum are made, ranging from $1: 10$ to $1: 50$. A drop of each of these dilutions is mixed with a drop of the typhoid culture or emulsion upon the center of a cover-slip and the coverslip inverted over a hollow slide. A control with normal serum and the same culture should always be made and also one with the culture alone to exclude the possibility of spontaneous clumping. Mixture 
with the typhoid culture, of course, each time doubles the dilutions so that, for instance, a drop of serum dilution $1: 10$, plus a drop of the typhoid culture, gives the final dilution of $1: 20$. The preparations may be examined with a high power dry lens or an oil immersion lens. In a positive reaction, the bacilli, which at first swim about actively, singly or in short chains, soon begin to gather in small groups and lose much of their activity. Within one-half to one hour, they will be gathered in dense clumps between which the fluid is clear and free from bacteria, and only upon the edges of the agglutinated masses may slight motility be observed. The degree of dilution and the time of exposure at which such a reaction may be regarded as of specific diagnostic value, have been largely a matter of empirical determination. It is generally accepted at present that complete agglutination within one hour in dilutions from $1: 40$ to $1: 60$ is definite prcof of the existence of typhoid infection. Exceptions, however, to this rule may occur. Agglutinations of typhoid bacilli in dilutions of $1: 40$, and over, have occasionally been observed in cases of jaundice and of tuberculosis, and these conditions must occasionally be considcred, though their importance was formerly exaggerated.

The method of making the Widal test from a drop of whole blood, dried upon a slide, is not to be recommended, as accuracy in dilution by this method is practically impossible.

As stated above, the agglutinin reaction rarely appears in typhoid fever before the beginning of the second week. It may continue during convalescence for as long as six to eight weeks and occasionally, in cases where there is a chronic infection of the gall-bladder, a Widal reaction may be present for years after an attack.

For very exact work, even in clinical cases, the microscopic agglutination method may be replaced by macroscopic agglutination, according to the technique described in another section (page 229).

In order to avoid both the necessity of keeping alive typhoid cultures for routine agglutination tests and also to preclude the danger of infection by the use of living culture, Ficker ${ }^{1}$ has recommended the use of typhoid bacilli killed by formalin. This method has no advantages for practical purposes and in scientific bacteriological work it is, of course, not to be considered in comparison with the other exact methods.

Precipitins. - The investigations of $\operatorname{Kraus}^{2}$ in 1897, by which the

1 Ficker, Berl. klin. Woch., xlviii, 1903, ' Kraus, Wien. klin. Woch., xxxii, 1897. 
precipitins were discovered, revealed specific precipitating substances, among others, also in typhoid immune sera. Since Kraus' original investigation, these substances have been studied by Norris ${ }^{1}$ and others. ${ }^{2}$

Opsonins.-A number of observers have shown that opsonins specific for the typhoid bacillus are formed in animals immunized with these organisms. Opsonins are formed also in patients suffering from typhoid fever, but exact opsonic estimations in all these cases are extremely difficult because of the rapid lysis which these bacteria may undergo both in the serum, and intracelluiarly after ingestion by the leucocytes. Klein ${ }^{3}$ has attempted in part to overcome this difficulty by working with dilutions of serum and at the same time using comparatively thick bacterial emulsions and exposures to the phagocytic action not exceeding ten minutes. Chantemesse ${ }^{4}$ has claimed that the opsonic index of typhoid patients was increased after treatment with a serum obtained by him from iminunized horses, and Harrison ${ }^{5}$ has reported similar results in patients treated by a modification of Wright's method of active immunization. Klein claims to have demonstrated that in typhoid-immune rabbits, after five injections, the opsonic contents of the blood were increased to an equal extent with the bactericidal substances. He concludes from this interesting observation that it may well be that the opsonins are quite as important in typhoid immunity as are the latter substances.

For diagnostic purposes in typhoid fever the estimation of the opsonic index, so far, has not been proven to be of great value.

Specific Therapy in Typhoid Fever.-The failure to produce a soluble toxin from typhoid cultures has naturally so far precluded the possibility of an antitoxic therapy, such as that which has been successful in diphtheria. In the light of our present knowledge of the poisonous products of the typhoid bacillus it seems but natural that attempts by earlier investigators to apply the principles of Behring's work to typhoid fever were doomed to fail. Attempts to employ specific bactericidal and bacteriolytic sera for therapeutic purposes in this disease have also been without favorable result.

Active Immunization.-We have seen that work by Pfeiffer and Kolle and subsequently by a large number of others has shown that it is com-

${ }_{1}$ Norris, Jour. of Inf. Dis., I, 3, 1904.

2 Barker and Cole, 22d Ann. Session, Assn. of Amer. Phys., Wash., 1897.

${ }^{3}$ Klein, Bull. Johns Hopkins Hosp., 1907.

${ }^{4}$ Chantemesse, 14th Internatl. Cong. for Hyg., Berlin, 1907.

${ }^{5}$ Harrison, Jour. Royal Army Med, Corps, 8, 1907. 
paratively easy to immunize animals actively against typhoid infection by the systematic injection of graded doses, at first of dead bacilli, later of fully virulent live cultures. Attempts to apply these principles prophylactically have been made recently on a large scale by Wright and his associates upon English soldiers in South Africa, and by German observers in German East Africa.

The first recorded experiment of this sort which was done upon human beings was that of Pfeiffer and Kolle, ${ }^{1}$ who in 1896 treated two individuals with subcutaneous injections of an agar culture of typhoid bacilli which had been sterilized at $56^{\circ} \mathrm{C}$. The first injection was made with two milligrams of this culture. Three or four hours after the injection the patient suffered from a chill, his temperature gradually rose to $105^{\circ} \mathrm{F}$., and there was great prostration and headache, but within twenty-four hours the temperature had returned to normal.

This experiment showed that such injections could be practiced upon human beings without great danger.

Simultaneously with the work of Pfeiffer and Kolle, Wright ${ }^{2}$ conducted similar experiments on officers and privates in the English army.

The actual number of persons treated directly or indirectly under Wright's ${ }^{3}$ supervision in an investigation covering a period of over four years comprised almost one hundred thousand cases. The methods employed by Wright have been modified several times by him and his collaborators in minor details; the principles, however, have remained consistently the same. In the first experiments Wright employed an agar culture three weeks old, grown at $37^{\circ} \mathrm{C}$., then sterilized at a temperature below $60^{\circ} \mathrm{C}$, and protected from contamination by the addition of five-tenths per cent of carbolic acid. Later, Wright ${ }^{4}$ employed bacilli grown in a neutral one-per-cent pepton bouillon in shallow layers or flasks. Great importance is attached both to the virulence of the typhoid strain, which may to a moderate extent be standardized by passage through guinea-pigs, and to care in using low temperatures for final sterilization. The temperature recommended by Harrison, ${ }^{5}$ working with Wright's method, is $52^{\circ} \mathrm{C}$, after which the cultures are carbolized. For the first dose in a human being, Wright recommends

1 Pfeiffer und Kolle, Deut. med. Woch., xxii, 1896; xxiv, 1898.

2 Wright, Lancet, Sept., 1896.

${ }^{3}$ Wright and Semple, Brit. Med. Jour., 1897; Wright and Leishman, Brit. Med. Jour., Jan., 1900.

${ }^{4}$ Wright, Brit. Med. Jour., 1901; Lancet, Sept., 1902; Brit. Med. Jour., Oct., 1903.

${ }^{5}$ Harrison, Jour. Royal Army Medical Corps, 1907. 
the quantity of bacilli fatal for 100 grams of guinea-pig. The dose may also, according to Wright, be regulated by making numerical counts of the emulsions used, by his usual method of counting against red blood corpuscles, and using for the first injection 750 to 1,000 millions of dead bacteria. The second injection, given after eleven days, should be double this quantity. Usually the first dose is followed by local inflammatory symptoms and the general systemic symptoms of toxemia. These, however, usually disappear after forty-eight hours.

Although the observations of Wright are extensive, it is nevertheless extremely difficult to tabulate satisfactory statistics from a mass of experiments which must of necessity be observed by a large number of individuals, in all of whom the personal equation modifies the results of the observations. On the whole, however, it seems fair to state that distinctly advantageous results followed the active immunization practiced by Wright. Wright's own estimation, in a careful attempt to present the subject fairly, gives a reduction of the morbidity from typhoid fever in the British army of fifty per cent, and a reduction of the mortality of those who became infected in spite of inoculations of fifty per cent also. Combining these two results, the actual reduction of the death rate by the method of vaccination would appear to amount to at least seventy-five per cent.

The method of Pfeiffer and Kolle, originally used by them in their experiments, has been extensively carried out by German observers upon the army taking part in the late East African campaign.

Roughly, the method consists in the injection of salt-solution emulsions of fresh agar cultures sterilized at $60^{\circ} \mathrm{C}$. The results reported from a large material were in general favorable, indicating that the morbidity of all the troops taking part was reduced by the inoculation and that the death rate among the inoculated persons was lower than that among normal individuals.

Recent extensive tests in the United States Army, carefully observed by Russell ${ }^{1}$ and others, seem to have removed any doubt which may have existed as to the efficacy of prophylactic typhoid vaccination. However, another point of importance in this connection has recently been raised by Metchnikoff and Besredka ${ }^{2}$. They vaccinated chimpanzees with typhoid bacilli and found that when emulsions of the clear bacteria were used, protection was only slight. Better results were ob-

${ }^{1}$ Russell, Am. Jour. of Med. Sc., cxlvi., 1913.

${ }^{2}$ Metchnikoff and Besredka, Am. de l'Inst. Past., 1911. 
tained-that is, apparently complete protection within 8 to 10 days, when living sensitized bacteria were injected. (Bacteria which had been exposed to the action of inactivated immune serum.) Broughton ${ }^{1}$ has applied this method to human beings. Gay ${ }^{2}$ has also prepared a sensitized dead typhoid vaccine which he has already used in a considerable number of cases. It will take some time, however, before a statistical estimation of the superiority of this method over the older vaccination with dead bacteria will be possible.

\section{BACILLUS FECALIS ALKALIGENES}

In 1896 Petruschky ${ }^{3}$ described a bacillus which is a not infrequent inhabitant of the human intestine, being found chiefly in the lower part of the small intestine and the large intestine. This organism, which he called Bacillus fecalis alkaligenes, is of little pathogenic importance, although Neufeld states that he has seen a case of severe gastroenteritis in which the watery defecations contained this bacillus in almost pure culture. As a rule, however, this organism can not be regarded as pathogenic, and is important chiefly because of the ease with which it may be mistaken for Bacillus typhosus.

Bacillus fecalis alkaligenes is an actively motile, Gram-negative bacillus, possessing, like the typhoid bacillus, numerous peritrichal flagella. On the ordinary culture media it grows like the typhoid bacillus. It does not coagulate milk. It produces no indol, and on sugar media in fermentation tubes produces no acid or gas. On potato, its growth, while somewhat heavier than that of the typhoid bacillus, is not sufficiently. so to permit easy differentiation. It differs from Bacillus typhosus in that it produces no acid on any of the sugar media, and is therefore easily differentiated by cultivation upon Hiss-serum-water media or on pepton waters containing sugars. On the Hiss semisolid tube-medium Bacillus fecalis alkaligenes, while clouding the medium throughout, grows most heavily on the surface where, eventually, it forms a pellicle.

${ }^{1}$ Broughton, C. R. de l'Acad. des Sc., cliv, 1911.

${ }^{2}$ Gay, Arch. of Int. Med., 1914.

${ }^{3}$ Petruschky, Cent. f. Bakt., I, xix, 1896. 


\section{CHAPTER XXVIII}

\section{BACILLI OF THE COLON-TYPHOID-DYSENTERY GROUP (Continued)}

\section{BACILLI INTER MEDIATE BETWEEN THE TYPHOID AND COLON ORGANIS MS}

\section{(Bacilli of Meat Poisoning and Paratyphoid Fever)}

THERE is an extensive group of Gram-negative bacilli which because of their morphology, cultural behavior, and pathogenic properties, are classified as intermediate between the colon and the typhoid types. The microorganisms belonging to this group have been described, most of them, within the last fifteen years, but few of them have been fully identified with one another. They have been variously designated as the "hog-cholera group," "the enteritidis group," the "paracolon group" or "paratyphoid group," because of the pathological conditions with which the chief members under investigation have been found associated.

Attempts to systematize the group by the comparative study of a large number of its members have been made, notably by Buxton ${ }^{1}$ and by Durham, ${ }^{2}$ and the work of these writers, based on cultural and agglutinative studies, has added materially to our knowledge of these organisms.

The microorganisms of this group are morphologically indistinguishable from the colon and typhoid bacilli. They are Gram-negative and possess flagella. Their motility is variable, but usually approaches that of the typhoid bacilli in activity. They correspond, furthermore, to the two other groups in their cultural characteristics upon broth, agar, and gelatin. On potato, they vary, some of them approaching in delicacy the typhoid growth upon this medium, others more closely approximating the heavy brownish growth of $\mathrm{B}$. coli. Indol is rarely formed by them, though this has not been absolutely constant in all descriptions. As a group, they are easily distinguished from Bacillus

1 Buxton, Jour. Med. Res., N. S., iii, 1900. ${ }^{2}$ Durham, Jour. Exper. Med., v, 1901. 
typhosus on the one hand, and from Bacillus coli on the other, by the following simple reactions tabulated by Buxton. ${ }^{1}$

\begin{tabular}{|c|c|c|c|}
\hline & B. coli. & Intermediates. & B. typhosus. \\
\hline Coagulation of milk. & + & - & - \\
\hline Production of indol......... & + & - & - \\
\hline Fermentation of lactose with gas & + & - & - \\
\hline Fermentation of dextrose with gas........ & + & + & - \\
\hline Agglutination in typhoid-immune serum... & - & - & + \\
\hline
\end{tabular}

The characteristics of the three groups as shown by fermentation tests may be tabulated as follows:

\begin{tabular}{|c|c|c|c|}
\hline & $\begin{array}{l}\text { Gas upon } \\
\text { Dextrose. }\end{array}$ & $\begin{array}{l}\text { Gas upon } \\
\text { Lactose. }\end{array}$ & $\begin{array}{l}\text { Gas upon } \\
\text { Saccharose. }\end{array}$ \\
\hline B. typhosus & - & - & - \\
\hline Intermediates & + & - & - \\
\hline B. coli communis..... & + & + & - \\
\hline B. coli communior. . . . . . . . . . & + & + & + \\
\hline
\end{tabular}

Pathogenically, the bacilli of this "intermediate group" have attracted attention chiefly in connection with meat poisoning, and with protracted fevers indistinguishable from mild typhoidal infections.

In 1888, Gärtner ${ }^{2}$ described a bacillus which he isolated from the meat of a cow, the ingestion of which had produced the symptoms of acute gastrointestinal catarrh in fifty-seven people. One of these died of the disease and the bacilli could be demonstrated in the spleen and in the blood of the patient.

This bacillus, called Bacillus enteritidis by Gärtner, was actively motile, formed no indol, but produced gas in dextrose media. Acute gastrointestinal symptoms could be induced by feeding the organisms to mice, guinea-pigs, rabbits, and sheep, and the bacilli could be recovered from the infected animals. An interesting observation, which has since become important in characterizing the group of these bacilli concerned in meat poisoning, was the fact that the bacterial bodies themselves were found by Gärtner to be extremely toxic, containing a poison which, in contradistinction to the endotoxins of many other microorganisms, was extremely resistant to heat. Sterilized cultures showed the same pathogenic effects as the living bacilli. Epidemics

${ }_{1}$ Buxton, loc. cit. $\quad{ }^{2}$ Gärtner, Corresp. Bl, d. Aerzt. Vereins, Turingen, 1888. 
of meat poisoning similar to the one described by Gärtner, in which similar bacteria were isolated, were those described by Van Ermengem, ${ }^{1}$ occurring at Morseele in 1891, the one described by Holst, ${ }^{2}$ the Rotterdam epidemic described by Poels and Dhont, ${ }^{3}$ the one described by Basenau, and many others.

Bacillus Morseele of Van Ermengem, Bacillus bovis morbificans of Basenau, ${ }^{4}$ and the bacilli isolated in similar epidemics by other observers, are, except for slight differences in minor characteristics, almost identical with Gärtner's microorganism.

In 1893, Theobald Smith and Moore, ${ }^{5}$ studying the diseases of swine, noted a great similarity between the so-called hog-cholera bacillus, the bacilli of the Gärtner group, and Bacillus typhi murium isolated by Loeffler. These observers first used the term "hog-cholera" group for the organisms under discussion.

In 1899 Reed and Carroll ${ }^{6}$ called attention to the fact that Bacillus icteroides, associated by Sanarelli with yellow fever, was culturally closely similar to the bacillus of hog cholera.

Meanwhile, other observers had been isolating bacilli, similar to those spoken of above, from cases of protracted fevers in human beings, often closely simulating typhoid infections. The first cases of this kind on record were those of Achard and Bensaude. ${ }^{7}$

In 1897, Widal and Nobecourt ${ }^{8}$ described a bacillus which they had isolated from an esophageal abscess following typhoid fever, which closely resembled Bacillus psittacosis of Nocard, ${ }^{9}$ and which, following a nomenclature previously suggested by Gilbert, ${ }^{10}$ they designated the paracolon bacillus. This microorganism, as well as Bacillus psittacosis, isolated from a parrot by Nocard, showed a close resemblance to bacilli of the Gärtner group.

In 1898, Gwyn ${ }^{11}$ reported a case occurring at the Johns Hopkins

${ }^{1}$ Van Ermengem, Bull. Acad. d. méd. de Belgique, 1892; "Trav. de lab. de l'univ. de Gand," 1892.

${ }^{2}$ Holst, Ref. Cent. f. Bakt., xvii, 1895.

3 Poels und Dhont, Holland Zeit. f. Tierheilkunde, xxiii, 1894.

${ }^{4}$ Basenau, Arch. f. Hyg., xx, 1894.

${ }^{5}$ Th. Smith and Moore, U. S. Bureau of Animal Industry Bull. vi, 1894.

${ }^{6}$ Reed and Carroll, Medical News, Ixxiv, 1899.

' Achard and Bensaude, Bull. de la soc. d. hôpitaux de Paris, Nov., 1906.

\& Widal et, Nobecourt, Semaine méd., Aug., 1897.

${ }^{9}$ Nocurd, Ref. Baungarten's Jahresh., 1896.

10 Gilbert, Semaine méd., 1895.

"Gwyn, Johns Hopkins Hosp. Bull., 1898. 
Hospital, which presented all the symptoms of typhoid fever, but lacked serum agglutinating power for Bacillus typhosus. From the blood of the patient, Gwyn isolated an organism, with cultural characteristics similar to those of the Gärtner bacillus, which he called a "paracolon bacillus." This bacillus was agglutinated specifically by the serum of the patient.

Cushing, ${ }^{1}$ in 1900 , isolated a similar microorganism from a costochondral abscess, appearing during convalescence from typhoid fever.

In the same year, Schottmüller ${ }^{2}$ reported five cases from which bacilli similar to those previously described were isolated. Careful cultural and agglutination studies of the microorganisms obtained from these cases showed that they could be divided into two similar, yet distinctly different types, one of them, the "Müller" organism, approaching closely to the typhoid type, especially in its growth upon potato; the other, the "Seeman" type, corresponding more closely to the Gärtner enteritidis bacilli. Similar cases were soon after reported by Kurth, ${ }^{3}$ Buxton and Coleman, ${ }^{4}$ Libman, ${ }^{5}$ and others.

The two types of organisms, paratyphoid A and B, described by Schottmüller and studied by many other observers, can be culturally differentiated though not without difficulty.

Type $A$ is more delicate in its growth on various media than $\mathrm{B}$, growing with almost invisible growth on potato, and differing from typhoid in its gas formation on dextrose broth only. Milk is not coagulated, but remains turbid, not being finally cleared by solution of the casein as in similar cultures of type B. Lactose whey is acidified and remains acid. This organism is not very important as a causative agent of human disease, and has been isolated from thenormal intestines of animals by Morgan. ${ }^{6}$ Kutscher for this reason suggests that essentially and except in rare instances this organism is a non-pathogenic saprophyte.

Type $B$ grows more heavily on all media than A, especially on potato (though this is not universally reported). Milk is slightly acid-

${ }^{1}$ Cushing, Johns Hopkins Hosp. Bull., 1900.

${ }^{2}$ Schottmüller, Deut. med. Woch., 1900; Zeit. f. Hyg., xxvi.

${ }^{3}$ Kurth, Deut. med. Woch., 1901.

${ }^{4}$ Buxton and Coleman, Proc. N. Y. Pathol. Soc., Feb., 1902.

${ }^{5}$ Libman, Jour. Med. Res., N. S., iii, 1902.

' Morgan, cited from Kutscher, Kolle und Wassermann, Handbüch.

Ergänzungs, I. 
ified at first, but eventually is rendered strongly alkaline and cleared up, possibly by casein solution. On lactose whey is alkalined and becomes strongly blue.

Eventual differentiation in doubtful cases must be made by agglutination. Infection with type B is not uncommon and far outstrips that with type $\mathrm{A}$ in importance.

Clinically, the diseases caused by the bacteria of this class may be divided into two main groups.

I. Those which fall into the category of meat poisoning, having a sudden and violent onset of gastroenteric symptoms directly following the ingestion of meat, and characterized by profound toxemia; and

II. Those in which the disease simulates a mild form of typhoid fever, differing from this only by the absence of the specific agglutination reaction for typhoid bacilli.

The differential diagnosis between the second type of case and true typhoid fever may be extremely difficult. However, careful studies by Lentz ${ }^{1}$ and others have revealed certain differences which though not conclusive are at least of some aid in determining the nature of the disease. In contradistinction to true typhoid the temperature reaction of this case may set in more abruptly and remain more irregular throughout the disease. Gastric symptoms, vomiting, and nausea are often more prominent than in typhoid fever and enlargement of the spleen is less regularly present than in the latter. Owing to the low mortality of paratyphoid fever (in 120 cases observed by Lentz less than 4 per cent, and in many other smaller epidemics no deaths have occurred), we have remained relatively ignorant concerning the pathologic anatomy of the disease. Longcope ${ }^{2}$ observed a case which was fatal after two weeks of illness in which there was no enlargement of Peyer's patches and no sign of even beginning ulceration. This seems to have been the experience of most other observers who have found less involvement of the lymphatics of the bowel than is found in typhioid fever. During the disease the bacteria can often be cultivated from the blood, and the serum of the patient may agglutinate specifically paratyphoid strains. In this way the diagnosis can often be made. Libmann ${ }^{3}$ has isolated the organism from the fluid aspirated from the gall bladder in a case operated on for cholecystitis.

Most of these microorganisms possess pathogenicity for mice, guinea-

\footnotetext{
${ }^{1}$ Lentz, Klin. Jahrb. xiv, 1914.

${ }^{2}$ Longcope, Amer. Jour. of Med. Sciences, cxxiv, 1902.

${ }^{3}$ Libmann, Jour. of Med. Res., viii, 1902.
} 
pigs, and rabbits, which exceeds that of the colon or typhoid bacilli. A number of the bacilli of this group, furthermore, especially those most closely similar to the original B. enteritidis of Gärtner, contain an endotoxin which shows a high resistance to heat, which may explain the fact that illness has occasionally followed the ingestion of infected meat even after preparation by cooking.

Bacteriological correlation of these bacilli has been attempted, as stated above, by Durham and by Buxton, and more recently by Kutscher and Meinicke. ${ }^{1}$ The subject is a difficult one and for ultimate clearness will require much further work.

Harding and Ostenberg ${ }^{2}$ have examined a series of organisms of the intermediate group on various sugars, and find that by the use of xylose and arabinose three definite groups can be established.

I. Those making aldehyd (red) on fuchsin-sulphite agar with both arabinose and xylose-both Schottmüller types A and B and strains of Bacillus enteritidis.

II. Red on arabinose and not on xylose-typhi murium, paratyphoid Gwyn, paratyphoid Loomis, and three others.

III. Red on xylose and not on arabinose-B. hog cholera.

This work was carefully carried out and may possibly point toward an ultimate classification. However, the strains employed were too few to permit definite conclusions at present.

Durham, ${ }^{3}$ on the basis of cultural and agglutinative studies, has formulated a classification of the Gram-negative bacilli of the typhoidcolon and allied groups, which, though hardly final, aids considerably in throwing light upon the interrelationships of the various species. Durham's divisions are as follows:

Division I. Typhoid-like Morphology (motile).

$A$. No sugars fermented. Type B. fecalis alkaligenes.

$B$. Acid in dextrose, but no gas. Type B. typhosus. Agglutination in typhoid serum.

$C$. Acid in dextrose, but gas only when other constituents are favorable. No acid or gas from lactose or saccharose. No agglutination in typhoid serum. Includes Bacillus "Gwyn" and Bacillus "O" of Cushing.

D. Acid and gas from dextrose. No acid or gas from lactose or

${ }^{1}$ Kutscher und Meinicke, Zeit. f. Hyg., lii, 1906.

${ }^{2}$ Harding and Ostenberg, Jour. of Inf. Dis., ii, 1912.

${ }^{3}$ Durham, loc. cit. 
saccharose. Grows more rapidly than typhoid. No agglutination in colon-immune serum. Slight reaction with some typhoid sera. Includes Gärtner's B. enteritidis, B. Morseele, Gunther's meat-poisoning bacillus, hog cholera bacillus, B. psittacosis, B. morbificans bovis, Durham's Bacillus "A," B. typhi murium.

Division II. Colon-like Morphology (motile).

$E$. Acid and gas from dextrose, none from lactose or saccharose. Rate of growth and colony appearance more like colon than typhoid.

$F$. Acid and gas from dextrose, and no gas from lactose. Types isolated by Durham.

G. Acid and gas from dextrose; acid, no gas, from lactose. Differ from $F$ in serum reactions.

H. B. coli communis. Acid and gas from dextrose and lactose; none from saccharose.

I. B. coli communior. Acid and gas from dextrose, lactose, and saccharose.

Division III. Non-motile. Polysaccharide splitters (starch). Type B. lactis aërogenes. Includes bacilli of mucosus capsulatus group, and Friedländer's bacillus. 


\section{CHAPTER XXIX}

\section{BACILLI OF THE COLON-TYPHOID-DYSENTERY GROUP \\ (Continued)}

\section{THE DYSENTERY BACILLI}

Although acute dysentery has been an extremely prevalent disease, occurring almost annually in epidemic form in some of the Fastern countries and appearing sporadically all over the world, its etiology was obscure until 1898 when Shiga ${ }^{1}$ described a bacillus which he isolated from the stools of patients suffering from this disease in Japan, and established with scientific accuracy its etiological significance. Since the discovery of Shiga's bacillus a number of other bacilli have been described by various workers, all of which, while showing slight biological differences from Shiga's microorganism, are sufficiently similar to it culturally and pathogenically to warrant their being classified together with it in a definite group under the heading of the "dysentery bacilli."

The manner in which Shiga made his discovery furnishes an instructive example of the successful application of modern bacteriological methods to etiological investigation. Many workers preceding Shiga had attempted to throw light upon this subject by isolations of bacilli from dysenteric stools, and by extensive animal inoculation. Shiga, following a suggestion made by Kitasato, approached the problem by searching for a microorganism in the stools of dysentery patients which would specifically agglutinate with the serum of these patients. His labors were crowned with success in that he found, in thirty-six cases, one and the same microorganism which showed uniform serum agglutinations. Further, he found that this bacillus was not present in the dejections of patients suffering from other diseases nor in those of normal men, and that when tested against the blood serum of such people it was not agglutinated.

Morphology.-Shiga's bacillus is a short rod, rounded at the ends,

1 Shiga, Cent. f. Bakt., xxiii, 1898; ibid., xxiv, 1898; Deut. med. Woch., xliii, xliv, and xlv, 1901 . 
morphologically very similar to the typhoid bacillus, and, like it, inclined to involution forms. The organism generally occurs singly, more seldom in pairs. It is decolorized by Gram's method of staining. With the ordinary anilin dyes it stains easily, showing a tendency to stain with slightly greater intensity at the ends. The organism is an aërobe and facultative anaërobe. Although described at first by Shiga as being motile, its motility has not been satisfactorily proven, and most observers agree in denying the presence of flagella and affirming the complete absence of motility.

Cultural Characteristics.-On agar the colonies are not characteristic, resembling those of the typhoid bacillus.

On gelatin, the colonies appear very much like typhoid colonies and the gelatin is not liquefied.

On potato, the growth, like that of typhoid, is at first not visible, but after about a week turns reddish brown.

In broth, there is clouding, with moderate deposits after some days. No pellicle is formed.

Milk is not coagulated. Litmus milk shows a slight primary acidity, later again becoming alkaline and taking on a progressively deeper blue color.

Indol is not formed in pepton water by all varieties.

No gas is formed in media containing dextrose, lactose, saccharose, or other carbohydrate.

While not delicately susceptible to reaction, the bacillus prefers slightly alkaline media.

Shiga differentiated his organism from the typhoid bacillus chiefly by supposed differences in colony characters and by the agglutination reaction.

Following the work of Shiga, a large number of investigators turned their attention to the subject of dysentery, with the result that many new forms were discovered and at first a considerable amount of confusion prevailed.

Flexner ${ }^{1}$ in 1899 investigated dysentery in the Philippines, and isolated a bacillus which, he considered, corresponded to Shiga's organism.

Strong and Musgrave $^{2}$ in 1900 described a bacillus isolated from

1 Flexner, Phila. Med. Jour., vi, 1900, and Bull. Johns Hopkins Hosp., xi, 1900.

Strong and Musgrave, Report Surg. Gen. of Army, Washington, 1900. 
dysentery cases in the Philippines which was essentially like that of Flexner.

Nearly simultaneously with the papers of Flexner and of Strong and Musgrave, Truse ${ }^{1}$ published investigations of an epidemic of dysentery occurring in Germany. His observations were of the greatest importance and largely formed the starting point of the further advances which have been made in the etiology of dysentery.

Kruse's organism was described as forming colonies on gelatin and agar, practically like those of Bacillus typhosus. Like this bacillus, no gas was formed from grape sugar, and the growth in milk and on potato, and even in Piorkowski's urine gelatin, resembled that of Bacillus typhosus. According to Kruse, this organism was absolutely without motility.

In 1901 Kruse $^{2}$ contributed a second paper. In this, besides confirming his previous observations, he described another class of organism coming from cases which he designated as "pseudo-dysentery of insane asylums." In the case of one patient, and at two autopsies, he isolated organisms which he could not distinguish morphologically or culturally from the true dysentery bacillus, but which showed differences in their serum reaction. By careful study of the behavior of these bacilli in the serum of patients and in immune serum from animals, he not only showed that they were different from his original cultures from cases of epidemic dysentery which, no matter what their source, were found to be alike, but that they showed differences among themselves and apparently fell into two or more varieties. One of these organisms culturally and by its serum reactions showed itself practically identical with one of the cultures he had received from Flexner.

Spronck ${ }^{3}$ in 1901 described an organism isolated in Utrecht from dysentery cases, which showed great similarity to the Shiga-Kruse organism; but, when tested in the serum of a horse immunized against true dysentery bacillus, showed practically no agglutination. He placed this organism in the group designated by Kruse as the "pseudo-dysentery bacilli." His communication is of importance, since it is the first reported instance in which any investigator had recognized and associated the so-called pseudo-dysentery bacilli with dysentery approaching the acute epidemic form in type.

Following this work a number of investigators, including Vedder

1 Kruse, Deut. med. Woch., xxvi, 1900.

${ }^{2}$ Kruse, Deut. med. Woch., xxvii, 1901.

3 Spronck, Ref. Baumgarten's Jahresber., 1901. 
and Duval, ${ }^{1}$ Flexner, and Shiga ${ }^{2}$ himself, published communications in which they claimed identity for the various forms previously described.

In 1902 Park $^{3}$ and Dunham described an organism which they found in a small outbreak of dysentery occurring in Maine. This organism differed from most of those previously described in that it was found to produce indol in pepton solutions.

In the same year Martini ${ }^{4}$ and Lentz published an article in which they attempted to differentiate various dysentery bacilli by means of agglutination. This research is of importance in that it supported the work of Kruse and of Spronck, indicating a difference between the agglutinative character of the Kruse organism and the so-called "pseudodysentery" type, in which Flexner's organisms were included. It is of further interest, since it indicated a marked difference between Flexner's Philippine cultures and the Philippine culture of Strong, the Strong organism refusing to agglutinate not only in "Shiga" immune serum, but also in "Flexner" immune serum.

Simultaneously with this article Lentz ${ }^{5}$ published the results of comparative cultural researches with dysentery and "pseudo-dysentery" bacilli, in which he made the important observation that the original Shiga-Kruse bacilli did not affect mannit, while the "pseudo-dysentery" bacilli, including Flexner's and Strong's Philippine cultures, fermented mannit, giving rise to a distinct acid reaction in the medium. The Flexner organisms and others of the "pseudo-dysentery" bacilli, however, fermented maltose, while the Shiga-Kruse type, as well as Strong's bacillus, left it unchanged at the end of forty-eight hours.

In January, 1903, Hiss and Russell ${ }^{6}$ described a bacillus ("Y") from a case of fatal diarrhea in a child, which by ordinary cultural test and absence of motility was found to resemble the Shiga-Kruse and Flexner bacilli. Immediately upon its isolation, it was found, however, to differ from the Kruse culture by its ability to ferment mannit. This observation was made independently of Lentz's work, which, at that time, had not become known in America. In the comparative study of Hiss and Russell on the fermentative abilities of various dysentery cultures, the serum water media (described on page 132) were used. By the use of

${ }^{1}$ Vedder and Duval, Jour. Exp. Med., vi, 1902.

${ }_{2}$ Shiga, Zeit. f. Hyg., 41, 1902.

${ }^{3}$ Park and Dunham, N. Y. Univ. Bull. of Med. Sci., 1902.

'Martini und Lentz, Zeit. f. Hyg., xli, 1902.

${ }^{5}$ Lentz, Zeit. f. Hyg., xli, 1902.

- Hiss and Russell, Med. News, Feb., 1903. 
these media, it was found that the Kruse culture, a culture of Flexner's bacillus from the Philippines, and Duval's "New Haven" culture fermented dextrose with the production of a solid acid coagulum, but did not affect mannit, maltose, saccharose, or dextrin. The culture of Hiss and Russell, on the other hand, fermented not only dextrose but also mannit with the production of acid and coagulation of the medium. Maltose, saccharose, and dextrin were not fermented. The "Y" bacillus, furthermore, was shown to differ entirely from the cultures of Shiga, Kruse, and "New Haven" in the serum of immunized animals. This serum had for bacillus " $\mathrm{Y}$ " a titer of $1: 500$ while the three other" abovenamed organisms did not agglutinate in it at any dilution. In normal beef serum, the Hiss-Russell organism was found to agglutinate as highly at $1: 320$, while the other three cultures gave no reaction in dilutions of over $1: 10$ or 20 .

Park and Carey, ${ }^{1}$ in March, 1903, described an epidemic of dysentery occurring in the town of Tuckahoe, near New York City, and isolated an organism which resembled the Shiga-Kruse bacilli in not fermenting mannit, but produced indol in pepton solution after five days. It corresponded in agglutination with the cultures "New Haven" and "Shiga" when tested in the serum of a goat immunized against the mannit-fermenting culture "Baltimore," i.e., did not react at 1:50, whereas Flexner's "Manila" and "Baltimore" cultures, Park and Dunham's "Seal Harbor" culture, and some New York cultures, all fermenting mannit, agglutinated up to two thousand dilution in the "Baltimore" serum.

The preceding review of a part of the literature, by which our knowledge of the dysentery bacilli was developed, demonstrates sufficiently that we have to deal in this group with a number of different microorganisms. This, as we have seen, was a fact first recognized by Kruse when he spoke of his true dysentery and his pseudo-dysentery strains. In spite of much confusion at first, the careful study of fermentation phenomena, of specific agglutinations, and, more recently, by Ohno ${ }^{2}$ and others, of the bacteriolytic phenomena in immune sera, has made it possible to distinguish sharply between a number of groups.

Basing the grouping of these microorganisms upon a careful study of fermentations, Hiss ${ }^{3}$ has divided them as follows:

${ }^{1}$ Park and Carey, Jour. Med. Res., ix, 1903.

?Ohno, Philippine Jour. of Sci., 1, ix., 1906.

'Hiss, Jour. Med. Res., N. S., viii, 1904. 


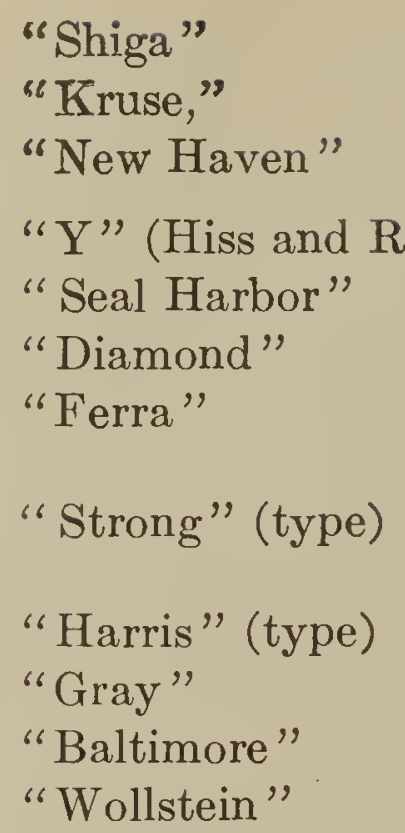

Ferment dextrose. Group I.

Ferment dextrose and mannit. Group II.

Ferments dextrose, mannit, saccharose Group III.

Ferment dextrose, mannit, maltose, saccharose, dextrin. Group IV.

It was noticed, it should be mentioned, however, that in the case of the "Y," "Diamond," and "Ferra" there was usually delayed acid fermentation of maltose, never any of dextrin.

In studying the agglutinative characters of these groups, furthermore, it was found that fermentation tests and agglutinations went hand in hand. The following table will illustrate this point: ${ }^{1}$

Serum of Rabbit immunized against Group I. (Shiga's culture). Bacilli of Group I.:

"Shiga" (homologous) . . . . . . . . . . . . . . . 20,000

"Kruse" . ............................. 20,000

"New Haven" ....................... 20,000

Bacilli of Group II.:

"Y" .................................... 200

"Ferra" ............................. 200

"Seal Harbor" .......................... 200

Bacilli of Group IV.:

"Baltimore" ............................ 800

"Harris" .............................. 800

"Gray" .............................. $\quad 800$

"Wollstein" ............................. 800

Serum of Rabbit immunized against Group II. ("Y" culture, Hiss and Russell).

Bacilli of Group I.:

"Shiga" ............................ less than 100

"Kruse"...................................... 100

"New Haven" " ........................... 100

${ }^{1}$ Hiss, Jour. of Med, Research, 13, N. S., viii, 1904. 
Bacilli of Group II.:

"Y" (homologous) ............................ 6, 6,400

"Ferra" .............................. 6,400

"Seal Harbor........................... 6,400

Bacilli of Group IV.:

"Baltimore" .............................. 1,600

"Gray" ............................... 1,600

"Harris" .................................... 1,600

"Wollstein" .............................. 1,600

Serum of Rabbit immunized against Group IV. ("Baltimore" culture).

Bacilli of Group I.:

"Shiga" . . . . . . . . . . . . . . . . . . . . less than 100

"Kruse" ............................... 100

"New Haven" .............................. 100

Bacilli of Group II.:

"Y" .................................... 400

"Ferra" .............................. 400

"জeal Harbor" ............................. 400

Bacilli of Group IV.:

"Baltimore" (homologous) . . . . . . . . . . . . . . . . . . 3,200

"Harris" . .................................. 3,200

"Gray" ................................ 3,200

"Wollstein" . ........................... 3,200

In common, all these groups possess an identical morphology, the Gram-negative staining characteristics, the lack of motility with close adherence to the line of inoculation in the Hiss tube medium, the inability to liquefy gelatin, the inability to form acid from lactose, and the inability to produce gas from any carbohydrate media.

Biological Considerations. - The dysentery bacilli in neutral broth or upon agar slants may remain alive without transplantation for periods of several months. They are aërobes and facultative anaërobes when proper sugars are present, preferring, however, the aërobic environment. They are easily destroyed by heat, an exposure to $60^{\circ} \mathrm{C}$. killing them usually in a short time (ten minutes). Against cold they show considerable resistance, surviving freezing for a period of several weeks. They show little resistance to the usual strengths of the common chemical disinfectants.

Pathogenicity.-There is practically no doubt at the present time as to the etiological connection between the bacilli of this group and the diseases clinically classified as acute dysentery. A more chronic form of 
dysentery due to a protozoan, the Amœba coli, though presenting much clinical resemblance to the bacillary dysenteries is, nevertheless, an entirely distinct disease.

Infection takes place, probably, entirely by ingestion of the bacteria with infected water or food contaminated from the feces of dysentery patients. A small epidemic occurring in a hospital in New York City and caused by the bacillus " $\mathrm{Y}$ " of Hiss and Russell was indirectly traced to milk by Zinsser. ${ }^{1}$

Endemic in a large part of the world, especially in the warmer climates, the disease most frequently occurs in epidemics of more or less definite localization, usually under conditions which accompany the massing of a large number of human beings in one place, such as those which occur in the crowded quarters of unsanitary towns, in institutions such as insane asylums, or in military camps. The mortality of such epidemics may be very large. According to Shiga, ${ }^{2}$ the disease in Japan frequently shows a mortality of over twenty per cent.

The disease in human beings usually begins as an acute gastroenteritis which is accompanied by abdominal pain and diarrhea. As it becomes more severe, the colicky pains and diarrhea increase, the stools lose their fecal character, becoming small in quantity and filled with mucus and flakes of blood. There is often severe tenesmus at this stage, and the bacilli are present in large numbers in the dejecta. Owing to the absorption of toxic products, symptoms referable to the nervous system, such as muscular twitching, may supervene, and if the disease is at all prolonged, there are marked inanition and prostration.

At autopsy in early stages there may be found only a severe catarrhal inflammation of the mucous membrane of the large intestine. In the later stages there are extensive ulcerations, and the bacteria are histologically found lodged within the depths of the mucosa and submucosa. Occasionally they may penetrate to the mesenteric glands, but as far as we know there is no penetration into the general circulation.

Poisonous Products of the Dysentery Bacilli.- The separate types of dysentery bacilli vary exceedingly in their powers to proluce toxic substances. Of all the various types which have been described, the strongest poisons have been produced with bacilli of the Shiga-Kruse variety, less regularly active ones with bacilli of the Flexner and of the " $Y$ " type. In fact, investigations carried out with the Shiga bacillus have tended to show that the disease itself is probably a true toxemia,

'Zinsser, Proc. N. Y. Path. Soc., 1907. ' 'Shiga, Cent. f. Bakt., xxiii, 1898. 
its symptoms being referable almost entirely to the absorption of the poisonous products of the bacillus from the intestine.

The earliest investigations, carried on chiefly upon rabbits, which are more susc jptible to this poison than any other animals, showed that even small doses of cultures of this bacillus administered intravenously or subcutaneously would produce death within a very short time. Conradi, ${ }^{1}$ Vaillard ${ }^{2}$ and Dopter, and others, finding that toxic symptoms were almost as pronounced when dead cultures were given as when the living bacilli were administered, came to the conclusion that the poisons of this bacillus were chiefly of the endotoxin type. More recently Todd, ${ }^{3}$ Kraus, ${ }^{4}$ and Rosenthal ${ }^{5}$ have claimed independently that they were able to demonstrate strong soluble toxins, similar in every way to diphtheria toxin. Kraus and Doerr ${ }^{6}{ }^{6}$ moreover, claim to have further corroborated this by producing specific antitoxins with these substances.

It is easy to obtain poisonous substances from dysentery cultures in considerable strength, both by extracting the bacilli themselves and by filtration of properly prepared cultures. It is therefore not unlikely that both types of poison are produced by the bacilli. Neisser and Shiga ${ }^{7}$ obtained toxins by emulsifying agar cultures in sterile salt solution, killing the bacilli at $60^{\circ} \mathrm{C}$., and allowing them to extract at $37.5^{\circ} \mathrm{C}$. for three days or more. The filtrates from such emulsions were extremely toxic. The simplest method of obtaining poisons from these bacilli is to cultivate them for a week or longer upon moderately alkaline meat-infusion broth. At the end of this time, the microorganisms themselves may be killed by heating to $60^{\circ}$ and the cultures filtered. According to Doerr, ${ }^{8}$ the toxins may be obtained in the dry state by precipitation with ammonium sulphate and re-solution of the precipitate in water.

The action of the dysentery toxin upon animals is extremely characteristic and throws much light upon the disease in man. The injection of a large dose intravenously into rabbits causes a rapid fall in temperature, marked respiratory embarrassment, and a violent

${ }_{1}^{1}$ Conradi, Deut. med. Woch., 1903.

${ }_{2}$ Vaillard et Dopter, Ann. de l'inst. Pasteur, 1903.

3 Todd, Brit. Med. Jour., Dec., 1903, and Jour. of Hyg., 4, 1904.

4 Kraus, Monatschr. f. Gesundheit, Suppl. 11, 1904.

${ }^{5}$ Rosenthal, Deut. med. Woch., 1904.

r Kraus und Doerr, Wien. klin. Woch., xlii, 1905.

7 Neisser and Shiga, Deut. med. Woch., 1903.

8 Doerr, "Das Dysenterietoxin," Jena, 1907. 
diarrhea. This is at first watery, later contains large amounts of blood. If the animals live a sufficient length of time, paralysis may occur, the animal may fall to one side or may drag its posterior extremities. It is a remarkable fact that intravenous inoculation gives rise to intestinal inflammation of a severe nature, unquestionably due to the excretion of the poison by the intestinal mucosa and limited, usually, to the cecum and colon, rarely attacking the small intestine. Flexner, ${ }^{1}$ who has experimented extensively upon this question, believes it probable that most of the pathological lesions occurring in the intestinal canal of $d y$ sentery patients are referable to this excretion of dysentery toxin, rather than to the direct local action of the bacilli.

Toxins from the Shiga-Kruse type are the most potent and those which cause paralysis.

Immunization with Dysentery Bacilli.-The immunization of small animals, such as rabbits and guinea-pigs, against dysentery bacilli, especially those of the Shiga type, is attended with much difficulty, owing to the great toxicity of the cultures. Nevertheless, successful results may be accomplished by the administration of extremely small doses of living or dead bacilli, increased very gradually and at sufficient intervals. Horses may be more easily immunized. The serum of such actively immunized animals contains agglutinins in considerable concentration and of a specificity sufficiently illustrated in the preceding section dealing with the identification of the various species. For diagnostic purposes in human beings, the agglutination reaction, according to the technique of the Widal reaction for typhoid fever, has been utilized by Kruse $^{2}$ and others. According to most observers, normal human serum never agglutinates dysentery bacilli in dilutions greater than one in twenty, while the serum of dysentery patients will often be active in dilutions as high as one in fifty.

Bactericidal substances have been demonstrated in the serum of immunized animals as well as in the serum of diseased human beings. These have been determined, in vitro, by Shiga, ${ }^{3}$ and by the intraperitoneal technique of Pfeiffer by Kruse. ${ }^{4}$ Bacteriolysis may take place in high dilutions of the serum, and has recently been used for the differentiation of the types of the dysentery bacilli by Ohno. ${ }^{5}$

True antitoxins in immune sera have been recently described by Kraus and Doerr. ${ }^{6}$

I Flexner, Jour. Exp. Med., 8, 1906.

3 Shiga, Zeit. f. Hyg., xli.

${ }_{5}^{5}$ Ohno, Philippine Jour. of Sci., vol. i, 1906.
2 Kruse, Deut. med. Woch., 1901

${ }^{4}$ Kruse, Deut. med. Woch., 1903.

${ }^{6}$ Kraus und Doerr, loc. cit. 


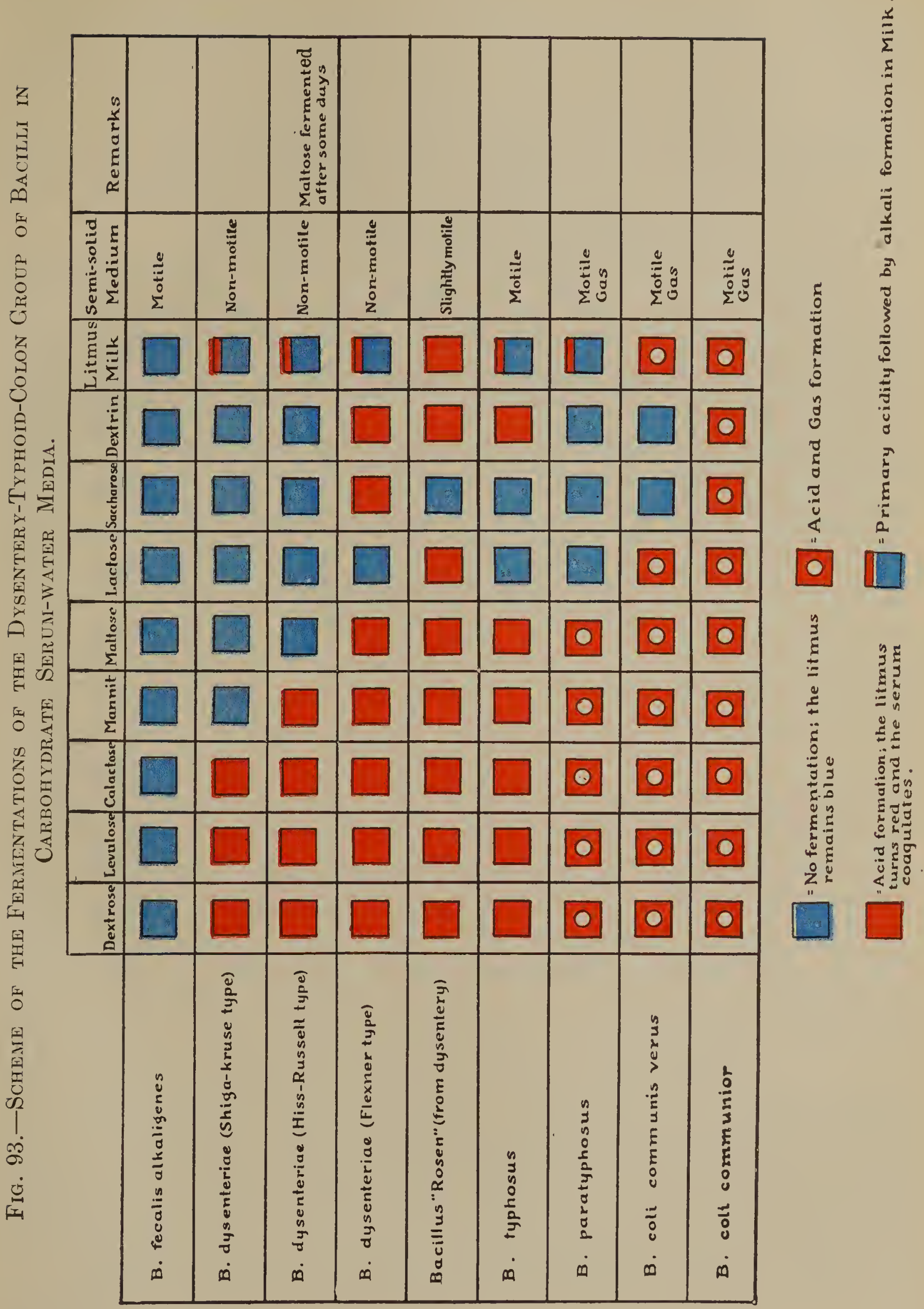


Passive immunization of animals and human beings with the serum of highly immunized horses has been variously attempted by Shiga, ${ }^{1}$ Kraus, ${ }^{2}$ Gay, ${ }^{3}$ and others. All these observers have reported distinct benefit to the patients and a reduction of the mortality by the use of such sera. Striking and rapid reductions of temperature and rapid convalescence, after a single injection, have occasionally been observed. The earlier workers were inclined to attribute the beneficial results of these sera entirely to their bactericidal value.

Todd has recently demonstrated that the mixture of such an immune serum with solutions of toxin and exposure of the mixture at $37.5^{\circ} \mathrm{C}$. for a half hour would produce almost complete neutralization of the poison, thus demonstrating that at least a large part of the beneficial action of the immune sera was due to a true antitoxic process. Because of the different varieties of dysentery bacilli, polyvalent serum has been recommended. Prophylactic vaccination of human begins with dead dysentery cultures has, so far, led to no practical result.

Shiga, Deut. med. Woch., 1901. 2 Kraus, loc. cit.

${ }^{3}$ Gay, Penn. Med. Bull., 1902. 


\section{CHAPTER XXX}

\section{BACILLUS MUCOSUS CAPSULATUS, BACILLUS LACTIS AËROGENES, BACILLUS PROTEUS}

\section{BACILLUS MUCOSUS CAPSULATUS}

\section{(Bacterium pneumonice, Friedländer's bacillus, Pneumobacillus)}

IN 1882, Friedländer ${ }^{1}$ announced the discovery of a microorganism which he believed to be the incitant of lobar pneumonia and which, in his original communications, he described as a "micrococcus."

A superficial morphological resemblance between Friedländer's microorganism and Diplococcus lanceolatus, now recognized as the most frequent cause of lobar pneumonia, led, at first, to much confusion, and it was not until several years later, owing to the careful researches of Fränkel ${ }^{2}$ and of Weichselbaum, ${ }^{3}$ that the "micrococcus" of Friedländer was recognized as a short, encapsulated bacillus which occurred in lobar pneumonia exceptionally only. Similar bacilli were subsequently found by other observers, bacilli which, mainly upon morphological grounds, are classified together as the "Friedländer group," or the "group of Bacillus mucosus capsulatus."

Morphology and Staining.-The Friedländer bacillus is a short, plump bacillus with rounded ends, subject to great individual variations as to size. Its average measurements are from 0.5 to 1.5 micra in width and 0.6 to 5 micra in length. Forms approaching both extremes may be met with in one and the same culture. The short, thick forms, frequently found in animal and human lesions, are almost coccoid and account for Friedländer's elror in first describing the bacillus as a micrococcus. The bacilli may be single, in diplo-form, or in short chains. They are non-motile and possess no flagella. Spores are not formed.

The bacillus is characteristically surrounded by a well-developed capsule which is most perfectly demonstrated in preparations taken directly from some animal fluid, such as the secretion or exudate from infected areas. It is also seen, however, in smears made from agar

1 Friedländer, Virchow's Arch., lxxxvii, 1882; Fort. d. Med., i, 1883; ibid., ii, 1884.

2 Fränkel, Zeit. f. klin. Med., x, 1886.

W Wichselbaum, Med. Jahrb., Wien, 1886. 
or gelatin cultures. The capsule is usually large, twice or three times the size of the bacillus itself. When seen in chains or in groups, several bacilli may appear to be inclosed in one capsule. Prolonged cultivation on agar or gelatin may result in disappearance of the capsule. The bacillus is easily stained with the ordinary dyes, but is decolorized when stained by the Gram-method. Capsules may often be seen when the more intense anilin dyes are employed. They are brought out with much regularity by any of the usual capsule stains.

Cultivation.-B. mucosus capsulatus is easily cultivated. It grows

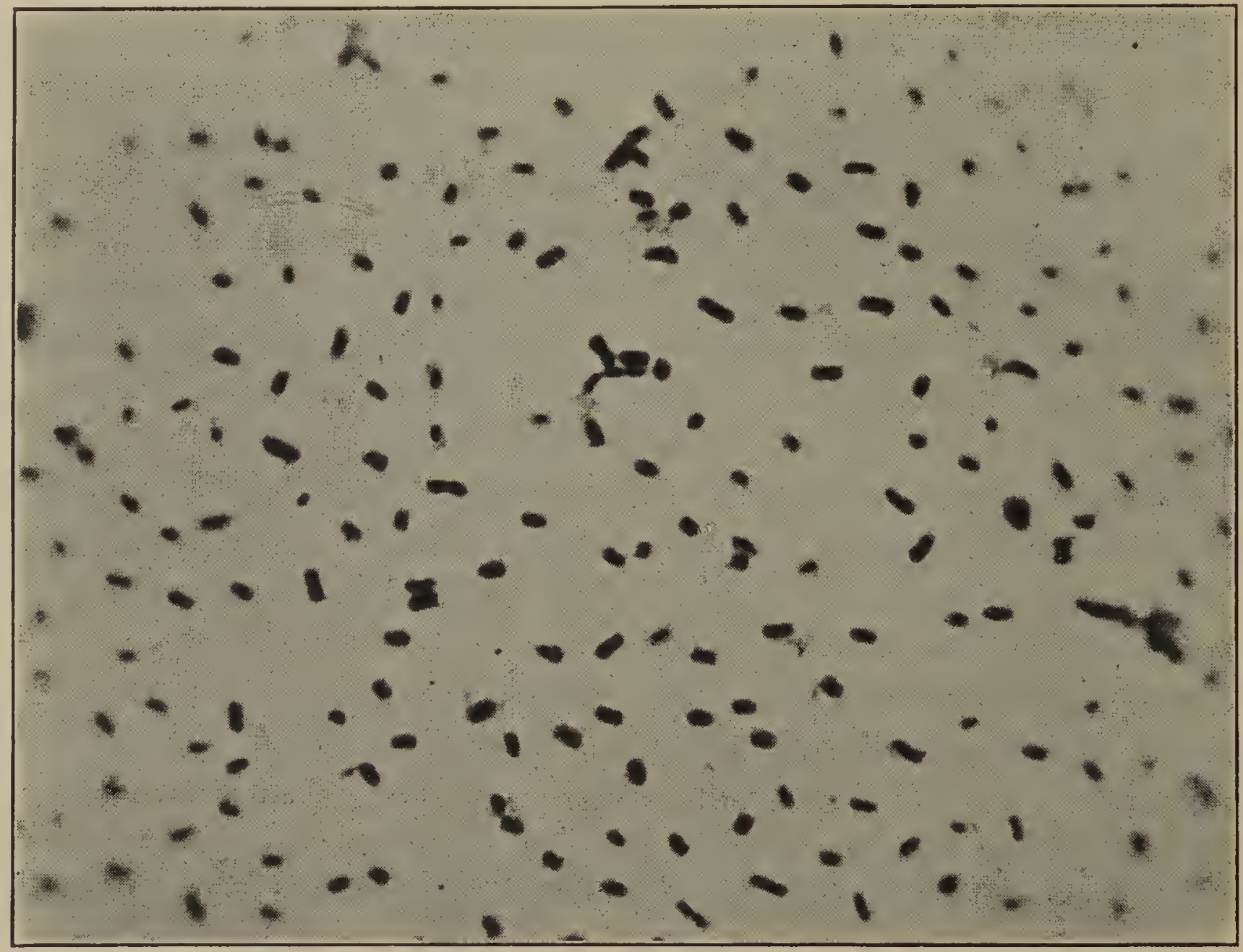

Fig. 94.-Bacillus mucosus capsulatus.

readily on all the usual culture media, both on those having a meatinfusion basis and on those made with meat extract. Growth takes place at room temperature $\left(18^{\circ}\right.$ to $\left.20^{\circ}\right)$ and more rapidly at $37.5^{\circ} \mathrm{C}$. A temperature of $60^{\circ} \mathrm{C}$. and over kills the bacilli in a short time. The thermal death-point according to Sternberg is $56^{\circ} \mathrm{C}$. Growth ceases below $10^{\circ}$ to $12^{\circ} \mathrm{C}$. Kept at room temperature and protected from drying, the bacillus may remain alive, in cultures, for several months.

The bacillus is not very fastidious as to reaction of media, growing 
equally well on moderately alkaline or acid media. It is aërobic and facultatively anaërobic; growth under anaërobic conditions, however, is not luxuriant.

On agar, growth appears in the form of grayish-white mucus-like colonies, having a characteristically slimy and semi-fluid appearance. Colonies have a tendency to confluence, so that on plates, after three or four days, a large part of the surface appears as if covered with a film of glistening, sticky exudate, which, if fished, comes off in a tenacious, stringy manner. It is often possible to make a tentative diagnosis of the bacillus from the appearance of this growth.

In broth, there is rapid and abundant growth, with the formation of a pellicle, general clouding, and later the development of a profuse, stringy sediment.

Stab cultures in gelatin show, at first, a white, thin line of growth along the course of the puncture. Soon, however, rapid growth at the top results in the formation of a grayish mucoid droplet on the surface, which, enlarging, gives the growth a nail-like appearance. This nail-shape was originally described by Friedländer and regarded as diagnostic for the bacillus. The gelatin is not fluidified. As the culture grows older the entire surface of the gelatin tube may be covered with growth, flowing out from the edges of the nail-head. The gelatin acquires a darker color and there may be a few gas bubbles below the surface. Microscopically, colonies on gelatin plates have a smooth outline and a finely granular or even homogeneous consistency.

On blood serum, a confluent mucus-like growth appears.

On potato, abundant growth appears, slightly more brownish in color than that on other media.

In pepton solutions, there is no indol formation.

In milk, there is abundant growth and marked capsule development. Coagulation occurs irregularly.

In considering the general cultural characteristics of the Friedländer bacillus, it must not be forgotten that we are dealing with a rather heterogeneous group, the individuals of which are subject to many minor variations. Capsule development, lack of motility, inability to fluidify gelatin, failure to form indol, and absence of spores, are characteristics common to all. In size, general appearance, gas formation, and pathogenicity, individual strains may vary much, one from the other. Strong ${ }^{1}$ has studied various races as to.gas formation and

${ }^{1}$ Strong, Cent. f. Bakt., xxv, 1899. 
concludes that most strains form gas from dextrose and levulose, but that lactose is fermented by some only. About two-thirds of the gas formed is hydrogen, the rest $\mathrm{CO}_{2}$. Acid formation, according to Strong, is also subject to much variation among different races. Similar studies by Perkins ${ }^{1}$ show that most of the ordinary cultural characteristics of bacilli of this group are extremely variable and can not serve as a basis for differentiation. Reactions on sugars, however, are more constant. Perkins suggests the following tentative division classes on this basis:

I. All carbohydrates fermented with the formation of gas.

II. All carbohydrates, except lactose, fermented with the formation of gas.

III. All carbohydrates, except saccharose, fermented with the formation of gas.

Type I. corresponds to B. aërogenes (Migula), Type II. to B. Friedländer or Bacterium pneumoniæ (Migula), and Type III. to Bacillus lactis aërogenes.

Differentiation by means of serum reactions has not proved satisfactory. ${ }^{2}$

Pathogenicity.-When Friedländer first described this microorganism, he assumed it to be the incitant of lobar pneumonia. Subsequent researches by Weichselbaum ${ }^{3}$. and others have shown it to be etiologically associated with pneumonia in about seven or eight per cent of all cases. The percentage in this country is probably lower. Such cases can often be diagnosed by the presence of the bacilli in the sputum, which is peculiarly sticky and stringy. Cases of Friedländer pneumonia are extremely severe and usually fatal. The bacillus has been found in cases of ulcerative stomatitis and nasal catarrh; in two cases of severe tonsillitis in children (Zinsser); in the pus from suppurations in the antrum of Highmore and the nasal sinuses (Fränkel and others), and in cases of fetid coryza (ozena), of which disease it is supposed by Abel ${ }^{4}$ and others to be the specific cause. Whether the ozena bacillus represents a separate

1 Perkins, Jour. of Infect. Dis., I, No. 2, 1904.

${ }^{2}$ J. G. Fitzgerald, who has récently made a careful study of the mucosus capsulatus group has concluded that present methods do not permit a subdivision of these organisms into separate species. He offers the following "tentative suggestion": It is conceivable that mutations based on the necessity of maintaining a parasitic existence have caused Gram-negative bacilli found normally in the body elsewhere than in the intestinal tract to develop capsules for protection and a new group has arisen which we designate B. mucosus capsulatus; and the varieties B. aërogenes and B. acidi lactici connect the group with the non-encapsulated colon group."

${ }^{3}$ Weichselbaum, loc. cit. ${ }^{4}$ Abel, Zeit. f. Hyg., xxi. 
species or not, can not at present be decided. The bacillus of Friedländer has been found in empyema fluid, in pericardial exudate (after pneumonia), and in spinal fluid. ${ }^{1}$ Isolated cases of Friedländer bacillus septicemia have been described. ${ }^{2}$ Being occasionally a saprophytic inhabitant of the normal intestine, it has been believed to be etiologically associated with some forms of diarrheal enteritis.

B. mucosus capsulatus is pathogenic for mice and guinea-pigs, less so for rabbits. Inoculation of susceptible animals is followed by local inflammation and death by septicemia. If inoculation is intraperitoneal, there is formed a characteristically mucoid, stringy exudate.

The question of immunization against bacilli of the Friedländer group is still in the stage of experimentation. Immunization with carefully graded doses of dead bacilli has been successful in isolated cases. Specific agglutinins in immune serum have been found by Clairmont, ${ }^{3}$ but irregularly and potent only against the particular strain used for the immunization.

\section{OTHER BACILLI OF THE FRIEDLANDER GROUP}

Bacillus of Rhinoscleroma.-This bacillus, discovered by v. Frisch 4 in 1882, is a plump, short rod, with rounded ends, morphologically almost identical with Friedländer's bacillus; it is non-motile and possesses a distinct capsule. Although at first described as Gram-positive, it has been shown to be decolorized with this method of staining. Culturally it is almost identical with B. mucosus capsulatus. It forms slimy colonies, has a nail-like appearance in gelatin stab cultures, and in pepton solutions produces no indol. It differs from B. mucosus capsulatus (Wilde ${ }^{5}$ ) in forming no gas in dextrose bouillon, in producing no acid in lactose bouillon, and in never coagulating milk.

Pathogenicity.-The bacillus of rhinoscleroma is but moderately pathogenic for animals delicately susceptible to the bacillus of Friedländer. Rhinoscleroma, the disease produced by this bacillus in man, consists of a slowly growing granulomatous inflammation, located usually at the external nares or upon the mucosa of the nose, mouth, pharynx, or larynx. It is composed of a number of chronic, hard, nodular swellings, which, on histological examination, show granulation tissue and productive inflammation. In the meshes of the abundant

${ }^{1}$ Jäger, Zeit. f. Hyg., xix.

${ }^{3}$ Clairmont, Zeit. f. Hyg., xxxix.
${ }^{2}$ Howard, Johns Hopkins Hosp. Bull., 1899.

${ }^{4} v$. Frisch, Wien. med. Woch., 1882.

${ }^{5}$ Wilde, Cent. f. Bakt., xx, 1896. 
connective tissue lie many large swollen cells, the so-called "Mikulicz cells." " The rhinoscleroma bacilli lie within these cells and in the intercellular spaces. They can be demonstrated in histological sections and can be cultivated from the lesions, usually in pure culture. Rhinoscleroma is rare in America. It is most prevalent in Southeastern Europe. The disease is slowly progressive and comparatively intractable to surgical treatment, but hardly ever affects the general health unless by mechanical obstruction of the air passages.

B. Ozænæ.-The work of $\mathrm{Abel}^{2}$ and others has shown that ozena, or

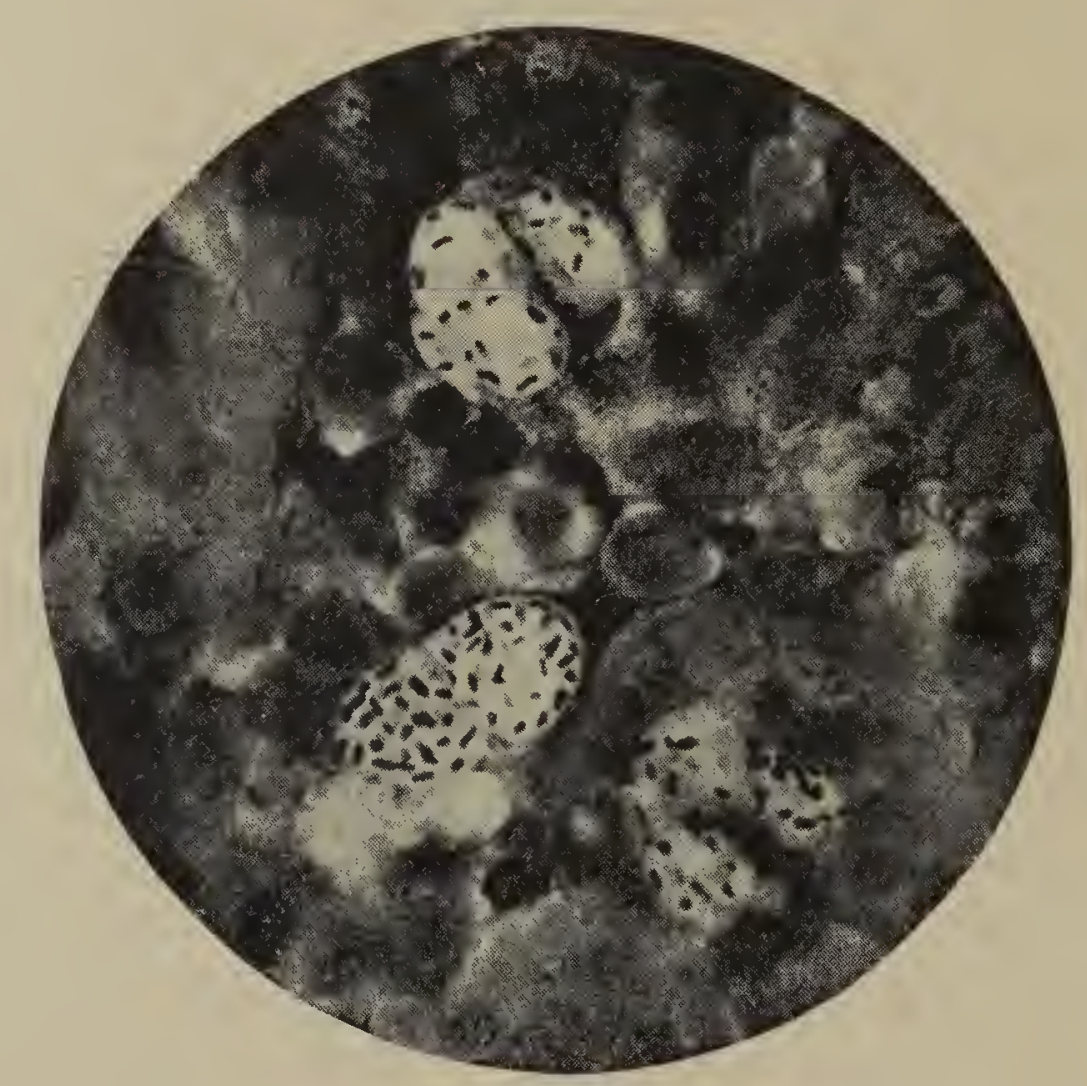

Fig. 95.-Bacillus of Rhinoscleroma. Section of tissue showing the microorganisms within Mikulicz cells. (After Fränkel and Pfeiffer.)

fetid nasal catarrh, is almost always associated with a bacillus morphologically and culturally almost identical with B. mucosus capsulatus. The bacillus can not be definitely separated from the latter. According to Wilde ${ }^{3}$ it forms no gas in dextrose bouillon and is less pathogenic for mice than B. Friedländer. Whether it is a separate species, or merely an atypical form changed by environment, can not be stated at present.

${ }_{1}$ Mikulicz, Arch. f. Chir., xx, 1876.

${ }^{2}$ Abel, Zeit. f. Hyg., xxi

3 Wilde, loc. cit. 


\section{BACILLUS LACTIS AËROGENES}

Bacillus lactis aërogenes is the type of a group which is closely similar to the colon group and of ten distinguished from it with difficulty. It was first described by Escherich ${ }^{1}$ in 1885 who isolated it from the feces of infants. Since then it has been learned that this bacillus is almost constantly present in milk, and, together with one or two other microorganisms, is the chief cause of the ordinary souring of milk. Apart from its occurrence in milk, moreover, the bacillus is widely distributed in nature, being found in feces, in water, and in sewage. It is distinguishable from the colon bacillus chiefly by the fact that it is non-motile, possesses no flagella, hardly ever forms chains, and, when cultivated upon suitable media, especially milk, it possesses a distinct capsule. It differs from the colon bacillus, furthermore, in that it is capable of fermenting polysaccharids, such as starch, and does not form indol upon pepton media. It is distinguishable from the bacillus of Friedländer (B. mucosus capsulatus), according to Wilde, ${ }^{2}$ by its more energetic gas formation in dextrose broth, its ability to produce acid on lactose media, and its invariable coagulation of milk.

The bacillus is about 0.5 to 1 micron in width and 2 to 4 micra in length. It grows easily upon the simplest media, is a facultative anaërobe, and grows most abundantly at a temperature between $25^{\circ}$ and $30^{\circ} \mathrm{C}$.

Upon agar and gelatin it grows readily with a heavy white growth, the colonies of which have a tendency to confluence and are distinctly more mucoid in appearance than are those of Bacillus coli.

In broth, it causes a general clouding and a pellicle. The cultures have a slightly sour or cheesy odor.

On potato, the growth is heavy and gas is formed.

On milk, there is rapid coagulation and acid formation. It is characteristic of this bacillus that it is capable of producing a large amount of acid, chiefly lactic, and of being able to withstand these large amounts of acid without being injured by them.

The pathogenicity of Bacillus lactis aërogenes for man is slight. Its chief claims to importance lie in its milk-coagulating properties and its almost constant presence in the human intestine. In infants, it may give rise to flatulence and it has been occasionally observed as the

${ }_{1}$ Escherich, Fort. d. Med., 16, 17, 1885. ${ }^{2}$ Wilde, Cent. f. Bakt., xx, 1896. 
sole incitant of cystitis. Among such cases rare instances have been observed in which it has formed gas in the bladder (pneumaturia). When this occurs the urine is not ammoniacal but remains acid.

Different strains of this bacillus vary much in their pathogenicity for animals. Wilde claims that it is more pathogenic for white mice and guinea-pigs than is the bacillus of Friedländer. He speaks of it as the most virulent member of this group. Kraus, writing in Fluegge's "Mikroorganismen," rates its pathogenicity less high.

Closely relatcd to this bacillus, as well as to those of the Friedländer group, is an encapsulated bacillus isolated from a case of bronchopneumonia by Mallory and Wright, ${ }^{1}$ which is strongly pathogenic for mice, guinea-pigs, and rabbits.

\section{BACILLI OF THE PROTEUS GROUP}

The bacilli of this group have little pathological interest, but are important because of the frequency with which they are encountered in routine bacteriological work. They may confuse the inexperienced because of a superficial similarity to bacilli of the colon-typhoid group. In form they may be short and plump or long and slender, staining easily with anilin dyes and decolorizing with Gram's method. They are actively motile and possess many flagella. The individuals stain irregularly, often showing unstained areas near the center. The type of the group is found in the so-called Bacillus proteus vulgaris described by Hauser ${ }^{2}$ in 1885.

Bacilli of this group are widely distributed, being found in water, soil, air, and wherever putrefaction takes place. In fact, proteus is one of the true putrefactive bacteria possessing the power to cause the cleavage of proteids into their simplest radicles.

Bacillus proteus vulgaris grows best at temperatures at or about $25^{\circ} \mathrm{C}$. and develops upon the simplest media. It is a facultative anaërobe and forms no spores.

In broth, it produces rapid clouding with a pellicle and the forma-. tion of a mucoid sediment.

In gelatin, the colonies are characteristically irregular, giving the name to this group.

Gelatin is rapidly liquefied. Liquefaction, however, is diminished or even inhibited under anaërobic conditions.

2 Hauser, "Ueber Fäulniss-Bakt.," Leipzigr, 1885. 
On agar and other solid media, as well as upon gelatin before liquefaction has taken place, characteristic colonies are produced. From the central flat, grayish-white colony nucleus, numerous irregular streamers grow out over the surrounding media, giving the colony a stellate appearance.

On potato, it forms a dirty, yellowish growth.

In milk, there is coagulation and an acid reaction at first; later the casein is redissolved by proteolysis.

Blood serum is often liquefied, but not by all races.

The pathogenic powers of proteus bacilli are usually slight. Large doses injected into animals may give rise to localized abscesses. In man proteus infections have been described as occurring in the bladder; in most cases, however, in combination with some other microorganism. The so-called Urobacillus liquefaciens septicus described by Krogius was probably a variety of this group. Epidemics ${ }^{1}$ of meat poisoning have been attributed to members of the proteus family by some observers. Thus Wesenberg ${ }^{2}$ was able to cultivate a proteus bacillus from putrid meat which had caused acute gastroenteritis in sixty-three individuals. Similar epidemics have been reported by Silberschmidt, ${ }^{3}$ Pfuhl, ${ }^{4}$ and others. In some of these the bacilli proved to be unusually toxic when injected into animals, but could not be recovered from the organs after death.

1 Schnitzler, Cent. f. Bakt., viii, 1890. ${ }_{2}^{2}$ Wesenberg, Zeit. f. Hyg., xxviii, 1898.

${ }^{3}$ Silberschmidt, Zeit. f. Hyg., xxx, 1899. ${ }^{4}$ Pfuhl, Zeit. f. Hyg., xxxv, 1900. 


\section{CHAPTFR XXXI}

\section{BACILLUS TETANI}

LockJAw or tetanus, though a comparatively infrequent disease, has been recognized as a distinct clinical entity for many centuries. The infectious nature of the disease, however, was not demonstrated until 1884, when Carlo ${ }^{1}$ and Rattone succeeded in producing tetanus in rabbits by the inoculation of pus from the cutaneous lesion of a human case. Nicolaier, ${ }^{2}$ not long after, succeeded in producing tetanic symptoms in mice and rabbits by inoculating them with soil. In connection with the lesions produced at the point of inoculation, Nicolaier described a bacillus which may have been Bacillus tetani, but which he was unable to cultivate in pure culture. Kitasato, ${ }^{3}$ in 1889 , definitely solved the etiological problem by obtaining from cases of tetanus pure cultures of bacilli with which he was able again to produce the disease in animals.

Kitasato succeeded where others had failed because of his use of anaërobic methods and his elimination of non-spore-bearing contaminating organisms by means of heat. His method of isolation was as follows: The material containing tetanus bacilli was smeared upon the surface of agar slants. These were permitted to develop at incubator temperature for twenty-four to forty-eight hours. At the end of this time the cultures were subjected to a temperature of $80^{\circ} \mathrm{C}$. for one hour. The purpose of this was to destroy all non-sporulating bacteria, as well as aërobic spore-bearers which had developed into the vegetative form. Agar plates were then inoculated from the slants and exposed to an atmosphere from which oxygen had been completely eliminated and hydrogen substituted. On these plates colonies of tetanus bacilli developed.

Morphology and Staining. - The bacillus of tetanus is a slender bacillus, 2 to 5 micra in length, and 0.3 to 0.8 in breadth. The vegetative forms which occur chiefly in young cultures are slightly motile and are

${ }^{1}$ Carlo e Rattone, Giornale d. R. Acad. d. Torino, 1884.

2 Nicolaier, Inaug. Diss., Göttingen, 1885 .

${ }^{3}$ Kitasato, Deut. med. Woch., No. xxxi, 1889. 
seen to possess ${ }^{1}$ numerous peritrichal flagella, when stained by special methods. After twenty-four to forty-eight hours of incubation, the length of time depending somewhat on the nature of the medium and the degree of anaërobiosis, the bacilli develop spores which are characteristically located at one end, giving the bacterium the diagnostic drumstick appearance.

As the cultures grow older the spore-bearing forms completely super-

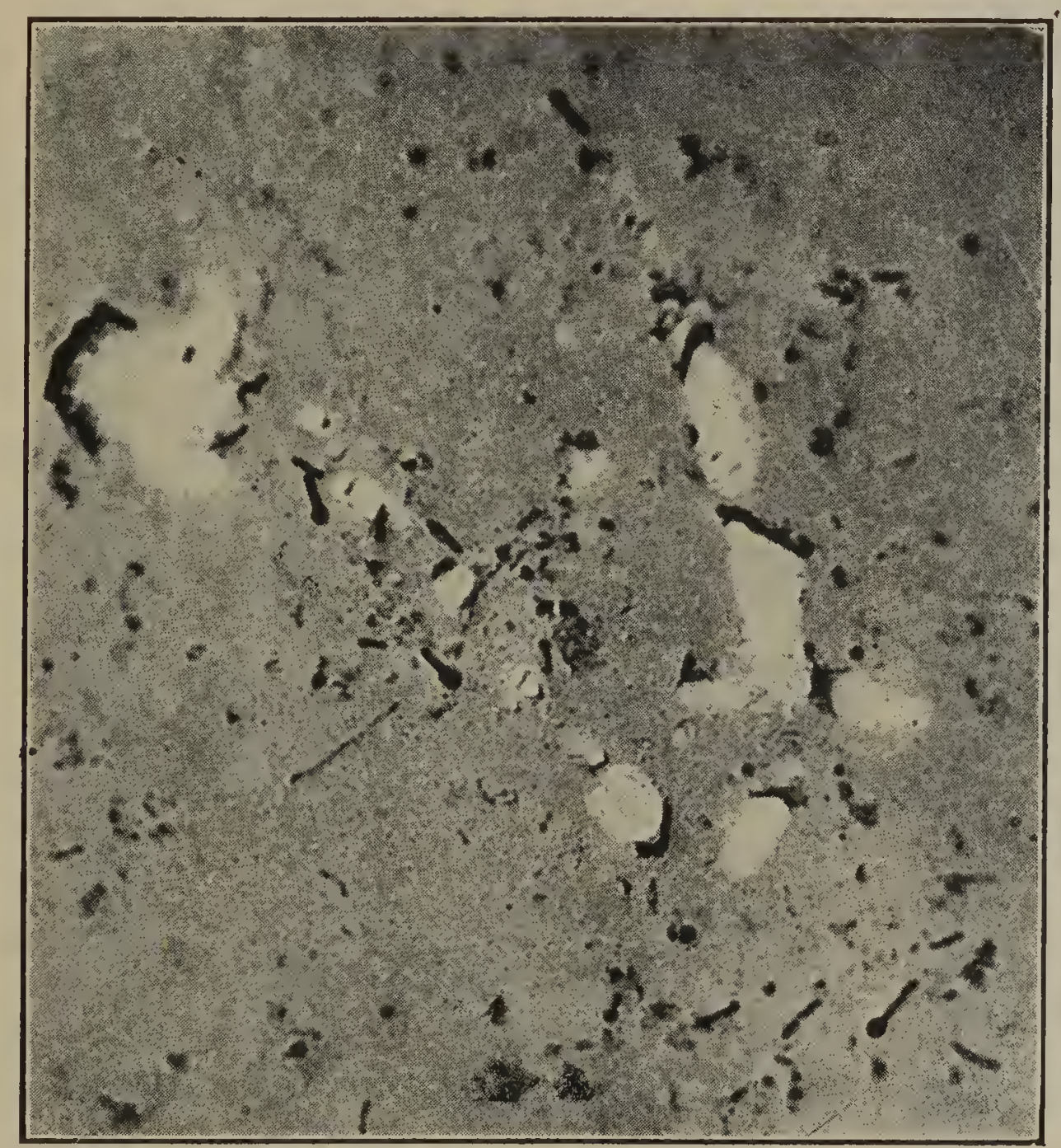

Fig. 96.-Bacillus tetani. Spore stain.

sede the vegetative ones. Very old cultures contain spore-bearing bacilli and spores only.

The tetanus bacillus is easily stained by the usual anilin dyes, and reacts positively to Gram's stain. Flagella staining is successful only when very young cultures are employed.

Distribution.-In nature, the tetanus bacillus has been found by Nicolaier and other's to occur in the superficial layers of the soil. The 
earth of cultivated and manured fields seems to harbor this organism with especial frequency, probably because of its presence in the dejecta of some of the domestic animals.

Biological Characteristics.-The bacillus of tetanus is generally described as an obligatory anaërobe. While it is unquestionably true that growth is ordinarily obtained only in the complete absence of

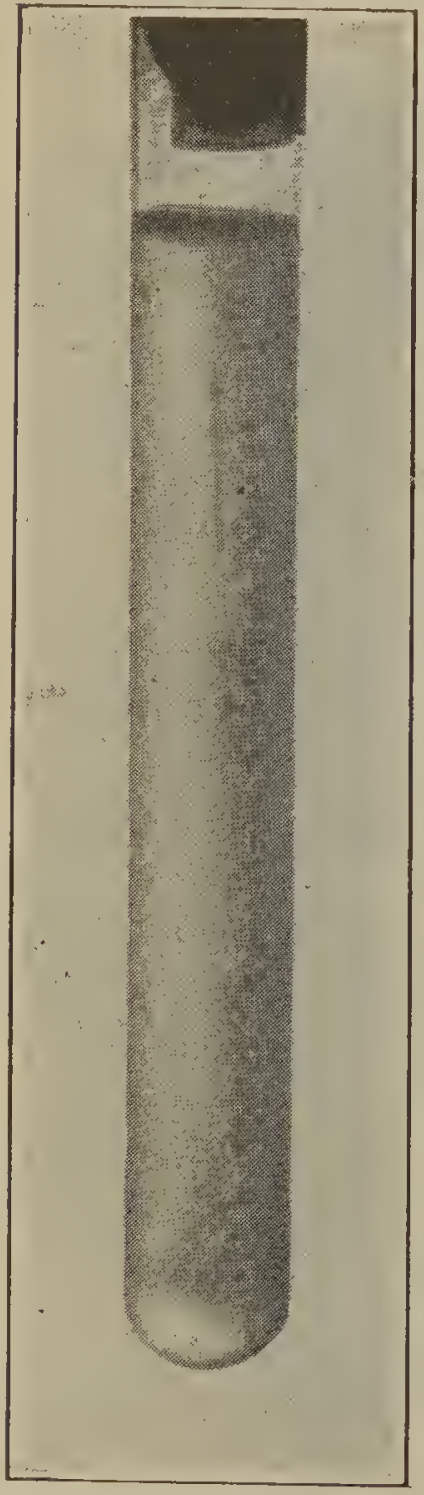

Fig. 97.-Young Tetanus Culture in Glucose Agar. oxygen, various observers, notably Ferran ${ }^{1}$ and Belfanti, ${ }^{2}$ have successfully habituated the bacillus to aërobic conditions by the gradual increase of oxygen in cultures. Habituation to aërobic conditions has usually been accompanied by diminution or loss of pathogenicity and toxin-formation. Anaërobic conditions may likewise be dispensed with if tetanus bacilli be grown in symbiosis with some of the aërobic bacteria. The addition to culture media of suitable carbohydrates, and of fresh sterile liver tissue, has also been found to render it less exacting as to absolute anaërobiosis. ${ }^{3}$

Anaërobically cultivated, Bacillus tetani grows readily upon meat-infusion broth, which it clouds within twenty-four to thirty-six hours. Anaërobic broth cultures may be simply made by covering the surface of the medium with a layer of albolin or any other oil, and removing the air by boiling.

Upon meat-infusion gelatin at $20^{\circ}$ to $22^{\circ} \mathrm{C}$. the tetanus bacillus grows readily, growth becoming visible during the second or third day. There is slow fluidification of the gelatin.

On agar, at $37.5^{\circ} \mathrm{C}$, growth appears within fortyeight hours. Colonies on agar plates present a rather characteristic appearance, consisting of a compact center surrounded by a loose meshwork of fine filaments, not unlike the medusa-head appearance of subtilis colonies. In agar stabs, fine radiating processes growing out in all directions from the central stab tend to give the culture the appearance of a fluff of cotton. Milk is a favorable culture medium and is not coagulated. On potato, growth is delicate and hardly visible.

${ }^{1}$ Ferran, Cent. f. Bakt., xxiv, No. 1.

${ }^{2}$ Belfanti, Arch. per le sci. med., xvi.

${ }^{3}$ Th. Smith, Brown, and Walker, Jour, Med. Res., N. S., ix, 1906. 
The most favorable temperature for the growth of this bacillus is $37.5^{\circ} \mathrm{C}$. Slight alkalinity or neutrality of the culture media is most advantageous, though moderate acidity does not altogether inhibit growth. All the media named may be rendered more favorable still by the addition of one or two per cent of glucose, maltose, or sodium formate. ${ }^{1}$ In media containing certain carbohydrates, tetanus bacilli produce acid.

In gelatin and agar, moderate amounts of gas are produced, consisting chiefly of $\mathrm{CO}_{2}$, but with the admixtures of other volatile substances which give rise to a characteristically unpleasant odor, not unlike that of putrefying organic matter. This odor is due largely to $\mathrm{H}_{2} \mathrm{~S}$ and methylmercaptan.

The vegetative forms of the tetanus bacillus are not more resistant against heat or chemical agents than the vegetative forms of other microorganisms. Tetanus spores, however, will resist dry heat at $80^{\circ} \mathrm{C}$. for about one hour, live steam for about five minutes; five per cent carbolic acid kills them in twelve to fifteen hours; one per cent of bichlorid of mercury in two or three hours. Direct sunlight diminishes their virulence and eventually destroys them. ${ }^{2}$ Protected from sunlight and other deleterious influences, tetanus spores may remain viable and virulent for many years. Henrijean ${ }^{3}$ has reported her success in producing tetanus with bacilli from a splinter of wood infected eleven years before.

Pathogenicity.-The comparative infrequency of tetanus infection is in marked contrast to the wide distribution of the bacilli in nature. Introduced into the animal body as spores, and free from toxin, they

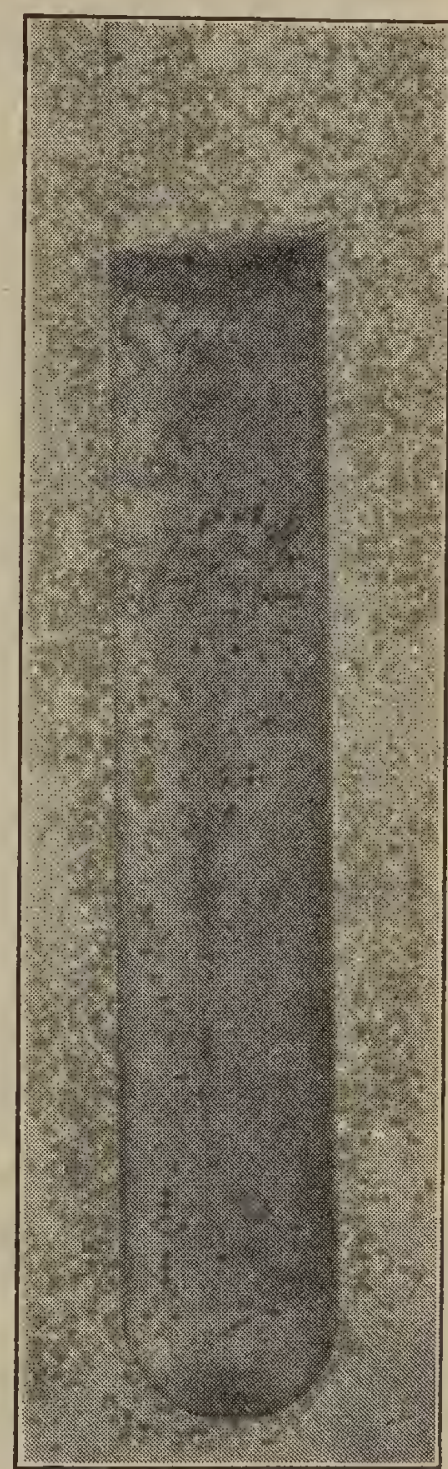

Frg. 98.-OLDER Tetanus Culture in Glucose Agar. may often fail to incite disease, easily falling prey to phagocytosis and other protective agencies before the vegetative forms develop and toxin is formed. The protective importance of phagocytosis was demonstrated by Vaillard and Rouget, ${ }^{4}$ who introduced tetanus spores inclosed in paper sacs into the animal body. By the paper cap-

1 Kitasato, Zeit. f. Hyg., 1891.

2 v. Eisler und Pribram, in Levaditi, "Handbuch," etc., Jena, 1907.

3 Henrijean, Ann. de la soc. méd. chir. de Liège, 1891.

4 Vaillard et Rouget, Ann. de l'inst. Pasteur, 1892. 
sules the spores were protected from the leucocytes, not from the body fluids. Nevertheless, tetanus developed in the animals. The nature of the wound and the simultaneous presence of other microorganisms seem to be important factors in determining whether or not the tetanus bacilli shall be enabled to proliferate. Deep, lacerated wounds, in which there has been considerable tissue destruction, and in which chips of glass, wood splinters, or grains of dirt have become embedded, are particularly favorable for the development of these germs. The injuries of compound fractures and of gunshot wounds are especially liable to supply these conditions, and the presence in such wounds of the common pus cocci, or of other more harmless parasites, may aid materially in furnishing an environment suitable for the growth of the tetanus bacilli. Apart from its occurrence following trauma, tetanus has been not infrequently observed after childbirth, ${ }^{1}$ and isolated cases have been reported in which it has followed diphtheria and ulcerative lesions of the throat. ${ }^{2}$

A definite period of incubation elapses between the time of infection with tetanus bacilli and the development of the first symptoms. In man this may last from five to seven days in acute cases, to from four to five weeks in the more chronic ones. Experimental inoculation of guinea-pigs is followed usually in from one to three days by rigidity of the muscles nearest the point of infection. This spastic condition rapidly extends to other parts and finally leads to death, which occurs within four or five days after infection.

Autopsies upon human beings or animals dead of tetanus reveal few and insignificant lesions. The initial point of infection, if at all evident, is apt to be small and innocent in appearance. Further than a general and moderate congestion, the organs show no pathological changes. Bacilli are found sparsely even at the point of infection, and have been but rarely demonstrated in the blood or viscera. Nicolaier succeeded in producing tetanus with the organs of infected animals in but eleven out of fifty-two cases. More recently, Tizzoni ${ }^{3}$ and Creite ${ }^{4}$ have succeeded in cultivating tetanus bacilli out of the spleen and heart's blood of infected human beings.

The researches of Tarozzi ${ }^{5}$ and of Canfora ${ }^{6}$ have shown also that spores may be transported from the site of inoculation to the liver, spleen, and other organs, and there lie dormant for as long as fifty-one days. If injury of the organ is experimentally practised and dead tissue

\footnotetext{
${ }^{1}$ Baginsky, Deut. med. Woch., 1893.

${ }^{4}$ Creite, Cent. f. Bakt., xxxvii.

${ }^{2}$ Foges, Wien. med. Woch., 1895.

${ }^{3}$ Tizzoni, Ziegler's Beit., vii.

5 Tarozzi, Cent. f. Bakt. Orig. xxxviii.

${ }^{6}$ Canfora, Cent. f. Bakt. Orig. xlv.
} 
or blood clot produced, the spores may develop and tetanus ensue. These experiments may explain cases of so-called cryptogenic tetanus.

Tetanus Toxin. - The pathogenicity of the tetanus bacillus depends entirely upon the soluble toxin which it produces. This toxin is produced in suitable media by all strains of virulent tetanus bacilli, individual strains showing less variation in this respect than do the separate strains of diphtheria bacilli. While partial aërobiosis does not completely eliminate toxin formation, anaërobic conditions are by far more favorable for its development.

The medium most frequently employed for the production of tetanus toxin is neutral or slightly alkaline beef-infusion bouillon containing fivetenths per cent $\mathrm{NaCl}$ and one per cent pepton. Glucose, sodium formate, or tincture of litmus may be added, but while these substances increase the speed of growth of the bacilli they do not seem to enhance the degree of toxicity of the cultures. Glucose is said even to be unfavorable for strong toxin development. It is important, too, that the bouillon shall be freshly prepared. ${ }^{1}$ There does not seem to be any direct relationship between the amount of growth and the degree of toxicity of the cultures. Under anaërobic conditions in suitable bouillon and grown at $37.5^{\circ} \mathrm{C}$., the maximum toxin content of the cultures is reached in from ten days to two weeks. After this time the toxin deteriorates rapidly.

Tetanus toxin has been produced without resort to anaërobic methods by several observers, notably by Debrand, ${ }^{2}$ by cultivating the bacilli in bouillon in symbiosis with Bacillus subtilis. By this method, Debrand claims to have produced toxin which was fully as potent as that produced by anaërobic cultivation.

The tetanus toxin, in solution in the bouillon cultures, may be separated from the bacteria by filtration through Berkefeld or Chamberland filters. Since the poison in such filtrates deteriorates very rapidly, much more rapidly even than diphtheria toxin, various methods have been devised to obtain the toxin in the solid state. The most useful of these is precipitation of the poison out of solution by oversaturation with ammonium sulphate. ${ }^{3}$ Very little of the toxin is lost by this method and, thoroughly dried and stocked in vacuum tubes, together with anhydrous phosphoric acid, it may be preserved indefinitely without deterioration. The precipitate thus formed is easily soluble in water or

1 Vaillard et Vincent, Ann. de l'inst. Pasteur, 1891.

2 Debrand, Ann. de l'inst. Pasteur, 1890, 1902.

${ }^{3}$ Brieger und Cohn, Zeit. f. Hyg., xv. 
salt solution, and therefore permits of the preparation of uniform solutions for purposes of standardization.

Brieger and Boer ${ }^{1}$ have also succeeded in precipitating the toxin out of broth solution with zinc chloride. Vaillard and Vincent ${ }^{2}$ have procured it in the dry state by evaporation in vacuo.

Brieger and Cohn, ${ }^{3}$ Brieger and Boer, ${ }^{4}$ and others have attempted to isolate tetanus poison, removing the proteids from the ammonium sulphate precipitate by various chemical methods. The purest preparations obtained have been in the form of fine yellowish flakes, soluble in water, insoluble in alcohol and ether. Solutions of this substance have failed to give the usual proteid reactions.

The toxin when in solution is extremely sensitive to heat. Kitasato ${ }^{5}$ states that exposure to $68^{\circ} \mathrm{C}$. for five minutes destroys it completely. Dry toxin is more resistant, ${ }^{6}$ often withstanding temperatures of $120^{\circ} \mathrm{C}$. for more than fifteen minutes. Exposure to direct sunlight destroys the poison in fifteen to eighteen hours. ${ }^{7}$

Interesting experiments as to the action of eosin upon tetanus toxin have been carried out by various observers. Flexner and Noguchi ${ }^{8}$ found that five per cent eosin added to the toxin would destroy it within one hour. This action is ascribed to the photodynamic power of the eosin.

The toxin exerts an extremely low osmotic pressure and is easily destroyed by electric currents.

Tetanus toxin is one of the most powerful poisons known to us. Filtrates of broth cultures, in quantities of $0.000,005$ c.c., will often prove fatal to mice of ten grams weight. Dry toxin obtained by ammonium sulphate precipitation ${ }^{9}$ is quantitatively even stronger, values of $0.000,001$ grams as a lethal dose for a mouse of the given weight not being uncommon. Brieger and Cohn ${ }^{10}$ succeeded in producing a dry toxin capable of killing mice in doses of 0.000,000,05 gram.

Different species of animals show great variation in their suscepti-

${ }^{1}$ Brieger und Boer, Zeit. f. Hyg., xxi.

${ }^{2}$ Vaillard et Vincent, Ann. de l'inst. Pasteur, 1891.

${ }^{3}$ Brieger und Cohn, loc. cit.

${ }^{4}$ Brieger und Boer, Zeit. f. Hyg., xxi.

${ }^{5}$ Kitasato, Zeit. f. Hyg., x.

${ }^{6}$ Morax et Marie, Ann. de l'inst. Pasteur, 1902.

${ }^{7}$ Fermi und Pernossi, Cent. f. Bakt., xv.

${ }^{8}$ Flexner and Noguchi, "Studies from Rockefeller Inst.," v., 1905.

${ }^{9}$ Brieger und Cohn., loc. cit.

${ }^{10}$ Brieger und Cohn., Zeit. f. Hyg., xv. 
bility to tetanus toxin. Human beings and horses are probably the most susceptible species in proportion to their body weight. The common domestic fowls are extremely resistant. Calculated for grams of body weight, the horse is twelve times as susceptible as the mouse, the guineapig six times as susceptible as the mouse. The hen, on the other hand, is 200,000 times more resistant than the mouse.

After the inoculation of an animal with tetanus toxin there is always a definite period of incubation before the toxic spasms set in. This peri od may be shortened by increase of the dose, but never entirely eliminated. ${ }^{1}$ When the toxin is injected subcutaneously, spasms begin first in the muscles nearest the point of inoculation. Intravenous inoculation, ${ }^{2}$ on the other hand, usually results in general tetanus of all the muscles. The feeding of toxin does not produce disease, the poison being passed through the bowel unaltered.

The harmful action of tetanus toxin is generally attributed to its affinity for the central nervous system. Wassermann and Takaki ${ }^{3}$ show that tetanus toxin was fully neutralized when mixed with brain substance. Other organs-liver and spleen, for instance-showed no such neutralizing power. The central origin of the tetanic contractions was made very evident by the work of Gumprecht, ${ }^{4}$ who succeeded in stopping the spasms in a given region by division of the supplying motor nerves.

The manner in which the toxin reaches the central nervous system has been extensively investigated, chiefly by Meyer and Ransom, and Marie and Morax. Meyer and Ransom ${ }^{5}$ from a series of careful experiments reached the conclusion that the toxin is conducted to the nerve centers along the paths of the motor nerves. Injected into the circulation, ${ }^{6}$ the toxin reaches simultaneously all the motor nerve endings, producing general tetanus. In this case too, therefore, the poison from the blood can not pass directly into the central nervous system, but must follow the route of nerve tracts.

These observations have been of great practical value in that they pointed to the desirability of the injection of tetanus antitoxin directly into the nerves and the central nervous system in active cases.

${ }^{1}$ Courmont et Doyen, Arch. de phys., 1893.

${ }^{2}$ Ransom, Deut. med. Woch., 1893.

${ }^{3}$ Wassermann und Takaki, Berl. klin. Woch., 1898.

${ }^{4}$ Gumprecht, Pflüger's Arch., 1895.

${ }^{5}$ Meyer und Ransom, Arch. f. exp. Pharm. u. Path., xlix.

${ }^{6}$ Marie et Morax, Ann, de l'inst. Pasteur, 1902. 
Tetanolysin.-Tetanus bouillon contains, besides the "tetanospasmin" described above which produces the familiar symptoms of the disease, another substance discovered by Ehrlich ${ }^{1}$ and named by him "tetanolysin." Tetanolysin has the power of causing hemolysis of the red blood corpuscles of various animals, and is an entirely separate substance from tetanospasmin. It may be removed from toxic broth by admixture of red blood cells, is more thermolabile than the tetanospasmin, and gives rise to an antihemolysin when injected into animals. For the production, standardization, and use of tetanus antitoxin, see p. 220 et seq.

${ }^{1}$ Ehrlich, Berl. klin. Woch., 1898. 


\section{CHAPTER XXXII}

\section{BACILLUS OF SYMPTOMATIC ANTHRAX, BACILLUS OF MALIGNANT EDEMA, BACILLUS AËROGENES CAPSULATUS, BACILLUS BOTULINUS}

\section{BACILLUS OF SYMPTOMATIC ANTHRAX}

(Bacillus anthracis symptomatici, Rauschbrand, Charbon symptomatique, Sarcophysematos bovis)

Sympтомaтic anthrax is an infectious disease occurring chiefly among sheep, cattle, and goats. It is colloquially spoken of as "quarterevil" or "blackleg." The disease has never been observed in man. It was formerly, and is often to-day, confused with true anthrax, largely because of a superficial similarity between the clinical symptoms of the two maladies. Bacteriologically, the two microorganisms are in entirely different classes.

Geographically, symptomatic anthrax is of wide distribution and infection is usually through the agency of the soil in which the bacillus is present, probably in the form of spores which may retain viability and pathogenicity for several years.

Morphology and Staining.-The bacillus of symptomatic anthrax is a bacillus with rounded ends, somewhat shorter and relatively thicker than the bacillus of malignant edema, being about four to six micra long, and five-tenths to six-tenths micra wide. It is usually seen singly and never forms long chains. The bacillus in its vegetative form is actively motile and possesses numerous flagella placed about its periphery. In artificial media it forms spores which are oval, broader than the rod itself, and placed near, though never actually at, the end of the bacillary body. This gives the bacillus a racket-shaped appearance.

It is readily stained with the usual anilin dyes, but is decolorized by Gram's method of staining.

Cultivation. - The bacillus is a strict anaërobe. It was obtained in pure culture first by Kitasato. ${ }^{1}$ Under anaërobic conditions it is easily 
cultivated upon the usual laboratory media, all of which are more favorable after the addition of glucose, glycerin, or nutrose. In all media there is active gas formation, which, owing to an admixture of butyric acid, is of a foul, sour odor. The bacillus is not very delicate in its requirements of a special reaction of media, growing equally well on those slightly acid or slightly alkaline.

On gelatin plates, at $20^{\circ} \mathrm{C}$., colonies appear in about twenty-four hours, usually round or oval, with a compact center about which fine radiating filaments form an opaque halo. The gelatin is fluidified.

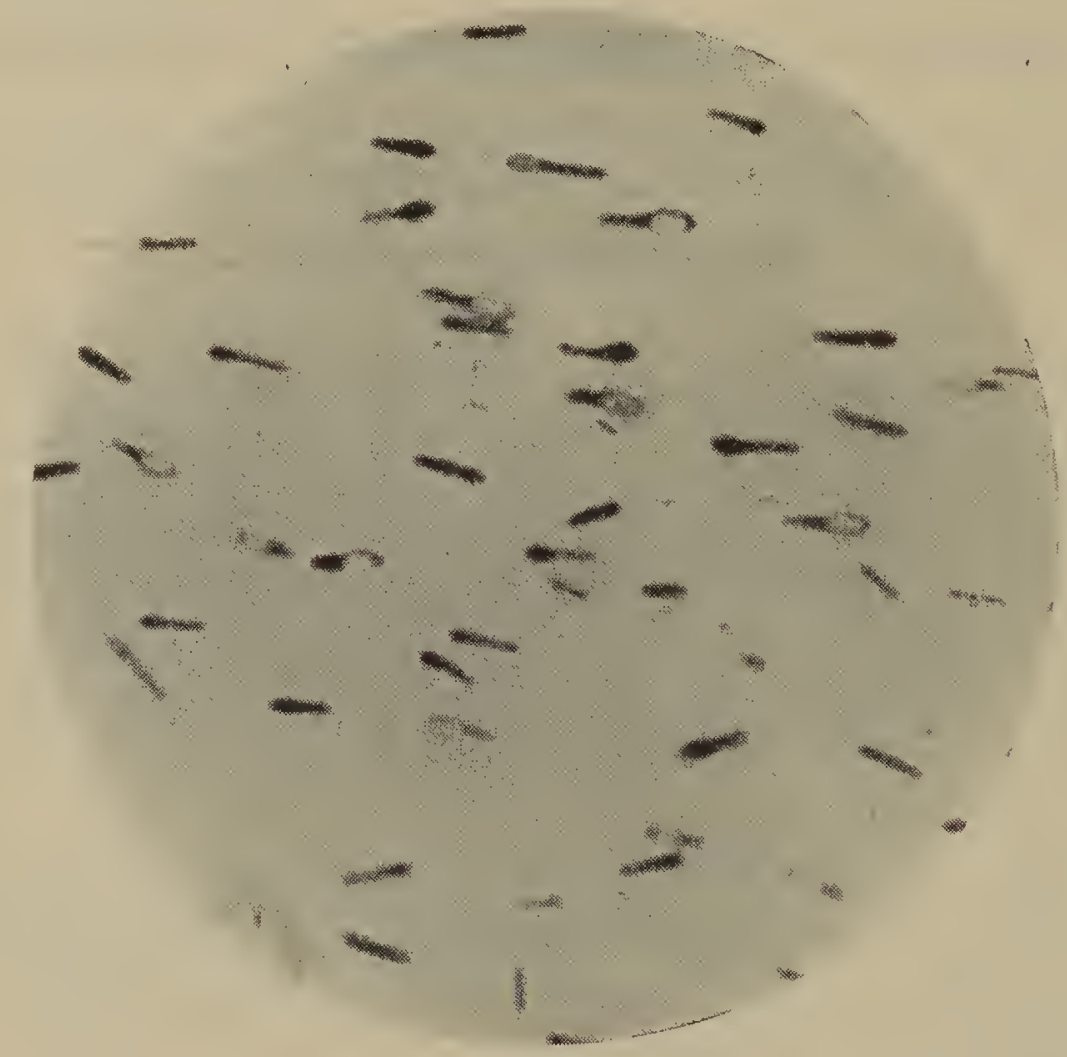

Fig. 99.-Bacillus of Symptomatic Anthrax. (After Zettnow.)

Surface colonies upon agar plates are circular and made up of a slightly granular compact center, from which a thinner peripheral zone emanates, containing microscopically a tangle of fine threads.

In agar stabs, at $37.5^{\circ} \mathrm{C}$, growth appears within eighteen hours, rapidly spreading from the line of stab as a diffuse, fine cloud. Gas formation, especially near the bottom of the tube, rapidly leads to the formation of bubbles and later to extensive splitting of the medium. In gelatin stab cultures growth is similar to that in agar stabs, though less rapid.

Pathogenicity.-Symptomatic anthrax bacilli are pathogenic for cattle, sheep, and goats. By far the largest number of cases, possibly the only spontaneous ones, appear among cattle. Guinea-pigs are very susceptible to experimental inoculation. Horses are very little suscep- 
tible. Dogs, cats, rabbits, and birds are immune. Man also appears to be absolutely immune. Spontaneous infection occurs by the entrance of infected soil into abrasions or wounds, usually of the lower extremities. Infection depends to some extent upon the relative degree of virulence of the bacillus-a variable factor in this species. Twelve to twenty-four hours after inoculation there appears at the point of entrance a soft, puffy swelling, which on palpation is found to emit an emphysematous crackling. The emphysema spreads rapidly, often reaching the abdomen and chest within a day. The course of the disease is extremely acute, the fever high, the general prostration extreme. Denth may result within three or four days after inoculation.

At autopsy the swollen area is found to be infiltrated with a thick exudate, blood-tinged and foamy. Subcutaneous tissue and muscles are edematous and crackle with gas. The internal organs show parenchymatous degeneration and hemorrhagic areas. The bacilli, immediately after death, are found but sparsely distributed in the blood and internal organs, but are demonstrable in enormous numbers in the edema surrounding the central focus.

If carcasses are allowed to lie unburied for some time, the bacilli will attain a general distribution, and the entire body will be found bloated with gas, the organs filled with bubbles. Practically identical conditions are found after experimental inoculation.

Toxins.-According to the investigations of Leclainche and Vallée, ${ }^{1}$ the bacillus of symptomatic anthrax produces a soluble toxin. It is not formed to any extent in ordinary broth, but is formed in considerable quantities in broth containing blood or albuminous animal fluids.

The best medium for obtaining toxin, according to the same authors, is the bouillon of Martin, ${ }^{2}$ made up of equal parts of veal infusion and a

${ }^{1}$ Leclainche et Vallée, Ann. de l'inst. Pasteur, 1900.

${ }^{2}$ Martin, Ann. de l'inst. Pasteur, 1898. 
pepton solution obtained from the macerated tissues of the stomachs of pigs. The toxin contained in filtrates of such cultures is quite resistant to heat, but rapidly deteriorates if free access of air is allowed.

Immunity.-Active immunization against the bacillus of symptomatic anthrax was first accomplished by Arloing ${ }^{1}$ and his collaborators by the subcutaneous inoculation of cattle with tissue-extracts of infected animals. The work of these authors resulted in a practical method of immunization which is carried out as follows:

Two vaccines are prepared. Vaccine I consists of the juice of infected meat, dried and heated to $100^{\circ} \mathrm{C}$. for six hours. Vaccine II is a similar meat-juice heated to $90^{\circ} \mathrm{C}$., for the same length of time. By the heating, the spores contained in the vaccines are attenuated to relatively different degrees. Vaccine $\mathrm{I}$ in quantities of 0.01 to 0.02 c.c. is emulsified in sterile salt solutions and injected near the end of the tail of the animal to be protected. A similar quantity of Vaccine II is injected in the same way fourteen days later.

This method has been retained in principle, but largely modified in detail by various worker's. Kitt ${ }^{2}$ introduced the use of the dried and powdered whole meat instead of the meat juice, and made only one vaccine, heated to $94^{\circ} \mathrm{C}$, for six hours. This method has been largely used in this country. ${ }^{3}$ Passive immunization with the serum ${ }^{4}$ of actively immunized sheep and goats has been used in combination with the methods of active immunization.

\section{BACILLUS OF MALIGNANT EDEMA}

\section{(Bacillus odematis maligni, Vibrion septique)}

In 1877, Pasteur ${ }^{5}$ described a bacillus which he had found in guineapigs and rabbits experimentally inoculated with putrefying animal tissues. This bacillus, which he named "Vibrion septique," he succeeded in cultivating only under anaërobic conditions and in an impure state, and described as its pathognomonic characteristics the formation of an extensive edema in and about the point of inoculation.

1 Arloing, Cornevin, et Thomas, "Le Charbon Sympt.," etc., Paris, 1887. Ref. from Grassberger und Schattenfroh, Kraus und Levaditi, "Handbuch," etc., vol. i, pt. 2.

${ }^{2}$ Kitt, Ref. from Grassberger und Schattenfroh, loc. cit.

${ }^{3}$ Report of Bureau of Animal Ind., Wash., 1902.

4 Arloing, Leclainche, et Vallée, loc. cit.

5 Pasteur, Bull. de l'acad. de méd., 1877, p. 793. 
Koch, ${ }^{1}$ who studied this infection in connection with his work upon anthrax in 1881, called attention to the fact that the bacillus described by Pasteur did not produce a true septicemia, and suggested the term "Bacillus of malignant edema," which is now in general use.

Gaffky $^{2}$ found that, apart from its presence in putrid material, the bacillus occurred in the upper layers of garden soil and in dust. It has since been found to be widely distributed in nature and in the intestines of animals and of man. Its wide distribution is unquestionably due to the great resistance of its spores.

Morphology and Staining.-The bacillus of malignant edema is a

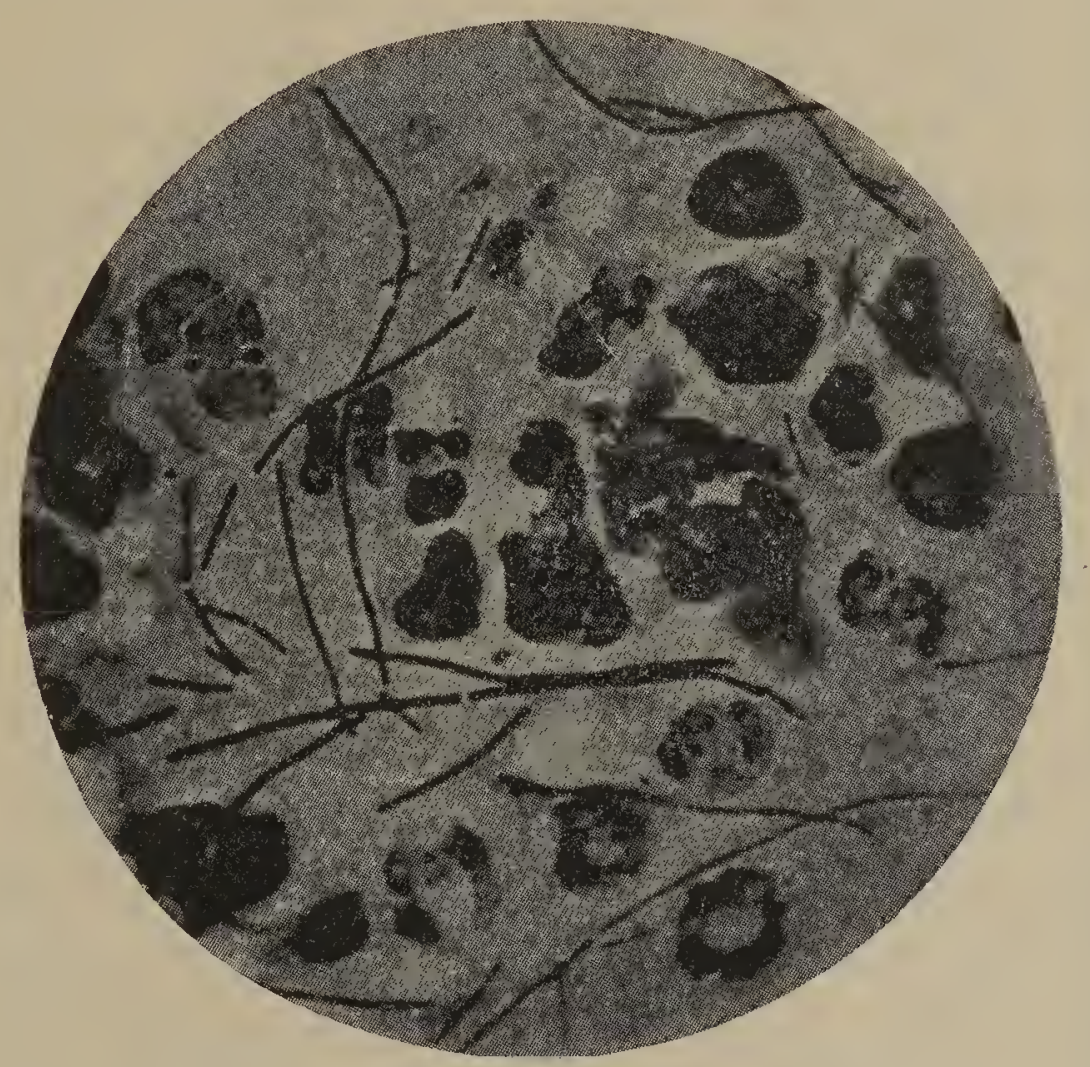

Fig. 101.-Baciludus of Malignant Edema. (After Fränkel and Pfeiffer.)

long slender rod, not unlike the anthrax bacillus, but decidedly more slender. Its average measurements are 1 micron in thickness and 3 to 8 micra in length. It usually occurs as single rods, but frequently appears in long threads showing irregular subdivisions. Often no subdivisions can be seen and the threads appear as long, homogeneous filaments. These threads are less frequently seen in preparations from solid media than in those from bouillon or edema fluid. The bacilli are motile and possess numerous laterally placed flagella. Their motility is never very marked and is often entirely absent. The bacillus

${ }_{1}$ Koch, Mitt. a. d. kais. Gesundheitsamt, i, 1881, p. 52 et seq.

${ }^{2}$ Gaffky, Mitt. a. d. kais. Gesundheitsamt, 1881. 
produces spores at temperatures above $20^{\circ} \mathrm{C}$., which are oval, irregularly placed either in the center or slightly nearer one or the other end, and cause a bulging of the bacillary body.

It is readily stained by any of the usual anilin dyes. Stained by Gram's method it is decolorized.

Cultivation.-Bacillus œedematis maligni is strictly anaërobic. Under anaërobic conditions it develops readily upon

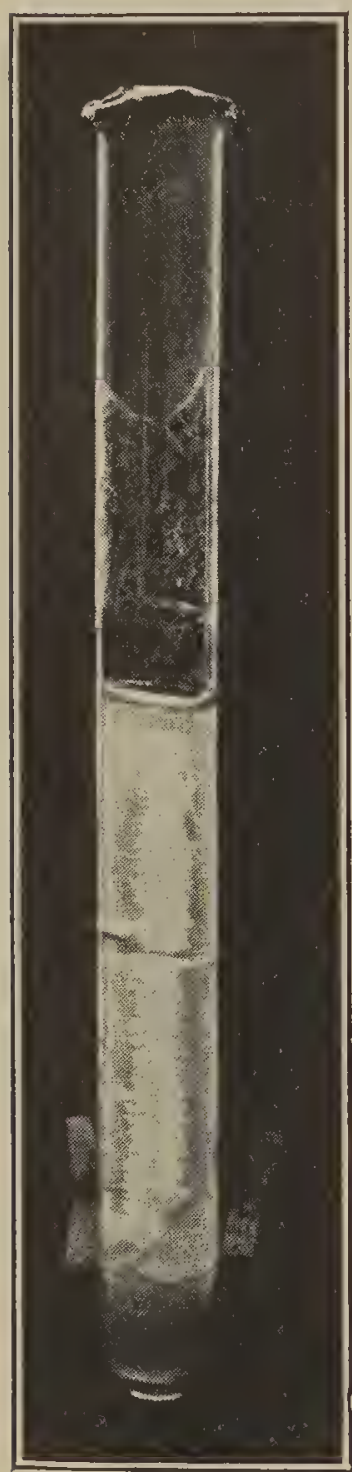

FIG. 102.-BACILLUS OF MALignant Edema. Culture in glucose agar. any of the usual artificial media. The bacillus is not very sensitive to the reaction of media and grows more luxuriantly in all media to which glucose has been added. In all media it forms, by the cleavage of proteids, putridlý offensive gases.

In gelatin at room temperature, colonies develop in about three days as small grayish spherical growths, which microscopically show an arrangement in radial filaments. The gelatin is fluidified.

In gelatin stab cultures growth begins as a white column extending to within a centimeter of the top of the medium. Soon irregularly radiating processes develop laterally and gas bubbles appear, breaking up the medium.

Stab cultures in agar show growth within twentyfour to thirty-six hours at $37.5^{\circ} \mathrm{C}$, appearing at first as a white line, but soon showing a cloud-like lateral extension along the entire line of the stab. If sugar is present bubbles appear throughout the medium.

In broth there is general clouding and a granular sediment; no pellicle is formed. Milk is slowly coagulated. On blood serum growth is very luxuriant. On potaio, a medium used in the earliest studies of the bacillus by Gaffky, the bacillus grows readily.

Isolation may be accomplished by any of the ordinary anaërobic plating methods. The bacillus can usually be obtained for subsequent isolation by injection of a susceptible animal with soil, especially that of gardens or manured fields.

Pathogenicity.-The bacillus is pathogenic for mice, guinea-pigs, rabbits, horses, dogs, sheep, pigs, some birds, and man. Cattle were formerly regarded as immune, an opinion which has since been found to be erroneous. 
Subcutaneous inoculation of pure culture into a susceptible subject produces, within twenty-four to thirty-six hours, an acute edematous inflammation about the point of inoculation. The edema extends throughout the subcuticular and deeper layers, and consists of thin, slightly bloody fluid. Neighboring lymph nodes become swollen and hemorrhagic. In the mixed infections of accidental inoculation, but more rapidly in experimental inoculations with pure cultures, gas is formed and consequent subcutaneous emphysema. Together with this there are symptoms of general toxemia. In the smaller test animals this disease is usually fatal. At autopsy the bacilli are found in the edema fluid about the local lesion. At autopsies done soon after death, the organisms are not found in the blood or internal organs. Later they may be generally distributed throughout the body. In mice only may the bacilli enter the blood stream before death. The internal organs of animals dead of this infection usually show parenchymatous degeneration and occasionally hemorrhages.

Malignant edema is not a frequent disease. It has been occasionally observed in horses, in cattle, and in sheep. In man the infection usually appears after traumatism or secondarily after compound fractures or upon the site of suppurating wounds. Isolated cases have been described as arising after hypodermic injections. One case has been reported as arising in the uterus after instrumental abortion.

Immunity.-Recovery from an infection with the bacillus of malignant edema produces immunity against subsequent infections. ${ }^{1}$ The bacillus in fluid media produces small amounts of a soluble toxin which in bacteria-free filtrates is capable of killing guinea-pigs. Relatively large quantities of filtrate must be employed. Roux and Chamberland, ${ }^{2}$ the first to work upon these toxins, were able, by means of them, to immunize guinea-pigs. Similar immunity could be produced by treatment with the toxic, filtered sera of animals dead of the disease. ${ }^{3}$

\section{BACILLUS AËROGENES CAPSULATUS}

Bacillus aërogenes capsulatus was first observed by Welch and fully described by Welch and Nuttall ${ }^{4}$ in 1892 . It is identical with a bacillus

1 Arloing et Chauveau, Ann. de méd. vét., 1884.

${ }^{2}$ Roux et Chamberland, Ann. de l'inst. Pasteur, 1887.

3 Sanfelice, Zeit. f. Hyg., xiv.

Welch and Nuttall, Bull. Johns Hopkins Hosp., iii, 1892, p. 81. 
later described by Fränkel, ${ }^{1}$ and named by him Bacillus phlegmonis emphysematosæ.

Similar, probably identical, bacilli have been found and reported subsequently by other observers, ignorant of the work of Welch and Nuttall. Such are the B. perfringens (Veillon and Zuber), and B. emphysematis vaginæ (Lindenthal, Wien. klin. Woch., 1897), and others.

Welch ${ }^{2}$ first obtained the bacillus from the intravascular blood of a case of ruptured aortic aneurysm, autopsied six hours after death, his attention being called to the blood particularly by the existence of air bubbles throughout the vessels.

Apart from its occurrence in infected subjects, the bacillus finds wide distribution in nature, being found in soil, dust, and brackish water, and in the normal intestinal tracts of human beings and mammals. ${ }^{3}$

Morphology and Staining.-Bacillus aërogenes capsulatus appears usually as a straight rod, not unlike the anthrax bacillus, but more variable in length and somewhat thicker in proportion than the latter. Occasionally bacilli are seen which are slightly curved, but these are rare. The bacillus averages 3 to 6 micra in length, but may be three or four times longer than this. More rarely the bacillus may appear so short as to be almost coccoid. In artificial cultures it is usually thicker and shorter than it is in animal tissues. The bacilli are generally single, but are often seen in short chains. Their ends are usually slightly rounded but, especially when in chains, may be almost square. Chainformation seems to occur chiefly in the blood, long chains never occurring in artificial media. This characteristic is regarded by Welch as a distinguishing feature in differentiating this bacillus morphologically from anthrax. In their first publication, Welch and Nuttall did not describe spores as appearing in these bacilli. Dunham, ${ }^{4}$ however, in 1895 , found spores in cultures grown upon blood serum. The spores seem to be formed only upon special media, rarely upon plain agar, never in the animal body. The spores are oval, and may be placed centrally or toward one end, one in each bacillus.

The bacillus is non-motile, and does not possess flagella. It pos-

${ }^{1}$ Fränkel, Cent. f. Bakt., xiii, 1893, p. 13.

${ }^{2}$ Welch, Bull. Johns Hopkins Hosp., xi, 1900, p. 185.

${ }^{3}$ Although B. aërogenes capsulatus and B. phlegmonis emphysematosæ are separately described in many books, notably Migula's "System d. Bakteriologie," the microorganisms have been shown beyond question to be identical and are acknowledged to be so by Fränkel himself.

4 Dunham, Johns Hopkins Hosp. Bull., viii, 1897, p. 68. 
sesses a capsule which, however, can not be constantly demonstrated. The capsules are best seen when preparations are made from animal fluids, but can often be demonstrated in those stained from artificial cultures. They are demonstrated best by one or the other of the ordinary capsule stains.

The bacillus is stained easily by the usual anilin dyes. In tissue preparations, the bacilli regularly retain the gentian-violet when stained by Gram's method. In smears from artificial culture media, while most of the bacilli stain by Gram, many will be seen wholly or partially decolorized, owing probably to the rapid production of involution forms.

Cultivation.-Bacillus aërogenes capsulatus is an obligatory anaërobe. The first cultivations by Welch and Nuttall were made in deep agar stabs. It grows well upon all the usual media, preferring a neutral or slightly alkaline reaction. All media are improved for the cultivation of this bacillus by the addition of glucose, lactose; or some other easily fermented carbohydrate.

Upon agar or gelatin plates, growth appears at $37.5^{\circ} \mathrm{C}$. within twenty-four hours, as a flat, grayish translucent round disk. The margins of colonies are slightly irregular and fringed. Gelatin is slowly liquefied by the large majority of cultures, but Welch states that occasionally liquefaction does not occur.

In deep agar stabs or in agar slant cultures, especially in those containing a carbohydrate, there is a rapid formation of gas bubbles, a characteristic which is especially well developed and lends the cultures of this bacillus their chief diagnostic feature.

In broth, growth is heavy and abundant. At first there is general clouding. Within forty-eight hours, however, a heavy, white, flocculent sediment is formed. Owing to the formation of gas, broth tubes if undisturbed usually show a light froth of bubbles on the surface.

On potato, growth is scanty and the medium possesses no advantages either for cultivation or diagnosis. On coagulated blood serum, growth is heavy and rapid and this medium is especially adapted for spore formation. There is slight peptonization of the blood serum. In milk, there is rapid coagulation, rapid acidification, and gas formation.

The carbohydrates, glucose, lactose, and saccharose, are fermented by this bacillus. Mannit is apparently not fermented.

Welch and Nuttall state that the bacillus is capable of producing gas from proteid matter. The gas formed, according to Dunham, ${ }^{1}$ consists of 
64 per cent of hydrogen, 28 per cent of $\mathrm{CO}_{2}$, and 8 per cent of a mixture of gases, chiefly nitrogen. The gas from the infected animal body is ignitable and burns with a bluish hydrogen flame.

Biological Considerations.-The bacillus, as stated, is anaërobic. Its anaërobic requirements, however, are less exacting than those of some other anaërobes, and in stab cultures it will often grow up to the surface of the stab. It grows best at $37.5^{\circ} \mathrm{C}$., but will also develop at room temperature $\left(20^{\circ}\right.$ to $22^{\circ} \mathrm{C}$. $)$.

Isolation.-The bacillus may, of course, be isolated by anaërobic plating methods. It is best isolated, however, from mixed cultures by animal inoculation. If, for instance, it is desired to obtain it from a mixed culture or from feces, a suspension of about 1 c.c. of the suspected material is made in 5 c.c. of sterile salt solution. This is thoroughly emulsified and filtered through a sterile paper. One to two c.c. of this suspension is then injected into the ear vein of a rabbit. After four or five minutes the rabbit is killed. It is then placed in the incubator for five to eight hours. At the end of this time, the animal is usually found tensely distended with gas. At autopsy, gas bubbles will be found distributed throughout the organs, most characteristically in the liver, where isolated bubbles are found covering the surface. From these bubbles cultures or smears may be taken for identification. Identification is easily made from its molphology, its capsule, lack of motility, and gas formation.

Pathogenicity.-Bacillus aërogenes capsulatus is highly pathogenic for guinea-pigs, but vely slightly for rabbits. Its virulence is subject to great variations, however, some strains showing little if any pathogenicity even for guinea-pigs. In general, its pathogenicity for the ordinary laboratory animals may be regarded as slight. In man, ${ }^{1}$ the bacillus has been isolated from numerous cases of so-called "emphysematous gangrene" 2 (gangrène foudroyant). The infection usually occurs upon the extremities and is characterized by a rapidly necrotizing inflammation, with which there occurs extensive subcutaneous emphysema. The infection usually follows traumatism," especially compound fractures, and is extremely grave. The bacillus has also been found in the uterus in puerperal infection, ${ }^{4}$ and in the fetus

I Wẹlch and Flexner, Jour. Exp: Med., 1, 1896.

${ }_{2}$ Mann, Ann. of Surgery, xix, 1894.

${ }^{3}$ Bloodgood, Progressive Med., 1899.

${ }^{4}$ Dobbin, Johns Hopkins Hosp. Bull., viii, 1897. 
dead in utero. It has been found in the blood before death, by Gwyn, ${ }^{1}$ in a case of chorea. The bacillus has also been found in infectious processes of various other parts of the body, such as the gastrointestinal and biliary tracts, the lungs, the pleura, and the meninges.

As stated above, this bacillus is frequently present in the normal intestinal contents. Its presence in abnormally high proportions, as indicated by a Gram stain of a smear of the feces, has been associated by a number of observers with various pathological conditions. Herter ${ }^{2}$ has recently studied this subject and believes that the abnormal proliferation of the bacillus in the gastrointestinal tract has in some way (probably by toxin absorption) an etiological relationship to pernicious anemia. This assertion, however, can in no way be regarded as conclusively proven.

\section{BACILLUS BOTULINUS}

Meat poisoning was formerly regarded as universally dependent upon putrefactive changes taking place in infected meat, resulting in the production of ptomaïns or other harmful products of bacterial putrefaction. It was not until 1888 that certain of these cases were definitely recognized as true bacterial infections, in which the preformed poison probably aided only in establishing the infection. Gärtner, in that year, discovered the Bacillus enteritidis, a microorganism belonging to the group of the paracolon bacilli, and demonstrated its presence both in the infecting meat and in the intestinal tracts of patients. The characteristics of this type of meat poisoning have been discussed more particularly in the section describing the bacillus of Gärtner and its allied forms.

There is another type of meat poisoning, however, which is not only much more severe (ending fatally in almost 25 per cent of the cases), but is characterized by a clinical picture more significant of a profound systemic toxemia than of a mere gastroenteric irritation. The etiological factor underlying this type of infection was first demonstrated by van Ermengem, ${ }^{3}$ in 1896, and named Bacillus botulinus. van Ermengem isolated the bacillus from a ham, the ingestion of which had caused disease in a large number of persons. Of the thirty-four individuals who had eaten of it, all were attacked, about ten of them

${ }^{1}$ Gwyn, Johns Hopkins Hosp. Bull., x, 1899.

2 Herter, "Bacterial Infection of the Intestinal Tract," New York, Macmillan, 1907.

3 van Ermengem, Cent. f. Bakt., xix, 1896; Zeit. f. Hyg., xxvi, 1897. 
very severely. van Ermengem found the bacilli in large numbers lying between the muscle fibers in the ham, and was able to cultivate the same microorganism from the stomach and spleen of one of those who died of the infection.

The results of van Ermengem have been confirmed by Römer, ${ }^{1}$ and others.

Morphology and Staining.-Bacillus botulinus is a large, straight rod with rounded ends, 4 to 6 micra in length by 0.9 to 1.2 micra in thickness. The bacilli are either single or grouped in very short chains. Involution forms are numerous on artificial media. The bacillus is slightly motile and possesses from four to eight undulated flagella, peripherally arranged. Spores are formed in suitable media, most regularly in glucose-gelatin of a distinctly alkaline titer. The spores are oval and usually situated near the end of the bacillus, rarely in its center. Spores are formed most abundantly when cultivation is carried out at $20^{\circ}$ to $25^{\circ} \mathrm{C}$., and are usually absent when higher temperatures are employed.

The bacillus is easily stained by the usual aqueous anilin dyes, and retains the anilin-gentian-violet when stained by Gram. It is necessary, however, in carrying out Gram's stain to decolorize carefully with alcohol since overdecolorization is easily accomplished, leaving the result doubtful.

Cultivation.-The bacillus is a strict anaërobe. In anaërobic environment it is easily cultivated on the usual meat-infusion media. It grows most readily at temperatures about $25^{\circ} \mathrm{C}$., less luxuriantly at temperatures of $35^{\circ} \mathrm{C}$. and over.

The bacillus is delicately susceptible to the reaction of media, growing only in those which are neutral or moderately alkaline.

In deep stab cultures in one per cent glucose agar, growth is at first noticed as a thin, white column, not reaching to the surface of the medium. Soon the medium is cracked and split by the abundant formation of gas. On agar plates, the colonies are yellowish, opalescent, and round, and show a finely fringed periphery.

On gelatin, at $20^{\circ}$ to $25^{\circ} \mathrm{C}$, growth is rapid and abundant, and differs little from that on agar, except that, besides the formation of gas, there is energetic fluidification of the medium. On glucose-gelatin plates, van Ermengem describes the colonies as round, yellowish, transparent, and composed of coarse granules which, along the periphery

${ }^{1}$ Römer, Cent. f. Bakt., xxvii, 1900. 
in the zone of fluidification, show constant motion. The appearance of the surface colonies on glucose-gelatin plates is regarded by the discoverer as diagnostically characteristic.

In glucose broth there is general clouding and large quantities of gas are formed. At $35^{\circ} \mathrm{C}$. and over, the gas formation ceases after four or five days, the broth becoming clear with a yellowish-white flocculent sediment. At lower temperatures this does not occur.

Milk is not coagulated and disaccharids and polysaccharids are not fermented.

The gas formed in cultures consists chiefly of hydrogen and methane. All cultures have a sour odor, like butyric acid, but this is not so offensive as that of some of the other anaërobic organisms.

The bacillus lives longest in gelatin cultures, but even upon these, transplantations should be done every four to six weeks, since the spores of this bacillus show less viability and resistance than do those of most spore-former's.

Pathogenicity.-Botulism or allantiasis, as noticed in human beings, is, as far as we know, always due to ingestion of infected meat, usually of ham, canned meats, or sausages (botulus = sausage). Symptoms appear only after a definite period of incubation which varies from twenty-four to forty-eight hours. The first definite symptoms are chilliness, trembling, and giddiness. These manifestations are soon followed by headache, occasionally by vomiting. In contradistinction to the meat poisonings caused by other microorganisms, those due to Bacillus botulinus may show few or no symptoms directly referable to the intestinal tract. The chief diagnostic characteristics of the disease are a group of symptoms referable to toxic interference with the cranial nerves. Loss of accommodation, dilated pupils, ptosis, aphonia, and dysphagia may occur. Fever is usually absent. Consciousness is rarely lost. The characteristic symptoms may be produced in various animals by injection of living cultures or culture filtrates, i.e., toxins. The most susceptible animals are guinea-pigs. These may be killed by the injection of minute quantities of bouillon cultures or of toxin. Preceding death, which occurs within twenty-four to thirty-six hours, there may be general motor paralysis, dyspnea, and hypersecretion of mucus from nose and mouth. Guinea-pigs may be infected per os as well as by hypodermic injections. Cats, mice, and monkeys are highly susceptible; rabbits are less so, but still favorable subjects for experimental studies. Birds, especially pigeons, are highly resistant, but react typically to large doses. Autopsies upon man or animals dead of botulism show 
general hyperemia of the organs with much parenchymatous degeneration and many minute hemorrhages.

The bacilli have been found in the spleen after death, ${ }^{1}$ but van Ermengem does not believe that they are generally distributed during the course of the disease. It is believed by most of those who have studied this disease that poisoning in the human subject is due to the toxins preformed in the infected meat by this bacillus. Experiments have shown that little or no poison is produced by the bacilli after gaining entrance to the human or animal body.

The Toxin of B. botulinus.-Bacillus botulinus produces disease chiefly by means of a strong soluble toxin secreted by it, and absorbed by the infected subject. This toxin is active in animals and presumably in man, not only when injected subcutaneously, but also when introduced through the gastrointestinal canal. The poison has been particularly studied by Brieger and his collaborators. It is obtained in filtrates of alkaline bouillon cultures. It has been precipitated out of the filtrate by Brieger and Kempner ${ }^{2}$ by means of a three per cent zinc (hlorid solution (2 volumes of 3 per cent $\mathrm{ZnCl}_{2}$ ). The toxin thus obtained was sufficiently powerful to kill a 250-gram guinea-pig in fifty hours.

Specific action of the toxin for the nerve-cells of the spinal ganglia has been shown by Marinesco. ${ }^{3}$ A specific antitoxin has been produced by Kempner and Pollack. ${ }^{4}$

1 Stricht, Quoted from van Ermengem, in Kolle und Wassermann.

${ }^{2}$ Brieger und Kempner, Deut. med. Woch., xxxiii, 1897.

3 Marinesco, Compt. rend. de l'acad. des sci., Nov., 1896.

* Kempner und Pollack, Deut. med. Woch., xxxii, 1897. 


\section{CHAPTER XXXIII}

\section{THE TUBERCLE BACILLUS}

IN view of the clinical manifestations of tuberculosis, it is not surprising that the infectious nature of the disease has been suspected for many centuries. Transmission by means of tuberculous material was first successfully accomplished by Klencke, in 1843, and, more elaborately, by Villemin, ${ }^{1}$ in 1865 . It was not until 1882 , however, that Koch ${ }^{2}$ succeeded in isolating and cultivating the tubercle bacillus. Baumgarten ${ }^{3}$ had previously seen the bacillus in tissue sections, but his researches were limited to purely morphological observations. Koch, in addition to demonstrating the bacillus in tuberculous tissues from various sources, produced characteristic lesions in guinea-pigs and other animals by infecting them with pure cultures, and established beyond doubt the etiological relationship of the bacillus to the disease.

Morphology.-Tubercle bacilli appear as slender rods, 2 to 4 micra in length, 0.2 to 0.5 micra in width. Their ends are usually rounded. The rods may be straight or slightly curved; their diameters may be uniform throughout; more often, however, they appear beaded and irregularly stained. The beaded appearance is due to different causes. Unstained spaces may occur along the body of the bacillus, especially in old cultures. These are generally regarded as vacuoles. The bodies of the bacilli, on the other hand, may bulge slightly here and there, often in three or four places, showing oval or rounded knobs which stain with great depth and are very resistant to decolorization. These thickenings were formerly regarded as spores, but in view of the fact that the bacilli are not more resistant against heat and disinfectants than other vegetative forms, this interpretation is probably incorrect. The bacilli are said to possess a cell membrane which confers upon them their resistance against drying and entrance of stains. This membrane gives a cellulose reaction and is believed to contain most of the waxy substances which can be extracted from the cultures.

1 Villemin, Gaz. hebdom., 1865.

${ }^{2}$ Koch, Berl. klin. Woch., 1882; Mitt. a. d. kais. Gesundheitsamt, 1884.

3 Baumgarten, Virchow's Arch., lxxxii. 
Various observers, notably Nocard and Roux, ${ }^{1}$ Mafucci, ${ }^{2}$ and Klein, ${ }^{3}$ have demonstrated branched forms of the tubercle bacillus. These observations, variously extended and confirmed, make it probable that Bacillus tuberculosis is not a member of the family of schizomycetes,

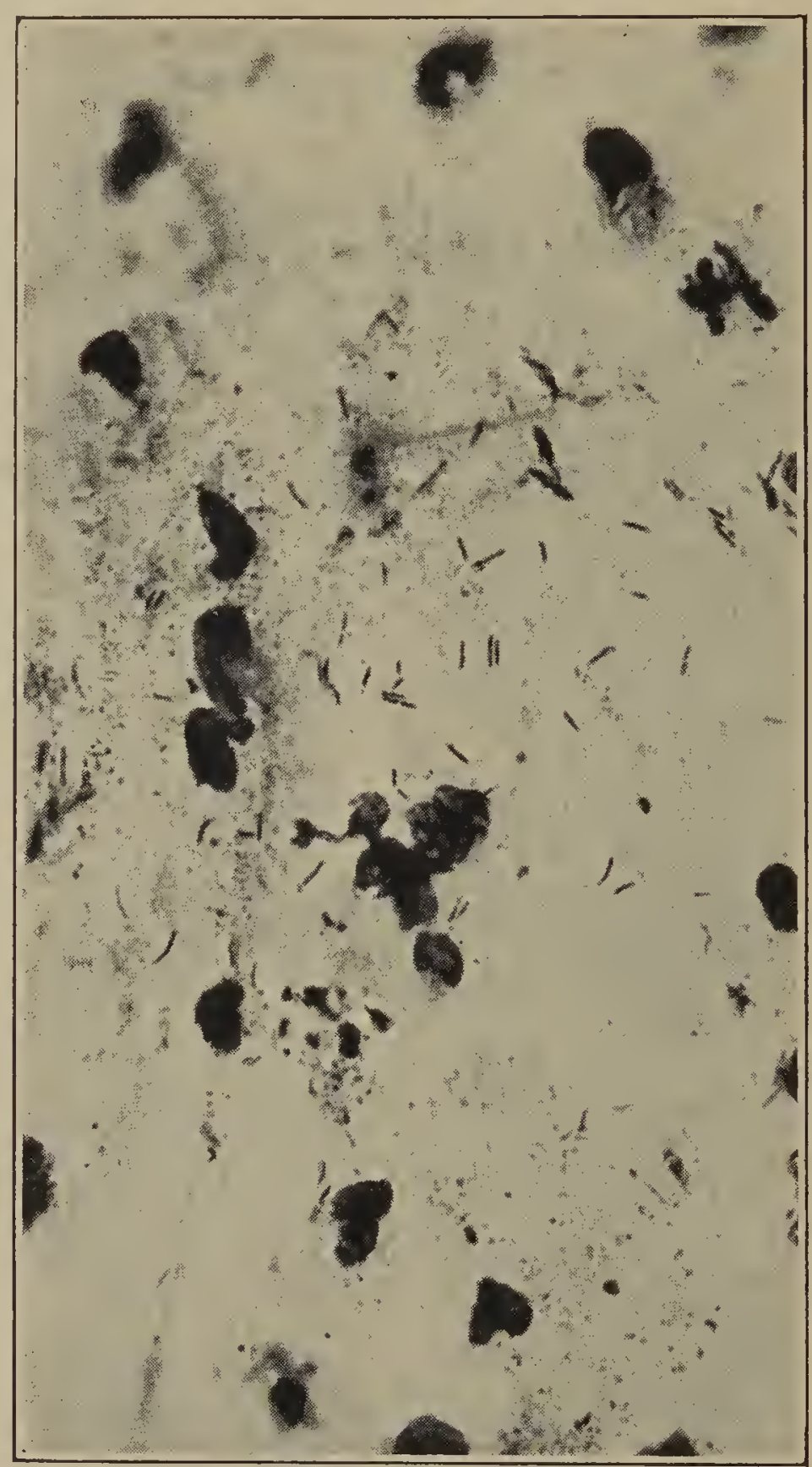

Fig. 103.-Tubercle Bacilli in Sputum.

but belongs rather to the higher bacteria, closely related to the actinomyces.

Staining.-Tubercle bacilli do not stain easily with the ordinary anilin dyes; to these they are made permeable only by long exposure or by heating of the staining solution. Once stained, however, the dye is tenaciously retained in spite of treatment with alcohol and strong

${ }^{1}$ Nocard et Roux, Ann. de l'inst. Pasteur, 1887. ${ }^{2}$ Mafucci, Zeit. f. Hyg., ii.

\footnotetext{
3 Klein, Cent. f. Bakt., 1890.
} 
acids. For this reason, this bacillus, together with some other bacteria to be mentioned later, is spoken of as "acid-fast." The acid-fast nature of the bacillus seems to depend upon the fatty substances contained in it, ${ }^{1}$ and has furnished the basis for differential staining methods. All the staining nethods devised for the recognition of the tubercle bacillus thus depend upon the use of an intensely penetrating staining solution, followed by vigorous decolorization which deprives all but the acid-fast group of their color. Counterstains of any of the weaker dyes may then be used to stain the decolorized elements. One of the first of the staining solutions to be of practical use was the anilin-water-gentianviolet solution of Ehrlich ${ }^{2}$ (11 c.c. saturated alcoholic gentian-violet to 89 c.c. 5 per cent anilin water). This dye, although of sufficient penetrating power, has the disadvantage of deteriorating rapidly and has in practice been almost entirely displaced by Ziehl's ${ }^{3}$ carbol-fuchsin solution. (Fuchsin $1 \mathrm{gm}$. in 10 c.c. alcohol absolute, added to 90 c.c. 5 per cent carbolic.) This staining solution is the one now in general use and is employed as follows: Thin smears, on slides or cover-slips, are covered with the dye and gently heated. In the case of cover-glasses, these may be floated, face downward, in staining dishes filled with the dye. The dye is allowed to act for about three minutes, steaming but not allowed to boil. At the end of this time the preparation is washed either with 5 per cent nitric acid, 5 to 20 per cent sulphuric acid, or 1 per cent hydrochloric acid, until most of the red color has disappeared (a few seconds), and the preparation appears pale pink. This results in decolorization of all microorganisms with the exception of members of the acid-fast group. Thorough washing in 80 to 95 per cent alcohol is now employed to complete the decolorization. The preparation is then rinsed in water and counterstained with 1 per cent aqueous methylene-blue.

Tubercle-bacillus staining has been further simplified by Gabbett, ${ }^{4}$ who combines decolorization and counterstaining. In this method preparations are stained with Ziehl's carbol-fuchsin as in the preceding; they are then rinsed in water and covered with a solution containing methylene-blue 1 gram, concentrated sulphuric acid 25 grams, and distilled water 100 c.c. This is allowed to act for from two to four

1 Bienstock, Fort. d. Med., 1886; Weyl, Deut. med. Woch., 1891.

${ }^{2}$ Ehrlich, Deut. med. Woch., 1882; Weigert, Deut. med. Woch., 1885.

з Ziehl, Deut. med. Woch., 1883; Neelsen, "Lehrb. d. allg. Path.," 1894

- Gabbett, Lancet, 1887. 
minutes, at the end of which time all elements in the preparation except the acid-fast bacilli will be decolorized and counterstained.

Tubercle bacilli in very young culture are often not acid-fast and it is not always possible to demonstrate acid-fast bacilli in pus from cold abscesses in sputum, in serous exudates, and in granulomatous lesions of the lymph nodes which can be shown by animal inoculation to be tuberculous. Much ${ }^{1}$ demonstrated in such material Gram-positive granules which lay singly in short chains or in irregular clumps, and which he believed to be non-acid-fast tubercle bacilli. He found similar granules in cultures of tubercle bacilli which showed on further incubation numerous acid-fast bacillary forms. His work has been repeatedly confirmed, and there seems little doubt but that these granules are really tubercle bacilli. Their demonstration is not, however, of great diagnostic value, as other bacilli form granules of the same appearance. Small rods and splinters are also found which stain by Gram's method, but not by carbol-fuchsin. ${ }^{2}$

To find "Much's granules," smears or sections are steamed in a solution of methyl violet B.N. (10 c.c. of saturated alcoholic solution of the dye in 100 c.c. of distilled water containing 2 per cent phenol). They are then treated with Gram's iodine solution 1-5 minutes; 5 per cent nitric acid 1 minute; 3 per cent hydrochloric acid 10 seconds; absolute alcohol and acetone equal parts, until decolorized. The granules may be stained by other modifications of Gram's method. Weiss ${ }^{3}$ has devised a combination stain. One part of Much's methyl violet is mixed with three parts of Ziehl's carbol-fuchsin and filtered; slides are stained for 24 to 48 hours in the mixture. They are then decolorized as in Much's method and counterstained with Bismarck brown or safranin 1 per cent. Both acid-fast and Gram-positive forms are stained by this method and in the red may be seen blue-black granules.

While the acid-fast group of bacteria is composed of a number of organisms to be mentioned later, a few only of these offer difficulties of differentiation from the tubercle bacillus. Those to be considered practically are the bacillus of leprosy and that of smegma. The latter bacillus, because of its distribution, is not infrequently found to contaminate feces, urine, or even sputum, and it is important to apply to suspected specimens one or the other of the stains devised for the differentiation of the smegma bacillus from Bacillus tuberculosis. The

\footnotetext{
1 Much, Berl. klin. Woch., 1908, xlv, 700.

${ }^{2}$ Liebermeister, Deutsche med. Woch., 1909, xxxv, 1324.

${ }^{3}$ Weiss, Berl. klin. Woch., 1909, xlvi, 1797.
} 
one most frequently employed is that of Pappenheim. ${ }^{1}$ The preparations are stained in hot carbol-fuchsin as before; the carbol-fuchsin is then poured off without washing and the preparation immersed in a solution made by saturating a 1 per cent alcoholic solution of rosolic acid with methylene-blue and adding 20 per cent of glycerin. In such preparations tubercle bacilli remain red, smegma bacilli appear blue.

Stained by Gram, tubercle bacilli retain the gentian-violet.

When tubercle bacilli are very sparsely present in sputum and other material it may be impossible to find them by direct examination, and often the only method of finding them will be animal inoculation. However, a number of methods have been devised by which the bacilli may be concentrated in such a way that they may be found even when a few only are present. One of these is to add peroxide of hydrogen to the sputum. By this the mucus is dissolved out and the solid particles settle or may be centrifugalized. A method very commonly employed to-day is that which depends on the use of "antiformin." This is a preparation used extensively for the cleansing of vats in breweries. It is described by Rosenau ${ }^{2}$ as consisting of equal parts of liquor sodæ chlorinatæ and a 15 per cent solution of caustic soda. The formula for liquor sodæ chlorinatæ he gives as:

Sodium carbonate ........................... 600

Chlorinated lime......................... 400

Distilled water $\ldots \ldots \ldots \ldots \ldots \ldots \ldots \ldots \ldots \ldots \ldots, 4,000$

If sputum is poured into a 10 to 15 per cent solution of antiformin and allowed to stand for several hours, most of the other elements of the sputum, cells, and bacteria, will dissolve out, and acid-fast bacilli be left in the residue. Strangely enough they are not killed by this process and if sufficiently washed may be cultivated or can produce lesions in guinea-pigs.

Isolation and Cultivation.-Tubercle bacilli are not easily cultivated. Their slowness of growth precludes their isolation by the usual plating methods. The first isolations by Koch ${ }^{3}$ were made upon coagulated blood serum from bits of tuberculous tissue smeared over its surface.

Isolation from tuberculous material may be greatly aided by inoculation into guinea-pigs. These animals will often withstand

${ }^{1}$ Pappenheim, Berl. klin. Woch., 1898.

${ }^{2}$ Rosenau, "Preventive Medicine and Hygiene," D. Appleton, N. Y., 1913; Uhlenhuth, Berl. klin. Woch., No. 29, 1908.

${ }^{3}$ Koch, loc. cit 
the acute infection which may be produced by the contaminating organisms and succumb at a later date (four to six weeks) to the tuberculous infection. The bacilli may then be obtained, after sterile dissection, by making cultivations from lymph nodes or other tuberculous foci which contain only tubercle bacilli. When isolation from sputum is attempted, whether directly or by means of animal inoculation, the sputum may be rendered comparatively free from contaminating bacteria by a process of washing devised by Koch. The sputum is thoroughly rinsed in running water to free it from its outer covering of pharyngeal mucus. It is then washed in eight or ten changes of sterile water. The material selected for cultivation is taken from the center of the washed mass, if possible from the small flakes of caseous material often visible in such sputum.

On blood serum at $37.5^{\circ} \mathrm{C}$., colonies usually become visible at the end of eight to fourteen days. They appear at first as small, dry, grayish-white, scaly spots with corrugated surfaces. After three or four weeks' cultivation, these join together, covering the surface of the medium as a dry, whitish, wrinkled membrane. Coagulated dog serum is regarded by Theobald Smith ${ }^{1}$ as one of the most favorable media for the growth of tubercle bacilli.

Slants of agar, to which whole rabbit's blood has been added in quantities of from 1 to 2 c.c. to each tube, make an excellent medium for this bacillus, both for isolation and continuous cultivation.

Cultivation methods were simplified by the discovery by Roux and Nocard that growth can be obtained upon media to which glycerin has been added. Upon glycerin-agar (glycerin 3 to 6 per cent), at $37.5^{\circ} \mathrm{C}$., colonies become visible at the end of from ten days to two weeks, at first as dry, white spots; later, as delicately corrugated membranes.

Glycerin bouillon (made of beef or veal with pepton one per cent, glycerin six per cent, and rendered slightly alkaline) is an extremely favorable medium. It should be filled, in shallow layers, into widemouthed flasks, since free access of oxygen is essential for growth. Transplants to this medium should be made by carefully floating flakes of the culture upon the surface. In this medium the bacilli will spread out upon the surface, at first as a thin, opaque, floating membrane. This rapidly thickens into a white, wrinkled, or granular layer, spreading out in all directions, and covering the entire surface of the fluid in from four to six weeks. Later, portions of the membrane sink to the

1 Th. Smith, Jour. Exp. Med., iii, 1898. 
bottom. In old cultures, the membrane assumes a yellowish hue. These cultures emit a peculiar aromatic odor.

Glycerin potato forms a favorable culture medium for the bacillus.

Hesse ${ }^{1}$ has devised a medium containing a proprietary preparation known as "Nährstoff Heyden," upon which tubercle bacilli are said to proliferate more rapidly than other bacteria. His method has yielded excellent results in the hands of other observers, both in isolation and in rapid cultivation. It is prepared as follows:

\begin{tabular}{|c|c|}
\hline "Nährstoff Heyden" 2 & 10 grams \\
\hline Sodium chloride.... & 5 \\
\hline Glycerin. & 30 \\
\hline Agar.... & 10 \\
\hline Normal sodium solution... & 5 c.c. \\
\hline A. $\alpha$ dest & $000 "$ \\
\hline
\end{tabular}

A variety of other culture media have been devised, none of them, however, possessing any marked advantages over those given.

Biological Considerations. - The tubercle bacillus is markedly dependent upon the free access of oxygen. The optimum temperature for its development is $37.5^{\circ} \mathrm{C}$. Temperatures below $30^{\circ}$ and above $42^{\circ} \mathrm{C}$. inhibit its growth. In fluid media, the bacilli are killed by a temperature of $60^{\circ}$ in fifteen to twenty minutes, by one of $80^{\circ}$ in five minutes, by one of $90^{\circ}$ in one

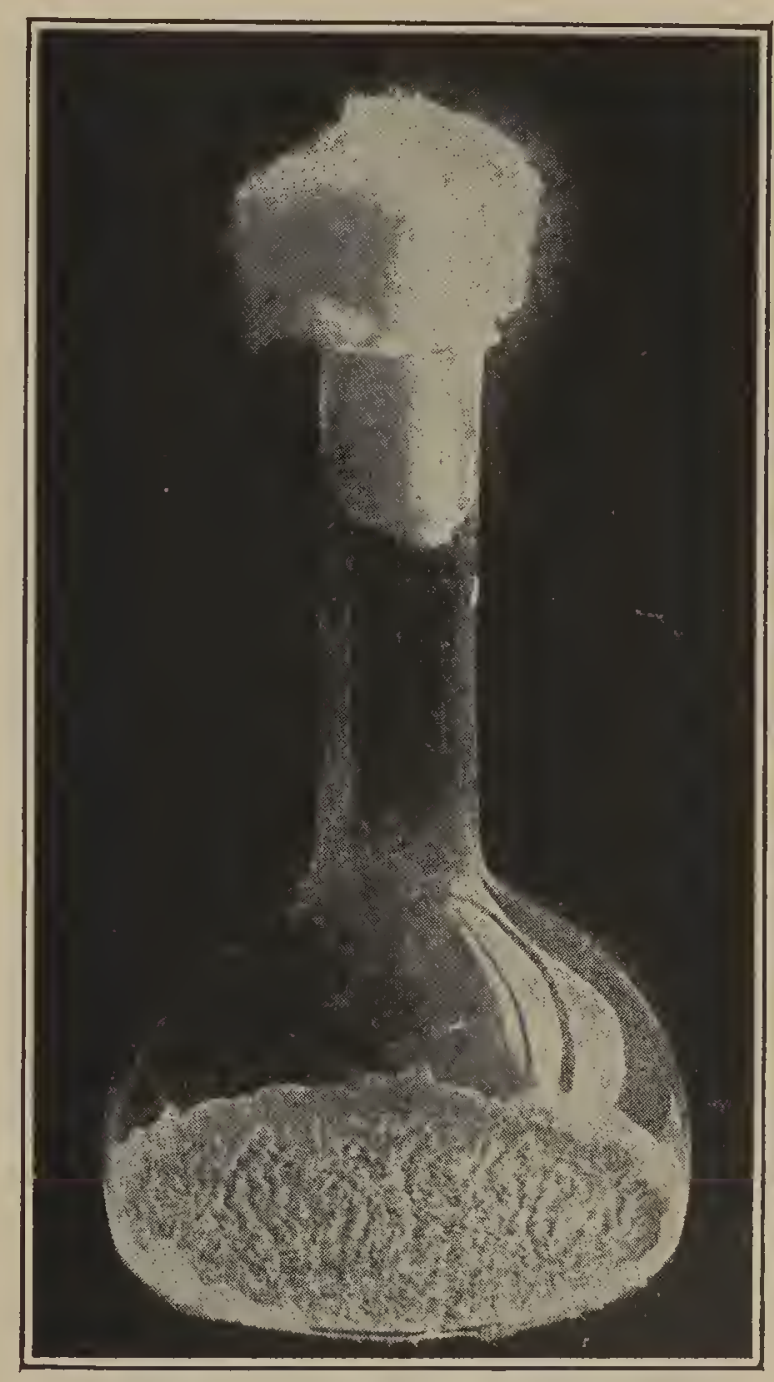

Fig. 104.-Culture of Bacillus Tuberculosis in Flask of Glycerin Bouillon. to two minutes. They will withstand dry heat at $100^{\circ} \mathrm{C}$. for one hour. They are resistant to cold. The comparatively high powers of resistance of the bacillus are attributed to the protective qualities of the waxy cell membrane. ${ }^{3}$

The natural life of cultures, kept in favorable environment, is from

${ }^{1}$ Hesse, Zeit. f. Hyg., xxxi.

2 "Nährstoff Heyden" is prepared in Germany. It is a white powder similar to nutrose.

${ }^{3}$ Th. Smith, Jour. Exper. Med., 1899; Grancher et Ledoux-Lebard, Arch. de méd. expér., 1892; Galtier, Compt. rend. de l'acad. des sci., 1887. 
two to eight months, varying to some extent with the nature of the culture medium. The viability of the bacilli in sputum is of great hygienic importance. In most sputum they may remain alive and virulent for as long as six weeks, in dried sputum for more than two months. ${ }^{1}$

Five per cent carbolic acid kills the bacilli in a few minutes. ${ }^{2}$ If used for sputum disinfection, however, where the bacilli are protected by mucus, complete disinfection by this method requires five to six hours. Bichloride of mercury is not very efficient for sputum disinfection because of the formation of albuminate of mercury.

For room disinfection, formaldehyde gas if thoroughly employed is efficient. Direct suniight kills tubercle bacilli in a few hours.

Pathogenicity.-The tubercle bacillus gives rise in man and susceptible animals to specific phenomena of inflammation which are so characteristic that a diagnosis of tuberculosis may usually be made on the basis of the histological examination of material, even without the finding of tubercle bacilli. The foci of inflommation known as tubercles have been systematically studied by Baumgarten ${ }^{3}$ and many others and descriptions of them may be found in any text-book of pathological anatomy.

In man, tuberculosis is by far the most common of diseases. Naegeli, ${ }^{4}$ in a large series of autopsies, found lesions of healed or active tuberculosis in an appalling percentage of cases. His figures are interesting in showing not only the frequency of the disease, but its relation to age. Before one year of age, he finds it very rare. From the first to the fifth year it is rare, but usually fatal when occurring. From the fifth to the fourteenth year, one-third of his cases showed tuberculous lesions; from the fourteenth to the eighteenth year, one-half of the cases. Between the ages of eighteen and thirty, almost all the cases examined showed some trace of tuberculous infection. Three-quarters of these were active, one-quarter healed. Two-fifths of all deaths occurring at these ages were due to tuberculosis. After the age of thirty, active lesions gradually diminished in number, healed lesions increased.

In 1900, at a public hearing of the New York Tenement House Commission, Pryor ${ }^{5}$ stated that the average yearly mortality from

${ }_{1}^{1}$ Schell und Fischer, Mitt. a. d. kais. Gesundheitsamt, 1884.

${ }^{2}$ De Toma, Ann. di med., 1886.

${ }^{3}$ Baumgarten, Berl. klin. Woch., 1901.

${ }^{4}$ Naegeli, Virchow's Arch., cix, 1900, p. 462.

5 Pryor, Med. News, lxxvii, 1900. 
tuberculosis in New York amounted to 6,000, and that in Manhattan alone there were constantly 20,000 persons suffering from the disease. Cornet ${ }^{1}$ estimates that in 1894 the deaths in Germany from all other infectious diseases amounted to 116,705 , while those from tuberculosis alone amounted to 123,904 . Similar statistics might be chosen at will from the health reports of any large city. While the disease is less common in rural districts than in large towns, the difference is not so striking as is generally supposed.

In man, pulmonary infection is by far the commonest type. Besides this, however, tuberculous processes may be found in the skin, the bones, the joints, the organs of special sense, and the abdominal viscera and peritoneum. No part of the human body is exempt from the danger of infection.

Infection in the human subject may take place by inhalation or through the skin or the digestive apparatus. V. Behring ${ }^{2}$ has within recent years expressed the belief that a large percentage of all cases of tuberculosis originate in childhood from infection by way of the intestinal tract. He determined, as have others since his publication, that tubercle bacilli may penetrate the intestinal mucosa without causing lesions. Behring's contention has caused a great deal of discussion, and the question he has raised is intimately bound up with the problem of the virulence of bovine tubercle bacilli for human beings, as he assumes that the infection is due to the use of infected milk.

The problem is plainly of the greatest importance hygienically, and for this reason has been diligently investigated during the last few years. The only reliable available method of approaching it has been to isolate the tubercle bacilli from large series of diseased human beings and determine for each case whether the guilty organism belonged to the human or the bovine type. These types, as we shall see presently, can be differentiated definitely by cultural characteristics and pathogenicity, and it is not likely, at least in the light of our present knowledge, that the type changes during the sojourn in the human body. Granted this permanence of type, it is naturally of much value in revealing the source of an infection, to determine whether or not a human being is harboring a bacillus of the human type or one of the bovine type.

One of the most valuable contributions made to this problem during the last three years is that of Park and Krumwiede. ${ }^{3}$ The accompanying

${ }^{1}$ Cornet, "Die Tuberculose," Wien, 1899, p. 1.

${ }^{2} v$. Behring, Deut. med. Woch., 39, 1903

${ }^{3}$ Park and Krumwiede, Jour. of Med. Res., Oct., 1910. 
tabulation is taken from their paper and represents a summary of their own cases and those reported by others.

Comibined Tabulation, Cases Reported and Own Series of Cases.

(From Park and Krumwiede, loc. cit.)

\begin{tabular}{|c|c|c|c|c|c|c|}
\hline \multirow[t]{2}{*}{ DIAGNOSIS. } & \multicolumn{2}{|c|}{$\begin{array}{l}\text { Adults } \\
16 \text { Years } \\
\text { and Over. }\end{array}$} & \multicolumn{2}{|c|}{$\begin{array}{l}\text { Children } \\
5 \text { to } 16 \text { Years. }\end{array}$} & \multicolumn{2}{|c|}{$\begin{array}{l}\text { Children } \\
\text { Under } 5 \\
\text { Years. }\end{array}$} \\
\hline & Human & Bovine & Human & Bovine & Human & Bovine \\
\hline Pulmonary tuberculosis. . & 568 & $1 ?$ & 11 & - & 12 & 一 \\
\hline Tuberculous adenitis, axillary or inguinal. & 2 & - & 4 & - & 2 & - \\
\hline Tuberculous adenitis, cervical. & 22 & 1 & 33 & 20 & 15 & 20 \\
\hline Abdominal tuberculosis. . & 15 & 3 & 7 & 7 & 6 & 13 \\
\hline Generalized tuberculosis alimentary origin. . & 6 & 1 & 2 & 3 & 13 & 10 \\
\hline Generalized tuberculosis . & 28 & - & 4 & 1 & 28 & 5 \\
\hline $\begin{array}{l}\text { Generalized tuberculosis, including meninges, } \\
\text { alimentary origin } \ldots \ldots \ldots \ldots \ldots \ldots \ldots \ldots\end{array}$ & - & - & 1 & - & 3 & 8 \\
\hline Generalized tuberculosis, including meninges. & 4 & - & 7 & 一 & 45 & 1 \\
\hline Tuberculous meningitis. . & - & - & 2 & - & 14 & 2 \\
\hline Tuberculosis of bones and joints. & 18 & 1 & 26 & 1 & 21 & - \\
\hline Genito-urinary tuberculosis. . & 11 & 1 & 1 & - & - & - \\
\hline Tuberculosis of skin. & 1 & - & 1 & - & 1 & 一 \\
\hline $\begin{array}{l}\text { Miscellaneous Cases: } \\
\text { Tuberculosis of tonsils......... }\end{array}$ & - & - & - & 1 & - & - \\
\hline Tuberculosis of mouth and cervical nodes.. & - & 1 & - & - & - & - \\
\hline Tuberculous sinus or abscesses. & 2 & - & 一 & 一 & 一 & 一 \\
\hline Sepsis, latent bacilli & - & - & - & 一 & 1 & - \\
\hline Totals. & 677 & 9 & 99 & 33 & 161 & 59 \\
\hline
\end{tabular}

Mixed or double infections, 4 cases. 
From this table it is evident that out of a total of 1,042 cases, 101 only were bovine in origin and over 50 per cent of these occurred in children under five years of age. Fifty-one out of the 59 cases occurring in the 161 infants were directly or indirectly traced to alimentary infection.

It seems reasonably accurate, therefore, to state the case as follows: Human adults are relatively insusceptible to bovine infection. Such infection can take place, but is unusual. Below 16 years of age the human race is relatively more susceptible and up to this age the danger of milk infection is unquestionably great, this source accounting for about one-third of the cases. Below 5 years the danger is greatest. This table alone should form sufficient evidence to silence absolutely any doubts as to the dangers of milk infection and remove any objections to the most rigid sanitary control of milk supplies.

On the other hand, it also shows that Behring's original claims were far too sweeping and can not be upheld.

Rosenberger ${ }^{1}$ has recently reported finding tubercle bacilli in the circulating blood of all cases of human tuberculosis which he examined. This announcement aroused much interest and has led to many investigations by other workers. Rosenberger's results were obtained by morphological examination of smears of citrated blood taken from the patients, dried upon slides and laked with distilled water. Many other observers have failed to zonfirm Rosenberger's results. Anderson ${ }^{2}$ examined 47 cases in which tubercle bacilli were found in the sputum and one case of joint tuberculosis. In none of these 48 cases was he able to obtain tubercle bacilli, neither by morphological examination nor by guinea-pig inoculation. Brem ${ }^{3}$ subsequently found that laboratory distilled water may frequently contain acid-fast saprophytes-a fact which may account in many cases for errors when morphological examination alone is relied upon and blood examined by the technique of Rosenberger. This, too, is suggested by the finding of acid-fast bacilli in the blood of perfectly healthy individuals. Therefore, although the bacilli may be present in the blood in a certain number of cases it does not seem likely that they are so distributed in anything like the high percentages found by Rosenberger. ${ }^{4}$

Bacillus tuberculosis (typus humanus) is pathogenic for guinea-

1 Rosenberger, Am. Jour. of Med. Sc., cxxxvii, 1909.

2 Anderson, U. S. P. H. Service, Hygienic Lab., Bull. 57, 1909.

${ }^{3}$ Brem, Jour. A. M. A., liii., 1909.

${ }^{4}$ Suzuki and Takaki, Centralbl. f. Bakt., Ixi, 1911. 
pigs, less markedly for rabbits, and still less so for dogs. It is slightly pathogenic for cattle, a question spoken of more extensively below.

Chemical Analysis of Tubercle Bacilli. ${ }^{1}$-Diligent efforts by many investigators to isolate the specific toxins which lend tubercle bacilli their pathogenic properties have led to careful chemical analysis of the organisms. About 85.9 per cent of the bacillus consists of water; 20 to 26 per cent of the residue can be extracted with ether and alcohol. This material consists of fatty acids and waxy substances (fatty acids in combination with the higher alcohols). The residue after alcoholcther extraction is composed chiefly of proteids. These can be extracted with dilute alkaline solutions, and consist chiefly of nucleo-albumins. A nuclein present in this fraction shows extremely high toxicity and has, ${ }^{2}$ therefore, been suspected of being the pathogenic principle of the bacillus. After these extractions the remainder contains "cellulose," supposed to represent the framework of the cell membrane, and an ash rich in calcium and magnesium.

Toxins of the Tubercle Bacillus.-The Tuberculins.-Filtrates of bouillon cultures of Bacillus tuberculosis ${ }^{3}$ will occasionally produce slight emaciation when injected into guinea-pigs, and when administered to tuberculous subjects in sufficient quantity will give rise to marked increase of temperature. It is likely, therefore, that the tubercle bacillus actually secretes a soluble toxin. ${ }^{4}$

The chief toxic principles, however, of Bacillus tuberculosis are probably endotoxins or bacterial proteins, bound during cell life to the body of the bacillus. Dead bacilli will produce sterile abscesses when injected into animals. Prudden and Hodenpyl, ${ }^{5}$ Straus and Gamaleia, ${ }^{6}$ and others, ${ }^{7}$ moreover, have shown that the injection of dead and carefully washed cultures of this bacillus will produce lesions histologically similar to those occurring after infection with the living germs, and will often lead to marasmus and other systemic symptoms of poisoning.

The hope of actively immunizing with substances obtained from

${ }^{1}$ Hammerschlag, Cent. f. klin. Med., 1891; Weyl, Deut. med. Woch., 1891; De Schweinitz and Dorset, Jour. Amer. Chem. Soc., 1895; Hammerschlag, loc. cit.

${ }^{2}$ Behring, Berl. klin. Woch., 1899.

${ }^{3}$ Straus and Gamaleia, Arch. méd. exp., 1891.

"Denys, "Le Bouillon Filtré," Louvain, 1905.

5 Prudden and Hodenpyl, N. Y. Med. Jour., June, 1891; Prudden, ibid., Dec. 5.

${ }^{6}$ Straus and Gamaleia, loc. cit.

${ }^{7}$ Mafucci, Cent. f. allg. Path., 1890. 
dead bacilli led Koch to employ various methods of extraction of cultures for the manufacture of tuberculin.

"Old Tuberculin"1 (Koch) ("T.A.K.").-The first tuberculin made by Koch is produced in the following manner: Tubercle bacilli are grown in slightly alkaline 5 per cent glycerin-pepton bouillon for six to eight weeks. At the end of this time, growth ceases and the corrugated pellicle of tubercle bacilli, which during growth has floated on the surface, begins, here and there, to sink to the bottom. The entire culture is then heated on a water-bath at about $80^{\circ} \mathrm{C}$., until reduced to one-tenth of its original volume. It is then filtered either through sterile filter paper or through porcelain filters. The resulting filtrate is a rich brown, syrupy fluid, containing the elements of the original culture medium and a 50 per cent glycerin extract of the tubercle bacilli. While the glycerin is of sufficient concentration to preserve it indefinitely, 0:5 per cent phenol may be added as an additional precaution. Dilutions of this fluid are used for diagnostic and therapeutic purposes.

"New Tuberculin" 2 (Koch) (TA, TO, TR).-Koch believed that the immunity resulting from treatment with the old tuberculin was purely an antitoxic immunity, devoid of all antibacterial action. The use of whole dead tubercle bacilli for immunization purposes, however, was impracticable; because, injected subcutaneously, they. were not absorbed, and introduced intravenously they were deposited in the lungs and gave rise to lesions. Koch was led, therefore, to resort to more energetic extraction of the bacilli in the hope of procuring a substance which could be easily absorbed and would at the same time give rise, when injected, to antibodies more definitely bactericidal. By extracting tubercle bacilli with decinormal $\mathrm{NaOH}$, for three days, filtering through paper and neutralizing, he obtained his TA (alkaline tuberculin). This preparation seemed to fulfil some of the hopes of its discoverer, but had the disadvantage of often producing abscesses at the points of injection. Koch then resorted to mechanical trituration of the bacilli. The method he subsequently followed for tuberculin production is now extensively used, and is carried out as follows: ${ }^{3}$

Virulent cultures of tubercle bacilli are dried in vacuo and thoroughly ground in a mortar. Grinding is continued until stained preparations reveal no intact bacilli. (This is done by machinery in all large manufactories.) One gram of the dry mass is shaken up in 100 c.c. of sterile distilled water. This mixture is then centrifugalized at high speed.

${ }^{1}$ Koch, Cent. f. Bakt., 1890; Deut. med. Woch., 1891.

${ }^{2}$ Koch, Deut. med. Woch., 14, $1897 . \quad{ }^{3}$ Ruppel, Lancet, March 28, 1908. 
The supernatant fluid, known as TO (Tuberculin-Oberschicht), contains the water-soluble constituents of the bacillus, gives no precipitate on the addition of 50 per cent glycerin, and has the same physiological action as the old tuberculin. The residue TR (Tuberculin-Rückstand), after pouring off $\mathrm{TO}$, is again dried and ground up, and again shaken in water and centrifugalized. This process is repeated several times, and eventually, after three or four repetitions, all the TR goes into emulsion. The total volume of water used for these TR extractions should not exceed 100 c.c. All of the TR emulsions are then mixed together. This gives TR a precipitate with 50 per cent of glycerin, and is supposed by Koch to contain substances important in producing an antibacterial immunity. For purposes of standardization, the amount of solid substance in 5 c.c. of the TR is determined by evaporation in vacuo and drying. To the rest are added a little glycerin and formaldehyde and enough water to allow each cubic centimeter of the solution to contain 0.002 grams of solid material. Thus the culture and the medium remaining the same, fairly accurate standardization is possible.

"New Tuberculin-Bacillary Emulsion." 1-In 1901, Koch combined "TO" and "TR" by putting forth a preparation referred to as "Bazillenemulsion." This consists of an emulsion of pulverized bacilli $1: 100$ in distilled water. After several days of sedimentation to remove the coarser particles, the supernatant fluid is poured off and fifty per cent volume of glycerin is added to it for purposes of preservation. This preparation contains 5 milligrams of solid substance in each cubic centimeter.

Bouillon Filtré (Denys). ${ }^{2}$ - This preparation consists of the filtrate (through Chamberland filters) of 5 per cent glycerin-pepton-bouillon cultures of Bacillus tuberculosis. Phenol 0.25 per cent is added to insure sterility. The filtered bouillon corresponds to the unconcentrated old tuberculin of Koch, but, not having been heated, is supposed by Denys to contain important soluble and possibly thermolabile secretory products of the bacillus.

T'uberculoplasmin (Buchner and Hahn). ${ }^{3}$-Buchner and Hahn, by crushing tubercle bacilli by subjecting them to a pressure of 400 atmospheres, obtained a cell-juice in the form of an amber fluid, to which they attributed qualities closely analogous to those of TR.

${ }^{1}$ Koch, Deut. med. Woch., 1901.

2 Denys, "Le Bouillon Filtré," Louvain, 1905.

${ }^{3}$ Buchner und Hahn, Münch. med. Woch., 1897; Hahn, ibid. 
Other tuberculins are those of Beraneck, ${ }^{1}$ highly recommended clinically by Sahli, ${ }^{2}$ that of Klebs, ${ }^{3}$ and the tuberculin produced from bovine tubercle bacilli by Spengler. ${ }^{4}$

Diagnostic Use of Tuberculin.-Subcutaneous Use.-The preparation usually employed for diagnostic purposes is Koch's "Old Tuberculin" (Alttuberculin). This preparation is administered by hypodermic injection of small quantities obtained by means of dilutions. The dilutions are best made with a 0.5 per cent aqueous carbolic acid solution. In practice a 1 per cent solution is made by pipetting 0.1 c.c. of tuberculin into 9.9 c.c. of the 0.5 per cent carbolic solution. A cubic centimeter of this then contains 0.01 c.c. of tuberculin. One c.c. of this solution added to 9 c.c. of 0.5 per cent carbolic acid gives a solution in whish each cubic centimeter contains 0.001 c.c., or 1 milligram of tuberculin.

The initial dosage in adults in Koch's ${ }^{5}$ early work, and as used by Beck ${ }^{6}$ on a large number of patients, was 1 milligram. This, according to present opinions, is too high, and most clinicians to-day prefer 0.1 to 0.2 of a milligram. If after three or four days no reaction has occurred, a second dose of 1 milligram is given. In the absence of reaction after three further days, a third dose of $5 \mathrm{mgm}$. may be given and, under similar conditions, a fourth of $10 \mathrm{mgm}$. This is the largest dose which should ever be given, and absence of a reaction to this dose may generally be regarded as proof that the patient is free from tuberculosis. Doses larger than $10 \mathrm{mgm}$. may give reactions in perfectly healthy subjects. Increase in dosage should be carried out only when the preceding dose has been entirely without reaction. In all cases it should be remembered that absolute rules of dosage can not be made and the condition and physique of each patient must be separately judged.

The reaction itself is recognized chiefly by the changes in temperature. In a positive reaction the patient's temperature will begin to increase within six to eight hours after injection, rising sharply within a few hours to 0.5 or $1.5^{\circ}$ higher than the temperature before injection. It then sinks more gradually than it rose, the reaction usually being complete within thirty to thirty-six hours. With the temperature there

${ }_{1}^{1}$ Beraneck, Compt. rend. de l'acad. des sci., 1903.

${ }^{2}$ Sahli, Corrbl. d. Schw. Aerzte, 1906.

${ }^{3}$ Klebs, Cent. f. Bakt., 1896; Deut. med. Woch., 1907.

${ }^{4}$ Spengler, Deut. med. Woch., xxxi, 1904; xxxi and xxxiv, 1905.

${ }^{5}$ Koch, Deut. med. Woch., 1890.

${ }^{6}$ Beck, Deut. med. Woch., 1899. 
may be nausea, a chill, rapid pulse, and general malaise. Locally visible tuberculous processes, such as lupus, lymph nodes, etc., may become more tender or swollen, and if the tuberculosis is pulmonary, there may be coughing and increased expectoration. The temperatures of persons subjected to the test should be taken regularly for three or four days before tuberculin is used.

Ophthalmo-Tuberculin Reaction.-Wolff-Eisner ${ }^{1}$ and, soon after him, Calmette ${ }^{2}$ proposed a method of using tuberculin for diagnostic purposes by instillation into the conjunctival sac. In tuberculous patients this process is followed by a sharp conjunctival congestion lasting from one to several days.

The preparation used for this purpose is produced in the following way:

"Old Tuberculin" is treated with double the quantity of 95 per cent alcohol, and the precipitate allowed to settle and the alcohol then filtered off through paper. The sediment is washed with 70 per cent alcohol until the filtrate runs clear, then pressed between layers of filter paper to remove excess of moisture, scraped into a dish, dried in vacuo over $\mathrm{H}_{2} \mathrm{SO}_{4}$, and broken up in a mortar under a hood.

Solutions of the powder are made in sterile normal salt solution, 1 per cent by weight, boiled and filtered. The solutions are used in strengths of 0.5 to 1 per cent, a drop of which is instilled into the conjunctival sac. ${ }^{3}$

Cutaneous Tuberculin Reaction.-Von Pirquet ${ }^{4}$ has suggested the cutaneous use of tuberculin for diagnostic purposes. A 25 per cent solution of "Old Tuberculin" is made in the following way:

Tuberculin......................... 1

Salt solution...................... 2

5 per cent carbolic acid in glycerin........... 1

After sterilization of the patient's forearm, two drops of this solution are placed upon the skin about $6 \mathrm{~cm}$. apart. Within each of these drops scarification is done, and the skin between them is scarified as a control. Within twenty-four to forty-eight hours, in tuberculous patients, erythema, small papules, and herpetiform vesicles will appear. The reaction is irregular and more reliable in children than in adults. Ac-

${ }^{1}$ Wolff-Eisner, Berl. med. Gesell., May 15, 1907.

${ }^{2}$ Calmette, Acad. des sci., June 17, 1907.

${ }^{3}$ Method in use at Saranac and kindly communicated by Dr. Baldwin.

${ }^{4}$ v. Pirquet, Berl. klin. Woch., xx, 1907; Med. Klinik, xl, 1907. 
cording to recent investigations, about 70 per cent of adults show a positive reaction and in such cases it is probable that an old healed tuberculosis may give rise to a positive test where absolutely no active process exists.

Recently, v. Pirquet has modified his procedure by using instead of the 25 per cent solution given above, the pure, undiluted "Old Tuberculin."

Moro ${ }^{1}$ has modified this by simply making a 50 per cent ointment of tuberculin in lanolin and rubbing it into the skin without scarification.

It is more simple and equally efficient to massage into the skin a drop of undiluted "Old Tuberculin."

The Tuberculin Test as Applied to Cattle.-In cattle, the symptoms of tuberculosis are not easily detected by methods of physical diagnosis until the disease has reached an advanced stage. In consequence, cows may be elements of danger without appearing in any way diseased to those who handle them. In consequence, routine examination of herds by the tuberculin test has become one of the necessary measures in public sanitation. According to Mohler, ${ }^{2}$ an accurate diagnosis may be established in at least 97 per cent of the cases. It is natural that a good deal of objection to the test is encountered on the part of dairy farmers and cattle raisers, and recently it has been publicly claimed that the cattle are injured by the test. There is, however, no scientific basis for this belief, if the test is carried out carefully and intelligently. As a matter of fact, the systematic use of the test would eventually be distinctly advantageous to the owners of the cattle themselves, since it has been shown that cows, even in the early stages of the disease, may expel tubercle bacilli, either during respiration or in the feces, and thus become a menace to healthy members of the herd.

The tuberculin test on cattle should be made as follows: (The directions given below are taken directly from the circular sent out from the Bureau of Animal Industry at Washington.)

1. Begin to take the rectal temperature at 6 A.M., and take it every two hours thereafter until midnight.

2. Make the injection at midnight.

3. Begin to take the temperature next morning at 6 A.M., and continue as on preceding day.

To those who have large herds to examine, or are unable to give the

1 Moro, Münch. med. Woch., 1906, p. 216.

${ }^{2}$ Mohler, Pub. H. and Mar. Hosp. Serv. Bull. 41, 1908. 
time required by the above directions, the following shortened course is recommended:

1. Begin to take the temperature at 8 A.M., and continue every 2 hours until 10 P.M. (omitting at 8 P.M., if more convenient); or take the temperature at 8 A.M., 12 M., and 10 P.M.

2. Make the injection at 10 P.M.

3. Take the temperature next morning at 6 or 8 A.M., and every 2 hours thereafter until 6 or 8 P.M.

Each adult animal should receive 2 c.c. of the tuberculin as it is sent from the laboratory. (The tuberculin sent out from the central laboratory at Washington is already diluted; 2 c.c. represents 0.25 c.c. of the concentrated "Old Tuberculin" of Koch.) Yearlings and two-year-olds, according to size, should receive from 1 to 1.5 cubic centimeters. Bulls and very large animals may receive three cubic centimeters. The injection should be made beneath the skin of the neck or shoulders behind the scapula, after washing the area with a weak carbolic acid solution.

There is usually no marked local swelling at the seat of the injection.

There are now and then uneasiness, trembling, and the more frequent passage of softened dung. There may also be slight acceleration of the pulse and of the breathing.

The febrile reaction in tuberculous cattle following the subcutaneous injection of tuberculin begins from six to ten hours after the injection, reaches the maximum nine to fifteen hours after the injection, and returns to normal eighteen to twenty-six hours after the injection.

A rise of two or more degrees Fahrenheit above the maximum temperature observed on the previous day should be regarded as an indication of tuberculosis. For any rise less than this a repetition of the injection after four or six weeks is highly desirable.

It is hardly necessary to suggest that for the convenience of the one making the test the animals should not be turned out, but fed and watered in the stable. It is desirable to make note of the time of feeding and watering and of any temperature fall after watering.

The tuberculin should not be used later than six weeks after the date on the bottle, nor if there is a decided clouding of the solution.

Therapeutic Uses of Tuberculin.-Tuberculin was first used therapeutically, shortly after its discovery, by Koch. ${ }^{1}$ Hailed with the most optimistic enthusiasm, its possibilities were overestimated and hopeless cases were treated unskilfully, with unsuitable dosage. The consequence was that harm was done, the method was attacked by Virchow

${ }^{1}$ Koch, Deut. med. Woch., iii, 1891. 
and others and the new therapy fell into almost complete neglect. At present, the use of tuberculin has again been revived, but with greater caution and with a thorough understanding of its limitations. The tendency has been toward smaller dosage and the limitation of the agent to early cases. No two institutions use tuberculin in exactly the same manner, and it is, therefore, impossible to do more than outline the general scheme of treatment. It must never be forgotten, however, that all forms of tuberculin treatment consist in an "active immunization" in which, for the time being, the toxemia of the patient is increased rather than neutralized. It is obvious, therefore, that only such cases are at all suitable for treatment in which the process is not a very acute one. The general principle of modern tuberculin therapy seems to lie in choosing doses so small that no marked general reaction shall follow. The preparations most frequently employed are Koch's "Alttuberculin," his "TR," his "Neu Tuberkulin-Bazillen Emulsion," and the Bouillon filtré of Denys. Initial doses of Alttuberculin range from 0.1 to 0.01 of a milligram. In case of successful avoidance of a reaction, the injection may be repeated, gradually increasing, about twice a week. The occurrence of a reaction should be the signal for a longer interval and a slower advance in the size of the dose.

The initial dose of "TR" is, as advised by Koch, ${ }^{1}$ about 0.002 mgm. This usually causes no reaction. The dose is doubled, at reasonable intervals, up to $1 \mathrm{mgm}$. After this, further increase is carefully gauged by the clinical indications. The maximum dose is about $20 \mathrm{mgm}$.

"Neu Tuberkulin-Bazillen Emulsion," 2 is begun with a dose of 0.001 mgm. Gradual increase as with the other preparations is then practiced. The maximum dose is about $10 \mathrm{mgm}$.

Bouillon filtré has been used chiefly by Denys ${ }^{3}$ and with apparently excellent results. Denys is very emphatic in advising the absolute avoidance of any reaction. He begins with a millionth or even the tenth of a millionth of a cubic centimeter of the bouillon and increases with extreme caution. His dilutions are made with glycerin broth.

Passive Immunization in Tuberculosis.-Numerous attempts have been made to immunize tuberculous subjects with the sera of actively

${ }^{1}$ Koch, Deut. med. Woch., xiv, 1897.

2 Bandelier und Roepke, "Lehrb. d. spezifisch. Tub. Ther.," Würzburg, 1908; Koch, Deut. med. Woch., 1901.

3 Denys, "Le Bouillon filtré," Louvain, 1905. 
immune animals. The most widely used method of producing such serum is that of Maragliano.

Maragliano's Serum.1-Maragliano believes that a toxalbumin is present in tubercle-bacillus cultures which is destroyed by the heating employed in the usual tuberculin production. He procures this substance by filtration of unheated cultures and precipitation with alcohol (tossina præcipitata). He furthermore makes an aqueous extract of the bacillary bodies. With these two substances he immunizes horses. He draws blood from these after four to six months of treatment. The serum is extensively used in Italy. Its value is, at present, very doubtful.

Marmorek's Serum. ${ }^{2}$ - Marmorek claims that the poisons produced by Bacillus tuberculosis depend largely upon the medium on which it is grown. He advanced the view in 1903 that the substances obtained in tuberculin were not the true toxins of the tubercie bacillus, that there was a marked difference between these and the poiscins elaborated by a younger (primitive) phase of the bacillus as it occurs only within the animal body or on media composed of animal tissue. He consequently grows his cultures on a medium composed of a leucotoxic serum (produced by inoculating calves with guinea-pig leucocytes) and liver tissue. Such cultures, he claims, contain no tuberculin. To the sera produced by immunization with these cultures he attributes high curative powers.

Bacilli Closely Related to the Tubercle Bacillus.-The Bacillus of Bovine Tuberculosis.-Tuberculosis of cattle (Perlsucht) was studied by Koch ${ }^{3}$ in connection with his early work on human tuberculosis. Koch did not fail to recognize differences between the reactions to infection in the bovine type of the disease and that of man. He attributed these, however, to the nature of the infected subject rather than to any differences in the infecting agents. This point of view met with little authoritative contradiction, until Theobald Smith, ${ }^{4}$ in 1898 , made a systematic comparative study of bacilli isolated from man and from cattle and pointed out differences between the two types. The opinion of Smith was fully accepted by Koch ${ }^{5}$ in 1901.

Since that time, the question, because of its great importance to prophylaxis, has been the subject of many investigations, most of them

1 Maragtiano, Berl. klin. Woch., 1899; Soc. de biol., 1897.

${ }^{2}$ Marmorek, Berl. klin. Woch., 1903, p. 1108; Med. Klinik, 1906.

${ }^{3}$ Koch, Arb. a. d. kais. Gesundheitsamt, 11, 1882.

${ }^{4}$ Th. Smith, Jour. Exp. Med., 111, 1898.

'Koch, Deut. med. Woch., 1901. 
confirming Smith's original work. Morphologically, Smith ${ }^{1}$ found that the bovine bacilli were usually shorter than those of the human type and grew less luxuriantly than these upon artificial media. He determined, furthermore, that, grown upon slightly acid glycerin bouillon, the bovine bacillus gradually reduces the acidity of the culture medium until the reaction reaches neutrality or even slight alkalinity. Fluctuations, after this, do not exceed 0.1 or 0.2 per cent on either side of neutrality. In the case of the human bacillus, on the other hand, there is but slight reduction of the acidity during the first weeks of growth; after this acidity increases and, though subject to fluctuations, never reaches neutrality. This behavior is probably due to action exerted upon the glycerin, since on ordinary bouillon no such differences between the two varieties can be noticed. These observations of Smith were confirmed by Ravenel, ${ }^{2}$ Vagedes, ${ }^{3}$ and others.

The cultural differences between the two types have been studied with especial care by Wolbach and Ernst, ${ }^{4}$ and Kossel, Weber, and Heuss. $^{5}$ All of these observers bear out Smith's contention that luxuriance and speed of growth are much more marked in the human than in the bovine variety. Marked differences, furthermore, have been shown to exist in the pathogenic qualities of these bacilli toward various animal species.

Guinea-pigs inoculated with the bovine type ${ }^{6}$ die more quickly and show more extensive lesions than those infected with human bacilli. The difference in the pathogenicity of the two organisms for rabbits is sufficiently striking to be of diagnostic value. The bovine bacilli usually kill a rabbit within two to five weeks; the human bacilli produce a mild and slow disease, lasting often for six months, and occasionally fail to kill the rabbits at all.

The practical importance of distinguishing between the two types, of course, attaches to the question as to whether the bovine and the human disease are mutually intercommunicable. Extensive attempts to infect cattle with bacilli of the human type have been made, ${ }^{7}$ for the most part with very little or no success. Infections of human beings with

1 Th. Smith, Jour. Exp. Med., 1905.

${ }^{2}$ Ravenel, Lancet, 1901; Univ. Penn. Med. Bull., 1902.

${ }^{3}$ Vagedes, Zeit. f. Hyg., 1898.

"Wolbach and Ernst, "Studies from the Rockefeller Inst.," 11, 1904.

${ }^{5}$ Kossel, Weber, und Heuss, Arb. a. d. kais. Gesundheitsamt, 1904 and 1905.

${ }^{6}$ Smith, loc. cit., and Medical News, 1902.

7 Beck, "Festsch. R. Koch," 1902; Smith, loc. cit. 
bovine bacilli, however, have been reported and proved beyond reasonable doubt, by Smith, ${ }^{1}$ Ravenel, ${ }^{2}$ Kossel, Weber, and Heuss, ${ }^{3}$ Park and Krumwiede, ${ }^{4}$ and others. Most of these infections have been in children. It is likely, therefore, that while cattle are to a considerable degree immune against the bacillus of the human type, human beings do not enjoy the same safeguard in respect to the bovine bacillus. During adult life, the danger of such infection, however, is far less than it is during infancy and early youth. This question has been discussed on p. 487 .

The Bacillus of Avian Tuberculosis.-A disease resembling in many features the tuberculosis of man is not uncommon among chickens, pigeons, and some other bird species. Koch was the first to discover in the lesions of diseased fowl bacilli much resembling Bacillus tuberculosis. It was soon shown, however, by the studies of Nocard and Roux, ${ }^{5}$ Mafucci, ${ }^{6}$ and others, that the bacillus of the avian disease represented a definitely differentiable species.

Morphologically, and in staining characteristics, the bacillus is almost identical with that of the human disease. In culture, however, growth is more rapid and takes place at a temperature of $41^{\circ}$ to $45^{\circ} \mathrm{C} .^{7}$ (the normal temperature of birds), while the human type is unable to thrive at a temperature above $40^{\circ}$.

Guinea-pigs, very susceptible to human tuberculosis, are very refractory to infection with the avian type; while, on the other hand, rabbits which are resistant to the human type, succumb rapidly to infection with avian tuberculosis. ${ }^{8}$ Prolonged cultivation and passage through the mammalian body is said to cause these bacilli to approach more or less closely to the mammalian type. Conversely, Nocard. ${ }^{9}$ succeeded in rendering mammalian tubercle bacilli pathogenic for fowl by keeping them in the peritoneal cavities of hens in celloidin sacs for six months.

Recently Koch and Rabinovitsch ${ }^{10}$ have isolated from the spleen of

I Smith, Trans. Assn. Amer. Phys., 1903.

${ }^{2}$ Ravenel, Univ. Penn. Med. Bull., 1902.

${ }^{3}$ Kossel, Weber, und Heuss, loc. cit.

${ }^{4}$ Park and Krumwiede, Jour. Med. Res., 1910.

${ }^{5}$ Nocard et Roux, Ann. de l'inst. Pasteur, 1887.

${ }^{6}$ Mafucci, Zeit. f. Hyg., xi.

${ }^{7}$ Mafucci, loc. cit.

${ }^{8}$ Straus et Gamaleia, Arch. de méd. expér., 1891; Courmont et Dor, Arch. de méd. exp., 1891.

${ }^{9}$ Nocard, Ann. de l'inst. Pasteur, 1898.

${ }^{10}$ Koch und Rabinovitsch, Virch. Arch., Beiheft to Bd. 190, 1907. 
a young man dead of tuberculosis, a microorganism which, culturally, morphologically, and in its pathogenic action upon birds, seemed to belong to the avian type. Löwenstein ${ }^{1}$ describes a similar organism cultivated from a human case which seems to be a transitional type. Observations of this order are, however, too few at the present time to be used as the basis of a definite opinion as to the relationship between the two varieties.

Tuberculosis in Cold-blooded Animals.-The bacillus isolated by Dubarre and Terre ${ }^{2}$ resembles Bacillus tuberculosis in morphology and in a certain degree of acid-fastness. It grows at low temperatures, $15^{\circ}$ to $30^{\circ} \mathrm{C}$. It is non-pathogenic for animals, but kills frogs within a month. Except for the acid-fastness it has little in common with Bacillus tuberculosis.

Similar acid-fast bacilli have been isolated from other cold-blooded animals (carp, frogs, turtles, snakes) by many observers.

There have been many attempts to show a close relationship between the tubercle bacilli of cold-blooded and those of warm-blooded animals. Moeller, Hansemann, Friedmann, Weber, Küster, and others have given this subject particular attention and it has gained especial interest because of the recent notorious claims of Friedmann that he has succeeded in obtaining, from turtles, a strain of acid-fast bacilli which can be successfully used in actively immunizing human beings. In 1903 Friedmann ${ }^{3}$ described two cases of spontaneous infection of a salt-water turtle (Chelone corticata) with acid-fast bacilli, presenting lesions in the lungs which simulated pulmonary tuberculosis in the human being (cavity formation and miliary nodules). The organisms cultivated from these lesions presented much similarity to those of the human type and, according to Friedmann, ${ }^{4}$ unlike other acid-fast bacilli of cold-blooded animals, could be grown at $37.5^{\circ} \mathrm{C}$. As a possible human origin for the turtle infections Friedmann mentions that the attendant who fed these turtles suffered from a double pulmonary tuberculosis.

Upon inoculation into guinea-pigs localized lesions only were produced, and dogs, rats, and birds were immune. The implication of Friedmann's work is that his culture represents a human strain attenu-

${ }^{1}$ Löwenstein, quoted from Koch and Rabinovitsch, loc. cit.

${ }^{2}$ Dubarre et Terre, Compt. rend. de la soc. de biol., 1897.

${ }^{3}$ Friedmann, D. Med. Woch., No. 2, Jan., 1903, 25.

${ }^{4}$ Friedmann, D. Med. Woch., No. 26, 464, 1903, and Centralbl. f. Bakt., I, xxxiv, 1903, also Zeitschr. f. Tuberkulose, iv, Heft 5, 1903. 
ated for man by passage through the turtle, although, as far as we are aware, no definite statement as to this has been made.

Summarizing the work of many investigators (Weber, Taute, Küster, Allegri, Bertarelli, and others) Küster ${ }^{1}$ makes a statement which is, in essence, as follows: In the carp, in snakes, turtles, and frogs spontaneous tuberculosis may occur. The organisms which cause these diseases are specific for cold-blooded animals, similar in many respects to the tubercle bacillus of warm-blooded animals, but in the latter do not produce progressive disease. Human, bovine, and avian tubercle bacilli inoculated into cold-blooded animals can produce lesions which histologically simulate tuberculosis. These microorganisms can remain a year in cold-blooded animals without losing their pathogenicity for guinea-pigs. Mutation of the tubercle bacillus of warm-blooded animals into cold-blooded ones has not been proven.

For these reasons it is quite impossible to exclude, in the apparently positive work of Friedmann and others, the isolation of a true "coldblooded" type organism, rather than a mutation form originally of the warm-blooded type. What Friedmann's present claims in this respect are for his culture has not been stated as far as we know. The possibility of a positive immunizing value of organisms isolated from cold-blooded animals in human beings, though remote, is not out of question. The problem is so serious and important, and the experience of many workers is, so far, so inconclusive that the time has not come for commercial exploitation and the cruel arousing of false hopes. The subject, however, deserves carefully controlled further investigations.

Bacillus of Timothy.-Moeller isolated from timothy-grass and from the dust in haylofts acid-fast bacilli, like Bacillus tuberculosis. They grow rapidly on agar, soon showing a deep red or dark yellow color.

Bacillus butyricus (Butter Bacillus).-Slightlyacid-fast bacilli resembling Bacillus tuberculosis have been isolated from milk and butter by Petri, ${ }^{2}$ Rabinovitsch, ${ }^{3}$ Korn, ${ }^{4}$ and others.

These bacilli are easily differentiated from Bacillus tuberculosis culturally. They are slightly pathogenic for guinea-pigs, but not for man.

Bacillus smegmatis and the bacillus of leprosy will be discussed in separate sections. The differentiation of these organisms by staining reactions has been discussed in the section on staining methods.

${ }^{1}$ Kolle und Wassermann's Handbuch, 2d edition, v, 767.

${ }^{2}$ Petri, Arb. a. d. kais. Gesundheitsamt, 1897.

${ }^{3}$ Rabinovitsch, Zeit. f. Hyg., 1897.

${ }^{4}$ Korn, Cent. f. Bakt., 1899. 


\section{CHÁPTER XXXIV}

\section{THE SMEGMA BACILLUS AND THE BACILLUS OF LEPROSY}

\section{BACILLUS S MEGMATIS}

IN 1884, Lustgarten ${ }^{1}$ announced that he had succeeded in demonstrating, in a number of syphilitic lesions, a characteristic bacillus, which he declared to be the etiological factor in the disease. The great importance of the subject of Lustgarten's communication caused numerous investigators to take up the study of the microorganisms found upon the genitals of normal and diseased individuals. As a result of these researches the presence of the Lustgarten bacilli upon the genitals of many syphilitics was confirmed; but at the same time bacilli, which in all essential particulars were identical with them, were found in the secretions about the genital organs and anus of many normal persons. The first to throw doubt upon the etiological significance of Lustgarten's bacillus, and to describe in detail the microorganism now recognized as Bacillus smegmatis, were Alvarez and Tavel. ${ }^{2}$ Similar studies were made soon afterward by Klemperer, ${ }^{3}$ Bitter, ${ }^{4}$ and others.

The smegma bacilli are now known to occur as harmless saprophytes in the preputial secretions of the male, about the external genital organs of the female, and within the folds of thighs and buttocks. They are usually found, in these situations, in clumps upon the mucous membrane, and occasionally in the superficial layers of the epithelium, intraand extra-cellularly.

Morphology.-The smegma bacilli are very similar to tubercle bacilli, but show greater variations in size and appearance than do the latter. In length the individuals may vary from two to seven micra. They are usually straight or slightly curved, but according to Alvarez and Tavel may show great polymorphism, including short comma-like forms, and occasional S-shaped spiral forms.

1 Lustgarten, Wien. med. Woch., 47, 1884.

2 Alvarez et Tavel, Arch. d. physiol. norm. et path., Oct., 1885.

${ }^{3}$ Klemperer, Deut. med. Woch., xi, 1885.

4 Bitter, Virchow's Arch., ciii. 
They are not easily stained, and though less resistant in this respect than the tubercle bacillus, they yet belong distinctly to the group of acid-fast bacilli. Once stained by the stronger dyes, such as carbolfuchsin or anilin-water-gentian-violet, they are tenacious of the dye, though less so than tubercle bacilli.

The identification of the smegma bacillus by staining methods has become of great practical importance since Fraenkel, ${ }^{1}$ Müller, ${ }^{2}$ and others have demonstrated the occasional presence of acid-fast bacilli, probably of the smegma group, in sputum, and in secretions from the tonsillar crypts and throat. The methods of differentiation which have been found most practical are those which depend upon differences in the retention of stain shown by these bacilli. While it may be stated as a general rule that the smegma bacilli are more easily decolorized than tubercle bacilli, it is nevertheless important that a control, as suggested by Wood, be marle with known tubercle bacilli whenever a slide of suspected smegma bacilli is examined. For the actual differentiation an excellent method is that of Pappenheim, described in detail in the section on Staining, page 106. This method depends upon the fact that prolonged treatment with alcohol and rosolic acid decolorizes the smegma bacilli but not the tubercle bacilli. Coles ${ }^{3}$ has stated that smegma bacilli will resist Pappenheim's decolorizing agent for four hours at the most, while tubercle bacilli will retain the stain, in spite of such treatment, for as long as twentyfour hours.

Although minor differences between the smegma bacillus and that of Lustgarten have been upheld by Doutrelepont ${ }^{4}$ and others, nevertheless, the etiological significance of Lustgarten's bacillus in syphilis has been finally discredited, and, if not identical with the smegma bacillus, it at least belongs to the same group.

The smegma bacilli have no pathogenic significance. They are found upon human beings as harmless saprophytes, and all attempts to infect animals have so far been unsuccessful. They are cultivated with great difficulty, first cultivations from man being successful only upon the richer media containing human serum or hydrocele fluid. After prolonged cultivation upon artificial media they may be kept alive upon glucose agar or ascitic agar. Their growth is slow;

1 Fraenkel, Berl. klin. Woch., 1898.

2 Müller, Deut. med. Woch., 1898.

${ }^{3}$ Coles, Jour. of State Med., 1904.

- Doutrelepont, quoted from Klemperer, loc. cit. 
and the colonies, appearing within five or six days after inoculation, are yellowish white, corrugated, and not unlike tubercle-bacillus colonies.

\section{BACILLUS LEPRA AND LEPROSY}

the bacillus of leprosy was first seen and correctly interpreted as the etiological factor in the disease in 1879, by G. Armauer Hansen, ${ }^{1}$ a Norwegian observer. Hansen found the bacilli in the tissues of the nodular lesions of patients, lying in small clumps, intra- and extracellularly, as well as in the serum oozing from the tissue during its removal. Hansen's observation was the fruit of over six years of careful study and as to his priority in making this great discovery, there can be no doubt. Almost simultaneously with his publication, however, Neisser ${ }^{2}$ published similar results, obtained by him during a brief stay at Bergen, during the preceding summer. The bacilli described by these workers are now recognized as being unquestionably the cause of the various forms of the disease known as leprosy.

Morphology and Staining.-The leprosy bacillus is a small rod measuring about 5 to $7 \mu$ in length and has a close morphological resemblance to Bacillus tuberculosis, except in that it is less apt to display the beaded appearance and is slightly less slender than the latter. It is non-motile, possesses no flagella, and forms no spores.

Like tubercle bacilli, furthermore, the leprosy bacilli belong to the class of so-called acid-fast bacteria, being stained with much difficulty; but when once stained they are tenacious of the color, offering considerable resistance to the decolorizing action of acids. It is necessary for differential diagnosis, however, to note that both the difficulty of staining and the resistance to decolorization are less marked in the case of this microorganism than in the case of Bacillus tuberculosis. It was this peculiar behavior to stains that caused the delay of several years in Hansen's publications, since he failed in obtaining good morphological specimens until the work of Koch upon bacterial staining had supplied him with proper methods. The bacillus is stained most easily with anilin-water-gentian-violet or with carbol-fuchsin solution. Stained by Gram's method, it is not decolorized and appears a deep blue. Differential staining by the Ziehl-Neelsen method shows the bacillus stained red unless decolorization by means of the acid and alcohol are prolonged

1 Hansen, Virch. Arch., 79, 1879.

2 Neisser, Breslauer ärztl. Zeitschr., 20, 1879. 
for an unusual time. A differentiation from tubercle bacilli by virtue of greater ease of decolorization is of value only in the hands of those having much experience with these bacilli, and follows no regular laws of acid-strengths or time of application which can be generally applied by the inexperienced. In tissues, the bacilli are easily stained by the methods used for staining tubercle bacilli. The sections are left in the Ziehl carbol-fuchsin solution either from two to twelve hours at incubator temperature or for twenty-four hours at room temperature. Subsequent treatment is that employed in the case of tuberculous tissue sections (see p. 112).

Cultivation.-Cultivation of the leprosy bacillus has not met with success. Hansen and others who have approached the problem with a thorough knowledge of the microorganism, combined with a competent bacteriological training, have failed in all their attempts. Numerous positive results reported by observers have always lacked adequate confirmation. Recently, Rost, ${ }^{1}$ of the British Army Medical Corps, has claimed success in cultivation of leprosy bacilli upon salt-free bouillon, his point of departure being the previous observation that salt-free media favored the growth of tubercle bacilli. His results have not been confirmed.

In 1909 Clegg ${ }^{2}$ succeeded in growing an acid-fast bacillus from leprous tissue, obtaining his results by inoculating leprous material upon agar plates upon which ameba coli had been grown in symbiosis with other bacteria. On such plates the acid-fast bacilli multiplied, and, subsequently, pure cultures were obtained by heating the cultures to $60^{\circ} \mathrm{C}$., which destroyed the ameba coli and other bacteria. These results were confirmed by other workers and, soon after that, Duval ${ }^{3}$ not only succeeded in repeating Clegg's experiments, but obtained cultures of an acid-fast bacillus directly from leprous lesions without the aid of ameba. He first observed that the leprosy organism would multiply around a transplanted piece of leprous tissue upon ordinary blood agar tubes upon which influenza bacilli and meningococci were grown. He concluded that such growth depended upon chemical changes in the media and believed the formation of amino-acids essential for the initial growth. The method he subsequently described depended upon supplying these substances either by adding tryptophan to nutrient agar or by pouring egg albumen and human blood serum in Petri dishes,

\footnotetext{
${ }^{1}$ Rost, Brit. Med. Jour., 1, 1905.

${ }^{2}$ Clegg, Philippine Jour. of Sc., iv, 1909.

${ }^{3}$ Duval, Jour. Exp. Med., xii, 1910, and ibid., 15, 1912.
} 
inspissating, at $70^{\circ} \mathrm{C}$., for three hours and, after inoculating with leprous tissue, adding a 1 per cent solution of trypsin. Indirectly the same result was obtained by employing culture media containing albuminous substances and inoculating with bacteria capable of producing amino-¿cids from the medium. After leprosy bacilli had been grown on this medium for several generations, they could easily be cultivated on agar slants without special additions or preliminary treatment.

In spite of extensive work upon this very important problem opinions are still divided as to the specific nature of the organisms cultivated by Clegg and by Duval. Animal experiments with these cultures have remained inconclusive. The cultures after prolonged preservation upon artificial media grow heavily, often lose their acid-fast characteristics, develop into streptothrix-like or diphtheroid forms and become markedly chromogenic, all these characteristics suggesting saprophytism.

In a recent communication, Duval and Wellman ${ }^{1}$ state their opinion as follows: From 29 cases of leprosy, 22 successive cultivations of acidfast bacilli were made; in 14 of them a chromogenic organism, similar to that of Clegg, was found. This grows either as a non-acid-fast streptothrix in subsequent cultivations or as non-acid-fast diphtheroid forms. From eight cases an organism distinctly different from the former was cultivated which grows only on specific media and by serological tests seems to give reaction which differentiates itfrom Clegg's organism. Duval believes that there is no reason to assume specific etiological relationship for the first organism mentioned. In the case of the second, he admits that not sufficient proof has been brought, but states his belief that its etiological significance is probable.

Pathogenicity.-Innumerable attempts to transmit leprosy to animals by inoculation have been unsuccessful. Nicolle, ${ }^{2}$ however, has recently claimed successful experiments upon monkeys (macacus) in whom inoculation with tissue from infected human beings was followed, in sixty-two days, by the development of a small nodule at the site of inoculation, in which, upon excision, leprosy bacilli were found. In most cases, however, inoculation has given rise merely to a transient inflammatory reaction.

Among human beings, leprosy has been a widely spread disease since the beginning of history, and much evidence is found in ancient literature which testifies to a wide distribution of the disease long before the Christian era and throughout the Middle Ages. At the present day,

1 Duval and Wellman, Jour. of Inf. Dis., xi, 1912.

${ }^{2}$ Nicolle, Sem. médicale, 10, 1905. 
leprosy is most common in the eastern countries, especially in India and China. In Europe the disease is found in Norway, in Russia, and in Iceland. In other European countries, while the disease occurs, it is not at all common. In the United States, there are, according to Osler, three important centers of leprosy situated in Louisiana, in California, and among the Norwegian settlers in Minnesota. The disease is also present in several provinces of Canada. In all countries in which segregation of lepers is rigidly practiced, the disease is diminishing. In Norway, according to Hansen, proper sanitary measures have reduced the number of lepers from 2,870 in 1856, to 577 in 1900 .

Clinically, the disease appears in two chief varieties, tubercular leprosy and the so-called anesthetic leprosy. In the former variety, hard nodular swellings appear, usually in the face, but often on other parts of the body as well. These lead to frightful disfigurement and are accompanied by a falling-out of hair and a loss of sensation in the affected areas. In the anesthetic form, there is usually at first pain in definite areas of the extremities and the trunk, which is soon followed by the formation of flat or slightly raised pigmented areas, within which there is absolute anesthesia with, later, atrophy and often secondary necrosis in the atrophied parts. The disease is usually chronic in its course.

The bacilli are found in large numbers in the cutaneous lesions. In the knobs of the nodular variety, they lie in clumps between the connective-tissue cells and within the large spheroidal cells which make up the nodules. They are found, also, in advanced cases, in the liver and in the spleen, lying within the cells, and, to a slighter extent, in the intercellular spaces. They have also been found within the kidneys, the endothelium of the blood-vessels, and in the testicles. ${ }^{1}$ In the blood, the bacilli have frequently been demonstrated, especially during the febrile attacks which occur during the disease. Westphal and Uhlenhut ${ }^{2}$ have found the bacilli within the central nervous system, and these observers, as well as others, have found them lying within the substance of the peripheral nerves, thus explaining the anesthesia. A fact of enormous importance to the question of transmission is the observation made by various observers, more especially by Sticker, that the bacilli are found with great regularity in considerable numbers in the nasal secretions of persons suffering from the disease. Sticker is inclined to regard the nose

\footnotetext{
${ }_{1}^{1}$ Sticker, Münch. med. Woch., 39, 1897.

${ }^{2}$ Westphal und Uhlenhut, Klin. Jahrb., 1901.
} 
as the primary path of infection. Whether or not this be true can not, at present, be decided. As a source of infection, however, the nasal mucus and, secondarily, the saliva, are certainly the vehicles by which large numbers of the bacilli leave the infected patient, and, therefore, tend to spread the disease.

The contagiousness of leprosy is far less than is that of most other bacterial diseases. Physicians and others who come into direct contact with large numbers of leprous patients, observing at the same time the ordinary precautions of cleanliness, rarely contract the disease. On the other hand, intimate contact with lepers without such precautions is the only possible means of transmission. The demonstration of leprosy bacilli in dust, soil, etc., must always be looked upon with suspicion, since, apart from actual human inoculation, there is no method of positively differentiating the bacilli from similar acid-fast organisms. Instances of transmission by contact are on record, not the least famous of which is the case of Father Damien, who contracted the disease while taking care of the lepers upon the island of Molokai. Hansen states that in his knowledge no case of leprosy can be found in which careful examination of the past history will not reveal direct contact with a previous case. Direct inoculation of the human being with material from a leprous patient has been successfully carried out by Arning, ${ }^{1}$ upon a Hawaiian criminal. In this case a piece of a leprous nodule was planted into the subcutaneous tissue of the left arm. One month after the inoculation, pain appeared in the arm and shoulder, and four and a half months later a typical leprosy nodule was formed. Four years after the inoculation, the patient was a typical leper.

Although our inability to cultivate the leprosy bacillus, and the lack of success attending animal inoculation, have made it impossible to study more closely the toxic action of this microorganism, there is, nevertheless, some evidence which points toward the production of a poisonous substance of some kind by the bacillus. Rost, ${ }^{2}$ who claims to have cultivated the bacillus, manufactured from his cultures, by the technique for the production of "Old Tuberculin," a substance which he called "leprolin," and which he employed therapeutically in the same manner in which tuberculin is employed in tuberculosis. As stated before, the results of Rost still lack confirmation. Of far greater importance, both in demonstrating the probability of the existence of a definite toxin as well as in indicating the close relationship between the leprosy bacillus 
and the Bacillus tuberculosis, are the investigations upon the action of tuberculin upon leprous patients. When tuberculin is administered to lepers, a febrile reaction occurs usually twenty-four or more hours after the administration. The fever differs from that produced by the use of the same substance in tuberculous patients in that it is of late occurrence and lasts considerably longer. At the same time, there may be marked redness and tenderness of the nodules. In isolated cases, Babes ${ }^{1}$ has noticed alarmingly high and prolonged fever together with systemic symptoms such as nausea, headache, and even unconsciousness, following the injection of tuberculin. The same writer claims to have extracted from the organs of lepers, which contained enormous numbers of bacilli, substances which showed an action similar to that of the tuberculin.

\section{RAT LEPROSY}

Stefansky ${ }^{2}$ first observed this disease among rats in Odessa, and since then it has been observed in Berlin (Rabinovitsch ${ }^{3}$ ), in London $\left(\right.$ Dean $\left.^{4}\right)$, in New South Wales (Tidswell ${ }^{5}$ ), and in San Francisco (Wherry ${ }^{6}$ and $\mathrm{McCoy}^{7}$ ). The disease occurs spontaneously among house rats and is characterized by subcutaneous induration, swelling of lymph nodes, with, later, falling out of the hair, emaciation, and sometimes ulceration. Its course is protracted and rats may live with it for six months or a year. When a rat suffering from this disease is dissected there is usually found, under the skin of the abdomen or flank, a thickened area which has the appearance of adipose tissue except that it is more nodular and gray and less shiny than fat. It is so like fat, however, that it is often possible to overlook it as evidence of disease by one unfamiliar with the condition. In this area acid-fast bacilli looking like the Bacillus lepræ are found in large numbers. These bacilli are also found in the lymph nodes and sometimes in small nodules which appear in the liver and lung.

${ }^{1}$ Babes, in Kolle und Wassermann, "Handbuch," etc., Erst. Ergänz. Bd., 1907.

2 Stefansky, Centralbl. f. Bakt., xxxiii, 481.

${ }^{3}$ Rabinovitsch, Centralbl. f. Bakt., xxxiii, 577.

${ }^{4}$ Dean, Centralbl. f. Bakt., xxxiv, 222; Jour. Hyg., xcix.

${ }^{5}$ Tidswell, cited by Brinkerhoff in "The Rat and Its Relation to Public Health," Treas. Dept., Wash., 1910.

${ }^{6}$ Wherry, J. A. M. A., June 6, 1908, p. 1903; Jour. Inf. Dis., dvii, Rep. U. S. P. H., and M. H. S., xxiii, 1841.

${ }^{7}$ McCoy, Rep. U. S. P. H. and M. H. S., xxiii, 981; Abstr. in J. A. M. A., Aug. 22, 19̣08, 690, 
The disease can be transmitted experimentally from rat to rat and probably is transmitted naturally from rat to rat by the agency of fleas (Wherry, McCoy). Although clinically not exactly like human leprosy the condition is sufficiently like it to arouse much hygienic interest. The distribution of the disease in various parts of the world does not correspond with the distribution of leprosy. A peculiar feature of its distribution is the fact that in San Francisco, as the writer was told by McCoy, almost all the rats that suffered from this disease came from the district in which the retail meat business is located, known as "Butchertown." The organisms were made to multiply in vitro by Zinsser and Cary in plasma preparations of growing rat spleen. Chapin has succeeded in cultivating them by a method analogous to the trypsinegg albumen method employed by Duval. In the experiments of Zinsser and Cary it was found that although the organisms may retain their acid-fast characteristics for many weeks within leucocytes they degenerate rapidly within the spleen cells, a fact which seems to have some bearing on the mechanism of resistance possessed by the body against acid-fast organisms. 


\section{CHAPTER XXXV}

\section{BACILLUS DIPHTHERIÆ, BACILLUS HOFFMANNI, AND BACILLUS XEROSIS}

\section{BACILLUS DIPHTHERI 尼}

Since 1821, when Bretonneau of Tours published his observations, diphtheria has been an accurately recognized clinical entity. Our knowledge of the disease in the sense of modern bacteriology, however, begins with the first description of Bacillus diphtheriæ by Klebs in 1883. Klebs ${ }^{1}$ had observed in the pseudomembranes from diphtheritic throats, bacilli which in the light of more recent knowledge we can hardly fail to recognize as the true diphtheria organism. His work, however, was purely morphological and, therefore, inconclusive. One year after this announcement, Loeffler ${ }^{2}$ isolated and cultivated an organism which corresponded in its morphological characters to the one described by Klebs. He obtained it from thirteen clinically unquestioned cases of diphtheria, and, by inoculating it upon the injured mucous surfaces of animals, succeeded in producing lesions which resembled closely the false membranes of the human disease. His failure to find the bacillus in all the cases he examined, his finding it, in one instance, in a normal throat, and his inability to explain to his own satisfaction some of the systemic manifestations of the infection which we now know to be due to the toxin, caused him to frame his conclusions in a tone of the utmost conservatism. The second and third publications of Loeffler, ${ }^{3}$ however, and the inquiry into the nature of the toxins produced by the bacillus, published in 1888 by Roux and Yersin, ${ }^{4}$ eliminated all remaining doubt as to the etiological relationship existing between this organism and the discase.

Innumerable observations, both clinical and bacteriological, by other workers, have, since that time, confirmed the early investigations,

${ }_{1}^{1}$ Klebs, Verh. d. 2. Kongr. f. inn. Medizin, Wiesbaden, 1883.

2 Loeffler, Mittheil. a. d. kais. Gesundheitsamt, 1884.

${ }^{3}$ Loeffler, Cent. f. Bakt., 1887 and 1890.

4 Roux and Yersin, Ann. de l'inst. Pasteur, 1888 and 1889. 
and it is to-day a scientific necessity to find the bacillus of Klebs and Loeffler in the lesion before a diagnosis of "diphtheria" can properly be made.

Morphology and Staining.-While Bacillus diphtheriæ presents certain characteristic appearances which facilitate its recognition, it is, at the same time, subject to a number of morphological variations with

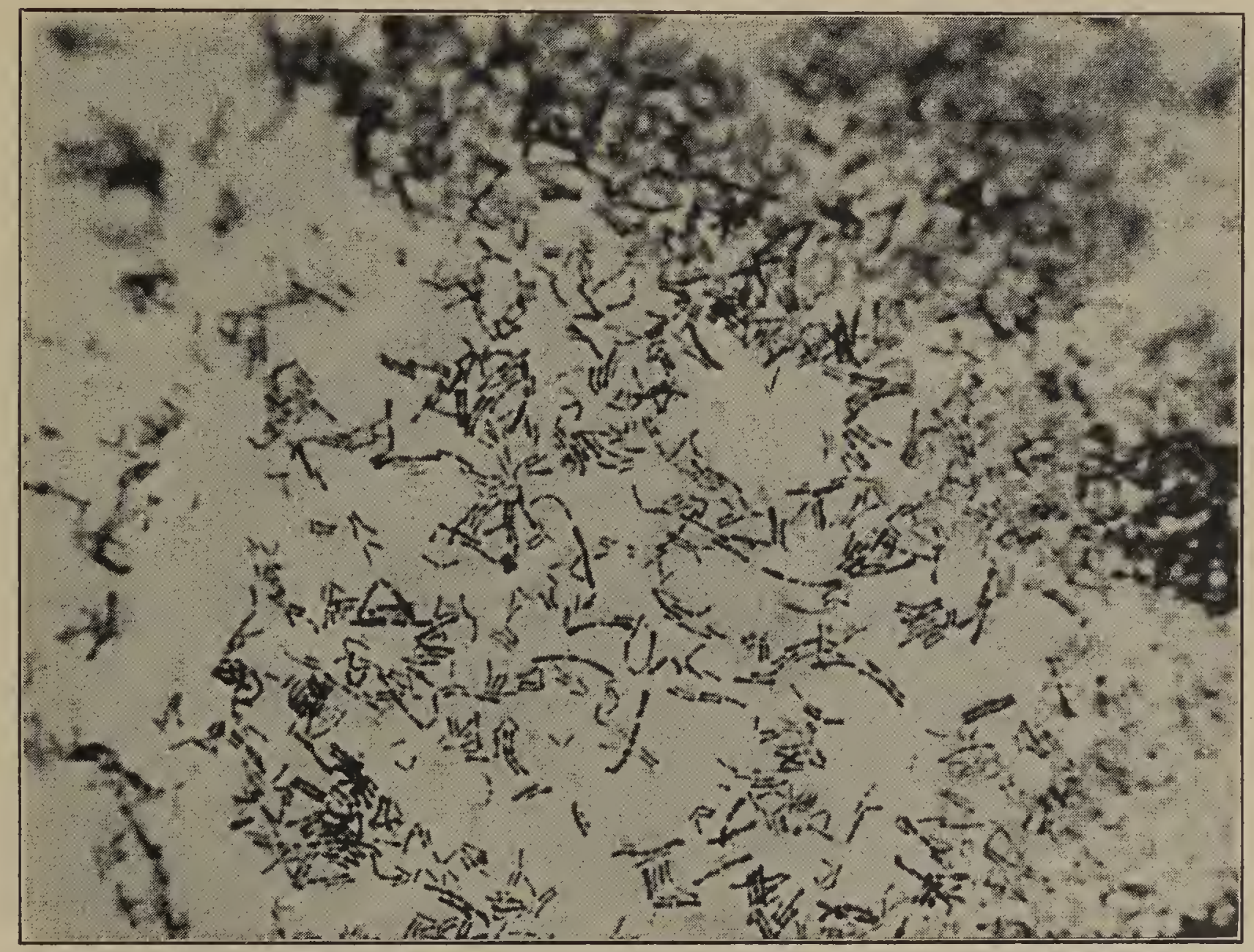

Fig. 105.-Bacillus Diphtheriæ.

all of which it is important to be familiar. These variations are, to a limited extent, dependent upon the age of the culture and upon the constitution of the medium on which it has been grown. These factors, however, do not control the appearance of the organism with any degree of regularity, and any or all of its various forms may occur in one and the same culture. It is likely that these different appearances represent stages in the growth and degeneration of the individual bacilli, but there does not seem to be any just reason for believing that, as several observers have stated, there is definite correlation between its microscopic form and its biological characteristics, such as virulence, toxicity, etc. 
The bacilli are slender, straight, or slightly curved rods. In length they vary from 1.2 micra to 6.4 micra, in breadth from 0.3 to 1.1. As seen most frequently when taken from the throat they are about 4 to 5 micra in length. They are rarely of uniform thickness throughout their length, showing club-shaped thickening at one or both ends. Occasionally they may be thickest at the center and taper toward the extremities. When thickened at one end only, a slendel wedge-shape results. Such forms are usually straight, of smaller size than their neighbors, and are more often stained with great uniformity. These are spoken of by Beck ${ }^{1}$ as the "ground type," and assumed, for insufficient reasons, to be the young individuals. Branched forms have been described by some investigators. They are rare and probably to be regarded as abnormal or involution forms due to unfavorable environment.

The organisms stain with the aqueous anilin dyes. A characteristic irregularity of staining which is of great aid in diagnosis is best obtained with Loeffler's "alkaline methylene-blue." (For preparation see section on Staining, p. 96.) Stained with this solution for five to ten minutes many of the bacilli appear traversed by unstained transverse bands which give them a striped or beaded appearance. The longer individuals often have a strong resemblance to short chains of streptococci. Others may appear unevenly granular. In cultures which are about eighteen hours old, many of the bacilli may show deeply stained oval bodies situated most frequently at the ends. These are the so-called "polar" or "Babes-Ernst" bodies." Special stains have been devised for the demonstration of these appearances. One of these was originated by Neisser, ${ }^{3}$ who claims for it differential value in distinguishing these organisms from pseudodiphtheria and xerosis bacilli.

His method requires two solutions:

1. Methylene blue (Grübler) $\ldots \ldots \ldots \ldots \ldots \ldots \ldots \ldots \ldots \ldots \ldots \ldots$ gram.

Alcohol, 96 per cent....................... 20 c.c.

Glacial acetic acid....................... 50 "

Water............................... 950 "

2. Bismarck brown $\ldots \ldots \ldots \ldots \ldots \ldots \ldots \ldots \ldots \ldots \ldots \ldots \ldots \ldots \ldots \ldots$ grams.

Water ..................................,

${ }_{1}$ Beck, in Kolle und Wassermann, ii, p. 773.

2 Babes, Zeit. f. Hyg., Bd. v, 1889.

3 Neisser, Zeit. f. Hyg., xxiv, 1897. 
The cover-slip preparation, after having been fixed, is stained with solution No. 1 for one to three seconds. It is then washed in water and immersed for from three to five seconds in solution No. 2. With this stain the bodies of the bacilli appear brown, the polar granules blue.

Another method which has been extensively used is that of Roux. The solutions required for this are:

1. Dahlia violet...................... 1 gram.

Alcohol, 90 per cent . . . . . . .

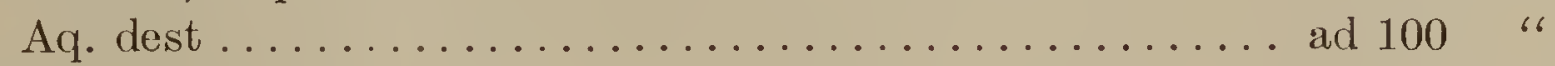

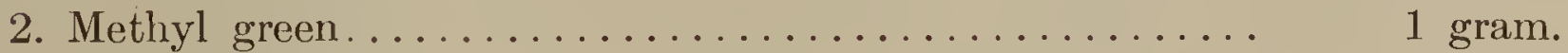

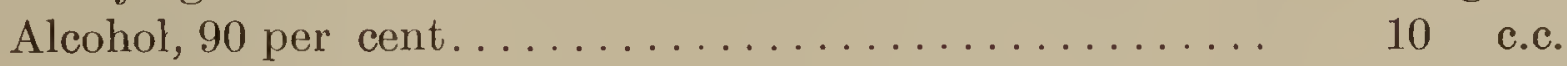

Aq. dest............................

'The two solutions are mixed, one part of 1 being added to three parts of 2. Preparations are stained in this mixture for two minutes. The polar bodies appear a dark violet. Other methods for the staining of polar bodies have been recommended. There is very little advantage in the use of these double stains and most bacteriologists employ for routine work the simple stain with Loeffler's alkaline methylene blue.

The significance of the polar bodies is not well understood. Their discoverer, Ernst, regarded them as bodies analogous to the spores of other organisms. The ease with which they are stained, however, and the low temperatures to which the bacteria succumb make this appear very unlikely. A more probable interpretation seems to be that of Escherich ${ }^{1}$ who regards them as chromatic granules.

Stained by Gram's method, the diphtheria bacilli retain the gentianviolet. Care must be used in carrying out this method and strict timing adhered to, since slight carelessness in this respect may lead to irregulạr results.

In stained smears from the throat or from cultures a characteristic grouping of the bacilli has been observed. They lie usually in small clusters, four or five together, parallel to each other, or at sharp angles. Two organisms may often be seen attached to each other by their corresponding ends while their bodies diverge to form a "V" or "Y" shape.

Biological Characteristics. - The diphtheria bacillus is a non-motile, non-flagellated, non-spore-forming aërobe. Its preference for oxygen is marked, but it will grow in anaërobic environment in the presence of suitable carbohydrates. It does not liquefy gelatin. The bacillus grows at temperatures varying between $19^{\circ} \mathrm{C}$. and $42^{\circ} \mathrm{C}$., the most favorable

${ }^{1}$ Escherich, "Aetiologie, etc., d. Diphth.," Wien, 1894. 
temperature for its development being $37.5^{\circ} \mathrm{C}$. Temperatures above $37.5^{\circ}$, while not entirely stopping its growth, impede the development of its toxin.

Resistance.-The thermal death point of this organism is $58^{\circ} \mathrm{C}$. for ten minutes, according to Welch and Abbott. Boiling kills it in about one minute. Low temperatures, and even freezing, are well borne. Desiccation and exposure to light are not so fatal to this organism as to most of the other pathogenic bacteria. Sternberg ${ }^{1}$ has found it alive in dried bits of the pseudomembrane after fourteen weeks. It is easily killed by chemical disinfectants in the strengths customarily employed. $\mathrm{H}_{2} \mathrm{O}_{2}$ seems especially efficacious in killing the organisms rapidly.

Cultivation.-The diphtheria bacillus grows readily on most of the richer laboratory media. It will grow upon nedia made of meat extract, but develops more luxuriantly on all those which have a meat infusion as their basis. While it will grow upon both acid and alkaline media, it is sensitive to the extremes of both, the most favorable reaction for its development being probably about 0.5 per cent alkalinity expressed in terms of $\frac{\mathrm{N}}{1} \mathrm{NaOH}$. Animal proteids added to the media, in the form of blood serum, ascitic fluid, or even whole blood, increase greatly the rapidity and richness of its growth. Horse serum is supposed by some to be especially favorable. ${ }^{2}$

Loeffer's Medium. - The most widely used medium for the cultivation of this bacillus is the one devised by Loeffler. This consists of:

Beef blood serum ................... 3 parts

One per cent glucose meat-infusion bouillon..... 1 part

'The mixture is coagulated at $70^{\circ} \mathrm{C}$. in slanted tubes and sterilized at low temperatures by the fractional method. Upon this medium the diphtheria bacillus in twelve to twenty-four hours develops minute, grayish-white, glistening colonies. These enlarge rapidly, soon outstripping the usually accompanying streptococci. The medium seems to possess almost selective powers for the bacillus and, for this reason, it is especially valuable for diagnostic purposes.

Meat-Infusion Agar.-Upon slightly alkaline meat-infusion agar the bacillus develops readily, though less so than on Loeffler's serum. Organisms which have been on artificial media for one or more generations may grow with speed and luxuriance upon this medium. When planted directly from the human or animal body upon agar, however,

$$
\text { 'Sternbery, "Manual Bac.," p. 455. 2 Michel, Cent. f. Bakt., } 1897 .
$$


growth may occasionally be slow and extremely delicate. Colonies on agar appear within twenty-four to thirty-six hours as small, rather translucent, grayish specks. The appearance of these colonies is quite characteristic and easily recognized by the practiced observer. Surface colonies are irregularly round or oval, showing a dark, heaped-up, nucleus-like center, fringed about by a loose, coarsely granular disk. The edges of these colonies have a peculiarly irregular, torn appearance which distinguishes them readily from the sharply defined chain-fringed streptococcus colonies. For these reasons agar is the medium most commonly used for purposes of isolation. The deep colonies in this medium are dense and sharply outlined.

The addition of dextrose 1 per cent, nutrose 2 per cent, or glycerin 6 per cent, renders agar more favorable for rapid growth, but unfits it for the preservation of cultures, the organism dying out more rapidly, probably because of acid formation.

Meat-Infusion Broth.-Upon beef or veal broth the diphtheria bacillus grows rapidly, almost invariably forming a pellicle upon the surface, -another expression of its desire for oxygen. The broth remains clear. Broth tubes with such growth, therefore, have a characteristic appearance.

Meat-infusion gelatin is a favorable medium for the Klebs-Loeffier bacillus, but growth takes place slowly because of the low temperature at which this medium must be kept. Gelatin is not fluidified.

Milk is an excellent medium, and for this reason may even occasionally be a vehicle of transmission. There is no coagulation of the milk.

Upon potato, B. diphtheriæ will grow only after neutralization of the acid. It is, at best, however, a poor nutrient mediun.

Upon the various pepton solutions the bacillus of diphtheria produces no indol.

Many special media have been recommended for the cultivation of this organism. The most important of these are the modification of Loeffler's serum devised by Beck, ${ }^{1}$ the horse-blood-fibrin cake used by Escherich, and Wassermann's ascitic-fluid-nutrose-agar, called by him "Nasgar." None of these has sufficient advantages over the simpler media, however, to make its substitution desirable.

Isolation.-Because of the comparative ease with which B. diphtheriæ is isolated from mixed cultures, it is not necessary to give in de-

${ }^{1}$ M. Beck, Kolle und Wassermann; Brit. Med. Jour. 
tail the different methods devised for this purpose. The one described below is the one most frequently employed, and is both simple and reliable.

Cultures are taken from throats upon Loeffler's blood serum. These are permitted to grow at $37.5^{\circ} \mathrm{C}$. for from eighteen to twentyfour hours. At the end of this time about 5 c.c. of bouillon are poured into the tubes and the growth is gently emulsified in the broth with a platinum loop. Two or three loopfuls of this emulsion are then streaked over the surface of glucose agar, serum agar, or nutrose

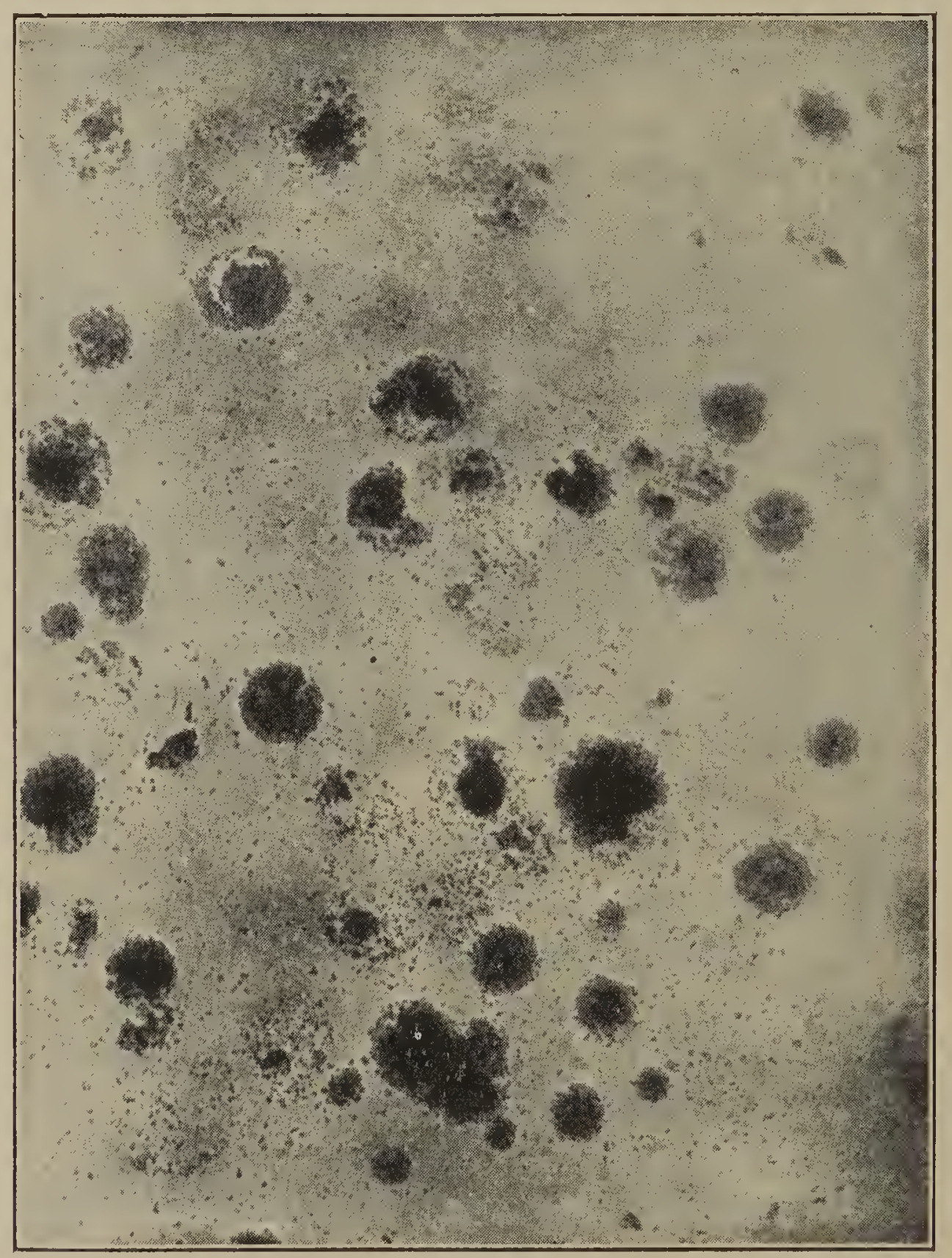

Fig. 106.-Colonies of Bacillus diphtheriæ on Glycerin Agar.

agar. After twenty-four hours' incubation these plates shcw characteristic colonies which can be easily fished and again transferred to Loeffler tubes or any other suitable medium. The same method 
is well adapted for the isolation of pseudodiphtheria and xerosis bacilli.

Pathogenicity.-Unlike most other microorganisms, Bacillus diphtheriæ causes a more or less specific local reaction in mucous membranes, which results in the formation of the so-called "pseudo-membranes." When these are characteristically present, infection with this bacillus should always be suspected. The consequent disease depends, in part, upon the mechanical disturbance caused by these false membranes and, in part, upon the systemic poisoning with the toxin which the bacilli produce. Although the diphtheria bacillus has been found after death in the spleen and liver, we have no data which would justify the assumption that a true diphtheria-septicemia may occur during life. It is probable that in those cases which Baginsky ${ }^{1}$ has called the septicemic form of diphtheria, Bacillus diphtheriæ has merely opened a path by which accompanying streptococci have gained access to the lymphatics and the blood stream. The most frequent sites of diphtheritic inflammation are the mucous membranes of the throat, larynx, and nose. They have also been found in the ear, upon the mucous membrane of the stomach and the vulva, and upon the conjunctiva and the skin. According to Loeffler, Strelitz, ${ }^{2}$ and others, the bacillus may, by extension from the larynx, give rise to a true diphtheritic bronchopneumonia.

For most of the usual laboratory animals the diphtheria bacillus is very pathogenic. Dogs, cats, fowl, rabbits, and guinea-pigs are susceptible. Rats and mice are resistant to all but extremely large doses. False membranes, analogous in every way to those found in human beings, have been produced in many animals of susceptible species, but only after inoculation with the bacillus had been preceded by mechanical injury of the mucosa. The lesions produced in animals by subcutaneous inoculation present many characteristic features which facilitate the bacteriological recognition of the diphtheria bacillus. Small quantities ( 0.5 to 1 c.c.) of a virulent broth culture, given subcutaneously to a guinea-pig, may produce the gravest symptoms and within six to eight hours the animal may show signs of great discomfort. Death occurs usually within thirty-six to seventy-two hours. Upon autopsy the point of inoculation is soggy with serosanguineous exudate; neighboring lymph-nodes are edematous. Lungs, liver, spleen, and kidneys are congested. There may be pleuritic and peritoneal exudates. Charac-

1 Baginsky, "Lehrbuch d. Kinderkrankheiten."

${ }_{2}$ Strelitz, Arch. f. Kinderheilk., 1891. 
teristic, and almost pathognomonic, is a severe congestion of both suprarenal bodies. The gastric ulcerations recently described by Rosenau and Anderson ${ }^{1}$ may occur, but are by no means regularly found (two out of fifty in our series ${ }^{2}$ ).

Diphtheria Toxin. ${ }^{3}$-Animals and man infected with B. diphtheriæ show evidences of severe systemic disturbances and even organic degenerations, while the microorganism itself can be found in the local lesion only. This fact led even the earliest observers to suspect that, in part at least, the harmful results of such an infection were attributable to a soluble and diffusible poison elaborated by the bacillus. The actual existence of such a poison or toxin was definitely proved by Roux and Yersin ${ }^{4}$ in 1889. They demonstrated that broth cultures in which B. diphtheriæ had been grown for varying periods would remain toxic for guinea-pigs after the organisms themselves had been removed from the culture fluid by filtration through a Chamberland filter.

Methods of Production of Diphtheria Toxin.-While toxin can be produced with almost all of the virulent diphtheria bacilli, there is great variation in the speed and degree of production, dependent upon the strain of organisms employed and upon the ingredients and reaction of the medium upon which they are grown. Most laboratories possess one or several strains of bacilli which are empirically known to be especially potent in this respect. One of the most extensively used, not only in this country, but in Europe as well, is the strain known as "Culture Americana," or "Park-Williams Bacillus No. 8," an organism isolated by Dr. Anna Williams of the New York Department of Health in 1894. Throughout more than ten years of cultivation this bacillus has retained its great power of toxin production.

Because of the severity of cases of diphtheria in which the diphtheria bacilli were associated with streptococci, many observers were led to believe that the presence of streptococci tended to increase the toxin-producing powel of B. diphtheriæ. Experiments by Hilbert, Theobald Smith, ${ }^{6}$ and others seem to have given support to this view.

The medium most frequently employed for the production of toxin is a beef-infusion broth. There are minor differences of opinion as to the

1 Rosenau and Anderson, Journ. Inf. Dis., iv, 1907.

2 Zinsser, Journ. Med. Res., xvii, 1907.

${ }^{3}$ Loeffler, Cent. f. Bakt., 1887.

Roux and Yersin, loc. cit.

${ }^{5}$ Hilbert, Zeit. f. Hyg., xxix, 1898.

${ }^{6}$ Smith, Medical Rec., May, 1896. 
most favorable constitution of this medium for the production of toxin. All agree, however, in recognizing the importance of pepton, without which, according to Madsen, ${ }^{1}$ no satisfactory toxin has yet been produced. This is added in proportions of from one to two per cent. The presence of sugars in the medium is not desirable in that it leads to acid production; L. Martin ${ }^{2}$ lemoves the sugars from the meat by fermentation with yeast. Smith ${ }^{3}$ accomplishes the same purpose with B. coli. According to Park and Williams, ${ }^{4}$ however, this is superfluous, the quantity of sugar present in ordinary butcher's meat not being sufficient to exert unfavorable influence.

Experience has shown that a primary alkaline reaction offers the most favorable conditions for toxin production. In all cultures of $\mathrm{B}$. diphtheriæ in non-sugar free broth, there is, at first, a production of acid and, while this continues, there is, as Spronk ${ }^{5}$ has shown, little or no evidence of toxin elaboration. It is only after this initial acidity has given way to alkalinity that cultures become decidedly toxic. Park and Williams, ${ }^{6}$ in an inquiry into the question of reaction, came to the conclusion that the best results are obtained with a broth to which, after neutralization to litmus, $\frac{\mathrm{N}}{1} \mathrm{NaOH}$ is added in an amount of 7 c.c. to the liter. In such a medium the largest yield of toxin is obtained after about five to eight days' growth at a temperature of $37.5^{\circ} \mathrm{C}$. If left at this temperature for a longer period a diminution in the strength of the toxin takes place.

Free access of oxygen to the culture medium during the growth of the organisms has been found to be of great importance. Roux obtained this by passing a stream of oxygen through the bouillon. The supply is quite sufficient for practical purposes, however, if the medium is distributed in thin layers in large-necked Erlenmeyer flasks.

Chemical Nature and Physical Properties of Diphtheria Toxrn.-The chemical composition of diphtheria toxin is not known. Brieger and Frankel, ${ }^{7}$ by repeated precipitation with alcohol, succeeded in extracting from toxic bouillon a white, water-soluble powder which possessed most of the poisonous proporties of the broth itself. This, in

1 Madsen, Kraus und Levaditi, "Handbuch d. Technic," etc., 1907.

${ }_{2}^{2}$ L. Martin, Ann. de l'inst. Pasteur, 1897.

${ }_{3}$ Th. Smith, Jour. Exp. Med., iv, 1899.

${ }^{4}$ Park and Williams, Journ. Exp. Med., 1897.

${ }^{5}$ Spronk, Ann. de l'inst. Pasteur, 1895.

- Park and Williams, Jour. Exp. Med., 1897.

7 Brieger und Frankel, Berl. klin. Woch., xi-xii, 1889. 
solution, gave many of the usual proteid reactions, but differed from proteids in failing to coagulate when boiled and in not giving precipitates when treated with magnesium sulphate, sodium sulphate, or nitric acid. It was believed by them to be closely related to the albumoses, bodies representing intermediate phases in the peptonization of albumins. Similar results have been obtained by Wassermann and Proskauer, ${ }^{1}$ Brieger and Boer, ${ }^{2}$ and others. Uschinsky, ${ }^{3}$ on the other hand, has disputed the proteid nature of toxins in general and has succeeded in producing. diphtheria toxin by growing the organism upon a medium entirely free from albuminous bodies. Uschinsky believes that the proteid reactions observed by other workers may be due to ingredients of the precipitates other than the toxin. It is not impossible, however, that the organisms may have produced proteid substances by synthesis from the simpler substances in Uschinsky's medium. The production of toxin from such a medium, therefore, is not a conclusive argument against the proteid nature of toxins. Accurate chemical isolation and analysis of diphtheria toxin have not yet been accomplished.

Diphtheria toxin is destroyed, ${ }^{4}$ when in the fluid form, by temperatures of $58^{\circ}$ to $60^{\circ} \mathrm{C}$. In the dry state, it may resist a temperature of $70^{\circ} \mathrm{C}$. and over, without noticeable change. Light and the free access of air produce rapid deterioration. Sealed, protected from light, and kept at almost freezing point, the toxin remains stable for very long periods. Electrical currents passed through toxic broth have little or no effect upon it.

Bacteria Similar to Bacillus Diphtheriæ.-Bacillus Hofrmanni (Pseudodiphtheria bacillus).--Hoffmann-Wellenhoff, ${ }^{5}$ in 1888, and, at almost the same time, Loeffler, ${ }^{6}$ described bacilli which they had cultivated from the throats of normal persons and in several instances from those of diphtheritic persons, which were in many respects similar to true B. diphtheriæ, but differed from this chiefly in being non-pathogenic for guinea-pigs. These organisms were at first regarded by some observers as merely attenuated diphtheria bacilli More recent investigations, however, prove them to be unquestionably a separate species, easily differentiable by proper methods. They differ from B. diphtheriæ in so

1 Wassermann und Proskauer, Deut. med. Woch., 1891, p. 585.

${ }^{2}$ Brieger und Boer, Deut. med. Woch., 1896, p. 783.

${ }^{3}$ Uschinsky, Cent. f. Bakt., xxi, 1897.

${ }^{4}$ Roux et $Y$ ersin, loc. cit.

${ }^{5}$ Hoffmann-Wellenhoff, Wien. med. Woch., iii, 1888.

${ }^{6}$ Loeffler, Cent. f. Bakt., ii, 1887. 
many important features, moreover, that the term "pseudodiphtheria bacillus" is hardly an appropriate one for them.

Morphology.-Bacillus Hoffmanni is shorter and thicker than Bacillus diphtheriæ. It is usually straight and slightly clubbed at one end, rarely at both: Stained with Loefier's blue it occasionally shows unstained transverse bands; unlike B. dliphtheriæ, however, these

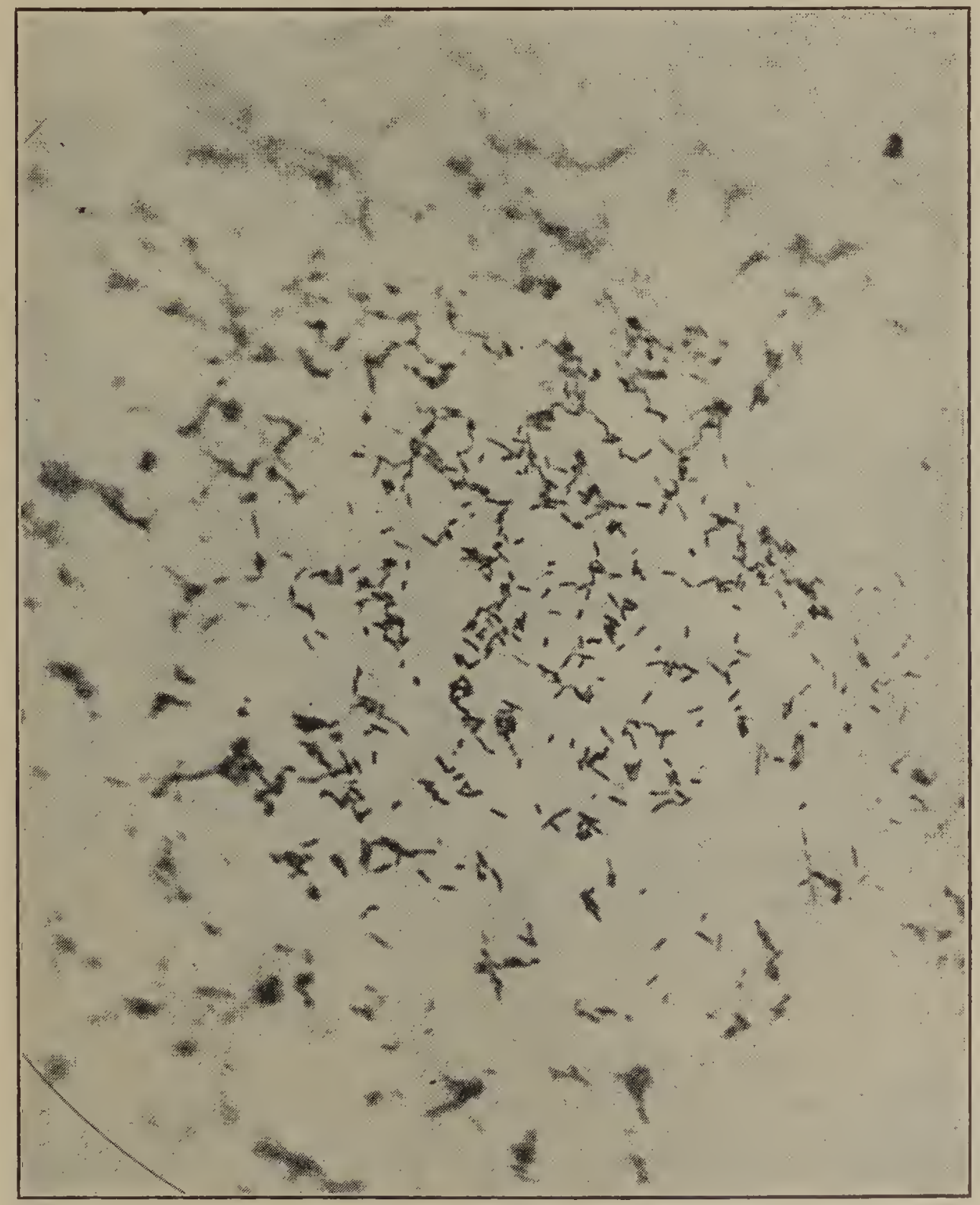

Fig. 107.-Bacillus Hoffmann.

bands hardly ever exceed one or two in number at most. In many cultures the single transverse band gives the bacillus a diplococcoid appearance.

Staining.-Stained by Neisser's or Roux's method, no polar bodies can be demonstrated. The bacillus forms no spores, is non-motile, and possesses no flagella. 
Cultivation.-On the usual culture media B. Hoffmanni grows more luxuriantly than B. diphtheriæ, developing even in first isolations from the human body upon the simple meat-extract media. On agar plates its colonies are larger, less transparent, and whiter than are those of true diphtheria bacilli. In fluid media there is even clouding

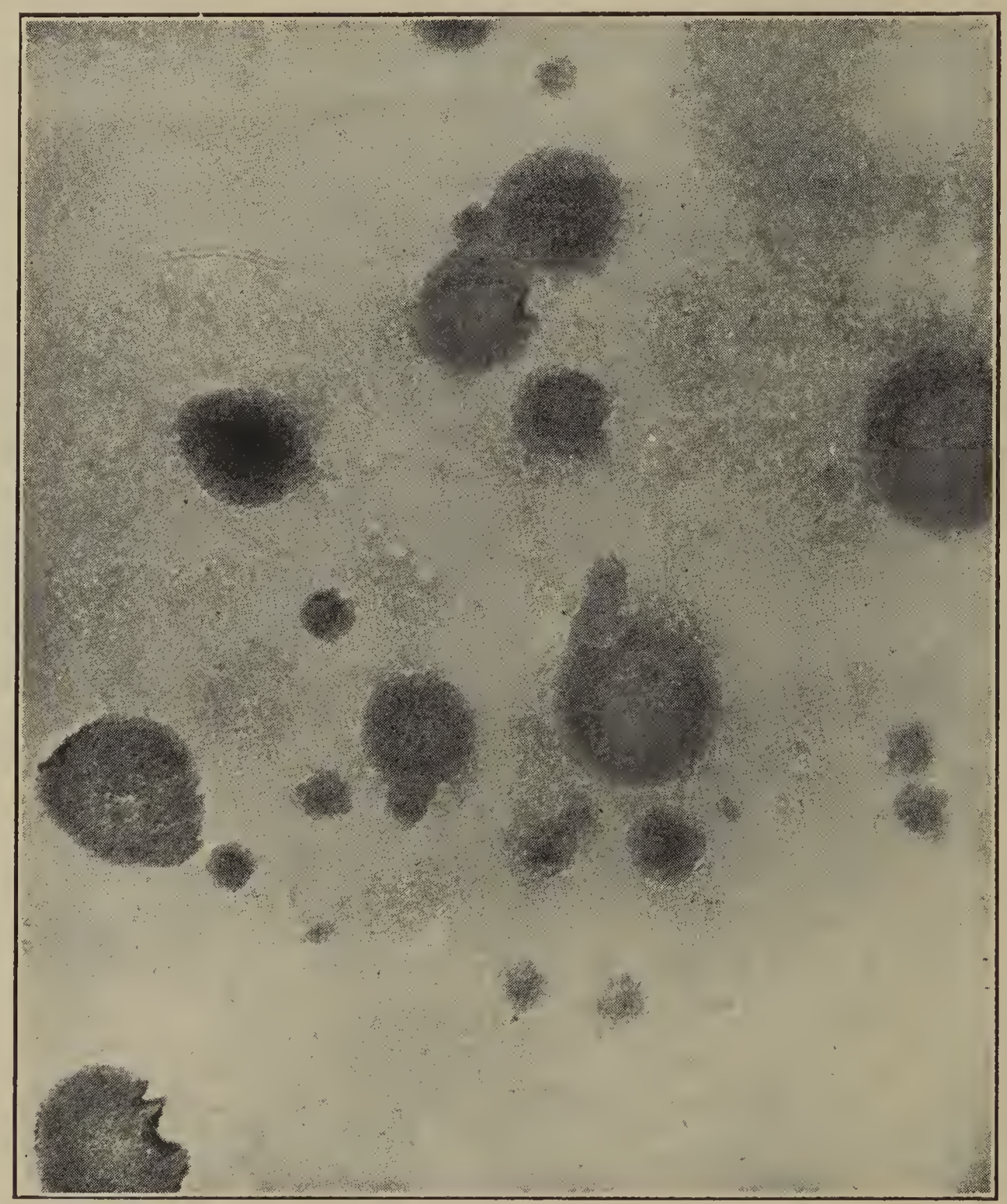

Fig. 108.-Colonies of Bacillus Hoffmanni on Agar.

and less tendency to the formation of a pellicle than with B. diphtherix. A positive means of distinction between the two is given by the inability of B. Hoffmanni to form acid upon various sugar media. The differentiation on a basis of acid formation was first attempted by Cobbett ${ }^{1}$ 
and has been recently worked out systematically by Knapp, ${ }^{1}$ and confirmed by various observers. ${ }^{2}$ The results of this work, carried out with the serum-water media of Hiss to which various sugars were added, show that B. Hoffmanni forms acid upon none of the sugars used, while B. diphtheriæ acidifies and coagulates media containing monosaccharids

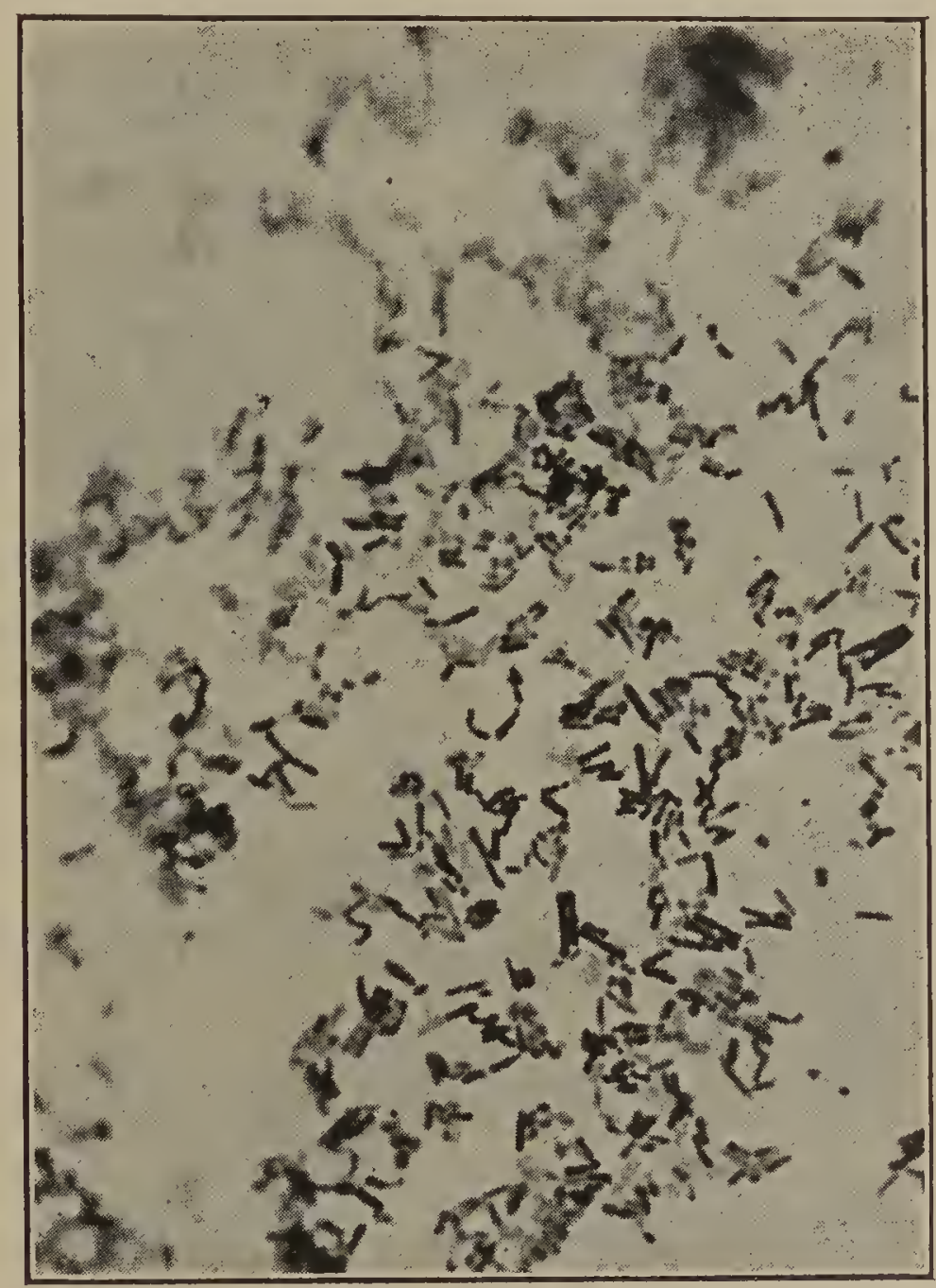

Fig. 109.-BACILLUS XEROSIS.

and several of the more complex sugars, as given in the diagram in the section following, dealing with $\mathrm{B}$. xerosis.

Differentiation can finally be made on the basis of animal pathogenicity, B. Hoffmanni being entirely innocuous to the ordinary laboratory animals. B. Hoffmanni forms no toxins, and animals immunized with it do not possess increased resistance to B. diphtheriæ.

Bacillus Xerosis. - In 1884, Kutschert and Neisser ${ }^{3}$ described a bacillus which they had isolated from the eyes of patients suffering from

Knapp, Jour. Med. Res., vii, 1904.

${ }^{2}$ Graham Smith, Jour. of Hyg., vi, 1906; Zinsser, Jour. Med. Res., xvii, 1907.

${ }^{3}$ Kutschert und Neisser, Deut, med. Woch., xxiv, 1884. 
a form of chronic conjunctivitis known as xerosis. This bacillus, which, morphologically, is almost identical with B. diphtheriæ, they believed to be the etiological factor of the disease. The frequency with which it has been isolated from normal eyes, however, precludes this etiological relationship, and it may safely be regarded as a harmless parasite which may indeed be more abundant in the slightly inflamed than in the normal conjunctiva.

Morphology.-B. xerosis resembles B. diphtheriæ closely. It is occasionally shorter than this, but on the whole no absolute morphological differentiation between the two is possible. It forms no spores and is non-motile. Polar bodies may occasionally be seen.

Cultivation.-On Loeffler's blood serum, on agar, glycerin agar, and in broth, its growth is very similar to that of $\mathrm{B}$. diphtheriæ, but more delicate throughout. It can not easily be cultivated upon the simple meat-extract media, nor will it grow on gelatin at room temperature. Its colonies on glycerin or glucose agar are microscopically identical with those of $\mathrm{B}$. diphtheriæ.

Differentiation.-It differs from B. diphtheriæ distinctly in its acidifying action on sugar media. These relations were first worked out by Knapp for various sugars and the alcohol mannit, and have been extensively confirmed by others. The differentiations resulting may be tabulated as follows:

\begin{tabular}{|c|c|c|c|}
\hline & $\begin{array}{c}\text { B. } \\
\text { Diphtherix. }\end{array}$ & $\begin{array}{c}\text { B. } \\
\text { Xerosis. }\end{array}$ & $\begin{array}{l}\text { B. } \\
\text { Hoff manni. }\end{array}$ \\
\hline Hiss serum-water media plus $1 \%$ & & & \\
\hline Dextrose ....................... & + & + & - \\
\hline Levulose......................... & + & + & - \\
\hline Galactose................. & + & + & - \\
\hline 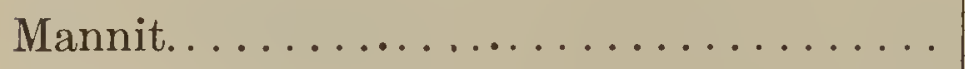 & - & - & - \\
\hline 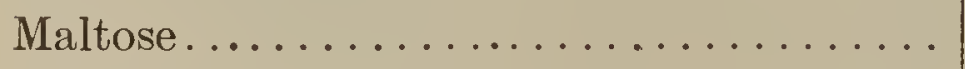 & + & + & - \\
\hline Lactose . . . . . . . . . . . . . . . . . & - & - & - \\
\hline Saccharose.................... & - & + & $\rightarrow$ \\
\hline Dextrin............ & + & - & - \\
\hline
\end{tabular}

A reference to the table shows that differentiation may be made by the use of two sugars-saccharose and dextrin. B. diphtherix forms acid from dextrin, not from saccharose; B. xerosis from saccharose, not from dextrin; B. Hoffmanni does not form acid from either.

B. xerosis is non-pathogenic to animals and forms no toxin. 
Other Bacilli Morphologically Resembling the Diphtheria Bacillus.Many bacilli have been described which have a slight morphological resemblance to Bacillus diphtheriæ but which have little or no pathological significance. Such organisms are met with in milk, air, and water, and as secondary invaders together with other bacteria in old discharging wounds. These bacilli are usually larger than the diphtheria bacillus and, although transversely striped, rarely show polar bodies. On the various media they grow heavily on even the simpler nutrient substances, with heavy, usually white or yellowish-white colonies. Culturally they show all the qualities of saprophytes. Inoculated into animals they produce at most a mild local reaction. In the literature these organisms have often been loosely spoken of as "Pseudodip.htheria" bacilli, a term which is inappropriate since they have nothing in common with the Klebs-Loeffler bacillus except a certain morphological resemblance. Differentiation is never difficult. 


\section{CHAPTER XXXVI}

\section{BACILLUS MALLEI}

\section{(Glanders Bacillus)}

GLANDERs is an infectious disease prevalent chiefly among horses, but transmitted occasionally to other domestic animals and to man. The microorganism causing the disease, though seen and described by several earlier authors, was first obtained in pure culture and accurately studied by Loeffler and Schütz ${ }^{1}$ in 1882 .

Morphology and Staining.-The glanders bacillus or B. mallei is a rather small rod with rounded ends. ${ }^{2}$ Its length varies from 3 to 4 micra, its breadth from 0.5 to 0.75 micron. Variation in size between separate individuals in the same culture is characteristic. The rods are usually straight, but may show a slight curvature. The bacillus is non-motile. There are no flagella and no spores are formed. The grouping of the bacilli in smears shows nothing very characteristic. Usually they appear as single bacilli lying irregularly parallel, often in chains of two or more. In old cultures, involution forms appear which are short, vacuolated, and almost coccoid.

While the glanders bacillus stains rather easily with the usual anilin dyes, it is so easily decolorized that especial care in preparing specimens must be observed. Stained in the usual manner with methylene-blue, it shows marked irregularity in its staining qualities; granular, deeply staining areas alternating with very faintly stained or entirely unstained portions. This diagnostically helpful characteristic has been variously interpreted as a mark of degeneration or a preparatory stage for sporulation. It is probably neither of the two, but an inherent irregularity in the normal protoplasmic composition of the bacillus, not unlike that c f $\mathrm{B}$. diphtheriæ. The bacillus is decolorized by Gram's method of staining.

Cultivation.-The glanders bacillus is easily grown on all of the

${ }^{1}$ Loeffler und Schütz, Deut. med. Woch., 1882.

${ }^{2}$ Loeffler, Arb. a. d. kais. Gesundheitsamt, 1886. 
usual meat-infusion media. It is practically indifferent to moderate variations in reaction, glowing equally well upon neutral, slightly acid, or slightly alkaline culture media. Glycerin or small quantities of glucose added to media seem to render them more favorable for the cultivation of this bacillus.

Upon agar the colonies show little that is characteristic. They appear after twenty-four hours at $37.5^{\circ} \mathrm{C}$. as yellowish-white spots, at first transparent, later more opaque. They are round, with an even

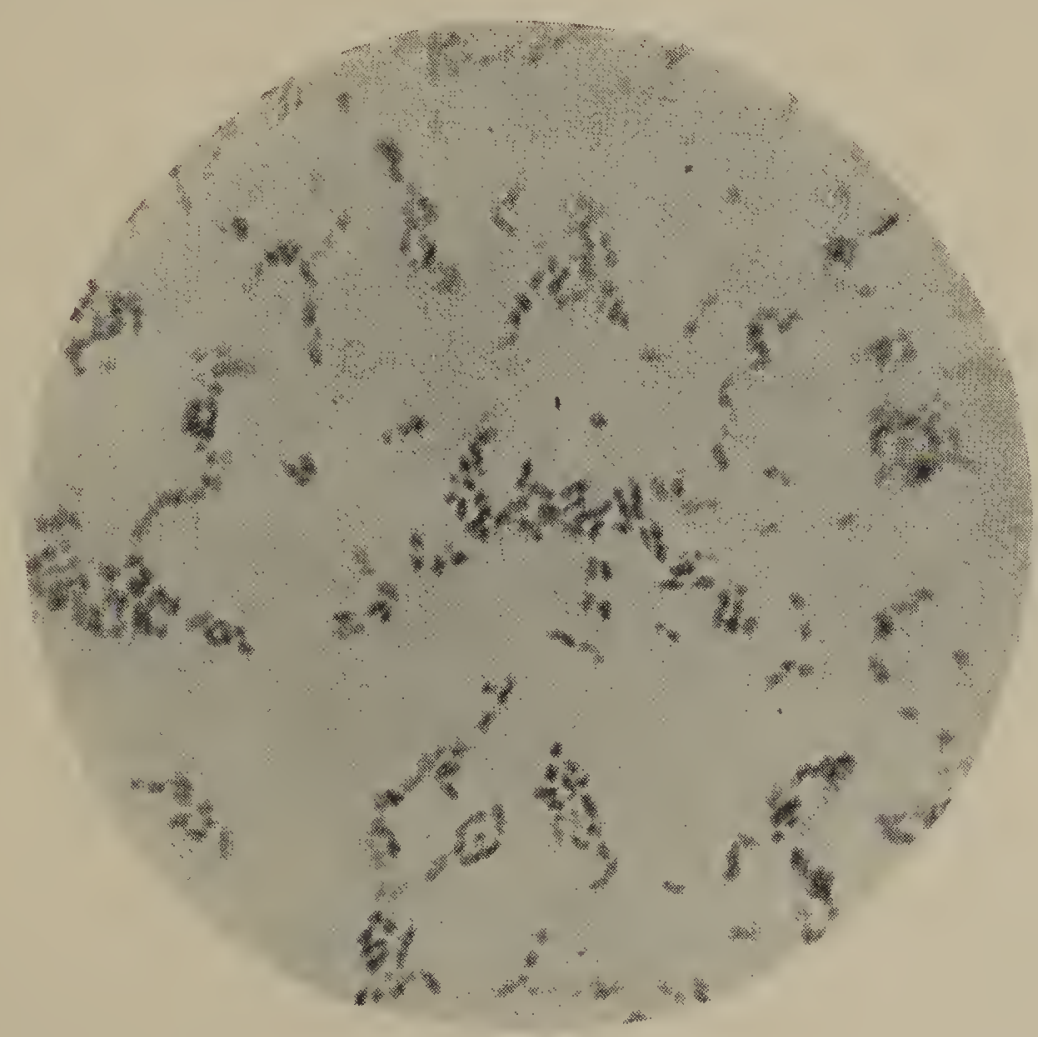

Fig. 110.-Glanders Baciluus. From potato culture. (After Zettnow.)

border, and microscopically appear finely granular. The older the cultures are, the more yellow do they appear.

On gelatin at room temperature, growth is slow, grayish-white, and no liquefaction of the gelatin occurs. Growth upon this medium is never abundant.

In broth, there is, at first, diffuse clouding, later a heavy, tough, slimy sediment is formed. At the same time the surface is covered with a similarly slimy pellicle. The broth gradually assumes a dark brown color.

In milk, coagulation takes place slowly. In litmus milk, acidification is indicated.

The growth upon potato presents certain features which are diagnostically valuable. On potatoes which are not too acid growth is abundant and within forty-eight hours covers the surface as a yellowish, trans- 
parent, slimy layer. This gradually grows darker until it has assumed a deep reddish-brown hue. In using this feature of the growth diagnostically, it must not be forgotten that a very similar appearance upon potato occurs in the case of B. pyocyaneus.

Biological Considerations.-Bacillus mallei is aërobic. ${ }^{1}$ Growth under anaërobic conditions may take place, but it is slow and impoverished. The most favorable temperature for its cultivation is $37.5^{\circ} \mathrm{C}$. It fails to develop at temperatures below $22^{\circ} \mathrm{C}$. or above $43^{\circ} \mathrm{C}$. On artificial media, if kept cool and in the dark, and in sealed tubes, the glanders bacillus will retain its viability for months and years. On gelatin and in bouillon, it lives for a longer time than on the other media. Exposed to strong sunlight it is killed within twenty-four hours. Heating to $60^{\circ} \mathrm{C}$. kills it in two hours, to $75^{\circ} \mathrm{C}$. within one hour. Thorough drying kills the glanders bacillus in a short time. In water, under the protected conditions that are apt to prevail in watering-troughs, the bacillus may remain alive for over seventy days. The resistance to chemical disinfectants is not very high. ${ }^{2}$ Carbolic acid, one per cent, kills it in thirty minutes, bichlorid of mercury, 0.1 per cent, in fifteen minutes.

Pathogenicity.-Spontaneous infection with the glanders bacillus occurs most frequently in horses. It occurs also in asses, in cats, and, more rarely, in dogs. In man the disease is not infrequent and is usually contracted by those in habitual contact with horses. Experimental inoculation is successful in guinea-pigs and rabbits. Cattle, hogs, rats, and birds are immune to experimental and spontaneous infections alike.

Spontaneous infection takes place by entrance through the broken skin, through the mucosa of the mouth or nasal passages. Infection in horses not infrequently takes place through the digestive tract. ${ }^{3}$ In all cases, so far as we know, previous injury to either the skin or to the mucosa is necessary for penetration of the bacilli and the development of the disease.

Glanders in horses may occur in an acute or chronic form, depending upon the relative virulence of the infecting culture and the susceptibility of the subject. The more acute form of the disease is usually limited to the nasal mucosa and upper respiratory tract. The more chronic type of the disease is often accompanied by multiple swellings of the

${ }_{1}$ Loeffler, loc. cit.

${ }^{2}$ Finger, Ziegler's Beitr., vi, 1889.

${ }^{3}$ Nocard, Bull. de la soc. centr. de méd. vét., 1894. 
skin and general lymphatic enlargement. This form is often spoken of as "farcy."

Acute glanders in the horse begins violently with fever and prostration. After two or three days there is a nasal discharge, at first serous, later seropurulent. At the same time there is ulceration of the nasal mucosa and acute swelling of the neighboring lymph nodes. These may break down and form deep pus-discharging sinuses and ulcers.

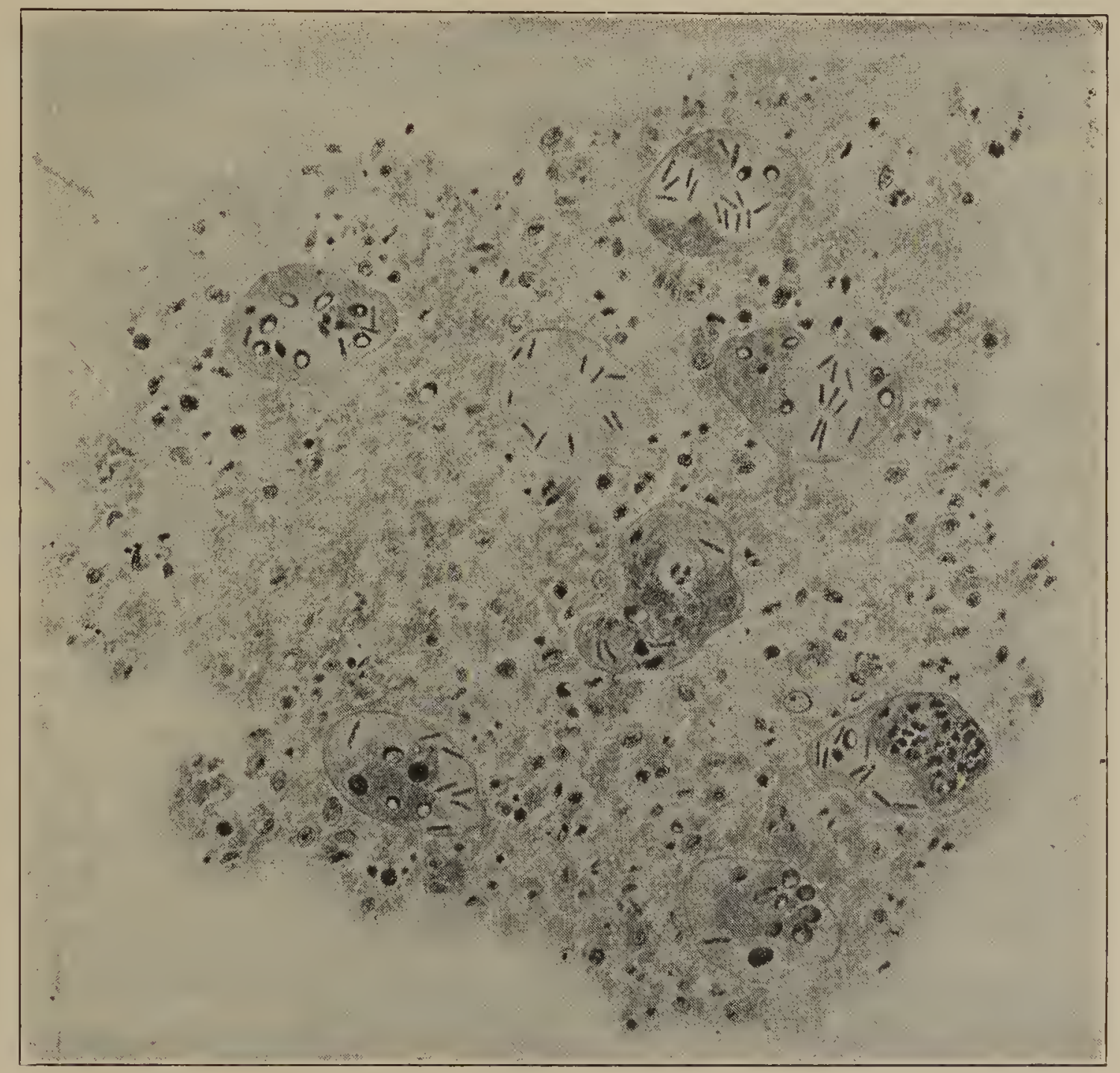

Fig. 111.-Glanders Bacilli in Tissue. (From a drawing furnished by Dr. James Ewing.)

Finally, there is involvement of the lungs and death within four to six weeks.

When the disease takes the chronic form the onset is more gradual. Concomitant with the nasal inflammation there is a formation of subcutaneous swellings all over the body, some of which show a tendency to break down and ulcerate. Together with this the lymphatics all over the body become enlarged. The disease may last for several years, and occasionally may end in complete cure. In horses the chronic form of 
the disease is by far the more frequent. In man the disease is similar to that of the horse except that the point of origin is more frequently in some part of the skin rather than in the nasal mucosa, and the clinical symptoms differ accordingly. The onset is usually violent, with fever and systemic symptoms. At the point of infection a nodule appears, surrounded by lymphangitis and swelling. A general papular eruption may occur. The papules may become pustular, and the clinical features may thus simulate variola. This type of the disease usually ends fatally in eight to ten days. The chronic form of the disease in man is much like that in the horse, but is more frequently fatal.

The histological appearance of the glanders nodules is usually one of diffuse leucocytic infiltration and the formation of young connective tissue which preponderates more and more as the discase becomes chronic. Virchow has classed these lesions with the granulomata. From the center of such nodules B. mallei may often be obtained in pure culture. The nodules may be generally distributed throughout the internal organs. The bacilli themselves are found, apart from the nodules, in the nasal secretions, and occasionally in the circulating blood. ${ }^{1}$

The bacteriological diagnosis of glanders may be made by isolating and identifying the bacilli from any of the above-mentioned sources. When superficial nodules can be opened for the purpose of diagnosis this may prove an easy task. The most diagnostically helpful medium in such cases is potato. In a majority of cases, however, isolation is extremely difficult and resort must be had to animal inoculation. The most suitable animal for this purpose is the male guinea-pig. Intraperitoneal inoculation of such animals with material containing glanders bacilli leads within two or three days to tumefaction and purulent inflammation of the testicles. Such an experiment, spoken of as the "Strauss test," 2 should always be reinforced by cultural examination of the testicular pus, the spleen, and the peritoneal exudate of the animals employed.

Toxin of Bacillus mallei.-The toxin of B. mallei, or mallein, belongs to the class of endotoxins. The toxic products have been invariably obtained by extraction of dead bacilli. ${ }^{3}$ Mallein differs from many other bacterial poisons in being extremely resistant. It withstands

1 Wassilieff, Deut. med. Woch., 1883.

2 Strauss, Arch. de méd. exp., 1889.

${ }^{3}$ Kresling, Arch. d. sci. biol., 1892; Preuser, Berl. thierär'zt. Woch., 1894. 
temperatures of $120^{\circ} \mathrm{C}$. and prolonged storage without noticeable loss of strength. ${ }^{1}$

In its physiological action upon healthy animals, mallein is not a powerful poison. It can be given in considerable doses without causing death. Mallein may be obtained by a variety of methods. Helman and Kalning, the discoverers of this toxin, used filtered aqueous and glycerin extracts of potato cultures. Roux ${ }^{2}$ cultivates virulent glanders bacilli in flasks containing 250 c.c. each of 5 per cent glycerin bouillon. Growth is allowed to continue at $35^{\circ} \mathrm{C}$. for one month. At the end of this time, the cultures are sterilized at $100^{\circ}$ for thirty minutes, and evaporated on a water bath to one-tenth their original volume. They are then filtered through paper. This concentrated poison is diluted ten times with 0.5 per cent carbolic acid before use. Concentration is done merely for purposes of conservation. The diagnostic dose of such mallein for a horse is 0.25 c.c. of the undiluted fluid.

At the Washington Bureau of Animal Industry, mallein is prepared by growing the bacilli for five months at $37.5^{\circ} \mathrm{C}$. in glycerin-bouillon. This is then boiled for one hour and allowed to stand in a cool place for one week. The supernatant fluid is then decanted and filtered through clay filters by means of a vacuum pump. The filtrate is evaporated to one-third its original volume on a water bath, and the evaporated volume resupplied by a 1 per cent carbolic acid solution containing about 10 per cent of glycerin.

Diagnostic Use of Mallein.-The injection of a proper dose of mallein into a horse suffering from glanders is followed within six to eight hours by a sharp rise of temperature, often reaching $104^{\circ}$ to $106^{\circ} \mathrm{F}$. $\left(40^{\circ} \mathrm{C} .+\right)$. The high temperature continues for several hours and then begins gradually to fall. The normal is not usually regained for several days. Locally, at the point of injection, there appears within a few hours a firm, hot, diffuse swelling, which gradually extends until it may cover areas of 20 to 30 centimeters in diameter. The swelling is intensely tender during the first twenty-four hours, and lasts for three to nine days. Together with this there are marked symptoms of general intoxication. In normal animals the rise of temperature following an injection is trifling, and the local reaction is much smaller and more transient. Injections are best made into the breast or the side of the neck.

1 Wladimiroff, in Kraus und Levaditi, "Handbuch," etc., 1908.

${ }_{2}$ Roux et Nocard, Bull. d. 1. soc. centr. vét., 1892. 
The directions given by the United States Government for using mallein for the diagnosis of glanders in horses are as follows:

"Make the test, if possible, with a healthy horse, as well as with one or more affected or supposed to be affected with glanders. Take the temperature of all these animals at least three times a day for one or more days before making the injections.

"The injection is most conveniently made at 6 or 7 o'clock in the morning, and the maximum temperature will then usually be reached by or before 10 P.M. of the same day.

"Use for each horse one cubic centimeter of the mallein solution as sent out, and make the injection beneath the skin of the middle of one side of the neck, where the local swelling can be readily detected.

"Carefully sterilize the syringe after injecting each horse by flaming. the needle over an alcohol lamp or, better, use separate syringes for healthy and suspected animals. If the same syringe is used, inject the healthy animals first, and flame the needle of the syringe after each injection.

"Take the temperature every two hours for at least eighteen hours after the injection. Sterilize the thermometer in a 5 per cent solution of carbolic acid, or a 0.2 per cent solution of corrosive sublimate, after taking the temperature of each animal.

"The temperature, as a rule, will begin to rise from four to eight hours after the injection, and reach its maximum from ten to sixteen hours after injection. On the day succeeding the injection take the temperature at least three times.

"In addition to the febrile reaction, note the size, appearance, and duration of any local swelling at the point of injection. Note the general condition and symptoms of the animal, both before, during, and after the test.

"Keep the solution in the sealed bottle and in a cool place, and do not use it when it is clouded or if it is more than six weeks old · when it leaves the laboratory of the Bureau it is sterile."

If the result of first injection is doubtful, the horse should be isolated and retested in from one to three months, when the slight immunity conferred by the first injection will have disappeared. The second injection into healthy horses usually shows no reaction whatever.

Mallein may cause reactions in the presence of other diseases than glanders, such as bronchitis, periostitis, and other inflammatory lesions and is not so specifically valuable as tuberculin for diagnosis. 
Immunity.-Recovery from a glanders infection does not confer immunity against a second inoculation. ${ }^{1}$ Artificial active immunization has been variously attempted by treatment with attenuated cultures, with dead bacilli, and with mallein, but without convincing results.

The serum of subjects suffering from glanders contains specific agglutinins. ${ }^{2}$ These are of great importance diagnostically if the tests are made with dilutions of, at least, 1 in 500, since normal horse serum may agglutinate $B$. mallei in dilutions lower than this.

1 Finger, Ziegler's Beiträge, vi, 1899. $\quad{ }^{2}$ Galtier, Jour. de méd. vét., 1901. 


\section{CHAPTER XXXVII}

\section{BACILLUS INFLUENZE AND CLOSELY RELATED BACTERIA}

THERE is no other epidemic disease which spreads over such enormous territories, and with such speed, as influenza. Epidemics have been numerous and reports of the disease, unquestionably recognizable, are extant even from the most remote times. The last serious epidemic occurred in the years 1889 to 1890, when the disease, spreading from the East, traveled through Russia and, pandemically, attacked all of Europe, then reached America, and eventually, having traveled eastward as well as westward from its point of origin, became prevalent in China, Japan, Australia, and Africa. Hundreds of thousands were attacked and the mortality of this epidemic was high. Its enormous scope and the rapidity of its spread were facilitated probably by the activity of modern international commerce.

The character of the disease pointed so definitely to a bacterial etiology that numerous attempts to isolate a specific microorganism were, of course, made. Pfeiffer ${ }^{1}$ finally, in 1892, described the bacillus which is at present definitely recognized as the etiological factor of influenza.

Morphology and Staining.-The bacillus of influenza (Pfeiffer bacillus) is an extremely small organism, about 0.5 micron long by 0.2 to 0.3 micron in width. They are somewhat irregular in length, but show rounded ends. They rarely form chains. They are non-motile, and do not form spores.

Influenza bacilli stain less easily than do most other bacteria with the usual anilin dyes, and are best demonstrated with 10 per cent aqueous fuchsin (5 to 10 minutes), or with Loeffler's methylene-blue (5 minutes). They are Gram-negative, giving up the anilin-gentianviolet stain upon decolorization. Occasionally slight polar staining may be noticed. Grouping, especially in thin smears of bronchial secretion, is characteristic, in that the bacilli very rarely form threads

1 Pfeiffer, Deut. med. Woch., ii, 1892; Zeit. f. Hyg., xiii, 1892; Pfeiffer und Beck, Deut: med. Woch., xxi, 1893. 
or chains, usually lying together in thick, irregular clusters without definite parallelism.

Isolation and Cultivation.--Isolation of the influenza bacillus is not easy. Pfeiffer ${ }^{1}$ succeeded in growing the bacillus upor serum-agar plates upon which he had smeared pus from the bronchial secretions of patients. Failure of growth in attempted subcultures made upon agar

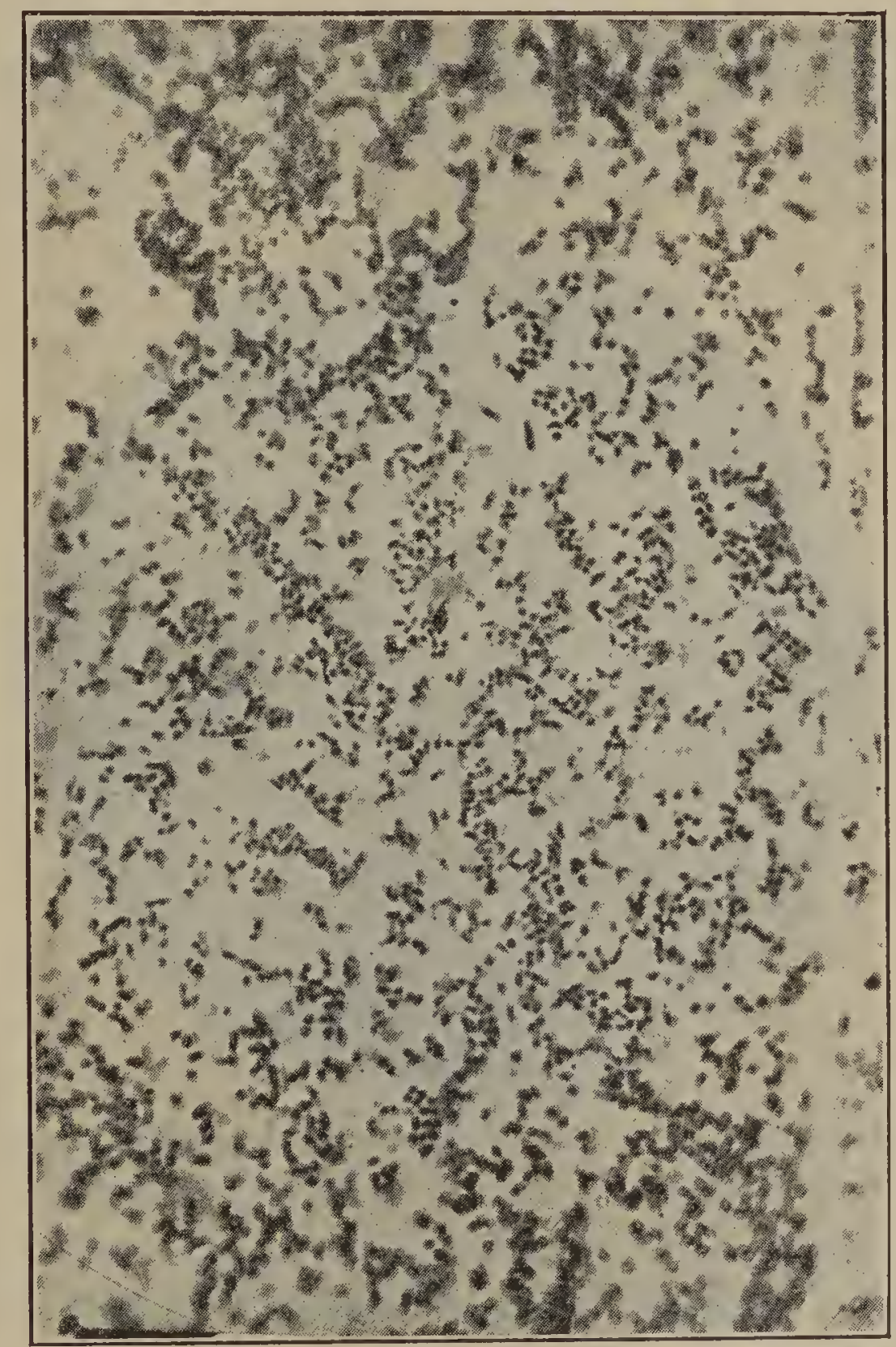

FIG. 112.-BaCillus INFLUENZ Smear from pure culture on blood agar.

and gelatin, however, soon taught him that the success of his first cultivations depended upon the ingredients of the pus carried over from the sputum. Further experimentation then showed that it was the blood, and more particularly the hemoglobin, in the pus which had made growth possible in the first cultures. Pfeiffer made his further cultivations 
upon agar, the surface of which had been smeared with a few drops of blood taken sterile from the finger. Hemoglobin separated from the red blood cells was found to be quite as efficient as whole blood. This method of Pfeiffer is still the one most frequently employed for isolation and cultivation. Whole blood taken from the finger may be either smeared over the surface of slants or plates, or mixed with the melted meat-infusion agar. In isolating from sputum, only that secretion should be used which is coughed up from the bronchi and is uncontaminated by microorganisms from the mouth. It may be washed in sterile water or bouillon before transplantation, to remove the mouth flora adhe-

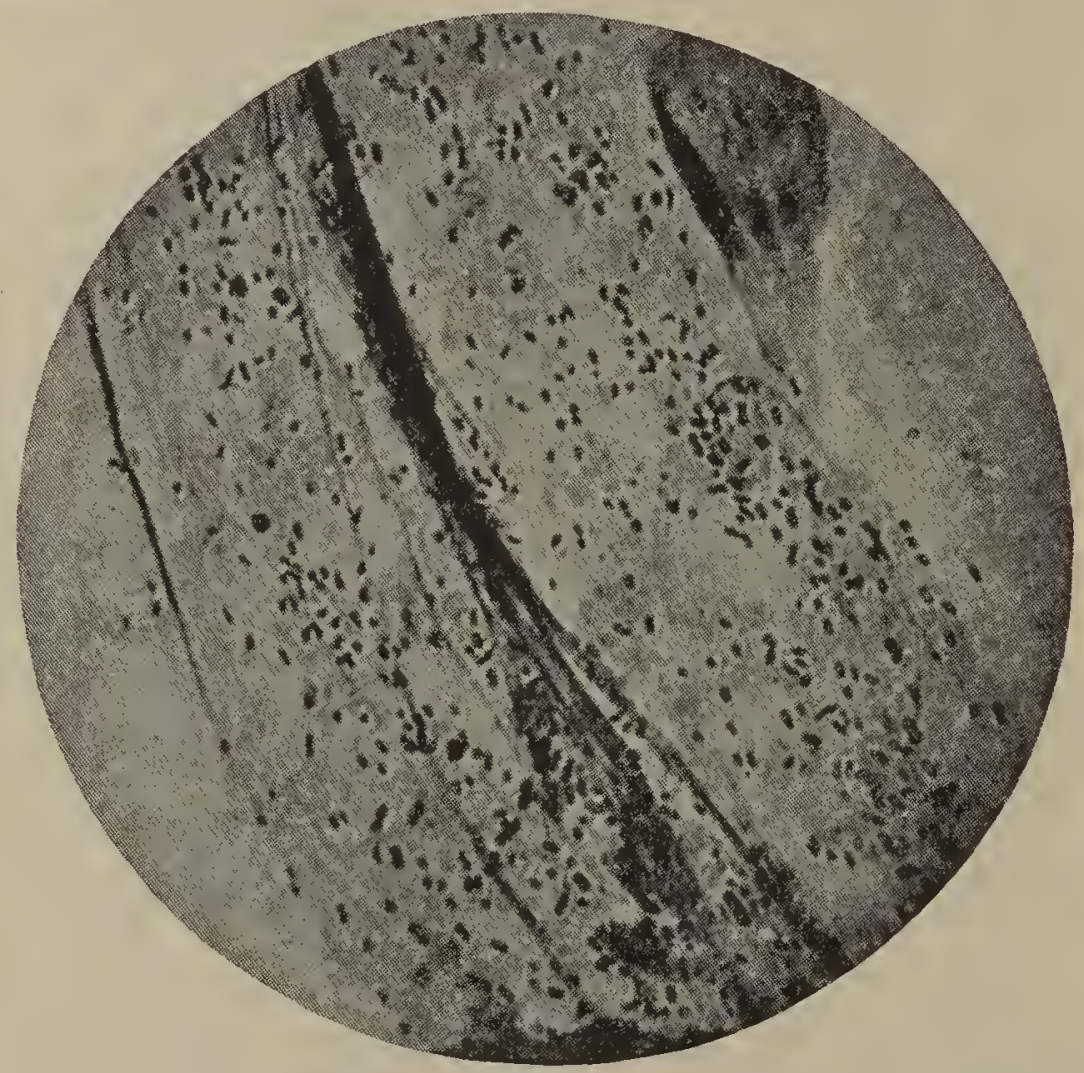

Fig. 113.-Bacillus influenz

rent to the outer surface of the little clumps of pus. The blood of pigeons or that of rabbits may be substituted for human blood. The former seems to be the more favorable of the two and even more so than human blood. Pigeons may be easily bled for this purpose from the large veins under the wing. Huber ${ }^{1}$ has succeded in cultivating influenza bacilli upon media containing a soluble hemoglobin derivative known as hematogen. This substance, however, offers some difficulties to sterilization and is not so favorable as whole blood. The absence of oxyhemoglobin from the hematogen, however, is theoretically important in that it shows that hemoglobin is suitable for the growth 
of this bacillus because of its nutrient qualities and not by virtue of its oxygen-carrying properties. Although the presence of hemoglobin seems to be a necessity for the successful cultivation of the bacillus, the quantity present need not be very large. Ghon and Preyss ${ }^{1}$ showed that an amount too small to be demonstrated spectroscopically sufficed for its growth.

Other substances which, added to neutral or slightly alkaline agar, have been used for the cultivation of influenza bacilli are the yolk of eggs $^{2}$ (not confirmed) and spermatic fluid. ${ }^{3}$ None of these, however, is as useful as the blood media. Symbiosis with staphylococci, ${ }^{4}$ too,

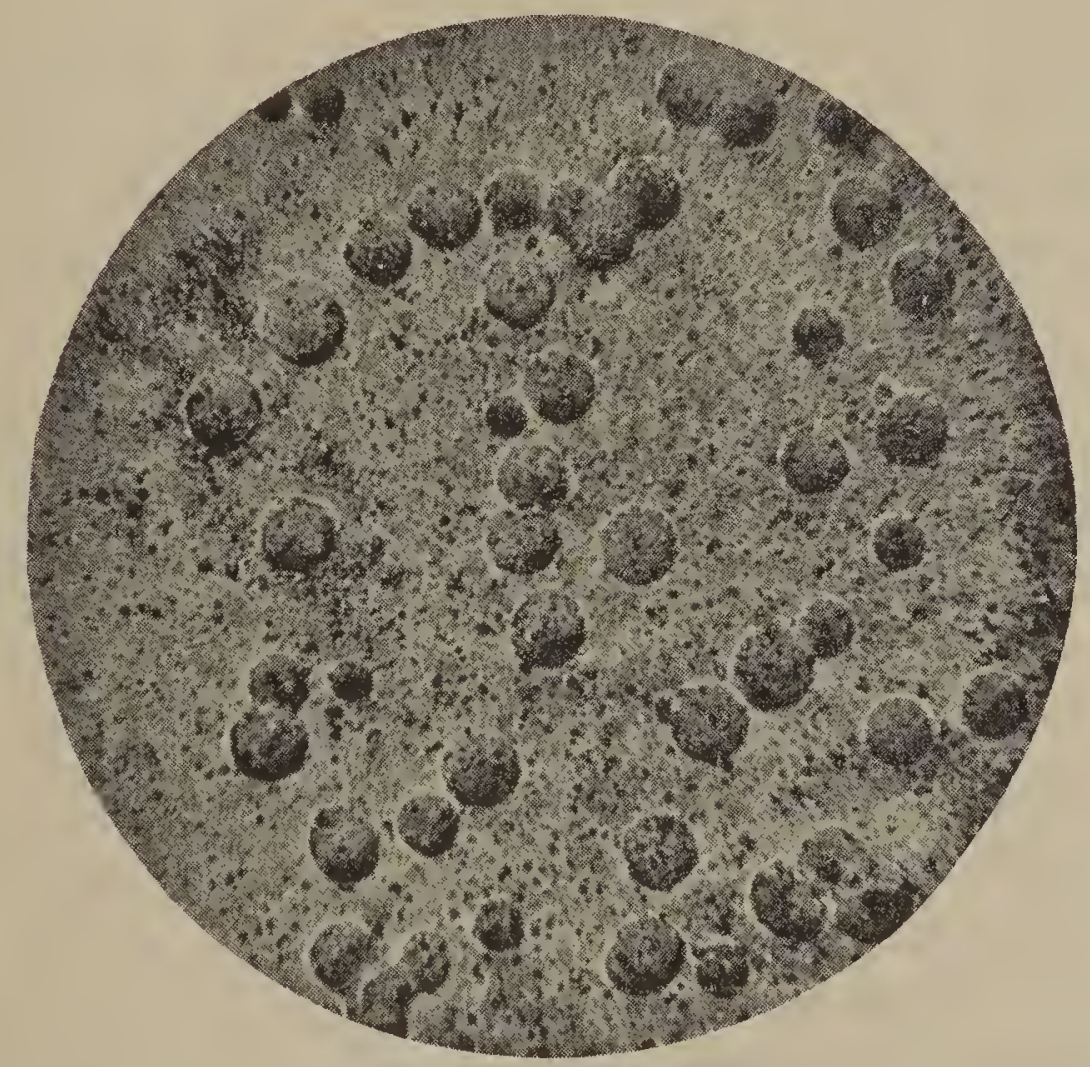

Fig. 114.-Colonies of Influtenza Baciluts on Blood Agar. (After Heim.)

has been found to create an environment favorable for their development.

Influenza bacilli do not grow at room temperature. Upon suitable media at $37.5^{\circ} \mathrm{C}$. colonies appear at the end of eighteen to twenty-four hours, as minute, colorless, transparent droplets, not unlike spots of moisture. These never become confluent. The limits of growth are reached in two or three days. To keep the cultures alive, tubes should

1 Ghon und Preyss, Cent. f. Bakt., xxxv, 1904.

${ }_{2}$ Nastjukoff, Cent. f. Bakt., Ref., xix, 1896.

- Cantani, Cent. f. Bakt., xxii, 1897.

${ }_{4}$ Grassberger, Zeit. f. Hyg., xxv, 1897. 
be stored at room temperature and transplantations done at intervals not longer than four or five days.

Biology.-The bacillus is aërobic, growing in broth-blood mixtures only upon the surface, hardly at all in agar stab cultures, and not at all under completely anaërobic conditions.

As it does not form spores, it is exceedingly sensitive to heat, desiccation, and disinfectants. ${ }^{1} \quad$ Heating to $60^{\circ} \mathrm{C}$. kills the bacilli in a few minutes. In dried sputum they die within one or two hours. They are easily killed even by the weaker antiseptics. Upon culture media the bacilli, if not transplanted, die within a week or less, the time depending to some extent upon the medium used.

Pathogenicity.-The relationship between the clinical disease known as influenza or grippe and the Pfeiffer bacillus has been definitely established by numerous investigators. ${ }^{2}$ During epidemics, the bacilli are found with much regularity in the nasal passages and bronchial secretions of those afflicted with the malady. The organs most frequently attacked in man are the upper respiratory passages and lungs. Here the disease most frequently takes the form of a broncho- or lobular pneumonia, and sections of the lung tissue of those who have died of the infection show innumerable bacilli upon and within the mucosa of the bronchioles. [Thin sections are stained for one-half to one hour in dilute carbol fuchsin and are then dehydrated in slightly acid alcohol (alcohol absolute z i, glacial acetic acid gtt. i-ij).j

Clinically, influenzal broncho-pneumonias are not essentially different from those due to other microorganisms, and it must always be left to the bacteriological examination to make the positive diagnosis. Pulmonary influenzal infection is not infrequently followed by abscess or gangrene of the lung, and occasionally develops into a chronic interstitial process. The bacilli have also been found in the middle ear, ${ }^{3}$ in the meninges, ${ }^{4}$ and in the brain and spinal cord. Bacilli in the circulating blood have never been satisfactorily demonstrated, although the general characters of the symptoms would suggest a septicemia. The short incubation period ${ }^{5}$ of the disease was involuntarily determined by Kretz, who fell ill twenty-four hours after accidentally breaking an agar plate of a pure culture which he was photographing.

The bacilli are said to remain in the bronchial secretions of conval-

${ }^{1}$ Kruse, in Flügge, "Die Mikroorg.," Leipzig, 1896.

${ }^{2}$ Weichselbaum, Wien. klin. Woch., 32, 1892; Baumler, Münch. med. Woch., 1894; Huber, loc. cit.

${ }^{3}$ Kossell, Charité-Annalen, 1893.

${ }^{4}$ Pfuhl, Berl. klin. Woch., xxxix, 1892.

${ }^{5}$ Quoted from Tedesco, Cent. f. Bakt., xliii, 1907. 
escents or even of normal individuals for many years. They are found for long periods in the lungs of those suffering from tuberculosis. To such sources, probably, are atiributable the sporadic cases developing constantly in crowded communities. Occasional reactivation of the influenzal infection may often aggravate the condition of phthisical patients. Cases of influenza observed apart from the large epidemics are rarely due to an unmixed Pfeiffer bacillus infection, but are usually due to a mixed infection, including with this bacillus, pneumococci, streptococci, and other secondary invaders. ${ }^{1}$ This may, in part, account for the frequently atypical courses of such attacks.

Dr. Anna Williams ${ }^{2}$ has recently studied hemoglobinophilic bacilli isolated from the eye in cases of trachoma. She believes that trachoma is probably caused by bacteria of this group. At first an acute infection or acute conjunctivitis occurs. Later when chronic productive inflammation supervenes the clinical picture is that of trachoma.

Experimental infection of animals reveals susceptibility only in monkeys. Pfeiffer and Beck ${ }^{3}$ produced influenza-like symptoms in monkeys by rubbing a pure culture of the bacillus upon the unbroken nasal mucosa. Intravenous inoculation in rabbits produced severe symptoms, but the bacilli do not seem to proliferate in these animals, the reaction probably being purely toxic. Cultures killed with chloroform may produce severe transient toxic symptoms in rabbits. ${ }^{4}$ Immunity produced by an attack of influenza, if present at all, is of very short duration.

Bacteria Closely Related to Influenza Bacillus.-Pseudo-INFLUEnzA BAcIluus. - In the broncho-pneumonic processes of children, Pfeiffer ${ }^{5}$ found small, non-motile, Gram-negative bacilli, which he was forced to separate from true influenza bacilli because of their slightly greater size, and their tendency to form threads and involution forms. These microorganisms are strictly aërobic and grow, like true influenza bacilli, only upon blood media. They are differentiated entirely by their morphology upon twenty-four-hour-old blood-agar cultures. Wollstein, ${ }^{6}$ who has made a careful study of influenza-like bacilli, both culturally and by agglutination tests, has come to the conclusion that these bacilli are so similar to the true influenza organisms that the term pseudo-influenza should be discarded. Strains of similar bacilli isolated from cases of

1 Tedesco, loc. cit.

${ }^{2}$ Dr. Anna Williams, Inter. Congress of Hygiene and Demography, Washington, 1912.

${ }^{3}$ Pfeiffer und Beck, Deut. med. Woch., xxi, 1893.

${ }^{4}$ Pfeiffer, loc. cit.

${ }_{5}$ Pfeiffer, Zeit. f. Hyg., xiii, 1892.

${ }^{6}$ Wollstein, Jour. Exp. Med., viii, 1906. 
pertussis, while differing from the others in some of their characteristics, could not properly be maintained as distinct species.

Koch-Weaks Baciluus. - Koch, ${ }^{1}$ in 1883 , Weeks ${ }^{2}$ and Kartulis, in 1857, described a small Gram-negative bacillus found in connection with a form of acute conjunctival inflammation, which occurs epidemically. The bacillus is morphologically similar to B. influenzæ, but is generally longer than this and more slender. The bacilli grow only at incubator temperature, but, unlike influenza bacilli, can be cultivated upon media of serum or ascitic fluid, without hemoglobin. In fact, growth upon serum-agar is more active than upon hemoglobin media. ${ }^{3}$

Bacillus of Pleuro-Pneumonia of Rabitis.-This is a small

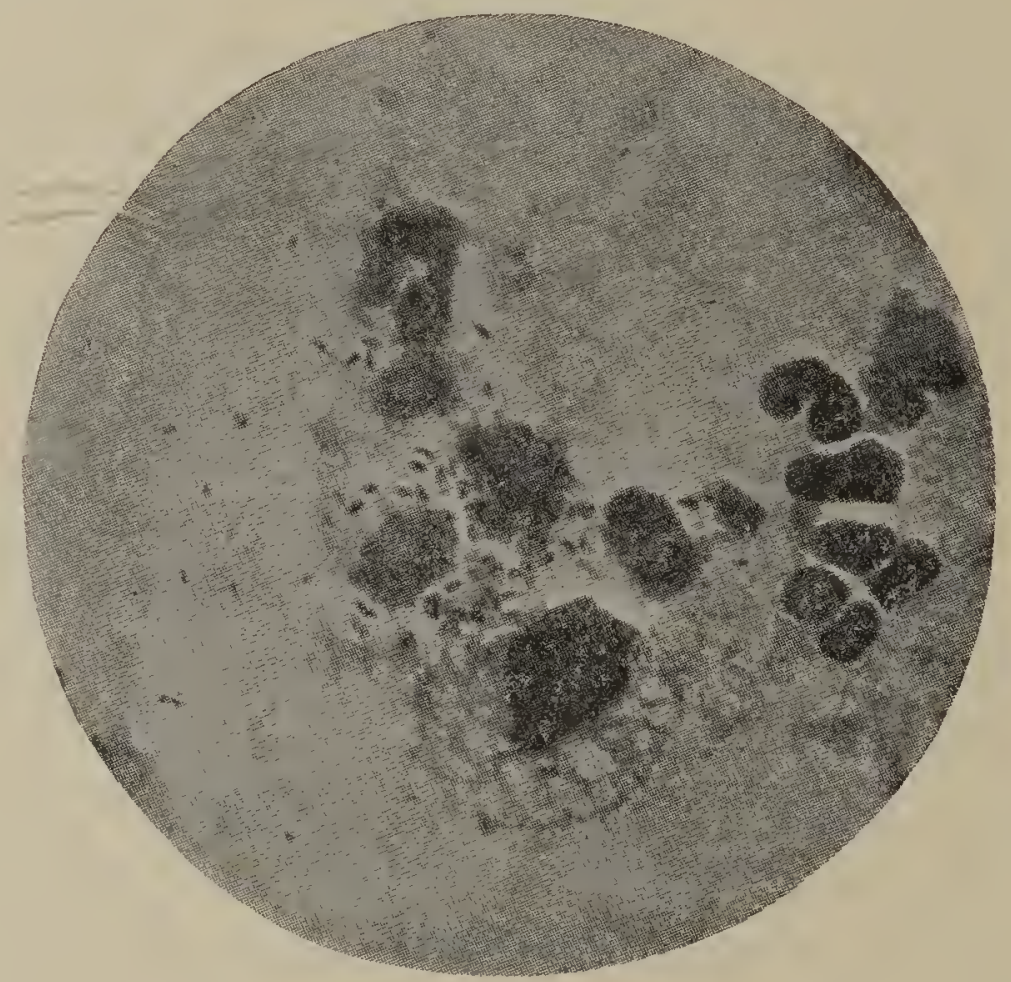

Fig. 115.-KOCH-WeEKS BACILLUS.

Gram-negative bacillus, described by Beck, not unlike that of influenza. These microorganisms are slightly larger than the Pfeiffer bacilli and grow upon ordinary media even without animal sera or hemoglobin.

BACILLUS MURISEPTICUS AND BACILLUS RHUSIOPATHIÆ.-While morphologically similar to the microorganisms of this group, these bacilli are culturally easily separated because of their luxuriant growth on simple media. (The last two microorganisms are more closely related to the groups of the bacilli of hemorrhagic septicemia. See page 551.)

${ }^{1}$ Koch, Arb. a. d. kais. Gesundheitsamt, iii; Cent. f. Bakt., 1, 1887.

2 Weeks, N. Y. Eye and Ear Infirmary Rep., 1895; Arch. f. Augenheilk., 1887.

3 Kamen, Cent. f. Bakt., xxv, 1899; Weichselbaum and Müller, Arch. f. Ophthalm., xlvii, 1899; Knapp, Studies from Dept. of Path., Coll. of P. and S., 1903. 


\section{CHAPTER XXXVIII}

BORDET-GENGOU BACILLUS, MORAX-AXENFELD BACILLUS, ZUR
NEDDEN'S BACILLUS, DUCREY BACILLUS

\section{BORDET-GENGOU BACILLUS}

\section{("Microbe de la Coqueluche," Pertussis bacillus, Bacillus of whooping- cough.)}

IN 1900 Bordet and Gengou ${ }^{1}$ observed in the sputum of a child suffering from pertussis a small ovoid bacillus which, though similar to the influenza bacillus, showed a number of morphological characteristics which led them to regard it as a distinct species. As they were at first unable to cultivate this organism, their discovery remained questionable until 1906, when cultivation succeeded and the biology of the microorganism was more fully elucidated.

Morphology.-The morphology of this organism is described by them as follows: The organism in the sputum, early in the disease, is scattered in enormous numbers indiscriminately among the pus cells, and at times within the cells. It is extremely small and ovoid, and frequently is so short that it resembles a micrococcus. Often its poles stain more deeply than the center. In general, the form of the organisms is constant, though occasionally slightly larger individuals are encountered. They are usually grouped separately, though occasionally in pairs, end to end.

Compared with the influenza bacillus in morphology, the bacillus of pertussis is more regularly ovoid and somewhat larger. It has, furthermore, less tendency to pleomorphism and involution.

Staining. - The Bordet-Gengou bacillus may be stained with alkaline methylene-blue, dilute carbol-fuchsin, or aqueous fuchsin solutions. Bordet and Gengou recommended as a staining-solution carbolated toluidin-blue made up as follows:

Toluidin-blue

5 gms.

Alcohol 100 c.c.

Water 500 c.c.

${ }_{1}^{1}$ Bordet et Gengou, Ann. de l'inst. Pasteur, 1906. 
Allow to dissolve and add 500 c.c. of 5 per cent carbolic acid in water. Let this stand one or two days and filter.

Stained by the method of Gram, the bacillus of Bordet and Gengou is decolorized.

Cultivation.-Early attempts at cultivation made by the discoverers upon ordinary ascitic agar or blood agar were unsuccessful. They finally obtained successful cultures from sputum by the use of the following medium:

One hundred grams of sliced potato are put into 200 c.c. of 4 per cent glycerin in water. This is steamed in an autoclave and a glycerin extract of potato obtained. To 50 c.c. of this extract 150 c.c. of 6 -percent salt solution and 5 grams of agar are added. The mixture is melted in the autoclave and the fluid filled into test tubes, 2 to 3 c.c. each, and

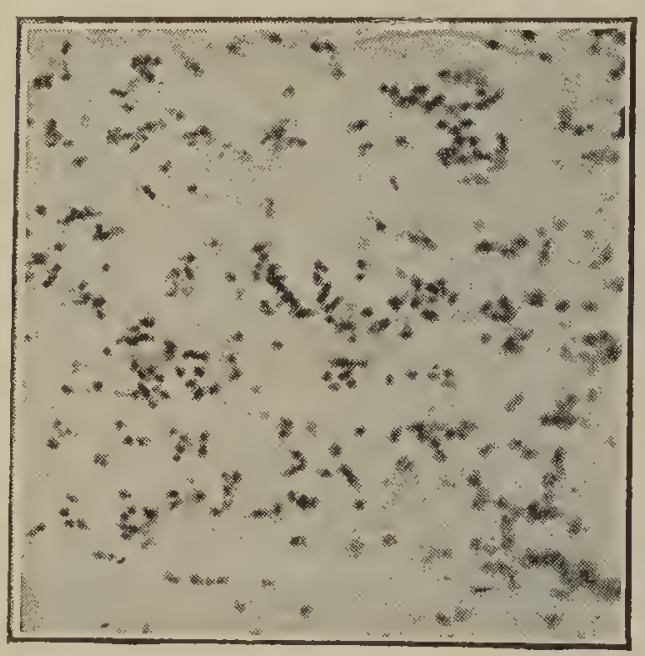

Fig. 116.-Bordet-Gengou Bacillus. sterilized. To each tube, after sterilization, is added an equal volume of sterile defibrinated rabbit blood or preferably human blood, the substances are mixed, and the tubes slanted.

On such a medium, inoculated with sputum, taken preferably during the paroxysms of the first day of the disease, colonies appear, which are barely visible after twenty-four hours, ${ }^{1}$ plainly visible after forty-eight hours. They are small, grayish, and rather thick. After the first generation the organisms grow with markedly greater luxuriance and speed. On the potato-blood medium, after several generations of artificial cultivation, they form a grayish glistening layer which, after a few days, becomes heavy and thick, almost resembling the growth of typhoid bacilli. In these later generations, also, they develop readily upon plain blood agar or ascitic agar and in ascitic broth or broth to which blood has been added. In the fluid media they form a viscid sediment, but no pellicle.

Culturally, the bacillus varies from B. influenzæ in growing less readily on hemoglobin media than the latter, on first cultivation from the sputum. Later it grows much more heavily on such media and shows less dependence upon the presence of hemoglobin than does B. influenzæ. It also grows rather more slowly than the influenza bacillus. It is 
strictly aërobic and in fluid cultures is best grown in wide flasks with shallow layers of the medium.

The Bordet-Gengou bacillus grows moderately at temperatures about $37.5^{\circ} \mathrm{C}$., but does not cease to grow at temperatures as low as $5^{\circ}$ to $10^{\circ} \mathrm{C}$. On blood agar and in ascitic broth it may remain alive for as long as two months (Wollstein).

Pathogenicity.-As regards the pathogenicity and etiological specificity of this organism for whooping-cough, no positive statement can as yet be made. The fact that it has been found in many cases in almost pure cultures during the early paroxysms, renders it likely that the organism is the specific cause of the disease. However, in early cases true influenza bacilli have been often found, and these latter seem to remain in the sputum of such patients for a longer period and in larger numbers than the bacillus of Bordet and Gengou. Endotoxins have been obtained from the cultures of the bacilli by Bordet and Gengou by the method of Besredka. ${ }^{1}$ The growth from slant cultures is washed up in a little salt solution, dried in vacuo, and ground in a mortar with a small, measured quantity of salt. Finally, enough distilled water is added to bring the salt into a solution of 0.75 per cent and the mixture is centrifugalized and decanted. One to two c.c. of such an extract will usually kill a rabbit within twenty-four hours after intravenous inoculation. Subcutaneous inoculation produces non-suppurating necrosis and ulceration without marked constitutional symptoms.

Inoculation of monkeys with the bacilli themselves by the respiratory path has failed to produce the disease.

Specific agglutinins may be obtained in immunized animals which prove absolutely the distinctness of this organism from Bacillus influenzæ. ${ }^{2}$ In the serum of afflicted children the agglutination is too irregular to be of value.

Specific complement fixation with the serum of patients is reported by Bordet and Gengou, but failed in the hands of Wollstein.

\section{MORAX-AXENFELD BACILLUS}

In 1896 Morax $^{3}$ described a diplo-bacillus, which he associated etiologically with a type of chronic conjunctivitis to which he applied the name "conjonctivite subaigue." Soon after this, a similar microorganism was found in cases corresponding to those of Morax by Axen-

1 Bordet, Bull. de la Soc. Roy. de Brux., 1907.

${ }^{2}$ Wollstein, loc. cit.

${ }^{3}$ Morax, Ann. de l'inst. Pasteur, 1896. 
feld. ${ }^{1}$ The condition which these microorganisms characteristically produce is a catarrhal conjunctivitis which usually attacks both eyes. The inflammation is especially noticeable in the angles of the eye, most severe at or about the caruncle. There is rarely much swelling of the conjunctiva and hardly ever ulceration. The condition runs a subacute or chronic course. Its diagnosis is easily made by smear preparations of the pus which is formed with especial abundance during the night.

Morphology.- In smear preparations from the pus, the microorganisms appear as short, thick bacilli, usually in the form of two placed end to end, but not infrequently singly or in short chains. Their ends

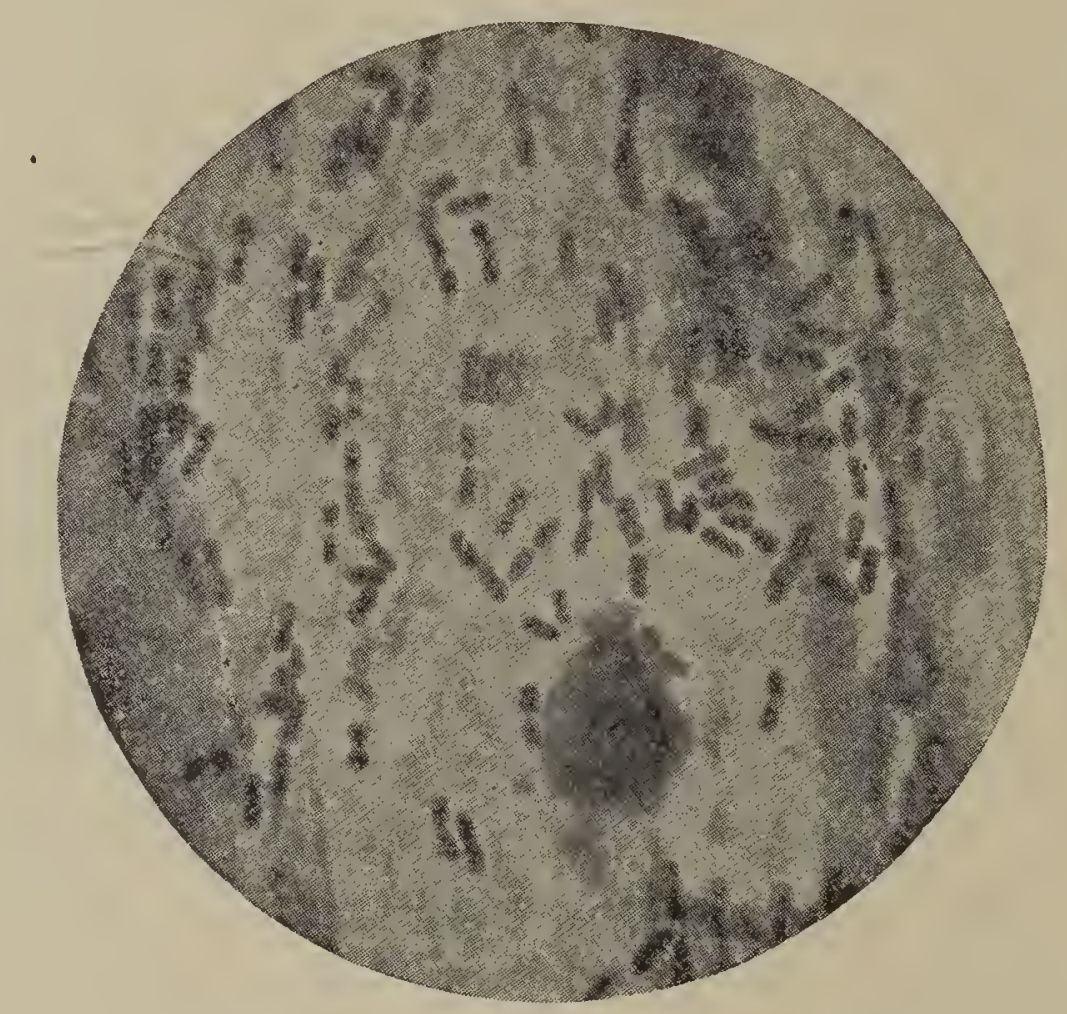

Fig. 117.-Morax-Axenfeld Diplo-Bacillus.

are distinctly rounded, their centers slightly bulging, giving the bacillus an ovoid form. They are usually about two micra in length.

They are easily stained by the usual anilin dyes, and, stained by the method of Gram, are completely decolorized.

Cultivation.-The Morax-Axenfeld bacillus can be cultivated only upon alkaline media containing blood or blood serum.

It grows poorly, or not at all, at room temperature.

Upon Loeffer's blood serum, colonies appear after twenty-four to thirty-six hours as small indentations which indicate a liquefaction of the medium. Axenfeld states that eventually the entire medium may 
become liquefied. Upon serum agar delicate grayish drop-like colonies are formed which are not unlike those of the gonococcus.

In ascitic bouillon general clouding occurs within twenty-four hours.

Pathogenicity. - Attempts to produce lesions in the lower animals with this bacillus have been universally unsuccessful. Subacute conjunctivitis, however, has been produced in human beings by inoculations with pure cultures.

\section{ZUR NEDDEN'S BACILLUS}

In ulcerative conditions of the cornea, Zur Nedden has frequently found a bacillus to which he attributes etiological importance.

The bacillus which he has described is small, usually less than one micron in length, often slightly curved, and generally found singly. It may be found in the diplo form but does not form chains. It is stained by the usual dyes, often staining poorly at the ends. Stained by Gram's method it is decolorized. The bacillus is non-motile.

Cultivation.-It is easily cultivated upon the ordinary laboratory media. Upon agar it forms, within twenty-four hours, transparent, slightly fluorescent colonies which are round, raised, rather coarsely granular, and show a tendency to confluence.

Gelatin is not liquefied.

Milk is coagulated.

Upon potato, there is a thick yellowish growth.

Upon dextrose media, there is acid formation, but no gas.

The bacillus forms no indol in pepton solutions.

Pathogenicity.-Corneal ulcers have been produced by inoculation of guinea-pigs.

\section{BACILLUS OF DUCREY}

The soft chancre, or chancroid, is an acute inflammatory, destructive lesion which occurs usually upon the genitals or the skin surrounding the genitals. The infection is conveyed from one individual to another by direct contact. It may, however, under conditions of surgical manipulation, be transmitted indirectly by means of dressings, towels, or instruments.

The lesion begins usually as a small pustule which rapidly ruptures, leaving an irregular ulcer with undermined edges and a necrotic floor which spreads rapidly. It differs clinically from the true or syphilitic chancre in the lack of induration and in its violent inflammatory 
nature. Usually it leads to lymphatic swellings in the groin which, later, give rise to abscesses, commonly spoken of as "buboes."

In the discharges from such lesions, Ducrey, ${ }^{1}$ in 1889 , was able to demonstrate minute bacilli to which he attributed an etiological relationship to the disease, both because of the regularity of their presence in the lesions and the successful transference of the disease by means of pus containing the microorganisms.

Morphology and Staining.-The Ducrey bacillus is an extremely small bacillus, measuring from one to two micra in length and about half a micron in thickness. It has a tendency to appear in short chains and in parallel rows, but many of the microorganisms may be seen irregularly grouped. It is not motile, possesses no flagella, and does not form spores.

Stained by the ordinary anilin dyes, it has a tendency to take the color irregularly and to appear more deeply stained at the poles. By the Gram method, it is decolorized. In tissue sections, it may be demonstrated by Loeffler's methylene-blue method, and in such preparations has been found within the granulation tissues forming the floor of the ulcers. In pus, the bacilli are often found within leucocytes.

Cultivation and Isolation.-Early attempts at cultivation of this bacillus were universally unsuccessful in spite of painstaking experiments with media prepared of human skin and blood serum. In 1900, Besançon, Griffon, and Le Sourd ${ }^{2}$ finally succeeded in obtaining growths upon a medium containing agar to which human blood had been added. They were equally successful when dog's or rabbit's blood was substituted for that of man. Since the work by these authors, the cultivation by similar methods has been carried out by a number of investigators. Coagulated blood, which has been kept for several days in sterile tubes, has been found to constitute a favorable medium. Freshly clotted blood can not be employed, probably because of the bactericidal action of the serum. Serum-agar has occasionally been used with success, but does not give results as satisfactory as those obtained by the use of the whole blood.

The best method of obtaining pure cultures upon such media consists in puncturing an unruptured bubo with a sterile hypodermic needle and transferring the pus in considerable quantity directly to the agar. If possible, the inoculation of the media should be made immediately

${ }^{1}$ Ducrey, Monatschr. f. prakt. Dermat., 9, 1889.

${ }^{2}$ Besançon, Griffon, et Le Sourd, Presse méd., 1900. 
before the pus has had a chance to cool off or to be exposed to light. When buboes are not available, the primary lesion may be thoroughly cleansed with sterile water or salt solution, and material scraped from the bottom of the ulcer or from beneath its overhanging edges with a stiff platinum loop. This material is then smeared over the surface of a number of blood-agar plates.

Upon such plates, isolated colonies appear, usually after forty-eight hours. They are small, transparent, and gray, and have a rather firm, finely granular consistency. The colonies rarely grow larger than pinhead size, and have no tendency to coalesce. At room temperature, the cultures die out rapidly. Kept in the incubator, however, they may remain alive and virulent for a week or more.

On the simpler media, glucose-agar, broth, or gelatin, cultivation is never successful. On moist blood-agar and in the condensation water of such tubes, the bacilli have a tendency to grow out in long chains. Upon media which are very dry, they appear singly or in short chains.

Pathogenicity.-Besançon, Griffon, and Le Sourd, and others, have succeeded in producing lesions in man by inoculation with pure cultures. Inoculation of the lower animals has, so far, been entirely without result.

\section{MICROCOCCUS MELITENSIS (MALTA FEVER)}

\section{(Bacillus melitensis)}

Malta fever is a disease occurring along the Mediterranean coast and its islands. It has been recently found to occur also in South America, South Africa, China, and in the West Indies. The disease is not very unlike typhoid fever, though more irregular and with a lower mortality. It is accompanied by joint pains, sweating, constipation, and occasionally orchitis. The spleen is almost always enlarged.

Recent investigations into the manner in which this disease is conveyed have revealed that it is primarily an infection of goats. A large percentage of the goats on Malta were shown to be infected and passed the organism with the milk. Forty per cent of the goats gave positive agglutination tests and the organisms have been found in the milk in about 10 per cent of the animals.

The most susceptible animals seem to be goats, but horses and cows are also susceptible. In guinea-pigs and rabbits the disease can be experimentally produced, but usually takes a protracted course. Monkeys 
are susceptible, and the disease produced in these animals is in many features identical with that of man.

Transmission seems to take place chiefly by the ingestion of infected milk. Direct cutaneous infection or through mucous membranes may also occur. In human beings, suffering from the disease, the organisms may be isolated from the blood stream during the entire course of the disease and as early as the second day. The disease is rarely fatal, death occurring in less than 2 per cent of the cases (Eyre, loc. cit.). ${ }^{1}$

The microorganism causing the disease was isolated in 1887 by Bruce, ${ }^{2}$ a British army surgeon.

Morphology.-Micrococcus melitensis is a minute bacterium appearing coccoid in smears from agar cultures, in broth cultures assuming the form of a short, slightly wedge-shaped bacillus resembling B. influenzæ. Babes ${ }^{3}$ regards it as unquestionably a bacillus. Eyre describes it as a minute coccus, and believes the bacillus-like individuals to represent involution forms. It appears in irregularly parallel groups, and occasionally forms short chains.

It is easily stained with the ordinary dyes, and is decolorized by Gram's method.

Cultivation.-Micrococcus melitensis can usually be cultivated from the spleens of those who have succumbed to the disease and from the blood stream in active cases. It grows on nutrient agar at $37.5^{\circ} \mathrm{C}$, forming small, pearly white colonies at the end of two or three days. It grows easily on all of the ordinary laboratory media.

Both in patients and in injected animals, infection with this bacterium produces specific agglutinins which are of great practical aid in diagnosis. ${ }^{4}$

${ }^{1}$ British Commission Report cited from Eyre in Kolle und Wassermann, Handbuch, etc., Ergänzungsband, Heft 2.

${ }^{2}$ Bruce, Practitioner, 1887.

${ }^{3}$ Babes, Kolle und Wassermann, iii, p. 443.

${ }^{4}$ Wright and Lamb, Jour. Path. and Bact., v, 1899. 


\section{CHAPTER XXXIX}

\section{THE BACILLI OF THE HEMORRHAGIC SEPTICEMIA GROUP AND BACILLUS PESTIS.}

Is many of the lower animals there occur violently acute bacterial infections characterized by general septicemia, usually with petechial hemorrhages throughout the organs and serous membranes and severe intestinal inflammations. These diseases, spoken of as the "hemorrhagic septicemias," are caused by a group of closely allied bacilli, first classified together by Hueppe ${ }^{1}$ in 1886 . Some confusion has existed as to the forms which should be considered within Hueppe's group of "hemorrhagic septicemia," a number of bacteriologists including in this class bacilli such as Loeffler's Bacillus typhi murium, and Salmon and Smith's hog-cholera bacillus, microorganisms which, because of their motility and cultural characteristics, belong more properly to the "Gärtner," "enteritidis," or "paratyphoid" group, intermediate between colon and typhoid.

The organisms properly belonging to this group are short bacilli, more plump than are those of the colon type, and showing a marked tendency to stain more deeply at the poles than at the center. They are non-motile, possess no flagella, and do not form spores. They grow readily upon simple media, but show a very marked preference for oxygen, growing but slightly below the surface of media. By some observers they are characterized as "obligatory aërobes," but this is undoubtedly a mistake.

While showing considerable variations in form and differences in minor cultural characteristics, the species characteristics of polar staining, decolorization by Gram, immobility, lack of gelatin liquefaction, and great pathogenicity for animals, stamp alike all members of the group. Its chief recognized representatives are the bacillus of chicken cholera, the bacillus of swine-plague (Deutsche Schweineseuche), 
and the Bacillus pleurosepticus which causes an acute disease in cattle and often in wild game.

Because of certain cultural and pathogenic characteristics, it seems best to consider the bacillus of bubonic plague with this group.

\section{BACILLUS OF CHICKEN CHOLERA}

(Bacillus avisepticus)

The bacillus of chicken cholera was first carefully studied by Pasteur ${ }^{1}$ in 1880 . It is a short, non-motile bacillus, measuring from 0.5 to 1 micron in length. Stained with the ordinary anilin dyes, it displays marked polar staining qualities, which often give it the appearance of being a diplococcus. It is decolorized by Gram's method. It does not form spores, but may occasionally form vacuolated degeneration forms, not unlike those nescribed for Bacillus pestis.

The bacillus is easily cultivated from the blood and organs of infected animals, it grows well upon the simplest media at temperatures varying from $25^{\circ}$ to $40^{\circ} \mathrm{C}$. In broth, it produces uniform clouding with later a formation of a pellicle. Upon agar it forms, within twenty-four to forty-eight hours, minute colonies, white or yellowish in color, which are at first transparent, later opaque. Upon gelatin, it grows without liquefaction. Upon milk, the growth is slow and does not produce coagulation. According to Kruse, ${ }^{2}$ indol is formed from pepton bouillon. Acid, but no gas, is formed in sugar broth.

Among barnyard fowl, this disease is widely prevalent, attacking chickens, ducks, geese, and a large variety of smaller birds. The infection is extremely acute, ending fatally within a few days. It is accompanied by diarrhea, often with bloody stools, great exhaustion, and, toward the end, a drowsiness bordering on coma. Autopsy upon the animals reveals hemorrhagic inflammation of the intestinal mucosa, enlargement of the liver and spleen, and often bronchopneumonia.

The specific bacilli may be found in the blood, in the organs, in exudates, if these are present, and in large numbers in the dejecta. Infection takes place probably through the food and water contaminated by the discharges of diseased birds. ${ }^{3}$

Subcutaneous inoculation or feeding of such animals with pure cultures, even in minute doses, gives rise to a quickly developing septicemia which is uniformly fatal. The bacillus is extremely patho-

"Pasteur, Comptes rend. de l'acad. des sci., 1880.

2 Kruse, in Flügge's "Die Mikroorganismen."

${ }^{3}$ Salmon, Rep. of the Com. of Agriculture, 1880, 1881, and 1882. 
genic for rabbits, less so for hogs, sheep, and horses, if infection is practiced by subcutaneous inoculation. Infection by ingestion does not seen to cause disease in these animals.

Historically, the bacillus of chicken cholera is extremely interesting, since it was with this microorganism that Pasteur ${ }^{1}$ carried out some of his fundamental researches upon immunity, and succeeded in immunizing chickens with attenuated cultures. The first attenuation experiment made by Pasteur consisted in allowing the bacilli to remain in a broth culture for a prolonged period without transplantation. With minute doses of such a culture (vaccin I) he inoculated chickens, following this, after ten days, with a small dose of a fully virulent culture. Although enormously important in principle, the practical results from this method, as applied to chicken cholera, have not been satisfactory. It was with this bacillus, furthermore, that Pasteur was first able to demonstrate the existence of a free toxin which could be separated from the bacteria by filtration.

\section{BACILLUS OF SWINE PLAGUE}

\section{(Bacillus suisepticus, Schweinesenche)}

This microorganism is almost identical in form and cultural characteristics with the bacillus of chicken cholera. It is non-motile, forms no spores, is Gram-negative, and does not liquefy gelatin. The bacillus causes an epidemic disease among hogs, which is characterized almost regularly by a bronchopneumonia followed by general septicemia. There is often a sero-sanguineous pleural exudate, a swelling of bronchial lymph glands and of liver and spleen. The gastrointestinal tract is rarely affected. The bacilli at autopsy may be found in the lungs, in the exudates, in the liver and spleen, and in the blood. The disease is rarely acute, but, in young pigs, almost uniformly fatal.

It is probable that spontaneous infection usually occurs by inhalation. Experimental inoculation is successful in pigs, both when given subcutaneously and when administered by the inhalation method. Mice, guinea-pigs, and rabbits are also susceptible, dying within three or four days after subcutaneous inoculation of small doses.

Active and passive immunization of animals against Bacillus suisepticus has been attempted by various observers. Active immunization, if carried out with care, may be successfully done in the laboratory. 
Passive immunization of animals with the serum of actively immunized horses has been practiced by Kitt and Mayr, ${ }^{1}$ Schreiber, ${ }^{2}$ and Wassermann and Ostertag. The last-named observers, working with a polyvalent serum produced with a number of different strains of the bacillus, have obtained results of considerable practical value. The researches of Kitt and Mayr have revealed a fact pointing to the interrelationship of the bacilli of the "hemorrhagic septicemia" group. They were able to show that the serum of horses immunized with chicken cholera bacilli was able to protect, somewhat, against Bacillus suisepticus.

Infection with the bacillus of swine plague, in hogs, is often accompanied by an infection with the hog-cholera bacillus (Schweinepest). The latter, as we have seen, is a microorganism belonging to the enteritidis group, intermediate between Bacillus coli and Bacillus typhosus, and differing from suisepticus in being actively motile, possessing flagella, not showing the polar staining, having a more slender morphology, and producing gas upon dextrose broth. A confusion between the two bacilli frequently occurs because of their nomenclature. Bacteriologically and pathogenically, they are quite distinct. Bacillus suisepticus produces an acute septicemia, accompanied by bronchopneumonia and usually not affecting the gastro-intestinal canal. The bacillus of hog cholera produces an infection localized in the intestinal canal.

\section{BACILLUS PESTIS}

\section{(Bacillus of Bubonic Plague)}

The history of epidemic diseases has no more terrifying chapter than that of plague. ${ }^{3}$ Sweeping, time and again, over large areas of the civilized world, its scope and mortality were often so great that all forms of human activity were temporarily paralyzed. In the reign of Justinian almost fifty per cent of the entire population of the Roman Empire perished from the disease. The "Black Death" which swept over Europe during the fourteenth century killed about twenty-five million people. Smaller epidemics, appearing in numerous parts of the world during the sixteenth, seventeenth, and eighteenth centuries, have claimed innumerable victims. In 1893, plague appeared in Hong Kong. During the epidemic which followed, Bacillus pestis, now recognized as the etiological factor of the disease, was discovered by

${ }^{1}$ Kitt und Mayr, Monatsh. f. prakt. Thierheilk., 8, 1897.

2 Schreiber, Berl. tierärztl. Woch., 10, 1899.

3 Hirsch. "Handb. d. histor.-geogr. Path.," 1881. 
Kitasato ${ }^{1}$ and by Yersin, ${ }^{2}$ independently of each other. By both observers the bacillus could invariably be found in the pus from the buboes of afflicted persons. It could be demonstrated in enormous numbers in the cadavers of victims. The constancy of the occurrence of the bacillus in patients, shown in the innumerable researches of many bacteriologists, would alone be sufficient evidence of its etiological relationship to the disease. This evidence is strengthened, moreover, by accidental infections which occurred in Vienna in 1898, with laboratory cultures.

Morphology and Staining.-Bacillus pestis is a short, thick bacillus with well-rounded ends. Its length is barely two or two and a half times

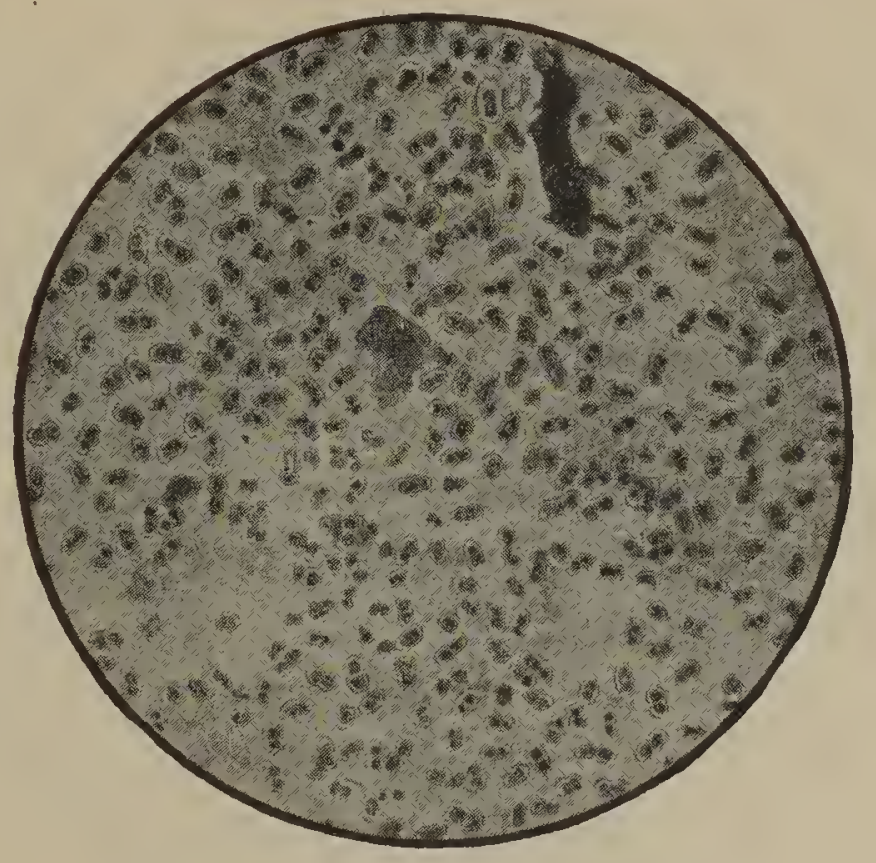

Fig. 118.-BAcillus Pestis. (After Mallory and Wright.)

its breadth (1.5 to 1.75 micra by 0.5 to 0.7 micron). The bacilli appear singly, in pairs, or, more rarely, in short chains of three or more. They show distinct polar staining. In size and shape these bacilli are subject to a greater degree of variation than are most other microorganisms. In old lesions or in old cultures the bacilli show involution forms which may appear either as swollen coccoid forms or as longer, club-shaped, diphtheroid bacilli. Degenerating individuals appear often as swollen, oval vacuoles. All these involution forms, by their very irregularity, are of diagnostic importance. They appear more numerous in artificial cultures than in human lesions.

According to Albrecht and Ghon, ${ }^{3}$ the plague bacillus may, by

Kitasato, Lancet, 1894.

2 Yersin, Ann. de l'inst. Pasteur, 1894.

3 Albrecht und Ghon, Wien, 1898. 
special methods, be shown to possess a gelatinous capsule. It does not possess flagella and does not form spores.

The plague bacillus is easily stained with all the usual anilin dyes. Diluted aqueous fuchsin and methylene-blue are most frequently employed. With these stains the characteristically deeper staining of the polar portions of the bacillus is usually easy to demonstrate. Special polar stains have been devised by various observers. Most of these depend upon avoidance of the usual heat fixation of the preparations, which, in some way, seems to interfere with good polar staining. Fixation of the dried smears with absolute alcohol is, therefore, preferable. The bacillus is decolorized by Gram's method.

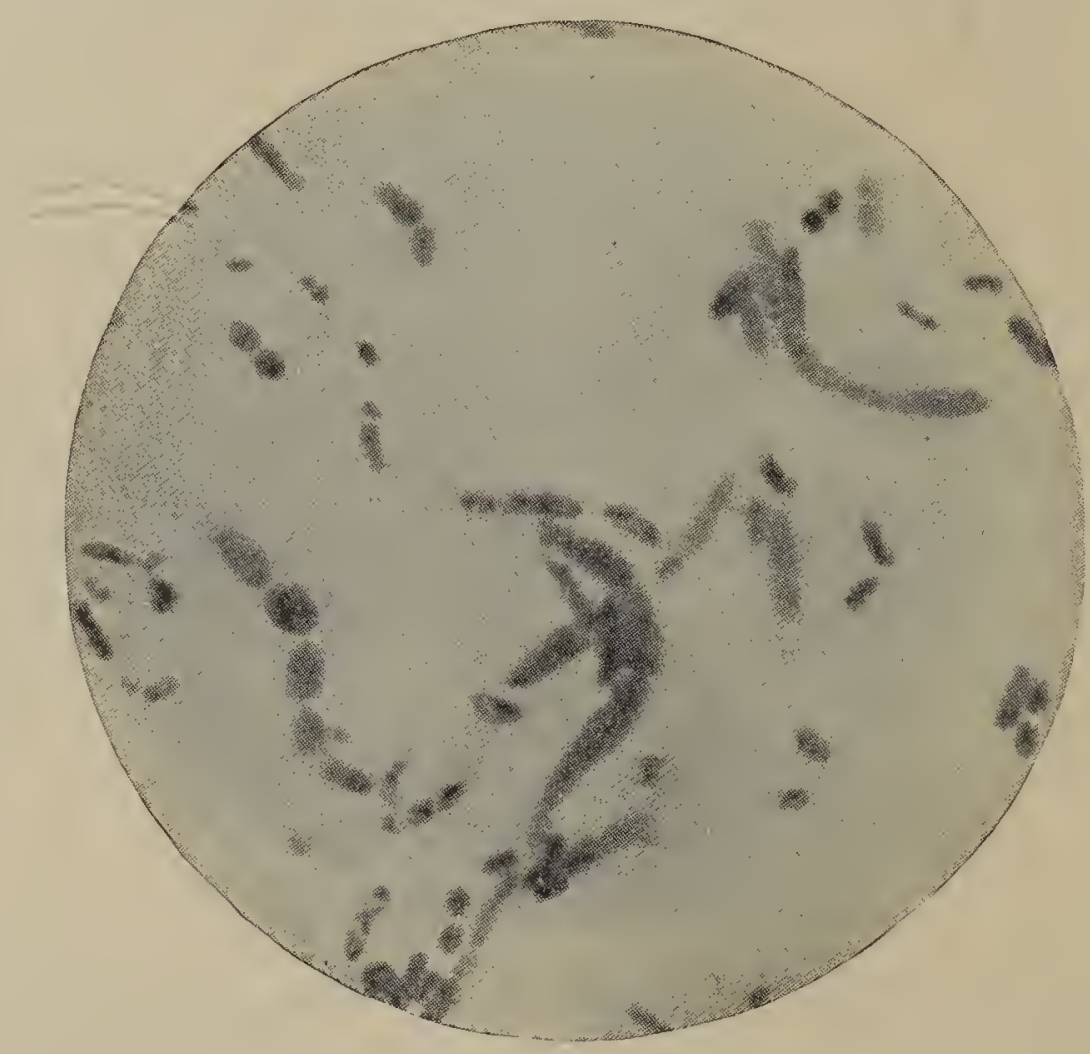

Fig. 119.-Bacillus pestis, Involution Forms. (After Zettnow.)

Isolation and Cultivation.-The bacillus is easily isolated in pure culture from the specific lesions of plague patients, during life or at autopsy. It grows readily and luxuriantly upon the meat-infusion media. The optimum temperature for its cultivation is about $30^{\circ} \mathrm{C}$. Below $20^{\circ} \mathrm{C}$. and above $38^{\circ} \mathrm{C}$., growth is sparse and delayed, though it is not entirely inhibited until exposed to temperatures below $12^{\circ} \mathrm{C}$, or above $40^{\circ} \mathrm{C}$. The most favorable reaction of culture media is neutrality or moderate alkalinity, though slight acidity does not prevent development.

On agar, growth appears within twenty-four hours as minute colonies with a compact small center surrounded by a broad, irregularly indented, granular margin. 
On gelatin, similar colonies appear after two or three days at $20^{\circ}$ to $22^{\circ} \mathrm{C}$. The gelatin is not liquefied.

In bouillon, the plague bacilli grow slowly. They usually sink to the bottom or adhere to the walls of the tube as a granular deposit and may occasionally form a delicate pellicle. Chain-formation is not uncommon. In broth cultures, moreover, a peculiar stalactite-like growth is often seen, when the culture fluid is covered with a layer of oil. Delicate threads of growth hang down from the surface of the medium into its depths like stalactites. Characteristic involution forms are brought out best when the bacilli are grown upon agar containing 3 per cent $\mathrm{NaCl}$.

Milk is not coagulated. In litmus-milk there is slight acid formation. On potato and on blood serum the growth shows nothing characteristic or of differential value. On pepton media no indol is formed.

Biological Considerations.-Bacillus pestis is aërobic. Absence of free oxygen is said to prevent its growth, at least under certain conditions of artificial cultivation. It is non-motile. Outside of the animal body the bacilli may retain viability for months and even years if preserved in the dark and in a moist environment. In cadavers they may live for weeks and months if protected from dryness. In pus or sputum from patients they may live eight to fourteen days. These facts are of great hygienic importance.

Complete drying in the air kills the bacilli within two or three days. ${ }^{1}$ Thoroughly dried by artificial means they die within four or five hours.

Dry heat at $100^{\circ} \mathrm{C}$. kills the bacillus in one hour. ${ }^{2}$ Live steam or boiling water is effectual in a few minutes. The bacilli possess great resistance against cold, surviving a temperature of $0^{\circ} \mathrm{C}$. for as many as forty days.

Direct sunlight destroys them within four or five hours. The common disinfectants are effectual in the following strengths: carbolic acid, one per cent kills them in two hours, five per cent in ten minutes; bichloride of mercury $1: 1,000$ is effectual in ten minutes.

In a recent communication to the New York Pathological Society, Dr. Wilson reported that plague cultures which he had kept sealed for as long as ten years in the ice chest were found living and virulent at the end of this time. This ability to go into a quasi latent stage under suitable conditions is of the greatest importance in connection with the problem of prevention. 
Pathogenicity.-In man, plague is acquired ${ }^{1}$ by entrance of the bacillus either through the skin or by the respiratory tract. The period of incubation is about three to seven days. Two distinct clinical types of the disease occur, depending upon the mode of infection. When cutaneous infection has occurred the disease is first localized in the lymph nodes nearest the point of inoculation. If the respiratory tract has been the portal of entrance the disease primarily takes the form of a pneumonia.

Infection may take place through the most minute lesions, hardly visible to the naked eye. Even the unbroken skin may admit the microorganisms if these are rubbed in with sufficient energy. From the primary lymphatic swellings, the bacilli enter the blood and may produce secondary foci.

The pneumonic form of plague usually begins with symptoms not unlike a typical pneumonia and is usually fatal within four or five or even fewer days. This form of the disease is especially menacing as a means of dissemination, because of the enormous numbers of plague bacilli in the sputum.

One of the chief characteristics of the general systemic plague infection is the very marked cardiac depression.

The bacteriological diagnosis during life may be made by finding the bacilli in the sputum or in aspiration fluid from a bubo. The microorganisms are identified morphologically, culturally, by animal experiment, and by agglutination reaction. Blood cultures from plague patients often yield positive results, especially when the blood is well diluted in neutral broth to prevent any inhibiting action of the antibodies in the serum.

At autopsy, in man, the bacilli are found in the primary lesions, in the blood, and in the spleen, the liver, and the lymphatics. There may be hemorrhages into the serous cavities. When pneumonia exists, it usually takes the form of a bronchopneumonia with extensive swelling of the bronchial lymph nodes.

In cases in which the disease is prolonged, there are often tuberclelike foci in the spleen, liver, and lungs. Histologically these foci show central necrosis surrounded by the usual inflammatory cell reactions. In more chronic cases connective-tissue encapsulation may appear.

Bacillus pestis is extremely pathogenic for rats, mice, guinea-pigs, rabbits, and monkeys. The most susceptible of these animals are rats 
and guinea-pigs, in whom mere rubbing of plague bacilli into the unbroken skin will often produce the disease. This method of experimental infection of guinea-pigs is of great service in isolating the plague bacillus from material contaminated with other microorganisms. For the same purpose, infection of rats subcutaneously at the root of the tail may be employed. Such inoculation in rats is invariably fatal.

The studies of $\mathrm{McCoy}^{1}$ upon guinea-pigs and white rats show that individual plague cultures may vary considerably in virulence. The size of the dose, always excepting enormous quantities such as a whole agar culture, seems to make little difference in the speed with which the animals die. There may be considerable variation in the susceptibility of individual animals. Prolonged cultivation on artificial media may gradually reduce the virulence of plague bacilli, though, as stated above, this has not been the experience of all observers.

In rats, spontaneous infection with plague is common and plays an important rôle in the spread of the disease. Rats become infected from the cadavers of plague victims or by gnawing the dead bodies of other rats dead of the disease. The pneumonic type of the disease is common in these animals and has been produced in them by inhalation experiments. During every well-observed plague epidemic, marked mortality among the domestic rats has been noticed.

Since the examination of rats for plague is an important phase of the study of epidemics, it may be well to review the typical lesions in these animals as described by an experienced American student of plague, George W. McCoy. ${ }^{2}$ McCoy, agreeing with the Indian Plague Commission, states that the naked eye is superior to the microscopical examination. There is engorgement of the subcutaneous vessels and a pink coloration of the muscles. The bubo when present is sufficient for diagnosis. Marked injection surrounds it and sometimes there is hemorrhagic infiltration. The gland itself is firm but usually caseous or occasionslly hemorrhagic. In the liver there is apparent fatty change, but this is due to necrosis. Pin-point spots give it a stippled appearance as though it had been dusted with pepper. Pleural effusion is an important sign. The spleen is large, friable, and often presents pinpoint granules on the surface. One or two per cent of rats may present no gross lesions. Cultures should of course be made. The method of examination consists in immersing the rat in any convenient antiseptic

${ }^{1}$ McCoy, Jour. of Inf. Dis., vi, 1909.

${ }^{2}$ George W. McCoy, Public Health Reports, July, 1912. 
to kill fleas and other ectoparasites. The rats are nailed by their feet to a shingle and the skin is reflected from the whole front of the body and neck so as to expose the cervical, axillary, and inguinal regions. The thoracic and abdominal cavities are then opened and examined.

Wherry, ${ }^{1} \mathrm{McCoy}{ }^{2}$ and others have found that the California ground squirrel was infected with plague, during the recent occurrence of plague on the Pacific coast, and several cases of plague in man were traced to this source. In studying these and other American rodents McCoy found that ground squirrels as a species were highly susceptible, never showing natural immunity. Field mice were but moderately susceptible. Gophers were highly resistant. McCoy has also described a case of spontaneous infection in a brush rat (Neotoma fuscipes). Rock squirrels were found by McCoy to be readily infected.

Wu Lion Teh (G. L. Tuck) ${ }^{3}$ has recently found that the Manchurian tarbagan or marmot (Arctomys bobac), an animal trapped for its fur, occasionally suffers from plague. The disease is never extensive and the animal of much less importance in spreading the disease than is the rat.

Plague is transmitted either by direct contact or inhalation, or indirectly by clothing, linen, and other objects worn or handled. The rôle in the transmission of the disease played by rats is probably of great importance. The animals vomit, defecate, and die in cellars, storerooms, etc., and thus set free vast numbers of plague bacilli for indirect accidental transmission to human beings. The actual mode in which this transmission takes place is by no means certain. The fact that in countries where plague is prevalent many of the natives go insufficiently shod or barefooted, may account for many infections.

Simond ${ }^{4}$ lays great stress upon transmission to man by means of fleas, the Indian rat-flea often being parasitic upon man. His conclusions are probably too far-reaching, though the possibility of such infection can not be denied. ${ }^{5}$

It is a curious fact observed by various bacteriologists that plague bacilli isolated from pneumonic cases are particularly apt to cause pneumonic lesions, having, as it were, acquired a selective pathogenicity for the lung. A most valuable contribution to our knowledge of pneu-

${ }^{1}$ Wherry, Jour. Inf. Dis., v, 1908.

${ }^{2}$ McCoy, Jour. Inf. Dis., vi, 1909; vii, 1910.

${ }^{3}$ Wu Lien Teh, Jour. of Hyg., xiii, 1913.

${ }^{4}$ Simond, Ann. de l'inst. Pasteur, 1893.

${ }^{5}$ Nuttall, Cent. f. Bakt., xxii, 1897; Nuttall, Hyg. Rund., ix, 1899. 
monic plague has recently been made by Strong, Teague, and Barber ${ }^{1}$ in their report of the American Red Cross Expedition to Manchuria during the plague epidemic of 1910-11. Their investigations were made with remarkable courage and skill under difficult conditions.

The chief points of interest in their reports may be summarized as follows: Expired air of plague patients rarely contains the bacilli; these are thrown out in coughing or hawking. Transmission is, in this form, direct from patient to patient and not indirect through animals. The first localization (Strong, Teague, and Crowell) is in the bronchi from which extension takes place. Septicemia soon follows the pneumonic process. Spreading occurs most likely in the wet and cold of winter, since the bacteria are rapidly destroyed by drying.

Toxin Formation.-The systemic symptoms of plague are largely due to the absorption of poisonous products of the bacteria. Albrecht and Ghon, ${ }^{2}$ Wernicke, ${ }^{3}$ and others were unable to obtain any toxic action with broth-culture filtrates and concluded that the poisons of B. pestis were chiefly endotoxins, firmly attached to the bacterial body. Kossel and Overbeck, ${ }^{4}$ however, on the basis of a careful investigation, came to the conclusion that, in addition to the endotoxin, there is formed in older broth cultures a definite and important true, soluble toxin.

Immunization.-A single attack of plague usually protects human beings from reinfection. A second attack in the same individual is extremely rare. Immunization in animals produces specific agglutinating and bacteriolytic substances which are of great importance in the bacteriological diagnosis of the bacillus. The agglutinating action of the serum of patients is clinically important in the diagnosis of the disease, even in dilutions of one in ten, since undiluted normal human serum has no agglutinating effect upon plague bacilli.

Active immunization of animals ${ }^{5}$ is accomplished by inoculation of the whole dead bacteria. Haffkine has attempted active immunization in human beings by subcutaneous treatment with sterilized broth cultures of B. pestis. Gaffky ${ }^{6}$ and his collaborators recommend, for similar purposes, forty-eight-hour agar cultures of a bacillus of standard virulence, emulsified in bouillon and sterilized at $65^{\circ} \mathrm{C}$.

${ }^{1}$ Strong, Teague, and Barber, Philippine Jour. of Sc.. Sect. B, vii, 1912, No. 3.

${ }^{2}$ Albrecht und Ghon, loc. cit.

${ }^{3}$ Wernicke, Cent. f. Bakt., Ref., xxiv, 1898.

${ }^{4}$ Kossel und Overbeck, Arb. a. d. Gesundh., xviii, 1901.

5 Yersin, Calmette, et Roux, Ann. de l'inst. Pasteur, 1895.

${ }^{6}$ Gaffky, Pfeiffer, Sticker, und Dieudonné, Arb. a. d. kais. Gesundheitsamt, xvi, 1899. 
The curative plague serum prepared by Yersin and others by the immunization of horses with plague cultures has been extensively used in practice and though often disappointing, a definitely beneficial influence on the milder cases has been noted. The sera are standardized by their protective power as measured in white rats.

\section{THE PLAGUE-LIKE DISEASE OF RODENTS (MCCOY) ${ }^{1}$}

\section{Bacterium Tularense (McCoy and Chapin) ${ }^{2}$}

McCoy has described a disease occurring in Californian ground squirrels (Citellus beechyi) which presents lesions very similar to those of plague in these animals. In fact the disease was noticed in the course of the systematic examination of rodents by McCoy at the Federal Laboratory in San Francisco. Although McCoy was able to transmit the disease to guinea-pigs, mice, rabbits, monkeys, and gophers, and plague-like lesions could be produced in most of the animals, he was at first entirely unable to cultivate any organism from these lesions. In 1912 McCoy and Chapin finally succeeded in growing the specific bacterium on an egg medium made entirely of the yolk. Morphologically it is a very small rod, 0.3 to 0.7 micron in length and often capsulated. The rods stain poorly with methylene blue, better with carbol fuchsin or gentian violet. They are found in large numbers in the spleens of animals dead of the disease.

${ }^{1}$ McCoy, U. S. Public Health Bull. 43, 1911.

${ }^{2}$ McCoy and Chapin, Jour. of Inf. Dis., x, 1912. 


\section{CHAPTER XI}

\section{BACILLUS ANTHRACIS AND ANTHRAX}

\section{(Milzbrand, Charbon)}

Anthrax is primarily a disease of the herbivora, attacking especially cattle and sheep. Infection not infrequently occurs in horses, hogs, and goats. In other domestic animals it is exceptional. Man is susceptible to the disease and contracts it either directly from the living animals or from the hides, wool, or other parts of the cadaver used in the industries.

The history of the disease dates back to the most ancient periods and anthrax has, at all times, been a severe scourge upon cattle- and sheepraising communities. Of all infections attacking the domestic animals no other has claimed so many victims as anthrax. In Russia, where the disease is most common, 72,000 horses are said to have succumbed in one year (1864). ${ }^{1}$

In Austro-Hungary, Germany, France, and the Eastern countries, each year thousands of animals and numerous human beings perish of anthrax. In England and America the disease is relatively infrequent. No quarter of the globe, however, is entirely free from it.

Especial historical interest attaches to the anthrax bacillus in that it was the first microorganism proved definitely to bear a specific etiological relationship to an infectious disease. The discovery of the anthrax bacillus, therefore, laid, as it were, the cornerstone of modern bacteriology. The bacillus was first observed in the blood of infected animals by Pollender in 1849, and, independently, by Brauell in 1857 . Davaine, ${ }^{2}$ however, in 1863, was the first one to produce experimental infection in animals with blood containing the bacilli and to suggest a direct etiological relationship between the two. Final and absolute proof of the justice of Davaine's contentions, however, was not brought until the further development of bacteriological technique, by Koch, ${ }^{3}$ had made it possible for this last observer to isolate the bacillus upon

1 Quoted from Sobernheim, Kolle und Wassermann., vol. ii.

2 Davaine, Comptes rend. de l'acad. des sci., lvii, 1863.

3 Koch, Cohn's "Beitr. z. Biol. d. Pflanzen," ii, 1876. 
artificial media and to reproduce the disease experimentally by inoculation with pure cultures.

Morphology and Staining.-The anthrax bacillus is a straight rod, 5 to 10 micra in length, 1 to 3 micra in width. It is non-motile. In preparations made from the blood of an infected animal, the bacilli are usually single or in pairs. Grown on artificial media they form tangles of long threads. Their ends are cut off squarely, in sharp con-

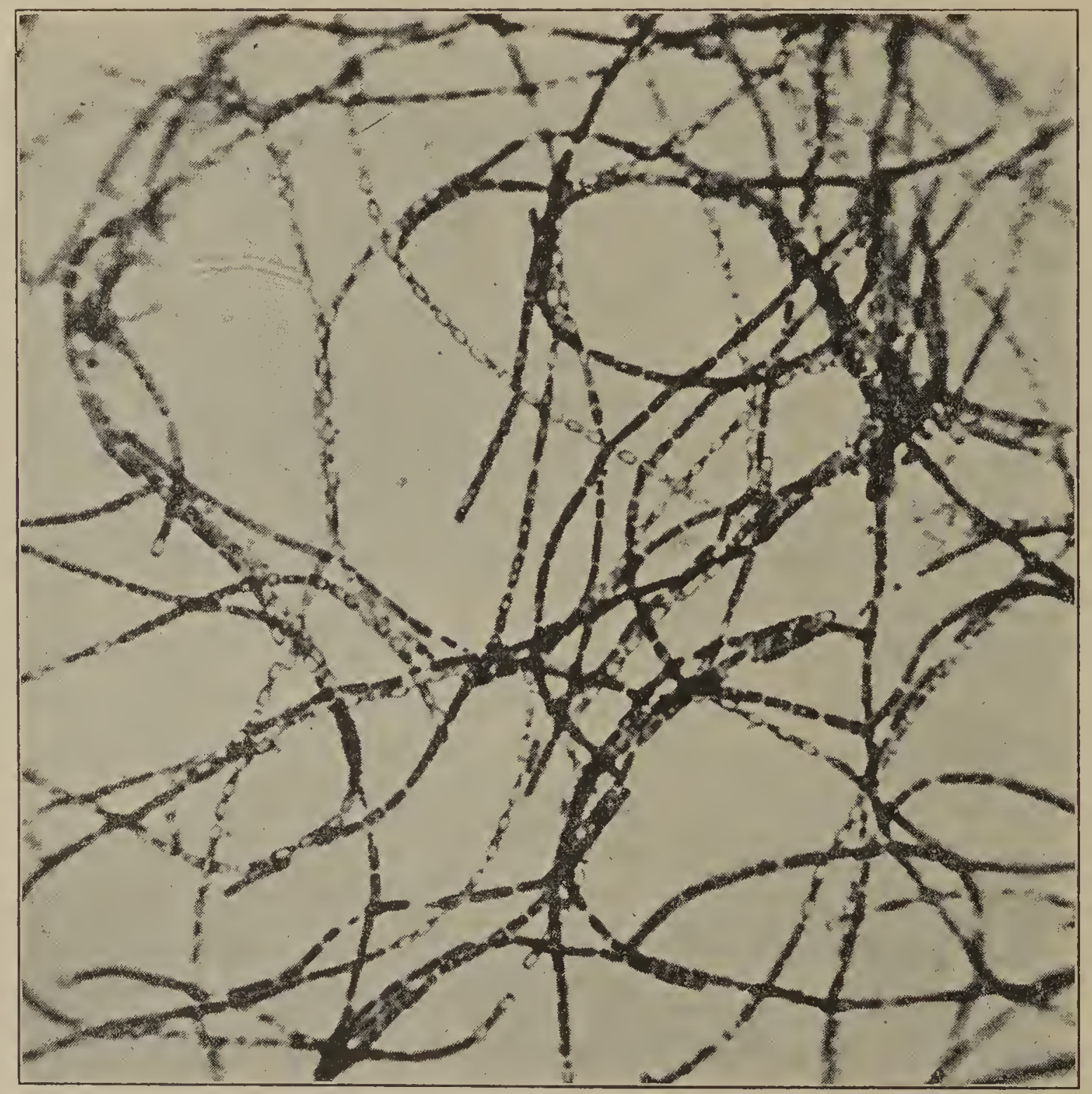

Fig. 120.-Bacillus anthracis. From pure culture on agar.

trast to the rounded ends of many other bacilli. The corners are often sharp and the ends of bacilli in contact in a chain often touch each other only at these points, leaving in consequence an oval chink between the ends of the organisms. The appearane of a chain of anthrax bacilli, therefore, has been not inaptly compared to a rod of bamboo. On artificial media the anthrax bacillus forms spores. Oxygen is necessary for the formation of these spores and they are consequently not found 
in the blood of infected subjects. The spores are located in the middle of the bacilli and are distinctly oval. They are difficult to stain, but may be demonstrated by any of the usual spore-staining procedures, such as Möller's or Novy's methods. The bacilli themselves are easily stained by the usual anilin dyes, and gentian-violet or fuchsin in aqueous solution may be conveniently employed. They are not decolorized by Gram's method.

In preparations from animal tissues or blood, stained by special pro-

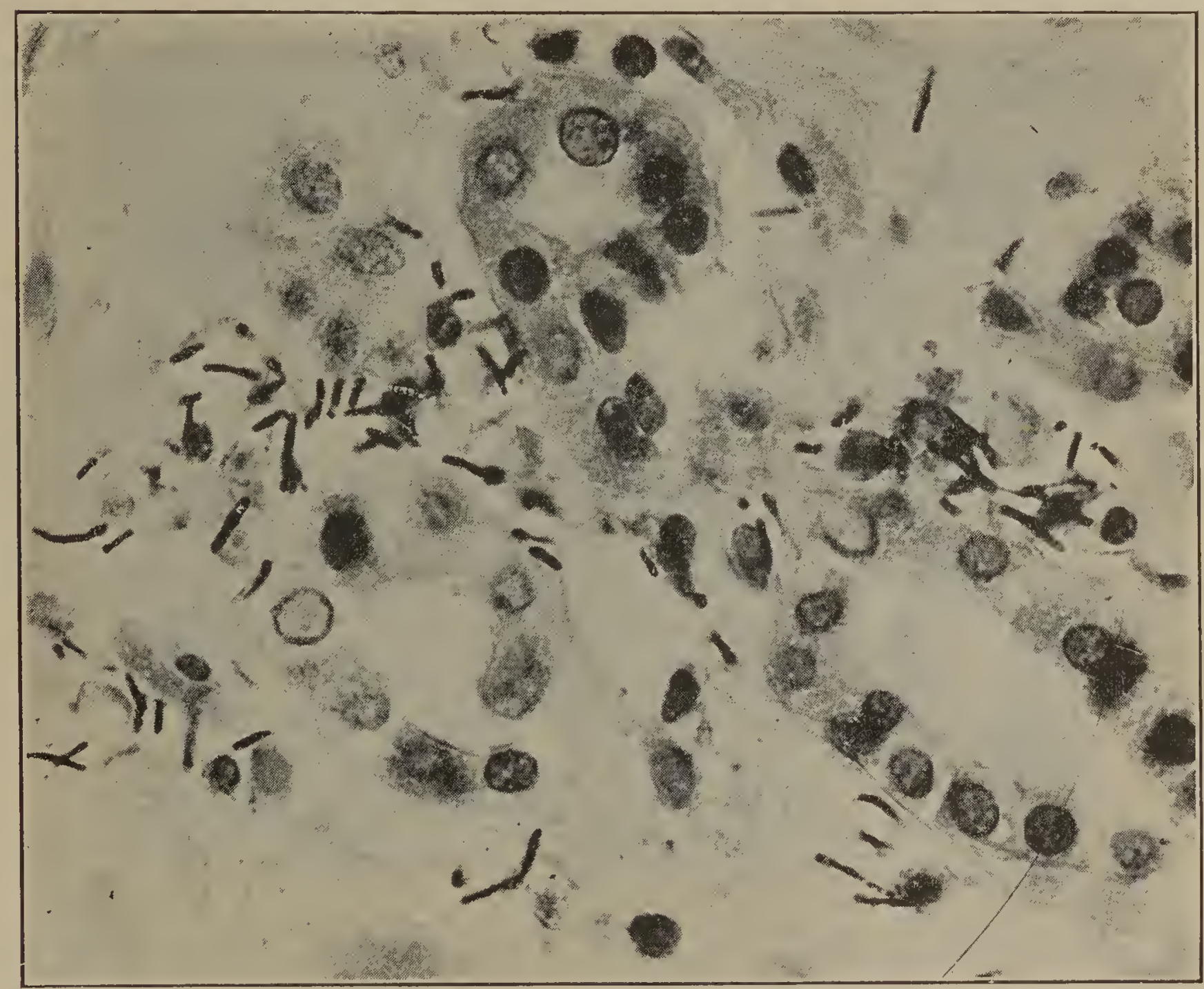

Fig. 121.-Bacildus anthracis. In section of kidney of animal dead of anthrax.

cedures, the anthrax bacillus may occasionally be seen to possess a capsule. The capsule is never seen in preparations from the ordinary artificial media. Some observers have demonstrated them in cultures grown in fluid blood serum. In chains of anthrax bacilli, the capsule when present seems to envelop the entire chain and not the individual bacteria separately.

Isolation.-Isolation of the anthrax hacillus from infected material 
is comparatively simple, both because of the ease of its cultivation and because of the sharply characteristic features of its morphological and cultural appearance.

Cultivation.--The anthrax bacillus is an aërobic, facultatively anaërobic bacillus. While it may develop slowly and sparsely under anaërobic conditions, free oxygen is required to permit its luxuriant and characteristic growth.

The optimum temperature for its cultivation ranges about $37.5^{\circ} \mathrm{C}$. It is not, however, delicately susceptible to moderate variations of tem-

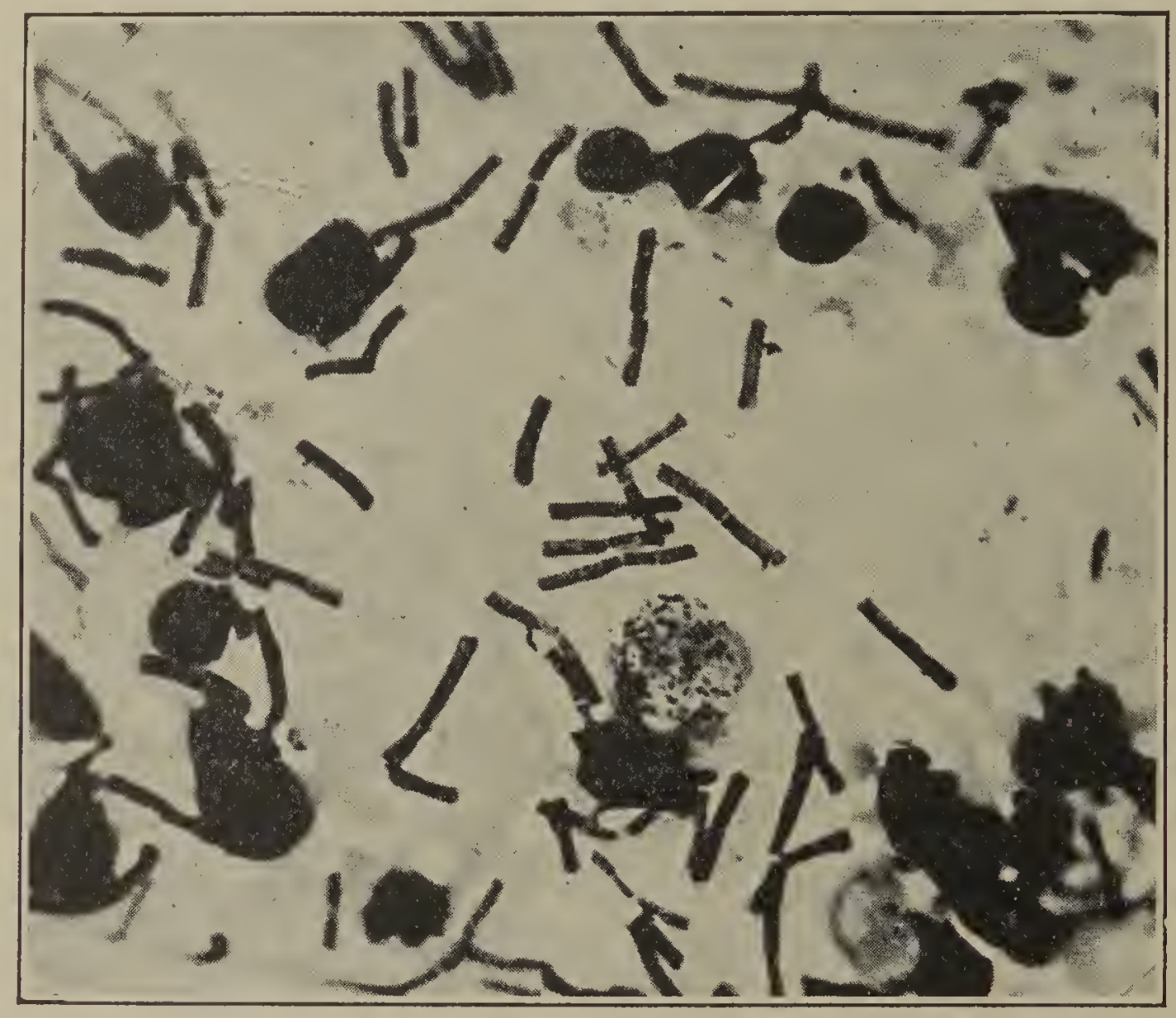

Fig. 122.-B B BllLUS ANTHRACIS. In smear of spleen of animal dead of anthrax.

perature and growth does not cease until temperatures as low as $12^{\circ} \mathrm{C}$. or as high as $45^{\circ} \mathrm{C}$. are reached. By continuous cultivation at some of the temperatures near either the higher or the lower of these limits, the bacillus may become well adapted to the new environment and attain luxuriant growth. ${ }^{1}$

The anthrax bacillus may be cultivated on all of the usual artificial media, growing upon the meat-extract as well as upon the meat-infusion media.

${ }_{1}^{1}$ Dieudonné, Arb. a. d. kais. Gesundheitsamt, 1894. 
It may be cultivated also upon hay infusion, various other vegetable media, sugar solutions, and urine. While moderate acidity of the medium does not prevent the growth of this bacillus, the most favorable reaction for media is neutrality or slight alkalinity.

Cn gelatin plates, colonies develop within twenty-four to forty-eight hours as opaque, white disks, pin-head in size, irregularly round and rather flat. As the colonies increase in size their outlines become less regular and under the microscope they are seen to be made up of a hair-like tangle of threads spreading in thin wavy layers from a more compact central knot. The microscopic appearance of these colonies has been aptly described as resembling a Medusa head. Fragments of a

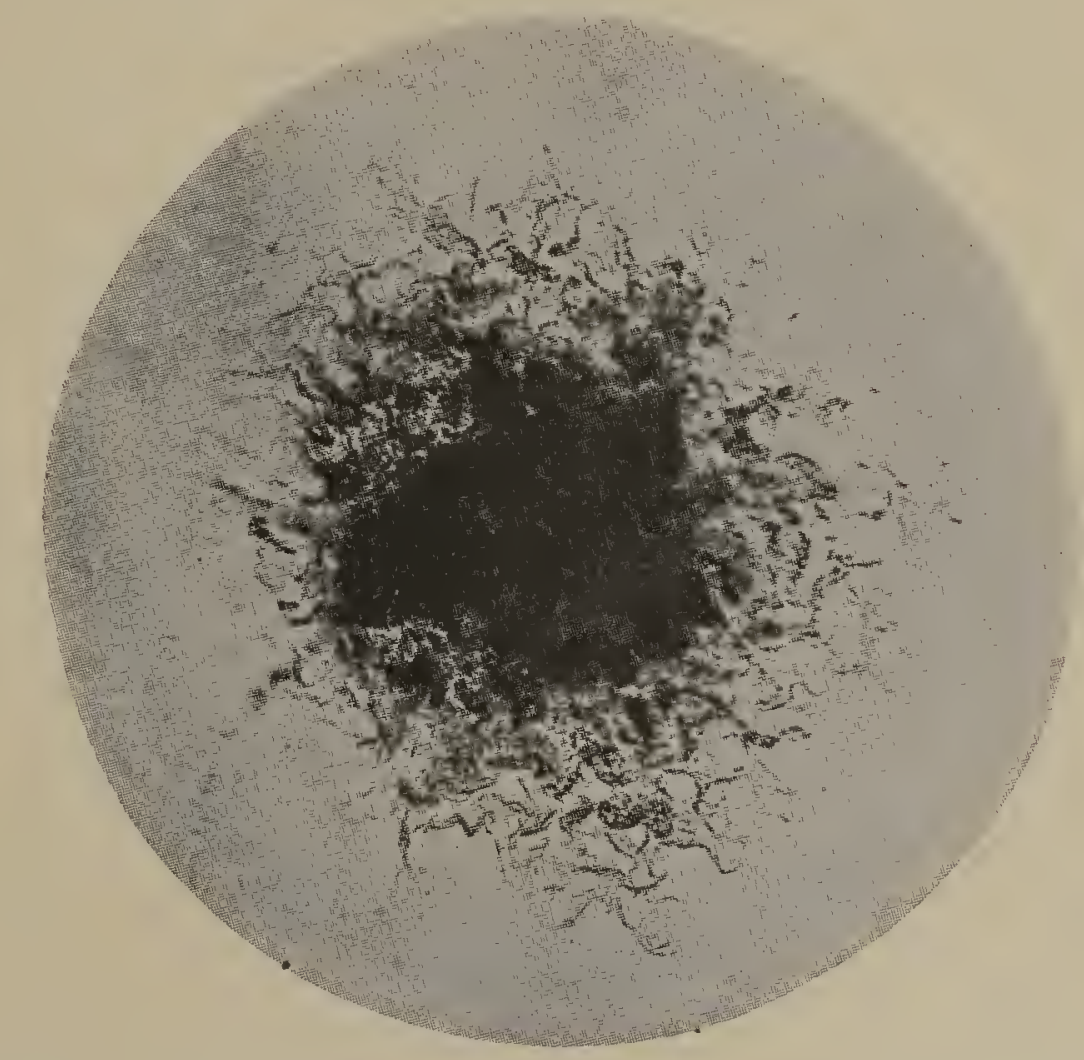

Fig. 123.-Anthrax Colony on Gelatin. (After Günther.)

colony examined on a slide with a higher power show the individual threads to be made up of parallel chains of bacilli.

After a day or two of further growth, the gelatin about the colonies becomes fluidified.

In gelatin stab cultures, growth appears at first as a thin white line along the course of the puncture. From this, growth proceeds in thin spicules or filaments diverging from the stab, more abundantly near the top than near the bottom of the stab, owing to more active growth in well oxygenated environment. The resulting picture is that of a small inverted "Christmas tree." Fluidification begins at the top, at first a shallow depression filled with an opaque mixture of bacilli and fluid. 
Later the bacilli sink to the bottom of the flat depression, leaving a clear supernatant fluid of peptonized gelatin.

In broth, growth takes place rapidly, but does not lead to an even, general clouding. There is usually an initial pellicle formation at the top where the oxygen supply is greatest. Simultaneously with this a slimy mass appears at the bottom of the tube, owing to the sinking of

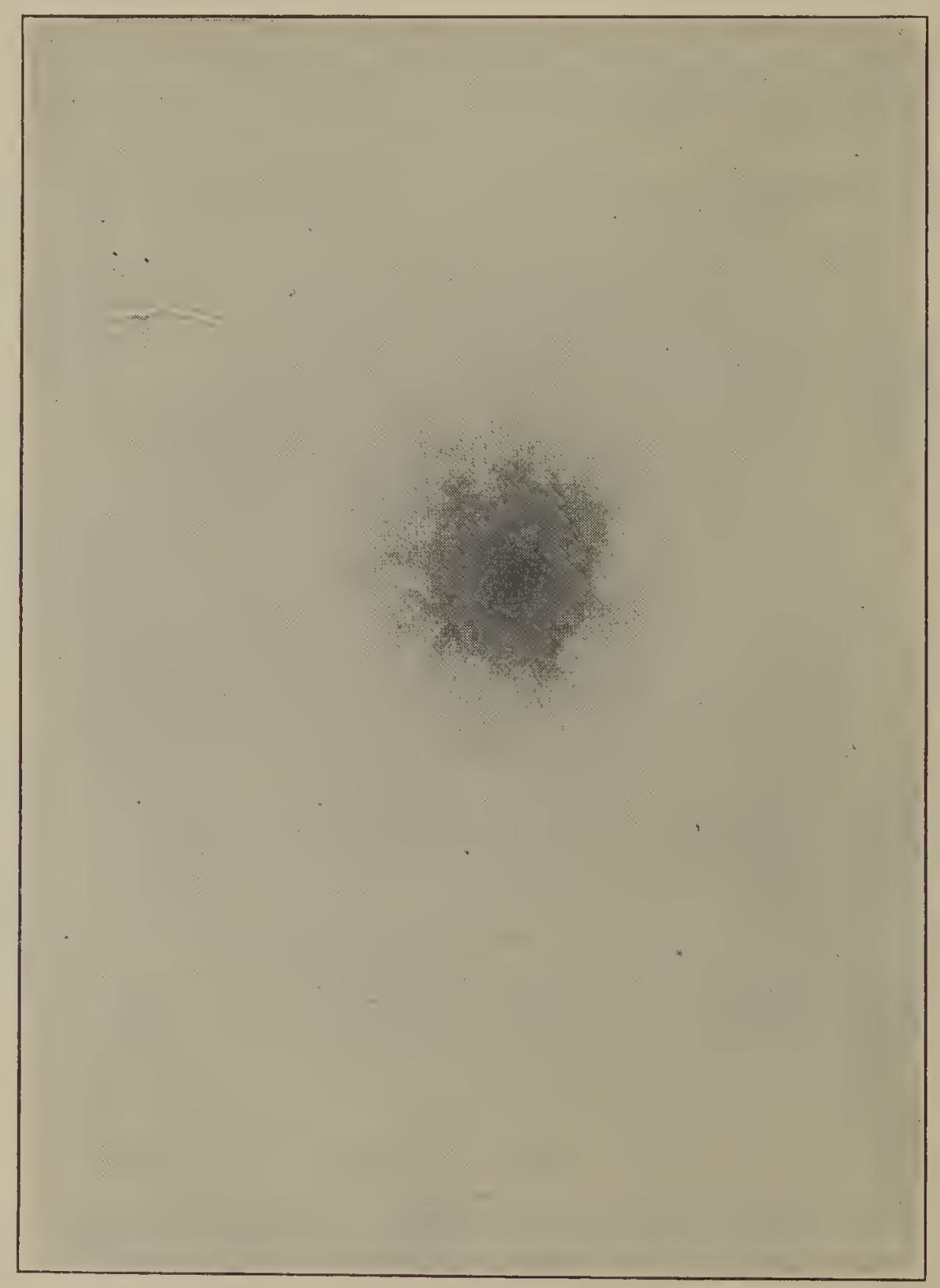

Fig. 124.-Anthrax Colony on Agar.

bacilli to the bottom. Apart from isolated flakes and threads the intervening broth is clear. Shaken up, the tube shows a tough, stringy mass, not unlike a small cotton fluff, and general clouding is produced only by vigorous mixing.

Upon agar plates, growth at $37.5^{\circ} \mathrm{C}$. is vigorous and colonies appear 
within twelve to twenty-four hours. They are irregular in outline, slightly wrinkled, and show under the microscope the characteristic tangled-thread appearance seen on gelatin, except that they are more compact than upon the former medium. The colonies are slightly glistening and tough in consistency.

On agar slants, the colonies usually become confluent, the entire surface soon being covered by a grayish, tough pellicle which, if fished, has a tendency to come away in thin strips or strands.

On potato, growth is rapid, white, and rather dry. Sporulation upon potato is rapid and marked, and the medium is favorable for the study of this phase of development.

Milk is slowly acidified and slowly coagulated. This action is chiefly upon the casein; very few, if any, changes being produced either in the sugars or in the fats of the milk. The acids formed are, according to Iwanow, ${ }^{1}$ chiefly formic, acetic, and caproic acids.

Biological Considerations. - The anthrax bacillus is aërobic and facultatively anaërobic. It is non-motile and possesses no flagella. In the animal body it occasionally forms capsules. In artificial cultures in the presence of oxygen, it sooner or later invariably forms spores. The spores appear after the culture has reached its maximum of development. Sporulation never occurs in the animal body, probably because of the absence of sufficient free oxygen. Spores are formed most extensively ${ }^{2}$ at temperatures ranging from $20^{\circ} \mathrm{C}$. to $30^{\circ} \mathrm{C}$. Spore formation ceases below $18^{\circ} \mathrm{C}$. and above $42^{\circ} \mathrm{C}$. For different strains these figures may vary slightly, as has been shown from the results of various observers. Spores appear most rapidly and regularly upon agar and potato media.

The spore-one in each bacillus-appears as a small, highly refractile spot in the center of the individual bacterium. As this enlarges, the body of the bacillus around it gradually undergoes granular degeneration and loses its staining capacity. ${ }^{3}$

If anthrax bacilli are cultivated for prolonged periods upon media containing hydrochloric or rosolic acid or weak solutions of carbolic acid ${ }^{4}$ cultures may be obtained which do not sporulate and which seem permanently to have lost this power, without losing their virulence to the same degree. Similar results may be obtained by continuous cul-

${ }_{1}$ I wanow, Ann. de l'inst. Pasteur, 1892.

${ }_{2}$ Koch, loc. cit.

3 Behring, Zeit. f. Hyg., vi and vii, 1889; Deut. med. Woch., 1889.

4 Chamberland et Roux, Comptes rend. de l'acad. des sci., xcvi, 1882. 
tivation at temperatures above $42^{\circ} \mathrm{C}$. By this procedure, however, virulence, too, is considerably diminished.

Resistance.-Because of its property of spore formation, the anthrax bacillus is extremely resistant toward chemical and physical environment. The vegetative forms themselves are not more resistant than most other non-sporulating bacteria, being destroyed by a temperature of $54^{\circ} \mathrm{C}$. in ten minutes. Anthrax spores may be kept in a dry state for many years without losing their viability. ${ }^{1}$ While different strains of anthrax spores show some variation in their powers of resistance, all races show an extremely high resistance to heat. Dry heat at $140^{\circ}$ C. kills them only after three hours. ${ }^{2}$ Live steam at $100^{\circ}$ kills them in five to ten minutes. Boiling in water destroys them in about ten minutes. Low temperatures have but little effect upon them. Ravenel ${ }^{3}$ found that, frozen by liquid air, they were still viable after three hours.

The variability shown by different strains of spores in their resistance to heat is even more marked in their behavior toward chemicals. ${ }^{4}$ Some strains will retain their viability after exposure to five-per-cent carbolic acid for forty days, ${ }^{5}$ while others are destroyed by the same solution in two days. Corrosive sublimate, $1: 2,000$, kills most strains of anthrax in forty minutes.

Direct sunlight destroys anthrax spores within six to twelve hours. ${ }^{6}$

Pathogenicity.--The anthrax bacillus is pathogenic for cattle, sheep, guinea-pigs, rabbits, rats, and mice. The degrees of susceptibility of these animals differ greatly, variations in this respect existing even among different members of the same species. Thus, the long-haired Algerian sheep show a high resistance, while the European variety are highly susceptible; and, similarly, the gray rat is much more resistant than the white rat. Dogs, hogs, cats, birds, and the cold-blooded animals are relatively insusceptible. For man the bacillus is definitely pathogenic, though less so than for some of the animals mentioned above.

While separate races of anthrax bacilli may vary much in their degree of virulence, a single individual strain remains fairly constant in this respect if preserved, dried upon threads or kept in sealed tubes, in

1 Surmont et Arnould, Ann. de l'inst. Pasteur, 1894.

${ }^{2}$ Koch und Wolfhügel, Mitt. a. d. kais. Gesundheitsamt, 1881.

3 Ravenel, Medical News, vii, 1899.

- Fränkel, Zeit. f. Hyg., vi, 1889.

${ }^{5}$ Koch, loc. cit.

6 Momont, Ann. de l'inst. Pasteur, 1892. 
a cold, dark place. Virulence may be reduced ${ }^{1}$ by various attenuating laboratory procedures which are of importance in that they have made possible prophylactic immunization. Heating the bacilli to $55^{\circ} \mathrm{C}$. for ten minutes considerably reduces their virulence. Similar results are obtained by prolonged cultivation at temperatures of $42^{\circ}$ to $43^{\circ} \mathrm{C}$., or by the addition of weak disinfectants to the culture fluids. ${ }^{2}$ Once reduced, the new grade of virulence remains fairly constant. Increase of virulence may be artificially produced by passage through animals.

Experimental infections in susceptible animals are most easily accomplished by subcutaneous inoculations. The inoculation is followed, at first, by no morbid symptoms, and some animals may appear perfectly well and comfortable until within a few hours or even moments before death, when they suddenly become visibly very ill, rapidly go into collapse, and die. The length of the disease depends to some extent, of course, upon the resistance of the infected subject, being in guineapigs and mice from twenty-four to forty-eight hours. The quantity of infectious material introduced, on the other hand, has little bearing upon the final outcome, a few bacilli, or even a single bacillus, often sufficing to bring about a fatal infection. Although the bacilli are not demonstrable in the blood until just before death, they nevertheless invade the blood and lymph streams immediately after inoculation, and are conveyed by these to all the organs. This has been demonstrated clearly by experiments where inoculations into the tail or ear were immediately followed by amputation of the inoculated parts without prevention of the fatal general infection. The bacilli are probably not at first able to multiply in the blood. At the place of inoculation and probably in the organs they proliferate, until the resistance of the infected subject is entirely overcome. At this stage of the disease, no longer held at bay by any antagonistic qualities of the blood, they enter the circulation and multiply within it. Autopsy upon such animals reveals an edematous hemorrhagic infiltration at the point of inoculation. The spleen is enlarged and congested. The kidneys are congested, and there may be hemorrhagic spots upon the serous membranes. The bacilli are found in large numbers in the blood and in the capillaries of all the organs.

The mode of action of Bacillus anthracis is as yet an unsettled point. It is probable that death is brought about to a large extent by purely

1 Toussaint, Comptes rend. de l'acad. des sci., xci, 1880; Pasteur, Chamberland et Roux, Comptes rend. de l'acad. des sci., xcii, 1881.

${ }^{2}$ Chamberland et Roux, ibid., xcvi, 1882. 
mechanical means, such as capillary obstruction. Neither a true secretory toxin nor an endotoxin has been demonstrated for the anthrax bacillus. The decidedly toxemic clinical picture of the disease, however, in some animals and in man, precludes our definitely concluding that such poisons do not exist. It is a matter of fact, however, that neither culture filtrates nor dead bacilli have any noticeable toxic effect upon test animals, and exert no appreciable immunizing action.

Spontaneous infection of animals takes place largely by way of the alimentary canal, the bacilli being taken in with the food. The bacteria are swallowed as spores, and therefore resist the acid gastric juice. In the intestines they develop into the vegetative forms, increase, and gradually invade the system. The large majority of cattle infections are of this type. Direct subcutaneous infection may also occur spontaneously when small punctures and abrasions about the mouth are made by the sharp spicules of the hay, straw, or other varieties of fodder.

When infection upon a visible part occurs, there is formed a diffuse, tense local swelling, not unlike a large carbuncle. The center of this may be marked by a black, necrotic slough, or may contain a pustular depression.

Infection by inhalation is probably rare among animals. Transmission among animals is usually by the agency of the excreta or unburned carcasses of infected animals. The bacilli escaping from the body are deposited upon the earth together with animal and vegetable matter, which forms a suitable medium for sporulation. The spores may then remain in the immediate vicinity, or may be scattered by rain and wind over considerable areas. The danger from buried carcasses, at first suspected by Pasteur, is probably very slight, owing to the fact that the bacilli can not sporulate in the anaërobic environment to which the burying-process subjects them. The disease, in infected cattle and sheep, is usually acute, killing within one or two days. The mortality is extremely high, fluctuating about eighty per cent.

In man the disease is usually acquired by cutaneous inoculation. It may also occur by inhalation and through the alimentary tract.

Cutaneous inoculation occurs usually through small abrasions or scratches upon the skin in men who habitually handle live-stock, and in butchers, or tanners of hides. Infection occurs most frequently upon the hands and forearms. The primary lesion, often spoken of as "malignant pustule," appears within twelve to twenty-four hours after inoculation, and resembles, at first, an ordinary small furuncle. Soon, however, its center will show a vesicle filled with sero-sanguineous, later sero- 
purulent fluid. This may change into a black central necrosis surrounded by an angry red edematous areola. Occasionally local gangrene and general systemic infection may lead to death within five or six days. More frequently, however, especially if prompt excision is practiced, the patient recovers. The early diagnosis of the condition is best made bacteriologically by finding the bacilli in the local discharge.

The pulmonary infection, known as "wool-sorter's disease," occurs in persons who handle raw wool, hides, or horse hair, by the inhalation or by the swallowing of spores. The disease is fortunately rare in this country. The spores, once inhaled, develop into the vegetative forms ${ }^{1}$ and these travel along the lymphatics into the lungs and pleura. The disease manifests itself as a violent, inregular pneumonia, which, in the majority of cases, leads to death. The bacilli in these cases can often be found in the sputum before death.

Infection through the alimentary canal may occasionally, though rarely, occur in man, the source of infection being usually ingestion of the uncooked meat of infected animals. This form of infection is rare, because in many cases the bacilli have not sporulated in the animal and the ingested vegetative forms are injured or destroyed by the acid gastric juice. When viable spores enter the gut, however, infection may take place, the initial lesion being localized usually in the small intestine. The clinical picture that follows is one of violent enteritis with bloody stools and great prostration. Death is the rule. The diagnosis is made by the discovery of the bacilli in the feces.

General hygienic prophylaxis against anthrax consists chiefly in the destruction of infected animals, in the burying of cadavers, and in the disinfection of stables, etc. The practical impossibility of destroying the anthrax spores in infected pastures, etc., makes it necessary to resort to prophylactic immunization of cattle and sheep.

Immunity against Anthrax.-Minute quantities of virulent anthrax cultures usually suffice to produce death in susceptible animals. Dead cultures are inefficient in calling forth any immunity in treated subjects. It is necessary, therefore, for the production of active immunity to resort to attenuated cultures. The safest way to accomplish such attenuation is the one originated by Pasteur, ${ }^{2}$ consisting in prolonged cultivation of the bacillus at $42^{\circ}$ to $43^{\circ} \mathrm{C}$. in broth. Non-spore-forming races are thus evolved.

The longer the bacilli are grown at the above temperature the greater 
is the reduction in their virulence. Koch, Gaffky, and Loeffler, ${ }^{1}$ utilizing the variations in susceptibilities of different species of animals, devised a method by means of which the relative attenuation of a given culture may be estimated and standardized. Rabbits are less susceptible than guineapigs, and virulent anthrax cultures, grown for two or three days under the stated conditions, lose their power to kill rabbits, but are less virulent for guinea-pigs. After ten to twenty days of further cultivation at $42^{\circ} \mathrm{C}$. the virulence for the guinea-pig disappears, but the culture is potent against the still more susceptible mouse. Even the virulence for mice may be entirely eliminated by further cultivation at this temperature.

The method of active immunization first practiced by Pasteur, and still used extensively, is carried out as follows: Two anthrax cultures of varying degrees of attenuation are used as vaccins. The premier vaccin is a culture which has lost its virulence for guinea-pigs and rabbits, and is potent only against mice. The deuxième vaccin is a culture which is still definitely virulent for mice and guinea-pigs, but not potent for rabbits. Forty-eight-hour broth cultures of these strains, grown at $37.5^{\circ} \mathrm{C}$, form the vaccin actually employed. Vaccin $I$ is subcutaneously injected into cattle in doses of 0.25 c.c., sheep receiving about half this quantity. After twelve days have elapsed similar quantities of Vaccin II are injected.

Pasteur's method has given excellent results and confers an immunity which lasts about a year.

Chauveau ${ }^{2}$ has modified Pasteur's method by growing the bacilli in bouillon at $38^{\circ}$ to $39^{\circ} \mathrm{C}$, at a pressure of eight atmospheres. Cultures are then made of races attenuated in this way, upon chicken bouillon and allowed to develop for thirty days. Single injections of 0.1 c.c. each of such cultures are said to protect cattle.

Active immunization of small laboratory animals is very difficult, but can be accomplished by careful treatment with extremely attenuated cultures.

Passive immunization by means of the serum of actively immune animals was first successfully accomplished by Sclavo. ${ }^{3}$

The subject of passive immunization has been especially investigated and practically applied by Sobernheim. ${ }^{4}$, The serum used is produced by actively immunizing sheep. It is necessary to carry immunization to an

1 Koch, Gaffky, und Loeffler, Mitt. a. d. kais. Gesundheitsamt, 1884.

${ }^{2}$ Chauveau, Comptes rend. de l'acad. des sci., 1884.

3 Sclavo, Cent. f. Bakt., xviii, 1895.

Sobernheim, Zeit. f. Hyg., xxv, 1897; xxxi, 1899. 
extremely high degree in order to obtain any appreciable protective. power in the serum. This is accomplished by preliminary treatment with Pasteur's or other attenuated vaccines, followed by gradually increasing doses of fully virulent cultures. Treatment continued at intervals of two weeks, for two or three months, usually produces an effective serum. Horses and cattle may also be used for the process, but they are believed by Sobernheim to give less active sera than sheep. Bleeding is done about three weeks after the last injection. The sera are stable and easily preserved.

Injections of 20 to 25 c.c. of such a serum have been found to protect animals effectually from anthrax and to confer an immunity lasting often as long as two months. Animals already infected are said to be saved by treatment with 25 to 100 c.c. of the serum.

Neither specific bactericidal nor bacteriolytic properties have, so far, been demonstrated in these immune sera. In fact, these properties are distinctly more pronounced against Bacillus anthracis in the normal sera of rats and dogs. Agglutinins have not been satisfactorily demonstrated in sera, partly because of the great technical difficulties encountered in the active chain-formation of the bacillus in fluid media. An increase of opsonic power of such serum over normal serum has not been satisfactorily demonstrated.

Bacteria Closely Resembling Bacillus anthracis.-In most laboratory collections there are strains of true anthrax bacilli so attenuated that they are practically non-pathogenic. These do not differ from the virulent strains in any morphological or cultural characteristics. Besides such strains there are numerous non-virulent bacteria culturally not identical with Bacillus anthracis, but resembling it very closely.

B. Anthracoides (Hueppe and Wood ${ }^{1}$ ).-A Gram-positive bacillus, morphologically different from $B$. anthracis in that the ends are more rounded. Culturally, somewhat more rapid in growth and more rapid in gelatin fluidification. Non-pathogenic. Otherwise indistinguishable from B. anthracis.

B. RAdicosus (Wurzel Bacillus).-Cultivated from water-city water supplies. Morphologically somewhat larger than Bacillus anthracis, and the individual bacilli more irregular in size. Very rapid fluidification of gelatin and growth most active at room temperature. Non-pathogenic.

B. subtilis (Hay Bacillus).--Although not very closely related to the anthrax group, this bacillus is somewhat similar and conveniently

${ }^{1}$ Hueppe und Wood, Berl. klin. Woch., xvi, 1889. 
described in this connection. It is of importance to workers with pathogenic bacteria, because of the frequency with which it is found as a saprophyte or secondary invader in chronic suppurative lesions.

Morphology.-Straightrod, 2 to 8 micra long, 0.7 micron wide. Spores formed usually slightly nearer one pole than the other. Grows in long chains and only in such chains are spores found. It does not decolorize by Gram's method. Is actively motile in young cultures in which

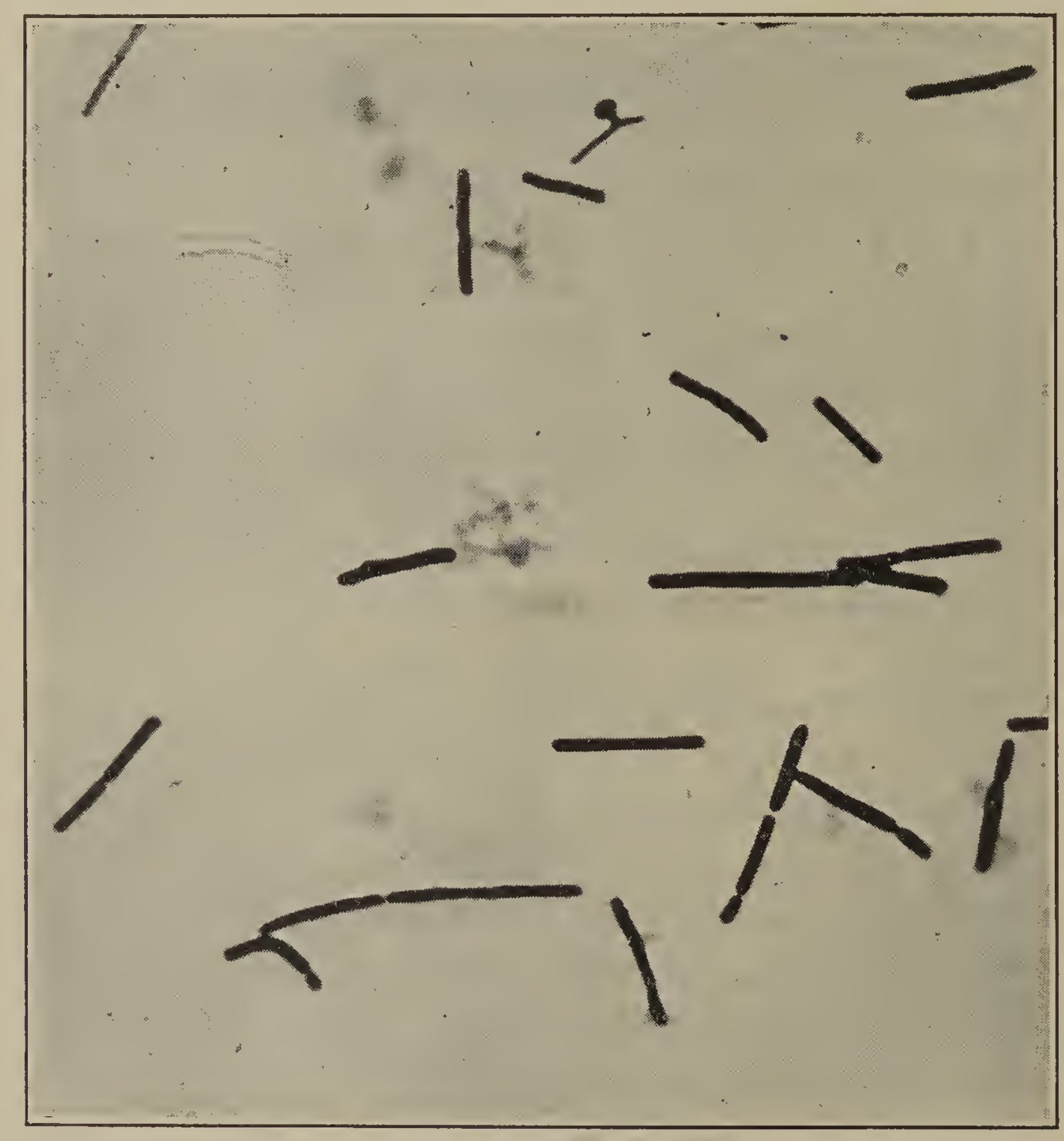

Fig. 125.-Bacillus Subtilis. (Hay Bacillus.)

the bacilli are single or in pairs. In older cultures chains are formed and the bacilli become motionless. Gelatin is liquefied. On gelatin and agar the bacilli grow as a dry corrugated pellicle. Microscopically, the colonies are made up of interlacing threads, being irregularly round with fringed edges. There is a tendency to confluence. The bacillus is found in brackish water, infusions of vegetable matter, etc., and is practically non-pathogenic, occurring onlý occasionally as a saprophyte in old sinuses and infected wounds. 


\section{CHAPTER XLI}

\section{BACILLUS PYOCYANEUS}

IT is a matter of common surgical experience that many suppurating wounds, especially sinuses of long standing, discharge pus which is of a bright green color. The fact that this peculiar type of purulent inflammation is due to a specific chromogenic microorganism was first demonstrated by Gessard ${ }^{1}$ in 1882 . The bacillus which was described by Gessard has since become the subject of much careful research and has been shown to hold a not unimportant place among pathogenic bacteria. ${ }^{2}$

Morphology and Staining.-Bacillus pyocyaneus is a short rod, usually straight, occasionally slightly curved, measuring, according to Flügge, about 1 to 2 micra in length by about 0.3 of a micron in thickness. The bacilli are thus small and slender, but are subject to considerable variation from the measurements given, even in one and the same culture. While ordinarily single, the bacilli may be arranged end to end in short chains of two and three. Longer chains may exceptionally be formed upon media which are especially unfavorable for its growth, such as very acid media or those containing antiseptics.

Spores are not found. The bacilli are actively motile and possess each a single flagellum placed at one end.

Bacillus pyocyaneus is stained easily with all the usual dyes, but is decolorized by Gram's method. Irregular staining of the bacillary body is common, but is always an indication of degeneration, and not a normal characteristic, as, for instance, in the diphtheria group.

Cultivation.-The pyocyaneus bacillus is aërobic and facultatively anaërobic. It can be adapted to absolutely anaërobic environments, but does not produce its characteristic pigment without the free access of oxygen. The bacillus grows readily upon the usual laboratory media and is not very sensitive to reaction, growing equally well upon moderately alkaline or acid media. Development takes place at temperatures as low as $18^{\circ}$ to $20^{\circ} \mathrm{C}$, more rapidly and luxuriantly at $37.5^{\circ} \mathrm{C}$.

${ }^{1}$ Gessard, Thèse de Paris, 1882.

2 Charrin, "La maladie pyocyanique," Paris, 1889. 
On agar slants, growth is abundant and confluent, the surface of the agar being covered by a moist, grayish or yellowish, glistening, even layer. The pigment which begins to become visible after about eighteen hours soon penetrates the agar itself and becomes diffused throughout it, giving the medium a bright green fluorescent appearance, which grows darker as the age of the culture increases.

In gelatin stabs, growth takes place much more rapidly upon the surface than in the depths. A rapid liquefaction of the gelatin takes place, causing a saucer-shaped depression. As this deepens, pigment begins to form in the upper layers, often visible as a greenish pellicle.

In gelatin plates, the colonies have a characteristic appearance. They are round and are composed of a central dense zone, and a peripheral, loosely granular zone, which extends outward into the peripheral fluidified area in a fringe of fine filaments. When first appearing, they are grayish yellow, later assuming the characteristic greenish hue.

In broth, growth is rapid and chiefly at the surface, forming a thick pellicle. Below this, there is moderate clouding. The pigment is formed chiefly at the top. In old cultures there is a heavy flocculent precipitate. In fluid media containing albuminous material, strong alkalinity is produced.

On potato, growth develops readily and a deep brownish pigment appears, which is not unlike that produced by $B$. mallei upon the same medium.

Milk is coagulated by precipitation of casein and assumes a yellowishgreen hue. In older cultures the casein may again be digested and liquefied

The pigment of Bacillus pyocyaneus has been the subject of much investigation. It was shown by Charrin ${ }^{1}$ and others that this pigment had no relation to the pathogenic properties of the bacillus. It is found in cultures as a colorless leukobase which assumes a green color on the addition of oxygen. Conversely, the typical green "pyocyanin," as the pigment is called, may be decolorized by reducing substances. This explains the fact that it is not found in cultures sealed from the air. Pyocyanin may be extracted from cultures with chloroform and crystallized out of such solution in the form of blue stellate crystals. These, on chemical analysis, have been found to belong to the group of aromatic compounds, with a formula, according to Ledderhose, of $\mathrm{C}_{14} \mathrm{H}_{14} \mathrm{~N}_{2} \mathrm{O}$.

Besides pyocyanin, Bacillus pyocyaneus produces another pig-

${ }^{1}$ Charrin, loc. cit.

Ledderhose, quoted from Boland, Cent. f. Bakt., xxv, 1889. 
ment which is fluorescent and insoluble in chloroform, but soluble in water. ${ }^{1}$ This pigment is common to other fluorescent bacteria, and not peculiar to Bacillus pyocyaneus. The reddish-brown color seen in old cultures $^{2}$ and supposed by some writers to be a third pigment, is probably a derivative from pyocyanin by chemical change.

Chloroform extraction of pyocyanin from cultures may serve occasionally to distinguish the pyocyaneus bacilli from other similar fluorescent bacteria. Ernst has claimed that there are two types of B. pyocyaneus, an $\alpha$-type which produces only the fluorescent, watersoluble pigment, and a $\beta$-type which produces both this and pyocyanin. ${ }^{3}$

Pathogenicity.-Bacillus pyocyaneus is one of the less virulent pathogenic bacteria. It is widely distributed in nature and may be found frequently as a harmless parasite upon the skin or in the upper respiratory tracts of animals and men. It has, however, occasionally been found in connection with suppurative lesions of various parts of the body, often as a mere secondary invader in the wake of another incitant, or even as the primary cause of the inflammation. In most cases where true pyocyaneus infection has taken place, the subject is usually one whose general condition and resistance are abnormally low. ${ }^{4}$ Thus pyocyaneus may be the cause of chronic otitis media in ill-nourished children. It has been cultivated out of the stools of children suffering from diarrhea, and has been found at autopsy generally distributed throughout the organs of children dead of gastro-enteritis. ${ }^{5}$ It has been cultivated from the spleen at autopsy from a case of general sepsis following mastoid operation. The bacillus has been found, furthermore, during life in pericardial exudate and in pus from liver abscesses. ${ }^{6}$

Brill and Libman, ${ }^{7}$ as well as Finkelstein, ${ }^{8}$ have cultivated B. pyocyaneus from the blood of patients suffering from general sepsis. Wassermann ${ }^{9}$ showed the bacillus to have bcen the etiological factor in an epidemic of umbilical infections in new-born children. Similar examples of B. pyocyaneus infection in human beings might be enumerated in large numbers, and there is no good reason to doubt that, under given

Boland, loc. cit.

${ }^{2}$ Gessard, Ann. de l'inst. Pasteur, 1890, 1891, and 1892.

3 Ernst, Zeit. f. Hyg., ii, 1887.

Rohner, Cent. f. Bakt., xi, 1892.

s Neumann, Jahrb. f. Kinderheilk., 1890.

S Kraunhals, Zeit. f. Chir., xxxvii, 1893.

7 Brill and Libman, Amer. Jour. Med. Sci., 1899.

- Finkelstein, Cent. f. Bakt., 1899.

-Wassermann, Virchow's Arch., clxv, 1901. 
conditions, fatal infections may occur. Such cases, however, are still to be regarded as depending mole upon the low resistance of the individual attacked than upon the great pathogenicity of B. pyocyaneus.

Many domestic animals are susceptible to experimental pyocyaneus infection, chief among these being rabbits, goats, mice, and guineapigs. Guinea-pigs are killed by this bacillus with especial ease. Intraperitoneal inoculation with a loopful of a culture of average virulence usually leads to the death of a young guinea-pig within three or four days.

Toxins and Immunization.-Emmerich and Löw have shown that filtrates of old broth cultures of B. pyocyaneus contain a ferment-like substance which possesses the power to destroy some other bacteria, apparently by lysis. They have called this substance "pyocyanase" and claim that, with it, they have succeeded in protecting animals from anthrax infection. During recent years pyocyanase has been employed locally for the removal of diphtheria bacilli from the throats of convalescent cases. Broth-culture filtrates evaporated to one-tenth their volume in vacuo are used for this purpose.

Pyocyanase is exceedingly thermostable, resisting boiling for several hours, and is probably not identical with any of the other toxins or peptonizing ferments produced by B. pyocyaneus.

The toxins proper of $B$. pyocyaneus have been the subject of much investigation, chiefly by Wassermann. ${ }^{1}$ Wassermann found that filtrates of old cultures were far more poisonous for guinea-pigs than extracts made of dead bacteria. He concludes from this and other observations that B. pyocyaneus produces both an endotoxin and a soluble secreted toxin. The toxin is comparatively thermostable, resisting $100^{\circ} \mathrm{C}$. for a short time. Animals actively immunized with living cultures of B. pyocyaneus give rise in their blood serum to bacteriolytic antibodies only. Immunized with filtrates from old cultures, on the other hand, their serum will contain both bacteriolytic and antitoxic substances. The true toxin of B. pyocyaneus never approaches in strength that of diphtheria or of tetanus. Active immunization of animals must be done carefully if it is desired to produce an immune serum, since repeated injections cause great emaciation and general loss of strength. Specific agglutinins have been found in immune sera by Wassermann ${ }^{2}$ and others. Eisenberg ${ }^{3}$ claims that such agglutinins are active also against some of the fluorescent intestinal bacteria.

1 Wassermann, Zeit. f. Hyg., xxii, 1896. ${ }^{2}$ Wassermann, Zeit. f. Hyg., 1902.

${ }^{3}$ Eisenberg, Cent. f. Bakt., 1903. 
Bulloch and Hunter ${ }^{1}$ have recently been able to show that old broth cultures of $B$. pyocyaneus contain a substance capable of hemolyzing the red blood corpuscles of dogs, rabbits, and sheep. This "pyocyanolysin" seems intimately attached to the bacterial body. Prolonged heating of cultures does not destroy it. Heating of hemolytic filtrates, however, destroys it in a short time. The filtration of young cultures yields very little pyocyanolysin in the filtrate. In old cultures, however, a considerable amount passes into the filtrate. Whether or not the hemolytic power is due to a specific bacterial product or is dependent upon changes in the culture fluid, such as alkalinization, etc., can not yet be regarded as certain.

Gheorghiewski ${ }^{2}$ claims to have found a leucocyte-destroying ferment in pyocyaneus cultures.

1 Bulloch und Hunter, Cent. f. Bakt., xxviii, 1900.

'Gheorghiewski, Ann. de l'inst. Pasteur, xiii, 1899. 


\section{CHAPTER XITT}

\section{ASIATIC CHOLERA AND THE CHOLERA ORGANISM}

\section{(Spirillum cholerce asiatica, Comma Bacillus)}

The organism of Asiatic cholera was unknown until 1883. In this year, Koch, ${ }^{1}$ at the head of a commission established by the German government to study the disease in Egypt and India, discovered the "comma bacillus" in the defecations of patients, and satisfactorily determined its etiological significance.

Koch's investigations were carried out on a large number of cases and many investigations have since then corroborated his results. The numerous morphologically similar spirilla which were later found in normal individuals and in connection with other conditions, have been shown by accurate bacteriological methods to be closely related, but not identical.

Apart from the evidence of the constant association of the cholera vibrio with the disease, the etiological relationship has been clearly demonstrated by several accurately recorded accidental infections occurring in bacteriological workers, and by the famous experiment of Pettenkofer and Emmerich, who purposely drank water containing cholera bacilli. Both observers became seriously ill with typical clinical symptoms of cholera, and one of them narrowly escaped death.

Morphology and Staining.- The vibrio or spirillum of cholera is a small curved rod, varying from one to two micra in length. The degree of curvature may vary from the slightly bent, comma-like form to a more or less distinct spiral with one or two turns. The spirals do not lie in the same plane, being arranged in corkscrew fashion in three dimensions. The spirillum is actively motile and owes its motility to a single polar flagellum, best demonstrated by Van Ermengem's flagella stain. Spores are not found. In young cultures the comma shapes predominate, in older growths the longer forms are more numerous. Strains which have been cultivated artificially for prolonged 
periods without passage through the animal body have a tendency to lose the curve, assuming a more bacillus-like appearance. The spirilla are stained with all the usual aqueous anilin dyes. They are decolorized by Gram's method. In histological section they are less easily stained, but may be demonstrated by staining with alkaline methylene blue.

Cultivation.-The cholera spirillum grows easily upon all the usual culture media, thriving upon meat-extract as well as upon meat-infusion

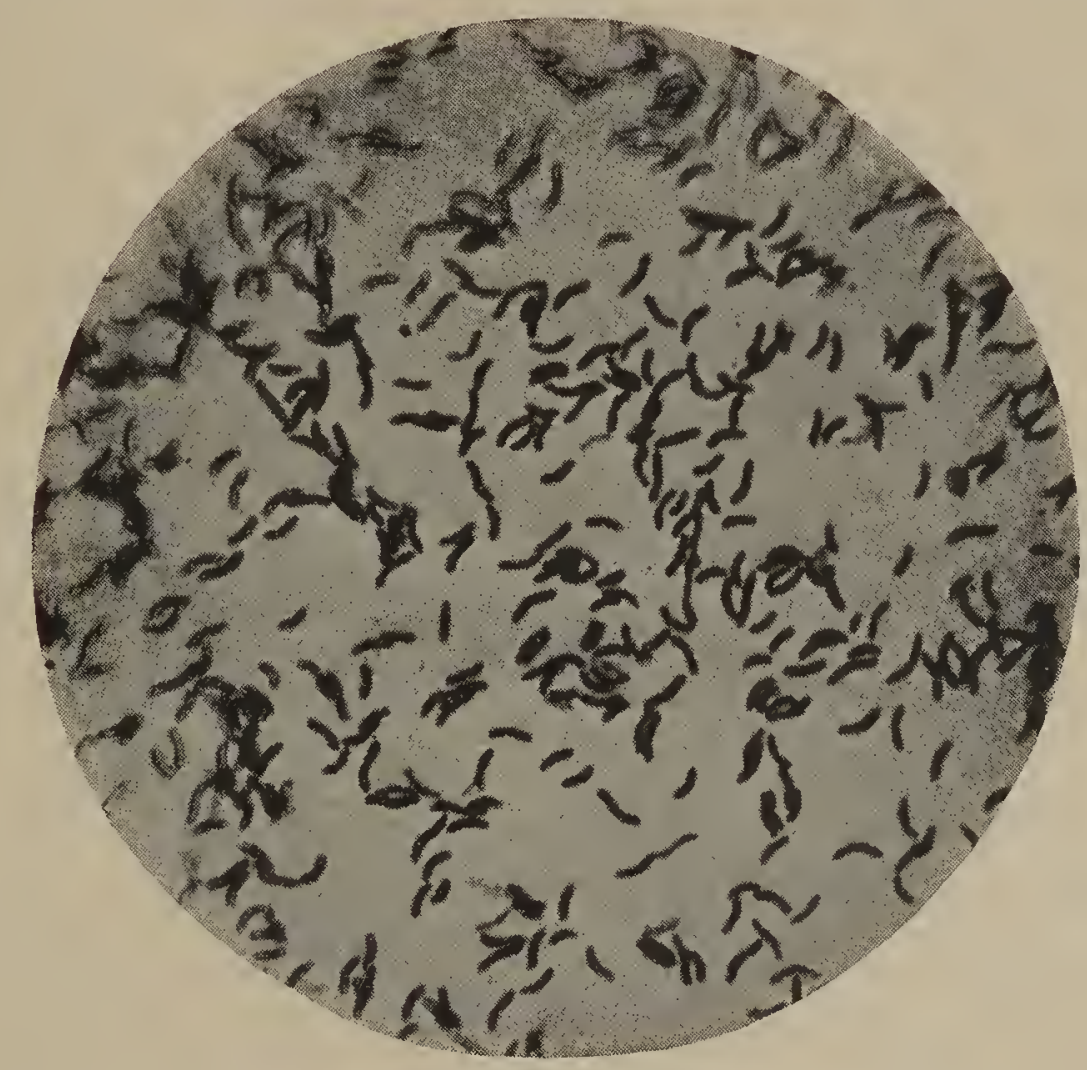

Fig. 126.-Cholera Spirillum. (After Fränkel and Pfeiffer.)

media. Moderate alkalinity of the media is preferable, though slight acidity does not prevent growth.

In gelatin plates growth appears at room temperature within twentyfour hours as small, strongly refracting yellowish-gray, pin-head colonies. As growth increases the gelatin is fluidified. Under magnification these colonies appear coarsely granular with margins irregular because of the liquefaction. Liquefaction, too, causes a rapid development in such colonies of separate concentric zones of varying refractive power. Old strains, artificially cultivated for long periods, lose much of their liquefying power.

In gelatin stab cultures fluidification begins at the surface, rapidly giving rise to the familiar funnel-shaped excavation.

Upon agar plates, within eighteen to twenty-four hours, grayish, opalescent colonies appear, which are easily differentiated by their 
transparency from the other bacteria apt to appear in feces. Agar plates, therefore, are important in the isolation of these organisms.

Coagulated blood serum is fluidified by the cholera vibrio. On potato, growth is profuse and appears as a brownish coarse layer. In milk, growth is rapid and without coagulation. In broth, general clouding and the formation of a pellicle result. The rapidity and luxuriance of growth of the cholera spirillum upon alkaline pepton solutions render such solutions peculiarly useful as enriching media in isolating this microorganism from the stools of patients. In pepton solution, too, the cholera spirillum gives rise to abundant indol, demonstrated in the so-called "cholera-red" reaction. This reaction has a distinct diagnostic value, but is by no means specific. ${ }^{1}$ In the case of the cholera vibrio the mere addition of strong sulphuric acid suffices to bring out the color reaction. This is due to the fact that, unlike some other indolproducing bacteria, the cholera organism is able to reduce the nitrates present in the medium to nitrites, thus itself furnishing the nitrite necessary for the color reaction. The medium which is most suitable for this test is that proposed by Dunham, ${ }^{2}$ consisting of a solution of 1 per cent of pure pepton and .5 per cent $\mathrm{NaCl}$ in water.

Dieudonné $^{3}$ has recommended a selective medium upon which cholera spirilla will grow well, but upon which the colon bacillus will grow either very sparsely or not at all. Its preparation is very simple. To 70 parts of ordinary 3 per cent agar, neutralized to litmus, there are added 30 parts of a sterile mixture of defibrinated beef blood and normal sodium hydrate.

The latter is sterilized by steam before being added to the agar. This pure alkali agar is poured out in plates and allowed to dry several days at $37^{\circ}$ or 5 minutes at $60^{\circ}$. The material to be examined is smeared upon the surface of these plates with a glass rod.

The principle of this medium is that cholera will grow in the presence of an amount of alkali which inhibits other fecal bacteria. The medium has been studied by Krumwiede, Pratt, and Grund, ${ }^{4}$ who have recommended a modification. They find the following combination satisfactory and an improvement upon Dieudonné's medium becauss transparent and more easily prepared. They prepare the following mixtures:

${ }^{1}$ See indol reaction, p. 167.

${ }^{2}$ Dunham, Zeit. f. Hyg., ii, 1887.

${ }^{3}$ Dieudonné, A., Cent. Bakt., 1., orig., 1909.

${ }^{4}$ Krumwiede, Pratt, and Grund, Jour. of Inf. Dis., x, 1912. 
Egg-White Medium.

A. White of egg and water a.a.

Sodium carbonate cryst. 12 per cent.

Mix in equal parts, steam in Arnold sterilizer for 20 minutes.

B. Meat pepton 3 per cent agar, neutral to litmus.

30 parts of $\mathrm{A}$ are added to 70 parts of $\mathrm{B}$.

Another modification recommended by them is as follows:

Whole-Egg Medium.

A. Whole egg and water a.a.

Sodium carbonate 12 to 13.5 per cent.

Mix in equal parts, steam for 20 minutes.

B. Meat free agar, viz., pepton, salt, and 3 per cent agar.

30 parts of $\mathrm{A}$ are mixed with 70 parts of $\mathrm{B}$ while the agar is boiling hot as above.

The medium is poured on the plates in a thick layer and allowed to stand open for 20 to 30 minutes and then the inoculation is carried out by surface streaking.

Isolation.-Isolation of the cholera vibrio from the feces, while easy in many cases, is occasionally attended with some difficulty owing to the large number of other bacteria present. The most satisfactory method of procedure is to inoculate a set of gelatin plates, another of agar plates, and a number of Dunham's peptonbroth tubes, with small quantities of the suspicious material. When the spirilla are numerous they can frequently be fished directly from suspicious colonies in the plates and isolated for further identification. When less numerous, they can usually be found in relatively increased numbers after eight or ten hours at $37.5^{\circ} \mathrm{C}$., in the topmost layers of the Dunham broth, which is an almost selectively favorable medium for these organisms. They collect at the surface where free oxygen is readily obtained. From the pepton broth, plate dilutions can then be prepared and colonies fished. ${ }^{1}$ Once isolated, the spirilla are identified by their morphology, by the appearance of their colonies, by their manner of growth upon gelatin stabs, by the cholera-red reaction, and, finally, by agglutinative and bacteriolytic tests in immune sera. Owing to the existence of other spirilla morphologically and culturally similar, the serum reactions are the only absolutely positive differential criteria. 
For isolation of the bacteria from water, it is, of course, necessary to use comparatively large quantities. Flügge ${ }^{1}$ and Bitter advise the distribution of about a liter of water in ten or twelve Erlenmeyer flasks. To each of these they add 10 c.c. of sterile pepton-salt solution (pepton ten per cent, $\mathrm{NaCl}$ five per cent). After eighteen hours at $37.5^{\circ} \mathrm{C}$. the surface growths in these flasks are examined both microscopically and culturally as before.

Biological Considerations.-The cholera spirillum is aërobic and facultatively anaërobic. It does not form spores. The optimum temperature for its growth is about $37.5^{\circ} \mathrm{C}$. It grows easily, however, at a temperature of $22^{\circ} \mathrm{C}$. and does not cease to grow at temperatures as high as $40^{\circ}$. Frozen in ice, these bacteria may live for about three or four days. Boiling destroys them immediately. A temperature of

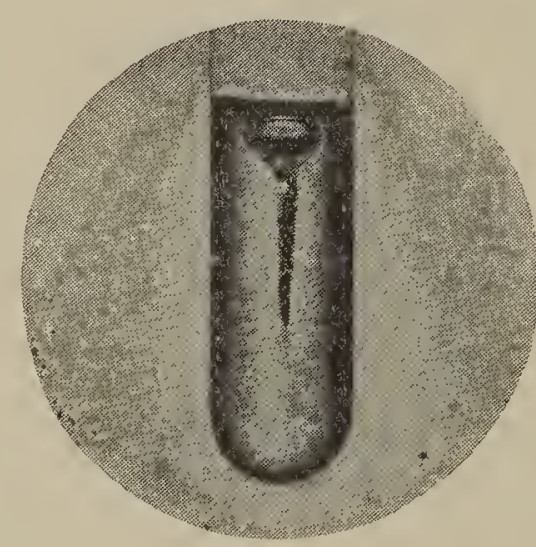

FIG. 127.

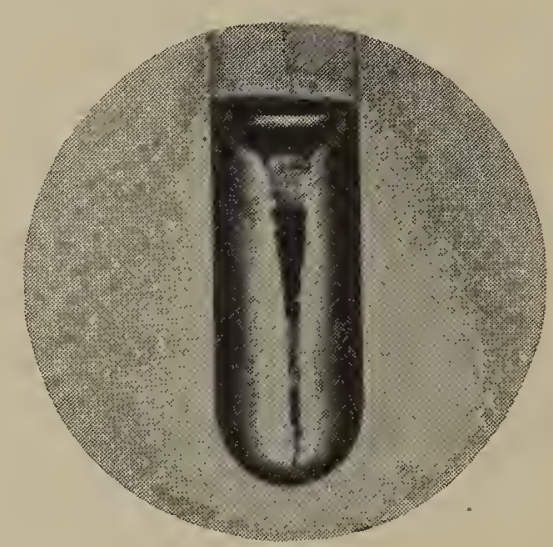

FIG. 128.

Fig. 127.-Cholera Sprrillum. Stab Culture in Gelatin, three days old.

Fig. 128.-Cholera Spirillum. Stab Culture in Gelatin, six days old. (After Fränkel and Pfeiffer.)

$60^{\circ} \mathrm{C}$. kills them in an hour. In impure water, in moist linen, and in food stuffs, they may live for many days. Associated with saprophytes in feces and other putrefying material, and wherever active acid formation is taking place, they are destroyed within several days. Complete drying kills them in a short time. The common disinfectants destroy them in weak solutions and after short exposures (carbolic acid, five-tenths per cent in one-half hour; bichlorid of mercury, $1: 100,000$ in ten minutes; mineral acids, $1: 5,000$ or 10,000 in a few minutes). ${ }^{2}$

Pathogenicity.-Cholera is essentially a disease of man. Endemic in India and other Eastern countries, it has from time to time epidemically invaded large territories of Europe and Asia, not infrequently assuming

${ }^{1}$ Flügge, Zeit. f. Hyg., xiv, 1893.

${ }^{2}$ Forster, Hyg. Rundschau, 1893. 
pandemic proportions and sweeping over almost the entire earth. ${ }^{1}$ Five separate cholera epidemics of appalling magnitude occurred during the nineteenth century alone; several of these, spreading from India to Asia Minor, Egypt, Russia, and the countries of Central Europe, reached even to North and South America. The last great epidemic began about 1883, traveled gradually westward, and in 1892 reached Germany where it appeared with especial virulence in Hamburg, and thence, following the highways of ocean commerce, entered America and Africa. During this epidemic in Russia alone 800,000 people fell victims to the disease.

In man the disease is contracted by ingestion of cholera organisms with water, food, or any contaminated material. The disease is essentially an intestinal one. The bacteria, very sensitive to an acid reaction, may often, if in small numbers, be checked by the normal gastric secretions. Having once passed into the intestine, however, they proliferate rapidly, often completely outgrowing the normal intestinal flora. Fatal cases, at autopsy, show extreme congestion of the intestinal walls. Occasionally ecchymosis and localized necrosis of the mucosa may be present and swelling of the solitary lymph-follicles and Peyer's patches. Microscopically the cholera spirilla may be seen to have penetrated the mucosa and to lie within its deepest layers close to the submucosa. The most marked changes usually take place in the lower half of the small intestine. The intestines are filled with the characteristically fluid, slightly bloody, or "rice-water" stools, from which often pure cultures of the cholera vibrio can be grown. The microorganisms can be cultivated only from the intestines and their contents, and the parenchymatous degenerations taking place in other organs must be interpreted as being purely of toxic origin.

In animals, cholera never appears as a spontaneous disease. Nikati and Rietsch ${ }^{2}$ have succeeded in producing a fatal disease in guinea-pigs by opening the peritoneum and injecting cholera spirilla directly into the duodenum. Koch ${ }^{3}$ succeeded in producing a fatal cholera-like disease in animals by introducing infected water into the stomach through a catheter after neutralization of the gastric juice with sodium carbonate. At the same time, he administered opium to prevent active peristalsis. A method of infection more closely analogous to the infec-

${ }^{1}$ Hirsch, "Handb. d. histor.-geogr. Path.," 1881.

${ }^{2}$ Nikati und Rietsch, Deut. med. Woch., 1884.

${ }^{3}$ Koch, Deut. med. Woch., 1885. 
tion in man was followed by Metchnikoff, ${ }^{1}$ who successfully produced fatal disease in young suckling rabbits by contaminating the maternal teat.

Subcutaneous inoculation of moderate quantities of cholera spirilla into rabbits and guinea-pigs rarely produces more than a temporary illness. Intraperitoneal inoculation, if in proper quantities, generally leads to death. It will be remembered that when working with intraperitoneal cholera inoculations the phenomenon of bacteriolysis was discovered by Pfeiffer. ${ }^{2}$

Different strains of cholera spirilla vary greatly in their virulence. The virulence of most of them, however, can be enhanced by repeated passages through animals. Most of our domestic animals enjoy considerable resistance against cholera infection, though under experimental conditions successful inoculations upon dogs, cats, and mice have been reported. Doves are entirely insusceptible. ${ }^{3}$

Hygienic Considerations. - The cholera spirillum leaves the body of the infected subject with the defecations only. Infection takes place, so far as we know, only by way of the mouth. From these two facts it follows that the chief source of danger for a community lies in infection of its water supply. As a matter of fact the bacteria have been frequently found in the wells, lakes, rivers, and harbors of afflicted territories, and in several cases it has been possible to define the limits of an epidemic almost precisely by the distribution of the contaminated water supply. A classic example of this is that of the Hamburg epidemic, during which Altona, a town as close to Hamburg as Brooklyn is to New York, with unrestricted interurban traffic but with separate water supply, was almost spared, while Hamburg itself was undergoing one of the most virulent epidemics of its history. It has been statistically noted, moreover, chiefly by Koch, that cholera in its spread not infrequently follows the water courses. Apart from infection through the water supply, cholera may be transmitted directly or indirectly by contact with contaminated linen, bedclothes, etc., the organism being conveyed to the mouth by the fingers, or by infected food. Epidemics due to this mode of infection alone, however, are apt to be more narrowly localized and more sporadic in their manifestations. It is probable that this mode of infection is of great importance in countries where the disease

${ }^{1}$ Metchnikoff, Ann. d. l'inst. Pasteur, 1894 and 1896.

${ }^{2}$ Pfeiffer, loc. cit.

${ }^{3}$ Pfeiffer und Nocht, Zeit. f. Hyg., vii, 1889. 
is endemic, but its significance in producing epidemics is limited owing to the fortunately low resistance of the spirillum to desiccation. The sudden appearance of cholera in a place far distant from the seat of a prevalent epidemic may be explained by the occasional presence of cholera spirilla in the dejecta of convalescents as late as two or three weeks after apparent recovery from the disease and consequent release from quarantine.

Cholera Toxin.-The absence of the cholera spirilla from the internal organs of fatal cases, in spite of the severe general symptoms of the disease, points distinctly to the existence of a strong poison produced in the intestine by the microorganisms and absorbed by the patient. It was in this sense, indeed, that Koch first interpreted the clinical picture of cholera. Numerous investigations into the nature of these toxins have been made, the earlier ones defective in that definite identification of the cultures used for experimentation were not carried out.

Pfeiffer, ${ }^{1}$ in 1892 , was able to show that filtrates of young bouillon cultures of cholera spirilla were but slightly toxic, whereas the dead bodies of carefully killed agar cultures were fatal to guinea-pigs even in small quantities. In consequence, he regarded the cholera poison as consisting chiefly of an endotoxin. ${ }^{2}$ The opinion as to the endotoxic nature of the cholera poison is not, however, shared by all workers. Metchnikoff, Roux, and Salimbeni, ${ }^{3}$ in 1896, succeeded in producing death in guinea-pigs by introduction into their peritoneal cavities of cholera cultures enclosed in celloidin sacs. Brau and Denier, ${ }^{4}$ and, more recently, Kraus, ${ }^{5}$ claim that they have succeeded not only in demonstrating a soluble toxin in alkaline broth cultures of cholera spirilla, but in producing true antitoxins by immunization with such cultures. It appears, therefore, that the poisonous action of the cholera organisms may depend both upon the formation of true secretory toxins and upon endotoxins. Which of these is paramount in the production of the disease can not be at present definitely stated. In favor of the great importance of the endotoxic elements is the failure, thus far, to obtain successful therapeutic results with supposedly antitoxic sera.

1 Pfeiffer, Zeit. f. Hyg., xi, 1892.

${ }^{2}$ Pfeiffer und Wassermann, Zeit. f. Hyg., xiv, 1893.

${ }^{3}$ Metchnikoff, Roux, et Salimbeni, Ann. de l'inst. Pasteur, 1896.

${ }^{4}$ Brau et Denier, Comptes rend. de l'acad. des sci., 1906.

${ }^{5}$ R. Kraus, Cent. f. Bakt., 1906. 
Cholera Immunization.-One attack of cholera confers protection against subsequent infection. Active immunization of animals may be accomplished by inoculation of dead cultures, or of small doses of living bacteria. In the serum of immunized animals specific bacteriolytic and agglutinating substances are found. The discovery of bacteriolytic immune bodies, in fact, was made by means of cholera spirilla. Both the bacteriolysins and the agglutinins, because of their specificity, are of great importance in making a bacteriological diagnosis of true cholera organisms.

Protective inoculation of man has been variously attempted by Ferran ${ }^{1}$ and others. Experiments on a large scale were done, more recently, by Haffkine, ${ }^{2}$ who succeeded in producing an apparently distinct prophylactic immunization by the subcutaneous inoculation of dead cholera cultures. Similar immunization with bacterial filtrates has been attempted by Bertarelli. ${ }^{3}$

\section{CHOLERA-LIKE SPIRILLA}

The biological group of the vibriones, to which the cholera spirillum belongs, is a large one, numbering probably over a hundred separate species. Most of these are of bacteriological importance chiefly because of the difficulties which they add to the task of differentiation, for while some of them simply bear a morphological resemblance to the true cholera vibrio, others can be distinguished only by their serum reactions and pathogenicity for various animals. Additional difficulty, too, is contributed by the fact that within the group of true cholera organisms occasional variations in agglutinability and bacteriolytic reactions may exist. Certain strains, too, the six El Tor cultures isolated by Gottschlich, while in every respect similar to true cholera spirilla, are considered as a separate sub-species by Kraus, ${ }^{4}$ because of their ability to produce hemolytic substances, a function lacking in other cholera strains.

Spirillum Metchnikovi. - This spirillum was discovered by Gamaleia ${ }^{5}$ in the feces and blood of domestic fowl, in which it had caused an intestinal disease. Morphologically and in staining reactions it is identical

1 Ferran, Comptes rend. de l'acad. des sciences, 1885.

${ }^{2}$ Haffkine, Bull. méd., 1892.

${ }^{3}$ Bertarelli, Deut. med. Woch., 33, 1904.

4 Kraus, Kraus und Levaditi, "Handbuch," vol. i, p. 186.

'Gamaleia, Ann. de l'inst. Pasteur, 1883. 
with Spirillum choleræ asiaticæ. It possesses a single polar flagellum, and is actively motile. Culturally it is identical with Vibrio choleræ except for slightly more luxuriant growth and more rapid fluidification of gelatin. It gives the cholera-red reaction in pepton media.

It is differentiated from the cholera vibrio by its power to produce a rapidly fatal septicemia in pigeons after subcutaneous inoculation of minute quantities. ${ }^{1}$ It is much more pathogenic for guinea-pigs than the cholera vibrio. It is not subject to lysis or agglutinated by cholera immune sera.

Spirillum Massaua.-This organism was isolated at Massaua by Pasquale ${ }^{2}$ in 1891 from the feces of a clinically doubtful case of cholera. Culturally and morphologically it is much like the true cholera vibrio, but in pathogenicity is closer to Spirillum Metchnikovi, in that small quantities produce septicemia in birds. It possesses four flagella. It does not give a specific serum reaction with cholera immune serum.

Spirillum of Finkler-Prior. ${ }^{3}$ - Isolated by Finkler and Prior from the feces of a case of cholera nostras. Morphologically it is like the true cholera spirillum, though slightly larger and less uniformly curved. Culturally it is much like the cholera vibrio, but grows more rapidly and thickly upon the usual media. It does not give the cholera-red reaction, nor does it give specific serum reactions with cholera immune serum.

Spirillum Deneke. ${ }^{4-A}$ vibrio isolated by Deneke from butter. Much like that of Finkler-Prior. It does not give the cholera-red reaction.

1 Pfeiffer und Nocht, Zeit. f. Hyg., vii, 1889.

${ }^{2}$ Pasquale, Giorn. med. de r. eserc. ed. R. Marina, Roma, 1891.

${ }^{3}$ Finkler und Prior, Ergänz. Hefte, Cent. f. allg. ges. Phys., 1884.

${ }^{4}$ Deneke, Deut. med. Woch., iii, 1885. 


\section{CHAPTER XLIII}

\section{DISEASES CAUSED BY SPIROCHATES (TREPONEMATA)}

The microorganisms known as spirochætes are slender, undulating; corkscrew-like threads which show definite variations both structurally and culturally from the bacteria as a class. Most important among them are the spirochæte of relapsing fever, Spirochæte pallida of syphilis, the spirillum of Vincent, Spirochæte refringens, Spirillum gallinarum, a microorganism which causes disease in chickens, Spirochæte anserina, which causes a similar condition in geese, and several species which have been found as parasites, both in animals and in man, without having definite etiological connection with disease.

The classification of these various species in one group is rather more a matter of convenience than one of scientific accuracy, since our knowiedge of them is not far advanced, and our inability to cultivate almost all of them has not permitted their detailed biological study. Formerly many of these organisms were regarded as bacteria belonging to the general group of the spirilla. Recently Schaudinn, ${ }^{1}$ the discoverer of the syphilis spirochæte, has claimed, upon the basis of a careful morphological study, that many of these forms are actually protozoa. He based this claim upon the observation that stainer preparations often showed undulating membranes extending along the long axis of the microorganisms and that definite nuclear structures were demonstrable. This observer also claimed that many of the spiral forms reproduce by cleavage along the longitudinal axis. Other observers have not agreed with this view, Laveran, ${ }^{2}$ Novy and Knapp, ${ }^{3}$ and others asserting that their own observations indicate a close relationship of these microorganisms to the true bacteria. Whatever the final conclusion may be, the question is more or less an academic one, in that our ideas as to the exact line of division between the unicellular animals and the unicellular plants is not by any means founded upon a sound basis. In common with the bacteria, most

1 Schaudinn, Arb. a. d. kais. Gesundheitsamt, 1904.

2 Laveran, Comptes rend. de l'acad. des sci., 1902 and 1903.

3 Novy and Knapp, Jour. of Infec. Dis., 3, 1906. 
of these microorganisms have the power of multiplication by transverse fission. They possess flagella and, in the case of some of them at least, definite immune bodies can be demonstrated in the serum of infected subjects similar to those produced by bacteria during infection. The undulating membranes and the definite differentiation between nucleus and cytoplasm claimed for them by some observers have not been uniformly confirmed, and their similarity to the trypanosomes has not therefore been established. On the other hand, none of these microorganisms has so far been successfully cultivated upon artificial media, with the exception of the spirilla which occur in Vincent's angina. For some of the diseases caused by this class of parasites, moreover, transmission by an intermediate host, in which the spirilla undergo multiplication, has been definitely shown, a fact which corresponds with the conditions observed in many protozoan infections. Upon a careful review of these various data, it seems to be fully justified, on the basis of our present knowledge, to group these microorganisms, as Kolle and Hetsch ${ }^{1}$ have done, in a class between bacteria and protozoa.

The terms spirochæete and spirillum have been indiscriminately used. In the original classification of Migula the difference between the two groups was based upon the rigidity of the cell body in the case of the spirilla and the sinuous or flexible nature of the cell in the case of the spirochætæ. Although the term spirillum is still colloquially used for some members of this group, merely because of past usage, it would be better to speak of all the microorganisms here grouped together by the term "spirochætes."

\section{SYPHILIS AND SPIROCHATA PALLIDA}

\section{(Treponema pallidum)}

The peculiar manifestations of syphilis, its mode of transmission, and the fact that its primary lesion was always located at the point of contact with a preceding case, have always stamped it as unquestionably infectious in nature. Until very recently the microorganism which gives rise to syphilis was unknown. Many bacteriologists had attacked the problem and many microorganisms for which definite etiological importance was claimed had been described. Most of these announcements, however, aroused little more than a sensational interest and received no satisfactory confirmation. A bacillus described

${ }^{1}$ Kolle und Hetsch, "Die experimentelle Bakt.," Berlin, 1906. 
by Lustgarten ${ }^{1}$ in 1884 seemed, for a time, to have solved the mystery. The Lustgarten bacillus was an acid-fast organism very similar to Bacillus tuberculosis, and found by its discoverer in a large number of syphilitic lesions. The observation, at first, aroused much interest and received some confirmation. Later extensive investigations, however, failed to uphold the etiological relationship of this bacillus to the disease and practically identified it with the smegma bacillus, so often a saprophyte upon the mucous membranes of the normal genitals.

In 1905, Schaudinn, ${ }^{2}$ a German zoologist, working in collaboration with Hoffmann, investigated a number of primary syphilitic indurations and secondarily enlarged lymph nodes, and in both lesions discovered a spirochæte similar to, but easily distinguished from, the spirochætes

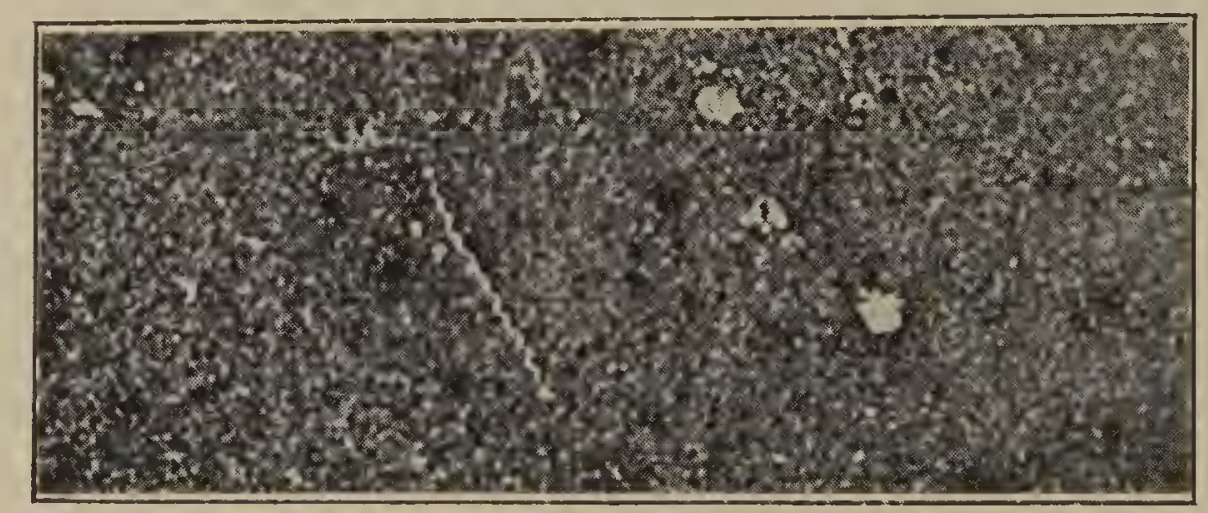

Fig. 129.-Spirocheteta Pallida. Smear preparation from chancre stained by the india-ink method.

already known. He failed to find similar microorganisms in uninfected human beings.

The microorganism described by him as "Spirochæta pallida" is an extremely delicate undulating filament measuring from four to ten micra in length, with an average of seven micra, and varying in thickness from an immeasurable delicacy to about 0.5 of a micron. It is thus distinctly smaller and more delicate than the spirochæte of relapsing fever. Examined in fresh preparations it is distinctly motile, its movements consisting in a rotation about the long axis, gliding movements backward and forward, and, occasionally, a bending of the whole body. Its convolutions, as counted by Schaudinn, vary from 3 to 12 and differ from those observed in many other spirochætes by being extremely steep, or, in other words, by forming acute, rather than obtuse, angles. The ends of the microorganism are delicately tapering and come to a point. In

${ }^{1}$ Lustgarten, Wien. med. Woch., xxxiv, 1884.

${ }^{2}$ Schaudinn und Hoffmann, Arb. a. d. kais. Gesundheitsamt, 22, 1905. 
his first investigations, Schaudinn was unable to discover flagella and believed that he saw a marginal undulating membrane similar to that noticed in the trypanosomes. Later observations by this observer, as well as by others, revealed a delicate flagellum at each end, but left the existence of an undulating membrane in doubt. Uncertain, in his later investigations, whether the microorganisms described by him could scientifically be classified with the spirochæte proper, Schaudinn suggested the name of "Treponema pallidum."

In the same preparations in which Spirochæta pallida was first seen, other spirochætes were present, which were easily distinguished from the former by their coarser contours, their flatter and fewer undulations, their more highly refractile cell bodies, and, in stained preparations, their deeper color. These microorganisms were not found regularly, and were interpreted merely as fortuitous and unimportant companions. To them Schaudinn gave the name of "Spirochæta refringens."

The epoch-making discovery of Schaudinn and Hoffmann was soon confirmed by many observers, and the etiological relationship of Spirochrta pallida to syphilis may now be regarded as an accepted fact. Although our inability to cultivate the microorganism has made it impossible to carry out Koch's postulates, nevertheless indirect evidence of such a convincing nature has accumulated that no reasonable doubt as to its caustive importance can be retained. The spirochætes have been found constantly present in the primary and secondary lesions of all carefully investigated cases, and, so far, have invariably been absent in subjects not afflicted with syphilis.

Schaudinn himself, not long after his original communication, was able to report seventy cases of primary and secondary syphilis in which these microorganisms were found. Spitzer ${ }^{1}$ found them constantly present in a large number of similar cases. Sobernheim and Tomasczewski ${ }^{2}$ found the spirochætes in fifty cases of primary and secondary syphilis, but failed to find them in eight tertiary cases. Mulzer, ${ }^{3}$ who found the microorganisms invariably in twenty cases of clinical syphilis, failed to find them in fifty-six carefully investigated non-syphilitic subjects. The voluminous confirmatory literature which has accumulated upon the subject can not here be reviewed. The presence of these spirochætes in the blood at certain stages of the disease has been demon-

1 Spitzer, Wien. klin. Woch., 1905.

${ }^{2}$ Sobernheim und Tomasczewski, Münch. med. Woch., 1905.

${ }^{3}$ Mulzer, Berl. klin. Woch., 1905, and Archiv f. Dermat. u. Syph., 79, 1906. 
strated by Bandi and Simonelli ${ }^{1}$ who found them in the blood taken from the roseola spots, and by Levaditi and Petresco ${ }^{2}$ who found them in the fluid of blisters produced upon the skin.

In tertiary lesions the spirochætes have been found less regularly than in the primary and secondary lesions, but positive evidence of their presence has been brought by Tomasczewski, ${ }^{3}$ Ewing, ${ }^{4}$ and others who succeeded in demonstrating them in gummata. Noguchi and Moore ${ }^{5}$ have recently found the Spirochæta pallida in the brain of patients dead of general paresis.

In congenital syphilis, many observers have found Spirochæta pallida in the lungs, liver, spleen, pancreas, and kidneys, and, in isolated cases, in the heart muscle. The organisms were always present in large numbers and practically in pure culture. These results more than any others seem to furnish positive proof of the etiological relationship between the spirochæte and the disease.

Demonstration of Spirochæta pallida.-In the living state the spirochætes have been observed in the hanging drop or under a coverslip rimmed with vaseline. It is extremely important, in preparing such specimens from primary lesions or from lymph glands, to obtain the material from the deeper tissues, and thus as uncontaminated as possible by the secondary infecting agents present upon the surface of an ulcer, and also as free from blood as possible. An ordinary microscope and condenser may be used, provided that the light is cut down considerably by means of the iris diaphragm. This method is, however, difficult and uncertain. It is better to employ a special device known as a "condenser for dark-field illumination" (Dunkel-KammerBeleuchtung). This apparatus is screwed into the place of the Abbe condenser. The preparation is made upon a slide and covered with a cover-slip as usual. A drop of oil is then placed upon the upper surface of the dark chamber and the slide laid upon it so that an even layer of oil, without air-bubbles, intervenes between the top of the dark chamber and the bottom of the slide. The preparation is then best examined with a high-power dry lens. An arc light furnishes the most favorable illumination. In such preparations the highly refractive cell-

${ }^{1}$ Bandi und Simonelli, Cent. f. Bakt., 40, 1905.

${ }^{2}$ Levaditi et Petresco, Presse méd., 1905.

3 Tomasczewski, Münch. med. Woch., 1906.

${ }^{4}$ Ewing, Proc. N. Y. Path. Soc., N. S., 5, 1905.

s Noguchi and Moore, Jour. Exp. Med., xvii, 1913. 
bodies stand out against the black background, and the motility of the organisms may be observed. ${ }^{1}$

The dark-field condenser is without question the easiest method of finding the Spirochæta pallida. Its use is easily learned and the apparatus is sufficiently cheap so that it lends itself to the use of the clinic and the office. With very little practice it is possible to detect the spirochæte in suspension if care is taken that not too much blood or other solid particles are mixed with the preparation. Should it be impossible to obtain the material scraped from syphilitic lesions in a sufficiently dilute condition it is best to emulsify it in a drop or two of human ascitic fluid.

Examination in Smears.--The Spirochæta pallida can not be stained with the weaker anilin dyes, and even more powerful dyes, such as carbol-fuchsin and gentian-violet, give but a pale and unsatisfactory preparation. The staining method most commonly used is the one originally recommended by Schaudinn and Hoffmann. This depends upon the use of Giemsa's azur-eosin stain employed in various modifications. The most satisfactory method of applying this solution is as follows:

Make smears upon slides or cover-slips, if possible from the depth of the lesions, as free as possible from blood.

Fix in methyl alcohol for ten to twenty minutes and dry.

Cover the preparation with a solution freshly prepared as follows:

Distilled water. . . . . . . . . . . . . . . . . . . . . . . 10 c.c.

Potassium carbonate $1: 1,000 \ldots \ldots \ldots \ldots \ldots \ldots \ldots \ldots, 5-10$ gtt.

Add to this:

Giemsa's solution (für Romanowski F'ärbung) ...........10-12 gtt.

This staining fluid is left on for one to four hours, preferably in a moist chamber.

Wash in running water.

Blot.

By this method Spirochæta pallida is stained characteristically with a violet or reddish tinge.

A rapid and convenient method for staining such smears consists in the use of azur I and eosin in aqueous solutions as recommended by

${ }^{1}$ For a critical summary of the various methods of dark-field illumination, the reader is referred to an article by Siedentopf, Zeit. f. wiss. Mikrosc., xxv, 1908. 
Wood (see section on Staining, page 109). The smears are fixed in methyl alcohol as before and are then flooded with the azur I solution. The eosin solution is then dropped on the preparation until an iridescent pellicle begins to form. Satisfactory preparations may be obtained by this method after ten or fifteen minutes of staining.

Goldhorn ${ }^{1}$ has prepared a stain which gives excellent results and is extremely rapid. He describes the preparation of his staining fluid as follows:

One gram of lithium carbonate is dissolved in 200 c.c. of water. To this are added 2 grams of methylene-blue and the mixture is carefully heated, filtered, and divided into two equal parts. To one of these parts is added 5 per cent acetic acid until acid to litmus. The two parts are then mixed, and a weak solution of eosin is added until a pale blue color is obtained. The fluid is then allowed to stand for a day and the precipitate which is formed is filtered off and allowed to dry without heat. One gram of this precipitate is dissolved in 100 c.c. of methyl alcohol. This stain is applied for five minutes or longer after methyl-alcohol fixation. Excellent results are usually obtained with this stain, but variations due to the difficulty of manufacturing it make it less reliable than the two methods previously mentioned.

Recently a rapid and extremely simple and reliable method for the demonstration of Spirochæta pallida in smears, by the use of India ink, has been described.

Smears are prepared in the following way: A drop of the fluid squeezed out of the syphilitic lesion, as free as possible from blood cells, is mixed, on a slide, with a drop of India ink (best variety is "Chin chin" Günther-Wagner Liquid Pearl ink), and the mixture smeared with the edge of another slide as in making blood smears. When the smear dries, which takes about a minute, it may be immediately examined with an oil-immersion lens. The organisms are seen unstained on a black background. (See Fig. 129, p. 594.)

Demonstration of SPIrochetes in Tissues.-Ordinary histological staining methods do not reveal the spirochætes in tissue sections. It is customary, therefore, to employ some modification of Cajal's silver impregnation. The technique most commonly employed is that known as Levaditi's method, ${ }^{2}$ which is carried out as follows:

${ }^{1}$ Goldhorn, Proc. N. Y. Path. Soc., N. S., 5, 1905.

${ }^{2}$ Levaditi, Comptes rend. de la soc. de biol., 59, 1905. 
The fresh tissue is cut into small pieces which should not be thicker than 2 to 4 millimeters.

Fix in ten-per-cent formalin (four-per-cent formaldehyde) for twentyfour hours.

Wash in water.

Dehydrate in 96-per-cent alcohol twenty-four hours.

Wash in water.

Place in a 3-per-cent silver-nitrate solution at incubator temperature $\left(37.5^{\circ}\right.$ C. $)$ and in the dark for three to five days.

Wash in water for a short time.

Place in the following solution (freshly prepared):

Pyrogallic acid....................... 2-4 grams.

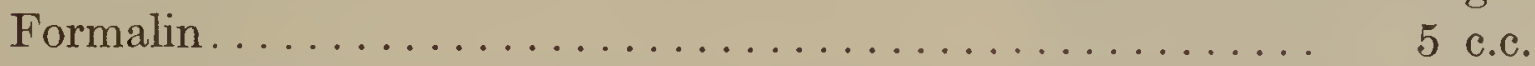

Distilled water..................... 100 "

Leave in this for twenty-four to forty-eight hours at room temperature.

Wash in water.

Dehydrate in graded alcohols.

Embed in paraffin and cut thin sections.

The sections may be examined without further staining, or, if desired, may be weakly counterstained with Giemsa's solution or hematoxylin.

A modification of this method which has been much recommended is that of Levaditi and Manouelian. ${ }^{1}$ The directions given by these authors are as follows:

Fix in formalin as in previous method.

Dehydrate in 96-per-cent alcohol twelve to twenty-four hours.

Wash in distilled water.

Place in a 1-per-cent silver-nitrate solution to which 10 per cent of pyridin has been added just before use.

Leave in this solution for two to three hours at room temperature and from four to six hours at $50^{\circ} \mathrm{C}$. approximately.

Wash rapidly in 10-per-cent pyridin.

Place in a solution containing 4 per cent of pyrogallic acid to which 10 per cent of C. P. acetone, and 15 per cent (per volume) of pyridin have been added just before use. Leave in this solution two to three hours.

${ }^{1}$ Levaditi et Manouelian, Comptes rend. de la soc. de biol., 60, 1906. 
Wash in water, dehydrate in graded alcohols, and embed in paraffin by the usual technique.

Examined after treatment by either of these methods, the spirochætes appear as black, untransparent bodies lying chiefly extracellularly. They are characteristically massed about the blood-vessels of the organs and only exceptionally seem to penetrate into the interior of the parenchyma cells.

Attempts at cultivating Spirochæta pallida were at first unsuccessful. Recently Schereschewsky ${ }^{1}$ has reported that he has succeeded in obtaining multiplication of the organisms on artificial media as

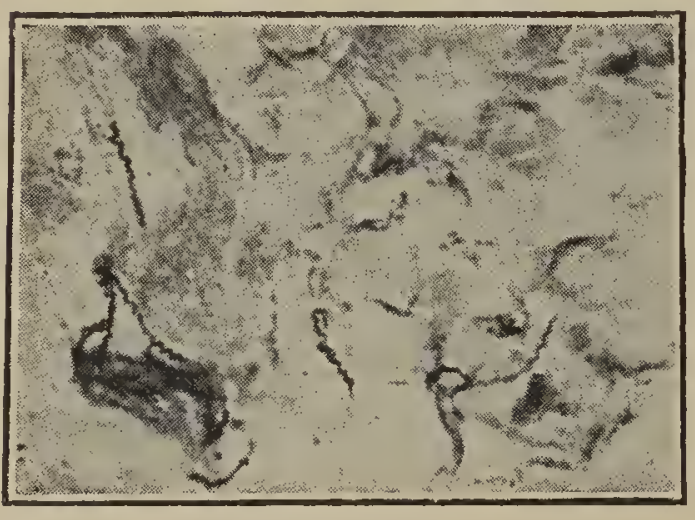

Fig. 130.-SPIRocheta PALLIDA. Spleen, congenital syphilis. (Levaditi method.) follows: Sterile horse serum in centrifuge tubes was coagulated at $60^{\circ} \mathrm{C}$. until it assumed a jelly-like consistency. It was then placed in the incubator at $37.5^{\circ} \mathrm{C}$. for three days before being used. The cultures were planted by snipping off a small piece of tissue from a syphilitic lesion, dropping it into such a tube, and causing it to sink to the bottom by means of centrifugalization. The tube was then tightly stoppered with a cork. In such anaërobic serum cultures Schereschewsky claims to have grown the organisms for several generations, though not in pure culture.

Mühlens also obtained growth of Spirochæta pallida in horse serum agar by a method which is very similar to that of Schereschewsky. None of these observers, however, succeeded in carrying out Koch's postulates with the cultures they obtained. This has recently been done in the splendid investigations of Noguchi. Noguchi ${ }^{2}$ began his work upon the Spirochæta pallida in 1910 and 1911. His first successful cultivations were made from the syphilis-infected testicles of rabbits, and after many unsuccessful attempts, with slightly varying media and technique, he finally succeeded in the following way: $\mathrm{He}$ prepared tubes $(20 \mathrm{~cm}$. high and $1.5 \mathrm{~cm}$. wide), containing 10 c.c. of a serum-water made of distilled water, three parts; and horse, sheep, or rabbit serum, one part. These were sterilized by the fractional method in the usual way (15 minutes each day). Into them was then placed a

1 Schereschewsky, Deut. med. Woch., N. S., xix and xxix, 1909.

${ }^{2}$ Noguchi, Jour. Exp. Med., xiv, 1911; xvii, 1913. 
small piece of sterile rabbit kidney or testicle and a bit of the testicle of a syphilitic rabbit, in which many spirochætes were present. The fluid was then covered with sterile paraffin oil and placed in an anaërobic jar. After 10 days at $33.5^{\circ} \mathrm{C}$. the spirochætes had multiplied considerably, in all but one case, together with bacteria. He obtained pure cultures from these initial cultivations after much difficulty, by a number of methods. At first he succeeded only by allowing the spirochætes to grow through Berkefeld filters, which they did on the fifth day. A better method more recently adopted by him consists in preparing high tubes of three parts of very slightly alkaline or neutral agar to which a piece of sterile tissue has been added. These tubes are then inoculated from the impure cultures with a long pipette. Close to the

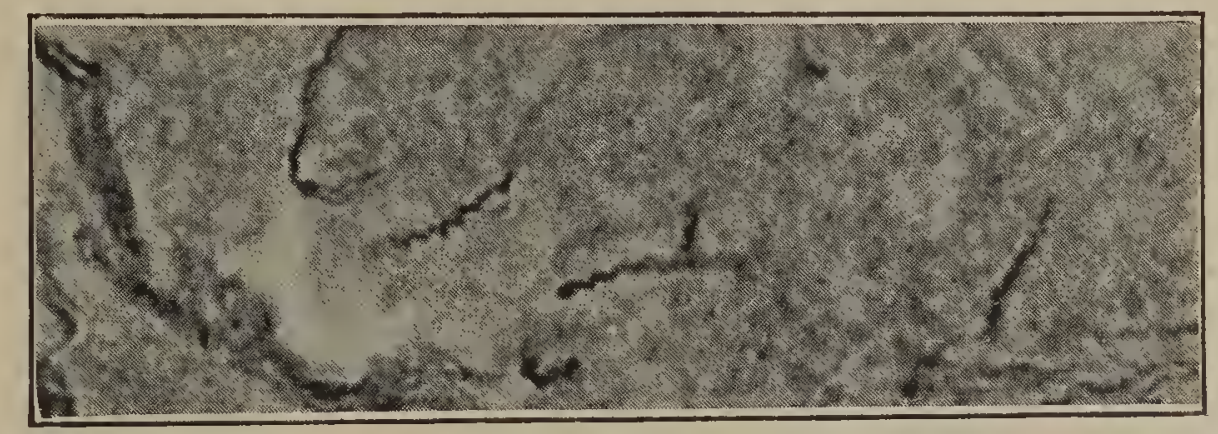

Fig. 131.-SPiroch eta PaLlida. Liver, congenital syphilis. (Levaditi method.)

tissue and along the stab the spirochætes and bacteria will grow and, after about ten days to two weeks, the spirochætes will have wandered away from the stab and will be visible as hazy colonies. They can then be fished, after cutting the tubes, and directly transplanted to other serum-agar-tissue tubes prepared as before, and eventually will grow in pure culture. By this method Noguchi has also cultivated pure cultures from lesions in monkeys, and has produced lesions both in rabbits and monkeys with his pure cultures. He has thus for the first time carried out Koch's postulates with syphilis and established beyond the shadow of a doubt the etiological significance of Spirochrta pallida in syphilis.

Animal Pathogenicity.-Until very recently, all experimental inoculation of animals was unsuccessful. During the year 1903 Metchnikoff and Roux ${ }^{1}$ finally succeeded in transmitting the disease to monkeys. The monkey first used by these observers was a female chimpanzee. At the point of inoculation, the clitoris, there appeared, twenty-six days

¿Metchnikoff et Roux, Ann. de l'Inst. Pasteur, 1903, 1904, and 1905. 
after inoculation, a typical indurated chancre, which was soon followed by swelling of the inguinal glands. Fifty-six days after the inoculation there appeared a typical secondary eruption, together with swelling of the spleen and of the lymph nodes. Similar successful experiments were made soon after this by Lassar. ${ }^{1}$ Soon after the experiments of Metchnikoff and Roux, successful inoculations upon lower monkeys (macacus) were carried out by Nicolle. ${ }^{2}$ Since that time, it has been found by various observers that almost all species of monkeys are susceptible. Simple subcutaneous injection is not sufficient to produce a lesion. The technique which has given the most satisfactory results consists in the cutaneous implantation of small quantities of syphilitic tissue obtained by excision or curetting of primary and secondary lesions. A small pocket is made under the mucous membrane of the genitals or of the eyebrows and the tissue placed in this under aseptic precautions. The inoculation may be made directly from the human being, but can also be successfully carried out from monkey to monkey for many generations. Attempts at transmission from tertiary lesions have so far been unsuccessful. The spirochætes can be demonstrated both in the primary lesions of the inoculated animal and in the secondarily enlarged glands. The successful inoculation of rabbits with syphilis has been recently performed by Bertarelli. ${ }^{3}$ He obtained ulcerative lesions by inoculation upon the cornea and into the anterior chamber of the eye and was able to prove the syphilitic nature of these lesions by finding the spirochæte within the tissue. In these animals, as well as in the lower monkeys, the disease usually remains localized.

In 1907, Parodi showed that syphilitic lesions could be produced by direct inoculation into the testicles of rabbits. This method of inoculation has been subsequently studied by many investigators, especially by Uhlenhuth and Mulzer. ${ }^{4}$ It is the easiest method of obtaining spirochæte in any quantity from lesions in man. The spirochæte-containing lesions may be either excised or scraped as conditions permit and rubbed up in a mortar with sterile sand, in a few centimeters of sterile human ascitic fluid. This emulsion is then injected directly into the substance of rabbit testicles. A swelling supervenes which is often noticeable after two weeks, and is usually at its height in 5 to 7 weeks. At this time the testicle is much larger than normal, some-

${ }^{1}$ Lassar, Berl. klin. Woch., xl, 1903.

${ }^{2}$ Nicolle, Ann. de l'inst. Pasteur, 1903.

${ }^{3}$ Bertarelli, Cent. f. Bakt., xli, 1906.

${ }^{4}$ Uhlenhuth und Mulzer, Arb. a. d. k. Gsndhtsamte., xxxiii, 1909. 
times evenly swollen and sometimes nodular, and of a firm elastic consistency. When taken out at castration it oozes a sticky fluid, both from testicle and the tunica, which is rich in actively motile spirochætes. By continuous transinoculation from one rabbit to another such a strain can be indefinitely carried along. It can be inoculated from rabbits to monkeys and vice versa. This method as well as Noguchi's cultivations have opened a new era of spirochæte investigation. It is stated by some observers that intravenous inoculation of rabbits may be followed by localization in the testis and occasionally gummatous infections in other parts of the body have been induced after such inoculation by Uhlenhuth, Mulzer, and others.

Immunization in Syphilis.-It is a well-known fact observed by clinicians that one attack of syphilis usually protects the infected individual at least from the development of another chancrous lesion. That this immunity develops quite rapidly was shown by Metchnikoff and Roux, who found that reinfection of a monkey was possible if attempted within two weeks of the first inoculation, but was unsuccessful if delayed beyond this period.

On the basis of this knowledge as to the actual development of an immunity, Metchnikoff, ${ }^{1}$ Finger and Landsteiner, ${ }^{2}$ and others have made extensive attempts to devise some method of active immunization. Working along the line of Pasteur's original attenuation of virus, these observers attempted to attenuate the syphilitic virus by repeated passage through monkeys. These experiments were entirely without success, the last-mentioned observers finding absolutely no attenuation after twelve generations of monkey inoculation.

The study of rabbits has permitted a little more definite formulation of our ideas on syphilis immunization. Bertarelli and others have shown that the production of a syphilitic lesion on the cornea of one eye does not protect against an inoculation subsequently done on the other eye. Apparently rabbits that have been inoculated with spirochæte material and that have not developed syphilitic disease can be successfully inoculated on subsequent attempts. The offspring of female rabbits with syphilis of the cornea are, according to Muhlens, not immune.

There is no evidence so far that specific therapy or treatment with spirochæte material has had favorable influence upon the disease except

1 Metchnikoff, Arch. gén. de méd., 1905.

2 Finger und Landsteiner, Sitzungsber. d. Wien. Akad. d. Wiss., 1905. 
in isolated cases reported by Uhlenhuth and Mulzer. Chemotherapy has had results analogous to those obtained in man. ${ }^{1}$

Attempts at passive immunization have been entirely without success.

The occurrence of a Wassermann reaction was formerly supposed to indicate the existence of specific syphilitic antibodies in the serum of patients. Our more recent information regarding this reaction seems to show that it depends upon the presence in the serum of syphilitic patients of substances produced indirectly because of the presence of syphilitic infection. It may be a relative increase of globulins or, as Schmidt has suggested, a change in the physical state of the globulins or other substances present in the serum. At any rate it has been found that the fixation of complement in the Wassermann reaction does not depend upon the occurrence of a specific antigen-antibody reaction. In the first place the antigens most commonly used, and successfully so, in the Wassermann reactions, are non-specific lipoidal extracts of normal organs. This alone would show that the specific spirochætal substance has no relation to the reaction. Again it has been demonstrated that extracts of cultures of the Spirochæta pallida as well as extractions from the testes of syphilitic rabbits do not furnish an antigen suitable for the Wassermann reaction. This has followed especially from the work of Noguchi, ${ }^{2}$ of Craig and Nichols, ${ }^{3}$ and of others. This forms a corollary to the other experiments previously mentioned and shows that, whatever the Wassermann reaction may be (and space does not permit us to review the theories, especially since none are definitely proven), it is not a specific complement fixation in the sense of Bordet and Gengou. It must be admitted, therefore, that our knowledge of syphilis immunity is in its infancy and that we know very little about the systemic reactions which follow infection with the Spirochæta pallida.

The fact that the syphilitic virus does not pass through a filter has been demonstrated by Klingmüller and Baermann, ${ }^{4}$ who inoculated themselves with filtrates from syphilitic material.

${ }^{1}$ Von Prowazek, "Handbuch der pathogenen Protozoen," i, 1912, Leipzig, Bartsch.

${ }^{2}$ Noguchi, Jour. Am. Med. Assoc., 1912.

${ }^{3}$ Craig and Nichols, Jour. Exp. Med., xvi, 1912.

${ }^{4}$ Klingmüller und Baermann, Deut. med. Woch., 1904. 


\section{THE SPIROCHATES OF RELAPSING FEVER}

The microorganisms causing relapsing fever were first observed in 1873 , by Obermeier, ${ }^{1}$ who demonstrated them in the blood of patients suffering from this distinct type of fever. Since his time extensive

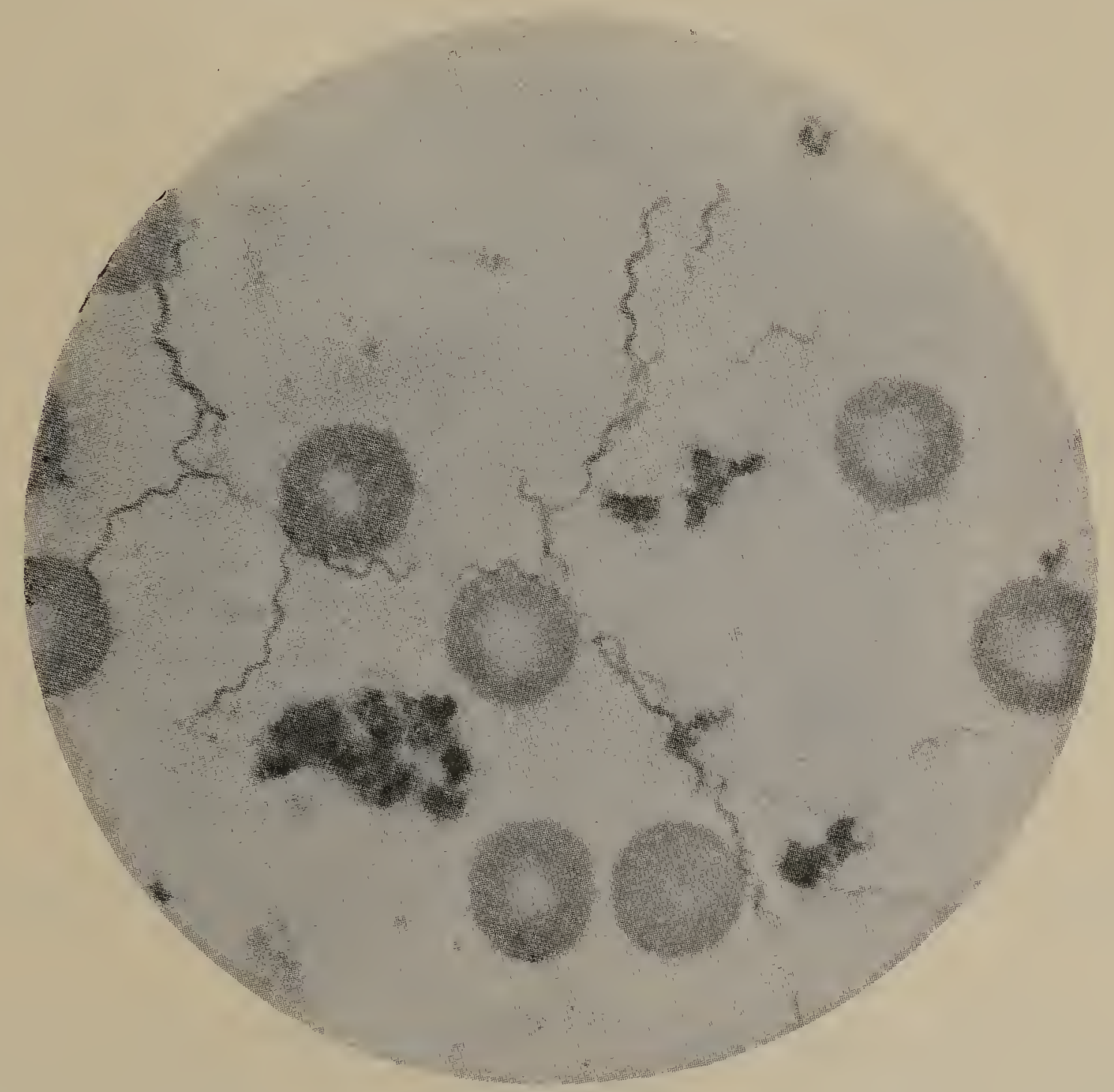

Fig. 132.-Spirochete of Relapsing Fever. (After Norris, Pappenheimer, and Flournoy.)

studies by many other observers have proven beyond question the etiological connection between the disease and the organisms.

Morphology and Staining.--The spirochæte of Obermeier is a delicate spiral thread measuring from 7 to 9 micra in length (Novy), and about 1 micron in thickness. While this is its average size, it may, according to some observers, be considerably longer than this; its undulations varying from 4 to 10 or more in number. Compared with the red blood

1 Obermeier, Cent. f, d. med. Wiss., 11, 1873. 
cells among which they are seen, the microorganisms may vary from one-half to 9 or 10 times the diameter of a corpuscle. In fresh preparations of the blood, very active corkscrew-like motility and definite lateral oscillation are observed. In stained preparations no definite cellular structure can be made out, the cell body appearing homogeneous, except in degenerated individuals, in which irregular granulation or beading has been observed. Flagella have been described by various observers.

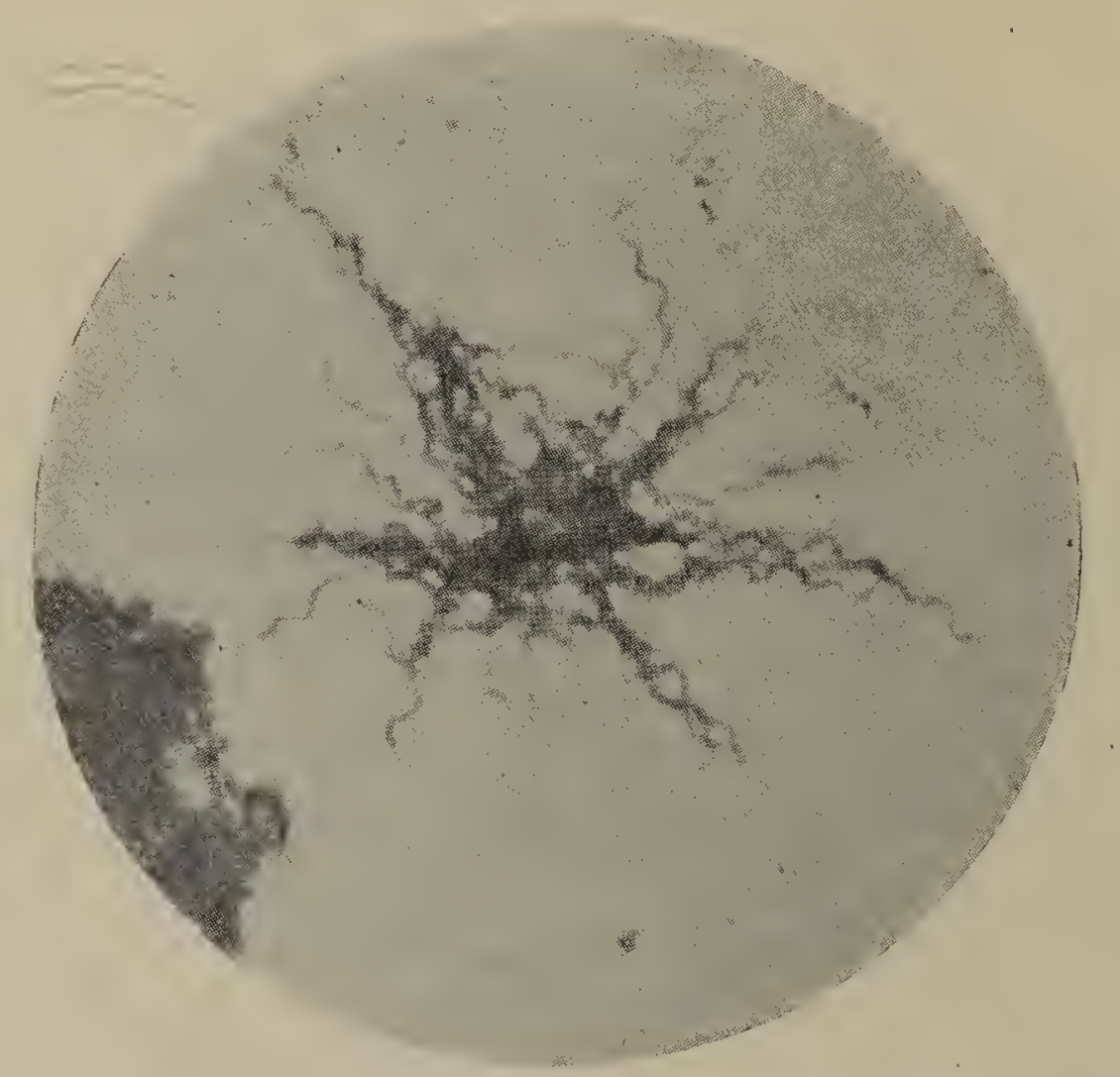

Fig. 133.-Spirochete of Relapsing Fever. Citrated normal rat blood. (After Norris, Pappenheimer, and Flournoy.)

Novy and Knapp ${ }^{1}$ believe that the organisms possess only one terminal flagellum. Zettnow, ${ }^{2}$ on the other hand, claims to have demonstrated lateral flagella by special methods of staining. Norris, Pappenheimer, and Flournoy, ${ }^{3}$ in smears stained by polychrome methods, have described long, filamentous tapering ends which they interpreted as bipolar, terminal flagella, never observing more than one at each end. Spores are not found.

Cultivation.-Innumerable attempts to induce these microorganisms to multiply upon artificial media have been made. Novy and Knapp succeeded in keeping the microorganisms alive and virulent in the

${ }^{1}$ Novy and Knapp, Jour. of Infec. Dis., 3, 1906.

${ }^{2}$ Zettnow, Deut. med. Woch., 32, 1906.

${ }^{3}$ Norris, Pappenheimer, and Flournoy, Jour, of Inf. Dis., 3, 1906. 
original blood for as long as forty days, and call attention to the fact that the length of time for which they may be kept alive depends to a great extent upon the stage of fever at which the blood is removed from the patient. They do not, however, believe that extensive multiplication, or, in other words, actual cultivation, had taken place in their experiments. Norris, Pappenheimer, and Flournoy, on the other hand, have obtained positive evidence of multiplication of the spirochætes in fluid media. They obtained their cultures by inoculating a few drops of spirochætal rat blood into 3 to 5 c.c. of citrated human or rat blood. Smears made from these tubes, after preservation for twenty-four hours at room temperature, showed the microorganisms in greater number

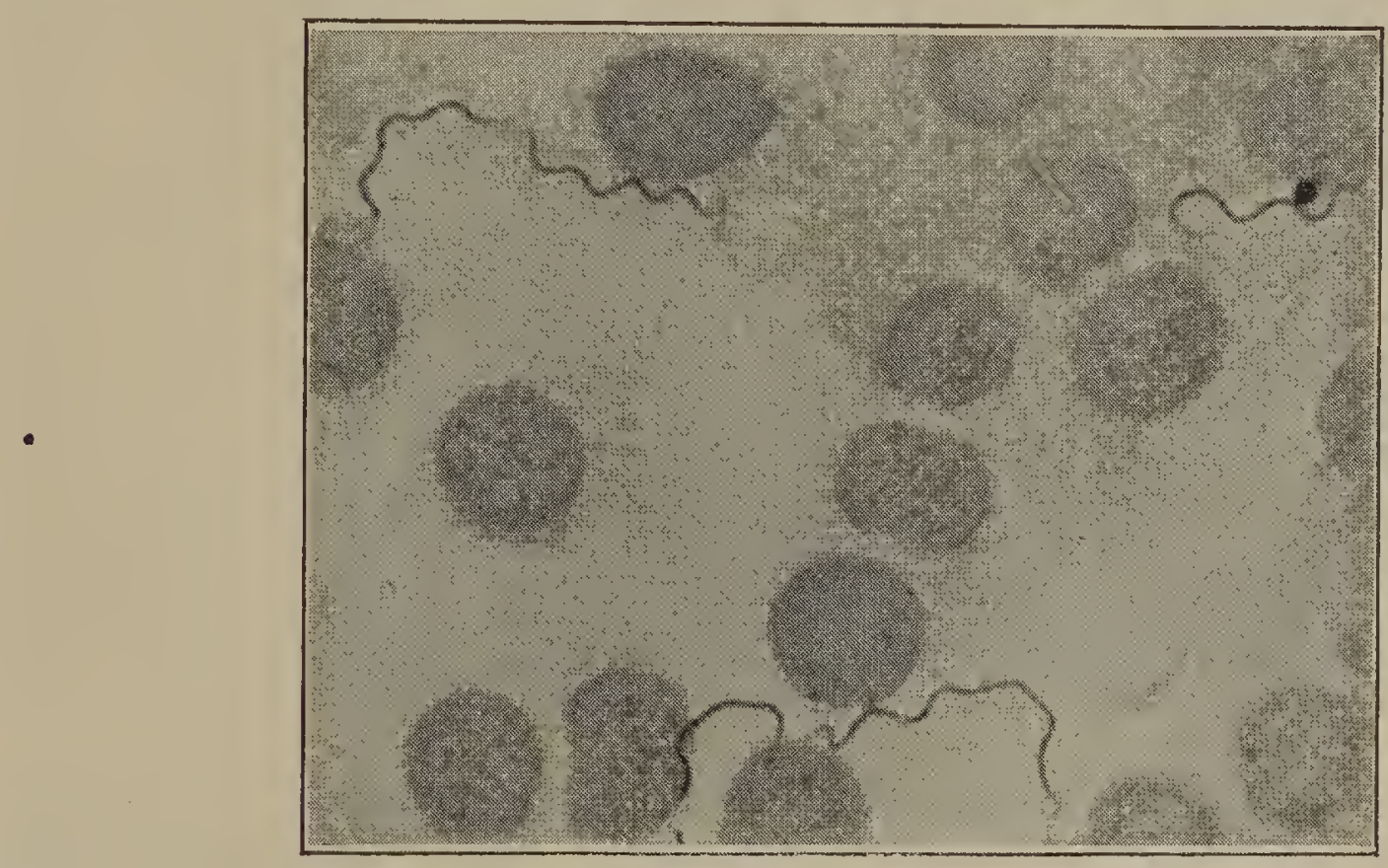

Fig. 134.- Spirochete of Relapsing Fever. (From preparation furnished by Dr. G. N. Calkins.)

than in the original infected blood. A similar multiplication could be observed in transfers made from these "first-generation" tubes to other tubes of citrated blood. Attempts at cultivation for a third generation, however, failed.

Noguchi ${ }^{1}$ has lately successfully cultivated the spirochæte of Obermeier in ascitic fluid containing a piece of sterile rabbit's kidney and a few drops of citrated blood under anaërobic conditions.

Four different, probably distinct varieties of spirochæte have been described in connection with relapsing fever, all of which have been cultivated by Noguchi by means of this method. The first is known as the spirochæte of Obermeier mentioned above. Probably distinct 
are the Spirochæta Duttoni, described by Dutton and Todd ${ }^{1}$ in 1905 , the Spirochæta Kochi, and the Spirochæta Novyi, ${ }^{2}$ the organism studied by Norris and Flournoy and Pappenheimer, and regarded as a different species by them.

Pathogenicity.-Inoculation with blood containing these spirochætes produces disease in monkeys, rats, and mice. Attempts to transmit the disease experimentally to dogs, rabbits, and guinea-pigs have so far been unsuccessful. The subcutaneous inoculation of monkeys is followed after from two to four days by a rise of temperature which occurs abruptly as is the case in the disease in man and which may last several days. During this time the spirochætes can be found in the blood of the animals just as it is found in that of infected human beings. The temperature subsides after a day or more, when it again rapidly returns to normal. As a rule, the paroxysms are not repeated. Occasionally, however, two or three attacks may supervene before immunity is established. In rats, an incubation time of from two to five days occurs. At the end of this time the spirochætes may be found in large numbers in the blood, and the animals show symptoms of a severe systemic infection. The attack lasts from four to five days, at the end of which time the microorganisms again disappear. Occasionally even in these animals relapses have been observed. Gross pathological changes are not found, with the exception of an enlargement of the spleen.

In man the disease caused by the spirochæte of Obermeier, commonly known as relapsing fever, is common in India, Africa, and most of the warmer countries. It has, from time to time, been observed epidemically in Europe, especially in Russia, and a few epidemics have occurred in the Southern United States. The disease comes on abruptly, beginning usually with a chill accompanied by a sharp rise of temperature and generalized pains. Together with the rise of temperature, which often exceeds $104^{\circ} \mathrm{F}$, there are great prostration and occasionally delirium. Early in the disease the spleen becomes palpable and jaundice may appear. The spirochætes are easily detected in the blood during the persistence of the fever, which lasts usually from three to ten days. At the end of this time the temperature usually drops as suddenly as it rose, and the general symptoms rapidly disappear. After a free interval of from one to three weeks a relapse may occur, which is usually less severe and of shorter duration than the original attack. Two, three, or

${ }^{1}$ Dutton and Todd, Brit. Med. Jour., 1905.

${ }^{2}$ Novy and Fraenkel, cited from Noguchi. 
even four attacks may occur, but the disease is not very often fatal. When patients do succumb, however, the autopsy findings are not particularly characteristic. Apart from the marked enlargement of the spleen, which histologically shows the changes indicating simple hyperplasia, and a slight enlargement of the liver, no lesions are found. The diagnosis is easily made during the febrile stage by examination of a small quantity of blood under a cover-slip or in the hanging-drop preparation.

Several types of relapsing fever have been described. In Africa the disease has long been prevalent in many regions and the investigations of Ross and Milne, ${ }^{1}$ Koch, ${ }^{2}$ Dutton and Todd ${ }^{3}$ and others have brought

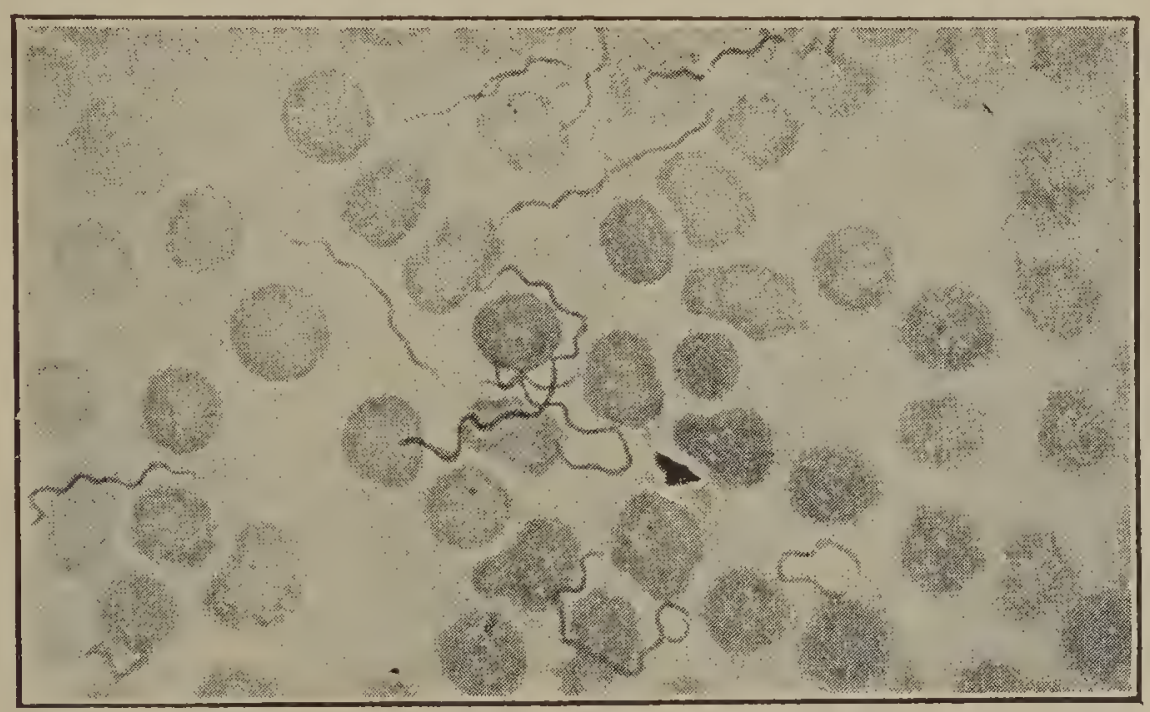

Fig. 135.-Spirochete of Dutton, African Tick Fever. (From preparation furnished by Dr. G. N. Calkins.)

to light that many conditions occurring among the natives, formerly regarded as malarial, are caused by a species of spirochæte. Whether or not the microorganisms observed in the African disease are exactly identical with the spirochæte observed by Obermeier is yet a question about which several opinions are held. Dutton and Todd believe that the same microorganism is responsible for both diseases. Koch, on the other hand, believes that the slightly smaller size of the African spirochæte and the milder course of the clinical symptoms indicate a definite difference between the two. Animal experiments made with the African organism, furthermore, usually show a much more severe infection than do similar inoculations with the European variety. The

${ }^{1}$ Ross and Milne, Brit. Med. Jour., 1904.

${ }^{2}$ Koch, Deut. med. Woch., xxxi, 1905.

${ }^{3}$ Dutton and Todd, Lancet, 1905, and Jour. of Trop. Med., 1905. 
spirochæte found in the African disease is usually spoken of at present as "Spirochæta Duttoni." Novy and Knapp, ${ }^{1}$ after extensive studies with the microorganisms from various sources, have come to the conclusion that, although closely related, definite species differences exist between the two types mentioned above, and that these again are definitely distinguished from similar organisms described by Turnbull ${ }^{2}$ as occurrin in a similar disease observed in India.

The mode of transmission of this disease is not clear for all types. Dutton and Todd, however, were able to show satisfactorily that, in the case of the African disease at least, transmission occurs through the intermediation of a species of tick. The conditions under which such intermediation occurs have been carefully studied by Koch. ${ }^{3}$ The tick (Ornithodorus moubata) infects itself when sucking blood from an infected human being. The spirochæte may remain alive and demonstrable within the body of the tick for as long as three days. Koch has shown, furthernore, that they may be found also within the eggs laid by an infected female tick. He succeeded in producing experimental infection in monkeys by subjecting the animals to the bites of the infected insects. For the European variety of the disease no such intermediate host has as yet been demonstrated.

Immunity.- It has long been a well-known fact that recovery from an attack of relapsing fever usually results in a more or less definite immunity. The blood of human beings, monkeys, and rats which have recovered from an attack of this disease show definite and specific bactericidal and agglutinating substances, and Novy and Knapp have demonstrated that the blood serum of such animals may be used to confer passive immunity upon others.

\section{VINCENT'S ANGINA}

The condition known as Vincent's angina consists of an inflammatory lesion in the mouth, pharynx, or throat, situated most frequently upon the tonsils. The disease usually begins as an acute stomatitis, pharyngitis, or tonsillitis, which soon leads to the formation of a pseudomembrane, which, at this stage, has a great deal of resemblance to that caused by the diphtheria bacillus. At later stages of the disease there may be distinct ulceration, the ulcers having a well-defined margin

${ }^{1}$ Novy and Knapp, loc. cit.

2 Turnbull, Indian Med. Gaz., 1905.

${ }^{3}$ Koch, Berl. med. Woch., 1906. 
and "punched-out" appearance, so that clinically they have often been erroneously diagnosed as syphilis. Apart from the localized pain, the disease is usually mild, but occasionally moderate fever and systemic disturbances have been observed. Unlike diphtheria and syphilis, this peculiar form of angina usually yields, without difficulty, to local treatment.

The nature of lesions of this peculiar kind was not clear until Plaut, ${ }^{1}$

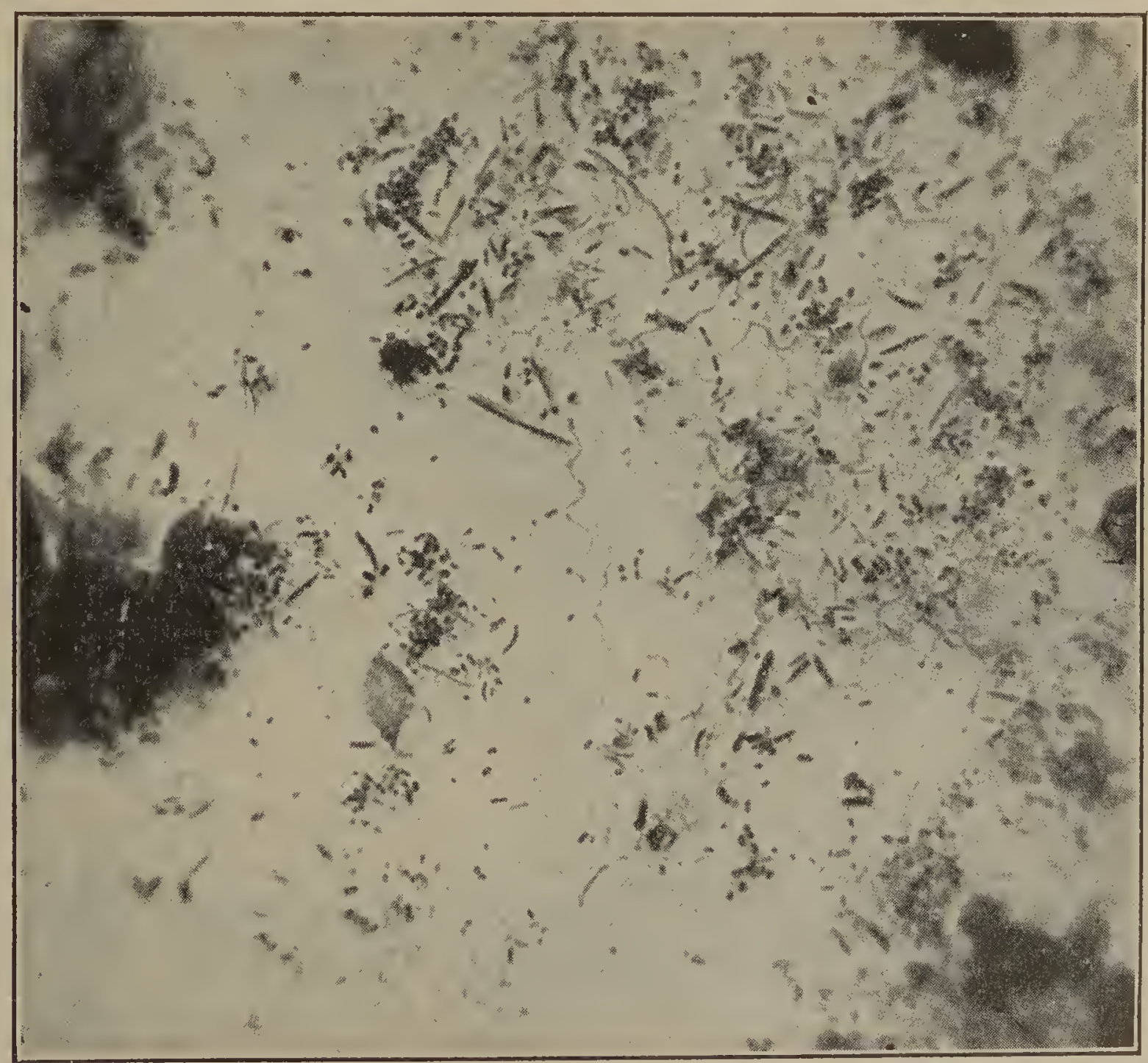

Fig. 136.- Smear from the throat of a Case of Vincent's Angina. Giemsa Stain.

Vincent, ${ }^{2}$ and others reported uniform bacteriological findings in cases of this description. These observers have been able to demonstrate in smears from the lesions a spindle-shaped or fusiform bacillus, together with which there is usually found a spirillum not unlike the spirillum of relapsing fever. The two microorganisms are almost

1 Plaut, Deut. med. Woch., xlix, 1894.

2 Vincent, Ann. de l'inst. Pasteur, 1896, and Bull. et mém. de la soc. méd. des hôp. de P., 1898. 
always found together in this form of disease and were regarded by the first observers as representing two distinct forms dwelling in symbiosis. More recently Tunnicliff, ${ }^{1}$ on the basis of experimental work, has claimed identity for the two forms, believing that they represent different developmental stages of the same organism.

The fusiform bacilli described by Vincent, Plaut, Babes, and others, are from 3 to 10 micra in length, and have a thickness at the center varying from 0.5 to 0.8 micron. From the center they taper gradually

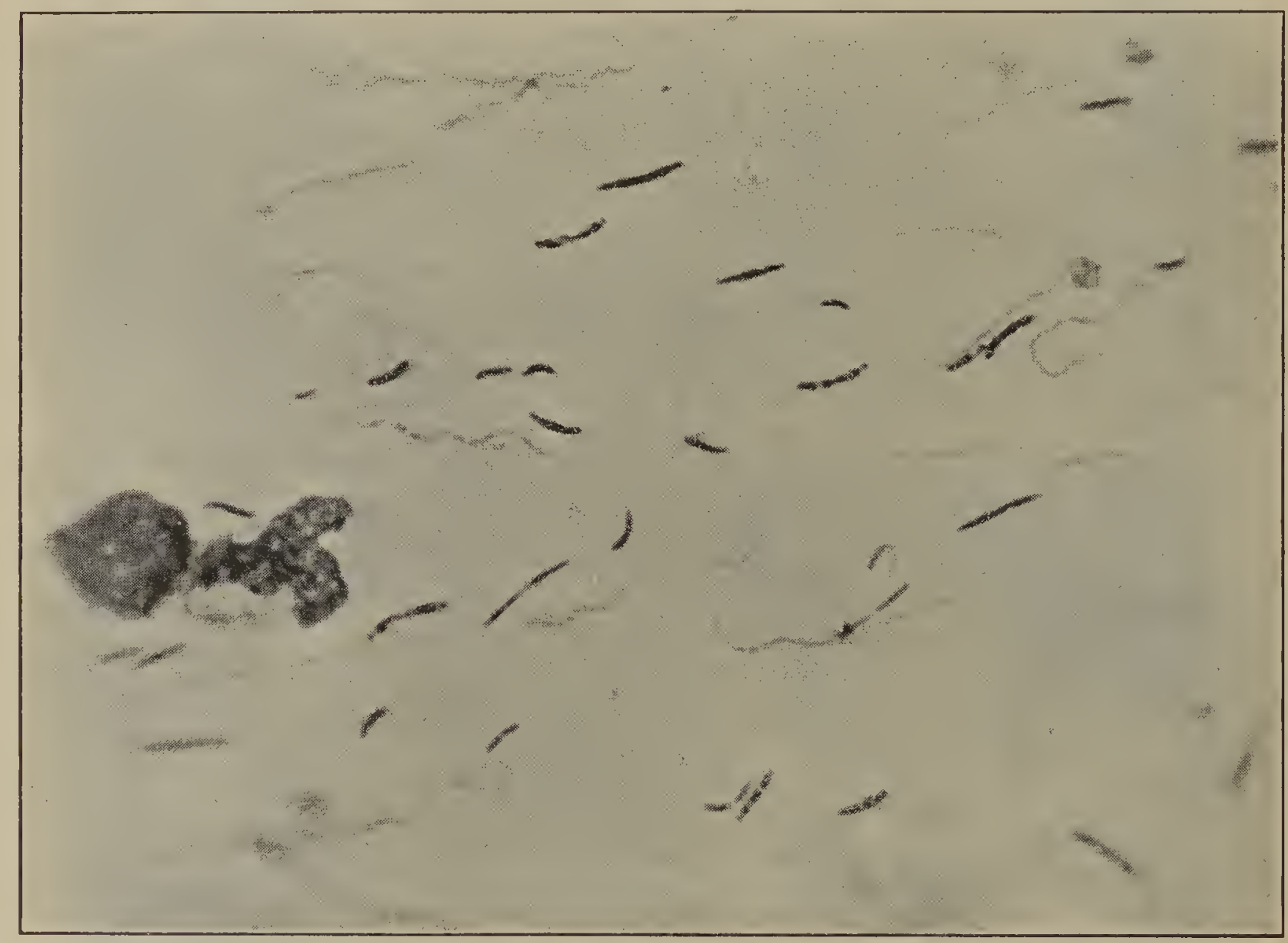

Fig. 137.-Throat Smear. Vincent's Angina. Fusiform bacilli and spirilla.

toward the ends, ending in blunt or sharp points. The length of these bacilli may vary greatly within one and the same smear preparation. They are usually straight, sometimes slightly curved. They do not stain very easily with the weaker anilin dyes, but are readily stained by Loeffler's methylene-blue, carbol-fuchsin, or better, by Giemsa's stain. Stained by Gram, they are usually decolorized, though in this respect the writers have found them to vary. Stained preparations show a characteristic inequality in the intensity of the stain, the bacilli being more 
deeply stained near the end, and showing a banded or striped alternation of stained and unstained areas in the central body. Their staining qualities in this respect are not unlike those of the diphtheria bacillus, and according to Babes ${ }^{1}$ the dark areas are to be interpreted as metachromatic granules. The bacilli are not motile.

The spirilla found in Vincent's angina are usually somewhat longer than the fusiform bacilli, and are made up of a variable number of undulations, shallow and irregular in their curvatures, unlike the more regularly steep waves of Spirochæta pallida. They are stained with even more difficulty than are the bacilli and usually appear less distinct in the preparations. The stain, however, is taken without irregularity, showing none of the apparent metachromatism observed in the bacilli.

By the earlier observers cultivation of these microorganisms was attempted without success. Recently, however, it has been shown that cultivation could be carried out under anaërobic conditions. Tunnicliff ${ }^{2}$ has cultivated the organisms anaërobically upon slants of ascitic agar at $37.5^{\circ} \mathrm{C}$. This observer found that in such cultures, before the fifth day, bacilli only could be found, that after this time, however, spirilla gradually appeared and finally constituted the majority of the organisms in the culture. It appeared to Tunnicliff from this study that the spirilla might be developed out of the fusiform microorganisms representing the adult form.

The microorganisms of Vincent's angina, when occurring in the throat, are rarely present alone, being usually accompanied by other microorganisms, such as staphylococci, streptococci, and not infrequently diphtheria bacilli. When occurring together with diphtheria, they are said, by some German observers, to aggravate the latter condition considerably. This frequent association with other microorganisms renders it impossible to decide conclusively that the fusiform bacilli and spirilla are the primary etiological factors in these inflammations. It has been frequently suggested that they may be present as secondary invaders upon the soil prepared for them by other microorganisms.

Animal inoculation with these microorganisms has led to little result.

Fusiform Bacilli other than those in Vincent's Angina.-Fusiform bacilli morphologically indistinguishable from those found in the angina of Vincent may frequently be found in smears taken from the gums,

${ }^{1}$ Babes, in Kolle und Wassermann, 1. Ergänzungsband, 1907.

${ }_{2}$ Tunnicliff, Jour. of Infec. Dis., 3, 1900. 
from carious teeth, and occasionally among the microorganisms in the pus from old sinuses. Several varieties of these bacilli have been described in connection with definite pathological conditions.

Babes, ${ }^{1}$ in 1893, observed spindle-shaped bacilli not unlike those described above, but somewhat shorter, in histological sections prepared from tissues from the gums of individuals suffering from scurvy. He found similar bacilli in rabbits intravenously inoculated with material from the patients and was able to cultivate the bacilli for several generations. His descriptions, however, of the microorganisms as found in the secondary cultures vary considerably from those of the original findings in the gums of the patients. His results are not convincing.

In noma, a gangrenous disease of the gums and cheeks, occurring occasionally in individuals who have been severely run down by acute infectious diseases or great hardship, Weaver and Tunnicliff have found spirilla and fusiform bacilli in large numbers. The organisms were present not only in smears from the surface, but were also found by histological methods, in large numbers, lying in the tissues beyond the area of necrosis. Here again it is not entirely certain whether these microorganisms were the primary etiological factors or whether they are to be regarded merely as secondary invaders of a necrotic focus.

Fusiform bacilli are cultivated with greater ease than formerly supposed; we have found it relatively simple to grow them together with Gram positive cocci in symbiosis in simple broth tubes covered with paraffin oil without the addition of any enriching substance and in similar symbiotic conditions on infusion agar plates under incomplete anaërobic conditions. In such plates they form curious colonies in which the fusiform bacilli and micrococci are intimately commingled. Krumwiede ${ }^{2}$ has had no difficulty in cultivating them in pure culture in anaërobic plates.

\section{SPIROCH府TA PERTENUIS}

In a disease known as "Frambosia tropica," or popularly "Yaws," occurring in tropical and subtropical countries and much resembling syphilis, Castellani, ${ }^{3}$ in 1905, was able to demonstrate a species of spirochæte which has a close morphological resemblance to Spirochæta pallida. The microorganism was found in a large percentage of the cases

\footnotetext{
1 Babes, Deut. med. Woch., xliii, 1893.

${ }^{2}$ Krumwiede, Jour. Inf. Dis., 1913.

${ }^{3}$ Castellani, Brit. Med. Jour., 1905, and Deut. med. Woch., 1906.
} 
examined both in the cutaneous papules and in ulcerations. Confirmatory investigations on a larger series of cases were later carried out by von dem Borne. ${ }^{1}$

The microorganism is from 7 to 20 micra in length with numerous undulations and pointed ends. Examined in fresh preparations, it has an active motility similar to that of Spirochæta pallida. In smears it is easily stained by means of the Giemsa method.

Both the clinical similarity between yaws and syphilis, as well as the similarity between the microorganisms causing the diseases, has opened the question as to the identity of the two microorganisms. According to most clinical observers, however, yaws, which is a disease characterized chiefly by a generalized papular eruption, is unquestionably distinct, clinically, from lues, and experiments of Neisser, Baermann, and Halberstadter, ${ }^{2}$ as well as of Castellani ${ }^{3}$ himself, have tended to show that there is a distinct difference between the immunity produced by attacks of the two diseases. The disease is transmissible to monkeys, as is syphilis, but it has been satisfactorily shown that monkeys inoculated with syphilitic material, while no longer susceptible to infection with Spirochæta pallida, may still be successfully inoculated with Spirochæta pertenuis.

\section{SPIROCH压TA GALLINARUM}

An acute infectious disease occurring among chickens, chiefly in South America, has been shown by Marchoux and Salimbeni ${ }^{4}$ to be caused by a spirochæte which has much morphological similarity to the spirochæte of Obermeier.

The disease comes on rather suddenly with fever, diarrhea, and great exhaustion, and often ends fatally. The spirochæte is easily demonstrated in the circulating blood of the animals by staining blood-smears with Giemsa's stain or with dilute carbol-fuchsin.

Artificial cultivation of the microorganism has not yet been accomplished. Experimental transmission from animal to animal is easily carried out by the subcutaneous injection of blood. Other birds, such as geese, ducks, and pigeons, are susceptible; mammals have, so far, not been successfully inoculated. According to the investigations of

1 Von dem Borne, Jour. Trop. Med., 10, 1907.

${ }^{2}$ Neisser, Baermann, und Halberstadter, Münch. med. Woch., xxviii, 1906.

${ }^{3}$ Castellani, Jour. of Hyg., 7, 1907.

${ }^{4}$ Marchoux et Salimbeni, Ann. de l'inst. Pasteur, 1903. 
Levaditi and Manouelian, ${ }^{1}$ the spirochætes are found not only in the blood but thickly distributed throughout the various organs.

Under natural conditions, infection of chickens seems to depend upon a species of tick which acts as an intermediate host and causes infection by its bite. The spirochæte, according to Marchoux and Sal-

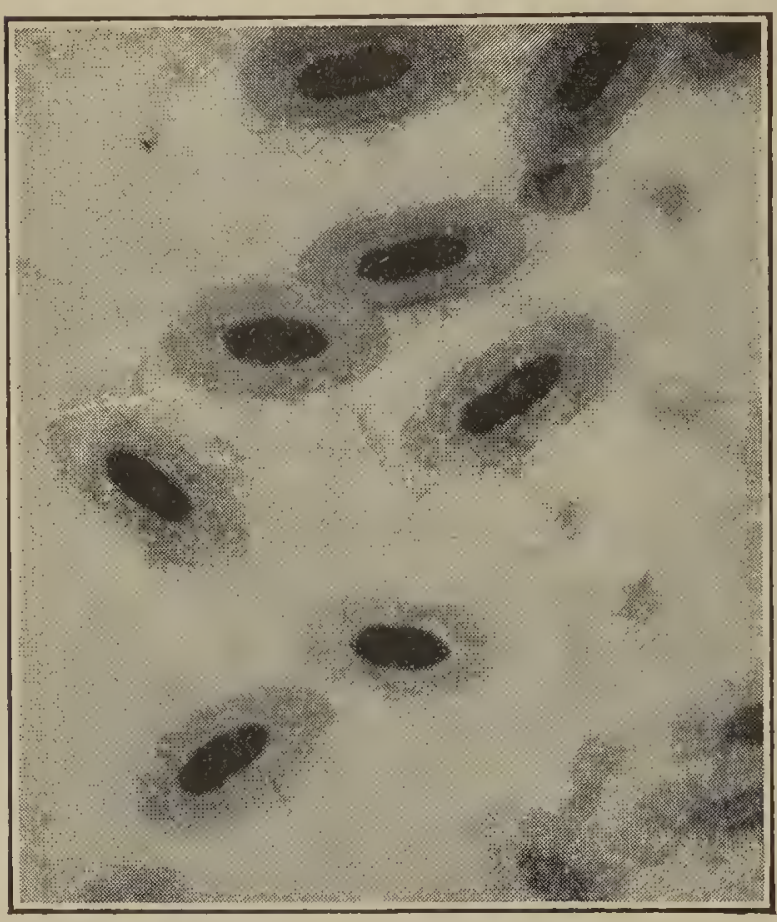

Fig. 138.-SPiroch mta gallinaRUM. (From preparation furnished by Dr. G. N. Calkins.) imbeni, may be found in the intestinal canal of the ticks for as long as five months after their infection from a diseased fowl.

In the blood of animals which have survived an infection, agglutinating substances appear and active immunization of animals may be carried out by the injection of infected blood in which the spirochætes have been killed, either by moderate heat or by preservation at room temperature. The serum of immune animals, furthermore, has a protective action upon other birds.

It is not impossible that the Spirochæta gallinarum may be identical with the Spirochæta anserina previously discovered by Sacharoff. ${ }^{2}$ This last-named microorganism causes a disease in geese, observed especially in Russia and Northern Africa, which both clinically and in its pathological lesions corresponds closely to the disease above described as occurring in chickens. The spirochæte is found during the febrile period of the disease in the circulating blood, is morphologically indistinguishable from the spirochæte of chickens, and can not be cultivated artificially. The similarity is further strengthened by the fact that Spirochæta anserina is pathogenic for other birds, but not for animals of other genera. Noguchi has succeeded in cultivating Spirochæta gallinarum by the same method by which he has cultivated the organisms of relapsing fever. Ascitic fluid tubes with a piece of sterile rabbit kidney were inoculated with a few drops of blood containing the spirochætes and cultivated at $37.5^{\circ} \mathrm{C}$. under anaërobic conditions.

Spirochæta phagedenis.-This is an organism cultivated by Noguchi

${ }^{1}$ Levaditi et Manouelian, Ann. de l'inst. Pasteur, 1906.

${ }^{2}$ Sacharoff, Ann. de l'inst. Pasteur, 1891. 
by his ascitic-fluid-tissue method from phagedenic lesions on human external genitals. It is probably a new species.

Spirochæta macrodentium.-Cultivated by Noguchi; ${ }^{1}$ is believed by him to be identical with the spirochæte found in Vincent's angina.

Spirochæta microdentium.-A similar organism with wide convolutions, cultivated by Noguchi from the tooth deposits chiefly in children. It was grown on mixtures of sheep serum water and sterile tissue in a way similar to that employed by him for other organisms of this group.

Spirochæta calligyrum.-Cultivated by Noguchi ${ }^{2}$ from condylomata -is probably a new species.

${ }^{1}$ Noguchi, Jour. Exp. Med., xv, 1912.

${ }^{2}$ Noguchi, Jour. Exp. Med., xvii, 1913. 


\section{CHAPTER XLIV}

\section{THE HIGHER BACTERIA}

\section{(Chlamydobacteriacece, Trichomycetes)}

STANDing midway between the true bacteria and the more complex molds or Hyphomycetes, there are a number of pathogenic microorganisms which offer great difficulties to classification. In the classification of Migula most of these forms have been placed in a rather heterogeneous group, the Chlamydobacteriaceæ. By other authors, notably Lachner-Sandoval, ${ }^{1}$ Berestnew ${ }^{2}$ and by Petruschky, ${ }^{3}$ the close relationship of these forms to the higher hyphomycetes has been emphasized and they have been grouped as a subdivision of the true molds under the family name of Trichomycetes.

Petruschky ${ }^{4}$ proposes the following clear schematization, which, even though possibly defective from a purely botanical point of view, is at least serviceable for the purposes of the bacteriologist.

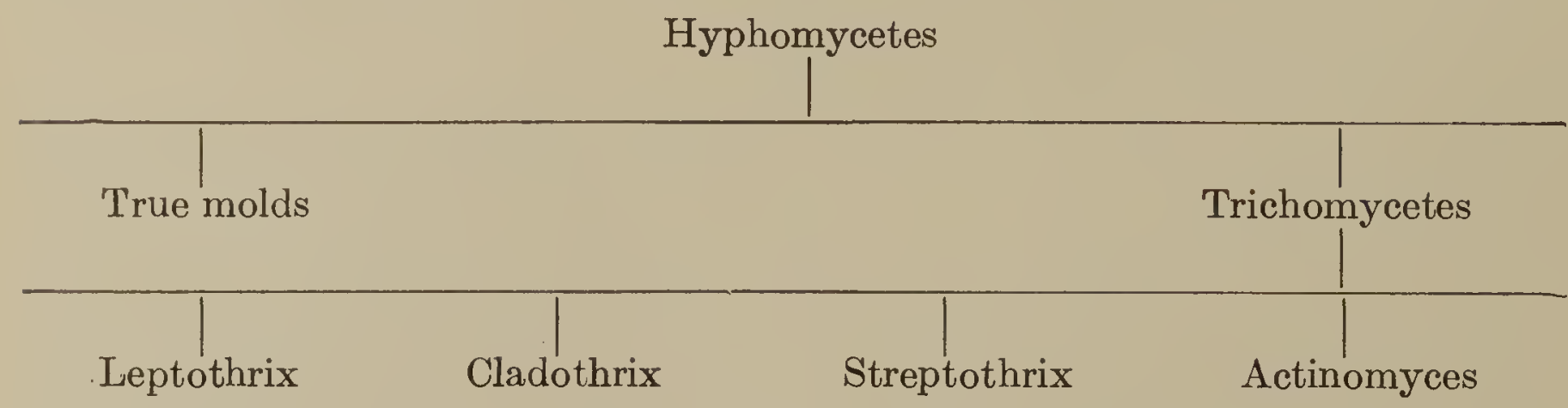

Leptothrix is used to designate those forms which appear as simple threads without branching.

Cladothrix is a thread-like form in which false branching may be recognized. By false branching is meant an appearance resulting from the fragmentation of threads. The terminal cell breaks away from the main stem, is set at an angle by the elongation of the thread itself, and,

\footnotetext{
${ }^{1}$ Lachner-Sandoval, "Ueber Strahlenpilze." Diss. Strassburg, 1898.

${ }^{2}$ Berestnew, Ref. Cent. f. Bakt., xxiv, 1898.

3 Petruschky, in Kolle und Wassermann, "Handbuch," etc.

Petruschky, loc. cit.
} 
as both continue dividing, the simulation of true branching is produced.

Streptothrix denotes forms with numerous true branches and spores which usually appear in chains.

Actinomyces is of more complicated structure, characterized by the formation of club-shaped ends and the stellate arrangement of its threads.

\section{LEPTOTHRIX}

Members of the leptothrix group have been observed in connection with inflammations of the mouth and pharynx by Fränkel, ${ }^{1}$ Michelson, ${ }^{2}$ Epstein, ${ }^{3}$ and others. In many of these cases the organism was identified by morphology chiefly, pure cultures not having been obtained. The disease in none of these cases was accompanied by severe systemic symptoms and it is likely that when found in human beings the organisms may be regarded simply as comparatively harmless saprophytes appearing in connection with some other specific inflammation.

Cultivation of the Leptothrices is not easy and has been successful only in the hands of Vignal ${ }^{4}$ and Arustamoff. ${ }^{5}$

\section{CLADOTHRIX}

Owing to much confusion in the differentiation of these forms from the streptothrices, it is not possible to determine whether cases of true cladothrix infection have been observed. It is likely that most cases ascribed to microorganisms of this class have really been due to streptothrix infection. The deciding criterion is, of course, the formation of branches and these seem to have been observed in most of the cases described. A closer differentiation, in the future, between true and false branching can alone determine whether or not cases of cladothrix infection proper may occur.

\section{STREPTOTHRIX}

Reports of cases of streptothrix infection of various parts of the body, in both animals and man, are abundant in the literature. The

1 Fränkel, Eulenburg's " Realencycl. d. gesam. Heilkunde," 1882.

${ }^{2}$ Michelson, Berl. klin. Woch., ix, 1889.

${ }^{3}$ Epstein, Prag. med. Woch., 1900.

\& Vignal, Ann. de phys., viii, 1886.

- Arustamoff, Quoted from Petruschky, loc. cit. 
earliest observations were made upon microorganisms isolated from the human conjunctiva. Nocard ${ }^{1}$ in 1888 described a member of this group as the etiological factor in a disease "farcies du bœuf" occurring among cattle in Guadeloupe. Eppinger ${ }^{2}$ found streptothrices in the pus of a cerebral abscess. Petruschlyy, ${ }^{3}$ Berestneff, ${ }^{4}$ Flexner, ${ }^{5}$ Norris and

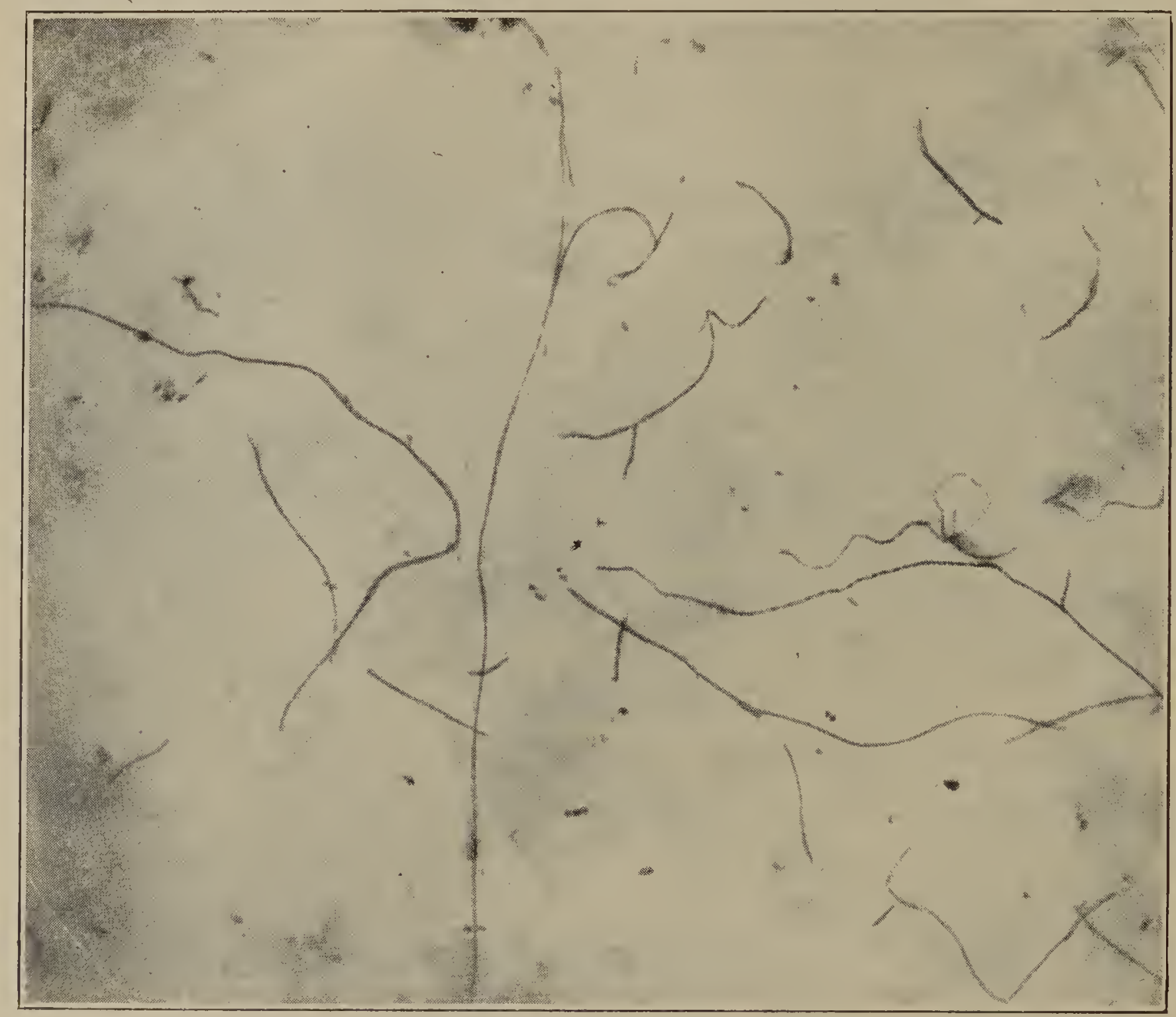

Hig 139.-Cladothrix. Showing Falge Branching.

Larkin, ${ }^{6}$ and a number of other observers have found these microorganisms in cases of pulmonary disease, simulating tuberculosis. Suppurations of bone and of the skin and the intestinal canal have been reported. The infection, therefore, is not very rare, but the diversc experiences of workers who have attempted to cultivate these micro-

I Nocard, Ann. de l'inst. Pasteur, ii, 1888.

2 Eppinger, Wien. klin. Woch., 1890.

3 Petruschky, Verhandl. d. Kongr. f. innere Mediz., 1898.

- Berestneff, Zeit. f. Hyg., xxix, 1898.

- Flexner, Jour. Exp. Med., iii, 1896.

- Norris and Larkin, Proc. of N. Y. Path. Soc., March, 1899. 
organisms seem to indicate that not all of the incitants described belonged to one and the same variety, but that probably a number of different types may exist.

Morphology.-Morphologically the streptothrices show considerable variation. In material from infectious lesions they have most often appeared as rods and filaments with well-marked branching. Occasionally the filaments are long and interwined, and branches have shown bulbous or club-shaped ends. In Norris and Larkin's case, the young cultures in the first generations seem to have consisted chiefly of rodshaped forms not unlike bacilli of the diphtheria group, showing marked metachromatism when stained with Loeffler's methylene-blue. They are

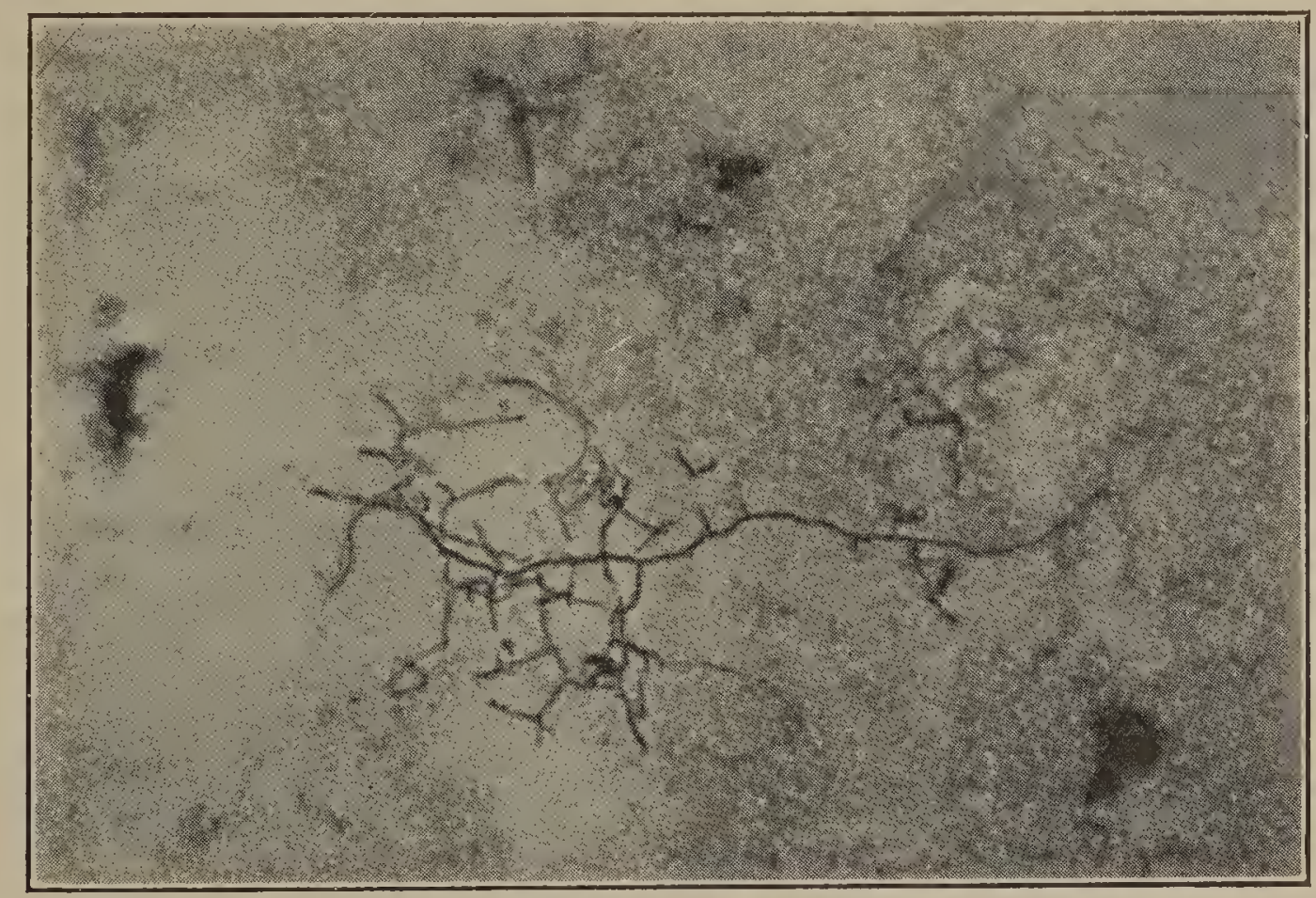

Fig. 140.--Streptothrix, Showing True Branching.

easily stained with this dye or with aqueous fuchsin. In tissue sections they may be demonstrated by the Gram-Weigert method.

Cultivation.-Direct cultivation upon agar and gelatin plates has occasionally been successful. At the end of four or five days grayishwhite, glistening, flat colonies may appear which attain a diameter of several millimeters within two weeks. The coloni later may take on a yellowish hue and begin to liquefy the gelatin. In bouillon flocculent precipitates and surface pellicles of interwined threads may form, without clouding of the medium. Norris and Larkin ${ }^{1}$ found much difficulty in cultivating, but finally succeeded by making smears of the infectious material upon fresh, sterile kidney-tissue of rabbits. The micro- 
organisms grew abundantly upon this, but failed to grow on any of the other tissues. After growth of several generations upon this medium, cultures were finally obtained upon agar plates and upon broth.

Inoculation of cultures into rabbits and guinea-pigs have given rise to subcutaneous abscesses, bronchopneumonia, and suppuration, according to the mode of infection.

\section{ACTINOMYCES}

Among the diseases caused by the Trichomycetes or higher bacteria, the most important is actinomycosis. Occurring chiefly in some of the domestic animals, notably in cattle, the disease is observed in man with sufficient frequency to make it of great clinical importance. In cattle the specific microorganism which gives ries to the disease was first observed by Bollinger ${ }^{1}$ in 1877 . In the following year Israel ${ }^{2}$ discovered a similar microorganism in human cases.

The parasites appear in the pus from discharging lesions as small granular bodies, plainly visible to the naked eye and somewhat resembling sulphur granules, of a grayish or of a pale yellow color. In size they measure usually a fraction of a millimeter. Ordinarily they are soft and easily crushed under a cover-slip, but occasionally, especially in old lesions, they may be quite hard, owing to calcification.

Microscopically they are most easily recognized in fresh preparations prepared by crushing the granules upon the slide under a cover-slip and examining them without staining. They may be rendered more clearly visible by the addition of a drop or two of 20 per cent potassium hydrate. When the granules are calcareous, the addition of a drop of concentrated acetic acid will facilitate examination. Fresh preparations may be examined after staining with Gram's stain. Observed under the microscope, the granules appear as rosette-like masses, the centers of which are quite opaque and dense, appearing to be made up of a closely meshed network of filaments. Around the margins there are found radially arranged striations which in many cases end in characteristically clubshaped bodies. Inside of the central network there are often scen coccoid or spore-like bodies which have been variously interpreted as spores, as degeneration products, and as separate cocci fortuitously found in symbiosis with the actinomyces. Individually considered,

? Bollinger, Deutsch. Zeit. f. Thiermed., iii, 1877.

? Israel, Virch. Arch, 74, 1878, and 78, 1879. 
the central filaments have approximately the thickness of an anthrax bacillus and are, according to Babes, ${ }^{1}$ composed of a sheath within which the protoplasm contains numerous and different sized granules.

About the periphery of the granules the free ends of the filaments become gradually thickened to form the so-called actinomycosis "clubs." These clubs, according to most observers, must be regarded as hyaline thickenings of the sheaths of the threads and are believed to represent a form of degeneration and not, as some of the earlier observers believed, organs of reproduction. They are homogeneous, and in the smaller and presumably younger granules are extremely fragile and soluble in water. In older lesions, especially in those of cattle, the clubs are more resistant and less easily destroyed.

They appear only in the parasites taken from active lesions in animals or man, or, as Wright ${ }^{2}$ has found, from cultures to which animal serum or whole blood has been added. In cultures from media to which no animal fluids have been added, such as glucose agar or gelatin, no clubs are found. In preparations stained by Gram's method the clubs give up the gentian-violet and take counter-stains, such as eosin.

The coccus-like bodies found occasionally lying between the filaments of the central mass, most observers now agree, do not represent anything comparable to the spores of the true hyphomycetes. In many cases they are unquestionably contaminating cocci; in others again they may represent the results of degeneration of the threads.

In tissue sections, the microorganisms may be demonstrated by Gram's method of staining or by a special method devised by Mallory. ${ }^{3}$ This is as follows for paraffin sections:

1. Stain in saturated aqueous eosin 10 minutes.

2. Wash in water.

3. Anilin gentian-violet, 5 minutes.

4. Wash with normal salt solution.

5. Gram's iodin solution 1 minute.

6. Wash in water and blot.

7. Cover with anilin oil until section is clear.

8. Xylol, several changes.

9. Mount in balsam.

Cultivation.-The isolation of actinomyces from lesions may be easy or difficult according to whether the pus is free from contamination

1 Babes, Virch. Arch., 105, 1886.

${ }^{2}$ J. H. Wright, Jour. Med. Res., viii, 1905.

3 Mallory, Method No. 1, Mallory and Wright, "Path. Technique," Phila., 1908. 
or whether it contains large numbers of other bacteria. In the latter case it may be almost impossible to obtain cultures. The descriptions of methods of isolation and of cultural characteristics given by various writers have shown considerable differences. The most extensive cultural work has been done by Bostroem, ${ }^{1}$ Wolff and Israel, and by J. H. Wright. Bostroem has described his cultures as aërobic, but Wolff and Israel ${ }^{2}$ and Wright ${ }^{3}$ agree in finding that the microorganisms iso-

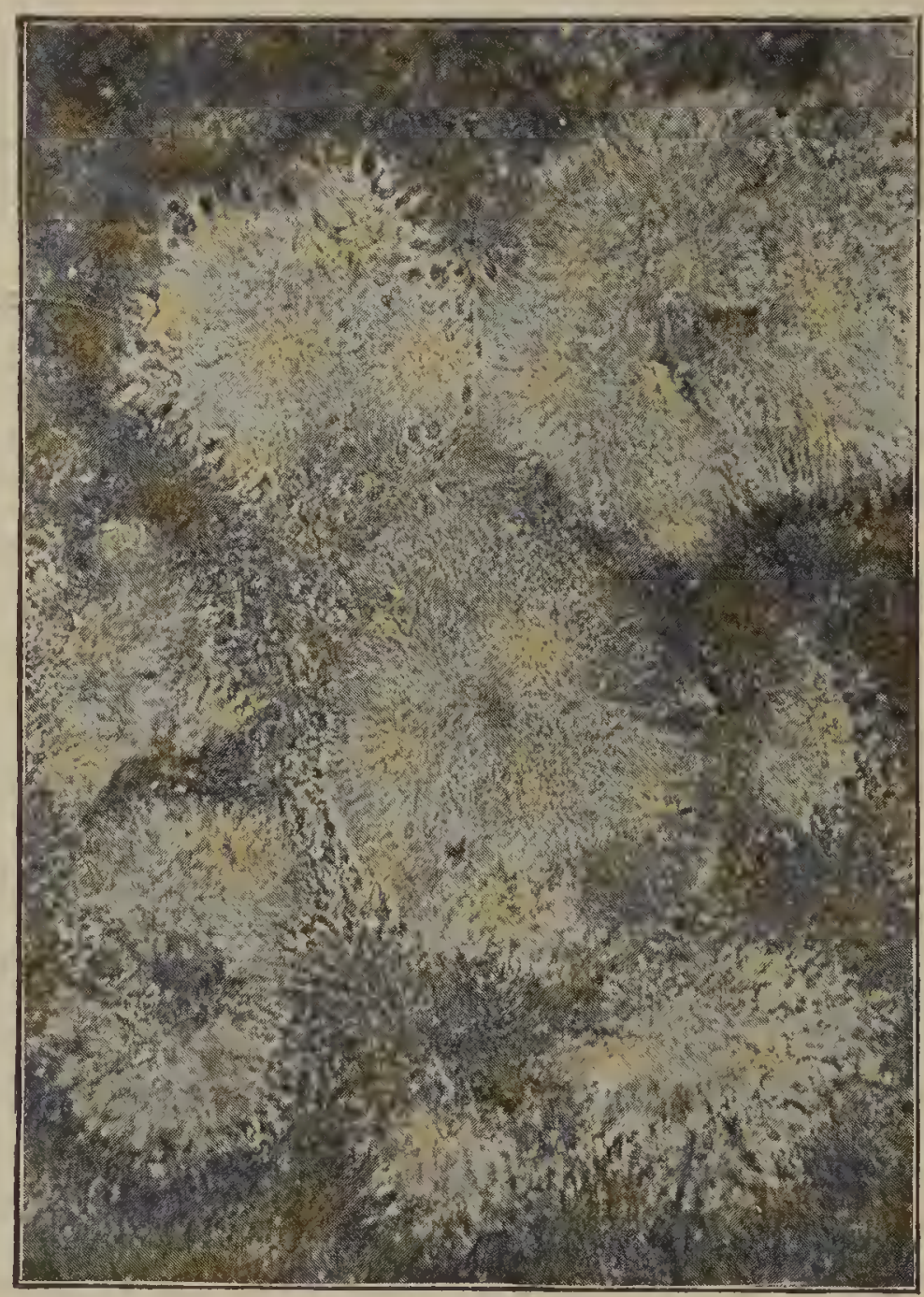

Fig. 141.-Actinomyces Granule Crushed Beneath a Cover-glass. Unstained. Low power. Shows radial striations. (After Wright and Brown.)

lated by them from actinomycotic lesions grow but sparsely under aërobic conditions and favor an environment which is entirely free from oxygen, or at least contains it only in small quantities. The method for isolation recommended by Wright is, briefly, as follows: Pus is obtained, if possible, from a closed lesion and washed in sterile water or broth. The granules are then crushed between two sterile slides and examined for

${ }_{1}^{1}$ Bostroem, Beitr. z. path. Anat. u. z. allg. Path., ix, 1890.

${ }^{2}$ Wolff und Israel, Virch. Arch., 126, 1891.

${ }_{3}^{3}$ J. H. Wright, Jour. Med. Res., viii, 1905. 
the presence of filaments. If these are present in reasonable abundance, the material is distributed in tubes of glucose agar, which are then allowed to solidify. If these first cultivations show a large number of contaminations, Wright recommends the preservation of other washed granules in test tubes for several weeks, in the hope that contaminating microorganisms may thus be killed by drying before the actinomyces lose their viability.

If cultivation is successful colonies will appear, after two to four days at $37.5^{\circ} \mathrm{C}$, as minute white specks, which, in Wright's cultures, appeared most abundantly within a zone situated 5 to 10 millimeters below the surface of the medium. Above and below this zone they are less numerous, indicating that a small amount of oxygen furnishes the best cultural environment. Upon the surface of agar slants, growth, if it takes place at all, is not luxuriant.

In alkaline meat-infusion broth growth takes place in the form of heavy, flocculent masses which appear at the bottom of the tubes. Surface growth and clouding do not take place.

Milk and potato have been used as culture media but are not particularly favorable.

Pathogenicity.-As stated above, actinomycosis occurs spontaneously most frequently among cattle and human beings. It may also occur in sheep, dogs, cats, and horses. Its locations of predilection are the various parts adjacent to the mouth and pharynx. It occurs also, however, in the lungs, in the intestinal canal, and upon the skin. When occurring in its most frequent location, the lower jaw, the

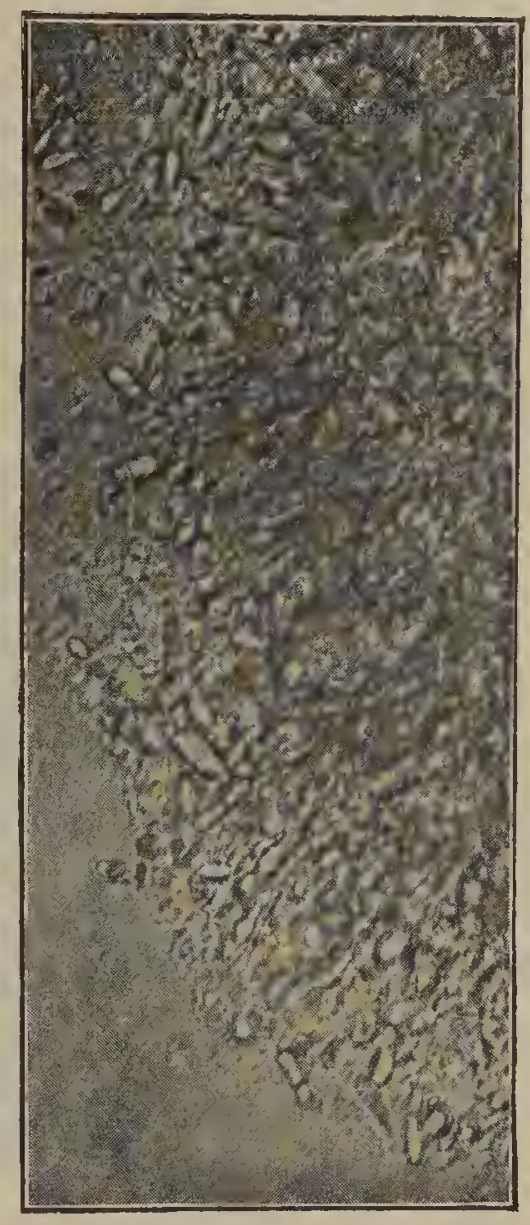

Fig. 142.-ACtinoM Y CES GRANULE Crushed Beneath a Cover-glass. U n stained. The preparation shows the margin of the granule and the "clubs." (After Wright and Brown.) disease presents, at first, a hard nodular swelling which later becomes soft because of central necrosis. It often involves the bone, causing a rarefying osteitis. As the swellings break down, sinuses are formed from which the granular pus is discharged. The neighboring lymph nodes show painless, hard swellings. Histologically, about the filamentous knobs or granules, there is a formation of epithelioid cells and a small round-cell infiltration. In older cases there may be an encapsulation in connective tissue and a 
calcification of the necrotic masses, leading to spontaneous cure. As a rule, this process is extremely chronic. Infection in the lungs or in the intra-abdominal organs is, of course, far more serious. When death occurs acutely, it is often due to secondary infection. The disease is acquired probably by the agency of hay, straw, and grain. Berestnew ${ }^{1}$ has succeeded in isolating actinomyces from straw and hay which he covered with sterile water in a potato jar and placed in the incubator. After a few days small white specks looking like chalk powder appeared upon the stalks, which, upon further cultivation, he was able to identify as the organism in question.

Animal inoculation, carried out extensively both with pus and with pure cultures by several observers, has yielded little result. Progressive

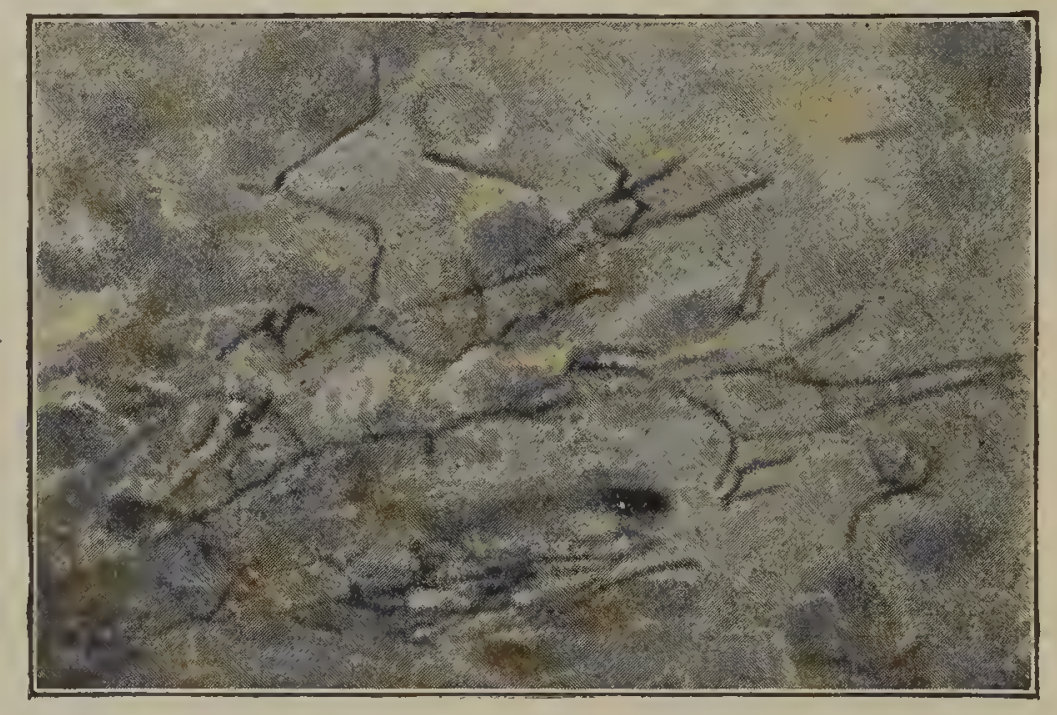

Fig. 143.-Branching Filaments of Actinomyces. (After Wright and Brown.)

actinomycotic lesions were never obtained, although occasionally small knobs containing colonies surrounded by epithelioid cells and connective tissue were observed, showing that the invading microorganisms were able to survive and grow for a short time, but were not sufficiently virulent to give rise to an extensive disease process. Transmission from animal to animal, or from animal to man directly, has not been satisfactorily proven.

Whether or not there are various forms of actinomyces must as yet be regarded as an open question. The investigations of Wolff and Israel, however, together with those of Wright, who alone observed thirteen different strains, seem to indicate that most, if not all, of the cases clinically observed are due to one and the same microorganism.

${ }_{1}^{1}$ Berestnew, Ref. Cent. f. Bakt., 24, 1898. 


\section{MYCETOMA (MADURA FOOT)}

The disease known by this name is not unlike actinomycosis. It is more or less strictly limited to warmer climates and was first recognized as a clinical entity, in India, by Carter. ${ }^{1}$ Clinically it consists of a chronic productive inflammation most frequently attacking the foot, less often the hand, very infrequently other parts of the body. Nodular swellings occur, which break down in their centers, leading to the formation of abscesses, later of sinuses. Often the bones are involved and a progressive rarefying osteitis results. From the sinuses a purulent fluid exudes, in which are found characteristic granular bodies. These may be hard, brittle, and black, resembling grains of gunpowder, or may be grayish-white or yellow and soft and grumous. According to the appearance of these granules, two varieties of the disease are distinguished, the "melanoid" and the "ochroid." Many observers believe that the yellow or ochroid variety is, in fact, actinomycosis. The black variety, which is certainly a distinct disease, is caused by a member of the hyphomycetes group. The parasite has been carefully studied by Wright, ${ }^{2}$ from whose description the following points are taken:

The small, brittle granules observed under the microscope show a dark, almost opaque center along the edges of which, filaments, or hyphæ, may be seen in a thickly matted mass. By crushing the granules under a cover-slip in a drop of sodium hypochlorite or of strong sodium hydrate, the black amorphous pigment is dissolved and the structural elements of the fungus may be observed. They seem to be composed of a dense meshwork of mycelial threads which are thick and often swollen, and show many branches. Transverse partitions are placed at short distances and the individual filaments may be very long. Spores were not observed by Wright. In a series of over fifty cultivations on artificial media from the original lesion, Wright obtained growth in a large percentage.

In broth, he obtained at first a rapid growth of long hyphæ which eventually formed a structure which he compares in appearance to a powder-puff.

On agar, growth appeared within less than a week and spread over the surface of the medium as a thick meshwork of spreading hyph

${ }^{1}$ Carter on Mycetoma, etc., London, 1874.

${ }^{2}$ Wright, Jour. of Exper. Med., 3, 1898. 
of a grayish color. In old cultures black granules appeared among the mycelial meshes.

On potato, he observed a dense velvety membrane, centrally of a pale brown, white at the periphery. Small brown droplets appeared on the growth in old cultures.

Animal inoculation with this microorganism has so far been unsuccessful. 


\section{CHAPTER XLV}

\section{THE YEASTS}

\section{(Blastomycetes, Saccharomycetes)}

THE yeasts or blastomycetes form a distinctive family among unicellular microorganisms, characterized essentially by their method of multiplication by budding. By this, they are sharply separated from the bacteria. Their differentiation from the higher fungi, the hyphomycetes, however, is less definitely established, since the chief characteristic of this latter class, the formation of hyphæ and mycelial threads, has occasionally been described for some of the forms otherwise identified with the yeasts. It is probable that a gradual transition between the two families exists, represented by a number of connecting forms, sometimes spoken of as oildia. For the practical purposes of the bacteriologist, the yeast family is sufficiently distinct, both morphologically and biologically, to make a separate classification extremely useful.

The yeast cell, as a rule, is oval, but among the wild yeasts, or "torulæ," spherical forms are common. In size, great variations occur, but in general the yeasts are much larger than bacteria, measuring usually from 10 to 20 micra in length with a width of about one-half or two-thirds of the long diameter. They possess a well-defined, doubly-contoured cell-membrane, composed chiefly of cellulose, and their body protoplasm, unlike that of the bacteria, shows definite structure. Within a mass of finely granul arcytoplasm, a number of highly refractive globules and vacuoles may be observed. Some of the globules have been interpreted as fat-droplets. Other granules, revealed by special staining methods, are interpreted as nuclear material.

When budding takes place, the mother cell sends out a small, globular evagination of the cell membrane into which maternal protoplasm flows. This bud gradually enlarges until it has attained approximately the same size as the original cell. Until that time, free intercommunication between the protoplasm of mother and daughter cell exists. Finally, by gradual narrowing of the isthmus connecting the two, the daughter cell becomes complete and free. By some observers 
definite karyokinetic changes in the nuclear structures have been described as accompanying the budding process. This observation, however, has not been generally confirmed. Under conditions of special stress, such as unfavorable environment or lack of nutrition, most yeasts possess the power of forming spores. These, called "ascospores," are formed within the yeast cell itself, each spore forming a separate membrane of its own, but all of them lying well protected within the original cell-membrane. The number of ascospores formed is constant for each species; and rarely exceeds four.

The yeasts have been studied most extensively in connection with fermentation and are industrially of great importance in the production

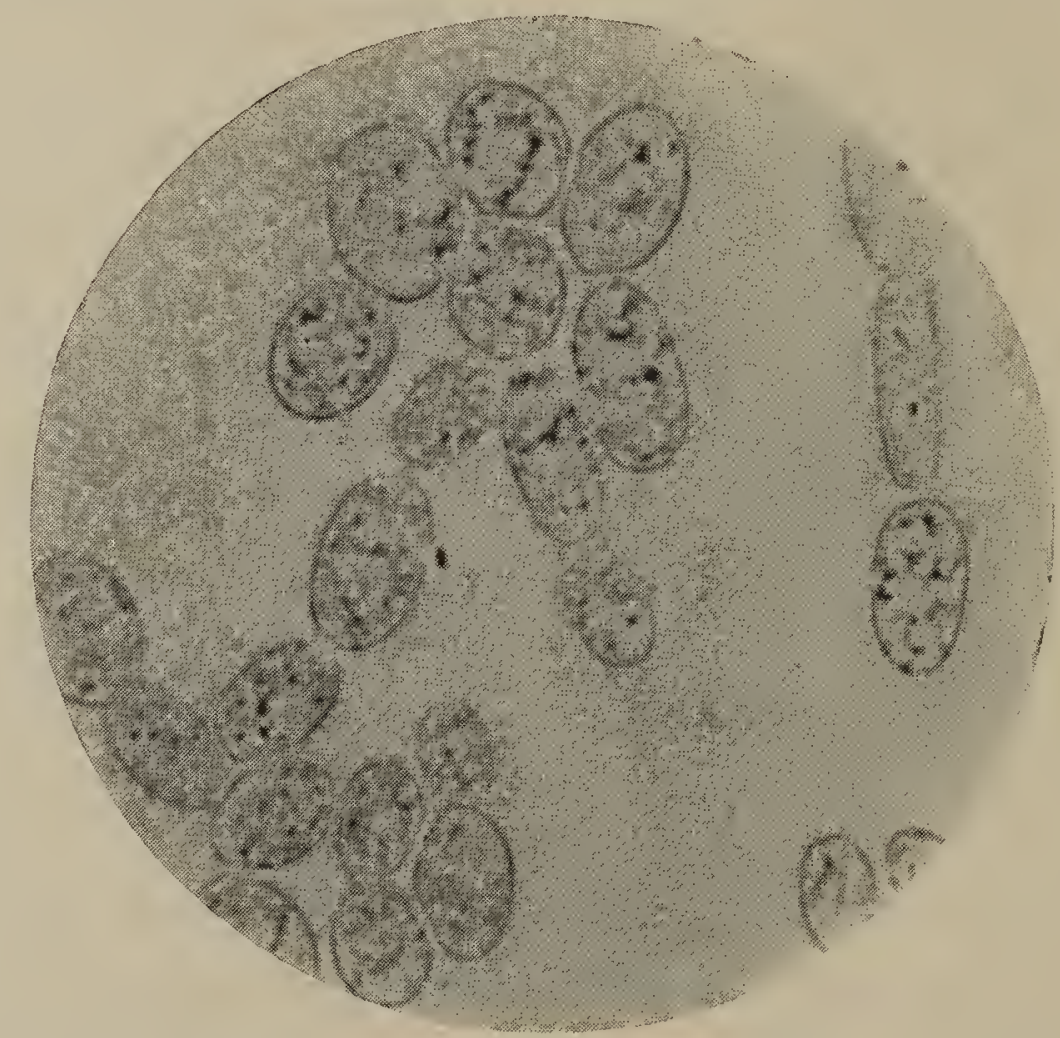

Fig. 144.-Yeast Celdis. Young culture unstained. (After Zettnow.)

of wine and beer. Although Schwann, as early as 1837, recognized the fact that many fermentations could not occur without the presence of yeast, it was not until considerably latel that the study of yeast fermentation was put upon a scientific basis. The typical fermentative action consists in the transformation of sugar into ethyl alcohol according to the following formula:

$$
\mathrm{C}_{6} \mathrm{H}_{12} \mathrm{O}_{6}=2 \mathrm{C}_{2} \mathrm{H}_{5} \mathrm{OH}+2 \mathrm{CO}_{2}
$$

The enzyme by which this fermentation is produced is known as " $z y-$ mase," and is, according to Buchner, in most cases, an endo-enzyme which may be procured from the cell by expression in a hydraulic press. In addition to this, however, the various yeasts also produce 
other ferments by means of which they may split higher carbohydrates, such as saccharose, maltose, and even starch, and prepare them for action of the zymase. The manner in which this is accomplished, and the by-products which are formed during the process, vary among different species, and it is for this reason that the employment of pure cultures is of such great importance in the wine and beer industries where differences in flavor and other qualities may be directly dependent upon the particular species of yeast employed for the fermentation. It is due to the work chiefly of Pasteur ${ }^{1}$ and of Hansen ${ }^{2}$ that the beer and wine industries have been carried on along exact and scientific lines.

As the incitants of disease in man, the yeasts have been much studied since 1894, when Busse ${ }^{3}$ reported a case of fatal, generalized yeast infection, beginning from a tibial bone abscess. The microorganism which was found in this case he named "Saccharomyces hominis." In morphological and biological characters it appeared to be a typical yeast, grew readily upon most artificial media, and produced active fermentation in sugars. Mycelia were not observed. When inoculated into animals, this yeast proved pathogenic for mice and rats. A peculiarity of Busse's culture, observed since then in the case of many pathogenic yeasts, was the formation of gelatinous capsules, of varying thicknesses, about the individual cells, developing with particular luxuriance in the animal lesions.

In 1896, Gilchrist ${ }^{4}$ described a peculiar skin disease, which he spoke of as pseudo-lupus vulgaris, in the lesions of which he demonstrated a large number of round, doubly-contoured bodies which, though differing somewhat from Busse's saccharomyces, were unquestionably members of, or closely related to, the family of blastomycetes.

In the same year, Curtis, ${ }^{5}$ in France, isolated a similar form from a myxoma of the leg. Ophüls ${ }^{6}$ has described a number of fatal cases occurring in California, which at first were wrongly interpreted as protozoan in origin, but later were determined by him to be caused by a species of blastomycetes. In a case observed by Zinsser ${ }^{7}$ similar microorganisms were isolated from an abscess of the back, which

1 Pasteur, "Ftudes sur la bière," Paris, 1876.

2 Hansen, "Prac. Studies in Fermentation," London, 1896.

3 Busse, Cent. f. Bakt., I, xvi, 1894, and Virch. Arch., 140, 1895.

${ }^{4}$ Gilchrist, Bull. Johns Hopkins Hosp., vii, 1896.

${ }^{5}$ Curtis, Ann. de l'inst. Pasteur, 10, 1896.

${ }_{6}^{6}$ Ophüls, Jour. Exp. Med., 6, 1901.

7 Zinsser, Proc. N. Y. Path. Soc., vii. 1907. 
in almost all respects corresponded to Gilchrist's cultures. Animal inoculation in rabbits and guinea-pigs proved positive in this case and the organism seemed to show selective action for the lungs and spleen. In the lungs of the animals, especially, lesions were found with surprising regularity even when the inoculation was made intraperitoneally.

Cases of blastomycotic infection in man have been reported in large numbers and appear to be less rare than they were formerly believed to be. The clinical course of the disease is by no means uniform. A welldefined clinical picture seems to characterize the cases of blastomycotic dermatitis first described by Gilchrist. The eruption is very chronic and begins usually as a small pimple or papule with moderate induration of the skin. Scabs and pustules then form, which discharge yellowishwhite pus. As the lesion slowly spreads, the older areas show a tendency to spontaneous healing. In Gilchrist's ${ }^{1}$ case, it took four years for the lesion to spread two inches. When not purely cutaneous, blastomycotic infection takes the form of chronic abscess formation occurring in various parts of the body. In the latter, metastatic lesions in the lungs have been occasionally observed, and in one case cited by Ophüls, ${ }^{2}$ the lung seemed to have been the primary focus.

The fact that blastomycetes have frequently been found in tumol tissue has led several Italian observers ${ }^{3}$ to assume an etiological relationship between these microorganisms and malignant growths. Absolutely no satisfactory evidence in favor of such a belief has been advanced, however, and the yeasts in these conditions must be regarded as purely fortuitous findings.

In animals, diseases caused by members of the yeast family have been reported by various observers. The most important communication of this kind is by Tokishige, ${ }^{4}$ who found these microorganisms in a nodular skin disease occurring among horses in Japan. Sanfelice ${ }^{5}$ has isolated similar microorganisms from the lymph glands of a horse which was apparently suffering from a primary carcinoma of the liver. The same author has described a member of this group which he obtained from a cheesy consolidation occurring in the lung of a hog.

Demonstration of the organisms offers little difficulty either in fresh preparations of the pus under a cover-slip, or in smears stained with

${ }^{1}$ Rixford and Gilchrist, Johns Hopkins Hosp. Rep., i, 1896.

2 Ophüls, loc. cit.

3 Sanfelice, Cent. f. Bakt., I, xxxi, 1902.

- Tokishige, Cent. f. Bakt., I, xix, 1896.

${ }_{5}^{5}$ Sanfelice, Cent. f. Bakt. I, xviii, 1895, and Zeitschr. f. Hyg., xxi, 1895. 
thionin, methylene-blue, or the polychrome stains. In fresh preparations the addition of a little $\mathrm{NaOH}$ in weak solution facilitates the search. When found, the organisms are easily recognized by their size, their highly refractive doubly-contoured cell-membrane, their vacuolated protoplasm, and, most important, by the discovery of budding forms. In tissue sections they are recognizable by the ordinary hematoxylineosin technique, but may be better demonstrated by the Loeffler methylene-blue method in use for bacterial tissue-staining. Excellent preparations are obtained by staining frozen sections with thionin, a method well adapted for the demonstration of capsules.

The cultivation of the blastomycetes is comparatively easy after they have once been obtained in pure culture. Their isolation, however, usually is difficult when they occur in material contaminated with bacteria. Growing more slowly than the bacteria, cultures taken from such contaminated material usually show very few yeast colonies. No special methods of facilitating the procedure have been devised, but success will often attend painstaking and oft-repeated plating of the mixed cultures in high dilution. The most favorable medium for this process is glucose agar. When once obtained in pure culture, the blastomycetes can readily be kept alive for indefinite periods by transplantation repeated every two or three months. On agar or glucose agar, colonies appear after about three or four days as minute, glistening, white hemispherical spots which are not unlike colonies of staphylococcus albus. Planted in agar stab cultures, the microorganisms indicate their preference for a well-oxygenated environment by growing but slightly along the course of the stab, but by heaping up in a thick, creamy layer upon the surface of the medium. This layer in old cultures may be a quarter of an inch high. At first white, the growth, after three or four weeks, may turn distinctly yellow or even brown. In broth, the microorganisms form a stringy, gelatinous, and uneven cloud. On Loeffer's blood-serum media, and upon gelatin, growth is easily obtained. The gelatin is not liquefied. Sugar media are fermented by few of the pathogenic blastomycetes, a fact which places them rather in distinct contrast with the fermenting yeasts used in the industries.

Great and fundamental differences seem to exist between the pathogenic species described by various observers, and attempts at systematizing the various members of the group, such as that by Rickets, ${ }^{1}$

Rickets, Jour. Med. Res., 6, 1902. 
have met with almost insurmountable obstacles. Some of the forms described, like that of Busse, have fermented sugars and have not formed mycelia, while organisms like that of Gilchrist did not cause fermentation in carbohydrate media, but, by their formation of mycelia under certain conditions, have indicated their close relationship or possibly their identity with the oïdia, transitional forms between the yeasts and the hyphomycetes. It is thus, in the light of our present knowledge of these microorganisms, quite impossible to establish within this group a distinct classification that is at all reliable.

In considering the possible origin of blastomycotic infection in animals and man, it is, of course, important that we should have some knowledge as to the pathogenic properties of the yeast met with and handled in daily life. Rabinowitsch, ${ }^{1}$ with this in view, has investigated the pathogenic properties of fifty different species of yeasts obtained from fruit, manure, dough, and other sources, and among them found only seven varieties that had any pathogenicity for rabbits, mice, or guinea-pigs. In most of her successful inoculations, however, the disease produced in the animals had but very little resemblance to the blastomycotic conditions observed in man.

${ }^{1}$ Rabinowitsch, Zeit. f. Hyg., xxi, 1895. 


\title{
CHAPTER XLVI
}

\author{
HYPHOMYCETES
}

(Eumycetes, Molds)

THE hyphomycetes or molds interest the bacteriologist chiefly because of the frequency with which they appear as contaminants during bacterial cultivation, and because they play the rôle of incitants in a few common diseases of the skin and mucous membranes.

Morphologically they are entirely distinct from and much more complex than the bacteria. To the yeasts they are more closely related, the gap between the two classes being bridged by certain forms often spoken of as "oïdia" which, though usually appearing in the budding yeast-form, may occasionally grow out in mycelial threads.

The characteristic feature of the hyphomycetes as a class is the formation of long, interlacing filaments or threads, known as mycelia. From these, branches come off which are spoken of as "hyphæ." Each mycelial thread possesses a well-marked, doubly-contoured sheath or cell-wall and a finely granular protoplasmic cell-body, which, in some of the forms, is multinucleated.

Two main classes of hyphomycetes are distinguished, the phycomycetes, and the so-called higher forms, or mycomycetes. The former class is characterized by the fact that no partitions exist within the mycelial threads or hyphæ, the entire meshwork of a single microorganism consisting of one multinucleated cell. This group, furthermore, possesses the power of reproduction by both a sexual and an asexual process. The second class, or the mycomycetes, possess a mycelial meshwork which is divided into numerous partitions, and reproduces usually by the asexual process only.

The process of reproduction, upon the basis of which the separation of groups within this class is determined, is best illustrated by citing a common example of each of the main divisions.

As an example of the phycomycetes, the division most commonly met with upon contaminated gelatin plates, or upon exposed and moist organic matter of any description, is the one spoken of as the muco- 
rince. Most forms belonging to this division appear, grossly, as a light, cotton-like fluff, spreading in a thin fur over the surface of the culture medium. Examined with the low power of a microscope, there may be seen a complicated network of fine mycelial threads, which show no septa and from which delicate hyphal branches arise. In the formation of the asexual spore organs near the tip of each hypha a septum appears. The tip of the hypha then gradually enlarges and furms a spherical capsule which is known as the sporangium. The unswollen

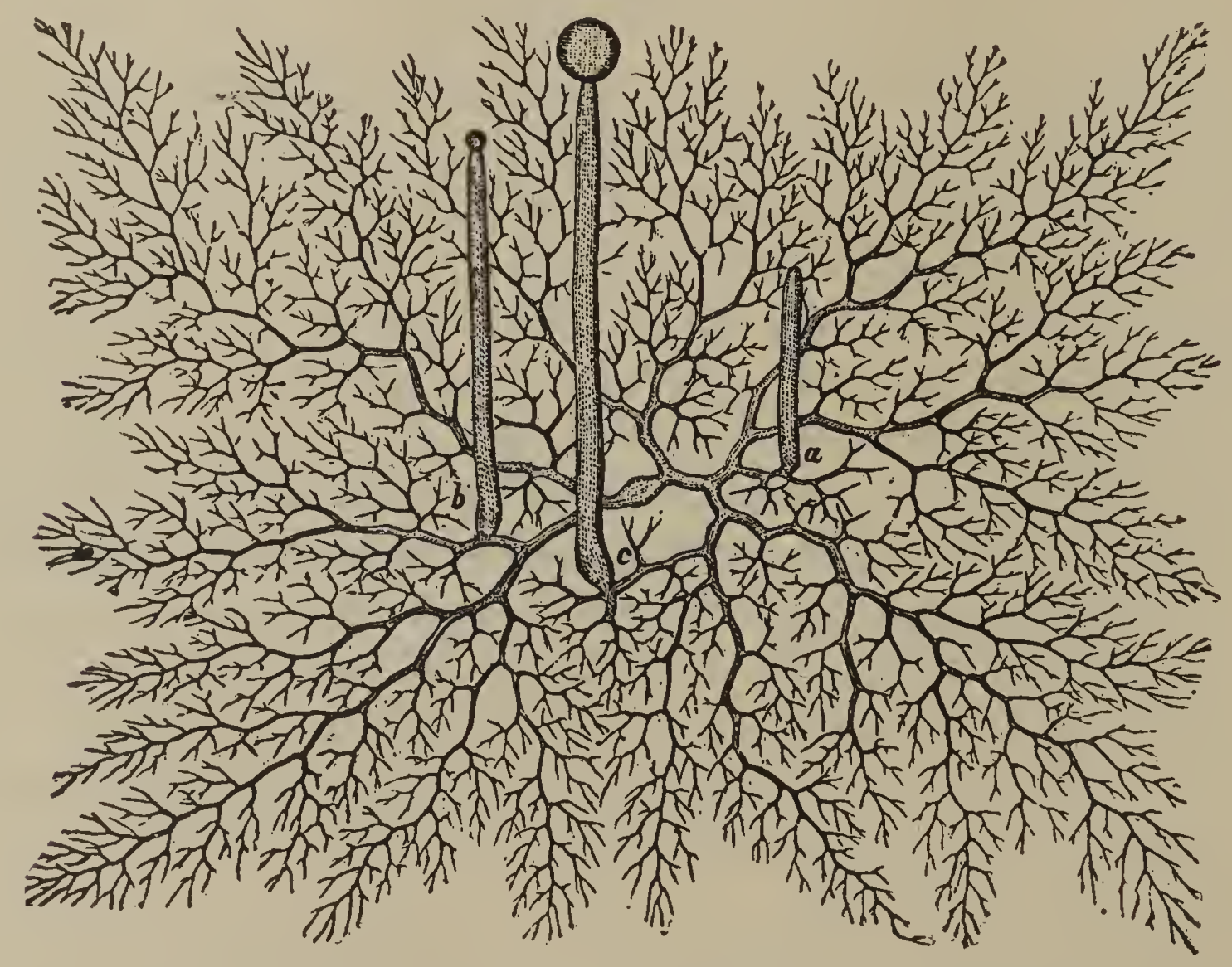

FIa. 145.--Mucor Mucedo. Single-celled mycelium with three hyphæ and one developed sporangium. (After Kny, from Tavel.)

portion of the hypha which projects into the sporangium is spoken of as the columella. Within the sporangium, a large number of small, round spores are formed. When these are ripe, the wall of the sporangium bursts and the spores escape. Upon suitable media, then, new mycelia develop from these spores. The sexual reproduction, which occurs in this group, takes place in the following way: From two hyphæ, placed in close apposition, lateral branches grow toward each other. These are spoken of as gametophores. The tips of the gametophores soon come in contact and, for a time, their protoplasm freely intercommunicates. Septa are then formed which cut off from the original hyphæ a central cell, the zygospore. This zygospore gradually enlarges 
and, under favorable conditions, sends out a branch which terminates in a non-sexual sporangium.

Among the higher molds, or mycomycetes, various methods of sporulation occur, but sexual reproduction does not usually take place.

One of the most commonly found genera is that of Penicillium. In this form the mycelial threads are partitioned off, by many transverse septa, into a number of separate cells. From these, hyphæ, also septate, are given off. From the ends of these hyphæ, germinating branches arise which are known as conidiophores. These conidiophores divide into two or three septate branches, the sterigmata. From the

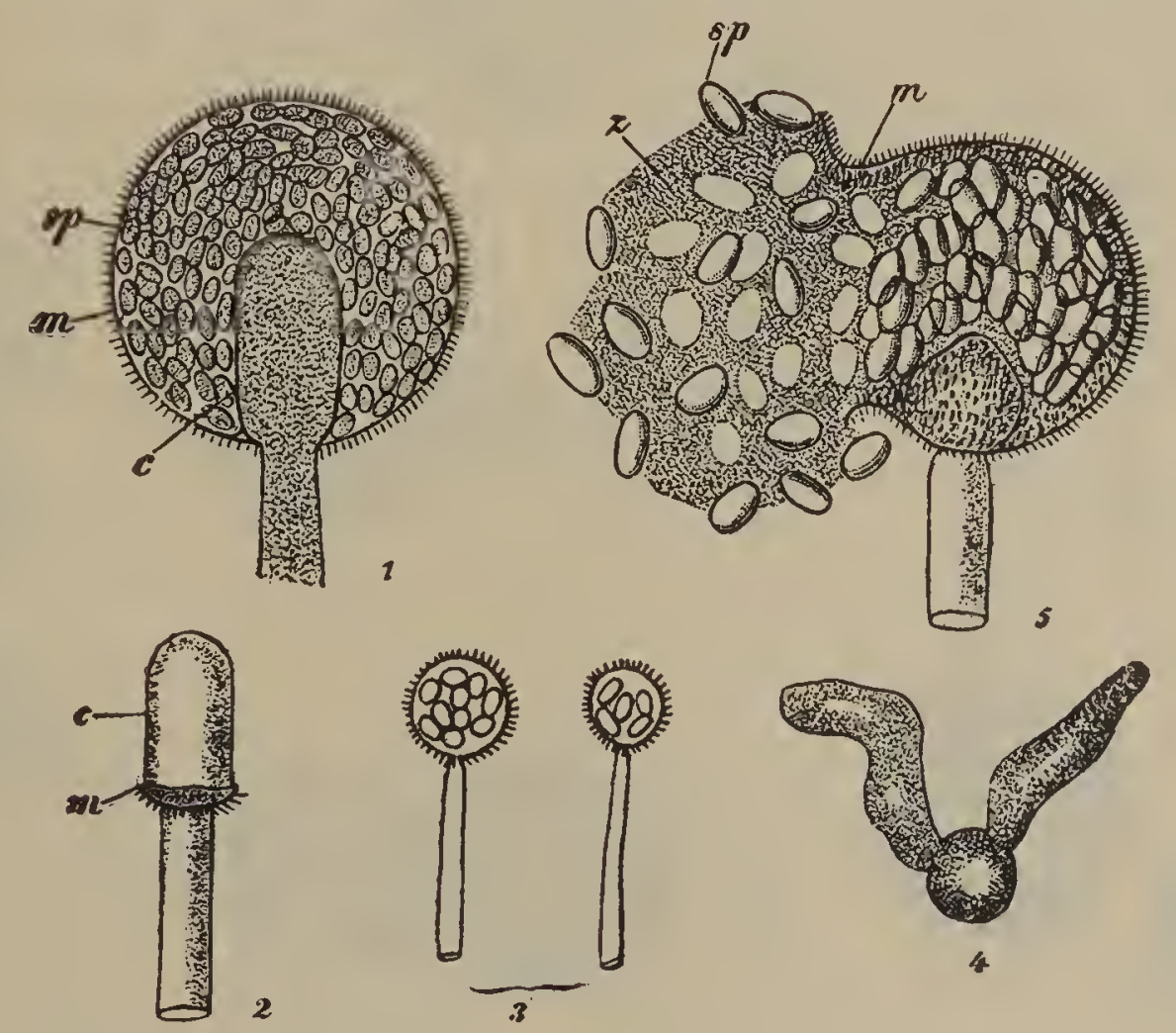

Fig. 146.-MUCOR MUCEDo. 1. Sporangium, c. columella, $m$. sporangium capsule, $s p$. spores. 2. Columella, after bursting of sporangium. 3. Poorly developed sporangia. 4. Germinating spore. 5. Emptying of sporangium. (After Brefeld.)

ends of these, other sterigmata may be given off and from the tip of each of these a single chain of spores or conidia are constricted off. The result is an appearance not unlike a hand in which the wrist represents the conidiophore; the metacarpal bones, the sterigmata; and the fingers, the long streptococcus-like chains of conidia.

Similar to and even more common than the penicillia are the varieties known as Aspergillus. These forms, like the preceding, form delicate mycelial meshworks from which branches or conidiophores about 3-10 $\mathrm{mm}$. in length, arise. These develop club-shaped expansions at their free ends and from these club-shaped expansions radially arranged sterig- 
mata arise. On the ends of these sterigmata spores or conidia develop similar to those developed by penicillium.

Other forms of sporulation occur within this group. 'Thus, tubular' spore capsules may be formed within the end segments of the hyphæ, known as ascospores. In other cases, within a mycelial thread, a swelling may take place into which protoplasm flows from the neighboring cells, at both ends. In this way, an oval spore case is developed

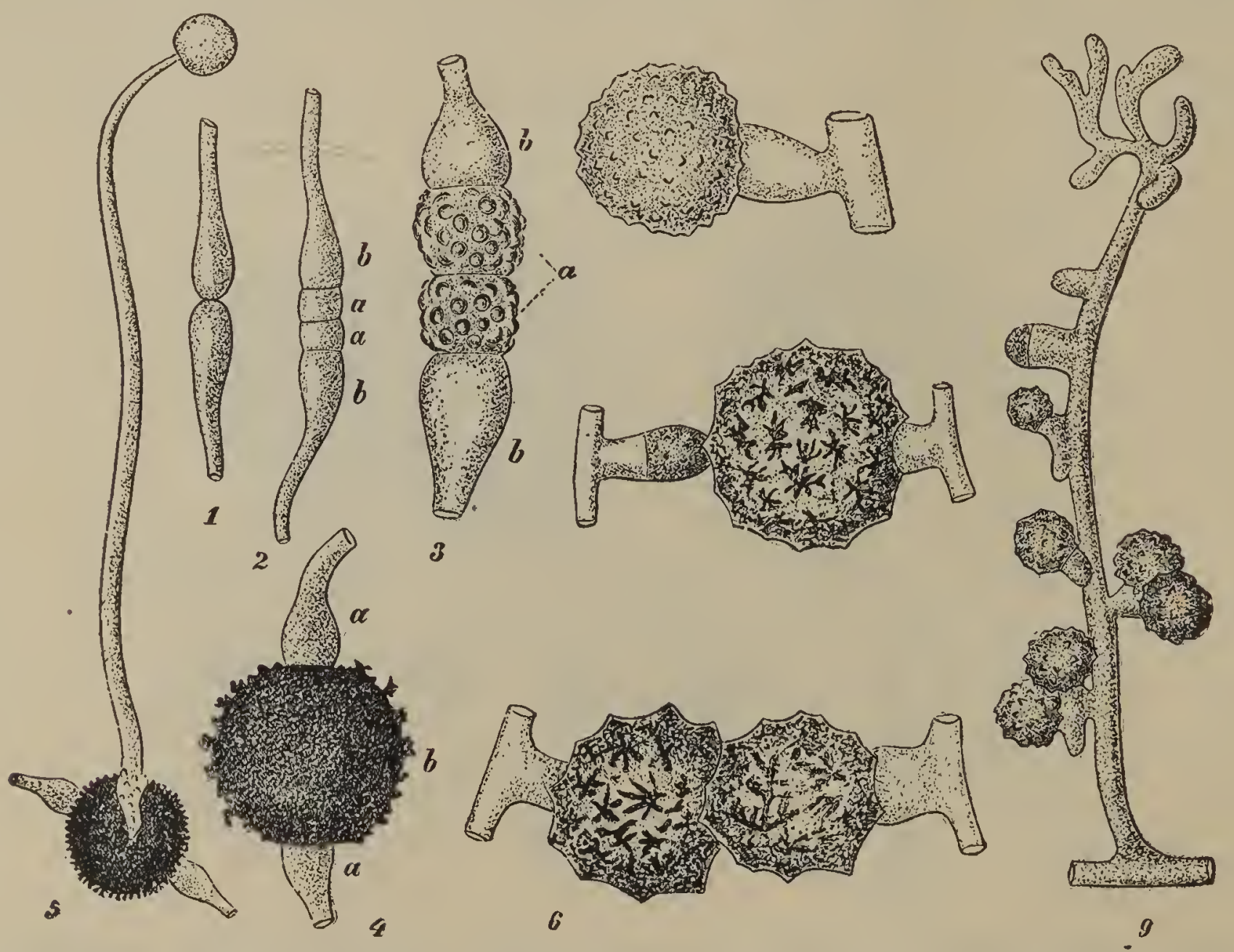

Fig. 147.-Mucor mucedo. Formation of zygospore. 1. Two branches coalescing. 2 and 3. Process of conjugation. 4. Ripe zygospore. 5. Germination of zygospore. 6 and 7. Mucor erectus. Azygo sporulation. No two branches meet, but form spores without conjugation. 8 and 9. Mucor tenuis. Azygo sporulation. The spores grow out from side branches without sexual union. (1-5 after Brefeld; 6-9 after Bainier, from Tavel.)

within the course of the mycelial thread. This is known as a chlamydospore. The segments on each side of the chlamydospore die out and the spore capsule is liberated from the mycelium.

The classification of the various divisions of the hyphomycetes is a problem requiring much study and great botanical insight, and can hardly be discussed in a general work on bacteriology.

Upon artificial media, the members of this group are not at all fastidious, growing easily upon organic matter of all kinds, provided 
moisture is present. In laboratories they are frequently found as contaminants, and in order to procure them for purposes of study it is only necessary to expose agar or gelatin plates in a dusty, dark corner. In households they are frequently found growing in store-rooms upon stale bread, shoes, leather trunks, and on remnants of food. Most of them prefer an acid environment and are dependent upon a free supply of oxygen. At room temperature they grow more readily than at the usual incubator temperature.

\section{DISEASES CAUSED BY HYPHOMYCETES}

Pityriasis versicolor (Microsporon furfur).--Pityriasis is a disease of the skin observed chiefly among persons living under conditions of uncleanliness, or among those who combine these conditions with a tendency to profuse perspiration. It begins as a small, light brown or yellowish patch upon the skin of the abdomen, breast, or back, is flat and barely raised from the cutaneous surface. It spreads and coalesces with similar spots until the entire area resembles strongly the figures of a map with irregular continents and islands. The disease does not penetrate into the skin itself, but consists, as Plaut has pointed out, of a simple saprophytism of the inciting agent upon the skin.

The condition is caused by a member of the group of Hyphomycetes, discovered in 1846 by Eichstedt, and later named Microsporon furfur. The microorganism consists of a dense meshwork of mycelial threads, from which septate hyphæ arise in large numbers. From the ends of these, spores arise in rows, after the manner depicted for

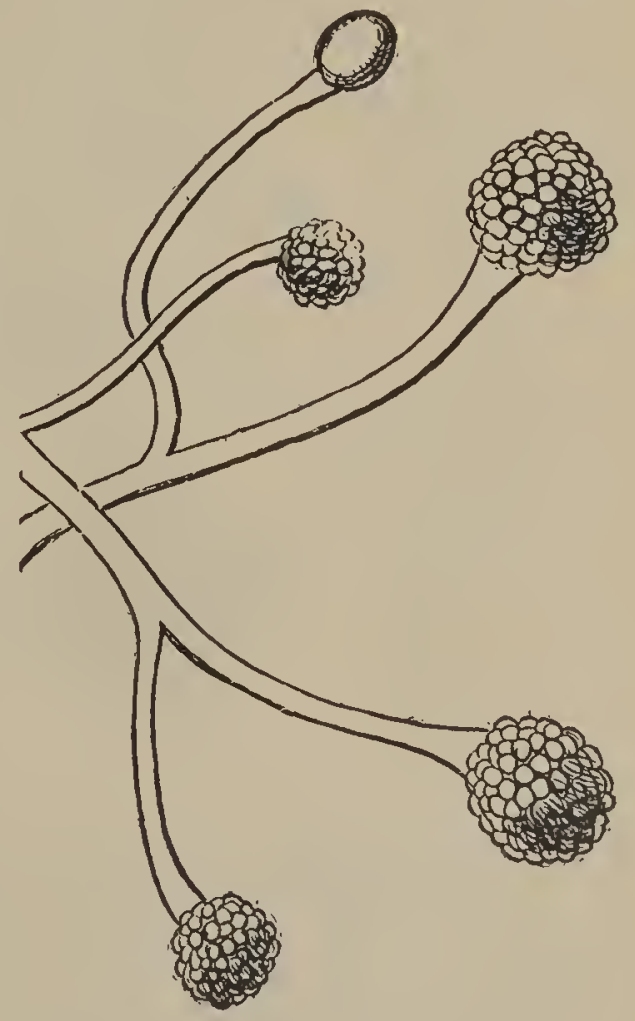

FIG. 148.-Mucor RAMOsus. Ripe sporangia on columellæ. (After Lindt.) penicillium (Fig. 149). The hyphæ, according to Unna, ${ }^{1}$ show a characteristic bending at right angles, due to a slight flattening of their diameters. Characteristic of the microsporon proper, in preparations made from cutaneous scrapings, are the fragments of bent hyphæ and the large numbers of free spores.

${ }^{1}$ Unna, "Histopathol. of Skin," transl., N. Y., Macmillan, 1896. 
Cultivation of Microsporon furfur has been successfully carried out by many observers. ${ }^{1}$ Growth is particularly characteristic upon potato, white or yellowish-white colonies appearing within four or five days and rapidly spreading over the entire surface of the potato.

Thrush (Soor or Muguet; Ö̈dium albicans).-Thrush is a localized disease of the mouth occurring most frequently in children suffering

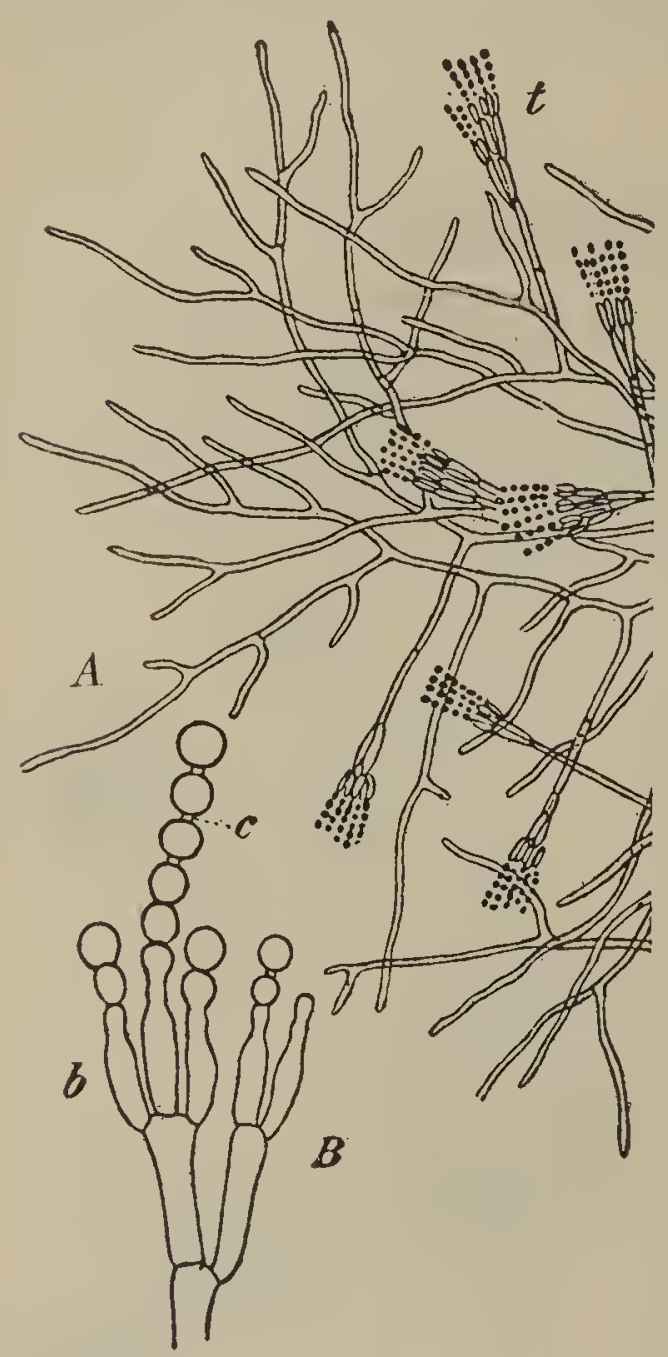

Fig. 149.-Penicillium GLAUCUM. A. Showing septate mycelia. $B$. End of a hypha -branching into two conidiophores, from which are given off the sterigmata. From the ends of these are developed the round conidia. (After Zopf.)

and in the presence of free oxygen, upon gelatin and agar.

Favus (Achorion Schoenleinii).-Favus is a disease attacking chiefly

${ }^{1}$ Kotjar, Ref. Baumgarten's Jahresbericht, 1892.

${ }^{2}$ Grawitz, Virch. Arch., 1886.

${ }^{3}$ Linossier et Roux, Comptes rend. de l'acad. des sci., 1889. ditions of uncleanliness, upon an area of catarrhal inflammation of the mucous membrane.

The microorganism which causes the condition was first described by Langenbeck in 1839, and has, since then, been studied by many observers. It was successfully cultivated by Grawitz ${ }^{2}$ in 1886 and recognized by him as belonging to the hyphomycetes. The most careful cultural studies which have been made upon the Oïdium albicans are those of Linossier and Roux. ${ }^{3}$

Morphologically, the oildium consists of budding cells, resembling those of yeast, which, under certain conditions, can produce mycelia and hyphæ from which spores are developed. Two main varieties are described, that which produces large spores and liquefies gelatin in culture, and that which gives rise to smaller spores and fails to liquefy gelatin. In many cases only the yeast-like budding cells can be found; these, however, when subjected to unfavorable cultural conditions, may be induced to send out hyphæ and form spores. Like yeasts, but to a lesser degree, the Oïdium albicans causes fermentation in sugars. It develops best under slightly acid conditions from malnutrition or it occurs, under con- 
the hairy portions of the body of man and some domestic animals. In man, it is found most frequently in undernourished children upon the scalp. It is a disease of extremely chronic course and is very resistant to treatment. Beginning as a small erythematous spot, it soon develops into small sulphur-yellow cupped crusts, which are placed usually about the base of a hair. These may spread and coalesce. The small indentated crust is spoken of as a scutulum. When such a scutulum is removed and examined under a microscope, teased out in a few drops of strong sodium hydrate solution (20 per cent), the incitant of the disease may be easily recognized and studied. In such a preparation the cen-

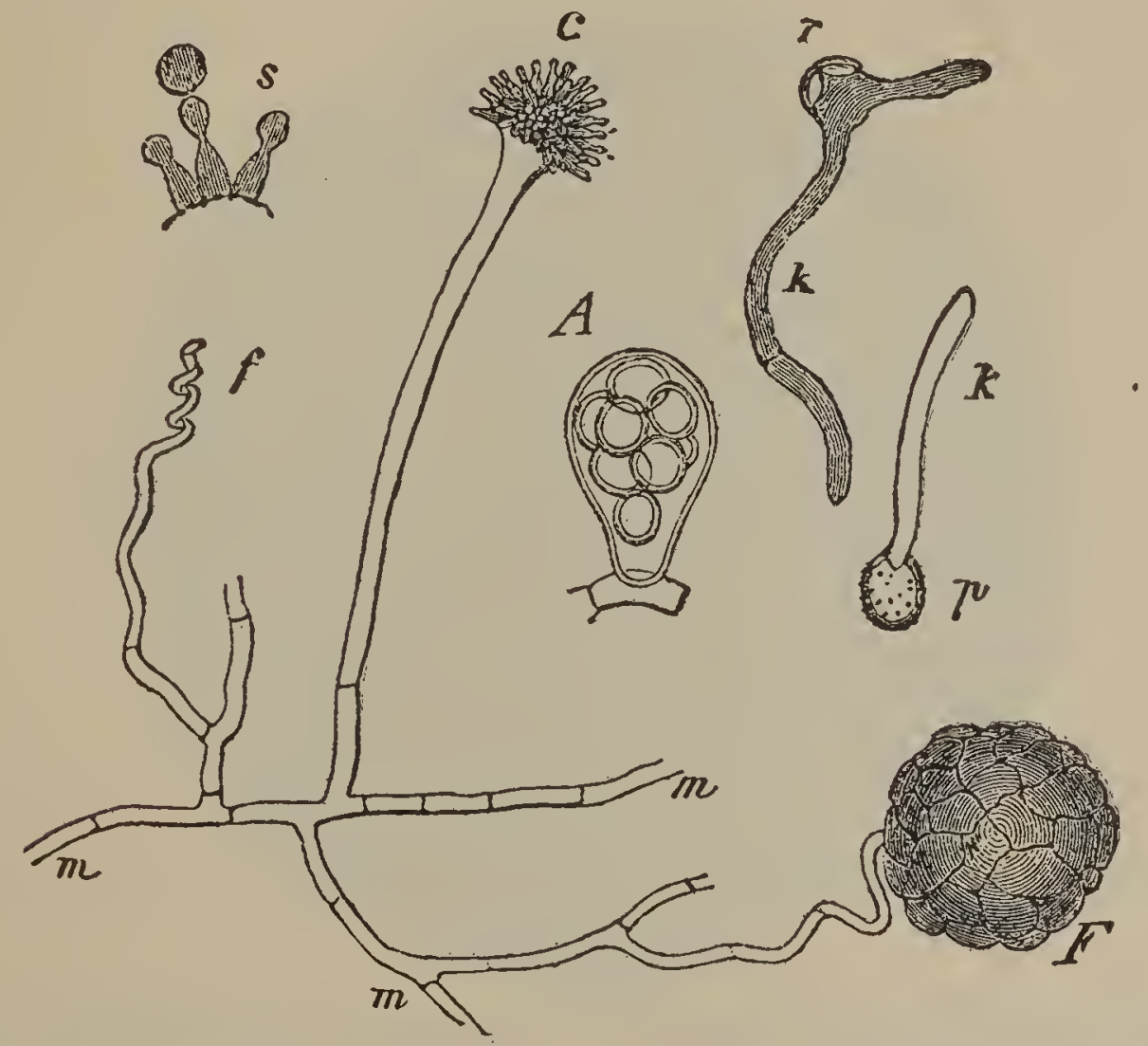

Fig. 150.-Aspergillus glaucus. m. Mycelial threads. s. Sterigmata.

$r$. Ascospore. $p$. Germinating conidium. A. Ascus. (After de Bary.)

ter of the scutulum is found to be made up chiefly of small doublycontoured spores which are irregularly oval or round, and may be arranged in chains, lying scattered among an extremely dense meshwork of fine mycelial threads. Toward the periphery of the scutulum, the spores are less numerous and the looser arrangement of the meshwork permits us to distinguish distinct filaments of mycelia which give off hyphæ, the ends of which are often swollen into small knobs. The interior of the scutulum usually contains a pure culture of the fungus.

Many varieties of achorion have been described, but Plaut ${ }^{1}$ believes that, at the present time, it is not possible to separate these from one

1 Plaut, in Kolle und Wassermann's "Handbuch," I. 
another, owing to the fact that selective cultivation has succeeded in altering many of the characteristics displayed by many of the strains. The same observer recommends the following method for obtaining pure cultures of this microorganism. As much of the material as can be conveniently obtained is gently rubbed up in a sterile mortar with fine sand or infusorial earth. The triturated material is then inoculated into fluid agar and plates are poured.

Ordinary streaked plates upon agar may also be used with success with material directly from the centers of scutula.

The achorion grows best upon acid agar at a temperature of $37.5^{\circ} \mathrm{C}$.

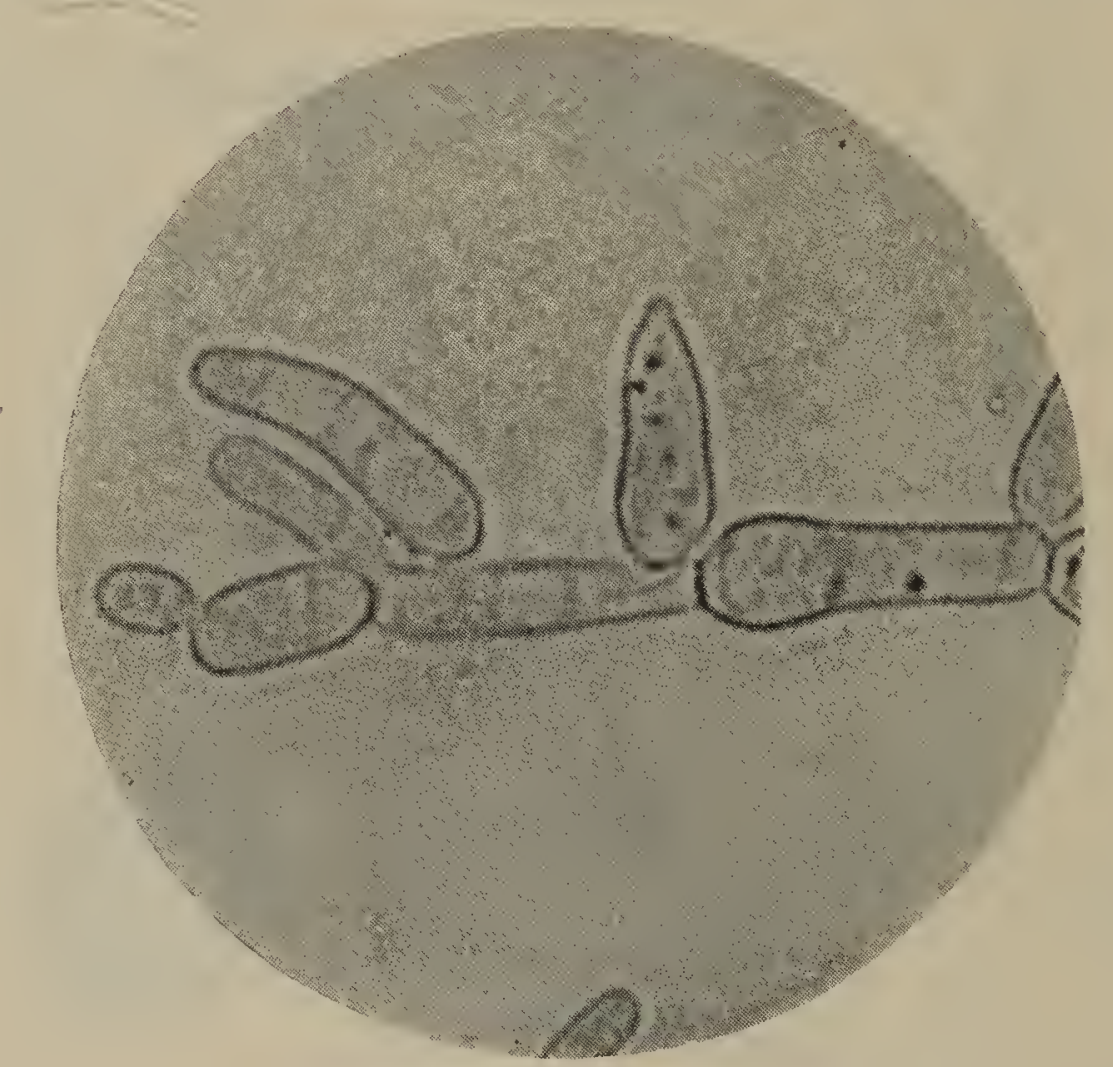

FIG. 151.-Thrush. Oidium albicans, unstained. (After Zettnow.)

Growth appears within from forty-eight hours to three days as yellowish disks, which occasionally may be slightly furred with aërial hyphæ.

Ringworm (Trichophyton tonsurans).-Ringworm, Tinea circinata, or Herpes tonsurans, is a contagious disease of the skin and hair, occurring most often in children and appearing upon both the haired portions of the body, as well as upon free skin. It is characterized by the formation of circular scaly patches, within which the hairs fall out.

The disease is caused by several species of the trichophyton, a genus of hyphomycetes. These microorganisms were first recognized as incitants of the disease by Gruby ${ }^{1}$ in 1841, and were most thoroughly

${ }^{1}$ Gruby, Comptes rend. de l'acad. des sci., 13, 1841. 
studied later, by Sabouraud. ${ }^{1}$ The fungi consist of finely interlaced narrow septate mycelia, within which characteristic swellings appear. From these swellings, chlamydospores develop. Hyphæ, both aërial and deep, grow out of the mycelial threads, on the ends of which ascospores may develop. In the diseased skin, the fungi grow chiefly within the hair sheath, causing an area of secondary inflammation about the base of the hair. The infection probabiy begins first in the epidermis surrounding the hairs, from which it then spreads into the hair bulb and thence grows up into the substance of the hair in which mycelial threads

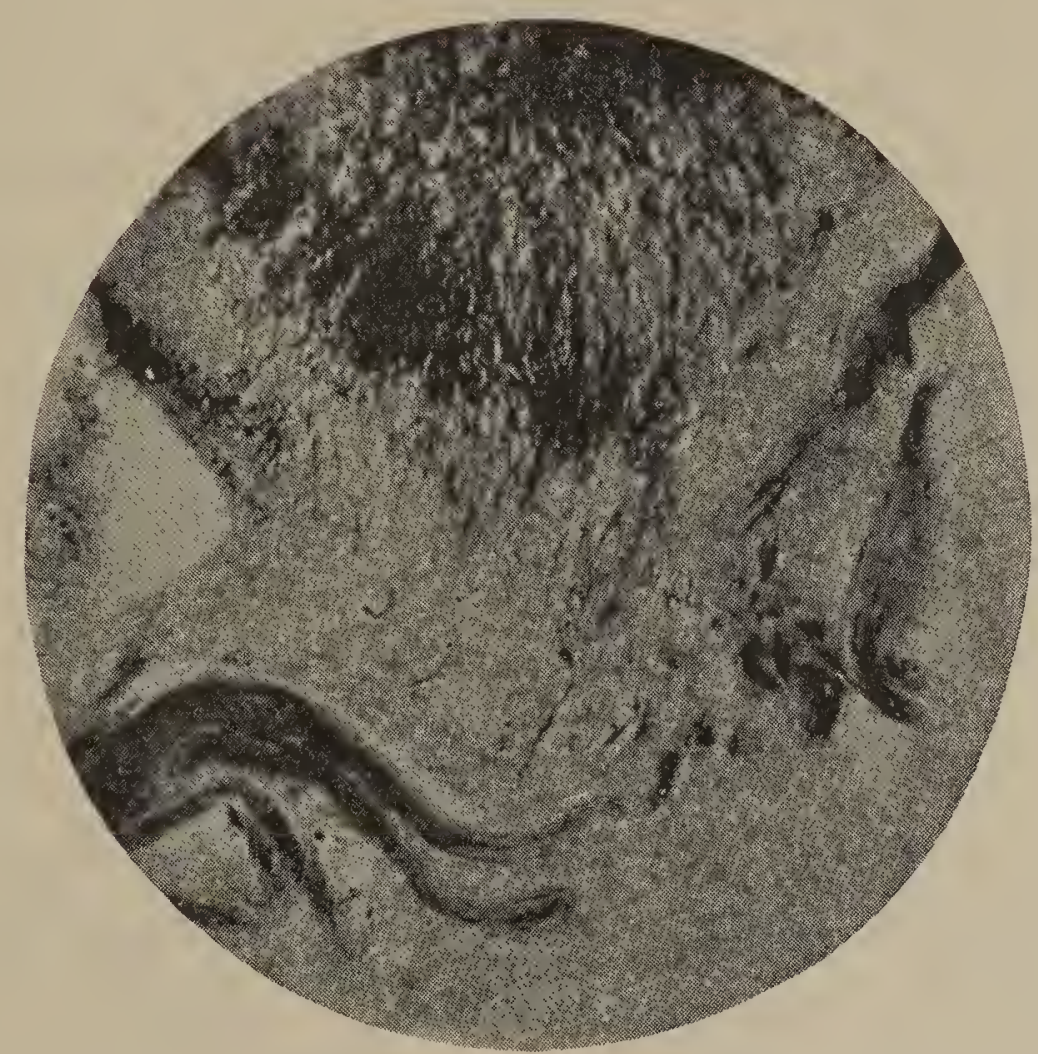

Fig. 152.-Achorion Schownleinir. Section of favus crust. Stained by Gram. (After Fraenkel and Pfeiffer.)

and spores develop in large numbers. The demonstration of the microorganism from a case can easily be accomplished by epilating an affected hair, making sure that the hair bulb has been removed. This is then immersed under a cover-slip in a drop of sodium hydrate or potassium hydrate solution and examined under the microscope. In this way enormous numbers of short mycelial threads and spores may be seen to lie within the bulb. Many varieties of these fungi have been described from different cases. Their interrelationship is not entirely clear. In general, a division is made between those which develop large spores and a more common small-spored variety.

1 Sabouraud, Ann. de dermat. et de syph., 3, 1892, and 4, 1893. 
Cultivation is comparatively simple and is best carried out upon acid glucose agar or gelatin. Upon such media, within five or six days, mycelial threads, which are septate and form chlamydospores, may be observed. Pigment, reddish or brown, is occasionally noted. Gelatin is liquefied. The disease may be produced with such cultures upon guinea-pigs. In man, the disease is usually acquired by infection from patient to patient.

Other Diseases in which Hyphomycetes have been Found.-A number of cases have been described in which members of this group have been found at autopsy in the lungs of persons dying of bronchopneumonia. ${ }^{1}$ In most of these cases, the fungus found belonged to the aspergillus group and was regarded as a merely secondary invader. A few cases, however, have been reported in which the fungus was regarded as the primary cause of the disease. A single case is on record, in which an intestinal infection with a member of the genus mucor resulted in a generalized infection with pulmonary and secondary cerebral abscesses. In birds, a disease of the lungs has long been known to be due to various species of aspergillus. In many domestic animals, diseases of the skin and hair occur which are caused by microorganisms similar to, or identical with, those occurring in man.

\section{SPOROTRICHOSIS}

Parasites closely allied to the blastomyces are the sporotrices which were first described by Schenck ${ }^{2}$ in this country and have been very thoroughly studied by De Beurmann and Gougerot. The parasites belong to the Fungi imperfecti. They occur in lesions as oval or cigarshaped spores (conidia) and grow in culture as branching septate mycelium with clusters of oval or spherical conidia about the ends of the hyphæ. According to some observers the conidia are attached to the mycelium by short pedicles. The conidia also occur along the sides of the hyphæ and are often grouped in whorls about the threads. Chlamydospores are also found in some cultures. The organisms are obligate aërobes and grow on all ordinary culture media, but better on those containing carbohydrates and of slightly acid reaction. The growth forms a thick, leathery, white coating on the surface of the medium which in older cultures becomes coffee-colored, and in some instances black.

${ }^{1}$ Sticker, Nothnagel, "Spez. Path. u. Ther.," 14, 1900.

2 Schenck, Johns Hopkins Hosp. Bull., 1898, 286. 
De Beurmann and Gougerot ${ }^{1}$ have described a number of species of sporotrices which are differentiated by variations in pigment production, in optimum temperature, and in profusion and morphology of the conidia in culture. Other observers believe all these organisms belong to the same species. The diagnosis may be made in some cases by finding conidia in the softened material from the lesions. These are best demonstrated by Gram's stain. In other cases it is necessary to resort to cultural methods, as the conidia can not always be found on direct examination.

Only a few cases of the disease have been reported in this country, but it is apparently common in France and has been reported in nearly every quarter of the globe. The lesions are usually subcutaneous, but visceral forms have been described. Numerous types of lesions are found. The commonest forms are disseminated nodules which resemble gummata. In other cases there are scattered subcutaneous abscesses which are usually associated with lymphangitis. There is also a papulo-vesicular form which usually leads to ulceration. The lesions are chronic in character and simulate the lesions of syphilis or tuberculosis, for which conditions many cases of sporotrichosis have probably been mistaken. Nodular lesions have also been found in the bones, in lymph nodes, and in the lungs and kidney. The lesions consist of foci of chronic granulation, the tissue containing numerous giant cells, which later undergo separation. There is as a rule little systemic disturbance associated with the disease. The lesions often heal spontaneously, leaving dense scars, but clear up very rapidly under iodide therapy.

The most susceptible laboratory animals are mice and rats which show lesions resembling those in man associated with marked cachexia, though the disease is seldom fatal. The disease has also been produced in rabbits, guinea-pigs, and dogs, though these animals are not susceptible to all strains. In making cultures De Beurmann and Gougerot recommend the use of Sabouraud's glucose pepton agar (water, 1,000 c.c.; pepton, $10 \mathrm{gm}$.; glucose, $40 \mathrm{gm}$.; agar, $18 \mathrm{gm}$.; not neutralized). Taylor ${ }^{2}$ recommends glycerin agar with the addition of dextrose and 1 per cent acetic or citric acid as the most favorable medium for these organisms. Tubes should be inoculated with large amounts of pus (1 c.c. if possible), and should be incubated for several days at room temperature.

${ }^{1}$ De Beurmann et Gougerot, "Traité des Sporotrichoses," Félix Alcan, Paris, 1912.

2 Taylor, Jour. A. M. A., 1913, lx, 1142. 


\section{SECTION IV}

\section{DISEASES OF UNKNOWN ETIOLOGY}

\section{CHAPTER XLVII}

\section{RABIES \\ (Hydrophobia, Rage, Lyssa, Hundswuth)}

RABIES is primarily a disease of animals, infectious for practically all the mammalia, but most prevalent among carnivora, dogs, cats, and wolves. It is said also to occur spontaneously among skunks of the southwestern United States, and is readily inoculable upon guinea-pigs, rabbits, mice, rats, and certain birds, chickens and geese being especially susceptible. Man is subject to the disease. Infection usually occurs as a consequence of the saliva of rabid animals gaining entrance to wounds from bites or scratches. The disease is prevalent to an alarming extent in all civilized countries except England, where the careful supervision of dogs, enforcement of muzzling laws, and rigid legislation regarding the importation of dogs, have caused a practical eradication of the disease in that country. A fair estimate of the prevalence of the disease may be obtained from the statistics of animals dying or killed because of rabies in differert countries. In Germany, according to Kolle and Hetsch, during the fifteen years ending in 1901, there were 9,069 dogs, 1,664 cattle, 191 sheep, 110 horses, 175 hogs, 79 cats, 16 goats, 1 mule, and 1 fox affected with rabies. In the eastern United States the disease is not uncommon. The statistics of the New York Department of Health, for a period of six months ending December 31, 1907, show 74 cases of rabies among dogs in the city of New York and vicinity. Among human beings the disease is no longer common in civilized countries, since early preventive treatment is successfully applied in almost all infected subjects.

Experimental infection in susceptible animals is best carried out by injections of a salt-solution emulsion of the brain or spinal cord of an 
afflicted animal, subdurally, through a trephined opening in the skull, but may also be accomplished by injection into the peripheral nerves, the spinal canal, or the anterior chamber of the eye. Intravenous and intramuscular injections are also successful, though less regularly so.

The time of incubation after inoculation varies with the nature of the virus used, the location of the injection, and the quantity injected. In accidental infections of man and animals the incubation is shortest and the disease most severe when the wounds are about the head, neck, and upper extremities and are deeply lacerated. This is explained by the fact that the poison is conveyed to the central nervous system chiefly by the path of the nerve trunks. This has been experimentally shown by di Vestea and Zagari ${ }^{1}$ who inoculated animals by injection into peripheral nerves, and showed that the nerve tissue near the point of inoculation becomes infectious more quickly than the parts higher up; thus the lumbar cord of an animal inoculated in the sciatic nerve is infectious several days before virus can be demonstrated in the medulla.

In man, infected with "street virus," that is, with the virus of a dog or other animal not experimentally inoculated, the incubation period varies from about forty to sixty days. Isolated cases have been reported in which this period was prolonged for several months beyond this.

The virulence of rabic virus may be markedly increased or diminished by a number of methods. By repeated passage of the virus through rabbits, Pasteur ${ }^{2}$ was able to increase its virulence to a more or less constant maximum. Such virus which had been brought to the highest obtainable virulence, he designated as "virus fixe." Inoculation of rabbits, dogs, guinea-pigs, rats, and mice with such virus usually results in symptoms within six to eight days. The same animals inoculated with street virus may remain apparently healthy for two to three weeks.

In dogs and guinea-pigs inoculation usually results first in a stage of increased excitability, restlessness, and sometimes viciousness. This is followed by depression, torpor, loss of appetite, inability to swallow, and finally paralysis. In rabbits the disease usually takes the form of what is known as "dumb rabies," the animals gradually growing more somnolent and weak, with tremors and gradual paralysis beginning in the hind legs.

In experimentally infected birds the disease is slow in appearing and

${ }^{1}$ di Vestea and Zagari, Ann. de l'inst. Pasteur, iii.

2 Pasteur's work on rabies. Compt. rend. de l'acad. des sciences, 1881, 1882, 1884, 1885, 1886, and Ann. de l'inst. Pasteur, 1887 and 1888. 
may show a course of gradually increasing weakness and progressive paralysis extending over a period of two weeks after the appearance of the first symptoms.

In man, the disease begins usually with headaches and nervous depression. This is followed by difficulty in swallowing and spasms of the respiratory muscles. These symptoms occur intermittently, the free intervals being marked by attacks of terror and nervous depression. Occasionally there are maniacal attacks in which the patient raves and completely loses self-control. Finally, paralysis sets in, ending eventually in death.

Pathological examination of the tissues of rabid animals and human beings reveals macroscopically nothing but ecchymoses in some of the mucous and serous membranes. Microscopically, however, many abnormal changes have been observed and were formerly utilized in histological diagnosis of the condition. Babes ${ }^{1}$ has described a disappearance of the chromatic element in the nerve cells of the spinal cord. This observation has been confirmed by others, ${ }^{2}$ but is no longer regarded as pathognomonic of rabies. The same observer has described a marked leucocytic infiltration which occurs about the blood-vessels of the brain and about the ganglia of the sympathetic system. These changes are not found in animals infected with virus fixe and are present only in animals and human beings inoculated with street virus.

In 1903, $\mathrm{Negri}^{3}$ of Pavia described peculiar structures which he observed in the cells of the central nervous system of rabid dogs. While present in all parts of the brain, these "Negri bodies" are most regularly present and numerous in the larger cells of the hippocampus major and in the Purkinje cells of the cerebellum. The presence of these structures in rabid animals and man has been confirmed by a large number of workers in various parts of the world, and the specific association of these bodies with the disease is now beyond doubt. In consequence, the determination of "Negri bodies" in the brains of suspected animals has become an extremely important method of diagnosis-more rapid and accurate than the methods previously known.

The demonstration of Negri bodies in tissues is carried out as follows: A small piece of tissue is taken from the cerebellum or from the center of the hippocampus major (cornu ammonis), and is fixed for twelve hours in Zenker's fluid. It is then washed thoroughly in water and

${ }^{1}$ Babes, Virch. Arch., 110, and Ann. de l'inst. Pasteur, 6, 1892.

${ }^{2}$ Van Gehuchten, Bull. de l'acad. de méd. et biol., 1900.

${ }^{3}$ Negri, Zeit. f. Hyg., xliii and xliv. 
dehydrated as usual in graded alcohols, embedded in paraffin, and sectioned. The sections are best stained by the method of Mann, as follows:

The sections, attached to slides in the usual way, are immersed in the following solution for from twelve to twenty-four hours:

Methylene-blue (Gruebler OO), 1 per cent........ 35 c.c.

Eosin (Gruebler BA), 1 per cent. . . . . . . . . . . 35 c.c.

Distilled water......................... 100 c.c.

They are then differentiated in:

Absolute alcohol...................... 30 c.c.

Sodium hydrate, 1 per cent in absolute alcohol .... 5 c.c.

In this solution blue is given off and the sections become red. After about five minutes the sections are removed from this solution, are washed in absolute alcohol, and are placed in water where they again become faintly bluish. It is of advantage to immerse them, now, in water slightly acidified with acetic acid. Following this they are dehydrated with absolute alcohol and cleared in xylol, as usual.

In preparations made in this way, the nerve cells are stained a pale blue, and in their cytoplasm, lying either close to the nucleus or near the root of the axis-cylinder process, are seen small oval bodies stained a deep pink. The bodies are variable in size, measuring from 1 to 27 micra in diameter. They are round or oval, show a more deeply stained peripheral zone which has been interpreted as a cell membrane, and, in their interior, often show small vacuole-like bodies. There may be more than one, often as many as three or four, in a single cell.

The rapid demonstration of Negri bodies in smears of brain tissue has recently been advocated by many observers and has been extensively used for diagnosis. It is carried out, according to Van Gieson, ${ }^{1}$ in the following way: A small pin-head-sized piece of brain tissue from the regions indicated above, is placed on one end of a slide under a coverglass and the cover is gently squeezed with the finger until the tissue is flattened out into a thin layer. The glass cover is then gently shifted across the slide until the brain tissue is smeared along the entire surface. These smears may be fixed in methyl alcohol and stained by the Giemsa method, as described in the chapter on Spirochæta pallida (see page 592).

Stained in this way, the Negri bodies are stained light blue, in contrast to the darker and more violet cell-bodies.

${ }^{1}$.Van Gieson, Proc. of N. Y. Pathol. Soc., N. S., iv, 1906. 
The smears may also be stained by a method originated by Van Gieson, which gives an excellent contrast stain and reveals more clearly the inner structure of the Negri bodies. Van Gieson's stain is prepared as follows:

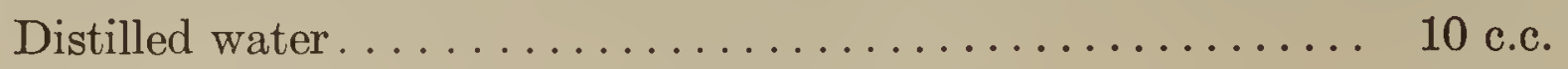

Saturated alcoholic solution of rosanilin violet.......... 2 drops.

Saturated aqueous solution of methylene-blue diluted one-half with water. . . . . . . . . . . . .

This method has been modified by Williams and Lowden, ${ }^{1}$ who add to 10 c.c. of distilled water 3 drops of saturated alcoholic basic fuchsin and 2 c.c. of Loeffler's methylene-blue. The slides are fixed in methyl alcohol, washed in water, and covered with the freshly prepared stain. The slide is held over the flame until the solution steams and is then rinsed in water and dried. The Negri bodies assume a brilliant hue and contain in their interior darkly stained, irregular particles which have been interpreted as chromatin bodies. As to the nature of the Negri bodies opinions are still divided. Their constant presence in rabic brain tissue is unquestioned and their diagnostic significance well established. Cultivation experiments, however, have been uniformly unsuccessful. A number of observers, Negri himself, Calkins, ${ }^{2}$ Williams and Lowden, ${ }^{3}$ and others, believe these bodies to be protozoa. The last-named authors base this opinion upon the definite morphology of the bodies, and their staining properties, which in many respects are similar to those of protozoa. These observers also claim that the morphology of the bodies shows a number of regular cyclic changes which are found accompanying different stages of the disease; these changes correspond, according to these workers, to similar cycles occurring among known protozoa of the suborders of the class Sporozoa. Many pathologists still look upon them as specific degenerations of the nerve cells similar to the changes observed by Babes.

It is not possible to decide absolutely from the facts at present at our disposal whether or not the Negri bodies should be regarded as parasites or as specific degeneration products. Their constant presence in rabic animals, and their apparent absence from normal brains and the brains of animals dead of other diseases, would tend to favor the parasitic view. To us it would seem that added to this the clear outlines, apparent regularity of structure, and curiously consistent grouping of the darkly

${ }^{1}$ Williams and Lowden, Jour. Inf. Dis., 3, 1906.

${ }^{2}$ Calkins, Discussion, Proc. N. Y. Pathol. Soc., N. S., vol. vi, 1906.

${ }^{3}$ Williams and Lowden, loc. cit. 
staining inclusions would add weight to such an assumption. We have triturated rabic tissue and shaken it up in anti-formin and seen many free Negri bodies apparently enucleated from the cells in consequence. Such complete extrusion from the cell also is seen in the ordinary smear preparations. It is at least unlikely that a cell-degeneration area would be expelled from the cytoplasm in so clearly outlined and morphologically unaltered a form. The fact that the virus is filtrable, as shown by Remlinger, ${ }^{1}$ Poor and Steinhardt, ${ }^{2}$ and others, would on the other hand seem to contradict the etiological importance of the Negri bodies unless, with some of the observers named, we assumed them to represent a large stage in the life-cycle of a protozoan parasite, which also occurred in smaller forms. It is a curious fact, also, that Negri bodies are scarce or absent in the spinal cord and cerebrum, though these areas are as virulent or more so than the hippocampus and cerebellum. They are small and hard to find in virus fixe, largest and most plentiful in cases in which the incubation period has been prolonged-as with street-virus infection. Much can be said on both sides, but in analyzing the present experimental facts, it seems fair to say that neither point of view is certain, though the parasitic nature of the Negri bodies seems very likely.

The cultivation of parasites from rabic tissues has of course been attempted by most bacteriologists who have studied the disease since Pasteur. In all attempts, until very recently, either no results were obtained or else the parasites described could be shown to be present because of extraneous contamination. Recently Noguchi announced that he has been able to cultivate the virus by employing a technique similar to his methods of cultivating Treponema pallidum and poliomyelitis virus. Into high tubes filled with ascitic fluid a bit of fresh sterile rabbit kidney and a small piece of rabic virus were placed. The ascitic fluid was covered with sterile oil and the tubes incubated at $37.5^{\circ} \mathrm{C}$. After five days' incubation cloudiness appeared and, with it, minute globoid bodies not unlike those seen in poliomyelitis. After several generations large highly refractile bodies with dark central spots appeared in the cultures, and these Noguchi ${ }^{3}$ regards as possibly the parasites and similar to Negri bodies. Opinions are still divided as to the significance of Noguchi's results. However, whatever may be one's opinion regarding the nature of the peculiar bodies visible in his cultures, he has accomplished the feat of preserving the virulence of the

${ }^{1}$ Remlinger, Ann. de l'inst. Past., xvii, 1903.

${ }^{2}$ Poor and Steinhardt, Jour. of Inf. Dis., xii, 1913.

${ }^{3}$ Noguchi, Jour. Exp. Med., xviii, 1913. 
virus through 21 generations on artificial media, a fact which alone would seem to prove that he had successfully cultivated it, even though his "nucleated bodies" do not eventually turn out to be anything more than cell degenerations. The possibility that he may have carried original virus through 21 generations and that it has remained virulent for about 100 days at $37.5^{\circ} \mathrm{C}$. can not be excluded as yet, but seems very remotè.

The Specific Therapy of Rabies.-The treatment which is now prophylactically applied to patients infected with or suspected of infection with rabies has been but little altered either in principle or in technical detail since it was first worked out by Pasteur. In principle it consists of an active immunization with virus, attenuated by drying, administered during the long incubation period in doses of progressively increasing virulence.

By the repeated passage of street virus through rabbits, Pasteur obtained a virus of maximum and approximately constant virulence which he designated as virus fixe. By a series of painstaking experiments he then ascertained that such virus fixe could be gradually attenuated by drying over caustic potash at a temperature of about $25^{\circ}$ C., the degree of attenuation varying directly with the time of drying. Thus, while fresh virus fixe regularly caused death in rabbits after six to seven days, the incubation time following the inoculation of dried virus grew longer and longer as the time of drying was increased, until finally virus dried for eight days was no longer regularly infectious and that dried for twelve to fourteen days had completely lost its virulence.

The method of active immunization, which Pasteur used, consisted in injecting, subcutaneously, virus of progressively increasing virulence, beginning with that derived from cords dried for thirteen days and gradually advancing to a strong virus. Thus the patient was immunized to a potent virus several weeks before the incubation time of his own infection had elapsed. Pasteur successfully proved the efficacy of his method upon dogs and finally upon human beings, the first human case being that of a nine-year-old child-Joseph Meister.

Technique of Rabies Therapy.-The technique developed by Pasteur is still, in the main, followed by those who treat rabies to-day.

I. As a preliminary, it is necessary to prepare or obtain virus fixe. This may generally be procured from an established laboratory or may be prepared independently by passing street virus through a series of young rabbits (weighing from 700 to 1,000 gms.). According to Högyes, ${ }^{1}$

${ }^{1}$ Högyes, quoted from Kraus and Levaditi, "Handb.," etc., I. 
the passage of the virus through twenty-one to thirty rabbits, in this way, will reduce its incubation time to seven or eight days. Babes claims to obtain a virus fixe more rapidly by passing the virus alternately through rabbits and guinea-pigs.

For purposes of inoculation, virus is prepared by emulsifying in sterile salt solution pieces of the medulla or cerebellum of animals dead of

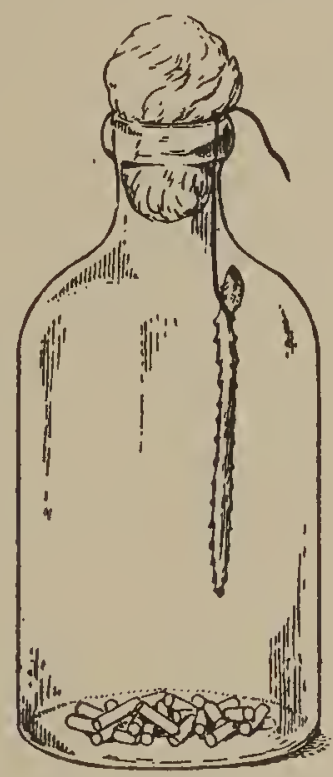

Fig. 153.-Method of Drying Spinal Cord of Rabbit for Purposes of Attenuation.

a previous inoculation. The brain tissue which is not emulsified may be preserved under sterile glycerin in a dark and cool place for further use.

II. Rabbits are inoculated with virus fixe by intracranial injection. A small incision is made in the shaved scalp in the median line, and the skin is retracted. With a small trephine or a round chisel, an opening is made in the skull in the angle between the coronary and sagittal sutures. Through this opening about 0.2 to 0.3 c.c. of the virus fixe is injected, either directly into the brain substance or simply under the dura.

As soon as a rabbit so inoculated has died it is autopsied. The animal before dissection should be washed in a disinfectant solution -lysol or carbolic acid. The skin is then removed and the animal, lying on its ventral surface, is fastened to a dissecting board. The spinal canal is then laid open with a pair of curved scissors and the spinal cord carefully removed. This is accomplished by cutting across the cord in the lumbar region, and lifting this with a forceps while the nerve roots are divided from below upward.

The cord is suspended by a sterile thread within a large bottle into the bottom of which pieces of potassium hydrate have been placed. The bottle is then set away in a dark room or closet, the temperature of 
which is regulated so as to vary little above $25^{\circ} \mathrm{C}$. Bacteriological controls as to the sterility of the cord should also be made.

After a suitable period of drying, pieces of the cord are prepared for injection. This is done in various ways at different laboratories. No attempt at exact dosage is made. At the New York Department of Health $1 \mathrm{~cm}$. of the cord is emulsified in 3 c.c. of sterile eight-tenths per cent salt solution, the dose for injection being usually 2.5 c.c. Marx ${ }^{1}$ emulsifies $1 \mathrm{~cm}$. of the cord in 5 c.c. of sterile bouillon or salt solution, using 1 to 3 c.c. of this for injection according to the age of the cord. For shipment an addition of 20 per cent of glycerin and 0.5 per cent of carbolic acid is made.

The scheme of treatment is also subject to variations according to the individual customs of various laboratories. The following scheme is the routine of the Pasteur Institute in Paris, as quoted in Kraus and Levaditi, "Handbuch für Immunitätsforschung," Vol. I, p. 713.

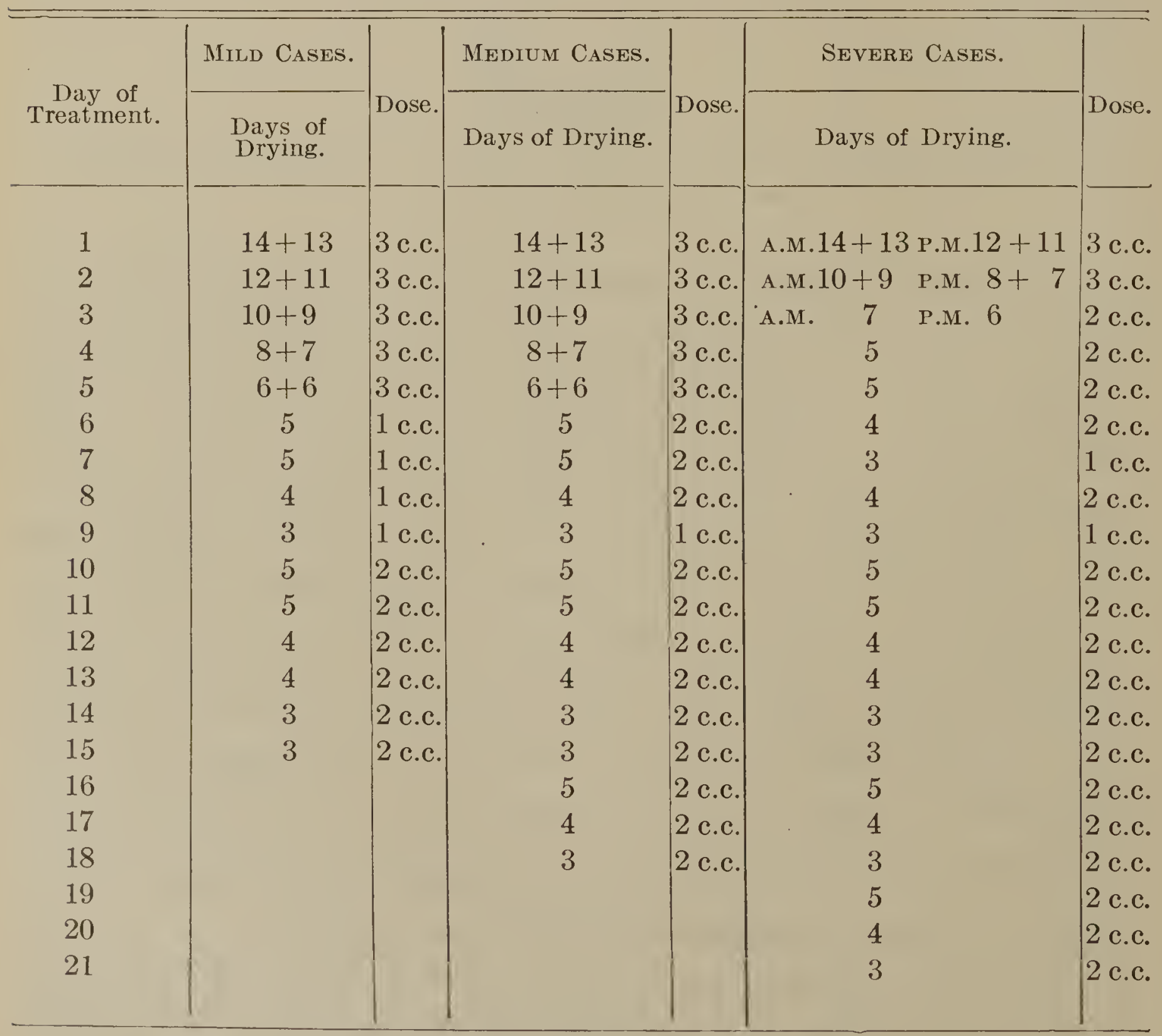

${ }^{1}$ Marx, Deut. med. Woch., 1899, 1900. 
The treatment at the New York Department of Health is as follows: 1

\begin{tabular}{|c|c|c|c|c|c|c|}
\hline \multirow{2}{*}{$\begin{array}{l}\text { Day of } \\
\text { Treatment. }\end{array}$} & Mild Cases. & \multirow{2}{*}{ Dose. } & Medium Cases. & \multirow[b]{2}{*}{ Dose. } & Severe Cases. & \multirow[b]{2}{*}{ Dose } \\
\hline & Days of Drying. & & Days of Drying. & & Days of Drying. & \\
\hline 1 & $14+13$ & 4 c.c. & 10 & 4 c.c. & A.M. $10+9$ P.M. $10+9$ & 4 c.c. \\
\hline 2 & $12+11$ & 4 c.c. & 9 & 4 c.c. & A.M. $8+7$ P.M. $8+7$ & 4 c:c. \\
\hline 3 & $10+9$ & 4 c.c. & 9 & 4 c.c. & 6 & 4 c.c. \\
\hline 4 & $8+7$ & 4 c.c. & $8+7$ & 4 c.c. & 4 & 4 c.c. \\
\hline 5 & 6 & 2 c.c. & 6 & 2 c.c. & 3 & 2 c.c. \\
\hline 6 & 5 & 2 c.c. & 5 & 2 c.c. & 4 & 2 c.c. \\
\hline 7 & 4 & 2 c.c. & 4 & 2 c.c. & 3 & 2 c.c. \\
\hline 8 & 3 & 2 c.c. & 3 & 2 c.c. & 2 & 2 c.c. \\
\hline 9 & 5 & 2 c.c. & 2 & 2 c.c. & 4 & 2 c.c. \\
\hline 10 & 4 & 2 c.c. & 5 & 2 c.c. & 1 & 2 c.c. \\
\hline 11 & 3 & 2 c.c. & 4 & 2 c.c. & 4 & 2 c.c. \\
\hline 12 & 5 & 2 c.c. & 3 & 2 c.c. & 3 & 2 c.c. \\
\hline 13 & 4 & 2 c.c. & 2 & 2 c.c. & 2 & 2 c.c. \\
\hline 14 & 3 & 2 c.c. & 4 & 2 с.с. & 4 & 2 c.c. \\
\hline 15 & 5 & 2 c.c. & 3 & 2 c.c. & 1 & 2 c.c. \\
\hline 16 & 4 & 2 c.c. & 2 & 2 c.c. & 4 & 2 c.c. \\
\hline 17 & & & 4 & 2 c.c. & 3 & 2 c.c. \\
\hline 18 & & & 3 & 2 c.c. & 2 & 2 c.c. \\
\hline 19 & & & 2 & 2 c.c. & 4 & 2 c.c. \\
\hline 20 & & & & & 3 & 2 c.c. \\
\hline 21 & & & & & 2 & 2 c.c. \\
\hline 22 & & & & & 4 & 2 c.c. \\
\hline 23 & & & & & 3 & 2 c.c. \\
\hline 24 & & & & & 2 & 2 c.c. \\
\hline 25 & & & & & 4 & 2 c.c. \\
\hline 26 & & & & & 3 & 2 c.c. \\
\hline
\end{tabular}

The severity or mildness of cases is estimated from the depth and degree of laceration of the wounds, also from their location-bites about the face and upper extremities being the most dangerous.

During the course of such treatment patients may show troublesome erythema about the point of injection and occasionally backache and muscular pains. Treatment need not be omittted unless these symptoms become excessive.

The efficiency of the Pasteur treatment in rabies is no longer problematical. According to Högyes, 50,000 people have been treated within ten years, with an average mortality of 1 per cent.

${ }^{1}$ Personal communication from Dr. Poor, of the New York Department of Health. 
Although the method described above is the one which is extensively used in all established institutes for the treatment of rabies, other methods have been elaborated and used to a slight extent. One of the most important of these is the "dilution method" of Högyes. This method is carried out as follows: A definite quantity of the spinal cord of a rabbit dead of virus fixe is emulsified in 100 c.c. of normal salt solution. Dilutions of this emulsion are made and the patient is injected at first with a dilution of $1: 1,000$, subsequent injections being made of gradually increasing concentration until a concentration of $1: 100$ is reached. This method, so far as it has been used, has been satisfactory, but it has not yet found extensive application.

Attempts to treat active rabies with the sera of immunized animals have so far been unsuccessful. 


\section{CHAPTER XLVIII}

\section{SMALLPOX}

SMALLPox or variola is one of the most virulent of infectious diseases. Throughout history it has been a severe scourge of mankind, prevailing in China and other Eastern countries many centuries before Christ and sweeping through medieval Europe, especially at the time of the Crusades, in a series of severe epidemics. All races of men are susceptible and no age from childhood to senility is exempt. In modern times the disease is endemic in most uncivilized countries, especially those of the East, and occurs sporadically in all parts of the globe. Owing to rigid enforcement of vaccination and of quarantine laws, however, the disease has been practically eradicated from civilized countries.

The etiological factor which causes smallpox is still unknown. Numerous researches aimed at the discovery of cultivatable microorganisms in the lesions or blood of infected patients have met with uniform failure. Streptococci, though often found in the smallpox vesicles and pustules, and often undoubtedly contributing materially to the fatal outcome of the disease, may be regarded as purely secondary in significance.

Communications which have claimed the discovery of a protozoan incitant of the disease have, on the other hand, been numerous and, in some cases, have seemed plausible. Yet absolute proof has always been lacking. The literature on this question is extensive and some of the earlier contributions, such as those of Grünhagen, ${ }^{1}$ of Van der Loeff, ${ }^{2}$ and of Pfeiffer, ${ }^{3}$ possess historical interest only. The work which, of recent years, has attracted the most serious attention to this subject is that published by Guarnieri ${ }^{4}$ in 1892 . This observer found, in the deeper cells of the epithelium covering the pustules, both of smallpox lesions and of vaccination lesions, small bodies which were easily stained by hematoxylin, safranin, or carmin. Similar bodies could be observed in

1 Grünhagen, Arch. f. Dermat. u. Syph., 1892.

${ }_{2}$ Van der Loeff, Monat. f. prakt. Dermat., iv.

3 L. Pfeiffer, Zeit. f. Hyg., xxiii.

- Guarnieri, Arch. per le sc. med., xxvi, 1892; Cent. f. Bakt., I, xvi, 1894. 
the cells of corneal lesions experimentally produced in rabbits. Guarnieri claimed that he distinguished both cytoplasm and nucleus in these bodies and described both binary division and reproduction by sporulation as in the sporozoa. He named the supposed protozoan "Cytoryctes variolæ." At about the same time Monti ${ }^{1}$ described similar bodies in the cells of the Malpighian layer of the skin covering smallpox lesions and, a few years later, Clarke ${ }^{2}$ confirmed the researches of Guarnieri. Subsequently, many researches were carried out on the same subject in this country, the most notable being those of Councilman, ${ }^{3}$ Magrath and Brinckerhoff, and of Calkins. ${ }^{4}$ The former authors came to the distinct conclusion that the bodies seen by Guarnieri were parasites, and the latter author even described a distinct life-cycle for these parasites comparable to that of some protozoa.

These researches, however, are by no means absolutely convincing, and Ewing, ${ }^{5}$ while admitting that the vaccine bodies are probably specific for variola, calls attention to the fact that specific cell-degenerations or inclusions are found in diphtheria, measles, glanders, rabies, and other infectious processes, which can not be regarded as in any way related to these diseases etiologically, and suggests the probability of a similar interpretation for the vaccine bodies. Much has been said on both sides of the question since that time, and the problem can not be regarded as settled. The burden of proof, of course, rests upon those who claim the discovery of a specific microorganism, and absolute proof will probably be lacking until our experimental methods are such as will permit of other than purely morphological demonstration.

Whatever the actual causative agent may be, it is certain that the disease is transmitted with extreme ease-actual contact, direct or indirect, with a patient being unnecessary for its transmission. For this reason the disease is often spoken of as being "air borne." While we have no certain knowledge of the portal of entry through which the virus invades the human body, many considerations have made it seem plausible that this may take place through the mucosa of the upper respiratory tract.

Our knowledge of the means of defense against the malady is fortunately more advanced than is that of its etiology. It has been known

'Monti, Cent. f. Bakt., I, xvi.

${ }^{2}$ Clarke, Brit. Med. Jour., 2, 1894.

${ }^{3}$ Councilman, Magrath, and Brinckerhoff, Jour. Med. Res., xi, 1904.

${ }^{4}$ Calkins, Jour. Med. Res., xi, 1904.

5 Ewing, Jour. Med. Res., xiii, 1905. 
for centuries that one attack of smallpox protects against subsequent attacks. This knowledge was made use of by the physicians of ancient China and India, who, during mild epidemics, exposed healthy children to infection, hoping that mild attacks would result which would confer immunity. While dangerous in the extreme, such "variolation," nevertheless, was not without some benefit and was even introduced into Europe in the eighteenth century by Lady Mary Wortley Montagu.

Such practices, however, were made unnecessary by the classical investigations of Jenner ${ }^{1}$ published in 1798. Jenner, as a student, had been impressed with the fact that country-people who had been infected with a disease known as cowpox, were usually immune against smallpox. His studies and observations came to a practical issue when, in 1796, he inoculated a boy, James Phipps, with pus from a cowpox lesion on the hand of an infected dairy-maid. Two months later the same boy was inoculated with material from a smallpox pustule without subsequent disease. With this experiment the principles of vaccination as in use at the present time were founded.

The question as to the identity of cowpox and smallpox has been the basis of a long controversy. Many observers claimed from the beginning that the two diseases, though closely related to each other, were essentially different. Others, on the contrary, and this seems to be the prevailing opinion among scientists at the present day, maintain that cowpox or vaccinia, as it is called when inoculated into a human being, represents merely an altered and attenuated variety of variola. This latter view is based on the following considerations, which we take from Haccius as quoted by Paul. ${ }^{2}$

1. Variola is invariably transmissible to cattle, when proper methods of inoculation are employed.

2. Variola carried through several animals, in the above way, becomes altered in character, approaching in nature typical vaccinia or cowpox.

3. Such virus, reinoculated into man, gives rise to purely local lesions which are mild and unlike smallpox.

4. Inoculation with such virus protects both man and animals against subsequent inoculation with cowpox, and, in the case of man, against smallpox as well.

It has been claimed, moreover, that cowpox originally was trans-

Ienner, "Inquiry into the Causes and Effects of the Variola-Vaccinx," London, 1798.

2 Paul, "Vaccination"; Kraus and Levaditi, "Handbuch," etc., I. 
mitted to cattle by human beings affected with smallpox. This seems likely both because of the comparative rarity of the former disease and because of its spontaneous occurrence almost invariably upon the teats of cows, although both males and females are equally susceptible to experimental inoculation.

The relationship of variola to chicken-pox or varicella has been more easily determined. Chicken-pox does not protect against smallpox nor is this the case vice versa. The two diseases are unquestionably quite distinct.

The Production of Vaccine.-During the early days of vaccination, it was customary to inoculate human beings with the matter obtained from the pustules of those previously vaccinated. While this method was perfectly satisfactory for the immediate purposes in view, practica? difficulties and the occasional accidental transmission of syphilis have rendered this practice undesirable. In consequence, at all institutes at which vaccine is produced for use upon man, the virus is obtained from animals. Horses and mules, both extremely susceptible to vaccine, have been employed, and goats have, at times, been chosen because of their insusceptibility to tuberculosis. Rabbits have also been used more recently by Calmette and Guerin. ${ }^{1}$

The animals almost exclusively employed at the present day, however, are calves, preferably at ages of from six months to two years. Very young suckling calves are unsuitable because of the great speed of development and small size of the lesions produced. The animals should be healthy and at some institutes (Dresden) are subjected, before use, to the tuberculin test; although, according to Paul, ${ }^{2}$ this produces a hypersusceptibility to the vaccine, and can be omitted without danger when careful supervision is observed. Some observers prefer to use lightcolored animals rather than dark-skinned or black ones, both for reasons of greater ease of cleanliness and because the former are supposed to be more susceptible than the latter. This contention is denied by others. The sex of the animals seems to be immaterial.

During the period of use, the calves are fed, according to age, with either an exclusive milk diet, or they are given, in addition, fresh hay. The greatest cleanliness in regard to the bedding and stalls must be observed and separate stables should be available for the animals under treatment and those under observation before treatment. These stables, if possible, should be so built that they can be easily scoured and flushed 
with water, and stalls should be disinfected after occupation. If possible, stables should be artificially heated and a comfortable temperature maintained. Halters and fastenings should be so arranged that the animals can not lick the scarified surfaces. Careful veterinary control before vaccination and during the period of treatment must be observed in order to eliminate animals with systemic disease or other complications.

The calves may be vaccinated with material taken from previously vaccinated animals. They may, on the other hand, be inoculated with "seed virus" obtained from the vesicles of human vaccinia. This method of using humanized virus for the inoculation of calves for vaccine production is preferred by many workers and is spoken of as "retrovaccination."

Actual vaccination of the animals is done as follows: Calves which have been kept under observation for at least a week are thoroughly washed and cleaned and the abdomen is clipped and shaved over an area extending from the ensiform cartilage to the pubic region, including the entire width of the belly and the inner folds of the thighs. It is best to shave the animal a day of two before vaccination so as to avoid fresh scratches and excoriations. Just before actual operation the animal is strapped to a specially constructed operating table in such a way as to allow free access to the shaved area. This area is now thoroughly washed with soap and water followed by alcohol, or, in some institutes, by a weak solution of lysol. If the latter is used, the field of operation must again be thoroughly rinsed with sterile water. About a hundred small scarifications are made in this area, preferably by crossed scratches, covering for each scarification an area of about 3-4 square centimeters. Into these areas the virus is rubbed, using for each small area a quantity about sufficient to vaccinate three children. Two to three centimeter spaces are left between the lesions. The lesions are then allowed to dry and may be covered with sterile gauze or, as in Vienna, ${ }^{1}$ with a paste made up of beeswax, gum arabic, rinc oxid, water, and glycerin. In some institutes the lesions are left entirely uncovered.

Ordinarily within about twenty-four hours after vaccination a narrow pink areola appears about the scratches. Within forty-eight hours the scratches themselves become slightly raised and papular, and within four or six days typical vaccinia vesicles have usually developed. 
To obtain the vaccine from such lesions, the entire operative field is carefully washed with warm water and soap, followed by sterile water. In some cases two per cent lysol is employed, but must again be thoroughly removed by subsequent washing with sterile water. Crusts, if present, are then carefully picked off and the entire contents of the vesiicle, sticky serum, and pulpy exudate removed by the single sweep of a spoon-curette. The curetted masses are caught in sterile beakers or tubes and to them is added four times their weight of a mixture of glycerin fifty parts, water forty-nine parts, and carbolic acid one part. ${ }^{1}$ German workers prefer a mixture of glycerin eighty parts, and water twenty parts, omitting the use of carbolic acid. The glycerinated pulp is allowed to stand for three or four weeks in order to allow bacteria, which are invariably present, to dic out. After preservation for such a length of time, moreover, thorough emulsification is obtained more easily than when this is attempted immediately after curettage. At the end of three or four weeks, the glycerinated pulp is thoroughly triturated, either with mortar and pestle or by means of specially constructed triturating devices. Pulp so prepared should remain active for at least three months if properly preserved in sealed tubes in a dark and cool place.

From the serum oozing from the bases of the lesions, after curettage, bone or ivory slips may be charged for vaccination with dry virus. The glycerinated pulp is put up in small capillary tubes, sealed at both ends, and distributed in this form. Park states that a calf should yield about 10 grams of pulp (which when made up should suffice to vaccinate one thousand five hundred persons), and, in addition, about two hundred charged bone slips.

The virus may be tested for its efficiency by a variety of methods. Calmette and Guerin ${ }^{2}$ inoculate rabbits upon the inner surfaces of the ears and estimate the potency of the virus from the speed of development and extensiveness of the resulting lesions. Guerin ${ }^{3}$ has estimated the potency of virus quantitatively by a method depending upon the inoculation of rabbits with a series of dilutions. Beginning with a mixture containing equal weights of glycerin and vaccine pulp, dilutions are made with sterile water ranging from 1 in 10 to 1 in 100 . Rabbits are shaved over the skin of the back and 1 c.c. of each of these dilutions is rubbed into the shaved areas. Fully potent virus should cause

1 Huddleston, quoted in Park, "Pathogenic Bacteria," N. Y., 1908.

${ }^{2}$ Calmette and Guerin, Ann. de l'inst. Pasteur, 1902.

-Guerin, Ann. de l'inst. Pasteur, 1905. 
closely approximated vesicles in a dilution of 1 in 500, and numerous isolated vesicles in a dilution as high as 1 in 1,000 .

Quantitative estimations of the bacteria in the glycerinated virus should be made by the plating method and the vaccine used only when after several weeks of preservation the numbers of the bacteria have been greatly diminishod. In glycerinated pulp the bacteria will often disappear entirely in the course of a month. The vaccine should also be tested for the possible presence of tetanus bacilli, by the inoculation of white mice. ${ }^{1}$

Vaccination of human beings is performed by slightly scarifying the skin of the arm or leg with a sharp sterile needle or lancet and rubbing into the lesion potent vaccine virus. The virus was formerly dried upon wood, bone, or ivory slips and moistened with sterile water before the operation. At the present day the glycerinated pulp is almost universally employed.

That vaccination is of incalculable benefit to the human race is no longer a question of opinion, and opposition to the practice is explicable only on the basis of ignorance. Statistical compilations upon this point are very numerous. It may suffice to select from the voluminous literature a single example, taken from Jürgensen, which embodies the statistics of death from smallpox in Sweden, during the periods immediately preceding and following the introduction of vaccination. In that country the first vaccination was done in 1801. By 1810 the practice was generally in use but not enforced. In 1816 it was legally enforced. The years from 1774 to 1855 can thus be divided into three periods.

1. Prevaccinal period, 1774-1801 (25 years). Deaths smallpox per million inhabitants. . . . . . . . . . . . . . . 2,050

2. Transitional period, $1801-1810(9$ years $) \ldots \ldots \ldots \ldots \ldots \ldots \ldots 680$

3. Vaccination enforced, $1810-1855$ (35 years) ............ 169

Prevaccinal period death rate 20.00 per mille.

Vaccinal period death rate $\quad 0.17$ per mille.

In considering the benefit of vaccination it must not be forgotten that revaccination is quite as important as the first vaccination, which confers immunity only for from seven to ten years. A child should therefore be vaccinated soon after birth or at least before the eighth month, and the process should be repeated every seven years thereafter. 


\section{CHAPTER XLIX}

\section{ACUTE ANTERIOR POLIOMYELITIS}

THe disease known as acute anterior poliomyelitis has long been recognized as an acute infectious condition, both because of the characteristics of its clinical manifestations and of its epidemic occurrence. For these reasons it was classified with acute infectious diseases by Marie and by Strümpell long before any experimental evidence of infection was obtainable.

Its contagiousness, while not a proven fact, seemed very likely from the evidence of its mode of spreading and has been removed from the sphere of mere conjecture by the careful study of a Swedish epidemic, comprising one thousand cases, made by Wickman. ${ }^{1}$

While acute anterior poliomyelitis is almost exclusively a disease of childhood, it is assumed by clinicians that it is etiologically closely related to, possibly identical with, certain diseases of the adult, characterized by bulbar paralysis and acute encephalitis. Into this category, also, some observers place the condition known as "Landry's paralysis." The basis for the identification of these conditions with poliomyelitis lies chiefly in the similarity of the pathological lesions and upon the fact that the last-named diseases occur most often during the course of poliomyelitis epidemics.

In consequence of the emphatically expressed opinion as to the infectious nature of acute poliomyelitis, the efforts to isolate specific microorganisms from cases have been many, and numerous microorganisms have been described as the causative agents of this disease. The outcome of all these investigations has been purely negative and the infectious agent of acute poliomyelitis still remains undiscovered. ${ }^{2}$

An important advance in the study of this disease was made in $\mathbf{1 9 0 8}$ when Landsteiner and Popper ${ }^{3}$ succeeded in transmitting it to two monkeys (Cyanocephalus hamadryas and Macacus rhesus). The trans-

1 Wickman, quoted from Landsteiner and Popper, Zeit. f. Immunitätsforch., ii, 1909.

${ }^{2}$ For literature, see Landsteiner and Popper, loc. cit.

Loc. cit. 
mission was accomplished by intraperitoneal injections of a sailine emulsion of the spinal cord of a child that had died during the fourth day of an attack of infantile paralysis-during the stage of acute fever. The first monkey injected became severely ill six days after the injection and died on the eighth day. The second animal became paralyzed seventeen days after the injection and was killed two days later. Cultural experiments with the substance injected were negative, as were also inoculation experiments carried out upon guinea-pigs, rabbits, and mice. The histological lesions produced in the inoculated monkeys were similar to those occurring in children afflicted with the disease.

An attempt to transmit the disease to another monkey with spinalcord substance of the animal that was killed resulted negatively.

Soon after the successful experiments of Landsteiner and Popper, a similar result was recorded by Knoepfelmacher. ${ }^{1}$ An attempt to transmit the disease from monkey to monkey was again negative.

Similar positive inoculation results were published, a little later than this, by Flexner and Lewis ${ }^{2}$ in November, 1909, and by Strauss and Huntoon ${ }^{3}$ in January, 1910.

Flexner and Lewis, in their work, moreover, succeeded in transmitting the disease through several inoculation-generations of monkeys, proving thereby that successful inoculation did not depend merely upon the transfer of an unorganized toxic body, but was due to a true infection. The same workers ${ }^{4}$ have ascertained that inoculation may be successfully applied not only by the intraperitoneal route but intracerebrally, subcutaneously, intravenously, and by the path of the larger nerves. They also proved that not only the brain and cord of afflicted animals contains the virus, but that this may be found, during the early days of the disease at least, in the spinal fluid, the blood, the nasopharyngeal mucosa, and lymph nodes near the site of inoculation.

Landsteiner and Levaditi, ${ }^{5}$ meanwhile, experimenting with the virus independently, succeeded in transferring the disease from one animal to others, demonstrated that the virus could pass through the pores of a Berkefeld filter, and showed that the virus was present in the salivary glands-a fact which may prove of great importance in possibly estab-

1 Knoepfelmacher, Mediz. Klinik, v, 1909.

2 Flexner and Lewis, Jour. Am. Med. Assn., 53, 1909.

3 Strauss and Huntoon, N. Y. Med. Jour., Jan., 1910.

4 Flexner and Lewis, Jour. Exp. Med., 12, 1909.

5 Landsteiner and Levaditi, Comptes rend. de la soc. de biol., Nov., 1909, and Dec., 1909. 
lishing a clew to the mode of contagion among human beings. The same authors, as well as Flexner and Lewis, were able to show that the virus was preservable under glycerin for as long as ten days and retained its virulence for from seven to eleven days when dried.

According to Flexner and Lewis the virus remains active, when frozen, for as long as forty days, but is extremely sensitive to heat, being destroyed by a temperature of from $45^{\circ}$ to $50^{\circ} \mathrm{C}$. maintained for thirty minutes.

Experiments aimed at the isolation or even morphological detection of a parasite in the virulent material have been entirely without success until recently. Bacteria which in the past have been isolated from nerve substance and spinal fluid in cases of poliomyelitis can of course be excluded from etiological significance by the recent determination of the filtrability of the virus as determined by Flexner and Lewis, and Landsteiner and Levaditi. Small coccoid forms in smears from the nerve tissue recently described by Proescher $^{1}$ are of very uncertain significance. The clouding of ascitic fluid after an incubation with poliomyelitis nerve substance has been found to be due to protein precipitation. The most important contribution which has been made in the solution of this problem is that of Flexner and Noguchi. ${ }^{2}$ These investigators placed small bits and emulsions of the brain of monkeys, dead of poliomyelitis, into high tubes containing human ascitic fluid together with a piece of fresh sterile rabbit kidney. In all essentials the method was that followed by Noguchi in his cultivation of Treponema pallidum, except that in the case of poliomyelitis anaërobic pus was unnecessary. It sufficed to cover the ascitic fluid with a layer of sterile alboline. It was necessary to use fresh unheated ascitic fluid. Heat sterilization rendered it unsuitable.

By this method, after five days opalescence appeared about the pieces of tissue. This increased until the tenth day when sedimentation began. Microscopical examination by Giemsa's method of staining revealed small globoid bodies measuring from 0.15 to 0.3 micron in diameter, arranged in pairs, short chains, and masses. Similar bodies could later be found in poliomyelitis tissues. Cultures were obtained from glycerinated as well as from fresh virus and from the filtered as well as the unfiltered material. Typical lesions and death have been produced in monkeys with such cultures even after the eighteenth generation on artificial media.

${ }^{1}$ Proescher, N. Y. Med. Jour., 1913.

${ }^{2}$ Flexner and Noguchi, Jour. of Exp. Med., xviii, 1913. 
We have few data which throw light upon possible immunity to the disease. Repeated attacks of the disease in the same human being have not been noted; but this, as Flexner and Lewis point out, may be due to the fact that the epidemics are rare, and individuals once afflicted have passed beyond the susceptible age by the time of the second epidemic. As a matter of fact, however, these workers have not succeeded in reinfecting monkeys that had recovered, and incline to the belief that one attack protects against subsequent infections.

Up to the present time monkeys and rabbits only have responded to experimental inoculation; numerous attempts made upon a variety of other animals have been without success.

In chickens a disease has been observed similar in many ways to poliomyelitis, but further study has shown this to be a polyneuritis of entirely different nature from infantile paralysis.

Of other animals besides monkeys, rabbits only have been successfully inoculated with this disease. Transmission to these animals was first reported by Kraus and Meinicke ${ }^{1}$ and later by Lentz and Huntemüller. ${ }^{2}$ Marks ${ }^{3}$ has studied the disease in rabbits thoroughly, and concludes that there is no doubt that the virus can be cultivated through a limited number of generations in rabbits. He was able to transmit to monkeys from rabbit material. The disease, however, does not resemble that of man or monkeys clinically and no definite lesions of the central nervous system are present. The rabbits seem perfectly well for six or seven days, when rapid weakness and death in convulsions occur.

\footnotetext{
1 Kraus und Meinicke, Deut. med. Woch., xxxv, 1909.

${ }^{2}$ Lentz und Huntemüller, Zeitschr. f. Hyg., lxvi, 1910.

${ }^{3}$ Marks, Jour. of Exp. Med., xiv, 1911.
} 


\section{CHAPTER L}

\section{YELLOW FEVER}

YELLOW fever is an acute infectious disease which prevails endemically in the tropical countries of the Western Hemisphere, but occurs also along the western coast of Africa and has exceptionally appeared, in epidemic invasions, in the north temperate United States and Europe. Guiteras, as quoted by Osler, classifies the distribution of the disease into three areas of infection.

1. The area in which the disease is never absent, including tropical South American ports and Havana.

2. The area of periodic epidemics, including sea-ports of the tropical Atlantic in America and Africa.

3. The area of accidental epidemics, extending from parallel $45^{\circ}$ north latitude to $35^{\circ}$ south latitude. In the United States severe epidemics have frequently occurred in Louisiana, Mississippi, and Alabama, and occasional but severe epidemics have occurred in Philadelphia and Baltimore.

The disease occurs spontaneously only in man, and experimental inoculation of lower animals has been successful only in the chimpanzee in a single case reported by Thomas. ${ }^{1}$

In man afflicted with the malady the clinical picture is one of a rapidly developing fever with severe gastrointestinal symptoms, vomiting of blood, albuminuria, and often active delirium. The mortality is usually high, often reaching eighty per cent or more in the severe epidemics.

Etiology and Method of Transmission.-The actual infective agent which causes yellow fever is, as yet, unknown. Numerous researches have been aimed at the elucidation of the problem, and microorganisms, for which etiological significance was claimed, have been isolated from the dejecta, the vomitus, and the secretions of afflicted patients. None of these claims has been supported by convincing proof and none of them has found subsequent confirmation.

A few of these claims only have historical importance because of the 
widespread interest they aroused among bacteriologists. Cornil and Babes, ${ }^{1}$ in 1883 , described chained cocci to which they attributed etiological significance, but their contentions have remained entirely unconfirmed. Sternberg, ${ }^{2}$ in 1897, described a colon-like organism, "bacillus X," for which he made very conservative claims, which he himself, later, withdrew.

The most active discussion was aroused by the announcement of Sanarelli, ${ }^{3}$ in 1897, that he had discovered, in the blood and tissues of patients dead of yellow fever, a Gram-negative bacillus, which he believed to be the etiological agent of the disease. He based his contention upon the facts that he had isolated the organism from seven cases of yellow fever, had produced symptoms similar to the disease of the human being by the inoculation of pure cultures into dogs, and had obtained agglutination of the bacillus in the serum of convalescent patients. Later he inoculated five human beings subcutaneously with sterilized cultures of this "Bacillus icteroides," and obtained symptoms which he believed simulated closely those of yellow fever. The claims of Sanarelli at first found much apparent confirmation, but later work by Durham and Myers, ${ }^{4}$ Otto ${ }^{5}$ Agramonte, ${ }^{6}$ and others has definitely refuted his original claims, and there is to-day no scientific basis for the assumption that the Bacillus icteroides has any etiological relationship to the disease. Protozoan incitants, also, have been described by Klebs, ${ }^{7}$ Schüller, ${ }^{8}$ Thayer, ${ }^{9}$ and others, without bringing conviction or even justifying extensive investigation of their claims.

While thus the causative agent of yellow fever remains undiscovered, some of its biological properties are known. Reed, Carroll, Agramonte, and Lazear ${ }^{10}$ were able to show that the infecting agent is prosent in the blood serum of patients during the first three days of the disease and that it could pass through the pores of Berkefeld filters. Such filtered serum remained infectious for human beings-a fact which demonstrates that the incitant is extremely small and possibly ultra-

\footnotetext{
1 Cornil and Babes, Comptes rend. de l'acad. des sci., 1883.

2 Sternberg, Cent. f. Bakt., I, xxii, 1897.

3 Sanarelli, Ann. de l'inst. Pasteur, 1897, and Cent. f. Bakt., I, xxii, xxvii, and xxix.

4 Durham and Myers, Thompson Yates Laboratory Reports 31902.

${ }^{5}$ Otto, Vierteljahrsch. f. gericht. Medizin, etc., 27, 1904.

- Agramonte, N. Y. Med. News, 1900.

7 Klebs, Jour. Am. Med. Assn., April, 1898.

8 Schüller, Berl. klin. Woch., 7, 1906.

9 Thayer, Med. Record, 1907.

10 Reed, Carroll, Agramonte, and Lazear, Phila. Med. Jour., 1900,
} 
microscopic. Blood serum, filtered or unfiltered, becomes non-infectious when heated to $56^{\circ} \mathrm{C}$. for ten minutes.

Mode of Transmission.-Until comparatively recent years the mode of transmission of yellow fever was not understood and many erroneous theories were prevalent. It was supposed that yellow fever was contagious, and transmitted from person to person by direct or indirect contact with those afflicted or by fomites. The first to make the definite assertion that yellow fever was transmitted by the agency of mosquitoes was Carlos Finlay. Finlay, ${ }^{1}$ as early as 1881, advanced the theory that mosquitoes were responsible for the transmission of this disease and, furthermore, recognized "Stegomyia fasciata" or "Stegomyia calopus" as the guilty species. Finlay's opinion, although later proved to be correct, was at first based only upon such circumstantial evidence as the correspondence of the yellow-fever zones with the distribution of this species of mosquito and the great prevalence of mosquitoes at times during which epidemics occurred. His theory was, therefore, received with much skepticism and was neglected by scientists until its revival in 1900 , when the problem was extensively investigated by a commission of American army surgeons.

Reed, Carroll, Agramonte, and Lazear were the members of this commission. The courage, self-sacrifice, and scientific accuracy which characterized the work of these men have made the chapter of yellow fever one of the most brilliant in the annals of American scientific achievement.

Their work was much facilitated by the experience of Gorgas ${ }^{2}$ and others, who had demonstrated the absolute failure of ordinary sanitary regulations to limit the spread of yellow fever.

They began their researches by investigating carefully the validity of Sanarelli's claims as to the etiological significance of his "Bacillus icteroides." The results of this work yielded absolutely no basis for confirmation.

They then proceeded to investigate the possibility of an intermediate host.

In August, 1900, the commission began its work on this subject by allowing mosquitoes, ${ }^{3}$ chiefly those of the stegomyia species, to suck

1 Finlay, Ann. Roy. Acad. d. Havana, 1881.

${ }^{2}$ Goryas, Jour. of Trop. Med., 1903.

${ }^{3}$ Reed, Carroll, Agramonte, and Lazear, Phila. Med. Jour., Oct., 1900; also Am. Pub. Health Assn: Rep., 1903; Agramonte, N. Y. Med. News, 1900; Reed, Jour. of Hyg., 1902; Reed, Carroll, and Agramonte, Am. Medicine, July, 1901. Boston Med. 
blood from patients, later causing the same insects to feed upon normal susceptible individuals. The first nine experiments were negative. The tenth, of which Carroll was the subject, was successful. Four days after being bitten by the infected insect Carroll became severely ill with an attack of yellow fever, by which his life was endangered, and from the effects of which he died several years later.

On the 13th of September, Lazear, while working in the yellow-fever wards, noticed that a stegomyia had settled upon his hand, and deliber-

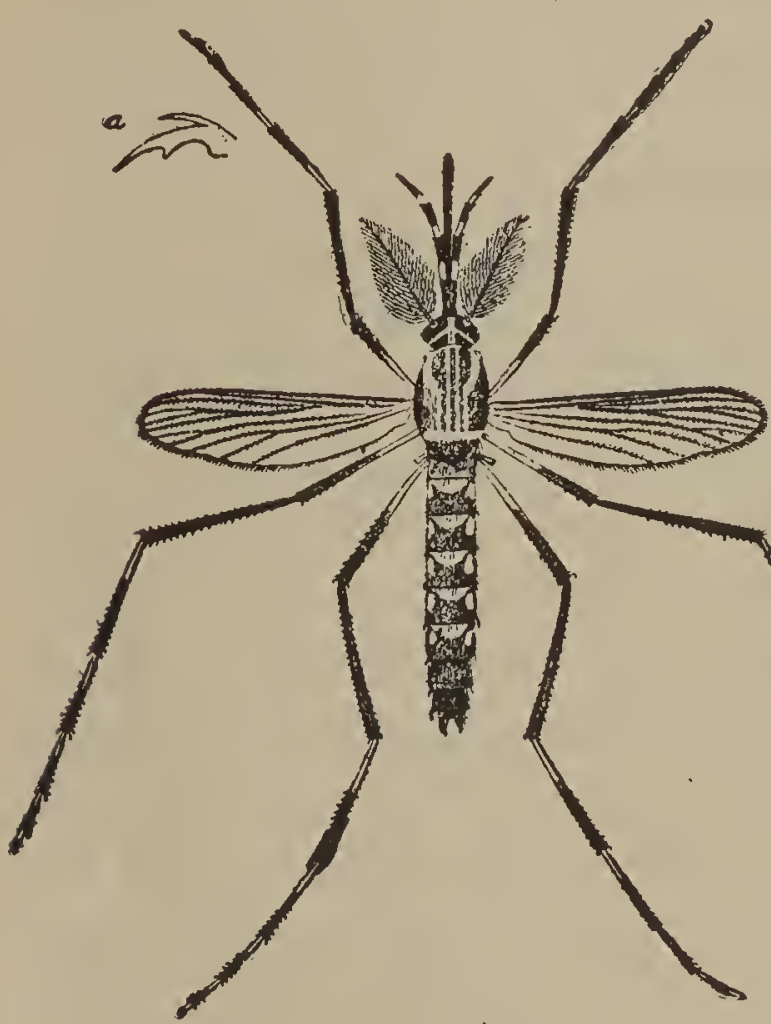

(a)

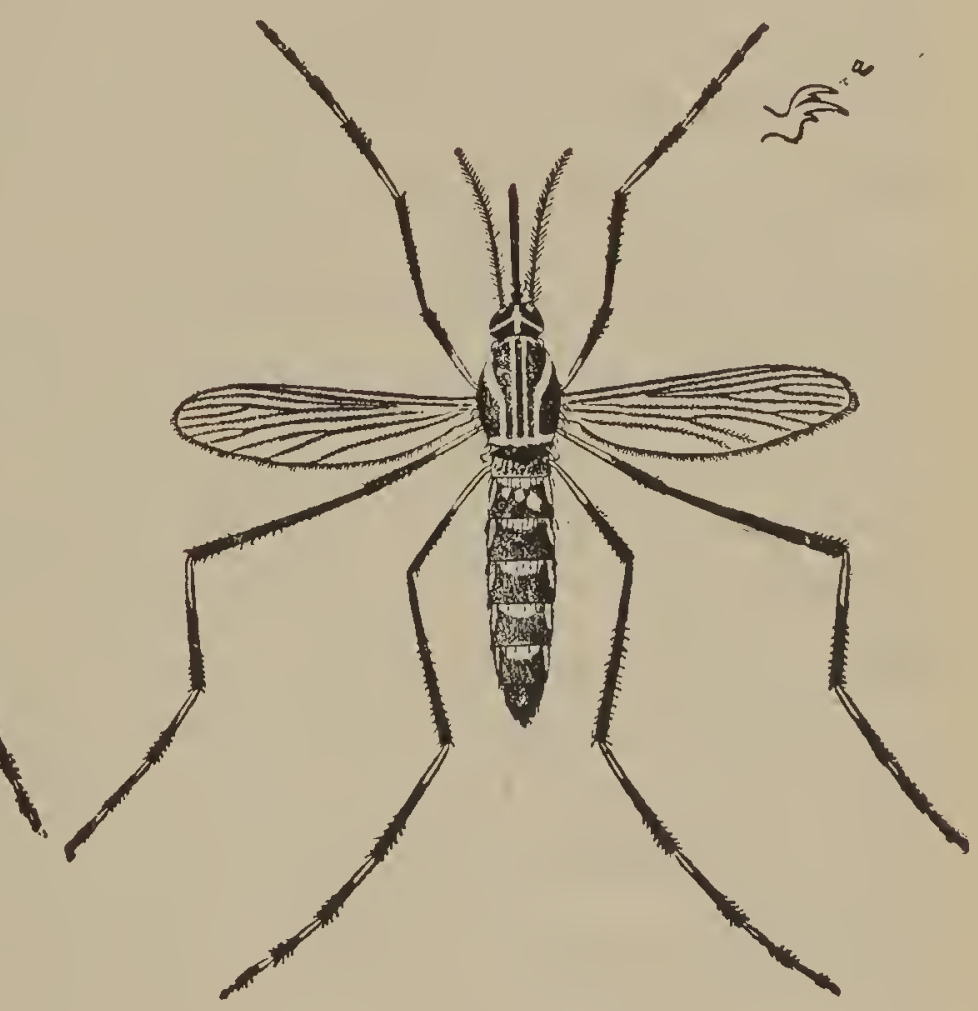

(b)

Fig. 155.--Stegomyia Fasciata. (a) Female. (b) Male. (After Carroll.)

ately allowed the insect to drink its fill. Five days later he became ill with yellow fever and died after a violent and short illness.

With these experiences as a working basis, the commission now decided upon a more systematic and thoroughly controlled plan of experimentation.

In November of the same year, 1900, an experiment station, "Camp Lazear," was established in the neighborhood of Havana, about a mile from the town of Quemados. The camp was surrounded by the strictest quarantine. Volunteers from the army of occupation were called for, and twelve individuals were selected for the camp, three immunes and nine non-immunes. Two of the latter were physicians. The immunes

and Surg. Jour., 14, 1901; Carroll, Jour. Am. Med. Assn., 40, 1903; Carrol, "Yellow. Fever" in Mense, "Handbuch der Tropen-Krankheiten," ii. 
and the members of the commission only were allowed to go in and out. All non-immunes who left the camp were prohibited from re-entering and their places taken by other non-immune volunteers. During December, five of the non-immune inmates were successfully inoculated with yellow fever by means of infected mosquitoes. During January and February five further successful experiments were made. Clinical observations were made by experienced native physicians, Carlos Finlay among them, and the patients, as soon as they were unquestionably ill with yellow fever, were removed to a yellow-fever hospital. This was done to prevent the possibility of the disease spreading within the camp itself. The mosquitoes used for the experiments were all cultivated from the larva and kept at a temperature of about $26.5^{\circ} \mathrm{C}$.

A further important experiment was now made A small house was erected and fitted with absolutely mosquito-prcof windows and doors. The interior was divided by wire mosquito netting into two spaces. Within one of these spaces fifteen infected mosquitoes were liberated. Seven of these had fed upon yellow-fever patients four days previously; four, eight days previously; three, twelve days previously; and one, twentyfour hours previously. A non-immune person then entered this room and remained there about thirty minutes, allowing himself to be bitten by seven mosquitoes. Twice after this the same person entered the room, remaining in it altogether sixty-four minutes and being bitten fifteen times. After four days this individual came down with yellow fever.

In the other room two non-immunes slept for thirteen nights without any evil results whatever.

It now remained to show that mosquitoes were the sole means of transmission and to exclude the possibility of infection by contact with excreta, vomitus, or fomites. For this purpose another mosquito-proof house was constructed. By artificial heating its temperature was kept above $32.2^{\circ} \mathrm{C}$. and the air was kept moist by the evaporation of water. Clothing and bedding, vessels, and eating utensils, soiled with vomitus, blood, and feces of yellow-fever patients were placed in this house and three non-immune persons inhabited it for twenty days. During this time they were strictly quarantined and protected from mosquitoes. Each evening, before going to bed, they unpacked and thoroughly shool clothing and bedding of yellow-fever patients, and hung and scattered these materials about their beds. They slept, moreover, in contact with linen and blankets soiled by patients. None of these persons contracted yellow fever. The same experiment was twice repeated by other non-immunes, in both cases with like negative results. 
All of the non-immunes taking part in these experiments were American soldiers. Four of them were later shown to be susceptible to yellow fever by the agencies of mosquito infection or blood-injection.

The results obtained by the investigations of this commission may be summarized, therefore, as follows:

Yellow fever is acquired spontaneously only by the bite of the Stegomyia fasciata. It is necessary that the infecting insect shall have sucked the blood of a yellow-fever patient during the first four or five days of the disease, and that an interval of at least twelve days shall have elapsed between the sucking of blood and the reinfection of another human being. Sucking of the blood of patients advanced beyond the fifth day of the disease does not seem to render the mosquito infectious, and at least twelve days are apparently required to allow the parasite to develop within the infected mosquito to a stage at which reinfection of the human being is possible.

The results of the American Commission were soon confirmed by Guiteras ${ }^{1}$ and by Marchoux, Salimbeni, and Simond. ${ }^{2}$ These latter observers, moreover, confirmed the fact that infection could be experimentally produced by injections of blood or blood serum taken from patients during the first three days of the disease. They showed that blood taken after the fourth day was no longer infectious: that 0.1 c.c. of serum sufficed for infection and finally that no infection could take place through excoriations upon the skin. They furthermore confirmed the observation of Carroll that the virus of the disease could pass through the coarser Berkefeld and Chamberland filters,-passing through a Chamberland candle " $\mathrm{F}$ " but held back by the finer variety known as " B."

The fundamental factors of yellow-fever transmission thus discovered, we are in possession of logical means of defense. The most important feature of such preventive measures must naturally center upon the extermination of the transmitting species of mosquito.

Stegomyia fasciata or calopus is a member of the group of "Culicidæ." It is more delicately built than most of the other members of the group culicidæ, is of a dark gray color, and has peculiar thoraxmarkings which serve to distinguish it from other species. The more detailed points of differentiation upon which an exact zoological recognition depends are too technical to be entered into at this place. Briefly described, they consist of lyre-like markings of the back,

1 Guiteras, Rev. d. méd. trop., Jan., 1901, and Am. Med., 11, 1901.

2 Marchoux, Salimbeni. and Simond, Ann. de l'inst. Pasteur, 1908. 44 
unspotted wings, white stripes and spots on the abdomen, and bandlike white markings about the metatarsi and tarsi of the third pair of legs. The peculiar power of transmitting yellow fever possessed by this species is explained by Marchoux and Simond ${ }^{1}$ by the fact that Stegomyia fasciata is unique among culicidæ in that the female lives for prolonged periods after sucking blood. Among other species -Culex fatigans, Culex confirmatus, and most others- the female lays its eggs within from two to eight days after feeding on blood and rarely lives longer than the twelfth day-the time necessary for the development of the yellow-fever parasite.

The limitation of yellow fever to tropical countries ${ }^{2}$ is explained by the fact that stegomyia develops only in places where high temperatures prevail. The optimum temperature for this species lies between $26^{\circ}$ and $32^{\circ} \mathrm{C}$. At $17^{\circ} \mathrm{C}$. it no longer feeds, and becomes practically paralyzed at $15^{\circ} \mathrm{C}$. In order to thrive, the species requires a temperature never going below $22^{\circ} \mathrm{C}$. at night and rising regularly above $25^{\circ} \mathrm{C}$. during the day. The females only are dangerous as sources of infection. The insect, like Anopheles, has the peculiarity of feeding chiefly at night.

Experiments done by Reed, Carroll, Agramonte, and Lazear, ${ }^{3}$ to ascertain whether the power of infecting was hereditarily transmissible from the mosquito to following generations, were negative. A positive result, however, has been reported by Marchoux and Simond. ${ }^{4}$ This question must still await more extensive research.

Immunity.-Natural immunity against yellow fever was formerly assumed to exist in the negro race. More recent investigations have not borne out this assumption. The negro soldiers of the American army in Cuba were afflicted equally with the white troops. The relative immunity of dark-skinned races, however, is explained possibly by the fact that the stegomyia prefers to attack light-colored surfaces.

A single attack seems to protect against subsequent infection throughout life.

Artificial immunization has, so far, been unsuccessful. Relative immunity was produced, however, by Marchoux, Salimbeni, and Simond, by injections of the serum of convalescents, serum heated to $55^{\circ} \mathrm{C}$, and of defibrinated blood preserved for eight days in vessels sealed with vaseline.

${ }^{1}$ Marchoux and Simond, Ann. de l'inst. Pasteur, 1906.

2 Otto, in Kolle und Wassermann, "Handbuch," etc., 11, Ergänzungsband.

' Loc. cit.

- Marchoux and Simond, Comptes rend. de la soc. de biol., 59, 1905. 


\section{CHAPTER LI}

\section{MEASLES, SCARLET FEVER, TYPHUS FEVER, AND FOOT- AND-MOUTH DISEASE}

\section{MEASLES}

The causative agent of measles is unknown to the present day, and it would be a thankless task to review the literature of the many attempts to isolate microorganisms from this disease, none of which has resulted in throwing any light on the etiology.

Attempts to produce the disease experimentally have frequently been made, the earliest recorded being those of Home of Edinburgh, published in 1759. ${ }^{1}$ Home took blood from the arms of patients afflicted with measles, and caught it upon cotton, and inoculated normal individuals by placing this blood-stained cotton on to wounds made in the arm. Home claimed that in this way he produced measles of a modified and milder type in fifteen individuals. Home's results, however, while at first accepted, were assailed by many writers and it is by no means certain that the disease produced by him was really measles.

A number of other observers after Home attempted experimental inoculation of this disease, and positive results were reported by Stewart of Rhode Island (1799), Speranza of Mantua (1822), Katowa of Hungary (1842), and McGirr of Chicago (1850).

The experiments of all these early writers, however, are unsatisfactory, owing to the necessarily unreliable technique of their methods.

In 1905, Hektoen ${ }^{2}$ succeeded in experimentally producing the disease in two medical students by subcutaneous injection of blood taken from measles patients at the height of the disease (fourth day). The experiments were carefully carried out and the symptoms in the subjects were unquestionable. They demonstrated beyond doubt that the virus of the disease is present in the blood. Attempts at cultivation carried out with the same blood were entirely negative. It was also shown by Hektoen's experiments that the virus of measles may be kept alive for at least twenty-four hours when mixed with ascitic broth.

${ }^{1}$ Home, "Medical Facts and Experiments," Edinburgh, 1759.

${ }^{2}$ Hektoen, Jour. Inf. Dis., ii, 1905. 


\section{SCARLET FEVER}

\section{(Scarlatina)}

The etiology of scarlet fever, like that of measles, is still obscure. Streptococci have been found with striking regularity in the throats of scarlet-fever patients, and a large number of investigations have seemed to furnish evidence for the etiological relationship of these microorganisms with the disease. According to von Lingelsheim, Crooke as early as 1885 demonstrated the presence of streptococci in the cadavers of scarlet-fever victims. Baginsky and Sommerfeld ${ }^{1}$ in 1900 examined a number of scarlatina cases with reference especially to streptococcus infection, and reported the presence of streptococci in the heart's blood of eight patients who had died after a very acute and short illness. They expressed the belief that the acuteness of the illness and the rapidity of death in these cases precluded the possibility of the streptococci being merely secondary invaders. A large number of other observers have expressed similar opinions, but we can not, as yet, justly conclude that streptococci are actually the etiological agents in this disease.

Class ${ }^{2}$ in 1899 described a diplococcus which he cultivated from a large number of scarlatina patients and with which he was able to produce exanthemata and acute fever in pigs. Subsequent investigations seem to show that Class was really working with a streptococcus.

Moser, ${ }^{3}$ working in Escherich's clinic, has recently reported the very favorable influence upon the course of scarlet fever of polyvalent streptococcus antisera. This is not really very strong evidence in favor of the streptococcus etiology of the disease, since there is, of course, no doubt that streptococcus infection complicates the disease, and it is to be expected that antistreptococcus serum should, therefore, benefit the patient's condition by combating this complication.

Mallory ${ }^{4}$ in 1904 published observations on four scarlatina cases on which he bases the belief that scarlatina is caused by protozoa. In the skin, between the epithelial cells, he found small bodies which were easily stained with methylene-blue and which because of their arrange-

\footnotetext{
${ }^{1}$ Baginsky and Sommerfeld, Berl. klin. Woch., 1900.

${ }^{2}$ Class, Phila. Med. Jour., iii, 1899.

3 Moser, quoted by Escherich, Wien. klin. Woch., xxiii, 1903.

* Mallory, Jour, Med, Research, x, 1904.
} 
ment and form he interpreted as parasites not very unlike the plasmodium of malaria. Subsequent investigations of Field ${ }^{1}$ and others have failed to substantiate Mallory's conclusions.

\section{TYPHUS FEVER}

Typhus fever is an infectious disease which is characterized by an incubation time of 5 days or more, high temperature, and a petechial rash. It has been characterized as peculiarly a disease of filth and has epidemically disappeared in most of the civilized countries, although it is still endemic in certain parts of Europe, North and South America, and occurs epidemically in Mexico under the name of Tabardillo. In New York it has recently been found to exist not infrequently. It was described as a new clinical entity by Brill, and has been spoken of as Brill's disease, but the work of Anderson and Goldberger has shown that Brill's disease is identical with typhus fever. Great advances have been made in the knowledge of the disease during the last few years.

In 1909, Nicolle ${ }^{2}$ successfully inoculated an anthropoid ape, and Anderson and Goldberger ${ }^{3}$ in the same year succeeded in inoculating lower monkeys, rhesus and capuchin. Similar successful monkey inoculations were made by Ricketts and Wilder, ${ }^{4}$ by Gavino and Girard. ${ }^{5}$ In these animals inoculation with blood from active cases is followed by a rapid rise of temperature after an incubation time of 5 days or more, and the fever remains high for 3 to 5 days, after which it comes down by lysis. Occasional recrudescences have been noticed in monkeys. Goldberger and Anderson have had a mortality of 2 per cent in their monkeys. The disease may be transmitted from monkey to monkey with the blood, which is infectious during the febrile period and may be so for as long as 32 hours after the temperature returns to normal. Ricketts and Wilder have described short bacilli looking like organisms of the hæmorrhagic septicæmia group in smears from the blood, but have not been able to cultivate them. Very recently Plotz ${ }^{6}$ has reported

${ }_{1}^{1}$ Field, Jour. Exper. Med., vii, 1905.

${ }^{2}$ Nicolle, Compt. rend. Acad. d. Sc., 1909, p. 157; Ann. de l'inst. Past., 1910, $1911,1912$.

${ }^{3}$ Anderson and Goldberger, Jour. A. M. A., 1912, p. 49; Jour. Med. Res., 1910, p. 469; N. Y. Med. Jour., 1912, p. 976.

${ }^{4}$ Ricketts and Wilder, Jour. A. M. A., Feb., 1910, p. 463; ibid., April 16, 1910 , p. 1304; ibid., April 23, 1910, p. 1373; ibid., July 23, 1910, p. 309.

5 Gavino and Girard, cited from Anderson and Goldberger.

${ }^{6}$ Plotz, Jour. of the A. M. A., Ixii, 1914, No. 20. 
the cultivation of a Gram-positive pleomorphic, anaërobic bacillus from the blood of six vases of Brill's disease and from an equal number of typhus cases. Complement fixation was obtained, when this organism was used as antigen, with the blood of typhus fever cases and it seems not unlikely, at the present time, that Plotz's bacillus may prove to be the etiological factor of typhus. His detailed report has not yet appeared at the present writing. Cultivation experiments by other writers have also been negative and the etiological significance of the organisms of Ricketts and Wilder is very doubtful.

Filtration experiments carried out by Ricketts and Wilder and by Anderson and Goldberger at first indicated that the virus did not go through Berkefeld filters. Nicolle, Conor, and Conseil ${ }^{1}$ noticed that inoculation with the filtered blood rendered monkeys refractory, an observation later recorded also by Wilder and Ricketts. Goldberger and Anderson report similar results. 'By the French investigators this fact has been interpreted as indicating that the virus is filtrable, and Goldberger and Anderson admit this as a possibility. It is likely therefore but not proven that the virus of typhus fever may have a filtrable stage.

By the work of Nicolle and his associates, and of Ricketts and Wilder, ${ }^{2}$ also of Anderson and Goldberger, ${ }^{3}$ it has been shown that the virus can be transmitted from human being to human being by the bites of the body louse (pediculus vestimenti); the flea and the bed bug apparently do not transmit the disease. The head louse (pediculus capitis) may possibly transmit it.

\section{FOOT-AND-MOUTH DISEASE}

This malady occurs chiefly in cattle, sheep, and goats, more rarely in other domestic animals. It is characterized by the appearance of a vesicular eruption localized upon the mucosa of the mouth and upon the delicate skin between the hoofs. In the females similar eruptions may appear upon the udders. With the onset of the eruption there may be increased temperature, refusal of food, and general depression. Usually the disease is mild; the vesicles go on to the formation of small ulcers and pustules, and gradually heal with a disappearance of systemic

${ }^{1}$ Nicolle, Conor, et Conseil, Compt. rend. Acad. d. Sc., Sept. 18, 1911.

${ }^{2}$ Wilder, Jour. Inf. Dis., July, 1911, p. 9.

${ }^{3}$ Goldberger and Anderson, Pub. Health Report, Wash., March, 1912; ibid., May 31, 1912. 
symptoms. Occasionally; however, the disease is complicated by catarrhal gastroenteritis or an inflammation of the respiratory tract. and death may ensue.

The disease is unquestionably transmitted from animal to animal by means of virus contained in the vesicular contents. It is also held that infection may take place through the agency of milk. It has been claimed, moreover, though on the basis of insufficient proof, that infection may take place through the air, without actual contact, direct or indirect, with lesions.

On rare occasions the disease may be transmitted to man. Such infection, when it does take place, occurs usually among the milkers and attendants in dairies, and is transmitted by direct contact. The course of the disease in man is usually very mild. Mohler states that the disease may be transmitted to man through the consumption of milk from infected animals. $\mathrm{He}^{1}$ adds, however, that in the United States the disease has been practically eradicated.

The causative agent of foot-and-mouth disease is unknown. A number of organisms have been cultivated from the vesicles and mucous membranes of afflicted animals, but none of these could be shown to have etiological significance. Loeffler and Frosch, ${ }^{2}$ moreover, have demonstrated that the virus contained in the vesicles may pass through the pores of a Berkefeld filter. It must be assumed that the causative agent of this disease is too small to be within the range of vision of our microscopes.

The virus of the disease is easily destroyed by heating to $60^{\circ} \mathrm{C}$. and by complete desiccation.

It has been observed that one attack of foot-and-mouth disease protects against subsequent attacks. This immunity in most cases lasts for years, though rare cases of recurrence within a single year have been reported. On the basis of such naturally acquired immunity, Loeffler has actively immunized horses and cattle with graded doses of virus obtained from vesicles and with the sera of such animals has produced passive immunity in normal subjects.

1 Mohler, Bull. No. 41, U. S. Pub. Health and Mar. Hosp. Serv., Wash., 1908.

${ }^{2}$ Loeffler und Frosch, Cent. f. Bakt., 1, 1908. 


\section{FILTRABLE VIRUS}

Recent investigations into the causation of disease have revealed that a considerable number of infections may be caused by organisms too small to be held back by filters through which even the smallest bacteria cannot pass. The earliest observations of such "filtrable virus" are probably those of Frosch (1898) in foot-and-mouth disease and of Beijerinck in the mosaic disease of tobacco. Since then similar investigations have shown that a large number of diseases are probably caused by such minute organisms; their investigation, long delayed by the belief in their invisibility by even the most powerful microscopic aid, and by our inability to cultivate them, has taken new impetus from the discovery of and the cultivation of minute globoid bodies from the virus of poliomyelitis by Flexner and Noguchi (see below). The following tabulation is based largely on the comprehensive summary published by .Wolbach. ${ }^{1}$

DISEASES CAUSED BY FILTRABLE VIRUS

\begin{tabular}{|c|c|c|c|}
\hline \multirow{2}{*}{ Disease } & \multicolumn{2}{|c|}{ Transmission } & \multirow{2}{*}{ Occurrence } \\
\hline & Direct & Indirect & \\
\hline Yellow fever.... & & Stegomya fasciata & Man \\
\hline Molluscum contagiosum. & Direct contact & & Man \\
\hline Dengue fever. . & & Culex fatigans & Man \\
\hline $\begin{array}{l}\text { Verruca vulgaris filtra- } \\
\text { bility? }\end{array}$ & $?$ & & Man \\
\hline Trachoma? filtrability. & Direct & & Man \\
\hline Poliomyelitis. & $\begin{array}{l}\text { Unknown; proba- } \\
\text { bly nasal, etc., } \\
\text { discharges }\end{array}$ & $\begin{array}{l}\text { Indirect by stable- } \\
\mathrm{fly}\end{array}$ & Man \\
\hline $\begin{array}{l}\text { Measles filtrability } \\
\text { claimed Goldberger and } \\
\text { Anderson }\end{array}$ & Direct & & Man \\
\hline $\begin{array}{l}\text { Typhus fever? Nicolle pos. } \\
\text { Ricketts neg. }\end{array}$ & & $\begin{array}{c}\text { Body louse (pedic- } \\
\text { ulus vestimenti) }\end{array}$ & Man \\
\hline
\end{tabular}

${ }^{1}$ Wolbach, Jour. of Med. Res., xxvii, 1912. 
FILTRABLE VIRUS

DISEASES CAUSED BY FILTRABLE VIRUS.-Continued

\begin{tabular}{|c|c|c|c|}
\hline \multirow{2}{*}{ Disease } & \multicolumn{2}{|c|}{ Transmission } & \multirow{2}{*}{ Occurrence } \\
\hline & Direct & Indirect & \\
\hline $\begin{array}{l}\text { Scarlet fever? filtrability } \\
\text { claimed by Cantacuzene } \\
\text { and Bernhardt but } \\
\text { doubtful }\end{array}$ & Probably direct & & $\begin{array}{l}\text { Man } \\
\text { Chimpanzee }\end{array}$ \\
\hline Foot-and-mouth disease. & Direct & & $\begin{array}{l}\text { Man, cattle, } \\
\text { and swine }\end{array}$ \\
\hline Rabies. & $\begin{array}{l}\text { Direct by bite with } \\
\text { saliva }\end{array}$ & & $\begin{array}{l}\text { Man and all } \\
\text { mammals; } \\
\text { birds can be } \\
\text { infected }\end{array}$ \\
\hline Vàriola and vaccinia... & Direct & 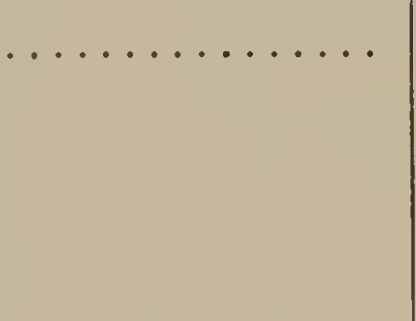 & $\begin{array}{l}\text { Man and cat- } \\
\text { tle; can be } \\
\text { transmitted } \\
\text { to monkeys } \\
\text { and rabbits }\end{array}$ \\
\hline $\begin{array}{l}\text { Pleuro-pneumonia of } \\
\text { cattle }\end{array}$ & Direct & & Bovine species \\
\hline African horse-sickness . & $\ldots \ldots$ & $\begin{array}{l}\text { Probably insects, } \\
\text { mosquitoes }\end{array}$ & Horses \\
\hline Sheep-pox....... & Direct & & Sheep \\
\hline Cattle plague. & $\begin{array}{c}\text { Food contaminated } \\
\text { with excreta }\end{array}$ & & Cattle \\
\hline Hog-cholera . . . . . . . . & Direct & & Hogs \\
\hline Swamp fever of horses. & $\ldots$ & $\begin{array}{l}\text { Probably indirect } \\
\text { by insects }\end{array}$ & Horses \\
\hline $\begin{array}{l}\text { Agalactia of sheep and } \\
\text { goats }\end{array}$ & Contact & & Sheep and goats \\
\hline "Blue tongue". & $?$ & $?$ & Sheep \\
\hline rus & $?$ & $?$ & Guinea-pigs \\
\hline
\end{tabular}


DISEASES CAUSED BY FILTRABLE VIRUS.-Continued

\begin{tabular}{|c|c|c|c|}
\hline \multirow{2}{*}{ Disease } & \multicolumn{2}{|c|}{ Transmission } & \multirow{2}{*}{ Occurrence } \\
\hline & Direct & Indirect & \\
\hline Guinea-pig paralysis... & $?$ & $?$ & Guinea-pigs \\
\hline Novy's rat disease... & $?$ & $?$ & Rats \\
\hline Fowl pest... & Feces & & $\begin{array}{l}\text { Pheasants, } \\
\text { sparrows, } \\
\text { geese }\end{array}$ \\
\hline Fowl diphtheria... & $\begin{array}{l}\text { Contact exudates, } \\
\text { etc. }\end{array}$ & & Fowl \\
\hline Rous's chicken sarcoma. & $?$ & $?$ & Chickens \\
\hline
\end{tabular}




\section{SECTION V}

\section{BACTERIA IN AIR, SOIL, WATER, AND MILK}

\section{CHAPTER LII}

\section{BACTERIA IN THE AIR AND SOIL}

\section{BACTERIA IN THE AIR}

BACTERIA reach the air largely from the earth's surface, borne aloft by currents of air sweeping over dry places. Their presence in air, therefore, is largely dependent upon atmospheric conditions; humidity and a lack of wind decreasing their numbers, dryness and high winds increasing them. Multiplication of bacteria during transit through the air probably does not take place.

Apart from these considerations the presence of bacteria in air also depends upon purely local conditions prevailing in different places. They are most plentiful in densely populated areas and within buildings, such as theaters, meeting halls, and other places where large numbers of people congregate. On mountain tops, in deserts, over oceans, and in other uninhabited regions, the air is comparatively free from bacteria. A classical illustration of this fact is found in the experiments which Pasteur carried out in his refutation of the doctrine of spontaneous generation. Tyndall also, in working upon the same subject, demonsirated this fact. From the surface of the ground and other places where bacteria have been deposited, they reach the air only after complete drying. It is a fact of much importance, both in bacteriological work and in surgery, that bacteria do not rise from a moist surface. From dry surfaces they may rise, but only when the air is agitated either by wind or by air-currents produced in other ways. In closed rooms, therefore, even when bacteria are plentiful and the walls and floors are perfectly dry, there is little danger of the inhalation of bacteria unless the air is agitated in some way. The most favorable conditions for the occurrence of many bacteria in air are the existence of a prolonged drought followed by a dry wind. Under such condi- 
tions, even the dark places and unlighted corners of streets and habitations are thoroughly dried out, and bacteria are taken up and carried about together with particles of dust. At such times the dangers from inhalation are much multiplied. By experiments made in balloons, it has been found that bacteria are plentiful below altitudes of about fifteen hundred feet and may be present, though much reduced in numbers, as high up as a mile above the earth's surface. The species of bacteria found in the air are, of course, subject to great variation, depending upon locality. Molds and spore-forming bacteria, being more regularly resistant to the effects of sunlight and drying than bacteria possessing only vegetative forms, are naturally more generally distributed.

Out of air thus laden with bacteria, they may again settle when the wind subsides and the air becomes quiescent. The process of settling, however, is extremely slow, since the weight of a bacterium is probably less than a billionth of a gram, and it may be held in suspension in air for considerable periods. Rains, snow, or even the condensation of moisture from a humid atmosphere, hastens this process considerably, and large quantities of bacteria may settle out from air, in a comparatively short time, in ice chests, in operating rooms, or in other places in which much condensation of water vapor takes place.

The importance of the air as a means of conveying disease is still a problem upon which much elucidation is needed. The importance of this manner of conveyance in smallpox, in measles, in scarlet fever, and in other exanthemata, can not be denied. As regards the diseases of known bacterial origin, conveyance by air is of importance in the case of tuberculosis, where infection by inhalation may take place, and in the case of anthrax, where inhaled anthrax spores may give rise to the pulmonary form of the disease. The importance of air conveyance for any great distance in pneumonia, in influenza, in diphtheria, and in meningitis is by no means clear and requires much further study. The expulsion of bacteria from the lungs and naso-pharynx does not take place during simple expiration, since an air-current passing over a moist surface is not sufficient to dislodge microorganisms. Expulsion of bacteria in these conditions must take place together with small particles of moisture carried out in sneezing, coughing, or any forced expiration. The bacteria thus discharged are then subject to the process of drying and often are exposed to direct sunlight for a considerable period before they are again taken up in the air.

The methods of estimating the bacterial contents of the air are not 
entirely satisfactory. The simple exposure of uncovered gelatin or agar plates for a definite length of time, and subsequent estimation of the colonies upon the plates, yield a result which is variable according to the air-currents and the degree of moisture in the atmosphere, and furnish no volume standard for comparative results. The methods which are in use at the present time depend upon the suction of a definite quantity of air by means of a vacuum-pump through some substance which will catch the bacteria. One of the first devices used for this purpose was that of Hesse, who sucked air through a piece of glass tubing, about $70 \mathrm{~cm}$. long and about $3.5 \mathrm{~cm}$. in diameter, the inner surface of which had been coated with gelatin in the manner of an Esmarch roll tube. This method is not efficient, since a large number of the bacteria may pass entirely through the tube without settling upon the gelatin. One of the most satisfactory methods at present in use is that in which definite volumes of air are sucked through a sand-filter. Within a small glass tube, a layer of sterilized quartz sand, about $4 \mathrm{~cm}$. in depth, is placed. The sand is kept from being dislodged by a small wire screen. After the air has been sucked through the filter the sand is washed in a definite volume of sterile water or salt solution, and measured fractions of this are planted in.agar or gelatin in Petri plates. The colonies which develop are counted. Thus, if two liters of air have been sucked through the filter, and the sand has been washed in 10 c.c. of salt solution, and 1 c.c. of this is planted, with the result of fifteen colonies, then the two liters of air have contained one hundred and fifty bacteria.

\section{BACTERIA IN SOIL}

Besides the normal bacterial inhabitants of the soil, bacteria reach the soil from the air, in contaminated waters, in the dejecta, excreta, and dead bodies of animals and human beings, and in the substance of decaying plants. It is self-evident, therefore, that the distribution of bacteria in soil depends largely upon the density of population and the use of the soil for agricultural or other purposes. Thus, bacteria are most plentiful in the neighborhood of cess-pools or in manured fields and gardens. Such conditions, however, may be regarded as abnormal. Even in uncultivated fields there is a constant bacterial flora in the soil which is of great importance in its participation in the nitrogen cycle, a phase of the bacteriology of soil which has been discussed in detail in another 
section. (See page 40.) There are, thus, regular and normal inhabitants of the soil which fulfil a definite function and may be found wherever plant life flourishes. In addition to these, innumerable varieties of saprophytes and pathogenic germs may be present, which vary in species and in number with local conditions. Numerous investigations into the actual numerical contents of the soil have been made. Houston ${ }^{1}$ found an average of 1,500,000 bacteria per gram in garden soil, and about 100,000 bacteria per gram in the arid soil of uncultivated regions. Fraenkel, ${ }^{2}$ in studying the horizontal distribution of bacteria in the earth, has found that they are most numerous near the surface, a gradual diminution occurring down to a depth of about two yards. Beyond this, the soil may be often practically sterile.

Pathogenic bacteria may at times be found in the surface layers, and these are often of the spore-bearing varieties. Most important among them from the medical standpoint are the bacillus of tetanus, of malignant edema, and the Welch bacillus. If a guinea-pig is inoculated subcutaneously with an emulsion of garden soil, death will result almost invariably with enormous bloating and swelling of the body due to gas production. This is due to the fact that the spore-bearing, gas-producing anaërobic bacilli are commonly present and are actively pathogenic for these animals. The frequent occurrence of tetanus in persons sustaining wounds of the bare feet and hands in fields and excavations, is a matter of common knowledge. Anthrax, also, may be easily conveyed by soil in localities where animals are suffering from this infection. It is not probable that pathogenic germs which are not spore-bearers survive in the soil for any great length of time. Unless the soil is specially prepared by the presence of defecations or other other organic material, the nutrition at their disposal is not at all suitable for their needs, since rapid decomposition of organic materials by saprophytes is always going on in the upper layers. Furthermore, in the deeper layers the conditions of temperature and possibly oxygen supply are not at all favorable for the growth of most pathogenic bacteria. Within a short distance from the surface the temperature of the soil usually sinks below $14^{\circ}$ or $15^{\circ} \mathrm{C}$. An interesting series of experiments by Fraenkel ${ }^{3}$ have demonstrated this point. This investigator buried freshly inoculated agar and gelatin cultures of cholera spirilla and of typhoid and anthrax bacilli at different levels, and examined them for growth after two weeks had elapsed.

${ }_{1}^{1}$ Houston, Report Med. Officer, Local Govern. Bd., London, 1897.

2 Fraenkel, Zeit. f. Hyg., ii, 1887.

${ }^{3}$ Fraenkel, Zeit. f. Hyg., xi, 1887. 
The anthrax bacilli hardly ever showed growth at a depth below about two yards, and cholera and typhoid developed colonies at these depths only during the summer months. Under natural conditions it must be remembered that, at these levels, suitable nutritive material is not found.

A consideration of practical importance in this connection is the possibility of infection by means of buried cadavers. An elaborate series of experiments has been carried out upon this subject in Germany, with results which demonstrate that the danger from the burial of persons dead of infectious diseases was formerly much exaggerated. Experiments ${ }^{1}$ usually failed to reveal the presence of cholera and typhoid bacilli within two to three weeks after burial, and tubercle bacilli were never found after three months had elapsed. It was only in the case of sporulating microorganisms, such as the anthrax bacillus, that the living incitants could be found for as long as two years after burial. The dangers of infection of human beings through the agency of soil, therefore, are chiefly those arising from the spore-bearing bacteria which are able to remain alive in spite of the unfavorable cultural conditions. It has been found by some observers, ${ }^{2}$ however, that, under special conditions, non-sporulating bacteria, more especially the typhoid bacillus, may remain alive in soil for several months. Although these bacteria, as well as those of cholera, diphtheria, etc., can not proliferate under the conditions found in the soil, the fact that they can remain viable for such prolonged periods in the upper layers suggests the possibility of danger from the use of unwashed vegetables, such as lettuce or radishes or other soil and sewage contaminated food products. The examination of soil for colon bacilli, while demonstrating the presence or absence of manure or sewage contamination, has no practical value, since colon bacilli are found in the dejecta of animals.

Examination of specimens of soil for their numerical bacterial contents is extremely unsatisfactory because the bacteria there found can hardly ever all be cultivated together under one and the same cultural environment. A large number are anaërobic, others again thrive at low temperatures, while again another class may require unusually high temperatures. When such examinations are made, however, specimens of the soil from the surface layer may be taken in a sterile platinum spoon. When taken from the lower levels, a drill,

1 Petri, Arb. a. d. kais. Gesundheitsamt, vii.

${ }^{2}$ Firth and Horrocks, Brit. Med. Jour., Sept., 1902. 
such as that devised by Fraenkel, may be used. This consists of an iron rod the lower end of which is pointed. Just above the point a movable collar is fitted. This collar has a slit-like opening. The rod beneath the collar has a deep longitudinal groove corresponding to the slit in the collar. A flange on the collar permits opening and closing of the groove while the instrument is below the ground. The drill is forced into the earth to the desired depth, the groove is opened and earth is forced into the chamber by twisting the rod. In the same manner the groove may be closed. The soil obtained in this way is taken out of the chamber and a definite quantity, say one gram, is dissolved and washed thoroughly in a measured volume of sterile water or sterile salt solution. Fractions of this are then mixed with the culture medium, plated, and cultivated aërobically or anaërobically as desired. 


\section{CHAPTER LIII}

\section{BACTERIA IN WATER}

ALL natural waters contain a more or less abundant bacterial flora. This fact, combined with our knowledge that the incitants of several epidemic diseases and a number of minor ailments of a diarrheal character are water borne, gives the bacteriological investigation of water a place of great importance in hygiene. In nature, there are very few sources of water supply which do not contain bacteria of one or another description. While pathogenic bacteria are usually not present except in those waters which are directly contaminated from human sources, a thorough understanding of the quantitative and qualitative bacterial contents of all natural waters is necessary in order that we may intelligently gather comparative data as to the fitness of any given water for human consumption.

The gross appearance of water is rarely, if ever, an indication of its danger. The turbid waters of running streams in sparsely populated agricultural districts may be safe, while perfectly clear well waters subjected to the dangers of contamination from neighboring sinks or cess-pools may contain large numbers of pathogenic germs.

The diseases which are known to be more directly connected with water supply are typhoid fever and cholera.

Typhoid germs discharged from the bowel or from the urine of typhoid patients or convalescents may be carried by the sewage or from the neighboring soil into a river or lake and lead to infection of the population deriving its drinking water from this source. There are a great many investigations on record in which severe typhoid epidemics have been traced to such sources.

In the case of cholera, where the germs are discharged from the bowels in enormous numbers, conveyance of the disease by water is even more spparent, and the discoverers of the cholera germ themselves, in their early work in Egypt and India, were able to isolate the bacteria from contaminated water supplies.

In regard to the less clearly understood diarrheal diseases, dysentery, cholera infantum, etc., the direct relation to water supply has not 
been so definitely proven, and can be deduced only from the diminution of such infections after the substitution of pure water for the previously used impure supply. It is thus seen that water bacteriology is one of the most important branches of the science of hygiene, and has led, and is constantly leading, to enormous diminution of the death rate in all communities where an intelligent study of the conditions has been made.

The bacterial purity of natural waters, although dependent upon special and local conditions in relation to possible contamination, differs widely, according to the source from which such waters are derived.

Rain water and snow water are usually contaminated with bacteria by the dust which they gather on their way to the ground, and are especially rich in bacteria when taken during the first few hours of a rain or snow storm when the air is still dusty and filled with floating particles. During the later hours of prolonged storms, rain water and snow water may be comparatively sterile. Miquel, ${ }^{1}$ who made extensive experiments in France on the bacterial contents of rain water, found that in country districts, where the air is less dusty, rain water contained an average of about 4.3 bacteria to the cubic centimeter.

The bacterial counts of snow water are usually somewhat higher than those of rain.

The waters of streams, ponds, and lakes are usually spoken of as surface waters, and these of all natural supplies contain the largest number of bacteria. In each case, of course, the quantitative and qualitative bacterial flora of such waters is intimately dependent upon the conditions of the surrounding country, the density of the population, and the relation of these waters to sewage. It is also, and to no less important degree, dependent upon weather conditions, the influence of light and temperature, and the food supply contained within the waters in the form of decayed vegetation. In all such surface waters there is constantly going on a process of self-purification. The chief factor in this process is sedimentation. In stagnant ponds and lakes with but sluggish currents there is a constant sedimentation of the heavier particles, which gradually but steadily leads to a diminution of the number of bacteria in the upper layers of the water. In rivers where sedimentation is to a certain extent prevented by rapidity of current, the effectiveness of such sedimentation is, of course, entirely dependent upon the speed of the current.

${ }^{1}$ Miquel, Revue d'hyg., viii, 1886. 
The influence of light in purifying surface waters is important chiefly in ponds, lakes, and sheets of water which expose a large surface to the sunlight, and where the surroundings are such that the sun has free access throughout the day. According to the researches of Buchner, ${ }^{1}$ the bactericidal effects of light penetrate through water to a depth of three feet.

The effects of temperature in purifying surface waters under natural conditions are probably not great. There is, however, a general tendency toward diminution of the bacterial flora as the temperature of such waters becomes lower.

The presence of protozoa in natural waters as purifying agents has recently been emphasized by Huntemüller, ${ }^{2}$ who claims that these organisms by phagocytosis greatly diminish the number of bacteria in any given body of water. It is self-evident that the number of bacteria in any of these waters is never constant, since all factors which tend to a diminution or increase in volume, such as drying up of tributary streams or the occurrence of heavy rains, would lead to differences of dilution which would materially change numerical bacterial estimations. The influence of rains, furthermore, may be a twofold one. On the one hand, heavy rain-falls, by washing a large amount of dirt into the rivers and lakes from the surrounding land, have a tendency to increase the bacterial flora. This influence would be especially marked in all bodies of water which are surrounded by cultivated land where manured fields and grazing-meadows supply a plentiful source of bacteria. On the other hand, in regions where arid, uninhabited lands surround any given river or lake, the rain would carry with it very little living contamination and would act chiefly as a diluent and diminish the actual proportion of bacteria in the water.

Another and extremely important source of water supply is that spoken of technically as "ground water." The "ground waters" include the shallow wells employed in the country districts, springs, and deep or artesian wells. The shallow wells that form the water supply for a large proportion of farms in the eastern United States are usually very rich in bacteria and are by no means to be regarded as safe sources, except in cases where great care is observed as to cleanliness of the surroundings. In such wells the filtration of the water entering the well may be subject to great variation according to the geological conditions of the surrounding ground. The proximity of barns and sinks may lead to dangerous contamination of such waters.

1 Buchner, Arch. f. Hyg., xvii, 1895. $\quad 2$ Huntemüller, Arch. f. Hyg., liv, 1905. 
Examinations by various bacteriologists have shown that such wells frequently contain as many as five hundred bacteria to the cubic centimeter.

Perennial spring waters are usually pure. Examinations by the Massachusetts State Board of Health ${ }^{1}$ in 1901 showed an average of about forty bacteria per cubic centimeter. As sources of water supply for general consumption, however, springs can hardly be very important because of the insignificant quantities usually derived from them.

Of much greater practical importance are deep artesian wells, which, under ordinary conditions, are largely free from bacterial contamination.

Quantitative Estimations of Bacteria.-The quantitative estimation of bacteria in water is of necessity inexact, because of the difficulty of always securing fair average samples from any large body of water, and because of the large variations in cultural requirements of the flora present in them. All these methods depend upon colony enumeration in plates of agar or gelatin, preferably of both. For the sake of gaining some basis of comparison for results which, at best, can never be entirely accurate, an attempt has been made by the American Public Health Association ${ }^{2}$ to standardize the methods of analysis.

Water for analysis should always be collected in clean, sterile bottles, preferably holding more than 100 c.c. If water is to be taken from a running faucet or a well supplied with piping, it is important that it should be allowed to run for some time before the sample is taken, in order that any change in bacterial content occurring inside of the pipes may be excluded. It is obvious that in water pipes through which the flow is not constant, bacteria may find favorable conditions for growth and such a sample would not represent fairly the supply to be tested.

When the water is taken from a pond, lake, or cess-pool, the bottle may be lowered into the water by means of a weight, or may be plunged in with the hand, great care being exercised not to permit contamination from the fingers to occur. A number of devices for collecting water have been originated, a very excellent one for small samples, by Sternberg, ${ }^{3}$ consisting of a small glass bulb with a capillary neck which is sealed while the bulb is hot. This is attached to a rod, and a wire noose is fastened to the neck of the bulb. When immersed in water the neck

${ }_{1}^{1}$ Mass. State Bd. of Health, 33d Annual Report for 1901.

${ }^{2}$ Fuller, Trans. Amer. Public Health Assn., xxvii, 1902. Report of Com. on Standard Methods of Water Analysis. Jour. Inf. Dis., Suppl. 1, 1905.

3 Sternberg, "Manual of Bact." 
may be broken off by means of the wire, and water will be forced into the bulb to satisfy the vacuum.

After the water has been collected it is important to plate it before the bacteria in it have a chance to increase. The changes taking place during transportation, even when packing in ice has been resorted to, have been found by Jordan and Irons ${ }^{1}$ to be considerable. It is imperative, therefore, that plating of the water, if possible, shall not be delayed for longer than one or two hours after collection.

Before plating, the bottle containing the sample should be shaken at least twenty-five times in order to distribute the bacterial contents evenly. The quantities to be plated will depend to a certain extent upon the probability of there being a large or small number of bacteria. If less than two hundred are suspected, 1 c.c. of the water should be taken out of the bottle with a sterile pipette and placed in a sterile Petri dish. To this is added 10 c.c. of gelatin at a temperature of about $30^{\circ} \mathrm{C}$., or of agar at a temperature of not over $40^{\circ} \mathrm{C}$. The water is thoroughly mixed with the medium by repeated tilting of the plate, and finally allowed to harden in the regular way. If unusual pollution or other data lead to a suspicion that the bacterial count is apt to be extremely high, it is advisable to dilute the sample of water with sterile water before plating. The reason for mixing water and medium in the Petri dish, rather than in the tube, as was formerly done, is the fact that in the pouring of the mixture from the tube a certain amount of residuum is left which naturally leads to a diminution in the actual number of colonies developing in the plate. The gelatin is incubated at $20^{\circ} \mathrm{C}$. the agar at $37.5^{\circ} \mathrm{C}$.

Neither the gelatin nor the agar alone can give an accurate estimation of the total bacterial contents of water. A better estimate can of course be made when both are used. However, since aërobic and anaërobic bacteria may be present and since many water organisms may require nutritive conditions and a reaction other than that of the standard media, it is quite likely that none of the methods in use can give an accurate total of organisms originally in the water. For sanitary purposes, however, the agar counts, in which the plates are incubated at $37.5^{\circ} \mathrm{C}$. or body temperature, are by far the most important since the bacteria which grow at this temperature are the ones likely to possess pathogenic significance, or at any rate to emanate from animal and human sources. It has been suggested by Rosenau ${ }^{2}$ and others that es-

${ }^{1}$ Jordan and Irons, Reports of the Amer. Pub. Health Assn., xxv, 1889.

${ }^{2}$ Rosenau, "Preventive Medicine and Hygiene," 755. D. Appleton \& Co., 1913. 
pecial value is attached to the organisms which grow at $40^{\circ} \mathrm{C}$. since the temperature excludes many non-pathogenic water bacteria, while it permits organisms of the colon-typhoid group to grow.

The gelatin and agar which are used should be made according to the standard methods recommended by the American Public Health Association. The gelatin should be made of meat infusion and not of meat extract, and should contain one per cent of Witte's pepton and ten per cent of the best so-called French brand of gelatin. It should not soften when kept at a temperature of $25^{\circ} \mathrm{C}$. The agar medium should also be made of meat infusion and of the highest grade of commercial thread agar. For general purposes the standard reaction of media should be one per cent acidity, but for long-continued work on water from the same source the optimum reaction should be ascertained and adhered to, and differences from the standard reaction should be mentioned in the report.

Incubation of the gelatin should be continued for forty-eight hours in an atmosphere saturated with moisture. When agar is used, incubation for twenty-four hours is usually sufficient, and it is advantageous to employ Petri dishes supplied with porous earthenware covers. ${ }^{1}$ Simple inversion of the Petri dishes when placed in the incubator obviates the necessity of using the porous covers.

In counting, the ordinary counting plates divided into $1 \mathrm{~cm}$. squares may be used, but, whenever possible, all the colonies in a plate should be counted.

The value of the quantitative estimation of bacteria in water is only a comparative one, and no arbitrary standards can be established for the purity of water on this basis. In general it may be said that water containing one hundred bacteria to the cubic centimeter or less is apt to be from a deep source and comparatively pure; that water containing five hundred bacteria to the cubic centimeter or over is open to suspicion, and that any water containing over one thousand to the cubic centimeter is probably from a polluted source. At the same time it is quite impossible to draw any direct conclusions from numerical colony counts, and all such results must be carefully weighed in the balance with qualitative analyses and chemical tests, and knowledge of environmental conditions.

Qualitative Bacterial Analyses of Water.-Of far greater importance than quantitative analysis is the isolation of bacteria either distinctly pathogenic, such as the cholera spirillum and the typhoid bacillus, 
or of other species probably emanating from contaminating sources, such as a B. coli. Unfortunately there are no reliable methods by which typhoid and cholera germs can be isolated from water with any degree of regularity or certainty. Although frequently the isolation of such organisms is possible, a negative result in these cases is by no means eliminative of their presence.

The isolation of typhoid bacilli from water is very difficult, chiefly because of the great dilution which contaminations undergo upon entering any large body of water. The difficulty of isolating typhoid bacilli, even from the stools of infected patients, makes it clear that such difficulties are enhanced when a considerable dilution of the excreta takes place. Furthermore, water is by no means a favorable medium for the typhoid bacillus. Russell and Fuller ${ }^{1}$ have shown that typhoid bacilli may die in water within five days, and it is unquestionable that the rate of increase of these bacteria is by no means equal to that of many other microorganisms for which polluted water at the temperature encountered in streams and lakes forms a much more favorable medium.

It is thus clear that even in infected waters the number of typhoid bacilli encountered can never be very great. ${ }^{2}$

A large number of methods for the isolation of the typhoid bacillus from water have been devised. Most of the media used are identical with those employed for the isolation of these bacteria from the stools. These media have been discussed in the chapter dealing with the typhoid bacillus.

Drigalski ${ }^{3}$ has reported a method for which he claims considerable success in isolating typhoid bacilli from water, which depends upon the motility of the organisms. One- to two-liter samples of water are taken and allowed to stand at room temperature in high jars for one or two days. Small quantities are then removed from the surface and planted on Wurtz's lactose litmus agar. The method depends upon the probability of the settling out of non-motile organisms and the possibility, therefore, of getting motile organisms only in the plates.

Parietti ${ }^{4}$ and others have attempted to eliminate other organisms by adding phenol and hydrochloric acid to neutral broth, in the hope that the high acidity and the antiseptic qualities of the phenol will destroy more delicate organisms than the typhoid bacillus. Inasmuch as B.

${ }^{1}$ Russell and Fuller, Jour. Inf. Dis., Suppl. 2, 1908.

${ }^{2}$ Laws and Anderson, Rep. of Med. Officer, London County Council, 1894.

${ }^{3}$ Drigalski, Arb. a. d. kais. Gesundheitsamt, xxiv, 1906.

${ }^{4}$ Parietti, Rev. d'igiene e san. pub., 1890. 
coli, however, withstands these reagents as well as B.typhosus, or even better, these methods have not met with great success.

A method which has proved useful in the hands of Adami and Chapin ${ }^{1}$ is one which depends upon the phenomenon of agglutination. These authors collect water in two-iter specimens and to each two liters add 20 c.c. of one per cent glucose broth. These samples are incubated at $37.5^{\circ} \mathrm{C}$. for twenty-four hours, and at the end of this time quantities of 10 c.c. are withdrawn and placed in test tubes. To each of these tubes potent typhoid serum, preferably diluted $1: 60$, is added, and whenever agglutination occurs the flocculi are washed and plated on various media for identification.

Vallet and others have attempted to precipitate typhoid bacilli out of water by chemical means. The purpose of these methods has been to entangle the bacteria in an inert precipitate, and thus concentrate the bacteria in water for purposes of cultivation. Vallet's method is as follows: To two liters of water add 20 c.c. of a 7.75 per cent solution of sodium hyposulphite and 20 c.c. of a 10 per cent solution of lead nitrate. When the precipitate has settled, the clear supernatant fluid is decanted and the precipitate dissolved in a saturated sodium hyposulphite solution. This clear solution is then plated. Willson ${ }^{2}$ has modified this method by adding to the water $0.5 \mathrm{gm}$. of alum to each liter. A precipitate is formed which may either be allowed to settle or may be brought down by centrifugalization. The supernatant fluid is removed and the precipitate plated.

In actual work many of the methods which are aimed purely at $B$. coli may lead to success in the isolation of B. typhosus, because of the similarity of the two organisms in their reaction to definite media. Thus, the method of Jackson, ${ }^{3}$ who employs one per cent of lactose in pure ox-bile for the isolation of B. coli, has occasionally led to the simultaneous isolation of $\mathrm{B}$. typhosus.

The isolation of the vibrio of cholera is less difficult than that of $\mathrm{B}$. typhosus, primarily because of the much greater numbers of these microorganisms discharged into sewage. The number of cholera spirilla in the excreta of cholera patients is enormously higher than is that of B. typhosus in the stools of typhoid-fever patients. It is not infrequent, therefore, that the source of a cholera infection may be directly traced to the water supply. Koch, ${ }^{4}$ the discoverer of the cholera vibrio,

\footnotetext{
${ }^{1}$ Adami and Chapin, Jour. Med. Res., xl, 1904.

${ }^{2}$ Willson, Jour. of Hyg., v, 1905.

${ }^{3}$ Jackson Journ. of Inf. Dis., Suppl. 2, 1907.

${ }^{4}$ Koch, Zeit, f. Hyg., xiv, 1893.
} 
has indicated a method which has frequently found successful application.

To 100 c.c. of the infected water are added one per cent of pepton and one per cent of salt. This mixture is then incubated at $37.5^{\circ} \mathrm{C}$., and after ten, fifteen, and twenty hours, specimens from the upper layers are examined microsopically and are plated upon gelatin. Upon the weak pepton solution cholera spirilla increase very rapidly at incubator temperatures, and then when plated in gelatin the detection of characteristic colonies is comparatively easy.

Because of the great difficulties outlined above in isolating specific pathogenic germs from polluted waters, bacteriologists have attempted to form an approximate estimate of pollution by the detection of other microorganisms which form the predominating flora of sewage. Chief among these is B. coli. The isolation and numerical estimation of $\mathrm{B}$. coli in polluted water has been for a long time one of the criteria of pollution. This so-called colon test, however, should always be approached with conservatism and should never be carried out qualitatively only. Careful quantitative estimation should be made in every case.

B. coli in water is by no means always the result of human contamination, since this bacillus is found in great abundance in the intestines of domestic animals. According to Poujol, B. coli does not even always point to fecal contamination, since this author was able to find the bacillus in the water of a number of wells where no possible contamination of any sort could be traced. Prescott ${ }^{1}$ explains this, as well as similar cases, by the fact that organisms of the colon group may occasionally be parasitic upon plants.

The opinions of hygienists are widely at variance as to the value of the colon test. While the discovery of isolated bacilli of the colon group may therefore be of little value, it is nevertheless safe to follow the opinion of Houston, ${ }^{2}$ who states that the discovery of B. coli in considerable numbers invariably points to sewage pollution, and that the absolute absence of $\mathrm{B}$. coli is, as a rule, reliable evidence of purity.

Rosenau states that a ground water should be condemned even if only a few colon bacilli are found, for, as he puts it, "these bacteria have no business in a soil-filtered and properly protected well or springwater." Surface waters, however, may easily contain a few colon bacilli without necessarily having been exposed to contamination by

${ }_{1}$ Prescott, Science, xv, 1903.

${ }^{2}$ Houston, Rep. Medical Officer, Local Gov. Board, London, 1900. 
human forces. The limit of safety, Rosenau states, is one colon bacillus per c.c. If more are present the water should be regarded as suspicious. If more than 10 per c.c. are found the water must be regarded as dangerous and unqualifiedly condemned.

For the purpose of isolating B. coli from water, a large number of methods have been devised. In examining sewage or other polluted waters in which the number of colon bacilli is comparatively large, the direct use of lactose litmus agar plates yields excellent results. Varying quantities of water may be added to this medium and the development of red colonies at incubator temperatures usually indicates the presence of bacilli of this species. These colonies may be fished and further identified. Copeland ${ }^{1}$ has proposed the addition of .2 per cent of phenol to this medium in order to inhibit other bacteria.

In water less grossly polluted, some method of enrichment must be employed in order to increase the number of bacteria so that they may be found in plates. For this purpose glucose bouillon in fermentation tubes, according to the method of Theobald Smith, may be employed. ${ }^{2}$ In this medium, at a temperature of $37.5^{\circ} \mathrm{C}$., the colon bacilli grow with great speed and transplants to plating media may be taken after eight or more hours' incubation.

A medium proposed by Jackson ${ }^{3}$ has been found successful. This consists of undiluted ox-bile, to which has been added 1 per cent of lactose.

For quantitative estimation of colon bacilli in water, Theobald Smith ${ }^{4}$ has proposed the use of dextrose bouillon in fermentation tubes, to which are added varying quantities of water, ranging from 0.001 to 1 c.c. The appearance of gas in any tube indicates the presence of B. coli, and the number can be approximately computed from the smallest quantity of water by which gas formation has been produced.

The presence of $\mathrm{B}$. coli in such fermentation tubes may be determined without isolation and cultivation, by estimating the comparative amount of $\mathrm{CO}_{2}$ in the gas. ${ }^{5}$ Whenever $\mathrm{CO}_{2}$ forms approximately 33 per cent of the gas present, Irons concludes that B. coli is present.

Jackson ${ }^{6}$ believes when lactose ox-bile is used that 25 per cent of gas within 72 hours may be regarded as positive for B. coli.

${ }^{1}$ Copeland, Jour. Boston Soc. Med. Sciences, 1901.

${ }^{2}$ Prescott, Science, xvi, 1902.

${ }^{3}$ Jackson, Jour. Inf. Dis., Suppl. 2, 1907.

${ }^{4}$ Th. Smith, 13th Ann. Rep. N. Y. S. Board of Health.

${ }^{5}$ Irons, Trans. Amer. Pub. Health Assn., xxvi, 1900.

${ }^{6}$ Jackson, "Biol. Studies of Pupils of W. T. Sedgwick," Boston, 1906. 


\section{CHAPTER LIV}

\section{BACTERIA IN MILK AND MILK PRODUCTS, BACTERIA. IN THE INDUSTRIES}

\section{BACTERIA IN MILK}

THE universal use of cows' milk as a food, especially for the nourishment of infants, has necessitated its close study by bacteriologists and hygienists. It furnishes an excellent culture medium for bacteria and is, therefore, pre-eminently fitted to convey the germs of infectious disease. The many changes which take place in milk, furthermore, and which add or detract from its nutritive value, are due largely to bacterial growth and have been elucidated by bacteriological methods.

Within the udder of the healthy cow, milk is sterile. If pyogenic or systemic diseases of bacterial origin exist in the cow, the milk may, under certain circumstances, be infected even within the mammary glands. In the milk ducts and in the teats, however, even in perfectly healthy animals, a certain number of bacteria may be found. For this reason, even when all precautionary measures are followed, the milk as received in the pail is usually contaminated. As a matter of fact, the anatomical location of the udder and the mechanical difficulties of milking make it practically impossible to collect milk under absolutely aseptic conditions, and, under the best circumstances, from 100 to 500 microorganisms per c.c. may usually be found in freshly taken milk. Withdrawn under conditions of ordinary cleanliness, the bacterial contents of milk are considerably higher than this. After the process of milking, in spite of all practicable precautions, the chances for the contamination of milk are considerable; but even could these be eliminated, the bacterial contents of a given sample would ultimately rapidly increase because of the rich culture medium which the milk provides for bacteria. Whether large increases shall take place or not depends, in the first place, upon the temperature at which milk is kept, and, in the second place, upon the length of time which intervenes before its consumption. Though fresh milk possesses slight bactericidal powers, ${ }^{1}$

1 Rosenau and McCoy, Jour. Med. Res., 18, 1908. 
these are by no means sufficient to be of practical importance in the inhibition of bacterial growth. Kept at or about freezing-point, the bacterial contents of milk do not appreciably increase. At higher temperatures, however, a rapid propagation of bacteria takes place which, especially during the summer months, speedily leads to enormous numbers. In a case reported by Park, ${ }^{1}$ where milk, containing at the first examination 30,000 microorganisms per cubic centimeter, was kept at $30^{\circ} \mathrm{C}$. $\left(86^{\circ} \mathrm{F}\right.$.) for twenty-four hours, the count at the end of this time yielded fourteen billions of bacteria for the same quantity.

It is of much importance, therefore, that the cleanliness of dairies, of cattle, and in the handling of milk should be reinforced by the utmost care in chilling and icing during shipment and before sale.

Because of its great importance, especially for the health of the children in large cities during the summer months, the milk question has, of recent years, received much attention from health officers. In the city of New York, the question has been made the subject of many careful studies by Park $^{2}$ and his associates. Commissions, working in Chicago, ${ }^{3}$ Boston, ${ }^{4}$ and other large towns, have placed the sale of milk under more or less exact bacteriological supervision. Park has determined that the milk, as sold in New York stores during the cold weather, not infrequently averages seven hundred and fifty thousand bacteria per cubic centimeter; during the hot summer months, the bacterial contents of similar milk not infrequently average one million and more, for the same quantity. ${ }^{5}$ In consequence of these and other researches, large dairies have introduced bacteriological precautions into their method of milk production. They have attempted the reduction of the bacterial contents of milk by scrupulous cleanliness of the barns and of the udders and teats of the cow, by the elimination of diseased cattle, by sterilization of the vessels in which the milk is received, and of the hands of the milker; also by the immediate filtering and cooling of the milk and the packing of the milk cans in ice, where they remain until. delivered to the consumer. In consequence of such measures, it is possible for cities to be supplied with milk containing no more, and often less, than fifty thousand bacteria to the cubic centimeter. A standard of cleanliness has been established in various towns by the

${ }^{1}$ Park, W. H., "Pathogenic Bacteria," New York, 1905, p. 463.

2 Park, Jour. of Hygiene, 1, 1901.

3 Jordan and Heinemann, Rep. of the Civic Federation of Chicago, 1904.

${ }^{4}$ Sedgwick and Batchelder, Bost. Med. and Surg. Jour., 126, 1892.

Escherich, Fort. d. Medizin, 16 and 17, 1885. 
introduction of the so-called "certified milk," which, by the New York Milk Commission, is required to contain no more than thirty thousand bacteria per cubic centimeter. Great stress is laid upon such numerical counts simply in that they are approximate estimates of cleanliness. Most of the bacteria, however, contained in milk are non-pathogenic, and numbers much larger than the maximum required for certified milk may be present without actual disease or harm following its consumption.

The various species of bacteria which may be found in milk include almost all known varieties. Whether there are special, so-called milk bacteria or not is a question about which investigators have expressed widely differing opinions. It is probable that many of the species, formerly regarded as specifically belonging to milk, are there simply by virtue of their habitual presence in fodder, straw, or bedding, or upon cattle. It is likely, furthermore, that some of these species are found with great regularity because of their power to outgrow other species under the cultural conditions offered them in milk.

Under normal conditions, milk always undergoes a process which is popularly known as souring and curdling. This is due to the formation of lactic acid from the milk sugar and is the result of the enzymatic activities of several varieties of bacteria commonly found in milk. Most common among these bacteria is the so-called Bacillus lactis aërogenes, an encapsulated bacillus closely related to the colon-bacillus group. (See page 451.) The transformation of the lactose into lactic acid may occur either directly by hydrolytic cleavage:

$$
\mathrm{C}_{12} \mathrm{H}_{22} \mathrm{O}_{11}+\mathrm{H}_{2} \mathrm{O}=4 \mathrm{C}_{3} \mathrm{H}_{6} \mathrm{O}_{3}
$$

or indirectly through a monosaccharid,

$$
\mathrm{C}_{12} \mathrm{H}_{22} \mathrm{O}_{11}+\mathrm{H}_{2} \mathrm{O}=2 \mathrm{C}_{6} \mathrm{H}_{12} \mathrm{O}_{6}=4 \mathrm{C}_{3} \mathrm{H}_{6} \mathrm{O}_{3} \text {. }
$$

Other microorganisms which may cause lactic-acid fermentation in milk are the so-called Streptococcus lacticus, the common pyogenic streptococcus, the Staphylococcus aureus, Bacillus coli communis and communior, and many other species. Most commonly concerned in lacticacid production, however, according to Heinemann, ${ }^{1}$ are the two firstmentioned, Bacillus lactis aërogenes and Streptococcus lacticus. The secret of the regularity with which some of these bacteria are present in sour milk is probably found in the ability of these varieties to withstand a much higher degree of acidity of the culture medium than other species.

1 Heinemann, Jour. of Inf. Dis., 3, 1906. 
In consequence, they are able to persist and develop when cultural conditions are absolutely unsuited to other bacteria.

Consequent upon acidification of the milk by lactic-acid formation, there is coagulation of casein. Casein precipitation, however, may also be due to a non-acid coagulation caused by a bacterial ferment. Casein precipitated in this way may be redissolved by a bacterial trypsin or casease, produced by the same or other bacteria, the milk again becoming entirely liquid, transparent, and of a yellowish color. The casein precipitated by lactic-acid formation, however, is never thus redissolved, because the high acidity does not permit the proteolytic ferments to act. ${ }^{1}$

Butyric-acid fermentation in milk, a common phenomenon, is also an evidence of bacterial growth. As a rule, it is produced by the anaërobic bacteria, and is a process developing much more slowly than other fermentations. A large number of bacteria have been described which are capable of producing such changes, the chemical process by which they are produced being, as yet, not entirely understood. It is probable that the process takes place after hydrolysis of the disaccharid somewhat according to the following formula:

$$
\mathrm{C}_{6} \mathrm{H}_{12} \mathrm{O}_{6}=\mathrm{C}_{4} \mathrm{H}_{8} \mathrm{O}_{2}+2 \mathrm{CO}_{2}+2 \mathrm{H}_{2} \text {. }
$$

Special bacteria have been described in connection with this form of milk fermentation, ${ }^{2}$ most of them non-pathogenic. It is unquestionable, however, that many of the well-known pathogenic bacteria, such as Bacillus aërogenes capsulatus, Bacillus cedematis maligni, possess the power of similar butyric-acid formation. While less commonly observed in milk, because milk is rarely kept long enough to permit of the action or development of these enzymes, the butyric-acid fermentation is of importance in connection with butter, where it is one of the causes producing rancidity.

Alcoholic fermentation may take place in milk as a result of the activities of certain yeasts. Upon the occurrence of such fermentations depends the production of kefyr, koumys, and other beverages which have been in common use for many years, especially in the region of the Caucasus. The characteristic quality of these beverages is contributed by the feeble alcoholic fermentation produced by members of the saccharomyces group, but side by side with this process lactic-acid forma-

${ }^{1}$ Conn, Exper. Stat. Rep., 1892.

2 Schattenfroh und Grasberger, Arch. f. Hyg., 37, 1900. 
tion also takes place. Beijerinck, ${ }^{1}$ who has carefully studied the socalled kefyr seeds, used for the production of kefyr in the East, has isolated from them a form of yeast similar in many respects to the ordinary beer yeast, and a large bacillus to which he attributes the lactic-acid formation.

Occasional but uncommon changes which occur in milk lead to the formation of the so-called "slimy milk," yellow and green milk, and bitter milk. These may be due to a number of bacteria. A microorganism which is commonly found in connection with the slimy changes in milk is the so-called Bacillus lactis viscosus. According to the researches of Ward, ${ }^{2}$ this microorganism is frequently derived from water and it is the water supply which should attract attention whenever such trouble occurs in dairies.

The so-called blue, green, and yellow changes are usually due to chromogenic bacteria, such as Bacillus cyanogenes, Bacillus prodigiosus, and others.

"Bitter milk," a condition which has occasionally been observed epidemically, is also the consequence of the growth of microorganisms. Conn, ${ }^{3}$ in 1891, isolated from a specimen of bitter cream a diplococcus which occasionally forms chains and which in sterilized milk develops rapidly, producing an extremely bitter taste. The organism of Conn differs from a similar diplococcus described by Wagmann ${ }^{4}$ in that it possesses the ability of producing butyric acid.

Milk in Relation to Infectious Disease.-As a source of direct infection, milk is second only to water, and deserves close hygienic attention. A large number of infectious diseases have been traced to milk, although the actual proof of the etiological part played by it in such cases has often been difficult to adduce and has necessarily been indirect. Nevertheless, even when indirect proof only has been brought, it has been sufficiently convincing to necessitate the most careful investigation into milk supplies whenever epidemics of certain infectious maladies occur.

Typhoid-fever epidemics have been frequently traced to milk infection, and, in this disease, milk is, next to water, the most frequent etiological factor. Schüder, ${ }^{5}$ in an analysis of six hundred and fifty typhoid epidemics, found four hundred and sixty-two attributed to

1 Beijerinck, Cent. f. Bakt., vi, 1889.

2 Ward, Bull. 165, Cornell Univ. Agri. Exp. Stat., 1899.

${ }^{3}$ Conn, Cent. f. Bakt., ix, 1891.

Wagmann, Milchztg., 1890.

schüder, Zeit. f. Hyg., xxxviii, 1901. 
water, one hundred and ten to milk, and seventy-eight to all other causes.

Trask ${ }^{1}$ compiled statistics of one hundred and seventy-nine typhoid epidemics supposed to have been caused by milk, in various parts of the world. In all such epidemics the origin of infection was generally traceable to diseased or convalescent persons employed in dairies, to contaminated well water used in washing milk utensils, or to the use of cans and bottles returned from dwellings where typhoid fever had existed. Actual bacteriological proof of the infectiousness of milk by the isolation of Bacillus typhosus is rare, but has been accomplished in isolated instances. In the case of one epidemic, Conradi ${ }^{2}$ isolated the bacillus from the milk on sale at a bakery at which a large number of the infected individuals had purchased their milk. The examination of market milk at Chicago, through a period of eight years, revealed the presence of typhoid bacilli but three times.

In spite of the few cases in which actual bacteriological proof has been brought, it is not unlikely that careful and systematic researches would reveal a far greater number, since many writers have shown that typhoid bacilli may remain alive in raw milk for as long as thirty days, ${ }^{3}$ and may actively proliferate in the milk during this time. One peculiarity of epidemics which may aid in arousing the suspicion that they have originated in milk is that, in such cases, women and children are far more frequently attacked than men. ${ }^{4}$

A feature which adds considerably to the dangers of milk infection is the unfortunate absence of any gross changes, such as coagulation, by the growth of typhoid bacilli.

Scarlet fever, ${ }^{5}$ though as yet of unknown etiology, has in many cases been traced indirectly to milk infection. Trask has collected fifty-one epidemics of scarlet fever presumably due to milk. In one epidemic occurring in Norwalk, Conn. ${ }^{6}$ twenty-nine cases were distributed among twenty-five families living in twenty-four different houses. The individuals affected did not attend the same school, and were of entirely different social standing, the only factor common to all of them being the milk supply.

\footnotetext{
1 Trask, Bull. No. 41, U. S. Pub. Health and Mar. Hosp. Serv., Wash.

${ }^{2}$ Conradi, Cent. f. Bakt., I, xl, 1905.

${ }^{3}$ Heim, Arb. a. d. kais. Gesundheitsamt, v.

+Wilckens, Zeit. f. Hyg., xxvii, 1898.

- Trask, loc. cit.

- IVerbert E. Smith, Rep. Conn. State Bd. of Health, 1897.
} 
Diphtheria has been frequently traced to the use of infected milk. In most of the epidemics reported as originating in this way, the proof has been necessarily indirect. In two out of twenty-three epidemics reported by Trask, however, Bacillus diphtheriæ was isolated from the milk directly. 'The ability of the Klebs-Ioeffler bacillus to proliferate and remain alive for a long while in raw milk has been demonstrated by Eyre ${ }^{1}$ and others.

Whether or not cholera asiatica may be transmitted by means of milk has been a disputed question. Hesse ${ }^{2}$ claims that cholera spirilla die out in raw milk within twelve hours. This statement, however, has not heen borne out by other observers. ${ }^{3}$ Unquestionable cases of direct transmission of cholera by means of milk have been reported by a number of writers, notably by Simpson. ${ }^{4}$

The relation of milk to the diarrheal diseases of infants has, of late years, received a great deal of attention. In large cities, during the summer months, numerous cases of infantile diarrhea among bottlefed babies occur, which, in many instances, are attributed to feeding with contaminated milk. Park and Holt, ${ }^{5}$ who have made extensive researches upon this question in New York City, have come to the conclusion that the harmful effects of contaminated milk upon babies can not be ascribed to any given single microorganism in the milk. Specifically toxic properties were found by these writers for none of the one hundred and thirty-nine different species of bacteria isolated from unsterilized milk. It is unlikely, therefore, that the diarrheal diseases among babies have a uniform bacteriological cause. Whether or not these diarrheal conditions depend entirely upon the bacterial contents of milk or, in a large number of cases at least, upon the inability of the child to digest cows' milk because of chemical conditions, must be left undecided. Park and Holt, in analyzing their extensive data, conclude that milk containing "over one million bacteria to the cubic centimeter is certainly harmful to the average infant."

The significance of the presence of streptococci in milk, as an element of danger, has recently received much attention in the literature. Heinemann, ${ }^{6}$ who has made a careful comparison of Streptococcus lacticus

1 Eyre, Brit. Med. Jour., 1899.

2 Hesse, Zeit. f. Hyg., xvii, 1894.

B Basenau, Arch. f. Hyg., xxiii, 1895.

- Simpson, Indian Med. Gaz., 1887.

- Park and Holt, Arch. of Ped., Dec., 1903.

- Heinemann, Jour. Inf. Dis., 3, 1906. 
(formerly spoken of as Bacillus acidi lactici [Kruse]), with other streptococci, has shown that, essentially, this streptococcus does not differ from streptococci from other sources, and is practically indistinguishable by cultural methods from Streptococcus pyogenes. Similar comparisons made by Schottmüller, ${ }^{1}$ Müller, ${ }^{2}$ and others have led to like results. Since streptococci may be found in milk from perfectly normal cows and are almost regularly associated with lactic-acid fermentation, it is unlikely that these microorganisms hold ordinarily any specific relationship to disease.

Recently, however, a number of epidemics of sore throat caused by streptococci have been traced to milk upon reasonably reliable evidence. Accounts of such epidemics in Chicago and in Baltimore have been published by Capps and Miller ${ }^{3}$ and by Hamburger. ${ }^{4}$

The presence of pus cells and leucocytes in milk, together with streptococci, was also formerly regarded as of great importance. Enumerations of leucocytes in milk were first made by Stokes and Weggefarth. ${ }^{5}$ Their method of enumeration consisted in centrifugalizing a definite volume of milk, spreading the entire sediment over a definite area on a slide, and counting the leucocytes found in a number of fields. Calculations from this may then be made as to the number of leucocytes per cubic centimeter. This method, and modifications of it, have been used by a large number of observers, but the value of the conclusions drawn from them has been much exaggerated. Normal milk may contain leucocytes in moderate numbers, and importance may be attached to such leucocyte counts only when their number largely exceeds that present in other specimens of perfectly normal milk. Whenever such high leucocyte counts are found, of course, a careful veterinary inspection and examination for pyogenic disease should be made.

Foot-and-mouth disease, an infectious condition prevailing among cattle, characterized by a vesicular rash on the mouth and about the hoofs, has, in a number of cases, been definitely shown to be transmitted to man through the agency of milk. Notter and Firth ${ }^{6}$ reported an epidemic occurring among persons supplied with milk from a single dairy in which foot-and-mouth disease prevailed among the cows. In this

${ }^{1}$ Schottmüller, Münch. med. Woch., 1903.

${ }^{2}$ Müller, Arch. f. Hyg., lvi, 1906.

${ }^{3}$ Capps and Miller, Jour. A. M. A., June, 1912, p. 1848.

${ }^{4}$ Hamburger, Bull. of the Johns Hopk. Hosp., xxiv, Jan., 1913.

5 Stokes and Weggefarth, Med. News, 91, 1897.

"Notter and Firth, quoted from Harrington, "Theory and Practice of Hygiene." 
epidemic, two hundred and five individuals were affected with vesiclar eruptions of the throat, with tonsillitis and swellings of the cervical lymph nodes. Similar cases have been reported by Pott. ${ }^{1}$

Although anthrax has never been definitely shown to have been conveyed by milk, Boschetti ${ }^{2}$ succeeded in isolating living anthrax bacilli from a sample of milk two weeks after its withdrawal from the cow.

Milk and Tuberculosis.-The question of the conveyance of tuberculosis by means of milk is a subject which, because of its great importance, has been extensively investigated by bacteriologists. A large number of observers have succeeded in proving the presence of tubercle bacilli in the milk of tuberculous cows by intraperitoneal inoculation of rabbits and guinea-pigs with samples of milk. Such positive results have been obtained by Bang, ${ }^{3}$ Hirschberger, ${ }^{4}$ Ernst, ${ }^{5}$ and many others. A number of these observers, notably Ernst, have shown that tubercle bacilli may be present in the milk without tuberculous disease of the udders. In an examination of the milk supply of Washington, D. C. ${ }^{6} 6.72$ per cent of the samples contained tubercle bacilli.

The path of entrance of the bacilli from the cow into the milk has long been a subject of controversy. That the bacilli may easily enter the milk, when tuberculous disease of the udder is present, stands to reason and is universally conceded. It is now believed also, on the basis of much experimentation, that in systemically infected cows tubercle bacilli may pass through the mammary glands into the milk, without evidence of local disease in the secreting gland. An experiment performed by the Royal British Tuberculosis Commission ${ }^{7}$ illustrates this point. A cow, injected subcutaneously with tubercle bacilli behind the shoulder, began to discharge tubercle bacilli in the milk within seven days after inoculation and continued to do so until death from generalized tuberculosis.

Milk may become indirectly contaminated, furthermore, with tubercle bacilli emanating from the feces of cows. It has been shown that tubercle bacilli are present in the feces of cattle so early in the disease that diagnosis can be made only by a tuberculin test. ${ }^{8}$

Whether or not contaminated milk is common as an etiological

${ }^{1}$ Pott, Münch. med. Woch., 1899.

${ }^{2}$ Boschetti, Giorn. med. vet., 1891.

${ }^{3}$ Bang, Deut. Zeit. f. Tierchem., xi, 1884.

${ }^{4}$ Hirschberger, Deut. Arch. f. klin. Med., xliv, 1889.

${ }^{5}$ Ernst, H. C., Amer. Jour. Med. Sci., xcviii, 1890.

${ }^{6}$ Anderson, Bull. No. 41, U. S. Pub. Health and Mar. Hosp. Serv., Wash., 1908.

${ }^{7}$ Quoted from Mohler, P. H., and Mar. Hosp. Serv. Bull. 41, 1908.

${ }^{8}$ Schroeder and Cotton, Bull. Bureau Animal Industry, Wash., 1907. 
factor in human tuberculosis, must be considered at present as an unsettled question. Behring, at the Congress of Veterinary Medicine, at Cassel, in 1903, advanced the view that pulmonary tuberculosis in adults may be a late manifestation of a milk infection contracted during infancy. He stated as his own opinion, moreover, that most cases of tuberculosis in man are traceable to this origin. The problem is as difficult of solution as it is important. In bottle-fed infants, infection by means of milk unquestionably occurs with considerable frequency. Smith, ${ }^{1}$ Kossel, Weber, and Huess, ${ }^{2}$ and others, have isolated tubercle bacilli of the bovine type from the mesenteric lymph nodes of many infected children. Animal experimentation has, furthermore, revealed that lesions in the mesenteric nodes, as well as later in the bronchial lymph nodes, may occur as a consequence of feeding tubercle bacilli, without any demonstrable lesions in the intestinal mucosa. It is thus certain that infection by the ingestion of tuberculous milk may occur, especially among young children who, as is well-known, are comparatively susceptible to bacilli of the bovine type. Whether or not such infection will account for many cases of tuberculosis in adults is a question which, for final solution, will require much more investigation. The sole reliable method of approaching it lies in determining the type, human or bovine, of the bacilli present in a large number of cases. Experience thus far seems to indicate that the bovine type is comparatively rare in the pulmonary disease of adults.

The value of the tuberculin reaction for diagnosis, and the elimination of all cattle showing a positive reaction, for the prevention of tuberculosis, can not be overestimated. The failure of the test in diseased animals is rare, and an accurate diagnosis can be established in over 90 per cent of diseased animals. ${ }^{3}$ The assertion that the cattle are permanently injured by tuberculin injections is without scientific basis. If this test were conscientiously carried out, and infected cattle eliminated, the dangers from bovine bacillus infection would be practically eliminated, for there are but few instances in which science has been able to furnish such definite information for absolute protection. It is needless to say, however, that the carrying out of such precautions is subject to great expense and great difficulties of organization.

Dairy inspection is practiced in the vicinity of many of our larger

1 Smith, Trans. Assn. Amer. Physic., 18, 1903.

${ }^{2}$ Kossel, Weber, and Huess, Tuberkul. Arb. a. d. kais. Gesundheitsamt, 1904, 1905, Hft. 1 and 3.

${ }^{3}$ Mohler, loc. cit. 
cities, and the movement is daily gaining ground. Until fully established, however, upon a financial basis which brings the best products within the means of the poorer classes, other inexpensive measures to render milk safe must often be resorted to.

Sterilization by high temperatures is objected to by pediatricians because of the physical and chemical changes produced in the milk which are said to detract from its nutritive value.

The development of scurvy and rickets in infants has often been attributed to the use of such milk. These objections, however, do not apply to the use of milk which has been subjected to the process of "pasteurization." By this term is meant the heating of any substance to $60^{\circ} \mathrm{C}$. for twenty to thirty minutes. The process, first devised by Pasteur for the purpose of destroying germs in wine and beer in which excessive heating was supposed to injure flavor, brings about the death of all microorganisms which do not form spores-in other words, of all the bacteria likely to be found in milk which can give rise to infection per os. At the same time the chemical and physical constitution of the milk is not appreciably changed, at least not to an extent which renders it less valuable as a food. Statistics by Park and Holt ${ }^{1}$ have shown strikingly the advantages of pasteurized over raw milk in infant feeding. Of fifty-one children fed with raw milk during the summer months, thirty-three had diarrhea, two died, and only seventeen remained entirely well. Of forty-one receiving pasteurized milk, but ten had diarrhea, one died, and thirty-one remained entirely well throughout the summer. The actual diminution of the living bacterial contents of milk by pasteurization is enormous, the milk so treated often containing not more than one thousand, usually less than fifteen thousand, living bacteria to each cubic centimeter.

Methods of Estimating the Number of Bacteria in Milk.-In estimating the number of bacteria in milk, colony counting in agar or gelatin plates is resorted to. Great care must be exercised in obtaining the specimens. If taken from a can, the contents of the can should be thoroughly mixed, since the cream usually contains many more bacteria than the rest of the milk. The specimen is then taken into a sterile test tube or flask. If the milk is supplied in an ordinary milk bottle, this should be thoroughly shaken before being opened, and the specimen for examination taken out with a sterile pipette. Dilutions of the specimen can then be made in sterile broth or salt solution. If an initial dilution

1 Park and Holt, loc. cit. 
of $1: 100$ is made, quantities ranging from 1 c.c. to 0.1 c.c. of this will furnish 0.01 c.c. to 0.001 c.c. of the milk, respectively. Inoculation of properly cooled tubes of melted neutral agar and gelatin, with varying quantities of these dilutions, are then made and plates poured. After twenty-four to forty-eight hours at room temperature or in the incubator, colony counting is done as described upon page 161, and the proper calculation is made. In samples in which few bacteria are expected, direct transference of $1 / 20$ or $1 / 40$ of a c.c. of the whole milk into the agar may be made. This saves time but is less accurate than the method given above.

Bacteria and Butter.-Butter is made from cream separated from milk either by standing or by centrifugalization. After this, the cream is agitated by churning, which brings the small fat-globules into mutual contact, allows them to adhere to each other and form elumps of butter. It has been a matter of common experience, however, that unless the cream is allowed to "ripen" for a considerable period before churning, the resulting butter lacks the particular quality of flavor which gives it its market value. The interval of ripening, at first a necessity upon. small farms where cream must be collected and allowed to accumulate, has now been recognized as an essential for the production of the best grades of butter, and it has been shown that the changes taking place in the cream during this period are referable to the action of bacteria. Cream, which before the ripening process contains but 50,000 bacteria to each cubic centimeter, at the end of a period of "ripening" will often contain many millions of microorganisms. At the same time, the cream becomes thick and often sour.

The species of bacteria which take part in this process and which, therefore, must determine to a large extent the quality of the end product, are various and, as yet, incompletely known. Usually some variety of lactic-acid bacilli is present and these, as in milk, outgrow other species and, according to Conn, ${ }^{1}$ are probably essential for "ripening."

It would be of great practical value, therefore, if definite pure cultures of the bacteria which favor the production of agreeable flavors could be distributed among dairies. In Denmark this has been attempted by first pasteurizing the cream and then adding a culture of bacteria isolated from "favorable" cream. These cultures, delivered to the dairyman, are planted in sterilized milk, in order to increase their quantity, and this culture is then poured into the pasteurized cream. In

'Conn, "Agricultural Bacterinlogy," Phila., 1901. 
most cases, these so-called "starters" are not pure cultures, but mixtures of three or more species derived from the original cream.

Adverse accidents in the course of butter-making, such as "souring" or "bittering" of butter, are due to the presence of contaminating, probably proteolytic, microorganisms in the cream during the process of "ripening."

As a means of transmitting infectious disease, butter is of importance only in relation to tuberculosis. Obermüller, ${ }^{1}$ Rabinowitch, ${ }^{2}$ Boyce, ${ }^{3}$ and others, have repeatedly found tubercle bacilli in market butter, and Mohler, ${ }^{4}$ Washburn, and Rogers have recently shown that these bacilli could remain alive and virulent for as long as five months in butter kept at refrigerator temperature. The acid-fast butter bacillus, described by Rabinowitch as similar to the true Bacillus tuberculosis, shows decided cultural and morphological differences from the latter.

Bacteria and Cheese.-The conversion of milk products into cheese consists in a process of proteid decomposition which, by its end products, leucin, tyrosin, and ammonia compounds, largely determines the cheeseflavors. The production of cheese, therefore, is due to the action of proteolytic bacterial enzymes ${ }^{5}$ and the variety of a cheese is largely determined by the microorganisms which are present and by the cultural conditions prevailing. The sterilization of cream, or the addition of antiseptics, absolutely prevents cheese production.

The organisms which are concerned in such processes have been extensively studied and attempts have been made, with moderate success, to produce a definite flavor with pure cultures.

In the production of cheese the two varieties, hard and soft cheeses, depend not so much upon the bacterial varieties as upon the differences in the treatment of the curds before bacterial action has begun. In the former case, a complete freeing of the curds from the whey furnishes a culture medium which is comparatively dry and of almost exclusively proteid composition; in the latter, retention of whey gives rise to cultural conditions in which more rapid and complete bacterial action may take place. The holes, which are so often observed in some of the hard cheeses, are due to gas production during the process of "ripening."

1 Obermüller, Hyg. Rundschau, 14, 1897.

${ }_{2}$ Rabinowitch, Zeit. f. Hyg., xxvi, 1897.

3 Boyce and Woodhead, Brit. Med. Jour., 2, 1897.

4 Mohler, U. S. P. H. and Mar. Hosp. Serv. Bull. 41, 1908.

s Freudenreich, Koch's Jahresbericht, etc., 135, 1891. 
As to the varieties of microorganisms present in various cheeses, much careful work has been done. Duclaux ${ }^{1}$ attributed the "ripening" of some of the soft cheeses to a microorganism closely related to Bacillus subtilis. V. Freudenreich ${ }^{2}$ in part substantiated this, but laid particular stress upon the action of Oidlium lactis, a mold, and upon several varieties of yeast. Conn, ${ }^{3}$ more recently, in a bacteriological study of Camembert cheese, has demonstrated that the production of this cheesi depends upon the united action of two microorganisms, one an oïdium, like the Oïdium lactis of Freudenreich, which is found chiefly in the interior softened areas, the other a mold belonging to the penicillium variety, found in a matted felt-work over the surface and penetrating but a short distance. In spite of the scientific basis upon which the work of these men and of others has seemed to place cheese production, attempts at uniformity in cheese production have met with almost insuperable obstacles because of the presence of a variety of adventitious microorganisms which, depending in species and proportion upon the local conditions under which the various cheeses have been produced, have added minor characteristics of flavor which have determined market value. Occasional failure of good results in cheese production ${ }^{4}$ is due to contamination with other chromogenic or putrefactive bacteria.

In its relationship to the spread of infectious disease, cheese is relatively unimportant except in regard to tuberculosis. Typhoid and other non-spore forming pathogenic germs can not survive the conditions existing during cheese-ripening for any length of time. Tubercle bacilli, both of the human and bovine types, have been found in cheese by Harrison ${ }^{5}$ and others, and Galtier has shown experimentally that tubercle bacilli may remain alive and virulent in both salted and unsalted cheese for as long as ten days.

\section{THE LACTIC-ACID BACILLI AND METCHNIKOFF'S BACTERIO- THERAPY}

A problem which has occupied clinical investigation for many years is that of gastrointestinal autointoxication. There are a number of conditions occurring in man, in which symptoms profoundly affecting

1 Duclaux, "Le Lait," Paris, 1887.

${ }^{2}$ V. Freudenreich, Cent. f. Bakt., II, i, 1895.

:Conn, Bull. Statis. Agri. Exp. Stat. 35, 1905.

4 Beijerinck, Koch's Jahresber, etc., 82, 189.

5 ILarrison and Galtier, quoted trom Mohler, U. S. Pub. H. and Mar. IIosp. Serv., II ygiene Lab. Bull. 41, 1908. 
the nervous system, the circulation, and, in a variety of ways, the entire body, can be clinically traced to the intestines, and can, in many cases, be relieved by thorough purgation and careful dict. In some of these conditions, specific microorganisms can be held accountable for the diseases (B. enteritidis, B. botulinus, etc.). In other cases, however, etiological investigations have met with but partial success because of the large variety of nicroorganisms present in the intestinal tract and because of the conplicated symbiotic conditions thereby produced. Intestinal putrefaction, recognized as the cardinal feature of such maladies, has been attributed to Bacillus proteus vulgaris, ${ }^{1}$ to Bacillus aërogenes capsulatus, to Bacillus putrificus, ${ }^{2}$ and to a number of other bactcria, but definite and satisfactory proof as to the etiological importance of any of these germs has not yet been advanced. The fact remains, however, that, whatever may be the specific cause, the disease itself, a grave and often fatal affliction, nay be clinically traced, in a number of cases, to the absorption of poisons from the intestinal canal, and it is more than likely that these poisons are the products of bacterial activity. Reason dictates, furthermore, that the bacteria primarily responsible for the production of these toxic substances do not beiong to the varieties which attack carbohydrates only, but must belong to that class of aërobic and anaërobic germs which possess the power of breaking up proteids - in other words, the bacteria of putrefaction.

On the basis of the mutual antagonism existing in culture between many acid-producing bacteria and those of putrefaction - a phenomenon recognized by some of the earliest workers in this field, many investigators have suggested the possibility of combating intestinal putrefaction by adding acid-forming bacteria together with carbohydrates to the diet of patients suffering from this condition. The first to suggest this therapy was Escherich ${ }^{3}$ who proposed the use, in this way, of Bacillus lactis aërogenes; with the same end in view, Quincke, ${ }^{4}$ a little later, suggested the use of yeasts - Oïdium lactis. The reasoning underlying these attempts was meanwhile upheld by experiments carried out both in vitro and upon the living patient. Thus Brudzinski ${ }^{5}$ was able to demonstrate that Bacillus lactis aërogenes, in culture, inhibited the development of certain races of the proteus species and succeeded in

1 Lesage, Rev. de méd., 1887.

2 T'issier, Ann, de l'inst. Pasteur, 1905.

3 Escherich, 'Therapeut. Monatshefte., Oct., 1887.

4 Quincke, Verhandl. des Congress f. Inn. Med., Wiesbaden, 1898.

; Brudzinski, Jahrbuch f. Kinderheilkunde, 52, 1900 (Ergänzungsheft). 
obtaining markedly favorable results by feeding pure cultures of Bacillus lactis aërogenes to infants suffering from fetid diarrhea. Similar experiments ${ }^{1}$ carried out with the Welch bacillus (aërogenes capsulatus) and Bacillus coli, however, had no such corroboratory results, since this anaërobe possesses a considerable resistance against an acid reaction. In considering the difficulties of the problems involved in this question,

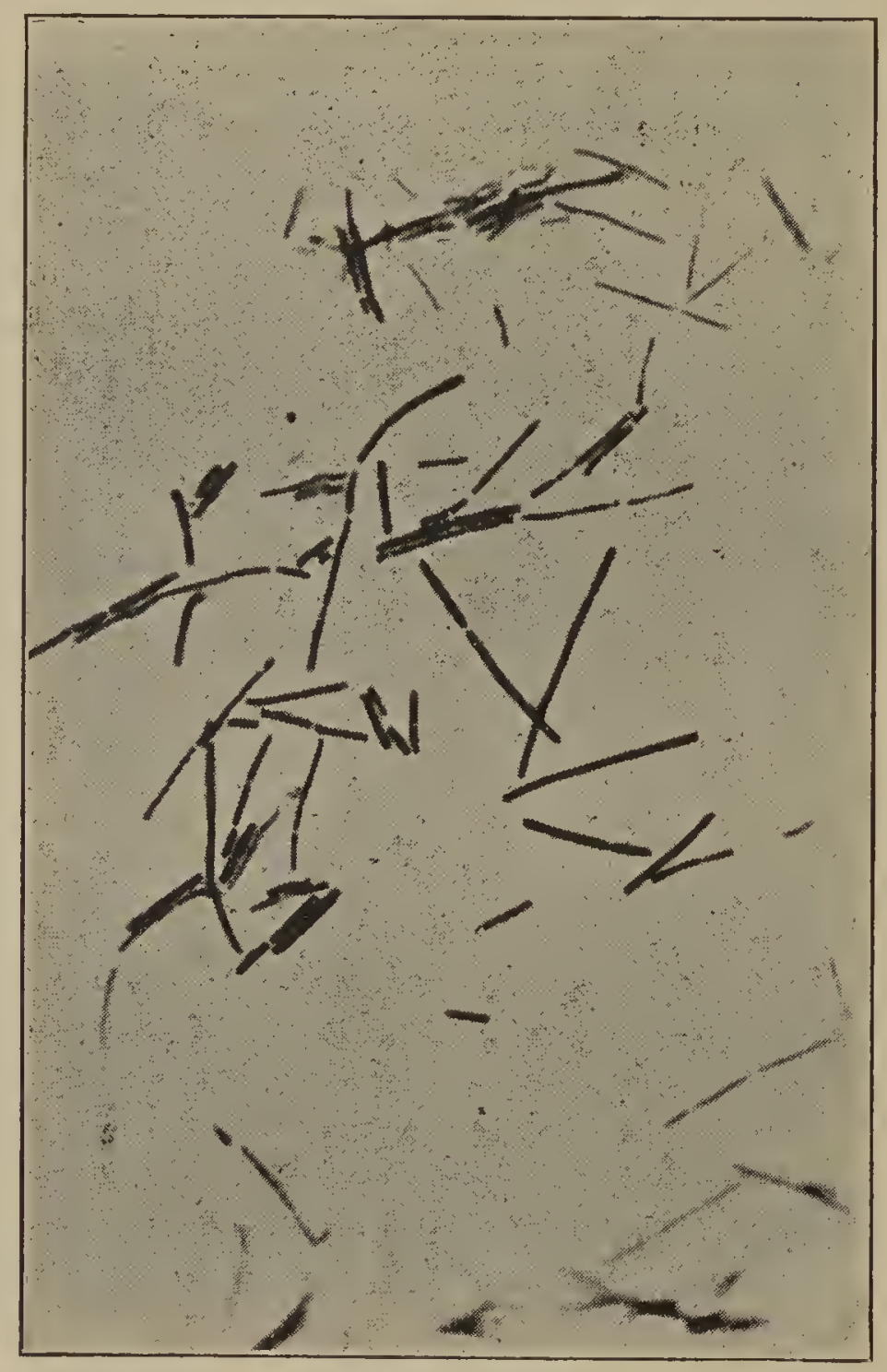

Fig. 156.-BaCillus BULGaricus.

it occurred to Metchnikoff ${ }^{2}$ that much of the practical failure of therapy, based upon the principles stated above, might be referred to insufficient powers of acid production on the part of Bacillus coli, Bacillus lactis aërogenes, and other germs previously used. In searching for more powerful acid producers, his attention was attracted to Bacillus bulgari-

1 Tissier and Martelly, Ann. de l'inst. Pasteur, 1906.

2 Metchnikoff, "Prolongation of Life," G. P. Putnam's Sons, N. Y.; also in "Bactériothérapie," etc. "Bibliothèque de thérapeutique," Gilbert and Carnot, Paris, 1909. 
cus, isolated from milk by Massol ${ }^{1}$ and Cohendy ${ }^{2}$ in 1905 . This bacillus, according to the researches of Bertrand and Weisweiller, ${ }^{3}$ produces as much as 25 grams of lactic acid per liter of milk. In addition to this, it manufactures, from the same quantity of milk, about 50 centigrams of acetic and succinic acids and exerts no putrefactive action upon proteids. Added to these characters, it is especially adapted to therapeutic application by its complete lack of pathogenicity.

The administration of the bacillus to patients suffering from intestinal putrefaction, first suggested by Metchnikoff in 1906, has, since that time, been extensively practiced and often with remarkable success. In spite of sharp criticism, especially by Luersen and Kühn, ${ }^{4}$ who deny much of the antiputrefactive activity of the bacillus, the treatment of Metchnikoff has found many adherents, upon the basis of purely clinical experiment. It is not possible to review completely the already extensive literature. Among the more valuable contributions may be mentioned the articles by Grekoff, ${ }^{5}$ by Wegele, ${ }^{6}$ and by Klotz. ${ }^{7}$ In Metchnikoff's experiments and in the work of his immediate successors, the bacillus was used either in milk culture or in broth in which it was induced to grow in symbiosis with other microorganisms.

Recently, North ${ }^{8}$ has suggested the use of Bacillus bulgaricus in parts of the body other than the digestive tract. His work was made feasible by the discovery that the bacillus could be cultivated in dextrose-pepton broth to which calcium carbonate has been added, after the manner recommended by Hiss. With such cultures, applied in the form of a spray, inflammations of the ear, nose, throat, genitourinary tract, etc., have been treated, many of them with success.

\section{BACTERIA IN THE INDUSTRIES}

Bacteria and Tobacco.- In the manufacture of tobacco, the harvested leaves are first dried and then heaped up in large masses in which the tobacco undergoes fermentation. During this fermentation, which

1 Massol, Revue médicale de la Suisse romande, 1905.

${ }^{2}$ Cohendy, Comptes rend. de la soc. de biol., 60, 1906.

3 Bertrand and Weisweiller, Ann. de l'inst. Pasteur, 1906.

${ }^{4}$ Luersen and Kühn, Cent. f. Bakt., II, xx, 1908.

${ }^{5}$ Grekoff, "Observations cliniques sur l'effet du lact. agri.," etc., St. Petersburg, 1907.

- Wegele, Deut. med. Woch., xxxiv, 1908.

7 Klotz, Zentralbl. f. innere Med., 1908.

8 North, Med. Record, March, 1909. 
goes on at temperatures varying from $50^{\circ} \mathrm{C}$. to $60^{\circ} \mathrm{C}$., carbohydrates are split up and much nicotin is destroyed. ${ }^{1}$ The end products consist largely of $\mathrm{CO}_{2}$ and various organic acids, butyric, formic, succinic, etc. During the fermentation, bacteria of many varieties are found in the heaps of tobacco leaves and many attempts have been made to determine flavors artificially by inoculating tobacco leaves of a poorer quality with cultures obtained from the finer Havana grades. Suchsland ${ }^{2}$ and others, who have attempted this, claim to have obtained marked improvements in domestic products by this method. The bacteria found in tobacco fermentation belong to many varieties. Some of these are closely related to the proteus and subtilis groups. Others are distinctly thermophilic, an attribute required by the high temperatures attained in the fermenting tobacco leaves. It is probable that the tobacco flavors can not be regulated by bacteriological methods alone, since it has been shown by Loew ${ }^{3}$ that an important factor in the tobacco fermentation is contributed by the leaf-enzymes, which, of course, depend intimately upon soil and climatic conditions.

Opium Productions.-In the preparation of opium for smoking purposes, the raw product is subjected to a prolonged period of fermentation by which the carbohydrates in the material are destroyed. According to various observers, the process is carried out in most cases by a species of aspergillus.

Indigo Production.-Indigo, which is obtained from the plants "Isatis tinctoria" and "Indigofer tinctoria," is not present, as such, in the plants. In some of these it is found in the form of indican, in others, as indoxyl. It has been shown by Alvarez and others that the oxidation of indican and indoxyl into indigo-blue is carried out largely by bacterial oxydases. Sterilized indigo plants do not produce the blue color. Alvarez ${ }^{4}$ has isolated a bacillus closely related to the Bacillus mucosus capsulatus group, to the action of which he attributes this oxidation.

Bacteria in the Tanning of Hides.-Raw animal hides are subject to decomposition until treated by a process known as tanning. This consists first in the depilation of the dried and salted skins, either by partial putrefaction in an atmosphere saturated with water vapor or by chemical treatment with solutions of milk of lime. After this, the

Behrens, quoted from Flügge, "Die Mikroorganismen," Bd. 1, Leipzig, 1896.

2 Suchsland, Ber. der Deut. botan. Ges., ix.

${ }^{3}$ Loew, Rep. U. S. Dep. Agriculture, 59, 1899.

- Alvarez, Comptes rend, de l'acad. des sci. vol. 105. 
tanning proper consists in subjecting the skins to prolonged immersion in solutions made up according to a large variety of formulæ- the principle of all of which, however, seems to be found in the mixing of various organic ingredients, such as bran-mash, oak-bark, and often dried animal excreta, in which fermentation and acid production occurs. According to Haenlein ${ }^{1}$ this acidification is the essential process by which the leather is sterilized and rendered soft and pliable. This author has described a microorganism, Bacillus corticalis, which he found regularly present in fir-tree bark and to which he ascribes the acid fermentation occurring in tanning liquors in which this ingredient is employed. Wood, ${ }^{2}$ who has worked extensively upon the subject, has attempted with some success to substitute pure cultures for the old uncertain chance mixtures employed. In spite of these investigations, however, while we must acknowledge the probable importance of bacteria in the tanning process, the subject is by no means upon a scientific or exact basis.

1 Haenlein, Cent. f. Bakt. II, i, 1895.

2 Wood, Jour. Soc. Chem. Industry, 1895, 1899. 


$$
=
$$




\section{INDEX OF AUTHORS}

Аввотт, 97, 326

Abel, 189, 450, 452, 557

Abel and Claudssen, 585

ACHARd ANd Bensaude, 430

ADAMI, 392

Adami and Chapin, 696

Agramonte, 669, 670

Albrecht ANd Ghon, 376, 377, 378, 555,561

Allegri, 502

Alva Rez, 716

Alvarez and Tavel, 503

Anderson, J. G., 707

Anderson and Goldberger, 677, 678

Anderson and McClintic, 80

Andrewes And Horder, 349

Aristotle, 2

Arloing, 63

Arloing and Chauveau, 471

Arloing, Cornevin and Thomas, 468

Arloing, Leclainche, and Vallée, 468

Arning, 509

Aronson, 91, 346, 347, 348, 350

Arruenius and Madsen, 211

Arthus, 297

Arustamoff, 619

Asakawa, 214

Ascoli AND Figari, 201

Axenfeld, 546

BABES, $11,510,514,550,613,614,623$, 648

BABES AND LEPP, 197

Baginsky, 343, 460, 519

Baginsky and Sommerfeld; 343, 344, 676

BAIL, 292, 293, 330

Bail and Petterson, 292

BAIL AND WEIL, 293

BALDWIN, 494

Bandelier and Roepke, 497
Bandi and Simonelli, 596

BANG, 707

BANZHAF, 219, 220

Barker aNd Cole, 424

Basenau, 430, 705

BAUER, 268

Baumgarten, 106, 479, 486

BAUMler, 540

BECK, 493, 499, 514

Beck, M., Kolle, and Wassermann, 517

BeckmanN, 78

Behrens, 716

v. Behring, 76, 77, 78, 79, 195, 205, 221, $295,487,490,569$

v. Behring and Kitasato, 196, 198

v. Behring and Kitashina, 295

v. Behring and Wernicke; 196, 198

BeiJerinck, 26, 27, 703, 712

Belfanti, 458

Belfanti and Carbone, 200

BeluaefF, 198

Beraneck, 493

Berestnew, 618, 620, 626

Bertarelli, 501, 590, 602, 603

Berthelot, 54

Bertrand and Weisweiller, 715

BESREdKA, 298, 302, 345, 416

Besredka and Steinhardt, 299

Beuermann and Gougerot, 645

BEZANÇON, 334

Bezançon, Griffon, and Le Sourd, 548

Bienstock, 393, 481

Biggs and Park, 231

Billroth, 7

Binaghi, 368

Birch-Hirschfeld, 192, 392

BitTer, 31, 168, 503

Blaise and Sambac, 64

BLOODGOOD, 474

Blumer, 411

Bogart and Bernard, 201 
BoLand, 579

Bollinger, 622

Bolton, 24

BoRdet, 200, 224, 225, 228, 232, 236, $240,242,243,337,346,367,545$

Bordet and Gay, 261

Bordet and Gengou, 245, 261, 262, 543

Bordoni-UFFrEDUZZI, 359

Borissow, 328

BoRsieKow, 139

BoscheTt, 707

Bostroem, 624

Boyce ANd WoOdhead, 711

Brau and Denier, 589

Brauell, 6

Brieger, 185, 195, 415

Brieger and Boer, 462, 522

Brieger and Cohn, 461, 462

Brieger and Frankel, 521

Brieger and Kempner, 478

Brill and Libman, 579

BRodghton, 427

BRUCE, 550

BRUCK, 215

BRUdzINSKI, 713

v. BRunn, 90

Buchṇer, 22, 63, 152, 186, 198, 203, 224, $225,389,691$

Buchner and Hahn, 492

Buchner and Meisenheimer, 50

BUdD, 399

BÜDINGER, 327

Buerger, 99, 349, 351, 355

BUFFon, 4

Bullock ANd Atrin, 282, 284

Bullock and Hunter, 581

BUllock and Western, 282

BUMm, 380, 381

Bunge and Trautenroth, 106

BuRKholtz, 63

Busse, 631

BüTsCHLI, 11, 12

Buxton, 428, 429

Buxton and Coleman, 180, 431

Cagniard-Latour, 3

Calkins, 650, 658

Calmetre, 199, 203, 494
Calmette and Guerin, 660, 662

Canalis, 189

Canfora, 460

Cantacuzène, 280

Cantani, 539

Capaldi, 134

Capps and Miller, 706

Carlo and Rattrone, 456

Carnot and Fournier, 363

CARrière, 47

Carroll, 671

CARTter, 627

Castellani, 234, 405, 614

Certes, 35

Chamberland and Roux, 194, 569, 571

Chantemesse, 412,424

Chantemesse and Widal, 418

Chapin, 562

Charrin, 577, 578

Charrin and Roger, 228

Chadvead, 195, 574

Christen, 67, 69

Christuas, 385

Chudiakow, 27

Churchman, 140

Churchman and Michael, 140

Citron, 273, 294

Clairmont, 449

Clatrke, 658

Class, 676

ClegGG, 506

Cobbetr, 524

Cohendy, 715

Conn, 5, 36

Cole, 366

Coleman and Buxton, 405

Coles, 504

Cons, 415, 702, 703, 710, 712

Conor, 678

Conradi, 137, 405, 443, 704

Conradi and Drigalski, 135

Conseil, 678

Copeland, 698

CoRnet, 342,487

Cornil and Babes, 669

Councilman, 658

Councilman, Mallory, and Wright, 374, 376, 377 
Courmont and Doyen, 463

Craig and Nichols, 604

Cramer, 21

Creite, 460

Crowell, 561

Curtis, 631

Cushing, 431

Cushing and Livingood, 392

D'Arsonville and Charrin, 65

Davaine, 6, 563

Davis, 343

DEAN, 283, 510

Debrand, 461

Delezenne, 201

Deneke, 591

Denys, 346, 490, 492, 497

Denys and LeClef, 281, 346

Denys and Marchand, 346

Denys and Van de Velde, 331

De Schweinitz and Dorset, 22, 490

Deslongchamps, 326

De Toma, 486

Deutsch and Feistmantel, 292

Dieudonnḱ, 64, 216, 566, 584

DobBin, 474

Dochez, 366

Doerr, 186, 299, 443

Doerr and Russ, 303

Donath and Landsteiner, 248

Dönitz, 188, 218

DOUTRELEPONT, 504

Dreyer and Madsen, 209, 215

Drigalski, 695

Drigalski and Conradi, 406

Dubarre and Terre, 501

DUClatux, 712

DUCREY, 548

v. Dungern, 201, 242, 411

Dunham, $386,472,473,584$

DURham, 428,434

Durham and Myers, 669

Dusch, 4

Dutton añd Todd, 608

Duval, 506

Duval and Wellman, 506

EBERTh, 399
Ehrenberg, 2

EhrLich, 7, 104, 187, 193, 199, 203, 204, $205,206,209,210,213,238,464,481$

Ehrlich, Kossel, and Wassermann, 205

Ehrlich and Morgenroth, 226, 241, $242,243,246$

Ehrlich ANd SAChs, 242, 243

EICHSTEDT, 639

EISENBERG, 580

Eisenberg and VolK, 420

v. Eisler and Pribram, 459

EJKMANN, 47, 49

ELSER, 377

Elser and Huntoon, 376, 378, 379, 387

ELSNER, 408

ENDO, 135

Engelmann, 27, 61

Eppinger, 573, 620

Epstein, 77, 178, 619

ERnst, 11, 579, 707

Escherich, 343, 389, 394, 453, 515, 700, 713

v. Esmarch, 68, 147, 149

Evans and RUssell, 92

Ewing, 596, 658

Eyre, 166, 550, 705

Eyre and Washburn, 360

FARNet, 413

Faure-Beaulieu, 384

Fehleisen, 336, 341, 342

Fermi and Pernossi, 462

Ferran, 28, 195, 458, 590

Ferri and Cetuli, 64

Ficker, 62, 231, 423

Field, 678

Finger, 530, 535

Finger and Landsteiner, 603

Finkelstein, 579

Finkler and Prior, 591

Finlay, 670

Firth and Horrocks, 687

Fischer and Proskauer, 88

Fisher, 9, 56, 59, 325

Fisher, A., 10, 19, 23, 24

FitzGerald, 450

Flexner, 197, 378, 413, 436, 444, 620 
Flexner and Jobling, 378

Flexner and LeW'S, 665

Flexner and Noguchi, 462

FLÜGGE, 84, 85, 198, 325, 385, 586

FOA, 360

Foa and Carbone, 362

V. Fodor, 198, 224

Foges, 460

Foote, 415

Forneaca, 334

Forster, F., 32, 586

Fracastor, 2

Fränkel, A., 353, 355, 360, 362, 404. $412,421,447,472,504,570,619,686$

FranzotT, 326

Freudenreich, 711, 712

Friedberger, 304

Friedberger and Hartoch, 303

Friedemann, 303

FriedLÄNDER, 352, 447

FriedmanN, 501

v. Frisch, 451

Frosch and Kolle, 339, 368

Fuhrmann, 44

Fuller, 692

FÜRBRINGER, 88

GaBBeT, 105, 481

GAFFKY, 333, 399, 400, 469

Gaffky, Pfeiffer, Sticker, and DieuDONNÉ, 561

Galtier, 485, 535

Gamaleia, 590

Garré, 327

Gärtner, 429

Gavino and Grrard, 677

GAY, 243, 244, 427, 446

Gay and Claypole, 405

Gay and Southard, 299, 300, 302

Gengou, 240, 244, 273

Geppert, 86

Gessard, 577, 579

Gheorghiewski, 581

Ghon and Pfeiffer, 376, 386

GiIon and Preyss, 539

Gibson, 219

Gibson and Collins, 219

Giemsa, 108
Gilbert, 430

Gilchrist, 631

Globig, 33

Goldberger, 677

GoLdHORN, 598

Goodwin and v. Sholly, 376

GoRdon, 350

Gorgas, 670

GotTsCHLich, 23, 557

Gougerot, 644

Gram, 102

Gran, 49

Grancher and Ledoux-Lebard, 485

Grassberger, 539

Grawitz, 640

GrekofF, 715

Gruber, 67, 78, 421

Gruber and Durham, 200, 228, 234

Gruby, 642

GRUND, 584

GrÜNHAGEN, 657

Guarnieri, 357, 359, 360, 657

Guerin, 662

Guiteras, 673

Gumprecht, 463

GÜNTHER, 352

GWYN, 430, 475

HaAs, 66

HAENLEIN, 717

HAFFKINE, 590

НАHN, 190, 191, 416, 492

Hallier, 7

HaMBURGER, 303

Hammarsten, 46

Hammerschlag, 22, 490

HaNel, 326

Hankin and LeUmann, 20

Hankin and Wesbrook, 168

Hansemann, 501

HaNSEN, 505, 631

Harding and Ostenberg, 136, 433

HARRINGTON, 91

Harrison, 425

Harrison and Galtier, 712

Ha RTMan, 340

Hauser, 151, 454

Heim, 704 
Herman, 381, 384

Heinemann, 701, 705

Hektoen, 283, 675

Hektoen ANd Ruediger, 282, 283

Hellimich, 22

Hellriegel and Wilfarth, 55,56

Henle, 3

HenRIJEAN, 459

Hericourt ANd Richet, 296

Herter, 177, 394, 475

Hesse, 27, 133, 485, 705

Hilbert, 31,520

HiLl, 86, 94, 694

Hirsch, 554, 587

Hirschberger, 707

Hiss, 13, 98, 132, 251, 289, 337, 338, 339, $340,347,349,351,354,355,356,357$, $358,364,365,369,407,408,411,439$, 440

Hiss and Atkinson, 198, 219

Hiss and Russele, 438

Hiss and Zinsser, 291, 366, 379

HofFman, 233

HoffMANN-WeLlenhoff, 522

HöGYES, 652, 655, 656

Holst, 430

Hoмe, 675

Hopkins and LANG, 349

Horton-SMith, 407, 411

Houston, 686, 697

Howard, 451

Howard and Perkins, 350, 369

HUBER, 538, 540

HuddLeston, 662

HuEPPE, 551

Hueppe ANd Wood, 575

Huntemüller, 691

Hunter, 198

IRONS, 698

ISAEFF, 363

ISRAEL, 622

IWANOW, 569 .

JACKSON, 138, 696, 698

JACKSON AND MELIA, 133, 138

J ̈̈̈GER, 373, 451

JENNER, 108, 193, 659
JOACHIM, 198

JoHNSTON, 404

JoNes, 75

Joos, 233

JoRdan AND Heinemann, 700

JoRdAN ANd Irons, 693

Jordan, Russell, and Zeit, 414

JoRgensen ANd MAdSEn, 233

Kamen, 394, 534

KAPPES, 21

KaRLINSKY, 411

KeLLY, 343

Kempner, 199

Kempner and Pollack, 478

Kempner and SChepilewsky, 215

KIRCHER, 1

Ḱrster and WolfF, 237

Krtasato, 456, 459, 462, 465, 555, 559

Kitasato and Weyl, 27

KitT, 468

KITT AND MaYR, 554

Klebs, 7, 352, 493, 512, 669

KLEIN, 176, 404, 424, 480

KLemperer, 503

KLemperer, G. and F., 362

KLingmüller and BaERmanN, 604

KLOTZ, 715

KNAPP, 525, 542

KNOEPFELMACHER, 665

ḰNORR, 205, 295, 340

Kobert and Stillmarck, 204

Косн, $7,63,73,77,84,86,89,321,335$, 352 , 404, 469, 479, 483, 490, 491, 492, 493, 496, 497, 498, 542, 563, 566, 569, $570,582,587,609,610,696$

Koch and Petruschky, 345

Koch and Rabinovitsch, 500

KoCh and WolfFH ÜGEL, 65,570

Koch, Gaffky, And Loeffrler, 65, 564

Kolle and Hetsch, 593

Kolle and Gtto, 331

Kolle and Wassermann, 189, 190, 191, 378, 502

KoRN, 502

KoRSCHUN, 291

KosSEL, 204, 532

Kossel ANd Overbeck, 551 
Kossel, Weber, and Heuss, 499, 500, 708

KotJAR, 628

KraUnhaLs, 569

KraUs, $186,200,235,237,328,329,423$, $443,446,589,590$

Kraus and Doerr, 441, 444

Krads and Löw, 232, 397

Kraus and v. Pirquet, 236

Kraus and Stenitzer, 416

Kresling, 532

KRETZ, 217

KRöNIG AND PAUL, 74, 77, 78, 80,

KRUMWiede, 614

Krumwiede and Pratt, 140

Krumwiede, Pratt, and Grund, 584

KRUSE, 292, 437, 444, 446, 540, 552

KURTH, 431

KÜsTER, 501

Kutscher, 342, 431

Kutscher and MeInjcke, 432

Kutschert and Neisser, 525

LAChNER-SaNdoval, 618

LANDSTEINER, 201

LANDSTEINER AND JAGIC, 240

LaANDSTEINER AND LeVAditi, 665

Landsteiner, Müller, and Poetzl, 263

Landsteiner and Popper, 664

LANG, 349

LANZ and Tavel, 343

LASSAR, 602

Laveran, 592

LaWs AND ANdERson, 695

Leclainche ANd Vallée, 467

LEDDERHOSE, 578

LEEUWENHOEK, 1

LEICHTENSTERN, 372

Leishman, 281

Lembke, 392

LENTZ, 438

LePIERRE, 377

Le Roy, Des Barres, and Weinberg, 368

LESAGE, 713

LEUChS, 137

Levaditi, 277, 280, 283

LEVAditi AND InMaNn, 283
Levaditi and Manouelian, 599, 616

Levaditi and Petresco, 596

LeWITH, 66

Libman, 339, 343, 431

Libman and Rosenthal, 370

LibORIUS, 149

v. LingeLSheIM, $328,331,337,340,346$, 348, 387

LiNossier AND RoUx, 640

LisTer, 6

LoEB, 324

LOEFFLER, 14, 100, 110, 136, 512, 520, $522,528,530$

LOEFFLER AND FrosCh, 678

LOEFFLER AND SCHÜTZ, 528

LoEw, 25, 716

LÖHLEIN, 282

LONGCOPE, 432

LowENSTEIN, 501

LUBARSCH, 190

LÜBBERT, 326

LUERSEN AND KüHN, 715

Lustgarten, 503, 594

MaAssen, 53

MACFADYen, 363

Macfadyen and Rowland, 416

Madsen, 218,521

MafUCCI, 480, 490, 500

MALLORY, 623,676

MalloRY AND Wright, 110, 112, 150, 454

MaNN, 96, 474

Maragliano, 498

Marbaix, 341, 342, 348

Marchiafava and Celli, 371

Marchoux and Salimbeni, 615

Marchoux, Salimbeni, and Simond, 673

Marchoux and Simond, 674

Marie and Morax, 463

MaRINESCO, 478

MARMIER, 344

MARMOREK, 344, 498

Marschal, 371

MarTin, L., 468, 521

Martin and Cherry, 204

Martini and Lentz, 438

MARX, 347 
MassoL, 715

McChintic, 80

McCoy, 510, 559, 560, 562

McCoy and Chapin, 562

Mennes, 362, 366

Mesnit, 232

Messea, 15

МетснNiкоFF, 188, 200, 201, 224, 225, $228,232,275,588,603,714$

Metchnikoff and BesRedKa, 426

Metchnikoff and Roux, 601

Metchnikoff, Roux, and Salimbeni, 589

MeYer, 187

Meyer and Ransom, 463

Michaelis, 96, 253, 263

Michel, 516

Michelson, 619

Mignesco, 63

Migula, 12, 36, 325, 472

MikUlicz, 452

MiLLER, 411, 706

Miquel, 32, 690

Mitchell, J. P., 167

Moeller, 98, 501

Mohler, 495, 679, 707, 708, 711, 712

MoLL, 198

Moltschanoff, 385

Momont, 570

MonTi, 658

Morax, 545

Morax and Marie, 462

Morgan, 404, 431

Morgentoth, 202

MorgenRoth ANd SAChS, 265

Moro, 492

Monpurgo, 189

Moser, 676

Mouton, 275

MoXter, 241

Мuch, 482

MÜHLENS, 600, 603

Muhlschlegel, 17

MÜLLER, 334, 504, 706

MüLLER, Fr., 325

MÜLLer, Otto Friedrich, 2

Müller, P. Th., 243, 254, 255, 272

MUlzer, 595, 602
Muntz, 25

MunTz And Schlossing, 57

Myers, 215, 253

NaEgeli, 486

NAKANISHI, $10,11,16,17$

NASTJUKOFF, 539

NeEDham, 4

NEGRI, 648

Neisser, 107, 329, 380, 505, 514

Neisser, BaErmann, aNd HalberSTADTER, 615

Neisser AND Sachs, 246, 273

Neisser ANd Shiga, 443

Neisser and Wechsberg, 201, 244, 329, 330,331

Nencki and Scheffer, 21

Neufeld, 345, 364, 365, 369, 412

Nedfeld and Haendel, 366

Nmufeld and Hüne, 283

NéUfeld ANd Rimpau, 282, 366

NEUfELd AND TöPFER, 283

NeUMANN, 411, 579

Nichols, 604

NicolaIER, 456

NicOLLE, 233, 298, 300, 506, 602

Nicolle, Conor, ANd Conseil, 678

Nicolle and Thenel, 233

Nielsen, 481

Nikati and Rietsch, 587

Nikolaysen, 385

NissEn, 76

NOCARD, 430, 500, 530, 620

Nocard ANd Roux, 10, 480, 500

NoguchI, 264, 265, 267, 270,600,601, $604,616,617,651$

NoRris, 237, 253, 424

NorRis AND LARKIN, 620, 621

Norris, Pappenheimer, and FlourNOY, 606

NoRTH, 715

NotTter AND Firth, 706

Novy and Freer, 64

Novy AND KNapP, 592, 606, 610

Nuttall, 198, 224, 237, 241, 253, 560

Nuttall AND ThieRfelder, 392

Obermeier, 6,605 
OBERMÜLleR, 711

OGston, 321, 335

Ohno, 439, 446

OMelianski, 49, 58

Omeltschenko, 79

OpHÜLs, 631, 632

Oppenheimer, 43, 47

Ostenberg, 136

Отто, 297, 298, 300, 669, 674

OtTolenghi, 359

Overton, 187

\section{PAlmer, 349}

PANE, 232, 362, 366

Papasotiriu, 391

Pappenheim, 106, 483

Parietti, 695

PARK, 89, 216, 217, 412, 700

Park and CAREy, 439

Park and Dunham, 438

Park and Holt, 705, 709

Park and Krumwiede, 487

Park and Throne, 220

Park and Williams, 366, 521

Parodi, 602

Pasquale, 338, 591

Passet, 326, 337

Pastedr, 5, 41, 189, 192, 193, 194, 196, $321,352,468,552,553,573,631,647$

Pasteur and Chamberland, 122

Pasteur, Chamberland, and Roux, 194,571

PAUL, 659, 660, 661, 663

Peabody and Pratt, 137, 409

Pearce, 202

Perkins, 448

Perrone, 339, 344

Petri, 502, 717

Petruschkx , 342, 404, 411, 426, 618, 620

Petterson, 291

Pfaundler, 231, 232, 234

Pfeffer, 54, 56

Pfeiffer, 195, 199, 230, 255, 279, 416, $536,537,541,588,589,657$

Pfeiffer and Beck, 536, 541

Pfeiffer and Friedberger, 241

Pfeiffer and Isaeff, 199, 224

Pfeiffer and Kolle, 231, 418, 425
Pfeiffer and Nocht, 588, 591

Pfeiffer and Wassermann, 589

PFLÜGER, 59

Pfuhl, 455, 540

Pick and Yamanouchi, 300

Pierrallini, 276

Piorkowski, 134

v. Pirquet, 494

v. Pirquet And Schick, 296, 301

PitT, 14

Plato, 381

Plaut, 611, 641

Plenciz, 2

Plotz, 677

Poels and Dhont, 430

Poels and Nolen, 368

Pollack, 427

Pollender, 6

Poor, 651

Porges, 13

Porges and Meier, 263

Portier And Richet, 296

Ротт, 706

Pratt, 140, 412, 584

Prescott, 391, 697, 698

Preuser, 532

Proscher, 331, 332

Proskauer and Beck, 29

Prudden, 404, 490

Prudden and Hodenpyl, 490

Pryor, 486

QUINCKE, 713

Rabinovitsch, 502, 510, 634, 711

RADZIEWSKY, 363

Ransom, 463

Ransome and Fullerton, 88

Ravenel, 500, 570

V. RECKLinghausen, 7

REDTENBACHER, 407

REED, 670

Reed and Carroll, 430

Reed, Cartroll, and Agramonte, 670

Reed, Carroll, Agramonte, and LaZEAR, 669, 670, 674

RemLinger, 651

Richardson, 64, 411, 412 
RICHET, 296, 302

Richet and Hericourt, 197, 331

Ricketts, 633

RicKetTS AND Wilder, 677

Rideal and WaLker, 80

RIEDER, 64

RINDFLEISCH, 7

Rixford and Gilchrist, 632

ROGER, 344

ROHNER, 579

RÖMER, 476

Rosenau and Anderson, 222, 297, 298, 520

Rosenau and McCoy, 699

RosenbaCH, 321, 337

Rosenberger, 489

Rosenthal, 441

Rosenow, 343, 370

Ross and Milne, 609

Rost, 506, 509

Rothberger, 403

Roux, 64, 149, 203

Roux and Chamberland, 471

Roux and Nocard, 533

Roux And Yersin, 107, 512, 520, 522

RUbNer, 24

RUPPEL, 22, 491

Russeld, 426

Russell and Fuller, 695

Sabouradd, 643

SACHAROFF, 616

SACHS, 188, 199, 202

SACHSE, 54

SAHLI, 493

SAlMon, 552

Salmon ANd Smith, 196

SANARELLI, 669

SANFElice, 471,632

SAderbeck, 294

SAUL, 77

SAVAGE, 231

Schaeffer and Steinschneider, 384

SCHAFER, 31

SChatTenFroh, 291, 328

Schattenfroh and Grasberger, 702

Schaudinn, 592

Schaudinn ANd Hoffmann, 594
Schell and Fischer, 486

SCHENCK, 644

Schereschewsky, 600

SCHering, 92

Scheuerlen And Spiro, 74,78

Schild, 392

Schimmelbusch, 327

Schlesinger, 345

Schlossmann, 90, 91

SCHNEIDER, 325

SCHNITZLER, 455

Scholtz, 380

Sснотtelius, 392

SChotтmÜller, 337, 344, 348, 350, 369, $405,431,706$

SCHREIBER, 546

Schroeder, 4

Schroeder and Colton, 707

Schroeter, 60

SCHÜDER, 414, 703

SCHÜLLER, 669

SCHULZE, 4

SснÜтZ, 341, 368

SCHÜTZe, 202, 236

Schwann, 3, 4

Sclavo, 357, 574

SEDGWick AND BATChELder, 700

Sharnosky, 103

ShatтoCk, 22

ShigA, 435, 438, 442, 444, 446

SiedentopF, 597

SigNoRELli, 140

SilBerschmidt, 455

Simon ANd Lamar, 286

Simon, Lamar, and Bispham, 286

Simond, 560

Simpson, 705

Simpson And Hewlett, 80

Sмітн, 101, 499, 500, 708

Sмith, Graham, 525

Smith, Herbert E., 704

Sмітн, Тн., 27, 34, 297, 484, 485, 498, $499,520,521,698$

Smith, Th., And Kilbourne, 195

Smith, Th., ANd Moore, 430

Smith, Th., Brown, and Waliker, 458

Sobernheim, 563, 574

Sobernheim ANd TomasczewsKi, 595 
Sommerville, 80

Spallanazani, Abbé, 4

SPENGLER, 493

Spilker and Gottstein, 65

SpItZer, 595

SPRONCK, 437

Spronk, 521

Stefansky, 510

Steinhardt, Dr., 651

Stern, 237, 421

Stern AND Korte, 259, 418

Sternberg, 34, 325, 340, 352, 359, 516, 669,692

Stevens and Myers, 204

STICKER, 508, 644

STOKES, 343

Stokes and Weggefarth, 706

Strauss, 532

Strauds and Gamaleia, 490, 500

Strauss and Huntoon, 652

Strelitz, 519

Stricht, 478

Strong, L. W., 449, 561

Strong and Musgrave, 436

Strong, Teague, and Barber, 561

Strong, Teague, and Crowell; 561

Suchsland, 716

Surmont, 201

Surmont and Arnould, 570

SuzUki ANd Takaki, 489

TACKe, 54

TALAMON, 353

Tarozzi, 460

Tavel and Krumbein, 368

TAYLOR, 645

Teague, 561

Tedesco, 540, 541

Terin, 327

Thayer, 669

Thomas, 668

Tidswell, 510

Tissier, 713

Tissier and Martelly, 392, 714

Tizzoni, 460

Todd, 329, 443

Tokishige, 632

Tomasczentuski, 596
ToRINI, 47

Torrey, 385

Totsuka, 419

Toussaint, 194, 571

Trask, 704

Trillat, 89

Tschistovitch, 236

TsikLinski, 32

TunNicliff, 612,613

Turnbulle, 610

UhLENHUTh, 237

Uhlenhuth AND MUlzer, 602

Ullmann, 384

UNNA, 639

Uschinsky, 28, 126, 522

VAGEdes, 499

VAILlaRd AND Dopter, 443

VaILlard and Rouget, 459

VAILlaRd AND VinCENT, 462

VALLERI-RADOT, 4

VALLET, 696

VAN DER LOEFF, 657

VAN de Velde, 329, 330, 342, 346

Van Ermengem, 14, 101, 430, 475

Van Gehuchten, 648

VAN GIESON, 649

VAUGHAN, 298, 304, 417

VAUGhan and WheEler, 302

Vedder and Duval, 438

VeEDER, 415

VeILlon, 333

Di Vestea ANd Zagari, 647

VigNAL, 619

Villemin, 479

VINCENT, 611

Voges, 29

VON DEM BORNE, 615

VotTALER, 457

WADSWORTh, 99, 249, 355, 357, 359, 361, 364,365

WAGMANN, 703

WATDEYER, 7

WALKER, 419

WARD, 64, 703

WASHBURN, 366 
WASSERMANN, 195, 199, 204, 236, 242, $383,385,579,580,604$

WASSERMANN AND BRUCK, 246, 262

Wassermann and Citron, 294

Wassermann, Neisser, and Bruck, 262

Wassermann and Proskauer, 522

Wassermann and Schütze, 237

WassermanN AND TAKAKI, 188, 214, 279,463

WASSILIEFF, 532

WEBER, 501

WECHSBERG, 242

WEEKS, 542

WEENY, 419

WEGELE, 715

WeichselbaUm, 333, 353, 372, 376, 386, $447,450,540$

Weichselbaum and MÜller, 542

Weiger'T, 7, 111, 214, 481

Weill-Hallé and Lemaire, 300

Weis, A. H., 328

Weiss, 482

WeLCH, 98, 129, 355, 357, 395, 472

Welch and Blachstein, 404

Welch and Flexner, 474

Welch and Nuttall, 177, 471

WERNICKE, 561

WeRTHEim, 382

Wesenberg, 455

Westphal and UhlenhUth, 508

WEYL, 22, 481, 490

WHERRY, 510

WICKMAN, 664

WIDAL, 421

Widal and Nobecodrt, 430

WidAl AND SiCARD, 421
WILCKENS, 704

WILDE, 451, 452, 453

WiLDER, 677

Willitams, 533

Williams and Lowden, 650

WILSON, 557

Willson, 696

WiltSCHOUR, 407

Winogradsky, 14, 54, 57

WinsLOW, 343

Winslow and Palmer, 349

WLAdimiroFF, 533

WoLBACH, 680

Wolbach ANd ERNst, 499

WoLFF, 294, 396

WOLFF AND ISRAEL, 624

WoLfF-EIsner, 301, 494

WOLFFHÜGEL, 89

Wollstein, 541, 544, 545

Wood, 95, 109, 359, 717

WORONIN, 55

Wright, 108，150，153，195，286，288, $425,623,624,627$

Wright and Douglas, 281, 282

Wright and LaMb, 550

Wu Lien Teh, 560

YERSIN, 555

Yersin, Calmette, and Roux, 561

ZEIT, 64

ZetTNow, 10, 11, 12, 606

ZIEHL, 97, 105, 481

ZinsSeR, 16, 155, 291, 412, 413, 442, 520, 525,631

Zinsser and CARY, 511 



\section{INDEX OF SUBJECTS}

Аввотт's method of staining spores, 97

Absorption method in study of agglutination reaction, 234

Achorion Schoenleinii, 640

cultivation of, 642

morphology of, 641

varieties of, 641

Acid formation by bacteria, 166

Acid-fast bacteria, stains for, 104

Acquired immunity, 192

definition of, 192

Actinomyces, 622

cultivation of, 623

discovery of, in cattle, 622

in $\operatorname{man}, 622$

morphology of, 619, 622

pathogenicity of, 625

in animals, 625

in $\operatorname{man}, 625$

parts of body infected in, 625

staining of, 623

varieties of, 626

Actinomycosis, 625

Active immunity. See under Immunity

Aërobic organisms, facultative, 26

obligatory, 25

non-infectiousness of, 183

respiratory processes of, 27

Agar for culture media, 127

lactose-litmus, 129

meat extract, 127

meat infusion, 128

Agar slants, cultivation of anaërobic bacteria on, 153

Agglutination reaction, 228

between agglutinin and agglutininstimulating substances, 233

clinical diagnosis by, in typhoid, 229

concentrated agglutinin in, 235
Agglutination reaction, differentiation of bacterial species by, 229

diluted agglutinins in, 235

group agglutination in, 234

immune or chief agglutinin in, 234

macroscopical observation of, 230

for bacterial differentiation, 231

major agglutinin in, 234

microscopical observation of, 229

for clinical diagnosis, 231

minor agglutinins in, 234

nature of, 228

of capsulated bacteria, 13

partial agglutinins in, 234

absorption method in study of, 234

proagglutinoid zone in, 235

proagglutinoids in, 235

pseudo-clumping in, 231

specificity of, 234

"thread-reaction" in, 231

upon dead bacteria, 231

upon living bacteria, 231

Agglutination tests, technique of, 250

macroscopic, 252

microscopic, 251

Agglutinins, 200, 228

action of, 240

agglutinin-stimulating substances and, 233

quantitative relations between, 233

reaction between, 233

bactericidal substances compared with, 231

cell. receptors in, 238

concentrated, failure of, to produce agglutination, 235

diluted, agglutination reaction with, 235

effect of heat on, 232

experimentation with, 231 
Agglutinins, in agglutination raction, chief or immune, 234

major, 234

minor, 234

partial, 234

in serum of glanders, 535

in staphylococcus immune sera, 331 nature of, 231

normal, 232

partial absorption method in study of, 234

production of, 232

in sera of animals, by injection of bacteria, 233

of culture extracts, 233

time of, 233

reaction of. See Agglutination reaction specificity of, 234

structure of (Ehrlich), 238

theoretical considerations concerning, 238

"thread-reaction" in, 231

Agglutinogen, 233

Aggressins (Bail's theory), 291

action of, 293

immunization with, 293

nature of, 293

occurrence of, 293

opposition to Bail's theory of, 294

Air, bacteria in, 673

dryness and high winds favorable to increase of, 683

estimation of numbers of, 684

occurrence of, in inhabited places, 683

scarcity of, in places high above earth, 684

settling of, with rain, snow, etc., 684 infectious material carried by, 684

Alcohol, as fixative in staining, 110

Alcoholic fermentation, 51

by yeasts, 52

in milk, 684

process of, 51

Alcohols as disinfectants, 77

Alexin, 198

action of, in blood serum, 224, 225

Alkali formation by bacteria, 166
Allantiasis, 477

Amboceptor and complement, quantitative relationship between, 244

Amboceptors, filtration of, 244 multiplicity of, in normal sera, 241

Amylase, 48 action of, 49 occurrence of, 49

Amylolytic ferment. See Amylase

Anaërobic cultivation of bacteria, 148 use of sterile tissue as an aid in, 156 See also under Cultivation of bacteria

Anaërobic organisms, facultative, 26

Gram-positive, in feces, 177

infectiousness of, 183

non-invasion of blood stream by, 183

obligatory, 26

respiratory processes of, 26

Anaphylactin, 302

Anaphylatoxin, 304

Anaphylaxis, 295

autopsy findings in, 299

definition of, 297

experimentation in, early, 295

immunity after, 299

in diphtheria antitoxin injections, 296

incubation during, 299

inherited, 300

observations in, fundamental, 296

by Arthus, 297

by Besredka, 298, 299

by Besredka and Steinhardt, 299

by Doerr, 299

by Gay and Southard, 299, 300

by Hericourt and Richet, 296

by Nicolle, 298, 300

by Otto, 297, 300

by Pick and Yamanouchi, 300

by Portier and Richet, 296

by Rosenau and Anderson, 297, 298,300

by Th. Smith, 297

by Vaughan and Wheeler, 298

by Weill-Hallś and Lemaire, 300

passive, 300

phenomena of, 295

"phenomenon of Arthus" in, 297

proteid injections in, 295 
Anaphylaxis, proteid injections in, mode of giving, 298

quantity of, 298

symptoms in, 299

theories concerning, 301

based on Ehrlich's receptor overproduction theory, 301

of Besredka, 302

of Doerr and Russ, 303

of Friedberger and Hartoch, 303

of Gay and Southard, 302

of Hamburger, 303

of v. Pirquet and Schick, 301

of Richet, 302

of Wolff-Eisner, 301

Anilin dyes, influence of, upon bacterial growth, 140

Animal alkaloids, 45

Animal experimentation, 169

animals used in, 169

cages for, 173

autopsies in, 173

inoculations in, 170

Antagonism of bacteria, 31

Anthrax, 563

bacterial causation of, 6

occurrence of, 563

Anthrax bacillus, 563

action of, 571

bacilli resembling, 575

Bacillus anthracoides, 575

Bacillus radicosus, 575

Bacillus subtilis, 575

biology of, 569

cultivation of, 566

early investigation of, 563

experimental inoculation with, 571

immunization against, 573

active (Pasteur's method), 574

attenuation in, 573

passive (Sobernheim's method), 574

in milk, 707

infection with, 572

by inhalation, 572

cutaneous, 572

pulmonary, 573

spontaneous, 572

through alimentary canal, 573
Anthrax bacillus, isolation of, 565

morphology of, 564

pathogenicity of, 570

prophylaxis against, 573

resistance of, 570

staining of, 565

susceptibility of animals to, 570

virulence of, 570

Anthrax, symptomatic, bacillus of, 465

cultivation of, 465

immunization against, 468

vaccines used in, 468

morphology and staining of, 465

occurrence of, 465

pathogenicity of, 466

autopsy findings in, 467

toxins of, 467

Anthropoid apes, blood of, distinguished

from human, 237

Antiaggressins, 293

Antiamboceptors, 242

Antianaphylaxis, 299

Antibodies, 197

experimentation and discovery of, 197

agglutinins, 200

alexin, 198

antiferments, 202

antitoxin, 198-199

bacteriolysins, 200

cytotoxins, 201

precipitins, 200

facts and theories concerning, 241

in sera, determination of, by complement fixation. See under Complement fixation.

Anticomplements, 242

Antiferments, 202

Antiformin, formula for, as given by Rosenau, 483

in examination of sputum for tubercle bacilli, 483

Antigen, definition of, 202

Antilab, 202

Antilactase, 202

Antileucocidin, 331

Antipepsin, 202

Antiricin, discovery and experimentation with, 204 
"Antisensibilisin," 302

Antiseptics, inhibition strengths of various, 84

values of, determination of, 80 table of, 80

Antistaphylolysin, 331

Antisteapsin, 202

Antistreptococcic sera, 346

Antitoxic sera, 196

Antitoxin, 198-199

diphtheria. See Diphtheria antitoxin

normal, 205

production of, a final test between toxin and endotoxin, 187

stability of, 206

tetanus. See Tetanus antitoxin

unit of, 205

valency of, for toxin, 210

Antivenin, 198

Arthrospores, 16

Ascospores, 630, 638

Ash in bacterial cell, 23

Asiatic cholera. See Cholera

Aspergillus, reproduction in, 637

Attenuated cultures in active immunization, 193

Autoclave, 71

technical details of, 72

Trillat's, 87

Autointoxication, gastrointestinal, 712

bacteria causing, 713

experimental combating of, by acid-producing bacilli, 713

Metchnikoff's treatment of, by means of Bacillus bulgaricus, 714-715

Autolysins, 248

Autopsies of infected animals, 173

Avian tuberculosis, bacillus of. See

Tubercle bacillus, bacilli related to

BABEs-Ernst granules, 11

Bacilli intermediate between typhoid and colon organisms, 428

bacterial correlation of, 431

bacilli of colon-like morphology in, 433
Bacilli intermediate between typhoid and colon organisms, bacterial correlation of, bacilli of typhoid--like morphology in, 433

non-motile bacilli in, 433

classification of, 432

differentiation of, from ty phoid and colon groups, 428

by cultural characteristics, 428

by morphology, 428

by motility, 428

hog-cholera bacilli in, 430

meat-poisoning bacilli in, 429

Bacillus enteritidis in, 429

Bacillus icteroides in, 430

Bacillus Morseele in, 430

paratyphoid bacilli in, 430

Bacillus psittacosis in, 430

"Müller" bacillus, 431

paracolon bacillus in, 430

"Seeman" bacillus, 431

pathogenicity of, 431

toxic products of, 431

Bacillus, 37

general description of, 9

Bacillus aërogenes capsulatus, 471

and pernicious anemia, 177

biological considerations of, 474

cultivation of, 473

isolation of, 474

morphology of, 472

occurrence of, 472,475

pathogenicity of, 474

staining of, 473

Bacillus anthracoides, 575

Bacillus avisepticus, 552

Bacillus botulinus, 475

antitoxin for, 199

cultivation of, 473

morphology of, 476

pathogenicity of, 477

staining of, 476

toxins of, 478

Bacillus bulgaricus, use of, by Metchnikoff for treatment of gastrointestinal autointoxication, 715

Bacillus butyricus, 502

Bacillus coli communior, 398 
Bacillus coli communis, 389

agglutinins for, 396

in immune serum, 396

in normal serum, 397

bladder diseases due to, 395

cholera infantum attributed to, 394

cholera nostras attributed to, 394

cultivation of, 390

differentiation of, from meat-poisoning and paratyphoid bacilli, 428

distribution of, 391

in animals, 392

in feces, 177

in man, 392

in milk, 391

in nature, 391

in water, 697

immunization with, 395

inflammatory conditions of liver and gall-bladder attributed to, 395

isolation of, 391

morphology of, 389

pathogenicity of, 393

peritonitis following perforation attributed to, 394

septicemia due to, 394

staining of, 389

toxic products of, 395

varieties of, 397

Winckel's disease in the newborn due to, 394

Bacillus diphtheriæ. See Diphtheria bacillus

Bacillus enteritidis, discovery and characteristics of, 429

Bacillus fecalis alkaligenes, 426

differentiation of, from typhoid bacillus, 427

in feces, 177

Bacillus Hoffmanni. See Diphtheria bacillus, bacilli similar to

Bacillus icteroides, 430

Bacillus influenzæ. See Influenza bacillus

Bacillus lactis aërogenes, 453

cultivation of, 453

morphology of, 453

occurrence of, 453

in feces, 177
Bacillus lactis aërogenes, pathogenicity of, 453

Bacillus lepræ. See under Leprosy

Bacillus mallei, 520. See also Glanders biological characteristics of, 530 cultivation of, 528

immunity against, 535

morphology of, 528

pathogenicity of, 530

bacteriological diagnosis in, 532

in horses, 530

in man, 532

nodules in, 532

spontaneous infection by, 530

staining of, 528

toxin of, 532

action of, 533

diagnostic use of, 533

directions of U. S. Government for, 534

obtaining and preparation of, 533

Bacillus melitensis. See Micrococcus melitensis

Bacillus mesentericus in feces, 177

Bacillus Morseele, discovery and characteristics of, 430

Bacillus mucosus capsulatus, 447 association of, with pneumonia, 450

with other diseases of mucous linings, $450-451$

cultivation of, 448

cultural characteristics of, 449

Fitzgerald's work on classification of, 450

immunization against, 451

inoculation of animals with, 451

morphology of, 447

pathogenicity of, 450

staining of, 448

Bacillus murisepticus, 542

Bacillus ozænæ, 452

Bacillus pestis. See Plague bacillus

Bacillus proteus vulgaris, 454

cultivation of, 454-455

Bacillus prodigiosus, quantitative chemical analysis of, 21

Bacillus psittacosis, 430

Bacillus pyocyaneus, 577 
Bacillus pyocyaneous, antitoxin against products of, 199

cultivation of, 577

favorable conditions for, 577

pigment in, 578

fluorescent variety of, 579

pyocyanin in, 578

immunization against, 580

filtrates of old cultures in, 580

pyocyanase in, 580

true toxin in, 580

morphology of, 577

occurrence of, in lesions and inflam-

matory affections, 579

pathogenicity of, 579

staining of, 577

susceptibility of animals to, 580

toxins of, 580

leucocyte-destroying ferment in, 581

pyocyanase in, 580

pyocyanolysin in, 580

true, 580

from filtrates of old cultures, 580

virulence of, 579

Bacillus radicicola, 55

Bacillus radicosus, 575

Bacillus rhusiopathiæ, 542

Bacillus smegmatis. See Smegma bacillus

Bacillus subtilis, 575

Bacillus suisepticus, 553

Bacillus tetani. See Tetanus, bacillus of

Bacillus tuberculosis. See Tubercle bacillus

Bacillus typhi abdominalis, 399

Bacillus typhi murium, 430

Bacillus typhosus. See Typhoid fever, bacillus of

Bacillus xerosis. See Bacillus diphtheriæ, bacilli similar to

Bacteria (see also Bacterial cell): acid and alkali formation by, 166

acid-fast stains for, 104

action of, in the body, 184

anabolic or synthetic activities of, 54

in root tubercles, 55

in soil, 54
Bacteria, animal experimentation with, 169

antagonism of, 31

biological activities of, 40, 164

chemical agents injurious to, 73 . See also Disinfectants

classes of, 182

bacilli, 9

cocci, 9

spirilla, 9

classification of, 35

based on organs of motility, 15

by Bail with regard to aggressins, 294

by Gram stain, 104

by Migula, 37

counting of, 161

cultivation of, 141

by anaërobic methods, 148 . See also under Cultivation of bacteria inoculation of media in, 141 dead, in active immunization, 193 degenerative forms of, 20

denitrifying, 52

destruction of. See Destruction of bacteria

differentiation of, by fermentation, 48 enzymes produced by, 168

diastatic, 169

inverting, 169

proteolytic, 168

gas formation by, 164. See also Gas formed by bacteria

Gram-negative, 104

Gram-positive, 104

in air. See Air, bacteria in

in industries, 715

in milk. See Milk, bacteria in

in soil. See Soil, bacteria in

in tissues, staining of, 110

in water. See Water, bacteria in

incubation of, 156. See also under Incubation of cultures

indol production by, 167

isolation of, methods of, 142

katabolic activities of, 41-53

by bacterial enzymes or ferments, 42 varieties of, 48 
Bacteria, katabolic activities of, by denitrifying bacteria, 52

by fat-splitting enzymes, 47

by lab enzymes, 46

by proteolytic enzymes, 43

liberation of energy by, 58

light production of, 59

microscopic study of. See Microscopic study

nitrifying. See Nitrifying bacteria

nutrition of, 25 . See also under

Nutrition of bacteria

occurrence of, in the body, 181

parasitic, 29

definition of, 182

pathogenic, 182

phenol production by, 167

physical agents injurious to, 62

pigment formation by, 59

protozoa and, differentiation of, 1

putrefactive, quantitative chemical analysis of, 21

reducing powers of, 167

relation of, to moisture, 35

to physical environment, 31

to pressure, 35

relationship of, to other plants, 35

reproduction of, 18

rate of, 18

varieties of, 18

saprophytic, 29

definition of, 182

size of, 9

staining of, methods of. See Staining of bacteria

sulphur. See Sulphur bacteria

symbiosis of, 31

thermal death points of, 34

variations in forms of, 19

virulence of, and infectiousness, 183

virulent, sublethal doses of, in active immunization, 195

Bacteriaceæ, 37

Bacterial cell, ash in, 23

Babes-Ernst granules in, 11

capsule of, 12

carbohydrates in, 23

chemical consitutents of, 21
Bacterial cell, chemical constit:.ents of, quantitative analysis of, 21

varieties of, 21

fats in, 22

globulin in, 22

membrane of, 12

metachromatic granules in, 11

morphology of, 100

motility of, 13

Brownian, 14

by flagella, 14

effect of temperature on, 15

molecular, 14

true, 14

nucleus in, 10

organs of locomotion of, 13

classification of bacteria based on, 15

osmotic properties of, 23

permeability of membrane of, 23

plasmolysis of, 23

plasmoptysis of, 24

polar bodies in, 11

proteids in, 22

refractive index of parts of, 24

specific gravity of forms of, 24

water in, 21

Bacterial enzymes or ferments, 42

action of, 42

environmental conditions on, 43

reversible, 43

similarity of, to ferments of specialized cells of higher plants and animals, 43

Bacterial forms, variations of, 19

Bacterial poisons, 184

action of, 187

ptomains and, 185

resistance of, to chemical action and heat, 187

summary of, 305

varieties of, 185

endotoxins, 185

proteins, 186

true toxins, 185

Bacterial products in active immunization, 195

Bacterial proteins, 186 
Bacterial spores, 15

formation of, 15

germination of, 17

position of, 17

varieties of, 15

arthrospores, 16

true, or endospores, 16

vegetative forms from, 17

Bactericidal action of blood serum, 224

Bactericidal strengths of common disinfectants, 85

Bactericidal substances compared with agglutinins, 231

Bactericidal tests, 257

in test tube, 257

technique of, 258

for typhoid fever, 258

in vivo, 255-7

Bacteriemia, definition of, 184

Bacteriological examination of blood cultures, 178

choice of media for, 179

results of, estimation of, 180

technique of obtaining material for, 178

from typhoid patients, 180

of exudates, 175

of feces, 176

of material from patients, 174

technique of collecting, 174

of urine, 176

Bacteriology, development and scope of, 1-8

Bacteriolysins, 200

immune, 225

Bacteriolytic tests, 255

Pfeiffer's test in, 255

determination of bacteriolytic power of serum against a known microorganism in vivo by, 255

identification of microorganism in known immune serum in vivo by, 257

Bacteriotropins, 282

Bacterium, 27

Bacterium tularense, 562

Bail, aggressin theory of, 292

opposition to, 294
Barsiekow's medium for colon-typhoid differentiation, 139

Bauer's modification of Wassermann test for syphilis, 268

"Bazillenemulsion," 492

Beggiatoa, genus, 38

Beggiatoaceæ, 38

Berkefeld filter, 120, 121

Bile medium for colon-typhoid differentiation, 138

Bile-salt agar, MacConkey's, for colontyphoid differentiation, 138

Bitter milk, bacteria causing, 703

Black Death, 554

Bladder diseases due to colon bacillus, 395

Blastomycetes. See Yeasts and Yeast cells

Blood, laked, 225

the seat of immunizing agencies, 198

Blood corpuscles, red, in Ehrlich's theory of lytic process in blood serum, 227

Blood cultures, bacteriological examination of, 178

results of, estimation of, 180

choice of media for, 179

technique of obtaining material for, 178

from typhoid patients, 180

Blood media, method of obtaining, 140

Blood serum, bactericidal action of, 224

Bordet's interpretation of lytic processes of, 225

immune, 198

reactivation of bactericidal power of, by normal serum, 225

reactivation of bacteriolytic powers of, by normal serum, 226

lytic processes of, 224

normal, 198

Bordet's lytic theory of constituents of, 225

alexin in, 225

"sensitizing substance" in, 225

obtaining of, from animals, 249

from man, 249

reactions with. See Serum reactions 
Blood serum reactions, method of obtaining, 139

Bollinger, discovery of actinomyces of cattle by, 622

Bordet and Gengou, discovery of whooping-cough bacillus by, 543

Bordet-Gengou bacillus, 543

cultivation of, 544

compared with that of influenza bacillus, 544

technique of, 544-545

morphology of, 543

compared with that of influenza bacillus, 544

pathogenicity of, 545

staining of, 543

toxins of, 545

Botulism, 477

Bouillon, malachite green, for colontyphoid differentiation, 137

Bouillon filtré (Denys), 492

Bovine tuberculosis, bacillus of. See Tubercle bacillus, bacilli related to

Broth used for culture media, 124 calcium carbonate, 126

glycerin, 125

meat extract, 124

meat infusion, 124

nitrate, 126

pepton-salt, 126

sugar-free, 125!

Uschinsky's proteid-free, 126

Bruce, discovery of Malta fever bacillus by, 550

Buboes, 548

Buchner, discovery of Bacillus coli communis by, 389

Buchner's method of pyrogallic absorption of oxygen in cultivation of anaërobic bacteria, 152

Wright's modification of, 153

Buerger's method of staining capsules, 99

Butter bacillus, 502

Butter, making of, 710

bacteria aiding, 710

transmission of infection by, 711

tubercle bacilli in, 711

Butyric-acid fermentation in milk, 702
Cadaverin, 45

Cages for animals, 171, 172, 173

Calcium-carbonate broth, 126

Capaldi's medium for colon-typhoid differentiation, 134

Capsule stains in staining of bacteria, 98

Carbohydrates in bacterial cell, 23

Carbolic acid as disinfectant, 77

Carbolic-acid coefficient, 80

Carbon dioxid formed by bacteria, 164

Carbon in nutrition of bacteria, 25

Casein, coagulation of, in milk, 702

Castelli, discovery of Spirochæta pertenuis by, 614

Cell-receptors, three forms of, in explanation of all known antibodies (Ehrlich):

haptines of the first order, 240

haptines of the second order, 240

haptines of the third order, 240

Cellulase, 49

Charbon. See Anthrax

Charbon symptomatique. See Anthrax, symptomatic, bacillus of

Cheese, making of, 711

bacteria aiding, 711, 712

pathogenic organisms in, 712

Chemotaxis, negative and positive, definition of, 277

Chicken cholera bacillus, 552

cultivation of, 552

immunization with, 553

morphology and staining of, 552

occurrence of, in animals, 552

pathogenicity of, 552

Chicken-pox, relation of, to smallpox, 660

Chlamydobacteriaceæ, 38, 618

classification of, 618

morphology of various forms of, 618

Chloride of lime as disinfectant, 76

Chlorine as disinfectant, 88

Cholera, Asiatic, 582

diagnosis of, 584

epidemics of, 586

immunization in, 590

active, 590

protective inoculation in, 590

in animals, 587 
Cholera, Asiatic, infection in, 587 pathological findings in, 587 spirillum of, 582

biological considerations of, 586 cultivation of, 583 diagnosis of, by "cholera-red" reaction, 584

Dieudonné's selective medium for cultivation of, 584

Dieudonné's selective medium for cultivation of, modified by Krumwiede and Pratt, 584

hygienic considerations of, 588 in feces, 177

isolation of, 585

from feces, 585

from water, 586,696

morphology of, 582

pathogenicity of, 586

in animals, 587

in man, 587

resistance of, 586

spirilla resembling, 590

Spirillum Deneke, 591

Spirillum Massaua, 591

Spirillum Metchnikovi, 590

Spirillum of Finkler-Prior, 591

staining of, 583

toxin of, 589

traced to milk, 705

Cholera, fowl, bacillus of, 7

Cholera infantum attributed to colon bacillus, 394

Cholera nostras attributed to colon bacillus, 394

Cholera-red reaction, 584

Chromo-bacteria, 59

Chromogenic Gram-negative cocci, 387

Cladothrix, 38, 619 morphology of, 618

Clearing of culture media, 119

by filtering, 120

with eggs, 119

Clostridium Pasteurianum, 54

Coagulins, 235

Cobra poison and its antitoxin, experimentation with, 204

Coccaceæ, 37
Cocci, description of, 9

Coefficient of inhibition, 80

Colon bacillus. See Bacillus coli communis

Colon bacillus group, 389

Colon test, for analysis of water, 697

Colon-typhoid differentiation, media for, 132

Barsiekow's, 138

bile, 137

Capaldi's, 134

Conradi-Drigalski, 135

Endo's, 135

Hesse's, 133

Hiss' plating, 133

Hiss' tube, 133

Jackson's lactose-bile, 138

Loeffler's malachite green, 136

MacConkey's bile-salt, 138

malachite green bouillon, 137

neutral-red, 138

Piorkowski's urine gelatin, 134

Colon-typhoid-dysentery group, bacilli of, 388

characteristics of, 388

Colonies in agar, 146

Colony-fishing, 146

Colony study of bacteria, 161

Color indicator in titration, 117

Comma bacillus. See Spirillum choleræ asiaticæ

Complement, deviation of, 244

filtration of, 244

fixation of. See Complement fixation in Ehrlich's theory of lytic process in blood serum, 226

in normal blood serum, 226

multiplicity of, in normal sera, 242

Complement fixation, action in, 245

by precipitates, 244

determination of antigen by, in serum reactions, 271

of antibodies in sera by, 261

principles of, 261

reaction in, 261

Wassermann test for. See Wassermann test for diagnosis of syphilis 
Complement fixation, proteid differentiation by, 273

Complementoids, 243

Conjonctivite subaigue, 545

Conradi-Drigalski medium, for colontyphoid differentiation, 135

isolation of typhoid bacillus in stools by, 408

Cotton used in filtering culture media, 120

Counting of bacteria, 161

Cowpox, relation of, to smallpox, 659

use of, in immunization against smallpox, 659

Crenothrix, 38

Creolin, 78

Cultivation of bacteria, anaërobic, 148

by absorption of oxygen by pyrogallic acid in alkaline solutions, 152

Buchner's method, 152

Wright's modification of, 153

combined with air exhaustion and hydrogen replacement, 153 on agar slants, 153

without use of hydrogen, 155

by displacement of air with hydrogen, 151

by mechanical exclusion of air, 148

Esmarch's method, 149

fluid media covered with oil, 150

Liborius' method, 149

Roux's method, 149

Wright's method, 150

Zinsser's apparatus for, 156

colony study in, 161

counting of bacteria in, 161

incubation in, 158. See also under

Incubation of cultures

Culture media, 124

agar for, 127

lactose-litmus, 129

meat extract, 127

meat infusion, 128

blood for, 140

blood serum for, 139

broth for, 124

calcium carbonate, 126

glycerin, 125
Culture media, broth for, meat extract, 124

meat infusion, 124

nitrate, 126

pepton-salt, 126

sugar-free, 125

Uschinsky's proteid-free, 126

clearing of, 119

by filtering through cotton, 120

through paper, 121

with eggs, 119

Dorsett egg, 130

enriching substances used in, 138, 139

fluid, covered with oil, in anaërobic cultivation of bacteria, 150

for colon-typhoid differentiation, 132

glassware preparation in, 113

glycerin' for, 126

ingredients of, 115

choice of, 116

lactose litmus-agar, 129, 135

litmus milk, 130

milk, 130

potato for, 130

glycerin, 130

preparation of, 113

general technique of, 113

process of, 124-40

reaction of, 119

serum, 131

Hiss' serum water, for fermentation tests, 132

Loeffler's, 131

slanting of, 123

special, 132

sterilization of, 121

filtration in, 122

heat in, 121

titration of, 117

color indicator in, 117

process of, 117

for alkaline media, 118

reaction of, 117

adjustment of, 119

tubing of, 121

Cultures, attenuated, in active immunization, 193 
Cultures, incubation of. See under Incubation of cultures

Cytase, 279

Cytoryctes variolæ, 658

Cytotoxins, 201

specific, injury to organs by, 201

DARK-FIELD condenser, 597

Decay, action of, 44

Defensive factors of animal organism, 189

Degenerative forms of bacteria, 20

Denitrifying bacteria, 52

activities of, 53

occurrence of, 53

Destruction of bacteria, 62

by chemical agents, 73 . See also Disinfectants

gaseous, 88

in solution, 73

inorganic, 73

organic, 77

by physical agents, 62

drying, 62

electricity, 65

heat, 65

light, 63

Diarrheal diseases traced to milk, 705

Diastase. See Amylase

Differential stains in staining of bacteria, 102

Diphtheria, tracing of, to milk, 705

Diphtheria antitoxin, 216

anaphylaxis in injections of, 296

normal, 205

production of, 216

horses used in, 216-17

technique of, 217

toxin for, 216

stable, 206

standardization of, 218

concentration and purifying in, 219

technique of, 218

unit for, 205

Diphtheria bacillus, 512

bacilli similar to, 522

Bacillus Hoffmanni, 522 cultivation of, 524
Diphtheria bacillus, bacilli similar to, Bacillus Hoffmanni, morphology of, 523

staining of, 523

Bacillus xerosis, 525

cultivation of, 526

differentiation of, from other bacilli, 526

other bacilli, 527

biological characteristics of, 515

cultivation of, 516

degenerative forms of, 19

differentiation of, from other forms,

107,522 , et seq.

grouping of, 515

isolation of, 517

morphology of, 513

"ground" type in, 514

occurrence of, in body, 518,519

pathogenicity of, 518

in animals, 519

"pseudo-membranes" in, 518

resistance of, 516

staining of, 574

Gram's method of, 507

polar or Babes-Ernst bodies in, 514

Neisser's stain for, 514

Roux's stain for, 515

toxin of, 520. See also Diphtheria toxin

chemical and physical properties of, 520

production of, 520

media employed in, 520

Park-Williams Bacillus No. 8 in, 520

resistance of, 522

Diphtheria toxin, analysis of (Ehrlich), 205-15

method of partial absorption in, 209 side-chain theory of, 212

summary of, 215

constitution of (Ehrlich), 210

graphic form of (Ehrlich), 211

views of Arrhenius and Madsen on, 212

epitoxoid in, 208

molecule of, haptophore group in, 207 
Diphtheria toxin, molecule of, toxophore group in, 207

normal solution of, 205

partial absorption of, 209

standardization of, Limes death in, 208

Limes zero in, 207

time changes in, 206

toxoid form of, 207

protoxoids in, 209

syntoxoids in, 209

toxon in, 209

unit for, 205

Diplococcus gonorrhœæ, 380

cultivation of, 381

conditions favorable to, 383

Wertheim's medium for, 381-2

differentiation of, from Micrococcus catarrhalis, 386

early work in, 380

infection by, in man, 384

morphology of, 380

pathogenicity of, in animals, 385

in man, 384

resistance of, 384

staining of, 381

Diplococcus lanceolatus. See Diplococcus pneumoniæ

Diplococcus mucosus, 387

Diplococcus pneumoniæ, 352

cultivation of, 355

differentiation of, from streptococcus, 357,367

cultural, 368

morphological, 367

by bile test, 369

by capsule, 367

by fermentations, 369

by grouping, 367

by growth in blood media, 367 , 369

by shape, 367

immunization against, 363

active, methods of, 363

agglutinins in immune sera in, 364

table of, 365

technique of, 364

opsonins in immune sera in, 366

precipitins in immune sera in, 365
Diplococcus pneumoniæ, immunization against, passive, with immune sera, 366

isolation of, 358

modes of inoculation with, 361

morphology of, 553

capsules in, 353-4

lancet-shape of cocci in, 353

pairing of cocci in, 353

pathogenicity of, 361

pneumonic complications and, 362

resistance of, 358

staining of, 355

susceptibility of animals to, 370

toxic products of, 362

virulence of, 360

in animals, 360

in man, 361

Disinfectants, 73

bactericidal strengths of common, 85

gaseous, 88

chlorine, 88

formaldehyde, 89

oxygen, 88

ozone, 88

sulphur dioxide, 88

testing of efficiency of, 79

by U. S. Public Health Service method, 80

carbolic-acid coefficient in, 80

determination of antiseptic values in, 83

of disinfectant values in, 86 table of, 85

factors in, 79

used in solution, 73

inorganic, 73

acids, bases, and salts, 74

halogens and derivatives, 75

chlorid of lime, 76

tetrachlorid of iodin, 76

oxydizing agents, 76

hydrogen peroxid, 76

potassium permanganate, 76

organic, 77

alcohols, 77

carbolic acid, 77

Cresol group, 78 
Disinfectants, used in solution, organic, essential oils, 78

formaldehyde, 78

iodoform, 77

Disinfection, practical, 86

of feces, 87

of hands, 87

of instruments, 87

of linen, etc., 87

of rooms, etc., 88

of sputum, 87

of urine, 87

Dissociation, electrolytic, 74

relation between degree of, and bactericidal powers of solutions, 74

Dorsett egg medium, 130

Drying in destruction of bacteria, resistance to, 62

$\mathrm{D}^{\prime} \mathrm{TN}^{1} \mathrm{M}^{250}$, definition of, 205

Ducrey bacillus, 547

cultivation and isolation of, 548

discovery of, 548

infection with, 547

pathogenicity of, 549

Dysentery, autopsy findings in, 442

occurrence of, 442

symptoms of, 442

Dysentery bacilli, 435

biological considerations of, 441

differentiation of, by Hiss, through agglutination tests, 440

through fermentation tests, 439

immunization with, 444

active, 444

agglutinins in, 444

bactericidal substances in, 444

true toxins in, 445

passive, 445

in feces, 178

investigation of, by Flexner, 436

by Hiss and Russell, 438

by Kruse, 437

by Lentz, 438

by Martini and Lentz, 438

by Ohno, 439

by Park and Carey, 439

by Park and Dunham, 438
Dysentery bacilli, investigation of, by Shiga, 435

by Spronck, 437

by Strong and Musgrave, 436

by Vedder and Duval, 437

pathogenicity of, 441

pseudo-dysentery bacillus and, 437, 438

Shiga's bacillus in, 435

cultural characteristics of, 436

morphology of, 435

toxic products of, 442

action of, on animals, 443

obtaining of, from cultures, 442

"Y" bacillus in, 438

EвеRтн, discovery of typhoid bacillus by, 399

Edema, malignant, bacillus of. See under Malignant edema

Eel-blood serum, toxic, 205

Eggs used in clearing culture media, 11.9

Ehrlich's analysis of diphtheria toxin, 205-215. See also under Diphtheria toxin

Eichstedt, discovery of Microsporon furfur by, 639

Electricity in destruction of bacteria, 65

Electrolytic dissociation, 74

relation between degree of and bac-

tericidal powers of solutions, 74

Elser and Huntoon, discovery of pseudomeningococcus by, 379

Elsner's potato-extract gelatin, isolation of typhoid bacillus in stools by, 407

Emphysematous gangrene, isolation of Bacillus aërogenes capsulatus from, 474 Endolysins, 291

Endo's fuchsin-agar, isolation of typhoid bacillus in stools by, 409

Endo's medium, for colon-typhoid differentiation, 135

Harding and Ostenberg's method of preparation of, 136

Endospores, 16

Endotoxins, 185, 306

compared with pigments, 186, 309 
Endotoxins of Staphylococcus pyogenes aureus, 328

of Streptococcus pyogenes, 345

summary of, 306

toxins distinguished from, 186

Environment and bacteria, 31

Enzymes, definition of, 42

katalyzers and, analogy between, 42

produced by bacteria, 168

diastatic, 169

inverting, 169

proteolytic, 168

varieties of. See under specific names

Epitoxoid in toxin, 208

Erlenmeyer flask, 114

Escherich, discovery of Bacillus lactis aërogenes by, 453

Esmarch roll tubes in isolation of bacteria, 147

Esmarch's method of anaërobic cultivation of bacteria, 149

Essential oils as disinfectants, 78

Exudates, bacteriological examination of. See Bacteriological examination

Eumycetes. See Hyphomycetes

Facultative aërobes, 26

Facultative anaërobes, 26

Farcy, 531

Fat-splitting enzymes, 47

action of, method of investigating, 47

varieties of, 47

Fats in bacterial cell, 22

Favus, 640

Feces, bacteriological examination of, 176

disinfection of, 87

number of bacteria in, 176

varieties of bacteria in, 177

Fermentation, alcoholic, 51

in milk, 702

butyric-acid, in milk, 702

enzymes of, 48,52

in development of bacteria, 26

inversion in, 42

lactic-acid, 50, 701

process of, 48
Fermentation tests, serum media for, 132

Filtering, in clearing of culture media, 102

through cotton, 120

through paper, 121

in sterilization of culture media, 122

Filters, varieties of:

Berkefeld, 120, 121

Kitasato, 123

Maassen, 124

Reichel, 122

Filtrable virus, 679

table compiled by Wolbach, 680

Filtration of immune body and complement, 244

Fishing, colony, 146

Fixation of complement, action in 246 by precipitates, 244

"Fixator," 279

Flagella, arrangement of, 15

structure of, 14

varieties of, 14

Flagella stains in staining of bacteria, 100

Florence flask, 114

Fluid media covered with oil, used in cultivation of bacteria, 150

Foot-and-mouth disease, 678

etiological factor of, 679

immunity in, 679

in milk, 706

pathology of, 678

transmission of, 679

Formaldehyde as disinfectant, 78, 89

generation of, by breaking up solid polymer with heat, 91

by Breslau method, 90

by direct evaporation, 89

by glycerin addition, 90

by lime method, 92

by potassium-permanganate method, 92

by Trillat method, 89

gauging amount of formalin in, 92

Trillat autoclave for, 89

Fractional sterilization, 70

at high temperatures, 70

at low temperatures, 71 
Frambosia tropica, 614

Friedländer, discovery of Bacillus mucosus capsulatus by, 447

Friedländer bacillus. See Bacillus mucosus capsulatus

v. Frisch, discovery of Bacillus of rhinoscleroma, 451

Fusiform bacilli, 614

from carious teeth, 613

from noma, 614

from scurvy, 614

of Vincent's angina, 612

GAFFKY, discovery of Micrococcus tetragenus by, 334

Gärtner, discovery of Bacillus enteritidis by, 429

Gas formed by bacteria, 164

analysis of, 164

carbon dioxide in, 164

hydrogen in, 165

hydrogen sulphide in, 165

quantitative, 166

Gas-pressure regulators for incubator's, 160

Moitessier's, 160

Gastrointestinal autointoxication, 712

bacteria causing, 713

experimental combating of, by acidproducing bacilli, 713

Metchnikoff's therapy of, by means of

Bacillus bulgaricus, 714-715

Gelase, 49

Gessard, discovery of Bacillus pyocyaneus by, 578

Glanders, bacteriological diagnosis of, 532 immunity in, 535

in horses, 530

acute form, 530

chronic form, 531

in man, 532

nodules of, 532

spontaneous infection in, 530

Glanders bacillus. See Bacillus mallei

Globulin in bacterial cell, 22

Glycerin, use of, for culture media, 126 meat extract, 126

meat infusion, 127
Glycerin broth, 125

Glycerin potato, 130

Gonococcus. See Diplococcus gonorrhœæ

Gram-negative bacteria, 104

Gram-negative cocci, chromogenic, 387

Gram-negative micrococci, table of diagnosis of, by differential value of sugar fermentation, 387

Gram-positive bacteria, 104

in feces, 177

Gram's method of staining bacteria, 102 classification of pathogenic bacteria by, 104

Paltauf's modification of, 103

Group agglutination, 234

Gruber-Widal reaction, 250

Gruby, discovery of Trichophyton tonsurans by, 642

Guarnieri's medium, Welch's modification of, 129

Halogens as disinfectants, 75

"Hanging block" method in study of bacteria, 94

"Hanging drop" method in study of bacteria, 93

Hansen, discovery of lepra bacillus by, 505

Haptophore group in toxin molecule, 207 in toxon molecule, 209

Haptophore groups in immune body, 228 complementophile, 228

cytophile, 228

Hauser, discovery of Bacillus proteus vulgaris by, 454

Heat, in destruction of bacteria, 65 dry and moist, comparison of, 66 effect of various degrees of, 65 moist, advantages of, 66

penetrating power of, 67 in sterilization of culture media, 121

Heat sterilization, 68 dry, 68

by burning, 68

by hot air, 68

moist, 69

by boiling, 69 
Heat sterilization, moist, by fractional sterilization, 70

by live steam, 60

by steam under pressure, 71

Hektoen and Ruediger on structure of opsonins, 283

Hemagglutinins, 235

Hematogen, 538

Hemolysin, immune, 226

Hemolysins of Staphylococcus pyogenes aureus, 329

of Streptococcus pyogenes, 344

specificity of, 247

varieties of:

autolysins, 247

heterolysins, 247

isolysins, 247

Hemolysis, 201, 225

Hemolytic tests, 259

obtaining blood for, 259

in large quantities, 260

in small quantities, 259

standard concentration of red blood cells for, 259

Hemolytic unit, definition of, 265

Hemorrhagic septicemia bacilli, 551

morphology of, 551

staining of, 551

varieties of:

bacillus of chicken cholera, 552

Bacillus pestis, 554

bacillus of swine plague, 553

Hepatotoxin, 201

Hesse's medium for colon-typhoid differentiation, 133

Heterolysins, 247

Hides, tanning of, bacteria in, 716

Hiss' agar-gelatin medium, isolation of typhoid bacillus in stools by, 407

Hiss' leucocyte extract theory and therapy, 289

Hiss' methods of staining capsules, 98

Hiss' plating media for colon-typhoid differentiation, 133

Hiss' tube medium for colon-typhoid differentiation, 133

Hiss' serum water media for fermentation tests; 132
"Hog-cholera" bacilli, 430

differentiation of, from swine plague bacillus, 554

Högyes, dilution method of, in treatment of rabies, 656

Horse meat, detection of, by precipitin tests, 254

Horses used in production of diphtheria antitoxin, 216-17

of tetanus antitoxin, 221

Howard and Perkins, discovery of Streptococcus mucosus by, 350

Hydrogen, formation of, by bacteria, 165

in nutrition of bacteria, 28

Hydrogen peroxid as disinfectant, 76

Hydrogen sulphid formed by bacteria, 165

Hydrophobia. See Rabies

Hypersusceptibility. See Anaphylaxis

Hyphomycetes, 635

conditions favorable to growth of, 638 diseases caused by, 639

favus, 640

pityriasis versicolor, 639

ringworm, 642

thrush, 640

diseases sometimes accompanied by, 644

morphology of, 635

reproduction of, 635

mycomycetes in, 636

Aspergillus variety in, 637

Penicillium variety in, 637

sporulation by other methods in, 637

phycomycetes in, Mucorinæ variety of, 635

sexual reproduction of, 636

structural classification of, 635

varieties of, 635

mycomycetes, 635

morphology of (typical), 635, 636 ascospores in, 638

conidia (or spores) in, 638

conidiophores in, 637

hyphal branches (septate) in, 637 
Hyphomycetes, varieties of, mycomycetes, morphology of, mycelial threads (septate) in, 637

sterigmata (septate) in, 637 morphology of less frequent

forms of, 637

ascospores in, 637

chlamydospores in, 638

phycomycetes, 635

morphology of (typical), 635-636

columella in, 636

hyphal branches in, 636

mycelial threads (non-septate) in, 636

sporangium in, 636

spores in, 636

morphology of sexual reproduction forms of, 636

gametophores in, 636

zygospores in, 636

"Immune Body" in blood serum, 226

in Ehrlich's theory of lytic process in blood serum, 226

haptophore groups of, 228

complementophile, 228

cytophile, 228

Immunity, absolute, 190

acquired, 192

definition of, 190, 192

active, 193

artificial, 193

definition of, 193

experimentation with attenuated cultures for, 193

with bacterial products for, 195

with dead bacteria for, 195

with sublethal doses of fully virulent bacteria for, 195

definition of, 189

natural, 190

individual, 191

of races, 191

of species, 190

passive, 196

relation of, to phagocytotic powers in animals, 279
Immunization, blood the seat of, 198

Incubation of cultures, 156 incubators in, 158 gas-pressure regulators for, 160 thermo-regulators for, 159

Indigo production, bacteria-in, 716

Individual immunity, 191 in higher animals, 192 in lower animals, 191

Indol production by bacteria, 167

Industries, bacteria in, 715

Infection, definition of, 181

fundamental factors in, 181

paths of, 183

inner, 184

outer, 183

Infectiousness, definition of, 182

due to number of bacteria, 182

due to variations in virulence, 183

Influenza, epidemic of, in 1889-90, 536 organs attacked in, 540

Influenza bacillus, 536

bacteria related to, 541

Bacillus murisepticus, 542

bacillus of pleuro-pneumonia, 542

Bacillus rhusiopathiæ, 542

Koch-Weeks bacillus, 542

pseudo-influenza bacillus, 541

biology of, 540

isolation and cultivation of, 537

on agar and gelatin, 537

blood added in, 537

hemoglobin added in, 537

hematogen added in, 538

morphology and staining of, 536

pathogenicity of, 540

in experimental inoculation of animals, 541

organs attacked in, 540

susceptibility of animals to, 541

Inhibition, coefficient of, 80

Inhibition strengths of various antiseptics, 84

Inoculation of animals, 170

intraperitoneal, 172

intravenous, 172

subcutaneous, 171

Inoculation of media, 141 
Inoculation of media, technique of transferring bacteria in, 141 virus used in transferring bacteria in, 141

Insusceptibility of cold-blooded animals, 190

Intravital method of Nakanishi in study of bacteria, 94

Inulin media, 132, 369

Invasion, paths of, 183

inner, 184

outer, 183

Inversion by fermentation, 42

Invertase, 49

Iodoform as disinfectant, 77

Iodine, tetrachloride of, as disinfectant, 76 tincture of, for skin sterilization, 76

Iron compounds in nutrition of bacteria, 29

Isolation of bacteria, 142

early methods of, 143

present methods of, 143

Esmarch roll tubes in, 147

Koch's plates in, 144

surface streaking in, 148

Isolysins, 247

Israel, discovery of actinomyces of man by, 622

JACKsON's lactose-bile medium for colontyphoid differentiation, 139

Jenner's discovery of immunization in smallpox by vaccinia, 659

Katalyzers, definition of, 42 enzymes and, analogy between, 42

Kefyr, 702

Kitasato, discovery of Bacillus tetani by, 456

discovery of plague bacillus by, 554

Kitasato filter, 123

Klebs, discovery of diphtheria bacillus by, 512

Koch, discovery of cholera spirillum by, 582

discovery of tubercle bacillus by, 479

Koch plates in isolation of bacteria, 144
Koch-Weeks bacillus, 542

Koumys, 702

$\mathrm{L}_{+}$, definition of, 208

$\mathrm{L}_{\mathrm{o}}$, definition of, 207

Lab enzymes, 46

Lactase, 50

Lactic-acid bacilli in therapy of gastrointestinal autointoxication, 714-715

Lactic-acid fermentation, 50 bacteria of, 50 in milk, 701

Lactose-bile medium, Jackson's, for colon-typhoid differentiation, 138

Lactose-litmus agar, 129

Laked blood, 225

Langenbeck, discovery of Oiddium albicans by, 640

Lautenschläger's thermo-regulator, 158, 159

Leprolin, 502

Leprosy, 505

bacillus of, 502,505

cultivation of, 506

by Clegg, 506

by Duval, 506

differentiation of, from tubercle bacillus by staining, 106, 506

inoculation with, 507, 509

morphology of, 505

occurrence of, in body, 508

pathogenicity of, 507

relation of, to tubercle bacillus, 509

staining of, 505

toxic products of, 509

clinical varieties of, 508

contagiousness of, 509

in rats, 510

occurrence of, 507

tuberculin administered in, results of, 510

Leptothrix, 619

morphology of, 618

Leucocidin of Staphylococcus pyogenes aureus, 329

action of, upon leucocytes, 330

discovery of, 329

leucotoxin differentiated from, 331 
Leucocidin of Staphylococcus pyogenes aureus, obtaining of, 330

Leucocyte extract, 289

effect of, upon infections in animals, 290

in man, 291

experimentation with, 291

obtaining of, 290

Leucotoxin, 201, 331

Liborius' method of anaërobic cultivation of bacteria, 149

Light in destruction of bacteria, 63 action of, 64

resistance to, 63

varieties of, 64

Lime, chloride of, as disinfectant, 76

Limes death, definition of, 208

Limes zero, 207

Linen, etc., disinfection of, 87

von Lingelsheim, discovery of Diplococcus mucosus by, 387

discovery of Micrococcus pharyngis siccus by, 387

Lipase, 47

Liver and gall-bladder, inflammatory conditions of, attributed to colon bacillus, 395

Lobar pneumonia, infectiousness of, 352

Loeffler and Schütz, discovery of glanders bacillus by, 528

Loeffler's malachite-green media, isolation of typhoid bacillus in stools by, 409

Loeffler's method of staining flagella, 100

Loeffler's serum medium, 131

Lustgarten, discovery of smegma bacillus by, 503

Lysins, 224

action of, Bordet's interpretation of, 225

compared with Ehrlich's, 225, 228 summary of, 228

Ehrlich's theory of, 226 compared with Bordet's, 226 complement in, 226 immune body in, 226
Lysins, action of, Ehrlich's theory of immune body in, formation of, 226

haptophore groups in, 228

complementophile, 228

cytophile, 228

red corpuscles in, 227

side chains or receptors in, 226

over-reproduction of, 226

in blood serum, 225

experimentation in, 224

in immune blood serum, reactivated

by normal serum, 226

in normal blood serum, Bordet's theory of, 225

alexin in, 225

sensitizing substance in, 225

investigation of, by Ehrlich, 226

Lysol, 78

Lyssa. See Rabies

MaAsSen filter, 124

MacConkey's bile-salt agar, for colontyphoid differentiation, 138

Macrophages, definition of, 276

Madura foot. See Mycetoma

Malachite-green media, for colon-typhoid differentiation, 136

bouillon, 137

Loeffler's, for isolation of typhoid bacillus in stools, 409

Malignant edema, bacillus of, 468

cultivation of, 470

early investigation of, 469

immunity in, 471

morphology of, 469

pathogenicity of, 470

staining of, 470

Mallein, 532

action of, 533

diagnostic use of, 533

directions of U. S. government for, 534

obtaining and preparation of, 533

Malta fever, 549

in domestic animals, 549

Maltase, 50

Maragliano's serum for tuberculosis, 498 
Marchiafava and Celli, discovery of meningococcus by, 371

Marmorek's serum for tuberculosis, 498

Measles, 675

investigation for virus of, 675

by Hektoen, 675

by Home, 675

Meat, determination of nature of, by precipitin tests, 254

used for culture media, 115 soluble, 116

Meat extract, 116

Meat-extract agar, 127

Meat-extract broth, 124

Meat-extract gelatin, 126

Meat-infusion agar, 128

Meat-infusion broth, 124

Meat-infusion gelatin, 127

Meat-poisoning bacilli, 429, 475

Meningitis, microorgartisms causing, 371 primary, 371

secondary, 371 serum therapy of, 378

Meningococcus. See Micrococcus intracellularis meningitidis

Metachromatic granules, 11

Metacresol as disinfectant, 77

Metchnikoff's therapy of gastrointestinal auto-intoxication by means of Bacillus bulgaricus, 714-715

by means of lactic-acid bacilli, 715

Mice, method of injecting, intravenously, 173

"Microbe de la coqueluche," 543

Micrococci, 321. See also Staphylococci

Micrococcus, 37

Micrococcus catarrhalis, 385

differentiation of, from gonococcus, 386 from meningococcus, 386

Micrococcus intracellularis meningitidis, 371

cultivation of, 374

oxygen in, 375

viability of organism in, 375

differentiation of, from Micrococcus catarrhalis, 386
Micrococcus intracellularis meningitidis, early observation of, 371-2

immunization against, 378 agglutinins in immune sera in, 378

modes of inoculation of, 377

morphology of, 373

pathogenicity of, 376

in animals, 377

in man, 376

pseudomeningococcus differentiated from, 379

resistance of, 376

staining of, 374

susceptibility of animals to, 377

viability of, 375

Micrococcus melitensis, 549

cultivation of, 550

morphology and staining of, 550

Micrococcus pharyngis siccus, 387

Micrococcus tetragenus, 333

cultivation of, 333

pathogenicity of, 333

Microorganisms, discovery of, 1

pathogenic, 321

Microphages, definition of, 276

Microscopic study of bacteria, 93

in fixed preparations, 94

process of, 94. See also under Staining

in living state, 93

by hanging block method, 94

by hanging drop method, 93

by intravital method of Nakanishi, 94

Microspira, 37

Microsporon furfur, 639

clinical picture of infection of, 639

cultivation of, 640

morphology of, 639

Milk, alcoholic fermentation in, 702

anthrax bacilli in, 707

bacteria in, 699

butter a means of transmitting, 711

butter-making aided by, 710

cheese-making aided by, 711

numbers of, 700

estimating of, 709 
Milk, bacteria in, propagation of, 700 sources of, 699

under ordinarily hygienic conditions, 699

varieties of, 701

"bitter," 703

butyric-acid fermentation in, 702

certified, 701

cholera traced to, 705

coagulation of casein in, 702

color changes in, 703

diarrheal diseases traced to, 705

diphtheria traced to, 705

foot-and-mouth disease virus in, 706

lactic-acid fermentation in, 701

pasteurization of, 709

pus cells and leucocytes. in, 706

relation of, to infectious diseases, 703

scarlet fever traced to, 704

"slimy," 703

streptococci in, 705

streptococcus throat infections con-

veyed by, 343, 706

supervision of supply of, 700

tubercle bacilli in, 707

infection from, 708

precautions against, 708

dairy inspection in, 708

Milk, tubercle bacilli in, precautions against, tuberculin test of cows in, 708

transmission of, from cow, 707

typhoid fever epidemics traced to, 703

Milk media, 130

Milzbrand, 563

Moeller's method of staining spores, 98

Moitessier's gas-pressure regulator, 169

Molds. See Hyphomycetes

Morax-Axenfeld bacillus, 545

cultivation of, 546

morphology and staining of, 546

pathogenicity of, 547

Motility of bacteria, 14

by flagella, 14

Brownian, 14

effect of temperature on, 15

inolecular, 14
Motility of bacteria, organs of, 13 true, 14

Mucorinæ, reproduction in, 635-636

Muguet. See Thrush

Multiplicity of amboceptors in normal sera, 241

of complement in normal sera, 242

Mycetoma, clinical picture of, 627

granules in, 627

melanoid, 627 cultivation of, 627-628

morphology of, 627

ochroid, 627

Mycomycetes, 635

NAKANISHI, "intravital" staining method of, in study of bacteria, 94

Negri bodies in central nervous system in rabies, 648

demonstration of, 648

diagnosis of rabies by, 650

explanation of, 650

significance of, 651

staining of, 108

Neisser, discovery of Diplococcus gonorrhœæ by, 380

discovery of lepra bacillus by, 505

Nephrotoxin, 201

Neufeld and Rimpau's discovery of opsonic substances, 282

Neurotoxin, 201

Neutral-red medium for colon-typhoid differentiation, 138

"New tuberculin" (Koch), 491

"New tuberculin-bacillary emulsion," 492

Nitrate-solution broth, 126

Nitrifying bacteria, 57 action of, 58

agricultural importance of, 58

Nitrogen fixation by bacteria, 54 microorganism of, 54

in root tubercles, 55

experimentation on, 56

microorganism of, 55

process of, 56

in soil, 54

Nitrogen in nutrition of bacteria, 28 
Nitrogen in nutrition of bacteria, sources of supply of, 28

Noguchi's modification of Wassermann test for syphilis, 270

Novy jar, 154

Nucleus in bacterial cell, 10

Nutrient media. See Culture media

Nutrition of bacteria, 25

carbon in, 25

hydrogen in, 28

nitrogen in, 25

oxygen in, 25

salts in, 29

Obermeier, discovery of spirochætes of relapsing fever by, 605

Obligatory aërobes, 25

Obligatory anaërobes, 26

Oidium albicans, 640

discovery of, 640

morphology of, 640

varieties of, 640

"Old tuberculin" (Koch), 491

Opium production, bacteria in, 716

"Opsonic coefficent of extinction," 286

Opsonic index, finding of, 286

Opsonic test, Wright's, 284

obtaining of bacterial emulsion for, 284

of blood serum for, 284

of leucocytes for, 284

opsonic index in, finding of, 286

parallel control test on normal serum in, 285

"pool" in, 285

technique of, 284

Simon, Lamar, and Bispham's technique of, 286

dilutions in, 286

opsonic coefficient of extinction in, 286

Opsonins, 281

decrease of phagocytic power upon introduction of bacteria without, 282

definition of, 282

increase of phagocytic power upon introduction of, 283
Opsonins, Neufeld and Rimpau's discovery of, 282

normal and immune, 282-3

specificity of, 282

structure of, according to Hektoen and

Ruediger, 283

Wright's test of. See Opsonic test

Wright's theory of, 282

Orthocresol as disinfectant, 78

Osmotic properties of bacterial cell, 23

Oxydases, 50

Oxygen as disinfectant, 88

in development of bacteria, 25

free, 26

absence of, 26

indirect supply of, 26

in nutrition of bacteria, 25

Ozone as disinfectant, 88

Paltauf's modification of Gram's stain, 103

Pancreascytotoxin, 201

Paper used in filtering culture media, 121

Paracolon bacillus, 430

Paracresol as a disinfectant, 78

Parasites, bacterial, 29

facultative, 30

media for growth of, 29

definition of, 182

infectiousness of, 182

pathogenicity of, 182

Paratyphoid bacilli, differentiation of, on sugar, 433

types $\mathrm{A}$ and $\mathrm{B}, 431$

Paratyphoid fever, differentiation of, from typhoid, 432

Passive immunity. See under Immunity Passive immunization, definition of, 196

Pasteur, discovery of bacillus of chicken cholera by, 552

discovery of bacillus of malignant edema by, 468

discovery of Diplococcus pneumoniæ by, 353

technique of, in rabies therapy, 652

Pasteurization of milk, 709

Pathogenic bacteria, 182, 321 
Pathogenicity, fundamental factors of, 181

of bacteria, 182

Penicillium, reproduction in, 637

Pepton-salt solution broth, 126

Pericardial exudates, bacteriological examination of, 175

Peritoneal exudates, bacteriological examination of, 175

Peritonitis following perforation attributed to colon bacillus, 394

Perlsucht, 498

Permanganate of potassium as disinfectant, 76

Pernicious anemia and Bacillus aërogenes capsulatus, 177

Peroxid of hydrogen as disinfectant, 76

Petri dish, 115, 144

Petruschky, discovery of Bacillus fecalis alkaligenes by, 427

Pfeiffer, discovery of influenza bacillus by, 536

discovery of Micrococcus catarrhalis by, 385

discovery of pseudo-influenza bacillus by, 541

Phagocytic index, 286

Phagocytosis, 275

cells active in, 276

"fixed," 276

macrophages, 276

microphages, 276

"wandering," 276

cells of animal origin in, 278

chemotaxis in, 277

complement or "cytase" in, 279

definition of, 275

dependence of, on opsonins, 281-2

diminution of, upon introduction of bacteria without opsonic serum, 282

immune body or "fixator" in, 279

immunity and, 279

in higher animals, 276

in protozoa, 275

increase of, upon the introduction of opsonic substances in serum, 282
Phagocytosis, macrophages in, 276

Metchnikoff's theory of, 276

opposition to, 279

microphages in, 276

process of, in the body upon introduction of bacteria, 277

upon introduction of nutrient broth, 276

susceptibility of various microorganisms to, 278

variety of phagocyte in, determined by

the bacterium, 278

Phenol production by bacteria, 167

Phosphates in the nutrition of bacteria, 29

Phosphorescence produced by bacteria, 59

Phragmidiothrix, 38

Phycomycetes, 635

Pigment, formation of, by bacteria, 59

chemical nature of, 59

cultural conditions on, 60

Piorkowski's urine gelatin for colon-typhoid differentiation, 134

Pityriasis versicolor, 639

clinical picture of, 639

microorganism causing. See Micro-

sporon furfur

occurrence of, 639

Plague, bacillus of, 554

biology of, 557

degeneration forms of, 20

immunization against, 561

active, 561

involution forms of, on salt agar, 557

isolation and cultivation of, 556

lesions in animals produced by, 559

morphology of, 555

pathogenicity of, 558

resistance of, 557

staining of, 556

transmission of, 560

toxins of, 561

variations in virulence of, 559

viability of, 557

epidemics of, 554

in animals, 558

in California ground squirrels, 560 
Plague, bacillus of, in animals, in Manchurian marmot, 560

inoculation in, 559

spontaneous infection in, 559

in man, 558

autopsy findings in, 558

bacteriological diagnosis of, in life, 558

infection in, 558

localized form of, 558

pneumonic form of, 558

transmission of, 560

Plague-like disease in rodents, 562

Planococcus, 37

Planosarcina, 37

Plasmolysis of bacterial cell, 23

Plasmoptysis of bacterial cell, 24

Plating in isolation of bacteria, 143

Pleural exudates, bacteriological examination of, 175

Pleuro-pneumonia, organism of, 542

Pneumobacillus. See Bacillus mucosus capsulatus

Pneumococcus, discovery of, 7 different types of, 336

Pneumococcus. See Diplococcus pneumoniæ

Pneumococcus-streptococcus group, mutation, 370

Pneumonia, complications of, 362

lobar, infectiousness of, 352 serum therapy for, 366

Poisons, bacterial. See Bacterial poisons

Polar bodies, 11

special stains for, 107

Poliomyelitis, acute anterior, 664 immunity in, 667

infectiousness of, 664

inoculation of animals with spinal substance of, 664

by Flexner and Lewis, 665, 666

by Knoepfelmacher, 665

by Landsteiner and Levaditi, 665

by Landsteiner and Popper, 665

resistance of virus of, 666

Polychrome stains in staining of bacteria, 107
Potassium permanganate as disinfectant, 76

Potato media, 130

glycerin, 130

Pour plate, technique of making, 145

Precipitin tests, 252

bacterial filtrates for, 254

technique of, 255

determining nature of meat by, 254

precipitating antisera for, 252

against albumin solutions, 253

technique of production of, 252

proteid solutions to be tested by, 254

Precipitins, 200, 235

agglutinins and, structure of (Ehrlich), 238

cell-receptors in, 238

theoretical considerations concerning, 238

bacterial differentiation by, 237

differentiation of proteids by, 237, 254

distinguishing blood of animal species by, 237

effect of heat on, 236

experimentation in, 235

group reaction of, 237

identity of, with sensitizers, 236

nature of, 236

specificity of, 237

Proagglutinoids, 235

Proteid differentiation by complement fixation, 273

substances necessary for, 273

technique of, 274

by precipitins, 237,254

Proteid injections, anaphylaxis in, 295.

See also Anaphylaxis

Proteids in bacterial cell, 22

Proteins, bacterial, 186

Proteolytic enzymes, 43

action of, 44

in breaking down animal excreta, 46

bacteria producing, 44

preteids necessary to, 44

ptomains produced by, 45 
Proteus group, bacilli of, 454

cultivation of, 454

morphology and staining of, 454

occurrence of, 454

pathogenicity of, 455

Protozoa and bacteria, differentiation of, 1

staining of, 108

Pseudo-dysentery bacillus, 437, 438

Pseudo-influenza bacillus, 541

Pseudo-membranes in diphtheria, 519

Pseudomeningococcus, 379

Pseudomonas, 37

Ptomains, 45, 185, 306

bacterial poisons and, 185

discovery of, 185

occurrence of, 185

toxins distinguished from, 45

varieties of, 45

Pus, bacteriological examination of, 175

Pus cells and leucocytes in milk, 706

Putrefaction, action of, 44

Putrefactive bacteria, quantitative analysis of, 21

Putrescin, 45

Pyemia, definition of, 184

Pyocyanase, 580

immunizing powers of, 580

Pyocyanin, 578

Pyocyanolysin, 581

Pyrogallic acid, use of, in cultivation of anaërobic bacteria, 152

\section{RABIES, 646}

course of, 647

in animals, 647

in men, 648

cultivation of organism of, by Noguchi, 651

diagnosis of, by presence of Negri bodies in central nervous system, 648-650

experimental infection of, 646

incubation in, 647

Negri bodies in central nervous system, 648

demonstration of, 648

by Van Gieson's method, 649
Rabies, Negri bodies in central nervous system, demonstration of, 'by Williams and Lowden's method, 650

staining in, 649

Mann's method of, 649

diagnosis by, 649-650

occurrence of, 646

pathology of, 648

specific therapy of (Pasteur's technique), 652

attenuation and preparation of virus fixe in, 652

inoculation of rabbits with virus fixe in, 652

spinal cord of inoculated rabbits in, desiccation of, 653

emulsification of, 654

treatment of cases with injections of spinal-cord solution in, 654

Högyes dilution method in, 656 scheme of, used at Pasteur Institute, 654

used in New York Department of Health, 655

virulence of virus of, 647

Racial immunity, 191

"Rage." See Rabies.

Rat leprosy, 510

relation of, to human leprosy, 511

Rauschbrand. See Bacillus of symptomatic anthrax

Receptors of toxin molecule in sidechain theory, 213

chemical action of, 213

over-production of, 214

Red blood cells, antibodies produced by, 200

Reducing powers of bacteria, 167

Refractive index of parts of bacterial cell, 24

Reichel filter, 122

Reichert's thermo-regulator, 158, 159

Relapsing fever, 605

immunity in, 610

symptoms of, 608

transmission of, 610

varieties of, 609 
Relapsing fever spirochæte, 605

cultivation of, 606

morphology and staining of, 605

pathogenicity of, 608

in animals, 608

in man, 608

symptoms of, 608

transmission of, 610

varieties of, 609

Reproduction of bacteria, 17

Resistance, definition of, 189

Rhinoscleroma, bacillus of, 451

Ricin, experimentation with, 204

Ringworm, 642

Root tubercles, 55

microorganism of nitrogen fixation in, 55

Roux's method of anaërobic cultivation of bacteria, 149

SacCharomycetes. See Yeasts and Yeast cells

Salts in nutrition of bacteria, 29

Saprophytes, bacterial, 29, 30 definition of, 182

Sarcina, 37

Sarcophysematos bovis. See Bacillus of symptomatic anthrax

Scarlatina. See Scarlet fever

Scarlet fever, 676

favorable influence of streptococcus antisera in, 676

streptococci present in, 676

traced to milk, 704

Schaudinn and Hoffmann, discovery of Spirochæta pallida by, 594

Schizomycetes, 37

Schweineseuche, 553

Scorpion poison, antitoxin for, 199

"Sensibilisin," 302

"Sensibilisinogen," 302

Septicemia, definition of, 184

diagnosis of, by isolation of bacteria from the blood, 178

due to colon-bacillus infection, 394

hemorrhagic. See Hemorrhagic septicemia

Serum media, 131
Serum media, Loeffler's, 131

Serum reactions, technique of, 249.

See also under individual tests

agglutination tests in, 250

antigen determined in, by complement fixation, 271

for typhoid fever, 271

obtaining of material for, 271

test in, 272

bactericidal and bacteriolytic tests in, 255

complement fixation in, for determination of antibodies, 261

for determination of antigen, 271

for proteid differentiation, 273

hemolytic tests in, 259

precipitin tests in, 252

proteid differentiation by complcment fixation in, 273

substances necessary for, 273

test in, 274

Wassermann test in, 262

modifications of, 268

"Serum sickness," 296

Serum water media for fermentation tests, 132

Shiga, discovery of dysentery bacillus by, 435

Shiga's bacillus, 435

cultural characteristics of, 436

morphology of, 435

Side-chain theory of toxin-antitoxin reaction, 212

chemical action in, 213

elements of molecules in, 213

atom group, 213

side chains or receptors, 213

over-production of receptors in, 214

Side chains, action of, in Ehrlich's theory of lytic process in blood serum, 226

Slanting of culture media, 123

"Slimy" milk, bacteria causing, 703

Smallpox, 657

etiological factor of, 657

immunization in, 658

by vaccination, 659,663

Jenner's discovery of, 659

technique of, 663 
Smallpox, immunization in, by vaccination, value of, 663

production of vaccine for, 647. See also under Vaccine

occurrence of, 657

protozoan incitant of, research for, 657

relation of chicken-pox to, 660

relation of cowpox to, 659

transmission of, 658

vaccine bodies in, discovery of, 657

explanations for, 658

Ewing's, 658

Smegma bacillus, 502, 503, 594

cultivation of, 504

morphology of, 503

occurrence of, 503

staining of, 504

identification of bacillus by, 504

tubercle bacillus and, differentiation between, by stains, 106

Smith's modification of Pitfield's method of staining flagella, 101

Snake poison, antitoxin for, 199

Soil, bacteria in, 685

from burial of infected cadavers, 687

in agricultural regions, 685

numerical estimation of, 687

pathogenic, in surface layers, 686

Solutions, saturated, for staining of bacteria, 95

staining-power of, 96

Soor. See Thrush

Species immunity, 190

differences in, 190

Specific gravity of forms of bacterial cell, 24

"Specific precipitates," 235-236

Spider poison, antitoxin for, 199

Spinal fluid, bacteriological examination of, 176

Spirillaceæ, 37

Spirillum, 38

description of, 9

Spirillum choleræ asiaticæ. See under Cholera

Spirillum Deneke, 591

Spirillum of Finkler-Prior, 539

Spirillum Massaua, 591
Spirillum Metchnikovi, 590

Spirochæta, genus, 38

Spirochæta anserina, 616

Spirochæta Calligyrum, 617

Spirochæta Duttoni, 610

Spirochæta gallinarum, 615

cultivation of, by Noguchi, 616

immunization against, 616

similarity of, to Spirochæta anserina, 616

transmission of, 615,616

Spirochæta macrodentium, 617

Spirochæta microdentium, 617

Spirochæta pallida, 593

animal pathogenicity of, 601

cultivation of, 600

dark-field examination of, 597

by Mühlens, 600

by Noguchi, 600

demonstration of, 596

in living state, 596

in smears, 597

by Goldhorn's method of staining, 598

by India-ink preparation, 598

by Schaudinn and Hoffmann's method of staining, 597

by Wood's method of staining, 597

in tissues, 598

by Levaditi's method, 598

by Levaditi and Manouelian's method, 599

immunization against, 603

active, 603

passive, 603

infection of animals by, 602

of cornea of rabbits, 602

of testes of rabbits, 602

morphology of, 595

observation of, 596-597

occurrence of, in syphilis cases, 595596

staining of, 108

Spirochæta pertenuis, 614

morphology of, 615

similarity of, to Spirochæta pallida, 615

Spirochæta phagedenis, 616 
"Spirochæta refringens," 595

Spirochæte of relapsing fever. See Relapsing fever spirochæte

Spirochæte of Vincent's angina. See Vincent's angina, spirochæte of

Spirochætes, cultivation of, 593 differentiation of, from spirilla, 593 diseases caused by, 592. See also under specific names reproduction in, 592 structure of, 592

Spirosoma, 37

Spore stains in staining of bacteria, 97 Spores, bacterial, 15

formation of, 15

germination of, 17

position of, 17

varieties of, 16

arthrospores, 16

true or endospores, 16

vegetative forms from, 17

Sporotrichosis, 644

Sporulation, physiological significance of, 17

process of, 16

Sputum, disinfection of, 87

Stable antitoxin, 206

Staining of bacteria, chemical principles in process of, 96

acid-fast bacteria stains, 104

Baumgarten's method, 106

Bunge and Trautenroth's method, 106

Ehrlich's method, 104

Gabbet's method, 105

Pappenheim's method, 106

Ziehl-Neelson method, 105

capsule stains, 98

Buerger's method, 99

Hiss' methods, 98 copper sulphate, 98 potassium carbonate, 98

Wadsworth's method, 99

Welch's method, 98

differential stains, 102

Gram's method, 102 classification by, 104 Paltauf's modification of, 103
Staining of bacteria, flagella stains, 100

Loeffler's method, 100

Smith's modification of Pitfield's method, 101

Van Ermengem's method, 101 polychrome stains, 107

Giemsa's method, 108

Jenner's method, 108

Wood's method, 109

Wright's modification of Leishman's method, 108

special stains for polar bodies, 107

Neisser's method, 107

Roux's method, 107

spore stains, 97

Abbott's method, 97

Moeller's method, 98

staining in tissues, 110

for actinomyces in sections, 112

for Gram-positive bacteria, 111

Gram-Weigert method, 111

in celloidin sections, 111

in paraffin sections, 111

for tubercle bacilli in sections, 112 in celloidin sections, 112 in paraffin sections, 112

Loeffler's method, 112 saturated solutions used in, 95 staining solutions in, power of, 96 steps in process of:

(1) smearing, 94

(2) drying, 95

(3) fixing, 95

(4) staining, 95

(5) washing, 95

(6) blotting, 95

(7) mounting, 95

Standardization of diphtheria antitoxin, 218

of tetanus antitoxin, 221

Staphylococci, 321. See also under individual staphylococci

definition of, 321

in feces, 177

Staphylococcus epidermidis albus, 332

Staphylococcus pyogenes albus, 332

Staphylococcus pyogenes aureus, 322

cultural characters of, 323 
Staphylococcus pyogenes aureus, immunization against, 331

active, 332

agglutinins in, 331

modes of inoculation with, 327

morphology of, 322

pathogenicity of, 326

in animals, 327

in man, 327

pigment formation of, 325

resistance of, 325

to chemicals, 326

to desiccation, 326

to heat and cold, 325

staining of, 322

susceptibility of animals to, 326

susceptibility of man to, 327

thermal death point of, 325

toxic products of, 328

endotoxins, 328

hemolysins, 328

leucocidin, 329. See also under

Leucocidin

virulence of, 326

Staphylococcus pyogenes citreus, 332

Steam in sterilization, 67

live, 69

saturated, 68

superheated, 68

Stegomyia fasciata, 673

Sterilization of culture media, 121

filtration in, 122

heat in, 121

Sternberg, discovery of Diplococcus pneumoniæ by, 353

Stimulins, 281

Streaking, surface, in isolation of bacteria, 148

"Street virus," 647

Streptococci, 37, 335

capsulated, description of organisms reported as, 367-369

classification of, 348

by Andrewes and Horder, 349

by carbohydrate fermentation powers, 348

by reactions to immune sera, 350

morphological, 348
Streptococci, classification of, morphological, Streptococcus longus seu erysipelatos in, 348

Streptococcus minor seu viridans in, 348

Streptococcus mucosus in, 350 definition of, 335

differentiation of, from pneumococci, 357,367

cultural, 368

morphological, 367

epidemic throat infections by, 343

in feces, 177

in milk, 705

in milk epidemics, 343

preparation of, for agglutination test, 251

pyogenic. See Streptococcus pyogenes

Streptococcus anginosus, 349

Streptococcus equinus, 349

Streptococcus erysipelatis, 342

Streptococcus fecalis, 349

Streptococcus longus seu erysipelatos, 348

Streptococcus mitior seu viridans, 348

Streptococcus mucosus, 350, 351

Streptococcus pyogenes, 335

brevis, 337, 338

cultivation of, 337

early experimentation with, 335

immunization against, 345

immune sera of infected animals in,

345

agglutinins in, 347

precipitins in, 348

specificity of, 347

standardization of, 347

leucocyte extracts in, 347

technique of, 346

longus, 337

modes of inoculation with, 341

in animals, 341

in man, 342

morphology of, 337

pathogenicity of, 340

in animals, 340

in man, 342 
Streptococcus pyogenes, resistance of, 339

staining of, 337

susceptibility of animals to, 341

toxic products of, 344

endotoxins, 344

hemolysins, 344

virulence of, 340

Streptococcus salivarius, 349

Streptothrix, 38, 619

cultivation of, 621

morphology of, 619,621

Sublethal doses of virulent bacteria in active immunization, 195

Sugar-free broth, 125

Sulphates in nutrition of bacteria, 29

Sulphur bacteria, 60

physiology of, 61

spectroscopic examination of, 61 varieties of, 60

Sulphur dioxid as disinfectant, 86

Sulphuretted hydrogen. See Hydrogen sulphid

Suprarenal cytotoxin, 201

Swine-plague bacillus, 553

differentiation of, from hog-cholera bacillus, 554

immunization against, 553

morphology of, 553

pathogenicity of, 553

Symbiosis of bacteria, 31

Symptomatic anthrax, bacillus of. See Anthrax, symptomatic

Syphilis, 593

in monkeys, 602

in rabbits, 602

microorganism of. See Spirochæta pallida

TANNING of hides, bacteria in, 716

Temperature, attained by application of various degrees of pressure, 72 effect of, on activity of bacteria, 15

high, 34

low, 34

relation of, to bacteria, 31

maximum, 32

minimum, 32
Temperature, relation of, to bacteria, optimum, 32

to cultures with spores, 33

to vegetative forms, 33

"Tetanolysin," 205, 464

"Tetanospasmin," 463

Tetanus antitoxin, 220

production of, 220

horses used in, 221

technique of, 221

toxin for, 220

standardization of, 222

unit of (Society of American Bacteriologists), 222

Tetanus bacillus, 456

autopsy findings in infections of, 460

biological characteristics of, 458

cultivation of, 458

distribution of, 457

early observation of, 456

favorable conditions for growth of, 459

incubation of, 460

isolation of, by Kitasato, 456

morphology of, 456

pathogenicity of, 459

following wounds, 460

relation of spores to, 459

resistance of, 459

staining of, 457

toxin of, 460

central nervous system attacked by, 463

mode of reaching, 463

incubation period of, 463

isolation of, 461

by chemical reaction, 462

by filtration, 461

by precipitation, 461

production of, 461

resistance of, 462

strength of, 462

susceptibility of animals to, 462

Tetanus spores, transportation of, to organs, 460

Thermal death points, 34

Thermo-regulators for incubators, 159

Lautenschläger's, 158, 159

Reichert, 158, 159 
Thiothrix, 38

Thrush, 640

microorganism causing, 640

Timothy, bacillus of, 502

Tissue sections, method of staining, 111

Gram-Weigert, 111

in celloidin sections, 111

in paraffin sections, 111

staining of bacteria in, 110

Titration of culture media, 117

color indicator in, 117

process of, 117

for alkaline media, 118

reaction of, 117

adjustment of, 119

Tobacco industry, bacteria in, 715

Torulæ, 617

Toxin, constitution of (Ehrlich), 210

graphic form of (Ehrlich), 211

views of Arrhenius and Madsen on, 212

diphtheria. See under Diphtheria toxin

endotoxin distinguished from, 186

epitoxoid in, 208

in side-chain theory, cell-nutrition in,

213

chemical action of, 213

elements of, 213

atom group, 213

side chains or receptors, 213

over-production of receptors in, 214

molecule of, haptophore group in, 207

toxophore group in, 207

partial absorption of, 209

standardization of, 207

Limes death in, 208

Limes zero in, 207

time changes in, 206

toxoid form of, 207

protoxoids in, 209

syntoxoids in, 209

toxon and, difference in action of, 209 toxon in, 209

used for production of diphtheria antitoxin, 216

valency of antitoxin for, 210
Toxin-antitoxin reaction, 203

side-chain theory in, 212

summary of, 215

theories as to process of, 203

by destruction of toxin by its specific antitoxin, 203

by direct union of toxin and antitoxin, 203

through mediation of tissue cells, 203

time element in, 204

Toxin solution, normal, 205

Toxin unit, 205

Toxins, 185

compared with pigments, 186,309

summary of, 305

Toxoid form of diphtheria toxin, 207

Toxoids, varieties of, 209

epitoxoid form in, 208

protoxoids, 209

syntoxoids, 209

Toxon, in diphtheria toxin, 209

toxin and, difference in action of, 209

Toxon molecule, 209

haptophore group in, 209

toxophore group in, 209

Toxophore group in toxin molecule, 207 in toxon molecule, 209

Trachoma, hemoglobinophilic bacilli in, 541

Trichomycetes. See Chlamydobacteriaceæ

Trichophyton tonsurans, 642

cultivation of, 644

demonstration of, 643

morphology of, 643

occurrence of, 642

Tricresol, 78

Trillat autoclave, 89

Tubercle bacillus, 479

bacilli related to, 498

Bacillus butyricus, 502

bacillus of avian tuberculosis, 500 cultivation of, 500

discovery of, 500 morphology and staining of, 500 susceptibility of animals to, 500

bacillus of bovine tuberculosis, 498 
Tubercle bacillus, bacilli related to, bacillus of bovine tuberculosis, early investigation of, 498 cultivation of, 499

differentiation of, from human type, 499

morphology of, 499

bacillus of fish tuberculosis, 495

bacillus of leprosy, 502, 509

bacillus of timothy, 502

bacillus of turtle tuberculosis, 501

Bacillus smegmatis, 502

biological considerations of, 485

chemical analysis of, 490

cultivation of, 483

"Nährstoff Heyden" in, 485

media for, 484

discovery of, 7

early investigation of, 479

examination for, by animal inoculation, 175

by Ziehl-Neelson staining method, 176

in circulating blood, 489

in feces, 178

in milk, 709

isolation of, 483

leprosy bacillus and, differentiation between, by stains, 106

methods of staining, 104, 105, 106

in sections, 112

celloidin, 112

paraffin, 112

morphology of, 479

Much granules, 482

pathogenicity of, 486

frequency in, 486

mode of infection in, 487

mortality in, 486

preparation of, for agglutination test, 251

quantitative analysis of, 22

smegma bacillus and, differentiation

between, by stains, 106

staining of, 480

differentiation of, from acid-fast group by Pappenheirn's method of, 482
Tubercle bacillus, staining of, Ehrlich's anilin-water-gentian-violet solution in, 481

Gabbet's decoloration and counterstaining in, 481

Ziehl's carbol-fuchsin solution in, 481 toxins of, 490

endotoxins in, 490

tuberculins in, 490

bouillon filtré (Denys), 492

"new tuberculin-bacillary emulsion" (Koch), 492

"new tuberculin" (Koch), 491

original method of making of, 491

present method of making of, 491

"old tuberculin" (Koch), 491

"tuberculoplasmin" (Buchner and Hahn), 492

use of antiformin in examination for, 483

Tuberculin. See under Tubercle bacillus, toxins of

"Tuberculoplasmin" (Buchner and Hahn), 492

Tuberculosis, frequency of, 486 immunization in, passive, 497

Maragliano's serum in, 498

Marmorek's serum in, 498

human and bovine types of bacilli in, in infections of man, 488 mode of infection in, 487 mortality of, 486 tuberculin in, diagnostic use of, 493 cutaneous reaction in, 494

in cattle, 495 ophthalmo reaction in, 494 subcutaneous injection of, 493 dosage and reaction in, 493

therapeutic uses of, 496

original, 496

present, 497

dosage in, 497

preparations employed in, 497

Tubing of culture media, 121

Typhoid bacillus. See under Typhoid fever 
Typhoid carrier state in rabbits, 404

Typhoid fever, bacillus of, 399

bacteriemia in, 405

biological conditions favorable to, 403

cultivation of, 399

differentiation of, from Bacillus fecalis alkaligenes, 427

from. meat-poisoning and paratyphoid bacilli, 428

discovery of, 7, 399

immunization against. See under

Typhoid fever, immunization in

in blood during disease, 405

obtaining cultures of, 405

in feces, 177

in gall-bladder, 411

in rose spots, 412

in sputum, 412

in stools, 406

examination in, 406

isolation of, 407

on Conradi-Drigalski medium, 408

on Elsner's potato-extract gelatin, 407

on Endo's fuchsin-agar, 409

on Hiss' agar-gelatin media, 407

on Loeffler's malachite-green media, 409

time of appearance in, 406

in urine, 411

in water, 694

inoculation of animals with, 404

with endotoxin of, 417

isolation of, 403

morphology of, 399

pathogenicity of, 404

in animals, 404

in man, 404

staining of, 339

suppurative lesions due to, 412

toxic products of, 415

obtaining of, 417

varieties of:

endotoxins, 415 .

true toxins, 416

typhoplasmin, 416
Typhoid fever, diagnosis in, by agglutinins in blood serum, 420

Widal test in, 421

obtaining blood for, 422

by bactericidal substances in blood serum, 419

by bactericidal tests in vivo, 258

by opsonic index, 424

epidemics of, traced to milk, 703

hygienic considerations in, 413

immunization in, 417

by inoculation with typhoid bacilli, 417

active, 424

technique of Pfeiffer and Kolle in, 425

of Wright in, 425

substances found in blood after, 418

agglutinins in, 419

chief or major, 420

group, 420

bactericidal, 419

bacteriolytic, 418

opsonins in, 424

precipitins in, 423

obtaining blood cultures in, 180

prophylactic measures in, 414

prophylactic vaccination in, 426

specific therapy in, 424

transmission of, 413,414

by flies, 415

from milk, 414

from oysters, 415

from water supply, 414

without intestinal lesions, 413

"Typhoplasmin," 416

Typhus fever, bacillus of Plotz, 678

bacillus of Ricketts and Wilder, 677

distribution of, 677

identity of, with Brill's disease, 677

inoculation of animals with, 677

URINE, bacteriological examination of, 176

Urobacillus liquefaciens, 455

Uschinsky's proteid-free medium, 126 
VACCINE production, for immunization in smallpox, 660

animals used in, 660

calves used for, 660

cleanliness observed in stabling of, 660

material used for vaccination of, 661 vaccination in, 661

preparation of field in, 661

scarifications in, 661

vaccinia vesicles developed in, 661 obtaining of vaccine from, 662

by curettage, 662

by ivory tips, 662

testing of vaccine in, for bacteria, 663 for efficiency, 662

Vaccine therapy of Wright, 286

dosage for, 288

opsonic curve in, 288

production of vaccines in, 286

standardization of emulsion in, 287

enumeration of bacteria against red

blood cells in, 287-8

sterilization of vaccine in, 288

Van Ermengem, discovery of Bacillus botulinus by, 475

Van Ermengem's method of staining flagella, 101

Variola. See Smallpox.

Vegetative forms from bacterial spores, 17

"Vibrion septique." See Malignant edema, bacillus of

Vincent's angina, 610

spirochæte of, 611

cultivation of, 613

fusiform variety of, 612

bacilli of other diseases resembling, 613. See also under Fusiform bacilli

other bacilli accompanying, 613

spirillum variety of, $6_{\text {. }}^{\gamma_{\text {e }}}$

symptoms of, 610

Vincent's spirilla, staining of, 108

Virulence, definition of, 183

variations in, and infectiousness, 183

Virulent bacteria, sublethal doses of, in immunization, 195

Virus fixe, in specific therapy of rabies, 652
WADSWORTH's method of staining capsules, 99

Wassermann test for diagnosis of syphilis, 262

antigen for, 262

determination of necessary quantity of, 264

obtaining of, from alcoholic extracts of syphilitic organs, 263

from alcoholic solution of normal organs, 263

from salt solution of syphilitic liver, 263

of syphilitic spleen, 262

preparation of, by Noguchi method, 264

complement in, 266

hemolytic serum in, 265

obtaining of, 265

potency of, 265

quantity of, 265

unit in, definition of, 265

determination of, 266

modifications of, 268

Bauer's, 268

Noguchi's, 270

performed with Spirochæta pallida antigen, 604

preparation for, 262

serum to be tested for syphilitic anti-

body in, 267

sheep corpuscles in, 267

technique of, 267

Water, bacteria in, 689

in ground waters, 691

in perennial springs, 692

in wells, 691

in rain and snow, 690

in surface waters, 690

influence of rain on; 690

light and temperature factors in purification of, 691

self-purification in, 690

pathogenic, 689

of cholera, 689

of diarrheal diseases, 689

of typhoid fever, 689

qualitative analysis of, 694 
Water, bacteria in, qualitative analysis of, isolation of cholera vibrio in, 696

Koch's method of, 696

isolation of colon bacillus in, 695

isolation of typhoid bacillus in, 695

Adami's and Chapin's method of, 696

Drigalski's method of, 695

Parietti's method of, 695

Vallet's method of, 696

quantitative estimations of, 692

collecting of specimens for, 692

colon bacilli in, 698

colon test in, 697

counting of bacilli in, 698

counting in, 694

incubation of specimens in, 694

plating of specimens in, 693

value of, 694

in bacterial cell, 21

Welch, discovery of Bacillus aërogenes capsulatus by, 471

Welch's method of staining capsules, 98

Welch's modification of Guarnieri's medium, 129

Wertheim's medium for cultivation of gonococcus, 381-2

Winckel's disease in the newborn due to colon bacillus, 394

Wires used in transferring bacteria, 141

Wolffhügel counting plate, 162

Wool-sorter's disease, 573

Wright, method of, of anaërobic cultivation of bacteria, 150

modification by, of Buchner's pyrogallic method of cultivation of anaërobic bacteria, 153

theory of opsonins of, 282

vaccine therapy of, See Vaccine therapy of Wright

Xerosis bacillus, 525

YAws, 603

Yeast cells, cultivation of, 633

demonstration of, 632

morphology of, 629

reproduction in, by budding, 629

by spore formation, 630
Yeasts, 629

differentiation of, from other microolganisms, 629

fermentation by, 630

industrial employment of, for fermentative purposes, 52

infection of, in animals, 632

in man, 631

clinical picture of, 632

pathogenic varieties of, 633

Yellow fever, 668

clinical picture of, 668

distribution of, 668

etiology of, 668

immunity in, 674

investigation of, by Guiteras and Marchoux, Salimbeni and Simond, 673

results of, 673

by Reed, Carroll, Agramonte, and Lazear, 670

results of, 673

microorganism of, biological properties of, 669

research for, 668

by Cornil and Babes, 669

by Sanarelli, 669

by Sternberg, 669

Stegomyia fasciata in, 673

description of, 673

power of transmission of infection by, reasons for, 674

tropical countries most favorable for, 674

transmission of, 670

by mosquitoes, 670

discovery of, by Finlay, 670

investigation and confirmation of,

by United States Commission, $67 \mathrm{fr}^{\mathrm{s}}$

Yersin, discuvery of plague bacillus by, 555

Zur Nedden's bacillus, 547 cultivation of, 547 morphology and staining of, 547 pathogenicity of, 547

Zymase, 51, 630 



\begin{tabular}{|c|}
\hline \hline A \\
TEXT-BOOK \\
OF \\
BACTERIOLOGY \\
Hiss \\
and \\
Rinser \\
\hline \hline
\end{tabular}






BRIGHAM YOUNG UNIVERSITY

1

31197210966468 
